\title{
CONTENTS
}

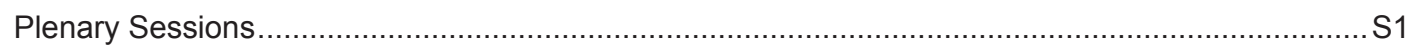

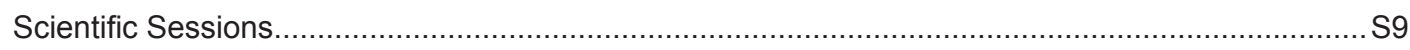

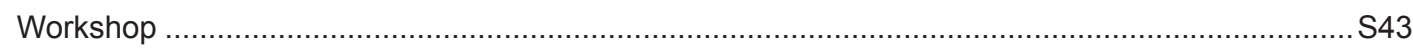

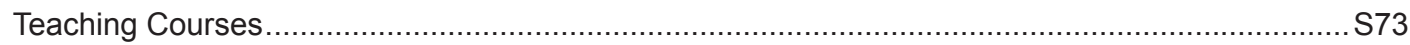

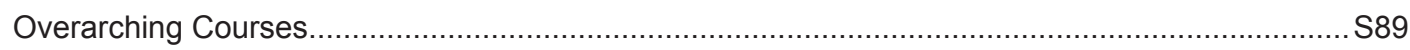

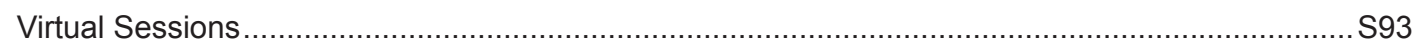

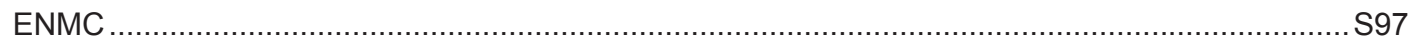

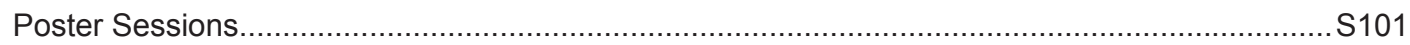

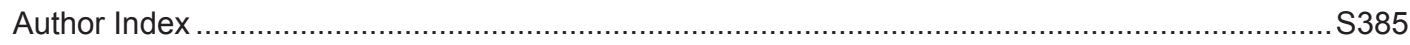





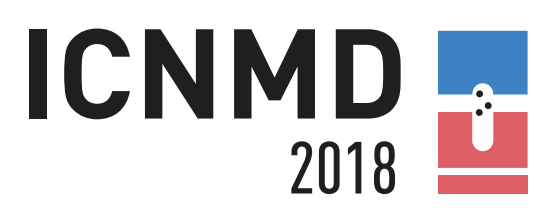

15TH INTERNATIONAL CONGRESS
ON NEUROMUSCULAR DISEASES

www.icnmd2018.org July 6-10, 2018 Vienna, Austria

\section{Plenary Sessions}





\section{PL 1.1 / \#155}

Topic: 01. Muscle

\section{PATHOMECHANISMS}

Volker Straub

John Walton Muscular Dystrophy Research Centre, Newcastle University, Institute of Genetic Medicine, Newcastle Upon Tyne, GB

Abstract: Traditionally a skeletal muscle is viewed as a distinct anatomical structure that is attached to bone by its tendons, is innervated by a nerve and generates movement through contraction. This simplistic view does highlight the most important physiological function of muscle, force generation through activity of its main molecular structure, the sarcomere. But rather than being defined by its anatomical structure, skeletal muscle is now viewed as a complex organ system consisting of about 700 muscles that make up roughly $40 \%$ of our body weight. The skeletal muscular system doesn't just permit movement of the body and maintains it posture, but supports vital metabolic functions, contributes to temperature control and circulation, interacts with focal and systemic hormonal pathways, and is a crucial contributor to respiratory function and the operation of our senses. Through the application of -omics technologies we have also learned that skeletal muscle tissue itself is far less homogenous than originally anticipated. Distinct skeletal muscles show different transcriptomes and proteomes, despite the fact that their mechanical functions may be very similar. The complexity of normal muscle function is reflected in the broad spectrum of clinical symptoms and muscle pathology when something goes wrong. Over the past 30 years, since the discovery of dystrophin and the characterisation of the $D M D$ gene, $>500$ different genes leading to about 900 distinct genetic neuromuscular disease have been described. Trying to identify and understand the pathomechanisms that specifically underlie genetic muscle diseases has helped to better characterize the relevant molecular pathways and subcellular structures that are involved in maintaining muscle fibre integrity and function.
Not surprisingly we distinguish metabolic muscle disease from structural muscle disease, including diseases that affect the integrity of the sarcolemma, the sarcomere or the nuclear lamina. But although classification systems based on pathomechanistic concepts have been helpful to both basic and clinical scientists, the application of a large array of new molecular imaging and-omics technologies have illustrated the complexity of disease mechanisms and continue to blur the boundaries between pathway-based modelling approaches and our anatomical view of muscle fibre architecture. The responsiveness of the muscular system to damage caused by pathogenetic mechanism is in the end limited and will result in muscle wasting and weakness, the main symptoms of genetic muscle diseases

\section{PL 1.2 / \#100}

Topic: 01. Muscle

\section{INFLAMMATORY MECHANISMS}

Andrew Mammen

NIAMS/NIH, Bethesda, US

Abstract not received.

\section{PL 1.3 / \#94}

Topic: 01. Muscle

\section{GENETICS / EPIGENETICS}

Hanns Lochmüller

Institute Of Genetic Medicine, Newcastle

University, International Centre for Life, Newcastle

Upon Tyne, US

Abstract not received. 


\section{PL 2.1 / \#140}

Topic: 02. Neuropathy

\section{INHERITED NEUROPATHIES: FROM GENES TO CLINICAL PHENOTYPE}

Mary Reilly

Queen Square, Institue Of Neurology, London, GB

Abstract: The inherited neuropathies are a heterogeneous group of disorders encompassing those disorders in which the neuropathy is the sole manifestation and those neuropathies where the neuropathy is part of a more complex neurological or multisystem disorder. The first group includes Charcot Marie Tooth disease (CMT) and the related disorders, the hereditary sensory neuropathies (HSN) and the distal hereditary motor neuropathies (HMN). The second group includes a wide range of disorders examples of which include the leuokodystrophies and the mitochondrial diseases. We now know that there is overlap between the causative genes for CMT, HSN and HMN and often use the term CMT to include all of these diseases. Next generation sequencing especially whole exome and whole genome analysis has also revealed that genes that were associated with other neurological diseases e.g. hereditary spastic paraparesis and the ataxias can sometimes cause CMT or start with a CMT like syndrome before evolving to a more classical disease. Traditionally we have approached the diagnosis of an inherited neuropathy with careful phenotyping to guide us in selecting genetic tests. Although this is still important, increasingly we are using phenotyping to validate variants identified by next generation sequencing. As well as clinical examination and neurophysiology, we use MRI increasingly both of the CNS in complex neuropathies and neuromuscular protocols of the limbs looking for patterns of denervation associated with certain neuropathies (e.g. sparing of adductors and semitendinosus in the thighs of patients with BICD2 and Dynein mutations). The phenotypes associated with individual genes are expanding rapidly but for the common genes certain patterns are common and therefore helpful in validating variants (e.g. early ankle plantar flexion weakness in HSPB1 and HSPB8 mutations). Understanding the detailed phenotypes is critical in therapy development. It is an exciting time in inherited neuropathy therapy development with the advent of genetic therapies including antisense, silencing RNA, viral delivered gene therapies and various gene editing therapies. All of these are either in preclinical or clinical trials for various neuropathies. For gene therapies knowing both what tissues need to be targeted for efficacy and which organs needed to be avoided to prevent side effects is important. We also need better ways to monitor disease progression so responsive outcome measures can be developed for clinical trials. While we may have started with detailed phenotyping of families many decades ago to identify causative genes, the mechanistic insights from these genes and the subsequent therapies being developed have led us back to the patients to delineate and understand the phenotypes better in order to be able to perform clinical trials and deliver efficacious therapies to our patients safely.

\section{PL 2.2 / \#167}

Topic: 02. Neuropathy

\section{IMMUNE MEDIATED NEUROPATHIES: AN EXPANDING FIELD OF TREATABLE NEUROPATHIES}

\author{
Pieter Van Doorn \\ Center For Lysosomal And Metabolic Diseases, \\ Erasmus MC, University Medical Center \\ Rotterdam, Rotterdam, $N L$
}

\begin{abstract}
Immune mediated neuropathies comprise an extending field of disorders with specific subgroups. Patients with these disorders need to be diagnosed because they are treatable. It ranges from the Guillain-Barré syndrome (GBS), a heterogeneous acute polyneuropathy to the more chronic often demyelinating -polyradiculoneuropathies, of which chronic inflammatory demyelinating polyneuropathy (CIDP) is the most prominent one. CIDP can be divided into the most often occurring symmetric sensory-motor variant, and the pure motor or sensory subgroups. Focal, or multifocal varieties of CIDP are known as Lewis-Sumner syndrome or multifocal acquired demyelinating sensory-motor neuropathy (MADSAM). There additionally is a variety named distal acquired demyelinating sensory neuropathy (DADS). Careful electrophysiological investigation (EMG) and the use of peripheral nerve ultrasound or MRI of the plexus can be helpful to
\end{abstract}


diagnose these patients. EAN/Peripheral Nerve Society guidelines for the diagnosis and treatment of GBS and CIDP are available or under construction. Intravenous immunoglobulin (IVIg) and plasma exchange (PE) are effective in GBS. There is now an indication that complement inhibition using eculizumab potentially potentially is effective in GBS, but additional studies are required. The results of the second-dose IVIg RCT (SID-GBS) conducted in GBS patients with a poor prognosis - based upon the mEGOS prognostic model - are eagerly awaited. An interesting new study that especially may be helpful in low-income countries studied the feasibility of small volume plasma exchange as an alternative and simple method for treatment of GBS.Patients within the spectrum of CIDP can be treated with IVIg, PE or steroids. If one of these proven effective treatments fails, one of these other treatments is still likely to be successful. A recent RCT showed that patients with CIDP can also be treated with subcutaneous IgG (ScIG). There is debate what treatment for CIDP is best. IVIg acts fast but is expensive, and steroids are cheap but can induce major side effects. Pulse steroid treatment however may have a higher chance to induce remission of disease, which is now investigated in new studies. Under- and over treatment are important, therefore it needs to be investigated at least once every 6-12 months if a patient still requires treatment because the disease can get into remission either therapeutically induced or spontaneously. Multifocal motor neuropathy (MMN) is a disorder that may mimic motor neuron disease, but is an IVIg treatable condition. Surprisingly these patients do not improve after steroids or PE. Other important immune-mediated neuropathies are related to the presence of a paraprotein or for example due to vasculitis.A comprehensive overview will be given and the latest developments will be discussed.

\section{PL 2.3 / \#55}

Topic: 02. Neuropathy

\section{ADVANCES IN THE TREATMENT OF PERIPHERAL NEUROPATHY}

David Cornblath

Johns Hopkins Medicine, Baltimore, US

Abstract not received.
PL 3.1 / \#97

Topic: 03. Motor Neurone

\section{EMERGING THERAPIES FOR MOTOR NEURONE DISEASES}

\author{
Albert Ludolph \\ Universitätsklinikum Ulm, Ulm, DE
}

Abstract not received.

\section{PL 3.2 / \#164}

Topic: 03. Motor Neurone

\section{CLINICAL CONCEPT OF ALS}

Leonard Van Den Berg

Department Of Neurology, University Medical

Center Utrecht, Utrecht, $N L$

Abstract not received.

\section{PL 3.3 / \#182}

Topic: 03. Motor Neurone

\section{ETHICAL ISSUES}

David J. Oliver

Tizard Centre, University of Kent, Canterbury, GB

Background: The care of a person with Motor Neurone Disease is often complex, as they face multiple issues - physical, psychosocial and spiritual. Within these issues are often complex ethical issues, involving difficult discussions with patient and family and within the multidisciplinary team. Careful discussion and consideration of the issues is needed, to enable patients and families to make the most appropriate decision. Methods: A review of the literature, combined with clinical experience, has been undertaken. Results: The ethical issues may occur throughout the disease progression: - At diagnosis The telling of the diagnosis may raise issues - particularly if the family is resistant to telling a patient, who has capacity and is asking for information, or the patient has lost capacity Many patients may now have genetic testing and there are issues as to how they react to knowing that they have a gene mutation. This may influence their future and family plans 
and they face a dilemma as to what to tell other family member who may be at risk of having the same mutation - When swallowing problems start and become more difficult consideration maybe given to the insertion of a gastrostomy. This discussion may occur when respiratory function is deteriorating. A gastrostomy may improve quality of life, and maybe survival but patients may find the change in feeding very difficult to cope with - Ventilatory support non-invasive ventilation and, on occasions, invasive ventilation with a tracheostomy - may be considered if respiratory failure develops. Although these interventions may improve symptoms the disease progresses and the patient faces increasing disability, and even the risk of becoming locked in - with no communication. These issues need to be discussed before starting the intervention - At the end of life patients may request treatment withdrawal, such as withdrawal of ventilatory support. There may be complex discussion between family members and team members, who may understand these ethical dilemmas in a different way - Some patients, and families, may ask about assisted dying. In some countries this may be an option - of euthanasia or physician assisted suicide - and there are ethical issues balancing the wishes of the patient and family. In many countries there is no option, but the issue raises discussion for all concerned. Throughout the care of a person with MND there is the need to assess mental capacity to make decisions. As there is increasing evidence that up to $15 \%$ of patients may develop fronto-temporal dementia and a further $35 \%$ may show evidence of frontal lobe dysfunction, which may affect decision making, these issues have profound implications. Conclusion: Ethical issues need to be considered carefully for all patients with MND throughout the disease progression and involves careful discussion between patient and family and all members of the wider multidisciplinary team.

\section{PL 4.1 / \#47}

Topic: 04. Neuromuscular Junction

\section{PHYSIOLOGY AND STRUCTURE}

$\underline{\text { Steve Burden }}$

Skirball Institute, Nyu Medical School, New York, US

Abstract not received.
PL 4.2 / \#121

Topic: 04. Neuromuscular Junction

\section{CONGENITAL MYASTHENIC SYNDROMES - NEW GENES AND BETTER TREATMENTS / ANTIBODIES}

\author{
Kinji Ohno \\ Nagoya University Graduate School of Medicine, \\ Nagoya, JP
}

Abstract not received.

\section{PL 4.3 / \#171}

Topic: 04. Neuromuscular Junction

\section{AUTOIMMUNITY}

Angela Vincent

Dep. Of Clinical Neuroscience, University of Oxford, Nuffield, Oxford, GB

Abstract: In the 1970s, myasthenia gravis was shown to be caused by antibodies to the acetylcholine receptors (AChRs) on the postsynaptic surface of the neuromuscular junction (NMJ). The mechanisms of AChR antibodies include internalisation of AChRs caused by divalent antibodies, complementmediated lysis of the postsynaptic membrane, and less common direct inhibition of AChR function. Together, and variably, these cause loss of AChR numbers or function. The weakness is caused by failure of the endplate potential to reach the critical threshold for activation of the compound muscle action potential. This is compounded by the loss of postsynaptic folds where the voltage gated sodium channels that initiate the action potential, are situated. Interestingly, the presynaptic nerve terminal and the postsynaptic muscle both "compensate" for the defect in transmission by upregulating acetylcholine release and AChR synthesis respectively. There are different subtypes of MG, with thymectomy of established benefit in the young-onset patients, but an increasing number of late-onset patients ( $>50$ years at onset) who often have co-morbidities and present a challenge. Some of the patients who do not have AChR antibodies by conventional assays, have antibodies that only bind detectably to "clustered AChRs" expressed on cell lines. This emphasises 
the importance of clustering of AChRs at the NMJ and for synaptic function in general. Some of these patients do not have EMG evidence of MG and are less severe but respond well to immunotherapies. In 2001, antibodies to MuSK were found in some of the myasthenia patients who were negative for AChR antibodies (seronegative MG); these patients often have marked bulbar and facial involvement which can be difficult to treat with the immunotherapies as used for AChR-MG. The MuSK antibodies are predominantly, but not exclusively, IgG4 and the anti-CD20 drug, rituximab, appears to work well in many MuSK-MG patients. More recently antibodies to another NMJ protein, LRP4, have been found in a small number of additional patients. MuSK and LRP4 are both postsynaptic membrane proteins that are responsible for the agrin-induced clustering of the AChRs at the neuromuscular junction. When LRP4 binds agrin, MuSK is activated, DOK7 binds and this begins a sequence of intracellular events that maintain the high density of AChRs at the NMJ. Anything that interferes with LRP4 or MuSK membrane molecules would be expected to lead to dispersal of AChRs, but the detailed mechanisms are still not fully explored. A better understanding could lead to novel pharmacological treatments as will be described. Koneczny I, Cossins J, Vincent A. The role of muscle-specific tyrosine kinase (MuSK) and mystery of MuSK myasthenia gravis. J Anat. 2014 Jan;224(1):29-35. Huda S, Cao M et al. Increased phosphorylation of MuSK provides a potential therapy for MuSK-Ab myasthenia gravis. 2018 submitted for publication. 



\section{ICNMD 2018}

15TH INTERNATIONAL CONGRESS ON NEUROMUSCULAR DISEASES

www.icnmd2018.org July 6-10, 2018 Vienna, Austria

Scientific Sessions 
SS 1.1 / \#38

Topic: 01. Muscle

\section{TREATMENT STRATEGIES IN GNE MYOPATHY}

\author{
Zohar Argov \\ Hebrew University of Jerusalem EKMD, Jerusalem, \\ IL
}

Background: Introduction: GNE myopathy ( previously known as HIBM or DMRV) is associated with mutations in the gne gene, a bifunctional key enzyme in synthesis of sialic acid (neuraminic acid).As a result a progressive distal myopathy with relative quadriceps sparing is observed. The typical histopathology is composed of 'rimmed vacuoles', which are autophagic vacuoles. Background: The degree of reduction in enzyme activity ranges between 30$60 \%$. Hyposialylation of muscle is observed in transgenic animal model, but is not consistently found in affected human muscle. It is possible that GNE myopathy is caused by additional mechanisms apart from metabolic deficiency. Methods: The degree of reduction in enzyme activity ranges between $30-60 \%$. Hyposialylation of muscle is observed in transgenic animal model, but is not consistently found in affected human muscle. It is possible that GNE myopathy is caused by additional mechanisms apart from metabolic deficiency. Results: 1 . In the mouse model supplementation with sialic acid or its metabolic derivatives led to improvement. 2. In a phase 3 trial in patients receiving 6 gr/day of sialic acid for 1 year, no change in muscle power was found. A phase 2 trial with the metabolic intermediate ManNac is ongoing. 3. An AAV-GNE construct was shown to deliver normal human GNE to muscle in normal and diseased animals, with persistence for prolonged period. A human trial is in preparation. Conclusion: Discussion: supplementation of sialic acid or its derivatives may not suffice to correct the muscle disease. Viral mediated gene therapy may be more suitable at this stage when basic mechanism is still controversial. In the meantime trials with medication affecting autophagy or other downstream processes should be encouraged.
SS 1.2 / \#283

Topic: 01. Muscle

\section{UPDATE OF RNA-BASED NMD THERAPIES}

\author{
Kanneboyina Nagaraju \\ Pharmaceutical Sciences, SUNY-Binghamton \\ University, Binghamton, NY, US
}

Background: Recent advances in genetics and genomics significantly enhanced our ability to manipulate gene expression at RNA level in vitro in cell culture conditions and in vivo in mouse models and in patients with neuromuscular diseases (NMDs). Methods: The US Food and Drug Administration (FDA) recently approved Exondys 51 (eteplirsen), a drug that specifically targets Exon 51 to induce its exclusion in dystrophin mRNA resulting in a truncated functional dystrophin protein. Efforts are currently underway to target different exons in Duchenne Muscular Dystrophy using different chemistries to enhance efficiency of dystrophin protein expression in skeletal and cardiac muscle. Results: This presentation will briefly cover various RNA targeting approaches (Exon exclusion, inclusion, exchange, altering mRNA decay and stop codon read through), stage of their current development and potential scientific, regulatory and ethical hurdles involved in developing these drugs for Neuromuscular diseases. Conclusion: The potential for RNA based approaches to treat NMDs is excellent.

\section{SS 1.3 / \#59}

Topic: 01. Muscle

\section{PROGRESS IN THE TREATMENT OF IDIOPATHIC INFLAMMATORY MYOPATHIES?}

Marianne De Visser

Department Of Neurology, Academic Medical

Centre, Amsterdam, NL

\begin{abstract}
Idiopathic inflammatory myopathies (IIMs) can be distinguished into subacute onset and treatable disorders (dermatomyositis (DM), necrotizing immune mediated myopathy (IMNM), antisynthetase syndrome (ASS), non-specific myositis (NSM)) and chronic inclusion body myositis (IBM).
\end{abstract}


The mainstay of treatment in the treatable IIMs is long-term oral glucosteroids, and second line immunosuppressants are often added not only to obtain better efficacy but also for its steroid-sparing effect. If patients do not respond quickly to oral steroids one might consider intravenous administration of methylprednisolone or add third line medication such as rituximab (RTX) of intravenous immunoglobuline (IVIg). A trial with RTX on DM/PM did not reach the primary and secondary outcome measures. However, most of the patients clearly improved. IVIg was investigated in two RCT's and various open-label prospective trials on refractory $\mathrm{DM} / \mathrm{PM}$ patients and was found to be clinically efficacious. Still, long-term outcome in most IIM patients is not favourable. IIM patients have a polycyclic or chronic continuous course in almost $70 \%$ of the cases with similar percentages of perceived disabilities and lower quality of life scores after long-term follow up. This requires other vigorous treatment modalities, which not only aim at suppressing inflammation but also at preventing muscle damage. Increased knowledge about the pathomechanisms of IIMs will likely advance new and targeted treatments. Recent data suggests that exercise could be an effective anti-inflammatory treatment albeit the underlying mechanisms are not fully understood. IBM is as yet considered to be treatment-resistant. Not only is IBM refractory to the conventional immunosuppressive and immunomodulating modalities, but recently a phase $2 b / 3$ study with bimagrumab, a monoclonal antibody that acts via the myostatin-mediated induction of muscle growth failed its primary endpoint. The results of a proof-ofconcept study with rapamycine were promising. Conducting clinical trials in IIMs is challenging for many reasons. First, classification seems to be a moving target with the ever-increasing number of myositis-specific autoantibodies giving rise to further subclassification. Second, consensus on assessment tools that are highly responsive to interventions is required. The International Myositis Assessment and Clinical Studies Group 'response criteria' are recently published and could serve as such. Third, patient reported outcome measures are indispensible to evaluate the efficacy of a therapy and currently, they are under development.
SS 2.1 / \#224

Topic: 11. Nerve Regeneration

\section{TISSUE ENGINEERING STRATEGIES FOR REPAIR OF PERIPHERAL NERVE}

$\frac{\text { Anthony Windebank }}{\text { Mayo Clinic, Rochester, US }}$

Abstract not received.

SS 2.2 / \#1019

Topic: 11. Nerve Regeneration

\section{USE OF PHYSICAL METHODS IN NERVE REGENERATION}

\author{
Thomas Hausner \\ Trauma Surgery, Lorenz Böhler Trauma Hospital, \\ Vienna, AT
}

\begin{abstract}
Regeneration and recovery of peripheral nerves after injury still today is a long-lasting procedure. Human nerves elongate with an average speed of $1 \mathrm{~mm}$ per day. Transection of a nerve at the level of the forearm e.g. with $25 \mathrm{~cm}$ distance to the finger-tips would stand for about 250 days of recovery, given that rapid surgery has been performed corresponding to the actual technical standard. Increasing the speed of elongation would be very helpful in the post-surgical treatment. Electric stimulation since long time is used in nerve regeneration, with quite good results. However, new physical methods to increase axonal recovery are in development. Extracorporeal shock wave treatment (ESWT) is an established method in wound regeneration, bone regeneration and integration of skin grafts as well as treatment of some painful conditions as plantar fasciitis or humero-radial epicondylitis. In the last few years ESWT has been examined extensively in the field of nerve regeneration. Different in vivo and in vitro studies have been performed using the Dermagold 100 device (MTS, Germany). It could be clearly shown that axonal elongation is increased by a single use of ESWT immediately after surgery in different rat experimental nerve injury models. ESWT in vitro enhances stemness and preserves multipotency of adipose derived stem cells. ESWT in vitro also increases Schwann cell survival and activation state. ESWT may augment and potentiate the axonal elongation and functional
\end{abstract}


recovery in a regenerating peripheral nerve segment. It also can be seen as a promising supporting tool for tissue engineering in peripheral nerve regeneration.

SS 2.3 / \#378

Topic: 11. Nerve Regeneration

\section{NEW DEVELOPMENTS IN CONDUITS}

David Hercher

Ludwig Boltzmann Institut, Vienna, AT

Abstract not received.

SS 3.1 / \#70

Topic: 08. Cancer

\section{THE ROLE OF HU PROTEINS IN THE DEVELOPMENT OF PARANEOPLASTIC NEUROPATHIES}

Bruno Giometto

Universita die Padova, Padova, IT

Abstract not received.

\section{SS 3.2 / \#79}

Topic: 08. Cancer

\section{PHYSIOPATHOLOGY OF PARANEOPLASTIC GANGLIONOPATHIES}

\section{Romana Hoeftberger}

Institute Of Neurology, Medical University of

Vienna, Vienna, $A T$

Background: Paraneoplastic ganglionopathies are acquired neuronopathies that are characterized by a primary degeneration either of sensory neurons in dorsal root ganglia (paraneoplastic sensory neuronopathy, PSN) or sympathetic and parasympathetic neurons in autonomic ganglia (paraneoplastic autonomic neuronopathy, PAN). Methods: Summary of relevant findings in paraneoplastic ganglionopathies with emphasis on physiopathology and clinical presentation. Results: The PSN is mainly associated with small cell lung cancer and antibodies directed against intracellular antigens, so-called onconeuronal antibodies. These antibodies can be found in up to $80 \%$ of cases, most frequently anti-Hu or anti-CV2/CRMP5, less common amphiphysin antibodies. The pathogenetic concept is considered to be an immune response that is initially triggered by an aberrant expression of the onconeuronal antigen by tumor cells and subsequently misdirected against the nervous system. Neuropathological investigation reveales moderate inflammation with diffuse endoneurial T-cell, B-cell- and plasma cell infiltration in the spinal ganglia. CD8-positive T-cells are tightly attached to the surface of neurons indicating a T-cell mediated attack resulting in irreversible neuronal death. Nerve biopsy usually shows an axonal neuropathy with loss of large myelinated fibers and interstitial macrophages containing myelin debris. The clinical presentation is characterized by a subacute onset and rapidly progressive disease course with asymmetrical or multifocal numbness in the upper or lower limbs, severe impairment of joint position and vibratory sensation, pain, paresthesias, and sensory ataxia. Cranial nerve involvement may present as loss of taste, numbness of the face or sensorineural hypoacusia. PSN associated with anti-Hu antibodies often presents with additional CNS involvement such as encephalomyelitis but may remain an isolated syndrome in about $25 \%$ of cases. PSN associated with anti-CV2/CRMP5 antibodies more often presents as a mixed axonal and demyelinating neuropathy, predominates in the lower limbs, may have motor involvement, and pain is less frequent. PSN associated with amphiphysin antibodies may present with additional symptoms of stiff person syndrome or encephalopathy. Patients with PAN may either present as chronic intestinal pseudo-obstruction due to affection of the enteric plexus, or less common as subacute pandysautonomia with dysfunction of the sympathetic and parasympathetic nervous system. Patients may harbor anti-Hu antibodies, up to $20 \%$ may have ganglionic acetylcholine receptor antibodies. The underlying tumor in PSN and PAN often remains occult for a long time and search for a malignancy should focus in particular on the detection of a SCLC. Conclusion: Early diagnosis of paraneoplastic ganglionopathy by the detection of onconeuronal antibodies is important to confirm the diagnosis and initiate tumor screening. Early treatment of the cancer gives the best chance of stabilizing the disorder. 
SS 3.3 / \#133

Topic: 08 . Cancer

\section{PARANEOPLASTIC MUSCLE DISORDERS}

Andrew Mammen

NIAMS/NIH, Bethesda, US

Abstract not received.

SS 3.4 / \#170

Topic: 08. Cancer

\section{LAMBERT-EATON-SYNDROME} (LEMS)

Jan Verschuuren

Leiden University Medical Center, Leiden, $N L$

Abstract not received.

SS 4.1 / \#159

Topic: 01. Muscle

UPDATE ON CLINICAL ASPECTS OF GLYCOGEN STORAGE DISORDERS

Antonio Toscano

University of Messina, Messina, IT

Abstract not received.

SS 4.2 / \#188

Topic: 01. Muscle

\section{NOVEL ENTITIES IN CONGENITAL} MYOPATHIES OF ADULT ONSET

Baziel Van Engelen

Radboud University Medical Centre, Nijmegen, NL

Abstract not received.
SS 4.3 / \#161

Topic: 01. Muscle

\section{NEW PHENOTYPES IN TITIN DEFECTS}

Bjarne Udd

Tampere Neuromuscular Center, Tampere, FI

Abstract not received.

SS 5.1 / \#1037

Topic: 13. Pediatric

STANDARDS OF CARE: WHAT WE

HAVE LEARNED FROM LONG

TERM-MANAGEMENT IN

\section{CHILDREN WITH NMD'S}

Guenther Bernert

Department Of Paediatrics, Kaiser Franz Joseph Hospital, Vienna, AT

Abstract not received.

SS 5.2 / \#832

Topic: 13. Pediatric

\section{FUTURE OF AAV-BASED GENE THERAPY APPROACHES FOR NMDS}

Thomas Voit

UCL Great Ormond Street Institute of Child Health, London, GB

Abstract: After more than a decade of animal experimentation, AAV-based clinical trials are now under way addressing Spinal Muscular Atrophy (SMA), Duchenne Muscular Dystrophy (DMD) and myotubular myopathy. They are addressing patients from infancy to young adults as well as different organ systems (CNS, skeletal and cardiac muscle). While too early to judge results across disease applications, there are important commonalities particularly regarding route of administration and maximum dosage in vector genomes $/ \mathrm{kg}$. At the same time, important questions remain, which need to be 
addressed in the future, both in animal experiments and in the human. These include the issues of hepatic and even potentially systemic toxicity of AAV capsids; pre-existing and treatment-induced immunity to AAV; the need for re-administration depending on the target tissue and the degree of genetic correction achieved. In some diseases such as SMA the route of administration remains an issue and has important consequences on the total dose, but potentially also on the biodistribution. In order to finetune these treatment approaches in the human biomarkers are becoming increasingly important to monitor the treatment effect over time. Finally, both in SMA and in DMD there is a potential to combine AAV-based gene therapy with chemical antisensetreatment, even if these technologies at the moment are largely developed in competition. These questions will be discussed in light of the most recent clinical and experimental data followed by a discussion of possible future steps.

SS 5.4 / \#856

Topic: 13. Pediatric

\section{TRANSITION FROM PEDIATRIC INTO ADULT CARE: A LONG WAY TO GO?}

Thomas Serjensen

Karolinska Institute, Solna, SE

Abstract not received.

SS $6.1 / \# 151$

Topic: 08. Cancer

\section{GENERAL PRINCIPLES AND CLINICAL ASPECTS OF CIPN}

Nathan Staff

Neurology, Mayo Clinic, Rochester, MN, US

Background: Chemotherapy-induced peripheral neuropathy (CIPN) is a common dose-limiting side effect that follows administration of several chemotherapeutic classes. While most CIPN is due to direct neurotoxicity of the chemotherapeutic, the recent introduction of check-point inhibitor cancer therapies has also resulted in a variety of immune-mediated neuromuscular side effects. CIPN occurs in up to $50 \%$ of all patients receiving neurotoxic chemotherapy and has been associated with both acute and long-term morbidities. Methods: Literature review was performed. Results: The clinical manifestations and pathomechanisms of CIPN from the different neurotoxic chemotherapy classes will be reviewed. The epidemiology and disease burden of CIPN will be explored by highlighting a recent study from Olmsted County, MN. Treatment and preventative strategies for CIPN, which have been largely unsuccessful, will also be described. Finally, a discussion surrounding the discovery and validation of CIPN susceptibility factors will be provided. Conclusion: CIPN continues to be a significant problem despite the development of more targeted cancer therapies. Key outstanding questions in CIPN are: 1) What are the pathomechanisms of CIPN? 2) Why is there variable susceptibility to CIPN? Answers to these key questions are expected to lead to rational predictive, preventative, and treatment strategies for this entity.

SS 6.2 / \#196

Topic: 08. Cancer

\section{BASIC MECHANISMS: AXONAL DEGENERATION AND CHEMOTHERAPY-INDUCED PERIPHERAL NEUROPATHY}

Ahmet Hoke

Johns Hopkins University School of Medicine, Baltimore, US

Abstract not received.

SS 6.3 / \#51

Topic: 08. Cancer

\section{ANIMAL MODELS OF CHEMOTHERAPY-INDUCED PERIPHERAL NEUROPATHY}

Guido Cavaletti

Head, Experimental Neurology Unit And Phd Program In Neuroscience School Of Medicine, University of Milano-Bicocca, Bicocca, IT

Abstract not received. 
SS $7.1 / \# 157$

Topic: 02. Neuropathy

\section{NEW GENES AND NEW MECHANISMS IN INHERITED NEUROPATHIES}

$\underline{\text { Vincent Timmerman }}$

Biomedical Sciences, University Of Antwerp, Antwerpen, BE

Background: New genes and new mechanisms in inherited neuropathies Vincent Timmerman Peripheral Neuropathy Research Group, University of Antwerp, Belgium Inherited peripheral neuropathies (IPN) are genetic conditions affecting 1 in 2,500 individuals. Charcot-Marie-Tooth (CMT) neuropathy is the best known form characterized by a vast phenotypic and genetic heterogeneity. The identification of mutations in more than 100 known genes through gene screening panels, whole exome or genome sequencing to find variants in novel disease causing genes, in combination with the increasing availability of cell and animal models, has deepened our understanding in the disease mechanism. The heterogeneity is complicated by the fact that CMT associated genes have diverse functions; they regulate myelination through Schwann cells, take care of axonal transport and neuronal metabolism, or have essential cellular functions by regulating autophagy and protein quality control. However, despite this knowledge there is still no effective therapy for most patients with CMT and related disorders. Methods: More recently novel models, such as the use of patient derived induced pluripotent stem cells, and neurons differentiated from these cells, in combination with omics approaches has opened novel opportunities for identifying common disease mechanisms and pathways. Results: These new methods will identify mode-of-actions and potential targets for therapy that are applicable for multiple CMT phenotypes. Conclusion: This information will complement IPN mutation databases, improve clinical and molecular diagnosis, and hopefully allow treatment strategies for some of these rare to ultra-rare IPN phenotypes.
SS 7.2 / \#148

Topic: 02. Neuropathy

\section{THERAPEUTIC OPTIONS IN CMT}

Michael Sereda

Neurogenetics, Max-Planck-Institute of

Experimental Medicine, Gottingen, DE

Abstract not received.

SS 7.3 / \#126

Topic: 02. Neuropathy

FAMILIAL AMYLOID NEUROPATHIES: A TREATABLE GENETIC NEUROPATHY

Davide Pareyson

Dept Of Clinical Neurosciences, IRCCS

Foundation, C.Besta Neurological Institute, Milan, IT

Abstract: Inherited transthyretin amyloidosis (ATTR) is an autosomal dominant disorder due to mutations of the transthyretin (TTR) gene. TTR is synthetized mainly by the liver and released in plasma as a tetrameric transport protein. Mutations in TTR, of which Val30Met is the most common worldwide, cause transthyretin tetramer dissociation, monomer misfolding, and aggregation into insoluble fibrillar proteins in different tissues. Orthotopic liver transplantation (OLT), by removing the main site of mutated TTR production, proved able to halt or slow neurological progression and was until few years ago the standard-of-care treatment in patients aged $<50$ years with Val30Met mutation. OLT is associated however with not negligible mortality rate and is not curative, since cardiac disease tends to progress and leptomeningeal amyloid deposition may become an issue. Tafamidis meglumine is a small molecule which kinetically stabilizes the TTR tetramer and prevents its dissociation into amyloidogenic monomers. It proved able to slow down disease progression particularly in the early disease phases and is currently approved in several countries for treatment of symptomatic patients with polyneuropathy in disease stage I (independent ambulation). Responders were also described among patients with late-onset disease, non-Val30Met mutations, and in 
later disease stages. The old nonsteroidal anti-inflammatory drug diflunisal has also been reported to be effective as a TTR tetramers stabilizer and produced significant slowing in disease progression in treated patients. The antibiotic doxycycline and the taurine conjugate form of ursodeoxycholic acid (TUDCA) have a synergistic effect on fibril disruption and their use is currently under investigation with encouraging results. Monoclonal antibodies directed against either the serum amyloid $\mathrm{P}$ component (SAP) or the amyloidogenic forms of transthyretin constitute another strategy to clear the amyloid deposits. Antisense Oligonucleotides (Inoserten) or interfering RNA lipid nanoparticles (Patisiran) binding to wild type and mutated TTR mRNA proved able to reduce TTR production by more than $75 \%$ and both proved very effective in two recently completed phase III trials. Both trials reached the primary endpoints and multiple measures were significantly better in treated as compared to untreated patients: stabilization or improvement occurred for a good proportion of treated patients. Inoserten was administered subcutaneously once a week for 15 months; thrombocytopenia and renal problems were adverse events which required monitoring of platelet and renal function. Patisiran, administered intravenously every 3 weeks for 18 months, was well tolerated with infusion-related reactions and peripheral edema as the most relevant reported side effects. Both treatments were effective independently from disease stage, presence of cardiopathy, type of mutation. The development of such novel therapies is changing the natural history of ATTR-neuropathy from a relentlessly progressive disorder inexorably leading to death into an effectively treatable disorder.

\section{SS 8.1 / \#1028}

Topic: 07. Cranial Nerves

\section{CRANIAL NERVES: WITHIN AND OUT OF THE SKULL, INCL. ANGIOSOMA}

\author{
Wolfgang Grisold $^{1}$, Anna Grisold ${ }^{2}$, Stefan Meng ${ }^{3}$ \\ ${ }^{1}$ Ludwig Boltzmann Institute for Experimental und \\ Clinical Traumatology, Vienna, AT; ${ }^{2}$ Dep. \\ Neurology, Allgemeines Krankenhaus Vienna, \\ Vienna, AT; ${ }^{3}$ Institute for Radiology, KFJ hospital, \\ Vienna, $A T$
}

Abstract: The course of cranial nerves (CN) is characterized by their intraparenchymal, intracranial part, the site of exit of the skull and their peripheral course. Thus dysfunction can be due to brainstem lesions, meningeal and CSF involvement, local changes at their exit of the skull, and a variety of conditions in their extracranial course. All parts of the cranial nerves are dependent on blood supply, which is provided by small arteries. The distribution of the blood supply follows the distribution of angiosomas, which follows a concept applicable for the whole body. According to this concept of blood supply, individual nerves receive the blood supply in different segments from adjacent angiosomas. As a practical consequence, intravascular interventions as embolizations of tumors of the base of the skull, can damage cranial nerves. As indirect signs of damage of motor nerves, also the MR visualization of muscles at the base of the skull is useful, which helps to detect asymmetries and neurogenic lesions. The tongue muscles as an example for strict unlilateral blood supply is a good example. Increasingly ultrasound can be used to demonstrate the extracranial parts of the nerves, where also thickening, and local tumors can be demonstrated.

\section{SS 8.2 / \#44}

Topic: 07. Cranial Nerves

\section{INFECTIOUS AND INFLAMMATORY CRANIAL NERVE LESIONS}

\author{
Nazha Birouk \\ Clinical Neurophysiology Rabat, Speciality \\ Hospital, Rabat, MA
}

\begin{abstract}
Cranial nerves can be involved in various infectious and inflammatory diseases either as a mononeuropathy or multicranial neuropathy. The diagnosis can be easily made in conditions with other expressions of the infection or inflammations within the nervous system or in extra-neurologic organs with in some conditions typical presentations. The diagnosis might be more challenging when the cranial neuropathy is the revealing symptom. The most frequent infection worldwide remains an active infectious ganglioneuritis caused by varicella zoster virus, producing shingles. The commonly associated cranial nerve neuropathies are: herpes zoster ophthalmicus ( $10 \%$ to $20 \%$ of all zoster cases), the
\end{abstract}


Ramsay Hunt syndrome (triad of ipsilateral facial paralysis, ear pain, and vesicles in the auditory canal and auricle) and optic neuritis. Facial nerve palsy can be due to Lyme borreliosis with a similar clinical presentation to Bell's palsy. This infection is to be searched in endemic areas, in the presence of skin rash or if the facial palsy is bilateral and/or recurrent. Cranial neuropathies in HIV occur at various stages of infection and are sometimes multifocal. Facial nerve palsy, for example, can develop at the time of seroconversion or later in the disease course secondary to opportunistic infections. In immunocompetent patients, many infectious conditions can be accompanied by cranial neuropathies such as meningitis due to tuberculosis, syphilis, listeriosis, cryptococcus and various other infectious agents. Cranial nerves might be impaired by contiguous infection as otitis or sinusitis. Cranial neuropathy can be associated to systemic inflammatory disorders such as sarcoidosis (mostly nerves VII, VIII and II), Sjogren syndrome (mostly nerves $\mathrm{V}$ and II) or ANCA vasculatis (nerves III, IV, VI, VII and X) and others. IgG4related disease has been newly identified with a wide spectrum of diseases that can involve nearly any organ system, including the central and peripheral nervous systems. The principal neurological manifestations result from orbital disease, pachymeningitis, and pituitary gland and stalk involvement. The common aspect is an inflammatory pseudotumor with possible impairment of oculomotor (as Tolosa Hunt syndrome), trigeminal and optic nerves. The diagnosis is based on the serum IgG4 elevation, the histological aspects with significant infiltration with IgG4 positive plasma cells, and the elimination of other inflammatory disorders like vasculitis. The disease responds well to treatment by corticosteroids. Specific inflammatory diseases of central nervous system such as multiple sclerosis and NMO imply cranial neuropathies, mostly optic neuritis but also other nerves such as oculomotor, facial and trigeminal nerves. We should keep in mind that an authentic Guillain Barre syndrome can rarely present with isolated bilateral facial nerve or multicranial nerves palsy.
SS 8.3 / \#230

Topic: 07. Cranial Nerves

\section{PAINFUL CRANIAL NERVE LESIONS}

Frank Thömke

Klinikum Worms gGmbH Fachbereich Neurologie, Worms, $D E$

Background: This presentation deals with cranial nerve palsies with persistent pain lasting days to weeks. Methods: Description of clinical signs, diagnosis and therapy. Results: Optic neuritis may be accompanied by persistent pain on the affected side, which occurs or increases with movements of the affected eye. Pain usually decreases with high does steroid treatment (usually $1000 \mathrm{mg}$ methylprednisolone for 3 days). MRI should be done since optic neuritis is a frequent initial symptom of multiple sclerosis. Moreoever, one should look for aquaporin-4 antibodies (NMO-IgG) to detect the beginning of a neuromyelitis optica. Painful ocular motor nerve lesions are often summarized as "painful ophthalmoplegia, a heterogenous group of diseases characterized by paresis of one or more ocular motor nerves associated with frontal or orbital pain. Vascular, inflammatory or tumorous diseases are main causes. Up to one third of ocular motor nerve palsies are attributed to microvascular nerve infarctions, a diagnosis by exclusion. Most of the patients complain ipsilateral pain, especially with $3^{\text {rd }}$ nerve palsies. This must be differentiated from $3^{\text {rd }}$ nerve palsies due to an aneurysm, which is easily done by MRI- or CT-angiography. Diagnosis of a Tolosa Hunt syndrome depends, among others, on the demonstration of granulomatous inflammation of the cavernous sinus, the superior orbital fissure or the orbit demonstrated by MRI or biopsy. Pain promptly resolves with steroid treatment (usually $1 \mathrm{mg}$ prednisolone or methylprednisolone per $\mathrm{kg}$ body weight). Abnormal eye movements recover within several weeks, which seem to be equally probable with or without steroids. Diagnosis of recurrent painful ophthalmoplegic neuropathy (,ophthalmoplegic migraine“) may be considered after two attacks and exclusion of an orbital, parasellar or posterior fossa tumor. In some patients, MRI may disclose gadolinium-enhancement of a thickened $3^{\text {rd }}$ nerve. Pain promptly decreases with steroid treatment (usually $1 \mathrm{mg}$ prednisolone or methylprednisolone per $\mathrm{kg}$ body weight). Abnormal 
eye movements recover within several weeks, which seem to be equally probable with or without steroids. Persistent pain with trigeminal nerve lesions may occur with acute or previous Herpes zoster, previous trauma, multiple sclerosis, or space occupying lesions. Herpes zoster may be diagnosed by laboratory findings, otherases by MRI or (CT). Herpes Zoster is treated with aciclovir or valaciclovir, and persistent pain due to multiple sclerosis with high dose steroids (usually $1000 \mathrm{mg}$ methylprednisolone for 3 to 5 days). Pain is a prominent symptom in facial palsy due to Herpes Zoster (Ramsay Hunt syndrome) and treated with aciclovir or valaciclovir (and steroids) added by analgetics and/or anticonvulsant substances such as carbamazepine or pregabalin. Bell's palsy may also be associated with retroauricular pain, which resolves with steroid treatment of Bell's palsy. Painful lesions of one or multiple of these nerves may occur with space occupying lesions or meningeosis neoplastica. They are often associated with diffuse headache and focal signs like hemiparesis or hemiataxia. Treatment depends on the etiology and involves neurosurgical procedures, steroid and radiation. Conclusion: MRI (or CT) should be done in most patients with painful cranial nerve lesions, and MRI- or CT-angiography in some of them. Steroids are an effective treatment in most cases.

\section{SS 8.4 / \#190}

Topic: 07. Cranial Nerves

\section{PAROXYSMAL DYSFUNCTIONS}

Friedrich Zimprich

\section{Department Of Neurology, Medical University of} Vienna, Vienna, AT

Abstract: Neuromuscular specialists are often required to evaluate patients displaying symptoms of transient (upper) cranial nerve dysfunctions, with ocular myasthenia gravis being a main differential diagnosis. In fact there is a wide range of different diseases which might manifest as oculomotor dysfunction with or without ptosis either as a consequence of cranial nerve palsies (III, IV or VI) or because they mimic these conditions. While many of these disorders may eventually result in a typical, i.e. clearly recognizable clinical picture the presentation in the initial stages or in mild cases can take the shape of fluctuating or paroxysmal, transient symptoms, which obviously stands in the way of finding a fast and definite diagnosis. The possible differential diagnoses range from ocular myasthenia to other myopathies (e.g. mitochondrial disorders), autoimmune diseases (e.g. thyroid-associated ophthalmopathy), inflammatory neuropathies, diseases affecting the intracranial pressure (intracranial hypotension or pseudotumor cerebri), vascular ischaemic diseases, diabetes associated cranial nerve palsies, inflammatory lesions of the central nervous system, nerve sheath tumors or other space occupying lesions along the nerves to ocular neuromyotonia. This differential diagnoses will be discussed and illustrative cases will be presented.

\section{SS 9.1 / \#1013}

Topic: 05. Autonomic

\section{PROTEINOPATHIES (THE AUTONOMIC VIEW)}

G Wenning

Department Of Neurology, Medical University

Innsbruck, Innsbruck, AT

Abstract not received.

\section{SS 9.2 / \#1014}

Topic: 05. Autonomic

\section{PROTEINOPATHIES (THE NEUROMUSCULAR VIEW)}

Walter Struhal

Department For Neurology, University Clinic Tulln, Karl Landsteiner University of Health Sciences, Tulln, $A T$

Abstract not received. 
SS 9.3 / \#1015

Topic: 05. Autonomic

\section{HEREDITARY NEUROPATHIES (THE AUTONOMIC VIEW)}

$\underline{\text { Max J. Hilz }}$

Neurology, Universitätsklinikum Erlangen, Erlangen, DE

Abstract not received.

SS 9.4 / \#1016

Topic: 05. Autonomic

\section{HEREDITARY NEUROPATHIES (THE NEUROMUSCULAR VIEW)}

Michaela Auer-Grumbach

Department Of Orthopaedics, Medical University of Vienna, Vienna, AT

Abstract not received.

SS 9.5 / \#1017

Topic: 05. Autonomic

\section{METABOLIC ANS DISEASE (DIABETES AND LIVER) (THE AUTONOMIC VIEW)}

$\underline{\mathrm{R} \text { Freeman }}$

Beth Israel Deaconess Medical Center, Boston, US

Abstract not received.

SS 9.6 / \#1039

Topic: 05. Autonomic

\section{METABOLIC ANS DISEASE (DIABETES AND LIVER) (THE NEUROMUSCULAR VIEW)}

Paola Sandroni

Mayo Clinic, Rochester, US

Abstract not received.
SS 10.1 / \#46

Topic: 02. Neuropathy

\section{ADA POSITION STATEMENT ON DIABETIC NEUROPATHY: DOES IT CHANGE YOUR PRACTICE?}

Vera Bril

Department Of Medicine (neurology), University Health Network, University of Toronto, Toronto, CA

Abstract not received.

SS 10.2 / \#144

Topic: 02. Neuropathy

\section{SCIENTIFIC DISCOVERY LEADS TO CLINICAL TRIALS IN DIABETIC NEUROPATHY}

James Russell

University of Maryland School of Medicine,

Baltimore, US

Abstract: A complex constellation of pathways are directly or indirectly affected in diabetic neuropathy. Dissecting out these pathways can lead to development of new approaches to reverse neuropathy and treat neuropathic pain. Despite the focus on glycemic control in diabetes, the evidence from randomized clinical trials that improved glycemic control reduces the prevalence of neuropathy in the majority of diabetics is unclear. The evidence for type I diabetes is strong but weak or absent for type 2 diabetes mellitus. While many potential pathways have been recognized as potential targets for therapy, this lecture will focus on mechanisms that currently offer or may offer immediate translation into clinical practice. While many clinical trials in diabetic neuropathy have failed, increasingly trials are being initiated by investigators that understand the complexities of mechanism and trial design. They appreciate the importance of sensitive and rapidly responsive efficacy measures in mild neuropathy observed early in the course of diabetes, when response to therapy is most likely to occur. There is considerable interest in the role of lifestyle interventions in reversing diabetic neuropathy. However, what is not always appreciated is that the dietary and exercise manipulations have clear and measureable effects on specific 
biological pathways similar to the effect of a medication. These interventions affect critical signaling pathways that regulate mitochondrial function, generation of reactive oxygen species, lipid metabolism, and growth factor production that may lead to nerve regeneration. Lipidomic characterization may lead to not only understanding how lipid pathways are implicated in diabetic neuropathy (pathway characterization) but also how manipulation of abnormal lipid metabolism may lead to therapy. Already, there is evidence that modification of lipid metabolism can result in improvement in diabetic small fiber neuropathy in humans. A novel approach to therapy has resulted from the observation that muscarinic receptor antagonists are implicated in protection from axonal degeneration both in vitro and in vivo. Neurite outgrowth from sensory neurons is in part mediated by muscarinic receptor-dependent regulation of mitochondrial function through the AMPK signaling pathway. Consistent with these observations in vitro, pharmacological blockade of the muscarinic receptor, M1R, using specific or selective antagonists, prevented or reversed peripheral neuropathy and reduced diabetes-induced mitochondrial dysfunction in vitro and in vivo. Work in vivo is currently being translated into clinical trials. Finally, the implications of specific drugs that target the Peroxisome proliferator-activated receptor-gamma co-activator 1alpha (PGC-1alpha) signaling pathway, which critically regulates mitochondrial and lipid metabolism, and the potential for translation to clinical trials will be discussed. Common polymorphisms of PGC-1alpha are associated with conversion from impaired glucose tolerance to diabetes and loss of PGC-1alpha causes neuropathy that is worsened by diabetes. Thus, understanding the critical mechanisms that lead to diabetic neuropathy may result in improved therapy and management of neuropathic symptoms.

\section{SS 10.3 / \#68}

Topic: 02. Neuropathy

\section{PAINFUL DIABETIC NEUROPATHY: A MANAGEMENT CENTERED APPROACH}

Eva Feldman

University of Michigan, Ann Arbor, US

Abstract: Pain is a persistent and morbid complication of diabetic polyneuropathy (DPN). There are no compelling data supporting the concept that glycemic control alone improves DPN pain, and most physicians and patients turn toward pharmacologic treatment. The American Diabetes Association, the American Academy of Neurology and the Canadian Pain Society are among the organizations that have issued pain management guidelines for DPN. DPN is controlled by different classes of agents: monoamine reuptake inhibitors and anticonvulsants are the main stay of therapy. Two agents, duloxetine (a selective norepinephrine and serotonin reuptake inhibitor) and pregabalin (a calcium channel $\alpha 2-\delta$ subunit ligand anticonvulsant) are approved by the FDA, Health Canada and the European Medicines Agency for painful DPN treatment. Other effective monoamine reuptake inhibitors, not officially approved, include the tricyclic antidepressant amitriptyline, the secondary amines, nortriptyline and desipramine and the dual reuptake inhibitor venlafaxine. Among the effective anticonvulsants that lack formal agency approval, gabapentin (another ligand of the calcium channel $\alpha 2-\delta$ subunit) is most commonly used, followed by topiramate and less commonly carbamazepine or phenytoin. Following guidelines, either duloxetine or pregabalin are used in the initial treatment of painful DPN; if neither single agent provides pain relief, the drugs are taken together, depending on side effect profiles. A common final dosage of duloxetine is either 60 or $120 \mathrm{mg} /$ day, depending on tolerability; pregabalin is $150 \mathrm{mg}$, either twice or thrice daily, again dependent on tolerability. When drug cost is an issue, an alternative to the above approach is to begin with gabapentin, reaching doses up to $1,800 \mathrm{mg}$ to $3,600 \mathrm{mg} /$ day in 3 to 4 divided doses. The tertiary amine amitriptyline can be added to gabapentin, or also begun as sole therapy, at nightly doses that range from 50 up to $100 \mathrm{mg}$. The secondary amines, nortriptyline and desipramine, are preferred over amitriptyline, if there is any history of cardiovascular disease or risk of urinary retention due to prostatism. While providing less pain relief that amitriptyline, the secondary amines have a more robust safety profile; finally, the dual reuptake inhibitor venlafaxine, also provides pain relief, although studies suggest pain reduction is less that with duloxetine. Dosages of 150 to $225 \mathrm{mg}$ /day can be used alone or in combination with an anticonvulsant drug. Importantly, duloxetine or venlafaxine therapy is best combined with anticonvulsant therapy; combination with the tricyclic antidepressants should be used only with caution secondary to adverse patient outcomes. Tapentadol is a centrally 
acting opioid with a dual mechanism of action: inhibition of noradrenaline reuptake and activation of the $\mu$-opioid receptor. While tapentadol is FDA approved, the International Association for the Study of Pain's Special Interest Group on Neuropathic Pain (NeuPSIG) completed a systematic meta-analysis and determined that the efficacy of tapentadol in the treatment of painful DPN was uncertain. The use of tapentadol is therefore not recommended as a first or second line treatment, and opioids as a class of drugs should be avoided in the treatment of painful DPN.

\section{SS $11.1 / \# 851$}

Topic: 12. Patient Issues

\section{JAMES LIND ALLIANCE PRIORITY SETTING PARTNERSHIP IN NEURO- ONCOLOGY (RESEARCH PRIORITIES)}

\author{
Kathy R. Oliver \\ International Brain Tumour Alliance (IBTA), \\ Tadworth, GB
}

Background: It is crucial that what patients value is reflected in research relating to treatment, care and support. This is particularly relevant in rare cancers, like brain and spinal cord tumours, where many clinical questions about these diseases remain unanswered. The James Lind Alliance (JLA - http://www. lindalliance.org/) was established in 2004 and is coordinated by the UK National Institute for Health Research (NIHR). It brings patients, caregivers and clinicians together in a 'Priority Setting Partnership' (PSP) to determine the top ten unanswered questions relating to a particular disease. Methods: The JLA PSP in neuro oncology (N-O) identified clinical research questions that were most important to people living with a brain or spinal cord tumour. The project's scope was: clinical uncertainties of interventions for primary brain or spinal cord tumours, in people of any age, from diagnosis to terminal stages. The methods for determining the top ten uncertainties were: 1. identification of stakeholder representatives (28) from a cross-section of the brain tumour community; 2. obtaining funding from four brain tumour charities, Cochrane Neuro-Oncology, and Edinburgh and Lothian Health Foundation; 3. developing and producing a protocol and website (www. neuro-oncology.org.uk); 4. publicising the JLA PSP $\mathrm{N}-\mathrm{O}$ survey to UK brain tumour communities and those abroad using multi-disciplinary professional and charity databases; 5 . analysing first round survey results and eliminating duplicate questions and those already answered by previous research; 6 . categorising and standardising questions into PICO (participants, interventions, comparisons, outcomes) format; 7. carrying out systematic Cochrane-style literature searches to ensure genuine uncertainties; 8. prioritising questions using standard JLA PSP methodology and unbiased facilitators; 9. conducting a second public survey on a short-list of prioritised questions; 10 . holding a workshop to determine the final top ten questions using a modified Delphi and Nominal Group technique. Results: Over 600 individual questions were generated from the initial survey, a patient forum and the Database of Treatment Uncertainties (DUETS). A second public survey was distributed (44 PICO questions created from uncertainties resulting from the initial survey). Over 200 people responded and voted for their top ten research priorities for brain and CNS tumours. The final top ten uncertainties were agreed. Much of the work of the JLA PSP N-O evolved to form a core activity of the UK National Cancer Research Institute (NCRI) Brain and CNS Clinical Studies Subgroup on Supportive and Palliative Care, which is approaching UK governmental, neuroscience and charity funders to support research into the top ten uncertainties identified. Conclusion: The JLA PSP $\mathrm{N}-\mathrm{O}$ collaboration was an enlightening and successful project. It will now be crucial to pro-actively promote the top ten research uncertainties for brain and CNS tumours in order to focus attention on what really matters to people affected by these diseases. It is vital to obtain reliable, evidenced-based information about these uncertainties through robust research focussed on the JLA PSP N-O top ten priorities.

\section{SS 11.2 / \#212}

Topic: 12. Patient Issues

\section{HOW TO DO INVESTIGATOR- INITIATED TRIALS: PCORI}

Richard J. Barohn

Neurology, University of Kansas Medical Center, Kansas City, KS, US

Abstract: In recent years there has been a great effort to get patients, families, community and patient advisory groups more involved in the clinical 
research process. When developing an idea for a clinical research project we get patients involved to identify their needs and what areas they believe we should focus on. We then place patients on the project steering committees, communication committees and Data Safety Monitoring Boards (DSMB). Outcome measures are increasingly becoming patient driven and the field of patient reported outcomes measures (PROM) is extremely important. We use various patient reported outcomes as either primary or secondary outcomes. In the USA such research is funded by the Patient Center for Outcomes Research Institute (PCORI). One such funding mechanism through PCORI is for comparative effectiveness research (CER). The goal is to compare the effectiveness of two or more interventions or approaches to health care by answering questions important to patients and other stakeholders. We have been successful in obtaining one of these awards to conduct a comparative effectiveness study of drugs for management of pain in cryptogenic sensory polyneuropathy (CSPN) called Patient Assisted Intervention for Neuropathy: Comparison of Treatment in Real Life Situations (PAIN CONTRoLS). We are comparing four medications (nortriptyline, duloxetine, pregabalin, mexiletine) at 45 sites in the USA and Canada. We enrolled 402 patients. The study assessed both effectiveness in reducing pain and quits due to various factors and utilized a novel Bayesian adaptive design. The results showed nortriptyline and duloxetine were more likely to have pain relief and fewer quits than pregabalin and mexiletine. We utilized patients and their caregivers in developing the concept and preparing the grant application. PCORI created an award that provides infrastructure in which the PCORI grants can operate through. This infrastructure called (PCORnet, the National PatientCentered Clinical Research Network) goal is to foster a wide range of experimental and observational patient-centered studies. This network is broken down to Clinical Data Research Networks (CDRNs). University of Kansas Medical Center has a CDRN called the Greater Plains Collaborative (GPC). One of the first accomplishments by the GPC was to deploy the development of the Healthcare Enterprise Repository for Ontological Narration (HERON) system at KUMC. This is an i2B2 software program that allows use of electronic medical records to survey for potential research subjects. One of the first projects was to develop a survey and utilizing the sites EMR, identify patients and utilize this list to send out the survey. We took an existing functional status form (the Amyotrophic Lateral Sclerosis Functional Rating Scale- Revised (ALSFRS-R)) and asked patients what they wanted to change or what additional questions they wanted to see poised to them regarding their functional status. We then modified the form and then submitted a protocol to be able to send out this survey.

SS 11.3 / \#213

Topic: 12. Patient Issues

\section{THE PATIENT VOICE IN MYASTHENIA GRAVIS TRIALS- PCORI, CER, AND MORE ALPHABET SOUP}

\author{
Pushpa Narayanaswami \\ Neurology, Beth Israel Deaconess Medical Center/ \\ Harvard Medical School, Boston, MA, US
}

Background: The Patient Centered Outcomes Research Institute (PCORI) is a nonprofit, nongovernmental organization in Washington, DC, that funds patient-centered comparative effectiveness research (CER). CER is the generation and synthesis of evidence that compares the benefits and harms of two or more interventions or approaches to manage a condition or improve healthcare. The US Food and Drug Administration defines patient reported outcomes (PROs) as "any report of the status of a patient's health condition that comes directly from the patient, without interpretation by a clinician or anyone else". PROs assess outcomes that are important to patients; they cannot be developed without actively engaging patients with the condition of interest. PROMISE-MG is a PCORI funded prospective, observational, comparative effectiveness trial of immunosuppressant treatments for myasthenia gravis (MG). We describe our patient engagement process to select a patient centered primary outcome measure. Methods: Patients were actively engaged to identify a PRO by 1 . Questionnaire survey of 58 people with self-identified MG at the annual Myasthenia Gravis Foundation of America meeting. 2. An online, web-based focus group of 13 people with $\mathrm{MG}$, using the results of the survey to develop the focus group script. 3. Selection of the outcome measure. 4. Evaluation of the measure in 30 patients with MG for completeness and accuracy in describing their experience with MG. Results: 1: Patient 
survey: Staying out of hospital was very important to $96 \%$ of respondents, followed by living independently (91.5\%). Ability to ambulate normally, drive, groom themselves and normal speech, chewing and swallowing were very important to $>80 \%$ of respondents. Fewer/less frequent side effects, fewer tablets/ less frequent dosing, less frequent physician visits/ laboratory monitoring were very important to over a third of respondents. Symptoms with the most significant impact were limb weakness (64\%), bulbar and ocular symptoms (36-38\%). The most burdensome adverse effects of treatment were weight gain (43\%), mood swings (36\%), insomnia (34\%) and diarrhea (30\%). 2. Focus group: the word "fatigue" was most frequently associated with MG, followed by "weakness" and "double vision". Participants endorsed frustration and isolation. Mobility limitations, visual symptoms and respiratory symptoms were most troublesome. Avoiding hospitalization was a high priority. The most concerning adverse effects were weight gain and long term risk of cancer. A good outcome was defined as "fewer medication side effects and $80 \%$ improvement in symptoms". 3: Based on the survey and focus group results, the MG-Quality of Life 15r scale (MGQOL15r) was thought to capture outcomes of greatest importance to patients. 4. 29/30 patients to whom the MG-QOL15r was administered responded that it completely and accurately described their experience with MG. Conclusion: MG-QOL is a 60 item scale which was developed with input from patients. The MGQOL-15 was subsequently developed with rigorous psychometric methods. Rasch analysis resulted in the MG-QOL15r, a three option modification, recently validated in a large cohort. We confirmed that the MG-QOL15r accurately and completely reflects the patient voice and selected it as the primary PRO for PROMISE-MG. Acknowledgements: Donald Sanders, MD, Co-Primary Investigator, PROMISE-MG; Myasthenia Gravis Foundation of America
SS 11.4 / \#308

Topic: 12. Patient Issues

\section{HOW CAN OUTCOMES OF CLINICAL STUDIES BE TRANSLATED FOR PATIENTS}

\author{
Angela Genge \\ Montreal Neurological Institute and Hospital, \\ Montreal, $C A$
}

Abstract not received.

\section{SS $12.1 / \# 127$}

Topic: 02. Neuropathy

\section{PATHOPHYSIOLOGY OF THE NODE OF RANVIER}

\author{
Antonino Uncini \\ Neuroscience, Imaging And Clinical Sciences, \\ University “G. d'Annunzio”, Chieti-Pecsra, Chieti, \\ IT
}

Background: The myelinated axons are organised in distinct domains characterised by specific molecular arrangements: nodes of Ranvier, paranodes, juxtaparanodes and internodes. The nodal region is a crucial evolutionary structure of the nervous system ensuring, by saltatory conduction, a fast transmission of impulses with the least expenditure of energy. It took almost eight decades, since the original description by Ranvier in 1871, to demonstrate that the nodes are the places where ionic currents generated action potentials for saltatory conduction, and only in the last two decades, the nodal region has been recognised as a possible site of specific autoimmune attack in peripheral neuropathies. For these neuropathies, the classification in demyelinating and axonal may be inadequate or even misleading, and the new category of nodo-paranodopathy has been recently proposed. Methods: In this lecture the process of saltatory conduction in myelinated and demyelinated axons will be firstly reviewed. Then the findings from serial electrophysiological studies, immunopathologic and ultrastructural evidence in humans, experimental models of autoimmune attack at the nodal region by antibodies against gangliosides, neurofascin 186, axoglial proteins (neurofascin 155, contactin 1, and contactin associated protein 1) will 
be examined. Results: The electrophysiological correlates of autoimmune attack at the excitable nodal axolemma and adhesion molecules at nodal and paranodal region ranging by a continuum from transitory nerve conduction failure to axonal degeneration, and the "demyelinating" features, explainable just by the paranodal involvement, will be discussed. Conclusion: At last the utility of the autoimmune nodo-paranodopathy category will be re-emphasized as the term pointing to the site of nerve injury, reminds specific pathophysiological mechanisms, reconciles apparently contrasting electrophysiological and pathological findings, and avoids misdiagnosis and taxonomic confusion.

\section{SS 12.2 / \#172}

Topic: 02. Neuropathy

\section{NEW PATHOGENETIC MECHANISMS UNDERLYING IMMUNE NEUROPATHIES}

\author{
Hugh J. Willison \\ Institute Of Infection, Immunity And Inflammation, \\ University of Glasgow, Glasgow, GB
}

Background: Guillain-Barré syndrome (GBS) is in part mediated by anti-GM1 ganglioside antibodies induced by preceding infections. Anti-GM1 antibodies target plasma membrane GM1 that is extensively distributed in both glial and axonal membranes, particularly at the node of Ranvier. Antibodies deposited at this site in models of GBS are associated with complement deposition, conduction block, structural disruption of ion channels and macrophage infiltration. The wide distribution of the GM1 ganglioside target leads to unwanted complexity in ascribing pathological outcomes to injury of cell-specific membranes, in particular unravelling the consequence of paranodal Schwann cell membrane injury on axonal function, and vice versa. Methods: To overcome this impasse, we have generated transgenic mice through glycosyltransferase manipulation that express GM1 exclusively in either neurons or glia, thus allowing us to very specifically target and injure axonal or glial membranes with a single antiGM1 ganglioside antibody. Through this route we can create mouse models of both the axonal and demyelinating forms of GBS, induced by a single antiGM1 antibody, thus creating otherwise highly comparable conditions. Results: Here, we show
anti-GM1 antibody binding is restricted to the nodal axolemma in GalNAcT ${ }^{-/}-\operatorname{Tg}$ (neuronal) mice and conversely to paranodal loops in GalNAcT $\mathrm{T}^{-/}-\mathrm{Tg}$ (glial) mice. When anti-GM1 antibody and a source of complement is added to a nerve-muscle ex vivo injury paradigm, there is a loss of axonal integrity (i.e. loss of neurofilament immunolabeling) when the neuronal membrane is targeted in GalNAcT ${ }^{-1-}-T g$ (neuronal). Conversely, axonal integrity is maintained when the paranodal membranes are decorated by antibody and complemnt products ex vivo in GalNAcT ${ }^{-/}-\mathrm{Tg}$ (glial) mice. In an passive immunisation model in vivo, Gal$\mathrm{NAcT}^{-/}-\mathrm{Tg}$ (neuronal) mice acutely develop weakness, respiratory dysfunction, associated complement deposition, and degenerative pathology in distal axons. In contrast, GalNAcT ${ }^{-/}-\mathrm{Tg}$ (glial) mice have significantly fewer abnormalities under the same acute conditions. Conclusion: These data indicate the high vulnerability of axonal membranes to acute injury and underline the importance of developing specific axonal projection strategies. In summary, targeting the nodal axolemmal or glial membranes allows us to study associated nodal pathology, and determine the downstream consequences on function and axon fate, currently a major area in GBS clinical research.

SS 12.3 / \#137

Topic: 02. Neuropathy

\section{ANTIBODIES TO THE NODE OF RANVIER IN CIDP: A NEW CLUE TO THERAPY}

$\underline{\text { Luis Querol }}$

Neuromuscular Diseases Unit, Hospital de la Santa Creu i Sant Pau, Barcelona, ES

Abstract not received.

SS 13.1 / \#216

Topic: 03. Motor Neurone

\section{OVERVIEW - DRUG TREATMENT FOR SMA}

Janbernd Kirschner

Dept. Of Neuropediatrics And Muscle Disorders, Medical Center - University of Freiburg,

Freiburg, DE 
Background: Spinal muscular atrophy (SMA) is an autosomal-recessive, neuromuscular disorder that is characterized by degeneration of the anterior horn cells of the spinal cord, resulting in muscle atrophy and proximal muscle weakness. SMA is caused by a homozygous deletion in the survival motor neuron 1 (SMN1) gene on chromosome 5q13. The SMN gene region also comprises a centromeric copy containing the SMN2 gene. The severity of the disease correlates with age of onset and SMN2 copy number and varies from a severe muscle weakness with tetraplegia in infants to a mild proximal muscle weakness in ambulant children and adults. Affected patients need a multidisciplinary treatment approach to address the different manifestation of the disease. In addition to symptomatic treatment including nutrition and ventilatory suuport new pharmaceutical approaches have been developed to address different aspects of the pathophysiology. Methods: Targets of the most advanced approaches include survival of motor neurons, increase of SMN protein production from the SMN2 gene by splicing modifiers, and introduction of the missing SMN1 gene with viral vectors. Results: Currently, nusinersen is the only drug that has been approved for the treatment of SMA. It is an antisense oligonucleotide that is administered by intrathecal injections and modifies the splicing of the SMN2 gene. Phase III trials have shown shown clear improvement of motor function in infants with SMA type 1 and in young children with SMA type 2. Long term safety and treatment effect in adolescent and adult still need further evaluation. Other splicing modifiers have been indentified through screening methods, they can be administered orally and are currently evaluated in clinical trials. A gene therapy approach using a AAV9 virus with the SMN1 gene has shown promising results in a phase I study and a larger European phase III trial for SMA tpye 1 will start this year. Olesoxime is a drug that has been developed to improve survival of motor neurons, but results of an international placebo-controlled trial were not sufficient for approval. Additional explorations of the pharmacokinetics and its effect on functional outcomes are ongoing. Conclusion: Clinical trials show promising results for the treatment of SMA with splicing modifiers and gene therapy. Early initiation of treatment seems particularly effective and warrants the implementation of newborn screening programs. As SMA is a rare disease with a broad spectrum of severity clinical trials cannot cover all age groups and all stages of the disease. Patient registries are an important tool to collect additional in- formation to document the long-term effect of any drug treatment in SMA.

SS 13.2 / \#98

Topic: 03. Motor Neurone

\section{TREATMENTS TO IMPROVE SURVIVAL OF MOTOR NEURONS}

\author{
Albert Ludolph \\ Universitätsklinikum Ulm, Ulm, DE
}

Abstract not received.

SS 13.3 / \#73

Topic: 03. Motor Neurone

\section{SPLICING MODIFICATION FOR SMA}

Nathalie Goemans

Treat-NMD Neuromuscular Network, Leuven, BE

Abstract not received.

SS 13.4 / \#48

Topic: 03. Motor Neurone

\section{GENE THERAPY FOR SMA}

Arthur H.m. Burghes ${ }^{1}$, Jerry Mendell ${ }^{2}$, Samiah Al-Zaidy ${ }^{2}$, Richard Shell ${ }^{3}$, W. D. Arnold ${ }^{4}$, Louise Rodino-Klapac ${ }^{2}$, Thomas W. Prior ${ }^{5}$, Linda Lowes ${ }^{2}$, Lindsay Alfano ${ }^{3}$, Kathleen Church ${ }^{3}$, John T. Kissel ${ }^{4}$, Sukumar Nagendran ${ }^{6}$, James L'Italien ${ }^{6}$, Douglas M. Sproule $^{6}$, Courtney Wells ${ }^{6}$, Kevin Foust ${ }^{6}$, Kathrin Meyer $^{2}$, Shibi Likhite' ${ }^{2}$, Vicki Mcgovern ${ }^{1}$, Stephen Kolb $^{4}$, Brian Kaspar ${ }^{6}$

${ }^{1}$ Biological Chemistry And Pharmacology, The Ohio State University, Columbus, OH, US: ${ }^{2}$ Center For Gene Therapy, Nationwide Children's Hospital, Columbus, OH, US, ${ }^{3}$ Department Of Pediatrics, Nationwide Children's Hospital, Columbus, $\mathrm{OH}$, US: ${ }^{4}$ Department Of Neurology, The Ohio State University, Columbus, OH, US, $;^{5}$ Department Of Pathology, The Ohio State University, Columbus, OH, US; ${ }^{\circ}$ AveXis, Inc., Bannockburn, IL, US 
Abstract: Spinal Muscular Atrophy (SMA) is an autosomal recessive disorder caused by mutation or loss of the survival motor neuron 1 gene (SMN1) gene and retention of SMN2. The SMN2 gene produces insufficient SMN but increased copy number of SMN2 modulates phenotype and does result in immune tolerance to an immune response against SMN when it is introduced from exogenous sources. Spinal muscular atrophy has been modeled in both mice and pigs. Untreated SMA mice live for 14 days and show loss of motor neurons including electrophysiological defects of the neuromuscular junction and compound muscle action abnormalities (CMAP) and MUNE. Knockdown of SMN in pig results in similar motor neuron abnormalities. scAAV9 has the ability to cross the blood brain barrier and efficiently transduce motor neurons with either delivery through the blood stream or via CSF delivery. Early scAAV9-SMN treatment of SMA mice and pigs results in correction of survival and motor neuron defects including CMAP and MUNE. Latter introduction especially in the pig results in correction of CMAP but MUNE is reduced indicating that motor neurons once lost cannot be recovered but those remaining can compensate by sprouting. A phase 1 gene therapy trial of scAAV9-SMN (AVXS101) was initiated in Type 1 SMA, the most severe form of SMA, with untreated children never gaining the ability to sit or maintain head control. All patients lacked SMN1 and had 2 copies of SMN2 and none contained the $\mathrm{C} 859 \mathrm{G}$ variant which increases incorporation of SMN2 exon 7. Patients received an intravenous dose of AVXS-101 at the low dose (Cohort $1, n=3$ ) or proposed therapeutic dose (Cohort 2, $\mathrm{n}=12$ ). AVXS-101 had a favorable safety profile and improved survival when compared to natural history studies. All 15 patients were alive and event-free at 20 months of age and did not require permanent mechanical ventilation, compared with $8 \%$ in published natural history. Patients in Cohort 2 demonstrated improvements in motor function: $11 / 12$ had achieved CHOP-INTEND scores $>40$ points and a mean increase of 24.6 points from a mean baseline of 28.2 points; $11 / 12$ were able to sit unassisted for at least 5 seconds, 10 for at least 10 seconds, and 9 for at least 30 seconds; $11 / 12$ achieved head control and 9 could roll over. Two patients were able to crawl, pull to stand, stand independently, and walk independently. None of the aforementioned motor function gains and milestone achievement is seen in untreated patients with SMA type 1 and 2 copies of SMN2. Asymptomatic elevated serum aminotransferase levels occurred in 4 patients and was attenuated by prednisolone treatment. (see Mendell et al this meeting for further details.) As in preclinical studies in animal models scAAV9-SMN (AVXS-101) has a dramatic impact on motor function and appeared to increase survival. This was particularly evident with early treatment in cohort 2 with achievement of motor milestones rarely or never seen in the type 1 SMA population.

\section{SS 14.1 / \#90}

Topic: 06. Mononeuropathy

\section{MR NEUROGRAPHY: NEW APPROACHES AND TECHNIQUES FOR DIAGNOSING MONONEUROPATHIES}

Jennifer Kollmer
Heidelberg University Hospital, Heidelberg, DE

Abstract not received.

SS 14.2 / \#189

Topic: 06. Mononeuropathy

\section{NEW ULTRASENSITIVE ULTRASOUND METHODS}

Stefan Meng

KFJ Hospital, Vienna, AT

Abstract not received.

SS 14.3 / \#63

Topic: 06. Mononeuropathy

\section{OCT}

Wolfgang Drexler

Medical University of Vienna, Vienna, AT

Abstract not received. 
SS $15.1 / \# 150$

Topic: 12. Patient Issues

\section{CHANGES IN THE NODAL COMPLEX IN PAINFUL NEUROPATHY}

\author{
Claudia Sommer \\ Neurologische Klinik Und Poliklinik, \\ Universitätsklinikum Würzburg, Würzburg, DE
}

Background: Autoantibodies to components of the paranodal protein complex have recently been described and are considered to characterize a subset of immune neuropathies that differs from other subtypes in clinical phenotype pathogenesis and response to therapy. Methods: We systematically screened sera of our patients with suspected immune neuropathies for the presence of autoantibodies to paranodal proteins. We analyzed skin and nerve biopsies for changes in the nodal/paranodal complex. Results: Pain was one of the predominant findings in two patients with anti-caspr antibodies, possibly reflected by binding of patients' IgG to transient receptor potential vanilloid 1 immunoreactive dorsal root ganglia neurons. Severe disruption of the paranodal and nodal architecture was detectable in teased fibres of the sural nerve biopsy and in dermal myelinated fibres, supporting the notion of the paranodes being the site of pathology. Nodal/paranodal architecture was disrupted in patients with anticontactin-1 antibodies, but pain was not a prominent feature in these patients. Conclusion: In contrast to other neuropathies with antibodies to the paranodal complex (anti-contactin-1, anti-neurofascin-155) two patients with auto-antibodies against caspr suffered from severe neuropathic pain. The mechanisms underlying this pain need to be uncovered.

\section{SS 15.2 / \#86}

Topic: 12. Patient Issues

\section{THE USE OF NERVE EXCITABILITY TESTING IN UNDERSTANDING ION CHANNEL DYSFUNCTION IN NEUROPATHY}

\author{
Matthew C. Kiernan \\ Brain \& Mind Centre, University of Sydney, \\ Sydney, $A U$
}

Abstract: Measurement of nerve excitability by threshold tracking provides complementary information to conventional nerve conduction studies and may be used to infer the activity of a variety of ion channels, energy-dependent pumps and ion exchange processes activated during the process of impulse conduction. While routine nerve conduction studies can document the presence of a neuropathy, they do not provide further insight into pathophysiology. This may be even more relevant across the range of neuropathic syndromes, where conventional nerve conduction studies are often normal. Following the validation of excitability testing protocols, clinical excitability techniques are being adopted to complement diagnostic nerve conduction studies. A number of recent clinical studies will be highlighted which have established mechanisms for neuropathy associated with systemic disease, likely to impact on the future management of patients, both acutely and chronically; provided insight into the mechanisms of painful neuropathies due to neurotoxicity associated with chemotherapy; determined mechanisms of hyperexcitability, further contributing to symptoms of neuropathy. While clinical nerve excitability studies are still in their relative infancy, and it is too early to know whether they have diagnostic value, there is growing evidence of their utility to provide novel insights into the pathophysiological mechanisms involved in a variety of neuropathic disorders.

\section{SS 15.3 / \#43}

Topic: 12. Patient Issues

\section{HUMAN PAIN CHANNELOPATHIES}

David Bennett

Nuffield Department Of Clinical Neuroscience, University of Oxford / Nuffield, Oxford, US

Abstract: There has been significant progress over the last decade in understanding the ion channels required by sensory neurons to transmit noxious stimuli. Over this same period we have recognized that mutations in such ion channels can result in primary neuropathic pain disorders. An excellent example is the voltage gated ion channel $\mathrm{Na}_{\mathrm{v}} 1.7$ encoded by the gene SCN9a. Loss of function mutations in this ion channel result in congenital inability to experience pain and gain of function mutations can cause a number of distinct neuropathic pain disorders including erythromelalgia, paroxysmal extreme pain 
disorder and small fibre neuropathy. There is a correlation between the impact of mutations on the biophysical properties of the ion channel and the severity of the clinical phenotype. The fact that mutations in such channels can cause monogenic pain disorders makes them attractive analgesic drug targets. Secondary neuropathic pain disorders such as traumatic neuropathy or painful diabetic neuropathy are also associated with altered expression and function of multiple ion channels which contribute to hyperexcitability. Finally we are now beginning to recognize that auto-antibodies to ion channels and proteins with which they complex can cause acquired pain channelopathies. We are trying to improve the stratification of neuropathic pain patients by linking the clinical phenotype with genotype with the ultimate aim of optimizing analgesic drug selection on an individualized basis.

\section{SS $16.1 / \# 138$}

Topic: 03. Motor Neurone

\section{NIV AND ALS}

Marianne De Visser

Department Of Neurology, Academic Medical

Centre, Amsterdam, NL

Abstract not received.

\section{SS 16.2 / \#109}

Topic: 03. Motor Neurone

\section{NUTRITION AND ALS}

\section{Esther Hobson}

University Of Sheffield, Sheffield Institute for

Translational Neurosciences, $H Q, G B$

Background: Malnutrition and weight loss are well recognised poor prognostic factors in amyotrophic lateral sclerosis. Body mass index (BMI) is an independent predictor of survival in ALS, with mild obesity having a protective effect and lower BMI associated with a worse prognosis. In patients with weight loss of $5 \%$ or more at diagnosis there is a twofold increase in risk of death, compared to those who have lost less than $5 \%$ of their usual pre-morbid weight. Poor nutrition in ALS is not simply as a result of bulbar dysfunction. Fatigue and poor upper limb dysfunction make feeding difficult whilst respiratory failure cause loss of appetite. As a consequence, eating is no longer a pleasurable and social activity, instead it becomes a burden on the patient and caregiver. The energy deficit caused by inadequate intake is compounded by observed hypermetabolism with resting energy expenditure observed to be, on average, $20 \%$ higher than healthy individuals. Patients with hypermetabolism have a greater level of lower motor neuron involvement, faster rate of functional decline and shorter survival compared with normometabolic patients with ALS. Methods: It is suggested that metabolic defects and the energy deficit are not simply a consequence of denervation and advanced disease but are an early feature of ALS pathology. They could therefore be potentially modifiable using dietary interventions. Patients themselves have not waited for evidence with more than half using some dietary supplementation. The consequences of these untested diets remains unclear but thereis some limited evidence that correction of the energy deficit with a high calorie diet extends life in an ALS mouse model.Moreover, a small pilot study of ALS patients indicated a potential survival advantage and tolerability of a high calorie diet. Future research will investigate the potential of calorie manipulation, particularly in the early stages of the disease. Results: Most patients later in the disease course opt for placement of a gastrostomy tube. Despite gastrostomy use being common practice, in the absence of comparative trials it remains difficult to determine the impacts for patient survival and quality of life. However, prospective and observational studies can provide some information. There are a variety of methods available for insertion, each likely to be broadly similar in its insertion risks. Longer term consequences and risk of tube damage or displacement are related to the robustness of the tube. Gastrostomy insertion with traditional feeding techniques alone does not typically reverse weight loss particularly in those who have already lost over $10 \%$ of their premorbid bodyweight, in whom, along with those in respiratory failure, the 30 day-mortality is significantly greater. Conclusion: This leads to the conclusion that gastrostomy insertion should be offered early in the disease. However there is often resistance to accepting this procedure due to various psychosocial factors, some of which may be overcome by better information (such as the www. mytube.mymnd.org website). What remains unclear is what is the best nutritional regime post-gastrostomy insertion, and how to communicate the individual risk and benefits, particularly those with a poorer prognosis or those without bulbar disease. 
SS 16.3 / \#52

Topic: 03. Motor Neurone

\section{NUTRITION NIV AND COGNITION IN ALS}

Vincenzo Silani

University of Milan Medical School, Milan, IT

Abstract not received.

SS $17.1 / \# 187$

Topic: 06. Mononeuropathy

\section{NEUROFIBROMATOSIS}

Katharina Wimmer

Medical University of Innsbruck, Innsbruck, AT

Abstract not received.

SS 17.2 / \#175

Topic: 06. Mononeuropathy

\section{PERIPHERAL NERVE TUMORS}

Gelareh Zadeh

University Health Network, Toronto, CA

Abstract not received.

SS 17.3 / \#106

Topic: 06. Mononeuropathy

INTRANEURAL PERINEURIOMA: MAKING THE DIAGNOSIS AND CLUES TO THE PATHOGENESIS

$\underline{\text { Michelle Mauermann }}$

Neurology, Mayo Clinic, Rochester, MN, US

Background: Intraneural perineurioma is a benign peripheral nerve neoplasm composed of whorls of perineurial cells surrounding nerve fibers and restricted to the boundaries of a peripheral nerve. It was previously unclear if these were caused by a genetic abnormality or were related to prior trauma. Methods: Toreviewtheclinical,electrophysiological, radiological and pathological features of intraneural perineurioma and its associated natural history. We will discuss how to differentiate these tumors from other benign nerve tumors and inflammatory nerve lesions. The recent advances in the underlying genetic causes will be discussed. Results: Intraneural perineurioma presents insidiously in children and young adults with a motor-predominant mononeuropathy or plexopathy. The sciatic nerve is most commonly affected. The electrophysiological findings demonstrate a chronic axonal process. The radiologic hallmark is fusiform nerve enlargement with T2 hyperintensity and contrast enhancement. Targeted biopsy of tumor demonstrating the typical pathological features is the gold standard for diagnosis. Pathologic features include nerve hypertrophy due to pseudo-onion bulb formations consisting of whorls of perineurial cells surrounding nerve axons that react for epithelial membrane antigen. A recent study led to the discovery of frequent recurrent missense somatic mutations in TRAF7. Rare patients have large somatic macrodeletions and duplications. Conclusion: Intraneural perineurioma is a benign hypertrophic peripheral nerve tumor that presents in young people with a motor-predominant mononeuropathy or plexopathy. It is slowly progressive and causes mild disability. Underlying genetic mutations in TRAF7 suggest a shared pathogenesis with intracranial meningiomas.

SS 17.4 / \#1029

Topic: 06. Mononeuropathy

\section{CANCER AND PERIPHERAL NERVES}

Wolfgang Grisold $^{1}$, Anna Grisold ${ }^{2}$, Stefan Meng ${ }^{3}$, Elisabeth Lindeck-Pozza ${ }^{4}$

${ }^{1}$ Ludwig Boltzmann Institute for Experimental und Clinical Traumatology, Vienna, AT; ${ }^{2}$ Dep.

Neurology, Allgemeines Krankenhaus Vienna, Vienna, AT; ${ }^{3}$ Institute for Radiology, KFJ hospital, Vienna, AT; ${ }^{4}$ Dep. Neurology, KFJ hospital, Vienna, $A T$
Abstract: Peripheral nerve tumors are rare and are conventionally classified according to the WHO classification 2016, which adds several new entities as hybrid tumors, perineuroma and malignant 
peripheral nerve sheath tumors (MPNST). In addition to this classification also mesenchymal, vascular and tumor like conditions have to be considered. The neoplastic involvement of peripheral nerves occurs in cancer, lymphoma, leukemia and even more rarely in plasmocytoma. In all cancer patients, MPNSTs can be a side effect of radiation therapy (RT). In solid tumor nerve metastasis, or circular compression of individual nerves is rare, however in the peripheral parts of the cranial nerves infiltration and spread can be observed. Infiltration and compression can also be seen in nerve roots and the nerve plexus. Lymphoma and leukemia can present with isolated appearance or diffuse infiltration, at any time of the disease, also as a recurrence. Also solid manifestations in leukemia termed chloroma are rarely reported. Tumorous infiltration of peripheral nerve by plasmocytoma is are, and also local amyloidomas have been described. For the different tumor entities also different morphological changes and mechanisms are described. Therapy depends on the tumor type and in addition to surgical and RT also chemotherapy or biological therapies depending on the tumor type are used. As in MPNST the prognosis is usually poor.

\section{SS 18.1 / \#85}

Topic: 03. Motor Neurone

\section{MND: THERAPEUTIC LANDSCAPES}

Matthew C. Kiernan

Brain \& Mind Centre, University of Sydney, Sydney, $A U$

Abstract: Recent evidence has emerged concerning unique pathophysiological processes linked to the development of motor neurone disease (MND), including glutamate-mediated excitotoxicity, which has resulted in development of novel diagnostic investigations and uncovered potential therapeutic targets. Advances in genetics, including the recently discovered $\mathrm{C} 9$ orf 72 gene, have radically changed the pathological mind-set, such that ALS forms part of a continuum with other primary neurodegenerative disorders, including frontotemporal dementia. At the simplest level, the management of MND remains focused on symptom control. Evidence-based management guidelines advise a multi-disciplinary model of care, led by a neurologist and clinical nurse consultant working together with physical therapists, occupational therapists, speech pathologists, respiratory physicians, gastroenterologists, psychologists and social workers to guide patient management, and such an approach has profoundly impacted patient quality of life and survival. In terms of emerging therapies, there have been several recent phase II/III trials that have recently shown promising results. Data concerning masitinib reported significant improvement from baseline to week 48 in the Revised ALS Functional Rating Scale scores (primary endpoint) and FVC (secondary endpoint). This new oral tyrosine kinase inhibitor regulates abnormal neuroglia activation and proliferation, processes linked to motor neurone death. A clinical trial of ultrahigh-dose methylcobalamin (E0302) significantly prolonged survival in a dose-dependent manner in MND patients who were treated within lyear of disease onset. Edaravone (3-methyl-1-phenyl2-pyrazolin-5-one), a potent free radical scavenger, is known to prevent neuronal damage inhibiting endothelial injury and is currently marketed for use after ischaemic stroke. Benefit from edavarone was established for a subgroup of MND patients, specifically younger patients with an FVC at least $80 \%$, and with recent onset of symptoms. Other potential treatment targets awaiting confirmation with human clinical trials include CNS copper treatment and anti-human endogenous retrovirus K (HERV K) treatment. Stem cell therapy remains a theoretical possibility: previous attempts to grow anterior horn cells have been abandoned. Now the overall goal is prevention of further neuronal loss by supporting residual motor neurones. The optimal route of administration and specific type of stem cells needed, remain unanswered questions that are currently being considered through several phase II studies.

\section{SS 18.2 / \#114}

Topic: 03. Motor Neurone

\section{TREATMENT STRATEGIES FOR HEREDITARY ALS}

Timothy Miller

Washington University, St. Louis, US

Background: Recent discoveries have defined the genetic cause in more than $60 \%$ of hereditary ALS. Along with this increased genetic discovery has 
emerged increased tools to specifically target genes and pathways that cause ALS. Methods: These therapeutic strategies include viral delivered siRNA, antisense oligonucleotides, antibodies, and small molecules. Results: Novel treatment strategies for hereditary ALS will be discussed with a particular focus on antisense oligonucleotides. Conclusion: Treatment strategies for hereditary ALS have tremendous potential to positively impact ALS.

\section{SS 18.3 / \#131}

Topic: 03. Motor Neurone

\section{DIAGNOSIS AND TREATMENT OF MOTOR NEUROPATHIES}

\author{
Alan Pestronk \\ Department Of Neurology, Washington University \\ School of Medicine, St. Louis, MO, US
}

Abstract not received.

\section{SS $19.1 / \# 139$}

Topic: 03. Motor Neurone

\section{DISTAL HEREDITARY MOTOR NEUROPATHIES (DHMN)}

\section{Mary Reilly}

Queen Square, Institue Of Neurology, London, GB

\begin{abstract}
The distal hereditary motor neuropathies (HMN) are a clinically and genetically heterogeneous group of slowly progressive neuropathies characterised by a distal predominant almost exclusive motor syndrome. There is overlap with the axonal forms of Charcot Marie Tooth disease (CMT2) such that causative genes are often described as causing both HMN and CMT2 (e.g. HSPB1 and HSPB8). The differentiation of these motor predominant syndromes into HMN and CMT2 is artificial as these conditions are motor predominant but especially with time there can be minimal sensory involvement. It may be more appropriate to use the term CMT to include HMN, CMT and the sensory predominant form, HSN, reflecting the spectrum of clinical disease from pure motor or motor predominant (HMN) through motor and sensory (CMT) to pure sensory or sensory predominant (HSN). As
\end{abstract}

multiple causative genes are identified for HMN, it is clear that there is also overlap with other inherited neurological conditions with some genes causing both HMN and hereditary spastic paraparesis (HSP) (e.g. REEP1) and others such as the mitochondrial gene MT-ATP 6 causing a spectrum of neurological conditions including a motor predominant CMT2 like phenotype. Although the traditional definition of distal HMN is a length dependent motor neuropathy starting in the lower limbs many clinical variations are now recognised. Many forms of distal HMN do not present with the typical weak ankle dorsiflexion with relatively preserved ankle plantarflexion reflecting the anterior lower limb muscle involvement seen with CMT and instead present with equal involvement of anterior and posterior calf muscle resulting in equal ankle dorsiflexion and plantarflexion weakness (e.g. this is seen with HSPB1 and HSPB8 mutations). We also now recognise with these same two genes (HSPB1 and HSPB8) that a myopathy can be present as well as a neuropathy. Other genes cause a non length dependent lower limb predominant motor neuropathy (e.g. with Dynein and BICD2). A number of genes (BSCL2, GARS, REEP1) cause a motor syndrome which is much more pronounced in the upper limbs and can involve the upper motor neurone fibers in some cases (BSCL2 can cause Silver syndrome which is characterised by amyotrophy of the hands with spasticity of the lower limbs). The involvement of upper and lower motor neurone fibres is important as it underlies that the pathogenesis of both upper motor neurone motor syndromes (e.g. HSP) and lower motor neurone syndromes (e.g. HMN) is likely to involve common pathways like axonal transport or protein misfolding disorders with the possibility of developing pathway therapies for all axonopathies rather than therapies for each individual genetic condition. This talk will focus on the evolving genotypes and phenotypes and the progress towards therapy development.

\section{SS 19.2 / \#32}

Topic: 03. Motor Neurone

\section{DISTAL MYOPATHIES}

Anthony Amato

Dep. Of Neurology, Brigham and Women's

Hospital, Boston, US 


\begin{abstract}
The distal myopathies are characterized clinically by progressive atrophy and weakness of distal arm or leg muscles and histologically by nonspecific myopathic features on muscle biopsy. The distal myopathies can be subdivided, based on the clinical features, age of onset, CK levels, muscle histology, and mode of inheritance. Myopathic causes of distal weakness are distal muscular dystrophies, of which we would include the myofibrillar myopathies and various, hereditary inclusion body myopathies, congenital myopathies, glycogen and lipid storage myopathies, and acquired forms such as seen in granulomatous myopathy.
\end{abstract}

\section{SS 19.3 / \#84}

Topic: 03. Motor Neurone

\section{ACQUIRED DISTAL MOTOR SYNDROMES}

\author{
Matthew C. Kiernan \\ Brain \& Mind Centre, University of Sydney, \\ Sydney, $A U$
}

Abstract: Distal or lower motor neuron syndromes are characterised by muscle atrophy, weakness and hyporeflexia without sensory involvement. These disorders may arise from disease processes affecting the anterior horn cell, the motor axon, or its surrounding myelin. Neuromuscular junction pathology and muscle disorders may mimic these disorders and form part of the differential diagnosis. Distal motor syndromes can be broadly classified as hereditary, sporadic or immune-mediated. Immunemediated neuropathies, such as multifocal motor neuropathy and chronic inflammatory demyelinating polyneuropathy are important to distinguish from sporadic and hereditary forms, as effective treatments are available. Lower motor neuronal presentations of motor neuron disease (MND) are most often sporadic, but several genetic mutations have been described. Hereditary forms include distal hereditary motor neuropathies and spinal muscular atrophy (SMA). With the latter, onset typically occurs during childhood or teenage years, although adult onset is not uncommon. The increasing availability of next-generation sequencing, including the ability for multiple genes to be sequenced in parallel, has resulted in an increase in the discovery of novel genetic mutations. The clinical evaluation of a patient presenting with a distal motor syndrome includes a directed assessment of disease onset and progression. This is particularly important to ascertain, as a rapid rate of decline may suggest a diagnosis of MND and remains an important factor in distinguishing MND from other relatively indolent conditions, such as SMA and immune neuropathies. The pattern of weakness may also suggest a specific diagnosis, with considerations including (1) symmetry versus asymmetry, (2) proximal versus distal involvement, (3) upper versus lower limb predominance and (4) presence versus absence of bulbar involvement. Nerve conduction studies and electromyography are essential to confirm that the disorder is neurogenic and should focus on assessing the pattern of involvement, including symmetry and length dependence; the presence of focal motor conduction block or demyelinating features; and the presence or absence of subclinical sensory abnormalities. Neuromuscular imaging, genetic testing, assessment of antibody markers and advanced neurophysiological techniques are all useful adjuncts to determine a specific diagnosis and the best therapeutic approach.

SS 20.1 / \#49

Topic: 04. Neuromuscular Junction

EVOLUTION OF MONOCLONAL AB TREATMENT APPROACHES IN MG

Ted M. Burns

Department Of Neurology, University of Virginia, Charlottesville, US

Abstract not received.

SS 20.2 / \#136

Topic: 04. Neuromuscular Junction

\section{OTHER NOVEL TREATMENT APPROACHES: EXERCISE}

Anna R. Punga

Neuroscience, Uppsala University, Uppsala, SE

Background: The benefits of physical exercise for healthy individuals are well established, in particular to reduce the risks of life-style disorders. Furthermore, physical exercise provides beneficial effects 
in several chronic diseases such as rheumatoid arthritis, chronic obstructive pulmonary disease and multiple sclerosis, and is therefore recommended as part of the treatment regimen. It is reasonable to assume that MG patients achieve at least similar benefits from physical exercise, as do healthy adults. Yet, exercise-related research in the field of MG is sparse and does not provide any guidelines on how MG patients should perform physical exercise to obtain desirable benefits without the risk of causing disease deterioration or more pronounced muscle fatigue. Two recent pilot studies reported that MG patients with mild disease activity could adhere safely to general exercise recommendations including resistance and aerobic training regimen without subjective or objective disease deterioration. Overall, though, there is an obvious lack of literature in the field of physical exercise and MG that hampers the possibility to individualize training programs that could be of particular importance to avoid development of secondary disease conditions. Methods: Two studies were performed: 1) To objectively evaluate functional skeletal muscle parameters in MG patients who conducted a 12-week supervised physical therapy program, focusing on aerobic and high-resistance strength training. 2) A follow-up study on patients for 6 months with an individually tailored program from a personal trainer. The primary hypothesis was that a physical exercise program based on general exercise recommendations improves muscle status in MG patients. The secondary hypothesis was that moderate aerobic and highresistance strength training improves the cardiovascular risk profile and self-assessed-wellbeing of MG patients. Both studies were prospective and unblinded, where each patient served as his/her own control. Outcome measures included clinical MG status, isometric muscle force, neurophysiological evaluations, neuromuscular ultrasound, physical performance-based measures, quality of life, fatigue severity and blood sample analysis. Wilcoxon signed-rank test was used to compare non-parametric data before and after training in each patient. A P-value $<0.05$ was considered significant. Results: After the training program, functional muscle parameters (neurophysiological parameters, isometric muscle force and ultrasound muscle thickness) improved in the proximal leg muscles. Further, physical performance-based measures improved as well as the clinical MG composite score. The longer follow-up study also indicated positive adherence to individualized programs and additional beneficial effects. Conclusion: These findings indicate that MG patients can indeed improve their functional muscle status as a result of aerobic and high-resistance strength training, especially in proximal leg muscles. This is important knowledge in order to establish both collective as well as individualized guidelines of physical exercise for MG patients.

\section{SS 20.3 / \#174}

Topic: 04. Neuromuscular Junction

\section{OVERVIEW OF TREATMENT GUIDELINES: GOING FORWARD}

Gil I. Wolfe

Neurology, University at Buffalo Jacobs School of

Medicine and Biomedical Sciences, Buffalo, NY, US

Background: The rarity of myasthenia gravis (MG) makes the planning and completion of randomized controlled trials (RCTs) logistically difficult with many trials failing to reach their recruitment goals. Due to restricted entry criteria that are often an essential element of well-designed RCTs, results from these studies may also have limited generalizability to the overall patient population. Furthermore, the majority of trials do not address the comparative effectiveness of different treatment modalities in a disease as heterogeneous as MG. As a result, most current treatments for MG are based on uncontrolled studies, with a high risk of bias. Recommendations based on a formalized consensus opinion of experts can help to guide treatment in such situations. Methods: To review treatment recommendation statements that have been produced recently by neuromuscular neurology experts in several European and Asian countries and to describe the process and results generated by an international panel that focused on several treatment domains related to MG. Plans for future revisions to the statements will also be presented. Results: Since 2012, treatment recommendations for MG have been established and published by several countries, beginning with Germany and including England, Japan and China. Of these national statements, only the Chinese employed a formal consensus process, using the RAND/UCLA appropriateness method (RAM). In October 2013, the Myasthenia Gravis Foundation of America (MGFA) appointed a Task Force to develop treatment guidance for $\mathrm{MG}$, and a 
panel of 15 international experts was convened. The panel also included a facilitator with extensive experience in consensus methodology and an observer representing patient interests from the MGFA. RAM methodology was used to develop consensus guidance statements. To frame the treatment guidance the following definitions were established by the panel: goals of treatment, minimal manifestations, remission, ocular $\mathrm{MG}$, impending crisis, manifest crisis, and refractory MG. An inperson meeting of the panel then determined 7 treatment topics to be addressed. Initial guidance statements were developed based on summaries of the literature for each topic. Three rounds of anonymous e-mail votes with modifications of the guidance statements, based on panel input were used to reach consensus. Guidance statements were developed for the following MG treatment domains: symptomatic and immunosuppressive treatments, intravenous immunoglobulin and plasma exchange, management of impending and manifest myasthenic crisis, thymectomy, juvenile $\mathrm{MG}, \mathrm{MG}$ associated with antibodies to muscle specific tyrosine kinase and MG in pregnancy. Conclusion: Both national and international treatment recommendations have helped define best practices in the management of MG. Although there are common threads across the various recommendations, differences do exist that reflect national practice and the availability or approval of treatment modalities. The formal international consensus can serve as a guide for clinicians who wish to optimize function and quality of life for their MG patients. It may be of particular benefit to those clinicians who practice in countries that do not have the resources to generate a national treatment statement. The international guidance is a living document that will require revision every few years to reflect developments in the field.

\section{SS 21.1 / \#67}

Topic: 09. Systemic Diseases

\section{NEUROLOGICAL COMPLICATIONS OF ZIKA VIRUS}

John D. England LSUHSC School of Medicine, New Orleans, US

Abstract: Zika virus (ZIV) is a mosquito-borne single-stranded RNA virus in the Flaviviridae family. On 1 February 2016 the World Health Organization
(WHO) declared ZIV infection a Public Health Emergency of International Concern (PHEIC) due to reports from Brazil of a cluster of microcephaly cases and other neurologic disorders termed Congenital Zika Syndrome as well as clusters of Guillain-Barre Syndrome (GBS) from French Polynesia and South America. Since that time an expanding spectrum of neurological complications of Zika virus infection have been described. ZIV is now recognized as a new neuropathological agent associated with a wide variety of neurological complications. These neurological sequelae of ZIV infection represent both direct effects of virus invasion and autoimmune responses to the virus. The neurological complications of ZIV include the following: 1) Congenital Zika Syndrome associated with infection of neural progenitor cells in the brain; 2) Guillain-Barre Syndrome (GBS), which is most likely caused by a secondary autoimmune response directed against components of peripheral nerve; 3) Sensory polyneuropathy, which may be associated with direct infection of peripheral neurons; 4) Encephalitis/meningoencephalitis and myelitis, which may be associated with direct viral infection/ inflammatory process in the central nervous system; 5) Acute Disseminated Encephalomyelitis; 6) Optic neuropathy; 7) Arterial Ischemic Stroke (AIS) in children due to probable vasculitis and possible direct ZIV infection of brain endothelial cells. Ongoing investigations are confirming these associations and exploring the pathogenesis of the complications of ZIV infection. Since there is no specific treatment for ZIV infection, public health measures are directed at prevention of primary infection.

\section{SS 21.2 / \#146}

Topic: 09. Systemic Diseases

\section{LEPROUS NEUROPATHIES}

Gerard Said

Institut Vernes, PARIS, FR

Abstract: Leprous neuropathy, which is due to infection of nerve cells by Mycobacterium leprae remains a health problem worldwide. In addition to being an infectious and potentially contagious disease, it induces irreversible damage to peripheral nerves, exposing patients to lifelong complications of sensory and motor denervation. The number of new cases reported globally in 2016 exceeds 200000 
with 135485 in India, 25218 in Brazil and 16826 in Indonesia. Nasal secretions and skin from untreated multibacillary leprosy (ML) cases can contaminate contacts. The incubation period may be as long as 20 years. Factors such as nutrition, hygiene and house crowding play an important role. M. leprae is an obligatory intracellular parasite with tropism for macrophages and Schwann cells. The bacillus attaches to Schwann cells via PGL-1. Once inside the Schwann cell, the mycobacterium will multiply very slowly, triggering a subacute to chronic inflammatory reaction, with replacement of functional parenchymal tissue by connective tissue and fibrosis. In the multibacillary type bacilli are easily found in the skin, and in nerve cells including Schwann cells, endothelial cells and macrophages. Conversely, in the paucibacillary (or tuberculoid) type a strong cell mediated immune reaction leads to granulomas formation and destruction of cells harbouring bacilli and nerve fibres nearby. In many cases treatment of patients with multibacillary leprosy is complicated by early or late reversal reaction, and further nerve damage. Nerve lesions lead to a symmetrical, pseudo-polyneuritic pattern, in most cases of multibacillary leprosy (ML), usually associated with typical skin lesions. In $10 \%$ of patients however skin lesions are missing, then only nerve biopsy permits diagnosis. The multifocal pattern, is more common in paucibacillar leprosy. Clinical manifestations of leprous neuropathy include 1 . Specific cutaneous lesions, which reveals the disease in half or more of the patients, depending on the type of leprosy. 2. Sensory loss is the most constant neurological finding, due to the involvement of mixed dermal nerves and nerve trunks. Proprioception is often preserved, so patients can still use their largely anaesthetic limbs effectively, which leads to painless trauma and trophic changes. The topographical distribution of sensory disturbances is extremely variable. 3. Motor involvement is a late event in the course of the disease. Amyotrophy and motor weakness usually progress pari passu. They predominate in the ulnar and median nerve territories, with characteristic claw hands, and in the territory of the peroneal nerve. 4. Tendon reflexes are often preserved 5. Nerve hypertrophy: Nerve trunks are palpably enlarged in one third of the patients with leprosy, sometimes before the occurrence of sensory loss in the corresponding territory. Doppler ultrasound can be useful in this setting. 6 There is no dysautonomia or sphincter disturbances 7 . Facial palsy with lagophthalmos of one or both eyes, with sparing of the other muscles supplied by the facial nerve is a classical feature. 8. Trophic disturbances are the most spectacular manifestations of leprosy. Severe sensory loss is always found in those areas where ulcers occur. 9. Symptomatic neuropathy, which is usually preceded by alteration of conduction velocity due to demyelination of nerve fibers, is invariably associated with major axonal damage and loss of more than $90 \%$ of nerve fibres in corresponding territory. Treatment is currently based on multidrug therapy with dapsone, rifampicin and clofazimine. The use of corticosteroids can reduce or prevent nerve damage in reversal reactions. Residual sensory loss which is extremely common in affected areas, can lead to trophic changes related to repeated traumas in painless areas.

\section{SS 21.3 / \#301}

Topic: 09. Systemic Diseases

\section{NEUROPATHIES IN INFECTIOUS DISEASES}

\author{
Nazha Birouk \\ Clinical Neurophysiology Rabat, Speciality \\ Hospital, Rabat, MA
}

\begin{abstract}
Infectious neuropathies are heterogeneous group of neuropathies regarding their multiple causes and numerous clinical presentations. They till represent an important world health issue. The clinical presentations are variable including symmetric distal neuropathy, mononeuropathy multiplex, radiculopathy or cranial neuropathy. The disorder process can be acute, sub acute or chronic axonal or demyelinating and is diagnosed by eletrodiagnostic or in some situations by nerve biopsy. The infectious agent can involve the peripheral nerve directly or indirectly by autoimmune and/or inflammatory process. The HIV related neuropathy became the major complication of HIV infection. The most frequent form is the painful distal and symmetric lent dependent polyneuropathy; it can be either HIV induced or antiretroviral toxic neuropathy. Inflammatory neuropathies such as acute or chronic polyradiculoneuropathies can be encountered at early stage of HIV infection. In the later stages, HIV infected patients can present with acute radiculopathies due to CMV infection. Peripheral neuropathy in patients with chronic infection with hepatitis $\mathrm{C}$ virus is mainly
\end{abstract}


due to associated cryoglobulinemia and the associated vasculitis that leads to acute or sub acute mononeuritis multiplex. The polyneuropathy in this context can be also induced by direct neurotoxic effect of the treatment. In Lyme disease the involvement of peripheral nerves is part of meningoradiculitis with frequent involvement of cranial nerves. The most frequent infectious neuropathy in the world remains an active infectious ganglioneuritis caused by varicella zoster virus, producing shingles. The main issue with this condition is the post-herpetic neuralgia that can be severe in some patients. Infectious diseases are an important part of differential diagnosis of peripheral neuropathies because most of them are potentially treatable. The systematic diagnosis for the most frequent ones such as HIV and hepatitis $\mathrm{C}$ is highly recommended in a patient with polyneuropathy. Other infections are discussed regarding particular clinical presentations and in some geographic areas.

\section{SS 21.4 / \#866}

Topic: 09. Systemic Diseases

\section{INFECTION RELATED NEUROMUSCULAR DISORDERS (NEUROPATHIES AND MYOPATHIES)}

\section{Chandrashekhar Meshram}

Neurology, Brain and Mind Institute, Nagpur, IN

Background: Various bacterial, viral and parasitic infections directly or indirectly affect peripheral nerves and muscles. Neuromuscular disorders can be caused by three mechanisms : direct infection, infection related but toxin mediated and post infectious syndrome. Involvement of nerves and muscles secondary to systemic infections is relatively rare phenomenon. Some of these infections are sporadic, while some occur in outbreaks. Some infections are predominantly observed in tropical countries. During outbreaks, cases are observed in clusters in affected regions and one gets the opportunity to study the disorders. Methods: Personal experience and unpublished personal communication was supplemented by search of published literature on neurological manifestations of systemic infections with involvement of peripheral nerves and/or muscles. Special attention was given to evaluate neuromuscu- lar involvement in Chikungunya, Dengue, Leptospirosis, Scrub Typhus and Rabies. Results: During the Chikungunya epidemic in India in 2006, Peripheral Neuropathy was observed in $36 \%$ patients with neurological involvement, entrapment neuropathy was seen in $10 \%$ while myositis was noted in $15 \%$ patients.In many patients there was combination of central and peripheral manifestations. Polyradiculoneuropathy ,lumbosacral plexopathy, GBS have been reported with Dengue virus infection. Autonomic symptoms are common and may occur in 30 $\%$ patients. These features are mainly immune mediated. Dengue associated hypokalaemic paralysis and myositis is also reported. A more benign and common, transient muscle weakness with moderately raised CPK is usually self limiting. But sometimes the myositis can be fulminant and require respiratory support. In Neuroleptospirosis, peripheral nervous system involvement is seen in $10 \%$ patients in form of myositis, rhabdomyolysis, GBS like presentation, brachial neuritis and mononeuritis multiplex. However CPK is raised in $30-50 \%$ patients. Neurological involvement occurs in $25 \%$ patients of Scrub Typhus. Neuromuscular involvement is in form of cranial nerve dysfunction, peripheral neuropathy, and myositis.In one study $39 \%$ patients of Scrub typhus had muscle involvement in form of myalgia,weakness, raised CPK and myopathic EMG. Twenty percent patients of Rabies present with ascending paralysis. Pain and paraethesia at the site of bite are common symptoms. Both upper and lower limbs are involved. Sometimes weakness is limited to the bitten limb. Conclusion: Neuromuscular manifestations associated with systemic infections, though uncommon should be considered in diffential diagnosis. Involvement is variable in severity and sometimes they occur in combination. Early detection and appropriate treatment helps in reducing the morbidity and mortality.

SS 22.1 / \#82

Topic: 10. History

THE HISTORY OF

NEUROMUSCULAR DISEASES AND THEIR TREATMENT IN HABSBURG COUNTRIES

Sonja Horn

University of Vienna, Vienna, AT 
Abstract: Research on neuromuscular diseases and their therapy in history is usually covered under the notion of "electrotherapy" - at least in the Habsburg inherited countries. Electrotherapy was used to treat soldiers of the Habsburg army already in the late $18^{\text {th }} \mathrm{C}$., however there are also distinctive reports about the frequent use of electrotherapy at the Vienna clinic during the mid $18^{\text {th }} \mathrm{C}$. Perhaps as a typical way of practicing medicine at Vienna, the treatment of patients at hospitals in was intensely linked to the training of physicians and surgeons, as well as to post mortem dissections and theoretical research. Therefore "electrotherapy" has to be seen as strongly linked to theoretical and experimental research on the function of nerves and muscles as well as on the anatomy of the brain and the "location" of intellectual functions. This concept of the production of medical and scientific knowledge was practiced not only at Vienna, but also at other universities in the Habsburg countries like Prague or Pavia, that are connected with researchers like Jan Evangelista Purkinje (1787 - 1869), Alessandro Volta (1745 - 1827) or Camillo Golgi (1843 - 1926). In my paper I will focus on concepts and topics of research on neuromuscular diseases, which is strongly linked to the practical use of electrotherapy.

\section{SS 22.2 / \#31}

Topic: 10. History

\section{HISTORY ON NM DISEASE}

$\underline{\text { Richard J. Barohn }}$

Neurology, University of Kansas Medical Center, Kansas City, KS, US

Abstract: MG was suspected as a disease probably since Thomas Willis first wrote about "the palsy" in the late 1600s. In the late 1800 s, MG was defined as a clinical entity by German neurologists; Wilhelm Erb, Samuel Goldflam, Herman Oppenheim, and Friedrich Jolly described the decremental muscle response on electrophysiologic testing. In the first half of the twentieth century. The two discoveries that led to a better understanding of MG involved the thymus gland's relation to MG and the drug treatment for MG using acetylcholinesterase inhibitors. Sauerbach first successfully removed an enlarged thymus gland in 1911. In the 1930s and 1940s, Blalock advocated and began performing thymectomy for MG patients with and without thymoma. Mary Walker in England was the first to observe the drugs physostigmine and neostigmine improved MG patients. In the second half of the twentieth century a number of discoveries occurred: (1) an understanding of the neuroanatomy and neurochemistry of the NMJ; (2) development of the animal model of experimental allergic MG; (3) the discovery of antibodies to the acetylcholine receptor (AChR) in humans with MG and passive transfer of MG to mice from human immunoglobulin of $\mathrm{MG}$ patients; (4) passive transfer of MG to mice from human immunoglobulin of MG patients; (5) the discovery that corticosteroids and other immunosuppressive agents, including plasmapheresis, can improve MG symptoms and signs; and finally, (6) the development of mechanical ventilation to keep patients alive when they go into respiratory failure from myasthenia crisis; (7) improvement in electrophysiologic techniques for diagnosing $\mathrm{MG}$, and the development of single fiber EMG. In the 1950s, the disorder known as LEMS was described, initially in patients with cancer and later in noncancer patients. In the 1970s, the first congenital myasthenic syndrome was described. In the current era, new autoantibodies are being discovered that are responsible for NMJ disorders (voltage-gated P-/Q-type calcium channels in LEMS; to LRP4, rapsyn, agrin, and cortactin in MG). We now have a great deal of experience in clinical research in $\mathrm{MG}$ and have refined the endpoint measurements and trial designs. With the completion and publication of the landmark thymectomy trial in $\mathrm{MG}$, we can now say with conviction that patients with MG who undergo thymectomy improve and their prednisone dose can be lowered more than patients who do not have a thymectomy. Prior to 2000, there were only seven randomized controlled published MG trials. As of 2018, 22 randomized clinical trials have been published for MG. Recently, this led to the US Food and Drug Administration approval of the complement inhibitor eculizumab (Soliris) for adult patients with generalized MG who are AChR antibody positive. Prior studies demonstrated increased complement in the serum of MG patients and the presence of complements at the NMJ in MG. MG indeed used to be a grave disease with $30 \%$ mortality. Now, with the advent of all the advances and others described above, everyone we see with MG should improve, and it should be a rare event indeed for a patient to die of MG. 
SS 22.3 / \#177

Topic: 10. History

\section{ANDALUSIAN NEUROLOGY OVER 700 YEARS}

$\underline{\text { Raad Shakir }}$

World Federation of Neurology, London, GB

\begin{abstract}
I will confine myself to the evolution of Medicine and Neurology in Andalusia 711-1492 AD. These centuries saw the massive proliferation of not only Sciences and Philosophy but were the vehicle of transfer of Greco-Roman literature back to Europe. There are numerous scholars and physicians who influenced our thinking and practice. Four will be discussed Averroes (1126-1198), Maimonides (1135-1209), Avenzoar (1094-1161) and Abulcasis (936-1013). The first three were contemporaneous and influenced each other. Each has contributed in a rather unique way to medicine with some emphasis on Neurology and the diseases of the nervous system. Averroes was a defender of the Aristotelian philosophy and is considered to be the father of secular thought in Europe. Maimonides in addition to being a first class physician and the private physician of Saladin was a most respected Torah scholar and theologian. Avenzoar was probably the first physician to practice surgery on animals before trying them on Humans. Abulcasis was a brilliant surgeon and his neurosurgical tools in treating hydrocephalus as an example, were ingenious to say the least. The Golden age of Andalusia was at its zenith during the $10^{\text {th }}$ to the $12^{\text {th }}$ century.
\end{abstract}

\section{SS 23.1 / \#42}

Topic: 04. Neuromuscular Junction

\section{THYMECTOMY FOR MYASTHENIA GRAVIS}

$\underline{\text { Richard J. Barohn }}$

Neurology, University of Kansas Medical Center, Kansas City, KS, US

Abstract: Thymectomy has a central role in the treatment of MG. In thymomatous MG, the tumor should be removed. Along with thymoma, the entirety of the thymus tissue should be removed. Improvement in myasthenic symptoms may or may not follow. A systematic review of the existing thymectomy literature emphasized this knowledge gap and recommended the MG field perform a randomized, controlled trial. (Gronseth and Barohn, 2000) However, owing to the difficulty of performing controlled trials involving thoracic surgery in a rare disease, high-quality evidence about thymectomy had been lacking. A recently completed landmark international, randomized, rater-blinded clinical trial controlling for medical treatment was designed to address this uncertainty. One hundred twenty-six recently diagnosed patients, ages 18 through 65 with AChR antibody-positive generalized MG were randomized to receive either extended transsternal thymectomy plus prednisone versus medical management with prednisone. Over a 3-year follow-up period, the time-weighted average QMG score was lower in the patients who underwent thymectomy ( 6.15 vs 8.99 ; $P<.001$ ) Similarly, the thymectomy group had a lower time-weighted alternate-day prednisone dose requirement (initially reported at $44 \mathrm{mg}$ vs $60 \mathrm{mg}$; $P<.001)$, which was later corrected to $32 \mathrm{mg}$ versus $54 \mathrm{mg}$ (95\% confidence interval, $12-32 \mathrm{mg} ; P<.001)$. Also in the thymectomy group, there were fewer patients requiring additional immunosuppression, fewer adverse events, and fewer admissions for myasthenic crises. These data provide support for thymectomy as a first-line treatment modality that can improve MG status and decrease the required dose and duration of immunotherapy in generalized MG. (Surprisingly, the effects of the thymectomy could be observed as early as 3 to 4 months and were maintained for the entire 3-year study.) Patients with persistent bulbar, respiratory, or limb weakness should be treated with PLEX before surgery. Thymectomy in MuSK, LRP4, and agrin antibody-positive patients is not supported by current evidence. Patients with MG with MuSK antibodies were not included in the recent thymectomy study. Nevertheless, MuSK and "double-negative" antibody patients have undergone thymectomy and anecdotally have done well. Similarly, there is limited evidence to support thymectomy in patients with ocular MG, although if the patient is AChR antibody positive, it may be considered in refractory cases. The recently completed thymectomy trial mandated a sternalsplitting procedure. Several new less invasive procedures are now being used for thymus removal. Video-assisted thoracoscopic surgery and robotic approaches to thymectomy such as robotic videoassisted thoracoscopic surgery offer shorter hospital durations of stay and limited morbidity have 
emerged as alternatives to the classic transsternal approach. There are no trials comparing these surgical techniques, however, and available reports suggest comparable results.

\section{SS 23.2 / \#173}

Topic: 04. Neuromuscular Junction

\section{RANDOMIZED CONTROLLED TRIAL OF THYMECTOMY: A DEEPER DIVE INTO DATA}

Gil I. Wolfe ${ }^{1}$, Henry J. Kaminski ${ }^{2}$, Inmaculada B. $\mathrm{Aban}^{3}$, Greg Minisman ${ }^{4}$, Hui-Chien Kuo ${ }^{3}$, Joshua R. Sonett ${ }^{5}$, Alexander Marx ${ }^{6}$, Cleo-Aron Weis ${ }^{6}$, Gary R. Cutter ${ }^{7}$

${ }^{1}$ Neurology, University at Buffalo, Buffalo, NY, US:'2Neurology, George Washington University, Washington, DC, US, ${ }^{3}$ Biostatistics, University of Alabama at Birmingham, Birmingham, AL, $U S ;{ }^{4}$ Univesity of Alabama at Birmingham, Birmingham, AL, US, ${ }^{5}$ General Thoracic Surgery, Columbia University Medical Center, New York, NY, US; ${ }^{6}$ Pathology, University Medical Centre Mannheim/University of Heidelberg, Mannheim, DE: ${ }^{7}$ Biostatistics, UNiversity of Alabama at Birmingham, Birmingham, AL, US

Background: MGTX, the Thymectomy Trial in Non-thymomatous Generalized Myasthenia Gravis Patients Receiving Corticosteroids, was the firstever randomized, rater-blinded study of thymectomy in myasthenia gravis (MG). The international trial addressed questions regarding the efficacy of thymectomy in non-thymomatous MG that had persisted for 75 years since use of the procedure was first reported by Blalock and colleagues in 1941. Methods: MGTX was designed to answer three questions: does the combination of alternate-day prednisone and extended transsternal thymectomy after 3 years compared to an identical dosing protocol of prednisone alone, (1) lead to better outcomes in $\mathrm{MG}$ as measured by a time-weighted average of the Quantitative MG Score (QMG), (2) reduce the time-weighted average alternate-day prednisone requirements, and (3) reduce side-effect burdens on patients primarily arising from medications used to treat the disease? Patients age 18 to 65 years with generalized non-thymomatous acetylcholine receptor antibody-positive MG of less than 5 years dura- tion and MG Foundation of America Clinical Classification 2 to 4 were enrolled. Dual primary outcomes were the time-weighted average of the Quantitative MG (QMG) score and prednisone dose over 3 years. Results: A total of $126 \mathrm{MG}$ patients underwent randomization at 36 sites. Subjects who underwent thymectomy showed significant improvements in MG status (time-weighted average QMG 6.15 vs. 8.99, $\mathrm{p}<0.001)$ and reduced alternateday prednisone requirements $(32 \mathrm{mg}$ vs. $54 \mathrm{mg}$, $\mathrm{p}<0.001)$ at 3 years with benefits observed as early as 9 to 12 months. The ability to withdraw completely from prednisone and remain off it at 3 years also favored the surgical arm (58\% vs. $20 \%$, $\mathrm{p}<0.01)$. The ability to withdraw from prednisone did not correlate with duration of disease, or age at MG onset or trial enrollment. Histological analysis of thymic follicular hyperplasia, seen in $67 \%$ of surgical patients, failed to correlate with the timeweighted QMG or prednisone exposure. However, intra-thymic fat content directly correlated with time-weighted prednisone exposure $(\mathrm{p}=0.004)$. On secondary outcomes, fewer patients who had thymectomy required immunosuppression with azathioprine $(17 \%$ vs. $48 \%, \mathrm{p}<0.001)$ or were hospitalized for exacerbations ( $9 \%$ vs. $37 \%, \mathrm{p}<0.001)$. The number of patients with treatment-associated complications did not differ significantly between groups $(p=0.73)$, but the thymectomy group had fewer treatment-associated symptoms $(\mathrm{p}<0.001)$ and lower distress levels related to symptoms $(\mathrm{p}=0.003)$. MG-Activities of Daily Living scale and the proportion of patients reaching Minimal Manifestation Status also significantly favored the surgical arm. Conclusion: MGTX provided a definitive answer sought by the MG community since the middle of the last century. Extended transsternal thymectomy performed within the first 5 years of disease improves clinical outcomes, reduces immunosuppressive medication requirements, and decreases side-effect burdens in generalized acetylcholine receptor antibody- positive MG patients without thymoma. This group represents the largest subpopulation of MG patients. Although the MGTX trial did not test less-invasive approaches for removing the thymus gland which have become increasingly popular over the last two decades, one can surmise that less invasive approaches that completely replicate the maximal tissue resection performed in an extended transsternal thymectomy would confer similar benefits for patients. 
SS 23.3 / \#104

Topic: 04. Neuromuscular Junction

\section{PATHOLOGY OF THE THYMUS GLAND IN MG}

$\underline{\text { Alexander Marx }}$

Pathology, University Medical Centre Mannheim/

University of Heidelberg, Mannheim, DE

Abstract not received.

SS 24.1 / \#214

Topic: 04. Neuromuscular Junction

\section{SYNAPTIC STABILITY IN CMS}

David Beeson

Nuffield Department Of Clinical Neurosciences,

University of Oxford, Oxford, GB

Abstract not received.

SS 24.2 / \#218

Topic: 04. Neuromuscular Junction

RECENT PROGRESS IN

UNDERSTANDING PRESYNAPTIC CONGENITAL MYASTHENIC SYNDROMES (CMS)

Hanns Lochmüller ${ }^{1}$, Emily O'Connor ${ }^{2}$, Grace Mcmacken $^{2}$, Yoshiteru Azuma², Sunitha Belaraju ${ }^{2}$, Teresinha Evangelista ${ }^{2}$, Roger Whittaker ${ }^{3}$, Ana Töpf ${ }^{4}$, Andreas Roos ${ }^{4}$, Rita Horvath ${ }^{4}$

${ }^{1}$ Department Of Neuropediatrics And Muscle Disorders, Freiburg University - Medical Center, Faculty of Medicine, Freiburg, DE: ${ }^{2}$ Institute Of Genetic Medicine, Newcastle University, Newcastle, GB; ${ }^{3}$ Institute Of Neuroscience, Newcastle University, Newcastle, GB $;{ }^{4} J$ ohn Walton Muscular Dystrophy Research Center, Newcastle University, Newcastle, GB

Background: Neuromuscular junction disorders, also called Myasthenic syndromes (MS), are a rare heterogeneous group of acquired (Myasthenia Gravis, MG) and inherited (Congenital Myasthenic Syndromes, CMS) neuromuscular disorders associated with distinctive clinical, electrophysiological, laboratory and ultrastructural abnormalities. The genetic defects in CMS either impair neuromuscular transmission directly or result in secondary impairments, which eventually compromise the safety margin of neuromuscular transmission and may affect proteins that are specific to the synapse or more widely expressed proteins. Methods: While the majority of patients with CMS harbours mutations in genes encoding postsynaptic proteins, defects in presynaptic proteins are increasingly discovered, often associated with additional symptoms of the PNS or CNS. Results: Mutations in SYT2, the gene encoding synaptotagmin 2 are dominantly inherited and show signs reminiscent of hereditary motor neuropathies and fatigability. Mutations in several presynaptic genes are causing severe childhood disorders with episodic apnea or intellectual disability. Loss of MYO9A, a recently identified CMS-associated protein, affects the neuronal cytoskeleton and leads to impaired transport of proteins, including agrin, which may provide a new and unexpected treatment option. Conclusion: We will cover the significant progress made in understanding the molecular pathogenesis of CMS, which is important for both patients and clinicians in terms of reaching a definite diagnosis and selecting the most appropriate treatment.

\section{SS 24.3 / \#225}

Topic: 04. Neuromuscular Junction

\section{CLINICAL ASPECTS OF CMS}

Hakan Cetin

Department Of Neurology, Medical University of Vienna, Vienna, $A T$

Background: Congenital myasthenic syndromes (CMS) are a heterogeneous group of inherited disorders of the neuromuscular junction (NMJ). They are caused by mutations in genes that code for proteins affecting NMJ structure and function. The genetic defect can be located at the presynaptic nerve terminal, the synaptic cleft and the postsynaptic membrane. More recently, next-generation sequencing techniques have also identified causative genes encoding ubiquitously expressed molecules, the functional defect of which, however, may be largely restricted to the NMJ. Methods: Postsynaptic NMJ defects including i) acetylcholine receptor (AChR) 
deficiency syndromes, ii) slow-channel CMS, iii) fast channel CMS, iv) low conductance CMS and v) CMS due to perturbation of the AChR clustering pathway are reviewed and correlated with clinical findings. Results: Mutations in the AChR subunit genes cause a primary AChR deficiency at the endplate. AChR deficiency syndromes show a recessive inheritance pattern. Disease severity is variable but weakness is usually evident at birth or within the first year of life. Feeding difficulties, ptosis, impaired eye movements and delayed motor milestones are frequent. The disease course is usually not progressive and the patients sometimes improve by early adulthood. Loss of endplate AChR may also be due to the recessive inheritance of RAPSN mutations. The more common "early onset" form of the disease is associated with hypotonia, bulbar dysfunction and joint contractures of hands and ankles. Affected neonates often require assisted ventilation. By contrast, "late onset" forms begin from early adulthood and are not associated with bulbar, speech and respiratory problems. They may be mistaken as seronegative myasthenia gravis, but weakness of ankle dorsiflexion may be a helpful clinical tool to differentiate CMS from autoimmune myasthenia gravis. Other AChR subunit mutations predominantly affect ion channel kinetics without a severe reduction of AChR numbers. Slow-channel CMS forms are characterised by longer AChR burst durations. This is associated with prolonged endplate potentials resulting in a depolarisation block of voltagegated sodium channels. Slow-channel CMS shows a dominant inheritance pattern but there can be variable penetrance, age of onset and disease severity. However, weakness of cervical and scapular muscles and finger extensors is often an early feature. Repetitive compound muscle action potentials to single nerve stimuli in electrodiagnostic studies are characteristic. Fast-channel CMS, by contrast, is characterised by shorter AChR burst-duration and endplate potentials that fail to elicit action potentials in the muscle cell. They have recessive inheritance. Feeding difficulties, ptosis, impaired eye movements and delayed motor milestones are often present at birth with the patients generally more severely affected than in AChR deficiency syndromes. Lowconductance CMS is also inherited recessively. It is very rare and the phenotype is similar to fast-channel syndrome. Mutations in the AChR clustering pathway including agrin, LRP4, MuSK and DOK7 are characterised by a limb-girdle distribution of weakness. Patients may achieve normal walking milestones but then start to experience falls associated with proximal muscle weakness. Conclusion: A deep understanding of disease mechanisms underlying CMS is crucial in order to correlate diagnostic and clinical findings with corresponding molecular defects and thus direct genetic screening and effective treatment.

\section{SS 24.4 / \#312}

Topic: 04. Neuromuscular Junction

\section{THERAPEUTIC STRATEGIES FOR CONGENITAL MYASTHENIC SYNDROMES}

\author{
Jacqueline Palace \\ Neurology, Oxford univeristy Hospitals Trust, DU, \\ $G B$
}

Abstract: The congenital myasthenic syndromes (CMS) are a heterogeneous group of genetic disorders which cause neuromuscular junction dysfunction. More than 30 different genes have been described as causing CMS and the treatment regimes vary, dependent upon the CMS subtype. For example pyridostigmine and 3,4-Diaminopyridine improve muscle strength in many genetic forms of CMS but make others worse. The mainstay of treatment remains anticholinesterases, in particular pyridostigmine, which improves strength in AChR deficiency syndromes due to epsilon subunit mutations, the fast channel syndromes and CMS due to mutations in RAPSN, CHAT, and glycosylation pathways genes. 3,4-diaminopyrodine (3-4DAP) can also be beneficial in the same conditions (although because acetylcholine is deficient in CHAT CMS increasing the presynaptic release with 3,4DAP may not be a good strategy). Oral salbutamol or ephedrine are beneficial in DOK7 and COLQ CMS and other forms of CMS where the AChR clustering pathway is impaired. It can also improve strength in forms of CMS where pyridostigmine is being taken at higher doses such as in patients with AChR epsilon mutations causing deficiency of the receptor, and in patients with fast channel CMS. Fluoxetine (usually at doses of 40-100mg daily) or quinidine are the main treatment options for slow channel syndrome CMS. Prolonged QT interval may limit the dose and needs monitoring. It is important to note that pyridostigmine (and 3,4-DAP) usually 
worsen slow channel syndromes, COLQ and DOK7 CMS and thus where CMS is suspected as a diagnosis, genetic screening of these genes should be undertaken prior to initiating anticholinesterase medication. 


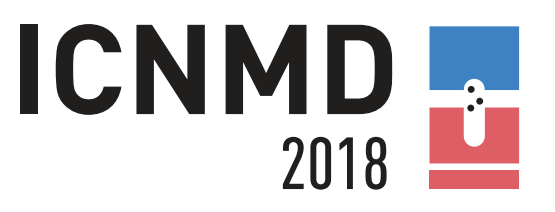

15TH INTERNATIONAL CONGRESS ON NEUROMUSCULAR DISEASES

www.icnmd2018.org July 6 - 10, 2018 Vienna, Austria

Workshop 
WS 1.1 / \#181

Topic: 06. Mononeuropathy

\section{CHEMOTHERAPY INDUCED PNP}

Anthony Windebank

Mayo Clinic, Rochester, US

Abstract not received.

\section{WS 1.2 / \#185}

Topic: 06. Mononeuropathy

\section{IATROGENIC MONONEUROPATHIES AFTER SURGERY AND TRAUMA}

Tatjana Paternostro-Sluga

Social Medical Center East - Donauspital, Institute of Physical Medicine and Rehabilitation,

Vienna, AT

Background: Iatrogenic mononeuropathies after surgery and trauma occur accidentally or may be part of the necessary treatment. It is of utmost importance to diagnose the lesions immediately and to start the appropriate treatment early. Even so iatrogenic mononeuropathies have always occurred and will occur in the future, the delayed timing of diagnosis and treatment is highly prevalent. Mononeuropathies may interfere significantly with activities of daily living and reduce the quality of life. Patients may recover completely from the primary disease, but the sequelae of the iatrogenic nerve lesion leads to long-lasting impairment and loss of social participation. Methods: The aim of the workshop is to increase the knowledge on iatrogenic nerve lesions and therefore improve diagnostic and therapeutic procedures for the patients. Results: Iatrogenic nerve lesions can result from different surgical procedures. In the upper extremity median nerve, accessory nerve, ulnar nerve, radial nerve, cervical and brachial plexus, axillary nerve and phrenic nerve may be typically affected. Lesions of the median nerve occur most frequently during carpal tunnel surgey, accessory nerve lesions result from lymph node biopsy in the posterior neck triangle and even this is common knowledge it still occurs. The ulnar nerve is frequently associated with supracondylar humeral fractures in children. This has to be taken into account when children with supracondylar humeral fractures are examined. Ulnar nerv palsy in small children may be overlooked at the beginning, especially when atrophy of the intrinsic muscles is not yet visible and finger abduction is done by radial innervated extrinsic finger extensors. In the lower extremity peroneal nerve, femoral nerve, ischiadic nerve, tibial nerve, ilioinguinal and genitofemoral nerves, lateral cutaneous femoral nerve, lumbosacral plexus, saphenous nerve and infra-patellar branch and sural nerve may be typically affected. Orthopedic and gynecological procedures may affect the ischiadic nerve, orthopedic procedures may also affect the femoral nerve and/or the gluteal nerves in total hip replacement. Knee surgery may affect the tibial nerve or the infra-patellar branch of the saphenous nerve. Tourniquet pressure at the thigh during knee surgery might be associated with femoral nerve palsy and a partial lesion might be overlooked. Weakness and insecurity of walking is wrongly attributed to the knee surgery. Conclusion: Prognosis of iatrogenic mononeuropathies depends on the degree of the lesion. The timing for nerve reconstruction is very important to optimize the outcome in high grade lesions. Diagnostic procedure include history, clinical examination, clinical electrophysiology, nerve ultrasound and magnetic resonance imaging. Conservative treatment has to include pain managment, maintenace of range of motion of joints and tendons, motor and sensory reeducation, minimizing trophic disturbances and regaining function. Surgical treatment includes primary nerve repair, nerve reconstruction, neurolysis. Secondary reconstructive procedures, eg muscle transposition may be applied if nerve regneration even after nerve surgery is not sufficient for regaining function. Knowledge, expertise and dedication in diagnosing and treatment of peripheral nerve lesions as well as interdisciplinary and multiprofessional teamwork are the landmarks of best practice in the treatment of iatrogenc nerve lesions. 
WS 2.1 / \#156

Topic: 01. Muscle

\section{DUX4, ITS ROLE IN DEVELOPMENT AND MUSCLE DISEASE}

\author{
$\underline{\text { Stephen J. Tapscott }}$ \\ Fred Hutchinson Cancer Research Center, Seattle, \\ US
}

Abstract not received.

WS 2.2 / \#152

Topic: 01. Muscle

\section{TRIALS READINESS IN FSHD: CLINICAL, IMAGING AND BIOMARKERS}

Jeffrey M. Statland

Neurology, University of Kansas Medical Center,

Kansas City, KS, US

Background: Facioscapulohumeral muscular dystrophy (FSHD) is one of the most common muscular dystrophies, and although it is slowly progressive, it can cause significant morbidity over the course of a lifetime, interfering with the ability to maintain a job or walk independently. There is at present no effective treatment for FSHD. Substantial evidence now implicates aberrant reactivation of DUX4 as the cause of FSHD providing a clear target for therapeutic intervention. The success in identifying the molecular and genetic mechanisms of FSHD provides a strong basis for drug development and therapeutic clinical trials. The key abnormality in FSHD, the aberrant expression of $D U X 4$, is potentially amenable to RNA therapies making possible similar strategies to drugs already approved for spinal muscular atrophy and Duchenne muscular dystrophy, or may be amenable to other disease-directed strategies targeting epigenetic changes in FSHD. In addition, drugs not specifically targeting DUX4 but designed to ameliorate downstream pathology, such as muscle inflammation or muscle atrophy and fibrosis, are either underway now or proposed to start in the next few years. Methods: A prospective study of FSHD was performed in the 1990s that determined the multisite reliability and sensitivity to disease progression of manual muscle testing, quantitative myometry, and timed functional outcomes. However limitations to using strength or functional outcomes for FSHD were identified: the first is that the clinical relevance of relatively small changes in strength is unknown; and the second is that demonstrating arrest of disease progression would require large numbers of participants or long follow up times, creating a significant patient and financial burden. To best inform clinical trial design, it is now necessary to identify and validate measures of disease activity and progression. A conceptual framework for thinking about outcome development in FSHD is built on expert opinion about molecular pathology, disease progression, natural history studies, recent large MRI studies, and patient surveys and registries. It is likely we do not need a single outcome measure for FSHD but rather a clinical trial toolbox: in which clinical outcomes and biomarkers will play an important role. Results: Biomarkers can provide proof of concept a drug is reaching its target, can provide strategies to inform inclusion criteria or stratification, and can provide early signals of disease activity which will predict a later functional change. Here we will review the current state of biomarker development in FSHD, looking at the current experience with: 1) molecular biomarkers in muscle tissue; 2) serum biomarkers; 3 ) the use of MRI as a measure of disease progression, or for stratification; and 4) other techniques like electrical impedance myography or muscle ultrasound for tracking early changes in muscle quality. Conclusion: Development of robust FSHD-relevant biomarkers can hasten therapeutic development, and form a cornerstone of clinical trial preparedness.

\section{WS 2.3 / \#125}

Topic: 01. Muscle

\section{FSHD'S CHANGING LANDSCAPE AND FUTURE PERSPECTIVES}

\author{
George W. Padberg \\ Dep. Of Neurology, Radboud University Medical \\ Center, Nijmegen, $N L$
}

Background: Facioscapulohumeral Dystrophy is an autosomal dominant disorder with a major myopathic phenotype and a variable, often incomplete minor phenotype. The myopathy has two components: an intrinsic muscle weakness, possibly congenital, on 
top of which develops a dystrophic proces with a specific descending sequence of muscle involvement which resembles a replay of embryological somite development. The age at onset and the rate of progression are extremely variable within and between families with FSHD and are used as measures of severity of the disease in individual cases. In earlier studies we estimated that $75 \%$ of the age at onset was related to environmental factors other than the primary gene defect. FSHD is caused by an epigenetic dysregulation of the D4Z4 macrosatellite repeat on $4 q 35$ which leads to an aberrant expression of the Double-Homeobox gene Dux4 embedded in the terminal repeat unit. Dux 4 can only be stably expressed if the repeat is followed by a PAS-sequence present in $4 \mathrm{qA}$ telomeric alleles to be found in $50 \%$ of the normal population. Dux4 is a double-homeodomain transcription factor normaly expressed in germ-cells and in the zygote-cleavage stage and repressed in later stages of development. Unknown at present is the rather basic question whether it has a function in myogenic stem-cells. The epigenetic repression of Dux4 has been related to the level of methylation proximal in the repeat. Hypomethylation is caused by a reduction of the number of repeats below 10 (FSHD1) or by mutations in SMCHD1 or DNMT3B (FSHD2), all leading to an identical muscle phenotype.The latter two are able to modulate FSHD1 as well. How the hypomethylation is established is presently under study but hypomethylation in itself appears not the only factor leading to Dux 4 mRNA transcription. The upstream factors and modifyers of Dux4 expression and its downstream effects and pathways have to be evaluated for their risks and benefits in emerging therapies. Methods: Review of recent genetic and myo-biological literature in relationship to clinical features against the perspective of emerging therapies. Results: Knowledge of the epigenetic factors preventing Dux4 expression are invaluable for counselling (non-penetrance) and might lead to therapies. The downstream effects have to take into account the inflammatory response in muscles (possibly the onset of the dystrophic process), the distal to proximal fatty infiltration pattern as seen on MRI, the atrophic processes of the muscles, the early involved versus the relatively spared muscles and their genetic make-up and metabolic properties, the characteristic asymmetry of muscle involvement, possible gender differences and their metabolic background, and retinal vascular, cochlear, skeletal and possibly CNS involvement. Review of these aspects will lead to a translational research agenda. Conclusion: All the clinical aspects of the disease have to be explained before we fully understand the possible consequences of therapeutic interventions for this, probably most prevalent genetic risk factor for muscular dystrophy and at the same time indispensible gene for the primate lineage.

\section{WS 3.1 / \#123}

Topic: 12. Patient Issues

\section{THE ROLE OF PALLIATIVE CARE $€$ - EARLY INVOLVEMENT OR END OF LIFE CARE?}

\section{David J. Oliver \\ Tizard Centre, University of Kent, Canterbury, GB}

Background: Palliative care is "an approach that improves the quality of life of patients and their families facing problems associated with life-threatening illness, through the prevention and relief of suffering, early identification and impeccable assessment and treatment of pain and other problems, physical, psychosocial and spiritual"(WHO 2002). Thus within this definition the timing of palliative care involvement is open and should be related to the needs of the patient, and family, as assessed by an holistic assessment by a multidisciplinary team. Methods: A review of the literature was undertaken, together with clinical experience. Results: Although palliative care is often associated with care at the end of life the role is much greater. For a patient with progressive neuromuscular disease, such as ALS, it can be argued that the care should be palliative in it approach from the time of diagnosis. For instance the way the diagnosis is told to the patient and family can have significant effect on the later stages of care - a patient who understands that death may be distressing, from choking or breathlessness, may continue to fear these issues, despite any further discussion and explanation that these risks are small. The care of any patient with neuromuscular disease will need to include the multidisciplinary team assessment of all aspects of care: Physical - the symptoms and mobility issues Psychological - the fears and concerns of the person Social - most patients are part of wider families and have concerns for them or are the cause of concern to them Spiritual - the deeper issues that all people consider, particularly as they face progressive illness, of the meaning of illness 
and life Thus all professionals involved in the care of people with neuromuscular disease need to provide palliative care - aiming to maximize the quality of life of patients and families and listen and respond to the issues they face as individuals. This is usually from a multidisciplinary team, and may improve not only quality, but length, of life. Some patients and families will need increased care, from a specialist palliative care team because of very complex symptoms or psychosocial issues., perhaps at particular times - such as with the distress of diagnosis, consideration of gastrostomy, commencement of ventilatory support and at the end of life in ALS. At other times the care may be provided by the multidisciplinary team providing palliative care. Conclusion: Close collaboration and communication is essential among all the teams involved always responding to the individual patient's needs. As the patient deteriorates and comes toward the end of life there may be increasing specialist palliative care, and hospice, involvement to support the patient, family, carers and professionals as death approaches. The care in the earlier stages will influence this time at the end of life and a careful team involvement, using specialist help as required, will allow patients and their families to approach death with confidence in their care and enable a less distressing terminal phase, and later bereavement for their family. Reference World Health Organization. Palliative care 2002 www. who.int/cancer/palliative/definition/en/

WS 3.2 / \#110

Topic: 12. Patient Issues

THE ROLE OF THE MULTIDISCIPLINARY TEAM IN NMD - THE EVIDENCE FROM ALS

Christopher Mcdermott

University Of Sheffield, Sheffield, US

Abstract not received.
WS 3.3 / \#169

Topic: 12. Patient Issues

\section{THE RECOGNITION OF THE END OF LIFE PHASE IN NMD - THE SUPPORT OF PATIENTS, FAMILIES AND PROFESSIONALS}

\author{
$\underline{\text { Stefan Lorenzl }}$ \\ Insitute of Nursing Science And Practice, \\ Salzburg, DE
}

Abstract not received.

WS 3.4 / \#854

Topic: 12. Patient Issues

\section{INTEGRATING PALLIATIVE CARE INTO SERVICE FOR ALS PATIENTS: REAL-LIFE EXPERIENCE}

$\underline{\text { Lev Brylev }}^{1}$, Vadim Parshikov ${ }^{2}$, Yaroslava Krasnaya $^{2}$, Ekaterina Dikhter ${ }^{2}$, Egor Larin ${ }^{3}$, Yana Batmanova $^{2}$, Vasiliy Shtabnitskiy ${ }^{4}$, Sergey Avdeykin ${ }^{5}$, Anastasia Ataulina ${ }^{5}$, Daria Puzanok ${ }^{2}$, Anna Kasianova ${ }^{2}$, Vera Fominyh ${ }^{1}$, Margarita Fominyh $^{2}$, Maria Ivanova ${ }^{6}$, Elena Lysogorskaya ${ }^{6}$, Anna Vorobyeva ${ }^{6}$, Vera S. Demeshonok ${ }^{7}$, Tatiana Leontyeva $^{2}$, Timur Ivanov ${ }^{8}$, Maria Ilchenko ${ }^{2}$, Alisa Apreleva $^{9}$, Inessa Zakroyshikova ${ }^{6}$, Kirill Gorbachev $^{1}$, Artur Volov ${ }^{2}$, Timur Mukhametov ${ }^{2}$, Sergey Korneychuk ${ }^{2}$, Natalia Semina ${ }^{8}$, Maria Zakharova $^{6}$

${ }^{1}$ Buyanov City Hospital, Moscow, RU; ${ }^{2} A L S$ center Moscow, Moscow, $R U,{ }^{3}$ Center of palliative medicine, Moscow, $R U,{ }^{4}$ Chaika, Moscow, $R U ;{ }^{5}$ Buyanov city hospital, Moscow, $R U ;{ }^{6}$ Research center of neurology, Moscow, $R U ;^{7}$ North-Western State Medical University named after I.I. Mechnikov, Saint Petersburg, $R U^{*}$ Aprel clinic, Moscow, RU; 9 Anglia Ruskin University, Cambridge, $G B$

Background: The first specialized multidisciplinary outpatient center for ALS patients in Moscow started in 2012 and it is now a part of ENCALS network. The clinic follows about 200 families with ALS every year and is funded by charity foundation "Live now". Before 2012 patients with ALS had no access 
to palliative care, because hospices would not accept them and the national legislation which first mentioned the provision of palliative care for non-cancer patientswas only passed in 2011. Methods: Experience has shown that the main needs of patients and their caregivers are information, symptom management, and social and psychological support. To meet these needs palliative care has been gradually introduced into everyday practice. As we had no specialized education in palliative care for neurological patients in Russia, foreign experts were invited to teach our team and a palliative care physician, who studied palliative care abroad, was invited to the multidisciplinary discussions. Multidisciplinary outpatient consultations were started and the palliative care approach, with the emphasis on patient autonomy, dignity and quality of life, changed our practice completely. Results: Analyzing our data from 672 patients we found out that median disease duration was 1188 days (range 791-1787), the time from first symptom to diagnosis was 365 days (range $191-587)$ and the time from diagnosis to referral to the team was 320 days (range 132 - 630) days. Patients on admission to our service had ALS for about 2 years and at this time the median vital capacity of $51 \%$ (range 38-80) and it was apparent that they had never talked about advance decision making and only $30 \%$ of them had ever talked to their families or physicians about prognosis and death. The training we received enhanced our communication skills and it was possible to talk with patients about prognosis and decisions about life sustaining interventions. This was particularly important if patient and families lack information and symptoms are inadequately controlled to prevent unwanted hospitalizations and prolonged ventilator support, that could affect quality of life. Moreover according to Russian legislation interventions, such as ventilation, cannot be withdrawn. One of the most prevalent end-of-life symptoms is dyspnea, $-40 \%$ of our patients have persistent dyspnoea with median Borg score 5 out of 10. Dyspnoea is also one of the main reason for admission to Intensive care, especially where there has been limited discussion of the use of life sustaining interventions. We were the first team in Russia to use morphine for dyspnea in ALS patients. Our experience shows that morphine may be used safely, with evidence that there is no increase in $\mathrm{pCO} 2$ in arterial blood, and effectively - patients evaluated the response as good for $50 \%$, partial for $40 \%$ and only $10 \%$ reported no help. Conclusion: There is continued close working between neurologists and pal- liative care specialists with the aim of integrating palliative care principles in standards of care for ALS patients. There are still many challenges on this way. The reported study was funded by "Live now" charity foundation and RFBR according to the research project № 18-315-00228мол_а

\section{WS 4.1 / \#33}

Topic: 01. Muscle

\section{UPDATE ON HISTOPATHOLOGICAL FEATURES OF INFLAMMATORY MYOPATHIES}

\author{
Anthony Amato \\ Dep. Of Neurology, Brigham and Women's \\ Hospital, Boston, US
}

\begin{abstract}
The major types of inflammatory myopathies (IM), including dermatomyositis (DM), polymyositis (PM), immune-mediated necrotizing myopathy (IMNM), antisynthetase syndrome (ASS), and inclusion body myositis (IBM) The characteristic histopathological abnormality on muscle biopsy in $\mathrm{DM}$ is perifasicular atrophy, however, this is present inperhapsonly $50 \%$ ofpatients. Immunohistochemical staining for myxovirus resistance protein $\mathrm{A}(\mathrm{MxA})$ is diagnostically more sensitive and highly specific. The inflammatory cell infiltrate is predominantly perivascular and in the perimysium and is composed primarily of macrophages, B cells, and plasmacytoid dendritic cells (PDCs). PM is a heterogeneous disorder and muscle pathology is variable. Most often, CD8+ T cells and macrophages are evident in perimysial and endomysial locations. These mononuclear inflammatory cells surround fibers with sarcolemmal MHC-I expression, however true invasion of non-necrotic muscle fibers is much more common in IBM than in PM. The major subtypes of IMNM are anti-SRP and anti-HMGCR necrotizing myopathies, though some are paraneoplastic, associated with other connective tissue diseases, or are idiopathic. The hallmark on muscle biopsy are the presence of multifocal necrotic and regenerating muscle fibers with a paucity of inflammatory cells. However, some patients with HMGCR myopathy have endomysial, macrophage-predominant infiltrates similar to what is seen in PM. Overexpression of MHC-I and membrane attack complex (MAC) deposition in capillaries may be
\end{abstract}


evident on sarcolemma of non-necrotic fibers. In ASS, muscle biopsies demonstrate a predilection for perimysial damage including perimysial fragmentation and staining with alkaline phosphatase, plasmacytoid dendritic cells and macrophages in the perimysium and around blood vessels, and MAC deposition on capillaries. Similar to DM there is perifascicular muscle fiber damage, but with AAS there is more perifascicular muscle fiber necrosis compared to DM, while in perifascicular atrophy is more prominent. MHC-1 and MAC deposits on muscle fibers may be seen on sarcolemma of perifascicular muscle fibers. EM may show actin filaments within myonuclei.

\section{WS 4.2 / \#101}

Topic: 01. Muscle

\section{UPDATE ON MYOSITIS SPECIFIC AUTOANTIBODIES / DISCUSSION ON UTILITY OF MSAS IN DIAGNOSIS AND PROGNOSIS}

Andrew Mammen

NIAMS/NIH, Bethesda, US

Abstract not received.

WS 4.3 / \#115

Topic: 01. Muscle

\section{UPDATE ON UTILITY OF SKELETAL MUSCLE MRI AND ULTRASOUND}

Jasper M. Morrow

Queen Square Centre For Neuromuscular Diseases, University College London, London, GB

Background: Recent advances in inherited muscle diseases have led to two major challenges. The development of next generation sequencing has made interpretation of genetic results very difficult; whilst the development of potentially effective gene therapies has emphasised the need for robust outcome measures to determine their efficacy. Qualitative and quantitative skeletal muscle imaging, in particular MRI is emerging as useful tools to address these challenges. Methods: The speaker will update recent literature on the topic, in addition to discussing his own research and clinical experience through illustrative case studies. Results: Pattern of muscle involvement on qualitative MRI or ultrasound has an important role in clinical phenotyping to help guide and interpret genetic testing in inherited muscle diseases; most notably in the congenital myopathies. Whilst most research has focussed on the lower limbs, whole body MRI is becoming more common. In acquired myopathies, MRI is useful in diagnosis, targeted biopsy and in aiding treatment decisions. The use of imaging in diagnosis will be illustrated through a number of clinical vignettes. Quantitative muscle MRI is able to measure both acute and chronic changes in skeletal muscle providing potential biomarkers in clinical trials. Fat-fraction quantification using methods such as three-point Dixon is the most robust marker of disease progression and has now been applied across a wide range of diseases in both natural history and interventional studies showing superior responsiveness to other outcome measures. Acute changes in muscle water distribution are sensitive to a number of quantitative techniques with T2 quantification being the most commonly used. Recent studies have shown that these changes are reversible with effective therapy, such as corticosteorids in Duchenne. Conclusion: Skeletal muscle MRI and ultrasound are rapidly becoming essential tools in neuromusuclar diseases. Potential avenues for furture research will be discussed.

WS 5.1 / \#120

Topic: 02. Neuropathy

\section{DIAGNOSIS AND TREATMENT OF CIDP: LESSON FROM THE DATABASES}

Eduardo Nobile-Orazio, Giuseppe Liberatore, Pietro Doneddu

Neuromuscular And Neuroimmunology Service, Humanitas Research Hospital, Milan University, Rozzano, Milan, IT

Background: Chronic inflammatory demyelinating polyradiculoneuropathy (CIDP) is a chronic disabling disease that often improves with immune therapy. A few variants of CIDP have been described but their frequency and possible evolution into typical CIDP are unsettled, possibly because of differences in the diagnostic criteria and in the time of 
follow-up. To date, the most reliable diagnostic criteria for CIDP are the 2010 EFNS/PNS revised criteria, with a reported sensitivity of $80 \%$ and specificity of $90 \%$. Methods: We implemented a web-based database to collect data from patients with CIDP followed by Italian Centers with expertise on CIDP to determine the frequency and characteristic of CIDP and it variants, the diagnostic criteria used for their diagnosis, the possible evolution into typical CIDP, the association with specific anti-nerve antibodies, and their response to therapy. All the patients were evaluated at the time of inclusion and will be followed for two years to monitor their outcome and response to therapy. Results: By February 2018 we enrolled 493 patients in the database, 41 of whom were excluded for the presence of an alternative diagnosis (23 patients) or insufficient data (18 patients). The diagnosis of typical CIDP was made in $368(81 \%)$ of the 452 included patients with 305 (83\%) fulfilling the EFNS/PNS criteria. Eighty-four patients $(19 \%)$ patients had a diagnosis of atypical CIDP, 64 (76\%) of whom fulfilled the EFNS/PNS criteria. Thirty-six of these patients $(8 \%$ of included patients) had distal acquired demyelinating symmetric neuropathy (DADS), 17 (4\%) purely motor CIDP, 17 (4\%) Lewis-Sumner syndrome (LSS) including two with focal CIDP, 14 (3\%) purely sensory including two with chronic immune sensory polyradiculopathy (CISP). Based on the symptoms ad disease onset and for at least one year, 39\% of the patients had an initial diagnosis of atypical CIDP that had evolved into typical CIDP in more than 50\% of the patients $(53.5 \%)$ at the time of inclusion after a mean disease duration of 6.5 years. Improvement after therapy was observed in $87 \%$ of the 289 treated patients with typical CIDP including response to IVIg in $74 \%$ of the patients, steroids in $52 \%$, plasma exchange in 53\% and immune suppressant in $37 \%$ with Rituximab being the most frequently effective (70\%). Patients with DADS and LSS had a less frequent response to therapy than those with typical CIDP ( $p=0.0006 ; p=0.0185)$, mainly reflecting a less frequent response to IVIg $(\mathrm{p}=0.0440 ; \mathrm{p}=0.0136)$. Patients with pure motor and sensory CIDP did not differ from typical CIDP. In 63 patients the diagnosis of CIDP was not supported by nerve conduction studies according to the EFNS/PNS diagnostic criteria. In $74 \%$ of them there were however at least two supportive criteria for the diagnosis of CIDP including response to immune therapies (94\%) and increased CSF proteins (82\%). Similarly to typical CIDP, $50 \%$ of the patients with clinical CIDP had a relapsing course increasing the reliability of the diagnosis for CIDP. Conclusion: This study on a large population of patients is providing useful information that may help to revise the current diagnostic criteria for CIDP.

\section{WS 5.2 / \#93}

Topic: 02. Neuropathy

\section{PARAPROTEINEMIC NEUROPATHIES: WHEN AND HOW TO TREAT}

Jean-Marc Léger

National Referral Center For Neuromuscular

Diseases, University Hospital Pitié-Salpêtrière, Paris, FR

\begin{abstract}
Since the first description 25 years ago of a high prevalence of monoclonal gammopathy (MG) in a context of peripheral neuropathy (PN), an increased number of such associations has been reported. However, neuropathies associated with MG have heterogeneous clinical, neurophysiological, neuropathological, and haematological features. The more pertinent relationship seems that found in distal acquired demyelinating sensory (DADS) neuropathy associated with IgM MG of unknown significance (MGUS), and the presence of serum auto-antibodies reacting with myelin-associatedglycoprotein (MAG). However in the absence of adequate data concerning relationship in most of cases, the following good practice points may be agreed: (1) Patients with demyelinating neuropathy (DNP) associated with MG should be investigated for a malignant plasma cell dyscrasia. The MG is more likely to be causing the neuropathy if it is IgM, autoantibodies (mainly directed to MAG) are present in serum or on nerve biopsy, or the clinical presentation is chronic distal sensory neuropathy. DNP associated with IgM MGUS sometimes responds to immune therapies. Their potential benefit should be balanced against their possible side-effects. Rituximab, an anti-CD 20 monoclonal antibody, seems a promising therapy. DNP associated with IgG or IgA MGUS is indistinguishable from chronic inflammatory demyelinating polyradiculoneuropathy (CIDP), on clinical, electrophysiologic, and therapeutic issues. For POEMS (polyneuropathy, organomegaly, endocrinopathy, M-protein, skin changes) syndrome, suited
\end{abstract}


therapy may be followed by dramatical improvement of the neuropathy. (2) Patients with chronic axonal polyneuropathy associated with $\operatorname{IgG}$ MG should be investigated for $\mathrm{Al}$ amyloidosis. However, the majority of chronic axonal polyneuropathies associated with IgG or IgM MGUS are indistinguishable from chronic idiopathic axonal polyneuropathies and do not need any treatment.

\section{WS 5.3 / \#107}

Topic: 02. Neuropathy

\section{POEMS SYNDROME: FROM DIAGNOSIS TO THERAPY}

$\underline{\text { Michelle Mauermann }}$

Mayo Clinic, Rochester, MN, US

Background: Monoclonal gammopathies affect $3-4 \%$ of the population older than 50 years and affect more than $5 \%$ of the population older than 70 years. They reflect a diverse group of disorders that share the secretion a monoclonal Immunoglobulin produced by the bone marrow. POEMS syndrome is a rare paraneoplastic syndrome associated with a monoclonal plasma cell disorder. It frequently presents with a subacute sensorimotor peripheral neuropathy that can progress to a severe polyradiculoneuropathy. The diagnostic criteria for POEMS syndrome require the presence of a monoclonal plasma cell proliferative disorder and a polyneuropathy. Other major criteria include elevated vascular endothelial growth factor (VEGF), Castleman disease and sclerotic bone lesions. There are several minor criteria including organomegaly, extravascular volume overload, endocrinopathy, skin changes, papilledema and thrombocytosis/polycythemia. Methods: In this course we will review the challenges in making the diagnosis of POEMS syndrome. We will also review the clinical, electrophysiological and pathologic features of the peripheral neuropathy associated with POEMS syndrome. We will discuss the rationale behind the treatments available for POEMS syndrome and the future directions in therapy. Results: POEMS syndrome typically presents with a demyelinating sensorimotor peripheral neuropathy that progresses to a polyradiculoneuropathy. It is often mistaken for chronic inflammatory demyelinating polyneuropathy (CIDP). Distinguishing features include older age of onset, infrequent cranial nerve involvement, more severe distal lower extremity involvement and frequent pain. The electrophysiological features show uniform demyelination with less frequent conduction block and temporal dispersion. Recent studies have showed the utility of blink reflexes in demyelinating neuropathies and its utility in assessing treatment responses in POEMS syndrome. The monoclonal plasma cell disorder associated with POEMS syndrome is lambda light chain restricted in greater than 95 percent of patients. Radiologic evaluation for the presence of sclerotic bone lesions is recommended, with $\mathrm{CT}$ providing increased sensitivity over skeletal survey. Currently there is no approved treatment for POEMS syndrome. Radiation is used for patients with limited bone lesions without evidence of systemic disease. For patients with greater numbers of bone lesions or bone marrow involvement, systemic therapy is recommended. Autologous stem cell transplantation has been shown to be beneficial in the improvement of overall survival and progression free survival. Studies of also shown that the peripheral neuropathy improves after autologous stem cell transplantation. In patients ineligible for radiation therapy or autologous stem cell therapy other systemic therapies are available and have been shown to be efficacious. Conclusion: POEMS syndrome typically presents with a subacute painful demyelinating polyradiculoneuropathy. It is differentiated from other monoclonal protein associated disorders by the presence of a lambda monoclonal protein, demyelinating features on nerve conduction studies as well as systemic symptoms. It is important to consider this diagnosis in cases of treatment refractory CIDP. Early diagnosis is necessary to prevent worsening disability. There are several therapies shown to be beneficial in the treatment of POEMS syndrome but there is lack of comparison between these treatments.

\section{WS 6.1 / \#195}

Topic: 07. Cranial Nerves

\section{ULTRASOUND IN CRANIAL NERVES}

$\underline{\text { Stefan Meng }}$

KFJ Hospital, Vienna, AT

Abstract not received. 
WS 6.2 / \#141

Topic: 07. Cranial Nerves

\section{CLINICAL AND MICROSURGICAL SIGNIFICANCE OF CRANIAL NERVE VISUALIZATION}

Andres Rodriguez

Uppsala University, Uppsala, SE

Abstract not received.

WS 6.3 / \#89

Topic: 07. Cranial Nerves

\section{HIGH RESOLUTION MRI OF CRANIAL NERVES}

Jennifer Kollmer

Heidelberg University Hospital, Heidelberg, DE

Abstract not received.

\section{WS 7.1 / \#71}

Topic: 08. Cancer

\section{CACHEXIA AND LATE EFFECTS OF CANCER (FROM AN ONCOLOGICAL PERSPECTIVE)}

Ioannis Gioulbasanis

Chemotherapy, Larissa General Clinic

"E Patsides", Larissa, GR

Background: Cancer cachexia is a syndrome characterized by progressive loss of weight and muscle wasting that eventually leads to functional decline and adversely affects survival. When fully developed, even patients themselves can easily recognize it, as it is associated with major changes in body image. However, a key prerequisite for a successful therapy is the timely initiation of any intervention before the syndrome becomes irreversible. An important limitation, however, is that there are no specific symptoms, signs or laboratory tests that could trigger well-timed clinical attention and diagnosis. Methods: According to our current perception, there is a pre-cachectic stage characterized by anorexia and "minor metabolic changes". Anorexia, however, is a non-specific symptom that may be present in about half of cancer patients at diagnosis and could be attributed to a variety of causative factors that may or may not be related with the pathogenesis of the syndrome. When the syndrome is progressing, reduced food intake (the unavoidable consequence of persistence anorexia) will lead to decrease energy intake. Moreover, systemic inflammation, measured in practice by increased levels of C-reactive protein, is usually present and is associated with increased metabolic rate and energy consumption. These conditions result in negative caloric and protein balance and they can be routinely identified in the context of a detailed nutritional assessment. They also lead to depletion of fat and muscle stores quantified as weight loss $>5 \%$ or $>2 \%$ when either body mass index is $<20 \mathrm{~kg} / \mathrm{m}^{2}$ or sarcopenia is present. Patients at this stage of cachexia may frequently experience fatigue, a symptom causally related to cachexia and, together with anorexia, they composed one of the most prevalent symptom clusters. Results: In the last (refractory) phase, the most characteristic feature is the deterioration of the performance status and patients commonly suffer from asthenia (a subjective sensation of tiredness without any effort) and/or depression. Moreover, because the tumor is no longer responsive to anticancer treatment, many of them experience uncontrolled symptoms like pain and dyspnea and their quality of life deteriorates further. Conclusion: While these aforementioned phases of the syndrome usually cannot be clearly distinguished from one another, most patients with advanced cancer with ultimately develop cachexia.

WS 7.2 / \#130

Topic: 08. Cancer

\section{SARCOPENIA}

Alan Pestronk

Washington University School of Medicine, Neuromuscular Clinic, St. Louis, US

Abstract not received. 
WS 7.3 / \#30

Topic: 08 . Cancer

\section{OUTLINE ON MYOPATHIES / NEUROMUSCULAR COMPLICATIONS ASSOCIATED WITH CANCER, INCL. STEROID MYOPATHY}

Anthony Amato

Dep. Of Neurology, Brigham and Women's

Hospital, Boston, US

\begin{abstract}
Weakness in patients with cancer is often multifactorial. Most common etiology is sarcopenia related to cachexia and disuse. Corticosteroids used to treat edema (brain tumors) can cause type 2 muscle fiber atrophy leading to weakness as well. Dermatomyositis, polymyositis, and autoimmune necrotizing myopathy as well as Lambert-Eaton myasthenic syndrome can be paraneoplastic and these disorders be the presenting manifestation. Checkpoint inhibitors have recently been shown to be associated with autoimmune myopathies and neuropathies. Only rarely have chemotherapies been associated with a direct toxic effect on muscle.
\end{abstract}

\section{WS 7.4 / \#103}

Topic: 08. Cancer

\section{CANCER REHABILITATION}

$\underline{\text { Christine Marosi }}$

Medical University of Vienna, Vienna, AT

Abstract not received.

\section{WS 8.1 / \#118}

Topic: 02. Neuropathy

\section{SPECTRUM OF PARAPROTEINEMIC NEUROPATHIES}

Eduardo Nobile-Orazio

Neuromuscular And Neuroimmunology Service, Humanitas Research Hospital, Milan University, Rozzano, Milan, IT

Background: A consistent proportion patients with neuropathy have a monoclonal gammopathy (or paraprotein) in their serum suggesting a possible pathogenetic link between the two abnormalities. In a series from an highly specialized Center for the diagnosis of neuropathy in the United States, 4\% of patients with neuropathy had a monoclonal gammopathy being the third leading cause of neuropathy after diabetes (31\%) and genetic disease (7\%). A similarly high frequency was observed in an unselected series of patients with neuropathy from Sweden where monoclonal gammopathy was only less frequent than diabetes (19\%), genetic diseases $(12 \%)$, alcohol $(10 \%)$, chronic inflammatory demyelinating polyradiculoneuropathy (CIDP) $(8 \%)$ other toxic neuropathies $(6 \%)$ and connective tissue disorders $(5 \%)$. On the other hand monoclonal gammopathy is frequent in the adult population being found in $3 \%$ of the population above 50 years of age and $7.5 \%$ above 85 years indicating that a coincidental association may also frequently occur. It is therefore important for the neurologist to clarify the pathogenetic significance of this association since the neuropathy may occur in patients with an otherwise asymptomatic monoclonal gammopathy not requiring for the hematologist any treatment. Methods: Neuropathy has been associated with several diseases characterized by the presence of a monoclonal gammopathy being reported in $3-14 \%$ of patients with multiple myeloma, $30-50 \%$ of those with Waldenström's macroglobulinemia (WM), 2-8\% with Lymphoma, and 10-20\% with light chain amyloidosis (AL). In these conditions the treatment is mainly addressed by the hematologist while neurologist are mainly involved in the initial diagnosis, since neuropathy may be the presenting symptom, or in assessing the possible occurrence of neuropathy induced by the chemotherapy used for the hematological disorder. More relevant for the neurologist is the occurrence of neuropathy in patients with monoclonal gammopathy of undetermined significance (MGUS), where the prevalence of symptomatic neuropathy range form $8 \%$ to $36 \%$. In all these studies the prevalence of neuropathy is higher in patients with IgM than with IgG or IgA MGUS, indicating that IgM monoclonal gammopathy are more likely to have a pathogenetic role in the neuropathy. This is also supported by the frequent occurrence of $\operatorname{IgM}$ antibodies against nerve antigens in patients with IgM monoclonal gammopathy that may be found in over $50 \%$ of the patients with neuropathy. Results: An additional condition presenting to the neurologist is the POEMS syndrome, a rare multisystem disease characterized by the presence polyneuropathy $(\mathrm{P})$, organomegaly $(\mathrm{O})$, endocrinopathy $(\mathrm{E})$, monoclonal 
gammopathy (M) and skin changes (S). The pathogenesis of POEMS syndrome is poorly understood but overproduction of vascular endothelial growth factor (VEGF) is considered to play a major role. A severe neuropathy with electrophysiological signs of both axonal and myelin involvement is the predominant manifestation of this syndrome and requires aggressive therapy to prevent a disabling clinical course. Conclusion: We will address in this lecture the therapy used by the neurologist in the treatment of these neuropathies and only briefly mention the therapies used by the hematologist to treat the malignant forms of monoclonal gammopathy.

\section{WS 8.2 / \#153}

Topic: 02. Neuropathy

\section{ANTI-MAG NEUROPATHY: CURRENT AND NOVEL TREATMENTS}

Andreas Steck

Department Neurology, Basel, $\mathrm{CH}$

\begin{abstract}
Anti-MAG (myelin-associated glycoprotein) neuropathy is a chronic disabling demyelinating peripheral neuropathy with an autoimmune etiology. Anti-MAG antibodies are detected in half of IgM paraproteinaemic neuropathy cases. Monoclonal IgM autoantibodies recognize the HNK-1 trisaccharide epitope that is present on MAG as well as on other glycoconjugates of the peripheral nervous system. Electrodiagnostic tests reveal a distal demyelinating polyneuropathy with marked prolonged distal motor latencies. Nerve biopsy shows demyelinating characteristics with pathognomonic features: IgM deposits in the myelin sheaths and widening of myelin lamellae. Anti-MAG antibodies are detected by ELISA. The goal of current therapies is the reduction of pathogenic autoantibodies, the decrease of expanded autoantibody-producing B cell clones, and/or the interference with antibody-effector mechanisms. We will review current and novel treatments. Cytostatics (e.g. cyclophosphamide), plasma exchange (for acute worsening), intravenous immunoglobulin, and the CD20+ B cell-depleting agent Rituximab have been used for the therapy of anti-MAG neuropathy. New anti- B cells drugs such as Ofatumumab, Ocrelizumab, Obinutuzumab and
\end{abstract}

Ibrutinib have been proposed. However, these approaches lack selectivity, and thus so far no satisfactory treatment is available. Because clinical improvement of patients' neuropathic symptoms correlates with reduced serum levels of anti-MAG antibodies and disease worsening is associated with increasing anti-MAG levels during treatment follow-up, a more efficient and safe therapy might be achieved with antigen-specific agents that selectively target anti-MAG IgM antibodies or antibody-producing $\mathrm{B}$ cells. We will present data showing that a synthetic glycomimetic of the natural HNK-1 glycoepitope efficiently remove pathogenic anti-MAG antibodies in an immunological mouse model for anti-MAG neuropathy thus opening a new avenue for treatment. References: Herrendorff R. et al. Selective in vivo removal of pathogenic anti-MAG autoantibodies, an antigen-specific treatment option for anti-MAG neuropathy. Proc Natl Acad Sci U S A. 2017;114(18):E3689-E3698 Pruppers M.H.J. et al. Improving future assessment and research in IgM anti-MAG peripheral neuropathy: A consensus collaborative effort. Neuromuscular Disorders 27 (2017) 1065-1072

WS 8.3 / \#111

Topic: 02. Neuropathy

\section{CREATING PROPER OUTCOME MEASURES}

\author{
Ingemar Merkies \\ Department Of Neurology, Maastricht University \\ Medical Center, Maastricht, $N L$
}

Abstract not received.

WS 9.1 / \#56

Topic: 06. Mononeuropathy

\section{NERVE FIBRE TRANSFER VERSUS NERVE RECONSTRUCTION}

Robert Schmidhammer

Ludwig Boltzmann Institute for Experimental and Clinical Traumatology, Vienna, AT

Abstract not received. 
WS 9.2 / \#81

Topic: 06. Mononeuropathy

\section{NERVE REGENERATION}

$\underline{\text { Ahmet Hoke }}$

Johns Hopkins University School of Medicine, Baltimore, US

Abstract not received.

WS 9.3 / \#179

Topic: 06. Mononeuropathy

\section{BRAIN PLASTICITY IN NERVE REGENERATION}

Thomas Hausner

Trauma Surgery, Lorenz Böhler Trauma Hospital,

Vienna, $A T$

Background: Injuries to the peripheral nervous system induces reorganization of the projections to the Central Nervous System (CNS). This reorganization occurs in the cortex and in different other levels of the CNS, such as the thalamus, brainstem relay nuclei, and spinal cord. In general, this reorganization is referred to as cerebral plasticity or as brain plasticity.Injuries to the peripheral nervous system induces reorganization of the projections to the Central Nervous System (CNS). This reorganization occurs in the cortex and in different other levels of the CNS, such as the thalamus, brainstem relay nuclei, and spinal cord. In general, this reorganization is referred to as cerebral plasticity or as brain plasticity. Methods: Functional recovery after nerve injury and repair relates to the plastic changes in the CNS. The reorganization of the cortex and subcortical structures occur very rapid and therefore influence the outcome of a peripheral nerve repair. To provide cortical reorganization different methods based on the phenomenon of transmodality - converging of a unimodal sensoric input to multimodal association areas of the cortex - have been established. Results: Rosen and Lundborg in 2007 published the "Sensor Glove System" which mediates impulses to the somatosensory cortex through the hearing sense initiated early after surgery. Schmidhammer and his group also in 2007 extended the sensoric input by using both the hearing and the optical sense starting the treatment the day after surgery. The same group could demonstrate recovery of primary activation of the affected hemisphere in a longitudinal functional magnetic resonance imaging (fMRI) observation of cortical somatosensory reorganization. Conclusion: The use of brain plasticity thus is one part of the complex treatment strategies in the repair and rehabilitation after peripheral nerve injuries. The author will give a review of the literature and report clinical experience and results in this field.

WS 10.1 / \#183

Topic: 02. Neuropathy

\section{HOW TRANSTHYRETIN BECOMES TOXIC}

Laura Obici

Fondazione IRCCS Policlinico San Matteo, Pavia, IT

Abstract not received.

WS 10.2 / \#198

Topic: 02. Neuropathy

\section{CHALLENGES IN DIAGNOSING AND MONITORING ATTR AMYLOIDOSIS}

Davide Pareyson

Dept Of Clinical Neurosciences, IRCCS

Foundation, C.Besta Neurological Institute, Milan, IT Abstract: Inherited transthyretin amyloidosis
(ATTR) has become a treatable disorder with effec-
tive therapies available. Early diagnosis is therefore
fundamental for preventing progression in this dis-
ease, lethal if left untreated. Diagnosis is easier in
familial cases in endemic regions, where Val30Met
is the most frequent mutation, degree of awareness is
high, and presentation is typical, with a predomi-
nantly small-fibre length-dependent sensory neurop-
athy with dysautonomia, only later involving motor
fibres. On the other hand, diagnosis is often delayed
in non-endemic region where the neuropathy occurs
late in life, the spectrum of mutations is much wider,
family history if often negative or misleading, signs 
of dysautonomia are subtle, presentation is atypical with a sensory-motor polyneuropathy involving all fibre types, and progression is faster. High degree of clinical suspicion, careful investigation of neuropathy features, of early autonomic manifestations, of carpal tunnel syndrome, and of cardiac, ocular or renal involvement allow prompt identification of the disease and early treatment. Careful monitoring of presymptomatic mutation carriers permits early detection of the first symptomatic phases of the disease. Systematic and homogenous follow up of treated patients, with common core protocols, will allow the collection of data useful to optimize treatment and select the best therapeutic options according to disease type and stage. Improvement of clinical and paraclinical outcome measures is a challenging task. The currently used types of Neuropathy Impairment Score (NIS) scores should be unified and simplified. Linear scales should be designed. Glomerular filtration rate (GFR) seems to be a reliable biomarker for disease staging and possibly for monitoring disease evolution, to be added to Brain Natriuretic Peptide (BNP) as a marker of cardiac disease. mBMI is another important parameter easy to calculate. Standardized nerve conduction studies, nerve echography, nerve MRI, skin biopsy, echocardiography, cardiac scintigraphy, cardiac MRI, autonomic function tests are all important tools for diagnosis and monitoring disease course during treatment.

\section{WS 10.3 / \#194}

Topic: 02. Neuropathy

\section{THE NOVEL SCENARIO OF ATTR AMYLOIDOSIS TREATMENT}

Teresa Coelho

Hospital de Santo Antonio, Porto, PT

Abstract not received.

WS 11.1 / \#918

Topic: 09. Systemic Diseases

\section{VITAMIN DEFICIENCIES AND NEUROPATHY}

Carrie K. Grouse

Neurology, University of California San Francisco, San Francisco, US
Background: Vitamin deficiencies may result in neurologic deficits, including neuropathy. Methods: Review of published literature on the topic of vitamin deficiencies and neuropathy. Results: Vitamin B12 (cobalamin), B1 (thiamine), B6 (pyridoxine), and vitamin $\mathrm{E}$ (alpha-tocopherol) are all essential vitamins that can become deficient, causing a peripheral neuropathy. Conclusion: It is important for neurologists to recognize the role of vitamin deficiencies in causing treatable sensory and motor neuropathies.

WS 11.2 / \#217

Topic: 09. Systemic Diseases

\section{MINERAL DEFICIENCIES AND NEUROPATHY}

\author{
$\underline{\text { Steven L. Lewis }}$ \\ Neurology, Lehigh Valley Health Network, \\ ALLENTOWN, PA, US
}

Background: Mineral deficiencies may result in various neurologic manifestations including neuropathy. Methods: Review of published literature on the topic of mineral deficiencies and neuropathy. Results: Copper deficiency may cause a primarily sensory axonal polyneuropathy in addition to a myelopathy; optic neuropathy can also occur. Hypophosphatemia, occurring due to intravenous hyperalimentation without inorganic phosphate, has been associated with an acute sensorimotor polyneuropathy resembling Guillain-Barre syndrome. Molybdenum deficiency has been reported to be associated with a motor neuron disorder in sheep. Conclusion: Although less common than vitamin deficiencies as a cause of neuropathy, it is important for neurologists to recognize the role of some mineral deficiencies in causing treatable peripheral and optic neuropathies. 
WS 11.3 / \#223

Topic: 09. Systemic Diseases

\section{VITAMIN AND MINERAL TOXICITIES AND NEUROPATHY}

\section{Nathan Staff}

Neurology, Mayo Clinic, Rochester, MN, US

Background: Peripheral nerves are uniquely susceptible to a variety of toxic insults, which is likely attributable to the metabolic demands on these neurons that span up to 1 meter in length. Peripheral neuropathy due to vitamin and mineral toxicities has been reported due a number of environmental, medicinal, and industrial exposures. While advances in public and occupational health have rendered many of these causes as very rare, these important and preventable etiologies may be overlooked in clinical practice if not considered in the differential diagnosis of peripheral neuropathy. Methods: Literature review was performed. Results: While most vitamins lead to neurological disease due to their deficiency, vitamin B6 leads to neurotoxicity from overuse and presents as a sensory-predominant axonal neuropathy or neuronopathy. Neurotoxicity from heavy metals arises from exposure to lead, mercury, inorganic arsenic, or thallium. Heavy metal neurotoxicity often has multisystem involvement, including abnormalities in the central nervous system (lead, mercury), integument (lead, arsenic, thallium), or gastrointestinal system (lead, arsenic, thallium). The peripheral neuropathy in heavy metal intoxication is variable and may range from motorpredominant (lead), sensory-predominant (mercury, arsenic, thallium), or polyradiculoneuropathy (arsenic, thallium). Treatment for heavy metal intoxication includes removal of exposure and chelation. Conclusion: Peripheral neuropathy due to vitamin and mineral toxicities continues to be a rare, but important cause of peripheral neuropathy. Oftentimes, these toxic neuropathies occur in the setting of multisystem disease, which may be a clue to the etiology. Furthermore, understanding the pathomechanisms of these rare neuropathies can provide important insights more broadly into peripheral nerve biology.
WS 12.1 / \#165

Topic: 03. Motor Neurone

\section{MULTIDISCIPLINARY CLINICS- WHAT IS THE EVIDENCE AND HOW CAN WE DO BETTER?}

Leonard Van Den Berg

Department Of Neurology, University Medical

Center Utrecht, Utrecht, $N L$

Abstract not received.

WS 12.2 / \#77

Topic: 03. Motor Neurone

\section{MEASUREMENT OF DISEASE PROGRESSION, CAN WE DO BETTER?}

Orla Hardiman

Trinity College Dublin, Dublin, IE

Abstract not received.

WS 12.3 / \#113

Topic: 03. Motor Neurone

\section{REHABILITATION INTERVENTIONS THAT CHANGE OUTCOME IN ALS/ MND}

Presenter TBD

WS 13.1 / \#1030

Topic: 08. Cancer

\section{CRANIAL NERVES}

Wolfgang Grisold $^{1}$, Anna Grisold ${ }^{2}$, Stefan Meng ${ }^{3}$

${ }^{1}$ Ludwig Boltzmann Institute for Experimental und Clinical Traumatology, Vienna, AT; ${ }^{2} D e p$.

Neurology, Allgemeines Krankenhaus Vienna, Vienna, AT; ${ }^{3}$ Institute for Radiology, KFJ hospital, Vienna, $A T$

Abstract: In cancer patients the cranial nerves (CN) can be affected by several mechanisms as infections, 
therapy related, and neoplastic. They can present as singular, or multiple lesions. Infections: Herpes Zoster is the most prominent infection in immune compromised patients. Usually the trigeminal nerve is involved resulting in sensory loss and pain. In caudal $\mathrm{CN}$ lesions local mucosatoxic effects need to be excluded. Therapy related effects can be classified into radiotherapy (RT) induced damage, damage by surgical interventions and embolization and a spectrum of chemotherapy induced effects as dysgeusia, which is prominent for hedgehog pathway inhibitors. Motor dysfunction is rare, but has been observed in vinka alkaloids. Neoplastic involvement within the skull is often caused by neoplastic CSF involvement, but also focal tumor seeding. Metastases at the base of the skull, and also in the major cavities present with pain in combination with $\mathrm{CN}$ lesions. Also skin tumor may spread retrogradely into the CNS. A very important model for $\mathrm{CN}$ lesions is the neoplastic spread via $\mathrm{CN}$, either anteroor retrogradely. This mechanism is most frequently observed in ENT tumors. In addition to clinical neurological examination imaging techniques are the most useful tool to determine the site and cause of the $\mathrm{CN}$ lesions. For the extracranial parts of the $\mathrm{CN}$, increasingly High frequency ultrasound is useful.

\section{WS 13.2 / \#64}

Topic: 08. Cancer

\section{PERIPHERAL NERVE LYMPHOMA}

\section{P. James B. Dyck}

Mayo Clinic, Rochester, US

Background: Lymphoma can involve almost any organ in the body including the nervous system. Neurolymphomatosis (NL) is a rare presentation of lymphoma involving the peripheral nerves. NL can be subdivided into those patients in whom the lymphoma began in the peripheral nervous system (primary NL) and those in whom it began in another organ system but spread to peripheral nerves (secondary NL). Methods: We have studied NL in terms of clinical, electrophysiological, radiographical and pathological features in a cohort of Mayo Clinic patients with NL proven by nerve biopsy. Results: We identified 48 NL patients (28 primary and $20 \mathrm{sec}-$ ondary). The most common presentations were asymmetric, painful radiculoplexus neuropathy
(37.5\%), polyradiculoneuropathy $(23 \%)$ and multiple or single mononeuropathy (18\%). Primary and secondary NL present similarly with primary NL having longer symptoms duration (10 vs. 6 months, $\mathrm{p}<0.001)$, single disease episodes $(96 \%$ vs. $35 \%$, $\mathrm{p}<0.001)$, more disease remission ( $58 \%$ vs. $20 \%$, $\mathrm{p}=0.056)$ and lower rates of mortality $(25 \%$ vs $67 \%$, $\mathrm{p}=0.054)$. 11 of the 42 patients had electrophysiology showing demyelination. Nerve biopsies diagnostic of lymphoma were distal cutaneous (sural 9/11) or proximal targeted fascicular (35/35) nerves. MRI was better identifying lymphoma than CT or PET ( $84 \%$ vs. $46 \%$ vs. $58 \%$ ). The lymphoma types were B-cell (46/48) and rarely T-cell (2/48) with diffuse large B-cell most common. Teased fiber showed increased rates of demyelination $(19 / 24)$, increased rates of axonal degeneration (18/24) and increased numbers of empty nerve strands $(9 / 24)$. The location of nerve lymphoma within the nerve was most commonly in the endoneurium (43/48), followed by epineurial (29/33). Clinical and pathological findings of primary and secondary NL were similar except secondary NL had a shorter duration of neuropathic symptoms ( 8.5 vs 4.5 months, $\mathrm{p}=0.01$ ), more frequent other organ involvement $(\mathrm{p}=0.045)$, more axonal degeneration $(31 \%$ vs. $7 \%, p=0.005)$, less favourable response to treatment and a higher mortality rate. Conclusion: 1) peripheral nerve lymphoma (NL) is a multifocal painful neuropathy that causes endoneurial inflammatory demyelination; 2) primary NL presents as a single episode whereas secondary NL often presents after remission of the lymphoma. These findings suggest that the nerve may act as a "safe-haven" for NL as most chemotherapeutic agents don't cross the blood nerve barrier and 3) primary NL is less severe with longer duration, less axonal degeneration, more favourable response to treatment and higher survival rates.

WS 13.3 / \#270

Topic: 08. Cancer

\section{CANCER-ASSOCIATED MYOSITIS}

Yves Allenbach

Department Of Internal Medicine And Clinical Immunology, APHP Pitié-Salpêtrière Hospital, Paris, FR

Abstract not received. 
WS 14.1 / \#143

Topic: 04. Neuromuscular Junction

MOLECULAR MECHANISMS

AFFECTING NEUROMUSCULAR JUNCTION DEVELOPMENT AND MAINTENANCE

Markus Rüegg

University Of Basel, Basel, CH

Abstract not received.

WS 14.2 / \#193

Topic: 04. Neuromuscular Junction

\section{SALBUTAMOL AND DOK-7 AS \\ POTENTIAL TREATMENT OPTIONS FOR NEUROMUSCULAR DISORDERS}

David Beeson

Nuffield Department Of Clinical Neurosciences, University of Oxford, Oxford, GB

Abstract not received.

\section{WS 14.3 / \#142}

Topic: 04. Neuromuscular Junction

\section{SYMPATHETIC INNERVATION OF THE NMJ; A POSSIBLE EXPLANATION FOR THE THERAPEUTIC EFFECT OF SYMPATHICOMIMETICS IN NMDS?}

Rüdiger Rudolf

Institute Of Molecular And Cell Biology, Mannheim University of Applied Sciences, Mannheim, DE

Background: Recently, sympathicomimetic drugs, such as albuterol or salbutamol, have been increasingly used to treat a wide range of congenital myasthenic syndromes. However, the rationale underlying the effectiveness of these drugs has remained elusive. Methods: The distribution of sympathetic neurons in skeletal muscle was investigated with immunofluorescence and confocal microscopy. Functional interaction between sympathetic neurons and skeletal muscle was investigated by combined electrical stimulation of sympathetic ganglia and in vivo two-photon microscopy. Effects of local chemical sympathectomy or congenital myasthenic syndrome on muscle atrophy and NMJ function and morphology as well as their rescue by systemic treatment with sympathicomimetics, were probed using in vivo confocal microscopy and measurement of compound muscle action potentials. Results: We found that sympathetic neurons form a dense network in skeletal muscle already in the newborn mouse and make close contact with neuromuscular junctions. Functionally, they seem to connect different targets including blood vessels, motor neurons, and muscle fibers. Electric stimulation of sympathetic neurons triggered immediate activation of muscle postsynaptic $\beta 2$-adrenoreceptor (ADRB2), cAMP production, and nuclear import of PGC1alpha. Systemic treatment with the sympathicomimetic clenbuterol rescued electrophysiological and morphological defects of neuromuscular junctions as well as muscle atrophy upon local chemical sympathectomy and in myasthenic mice. Conclusion: In summary, this work shows intense sympathetic innervation of skeletal muscle that plays functions beyond the control of blood flow. Indeed, it appears to be of fundamental importance for muscle trophism and regulates the functionality and maintenance of neuromuscular junctions. Sympathetic innervation of skeletal muscle could be a link to the understanding of the therapeutic effects of sympathicomimetics in congenital myasthenic syndromes.

WS 15.1 / \#178

Topic: 01. Muscle

\section{PLECTIN: OVERVIEW AND LESSONS FROM MOUSE MODELS}

Gerhard Wiche

Biochemistry And Cell Biology, University of

Vienna-MFPL, Vienna, AT

Background: Plectin is the prototype of a family of proteins known as cytolinkers, or cytoskeletal linker proteins. Mainly associating with intermediate filaments (IFs) and acting as a cytoskeletal crosslinker, plectin strengthens cells mechanically. Plectin's 
large size of $>500 \mathrm{kDa}$, multi-modular surface structure, and oligomerization potential enable it to undergo a broad spectrum protein-protein interactions that dictate its spatial localization, regulate cellular dynamics, and create cytoskeleton based signaling scaffolds. Plectin binds not only to all types of IFs, the actomyosin network system, and microtubules, but also to transmembrane receptors, proteins of the subplasma membrane protein skeleton, components of the nuclear and mitochondrial envelopes, and signaling proteins such as kinases with key roles in migration, proliferation, and energy metabolism of cells. Due to alternative splicing, plectin is expressed as multiple isoforms bearing distinct $\mathrm{N}$-terminal head domains that dictate the differential subcellular targeting of the isoforms. Through specific interactions with proteins at their target sites and their highaffinity binding to IFs of all types, distinct plectin isoforms provide site-specific and robust IF-docking sites. Plectin isoforms are thus central to IF network organization, compartmentalization, and connectivity. In fact, the functional repertoire of IF networks in different cell types is largely dependent on the type and expression levels of individual plectin isoforms. IF anchorage has profound effects on the cytoplasmic microenvironment, including the redirection of actomyosin-driven forces and the repression of microtubule networks. Moreover, plectin isoform-mediated linkage of peripheral junctional complexes, interior organelles, and specialized cellular machineries (e.g. the contractile apparatus of muscle cells) to the IF network creates a tensionsensitive communication system which is key to mechanotransduction. Methods: To decipher plectin's diverse functions and role in disease, we have generated a large collection of genetically altered mouse lines, comprising full-knockouts (null-KOs), tissue/cell type-restricted conditional-KOs, isoformspecific KOs, double-KOs, and knock-ins. This unique assemblage of mouse models serves as a powerful tool for studying consequences of plectin gene defects on the organismal, cellular, and molecular levels. Results: As constituent and interaction partner of vital cellular elements, dysfunctional plectin leads to multiple disorders and diseases, some of which are multi-systemic, i.e. affecting more than one tissue and cell type. The most common disease caused by plectin deficiency is epidermolysis bullosa simplex (EBS)-MD, an autosomal-recessive skin blistering disorder with late-onset muscular dystrophy. Other subtypes of EBS, EBS-PA and EBS-Mys, manifest with pyloric atresia and congenital myasthenia, respectively, and a dominant subtype, EBS-Ogna, results in a skinonly phenotype. Mutations in isoform-specific sequences of the plectin gene can cause either muscular dystrophy without skin phenotype, or skin blistering without muscular phenotype, in accordance with the isoforms' specific functions. Conclusion: The analysis of mouse mutants revealed plectin to play an essential role in maintaining the integrity of the neuromuscular system, including the contractile apparatus of myofibers, the neuromuscular synapse, myonuclei and mitochondrial networks, as well as motor neuron and Schwann cell dependent functions. Furthermore, the role and pathomechanisms involving individual isoforms could be dissected and advances were made towards the development of treatment and therapeutic strategies of musclerelated plectinopathies using mutant mice-derived immortalized cell cultures.

\section{WS 15.2 / \#184}

Topic: 01. Muscle

\section{HUMAN PLECTIN AND CLINICAL ASPECTS}

\author{
Rolf Schröder \\ Neuropathology, University Hospital Erlangen, \\ Erlangen, $D E$
}

Background: Plectin, a multi-functional linker protein of exceptionally large size $(>500 \mathrm{kDa})$, is abundantly expressed in a wide range of mammalian cells and tissues, most prominently in muscle, brain and stratified squamous epithelia. Mutations in the human plectin gene (PLEC) on chromosome 8q24 cause a variety of rare human disorders (referred to as "plectinopathies"), namely autosomal recessive epidermolysis bullosa simplex with muscular dystrophy (EBS-MD), EBS-MD with myasthenic features (EBS-MD-MyS), limb girdle muscular dystrophy (LGMD) type 2Q (LGMD2Q), EBS with pyloric atresia (EBS-PA), and the autosomal dominant variant EBS-Ogna. Methods: Clinical, genetic, myopathological and biochemical analyses of patients with plectinopathies. Results: In addition to an overview on the broad spectrum of clinical presentations of plectinopathies, this lecture will focus on the essential features of plectin-related human skeletal muscle pathology. Furthermore, recent 
advances in our current understanding of the molecular pathogenesis of human plectinopathies will be discussed in relation to future targeted treatment concepts. Conclusion: Plectin has an essential role for the structural and functional integrity of human skeletal muscle tissue. To date, mutations in the plectin gene account for three distinct forms of human myopathies. Given the recent advances of next generation sequencing approaches, more plectin-related myopathies and cardiomyopathies are likely to be discovered.

\section{WS 15.3 / \#191}

Topic: 01. Muscle

\section{PLECTIN AND MITOCHONDRIA (MICE AND MEN)}

\author{
Lilli Winter \\ Center For Anatomy And Cell Biology, Medical \\ University of Vienna, Vienna, AT
}

Background: Plectin, a 500-kDA multifunctional cytolinker protein, interlinks intermediate filaments (IFs) with each other and anchors them to sites of strategic importance for the organization and function of cells. Plectin's versatility is in part due to complex splicing events in the $\mathrm{N}$-terminal region of its gene (PLEC), giving rise to a multitude of alternatively spliced isoforms containing different first exons. In skeletal muscle, plectin is essential for myofiber integrity and function: by specifically targeting and anchoring desmin IFs to Z-disks (plectin 1d, P1d), costameres (P1f), mitochondria (P1b), and the nuclear/ER membrane system (P1), the musclespecific set of plectin isoforms determines the cytoarchitecture of the IF network and thereby ensures the precise alignment of sarcomers. Accordingly, mutations in the human plectin gene cause epidermolysis bullosa simplex with muscular dystrophy (EBS-MD), an autosomal recessive skin blistering disorder associated with progressive muscle weakness. In addition, PLEC mutations lead to EBS-MD with myasthenic syndrome, EBS with pyloric atresia, limb-girdle muscular dystrophy type $2 \mathrm{Q}$, or EBS-Ogna. With skeletal muscles harboring desmin-positive protein aggregates, degenerative changes of the myofibrillar apparatus, and mitochondrial abnormalities, most plectinopathies can be annotated among the expanding group of myofibril- lar myopathies (MFM). All MFM show a progressive clinical course, lead to severe physical disability and premature death. The knowledge of the precise molecular mechanisms that translate MFM-causing gene mutations into the myopathic phenotype is still limited, but critical for the understanding of patients' needs and the development of treatments. Methods: To assess the mitochondrial pathology of plectinopathy patients, skeletal muscle tissues derived from three EBS-MD patients were analyzed for mitochondrial abnormalities, including altered subcellular localization and expression of respiratory chain enzymes. In addition, to better understand the mechanisms behind mitochondrial defects in plectinopathies and to dissect the role of individual plectin isoforms, we comparatively analyzed morphological and functional characteristics of mitochondrial networks in skeletal muscle tissues and teased muscle fibers derived from wild-type, muscle-specific conditional plectin knockout (MCK-Cre/cKO), and plectin-isoform-specific knockout mice, lacking just one isoform (either P1b or P1d) while expressing all others. Results: In muscle sections from EBS-MD patients and plectin-deficient mice, mitochondrial content appeared reduced. Mitochondria were abnormally distributed within muscle fibers, aggregated, and no longer associated with the Z-disks. Mitochondrial clusters were observed in sarcoplasmic and subsarcolemmal regions. Additionally, decreased citrate synthase activity, decreased mitochondrial respiratory functions, and altered ADP kinetics were detected in plectin-deficient mouse muscles. By comparing mitochondrial networks in muscle sections and teased muscle fibers derived from isoform $\mathrm{P} 1 \mathrm{~b}$ or $\mathrm{P} 1 \mathrm{~d}$-specific knock-out mice, we found plectin-isoform-dependent morphological alterations of mitochondria. In P1d-KO muscle, where Z-disks appeared misaligned, mitochondria lost their regular cross-striated staining pattern, but remained in close contact to the Zdisks. In contrast, mitochondria in P1b-KO muscle were more elongated and loosely arranged around the Z-disks; however, no Z-disk misalignment occurred in this case. Conclusion: Plectin (isoform) mutations dramatically affect the subcellular distribution and biochemical properties of mitochondria in men and mice. The depletion of distinct plectin isoforms affected mitochondrial network organization in different ways. 
WS 15.4 / \#192

Topic: 01. Muscle

\section{PLECTIN, CARDIOMYOPATHIES, AND THERAPEUTIC APPROACHES (MICE)}

\author{
Oliver Mueller \\ University Of Kiel, Kiel, DE
}

Abstract not received.

WS 16.1 / \#292

Topic: 01. Muscle

\section{THE CLINICAL PHENOTYPES OF CALPAINOPATHY}

Andoni Urtizberea

Aphp - Filnemus, Hendaye, FR

Abstract: Primary calpain deficiency, or LGMD2A, is characterized by a selective, slowly progressive involvement of proximal muscles of pelvic and scapular girdles. In most LGMD2A patients, the typical overall picture corresponds to the clinical phenotype originally described by W. Erb in 1884 and revisited by M. Fardeau in the 1980s in a genetic isolate of patients living in the Reunion Island. The onset of weakness occurs in the early teens, in most cases in the lower limbs rather than in the upper limbs. Difficulties in running or in climbing stairs, tiptoe walking and scapular winging are classical symptoms/signs at presentation. Marked contractures in elbows or ankles, and muscle pseudo-hypertrophy may exist but are rarely observed in the first years. Muscle weakness and wasting are noted predominantly in the the scapular fixators, the biceps brachialis, the glutei muscles as well as in the posterior compartment of legs and thighs suggesting a high selectivity, a clue that is usually confirmed on muscle imaging. A gradual drop of the forced vital capacity (FVC) may be noted i a few patients especially after they turn wheelchair bound (on average 20-30 years after onset). Primary cardiac compromise has never been reported to date allowing these patients to live long. The pace of functional deterioration remains variable but overall seems slow as established by a natural history of disease studied on a four-year duration. A number of clinical variants of LGMD2A have been described in the literature. Pseudometabolic forms include exercice intolerance and myalgias. Unusual cases have been reported at both ends of the age spectrum: benign cases can present with very mild, late-onset non-progressive muscle weakness and moderately high CK levels whereas early onset, even before age 5 years, and rapid loss of ambulation are also observed. Eosinophilic myositis presentation is rare, often misleading and most probably not specific to LGMD2A. Although an autosomal recessive mode of inheritance remains the rule, a very few cases of dominantly transmitted calpainrelated LGMD have recently been reported. Similarly cases where marked intra-familial variability are not that exceptional. Likewise, when it comes to compare the phenotypes between families harbouring the very same genotype suggesting the existence of modifying and/or epigenetic factors. With a wider, easier access to NGS studies, it is likely that the spectrum of these clinical exceptions to the rule will expand over time.

\section{WS 16.2 / \#54}

Topic: 01. Muscle

\section{MUSCLE PATHOLOGY OF LIMB- GIRDLE MUSCULAR DYSTROPHIES (LGMD)}

Kristl G. Claeys

Neurology, University Hospitals Leuven and $K U$

Leuven, Leuven, BE

\begin{abstract}
Limb-girdle muscular dystrophies (LGMD) are a heterogeneous group of rare, genetic and progressive muscle disorders, characterized by weakness and atrophy of predominantly pelvic and shoulder girdle muscles. The inheritance pattern in LGMD is mostly autosomal recessive (LGMD2), and less frequently autosomal dominant (LGMD1). So far, 26 subforms of LGMD2 (LGMD2A-2Z) and 8 subtypes of LGMD1 (LGMD1A-1H), caused by respectively two or one mutation(s) in mostly distinct genes, have been identified. In most LGMD patients, creatine kinase (CK) level in serum is elevated. To date, pathomechanisms in LGMD are not well understood and curative treatments do not exist. Muscle biopsy in LGMD usually shows dystrophic changes consisting of necrotic and regenerating
\end{abstract}


muscle fibers. Further histopathological abnormalities are increased variability in fiber diameter, elevated number of internalized nuclei and increased amount of endomysial connective tissue. Besides the general findings of necrosis and regeneration, additional pathological features can be identified in the muscle biopsy of some LGMD subforms, such as lobulated fibers in LGMD2A, eosinophils in LGMD2A, amyloid deposits in myofibers and the perivascular area in LGMD2B and LGMD2L, rimmed vacuoles in LGMD2G, LGMD1A, LGMD1D, LGMD1F and LGMD1G, and protein aggregates in LGMD1D. Perivascular and/or endomysial inflammation can be observed in some biopsies of LGMD2A, LGMD2B, LGMD2C, LGMD2I or LGMD1A patients. In some cases, the inflammatory infiltrates can even be associated with up-regulation of the major histocompatibility class 1 antigen (MHC-I), which might lead to erroneous treatment of these genetic muscle diseases with immunosuppressive agents. Also the presence of mitochondrial abnormalities in some LGMD subforms, such as LGM$\mathrm{D} 1 \mathrm{H}$, can lead to a wrong diagnosis of mitochondrial disease. Immunohistochemistry and immunoblotting with antibodies directed against distinct affected proteins are very helpful in the diagnosis of some LGMD subforms. Furthermore, ultrastructural findings can also add important information to the diagnosis in a few LGMD subtypes, such as the presence of sarcolemmal defects with replacement of the membrane by layers of small vesicles in LGMD2B and LGMD2L, or a reduction in caveolae in LGMD1C. With the novel massive parallel sequencing technologies rapidly entering clinical practice, there is more and more a shift in the order of diagnostic examinations to be expected. In the traditional diagnostic work-up of patients with muscle disease, a muscle biopsy is performed followed by Sanger sequencing of single candidate genes. Because of the high diagnostic rates of neuromuscular gene panels and wholeexome sequencing (WES) and their favorable costeffectiveness, these novel massive parallel sequencing technologies are increasingly being used even before a muscle biopsy is performed. However, muscle histopathology will remain very important to study pathogenicity of the large number of variants that frequently evolve from these large genetic analyses.
WS 16.3 / \#117

Topic: 01. Muscle

\section{THE ADVENT OF NGS IN LGMD DIAGNOSIS}

\author{
Vincenzo Nigro $^{1}$, Marco Savarese ${ }^{2}$, Annalaura \\ Torella $^{1}$ \\ ${ }^{1}$ Precision Medicine, Università degli Studi della \\ Campania "Luigi Vanvitelli", Naples, IT; ${ }^{2}$ Medical \\ Genetics, Medicum, Folkhalsan Institute of \\ Genetics, Uni Helsinki, Helsinki, FI
}

Background: Next generation sequencing (NGS) has revolutionized our approach to genetic disorders, from a single gene testing, to genomic studies. This is especially true for heterogeneous conditions with overlapping phenotypes, like limb girdle muscular dystrophies. Methods: Three different NGS strategies for DNA sequencing have been developed: a targeted re-sequencing of specific regions/ genes of interest captured by specific probes or amplified by PCR; a whole exome sequencing (WES) targeting the whole set of coding regions (about $1.5 \%$ of the human genome); and a whole genome sequencing (WGS) which has no preselection of DNA fragments. Different targeted approaches have been described so far. In particular, we originally developed a strategy based on Haloplex selection MotorPlex) and capture of 89->270 genes characterized by a megabase size of the targeted regions. Using that approach about $50 \%$ of $>500$ undiagnosed LGMD were solved. However, the further reduction of sequencing costs provides us the opportunity to move towards WES: a unique test able to investigate, at the same time, both disease-genes and novel candidate ones. Results: Patients with undiagnosed LGMD undergo a standardized annotation using Phenotips and selected cases are recruited for trio/ quartet whole exome analysis. We adopted a complete whole exome enrichment with a target of $\sim 69 \mathrm{Mb}$ and a coverage of $\sim 200 \mathrm{x}$. Results are shared mainly using Phenome Central to recognize similar patients with the same genetic disease. We also optimized the bioinformatic pipeline thank to a collaboration with the Telethon Undiagnosed Program. In addition, we developed a next approach for the still negative cases. We designed a "ultra exome" probe collection to be used in connection with the $10 \mathrm{x}$ Genomics. This produces a partitioning of high molecular weight DNA fragments (HMW-gDNA) into 
micelles, along with an adapter molecule and a barcode sequence. With this strategy, WES are fully covered and phased and even small structural variations may be detected. Conclusion: Unbiased NGS methods (like WES or WGS) may represent the first step of a comprehensive diagnostic workflow for LGMD, including traditional investigations, such as muscle biopsies and electromyography, and more specialized procedures like ultrasound muscle imaging and magnetic resonance imaging.

\section{WS 16.4 / \#35}

Topic: 01. Muscle

\section{PERSPECTIVES IN CLINICAL TRIALS OF RECESSIVE AND DOMINANT LGMD}

\section{Corrado Angelini \\ IRCCS S.Camillo Hospital, Venice, IT}

Background: The term Limb Girdle Muscular Dystrophy(LGMD) defines a progressive weakness with onset in the proximal limb girdle muscles, with age at onset of symptoms varying from early childhood(not congenital)to late adulthood. The progression of muscle weakness is usually symmetrical and variable among individuals and genetic type. These disorders present a wide spectrum of muscle involvement and wasting, spanning from very severe forms with childhood onset and rapid progression, to relatively benign forms with late onset. Methods: A consortium meeting, under the auspices of the European Neuromuscular Center, introduced a classification of LGMD based on molecular/genetic criteria. The autosomal dominant loci were designated as LGMD1, and the autosomal recessive loci were designated as LGMD2. LGMD nomenclature adopted a progressive alphabetical letter indicating the order of gene mapping identification. A new classification was proposed in an ENMC meeting held in 2017 naming autosomal dominant LGMD D and recessive forms $\mathrm{R}$ and numbering them. This new classification has included dystrophies with proximal or distoproximal features, high $\mathrm{CK}$ presentation with histopathological evidence at biopsy of fiber degeneration/regeneration, fiber splitting, MRI imaging consistent with degenerative changes, fibro-fatty infiltration. In differential diagnosis appear the inflammatory myopathies, myofibrillar myopathies and metabolic myopathies, which can be excluded on the basis of clinical features muscle histopathology and laboratory exams. Results: LGMDs constitute a considerable fraction of all dystrophic patients and their prevalence ranges from about 8 to 70 cases per million inhabitants(1:123,000-1:14,500) depending on the geographical area and ethnic origin. The frequency of each form of LGMD is variable in different populations Calpainopathy is the most prevalent form in the majority of countries, its frequency ranges from about $10 \%$ of LGMD cases in the United States , to $80 \%$ in Basque country. Calpainopathy and Dysferlinopathy account for about $50 \%$ of total cases and primary sarcoglycanopathies are about 10$15 \%$ of cases.LGMD2I is the most common form in Northern Europe. To perform clinical trials similar pathomechanisms and homogeneous phenotypes are required. The clinical phenotypes due to mutation in the LGMD genes include instead severe childhoodonset forms, distal and proximal myopathies, pseudo-metabolic myopathies, eosinophilic myositis, and hyperCKemia. It is crucial to identify suitable selection criteria to be used in trials when treating patients after identifying defective for genes responsible for LGMD. Nowadays therapy is still an unresolved problem in LGMD mostly limited to rehabilitation, clinical follow-up of cardiological and respiratory complications.Several drugs have been tested such as corticosteroids and myostatin inhibitors with variable success. There is active pursue of new drugs and of genetic and cell therapy and a number of clinical trials are undergoing development. Conclusion: LGMDs are relatively rare and clinical trials should be done in homogeneous groups of patients after careful study of outcomes. About $8 \%$ of patients with a diagnosis of LGMD may actually have FSHD, and misdiagnosis can occur. Muscle imaging (CTscan and MRI)may be helpful to characterize the severity at the pattern and distribution of muscle wasting and in follow up of LGMDs. Outcome measures for each clinical subtype should be carefully studied and tailored for each clinical trial.

\section{WS 17.1 / \#76}

Topic: 09. Systemic Diseases

\section{PRIMARY AND SECONDARY VASCULITIC NEUROPATHY}

Gerard Said

Institut Vernes, PARIS, FR 
Abstract: Ischemic nerve lesions occur in primary or secondary vasculitis. The consequences of vascular inflammation and occlusion depend on the size and number of blood vessels affected. Clinical neuropathy occurs in more than 75 per cent of the patients with systemic vasculitis of the PAN group. Typically the clinical picture is that of an acute or subacute mononeuritis multiplex, with successive or simultaneous involvement of multiple nerve trunk territories over days, weeks or months. Distal symmetrical sensory or sensorimotor neuropathy also occurs. The peroneal nerve is the most commonly affected nerve. The onset of the neuropathy is typically abrupt and the deficit severe, but in many cases only partial deficit in a nerve territory is observed. Recovery from motor deficit due to ischemic neuropathy takes months, because of the axonal pattern of nerve lesions. Residual pains may be difficult to differentiate from relapses of the neuropathy. In polyarteritis nodosa (PAN) the ischemic neuropathy induced by NA can be observed as an isolated manifestation, or in the context of a multisystemic disorder. In classic PAN cutaneous vasculitis is the most common non neurological manifestation. Arthritis, renal involvement and asthma are present in 10\% of patients on average. Biological markers of inflammation including CRP and ESR, increased platelet and white blood cell count with eosinophilia are often markedly elevated, but remain normal in nearly $30 \%$ of the patients seen in neurology. Churg and Strauss syndrome : In the CSS, disseminated necrotizing vasculitis occurs among asthmatic patients, with fever, eosinophilia, and a fulminant multisystem disease with pathology of NA, eosinophilic infiltration and extravascular granulomas. The vascular and nerve lesions observed in nerve biopsies from patients with this syndrome and response to treatment are actually similar to those observed in PAN, with sometimes presence of elevated ANCA. The occurrence of NA in the context of rheumatoid arthritis is associated with a poor outcome Granulomatous vasculitis $(\mathrm{GV})$ is characterised by vasculitis of upper and lower respiratory tract with or without glomerulonephritis. Peripheral neuropathy has been observed in $25 \%$ of the patients with GV but peripheral neuropathy seldom is the first manifestation of the disease. Of major interest to neurologists is the occurrence of peripheral neuropathy as the only clinical manifestation of vasculitis. General signs or symptoms, usually minor, including fever and loss of weight are present in half of them. One fourth of the patients presented with a distal symmetrical neuropathy. The diagnosis of NA had seldom been considered before the results of the nerve and muscle biopsies. One third of patients with the so-called non systemic vasculitis subsequently developed systemic manifestations within an average 6 years, but the overall outcome remains better than in classic PAN. Vasculitic neuropathy is a frequent factor of disability in the elderly. The diagnosis of NA needs histological confirmation: the specific lesion can be found in the muscle, in the nerve, or both in the nerve and the muscle specimens, which must be studied on serial sections because NA is segmental. Nerve ischemia due to vasculitis neuropathy induces acute axonal degeneration. Secondary vasculitis: Symptomatic viral infection, by HIV infection, $\mathrm{CMV}$; $\mathrm{B}$ and $\mathrm{C}$ virus hepatitis, and HTLV-1, can be associated with neuropathy and necrotizing vasculitis. Angiitis has been recognized at autopsy in the CNS and occasionally in patients with sarcoid neuropathy. A small proportion of diabetic patients over the age of 50, may present proximal neuropathy of the lower limbs characterised by a variable degree of pain and sensory loss associated with uni- or bilateral proximal muscle weakness and atrophy. Treatment: Prednisone is usually started at $1 \mathrm{mg} / \mathrm{Kg}$ /day. Simultaneous treatment with cyclophosphamide, 2 $\mathrm{mg} / \mathrm{Kg} /$ day, or Imuran may help reducing the doses of corticosteroids. Full dose of steroids is prescribed for 6-8 weeks and then tapered. Markers of disease activity must be checked periodically to monitor the treatment. Half of the patients relapse during tapering of prednisone or after treatment has been stopped. In some patients treatment with low dose prednisone must be pursued indefinitely. Spontaneous remissions of several years duration occur.

\section{WS 17.2 / \#102}

Topic: 09. Systemic Diseases

\section{STATIN NEUROPATHIES}

\section{Hadi Manji \\ Mrc Centre For Neuromuscular Diseases, National Hospital for Neurology, London, GB}

Background: In the United States, 37 milllion people are on statins; in the UK $13 \%$ of the population are on statins. Despite this huge number of patients taking this class of drug, reports of a peripheral neuropathy due to statins are relatively sparse when 
compared to the well-known myopathic side effects. It was the publication by Gaist et al, in Neurology in 2002, that the first red flag was raised regarding the risk of neuropathy with statins. This community based case control study found that stain users were $4-14$ times more likely to develop a polyneuropathy. ${ }^{1}$ The study was subsequently critisised on the grounds of patient selection and the criteria used for a diagnosis of neuropathy. In 2017, Gaist and colleagues published their results from a similar but larger cohort study and concluded "statin use was not associated with an increased risk of idiopathic neuropathy. ${ }^{2 "}$ Nevertheless, anecdotal reports of statin related neuropathic syndromes continue to be published in the literature which are difficult to ignore. For example, Rajabally et al reported a well studied case of "a disorder resembling GuillainBarre syndrome on initiation of statin therapy."3 On the contrary, the Fremantle Diabetes Study into lipid lowering therapy and peripheral sensory neuropathy in type 2 diabetes suggested that statins may in fact protect patients against the development of diabetic neuropathy. ${ }^{4}$ Similarly, in HIV infected patients on anti-retroviral drugs, again statins were associated with a protective effect against the development of a neuropathy. ${ }^{5}$ More recent evidence suggests that statins may have a role to play in the treatment of neuropathic pain. Zhang et al generated experimental models in mice and rats that mimicked trauma associated neuropathic pain. Their results indicated that simvastatin and rosuvastatin could prevent or reverse mechanical and thermal hypersensitivity in a dose dependant manner. ${ }^{6}$ The underlying mechanism of action is hypothesized to be related to the reduction of the expression of proinflammatory cytokines and reducing the activation of spinal microglia and astrocytes. Conclusion: The risk of statin induced neuropathy is low although it is not always possible to exclude individual idiosyncratic reactions. Statins may have a role in the prevention of neuropathy in some situations such as diabetes and HIV infection. Statins may help in the treatment of neuropathic pain as a results of their anti-inflammatory properties. References: 1) Gaist D et al. Neurology 2002;58:1333-1337 Statins and risk of polyneuropathy. 2) De Koning Svendsen et al. British Journal of Clinical Pharmacology 2017(83) 2087-2095 Statins and polyneuropathy revisited: case-control study in Denmark, 1999-2013. 3) Rajabally Y et al. Muscle and Nerve 2004:30:663-666. Disorder resembling Guillain Barre Syndrome on initiation of statin therapy. 4) Davies TME et al Dia- betologia 2008:51:562-566 Lipid lowering therapy and peripheral neuropathy in type 2 diabetes: the Fremantle study 5) Chen $\mathrm{H}$ et al. Peripheral neuropathy in ART -experienced patients: Prevalence and risk factors. J Neurovirol 2013:19(6):557-564. 6) Xiang Qun Shi et al. Pain 2011 (152): 1033-1043. Statins alleviate experimental nerve injury-induced neuropathic pain.

\section{WS 17.3 / \#154}

Topic: 09. Systemic Diseases

\section{MGUS ASSOCIATED PERIPHERAL NEUROPATHY: DIAGNOSIS AND TREATMENT}

Andreas Steck

Department Neurology, Basel, $\mathrm{CH}$

\begin{abstract}
A monoclonal gammopathy of undetermined significance (MGUS) is a frequent diagnosis in an elderly patient with a peripheral neuropathy (PN). It is important for the clinician to be able to determine whether an association exists between the paraprotein and the PN. The clinical phenotypes of the neuropathy, as well as the type of monoclonal protein are important clues for the diagnosis and optimal management. The prevalence of $\mathrm{PN}$ in patients with MGUS is about 5\% in $\operatorname{IgG}, 15 \%$ in $\operatorname{IgA}$ and up to $30-50 \%$ in IgM MGUS. We first discuss the broad concepts of diagnostic evaluation, before presenting specifics relating to each diagnosis. The evaluation for a monoclonal protein in a patient with a peripheral neuropathy should include a serum protein electrophoresis and serum immunofixation electrophoresis and quantitative immunoglobulins, and serum free light chains should be obtained. The nature of the symptoms, clinical course, and involvement of motor, sensory or autonomic systems helps in the differential diagnosis. Electrophysiology delineates specific features of the different paraprotein- associated PN. Anti-MAG and anti-ganglioside antibodies (GQ1b, GM1, GD1a, GD1b) are useful tests. We will discuss and present clinical vignettes of the major clinical syndromes such as the NP associated with anti-myelin-associated glycoprotein IgM antibodies and the chronic sensory ataxic neuropathy with monoclonal IgM anti-disialosyl antibodies. NP associated with IgG and IgA MGUS often present with a CIDP phenotype. Treatment strategy for these
\end{abstract}


disorders will be presented. Finally important differential diagnoses will be mentioned. This clinically oriented presentation should help neurologists improve their knowledge and stay abreast of recent advances in the field of MGUS PN.

\section{WS 18.1 / \#69}

Topic: 04. Neuromuscular Junction

\section{AUTOANTIBODIES IN MYASTHENIA GRAVIS}

Nils Erik Gilhus

Department Of Clinical Medicine, University of

Bergen, Bergen, NO

Abstract: Myasthenia gravis (MG) is an autoimmune disease characterized by autoantibodies. The autoantibodies are directed against acetylcholine receptors (AChR) in the postsynaptic muscle membrane, or alternatively against molecules nearby that interact with AChR. The antibodies bind in vivo leading to dysfunction and reduction in AChR and defective postsynaptic signaling in muscle. MG patients should always be classified into subgroups. This classification depends on autoantibody detection, as well as age, clinical manifestations and thymus pathology.

\begin{tabular}{llll}
\hline MG subgroup & Antibody & $\begin{array}{l}\text { Age at } \\
\text { onset }\end{array}$ & Thymus \\
\hline Easrly onset & AChR & $<50$ years & Hyperplasia common \\
Late onset & AChR & $>50$ years & Atrophy common \\
Thymoma & AChR & Any & Lymphoepithelioma \\
MuSK & MuSK & Any & Normal \\
LRP4 & LRP4 & Any & Normal \\
Seronegative & None & Any & Variable \\
& detected & & \\
Ocular & Variable & Any & Variable \\
\hline
\end{tabular}

Antibodies against muscle-specific kinase appear in $1-10 \%$ of patients, and against LRP4 in $1-3 \%$. MG in childhood is nearly always of the early onset or ocular type. Detection of AChR, MuSK or LRP4 antibodies has a near $100 \%$ specificity for MG. The sensitivity is around $80 \%$, highest for thymoma and lowest for ocular MG. More sensitive techniques have been developed for antibody detection, reducing the seronegative MG subgroup with one half. However, it is not known if the most sensitive assays have the same high MG specificity, and these assays are not commercially available. There is no correlation between antibody concentration and MG severity. This reflects that pathogenicity varies between antibody isotypes and idiotypes. There is a tendency for some correlation between changes in antibody concentration and clinical development in the same patient over time, so that repeated antibody measurements can be of value. Some MG patients have additional antibodies against intracellular muscle antigens, titin and ryanodine receptor in particular. Presence of such antibodies indicates thymoma or late onset MG. Patients with the antibodies tend to have more severe disease and a greater need for long-term immunosuppressive treatment. Antibodies against agrin, cortactin, and collagen $\mathrm{Q}$ in muscle have been detected in some MG patients without other antibodies. The MG specificity of these antibodies is not known in detail. The disease-inducing antibodies against AChR, MuSK and LRP4 all pass placenta and into the fetus during pregnancy. In approximately $20 \%$ of the newborn of MG mothers they lead to a transient neonatal myasthenia, usually with only mild symptoms for some days or a few weeks. Arthrogryposis and spontaneous abortions induced by mother's AChR antibodies have been described in rare cases. MG is the prototype of an antibody-mediated autoimmune disease. It usually responds well to immunosuppressive therapy, so that the great majority of MG patients have rather mild disease or are in remission.

WS 18.2 / \#135

Topic: 04. Neuromuscular Junction

\section{CIRCULATING MICRO-RNA AS BIOMARKERS IN MYASTHENIA GRAVIS}

Anna R. Punga

Neuroscience, Uppsala University, Uppsala, SE

Background: MicroRNAs (miRNAs) are small noncoding RNA molecules, which bind specifically to different mRNA targets and thereby regulate several important intracellular biological processes. Circulating (extracellular) miRNAs can be measured in most biofluids, and therefore they have emerged as easily accessible markers in various body fluids, including blood serum and plasma, with 
disease-specific profiles for example in autoimmune conditions. In myasthenia gravis (MG), there is a great need for reliable objective biomarkers to monitor the disease course and therapeutic response. Recent studies in MG patients have focused on early onset MG with generalized disease phenotype. In acetylcholine receptor antibodypositive $(\mathrm{AChR}+)$ MG, levels of the miRNAs miR-150-5p and miR21-5p were elevated. Further, miR-150-5p levels were lower in patients receiving immunosuppression and those having undergone thymectomy. In $\mathrm{MuSK}+\mathrm{MG}$, another profile of circulating miRNAs is present, including upregulation of the let-7 family of miRNAs. Methods: The aim was to determine disease specific pattern of miRNAs in other subgroups of $\mathrm{MG}$, including late onset $\mathrm{MG}$ (LOMG) and ocular MG. Different discovery cohorts consisted of patients with generalized LOMG and ocular MG as well as age- and gender-matched healthy controls (HCs). We also included longitudinal validation sets of LOMG patients to correlate disease over time with miRNA pattern. For the ocular MG cohort, we had longitudinal data and aimed at determining whether certain miRNA levels would predict generalization of MG weakness. Total RNA was isolated from $200 \mu \mathrm{l}$ blood serum and cDNA synthesis was performed by reverse transcription, followed by real-time PCR amplification. MicroRNA expression was analyzed using the Serum/Plasma Focus microRNA PCR Panel (Exiqon) in the discovery cohorts and in the validation cohorts we added selected primers detected from the discovery cohort to the qPCR plates. Internal technical controls included interplate calibration with UniSp3, quality of RNA isolation with UniSp2 and UniSp4, cDNA synthesis control with UniSp6 and hemolysis control $\Delta \mathrm{Cq}$ (miR-23a - miR-451a). miRNA data were converted to the logarithmic scale and paired two-tailed T-test or the Wilcoxon matched paired test was used to compare the values of miRNA over time. Spearman Rank correlation was performed to find correlation between continuous factors (age, disease duration, disease severity) and different miRNAs. Statistical significance was defined as $p<0.05$. Results: A pattern of miRNAs was found to be strongly elevated in the LOMG cohort and in the ocular MG cohort another pattern of elevated miRNAs was found. A few miRNAs correlated with the clinical MG composite score (MGC) and were reduced in parallel with initiation of immunosuppression and clinical improvement at 1-year follow-up. Further, levels of some miRNAs differentiated generalized from ocular MG.
Conclusion: In conclusion, we found a specific pattern of miRNA expression in the subgroup of MG patients with LOMG and in the cohort of ocular MG. The correlation between some of these miRNAs with the MGC score and the clinical course strengthen the potential role of these miRNAs as biomarkers in follow-up of MG.

WS 18.3 / \#105

Topic: 04. Neuromuscular Junction

\section{THYMUS BIOMARKERS AND CLINICAL RELEVANCE}

Alexander Marx

Pathology, University Medical Centre Mannheim/

University of Heidelberg, Mannheim, DE

Abstract not received.

WS 19.1 / \#147

Topic: 01. Muscle

\section{MUSCLE BIOPSIES}

Rolf Schröder $^{1}$, Monika Hofer ${ }^{2}$
${ }^{1}$ Neuropathology, University Hospital Erlangen,
Erlangen, DE; ${ }^{2}$ Oxford University Hospitals,
Oxford, GB

Background: Although next generation sequences approaches have certainly revolutionised the diagnostics of human muscle and nerve diseases, there is still an essential role for muscle biopsies in the diagnostic work-up of patients with neuromuscular symptoms. However, appropriate indications, careful choice of muscle to biopsy and appropriate handling and work-up of the muscle tissue are of paramount importance to ensure a high diagnostic yield. Methods: Light and electron microscopic analysis of skeletal muscle tissue in conjunction with immunohistochemistry of essential disease-related muscle proteins. Results: Our talk will address the following issues: 1 . Indications and contraindications 2. Choosing the right muscle for biopsy 3. Essentials for handling and further work-up of muscle tissue 4. Standard histochemical staining panel and basic interpretation of results 5. Standard immunohistochemical staining panel and basic interpretation 
of results 6. Indications for electron microscopy analysis and basic interpretation Conclusion: Diagnostic muscle biopsies are still an invaluable tool in the diagnostic work-up of neuromuscular patients. This presentation discusses the aspects mentioned above and is primarily aimed at neurologists looking after muscle patients. Selected cases will be used to demonstrate the principles.

\section{WS 19.2 / \#65}

Topic: 01. Muscle

\section{NERVE BIOPSIES: THERE IS STILL A PLACE FOR NERVE BIOPSY IN MODERN CLINICAL PRACTICE}

\author{
P. James B. Dyck \\ Mayo Clinic, Rochester, US
}

Background: Nerve biopsy is a useful tool for the diagnosis and treatment of peripheral neuropathies but the need for its continued use in the future has been questioned. With improvement in laboratory, radiographic and electrophysiological evaluations, nerve biopsy use has been on the decline. With better understanding of the pathophysiology, some neuropathies no longer need to be biopsied (inherited) whereas others can be recognized by the disease pattern (CIDP). Methods: Herein, we review our approach to doing nerve biopsies including selection of appropriate patients and nerve biopsy sites. Results: When deciding to do a nerve biopsy, there are some features that are helpful. A thorough neurological evaluation should be completed before nerve biopsy is performed. Patients with rapidly worsening, progressive neuropathies who have major clinical deficits are good biopsy candidates. Traditionally, whole distal cutaneous nerve biopsies are performed. These are taken from sensory nerves (e.g. sural, superficial peroneal, saphenous, superficial radial, etc.) and so are only useful in sensorimotor or pure sensory neuropathies. The nerve needs to be clinically affected in order for a nerve biopsy to show findings. Nerve biopsies laboratories should do a wide variety of preparations including teased fibers, paraffin and epoxy sections, immunohistochemistry and electron microscopy. Reports on the diagnostic yield of nerve biopsy vary and the findings are hard to validate because the quality of the evaluations and of the pathological specimens vary. In our experience, nerve biopsies from carefully selected patients frequently show important findings that change clinical management. For motor predominant, focal or proximal neuropathies the traditional distal cutaneous biopsy may be inadequate. With improved resolution of MRI, focal lesions along the nerve can now be identified and biopsied. We have extensive experience with targeted fascicular nerve biopsies and find that about $84 \%$ of these targeted biopsies reveal meaningful pathology from which $76 \%$ are potentially treatable. Such cases usually are focal or multifocal, proximal or motor predominant. The causes include inflammatory demyelinating, vasculitis, sarcoidosis, amyloidosis, leprosy, lymphoma, metastatic tumors, vascular malformations and others. Conclusion: There is strong evidence that nerve biopsy should still be performed in selected patients in modern neurological practice. For most typical length-dependent neuropathies whole distal cutaneous biopsy is the procedure of choice. In contrast, for focal motor predominant or proximal neuropathies targeted fascicular nerve biopsy is a superior approach. Patients should be carefully screened and have a thorough evaluation and only patients with severe neuropathies that have a high likelihood of being treatable should be biopsied to offset potential side effects. Biopsies should be performed at centers with expertise in peripheral nerve diseases with specialized neurologists, radiologists, peripheral nerve surgeons and pathologists and laboratories that offer a variety of nerve preparations to ensure maximal benefit with minimal morbidity.

WS 19.3 / \#91

Topic: 01. Muscle

\section{SKIN BIOPSIES}

Giuseppe Lauria

"Carlo Besta” Neurological Institute, Milan, IT

Abstract not received. 
WS 20.1 / \#99

Topic: 09. Systemic Diseases

\section{DIABETIC CRANIAL NEUROPATHIES}

Tudor Lupescu

Department Of Neurology, "Agrippa Ionescu”

Hospital,, Bucharest, RO

Background: Diabetes mellitus is a frequent condition representing nowadays practically a pandemic with a prevalence of almost 1 in every 10 people. There is also a high prevalence of neuropathy among diabetic patients, of about $50 \%$. The cranial nerves affected in diabetes are the oculomotor (3rd, 4th, and 6th) and the facial. Although cranial neuropathies represent $1-2 \%$ of the total diabetic neuropathies, due to the very high number of diabetic patients, most neurologists will encounter such cases. Methods: The presentation will deal with the two most frequent types of cranial neuropathies, namely those that involve the oculomotor and the facial nerves. Results: All the oculomotor nerves can be involved, but neuropathy of the 3rd cranial nerve is the most frequent. It is followed by involvement of the abducens and the trochlear nerve. The common oculomotor diabetic neuropathy is characterized by sudden onset of palpebral ptosis, diplopia, divergent strabismus, usually preceded by ocular pain. Sparing of the pupillary function is quite typical for diabetic palsy of the third cranial nerve. This feature is explained by the ischemic mechanism of this neuropathy, with involvement of the centrofascicular portion of the nerve, leaving intact the fibres concerned with pupillary function that are situated more peripherally. Abducens nerve palsy can be recognized by the convergent strabismus. Trochlear nerve palsies are rare. The differential diagnosis of these cranial neuropathies is very important, since other conditions in which the oculomotor nerves are affected can be very significant and dangerous. We must exclude an aneurysm of the posterior communicating artery, midbrain ischemia, or tumour. Therefore a thorough clinical examination and neuroimaging are very important in the management of these cases. The prognosis for recovery is good. The diabetic facial palsy is more frequent in older diabetics than in non-diabetic patients. The clinical presentation is similar to the common Bell's palsy. There is an acute onset of unilateral weakness of facial muscles, often preceded by retroauricular pain. A study showed that in the majority of diabetic patients, there was no taste abnormality. Therefore it was assumed that the lesion in diabetic facial palsies is most often distal to the chorda tympani. Of course it is important to see that other signs of central nervous involvement are absent, otherwise we might misdiagnose important clinical conditions (stroke, multiple sclerosis). The clinical examination and neuroimaging are useful here. Sometimes, electrodiagnosis (nerve conduction studies, blink reflex) can add important functional data. As in idiopathic facial palsy, the prognosis for recovery is good. There are situations in which cranial nerve palsies accompany more serious conditions, associated or not with diabetes. There are cases with oculomotor nerve involvement in mucormycosis, and also cases with facial and cervical radiculoplexus neuropathy. Conclusion: Although rare among associated neuropathies, the high prevalence of diabetes mellitus provides a significant number of cases with cranial nerve neuropathies. Thus, knowledge regarding these conditions is important, since they present with an acute onset, pain, and may mimic other important and sometimes life-threatening diseases.

\section{WS 20.2 / \#75}

Topic: 09. Systemic Diseases

\section{MUSCULOSKELETAL COMPLICATIONS OF DIABETES ARE COMMON AND UNDERDIAGNOSED}

\section{Anna Grisold \\ Medical University of Vienna, Vienna, AT}

Background: Diabetes mellitus (DM) is a disease with an enormous socio-economic impact and a still increasing prevalence worldwide. The underlying pathophysiology of type I DM is an autoimmune destruction of pancreatic cells, whereas in type II DM insulin resistance, hyperglycemia and inadequate insulin secretion are the main mechanisms. Persistent hyperglycemia subsequently leads to microvascular complications such as nephropathy, neuropathy and retinopathy and macrovascular complications like stroke, coronary and peripheral artery diseases. Further, musculoskeletal disorders represent a broad spectrum of still underestimated comorbidities in diabetes. The exact pathomechanisms of musculoskeletal complications still remain elusive. Muscle 
inflammation, fibrosis, infarction, hemorrhage, necrosis, neuropathy and vasculopathy have been discussed as concomitant structural alterations. From an etiological point of view, disease duration as well as disease control, age and comorbidities play a crucial role in the development of musculoskeletal complications. Musculoskeletal complications in DM: Regarding complications of the skeleton, upper extremities are more often affected than lower extremities. Common manifestations include "frozen shoulder" syndrome due to adhesive capsulitis, but also limited joint mobility, Dupuytren's contractures, flexor tenosynovitis, carpal tunnel syndrome and diffuse idiopathic skeletal hyperostosis. In the lower extremities, especially in the feet, skeletal complications are usually more disabling, for example Charcot's joint of the ankle and foot. Apart from the aforementioned skeletal involvement, also muscle tissue might be affected by DM. Sarcopenia and muscle atrophy as generalized presentations, but also painful muscle infarcts are characteristic. Additionally, infectious and inflammatory myositis may be observed. Given their generally impaired metabolic state, diabetic patients are particularly prone to infectious complications in articular structures. Also a decreased bone density has been described in the literature. Therapy: Glycemic control due to pharmacotherapy and diet are the most important therapeutic options. Besides, the anti-inflammatory and immune-mediating effect of corticosteroids may be used in the treatment of musculoskeletal disorders in DM patients. However, the indication has to be chosen carefully in consideration of potentially resulting metabolic perturbations. A regular and sensible physiotherapy program is an important cornerstone of DM management to prevent further musculoskeletal complications and strengthen the patient. Conclusion: Musculoskeletal complications are frequent, but still underestimated in patients with DM. They often present with reduced mobility and pain, which might be difficult to distinguish from peripheral nerve lesions. This lecture points out the most important and frequent musculoskeletal complications in patients with DM.
WS 20.3 / \#128

Topic: 09. Systemic Diseases

\section{ATYPICAL NEUROPATHIES IN DIABETES: DIAGNOSIS AND MANAGEMENT}

\author{
Amanda Peltier \\ Vanderbilt University, Nashville, US
}

Abstract not received.

\author{
WS 20.4 / \#50
}

Topic: 09. Systemic Diseases

\section{TREATMENT INDUCED AND INFLAMMATORY NEUROPATHIES IN DIABETES}

\author{
Brian Christopher Callaghan \\ University of Michigan School of Public Health, \\ Ann Arbor, US
}

\begin{abstract}
Patients with diabetes may experience multiple types of periperhal neuropathies. The most common is distal symmetric polyneuropathy; however, other less common neuropathies such as treatment induced neuropathy (TIND), chronic inflammatory demyleinating polyneuropathy (CIDP), and diabetic lumbosacral radiculoplexus neuropathy (LSRPN) may also occur. In patients with diabetes, TIND is often unrecongnized. The neuropathy predominantly affects small nerve fibers leading to a length dependent, painful neuropathy as well as an autonomic neuropathy. This neuropathy usually occurs when the HA1C falls 3 or more points in 3 months. Patients with type 1 and type 2 diabetes can both have this complication. Recurrent events can lead to large fiber injury and worse patient outcomes. The more autonomic involvement that occurs, the less likely the disease is to be recognized. Only $5 \%$ of mild TIND and $41 \%$ of severe neuropathy is recognized by treating physicians. TIND should be considered in patients with diabetes that have abrupt changes in pain and/or autonomic symptoms. Typical diabetic polyneuropathy presents slowly; therefore acute/subacute presentations are a red flag for an atypical neuropathy such as TIND. Inflammatory neuropathies, such as CIDP may also be more common in patients with diabetes. Multiple
\end{abstract}


groups have shown that the prevalence of CIDP is higher in patients with diabetes than in those without diabetes. Furthermore, another study demonstrated that the treatment responsiveness is not different between patients with CIDP with and without diabetes. However, patients with CIDP and diabetes require more EFNS criteria to have the same treatment responsiveness as those without diabetes. Fulfilling more EFNS criteria increases the likelihood of treatment response in those with diabetes. A separate study found that patient with CIDP and diabetes are less likely to be treatment than those without diabetes. Despite these studies, other investigators have found that the prevalence of CIDP is not higher in those with diabetes than those without. This group has also reported patients with painless motor neuropathy in diabetes that they believe is a LSRPN and not CIDP. LSRPN also occurs more commonly in patients with diabetes compared to those without. This peripheral neuropathy usually presents with severe pain, followed by weakness, followed by some degree of recovery. Patients typically have an asymmetric presentation with proximal weakness in one leg more than the other. The only clinical trial did not show benefit of weekly IV steroids on the primary outcomes, but did lead to reduced pain. A cervical variant of this condition also occurs, but is less common. Methods: Review of the literature Results: summarized in the background. Conclusion: Diabetes is associated with many manifestations of peripheral nerve injury. In addition, to distal symmetric polyneuropathy, TIND, CIDP, and LSRPN can also occur. 


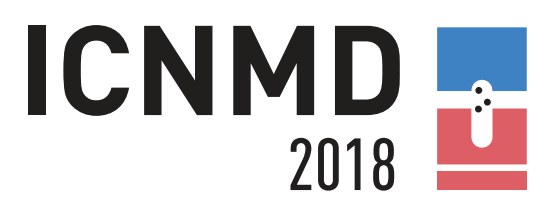

15TH INTERNATIONAL CONGRESS ON NEUROMUSCULAR DISEASES

www.icnmd2018.org July 6-10, 2018 Vienna, Austria

Teaching Courses 
TC $1.1 / \# 58$

Topic: 01. Muscle

\section{CLINICAL PHENOTYPES AND HOW TO MANAGE PATIENTS WITH HIGH HYPERCKEMIA, STATIN MYOPATHY}

\author{
Marianne De Visser \\ Department Of Neurology, Academic Medical \\ Centre, Amsterdam, NL
}

Abstract not received.

\section{TC 1.2 / \#145}

Topic: 01. Muscle

\section{HOW TO DIAGNOSE A PATIENT WITH HYPERCKEMIA}

\author{
$\underline{\text { Sabrina Sacconi }}$ \\ Centre de référence des Maladies \\ Neuromusculaires, Hôpital Archet, France. CNRS \\ UMR7277, INSERM U1091, IBV - Institute of \\ Biology Valrose, UNS Université Nice Sophia- \\ Antipolis, Faculté de Médecine, Nice, FR
}

\begin{abstract}
Rhabdomyolysis ranges to an asymptomatic illness to a life-threatening condition associated with electrolyte imbalances, acute renal failure and disseminated intravascular coagulation. It results from skeletal muscle injury followed by the release of muscle contents into the plasma. The most sensitive laboratory finding of muscle injury is an elevated plasma creatine kinase level. Creatine kinases (CK) is a dimeric enzyme catalyzing the reversible phosphorylation of creatine by adenosine triphosphate. The CK enzyme is composed of subunits derived from either muscle (M) or brain (B). Three isoenzymes have been identified, and they are present in several tissues: striated muscle (MM), heart tissue (MB), and brain (BB). As a consequence of cardiac or brain injury $\mathrm{MB}$ and $\mathrm{BB}$ isoforms may be elevated. Increase in CK can be also seen in several physiological conditions: CK levels transiently rise after exercise or heavy manual labor; they vary significantly by sex and race, possibly in relation to differences in muscle mass or total body mass, and/or inherited differences in the permeability of the sarcolemma to CK. There is also a small reduction in
\end{abstract}

CK levels as people age. In pathological conditions, weakness, myalgia and tea-colored urine can be associated to increase in CK. Muscular trauma is one of the most common non-neuromuscular causes of rhabdomyolysis. Less common causes include electrolyte abnormalities, vessels occlusion, shock state, infectious causes, drugs, toxins, endocrinopathies, auto-immune diseases. A large spectrum of neuromuscular conditions can be diagnosed in presence of acute recurrent or chronic rhabdomyolysis including inflammatory and necrotizing myopathies, metabolic diseases as glycogenosis, B oxidation disorders, mitochondrial myopathy, lipidosis, pseudo metabolic presentation of muscular dystrophy or congenital myopathies. The diagnostic work-up may be complicated by the fact that, among the patients displaying increased $\mathrm{CK}$, asymptomatic or pauci symptomatic carriers as well as early stages myopathies may be diagnosed. High CK can also be found in patients suffering from peripheral nervous system or motor neuron disease associated to extensive muscle wasting. The objective of this teaching course is to describe the aetiological spectrum of acute recurrent and chronic rhabdomyolysis and to provide an appraisal of the current strategies available to facilitate early diagnosis of this condition. Early diagnosis will allow early therapy when available, and prevention and/or the prompt management of acute and chronic complications. On this purpose, muscle biopsy is still the key exam in order to solve the large majority of myopathic-related causes of rhabdomyolisis, but it appears the more and more evident that new technologies are changing the way to address this issue and may, in the next future, reduce the need of this invasive approach. We will discuss the role of these new and powerful technologies, especially when unraveling genetic associated diseases, combined with more traditional approaches including an accurate clinical examination, standard biological exams, electrophysiology and muscle MRI, that are, despite all that, still necessary to guide the interpretation of the results. Since the field of new technologies is rapidly evolving, future researches are required to establish algorithms for cost-effective medical care. 
TC 1.3 / \#36

Topic: 01. Muscle

\section{HYPERCKEMIA IN MUSCULAR DISTROPHIES AND METABOLIC MYOPATHIES}

Corrado Angelini

IRCCS S.Camillo Hospital, Venice, IT

Background: Hyperckemia is a common finding in asymptomatic and paucisymptomatic muscle patients, that can precede the onset of several myopathies. The first description was by Rowland in MDR Symposium held in Venice in 1980 and this generated an interest by clinicians and patients with either high CK or paucisymptomatic clinical presentation such as cramps or exercise intolerance. Methods: A literature review was performed using the following keywords: metabolic myopathies, Pompe disease, Mc Ardle disease, disorder of lipid metabolism, high CK, biopsy, MRI. Since then this entity has been covered by international guidelines in order to handle such patients and perform exams and in such guidelines need to include nowdays muscle CT/MRI imaging. Results: The most common muscular dystrophies that appear first as isolated hyperckemia are calpainopathy, disferlinopathy or alpha-sarcoglycanopathies. High CK in females evokes as first suspicion the carrier status for dystrophinopathy. An increase of $\mathrm{CK}$ in pauci/asymptomatic male can be due to juvenile onset Pompe disease, that is the first hereditary myopathy for which a registered treatment is available, it is therefore important to diagnose such cases. A similar screening was done in the LOPED study by DBS assay, that identified numerous late onset Pompe phenotypes presenting with hyperckemia or mild LGMD phenotype that subsequently were found to have pathogenetic mutations in GAA gene and underwent ERT treatment. Mc Ardle patients suffer from cramps and exercise intolerance. The timing of onset of symptoms in relation to exercise provides a clue to the nature of the underlying problem and guides further investigation. Glycolysis is the main source of energy at rest and during sustained moderate exercise. In early exercise, particularly during high-intensity exercise such as weight lifting or sprinting, and before adaptive changes, such as increased respiration and blood flow, muscle energy demands are met primarily by anaerobic glycolysis. In muscle glycogenolytic disorders myalgia develops early during exercise, in most cases the onset of symptoms starts in childhood. In these conditions, fixed weakness may develop later in life. Recurrent myoglobinuria is common in all these disorders, a history of a 'second wind' phenomenon is suggestive of McArdle disease. This disorder is caused by a lack of glycogen breakdown due to mutations in the muscle glycogen phosphorylase(myophosphorylase). The nonsense p.R50X stop codon mutation accounts for $43 \%$ of cases of myophosphorylase deficiency in caucasian populations and ACE I/D polymorphism influences severity of disease expression. Fatty acids are the major substrate at rest and in prolonged low-intensity exercise, such as during a marathon. In lipid storage disorders elevated $\mathrm{CK}$ can be observed in carnitine deficiency, in ETF dehydrogenase deficiency. Myalgia or myoglobinuria in fatty acid oxidation defects occur later in exercise than in glycogenolytic disorders. Conclusion: Serum CK varies greatly depending on prior physical activity level and is typically elevated in patients with complete enzymatic blocks (myophosphorylase and phosphofructokinase deficiency)or in muscular dystrophies. In fatty acid disorders recurrent myoglobinuria following prolonged aerobic exercise is common in a number of lipid disorders, especially if preceded by fasting. Between attacks CK is usually normal at difference with muscular dystrophy.

\section{TC 1.4 / \#87}

Topic: 01. Muscle

\section{DIAGNOSTIC APPROACH AND GUIDELINES FOR OLIGOSYMPTOMATIC HYPERCKEMIA}

Theodore Kyriakides

Clinic A, Cyprus Institute of Neurology and Genetics, Nicosia, CY

Background: Increased serum creatine kinase (CK) above the upper limit of normal (ULN) or hyperCKemia is a frequent finding among the normal population and prevalence rates of persistent hyperCKemia of $1.3 \%$ have been reported. The overwhelming majority of these individuals are oligo/asymptomatic and hyperCKemia is a chance finding. The dilemma often arises as to whether there is an underlying 
myopathy which could potentially evolve into clinically significant weakness in the future or whether such an individual could be at risk by certain drugs such as statins. Current evidence suggests that on a population basis the likelihood of discovering a significant cause for oligo/asymptomatic and hyperCKemia is extremely low and routine investigation of such individuals is not cost-effective. Potential tools for investigation include the muscle biopsy, specific DNA testing and muscle panel DNA testing made possible by Next Generation Sequencing. Methods: In the absence of RCTs to provide a diagnostic strategy, case-based observational studies could be interrogated in an attempt to provide posItive and negative predictive values of certain parameters so selected patients could be investigated.Such parameters could be ; age of the patient, symptoms, level of hyperCKemia and the EMG. Results: Data will be presented on the positive and negative predictive value on reaching a diagnosis in patients with hyperCKemia following biopsy according to patient's age, level of hyperCKemia, symptoms and EMG result. Conclusion: Randomised control studies are not available to guide a diagnostic strategy in oligosymptomatic patients with hyperCKemia. An approach based on available evidence from observational studies has to be used to optimise investigations of such patients.

\section{TC $2.1 / \# 132$}

Topic: 04. Neuromuscular Junction

\section{SYNAPTIC SIGNALLING PATHWAYS IN NEUROMUSCULAR JUNCTION DEVELOPMENT AND PATHOPHYSIOLOGY}

William D. Phillips

Physiology, The University Of Sydney, Sydney, $N S W, A U$

\begin{abstract}
Myasthenia gravis provides the clearest example of impaired neuromuscular transmission, but compromised nerve-muscle communication can contribute to weakness in diverse diseases and conditions. Mouse models of Duchenne muscular dystrophy reveal relatively mild impairments of neuromuscular transmission. In contrast, loss of nerve terminals from motor endplates seems to begin early in the progression of motor neuron disease. A
\end{abstract}

slower, progressive loss of neuromuscular connections occurs in old age. In elderly mice, at least, this loss of neuromuscular connections could be slowed by exercise or dietary restriction. Mouse studies also show that failure of two-way synaptic communication can contribute to failure of neuromuscular transmission, and/or loss of nerve-muscle connections. Most of what we know about the interactions between nerve and muscle has come from studying embryonic development of the neuromuscular junction (NMJ). Muscle specific kinase (MuSK), a transmembrane tyrosine kinase, is a central player in formation of the NMJ. A small patch of MuSK in the central region of the embryonic muscle fibre marks the site for the future NMJ. The intrinsic kinase activity of MuSK recruits acetylcholine receptors $(\mathrm{AChR})$ and the cytoplasmic adaptor protein, rapsyn, so as to form specialized postsynaptic membrane. A cytoplasmic protein called DOK7 binds directly to MuSK dimers. Higher levels of DOK7 expression elevate the intrinsic kinase activity of MuSK. As motor axons invade embryonic muscles they secrete a proteoglycan called neural agrin. Neural agrin binds a complex of LRP4 and MuSK, which further increases MuSK activity. The agrin-LRP4-MuSKrapsyn pathway stabilizes the AChR-rich postsynaptic membrane beneath the nerve terminal. Acetylcholine acts via postsynaptic AChR and calcium-activated proteases to drive an opposing system that tends to remove AChRs from the AChR patch. During normal development, agrin-driven AChR stabilization and acetylcholine-driven AChR disassembly balance each other. This helps to align postsynaptic AChRs beneath the growing presynaptic nerve terminal. Meanwhile postsynaptic LRP4 and synaptic-cleft laminins signal back to the overlying axon membrane to induce the assembly of presynaptic acetylcholine release machinery. The same molecular mechanisms that control development of the embryonic NMJ remain vital for sustaining effective neuromuscular connections through adult life. Lossof-function mutations that affect neural agrin, LRP4, MuSK, DOK7, or rapsyn can each give rise to congenital myasthenias. A subset of myasthenia gravis patients display IgG4-type MuSK autoantibodies that inhibit the activation of LRP4-MuSK by neural agrin. When injected into adult mice these antibodies cause disassembly of the postsynaptic AChRs, leading to myasthenic weakness. The MuSK antibodies also disrupt the adaptive increase in presynaptic acetylcholine release. In this mouse model, where MuSK function is impaired, the acetylcholine-driven 
AChR disassembly pathway can be perilous. Pyridostigmine (a first line treatment for anti-AChR myasthenia gravis) exacerbated the loss of postsynaptic AChRs. Studies in mouse models neuromuscular disease suggest that enhancing MuSK activity can help prevent failure of neuromuscular control. A better understanding of how the MuSK system operates in mature muscle will be needed before the therapeutic potential of MuSK can be properly understood.

\section{TC 2.2 / \#524}

Topic: 04. Neuromuscular Junction

\section{NEUROMUSCULAR TRANSMISSION AND ITS ASSESSMENT}

\author{
Clarke Slater \\ Institute Of Neuroscience, Newcastle University, \\ Newcastle Upon Tyne, GB
}

\begin{abstract}
Neuromuscular transmission provides the essential link between neural commands and the coordinated muscle contractions that turn them into useful actions. In a clinical setting, the efficacy of transmission can be assessed by recording the summed electrical activity of many muscle fibers, using either large electrodes on the muscle surface or needle electrodes inserted into the muscle. In both cases one records compound muscle action potentials (CMAPs) which reflect the activity of many muscle fibers. In normal subjects, during repetitive nerve stimulation at frequencies of up to $100 \mathrm{~Hz}$, the amplitude of the CMAP remains constant, but in some patients there is a significant $(>10 \%)$ 'decrement' of the response, indicating that some of the neuromuscular junctions (NMJ) are unable to follow faithfully the pattern of stimulation. To investigate the basis of such failure in detail, it is necessary to make intracellular recordings from individual NMJs in isolated nerve-muscle preparations. When the electrodes are placed within $1 \mathrm{~mm}$ or so of an NMJ, one records both evoked and spontaneous depolarizations from the resting potential of about $-75 \mathrm{mV}$. The evoked endplate potentials (EPPs) are typically about $40 \mathrm{mV}$ in amplitude, but to record them special precautions must be taken to block action potentials and contractions. Spontaneous miniature EPPs (mEPPs) occur at a frequency of about $1 \mathrm{~Hz}$. These result from the action of multimolecular 'quanta' of acetylcholine (ACh), the contents of individual
\end{abstract}

presynaptic vesicles (SVs), on the muscle fiber surface. A nerve impulse causes entry of $\mathrm{Ca}^{2+}$ into the nerve terminal which normally triggers the release of 20-200 quanta whose effects sum to make up the EPP. Because the effects of mEPPs do not add linearly, the number of quanta released, or 'quantal content' (QC), is best estimated from recordings of the synaptic currents (EPCs and mEPCs). This is done using 2 intracellular electrodes, one to record voltage and the other to pass current, in a 'voltageclamp' arrangement. Usually substantially more quanta are released in response to a single nerve impulse that are required to activate the muscle fiber, giving the process of transmission a high 'safety factor'. Muscles are normally activated by bursts of 5-10 nerve impulses at up to $100 \mathrm{~Hz}$ or longer trains at lower frequency. In response to repetitive stimulation, the QC at individual NMJs declines, but the high safety factor of transmission means that the EPP normally remains large enough to trigger an action potential. In patients with impaired neuromuscular transmission, the EPP may fall below threshold, resulting in a failure of muscle fiber activation. Different factors may cause a reduction of EPP amplitude. These include; reduced AChR density (e.g. $\mathrm{MG}, \mathrm{AChR}$ deficiency), reduced QC resulting from impaired quantal release (e.g. LEMS, various forms of CMS), and reduced QC resulting from reduced NMJ area (e.g. DOK-7 myasthenia).

\section{TC 2.3 / \#158}

Topic: 04. Neuromuscular Junction

\section{PRESYNAPTIC RECEPTORS AND NEUROMUSCULAR TRANSMISSION}

\author{
Ana M. Sebastião \\ Faculty Of Medicine, Institute Of Pharmacology \\ And Neurosciences And Institute Of Molecular \\ Medicine, University of Lisbon, Lisboa, PT
}

Background: The neuromuscular junction has unique properties to evaluate presynaptic mechanisms and receptors: 1 . It has a relatively simple and well known structure. 2 . There is only one nerve terminal per each skeletal muscle fibre, each synaptic response thus corresponding to the activity of a single nerve terminal. 3 . There is only one type of neurotransmitter being released, acetylcholine, though several neuromodulators are also released to 
control synaptic function, as it is the case of ATP and adenosine. Acetylcholine itself can also act as a neuromodulator, controlling its own release and function. 4. It is possible to record from the synaptic region, in contrast with what occurs in most central nervous system synapses. 5 . Changes in the amount of neurotransmitter released per nerve impulse (quantal content) can be easily quantified. Methods: Using the neuromuscular junction as a model, it could be established that: Results: 1. ATP, which is released together with acetylcholine as well as from peri-synaptic Schwann cells (PSCs), is extracellularly converted into adenosine through a cascade of enzymes, denominated ecto-nucleotidases 2. Adenosine, by acting through G-protein coupled receptors, can either inhibit or facilitate acetylcholine release, through A1 (inhibitory) or A2A (excitatory) receptors that can co-exist in the same nerve terminal. 3. At low frequencies of stimulation the influence of adenosine is inhibitory, but at higher stimulation frequencies it can become excitatory. 4 . Adenosine is removed from the synaptic cleft through equilibrative adenosine transporters. 5. ATP itself acts on PSCs through Gq-coupled ATP receptors, leading to calcium signals that induce the release of more ATP from these cells. 6. Calcium signals in PSCs can also be evoked by acetylcholine through Gq-coupled muscarinic receptors. 7. Muscarinic and nicotinic acetylcholine receptors are also present in the nerve terminal to control ACh release. During disease states this fine-tune control of neurotransmission may become dysfunctional, as it is the case of Amyotrophic Lateral Sclerosis. Using disease models, it was found that at very early stages, there is an unbalanced modulation of calcium homeostasis in the nerve terminal and PSCs, which reflects in enhanced but asynchronous $\mathrm{ACh}$ release. Concomitantly, there are alterations in muscarinic receptor function in PSCs as well as A1 and $\mathrm{A} 2 \mathrm{~A}$ receptor function in motor nerve terminals, which are already evident in the pre-symptomatic stage. Interestingly, some of these excitatory alterations fade away at symptomatic states of the disease, where a global inhibition of neuromuscular transmission becomes evident. Conclusion: Summing up, the simplicity of the neuromuscular junction allowed to unravel further levels of synaptic complexity, which extend to non-neuronal components and allow fine-tune modulation of synaptic activity. This fine-control fails in several pathologies, as we found in Amyotrophic Lateral Sclerosis model. Initially there is an exacerbated and unbal- anced excitatory activity, which collapses at a later stage of disease progression, leading to a progressive failure of neuromuscular transmission. Work supported by LISBOA-01-0145-FEDER-007391, co-funded by FEDER through POR Lisboa 2020 (Programa Operacional Regional de Lisboa) from PORTUGAL 2020 and Fundação para a Ciência e Tecnologia (FCT) and by a Twinning action (SynaNet) from the EU H2020 programme (project number: 692340).

\section{TC 2.4 / \#95}

Topic: 04. Neuromuscular Junction

\section{DIAGNOSIS AND THERAPY FOR NEUROMUSCULAR TRANSMISSION DISORDERS - MYASTHENIA GRAVIS AND CONGENITAL MYASTHENIC SYNDROMES}

\author{
Hanns Lochmüller \\ Department Of Neuropediatrics And Muscle \\ Disorders, Freiburg University - Medical Center, \\ Faculty of Medicine, Freiburg, DE
}

Background: Neuromuscular junction disorders, also called Myasthenic syndromes (MS), are a rare heterogeneous group of acquired (Myasthenia Gravis, MG) and inherited (Congenital Myasthenic Syndromes, CMS) neuromuscular disorders associated with distinctive clinical, electrophysiological, laboratory and ultrastructural abnormalities. Methods: MG has been associated with auto-antibodies against the acetylcholine receptor (AChR) or MuSK, and more recently with other antibodies in rare cases. Therapy is based on esterase inhibitors, immune therapies and thymectomy. Recent advances in understanding and clinical research have led to treatment guidelines, which were developed by a subcommittee of the American Academy of Neurology which are under consideration for adoption by the European Reference Network for Rare Neuromuscular Disorders (EURO-NMD). Results: The genetic defects in CMS either impair neuromuscular transmission directly or result in secondary impairments, which eventually compromise the safety margin of neuromuscular transmission. More recently, we have identified two genes (DOK7, GFPT1) that cause fatigable weakness of muscles in a limb-girdle distribution, but rarely affecting facial or eye muscles. Next-generation 
sequencing and deep phenotyping, in combination with international data sharing, reveals new genetic causes of CMS, but also unusual, overlapping clinical phenotypes which blur the boundaries with primary myopathies and motor neuropathies (SYT2, $G M P P B)$. Conclusion: We will cover the significant progress made in understanding the molecular pathogenesis of CMS and MG, which is important for both patients and clinicians in terms of reaching a definite diagnosis and selecting the most appropriate treatment. Moreover, new avenues of clinical and translational research will be addressed.

\section{TC $3.1 / \# 40$}

Topic: 02. Neuropathy

\section{CMT NEUROPATHIES IN THE ELDERLY}

Michaela Auer-Grumbach

Department Of Orthopaedics, Medical University

of Vienna, Vienna, AT

\begin{abstract}
Axonal neuropathies are frequently observed in the elderly and may lead to progressive disability. The underlying causes often remain elusive. However, recent studies have identified pathogenic variants in genes known to be involved in CharcotMarie-Tooth neuropathy type 2 (CMT2) disease in patients with and without family history. We present clinical and genetic data of 126 index patients with unexplained progressive axonal neuropathies and disease onset after age 35 years. Whole exome sequencing has been carried out in all probands. In more than $20 \%$ of the patients we observed one or two variants in MME, encoding the metalloprotease neprilysin. We detected heterozygous, homozygous and compound heterozygous loss-of-function and missense mutations in our probands and therefore confirm that MME mutations may segregate in an autosomal dominant and autosomal recessive fashion. Whenever possible, neprilysin levels in plasma were determined and correlated with particular variants and associated phenotypes. In further patients we identified pathogenetic or likely pathogenic variants in other genes known to cause late onset neuropathies (MPZ, LRSAM1, TTR, GARS, HARS, AARS, HMBS, HSPB8, MFN2, DHTKD1, WARS). Still more than $50 \%$ of the probands did not receive a genetic diagnosis and remain unexplained but further evaluation of WES data is ongoing.
\end{abstract}

TC 3.2 / \#180

Topic: 02. Neuropathy

\section{ESSENTIAL CLUES TO EARLY RECOGNITION OF AMYLOID NEUROPATHIES}

\author{
Davide Pareyson \\ Dept Of Clinical Neurosciences, IRCCS \\ Foundation, C.Besta Neurological Institute, \\ Milan, IT
}

\begin{abstract}
Inherited transthyretin amyloidosis (ATTR) is an autosomal dominant disorder due to mutations of the transthyretin (TTR) gene. TTR is synthetized mainly by the liver and released in plasma as a tetrameric transport protein. Mutations in TTR, of which Val30Met is the most common worldwide, cause transthyretin tetramer dissociation, monomer misfolding, and aggregation into insoluble fibrillar proteins in different tissues. Peripheral nerves and heart are the most frequently affected organs, but also eye, leptomeninges and
\end{abstract} kidneys can be involved. As there are effective treatments which are already available or will soon be marketed, and such treatments are more effective in the first disease phases, early diagnosis is fundamental for preventing disease progression in this otherwise lethal disorder. Early diagnosis is easier in familial cases in endemic regions, where Val30Met is by far the predominant mutation, degree of awareness is high, and presentation is typical, i.e., a predominantly small-fibre length-dependent sensory neuropathy with dysautonomia, only later involving motor fibres. Diagnosis is often delayed in non-endemic region where onset occurs much later in life, family history if often negative or misleading, dysautonomia is more subtle, presentation is atypical with a sensory-motor polyneuropathy involving all fibre types (or with other atypical presentations), and progression is definitely faster. ATTR-neuropathy must be considered in the differential diagnosis of any rapidly progressive neuropathy, particularly if later in life. Red flags are: association with carpal tunnel syndrome, early dysautonomia (particularly impotence in males), gastrointestinal involvement, neuropathic pain, loss of weight, fasciculations. When dealing with a patient with a possible TTRrelated neuropathy, the clinician should look for evidence of clinical or subclinical involvement of other target organs: ocular involvement with vitreal opacities, glaucoma, pupillary anomalies; cardiac 
involvement with arrhythmia, conduction blocks, interventricular septum hypertrophy, overt cardiomyopathy; proteinuria, renal failure, recurrent urinary tract infections. Ecocardiography, Technetium-99m-diphosphonate (99mTc-DPD) scintigraphy showing abnormal cardiac tracer accumulation, cardiac MRI, tilt test, sudomotor function testing are all ancillary investigation which may give clues to amyloidosis. Increased brain natriuretic peptide (BNP) levels may reveal subclinical cardiopathy, glomerular filtration rate is an important disease biomarker. Biopsies can be helpful by demonstrating tissue amyloid accumulation in nerve, abdominal fat, salivary glands, skin, and gastro-intestinal tract, but may also fail in detecting amyloid. A careful nerve conduction study with EMG is important but results may be aspecific; nerve conduction slowing is not infrequent and may be misleading. CSF protein content may be increased, explaining the high rate of misdiagnosis with CIDP. Other incorrect diagnoses include lumbar stenosis and Charcot-Marie-Tooth disease. However, if the clinical picture is compatible with ATTR-neuropathy the clinician should ask for genetic testing, which provides a definite diagnosis.

\section{TC 3.3 / \#78}

Topic: 02. Neuropathy

\section{SENSORY AND AUTONOMIC HEREDITARY NEUROPATHIES}

\author{
Max J. Hilz \\ Neurology, University of Erlangen-Nuremberg, \\ Erlangen, DE
}

Abstract: Hereditary sensory and autonomic neu-
ropathies (HSAN), Fabry disease, transthyretin-re-
lated familial amyloid polyneuropathy (TTR-FAP)
and acute intermittent porphyria are examples of
hereditary autonomic dysfunction. So far, eight
HSANs have been classified. HSAN I presents in
adulthood with prominent ulcers/mutilations of the
lower extremities, stress-fractures, osteomyelitis,
osteolysis, distal anhidrosis, low-normal nerve con-
duction velocities, impaired cold and heat pain-per-
ception. Already during infancy, HSAN II-patients
have acral anhidrosis, tonic pupils, eating/swallow-
ing difficulties, constipation, apneic episodes, and
develop deformed distal phalanges, paronychia, fin-
ger and plantar ulcers, unrecognized fractures and mutilating acropathy. They have severely impaired sensory-perception including vibratory and thermal perception, abnormal sensory but normal motor nerve conduction. Autosomal-recessive HSAN III (Familial Dysautonomia) afflicts Ashkenazi Jewish children, is characterized by diminished deep tendon reflexes, absent overflow tears, fungiform papillae of the tongue and axon flare response following intradermal histamine injection, pronounced central and peripheral autonomic dysregulation, including severe orthostatic hypotension $(\mathrm{OH})$ and autonomic crises with excessive arterial hypertension, profuse sweating, skin blotching, puffy hands and behavioral abnormalities. Children with HSAN IV (Congenital Insensitivity to Pain with Anhidrosis) have episodic high fever with anhidrosis, pain-insensitivity, unnoticed injuries, multiple fractures, neuropathic joints and limb mutilation. Children with $H S A N V$ have a selective loss of pain-perception. Autosomal-recessive HSAN VI is due to a DST (Dystonin) gene mutation, presents with neonatal hypotonia, poor feeding, respiratory problems, joint contractions, dysmorphic features, early death by age 2 and was reported in one Ashkenazi Jewish family. HSAN VII was reported in three patients with mutations in the SCN11A (Sodium Voltage-Gated Channel Alpha Subunit 11) gene which is also involved in Episodic Pain Syndrome. Patients have pain-insensitivity, multiple injuries, fractures, muscle-weakness, gastrointestinal-dysfunction and hyperhidrosis. Autosomal-recessive HSAN VIII is due to mutations in the PRDM12 gene (encoding a protein regulating pain-perception) and presents with pain- and temperature-insensitivity, self-mutilation, reduced sweating and tear-formation, absent corneal reflex, etc. Neurological manifestations of Fabry disease include severe lancinating pain, burning paraesthesias in the extremities, often triggered by temperature changes, anhidrosis, gastrointestinal-problems, vasomotor and cardiovascular autonomic dysfunction, baroreflex alteration, etc. Early diagnosis and enzyme replacement therapy can be beneficial. Autosomal-dominant TTR-FAP causes life-threatening autonomic dysfunction, particularly in early-onset patients, and length-dependent peripheral polyneuropathy with prominent small nerve fiber dysfunction causing impaired thermal and pain-perception, neuropathicpain, allodynia, painless injuries, Charcot joints, carpal tunnel syndrome, and cardiac conduction failure. Patients have peripheral blood flow and blood pressure dysregulation with $\mathrm{OH}$, altered heart rate 
variability, and cardiac arrhythmia resulting in syncope and sudden death. Gastrointestinal dysfunction includes constipation and/or diarrhea, nausea, vomiting. There may be bladder and erectile dysfunction. Autosomal-dominant acute intermittent porphyria due to haem-biosynthetic enzyme hydroxymethylbilane synthase deficiency, causes life-threatening acute attacks with autonomic symptoms, particularly painful-abdominal crises, peripheral-neuropathy progressing to respiratory paralysis, and cerebral involvement including psychosis, seizures or posterior reversible encephalopathy syndrome. Splanchnic autonomic dysfunction causes abdominal pain, with/ without constipation or diarrhoea. There may be nausea and vomiting, arterial hypertension or $\mathrm{OH}$, excessive sweating, bladder-sphincter dysfunction and other features of autonomic dysfunction. Autopsies show demyelination/axonal degeneration of vagus nerve and loss of sympathetic ganglion cells.

\section{TC 3.4 / \#66}

Topic: 02. Neuropathy

\section{NEUROPATHIC MANIFESTATIONS IN PORPHYRIA}

\section{P. James B. Dyck}

Mayo Clinic, Rochester, US

Background: Porphyrias are rare disorder of heme metabolism that presents in different types and each of the different types are characterized by a defect in an enzyme required for heme synthesis. These disorders can produce disturbances of multiple organ systems including the skin, liver, and central and peripheral nervous systems. Methods: We present information on the natural history, laboratory findings, evaluation and treatment of the neurological syndromes occurring in porphyria patients. Results: The types of porphyria that typically cause neurological disease are acute intermittent porphyria, hereditary coproporphyria and variegate porphyria, which are all autosomal dominant inherited conditions. The porphyrias generally present with attacks of illness. These attacks are characterized by neuropsychiatric symptoms with mood disorder or psychosis, motor predominant peripheral neuropathy (which often involve proximal and distal nerve segments and so resemble CIDP with severe weakness). Autonomic involvement (tachycardia, constipation, hypertension) and gastrointestinal disturbance (abdominal pain) are common, and (in the case of variegate porphyria and some hereditary coproporphyria) photosensitivity and cutaneous manifestations occur. Classically, there is a reddish discoloration of urine during an attack (after prolonged observation oxidation of porphyria precursors occurs). Attacks in porphyria can be induced by hormonal factors, nutritional factors, alcohol and use of drugs which induce the heme production pathway leading to an overabundance of heme precursors. An accurate diagnosis can be made through routine testing for overproduction of porphyria during an acute attack but this is more difficult in the quiescent phase. The most important factor in making the diagnosis is a high index of suspicion and an awareness of the diversity of clinical manifestations. DNA testing is available for the diagnosis. The best management of the disorder is through the prevention of acute attacks (avoidance of cytochrome P450-inducing agents and avoidance of periods of fasting) but intravenous hematin and glucose are helpful and should be considered during an acute attack. Introduction of new agents for the treatment of the porphyrias is in development. Conclusion: The neurological manifestation of porphyria generally present with attacks of neuropsychiatric symptoms, motor predominant peripheral neuropathy, abdominal pain and autonomic symptoms. They occur in patients with acute intermittent porphyria, hereditary coproporphyria and variegate porphyria. The diagnosis is often made by measuring excess porphyrins during an attack.

TC 4.1 / \#1033

Topic: 13. Pediatric

\section{EPIDEMIOLOGY AND DIFFERENTIAL DIAGNOSIS}

Guenther Bernert

Department Of Paediatrics, Kaiser Franz Joseph Hospital, Vienna, AT

\section{Abstract not received.}


TC 4.2 / \#1034

Topic: 13. Pediatric

\section{CLINICAL ASSESSMENT AND MUSCLE IMAGING: TECHNIQUES AND PITFALLS}

Presenter TBD

TC 4.3 / \#1035

Topic: 13. Pediatric

\section{FLOPPY INFANT SYNDROME AND THE ROLE OF "NEXT GEN"}

James J. Dowling

Hospital for Sick Children, Toronto, ON, CA

Abstract not received.

TC 4.4 / \#858

Topic: 13. Pediatric

\section{WHAT YOU MAY MISS WITHOUT EMG}

Matthew Pitt

Department Of Clinical Neurophysiology, Great Ormond Street Hospital for Children NHS

Foundation Trust, London, GB

Abstract not received.

\section{TC $5.1 / \# 45$}

Topic: 06. Mononeuropathy

\section{ENTRAPMENT SYNDROMES IN THE UPPER EXTREMITY - ELECTROPHYSIOLOGY}

$\underline{\text { Christian Bischoff }}$

Neurologische Gemeinschaftspraxis, München, DE

Abstract not received.
TC 5.2 / \#163

Topic: 06. Mononeuropathy

\section{ENTRAPMENT SYNDROMES IN THE UPPER EXTREMITY - SONOGRAPHY}

Nens Van Alfen

Radboudumc, Nijmegen, $N L$

Background: Nerve ultrasound is a proven technique for assessment of peripheral neuropathies, with excellent resolution in superficial tissue layers. In experienced hands it is more sensitive than MRI in detecting peripheral nerve pathologies $(93 \%$ versus $67 \%)$ and has comparable specificity $(86 \%)$. Ultrasound is a valuable adjunct to electrophysiology for assessing entrapment neuropathies. It has similar sensitivity for detecting entrapments as EMG, but both techniques complement eachother by adding different types of information about the lesion, so one can describe both form and function. Methods: For nerve size measurement, the workhorse is the transverse cross-sectional area (CSA). Because peripheral nerves can have different shapes, for example, round, oval, oblong, or triangular, depending on their anatomic location, transverse CSA is more reliable and reproducible than nerve diameter measurement, and is the most robust measure to confirm nerve pathology. The CSA should be measured within the hyperechoic epineurial rim that encircles the nerve fascicles. As it does not take much time to scan along the length of a nerve, it is strongly advised to scan the whole nerve during an exam, instead of just scanning a few predefined points along its course. Results: It is important to use the right reference value for the right patient. Ideally, a disease specific reference is available for the clinical question that needs to be answered, but if not, a population-based reference such as the p95 for CSA can also be used. Reference values for different nerves and populations are available in the literature. Conclusion: This talk will focus on the use of nerve ultrasound in entrapment syndromes of the upper extremities, including carpal tunnel syndrome, interosseus anterior syndrome, ulnar entrapment at the wrist and elbow, Martin Gruber anastomosis, radial nerve compression in the upper arm and supinator region, the incisura scapulae syndrome and neurogenic thoracic outlet syndrome. Case examples will show scanning technique, and diagnostic values will be presented where available. 
TC 5.3 / \#134

Topic: 06. Mononeuropathy

\section{ENTRAPMENT SYNDROMES IN THE LOWER EXTREMITY - ELECTROPHYSIOLOGY}

$\underline{\text { Simon Podnar }}$

University Medical Centre Ljubljana, Ljubljana, SI

Abstract not received.

\section{TC 5.4 / \#74}

Topic: 06. Mononeuropathy

\section{ENTRAPMENT SYNDROMES IN THE LOWER EXTREMITY $€$}

\author{
Alexander Grimm \\ Neurology, Tuebingen University Hospital, \\ Tuebingen, $D E$
}

\begin{abstract}
Entrapment syndromes in the lower limbs are rare compared to those of the upper limbs. However, to accurately diagnose them is even more difficult. Gold standard of diagnosis are nerve conduction studies and electromyography, e.g. in peroneal or tibial palsy. Nevertheless, its sensitivity in tarsal tunnel syndrome or meralgia paresthetica is restricted. Second, as already known from ulnar nerve palsy or carpal tunnel syndrome, anatomical variants and secondary reasons of entrapment cannot be detected by electrophysiology. In these cases, nerve ultrasound has proven its validity. With high resolution probes ranging from $10 \mathrm{MHz}$ (for femoral and ischiatic nerve) to $20 \mathrm{MHz}$ (for sural or superficial peroneal nerve) we can accurately visualize the nerve and its fascicles. Thereby one can not only perform cross-sectional area measurements, but also elucidate fascicular anatomy and echointensity. Further, surrounding tissue can be demonstrated, e.g. vessels, tendons, muscles, scare tissue, ganglion cysts and others. E.g. in peroneal palsy, one might often find intra- or extraneural ganglia as reasons of pathology. Consequences, which might arise out of the imaging, are outstanding. In my talk, I want to go on further details concerning common entrapments as tarsal tunnel syndrome and fibular head palsy of the peroneal nerve, but also demonstrate some interesting and astonishing cases of rare nerve pathology,
\end{abstract}

in which ultrasound could visualize the underlying pathology and prepare the way to surgical or conservative procedure. Methods: Nerve ultrasound, electrophysiology. Results: Examples of secondary reasons of entrapments. Conclusion: Nerve ultrasound might be a useful technique to further analyze mononeuropathies

TC $6.1 /$ \#41

Topic: 01. Muscle

\section{A PATTERN RECOGNITION APPROACH TO PATIENTS WITH A SUSPECTED MYOPATHY}

\author{
Richard J. Barohn \\ Neurology, University of Kansas Medical Center, \\ Kansas City, KS, US
}

\begin{abstract}
Myopathies are disorders affecting the channel, structure, or metabolism of skeletal muscle. Myopathies can be distinguished by characteristic clinical and laboratory features. The pattern recognition approach involves asking key questions and then based on the answer putting the patient in one of the ten patterns. The key questions are: 1.) Does the patient have "negative" or "positive" symptoms and signs? 2.) What is the temporal time line? What is the distribution of weakness or stiffness? 4.) Are there triggering events for episodic weakness, pain, stiffness? 5.) Is there a family history of a myopathic disorder? 6.) Are there associated systematic symptoms/signs? The ten patterns are: 1.) Proximal "limbgirdle" Weakness 2.) Distal Weakness 3.) Proximal arm/distal leg weakness (Scapuloperoneal) 4.) Distal arm/proximal leg weakness 5.) Eyeball Weakness-Ptosis/ophthalmoplegia 6.) Prominent neck and trunk extensor weakness 7) Bulbar / diaphragm weakness 8.) Episodic pain, weakness, dark urine 9.) Episodic weakness delayed or unrelated to exercise 10.) Stiffness/decreased ability to relax. Once a pattern has been identified, the next step is determining what laboratory tests are needed.
\end{abstract}


TC 6.2 / \#61

Topic: 01 . Muscle

\section{LABORATORY APPROACH TO MUSCLE DISORDERS}

Mazen Dimachkie

The University of Kansas Medical Center, Kansas City, US

Abstract not received.

TC 6.3 / \#116

Topic: 01. Muscle

\section{GENETIC APPROACH TO MUSCLE DISORDERS}

Tahseen Mozaffar

Uc Irvine-mda Als And Neuromuscular Center,

University of California, Irvine, Orange, US

Abstract not received.

TC 6.4 / \#1031

Topic: 01. Muscle

APPROACH TO TRANSLATIONAL RESEARCH AND CLINICAL TRIALS IN MUSCLE DISEASE

Michael Hanna

UCL Institute Of Neurology, London, GB

Abstract not received.

TC $7.1 / \# 34$

Topic: 03. Motor Neurone

\section{HOW DO WE DISCUSS THE} GENETIC IMPLICATIONS WITH

\section{ALS}

Ryuji Kaji

Tokushima University Graduate School of Medicine, JP

Abstract not received.
TC 7.2 / \#96

Topic: 03. Motor Neurone

\section{COGNITIVE CHANGE - RECOGNITION AND MANAGEMENT}

Albert Ludolph

Universitätsklinikum Ulm, Ulm, DE

Abstract not received.

TC 7.3 / \#53

Topic: 03. Motor Neurone

\section{BREAKING THE NEWS OF THE DIAGNOSIS}

Dorothée Lulé

University of Ulm, Ulm, DE

Abstract not received.

TC 7.4 / \#108

Topic: 03. Motor Neurone

THE ROLE OF MULTIDISCIPLINARY CARE IN ALS

Christopher Mcdermott

University Of Sheffield, Sheffield, US

Abstract not received.

TC 8.1 / \#72

Topic: 02. Neuropathy

\section{MRI AND US IN THE DIAGNOSIS OF NEUROPATHY}

Stephan Goedee

Brain Centre Rudolph Magnus, Umc Utrecht, Utrecht, NL

Background: Diagnosis of chronic inflammatory neuropathies rely heavily on electrodiagnosis. In order to demonstrate sufficient proof of demyelination, elaborate studies are needed since the electrodiag- 
nostic findings of demyelination are focal and patchy distributed. Neuro-imaging may complement this, as even extensive EDX studies may fail to identify these potentially treatable neuropathies. MRI imaging has been aknowledged as an important adjunctive diagnostic tool in the current sets of diagnostic criteria. More recently, ultrasound has gained importance as a powerful and practical bedside tool, that allows reliable identification of chronic inflammatory neuropathies. Methods: The role of MRI and ultrasound imaging in the diagnosis of neuropathy wil be discussed, including their part in complementing electrodiagnosis in the appropriate cinical context. Results: Standardization of MRI protocols, including diagnostically relevant sequences (T2/DIXON) and parameters (enlargment, T2 hyperintense signal nerve(root)), and recent developments such as DTI imaging and neurography will be discussed in detail. Ultrasound evaluation and diagnosis of chronic inflammatory neuropathies will also demonstrated in detail, including proposed scoring systems and a practical sonographic protocol en prediction rule.
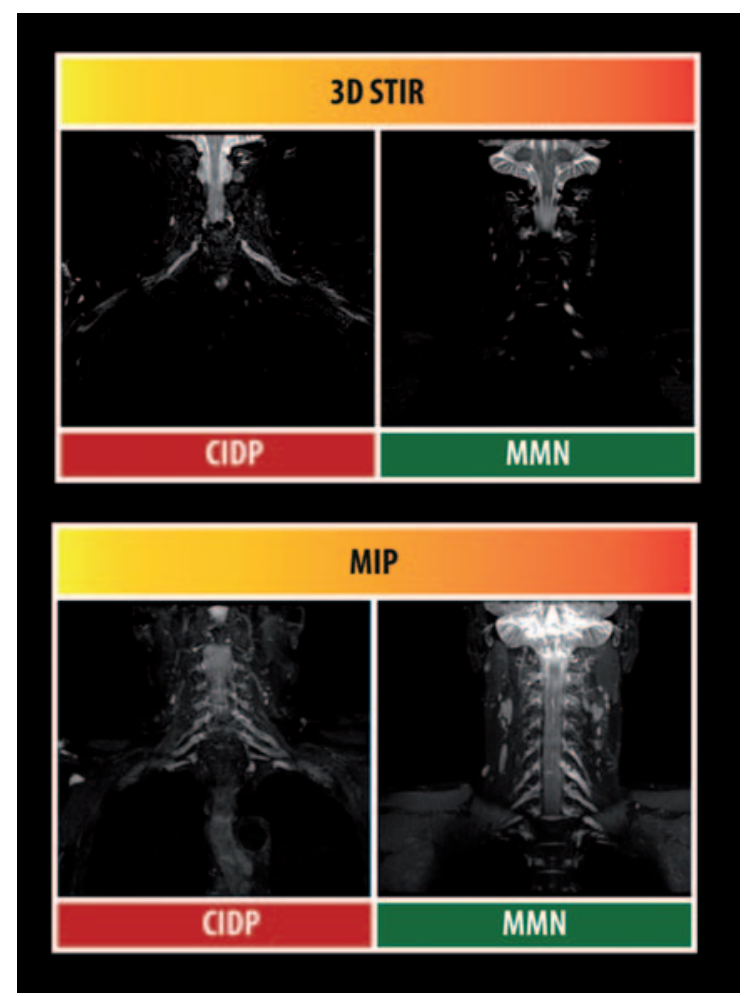

Conclusion: MRI and ultrasound imaging are important emerging diagnostic tools in neuromuscular practice, that can aid subtantially in the distinction of potentially treatable neuropathies.
TC 8.2 / \#92

Topic: 02. Neuropathy

\section{SKIN BIOPSY: NOT JUST FOR DIAGNOSIS}

Grazia Devigili

Fondazione Irccs Istituto Neurologico Carlo Besta, Milan, IT

Abstract not received.

TC 8.3 / \#112

Topic: 02. Neuropathy

\section{OUTCOME MEASURES IN NEUROPATHY}

Ingemar Merkies

Department Of Neurology, Maastricht University

Medical Center, Maastricht, $N L$

Abstract not received.

TC 8.4 / \#166

Topic: 02. Neuropathy

\section{ROLE OF ELECTROPHYSIOLOGY IN THE CHARACTERIZATION AND DIAGNOSIS OF NEUROPATHIES: NEW INSIGHTS}

\author{
Peter Van Den Bergh \\ Neuromuscular Reference Centre, University \\ Hospital Saint-Luc, Brussels, BE
}

\begin{abstract}
Electrophysiology plays a crucial role in the characterization and diagnosis of peripheral neuropathies. It provides insight in the type and mechanism of peripheral neuropathy by giving information on the spatial pattern (generalized, multifocal, focal), the fibre type involved (motor, sensory), pathology (axonal, demyelinating), and the severity and time course (acute, ongoing, chronic). Electrophysiological studies are key in the early detection and characterization of inflammatory demyelinating neuropathies and in differentiating these from primary axonal neuropathies.
\end{abstract}


Inflammatory demyelinating neuropathies constitute a significant proportion of the acquired peripheral neuropathies. They include Guillain-Barré syndrome (GBS), chronic inflammatory demyelinating polyradiculoneuropathy (CIDP), multifocal motor neuropathy (MMN), multifocal demyelinating neuropathy with persistent conduction block (Lewis-Sumner syndrome), and paraproteinemic neuropathies. A proper diagnosis as early as possible is very important because timely immune treatment can largely reduce morbidity and disability. The diagnosis is based on a constellation of clinical and laboratory features, including electrophysiological studies, spinal fluid examination, and in selected cases serological studies and peripheral nerve biopsy. In CIDP, electrophysiological criteria for demyelination are designed to exclude abnormalities that can be explained by axonal degeneration (Eur J Neurol 2010). Therefore, lesser degrees of demyelination cannot be defined with certainty. Optimised electrophysiological criteria are capable, however, to support the diagnosis with different levels of probability (possible, probable, definite) in the very large majority of cases. In GBS, much effort has gone into developing criteria which can distinguish axonal and demyelinating subtypes. The discovery of reversible conduction failure (RCF) has led to the concept of nodopathy/ paranodopathy, where conduction slowing and conduction block are due to the immune attack mainly at the nodal axolemma level. There is no actual demyelinisation as defined pathologically and if the immune attack continues, conduction failure may not reverse and axonal degeneration will ensue. Recent electrophysiological studies support this pathophysiological mechanism and show that the dichotomous distinction between axonal and demyelinating in GBS is not tenable (Muscle nerve, 2018).

TC $9.1 / \# 1021$

Topic: 01. Muscle

\section{REQUIREMENTS AND PITFALLS FOR SMALL MOLECULES}

Mike Kelly

Rush University Medical Center, Chicago, US
TC 9.2 / \#1022

Topic: 01. Muscle

\section{REQUIREMENTS AND PITFALLS FOR GENE THERAPY}

Isabelle Richard INTEGRARE, Généthon, Inserm, Univ Evry, Université Paris-Saclay, Evry, FR

Abstract not received.

TC 9.3 / \#1023

Topic: 01. Muscle

\section{REQUIREMENTS AND PITFALLS FOR STEM CELLS}

Miranda Grounds

University of Western Australia, Perth, $A U$

Abstract not received.

TC 9.4 / \#1059

Topic: 01. Muscle

\section{PRECLINICAL TRIAL DESIGN}

Annamaria De Luca

Dep Pharmacy \& Drug Sciences, University of

Bari, Bari, IT

Abstract not received.

TC 9.5 / \#1024

Topic: 01. Muscle

\section{STATISTICAL POWER EVALUATION}

Heather Gordish-Dressman

George Washington University School of Medicine and Health Sciences, Washington, DC, US

Abstract not received.

Abstract not received. 
TC 9.6 / \#732

Topic: 01. Muscle

DATA INTERPRETATION, TRANSLATIONAL MEANING

Annamaria De Luca

Dep Pharmacy \& Drug Sciences, University of Bari, Bari, IT

Abstract not received.

TC 9.7 / \#1036

Topic: 01. Muscle

\section{REGULATORY REQUIREMENTS}

Didier Caizergues

Genethon, Paris, FR

TC 9.8 / \#1020

Topic: 01. Muscle

\section{CLINICAL TRIAL READINESS}

Kathryn R. Wagner

The Johns Hopkins School of Medicine, Baltimore, $M D$, US

Abstract not received.

TC 9.9 / \#1025

Topic: 01. Muscle

\section{CARE ASPECTS IN CLINICAL}

\section{TRIAL PLANNING}

Anna Mayhew

Newcastle University, Newcastle Upon Tyne, GB

Abstract not received.
TC 9.10 / \#1026

Topic: 01. Muscle

\section{PATIENT PERSPECTIVE}

Elizabeth Vroom

Duchenne Parent Project Netherlands, Veenendaal, $N L$

Abstract not received.

TC 9.11 / \#1027

Topic: 01. Muscle

\section{BUSINESS MODEL}

Cristina Csimma

Csimma Llc, Lincoln, US

Abstract not received. 



\section{ICNMD - 15TH INTERNATIONAL CONGRESS 2018 ON NEUROMUSCULAR DISEASES}

www.icnmd2018.org July 6 - 10, 2018 Vienna, Austria

Overarching Courses 
OA $1.1 / \# 129$

Topic: Overarching Topic

\section{STEM CELLS AS TOOLS FOR RESEARCH AND THERAPY IN NEUROMUSCULAR DISORDERS}

Rita Perlingeiro

University of Minnesota, Minneapolis, US

Abstract not received.

OA 1.2 / \#176

Topic: Overarching Topic

\section{STEM CELLS IN MUSCULAR DYSTROPHIES}

Mayana Zatz

University Of São Paulo, São Paulo, BR

Abstract not received.

\section{OA 1.3 / \#83}

Topic: Overarching Topic

\section{STEM CELLS IN ALS}

Dimitrios Karussis

Hadassah Hmo, Jerusalem, IL

Abstract not received.

\section{OA $2.1 / \# 60$}

Topic: Overarching Topic

\section{NEUROMUSCULAR DISORDERS WITH DYSPHAGIA: AN OUTLINE}

\author{
Marianne De Visser \\ Department Of Neurology, Academic Medical \\ Centre, Amsterdam, NL
}

Abstract not received.
OA 2.2 / \#39

Topic: Overarching Topic

\section{HOW TO ASSESS AND MANAGE DYSPHAGIA}

\author{
Zohar Argov \\ Hebrew University of Jerusalem EKMD, \\ Jerusalem, IL
}

Background: The topic of dysphagia management and swallowing assessment receives low attention in the neuromuscular field. The main risks of dysphagia are reduced food intake and recurrent aspirations. Treatment trials in at least two muscle disorders (OPMD and sIBM) looked at dysphagia as an outcome, but there are no agreed upon clinical and investigational guidelines. Aims To review tests that can serve as treatment assessments for either clinical follow up or therapy trials and to present the main dysphagia management modalities. Observations 1 . There are several patient reported outcome measures that were validated for dysphagia (e.g. EAT10 SWAL-QoL). 2. Drinking tests include the 'classical' 80 cc cold water test (useful and easy to use bedside test that has served as an outcome in OPMD trials). Its main shortcomings are test-test variability and lack of strong correlation with aspiration risk. Using different fluid consistencies may resolve these issues. 3. Videofluoscopy (VFS) of barium swallow using an 8 point penetration- aspiration scale has been practiced in neuromuscular and other neurological conditions. It was validated in some disorders, its main deficiency is the semi quantitative nature of the test and the inconsistency between swallow attempts. VFS can monitor and quantify the several stages of the swallowing process. It requires high technical skills and dedicated equipment. Radionuclide swallow test has been developed and is less technically complicated yet limited data are available. 4. Dysphagia management includes: change of diet and eating habits, mechanical dilatation (which usually needs to be repeated), Botox injection (minimal observations), cricopharyngeal myotomy (needs a very experienced surgeon using the right technique for prolonged effects) and feeding tubes. Conclusion: Neuromuscular experts should become more familiar with dysphagia assessment and management. New therapeutic trials, now being planned for several conditions, mandate the development of clinical and investigational guidelines. 
OA 2.3 / \#122

Topic: 03. Motor Neurone

\section{ETHICAL ISSUES IN DYSPHAGIA TREATMENTS}

David J. Oliver

Tizard Centre, University of Kent, Canterbury, GB

Background: Dysphagia is a common symptom for people with neuromuscular disease - up to $90 \%$ in ALS. It is a distressing symptom for patients who face frustration at mealtimes, reduced nutrition, anxiety and fear of starvation, increased risks of aspiration and drooling. Moreover it is often a family issues as family carers experience the same emotional reaction and fears. Methods: A review of the literature has been undertaken. Results: There are several aspects of managing the symptoms - speech and language assessment allowing nutrition and hydration to be continued by careful feeding of foods with the most appropriate consistency; careful feeding by hand at risk, acknowledging the risk of aspiration but allowing the patient to retain the taste and activity of eating; consideration of enteral feeding, by naso-gastric tube or gastrostomy; intravenous or subcutaneous hydration to allow hydration. However there are many ethical issues raised in the assessment and discussion and management of these patients. In particular: - Whether to consider and use at risk feeding by hand - this may be appreciated by the patient and family but there is a risk of aspiration and possible deterioration and death - Whether to consider and discuss enteral feeding. Nasogastric tubes have a small risk of causing harm, and there is an appreciable morbidity and mortality of the placement of a gastrostomy. This may need to be considered earlier in the patient's progression, as deterioration in the respiratory function may be a factor with greater importance for the safety of the procedure. - Whether to with-hold treatment - either the procedure such as gastrostomy, or the use of feeds and hydration - or withdraw treatment as the patient deteriorates and comes towards the end of life. - In some countries, where assisted dying is permitted, dysphagia may be one of the symptoms that can lead to patients requesting a hastened death, from physician assisted suicide or euthanasia. Conclusion: These issues are complex and require careful assessment and communication within teams, and closely involving patient and family.
OA $3.1 / \# 37$

Topic: 01. Muscle

\section{IMPACT AND EVOLUTION OF COGNITIVE IMPAIRMENT IN MUSCULAR DYSTROPHIES}

\author{
Corrado Angelini \\ Irccs S.Camillo Hospital, Venice, IT
}

Background: Cognitive involvement is an important feature in several types of muscular dystrophies such as myotonic dystrophy (DM1) or limb girdle dystrophies (LGMD) and needs to be addressed by physicians. DM1 is a multisystemic disease characterized by muscle, heart and CNS dysfunction. There are at least five sub-types of DM1 classified as congenital (onset at birth), childhood (1-10 years),juvenile (10-20 years), adult (20-40 years), late onset( $>40$ years). Methods: A literature review was performed using the following keywords: DM1, LGMD, cognitive impairment, MRI and white matter abnormalities. Results: Congenital DM1 presents at birth with polyhydramnios, reduced fetal movements; after birth the child has severe weakness, muscle hypotonia, respiratory involvement. There is then a gradual improvement although these children present cognitive deficit and learning abnormalities. Apathy seems to be associated with impaired cognitive status, fatigue, motor disability. Fatigue is a prominent sign and a major complaint in DM1. Besides cognitive and behavioural features reduced awareness of disease is defined as "anosognosia", i.e.lack of awareness of disease burden can be studied by correlating patient's perception of disease status to caregiver observation. A significant number of brain imaging studies were done in DM1. In young/adult patients a significant structural alteration is present in white matter with relevant abnormalities in frontal, temporo-parietal polar and occipital regions. Atrophy of cerebral grey matter was also observed suggesting a global network and neuronal impairment. In congenital DM1 hydrocephalus might be present on MRI. The juvenile form of DM1 with age of onset after 10 years has under-recognized school learning problems beside the classical signs of grip myotonia distal muscle weakness and atrophy,. In adult onset DM1 nocturnal episodes of natural apnoea and day-time sleepness are common. Loss of serotonin-containing neurons might be in relation to hypersomnia. In late onset forms there are behavioural abnormalities that 
range from inappropriate behaviour to depression. In DM1 the evolution of cognitive and white matter abnormalities are a matter of research seem to progress with time and studies report after 5-9 years a progressive dysfunction for verbal memory, attention tasks and visual-spatial construction. White matter abnormality seems to progress although with variable extent on tested patients and families. Few longitudinal studies have attempted to correlate MRI imaging with neuropsychological changes, further research on longitudinal DM1 cohorts are needed. In LGMD due to a dystroglycan involvement (LGMD 2I)important cognitive deficits and visual-spatial construction difficulties are present. In an observational study we found several changes in nine LGMD 2I patients who underwent brain MRI scanning: four showed non-specific white matter abnormalities; two showed moderate ventriculomegaly; three showed mild enlargement of subarachnoid spaces; cerebellar atrophy was present in one patient. Conclusion: These observations point-out to the need for of developing clinical protocols for LGMD and DM1 patients that address the CNS dysfunction and quality of life. Physician should remain vigilant about personal hygiene, child neglect, financial needs in muscular dystrophy patients. The use of drugs such as modafinil might help to keep DM1 patients in a better vigilance status. Behaviour cognitive abnormalities might be approached both with drugs or individual cognitive strategies.

\section{OA 3.2 / \#57}

Topic: Overarching Topic

\section{BRAIN IMAGING TECHNIQUES FOR THE NEUROMUSCULAR PATIENTS}

Nicola De Stefano

University Of Siena, Siena, IT

Abstract not received.
OA 3.3 / \#62

Topic: 01. Muscle

\section{THE GAP BETWEEN COGNITIVE ASSESSMENT AND BRAIN IMAGING: LESSONS FROM DMD}

Nathalie Doorenweerd

\author{
C.j. Gorter Center For High Field Mri, Leiden \\ University Medical Center, Leiden, $N L$
}

Background: Cognitive assessments have demonstrated cognitive impairment, learning and behaviour difficulties and/or increased comorbidity of neuropsychiatric disorders in several different neuromuscular dystrophies. Neuroimaging has been used to elucidate the development of the healthy brain throughout a person's lifespan and its relation to cognitive function. However, when applied to Duchenne muscular dystrophy patients, who have a range of cognitive abilities and also a range of quantified structural cerebral alterations, the relationship between the two is anything but straightforward. Methods: On a group level, the power of imaging to detect patterns in altered structure or metabolism that point towards the involvement of specific mechanistic pathways is remarkable. When the function of a protein or gene within the brain is largely unknown, this is an important relationship. For an individual assessment, however, it is important to note the variability in absolute values of quantitative MRI measures, and the partial overlap with values associated with healthy controls. Such variability is not restricted to neuromuscular dystrophies, but rather a common theme in neuroscience. Results: For example, cerebral atrophy has been reported in cognitively-normal Alzheimer's patients, and a patient with severe autism can have a normal-appearing anatomical MRI. Conclusion: To discuss the gap between cognitive assessment and brain imaging, we will provide examples from our own research into the brain involvement in Duchenne muscular dystrophy. We will also review the recent literature and show examples from Myotonic Dystrophy and specific Congenital muscular dystrophies that highlight the wide spectrum of both cognitive ability and MRI abnormalities. 


\title{
ICNMD 2018 므
}

www.icnmd2018.org

\section{TH INTERNATIONAL CONGRESS ON NEUROMUSCULAR DISEASES} July 6 - 10, 2018 Vienna, Austria

\author{
Virtual Sessions
}




\section{ID:840}

Topic: 02. Neuropathy

\section{NEUROPATHIES IN AFRICA}

$\underline{\text { Riadh Gouider }}$

Neurology, Razi Hospital, Tunis, TN

Background: Systematic studies about peripheral neuropathies (PN) in Africa are lacking. PN are common and their incidence was estimated at 2.1 to 31 per thousand in this continent. Methods: We reviewed epidemiology and etiologies of PN in Africa. We searched Medline, Scopus and science direct using the following search terms "Neuropathies", "Africa", "epidemiology" and "etiology". Results: Diabetic neuropathy, one of the leading causes of PN worldwide, is becoming an increasing burden in African countries because of the poor quality of diabetes control and care. Conversely, etiologies of neuromuscular disorders vary considerably according to the African region considered. In Sub-Saharan Africa, infectious diseases are very prevalent causes. Neuropathies related to HIV, are frequently observed in South of Africa. They may be due to the direct effects of HIV, exposure to antiretroviral medications, advanced immune suppression and/or comorbid tuberculosis infection and exposure to antituberculosis medications. On another hand, leprosy is one of the main causes of PN in endemic countries such as in east and tropical Africa. Neuropathy is often clinically silent in its evolution making early diagnosis exceptionally challenging. Elsewhere, both deficiency in micronutrients related to malnutrition and toxin ingestion are mostly represented in western Africa. The occurrence of tropical ataxic neuropathy (TAN), due to the ingestion of poorly processed cassava, continues at a lesser prevalence than reported thirty years ago.Finally, hereditary neuropathies, especially Autosomal recessive forms of Charcot-Marie-Tooth disease (AR CMT) are frequent in North Africa because of high consanguinity in this region. They are characterized by an early onset, and quiet severe phenotype.Genetic analysis of large African families allowed discovering many new genes mutations such as LMNA/C gene mutations with variable phenotypes (CMT or Muscular dystrophy) and MTMR13 gene mutations with some clinical and histological characteristics as glaucoma with focally folded myelin sheaths aspect. Conclusion: Given this particular distribution of PN etiologies among regions in Africa, it is difficult to draw a diagnostic strategy without considering the patient origin. Lack of neurologists in some countries and of tools of diagnosis such an EMG in several countries makes the situation even more challenging.Although the handicap generated by such diseases, many patients still not accessing the level of health and social care services that could improve their health related quality of life.

\section{ID:857}

Topic: 02. Neuropathy

\section{VIRTUAL SESSION: HIV-} ASSOCIATED NEUROMUSCULAR MANIFESTATIONS: EXPERIENCE FROM CAPE TOWN

Jeannine M. Heckmann, Kathleen J. Bateman, Melody Asukile, Sarvani Chetty, Herman Ekea, Saara Neshuku

Medicine (neurology), University of Cape Town, Cape Town, ZA

Background: HIV infection can affect multiple levels of the nervous system at the same time and at all stages of infection. This also applies to the neuromuscular system. South Africa remains a high prevalence region with $>5$ million people on government sponsored antiretroviral treatment (ART) programs. Methods: The aim of this virtual session is to present interesting cases which we have encountered in our resource-constrained, high HIV prevalence setting. In addition, we invite discussion of more commonly encountered management issues. Results: The commonest manifestation, distal sensory polyneuropathy shows increasing incidence with advancing age, despite effective antiretroviral therapy, and has been shown to associate with increasing functional disability when compared to those with one or less neuropathic signs. Acute inflammatory demyelinating polyradiculoneuropathy (AIDP) occur in patients with CD4 counts between 45 and 652 cells $/ \mathrm{mm}^{3}$ and have a similar response to therapy compared to those with HIV-unaffected AIDP. Mimics of AIDP in our setting have included tuberculous lumbosacral radiculopathy, which may manifest as an immune reconstitution syndrome, diffuse infiltrative lymphocytic syndrome (DILS), and, rarely, syphilitic meningitis with radiculopathy. Since the 
earlier roll-out of ART, lower motor neuron syndromes are less frequently encountered including the segmental inflammatory motor radiculopathy, lymphomatous or cytomegalovirus radiculopathies. HIV-associated chronic inflammatory demyelinating polyradiculoneuropathy responds well overall to treatment with corticosteroids and antiviral agents, and may be monophasic, but second-line treatment may be required in the minority. Subacute HIV-associated "motor neuron disease" remains very rare. Myasthenia gravis is seen in patients infected with HIV, presenting either before or after HIV infection, but these cases are treated similarly to those without HIV. Several cases of HIV-associated inflammatory myopathy have been encountered and mostly respond to immune-therapy and ART. Although uncommon, we will also discuss a sporadic steroid-responsive, but steroid-dependent nemaline myositis. Conclusion: We describe a range of neuromuscular manifestations associated with HIV-infection. As has been observed in first world countries, the earlier initiation of ART has resulted in an overall decline in neuromuscular complications associated with more advanced HIV-infection. 



\section{ICNMD $2018=$}

www.icnmd2018.org July 6-10, 2018 Vienna, Austria

\section{ENMC}




\section{ID:321}

Topic: European Neuromuscular Centre (ENMC)

\section{WHY OPT FOR AN ENMC WORKSHOP?}

\author{
George W. Padberg ${ }^{1}$, Baziel Van Engelen ${ }^{2}$ \\ ${ }^{1}$ Dep. Of Neurology, Radboud University Medical \\ Center, Nijmegen, NL
}

Abstract not received.

\section{ID:322}

Topic: European Neuromuscular Centre (ENMC)

\section{THE IMPACT OF 25 YEARS ENMC WORKSHOPS}

\section{$\underline{\text { Raffaella Willmann }}$}

\section{Enmc Executive Committee, Swiss Foundation for}

Research on Muscle Diseases, Zürich, CH

Background: The European NeuroMuscular Centre (ENMC) was constituted in 1992 to encourage and facilitate collaborative research in Europe on neuromuscular disorders (NMD) to contribute to the eradication of these diseases. During its 25 years of activity, the ENMC established a network of more than 2500 clinicians and scientists from over 65 different countries and hosted/organized 236 workshops. Discussion topics, agreements and deliverables of the workshops have been regularly summarised in scientific reports, published on Neuromuscular Disorders and actively cited in the scientific community. During its first 10 years of activity (with about 100 workshop sponsored), the ENMC contributed to the achievement of several goals, such as the creation of several disease-specific consortia, publication of books on diagnostic criteria, definition of clinical and molecular features of NMD. These activities reflected the most urgent needs of the NMD community, but then it was also felt that future policies had to include other initiatives to improve neuromuscular cure and care (Rüdel et al., Neuromusc. Dis., 2000, 10:75-82; Emery et al., Clinical Medicine, 2001, 1:200-202). Particularly, the founders encouraged the ENMC to engage in additional activities in order to foster the development of protocols for therapeutic trials and management studies, to promote training and to establish new cooperative networks for clini- cal trials. Therefore, while continuing its support to the NMD community through the financing of focused workshops, the ENMC also invested in the promotion of new initiatives, such as the "Clinical Trial Network", aimed at providing the evidence and guidance for developing treatments and for management of NMD. This activity contributed to set the ground for the TREAT-NMD network, funded from 2007 to 2011 within the EU FP6 Health program, to which ENMC was part, now continuing as TreatNMD Alliance (www.treat-nmd.eu). In the most recent years, the ENMC refreshed the structure of its sponsored workshops, paying particular attention to the value of involving people affected by neuromuscular conditions in every meeting. Giving voice to patients often resulted in important contributions during the discussion and is now an essential component of each workshop's program. In addition, the ENMC started to actively involve young researchers and clinicians in the workshops, thus contributing to the development of the next generation of professionals. Methods: To evaluate the long-term vision of improving quality of life of people affected by NMD, the ENMC conducted a survey and a detailed analysis of workshop topics and citation index in the last 25 years. Moreover, it retrospectively monitored the implementation in the NMD community of agreements, decisions and other deliverables derived from a recent five-years-cohort of ENMC workshops. Results: The evaluation identified and quantified pitfalls and challenges in fulfilling deliverables and goals, highlighting possible reasons for success or for delays or failure in translating the workshop outcomes into actions to improve patients' quality of life. Conclusion: These data will be presented in occasion of the ENMC $25^{\text {th }}$ anniversary symposium at the ICNMD meeting and will be used in future to support effective and constructive workshops for implementable research progresses.

ID:323

Topic: European Neuromuscular Centre (ENMC)

\section{CHARCOT-MARIE-TOOTH DISEASE AT ENMC WORKSHOPS}

\author{
Mary Reilly \\ Institue Of Neurology, London, GB
}

Abstract: Charcot Marie Tooth disease (CMT) is 
the commonest inherited neuromuscular disorder affecting 1 in 2,500 people. It characteristically starts in the first two decades of life with distal wasting, weakness, foot deformity and sensory loss and progresses at different rates depending on the subtype. There are over 100 causative genes but in northern Europe, UK and the US about $50 \%$ of patients have the common CMT1A genotype due to a 1.4 megabase duplication of chromosome 17 containing the peripheral myelin protein 22 gene (PMP22). This is also the commonest genotype worldwide. The chromosome 17 duplication was the first causative gene to be identified for CMT in 1991 and the 25 years since then has seen an explosion in gene identification followed by natural history studies, outcome measure development, guideline development and clinical trials. Since it was founded in 1992, the ENMC has played a crucial role in all of the above developments in CMT and has enabled collaborative research and progress which would have been much more difficult without the CMT ENMC workshops. The ENMC has not only brought European researchers and patients together but it has also facilitated the inclusion of people from further afield including the US and Australia when beneficial. The ENMC was ahead of its time by truly giving patients a voice in research and developing therapies for their disease. The initial ENMC meetings facilitated gene discovery by enabling carefully phenotyped family details to be gathered, discussed and combined for further genetic studies. Many new types of CMT were identified in this way. In the early 2000 s pre clinical studies suggested that ascorbic acid might be a beneficial therapy for CMT1A. A very important ENMC meeting on CMT1A in 2005 allowed us to look at the evidence and critically to agree a protocol for trials. This meant all three of the 2 year international trials (French, UK/Italian and US) used the same primary outcome measure (CMTNS) which allowed all the results to eventually be considered together in a Cochrane review. Although all the trials were negative, I suspect this may have been the first time all trials of a new therapy used an identical primary outcome measure. Following this a further ENMC meeting in 2009 to look critically at our outcome measures resulted in both agreeing modifications to the adult measure (CMTNS) and laid the grounds for developing the paediatric outcome measure (CMTPeds), which has since been published and validated. Most recently in response to the lack of evidence for the varying types of foot surgery for CMT, an ENMC meeting in 2016 resulted in the publication of pre- liminary guidelines and the identification of areas needing further research. CMT clinicians, researchers and most of all patients owe a lot to the ENMC.

\section{ID:324}

Topic: European Neuromuscular Centre (ENMC)

\section{ENMC TRANSLATIONAL RESEARCH WORKSHOP}

\section{Kanneboyina Nagaraju}

School Of Pharmacy And Pharmaceutical Sciences, SUNY-Binghamton University, Binghamton, NY, US

Background: There are very few specific therapies for neuromuscular diseases (NMD) despite significant basic sciences advances for the last 2-3 decades in this field. There are several reasons why basic science research failed to translate into new therapies for these disorders. One of the reasons is the lack of rigor and reproducibility of preclinical research in animal models of these diseases. One way to address this issue is to bring various stakeholders such as basic scientists, pre-clinical scientists, journal editors, clinicians, clinical trialists, industry, funding agencies, parent groups, and regulatory agencies to formulate guidelines that enhance reproducibility of preclinical animal research in NMDs. Methods: The ENMC workshop assembled 27 stakeholder representatives from seven different countries (USA, Italy, Switzerland, Netherlands, Belgium, United Kingdom, Australia) in Heemskerk in the Netherlands, on the weekend of the 10th-11th of February 2017 to help finalize a plan to guarantee quality in translational research for neuromuscular diseases. Results: After extensive one and half day discussions the participants agreed that there are significant gaps in a) understanding translational research both among basic scientists and clinicians, b) evaluating grant applications by funding agencies and c) publication of peer reviewed translational research in journals. Workshop participants agreed to 1) organize a teaching workshop for clinicians and basic researchers interested in the planning and conduct of NMD clinical trials, 2) promote rigorous scientific evaluation of grant applications and to enforce the requirement for strong if possible independent validation of pre-clinical data before human clinical trials and 3) encourage journal editors to implement the ARRIVE guidelines and publish negative data. 
Conclusion: This workshop for the first time highlighted that promoting robust and reproducible translational research would enhance success of future treatments for NMDs. By facilitating these discussions and by offering the adequate format, ENMC greatly contributed and continues to contribute to the progress in research for neuromuscular diseases.

\section{ID:326}

Topic: European Neuromuscular Centre (ENMC)

\section{THE POSITION OF NEUROMUSCULAR PATIENTS IN SHARED DECISION MAKING}

Anna Ambrosini

Research And Development, Fondazione Telethon, Milan, IT

Background: In occasion of its $25^{\text {th }}$ anniversary, the European Neuromuscular Centre (ENMC) organised a special workshop involving 45 participants from 15 countries, representing patients and patient organizations, health professionals, clinical researchers, regulatory agencies, social scientists, ethicists and industry. Aim of the meeting was the assessment of patient participation in research and care options for neuromuscular diseases, using Shared Decision Making (SDM) as a tool to drive discussions. Methods: SDM has been defined as "an approach where clinicians and patients share the best available evidence when faced with the task of making decisions, and where patients are supported to consider options, to achieve informed preferences". In practice, SDM applies to the communication between a health professional and a patient about the options of prevention, screening, diagnostic tests and treatment, including the option of not intervening; the goals of the method being better-informed patients, more confidence in and satisfaction about the treatment, and more compliance. Results: During the meeting, the SDM concept was discussed at first in relation to its natural field of application, the bilateral interaction between patients and their clinician. Different moments of the delivery of care were addressed, from genetic screening and diagnosis, to the management of transition from child to adult, and implementation of standards of care that have major impact on daily life. Then, the participants borrowed the SDM concepts to investigate the position of patient/patient organizations with respect to their level of participation in decision processes dealing with clinical research, in particular, in relation to patient registries and biobanks, research on quality of life, clinical trials design, and regulatory and consenting processes. Expectations are that, thanks to a better knowledge and empowerment, the patient community is able to take an increasingly decisive role in clinical trials, dissemination, and relationship with pharmaceutical industry and public health institutions. For each field addressed during the workshop, working groups including both professionals and patient representatives analysed: i) opportunities and limitations of patient participation as well as wishes of the patient community; ii) positive and negative examples and availability of best practices; iii) feasibility of implementation of a greater patient involvement; iv) next steps to be taken. In the end, some common features among all topics became evident. In order to be active partners, patients need to be properly informed, hence formation is key. However, education and training concern all stakeholders, with coaching and proficient communication about wishes and challenges of implementing patients' participation in the various areas addressed. The roles and preferences of all stakeholders should be made more explicit. Moreover, structural changes are necessary in order to include patient representatives/organizations in advisory boards, ethics committees and in every stage of clinical trials. Conclusion: Overall, the active inclusion of patients in delivery of care and research processes requires changing attitudes, and therefore, ambassadors are needed who inspire and empower others about the benefits of patients' participation. It was emphasized that exchange at the European level is also needed. An ENMC white paper will identify more specific actions and goals for the six topics discussed. 


\section{ICNMD 2018 므}

www.icnmd2018.org

\section{TH INTERNATIONAL CONGRESS ON NEUROMUSCULAR DISEASES}

July 6 - 10, 2018 Vienna, Austria

\section{Poster Sessions}




\section{PS1Group1-001 / \#342}

Topic: Group 1 - Muscle Diseases of Genetic Origin and Acquired Myopathies: Clinical Features, Pathophysiology, Therapy

\section{A FAMILY-BASED STUDY INTO PENETRANCE IN FACIOSCAPULOHUMERAL MUSCULAR DYSTROPHY TYPE 1}

\author{
Mariëlle Wohlgemuth $^{1}$, Richard J. Lemmers ${ }^{2}$, \\ Marianne A. Jonker ${ }^{3}$, Elly L. Van Der Kooi ${ }^{4}$, \\ Corinne G. Horlings ${ }^{5}$, Baziel G. Van Engelen ${ }^{5}$, \\ Silvere M. Van Der Maarel ${ }^{2}$, George W. Padberg' \\ Nicol C. Voermans ${ }^{5}$ \\ ${ }^{1}$ Neurology, Radboud University Medical Center, \\ Nijmegen, NL, ${ }^{2}$ Human Genetics, leiden University \\ medical Center, Leiden, NL,; Health Sciences, \\ Radboud University Medical Center, Nijmegen, \\ $N L ;{ }^{4}$ medical center leeuwarden, leeuwarden, \\ NL, ${ }^{5}$ Neurology, Radboud University Medical \\ Center, Nijmegen, $N L ;{ }^{6}$ Dep. Of Neurology, \\ Radboud University Medical Center, Nijmegen, NL
}

Background: Objective: An observational crosssectional study in a national FSHD expertise center to estimate the penetrance of facioscapulohumeral muscular dystrophy (FSHD) 1 and to evaluate phenotype-genotype correlations. Methods: Ten FSHD1 probands carrying 4-9 D4Z4 units alleles and 140 relatives were examined. All 150 subjects were genetically characterized, including D4Z4 methylation levels in the mutation carriers. Mutation carriers were classified as 1) symptomatic: with symptoms of muscle weakness on history and muscle FSHD signs on examination; 2) asymptomatic: without symptoms of muscle weakness but with muscle FSHD signs on examination; and 3) non-penetrant: without symptoms of muscle weakness on history and without muscle FSHD signs on examination. We assessed the relationship between age-corrected clinical severity score and repeat size, sex and D4Z4 methylation levels. Results: The maximum likelihood estimates of symptomatic and those of symptomatic plus asymptomatic FSHD showed that penetrance depends on repeat size and increases until late adulthood. We observed many asymptomatic carriers with subtle facial weakness with or without mild shoulder girdle weakness $(25 \%(17 / 69))$. Non-penetrance was observed less frequently than in recent population studies $(17 \%(12 / 69))$, and most asymptomatic patients reported some shoulder pain. Methylation tended to be lower in moderately to severely affected mutation carriers with longer repeats. Conclusion: This family-based study detected a lower overall non-penetrance than previously observed, probably due to many asymptomatic mutation carriers identified by careful examination of facial and shoulder muscles. The recognition of asymptomatic mutation carriers is essential for selection of participants for future trials, and the likelihood estimates are helpful in counseling.

\section{PS1Group1-002 / \#482}

Topic: Group 1 - Muscle Diseases of Genetic Origin and Acquired Myopathies: Clinical Features, Pathophysiology, Therapy

\section{EVALUATING THE USEFULNESS OF NEW LINE IMMUNOASSAYS FOR MYOSITIS ANTIBODIES IN CLINICAL PRACTICE: A RETROSPECTIVE STUDY}

\author{
Federica Montagnese $^{1}$, Haris Babacic ${ }^{2}$, Peter \\ Eichhorn $^{3}$, Benedikt Schoser ${ }^{4}$ \\ ${ }^{1}$ Neurology, Friedrich Baur Institut LMU, Munich, \\ $D E ;{ }^{2}$ Neurology, Friedrich Baur Institut LMU, \\ Munich, DE:3 Institute Of Laboratory Medicine, \\ Ludwig Maximilian University, Munich, \\ DE: $;{ }^{4}$ Friedrich-baur Institute, Departement Of \\ Neurology, Ludwigs-Maximilians University of \\ Munich, Munich, DE
}

Background: The diagnosis of idiopathic inflammatory myopathies (IIM) relies on clinical and histological (inflammatory infiltrates on muscle biopsy) criteria. However, an increasing number of myositisassociated (MAA) and myositis-specific antibodies (MSA) are being detected in sera of IIM patients and are considered useful diagnostic biomarkers. New line immunoassays are nowadays widely used for MSA/MAA detection though some concerns on their reliability arise due to limited validation in comparison with the gold standard immunoprecipitation. Furthermore, the disease specificity of these antibodies has been mainly studied in myositis patients and data on screening in control patients are often limited. The aim of our study was to assess the accuracy of MSA and MAA in diagnosing IIM in our large cohort of patients. Methods: We have retrospectively analysed the sera of patients that between 2014 and 2017 have been tested for myositis 
antibodies. The "Euroline: myositis 16 Ag" Kit by EUROIMMUN has been used to assess the presence of the following MSA/MAA: Mi-2alpha,Mi-2beta,T IF 1 g a m ma, MD A 5, NXP 2, S A E 1, Ku, P M S c 1100 , P M - S c 175 , J o - 1, S R P, P L - 7, P L 12,EJ,OJ,Ro-52. Additional clinical data as symptoms at onset, CK, muscle biopsy and conclusive diagnosis were also analysed. We have calculated the sensitivity (Se), specificity (Sp), positive/ negative predictive values (PPV, NPV) and positive/ negative likelihood ratios (LR+, LR-) for MSA and MAA as well as group as for the single antibodies. Results: 1232 patients (1727 serum samples) were identified. Muscle biopsy was performed in 583 patients $(47 \%)$. Conclusive diagnoses were: myopathy $(\mathrm{n}=356)$, IIM $(\mathrm{n}=148)$, no evidence of neuromuscular diseases $(\mathrm{n}=587)$ and other neuromuscular diseases (e.g. ALS, neuropathies; $n=141$ ). The overall Sp was for MSA $95 \%$ and for MAA $89 \%$, whereas the Se was $21 \%$ and $22 \%$ for MSA and MAA respectively. Further analyses were performed considering only individuals who underwent muscle biopsy as gold standard for IIM diagnosis. This group comprised 134 myositis and 446 controls (55\% myopathy, $11 \%$ other neuromuscular disease and $35 \%$ no neuromuscular diseases). In this subgroup of patients the Se and Sp were equal to those observed in the whole cohort. The PPV was higher for MSA (54\%) compared to MAA (37\%) whereas the NPV was the same for both MSA/MAA (80\%). A positive test increases the post-test probability that the patient has myositis of about $30 \%(\mathrm{LR}+=4)$ for MSA and $15 \%(\mathrm{LR}+=2)$ for MAA, whereas a negative test does not significantly decrease the probability that the patient has a myositis (LR- $=0,8$ for MSA and 0,9 for MAA). In 154 patients the test was repeated at least twice, here we found a high agreement between repeated measurements as in only $18 \%$ of patients the test retrieved discrepant results. Conclusion: This study may help clinicians to understand the significance and proper use of MSA/MAA in clinical practice. Commercial immunoassays for myositis antibodies show low sensitivity and high specificity, supporting the claim that they should be used for confirmatory rather than screening purposes. Repeating the test does not seem necessary, considering the high agreement in repeated measurements. Combining antibody findings with clinical features will help building up specific diagnostic algorithms for IIM

\section{PS1Group1-003 / \#853}

Topic: Group 1 - Muscle Diseases of Genetic Origin and Acquired Myopathies: Clinical Features, Pathophysiology, Therapy

\section{EXPRESSION OF DP116 IS A PREDISPOSING FACTOR FOR CARDIAC DYSFUNCTION IN DUCHENNE MUSCULAR DYSTROPHY}

Tetsushi Yamamoto ${ }^{1}$, Awano Hiroyuki ${ }^{1}$, Takamitsu Imanishi $^{1}$, Yuji Nakamachi ${ }^{1}$, Masafumi Matsuo ${ }^{2}$, Kazumoto Iijima $^{3}$, Jun Saegusa ${ }^{1}$

${ }^{1}$ Department Of Clinical Laboratory, Kobe

University Hospital, --, Kusunoki-cho, Chuo-ku, Kobe, JP,'2Department Of Physical Therapy, Faculty Of Rehabilitation, Kobe Gakuin University, kobe, JP, ${ }^{3}$ Department Of Pediatrics, Kobe University Graduate School of Medicine, kobe, JP

Background: BACKGROUND: Duchenne muscular dystrophy (DMD), the most common inherited muscular disease in childhood, is caused by dystrophin deficiency because of mutations in the $D M D$ gene. Cardiomyopathy is the most important nonmuscular symptom threatening the life of DMD patients. The correlation between cardiac involvement and dystrophin mutation has been not fully understood since it was analyzed only in the view of mutation position in the $D M D$ gene. In this study, we focused dystrophin isoforms produced by tissuespecific promoters and analyzed relationship between cardiac involvement in DMD patients and disrupted dystrophin isoform. Methods: METHODS: The medical records of DMD patients registered at the Department of Pediatrics of Kobe University Hospital from August 2007 to January 2017 were retrospectively reviewed. All echocardiograms were obtained by 1 examiner (T. Yamamoto), with considerable experience in imaging of patients with DMD, using a commercially available echocardiographic system (Aplio XG; Toshiba Medical Systems, Tochigi, Japan). Total 1109 echocardiographic data were obtained from 181 DMD patients with confirmed mutations in the $D M D$ gene. For KaplanMeier analysis, to estimate a cardiac dysfunctionfree survival rate, 19 patients were excluded due to existing cardiac dysfunction at the first examination. As a result, the remaining 162 patients were analyzed. Results: RESULTS: A Kaplan-Meier curve 
revealed that at the age of 14 years, cardiac dysfunction (left ventricular ejection fraction $<53 \%$ ) was observed in nearly half of the DMD patients. To examine the impact of dystrophin isoforms on a cardiac dysfunction, patients were divided by patterns of dystrophin isoform deficiency into 5 groups based on the mutations affecting dystrophin isoforms. The Dp427 group consisted of all 162 patients who had mutations at any position in the $D M D$ gene. The Dp260, Dp140, Dp116, and Dp71 groups consisted of $120,102,19$, and 11 patients, respectively. The cardiac dysfunction-free survival rates of the Dp260 group was similar to that of the other. Furthermore, the survival rates of the Dp140 and Dp71 groups did not differ significantly from in the others. However, the survival rate was significantly higher in the Dp116 group than in the others (log-rank test, $\mathrm{P}=0.022$ ). Moreover, patients in the Dp116 group were at lower risk of cardiac dysfunction than the other patients (hazard ratio, 2.89; 95\% confidence interval, 1.038-8.045; $\mathrm{P}=0.042$ ). At age 25 years, the survival rate was 0.6 in the Dp116 group, and 0.15 in the other patients. Left ventricular dilationfree survival rates were estimated in the 5 DMD patient groups and compared with those in other patients. None of these comparisons showed significant differences, including a comparison of patients in the Dp116 group with all other patients (log-rank test, $\mathrm{P}=0.23$ ). Conclusion: CONCLUSIONS: Cardiac dysfunction was less frequent in mutation of Dp116 coding region than other patients with DMD. These results indicate that Dp116 is a predisposing factor for cardiac dysfunction in DMD.

\section{PS1Group1-004 / \#348}

Topic: Group 1 - Muscle Diseases of Genetic Origin and Acquired Myopathies: Clinical Features, Pathophysiology, Therapy

\section{RIMEPORIDE: RESULTS FROM A PHASE IB STUDY IN PATIENTS WITH DUCHENNE MUSCULAR DYSTROPHY}

Teresa Gidaro ${ }^{1}$, Laurent Servais ${ }^{1}$, Stefano Previtali ${ }^{2}$, Alberto Zambon', Jackie Pitchforth ${ }^{3}$, Kate Maresh', Jordi Diaz-Manera ${ }^{4}$, Pierre G. Carlier ${ }^{5}$, Pierre Yves Baudin $^{6}$, Benjamin Marty ${ }^{5}$, Stéphanie Carnesecchi ${ }^{7}$, Christian Laveille ${ }^{8}$, Julian Gray ${ }^{9}$, Florence Porte $\underline{\text { Thome }}^{9}$, Delphine Labolle ${ }^{9}$, Melanie Annousamy ${ }^{1}$,
Virginie Chê ${ }^{1}$, Maria Grazia Natali Sora ${ }^{2}$, Simonetta Gerevini $^{10}$, Nuria Vidal ${ }^{4}$, Katie Groves ${ }^{11}$, Francesco Muntoni $^{3}$

${ }^{1}$ I-motion, Hopital Trousseau, PARIS, $F R ;{ }^{2}$ Neurology, San Raffaele Scientific Institute, Milano, IT; ${ }^{3}$ Dubowitz Neuromuscular Unit, UCL Great Ormond Street Institute of Child Health, London, GB; ${ }^{4}$ Neuromuscular Disorders Unit, Hospital de la Santa Creu i Sant Pau, Barcelona, ES: ${ }^{5}$ Aim \& Cea Nmr Laboratory, Neuromuscular Investigation Center, Institute of Myology, Paris, FR $;{ }^{6}$ CRIS, Froyennes, BE; $;{ }^{7}$ Department Of Pathology And Immunology, Faculty of Medicine, Geneva, $\mathrm{CH} ;{ }^{8}$ Calvagone, Lyon, $F R ;{ }^{9} \mathrm{R} \& d$, EspeRare, Plan les Ouates, $\mathrm{CH} ;{ }^{10}$ Department $\mathrm{Of}$ Neuroradiology, San Raffaele Scientific Institute, Milano, IT; ${ }^{11}$ Great Ormond Street Hospital For Children, Somers Clinical Research Facility, London, GB

Background: Duchenne muscular dystrophy (DMD) is an orphan disease affecting approximately 1 in 3500 male births worldwide. Children typically lose their ability to walk between 7-13 y and this is followed by progressive respiratory muscle weakness and complications. Cardiac disease is an increasingly important source of morbidity and mortality in these patients. Rimeporide is a first-inclass NHE-1 inhibitor originally developed for congestive heart failure (CHF) that is being repositioned for patients with DMD. Rimeporide demonstrated a beneficial effect in mdx mice and hamster with dilated cardiomyopathy. Its safety, tolerability, and pharmacokinetics were examined in 7 clinical pharmacology studies involving 145 healthy subjects and 21 patients with CHF treated. Rimeporide was well tolerated in healthy male subjects and in patients with CHF as single and multiple doses up to $600 \mathrm{mg}$. Methods: Rimeporide was tested in a multicentre, European, open-label, Phase 1b study in 6-11 years DMD boys to assess its safety, tolerability and pharmacokinetics and to explore effects on biomarkers (serum and MRI) after a 4-week treatment. 4 dose levels were assessed sequentially through 4 ascending dose cohorts (from 75 to 900 $\mathrm{mg}$ daily dose). Dosing in the next cohort being started only after safety was deemed satisfactory at the preceding dose by an independent Safety Monitoring Committee. The trial sites were in Paris, Milan, London and Barcelona. 20 patients were enrolled and completed the study. Results: Over the course of the study, there has been no significant safety 
signal. Safety and tolerability were good in all dose cohorts. Adverse events observed were mild, shortlived, and considered unrelated to study medication. There was one serious adverse event, (loose stools and vomiting requiring overnight hospitalisation in an $8 y$ old patient in cohort 3 receiving $600 \mathrm{mg}$ ) on the last day of the dosing period. The SAE was considered to be due to food poisoning and unrelated to Rimeporide. No significant new risks associated with treating young patients with DMD (6 to 11 years old) were identified. The pharmacokinetic profile was in line with the plasma concentrations observed in adults. Rapid absorption, dose related increase in concentration and no accumulation were observed. Serum biomarkers of inflammation and cardiac/skeletal muscle are currently measured. In addition, skeletal muscle MRIs taken at baseline and end of study are currently being analysed centrally. Conclusion: Rimeporide was shown to provide beneficial effects in animal models of DMD \& cardiomyopathy and was shown to be well tolerated in healthy adults and now in DMD boys. It has the potential to be a muscle-sparing agent that may alleviate long-term accumulated skeletal and cardiac muscle damage and inflammation. In contrast to many therapeutics under development, rimeporide is potentially applicable to all DMD patients, regardless of the mutation. As it was already shown to be well tolerated in adults and now in DMD boys, it is anticipated that it could be safely combined with other therapies (e.g. corticosteroids, exon skipping, ACE inhibitors..) to achieve synergistic effect. The next milestone is a phase II study to evaluate the efficacy of rimeporide in a larger group of patients.

\section{PS1Group1-005 / \#603}

Topic: Group 1 - Muscle Diseases of Genetic Origin and Acquired Myopathies: Clinical Features, Pathophysiology, Therapy

\section{A LONG TERM PREVENTIVE EFFICACY STUDY WITH RIMEPORIDE, A SODIUM-PROTON EXCHANGER INHIBITOR, IN GRMD DOGS}

Inès Barthélémy ${ }^{1}$, Jinbo $\mathrm{Su}^{2}$, Yves Fromes ${ }^{3}$, Stéphanie Carnesecchi ${ }^{4}$, Florence Porte Thome ${ }^{5}$, Pierre G. Carlier ${ }^{3}$, Bijan Ghaleh ${ }^{2}$, Stephane Blot ${ }^{1}$ ${ }^{1}$ U955 - Imrb, Inserm, Ecole Nationale Vétérinaire
d'Alfort, UPEC, Maisons-Alfort, FR ${ }^{2}$ Inserm Umr 955, Institut Mondor de Recherche Biomédicale, Créteil, FR ${ }^{3}$ Aim \& Cea Nmr Laboratory, Neuromuscular Investigation Center, Institute of Myology, Paris, FR $;{ }^{4}$ Department Of Pathology And Immunology, Faculty of Medicine, Geneva, $\mathrm{CH} ;{ }^{5} \mathrm{R} \&$, EspeRare, Plan les Ouates, $\mathrm{CH}$

Background: While the concept around the NHE-1 exchanger inhibition and its cardioprotective effect in models of heart failure is known for over a decade, it is a new concept in Duchenne Muscular Dystrophy (DMD). Cardiac disease is the leading cause of death in DMD and myocardial fibrosis is a key pathology predicting worsening of heart function and cardiac events. Rimeporide, working through inhibition of NHE-1, is cardioprotective in cardiomyopathic hamsters and $\mathrm{mdx}$ mice with reduction in cardiac pathology including fibrosis. Rimeporide also showed positive effects on skeletal muscles and diaphragm in mdx mice. Golden Retriever Muscular Dystrophic (GRMD) dogs reproduce the full spectrum of DMD and allow the study of skeletal, respiratory and cardiac outcomes with technologies translatable to patients. This study was designed to collect further knowledge on the co-evolution of cardiac, respiratory and locomotion defects. Methods: This is the largest study ever conducted in GRMD dogs. 24 GRMD dogs received Rimeporide at 20 $\mathrm{mg} / \mathrm{kg} /$ day or placebo. At 2 months, dogs were stratified using a previously validated method between severe and moderate phenotype. Treatment was blinded, started from the age of 2 months until the age of 10 months. The gait quality was assessed using 3D-accelerometry recording during spontaneous locomotion. Validated clinical scoring analogous to motor scores for DMD patients were performed monthly. Respiratory function was also evaluated monthly using respiratory inductance plethysmography and non-invasive tools including fluoroscopic assessments. Echocardiography, including Tissue Doppler Imaging and speckle tracking techniques (at 2, 4, 6, 9 and 12 months). MRIs (at 2, 6 and 12 months with Gadolinium enhancement for cardiac) were also used for cardiac and skeletal muscles assessments. Serum biomarkers and histopathology analysis are conducted in search of non-invasive biomarkers to be used in patients. Results: A pharmacokinetic study was conducted to select the dose prior to the study initiation. Based on cardiomyopathic hamster and mdx mice studies, therapeutic exposure is above $500 \mathrm{ng} / \mathrm{mL}$. GRMD dogs received 
$10 \mathrm{mg} / \mathrm{kg}$ of Rimeporide as a capsule in the morning and in the evening. Despite a high variability in absorption and elimination, it was possible to confirm that $20 \mathrm{mg} / \mathrm{kg} /$ day given as 2 doses of $10 \mathrm{mg} / \mathrm{kg} /$ day was maintaining plasma concentration above the therapeutic exposure. 24 GRMD dogs were enrolled and subsequently stratified between moderate and severe forms. At 2-month of age, 6 GRMD dogs were found with decreased stride frequency and a reduced spontaneous speed together with an elevation number of circulating CD4+/CD49dhi T cells and were subsequently assigned as severe forms. 18 additional GRMD dogs were classified as moderate forms. Over the 10-month treatment period, the tolerability of rimeporide was good. As observed in toxicology studies, there was no safety flags. Analyses of skeletal, cardiac, respiratory functions are ongoing and will be discussed in the poster. Conclusion: Results from this translational study in GRMD dogs will contribute to design phase II/III pivotal study by guiding outcome criteria, dose and biomarker selections as well as increasing the understanding of the efficacy profile of Rimeporide in skeletal, respiratory and cardiac muscle and in general supporting DMD disease understanding.

\section{PS1Group1-006 / \#458}

Topic: Group 1 - Muscle Diseases of Genetic Origin and Acquired Myopathies: Clinical Features, Pathophysiology, Therapy

\section{PREDICTORS OF EARLY LEFT VENTRICULAR SYSTOLIC DYSFUNCTION IN DMD PATIENTS}

Raphael H.D. Cirino ${ }^{1}$, Rosana H. Scola ${ }^{2}$, Renata D. $\underline{\text { Ducci }}^{3}$, Ana C.C. Wermelinger ${ }^{1}$, Claudia S.K. Kay ${ }^{2}$, Paulo J. Lorenzoni ${ }^{2}$, Lineu C. Werneck ${ }^{2}$, Eliane R. Carmes $^{4}$, Claudio L.P. Da Cunha ${ }^{1}$

${ }^{1}$ Cardiology, Hospital de Clínicas - Universidade Federal do Paraná, Curitiba, BR, ${ }^{2}$ Neurology, Hospital de Clínicas - Universidade Federal do Paraná, Curitiba, BR $;{ }^{3}$ Neurology, Hospital de Clínicas - Universidade Federal do Paraná, Curitiba, BR $;{ }^{4}$ Internal Medicine, Hospital de Clínicas - Universidade Federal do Paraná, Curitiba, BR

Background: The life expectancy of Duchenne muscular dystrophy (DMD) patients has improved in the last years, but the management of cardiac complications remains a challenge in DMD therapy. Most DMD patients develop cardiomyopathy, but the symptoms can be masked by severe musculoskeletal weakness. Myocardial strain is an emerging technique that measures myocardial deformation and is able to diagnose left ventricular systolic dysfunction (LVSD) even in the absence of reduced left ventricular ejection fraction (LVEF). Early detection of left ventricular systolic dysfunction (LVSD) is important for therapeutic strategies for DMD patients. We analyzed the myocardial strain by echocardiography as a main tool for the early detection of LVSD and determined the predictors of early LVSD. Methods: Cross-sectional study of forty DMD patients with normal LVEF followed at the Neuromuscular Disorders Service of the Hospital de Clínicas of the Federal University of Parana in Curitiba, Brazil between January 2014 and June 2016. LVEF was measured by 3-dimensional transthoracic echocardiography. Global longitudinal strain (GLS) was used to analyse subtle disturbances in the longitudinal contraction of the myocardium. Patients were determined to have early LVSD [GLS $>-18$ ] or normal left ventricular systolic function [GLS $\leq-18]$. Independent samples Student's t-test was used to compare continuous variables in the studied groups. For qualitative variables, the comparison was made using Fisher's exact test. We adjusted bivariate logistic regression models for variables with $\mathrm{p}<0.05$ in the univariate analysis, when possible, including age as covariate. After adjustment, the Wald test was used to assess the significance of the variables included in the model, estimating odds ratios with $95 \%$ confidence intervals. P values $<0.05$ were considered statistically significant. The study was approved by the Ethics Committee. Results: The study population consisted of 40 DMD male patients aged 2 to 19 years (median: 10.5 years; mean: $10.7 \pm 3.9$ years). Only 18 patients were still walking. Thirtyone patients used corticosteroids. Twenty $(50.0 \%)$ patients had early LVSD. The remaining 20 patients had normal LVSF as determined by GLS measurements. Patients who had early LVSD were older and had a longer disease duration and a higher frequency of gait loss, corticosteroid therapy and of mutations in exons 45, 46, 47, 48, 49, 50 and 52. The logistic regression model was adjusted for the analysis of exons 45 (OR 9.6, 95\% CI 0.90-102.8, $\mathrm{p}=0.053$ ) and 50 (OR 36.1, 95\% CI 2.15-604.0, $\mathrm{p}=0.010$ ). It was not possible to adjust the bivariate model for the other variables. Conclusion: Echocardiographic myocardial strain analysis can detect LVSD before 
the reduction of the LVEF in a large number of DMD patients. The importance of the early detection of LVSD lies in the fact that early initiation of specific treatments could prevent the progression of cardiomyopathy and its complications. The results of this study also suggest that older age, longer disease duration, gait loss, use of corticosteroids and mutations within the "hot spot" region of the DMD gene are predictors of early LVSD.

\section{PS1Group1-007 / \#736}

Topic: Group 1 - Muscle Diseases of Genetic Origin and Acquired Myopathies: Clinical Features, Pathophysiology, Therapy

\section{GENE THERAPY BY CRISPR-CAS9 MEDIATED EXON SKIPPING IN A PRE-CLINICAL MODEL OF DMD, THE GRMD DOG}

$\underline{\text { Isabel Punzón }}^{1}$, Inès Barthélémy ${ }^{1}$, Frédéric Auradé2, Nicolas Blanchard-Gutton ${ }^{1}$, France Piétri-Rouxel ${ }^{3}$, Frédéric Relaix ${ }^{2}$, Stéphane Blot ${ }^{1}$

${ }^{1}$ Neurobiology, U955 - IMRB, INSERM, Ecole Nationale Vétérinaire d'Alfort, UPEC, Maisons Alfort, FR $;^{2}$ Henri Mondor Hospital School Of Medicine, INSERM-UPEC IMRB U955-E10, Créteil cedex, FR, ${ }^{3}$ Sorbonne Université-UMRS974Inserm-Institut de Myology, Paris, FR

Background: Duchenne muscular dystrophy (DMD) is a rare degenerative disease without any cure at present. It is an X linked disease due to mutations into the dystrophin gene, that causes the absence of the protein and the progressive degeneration of muscles including heart and diaphragm. Golden Retriever Muscular Dystrophy dog (GRMD) mimics perfectly the DMD and is the accepted pre-clinical large animal model for this disease. The GRMD presents a spontaneous splice site single mutation in the dystrophin gene that leads to the skipping of exon 7 , disruption of the reading frame, absence of protein and consequently progressive muscle degeneration, weakness, heart and respiratory failure. A promising approach to cure DMD is gene therapy and specifically exon skipping which efficacy has already been proved restoring partially functional variants of dystrophin. Drugs start to be commercialized to skip exon 51, but a personalised medecine would be preferable. Our group showed that exon skipping strategy using antisense sequences vectorized into adenoassociated vectors (AAV) could rescue the dystrophin absence and significantly improves muscle function in GRMD dogs. However a longterm follow-up showed a decrease in dystrophin expression parallel to a loss of viral genome. To avoid the need of repeated injections of molecules for the exon skipping, with the associated inconvenience for patients and the possible immunoreactions side effects we propose the use of CRISPR tool. This emerging tool for genome editing, appears powerful to perform exon skipping therapy in a permanent way (genome edition of satellite cells) and it has been already successfully proved in mice and human cells. Methods: We started a translational study of genome edition based exon-skipping therapy in GRMD dog, using AAV. 2 pairs of RNA guides were cloned into an AAV-9 vector with tropism for skeletal muscles and heart. A CMV promoter was used for driving the Cas9 and mCherry expression and human and mouse U6 promoters for both gRNAs. A 7 month-old GRMD male dog was intramuscularly injected with an AAV vector containing two RNA guides flanking exons 6 and 9. Results: First, we showed efficacy of CRISPR-exon skipping (6 to 9) in vitro on primary GRMD myoblasts. In vivo, after intramuscular injections, the skipped transcript was detected and associated with dystrophin expression, in biopsies performed 2 months after AAV injection. This is the first proof of principle that genome editing-mediated multi-exon skipping can be performed in a large animal model of DMD, leading to an in-frame transcript and dystrophin expression. Conclusion: Further steps will include systemic delivery of AAVs, to evaluate the levels of dystrophin in targeted muscles and to correlate this to clinical and functional improvements. The genome edition of satellite cells, which would allow a sustained expression of dystrophin, will also be assessed. We also expect to answer crucial preclinical questions of feasibility, bio distribution, efficiency and safety before translation to humans. The GRMD model offers the unique opportunity to study long-term effects (several years) of such a strategy in a dystrophic context and will allow a relevant comparison of the CRISPR based strategy with pre-existing ones. 


\section{PS1Group1-008 / \#734}

Topic: Group 1 - Muscle Diseases of Genetic Origin and Acquired Myopathies: Clinical Features, Pathophysiology, Therapy

\section{IN VIVO CELL TRACKING OF CANINE MYOBLASTS BY SODIUM/ IODIDE SYMPORTER GENE EXPRESSION}

$\underline{\text { Isabel Punzón }}^{1}$, David Mauduit ${ }^{1}$, Inès Barthélémy ${ }^{1}$, Nicolas Blanchard-Gutton ${ }^{2}$, Jean-Laurent Thibaud ${ }^{3}$, Pauline De Fornel ${ }^{3}$, Bryan Holvoet ${ }^{4}$, Jean-Thomas Vilquin $^{5}$, Maurilio Sampaolesi ${ }^{6}$, Stéphane Blot ${ }^{2}$ ${ }^{1}$ Enva, Neurobiology, U955 - IMRB, INSERM, Maisons Alfort, FR; ${ }^{2}$ Enva, Neurobiology, U955IMRB, INSERM, Maisons Alfort, FR, ${ }^{3} 58$ Rue Auguste Perret,, MICEN Vet,, Créteil, FR: ${ }^{4} \mathrm{Ku}$ Leuven, Belgium, Translational Cardiomyology Lab, Stem Cell and Embryo Biology, Dept Development \& Regeneration, Leuven, BE: ${ }^{5}$ Myology Research Center, Pitié Salpêtrière Hospital, Sorbonne Université UPMC Univ Paris 06, Inserm UMRS974, CNRS FRE3617, Myology Research Center, Paris, FR; ${ }^{6}$ Translational Cardiomyology Lab, Stem Cell and Embryo Biology, Dept Development \& Regeneration, Leuven, $B E$

Background: Cell therapy emerged as a promising approach for the treatment of degenerative muscular diseases but due to disappointing results of clinical trials, efforts need to be done for the identification of the potential therapeutic cell (survival, homing to skeletal muscle, bio distribution, muscle regeneration...). For cell therapy improvement through a better understanding of therapeutic stem cells, we propose a non-invasive cell monitoring by medical imaging that would improve the understanding of the behavior of the cells following transplantation. Methods: We demonstrated the feasibility to track canine $\mathrm{NIS}^{+}$myoblasts by SPECT/CT with specific uptake of ${ }^{99 \mathrm{~m}} \mathrm{Tc}$-pertechnetate $\left({ }^{99} \mathrm{mcO}_{4}^{-}\right)$in the skeletal muscle of a large animal. The sodium/iodine symporter (NIS), a natural symporter of iodide in thyroid, salivary glands and stomach was used as a reporter gene. We used a lentiviral vector expressing canine NIS to transduce primary canine myoblasts. Results: NIS did not interfere with myotubes formation, and only cells expressing NIS (myoblasts and myotubes) were able to uptake radioactivity both in vitro and in vivo. We performed whole body scintigraphy imaging after intramuscular injections of different amounts of $\mathrm{NIS}^{+}$and $\mathrm{NIS}^{-}$expressing myoblasts. SPECT/CT acquisitions at different timepoints until 30 days post cells' injection were done and biopsies were taken at day 32 for histological analysis. Three millions of NIS expressing myoblasts were detected by scintigraphic imaging only at $48 \mathrm{~h}, 10$ millions were detected until one week, and 20 millions were still detected after one month. Histological analysis of injected muscles showed cNIS expression 1 month after cell injection and myoblasts expressing NIS fused with muscle fibers. Conclusion: We demonstrate that this is a non-invasive tool to track cells in vivo, that only functional cells trap radioactivity and that a longterm follow-up is possible being repeatable as many times as necessary. We propose a follow-up study using non-invasive cell tracking by scintigraphy imaging, in combination with functional studies to determine the migratory behavior and therapeutic properties of cells: muscle colonization, dystrophin expression, influences of cells' environment on their migration and differentiation.

\section{PS1Group1-009 / \#928}

Topic: Group 1 - Muscle Diseases of Genetic Origin and Acquired Myopathies: Clinical Features, Pathophysiology, Therapy

\section{CLINICAL OUTCOME STUDY OF DYSFERLINOPATHY: RELATIONSHIP BETWEEN MUSCLE MRI AND PHYSIOTHERAPY OUTCOME MEASURES}

Jordi D. Manera ${ }^{1}$, Roberto Fernández-Torrón ${ }^{2}$, Meredith James², Anna Mayhew², Michele Eagle², Robert Muni Lofra ${ }^{2}$, Fiona Smith ${ }^{3}$, Helen Sutherland ${ }^{2}$, Anne Marie Sawyer ${ }^{4}$, Carolina Tesi Rocha ${ }^{5}$, John W. Day ${ }^{5}$, Anthony Peduto ${ }^{6}$, Kirsti J. Jones $^{7}$, Eva M. Coppenrath ${ }^{8}$, Maggie C. Walter ${ }^{9}$, Pierre G. Carlier ${ }^{10}$, Tanya Stojkovic ${ }^{11}$, Andrew Blamire $^{3}$, Kate Bushby ${ }^{2}$, Volker Straub ${ }^{12}$ ${ }^{1}$ Neuromuscular Unit, Neurology Department, Hospital de la Santa Creu i Sant Pau, Barcelona, ES, ${ }^{2}$ The John Walton Muscular Dystrophy Research Centre, Newcastle Hospitals Trust, Newcastle University, Newcastle Upon Tyne, GB $;^{3}$ Magnetic Resonance Centre, Institute For Cellular Medicine, Newcastle University, Newcastle Upon Tyne, 
$G B,{ }^{4}$ Lucas Centre For Imaging, Stanford University School of Medicine, Stanford, CA, US, ${ }^{5}$ Department Of Neurology And Neurological Sciences, Stanford University School of Medicine, Stanford, CA, US; ${ }^{\circ}$ Department Of Radiology, Westmead Hosptial, Faculty Of Health Sciences, University of Sydney, Sydney, AU; ${ }^{7}$ Institute For Neuroscience And Muscle Research, Children's Hospital At Westmead, University of Sydney, Sydney, $A U ;{ }^{8}$ Department Of Clinical Radiology, Ludwig-Maximilians-University Munich, Munich, $D E ;{ }^{9}$ Friedrich-baur Institute, Departement Of Neurology, Ludwigs-Maximilians University of Munich, Munich, DE: ${ }^{10}$ Aim \& Cea Nmr Laboratory, Neuromuscular Investigation Center, Institute of Myology, Paris, FR; ${ }^{11}$ Ap-hp, G.h. Pitié-salpêtrière 47-83, Boulevard De L'hôpital, Institut de Myologie, Paris, FR $;{ }^{12}$ John Walton Muscular Dystrophy Research Centre, Newcastle University, Institute of Genetic Medicine, Newcastle Upon Tyne, GB

Background: Dysferlinopathies are caused by mutations in the DYSF gene. Patients may present with isolated hyperCKemia, limb girdle muscle weakness or predominant weakness of the lower legs. The Jain Foundation is funding The Clinical Outcome Study for Dysferlinopathy, a multi-centre natural history study in a cohort of 203 dysferlinopathy patients. Previous smaller studies have reported an involvement of scapular muscles on muscle MRI but no longitudinal studies have been reported for upper limb involvement in muscle MRI in relation to physiotherapy functional outcome measures. Methods: Here we describe baseline and year 3 upper limb muscle MRI data in a large cohort of dysferlinopathy patients. To examine the longitudinal correlation between muscle MRI semiquantitative scoring and functional outcome measures. 203 patients (11 to 86 years old) were enrolled in the JAIN COS study across 15 centers (Europe, USA, Australia, Japan). Patients underwent physiotherapy, medical and MRI assessments. 74 patients underwent upper limb muscle MRI at the time of enrollment and 61 patients had upper limb muscle MRI at year 3 at the point this analysis was completed. Scans were acquired from different systems and manufacturers at $1.5 \mathrm{~T}$ and 3T. Muscles were scored on axial T1-weighted sequences with the semiquantitative Mercuri visual scale modified by Fisher. Physiotherapy assessments included muscle strength (manual muscle testing; hand held dynamometry ) and functional ability evaluations (Performance of Upper limb, Brooke test). Change between baseline and year 3 muscle MRI was assessed using Wilcoxon's Signed Rank Tests and statistical significance was set at $\mathrm{p}=0.05$. Results: The subscapularis $(80.8 \%)$, latissimus dorsi $(82.6 \%)$, infraspinatus (73.8\%) and supraspinatus $(72.8 \%)$ were the most affected scapular muscles at baseline. The subscapularis muscle was involved in some patients without proximal upper limb symptoms or dysfunction. The biceps brachii $(57.1 \%)$ and the anterior muscles of the forearm $(53.8 \%)$ were the most affected muscles from the arm and forearm at baseline. Baseline data identified the tongue (34.2\%) and the cervical paraspinal muscles $(24.6 \%)$ as the most commonly involved cranial and cervical muscles. Cranial and cervical muscles did not show longitudinal change apart for the tongue and cervical paraspinal muscles. Conclusion: Muscle semiquantitative scoring correlated with functional outcome measures in upper limb in dysferlinopathy patients.

\section{PS1Group1-010 / \#10}

Topic: Group 1 - Muscle Diseases of Genetic Origin and Acquired Myopathies: Clinical Features, Pathophysiology, Therapy

\section{MUSCLE IMAGING AND CLINICAL OUTCOME MEASURES IN OCULOPHARYNGEAL MUSCULAR DYSTROPHY}

H. M.j.m. Kroon ${ }^{1}$, Corinne G. Horlings ${ }^{2}$, J. G. Kalf $^{3}$, Baziel G. Van Engelen ${ }^{2}$

${ }^{1}$ Rehabilitation, Radboud University Medical

Centre, Nijmegen, NL; ${ }^{2}$ Neurology, Radboud

University Medical Centre, Nijmegen,

NL; ${ }^{3}$ Rehabilitation, Radboud University Medical

Centre, Nijmegen, $N L$

Background: Oculopharyngeal muscular dystrophy (OPMD) is a rare late onset progressive neuromuscular disorder. Currently, there is a profound lack of knowledge on muscle abnormalities in OPMD. Therefore we collected a large data set to present for the first time in OPMD (1) quantitative muscle ultrasound (QMUS) of the head-neck region and legs and its relation to clinical outcome measures and (2) a comparison between QMUS and muscle MRI. Methods: Forty eight OPMD patients (24 Women, 
mean age 60.5 , SD 8.7) including asymptomatic patients, participated. MRI (DIXON, quantitative fat fractions) was used to measure muscle fatty infiltration of the rectus femoris, gastrocnemius, soleus and biceps femoris muscles. QMUS (echo-intensities) was used to measure fatty infiltration of the same leg muscles and of the tongue muscle and masseter muscle. Also, all participants were clinically tested by the Motor Function Measure (MFM) and muscle strength measures (MRC scale and dynamometry) of knee extension, knee flexion, and foot plantar flexion and by measuring the maximum isometric pressure of the tongue (MIP) and maximum bite force (Bite Force Gauge). Correlations were calculated between the echo intensities (EI) and the clinical measurements and between the EI and the mean MRI fat fractions, with accepting $r<.30$ being a weak, r.30.70 being a moderate and $r>.70$ being a strong correlation. Results: Mean EI of all muscles correlated moderately with the MFM $(r=-.584 ; p=.00)$. EI of rectus and biceps femoris correlated moderately with knee extension and knee flexion strength $(r=-.660$; $\mathrm{p}=.00$ and $\mathrm{r}=-.526 ; \mathrm{p}=.00$ ), and moderately with dynamometry of knee extension and flexion $(\mathrm{r}=$ $-.661 ; p=.00$ and $r=-.363 ; p=.02$ ). EI of the soleus and gastrocnemius did not correlate with foot plantar flexion $(r=-.241 ; p=.11)$. EI of tongue correlated moderately with the MIP $(r=-.633 ; p=.00)$, but EI of the masseter did not correlate with the maximum bite force $(\mathrm{r}=-.271 ; \mathrm{p}=.06)$. Mean EI and the mean MRI fat fractions are moderately correlated biceps femoris $(\mathrm{r}=.354 ; \mathrm{p}=.02)$, rectus femoris $(\mathrm{r}=.502 ; \mathrm{p}$ $=.00)$ and gastrocnemius $(\mathrm{r}=.544 ; \mathrm{p}=.00)$, except the soleus $(r=.193 ; p=.23)$. Conclusion: In OPMD patients echo intensity correlates moderately with the measures of maximum motor functioning of the legs and the tongue, but not when using dynamometry or bite force, suggesting that the latter measures are less useful in OPMD. QMUS and MRI of the legs show moderate correlations, suggesting that QMUS of the leg muscles could be used instead of more invasive and expensive MRI.

\section{PS1Group1-011 / \#444}

Topic: Group 1 - Muscle Diseases of Genetic Origin and Acquired Myopathies: Clinical Features, Pathophysiology, Therapy

\section{DIGENIC SQSTM1-TIA1 MYOPATHY: CLINICAL AND PATHOLOGICAL FEATURES}

\author{
Zhiyv Niu ${ }^{1}$, Carly S. Pontifex ${ }^{2}$, Sarah Berini ${ }^{1}$, \\ Leslie E. Hamilton ${ }^{2}$, Elie Naddaf ${ }^{1}$, Eric Wieben ${ }^{1}$, \\ Ross A. Aleff', Kristina Martens ${ }^{2}$, Angela Gruber ${ }^{3}$, \\ Andrew G. Engel ${ }^{1}$, Gerald Pfeffer $^{2}$, Margherita \\ $\underline{\text { Milone }}^{4}$ \\ ${ }^{1}$ Mayo Clinic, Rochester, MN, US, ${ }^{2}$ Clinical \\ Neurosciences, University of Calgary, Calgary, AB, \\ CA, ${ }^{3}$ PreventionGenetics, Marshfield, WI, \\ US $;{ }^{4}$ Neurology, Mayo Clinic, Rochester, MN, US
}

Background: T-cell intracellular antigen-I (TIA1, chr.2) is a largely expressed RNA-binding protein required for the cytoplasmic stress granules formation, which is a crucial step in preventing misfolded protein accumulation. To date, a single TIA1 mutation (p.Glu384Lys) is known to cause a late-onset myopathy with rimmed vacuoles. TIA1 mutations were recently identified in amyotrophic lateral sclerosis (ALS) / frontotemporal dementia (FTD). Sequestosome-1 (SQSTM1, chr.5) is a scaffolding protein participating in apoptosis, cell survival, autophagy, and regulation of ubiquitinated protein turnover. Mutations in SQSTMI can cause a multisystem proteinopathy manifesting as Paget disease of bone (PDB), ALS, FTD and myopathy with rimmed vacuoles but have incomplete penetrance. Methods: Review of clinical, serological, electrophysiological, muscle morphological, cardiac and radiological findings of three unrelated probands with late-onset distal myopathy and their affected and unaffected family members, including the affected fraternal twin of a proband. Next Generation Sequencing panel, whole exome sequencing, and targeted Sanger sequencing analyses of family members were performed to investigate the molecular genetic etiology of the myopathy. Results: Genetic analyses detected two variants in genes located on different chromosomes responsible for the myopathy in the three unrelated probands: a heterozygous pathogenic mutation in SQSTM1 (c.1175C > T, p.Pro392Leu) and a heterozygous variant in TIA1 (c.1070A $>$ G, p. Asn357Ser). The affected fraternal twin of a proband carries both variants; the unaffected family members 
have one or no variants. Two probands have a distal myopathy with rimmed vacuoles that presented with weakness of the index extensor; the other proband has a distal myopathy with myofibrillar pathology accompanied by hypercapnic respiratory insufficiency. Some of the affected individuals in a family have cognitive impairment. Conclusion: 1) The affected individuals of three unrelated families have a myopathy associated with both variants, one in SQSTM1 and one in TIA1 (genes located on different chromosomes), suggesting that the myopathy is digenic. 2) The combined SQSTM1 and TIA1 variants can cause myofibrillar myopathy, and therefore, analysis of both genes should be considered in the molecular investigation of myopathy with rimmed vacuoles as well as in the genetic characterization of myofibrillar myopathy. 3) Respiratory insufficiency can occur in SQSTM1-TIA1 myopathy and, therefore, patient's respiratory status should be closely monitored. 4) The cognitive impairment observed in a family is in keeping with the known deleterious effects of TIA1 and SQSTM1 mutations on central nervous system, previously reported in the form of FTD.

\section{PS1Group1-012 / \#26}

Topic: Group 1 - Muscle Diseases of Genetic Origin and Acquired Myopathies: Clinical Features, Pathophysiology, Therapy

\section{PATHOLOGICAL AND GENETIC STUDIES IN PATIENTS WITH MENDELIAN DISORDERS OF MTDNA MAINTENANCE}

Diana Lehmann $^{1}$, Amy E. Vincent ${ }^{2}$, Hannah Rosa ${ }^{2}$, Mariana Rocha², Stephan Zierz ${ }^{3}$, Robert W Taylor ${ }^{2}$, Douglass M. Turnbull ${ }^{2}$

${ }^{1}$ Department Of Neurology, University of Ulm, Ulm, DE: ${ }^{2}$ Institute Of Neuroscience, Newcastle University Wellcome Trust Centre for Mitochondrial Research, Newcastle upon Tyne, $G B ;{ }^{3}$ Department Of Neurology, University of Halle/S., Halle/S., DE

Background: The underlying genetic defect in patients with mitochondrial PEO is either a primary mutation of the mitochondrial genome (single, large-scale mtDNA deletion or mtDNA point mutation) or recessively and dominantly-inherited mutations in nuclear genes involved in mtDNA maintenance leading to clonally-expanded multiple mtDNA deletions in muscle. The nuclear disease genes are largely implicated in the replication and stability of mtDNA, and as such a pathogenic mutation leads to secondary instability of the mitochondrial genome. Causal mtDNA deletions can be found in a heteroplasmic (mixture of mutated and wild type mtDNA) state. However, each tissue/cell has its own genetic defect and biochemical threshold of mutated mtDNA load which needs to be exceeded before focal respiratory chain deficiency becomes evident. Methods: To investigate this, muscle biopsies of 16 patients with genetically- and clinically-characterized mitochondrial disease of nuclear origin (9POLG, 4 TWNK, 2 RRM $2 B$ and 1 $S L C 25 A 4$ (ANT1)) and 4 healthy controls were analysed using quadruple OXPHOS immunohistochemistry, quantifying the biochemical phenotype in individual muscle fibres of patient muscle biopsies. This technique is based on quadruple immunofluorescence to detect structural components of complexes I (NDUFB8) and IV (COXI), as well as porin (a marker of mitochondrial mass) and laminin (a cell membrane marker to define the boundaries of muscle fibres). Further studies on 6/17 patients (2 POLG, 2 RRM2B, 1 TWNK, 1 SLC25A4 (ANT1)) included the correlation of the biochemical deficiency with the mtDNA abnormality in individual cells, following laser microcapture and determination of the size and level of clonally-expanded mtDNA deletion within fibres by real-time PCR, long-range PCR and sequencing of breakpoints. Results: The data from quadruple immunocytochemical studies show that the muscle biochemical phenotype is different in patients with multiple mtDNA deletions compared to other mtDNA mutations, but showed no difference between genotypes. Real-time PCR showed, that the level of deletion is increasing with the biochemical defect. In all patients, the levels of deletion of ND4/D Loop and ND4/ND1 are significantly increasing with the MRC profile. However, in all groups of fibres there seem to be very low levels of ND1 deletions. Conclusion: It has already been shown, that patients harbouring multiple deletions have a distinct MRC- (muscle respiratory chain) profile. Three different groups of fibres were found in these patients: cells without deficiency, cells with isolated complex I deficiency and cells with combined complex I and complex IV deficiency. The correlation of biochemical deficiency with level of deletion is arguing against any point mutations as cause of the deficiency. 


\section{PS1Group1-013 / \#451}

Topic: Group 1 - Muscle Diseases of Genetic Origin and Acquired Myopathies: Clinical Features, Pathophysiology, Therapy

\section{EPILEPSY CHARACTERIZATION IN LAMA2-RELATED CONGENITAL MUSCULAR DYSTROPHY}

\author{
Daniel Natera De Benito ${ }^{1}$, Debora Itzep ${ }^{2}$, Jordi \\ Muchart $^{2}$, Alia Ramirez ${ }^{2}$, Delia Yubero ${ }^{2}$, Laura \\ Carrera $^{2}$, Javier Aparicio ${ }^{2}$, Cristina Jou ${ }^{2}$, Cecilia \\ Jimenez Mallebrera ${ }^{2}$, Jaume Colomer ${ }^{2}$, Carlos \\ Ortez $^{2}$, Andres Nascimento ${ }^{2}$, Victoria San Antonio \\ Arce $^{2}$ \\ ${ }^{1}$ Neuromuscular Disorders Unit, Hospital Sant \\ Joan de Déu, Barcelona, ES; ${ }^{2} H o s p i t a l$ Sant Joan de \\ Déu, Barcelona, ES
}

Background: $L A M A 2$-related congenital muscular dystrophy (CMD), is an autosomal recessive neuromuscular disorder resulting in deficiency of the laminin a2 chain, a component of the skeletal muscle extracellular matrix laminin-2. Laminin-2 is expressed in basement membranes of striated muscle, but also in the neuromuscular synapses, schwann cells and in brain where it is thought to have a role in neuronal migration. Although it is a muscular disease, it may be associated with peripheral neuropathy and a diversity of cerebral manifestations. The risk of epileting seizures is described in the literature up to $20 \%$ of the cases, even without cerebral structural anomalies on MRI. Although both focal and generalized seizures have been reported, the epilepsy phenotypic spectrum has not been defined. In this study we describe the epilepsy phenotype in a cohort of patients with $L A M A 2$-related CMD who have experienced seizures in order to clarify the spectrum of phenotypes that may occur. Electroclinico-radiologic presentation, response to treatment, and long-term outcome are described. Methods: All patients with genetically and/or pathologically confirmed diagnosis of LAMA2-related CMD followed-up in our hospital were included. Medical records of patients with demographic information, clinical data, EEG records, and MRI studies were compiled. Seizures and epilepsy were described and classified according to the ILAE classifications. Results: Epilepsy was observed in eight of twenty patients with $L A M A 2$-related CMD. A partial merosin deficiency was detected in five patients and a complete merosin deficiency in three. The mean age at the time of the study was 19.5 years (range 10-32 years). Mean age at seizure onset was 8 years (range 2-20 years). The most common presenting seizure type was focal onset seizure with or without impaired awareness in 5 of 8 subjects. Visual aura was described in four patients. Vomiting was also frequently reported (4/8).Three subjects had motor seizures with an unknown onset. Two subjects also had bilateral tonic-clonic seizures. One case had atypical absences and drop attacks. Longterm seizure control was assessed based on the Brodie classification: six patients $(75 \%)$ had ongoing refractory seizures (D), and two (25\%) had fluctuating course $(\mathrm{C}$ : periods of at least 12 months seizurefree, interspersed with relapses). Interictal EEGs showed focal or multifocal epileptiform abnormalities in seven of eight cases. Focal occipital abnormalities were the most commonly observed. Background activity was normal in six patients, and generalized background slowing was reported in two cases An increased signal intensity of subcortical and periventricular white matter on T2-weighted MRI was reported in all the patients. Four patients $(50 \%)$ had suspected focal cortical dysplasia. Conclusion: The epilepsy in LAMA2-related CMD typically presents with childhood-onset focal seizures that are not usually prolonged and have prominent visual and autonomic features. EEG show focal interictal abnormalities that predominate in the posterior quadrants. MRI brain demonstrates a constellation of structural abnormalities, including focal cortical dysplasia, in addition to the wellknown increased signal intensity of white matter on T2-weighted MRI.

\section{PS1Group1-014 / \#786}

Topic: Group 1 - Muscle Diseases of Genetic Origin and Acquired Myopathies: Clinical Features, Pathophysiology, Therapy

\section{A PHASE 1B/2 STUDY OF THE ANTI- MYOSTATIN ADNECTIN RG6206 (BMS-986089) IN AMBULATORY BOYS WITH DUCHENNE MUSCULAR DYSTROPHY}

Kathryn R. Wagner ${ }^{1}$, Brenda L. Wong ${ }^{2}$, Barry Byrne $^{3}$, Lee Sweeney ${ }^{3}$, Leslie Jacobsen ${ }^{4}$, Giridhar Tirucherai ${ }^{4}$, Michael Rabbia ${ }^{5}$, Jeppe Buchbjerg ${ }^{6}$, Dukart Juergen ${ }^{6}$, Michelle Krishnan ${ }^{6}$, Clifford Bechtold $^{4}$ 
The Johns Hopkins School of Medicine, Baltimore, MD, US $;{ }^{2}$ Neurology, Cincinnati Children's Hospital Medical Center, Cincinatti, OH, US: ${ }^{3}$ University of Florida, Gainesville, FL, US: ${ }^{4}$ Bristol-Myers Squibb, Princeton, NJ, US, ${ }^{5}$ Roche Translational and Clinical Research Center, New York, NY, US; ${ }^{6}$. Hoffmann-La Roche Ltd, Basel, CH

Background: Pharmacologic inhibition of myostatin, a negative regulator of muscle growth, has been shown to increase skeletal muscle mass in several species, including humans. RG6206 is a potent, novel anti-myostatin adnectin previously shown to inhibit myostatin activity in healthy adults. This Phase 1b/2 study (NCT02515669) assessed the safety, tolerability, pharmacokinetics and pharmacodynamics of the novel anti-myostatin adnectin RG6206 (BMS-986089) in ambulatory boys with Duchenne muscular dystrophy (DMD). Methods: Forty-three ambulatory boys with DMD aged 5-10 years were randomized to receive weekly subcutaneous doses of RG6206 (4-50 mg) or placebo. The primary endpoint was safety over 24 weeks. Secondary endpoints included pharmacokinetics of RG6206, frequency of anti-drug antibodies and pharmacodynamic effect on serum myostatin levels. Exploratory outcome measures included 4-stair climb velocity (4SC), 6-minute walk test (6MWT) and North Star Ambulatory Assessment (NSAA) score. Muscle volume and lean body mass were assessed by dual-energy X-ray absorptiometry (DXA) or magnetic resonance imaging (MRI). Results: No clinically significant changes in laboratory values, vital signs or echocardiogram parameters were observed over 24 weeks. The most common adverse events considered to be related to study drug were cutaneous injection site reactions (RG6206 $\mathrm{n}=10,31.3 \%$; placebo $\mathrm{n}=1,9.1 \%$ ), which were mild (except one event of moderate injection site discomfort). One patient tested positive for anti-drug antibodies; however, this patient had a positive titer at baseline. RG6206 serum concentrations increased with dose and were accompanied by dose-dependent reduction in free myostatin, with a $\geq 95 \%$ reduction from baseline with the highest dose. The study was not powered to demonstrate effects on exploratory functional endpoints and, as such, no efficacy on 4SC, 6-MWT and NSAA score was observed. However, DXA imaging data showed an increase in lean body mass in boys receiving RG6206 compared with placebo. In addition, MRI imaging of the right thigh showed positive effects on muscle composition due to RG6206, with an increase in contractile, and decrease in non-contractile, tissue. Conclusion: RG6206 was well tolerated by ambulatory boys with DMD, with no clinically significant safety concerns. Robust antimyostatin target engagement was demonstrated, and DXA and MRI imaging outcomes suggest a positive effect of RG6206 on muscle over the 24-week study. A 48-week open-label phase of this study is ongoing and a Phase $2 / 3$ study is underway to further evaluate RG6206 in ambulatory boys with DMD (NCT03039686). Acknowledgements Study sponsored by F. Hoffmann-La Roche.

\section{PS1Group1-015 / \#350}

Topic: Group 1 - Muscle Diseases of Genetic Origin and Acquired Myopathies: Clinical Features, Pathophysiology, Therapy

\section{IN VITRO FUNCTIONAL CHARACTERIZATION OF FKRP PATIENT MISSENSE MUTATIONS}

Sara F. Dias Henriques, Evelyne Gicquel, Justine

Marsolier, Isabelle Richard

INTEGRARE, Généthon, Inserm, Univ Evry, Université Paris-Saclay, Evry, FR

Background: Muscular dystrophies are monogenic diseases affecting the skeletal muscle, characterized by a progressive loss of the muscle mass, can lead to premature death by heart or respiratory complications and are currently without treatment. One group of muscle dystrophies termed "dystroglycanopathies" encompasses different genetic pathologies that lead to a change in the glycosylation status of the protein alpha-dystroglycan (alpha-DG). This protein is a crucial component of the dystrophin-associated glycoprotein complex (DGC) at the skeletal muscle whereby, through a complex and specific glycosylation, the plasma membrane is anchored to the extracellular-matrix. Loss of this glycosylation weakens this link and leads to the death of muscle fibers. Many proteins cooperate in the assembly of the final glycosylation moiety such as the FukutinRelated Protein (FKRP). Mutations in FKRP lead to different pathologies, from the severe Congenital Muscular Dystrophy type 1C (MDC1C) to the mild Limb-Girdle Muscular Dystrophy type 2I (LGMD2I) and one of the most prevalent of the LGMD group. Most patient-mutations are missense mutations, some of which leading to a misfolded protein 
that is retained in the endoplasmic reticulum (ER) and prematurely degraded before reaching the functionality compartment, the Golgi (Esapa et al., 2002 and 2005; Torelli et al., 2005; Keramaris-Vrantsis et al., 2007). We have shown that some LGMD-associated misfolded proteins still retain partial function when rescued to the correct compartment (Soheili et al., 2011). It is therefore crucial to assess the functionality of FKRP mutant proteins in line with potential corrective treatments. Methods: Through transgene overexpression in a FKRP knockout (KO) cell line, we have characterized different patterns of cellular localization of the mutant proteins, some of which never described before. Results: Combination of these localization results with functional assays lead to the identification of some mutants that still bear function when properly addressed to the Golgi. Interestingly we have also identified mutants that, although correctly localized in the Golgi, where nonetheless severely functionally impaired, revealing that accurate cellular localization is not directly linked with function. Structural analysis is currently being pursued in understanding the function impairment of these mutants. Conclusion: Our results clearly show that cellular localization studies are not enough to fully understand the consequences of the mutations and a complementary functional assessment of the mutated proteins is required when curative treatments are envisioned.

\section{PS1Group1-016 / \#528}

Topic: Group 1 - Muscle Diseases of Genetic Origin and Acquired Myopathies: Clinical Features, Pathophysiology, Therapy

\section{IDENTIFICATION OF MUTATIONS IN A COHORT OF UNCLASSIFIED INHERITED MUSCLE DISORDERS BY TARGETED NEXT GENERATION SEQUENCING}

$\underline{\text { Kiran Polavarapu }}{ }^{1}$, Aditi Joshi², Veeramani

Preethish-Kumar ${ }^{1}$, Aradhna Mathur ${ }^{2}$, Sushmita

Nayak $^{2}$, Sakshi Ambawat ${ }^{2}$, Saraswati Nashi ${ }^{3}$, Seena

Vengalil $^{3}$, Rashmi Santhosh ${ }^{4}$, Gayathri

Narayanappa ${ }^{4}$, Mohammed Faruq ${ }^{2}$, Atchayaram

Nalini $^{5}$

${ }^{1}$ Clinical Neurosciences, Neurology, National

Institute of Mental Health and Neurosciences

(NIMHANS), Bangalore, IN;'2Institute of Genomics and Integrative Biology, New Delhi, IN, ${ }^{3}$ Neurology, National Institute of Mental Health and

Neurosciences (NIMHANS), Bangalore,

IN: ${ }^{4}$ Neuropathology, National Institute of Mental

Health and Neurosciences (NIMHANS), Bangalore,

IN $;{ }^{5}$ Neurology, National Institute of Mental Health and Neurosciences, IN

Background: Inherited myopathies and muscular dystrophies are a genetically heterogenous group of neuromuscular disorders which often present with overlapping clinical and pathological phenotypes. Although muscle histochemistry, immunostaining, western-blot and electron microscopy techniques offer important clues, these are often found insufficient to provide a conclusive diagnosis. With rapid evolution of gene discovery, accurate molecular diagnosis is essential for these disorders to provide appropriate genetic counselling, prognostication and identification of disease burden essential for future therapeutic research. Targeted Next generation sequencing (NGS) with gene panels are emerging as quick low-cost methods to diagnose mendelian disorders.

Methods: Randomly selected patients with clinical features suggestive of Limb girdle muscular dystrophies (LGMD), distal myopathies (DM) and congenital myopathies (CM) evaluated over last 10 years in our Neuromuscular clinic were taken up for genetic analysis by Targeted NGS with a gene panel constituting 53 known muscular dystrophy and myopathy genes. DNA extracted from blood was utilized for library preparation as per Agilent SureselectQXT protocol followed by sequencing on HIseq Illumina platform.

Results: We performed Target NGS in 45 patients with mean age of $21 \pm 11.7$ (3-49 years) and male/female ratio of 3:2. Broad clinical phenotypes included LGMD: 31 (68.9\%), CM: 9 (20\%), DM: 5 $(11.1 \%)$. Majority of cases were sporadic: 33 $(73.3 \%)$, while family history was present in 12 (26.7\%): Autosomal dominant inheritance in 7, recessive history in 3 and suspected X-linked inheritance in 2 families. Serum Creatine kinase was elevated in $82.2 \%$ ( $>5$ times: $22,<5$ times: 15 ). Muscle biopsy reports available in 42 patients showed muscular dystrophy in 25 (60.9\%). Myopathic features were reported in 17 patients showing varying degree of cytoplasmic inclusions in 9 cases and rimmed vacuoles in 4 . Putative disease-causing variants in 17 genes were identified in 31 patients $(68.8 \%)$ and diagnosis was possible in $21(46.66 \%)$ 
where clinical suspicion was supportive, and variants were either reported pathogenic or novel rare variants $(\mathrm{MAF}=0)$ recognized as damaging/probably damaging by in-silico predictions (Table 1). In another 10 cases, variants were identified with uncertain significance/not exactly correlating with phe- notype and need to be further characterized. Most common genes affected were CAPN3 (Calpain-LGMD2A) in 5 patients followed by DYSF (DysferlinLGMD2B), EMD (Emerin-EDMD) in 4 cases each, RYR1 (Ryanodine receptor1-CM) in 3 and GMPPB (LGMD 2T), LMNA (LGMD 1B/EDMD) in 2 each.

\begin{tabular}{|c|c|c|c|c|c|c|c|}
\hline S.No & Gender & Age & Clinical phenotype & Gene & Variant & Zygosity & Type \\
\hline 1 & $\mathrm{~F}$ & 19 & $\begin{array}{l}\text { LGMD 2A (Agarwal } \\
\text { community) }\end{array}$ & CAPN3 & $\begin{array}{l}\text { exon19:c.2051-1G>T, exon22:c. } \\
\text { G2338C:p.D780H }\end{array}$ & $\begin{array}{l}\text { Compund } \\
\text { heterozygous }\end{array}$ & Reported \\
\hline 2 & $\mathrm{~F}$ & 15 & LGMD & CAPN3 & exon17:c.1962delC:p.R655Gfs*6 & Homozygous & Novel \\
\hline 3 & M & 17 & $\begin{array}{l}\text { LGMD (consanguinity with } \\
\text { dominant family history) }\end{array}$ & CAPN3 & exon17:c.1962delC:p.R655Gfs*6 & Homozygous & Novel \\
\hline 4 & M & 18 & LGMD & CAPN3 & $\begin{array}{l}\text { exon10:c.G1343A:p.R448H, exon20:c. } \\
\text { A2120G:p.D707G }\end{array}$ & $\begin{array}{l}\text { Compund } \\
\text { heterozygous }\end{array}$ & Reported \\
\hline 5 & $\mathrm{~F}$ & 14 & $\begin{array}{l}\text { LGMD 2A (Agarwal } \\
\text { community) }\end{array}$ & CAPN3 & $\begin{array}{l}\text { exon19:c.2051-1G>T, exon22:c. } \\
\text { G2338C:p.D780H }\end{array}$ & $\begin{array}{l}\text { Compund } \\
\text { heterozygous }\end{array}$ & Reported \\
\hline 6 & $\mathrm{~F}$ & 26 & $\begin{array}{l}\text { LGMD, early contractures, } \\
\text { CK: Mild elevated }(2 \mathrm{X})\end{array}$ & COL6A1 & exon29:c.1822+2T $>C$ (splice site) & Homozygous & Novel \\
\hline 7 & $\mathrm{~F}$ & 12 & $\begin{array}{l}\text { Cong. Myopathy with Dilated } \\
\text { Cardiomyopathy }\end{array}$ & DES & exon1:c.C448T:p.R150X & Homozygous & Novel \\
\hline 8 & M & 20 & $\begin{array}{l}\text { ?BMD (X-linked history- } \\
\text { multiple males affected in } 3 \\
\text { generations) }\end{array}$ & DMD & exon72:c.C10255T:p.Q3419X & Hemizygous & Reported \\
\hline 9 & M & 29 & $\begin{array}{l}\text { HyperCkemia with mild limb } \\
\text { girdle weakness }\end{array}$ & DYSF & exon18:c. $1638+1 \mathrm{G}>\mathrm{A}$ (splice site) & Homozygous & Novel \\
\hline 10 & $\mathrm{~F}$ & 24 & LGMD & DYSF & exon9:c.G895A:p.G299R & Homozygous & Reported \\
\hline 11 & M & 20 & EDMD & EMD & exon2:c.C123A:p.Y41X & Hemizygous & Novel \\
\hline 12 & M & 21 & EDMD (X-linked history) & EMD & exon6:c.651_655del:p.Q219Sfs*28 & Hemizygous & Novel \\
\hline 13 & M & 9 & EDMD & EMD & exon4:c.266-2A >G (splice site) & Hemizygous & Reported \\
\hline 14 & M & 13 & EDMD & FHL1 & exon7:c.C862G:p.R288G & Hemizygous & Novel \\
\hline 15 & M & 8 & LGMD & FKRP & Exon4: c.C638T: p.P213L & Homozygous & Reported \\
\hline 16 & $\mathrm{~F}$ & 35 & LGMD with fatigability & GMPPB & exon9:c.G1000A:p.D334N & Homozygous & Reported \\
\hline 17 & M & 49 & LGMD & GMPPB & exon9:c.G1000A:p.D334N & Homozygous & Reported \\
\hline 18 & M & 38 & $\begin{array}{l}\text { Proximo-distal weakness, } \\
\text { Muscle MRI: severe } \\
\text { involvement of medial } \\
\text { gastrocnemius with sparing of } \\
\text { soleus and rectus femoris }\end{array}$ & LMNA & $\begin{array}{l}\text { exon2:c.369delG:p.E124Rfs*3, } \\
\text { exon2:c.G370C:p.E124Q }\end{array}$ & $\begin{array}{l}\text { Compund } \\
\text { heterozygous }\end{array}$ & Novel \\
\hline 19 & M & 20 & $\begin{array}{l}\text { LGMD with axial contractures, } \\
\text { Cardiomyopathy (AD family } \\
\text { history), Muscle MRI: severe } \\
\text { involvement of medial } \\
\text { gastrocnemius with sparing of } \\
\text { soleus and rectus femoris. }\end{array}$ & LMNA & exon7:c.G1318A:p.V440M & Heterozygous & Reported \\
\hline 20 & $\mathrm{~F}$ & 10 & Cong. Myopathy & RYR1 & $\begin{array}{l}\text { exon27:c.C3725T:p.P1242L, exon39:c. } \\
\text { G6502A:p.V2168M (reported) }\end{array}$ & $\begin{array}{l}\text { Compund } \\
\text { heterozygous }\end{array}$ & Novel \\
\hline 21 & $\mathrm{~F}$ & 19 & $\begin{array}{l}\text { Cong. Myopathy, Muscle } \\
\text { MRI: Selective semitendinosus } \\
\text { involvement }\end{array}$ & TTN & $\begin{array}{l}\text { exon308:c.98702_98703del:p. } \\
\text { S32901fs*0 }\end{array}$ & Homozygous & Novel \\
\hline
\end{tabular}


Conclusion: Targeted gene panel NGS offers a quick cost-effective strategy with good diagnostic yield in inherited muscle disorders, when complimented with proper clinical phenotyping.

\section{PS1Group1-017 / \#849}

Topic: Group 1 - Muscle Diseases of Genetic Origin and Acquired Myopathies: Clinical Features, Pathophysiology, Therapy

\section{L-CITRULLINE AND METFORMIN DELAY MUSCLE DEGENERATION IN DUCHENNE MUSCULAR DYSTROPHY: RESULTS FROM A ANDOMISED CLINICAL TRIAL}

Patricia Hafner $^{1}$, Ulrike Bonati ${ }^{1}$, Andrea Klein ${ }^{2}$, Daniela Rubino ${ }^{1}$, Vanya Gocheva ${ }^{1}$, Simone Schmidt $^{1}$, Vincent Laugel ${ }^{3}$, Andrea Capone ${ }^{4}$, Monika Gloor ${ }^{5}$, Oliver Bieri ${ }^{5}$, Thomas Zumbrunn ${ }^{6}$, Nuri Gueven ${ }^{7}$, Dirk Fischer ${ }^{8}$

${ }^{1}$ Division Of Paediatric Neurology, University Children's Hospital Basel, Basel, $\mathrm{CH} ;{ }^{2}$ Division Of

Paediatric Neurology, University of Berne Hospital, Berne, CH; ${ }^{3}$ Strasbourg University Hospital, Strasbourg, FR; ${ }^{4}$ Division Of Paediatric Neurology, Children's Hospital Aarau, Aarau, $\mathrm{CH} ;{ }^{5}$ Department Of Radiology, University of Basel Hospital, Basel, CH; ${ }^{6}$ Department Of Clinical Research, University of Basel Hospital, Basel, $\mathrm{CH} ;{ }^{7}$ Pharmacy, School Of Medicine, University of Tasmania, Hobart, AU; ${ }^{8}$ Division Of Neurology, Kantonsspital Bruderhlz, Bruderholz, $\mathrm{CH}$

Background: Sufficient therapeutic efficacy in Duchenne muscular dystrophy (DMD) has not been achieved yet and current therapeutic management still remains supportive. Recent evidence suggests that the loss of dystrophin in DMD not only disturbs cytoskeletal integrity but also cell metabolism resulting in reduced intracellular nitric monoxide (NO) levels. This phase IIa clinical trial assessed the efficacy and safety of the NO enhancing components Lcitrulline and metformin to protect against muscle degeneration in ambulant patients with DMD. Methods: 49 ambulant DMD patients aged between 6.5 and 10 years were screened and 47 randomly allocated in a 1:1 ratio to receive daily treatment with oral L-citrulline (2.5g three times a day) and metformin (250mg three times a day) or placebo for 26 weeks. Co-medication with glucocorticoids was allowed if started 6 months prior to randomisation. Primary endpoint was the change of motor function measure (MFM) D1 subscore from baseline to week 26 in the modified intention to treat (mITT) patient set. Secondary endpoints included the total MFM score and its subscores; 6 min walking distance; timed function tests, quantitative muscle testing, quantitative muscle MRI assessments and biomarker laboratory analysis. Two subgroups were defined by a 6 min walking distance (6MWD) of $\geq 350 \mathrm{~m}$ (stable) and $<350 \mathrm{~m}$ (unstable) at baseline. Results: 45 of the 47 randomised patients (mean age 8.20 [1.07] years; 45 [100\%] boys) completed the study. At week 26, L-citrulline and metformin treatment improved the MFM D1 decrease compared to placebo non-significantly (mean difference $5.54 \%$ [ $95 \%$ CI -1.00 to 12.09 ]; $P=.095$ ). Significant differences in favor of the L-citrulline and metformin treatment were observed in the stable subgroup with a baseline $6 \mathrm{MWD}$ of $\geq 350 \mathrm{~m}$ (mean MFM D1 difference of $6.81 \%$ [95\% CI 1.00 to 12.26]; $P=.024$ ) and on both quantitative MRI sequences to assess muscle degeneration (mean fat fraction difference -1.79 [95\% CI -3.26 to -0.32$] ; P$ $=.018$; mean $\mathrm{T} 2$ relaxation time difference -1.33 [95\% CI -2.40 to -0.26$] ; P=0.016)$. The treatment with L-citrulline and metformin was well tolerated, with stomach-ache, a known side effect of metformin, as the most frequent adverse event. Conclusion: The non-significant difference of the D1 in the mITT in favor of L-citrullin and metformin is clinically meaningful, but the study is underpowered.

\section{PS1Group1-018 / \#814}

Topic: Group 1 - Muscle Diseases of Genetic Origin and Acquired Myopathies: Clinical Features, Pathophysiology, Therapy

\section{MUTATION ANALYSIS IN MLPA NEGATIVE DUCHENNE MUSCULAR DYSTROPHY: NGS AS A DIAGNOSTIC TOOL PRIOR TO MUSCLE BIOPSY}

$\underline{\text { Kiran Polavarapu }}^{1}$, M K. Saroja ${ }^{2}$, Veeramani Preethish-Kumar ${ }^{1}$, Deepah Sekar ${ }^{3}$, Saraswati $\mathrm{Nashi}^{4}$, Seena Vengalil ${ }^{4}$, Priya T. Thomas 5 , Sudha N. $\mathrm{Rao}^{2}$, Atchayaram Nalini ${ }^{6}$

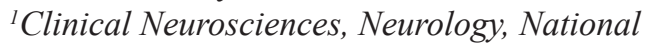
Institute of Mental Health and Neurosciences (NIMHANS), Bangalore, $I N ;{ }^{2} R \& d$ Division, Genotypic Technology, Bangalore, IN; ${ }^{3}$ NIMHANS, 
Bengaluru, Bengaluru, IN; ${ }^{4}$ Neurology, National Institute of Mental Health and Neurosciences (NIMHANS), Bangalore, IN; ${ }^{5}$ Department Of Psychiatric Social Work, National Institute of Mental Health and Neuro Sciences, Bangalore, $I N ;{ }^{6}$ Department Of Neurology, National Institute of Mental Health and Neuro Sciences, Bangalore, IN

Background: Duchenne muscular dystrophy (DMD) is the most severe and common form of childhood muscular dystrophies resulting from mutations in Dystrophin gene (Xp21), affecting 1 in 3500 boys worldwide. While $75 \%$ patients have deletions/duplications of one or more exons, smaller mutations - point mutations, insertion/deletions (INDELs) etc., occur in $20-25 \%$ who require direct sequencing of entire gene or invasive muscle biopsy for diagnostic confirmation. Next generation sequencing (NGS) offers a cheaper and higher throughput alternative to traditional Sanger sequencing and can be developed as a single diagnostic platform to identify both copy number variations (CNVs) and small mutations in DMD patients. Methods: Clinically suspected and/or biopsy confirmed DMD children in whom MLPA (Multiplex ligation-dependent probe amplification) did not identify deletions/duplications of exons were recruited from our Neuromuscular clinic. Custom probe design for DMD gene was created using Agilent's SureDesign ${ }^{\mathrm{TM}}$ tool with a total capture size of $2.077 \mathrm{Mbp}$ to cover entire gene (exons, introns and promoter regions) at least twice. After obtaining informed consent/assent, DNA extracted from blood was used to prepare libraries and sequenced on NextSeq ${ }^{\mathrm{TM}}$ (Illumina). Results: Mutational analysis was performed in 64 MLPA negative DMD children with mean age of 7.87 \pm 2.3 (range:3-13 years). Majority were sporadic cases and family history (X-linked recessive) is present in only six cases (9.37\%). Biopsy was performed in 51 boys (79.7\%) and showed loss of Dystrophin on immunohistochemistry. NGS identified hemizygous mutations of DMD gene in 58/64 children (90.6\%). Nonsense mutations were most common at $54.7 \%(35 / 64)$ followed by frameshift mutations due to small INDELs $21.9 \%(14 / 64)$. Small INDELs of up to 10 bases were identified accurately. Variants affecting splicing occurred in 8/63 (12.5\%) out of which six involved invariant GT and AG dinucleotides at the 5' end (splice donor) and 3' end (splice acceptor) of introns. Missense mutation was identified in only one patient. Except for one splice mutation where variation occurred nine bases upstream of exon 12, all other vari- ants were classified as pathogenic (55) or likely pathogenic (2) as per ACMG guidelines. In total there were 57 unique variants among which $60 \%$ (34) were novel and only one mutation (p.Arg539*) recurred in two unrelated patients. Unlike larger CNVs, which occur predominantly in exons 45-55 and 2-10 for deletions and duplications respectively, small mutations lacked any hot spot regions and more uniformly spread across coding region with exons 30 and 44 having most number of mutations (4 each). In thirteen patients who did not undergo biopsy and clinical suspicion was high, we were able to accurately identify pathogenic mutations. Mutations were not identified in only six cases $(9.5 \%)$, where possibility of deep intronic variants/complex rearrangements should be considered. Conclusion: In this study we describe spectrum of smaller mutations in a large cohort of DMD children from India. Accurate diagnosis of these mutations is important to identify potential cases who can benefit from mutation specific therapies like nonsense read-through and NGS offers a valuable diagnostic screening tool before contemplating invasive muscle biopsy in clinically suspected DMD patients. Acknowledgement: This study is funded by DST-SERB (Govt. of India) project: EMR/2014/000943

\section{PS1Group1-019 / \#756}

Topic: Group 1 - Muscle Diseases of Genetic Origin and Acquired Myopathies: Clinical Features, Pathophysiology, Therapy

\section{EZUTROMID SIGNIFICANTLY REDUCES MUSCLE DAMAGE IN DUCHENNE MUSCULAR DYSTROPHY}

Francesco Muntoni ${ }^{1}$, Gary Layton ${ }^{2}$, Indranil Bhattacharya $^{3}$, Krista Vandenborne ${ }^{4}$, Crystal Faelan $^{5}$, Anne Heatherington ${ }^{3}$, David Roblin ${ }^{6}$, Ruth Osahon $^{6}$, Jon Tinsley ${ }^{7}$, Kay Davies ${ }^{8}$

${ }^{1}$ Dubowitz Neuromuscular Unit, UCL Great Ormond Street Institute of Child Health, London, GB; ${ }^{2}$ Summit Therapeutics Inc., Abingdon, AL, GB; ${ }^{3}$ Summit Therapeutics Inc., Cambridge, MA, $U S:{ }^{4}$ University of Florida, Gainsville, FL, US; ${ }^{5}$ Flagship Biosciences, Westminster, CO, US, ${ }^{6}$ Summit Therapeutics Inc., Abingdon, GB, ${ }^{7}$ Summit Therapeutics Plc, Abingdon, $G B ;^{8}$ university of oxford, oxford, $G B$ 
Background: To share interim 24-week safety and efficacy results from PhaseOut DMD evaluating ezutromid, a potential first-in-class utrophin modulator for the treatment of all patients with Duchenne muscular dystrophy (DMD). Utrophin is a naturally occurring protein that may act as a functional replacement for dystrophin. Increasing the production of utrophin in mature muscle fibres protects and improves outcome in preclinical models, and could modify the course of DMD. Methods: PhaseOut DMD is a Phase 2 open-label study of ezutromid administered to 40 ambulatory patients with DMD. Primary endpoints (48 week) are magnetic resonance spectroscopy (MRS) assessments. Key secondary endpoints are evidence of target engagement and quantification of muscle damage on muscle biopsies; collected at baseline $(\mathrm{n}=40)$ and week 24 $(n=25)$ or $48(n=15)$. Safety, pharmacokinetics, and functional measures are monitored throughout. Safety monitoring included collection of treatment emergent adverse events (TEAE), electrocardiogram (ECG), echocardiogram (ECHO), and laboratory parameters. An interim 24-week analysis was planned to assess efficacy and safety compared to baseline, with a focus on changes in the degree of muscle pathology as monitored by muscle biopsy. Results: A statistically significant reduction in \%fibres expressing developmental myosin (MHCd) was observed (from $11.37 \%$ to $8.76 \%$, least mean square difference (LSMD) $-2.62 \%$ CI, $-4.33,-0.90$ ); a relative reduction of $23 \%$. Mean utrophin intensity increased (relative $+7 \%$ ) ( 0.370 to 0.396 , LSMD 0.026 $95 \% \mathrm{CI},-0.005,0.058)$. MRS indicated a statistically significant decrease in water relaxation time (T2) in the soleus muscle: the average reduction was -0.86 milliseconds ( $95 \%$ confidence intervals -1.44 , -0.28), which was from 31.85 milliseconds at baseline to 30.99 milliseconds at 24 weeks, $(n=38)$. Further, an increase in mean fat fraction (vastus lateralis: from $14.7 \%$ to $18.5 \%, n=37$ ) was noted. No patient lost ambulation and there were no meaningful changes in functional performance (eg 6MWD: $404 \mathrm{~m}$ at baseline, $395 \mathrm{~m}$ at 24 weeks, $\mathrm{n}=39$ ). All patients achieved ezutromid plasma levels sufficient to modulate utrophin. Up to Week 24, none of the subjects had any clinically significant TEAE, and no subject withdrew due to safety issues. Only one subject experienced an SAE, which resolved within a day with no dosage day missed, whilst the subject remained in the study. In addition, there was no relevant prolongation of the corrected QT interval on the ECG, or deterioration of left ventricular ejection fraction or fractional shortening. There were no clinically significant laboratory abnormalities related to ezutromid use. Ezutromid continues to be safe and well tolerated. Conclusion: Decreased $\mathrm{MHCd}$, a marker of regeneration, indicates reduction in muscle damage. Utrophin levels were increased which, with decreasing MHCd, provides evidence of target engagement. MRS T2 results in this age group are consistent with a reduction in muscle inflammation and/or damage. Ezutromid has continued to be safe and well-tolerated after prolonged dosing across a wide exposure range. These interim results support utrophin modulation as a potential treatment for DMD patients irrespective of their mutation.

\section{PS1Group1-020 / \#698}

Topic: Group 1 - Muscle Diseases of Genetic Origin and Acquired Myopathies: Clinical Features, Pathophysiology, Therapy

\section{NUMBER NEEDED TO TREAT IN SPINAL MUSCULAR ATROPHY TYPE 1 WITH AVXS-101 RELATIVE TO NUSINERSEN}

Omar Dabbous $^{1}$, Martin Cloutier ${ }^{2}$, Annie Guerin $^{2}$, Irina Pivneva ${ }^{2}$, Eric Q. Wu ${ }^{2}$, Douglas M. Sproule ${ }^{3}$ ${ }^{1}$ AveXis, Inc., Bannockburn, AL, US, ${ }^{2}$ Analysis Group, Inc., Boston, MA, US $;^{3}$ AveXis, Inc., Bannockburn, IL, US

Background: To assess the number needed to treat (NNT) to prevent death and use of permanent assisted ventilation and to improve motor function with AVXS-101 compared to nusinersen in patients with spinal muscular atrophy type 1 (SMA1). Methods: Patients diagnosed with SMA1 were treated in clinical trials with AVXS-101 (NCT02122952; study cohort 2; $\mathrm{N}=12$ ) or nusinersen (NCT02193074; $\mathrm{N}=80$ ). Trial duration was up to 24 months for AVXS-101 (median $=24.1$ months) and up to 13 months for nusinersen (median $=9.2$ months). NNT with AVXS-101 compared to nusinersen was assessed for survival and event-free survival (absence of death and use of permanent assisted ventilation) at last visit, and for motor function (increase of $\geq 4$ points in Children's Hospital of Philadelphia Infant Test of Neuromuscular Disorders [CHOP-INTEND] score from baseline) at last visit and at a median of 9 months. For the nusinersen trial, CHOP-INTEND score was measured for patients with $\geq 6$ months of 
follow-up ( $\mathrm{N}=73)$. The NNT to prevent one death, one event (death or use of permanent assisted ventilation), or for one patient to improve motor function relative to nusinersen was calculated as the reciprocal of the difference between AVXS-101 and nusinersen in event rates or motor function achievement rates. Results: Patient mean age at first dose was 3.4 (0.9-7.9) and 5.3 (1.7-7.9) months in the AVXS-101 and nusinersen trials. NNT analyses showed that treating 6.2 patients with AVXS-101 instead of nusinersen would prevent 1 more death over the course of the study interval; treating 2.6 patients with AVXS-101 versus nusinersen would prevent 1 more event; and treating 3.5 patients with AVXS101 versus nusinersen would allow 1 more patient to improve motor function (at last visit and at a median of 9 months). Conclusion: Efficacy in preventing death and use of permanent assisted ventilation and in improving motor function is much higher with AVXS-101 versus nusinersen.

\section{PS1Group1-021 / \#821}

Topic: Group 1 - Muscle Diseases of Genetic Origin and Acquired Myopathies: Clinical Features, Pathophysiology, Therapy

\section{A NOVEL NEXT-GENERATION THERAPY FOR POMPE DISEASE WITH IMPROVED EFFICACY IN MICE}

$\underline{\mathrm{Su} X u}$, Yi Lun, Rebecca Soska, Anju Nair, Michelle Frascella, Anadina Garcia, Abdul S. Ponery, Jessie Feng, Cecilia Della Valle, Russell Gotschall, Hung Do, Kenneth J. Valenzano, Richie Khanna Amicus Therapeutics, Cranbury, US

Background: Pompe disease is a lysosomal storage disorder that results from deficiency in acid $\alpha$-glucosidase (GAA) activity, and is characterized by progressive accumulation of lysosomal glycogen in cardiac and skeletal muscles. Currently, the only approved treatment is enzyme replacement therapy using biweekly infusions of a recombinant human GAA (rhGAA), alglucosidase alfa. Although alglucosidase alfa provides some clinical benefits, the infused enzyme shows insufficient uptake into key disease-relevant skeletal muscles, mainly due to sub-optimal levels of mannose-6-phosphate (M6P), a carbohydrate that binds cation-independent M6P receptors at the cell surface, resulting in enzyme internalization and lysosomal targeting. Methods: We have developed a proprietary, novel rhGAA, ATB200, that has optimized M6P content due to its combination of a specialized cell line and an optimized manufacturing/purification process. Moreover, we are developing ATB200 to be co-administered with a small molecule pharmacological chaperone, AT2221 (miglustat, N-butyl-deoxynojorimycin), as a next-generation therapy for Pompe disease. Results: ATB200 use led to substantially greater lysosomal targeting compared to alglucosidase alfa following intravenous injection to $\mathrm{Gaa}$ knock-out (KO) mice. Our data indicate that AT2221 stabilizes ATB200 in blood ex vivo and improves its pharmacokinetic properties in vivo. In Gaa KO mice, co-administration of ATB200 with AT2221 (ATB200/AT2221) showed significantly greater glycogen reduction in key disease-relevant muscles compared to alglucosidase alfa. Repeat administrations of ATB200/AT2221 also substantially reduced lysosomal proliferation, reversed autophagic buildup, and increased muscle fiber size. The effects seen at the biochemical and cellular levels ultimately translated into improved muscle function in Gaa KO mice compared to the standard of care. Conclusion: Collectively, these data demonstrate the superiority of ATB200/AT2221 over the current approved therapy in these preclinical studies, warranting further investigation as a treatment of Pompe disease.

\section{PS1Group1-022 / \#360}

Topic: Group 1 - Muscle Diseases of Genetic Origin and Acquired Myopathies: Clinical Features, Pathophysiology, Therapy

\section{THE MULTIPLE FACES OF ANTI- HMGCR ANTIBODY-RELATED MYOPATHIES}

\author{
Pegah Masrori ${ }^{1}$, Willem De Ridder ${ }^{2}$, \\ Jonathan Baets ${ }^{1}$ \\ ${ }^{1}$ Neuromuscular Reference Centre, Departement Of \\ Neurology, Antwerp University Hospital, Edegem, \\ $B E ;^{2} N e u r o m u s c u l a r$ Reference Centre, \\ Departement Of Neurology, Antwerp University \\ Hospital, Edegem, BE
}

Background: Anti-HMGCR antibody-associated myopathies define a distinct and clinically diverse subset of idiopathic inflammatory myopathies (IIM). The most typical clinical presentation consists of 
subacute progressive proximal muscle weakness with moderately to severely elevated serum creatine kinase (CK) levels and myopathic electromyography findings. The muscle biopsy usually shows features of a necrotising myopathy, evident by myofibre necrosis and regeneration, with minimal or absent lymphocytic infiltrates and major histocompatibility complex I (MHC-I) expression on non-necrotic muscle fibres. As for most other IIMs, high-dose corticosteroids is considered the first-line treatment but most cases are refractory to conventional steroid monotherapy. Other immunosuppressive therapy such as methotrexate, azathioprine, rituximab and cyclophosphamide are increasingly being used. Intravenous immunoglobulin (IVIg) and plasmapheresis may be required. Associations have been described with statin exposure and some genetic risk factors. Exact pathomechanisms and potential triggers of the disorder particularly still need to be identified in patients not exposed to statins. Methods: Myositis specific antibodies (immunodot assay covering 16 auto-antibodies, including anti-HMGCR) were systematically assessed in a cohort of 40 IIM patients. Patients with anti-HMGCR antibodies were selected for specific follow-up. CK levels were repeatedly measured as well as auxiliary laboratory findings and an electromyography (EMG) was performed. Muscle biopsies were obtained for all patients from quadriceps muscle and analysed following standard histologic and immunohistochemical techniques for light microscopy. Wholebody muscle magnetic resonance imaging (MRI) was performed on a $1.5 \mathrm{~T}$ MRI platform. Results: We present five patients with an anti-HMGCR antibody-related myopathy. Firstly, we found that the presenting symptoms are highly variable ranging from subacute severe proximal weakness to more insidious complaints linked to subtle proximal weakness. Statin use is only associated in one patient but viral infection preceding the myopathy is noted in three patients. CK levels are consistently highly elevated (above $>5000 \mathrm{U} / \mathrm{L}$ ). EMG confirmed myopathic findings, muscle MRI imaging illustrated oedema in different muscle groups and muscle biopsy showed often relatively subtle abnormalities mainly consisting of fibre necrosis with myophagic reaction in the absence of florid inflammatory changes. The immune-mediated aetiology of the disease could however be confirmed immunohistochemically in all patients. Initial treatment was based on conventional steroid monotherapy with highly variable response among the patients reported ranging from significant clinical and biochemical improvement to complete refractoriness in others. As needed, therapy was escalated with second and third-line agents and the responses will be discussed in detail. Conclusion: Our findings highlight the clinical, histopathological and prognostic heterogeneity of anti-HMGCR antibody-related myopathies and point-out potential diagnostic and therapeutic pitfalls of this treatable myopathy. Based on our findings, we explore the role of viral infections in the pathophysiology of this disorder.

\section{PS1Group1-023 / \#1010}

Topic: Group 1 - Muscle Diseases of Genetic Origin and Acquired Myopathies: Clinical Features, Pathophysiology, Therapy

\section{A PLURIPOTENT STEM CELL- DERIVED MODEL OF MEROSIN DEFICIENT CONGENITAL MUSCULAR DYSTROPHY}

Kenyon Lyon ${ }^{1}$, Amanda Rickard ${ }^{2}$, Monica Hayhurst Bennett $^{2}$, Uli Schmidt ${ }^{2}$

${ }^{1}$ College Of Engineering, California Polytechnic

State University, San Luis Obispo, US ${ }^{2}$ Genea

Biocells, San Diego, CA, US

Background: Congenital muscular dystrophies (CMD) are a group of early onset, often very severe forms of muscle disorders. Congenital muscular dystrophy type 1A (MDC1A) accounts for about $40 \%$ of cases of CMD and has an estimated prevalence of 7/1,000,000. MDC1A is an autosomal recessive disease caused by mutations in the LAMA2 gene encoding the $\alpha 2$ chain of laminin-211. Laminin-211 is a major component of the skeletal muscle basement membrane that stabilizes muscle and facilitates extracellular signaling. MDC1A is a lifethreatening disease characterized by hypotonia, muscle weakness, scoliosis, joint contractures and respiratory difficulties. Currently, there is no treatment available for MDC1A. Human pluripotent stem cells (hPSCs) represent a renewable source of skeletal muscle cells for disease modeling and therapeutics screening and serve as an attractive alternative to primary cultures which are more variable and only undergo limited population doublings. In these studies, MDC1A affected hPSCs were differentiated to skeletal muscle and examined for disease-relevant 
phenotypes. Methods: Genea Biocells' published protocol was used to differentiate both healthy and MDC1A affected hPSCs into mature skeletal muscle myotubes and examined for MDC1A-specific defects. Results: MDC1A-affected hPSCs differentiated into multinucleated myotubes and expressed myogenic markers. We have identified a variety of clinically relevant MDC1A phenotypes that are amenable to high-throughput screening and therapeutics testing, including impaired metabolic function and mitochondrial defects. We have also detected increased activation of apoptosis-related markers in MDC1A-affected cells. Moreover, we detected defects in myogenesis through high-content imaging for myogenic markers throughout the differentiation process. Conclusion: We have developed and characterized the world's first pluripotent stem cell-derived model of MDC1A, a powerful tool for studying MDC1A pathophysiology and for use in the development of effective treatments for CMDs.

\section{PS1Group1-024 / \#1006}

Topic: Group 1 - Muscle Diseases of Genetic Origin and Acquired Myopathies: Clinical Features, Pathophysiology, Therapy

\section{AN IN VITRO MODEL OF MYOTONIC DYSTROPHY TYPE 1 USING HUMAN EMBRYONIC STEM CELL-DERIVED SKELETAL MUSCLE}

Ernesto Solana-Guizar ${ }^{1}$, Samuel Labarge ${ }^{2}$, Monica Hayhurst Bennett ${ }^{2}$, Uli Schmidt ${ }^{2}$

${ }^{1}$ California State University San Marcos, San Marcos, CA, US; ${ }^{2}$ Genea Biocells, San Diego, CA, US

Background: Myotonic Dystrophy Type 1 (DM1) is the most common form of adult-stage onset muscular dystrophy with an estimated prevalence of $1 / 8,000$ worldwide. Patients affected by DM1 display a range of symptoms (e.g. muscle wasting, intellectual disability, and infertility). DM1 is an autosomal-dominant, multisystemic genetic disorder characterized by CTG triplet repeat expansion in the 3' untranslated region of the dystrophia myotonica protein kinase (DMPK) gene, with larger repeat size directly correlating to higher disease severity. The CTG repeat expansion leads to sequestration of splicing regulators (such as Muscleblind Like Splicing Regulator 1 [MBNL1]) in nuclear foci containing DMPK mRNA, and subsequent splicing defects (e.g. exon inclusions and exclusions). In this study, we differentiated unaffected control and DM1-affected human pluripotent stem cell lines to skeletal muscle using Genea Biocells' published protocol and investigated their phenotypes with the aim to establish a clinically relevant stem cell-based disease model of DM1. Methods: Unaffected control and DM1-affected (Genea067 [1626/1773 CTG], Genea157 [1060 CTG], Genea158 [180 CTG]) human embryonic stem cell lines were differentiated into skeletal muscle (i.e. myoblasts and myotubes) according the manufacturer's protocol and analyzed for the following: (1) Lineage commitment and differentiation kinetics of myoblasts to terminally mature myotubes was examined and quantified. (2) MBNL1 nuclear foci were visualized and quantitated by high content imaging. (3) At the molecular level, splice variants were analyzed with semi-quantitative PCR and Nanostring gene expression analysis technology. (4) We investigated cellular metabolism and energetics using Seahorse Technologies' XF Cell Mito Stress test to understand ATP production, as well as basal and maximal respiration. Results: (1) We found that affected cells display aberrant myotube formation, as previously reported in animal models and the congenital (infant-onset) form of DM1. (2) We observed a direct correlation between disease severity and MBLN1 foci intensity. (3) We observed impaired splicing in ALPK3, ATP2A1, and CACNA1S between unaffected and Genea067 day 3 myotubes. (4) We found an inverse relationship between disease severity, i.e. number of CTG repeats, and mitochondrial respiration. Conclusion: Together, this work demonstrates the usefulness of DM1-affected human pluripotent stem cell-derived skeletal muscle to accurately model myotonic dystrophy type-1 in vitro, recapitulating known phenotypes that correlate with disease severity as well as tissue-specific mechanisms. This model represents a new and attractive tool to develop and test DM-1 muscle-targeted therapeutics. 


\section{PS1Group1-025 / \#827}

Topic: Group 1 - Muscle Diseases of Genetic Origin and Acquired Myopathies: Clinical Features, Pathophysiology, Therapy

\section{DIMETHYLFUMARATE (DMF) DOWNMODULATES INFLAMMATION IN SKELETAL MUSCLE}

$\underline{\text { Karsten Schmidt }}^{1}$, Simranjeet Kaur ${ }^{1}$, Alice Faust ${ }^{1}$, Peter Balcarek ${ }^{2}$, Jens Schmidt ${ }^{1}$

${ }^{1}$ Department Of Neurology, University Medical Center Göttingen, Göttingen, DE; ${ }^{2}$ Department Of

Trauma Surgery, Orthopaedics And Plastic Surgery, University Medical Center Göttingen, Göttingen,

$D E$

Background: The pathogenesis of IBM is caused by degenerative and inflammatory mechanisms. Various immunosuppressive therapies have failed to show a significant beneficial effect in clinical studies. DMF is an anti-inflammatory drug used in multiple sclerosis and psoriasis. It is known to diminish the cellular stress response to pro-inflammatory stimuli and some of these effects are mediated by activation and nuclear translocation of NRF-2. This study aims to evaluate the effect of dimethylfumarate (DMF) in in vitro and in vivo model systems for inclusion body myositis (IBM). Methods: The effect of DMF was studied in vitro in a well-established pro-inflammatory cell culture model system originated from a myoblast cell line and cultured primary muscle cells on RNA and protein level using quantitative PCR, immunocytochemistry and western blotting. For in vivo studies, a previously characterized double-transgenic mouse strain was used that harbours human mutated amyloid precursor protein (APP) and presenilin (PS)-1 under muscle specific control via the creatin kinase (CK) promotor. The mice were fed by oral gavage with 15 , 30 or $45 \mu \mathrm{g}$ DMF or vehicle twice per day for 14 days, followed by a pro-inflammatory stimulus via injection of LPS compared to saline into the calf. Animals were sacrificed after 48 hours and the calf muscles were analyzed by qPCR, immunohistochemistry and ex vivo flow cytometry. Results: In muscle cell cultures, DMF led to an upregulation and nuclear translocation of NRF-2. DMF diminished the muscle inflammation in the mouse model of IBM (APP/PS1 transgenic mice) upon intramuscular injection of LPS compared to controls. mRNA expression analysis by qPCR demonstrated a modest effect on pro-inflammatory cytokines in DMF treated mice compared to controls. Conclusion: The data suggest that DMF is active in skeletal muscle by translocation of NRF-2 and upregulation of associated genes. Moreover, a significant reduction of LPS-induced inflammation was noted in mice treated with DMF compared to controls in double-transgenic mice (APP/PS1).

\section{PS1Group1-026 / \#878}

Topic: Group 1 - Muscle Diseases of Genetic Origin and Acquired Myopathies: Clinical Features, Pathophysiology, Therapy

\section{TAMOXIFEN IN DMD: RATIONALE AND PROTOCOL FOR A MULTICENTRE, RANDOMISED, DOUBLE-BLIND, PLACEBO- CONTROLLED, PHASE 3 TRIAL}

$\underline{\text { Simone Schmidt }}^{1}$, Anna-Lena Orsini ${ }^{1}$, Patricia

Hafner $^{1}$, Daniela Rubino-Nacht ${ }^{1}$, Olivier M. Dorchies $^{2}$, Andres Nascimento ${ }^{3}$, U Schara ${ }^{4}$, Stefan Spinty $^{5}$, Haluk Topaloglu ${ }^{6}$, Dirk Fischer ${ }^{1}$ ${ }^{1}$ University Children's Hospital Basel, Basel, $\mathrm{CH} ;{ }^{2}$ School Of Pharmaceutical Sciences, University of Geneva, Geneva, $\mathrm{CH},{ }^{3}$ Hospital Sant Joan de Déu, Barcelona, ES, ${ }^{4}$ Universitaetsklinikum, Essen, DE: $;^{5}$ Alder Hey Children's Hospital, Liverpool, GB; ${ }^{6}$ Hacettepe Children's Hospital, Ankara, TR

Background: Currently, only symptomatic treatment with glucocorticoids is available in Duchenne muscular dystrophy (DMD) which have limited efficacy but many adverse effects. Most current research on therapeutics of DMD focuses on correcting the gene defect. However, as there are more than 250 mutations in the human dystrophin gene, this approach will treat only a small percentage of patients and will be expensive. Using the mouse DMD model it could be shown that tamoxifen (TAM), given orally for periods of 2 or 15 months at doses as low as $0.3 \mathrm{mg} / \mathrm{kg} / \mathrm{day}$, results in almost full recovery of force and structure of muscles. TAM is one of the most efficacious drugs ever investigated in an animal model of DMD. Our aim is to investigate whether TAM treatment, compared to placebo, reduces the disease progression in DMD patients. Methods: We 
are setting up an international (France, Germany, Greece, Spain, Switzerland, United Kingdom, The Netherlands, Turkey) randomised double blind placebo controlled 48-week clinical trial with a core population (group A) of 79 ambulant 6.5 to 12 years old DMD patients that are on a stable standard treatment with glucocorticoids. Furthermore, we will include 16-20 non-ambulant patients age 10 to 16 years who do not receive glucocorticoids (parallel group B) to obtain efficacy and safety data in a broader DMD population. All patients will receive $20 \mathrm{mg}$ of TAM or placebo once daily over 48 weeks. The primary outcome for ambulant DMD patients (group A) is the motor function measure (MFM) subscore D1 (standing and transfer). In the second patient population (non-ambulant patients, group B) the MFM D2 subscore is the primary endpoint, allowing extrapolation of D2 data from the group A (ambulant patient) population. Secondary outcomes include the total MFM and subscores D2 and D3, the North Star Ambulatory Assessment (NSAA), timed function tests (TFT) including 6 minute walking distance (6MWD), proximal upper limb function (PUL), and quantitative muscle testing (Grip force). In addition, to investigate whether longterm 48week TAM treatment can slow muscle degeneration, quantitative thigh muscle magnetic resonance imaging will be performed. Results: The study aims to describe an efficacy and safety profile for tamoxifen in the treatment of DMD patients. Conclusion: According to the recommendations for trials in Duchenne and Becker muscular dystrophy released by the Committee for Medicinal Products for Human Use (CHMP) of the European Medicinal Agency (EMA/ CHMP/236981/2011, Corr. 1), muscle function and muscle force should be evaluated to assess the effect of any studied intervention in muscular dystrophies. The purpose of this study is to evaluate if tamoxifen shows positive effects on muscle function and muscle force in comparison to placebo in patients with DMD. Recruitment is expected to start in May 2018.
PS1Group1-027 / \#494

Topic: Group 1 - Muscle Diseases of Genetic Origin and Acquired Myopathies: Clinical Features, Pathophysiology, Therapy

\section{MUSCLE HISTOPATHOLOGY IN INFANTILE DNM1L-RELATED MITOCHONDRIAL EPILEPTIC ENCEPHALOPATHY IS KEY FOR CLINICAL DIAGNOSIS}

$\underline{\text { Enrico Bertini }}^{1}$, Daniela Verrigni ${ }^{2}$, Domenica

Battaglia $^{3}$, Lucia Fusco ${ }^{4}$, Lorenzo Figà Talamanca ${ }^{5}$, Rosalba Carrozzo ${ }^{2}$, Daria Diodato ${ }^{2}$, Adele D’Amico ${ }^{2}$, Laura Papetti ${ }^{4}$, Daniele Ghezzi ${ }^{6}$, Costanza Lamperti ${ }^{6}$

${ }^{1}$ Unit Of Neuromuscular Disorders, Laboratory of Molecular Medicine, Bambino Gesu' Children's Hospital, Rome, IT; ${ }^{2}$ Unit Of Neuromuscular Disorders, Laboratory Of Molecular Medicine, Bambino Gesu' Children's Hospital,, Rome, IT, ${ }^{3}$ Infantile Neuropsychiatric Unit, Fondazione Policlinico Universitario Agostino Gemelli, Rome, IT; ${ }^{4}$ Division Of Neurology, Bambino Gesu' Children's Hospital, Rome, IT,; Division Of Neuroradiology, Bambino Gesu'Children's Hospital,, Rome, IT; ${ }^{6}$ Division Of Molecular Neurogenetics, Istituto Neurologico C. Besta, Milano, IT

Background: DNM1L (dynamin 1 like) encodes a member of the dynamin superfamily of GTPases and mediates membrane remodeling during a variety of cellular processes; in fact DNM1L has an important role in the maintenance of mitochondrial and peroxisomal morphology. Dysfunction of DNM1L is implicated in several neurological disorders and associated with either de novo-dominant mutations or compound heterozygous DNMIL mutations (MIM \#603850), as well. Many patients develop refractory seizures, consistent with an epileptic encephalopathy, and thereafter show neurologic decline. The age at onset, features, and severity are variable, and some patients may not have clinical evidence of mitochondrial or peroxisomal dysfunction. Following the first description there has been increasing reports in the last 2 years on sporadic patients all affected by early onset encephalopathy with acquired microcephaly and drug-resistant seizures, progressive brain atrophy or abnormal brain development, optic atrophy, and persistent lactic acidemia 
related to de novo dominant-negative mutations. Methods: We collected 5 sporadic patients affected by early onset epileptic encephalopathy. Patients were studied by neuroimaging, skin and muscle biopsy and genomic DNA was analyzed by highthroughput sequencing using TruSight One panel (Illumina (San Diego, CA) in 3 patients while the remaing 2 patients were were analyzed using a custom gene panel for the screening of 230 genes associated with mitochondrial diseases.The muscle biopsies and cultures fibroblasts were processed by routine stainings, and immunofluorescence to detect the mitochondrial network arrangement. Results: Molecular genetic analysis disclosed heterozygous de novo DNML1 mutations in all 5 patients, and 3 of these mutations were novel. Unreported mutations were validated in a yeast model. Summarizing the clinical reports of the 5 patients, in all first symptoms appeared between the second and fifth year of life after normal developmental milestones occurring in the first year of life. The first manifestation consisted in prolonged treatment-resistant.generalized clonic seizures or hemiclonic seizures that shortly progressed into frequently recurrent epileptic mal status. The brain MRI showed at the beginning T2 hyperintensities in the posterior white matter regions and transient $\mathrm{T} 2$ hyperintensities in the cortical areas in 2 patients when performed serially. With time, the MRI showed moderate to severe global cerebral atrophy in all patients Histopathology of the muscle biopsy was revised in 4 patients and showed peculiar features: scattered fibers with a partial reduction of cytochrome $c$ oxidase (COX) and succinate dehydrogenase (SDH) stain with aspects of polymorphic core like areas. These areas corresponded to reduced immunoreactive areas displayed by immunohistochemistry with antibodies recognizing TOMM20, defining abnormally distributed mitochondria. Biochemical spectrophotometric assay of the mitochondrial OXPHOS enzymes showed normal activities. In cultures fibroblasts we observed an increased filamentous network in mutant fibroblasts in 3 patients, while in one patient the mitochondrial network appeared as normal controls and in the last patient we observed a "chain-like" structure, not observed in controls but already reported in patients with biallelic mutations in DNM1L. Conclusion: We describe 5 sporadic patients affected by severe mitochondrial treatment-resistant seizures correlated with de novo heterozygous mutations in DNML1. The most consistent finding in all patients was a histopathological peculiar pattern
PS1Group1-028 / \#731

Topic: Group 1 - Muscle Diseases of Genetic Origin and Acquired Myopathies: Clinical Features, Pathophysiology, Therapy

\section{EFFECT OF A LONG-TERM TREATMENT WITH METFORMIN IN DYSTROPHIC MDX MICE: A RECONSIDERATION OF ITS THERAPEUTIC INTEREST IN DMD}

Paola Mantuano $^{1}$, Roberta F. Capogrosso ${ }^{1}$, Francesca Sanarica $^{1}$, Maria Grazia Morgese ${ }^{2}$, Michela De Bellis ${ }^{1}$, Anna Cozzoli ${ }^{1}$, Adriano Fonzino $^{1}$, Elena Conte ${ }^{1}$, Giulia Maria Camerino ${ }^{1}$, Luigia Trabace $^{2}$, Annamaria De Luca $^{1}$

'Pharmacy - Drug Sciences, University of Bari “Aldo Moro”, Bari, IT; ${ }^{2}$ Experimental And Clinical Medicine, University of Foggia, Foggia, IT

Background: The pharmacological stimulation of AMP-activated protein kinase (AMPK) via metabolic enhancers has recently emerged as a potential therapeutic strategy for Duchenne muscular dystrophy (DMD). Here we focused on metformin, a widely-prescribed anti-hyperglycemic drug which indirectly activates AMPK by inhibiting mitochondrial respiratory chain complex I. Recently, the potential synergistic action of metformin and NO-precursors has been proposed in clinical trials on DMD patients; a combination of metformin and L-arginine exerted positive effects on mitochondrial protein expression, in parallel slowing disease progression (NCT02516085). Despite these encouraging results, preclinical data supporting the rationale of metformin use in DMD are still poor. In addition, controversial actions of metformin on skeletal muscle have been reported. These observations made us questioning about the actual therapeutic potential of metformin as metabolic enhancer on dystrophic muscle in $m d x$ mice and humans. In the present study, we assessed the effects of a long-term treatment with metformin in the exercised $m d x$ mouse, characterized by severe mechanical-metabolic uncoupling and functional maladaptation, in order to gain more insight into the mechanisms of action of the drug and its potential efficacy in a metabolic state of dystrophic muscle closer to that observed in patients. Methods: Metformin was administered in drinking water at a dose of $200 \mathrm{mg} / \mathrm{kg}$ for 20 weeks. A validated multidisciplinary in vivo and ex vivo 
approach was used to assess the impact of drug treatment on disease-related primary readouts. Results: We disclosed the ability of metformin to markedly ameliorate histopathology of $m d x$ gastrocnemius (GC) muscle, significantly reducing total area of damage and, specifically, non-muscle area. This was accompanied by a significant reduction of pro-fibrotic TGF- $\beta 1$ in GC muscle (recovery score, r.s., $106 \%$ ) and by a marked decrease of plasma MMP-9 level (r.s. 43\%), both measured by ELISA tests. In parallel, metformin partially counteracted in vivo $m d x$ weakness, detected by grip strength test, and significantly increased ex vivo specific twitch and tetanus of diaphragm (r.s. 28\%), but not of EDL muscle, although this latter was partially protected by the treatment with respect to mechanical threshold, electrophysiological index of excitation-contraction coupling. However, these improvements were not related to a protective metformin action on dystrophic muscle metabolism, as shown by the modest effects exerted on the increase of pAMPK/ AMPK ratio measured by western blot, on the expression of genes involved in mechanical-metabolic response measured by Real-time PCR, and by the lack of fast-to-slow fiber-type shift revealed by SDH staining on tibialis anterior muscle. Similar results were obtained in the milder phenotype of sedentary $m d x$ mice. This limited efficacy could be related to the inability of metformin to increase the low muscle levels of L-arginine, L-citrulline and taurine in nonexercised/exercised $m d x$ mice, as shown by HPLC analysis, suggesting the need of a synergic action on metabolic pathways for an efficient genetic or epigenetic effect of metformin. Conclusion: Our findings encourage the need to improve our knowledge on alternative, metabolism-independent mechanisms of action of metformin, to differently repurpose this drug for the treatment of DMD, further supporting its clinical use in combination with metabolism enhancers such as NO precursors.
PS1Group1-029 / \#366

Topic: Group 1 - Muscle Diseases of Genetic Origin and Acquired Myopathies: Clinical Features, Pathophysiology, Therapy

\section{IDENTIFICATION OF LATE-ONSET POMPE DISEASE AMONG HIGH- RISK POPULATION WITH NATIONWIDE SCREENING STUDY IN JAPAN}

Katsuhisa Ogata ${ }^{1}$, Motomichi Kosuga ${ }^{2}$, Eri Takeshita $^{3}$, Tsuyoshi Matsumura ${ }^{4}$, Keiko Ishigaki $^{5}$, Shiro Ozasa ${ }^{6}$, Hajime Arahata ${ }^{7}$, Kazuma Sugie ${ }^{8}$, Toshiaki Takahashi ${ }^{9}$, Satoshi Kuru ${ }^{10}$, Michio Kobayashi $^{11}$, Hiroto Takada ${ }^{12}$, Ayako Hattori ${ }^{13}$, Masanori P. Takahashi ${ }^{14}$, Nobuyuki Tanaka ${ }^{15}$, Takashi Kimura $^{16}$, Michinori Funato ${ }^{17}$, Torayuki Okuyama $^{18}$, Hirofumi Komaki ${ }^{19}$

${ }^{1}$ Institute Of Clinical Research / Department Of

Neurology, National Hospital Organization

Higashisaitama Hospital, Hasuda, Saitama, $J P ;{ }^{2}$ Center For Lysosomal Storage Diseases / Department Of Clinical Laboratory Medicine I Division Of Medical Genetics, National Center for Child Health and Development, Setagaya, Tokyo, $J P,{ }^{3}$ Department Of Child Neurology, National Center of Neurology and Psychiatry, Kodaira, Tokyo, JP: ${ }^{4}$ Neurology, National Hospital Organization Toneyama National Hospital, Toyonaka, JP, ${ }^{5}$ Department Of Pediatrics, Tokyo Women's Medical University, Shinjuku, Tokyo, $J P,{ }^{6}$ Department Of Pediatrics, Kumamoto University, Kumamoto, JP; ${ }^{7}$ Department Of Neurology, National Hospital Organization Omuta Hospital, Omuta, Fukuoka, JP, ${ }^{8}$ Department Of Neurology, Nara Medical University, Kashihara, Nara, JP; Department Of Neurology, National Hospital Organization Sendai-Nishitaga National Hospital, Sendai, Miyagi, JP: ${ }^{10}$ Department Of Neurology, National Hospital Organization Suzuka National Hospital, Suzuka, Mie, JP; ${ }^{11}$ Department Of Neurology, National Hospital Organization Akita National Hospital, Yurihonjo, Akita, JP; ${ }^{12}$ Department Of Neurology, National Hospital Organization Aomori Hospital, Aomori, JP:13 Department Of Neonatology And Pediatrics, Nagoya City University, Nagoya, Aichi, JP; ${ }^{14}$ Department Of Neurology, Osaka University, Suita, Osaka, JP;'15Department Of Neurology, National Hospital Organization Shimoshizu 
National Hospital, Yotsukaido, Chiba, $J P ;{ }^{16}$ Department Of Neurology, National Hospital Organization Asahikawa Medical Center, Asahikawa, Hokkaido, JP; ${ }^{17}$ Department of Pediatrics, National Hospital Organization Nagara Medical Center, Gifu, JP:18Center For Lysosomal Storage Diseases / Department Of Clinical Laboratory Medicine, National Center for Child Health and Development, Setagaya, Tokyo, $J P ;{ }^{19}$ Department Of Clinical Research Promotion / Department Of Child Neurology, National Center Hospital, National Center of Neurology and Psychiatry, Kodaira, Tokyo, JP

Background: Late-onset Pompe disease (LOPD) is a rare, but treatable metabolic myopathy caused by deficiency of acid alpha-glucosidase (GAA). LOPD is characterized by progressive muscle weakness and/or respiratory failure, but some LOPD patients could be overlooked due to lack of typical signs. To evaluate the utility of dried blood spots (DBS) in the diagnostic work-up and assess the prevalence of LOPD within a Japanese high-risk population, we prospectively screened for LOPD in a Japanese cohort of undetermined myopathy patients aged one year or older with muscle weakness and/or elevated serum creatine kinase levels. Methods: Sixteen neuromuscular center hospitals, members of Muscular Dystrophy Clinical Trial Network (MDCTN), joined the Pompe disease high risk screening study in Japan (PHiRS-J). We tested GAA activity with DBS as first screening method. Patients with reduced GAA activity in DBS were re-analyzed for enzyme activity in lymphocytes, and investigated for GAA gene mutations. Results: Among 164 patients enrolled, two were incompatible with inclusion criteria. Eleven patients showed reduced GAA activity with DBS. Of these patients, three showed normal GAA enzyme activity in lymphocytes; five were revealed as pseudodeficiency; three with confirmed reduction of GAA activity in lymphocytes were identified to have homozygous or compound heterozygous mutations of the GAA gene. Conclusion: In a Japanese highrisk cohort, we found a prevalence of LOPD around $2 \%$. This study suggests that screening of GAA activity with DBS is useful to diagnose patients with LOPD in a high-risk population.
PS1Group1-030 / \#496

Topic: Group 1 - Muscle Diseases of Genetic Origin and Acquired Myopathies: Clinical Features, Pathophysiology, Therapy

\section{CLINICAL COURSE OF ADULT POMPE DISEASE PATIENTS WHO DID NOT START OR DISCONTINUED ENZYME REPLACEMENT THERAPY}

Harmke A. Van Kooten ${ }^{1}$, Laurike Harlaar ${ }^{1}$, Nadine A.m.e. Van Der Beek ${ }^{1}$, Pieter A. Van Doorn ${ }^{1}$, Ans T. Van Der Ploeg ${ }^{2}$, Esther Brusse ${ }^{1}$

${ }^{1}$ Department Of Neurology, Center For Lysosomal And Metabolic Diseases, Erasmus MC, University Medical Center Rotterdam, Rotterdam, NL, ${ }^{2}$ Department Of Paedicatrics, Center For Lysosomal And Metabolic Diseases, Erasmus MC, University Medical Center Rotterdam, Rotterdam, $N L$

Background: Since the introduction of enzyme replacement therapy (ERT) for Pompe disease in 2006, the disease course of patients who did not start ERT, or who discontinued ERT has rarely been described. However, insight in the clinical course of these patients adds to the implementation and further development of guidelines on starting and stopping of ERT in adult Pompe patients. Methods: From our cohort of adult Pompe patients ( $\mathrm{n}=127)$, we describe the clinical course of two groups; 1) those who have never been treated with ERT and 2) patients in whom ERT was discontinued for various reasons.

Results:

Figure 1. Flowchart of the study population

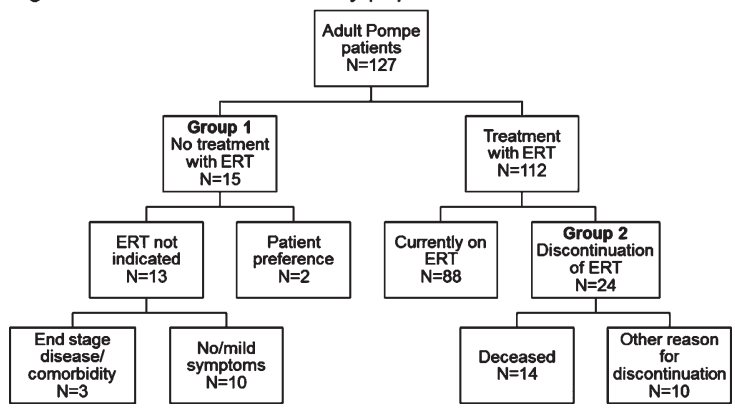

Figure 1 shows an overview of the study population. In group 1 (no ERT; $\mathrm{n}=15$ ) 10 patients had no or only very mild symptoms; these patients remained clinically stable during follow-up (median 5.8 years, 
range $0.6-11.4$ years). Two patients with moderate symptoms of Pompe disease preferred not to start ERT and three patients were too severely affected to start ERT or had life threatening comorbidity. In group 2 (discontinuation of ERT; n=24) ERT was ceased in 14 patients after death. In 10 patients, ERT was discontinued for other reasons: in five patients because of infusion related reactions, two patients chose to stop ERT because they experienced a high burden of the treatment regime, in one severely affected patient we stopped because of continued clinical deterioration under ERT, one patient stopped ERT to undergo treatment for lymphoma and in one patient we ended ERT because of non-compliance. Median treatment duration of the 10 patients who discontinued ERT was 2.1 years (range 0.3 - 14.6 years). ERT had been effective, improving or stabilizing motor and pulmonary function, in eight of them. For three patients sufficient follow up data after treatment cessation was available, with follow up duration of $1.3-8.0$ years. After discontinuation of ERT we did not observe accelerated disease progression beyond the estimated natural progression rate in these three patients. Conclusion: Pompe patients with no or mild symptoms may show a stable disease course for many years, indicating that starting ERT could likely be postponed in this group of patients, under careful follow up. Importantly, in patients who discontinued ERT for various reasons, we did not observe accelerated disease progression beyond estimated disease progression rate. Both findings facilitate the process of shared decision making when considering start or discontinuation of ERT in adults with Pompe disease.

\section{PS1Group1-031 / \#314}

Topic: Group 1 - Muscle Diseases of Genetic Origin and Acquired Myopathies: Clinical Features, Pathophysiology, Therapy

\section{PROLONGED EXERCISE TEST IN PATIENTS WITH HISTORY OF THYROTOXICOSIS}

Cheng-Yin Tan ${ }^{1}$, Hui-Ting Tan², R. Jeyakantha

Ratnasingam ${ }^{2}$, Khean-Jin Goh ${ }^{2}$

${ }^{1}$ University of Malaya, Kuala Lumpur, MY;

${ }^{2}$ University of Malaya, Kuala Lumpur, MY

Background: Thyrotoxic periodic paralysis (TPP) is characterised by recurrent episodes of reversible, severe proximal muscle weakness associated with hypokalaemia and hyperthyroidism. TPP is common in Asians and is a secondary hypokalaemic periodic paralysis, with clinical and electrophysiological characteristics similar to primary hypokalaemic periodic paralysis. Prolonged exercise test (PET) is an easy, non-invasive test evaluating abnormal muscle membrane excitability in periodic paralyses. Results of PET studies in TPP patients on their thyroid status have been variable with one study showing normalization of response while another did not. We aim to evaluate patients with history of thyrotoxicosis with PET and correlate it with the thyroid status. Methods: We prospectively recruited 35 patients with history of thyrotoxicosis (regardless of whether they had TPP) from Endocrinology Clinic. Of the $35 \mathrm{pa}-$ tients, 18 were hyperthyroid, 17 euthyroid or hypothyroid. The thyroid status was determined biochemically from the latest thyroid function test. All patients underwent PET as described by McManis. Compound muscle action potential (CMAP) amplitudes post-exercise were compared against pre-exercise amplitudes and recorded as percentage of mean baseline CMAP amplitude. Results: Compared to 17 euthyroid or hypothyroid patients, significant time-dependent declines in CMAP amplitudes at 20 minutes $(88.3 \pm 11.9 \%$ vs $96.7 \pm 5.2 \%$; $\mathrm{p}=0.012), 25$ minutes $(88.8 \pm 12.9 \%$ vs $96.4 \pm 8.6 \%$; $\mathrm{p}=0.047)$ and 30 minutes $(88.1 \pm 13.0 \%$ vs $97.1 \pm 10.3 \%$; $\mathrm{p}=0.030$ ) after exercise were observed in hyperthyroid patients. The calculated mean greatest decrement from immediate post exercise CMAP amplitudes were $22.5 \pm 13.0 \%$ in hyperthyroid patients and $15.5 \pm 13.0 \%$ in euthyroid/hypothyroid patients. Conclusion: CMAP decrement on PET was significantly greater in hyperthyroid patients compared to euthyroid/hypothyroid patients even without a history of TPP. Muscle membrane excitability is highly influenced by thyroid hormone level. TPP results from increased levels of thyroid hormone activity in genetically susceptible patients. 


\section{PS1Group1-032 / \#882}

Topic: Group 1 - Muscle Diseases of Genetic Origin and Acquired Myopathies: Clinical Features, Pathophysiology, Therapy

\section{CLINICAL AND}

\section{HISTOPATHOLOGICAL FINDINGS} IN MYOTONIC MUSCULAR

\section{DYSTROPHY TYPE 2:}

\section{RETROSPECTIVE REVIEW OF 50 DNA-CONFIRMED CASES}

\author{
Bhaskar Roy $^{1}$, Qian $\mathrm{Wu}^{2}$, Charles Whitaker ${ }^{3}$, Kevin \\ Felice $^{3}$ \\ ${ }^{1}$ Neurology, Yale University, New Haven, CT, US; ${ }^{2}$ \\ Pathology, University of Connecticut, Farmington, \\ CT, US: $;^{3}$ Neurology, Hospital for Special Care, New \\ Britain, CT, US
}

Background: Myotonic dystrophy type 2 (DM2) is an autosomal dominant multisystem disorder causing a progressive myopathy and myotonia due to (CCTG)n repeat expansion in intron 1 of the CNBP gene in chromosome $3 \mathrm{q} 21$. Focused study on a USbased population is limited. Methods: We reviewed the clinical presentation, muscle strength grading by MRC, Jamar hand grip dynamometry, electromyography (EMG), creatine kinase (CK) values, forced vital capacity (FVC), muscle histopathology, CCTG repeat expansion (bp) size for DM2 patients from 1992 to 2015 evaluated at the Muscular Dystrophy Care Center at Hospital for Special Care, CT, USA. Muscle histopathology was graded based on myofiber size variability, internal nucleation, pyknotic nuclear clumps, necrosis, myofiber hypertrophy and atrophy. Results: Fifty patients with DM2 were included in this study. Forty-six patients had positive genetic study and 4 were affected family members. Age of symptom onset ranged from 15 to 72 years $(48.58 \pm 13.12)$. Duration between symptom-onset and initial evaluation ranged from 1 to 42 years $(7.3$ $\pm 9.37)$. Majority of the patients $(64 \%)$ had proximal lower extremity symptoms as the initial presentation. Ten patients did not have any weakness and 17 patients had severe weakness based on a composite MRC scoring system. Hip flexors were the most frequently and severely affected muscles, followed by knee extensors and elbow extensors. About $30 \%$ patients had finger muscles involvement or ankle dorsiflexion weakness. Though most patients had a milder and stable disease course, we noted significant progression of weakness in a few patients. For composite MRC score, the rate of mean decline per year was $0.91 \pm 0.79$. For hip flexors, the rate of mean decline in MRC score per year was $0.21 \pm 0.19$. FVC (\% predicted) in $32(64 \%)$ patients ranged from 36 to $100 \%$ (mean, $81.1 \pm$ $18.3 \%$ ). CK values in 36 patients ranged from 20 to 2000 units/L (mean, $581.7 \pm 449.3$ ). Nine patients had clinical myotonia and 35 patients had myotonia on EMG. Among the muscles examined, biceps and tibialis anterior had myotonic discharges most frequently, $91.6 \%$ and $85.7 \%$ times respectively. No association was noted between the presence of myotonia in EMG and muscle weakness. We noted significant correlation of severity of histopathology with weakness. There was no correlation between CCTG repeat numbers with age of onset, weakness, disease progression, $\mathrm{CK}$ value, hand grip strength or histopathological severity. Among systemic involvement, 24 patients had cataracts $(48 \%)$ and 7 patients had hearing loss. Five patients had symptomatic cardiac arrhythmia and 3 of them required pacemaker. Eleven patients had diabetes and 8 patients had hypothyroidism. Conclusion: This large cohort of DM2, evaluated over a 24-year-period in a single center, showed variation in clinical presentation and disease severity.

\section{PS1Group1-033 / \#327}

Topic: Group 1 - Muscle Diseases of Genetic Origin and Acquired Myopathies: Clinical Features, Pathophysiology, Therapy

\section{ASCERTAINMENT OF THE ADULT PATIENT COHORT WITH MITOCHONDRIAL DISEASE IN GLASGOW}

Maria Elena Farrugia $^{1}$, Cheryl Longman ${ }^{2}$, Lesley

Snadden $^{2}$, Grainne S. Gorman ${ }^{3}$, Andrew M.

Schaefer ${ }^{3}$, Robert W Taylor ${ }^{3}$, Yi Shiau $\mathrm{Ng}^{3}$, Douglass M. Turnbull ${ }^{3}$, Robert Mcfarland ${ }^{3}$, Richard K. Petty ${ }^{4}$

${ }^{1}$ Neurology, Institute of Neurological Sciences, Glasgow, GB; ${ }^{2}$ Genetics, West of Scotland Regional Genetics Service, Glasgow, GB $;^{3}$ Institute Of Neuroscience, Newcastle University Wellcome Trust Centre for Mitochondrial Research, Newcastle upon Tyne, GB; ${ }^{4}$ Neurology, Institute of Neurological Sciences, Glasgow, GB 
Background: Mitochondrial disorders are caused by gene mutations in nuclear or mitochondrial (mt) DNA, encoding structural mitochondrial proteins or proteins involved in mitochondrial function. Patients with mitochondrial disease have diverse phenotypes. Patients may present with ptosis and ocular motility disorders (which may be misdiagnosed as ocular myasthenia). Some suffer from myopathy, diabetes, migraine, deafness and seizures (common red flags for general neurologists). Others may present with ataxia, neuropathy, cognitive impairment, psychosis, or intestinal pseudo-obstruction. These symptoms may not be immediately recognised as part of the disease spectrum and may lead to mis-management. The muscle clinic in Glasgow captures a population of circa 2 million. We have a longstanding collaboration with the NHS Highly Specialised Service for Mitochondrial Disease and Wellcome Centre for Mitochondrial Research in Newcastle, where we refer muscle pathology and patients to help us with the molecular diagnoses and patient management. We wanted to determine the population of molecularly confirmed mitochondrial patients attending the adult muscle clinic in Glasgow and characterise their phenotypes. Methods: We retrospectively identified 57 adult patients (20 families) with molecularly confirmed mitochondrial mutations from a neuromuscular database held in Glasgow. Results: The cohort included 25 patients with $\mathrm{m}$. $3243 \mathrm{~A}>\mathrm{G}$ mutation (14 families), 9 with the m. $8344 \mathrm{~A}>\mathrm{G}$ mutation (2 families), 11 with sporadic large-scale mtDNA deletions, 3 with $P O L G$ dominant mutations and 2 with YARS2 recessive mutations (reported in previous publication; Sommerville et al, JAMA Neurol 2017). Another 7 had various mutations (including mutations in mt-transfer RNA, defects in complex I, complex III, mt-ATP 6, a patient with $\mathrm{m} .7497 \mathrm{G}>$ A mutation with a contractural limb-girdle phenotype, and a patient with multiple mitochondrial deletions for which no nuclear defect has been identified). The common symptoms within the m. $3243 \mathrm{~A}>\mathrm{G}$ cohort included diabetes, deafness and migraine. Four patients had sustained stroke-like episodes (including 2 from the same pedigree with recurrent events and including one which led to her demise). Within the m. 8344 $\mathrm{A}>\mathrm{G}$ cohort, symptoms included hearing loss, myopathy and exercise intolerance. Two patients (mother and son) had abnormal EEGs with lateralising epileptiform features but no clear clinical seizures or myoclonus. Within the single large-scale mtDNA deletions all had ptosis with progressive external ophthalmoplegia (PEO), 2 had pacemakers, 2 had cognitive difficulties and one required feeding through a gastrostromy tube. All 3 patients with $P O L G$ mutations had ptosis with PEO and myopathy. One patient had an episode of intestinal pseudoobstruction which settled with conservative management. We finally discuss the case of a lady with $\mathrm{m} .3243 \mathrm{~A}>\mathrm{G}$ mutation, who succumbed to complications from a stroke-like episode and the difficulties pertaining to her management. Such patients can present with super-refractory epileptic seizures, requiring aggressive management. We discuss the difficult decisions pertaining to prophylactic anti-seizure medication for her family members, who harbour the mutation at high heteroplasmy levels in urine and muscle. Conclusion: Patients with mitochondrial disorders suffer from a complex multi-system disease. Awareness of this is pertinent when assessing mitochondrial patients, discussing diagnosis and prognosis with patients and their families. Their management requires a multidisciplinary approach.

\section{PS1Group1-034 / \#373}

Topic: Group 1 - Muscle Diseases of Genetic Origin and Acquired Myopathies: Clinical Features, Pathophysiology, Therapy

\section{RESTING-STATE FMRI SHOWS ALTERED DEFAULT-MODE NETWORK FUNCTIONAL CONNECTIVITY IN DUCHENNE MUSCULAR DYSTROPHY PATIENTS}

\author{
Nathalie Doorenweerd $^{1}$, Mischa De Rover ${ }^{2}$, Chiara \\ Marini-Bettolo ${ }^{3}$, Kieren G. Hollingsworth ${ }^{4}$, Erik H. \\ Niks $^{5}$, Jos G.m. Hendriksen ${ }^{6}$, Hermien E. Kan ${ }^{1}$, \\ Volker Straub ${ }^{7}$ \\ ${ }^{1}$ C.j. Gorter Center For High Field Mri, Leiden \\ University Medical Center, Leiden, $N L,{ }^{2}$ Clinical \\ Psychology Unit, Leiden University, Leiden, \\ NL; J John Walton Muscular Dystrophy Research \\ Center, Newcastle University, Newcastle Upon \\ Tyne, GB; ${ }^{4}$ Newcastle Magnetic Resonance Centre, \\ Newcastle University, Newcastle Upon Tyne, \\ GB: ${ }^{5}$ Neurology, Leiden University Medical Center, \\ Leiden, $N L ;{ }^{6}$ Neurological Learning Disabilities, \\ Kempenhaeghe Epilepsy Center, Heeze, NL; ${ }^{7}$ John \\ Walton Muscular Dystrophy Research Centre, \\ Newcastle University, Institute of Genetic Medicine, \\ Newcastle Upon Tyne, GB
}


Background: Duchenne muscular dystrophy (DMD) is an X-linked neuromuscular disorder caused by mutations in the dystrophin encoding $D M D$ gene. Dystrophin is expressed in muscle but also in brain and difficulties with attention/inhibition, working memory and learning are well described. Reduced functional connectivity in the motor cortex has been reported in DMD. However, the default mode network (DMN) remains unexplored despite being involved in switching between different stages of attention as well as information processing and self-reflection. Therefore, the aim of this study was to determine if there are differences in DMN connectivity between DMD and controls. Methods: Thirty-three DMD and twenty-four male age matched controls were recruited and scanned at two clinical sites (LUMC and NU). T1-weighted anatomical scans and resting state functional scans were obtained on a 3 Tesla Philips Achieva. FSL v.5.0.8 was used for all analyses which were performed in accordance with recent FSL recommendations, including ICA-AROMA motion correction. The DMN was first identified in the controls. Statistics were performed with FSL RANDOMISE to test for differences between DMD and controls in the DMN and the visual network, with age as covariate and threshold-free cluster enhancement multiple comparison correction. Results: There was significant hyper-connectivity in the precuneus and hippocampus and additional connections with the right thalamus in relation to the DMN in DMD compared to controls. This may be related to the often impaired learning abilities in DMD. Additional connectivity is also found in the sensori-motor cortex. As DMD is hallmarked by progressive muscle wasting, altered connectivity in this region is not surprising but the relationship with the default mode network is un-

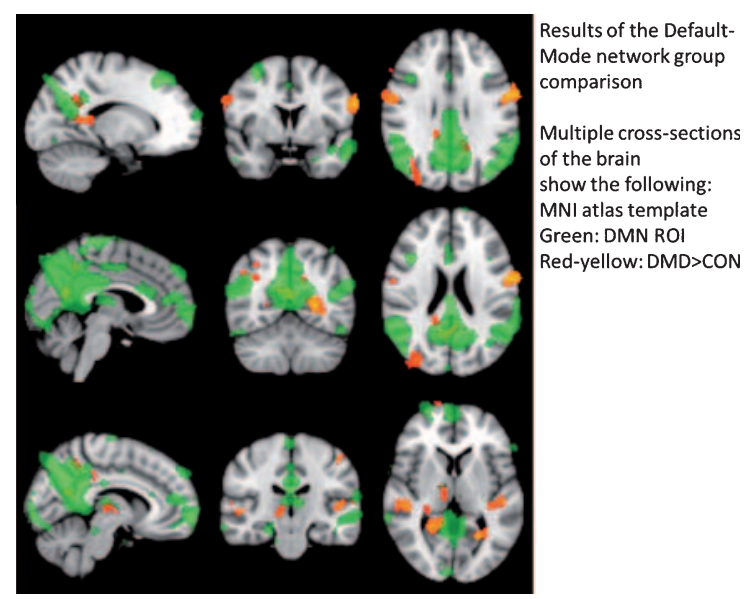

clear. Reduced grey matter volume and perfusion known to be present throughout the brain in DMD could lead to lower signal to noise ratio which could have a confounding effect on the comparison. Therefore, the visual network was assessed as negative control and indeed, no significant differences were found between DMD and controls. Conclusion: Altered DMN connectivity was found in DMD with both hyper-connectivity within the DMN and reconfiguration of the network to include additional brain regions. As the DMN in general is active during rest and inactive during tasks, it will be important to assess in DMD if this extended DMN, which includes greater parts of the precuneus, hippocampus and thalamus, is also inactivated when a task is performed. If so, this may help explain the incidence of attention/inhibition, working memory and learning difficulties in DMD.

\section{PS1Group1-035 / \#930}

Topic: Group 1 - Muscle Diseases of Genetic Origin and Acquired Myopathies: Clinical Features, Pathophysiology, Therapy

\section{SPECIFIC MUTATIONS IN MYBPC1 CAUSE MYOPATHY AND "MYOGENIC TREMOR"}

Sandra Jackson ${ }^{1}$, Jochen Schaefer ${ }^{1}$, Annika Saak ${ }^{1}$, Janis Stavusis ${ }^{2}$, Baiba Lace ${ }^{3}$, Janelle Geist ${ }^{4}$, Inna Inashkina $^{2}$, Dita Pelnena ${ }^{2}$, Sander Pajusalu ${ }^{5}$, Nathan Wright $^{6}$, Aikaterini Kontrogianni-Konstantopoulos ${ }^{4}$, Carsten G. Bönnemann ${ }^{7}$

${ }^{1}$ Neurology, Uniklinikum C.G.Carus, Dresden, DE: ${ }^{2}$ Latvian Biomedical Research Centre, Riga, $L V ;{ }^{3}$ Centre Hospitalier Universitaire de Quebec, Quebec, CA; ${ }^{4}$ University of Maryland School of Medicine, Baltimore, US, ${ }^{5}$ Tartu University

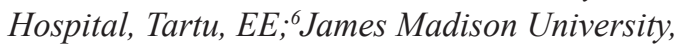
Harrisonburg, US, ${ }^{7} \mathrm{NIH}$ Porter Neuroscience Research Center, Bethesda, MD, US

Background: Myosin-binding protein $\mathrm{C} 1$, a sarcomeric protein, is thought to stabilize the thick filaments interacting with myosin, actin and creatine kinase. To date, only few MYPBC1-mutations have been reported in human disease and lead to autosomal-dominant distal arthrogryposis and autosomalrecessive lethal contracture syndrome. Methods: We describe two multi-generation families with non-progressive, proximal and axial weakness, mild 
spinal rigidity and myalgia. In both families, postural fast-frequency tremor of hands and tongue segregated with the disease. Pathogenic mutations identified by panel sequencing were introduced into truncated GST-tagged recombinant mutant and wildtype proteins. Binding of the mutant protein to myosin was assessed with overlay and immunoblotting assays, and 3D-modelling of the MYBPC1-myosin interaction was performed. Results: Two mutations located in adjacent codons in the M-motif of MYPBC1 and segregating with the disease in both families, demonstrated significantly increased binding of the mutant to myosin compared to the wildtype protein. 3D-modelling and MD-simulation implicated increased electrostatic interactions of MYBPC1 with myosin. Neurophysiological testing excluded a peripheral generator of the tremor. Conclusion: The mutations, which segregated with the disease in both families were predicted by MD-modelling to increase the binding affinity of MYBPC1 to myosin. This was confirmed with in vitro bindingassays. Presumably, cross-bridge cycling of actin and myosin will be affected thus reducing force generation. The tremor associated with the disease was shown to be neither of peripheral nor of central origin, because $M Y B P C 1$ is exclusively expressed in muscle sarcomeres. Thus we postulate a novel class of tremor which we designated "myogenic tremor".

\section{PS1Group1-036 / \#957}

Topic: Group 1 - Muscle Diseases of Genetic Origin and Acquired Myopathies: Clinical Features, Pathophysiology, Therapy

\section{DIAGNOSTIC APPROACH TO CHRONIC PROGRESSIVE EXTERNAL OPHTHALMOPLEGIA - FROM CLINICAL EVALUATION TO GENETIC CONFIRMATION}

Biruta Kierdaszuk $^{1}$, Magdalena Kaliszewska ${ }^{2}$, Katarzyna Tonska², Ewa Bartnik², Anna M. Kaminska ${ }^{1}$, Anna Kostera-Pruszczyk ${ }^{1}$ ${ }^{1}$ Department Of Neurology, Medical University of Warsaw, Warsaw, PL;'2Institute Of Genetics And Biotechnology, Faculty Of Biology, University of Warsaw, Warsaw, PL

Background: Mitochondrial encephalomyopathies comprise a group of heterogeneous disorders which result from impaired oxidative phosphorylation. The respiratory chain complexes present in mitochondria are dependent both on mitochondrial (mtDNA) and nuclear genome (nDNA). Different age of onset, various clinical presentations and individual course of the disease make the diagnosis of mitochondrial disorders challenging. From a variety of symptoms progressive external ophthalmoplegia (PEO) seems to be the most common. The aim of this study was the clinical and genetic characteristics of Polish patients with progressive external ophthalmoplegia. Methods: Clinical, electrophysiological, neuroradiological and morphological data of 72 patients aged 11 to 76 years were analyzed. The muscle specimens were assessed on light and electron microscopy using routine procedures. Genetic studies of mtDNA were performed in all patients. Among nDNA genes $P O L G$ was studied in 26 patients, TWNK (C10orf2) in 3 patients and RNASEH1 in 2 patients. Results: 24 patients with ptosis and PEO were included to chronic progressive external ophthalmoplegia (CPEO) group and 19 with ptosis, PEO and limb or trunk muscles' weakness to CPEO+ group. There were 22 patients with PEO and the central nervous system impairment classified as mitochondrial encephalomyopathy (ME), 6 patients with Kearns-Sayre syndrome (KSS) and one patient with sensory ataxic neuropathy, dysarthria, ophthalmoparesis (SANDO) syndrome. 7 patients had relatives with similar symptoms. Among various diagnostic procedures electromyography showed myopathic changes in the majority of cases. Ragged-red fibers were present in most of muscle biopsies. Magnetic resonance imaging showed atrophied ocular muscles in patients with pure myopathic phenotype and revealed cortical and subcortical atrophy in those with the central nervous system involvement. In single cases magnetic resonance spectroscopy showed elevated lactates in the brain. Genetic studies of $\mathrm{mtD}$ NA proved already known single or multiple mtDNA deletions in all patients and in most cases they were detected in the muscle tissue. Genetic analysis of nDNA genes confirmed mutations in $P O L G$ gene in 6 patients. There were 3 CPEO patients with p. [Arg309Leu];[Gln968Glu], p.[Ala518Thr];[=] and p.[Trp748Ser];[Ser998Pro] mutations, and 2 CPEO+ patients with p.[Thr251Ile;Pro587Leu];[Thr251Ile; Pro587Leu] and p.[Thr251Ile;Pro587Leu];[Lys1191 Asn] mutations. In patient with SANDO syndrome the mutation p.[Arg290Cys]; $[\operatorname{Arg} 309 \mathrm{Cys}]$ in $P O L G$ gene was confirmed. Additionally the analysis of the TWNK (C10orf2) gene proved the mutation p.[Arg- 
374Gln]; [=] in 2 CPEO patients and one CPEO+ patient. Assessment of RNASEH1 gene revealed homozygous variant p.[Gly165Arg];[Gly165Arg] in two siblings with ME. Conclusion: Detailed patients' history and careful assessment of family history are essential in the diagnostic work-up. Morphological evaluation of the muscle biopsy as well as magnetic resonance imaging are also important. Genetic studies of both mtDNA and nDNA are necessary for final diagnosis of chronic progressive external ophthalmoplegia and its genetic counseling.

\section{PS1Group1-037 / \#473}

Topic: Group 1 - Muscle Diseases of Genetic Origin and Acquired Myopathies: Clinical Features, Pathophysiology, Therapy

\section{FEMALE FERTILITY IN MYOTONIC DYSTROPHY TYPE 1 AND 2}

Olesja Parmova ${ }^{1}$, Iva Srotova $^{1}$, Monika Hulova ${ }^{1}$, Eva Vlckova $^{1}$, Livie Mensova ${ }^{2}$, Martina

Podborska $^{3}$, Petra Stradalova ${ }^{1}$, Eva Kralickova ${ }^{1}$, Igor Crha ${ }^{4}$, Radim Mazanec ${ }^{2}$, Stanislav Vohanka ${ }^{1}$, Josef Bednarik ${ }^{1}$

${ }^{1}$ Neurology, University Hospital Brno, Brno, CZ; ${ }^{2}$ Neurology, Motol University Hospital, Prague,

CZ; ${ }^{3}$ Clinical Biochemistry, University Hospital Brno, Brno, CZ; ${ }^{4}$ Obstetrics And Gynecology,

University Hospital Brno, Brno, CZ

Background: Myotonic dystrophy (DM) represents the most common type of muscular dystrophy in adult age. Clinical signs and symptoms in type 1 (DM1) and type 2 (DM2) overlap. DM is a multisystem disorder that, among others, affects endocrine system and thus may have a negative impact on fertility. Female fertility impairment has already been shown in patients with DM1, while only few data are available on DM2 patients. The aim of our study was to compare the fertility characteristics in women with DM1, DM2 and healthy volunteers. Methods: Patients: 22 reproductive-age females (mean age $34.8 \pm 8.0$ ) with DM2 and 18 reproductive-age females with DM1 (mean age 33.9 \pm 6.8 ) were included in this case control study. With respect to significant differences between these two groups in several relevant parameters (e.g. number of pregnancies), particular control groups were created for each of them: DM2 control group consisted of 22 age-matched healthy controls (mean age $34.1 \pm 7.5$ ) and DM1 control group of 18 age-matched healthy controls (mean age $33.2 \pm 7.0$ ). Methods: All participants completed a set of questionnaires used to evaluate fertility. Results: The time to get pregnant was significantly longer in DM1 females when compared with appropriate controls $(p<0.01)$ and the miscarriages were more frequent in this DM group $(p=0.02)$. Females with DM1 also had more often irregular menstrual cycle $(\mathrm{p}=0.02)$ which was more frequently controlled by oral contraceptives in this group. Furthermore, patients with DM1 had higher incidence of uterine myomas and more frequently underwent abdominal gynecologic surgery $(p<$ 0.01 ). In comparison with healthy controls, an unsignificant trend to more frequent investigation of fertility problems and their treatment was observed in women with DM1 $(p=0.08)$. Females with DM2 have more frequent miscarriages $(p=0.04)$ as the only statically significant difference when compared with healthy controls. Conclusion: Our study confirmed clear fertility impairment in women with DM1 and showed less pronounced (but still significant) fertility changes also in female patients with DM2.

\section{PS1Group1-038 / \#340}

Topic: Group 1 - Muscle Diseases of Genetic Origin and Acquired Myopathies: Clinical Features, Pathophysiology, Therapy

\section{EFFECT OF AHK, A NOVEL MODULATOR OF RYANODINE RECEPTORS, IN DUCHENNE MUSCULAR DYSTROPHY}

\author{
Haizpea Lasa Fernandez ${ }^{1}$, Garazi Aldanondo², \\ Jaione Lasa Elgarresta ${ }^{2}$, Aitziber Irastorza ${ }^{3}$, Jose \\ Ignacio Miranda ${ }^{3}$, Jesus Maria Aizpurua ${ }^{3}$, Adolfo \\ López De Munain ${ }^{4}$, Ainara Vallejo Illarramendi ${ }^{2}$ \\ ${ }^{1}$ Department Of Neuroscience, University of the \\ Basque Country, Leioa, ES; ${ }^{2}$ Neuroscience Area, \\ Biodonostia Research Institute, Donostia, \\ ES; ${ }^{3}$ Faculty Of Chemistry, University of the Basque \\ Country, Donostia, ES $;{ }^{4}$ Neurology, Hospital \\ Donostia, Donostia, ES
}

Background: In the mdx mouse model of Duchenne muscular dystrophy (DMD) the sarcoplasmic reticulum ryanodine receptor (RyR) is abnormally nitrosylated and this leads to calstabin depletion from the 
protein complex and subsequent calcium leak through the channel. RyR stabilizers, such as S107, enhance RyR-calstabin binding preventing calcium leak and the consequent damage. Methods: To study the in vitro effect of AHK we have evaluated RyRcalstabin colocalization using in situ proximity ligation assay (PLA) in dystrophin-deficient human myotubes treated overnight with AHK150 nM. We also have determined intracellular calcium levels in these myotubes using Fura-2AM, a ratiometric calcium indicator. For the in vivo studies, we have administered AHK to male $\mathrm{mdx}$ mice in the drinking water for 5 and 12 weeks. We have determined forelimb strength using a grip strength meter. Diaphragm muscle sections were used for histopathological analysis using immunofluorescence. The percentage of central nucleation was determined as a marker of muscle degeneration/regeneration. To study cardiac and cognitive deficits in vivo, we have used 4 monthold mdx mice. We have analyzed cardiomyocyte damage after beta-isoproterenol challenge, using Evans blue dye (EBD) uptake, as an indicator of cardiomyocyte plasma membrane integrity. Finally, we have measured mdx cognitive deficits by tracking the movement after an acute stress produced by a brief restrain. Results: The present work shows that treatment of 1 month-old mdx mice during 5 and 12 weeks with AHK, a novel RyR modulator, improves the dystrophic phenotype of mdx mice. After the treatment during 5 weeks the histopathological and biochemical evidence of skeletal muscle damage is reduced and the strength is improved. Since cardiac and cognitive impairments manifest in older mice, the treatment is prolonged for 12 weeks. Once we characterized the behavioral phenotype of $\mathrm{mdx}$ mice, we observe improvements in treated mice regarding to heart function and behavioral deficits. Conclusion: Overall, our results demonstrate that AHK is effective in improving not only skeletal muscle dystrophic phenotype but also the impairments observed in cardiac muscle and CNS in the mdx mouse model of DMD. Thus, they support AHK as a potential therapeutic drug to prevent the progression of muscular, cardiac and neurological defects in DMD patients.
PS1Group1-039 / \#380

Topic: Group 1-Muscle Diseases of Genetic Origin and Acquired Myopathies: Clinical Features, Pathophysiology, Therapy

\section{ASPIRO PHASE 1/2 GENE THERAPY TRIAL IN X-LINKED MYOTUBULAR MYOPATHY (XLMTM): PRELIMINARY SAFETY AND EFFICACY FINDINGS}

\author{
Nancy Kuntz ${ }^{1}$, Perry B. Shieh², Barbara Smith ${ }^{3}$, \\ Carsten G. Bönnemann ${ }^{4}$, James J. Dowling 5 , \\ Michael W. Lawlor ${ }^{6}$, Wolfgang Müller-Felber ${ }^{7}$, Mo \\ Noursalehi $^{8}$, Sal Rico $^{8}$, Laurent Servais ${ }^{9}$, Suyash \\ Prasad $^{8}$ \\ ${ }^{1}$ Ann \& Robert H Lurie Children's Hospital of \\ Chicago, Chicago, IL, US, ${ }^{2}$ University of California, \\ Los Angeles, Los Angeles, CA, US: ${ }^{3}$ University of \\ Florida, Gainesville, FL, US: ${ }^{4}$ NIH Porter \\ Neuroscience Research Center, Bethesda, MD, \\ US: ${ }^{5}$ Hospital for Sick Children, Toronto, ON, \\ CA, ${ }^{6}$ Medical College of Wisconsin, Milwaukee, WI, \\ US $;^{7}$ Klinikum der Universität München, Munich, \\ DE; ${ }^{8}$ Audentes Therapeutics, San Francisco, CA, \\ US, ${ }^{9}$ Hopital Armand Trousseau, Paris, FR
}

Background: X-linked myotubular myopathy (XLMTM) is a rare disease caused by mutations in the MTM1 gene and characterized by profound muscle weakness, respiratory failure and early death. A retrospective chart review of 112 boys with XLMTM (RECENSUS study [Beggs, 2017]) showed overall mortality of $44 \%$. Additional natural history data are being collected in an ongoing, prospective, non-interventional, run-in study of male patients with XLMTM (the INCEPTUS study), to serve as a longitudinal baseline and within-patient control for the ASPIRO clinical study. Methods: Phase 1/2, open-label, randomized, ascending dose study to evaluate the safety and preliminary efficacy of an investigational gene therapy product (AT132) in patients with XLMTM. AT132 (rAAV8-Des-hMTM1) is designed to deliver functional copies of the MTM1 gene to skeletal muscle cells. Approximately 12 XLMTM patients less than 5 years of age are planned to be randomized into three ascending dose cohorts $(\mathrm{n}=4$ per cohort $)$ to receive a single AT132 administration $(n=3)$ or to act as a delayed treatment control $(\mathrm{n}=1)$. Treatment-randomized Cohort 1 patients received an intravenous infusion of $1 \times 10^{14}$ vector genomes (vg) per kg of AT132. 
Results: A total of 6 adverse events (AEs) were reported, of which 3 were deemed probably or possibly related to drug and 2 were deemed serious AEs (SAEs). Both SAEs occurred in Patient 3, who is responding to intravenous steroids and supportive care. Efficacy assessments have shown notable improvements in both the Children's Hospital of Philadelphia Infant Test of Neuromuscular Disorders (CHOP-INTEND) scale and maximal inspiratory pressure (MIP) (Table 1). Whereas age-appropriate motor milestones had not been achieved at baseline, by week 12, Patient 1 (aged 1 year) had acquired several new developmental skills, including the ability to control head movements, roll over by himself and sit unassisted for $>5$ seconds. Patient 2 was observed for 8 weeks, and Patients 3 and 4 (Control) were observed for 4 weeks. All treated patients have demonstrated improvements in airway clearance and secretion management as evidenced by changes in the Parental Global Impression of Secretion Improvement and Severity Scales. Clinical Global Impression of Improvement reports demonstrate increased limb and trunk strength, improved velocity and accuracy of movement and increased loudness during vocalization and crying, improving their ability to communicate. Conclusion: Preliminary data from cohort 1 of the ASPIRO study are encouraging and based on these observations, the current plan is to enroll three additional patients at this dose level.

\section{PS1Group1-040 / \#442}

Topic: Group 1-Muscle Diseases of Genetic Origin and Acquired Myopathies: Clinical Features, Pathophysiology, Therapy

\section{MR IMAGING OF RESPIRATORY MUSCLE DYSFUNCTION IN POMPE DISEASE}

$\underline{\text { Laurike Harlaar }}^{1}$, Pierluigi Ciet ${ }^{2}$, Alice Pittaro², Piotr A. Wielopolski², Harmke A. Van Kooten ${ }^{1}$, Nadine A.m.e. Van Der Beek ${ }^{1}$, Esther Brusse ${ }^{1}$, Ans T. Van Der Ploeg ${ }^{3}$, Marleen De Bruijne ${ }^{4}$, Harm A.w.m. Tiddens ${ }^{5}$, Pieter A. Van Doorn ${ }^{1}$ ${ }^{1}$ Department Of Neurology, Center For Lysosomal And Metabolic Diseases, Erasmus MC, Rotterdam, $N L ;{ }^{2}$ Department Of Radiology And Nuclear Medicine, Erasmus MC, Rotterdam, $N L,{ }^{3}$ Department Of Paediatrics, Division Of Metabolic Diseases And Genetics, Center For Lysosomal And Metabolic Diseases, Erasmus

Table 1: ASPIRO Cohort 1 Baseline Characteristics and Preliminary Efficacy Results

\begin{tabular}{|c|c|c|c|c|c|}
\hline Patient \# & & 1 & 2 & 3 & $\begin{array}{c}4 \text { (Delayed } \\
\text { Treatment Control) }\end{array}$ \\
\hline Age at Baseline (Years) & & 0.8 & 4.1 & 2.6 & 4.0 \\
\hline Ventilator Support & & $12 \mathrm{~h} /$ day BiPAP & $\begin{array}{c}17 \mathrm{~h} / \text { day invasive } \\
\text { ventilation }\end{array}$ & $\begin{array}{c}24 \mathrm{~h} / \text { day invasive } \\
\text { ventilation }\end{array}$ & 12 h/day BiPAP \\
\hline \multirow{4}{*}{$\begin{array}{l}\text { CHOP-INTEND Score (a } \\
\text { maximum score of } 64 \text { is } \\
\text { typically attained in healthy } \\
\text { infants between } 3-6 \text { months } \\
\text { of age) }\end{array}$} & $\begin{array}{l}\text { Median Score in } \\
\text { INCEPTUS }\end{array}$ & 29 & 45 & 28 & 49 \\
\hline & $\begin{array}{l}\text { Baseline Score in } \\
\text { ASPIRO }\end{array}$ & 29 & 45 & 34 & 49 \\
\hline & $\begin{array}{l}\text { Most Recent Score in } \\
\text { ASPIRO }\end{array}$ & 56 (week 12) & 56 (week 8) & 36 (week 4) & 46 (week 4) \\
\hline & $\begin{array}{l}\text { Change from } \\
\text { Baseline (\%) }\end{array}$ & $27(93 \%)$ & $11(24 \%)$ & $2(6 \%)$ & $3(-6 \%)$ \\
\hline \multirow{4}{*}{$\begin{array}{l}\text { Maximum Inspiratory } \\
\text { Pressure }\left(\mathrm{cm} \mathrm{H}_{2} \mathrm{O} \text {; normal }\right. \\
\text { minimal pressures in } \\
\text { healthy children }<5 \text { years } \\
\text { are } \geq 80 \mathrm{~cm} \mathrm{H} 2 \mathrm{O})\end{array}$} & $\begin{array}{l}\text { Median Pressure in } \\
\text { INCEPTUS }\end{array}$ & 29 & 34 & 24 & 65 \\
\hline & $\begin{array}{l}\text { Baseline Pressure in } \\
\text { ASPIRO }\end{array}$ & 33 & 44 & 26 & 58 \\
\hline & $\begin{array}{l}\text { Most Recent } \\
\text { Pressure in ASPIRO }\end{array}$ & 80 (week 12) & 77 (week 8) & 44 (week 4) & NA \\
\hline & $\begin{array}{l}\text { Change from } \\
\text { Baseline (\%) }\end{array}$ & $47(142 \%)$ & $33(76 \%)$ & $18(70 \%)$ & NA \\
\hline
\end{tabular}


MC-Sophia, Rotterdam, NL; ${ }^{4}$ Biomedical Imaging Group Rotterdam, Departments Of Radiology And Medical Informatics, Erasmus MC, Rotterdam, $N L,{ }^{5}$ Department Of Paediatrics, Respiratory Medicine And Allergology, Department Of Radiology And Nuclear Medicine, Erasmus MC, Rotterdam, $N L$

Background: Adult patients with Pompe disease present with a spectrum of symptoms, mainly characterized by progressive limb-girdle weakness and decreased pulmonary function. Limb-girdle weakness mostly improves or stabilizes after start of enzyme replacement therapy (ERT). Pulmonary function often benefits less well from ERT and may continue to deteriorate. Standard pulmonary function tests only give limited insight in the pathophysiological mechanisms of the pulmonary insufficiency. MRI enables to visualize the diaphragmatic and chest wall movements simultaneously. In a previous study, MRI showed very limited movement of the diaphragm in ten Pompe patients with severe respiratory muscle weakness. Our aim is to study diaphragmatic function in a large group of Pompe patients and to investigate the best outcome measures to evaluate the function of the diaphragm. Methods: We perform spirometer-controlled MRI scans in patients with Pompe disease and sex- and age-matched healthy controls. We acquired dynamic sagittal 2D images in left and right mid hemi-diaphragm during forced inspiration. After manual segmentation of these images, we measured the cranial-caudal and anterior-posterior distance, lung area, diaphragm height, diaphragmatic shape factor and diaphragm slope (figure). We calculated the ratios between maximal inspiration and maximal expiration. We tested intra- and interobserver variability of the measurements, and differences between Pompe patients and controls. Results: In our ongoing study we have now included 34 patients with Pompe disease (age range 15-70 years) with varying disease severity, and 18 controls. All thorax-related measurements showed a high reliability (intraclass correlation coefficient $>0.9$ ). Diaphragm-related measurements showed a larger intra- and interobserver variability. On the right side, mean cranialcaudal ratio was 1.4 in Pompe patients and 1.7 in controls $(p<0.01)$; mean lung area ratio was 2.1 in Pompe patients and 2.5 in controls $(p<0.01)$. On the left side, mean cranial-caudal ratio was 1.4 in Pompe patients and 1.6 in controls $(\mathrm{p}<0.01)$; mean lung area ratio was 1.9 in Pompe patients and 2.1 in controls $(p=0.03)$. In anterior-posterior ratio we found no significant difference between Pompe patients and controls. Conclusion: MRI has good possibilities to evaluate simultaneously diaphragmatic and chest wall movements, which both contribute to pulmonary function. Cranial-caudal ratio and lung area ratio are decreased in the group of patients with Pompe disease compared to controls. We are currently exploring more specific diaphragmatic measures to see whether these can serve as a biomarker for diaphragmatic function in the evaluation of disease progression and response to therapy.

\section{Thorax-related outcomes}

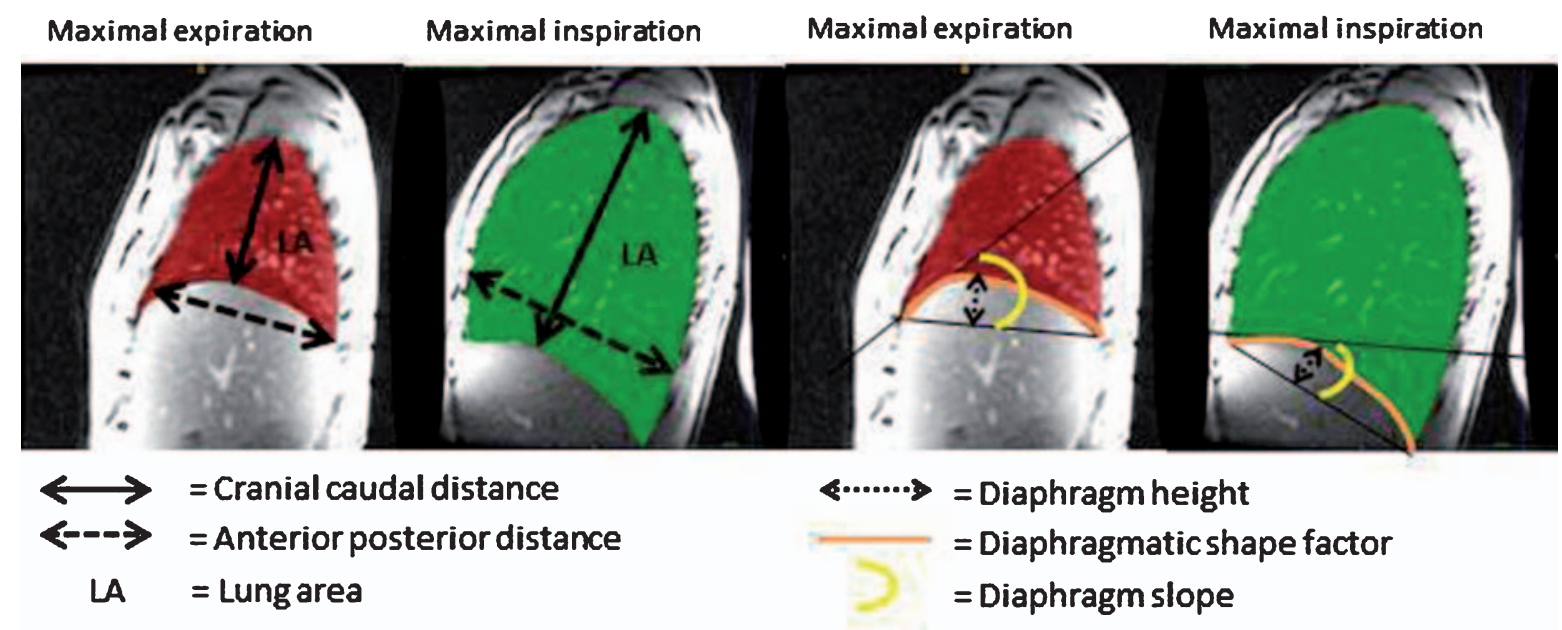




\section{PS1Group1-041 / \#478}

Topic: Group 1 - Muscle Diseases of Genetic Origin and Acquired Myopathies: Clinical Features, Pathophysiology, Therapy

\section{A CASE OF VLCAD DEFICIENCY MYOPATHY WITH NEW MUTATION AND FAVORABLE RESPONSE TO L-CARNITINE, RIBOFLAVIN, AND COQ10}

\author{
Farzad Fatehi $^{1}$, Yalda Nilipour ${ }^{2}$, Shahriar Nafissi ${ }^{1}$ \\ ${ }^{I}$ Neurology, Tehran Univeristy of Medical Sciences, \\ Tehran, IR ${ }^{2}$ Pathology, Mofid Hospital, Tehran, IR
}

Background: Very long-chain acyl-CoA dehydrogenase (VLCAD) deficiency is a very rare condition preventing the body from transforming very longchain fatty acids (VLCAs) to energy, principally during periods of fasting. Signs and symptoms of VLCAD deficiency classically manifest during infancy or early childhood with hypoglycemia, lethargy, muscle weakness, and episodes of dark urine. There is no approved treatment for such patients. Herein, we describe a patient with a new mutation favorably responding to the treatment. Methods: A 38-year-old man was referred for a very slowly progressive lower limb weakness since childhood with the inability to climb upstairs since two years before. He reported episodes of dark urine after exercise. On examination, he had the weakness of neck flexion, bilateral arms abduction (MRC grade 3), bilateral elbows flexion (MRC grade 4), bilateral hips flexion (MRC grade 4), and bilateral knees extension (MRC grade 4) with normal deep tendon reflexes. The CK was $5400 \mathrm{IU} / \mathrm{L}$; cardiac exams were normal; and electromyography demonstrated a myopathic pattern in proximal muscles of upper and lower limbs. Muscle MRI revealed moderate fat deposition in paraspinal muscles, adductor and vastus muscles as well as medial heads of gastrocnemius muscles. On muscle biopsy, myopathic atrophy of mainly type I fibers with multiple necrotic/regenerative fibers and fine lipid droplets in muscle fibers in ORO stain was seen. For genetic testing, DNA was extracted from blood cells and targeted next generation sequencing (NGS) was performed for this individual. The variants located in coding regions of all the 42 genes in this NGS panel for Metabolic Myopathies were evaluated. The obtained result was confirmed with the help of direct DNA sequencing of the candidate variant in the proband. Results: Targeted next-generation sequencing revealed a pathogenic variant defined as c.900G $>$ A (p. Met300Ile) in exon 10 of ACADVL gene due to VLCAD deficiency (MIM\#201475) not described previously. Segregation analysis for the parents confirmed the heterozygote status of both father and mother (carrier status) for ACADVL gene. We started L-carnitine 2 grams daily, CoQ10 $300 \mathrm{mg}$ daily and riboflavin $300 \mathrm{mg}$ daily with a favorable response, and after one month the patient was able to climb upstairs without aid. Conclusion: In sum, it seems that the cocktail of L-carnitine, CoQ10, and riboflavin may be an optimal option for this very rare form of lipid storage myopathy.

\section{PS1Group1-042 / \#713}

Topic: Group 1 - Muscle Diseases of Genetic Origin and Acquired Myopathies: Clinical Features, Pathophysiology, Therapy

\section{IMPAIRED INSULIN SIGNALLING IN SKELETAL MUSCLE OF MYOTONIC DYSTROPHY PATIENTS}

Laura V. Renna ${ }^{1}$, Francesca Bose ${ }^{1}$, Elisa Brigonzi ${ }^{2}$, Giovanni Meola ${ }^{2}$, Rosanna Cardani ${ }^{3}$

${ }^{1}$ Laboratory Of Muscle Histopathology And Molecular Biology, IRCCS Policlinico San Donato, San Donato Milanese (MI), IT; ${ }^{2}$ Department Of Biomedical Sciences For Health, University of Milan, San Donato Milanese (MI), IT; ${ }^{3}$ Laboratory Of Muscle Histopathology And Molecular Biology, IRCCS-Policlinico San Donato, San Donato Milanese (MI), IT

Background: Myotonic dystrophy type 1 (DM1) and type 2 (DM2) are neuromuscular multisystemic disorders caused by expanded CTG or CCTG repeats which lead to nuclear accumulation of mutant transcripts deregulating the activity of some splicing regulators and leading to aberrant alternative splicing of different genes. DM are characterized by metabolic dysfunctions such as insulin resistance, hyperinsulinemia and a fourfold higher risk of developing Diabetes mellitus type 2 (T2DM). Since it is known that insulin resistance is a risk factor for cardiovascular disease, neuropathy and loss of muscle mass, metabolic changes in DM patients might contribute to worsen some aspects of the disease at 
heart, skeletal muscle and brain level. Splicing alteration of insulin receptor (IR) gene is considered one of the causes of insulin resistance in DM patients, however it cannot be excluded that post-receptor signalling abnormalities could also contribute to this feature. The aim of this study is to investigate whether post-receptor abnormalities contribute to the peripheral insulin resistance in DM patients and whether deregulated metabolism pathways may be involved in muscle alterations, thus contributing to muscle atrophy and weakness in DM patients. Methods: Insulin pathway activation has been investigated in insulin stimulated skeletal muscle biopsies obtained from 9 DM1, 2 DM2, 5 healthy subjects (CTR) and 1 T2DM patients (ex vivo analysis). Activation of IRS1, AKT, p70S6K, GSK3 $\beta$, ERK $1 / 2$ and AMPK have been analysed by western blot. ERK1/2 involvement in DM insulin resistance has been investigated in human myotubes treated with the MEK inhibitor U0126 $(25 \mu \mathrm{M})$. Results: Our results indicate that DM skeletal muscle exhibits high basal phosphorylation of AKT, GSK3 $\beta$ and ERK1/2. Moreover, ex vivo analysis of insulin pathway activation has shown that insulin action appears to be impaired in DM samples as compared to CTR. Glucose uptake performed in human myotubes in the absence or presence of U0126 has shown that the specific decrease of ERK activity can lead to an increase in insulin dependent glucose uptake in both DM1 and DM2 muscle cells. Conclusion: In conclusion, ex vivo and in vitro studies show an alteration in activation of several proteins of insulin pathway that might contribute to DM insulin resistance. Results on the correlation between metabolic dysfunction and skeletal muscle impairment will be presented at the meeting. The results of this study may contribute to the identification of novel biomarkers that could be target for therapeutic intervention on insulin resistance and thus to improve the quality of life of DM patients.
PS1Group1-043 / \#843

Topic: Group 1 - Muscle Diseases of Genetic Origin and Acquired Myopathies: Clinical Features, Pathophysiology, Therapy

\section{DISTAL MYOPATHIES WITH RIMMED VACUOLE IN IRAN, A CLINICAL, HISTOPATHOLOGICAL AND GENETIC REPORT OF A LARGE GROUP}

Yalda Nilipour $^{1}$, Shahriar Nafissi ${ }^{2}$, Farzad Fatehi ${ }^{3}$, Yalda Ashoorian ${ }^{4}$, Nahid Beladi Moghadam ${ }^{5}$, Reza Boostani ${ }^{6}$, Mohammad Rohani ${ }^{7}$, Bahram Haghi Ashtiani $^{8}$, Babak Zamani ${ }^{9}$, Keyvan Basiri ${ }^{10}$, Fereshteh Ashtari ${ }^{11}$, Davood Fathi ${ }^{3}$, Hosein Shamshiri ${ }^{2}$

${ }^{1}$ Pathology, Pediatric Pathology Research Center, Research Institute for Children Health, Shahid Beheshti University of Medical Sciences, Tehran, IR $;{ }^{2}$ Neurology, Tehran Univeristy of Medical Sciences, Tehran, IR $;^{3}$ Neurology, Iranian center of neurological research, Neuroscience institute, Shariati Hospital, Tehran Univeristy of Medical Sciences, Tehran, IR, ${ }^{4}$ Pathology, Shahid Beheshti university of medical sciences, Tehran, IR $;{ }^{5}$ Neurology, Shahid Beheshti university of medical sciences, Tehran, IR ${ }^{6}$ Neurology, Mashhad University of medical sciences, Mashhad, IR $;{ }^{7}$ Neurology, Hazrat Rasool Hospital, Iran university of medical sciences, Tehran, $I R ;{ }^{8}$ Neurology, Firoozgar hospital, Iran university of medical sciences, Tehran, IR; ${ }^{9}$ Iran University of Medical Sciences, Tehran, IR, ${ }^{10} I$ Sfahan University of Medical sciences, Isfahan, IR; ${ }^{11}$ Neurology, Isfahan University of Medical sciences, Isfahan, IR

Background: Distal myopathies are a rare group of neuromuscular disorders. Rimmed vacuoles are a feature of several myopathies, most of them predominantly affects the distal muscles. The presence of vacuoles in muscle biopsy associated with other pathological findings and the clinical phenotype of patients, age of onset, mode of inheritance and muscle imaging are important diagnostic clues. Rimmed vacuole myopathy is the most common type of distal myopathy in Iran and the highest prevalence of GNE myopathy was reported in Persians Jewish population. Methods: In this study, we retrospectively evaluate the clinical and histopathological characteristics of 75 Iranian patients (34 male and 41 female) 
from 75 different families with clinical suspicion of hereditary inclusion body myopathy, reported to have rimmed vacuoles in their muscle biopsies. We managed to perform direct sequencing for coding exons 1 to 12 of the GNE gene of 23 patients of this group. Results: Seventeen patients out of 75 had positive family history of muscle weakness. Age of onset of symptoms was 13 to 52 years. Nine patients did not show quadriceps sparing phenotype and 4 patients had loss of ambulation at the time of biopsy which was happened 4 to 13.5 years after onset of their symptoms. Maximum Creatine Kinase detected level was 3061 U/I. In microscopic evaluation, group atrophy was a common finding and some foci of chronic inflammation were identified in 12 cases. The number and the shape of the rimmed vacuoles was observed to be in a wide variety. Thirty-two cases showed necrotic fibers in their muscle biopsies and no congophilic inclusion was seen in 35 cases. Endomysial fibrosis and adipose tissue replacement were identified in 51 and 32 cases respectively. Intermyofibrillar network disruption as core-like lesions and moth-eaten fibers were seen in 41 cases and lobulated fibers were observed in ten muscle samples. Fiber type grouping was noted in 16 patients. No pathogenic mutation in GNE gene was found in 4 patients. Twelve patients were found to be homozygote for pathogenic mutations among which the most common one was c.2228T $>\mathrm{C}$ p. (Met743Thr) mutation in exon 12 observed in 8 cases and two of them had homozygote mutations in exon 4 followed by 1 in exon 3 and 1 in exon 9. One case found to be heterozygote for the GNE c.2228T $>$ C pathogenic mutation. Four patients found to be heterozygote for one pathogenic mutation and one variant of uncertain clinical significance in different exons and 2 patients found to be putative homozygotes for same variant in exon 4 which was of unknown clinical significance. Conclusion: This study is the largest group study of 75 patients suffering from distal myopathy with rimmed vacuoles from Iran. Our findings emphasize the clinical, pathological and genetic heterogeneity of the disease. GNE myopathy is the most common early adult onset autosomal recessive distal myopathy in Iran. More extensive genetic studies such as whole exome sequencing on a larger group of patients may find new genes and will expand our knowledge.

\section{PS1Group1-044 / \#896}

Topic: Group 1 - Muscle Diseases of Genetic Origin and Acquired Myopathies: Clinical Features, Pathophysiology, Therapy

\section{GMPPB HOMOZYGOUS VARIANT IN ADULT ONSET LIMB GIRDLE MYASTHENIC SYNDROME: A LIKELY FOUNDER MUTATION}

Veeramani Preethish-Kumar ${ }^{1}$, Ana Töpf ${ }^{2}$, Kiran Polavarapu $^{3}$, Aditi Joshi ${ }^{4}$, Sunitha Balaraju ${ }^{2}$, Andreas Roos ${ }^{2}$, Rita Horvath ${ }^{2}$, Saraswati Nashi ${ }^{5}$, Seena Vengalil ${ }^{6}$, Aradhna Mathur ${ }^{4}$, Sushmita Nayak $^{4}$, Sakshi Ambawat ${ }^{4}$, Mohammed Faruq ${ }^{4}$, Atchayaram Nalini ${ }^{6}$, Hanns Lochmüller ${ }^{7}$ ${ }^{1}$ Clinical Neurosciences, Neurology, National Institute of Mental Health and Neurosciences (NIMHANS), Bengaluru, IN; $;^{2}$ John Walton Muscular Dystrophy Research Center, Newcastle University, Newcastle, GB $;^{3}$ Clinical Neurosciences, Neurology, National Institute of Mental health and Neurosciences (NIMHANS), Bengaluru, IN; ${ }^{4}$ Institute of Genomics and Integrative Biology, New Delhi, IN; ${ }^{5}$ Neurology, National Institute of Mental Health and Neurosciences (NIMHANS), Bangalore, IN; ${ }^{\circ}$ Neurology, National Institute of Mental Health and Neurosciences, Bengaluru, IN $;{ }^{7}$ Department Of Neuropediatrics And Muscle Disorders, Freiburg University - Medical Center, Faculty of Medicine, Freiburg, DE

Background: Mutations in GMPPB encoding GDPmannose pyrophosphorylase $\mathrm{B}$ affects glycosylation of $\alpha$-dystroglycan resulting in Congenital muscular dystrophies (CMD) and Limb girdle muscular dystrophies (LGMD). To date there are few genetically verified individuals with GMPPB-associated dystroglycanopathiess. Recently, variants in GMPPB were reported to cause congenital myasthenic syndrome. Methods: Ten patients belonging to 4 south Indian families with late onset limb girdle syndrome and easy fatigability are described. Evaluation included clinical phenotyping, serum Creatine Kinase (CK), Repetitive nerve stimulation, and muscle biopsy. Six patients underwent genetic testing. Results: All were born to consanguineous parents. Five patients were evaluated at hospital and all had gradually progressive fatigable limb girdle weakness, prominent truncal weakness with calf hypertrophy and retained 
tendon reflexes (Table 1). CK levels were elevated in all $(650-4547 \mathrm{IU} / \mathrm{L})$. Muscle biopsy in 4 patients was suggestive of muscular dystrophy. RNS showed decrement response in probands from family 3 and 4. Pyridostigmine and Salbutamol were started in family 4, and they showed significant improvement in fatigue and weakness. Genetic analysis revealed identical missense mutation c.1000G $>A$ (p.Asp334Asn) in exon 9 of GMPPB gene in 6 patients tested of all four families. This mutation was previously reported in a compound heterozygous form in two patients of Asian origin (Pakistan and India) in published literature. However, the phenotype described in these two cases was Congenital muscular dystrophy with CNS involvement. While, in all our patients the mutation was present in homozygous form and had a milder, slowly progressive phenotype consistent with Limb girdle myasthenic syndrome. This indicates the milder effect of the mutation which may require a different mutation to cause severe phenotype. As of now this variant has not been reported from other geographical regions. Furthermore, the allele frequency of c.1000G $>A$ among south Asian population is 0.0005 and has zero frequency in other populations in ExAC database. Hence, we suspect a possible founder affect for this mutation in South Asia and needs to be further explored. Table 1: Clinical features. Conclusion: This report further expands the emerging phenotypic spectrum of GMPPB associated dystroglycanopathies and indicates a probable South Asian founder mutation with milder effect in its homozygous form.
PS1Group1-045 / \#480

Topic: Group 1 -Muscle Diseases of Genetic Origin and Acquired Myopathies: Clinical Features, Pathophysiology, Therapy

\section{NFAT5 AND P38 MAPKS INTERACT IN MUSCLE CELLS RESPONDING TO OSMOTIC AND INFLAMMATORY STRESS AND IN POLYMYOSITIS}

Boel De Paepe $^{1}$, Sandrine Herbelet ${ }^{2}$, Jens Schmidt ${ }^{3}$, Jan De Bleecker ${ }^{4}$

${ }^{1}$ Neuromuscular Reference Center, Ghent University Hospital, Ghent, $B E ;^{2}$ Ghent University, Ghent, BE; $;^{3}$ Neurology, University of Goettingen, Goettingen, DE; ${ }^{4}$ Neurology, Ghent University, Ghent, $B E$

Background: The transcription factor Nuclear Factor of Activated T-cells 5 (NFAT5) is the key regulator of cells' responses to osmotic stress, but is also implicated in inflammatory muscle disease. NFAT5 most likely is regulated by Mitogen-activated protein kinases (MAPKs) and the four members of the p38 family of MAPKs, termed MAPK11 (p38ß),

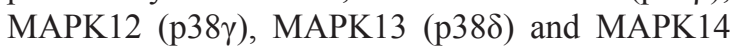
(p38 $\alpha$ ), have known associations with inflammation. Methods: We study MAPKs mRNA expression and protein activation in an in vitro muscle inflammation model, and in muscle from polymyositis patients. Results: We observe that in muscle cells in culture, exposure to increased salt concentrations and

\begin{tabular}{|c|c|c|c|c|c|c|c|c|}
\hline Family & Year & Gender & Age & Onset & Clinical features & $\begin{array}{l}\text { Creatine } \\
\text { kinase }\end{array}$ & RNS & $\begin{array}{l}\text { Family } \\
\text { History }\end{array}$ \\
\hline 1 & 2004 & M & 50 & 42 & $\begin{array}{l}\text { Weakness: Proximal>distal, truncal } \\
\text { weakness; Fatigue +; Scapular winging; } \\
\text { Calf hypertrophy; Fatigue +; DTRs: } 2+\end{array}$ & 650 & $\begin{array}{l}\text { Significant } \\
\text { decrement } \\
(>20 \%)\end{array}$ & $\begin{array}{l}3 \text { siblings } \\
\text { affected }\end{array}$ \\
\hline 2 & 2006 & $\mathrm{~F}$ & 35 & 30 & $\begin{array}{l}\text { Weakness: Proximal; fatigue +; calf } \\
\text { hypertrophy; DTRs } 2+\end{array}$ & 1583 & & $\begin{array}{l}\text { elder sister } \\
\text { affected }\end{array}$ \\
\hline 3 & 2016 & $\mathrm{~F}$ & 32 & 22 & $\begin{array}{l}\text { Weakness: Proximal; fatigue +; calf } \\
\text { hypertrophy; DTRs } 2+\end{array}$ & 1790 & $\begin{array}{l}\text { Significant } \\
\text { decrement } \\
(>20 \%)\end{array}$ & Negative \\
\hline \multirow[t]{2}{*}{4} & 2017 & F (younger sister) & 45 & 28 & $\begin{array}{l}\text { Weakness: Proximal and truncal weakness; } \\
\text { chewing difficulty; fatigue +; mild ptosis } \\
\text { (fatigable) and facial weakness; calf } \\
\text { hypertrophy; DTRs } 2+\end{array}$ & 4547 & $\begin{array}{l}\text { Mild } \\
\text { Decrement } \\
(<10 \%)\end{array}$ & \multirow{2}{*}{$\begin{array}{l}\text { Maternal } \\
1 \text { st cousin } \\
\text { affected } \\
(\mathrm{M} / 44 ; \\
\text { onset: } \\
18 \text { yrs })\end{array}$} \\
\hline & 2017 & F (elder sister) & 46 & 36 & $\begin{array}{l}\text { Weakness: Proximal and truncal weakness; } \\
\text { chewing difficulty; fatigue +; facial } \\
\text { weakness; calf hypertrophy; DTRs } 2+\end{array}$ & 2107 & $\begin{array}{l}\text { Significant } \\
\text { Decrement } \\
(>20 \%)\end{array}$ & \\
\hline
\end{tabular}


pro-inflammatory cytokines influence NFAT5 mRNA expression and translocation to the nucleus. A maximal 4-fold increase of NFAT5 messenger levels in myotubes treated with IL1 $\beta$ and IFN $\gamma+\operatorname{IL} 1 \beta$ for $24 \mathrm{~h}$ is detected, in the latter condition accompanied by a moderate increase of MAPK12 and MAPK13 phosphorylation. Neither MAPK14 expression nor phosphorylation is substantially altered by cytokines and increased $\mathrm{NaCl}$ concentrations. Longer exposure of myotubes in culture to cytokines does not increase NFAT5 nor MAPK14 expression, yet hyperosmotic conditions lead to a time-dependent increase of expression, reaching 9-fold (NFAT5) and 18-fold (MAPK14) after 72h. At this stage, culture densities decrease substantially, disfavoring the differentiated multinucleated cells and leaving myoblasts as the remaining cell developmental stage. In muscle tissues, levels of phosphorylated MAPK11/12/14 relative to actin content, increase from $0.52 \pm 0.24$ in normal $(\mathrm{n}=4)$, to $0.87 \pm 0.23$ in polymyositis $(n=4)$, but this difference does not reach statistical significance $(\mathrm{p}=0.08)$. Immunofluorescent localization studies show expression in blood vessel endothelium and in a subset of small, most often CD56+ (regenerating) muscle fibers. Conclusion: We identify p38 MAPKs as possible phospho-activators of NFAT5 in muscle cell's responding to inflammatory stress, and put forward MAPK12 as a likely regulator relevant to inflammatory myopathy.

\section{PS1Group1-046 / \#551}

Topic: Group 1-Muscle Diseases of Genetic Origin and Acquired Myopathies: Clinical Features, Pathophysiology, Therapy

\section{IMPLICATION OF THE BREAKPOINTS POSITION IN PATIENTS WITH THE MACRODELETION OF EXONS 45 TO 55}

Javier Poyatos Garcia ${ }^{1}$, Clara Gomis Coloma ${ }^{2}$, Nuria Muelas ${ }^{3}$, Pilar Martít ${ }^{4}$ Juan J. Vílchez ${ }^{4}$ ${ }^{1}$ Neurology, Instituto de Investigación Sanitaria la Fe, Valencia, ES $;^{2}$ Neurology, INSTITUTO DE INVESTIGACIÓN SANITARIA LA FE, Valencia, ES $;{ }^{3}$ Neurology, Hospital Universitari i Politècnic La Fe, Valencia, ES: ${ }^{4}$ Neurology, Hospital Universitari i Politècnic La Fe, Universitat de València and CIBERER, Valencia, ES
Background: Duchenne muscular dystrophy (DMD) is a severe myopathy that affects to 1 out of $3500-5000$ newborn boys and it is responsible of severe disability and early death, caused by mutations in the DMD gene. Becker muscular dystrophy, is an allelic form, which manifest a benign phenotype. Sixty five per cent of the mutations in the DMD consist on intragenic deletions that disrupt the reading frame of the gene, while in BMD the reading frame is preserved. An emerging gene therapy in DMD patients consist on inducing skipping of an exon that recover the reading frame in a restrictive group of cases. Multiexon skipping is another approach that can be achieved with the new Crispr-Cas9 technology. In particular, $63 \%$ of DMD mutations are located between exons 45 to 55 and it has been observed the existence of a spontaneous deletion spanning that region in asymptomatic subjects or variable Becker phenotype. Our aim is to analyze the diverse clinical profile and to refine the underlying molecular mechanisms in a cohort of subjects with a 45-55 deletion. Our hypothesis is that the different location of the intronic breakpoint positions of these patients might be responsible of their clinical phenotype. Methods: We analyzed the exact position of the intronic breakpoints in 8 index patients with this deletion. We performed an array, and multiple PCRs in order to restrict the area of the breaking points in the introns 44-45 and 55-56, following by amplification and sequencing of the deletion junction to detect the exact breakpoint We also checked the clinical features of the patients in an attempt to correlate them with their sequences. Results: A group of 6 patients shared the same breakpoints in both introns. These patients presented variable clinical manifestation (2 asymptomatics, 2 mild BMD, 1 BMD and 1 with cardiomyopathy). In one of the other 2 patients, the deletion affected the regulatory regions of the dystrophin isoform Dp140 and in fact the patient presented ADHD (attention deficit hyperactivity disorder), dyslexia and dysgraphia. The last patient presented a proximal insertion of a stretch from a deleted region in the 44-45 intronic breakpoint. This patient showed an early onset cardiomyopathy. Conclusion: We cannot certainly affirm that $t$ the position of the intronic breakpoints in patients with deletion 45-55 is related with the clinical features of these patients, nevertheless we found some associations. We detected a major hotspot, where 6 of 8 patients share the same breakpoints and present variable clinical manifestations, where we can find asymptomatic patients (suggesting that there are other factors that may have 
a greater impact on the clinical manifestation of the disease, such as age or genetic context). The impairment of the regulatory regions of Dp140 in our patient has a consequence in his cognitive status. Moreover the other type of deletion may be associated with an early apparition of cardiomyopathy, nevertheless more patients with the last 2 deletion categories must be analyzed in order to stablish these hypothesis.

\section{PS1Group1-047 / \#627}

Topic: Group 1 - Muscle Diseases of Genetic Origin and Acquired Myopathies: Clinical Features, Pathophysiology, Therapy

\section{THE STUDY OF ALISKIREN IN MDX DYSTROPHIC MICE}

Thainá A. Marin ${ }^{1}$, Bruno M. Bertassoli ${ }^{1}$, Giuliana Petri $^{1}$, Jose F.R. Santos ${ }^{1}$, Vinicius G. Silva ${ }^{2}$, Fernando L.A. Fonseca ${ }^{3}$, Alzira A.S. Carvalho ${ }^{4}$, David Feder ${ }^{1}$

${ }^{1}$ Pharmacology, Faculdade de Medicina do ABC, Santo Andre, BR; ${ }^{2}$ Faculdade de Medicina do ABC, Santo Andre, BR, ${ }^{3}$ Laboratory Analysis, Faculdade de Medicina do ABC, Santo Andre, BR; ${ }^{4}$ Neurology, Faculdade de Medicina do ABC, Santo Andre, BR

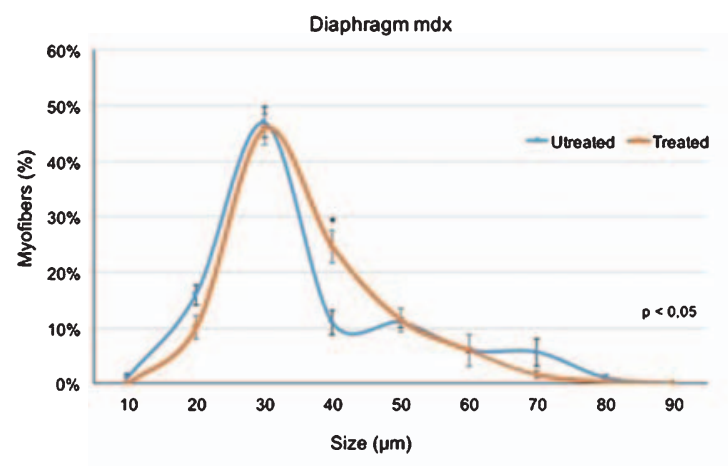

Background: Duchenne Muscular Dystrophy (DMD) is a genetic neuromuscular disease linked to the $\mathrm{X}$ chromosome, caused by mutation of dystrophin gene (DYS) that encodes the dystrophin protein, responsible for the stability of the sarcolemma. In the absence / deficiency of dystrophin, the muscle cells are susceptible to damage induced by continuous cycles of degeneration and limited regeneration. Inflammation, necrosis and fibrosis are the pathophysiological characteristics that lead to the main consequence of DMD, the loss of muscle function. Aliskiren is one of the newer drugs that interact with the Renin Angiotensin Aldosterone System (RAAS), directly inhibiting renin. High levels of circulating renin activate the pathologic signaling pathway of fibrosis in several diseases through stimulation of the pro-renin receptor, whose mechanism is completely independent of both Angiotensin II (ang II) production and stimulation of Ang II type 1 receptor. Methods: We treated 8-weeks-old male mdx mice. 8 mice received $25 \mathrm{mg} / \mathrm{kg}$ of Aliskiren daily for 5 weeks and 8 mice received $0,2 \mathrm{ml}$ of saline solution $(0,9 \%)$, both by gavage. Measure muscle strength (Kondziela test) was performed weekly, as well as the weighing of each animal. After 5 weeks, all animals were euthanized, followed by muscles biopsy of extensor digitorum longus (EDL), tibialis anterior (TA) and diaphragm muscles in order to

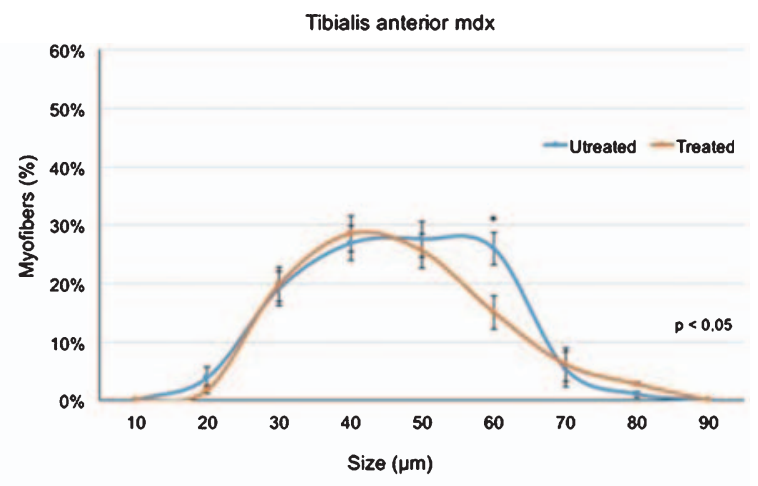

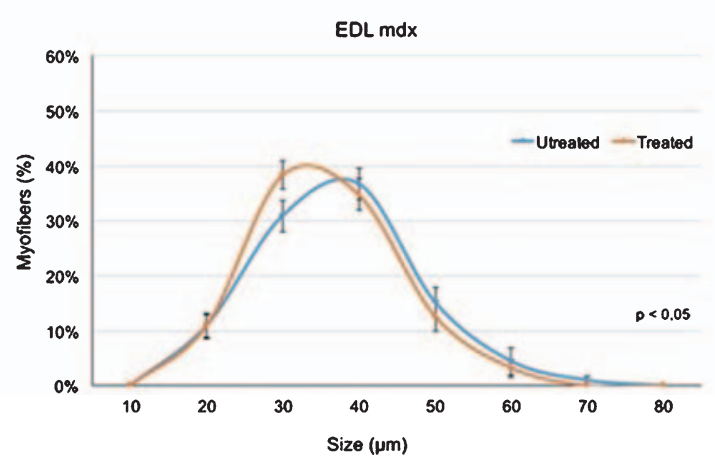


analyse morphological features. The creatine kinase (CK) was also performed. Results: No changes were observed in the animals weight during the study. The hold impulse calculated by the Kondziela Test did not show significant differences between treated and untreated animals. CK values between the groups did not differ significantly. Regarding the Feret's diameter, a significant increase of fibers'diameter higher than 40um was observed in diaphragm muscle of treated group $(\mathrm{p}<0.05)$. In addition, TA of treated group showed a significant increase in fibers" diameter higher than 60um (p $<0.05$ ) was also demonstrated. In contrast, EDL did not show difference in fiber diameter of both groups. Conclusion: Treatment with aliskiren in mdx dystrophic mice for 5 weeks did not change the weight of animals. The strength was also unaffected in treated group. The preliminary analyzes of the morphological muscles findings not show some beneficial effect of aliskiren in this model. Long-term drug studies already under way, and measure of fibrosis and inflammatory cytokines will be very helpful to determine the real effect of aliskiren in DMD.

\section{PS1Group1-048 / \#691}

Topic: Group 1 - Muscle Diseases of Genetic Origin and Acquired Myopathies: Clinical Features, Pathophysiology, Therapy

\section{DUCHENNE MUSCULAR DYSTROPHY: DO BOYS WITH A SHORTER STATURE MAINTAIN AMBULATION LONGER?}

Léa Labove ${ }^{1}$, Magdalena Jaworski ${ }^{1}$, Cam-Tu Emilie Nguyen $^{2}$

${ }^{1}$ CHU Sainte-Justine, Montreal, $Q C, C A ;{ }^{2}$ Pediatric Neurology, CHU Sainte-Justine, Montreal, QC, CA

Background: Duchenne muscular dystrophy (DMD) is an X-linked progressive neuromuscular disorder caused by mutations in the DMD gene and affecting approximately 1:5000 male births, leading to loss of ambulation and premature death. While many promising therapies are currently being developed, corticosteroids remain the cornerstone of pharmacological management in DMD. Stunted growth is a frequent side effect of steroids in DMD patients. It is not uncommon for families and patients to voice concerns about this "visible" side effect of corticotherapy. Some clinicians have ventured that a shorter stature may confer a mechanical advantage in $\mathrm{DMD}$, delaying the loss of ambulation in the shorter boys. Methods: Our aim was to study the relationship between height and the age at loss of ambulation in our population of DMD boys through a single-center retrospective chart review. Seventy boys with DMD were identified and their charts reviewed. The median age at diagnosis was 4.2 years, and median age of corticosteroid initiation was 7.5 years. Out of these 70 boys, 40 had lost ambulation at the time of this study, at a mean age of 11.6 years. We separated the boys who had lost ambulation in two groups: those who lost ambulation before 11.6 years and those who lost ambulation after 11.6 years, and compared these two groups in terms of height, using the Student's T-test. Results: At age 10, the boys who lost ambulation after 11.6 years were on average $11.9 \mathrm{~cm}$ shorter than the boys who lost ambulation before 11.6 years $(p<0.0001)$, a difference that is both statistically and clinically significant. When we looked at the height at age 7 instead of 10, the difference between the two groups was not statistically significant $(+1.5 \mathrm{~cm}$ for the boys who lost ambulation before 11.6 years; $p=0.3731$ ). Given that the difference in average height between the two groups appears to develop between the ages of 7 and 10 , we hypothesize that the height differential is due to the effect of corticosteroids, which were initiated at a median age of 7.5 years. Conclusion: In our cohort, DMD boys who lost ambulation at a later age were significantly shorter than those who lost ambulation earlier. It is not yet clear if this is due to a mechanical advantage related to the shorter stature, or if the shorter stature could represent a biomarker of optimal corticotherapy response in DMD. This important finding should be validated in multicentric DMD cohorts. 


\section{PS1Group1-049 / \#898}

Topic: Group 1 - Muscle Diseases of Genetic Origin and Acquired Myopathies: Clinical Features, Pathophysiology, Therapy

\section{LOW LEVELS OF DYSTROPHIN PROTEIN ASSOCIATED WITH ATTENUATION OF DUCHENNE MUSCULAR DYSTROPHY PROGRESSION}

Johannes Dworzak $^{1}$, Frederick Schnell ${ }^{2}$, Jay S. Charleston $^{1}$, Karin Lucas ${ }^{2}$, Doug Sanders ${ }^{1}$, Diane Frank $^{2}$

${ }^{1}$ Sarepta Therapeutics, Inc., Cambridge, MA, US; ${ }^{2}$ Sarepta Therapeutics Inc, Cambridge, MA, US

Background: Duchenne muscular dystrophy (DMD) is a degenerative, fatal, $\mathrm{X}$-linked recessive disorder affecting 1 in 3500 to 5000 male births worldwide. It is marked by an absence of dystrophin in muscle fibers stemming from $D M D$ gene mutations. Absence of dystrophin leads to progressive loss of muscle function. The precise amount of dystrophin associated with clinically meaningful benefit in DMD patients is unclear, although numerous findings indicate that small amounts of dystrophin, associated with natural mechanisms such as spontaneous exon skipping, may lead to slower disease progression. We explored potential associations between low dystrophin levels in DMD patients and attenuation of disease progression. Methods: A literature search was conducted to identify recent relevant articles evaluating the natural occurrence of exon skipping and clinical disease progression in DMD patients. Results: A body of evidence indicates that DMD patients with mutations amenable to exon 44 skipping experience a milder disease course than other DMD subgroups. In one natural history study, patients with mutations amenable to exon 44 skipping experienced a slower rate of disease progression, with loss of ambulation (LOA) 2 years later than those with other mutations, yet traces of dystrophin were detected in only 3 of 6 patients by immunohistochemistry and in 0 of 4 patients by Western blot analysis using clinical diagnostic methods. Notably, in a subanalysis of corticosteroid-treated patients, those with mutations amenable to exon 44 skipping did not achieve a median age of LOA during follow-up, while those with mutations amenable to exon 51 skipping had a median age at LOA of 13.0 years. In a separate analysis of corticosteroid-treated DMD patients, the median age at LOA was 15.2 years in patients amenable to exon 44 skipping and 10.5 years in patients amenable to exon 51 skipping. Spontaneous low-level exon 44 skipping in these patients is thought to facilitate restoration of the messenger ribonucleic acid reading frame, facilitating dystrophin expression and/or the presence of dystrophin-positive muscle fibers. An analysis utilizing the NorthStar Ambulatory Assessment demonstrated that patients with mutations amenable to exon 44 skipping declined less rapidly over 2 years than patients with mutations amenable to exon 53 or 51 skipping. A separate study showed that patients with mutations amenable to exon 44 skipping had higher baseline values on the 6-minute walk test and more modest declines at 12 months than those with mutations amenable to skipping exons 45 or 53 (differences were not statistically significant). Some evidence suggests that DMD patients with deletions of exons 3-7 also experience a milder phenotype and express low levels of dystrophin. A recent case study described a patient aged 10 years with a nonsense mutation in exon 42 and a mild phenotype that was presumably the result of spontaneous exon 42 skipping and subsequent dystrophin production. Conclusion: These findings demonstrate that spontaneous exon skipping and production of low dystrophin levels may impact the clinical course of the disease, and offer insights regarding the interpretation of results from clinical trials evaluating therapeutic interventions designed to restore dystrophin in DMD patients.

\section{PS1Group1-050 / \#934}

Topic: Group 1 - Muscle Diseases of Genetic Origin and Acquired Myopathies: Clinical Features, Pathophysiology, Therapy

\section{TAMOXIFEN PROLONGS} SURVIVAL, IMPROVES MOTOR FUNCTION AND REDUCES LEVELS OF DNM2 IN MTM1-NULL MICE, A MODEL OF XLCNM

Elinam Gayi ${ }^{1}$, Laurence A. Neff ${ }^{1}$, Hesham M. Ismail $^{1}$, Xènia Massana-Muñoz ${ }^{2}$, Belinda $\mathrm{S}$. Cowling ${ }^{2}$, Jocelyn F. Laporte ${ }^{2}$, Leonardo Scapozza ${ }^{1}$, Olivier M. Dorchies ${ }^{1}$

${ }^{1}$ School Of Pharmaceutical Sciences, University of Geneva, Geneva, $\mathrm{CH} ;{ }^{2}$ Department Of Translational Medicine And Neurogenetics, University of

Strasbourg, Illkirch, FR 
Background: X-linked centronuclear myopathy (XLCNM) is a rare and severe congenital myopathy characterised by generalised muscle weakness and abnormal nuclei positioning. Most affected boys die in their first year of life and survivors fail to achieve independent ambulation. It is caused by mutations in the Mtm1 gene encoding myotubularin, a ubiquitously expressed phosphoinositide phosphatase. No cure exists and very few pharmacological avenues are being explored. Here, Mtm1-null mice were treated with tamoxifen (TAM), a drug that modulates oestrogen actions and that we have previously shown to be efficacious in dystrophic $\left(\mathrm{mdx}^{5 C v}\right)$ mice, a model of Duchenne muscular dystrophy (DMD). We report that TAM is also effective in Mtm1-null mice, a model of XLCNM. Methods: Wild type and Mtm1-null mice were given normal chow or a TAM supplemented chow starting at weaning. Survival was monitored, and mice underwent weekly gridtests, weigh-ins and clinical scorings to evaluate disease progression. Mice were kept until Day 42, 84 or $>200$; at each of these time points in-vivo force recordings were performed in the triceps. Hind leg muscles were used for histological, ultrastructural and biochemical analyses. Results: Non-treated Mtm1-null mice died at around 45 days. By contrast, about half of the Mtm1-null mice treated with clinically relevant doses of TAM survived beyond 290 days of age. Clinical scoring showed that the motor function of the affected mice was markedly improved. In vivo force recordings performed at D42 and D84 revealed that the force of treated Mtm 1-null was significantly improved after as short as 3 weeks of treatment. Histological and electron microscopy analyses show partial rescue of muscle structure and triads, consistent with improved calcium homeostasis in FDB fibres and better excitation-contraction coupling. Quantitative PCR and western blots demonstrated reduction of DNM2, a disease modifier in XLCNM. Conclusion: We found that tamoxifen extends the lifespan of Mtm1-null mice more than 6-fold, rescues their motor skills and corrects levels of known disease modifiers. These results suggest that oestrogen signalling may be a key pathway that modifies disease severity in unrelated myopathies such as XLCNM and DMD.

\section{PS1Group1-051 / \#961}

Topic: Group 1 - Muscle Diseases of Genetic Origin and Acquired Myopathies: Clinical Features, Pathophysiology, Therapy

\section{ARE THERE DIFFERENT CLINICAL ENTITIES WITH DISTINCT DISEASE COURSE AMONG D4Z4 REDUCED ALLELE CARRIERS?}

Giulia Ricci ${ }^{1}$, Fabiano Mele $^{2}$, Lucia Ruggiero ${ }^{3}$, Elisabetta Bucci ${ }^{4}$, Lorenzo Maggi ${ }^{5}$, Monica Govi², Francesco Sera ${ }^{6}$, Liliana Vercelli ${ }^{7}$, Lucio Santoro ${ }^{3}$, Tiziana Mongini ${ }^{7}$, Luisa Villa ${ }^{8}$, Maurizio Moggio ${ }^{8}$, Massimiliano Filosto ${ }^{9}$, Marina Scarlato ${ }^{10}$, Stefano C. Previtali ${ }^{10}$, Michelangelo $\mathrm{Cao}^{11}$, Elena Pegoraro $^{12}$, Roberta Telese ${ }^{13}$, Antonio Di Muzio ${ }^{13}$, Carmelo Rodolico ${ }^{14}$, Giovanni Antonini ${ }^{4}$, Maria Grazia D'Angelo ${ }^{15}$, Angela Berardinelli ${ }^{16}$, Rachele Piras $^{17}$, Maria Antonietta Maiolii ${ }^{17}$, Giuliano Tomelleri $^{2}$, Corrado Angelini ${ }^{18}$, Gabriele Siciliano ${ }^{1}$, Rossella Tupler ${ }^{2}$

${ }^{1}$ Department Of Clinical And Experimental Medicine, University of Pisa, Pisa, IT; ${ }^{2}$ Department Of Life Sciences, University of Modena and Reggio Emilia, Modena, IT; ${ }^{3}$ Department Of Neurosciences, Reproductive And Odontostomatological Sciences, University Federico II of Naples, Naples, IT; ${ }^{4}$ Department Of Neuroscience, Mental Health And Sensory Organs, S. Andrea Hospital, University of Rome "Sapienza", Rome, IT; ${ }^{5}$ Besta Neurological Institute, Milan, IT; ${ }^{6}$ Mrc Centre Epidemiology For Child Health, UCL Institute of Child Health, London, GB; $;^{7}$ Department Of Neuroscience, Center For Neuromuscular Diseases, University of Turin, Turin, IT; ${ }^{8}$ Fondazione Irccs Ca' Granda Ospedale Maggiore Policlinico, Dino Ferrari Center, University of Milan, Milan, IT; ${ }^{9}$ Neurology Clinic, Spedali Civili Hospital, University of Brescia, Brescia, IT; ${ }^{10}$ Inspe And Division Of Neuroscience, IRCCS San Raffaele Scientific Institute, Milan, IT; ${ }^{11}$ Department Of Neurosciences, University of Padova, Padova, IT: ${ }^{12}$ University of Padova - Azienda Ospedaliera di Padova, Padova, IT, ${ }^{13}$ Center For Neuromuscular Disease, Cesi, University “G. D'Annunzio”, Chieti, IT: ${ }^{14}$ Department Of Neurosciences, Policlinico " $g$.

Martino",, University of Messina, Messina, IT; ${ }^{15}$ Department Of Neurorehabilitation, IRCCS Institute Eugenio Medea, Bosisio Parini, Bosisio Parini, IT, ${ }^{16}$ Unit Of Child Neurology And Psychiatry, IRCCS"C. Mondino" Foundation, Pavia, Pavia, IT; ${ }^{17}$ ASL8, Centro Sclerosi Multipla, Cagliari, IT; ${ }^{18}$ IRCCS S.Camillo Hospital, Venice, IT

Background: The phenotypic classification of patients with facioscapolohumeral muscular dystrophy 
(FSHD) is crucial for genetic studies and the definition of outcome measures toward trial readiness. The Italian Clinical Network for FSHD has recently proposed a new FSHD Comprehensive Clinical Evaluation Form (CCEF) that defines nine clinical categories aimed at capturing clinical diversity. In particular category A, subcategories A1-A3, identifies subjects with facial and scapular girdle weakness, subcategory B1 identifies patients showing scapular muscle weakness without facial muscle involvement. Methods: We applied the CCEF on 814 index cases carrying of contracted alleles with 1-10 D4Z4 repeats from the Italian National Registry for FSHD. Results: Our analysis suggests that the facial-sparing FSHD phenotype (subcategory B1), representing $10,2 \%$ of index cases, is associated with milder motor disability and slower progression of muscle weakness when compared with the classical FSHD phenotype (category A), regardless the size of the D4Z4 contracted allele. Conclusion: These results highlight the importance of building a precise phenotypic classification of patients and families for a correct stratification of patients to identify the best for trials.

\section{PS1Group1-052 / \#285}

Topic: Group 1 - Muscle Diseases of Genetic Origin and Acquired Myopathies: Clinical Features, Pathophysiology, Therapy

\section{A PH1/2 STUDY OF ENA ${ }^{\circledR}$ ANTISENSE OLIGONUCLEOTIDE (DS-5141B) WITH EXON 45 SKIPPING ACTIVITY IN PATIENTS WITH DMD}

\author{
$\underline{\text { Masafumi Matsuo }}^{1}$, Hiroyuki Awano ${ }^{2}$, Setsuo \\ Hasegawa $^{3}$, Satoru Inoue ${ }^{4}$, Naoyuki Maeda ${ }^{4}$, \\ Hirofumi Komaki ${ }^{5}$ \\ ${ }^{1}$ Department Of Physical Therapy, Faculty Of \\ Rehabilitation, Kobe Gakuin University, Kobe, \\ $J P ;^{2}$ Pediatrics, Kobe University Graduate School \\ of Medicine, Kobe, JP, ${ }^{3}$ Pharmaspur Inc., Tokyo, \\ JP; ${ }^{4}$ Daiichi Sankyo Co., Ltd., Tokyo, JP, ${ }^{5}$ Child \\ Neurology, National Center of Neurology and \\ Psychiatry, Tokyo, JP
}

Background: Duchenne muscular dystrophy (DMD) is a progressive, lethal neuromuscular disorder caused by the absence of dystrophin due to mutation of the DMD gene. Antisense oligonucleotide (AO)-mediated exon skipping allows restoration of the dystrophin reading frame, resulting in the synthesis of partially functional dystrophin for DMD patients who have out-of-frame deletions. This exon skipping treatment strategy for DMD is now being studied using various modified nucleic acids. The most important drawback of AOs that target $D M D$ exon skipping is not delivered into cardiac muscle. DS-5141b is an AO consisting of 2'- $O, 4$ '- $C$-ethylene-bridged nucleic acids $\left(\mathrm{ENA}^{\circledR}\right)$ and 2'-O-methyl RNA (2'-OMe) and induces $D M D$ exon 45 skipping. Our ENA ${ }^{\circledR}$ oligonucleotides have suitable characteristic for nucleic acid therapeutics. They can form more thermodynamically stable duplexes with complementary RNA than other oligonucleotides and are highly stable in plasma. In pre-clinical studies, the potent efficacy and safety of DS-5141b was observed. DS-5141b at doses over $3 \mathrm{mg} / \mathrm{kg}$ induced distinct exon 45 skipping in anterior tibial muscles, diaphragm and heart in $\mathrm{mdx}$ mice. On the other hand, 2'-OMe AO and a phosphorodiamidate morpholino oligomer (PMO) AO with the same nucleotide sequence as DS-5141b did not show the clear exon-skipping activity at doses up to $30 \mathrm{mg} / \mathrm{kg}$. It was remarkable that the exon 45 skipping was detected in heart at such a low dose suggesting cardiac improvement (Neuromuscular Disorders Vol. 27 Suppl. 2, 2017, Page S216). Methods: To assess the safety, tolerability, efficacy, and pharmacokinetics of DS-5141b in patients with DMD, a phase $1 / 2$ study has been conducted as a multicenter, uncontrolled, open-label study in Japan (NCT02667483). This study was a first-in-human study consisted of 2 parts. Part 1 was a sequential dose escalation part to examine 4 dose levels $(0.1,0.5,2.0$ and $6.0 \mathrm{mg} / \mathrm{kg}$ ). DS-5141b was administered subcutaneously at 2 dose levels each in 2 cohorts and administration of each dose level was once weekly for 2 weeks (Cohort 1: 0.1 and $2.0 \mathrm{mg} / \mathrm{kg}$, Cohort 2: 0.5 and $6.0 \mathrm{mg} /$ $\mathrm{kg}$ ). In Part 2, subcutaneous administration of DS$5141 \mathrm{~b}$ was conducted for 12 weeks at 2 dose levels $(2.0$ and $6.0 \mathrm{mg} / \mathrm{kg})$. Eligible subjects were ambulant DMD male patients aged 5-10 years with a mutation amenable to exon 45 skipping. Safety assessments include adverse event, laboratory tests, change of weight, vital sign, and ECG. The primary efficacy endpoint of this study is dystrophin expression and the secondary is detection of in-frame DMD mRNA harboring exon 45 skipping in muscle tissue. Results: A total of 7 subjects had received DS-5141b and all of them had completed administration for 12 weeks in Part 2. No serious adverse events and adverse events resulting in discontinued 
administration have been reported. Conclusion: Results of the Ph1/2 study and properties of DS-5141b will be presented.

\section{PS1Group1-053 / \#517}

Topic: Group 1 - Muscle Diseases of Genetic Origin and Acquired Myopathies: Clinical Features, Pathophysiology, Therapy

\section{MOST LGMD2L MUTATIONS IN THE ANO5 GENE RESULT IN DECREASED PROTEIN LEVELS IN ANOCTAMINOPATHY PATIENTS}

\author{
Anna Vihola ${ }^{1}$, Helena Luque ${ }^{2}$, Marco Savarese ${ }^{2}$, \\ Sini Penttilä ${ }^{3}$, Giorgio Tasca ${ }^{4}$, Bjarne $\mathrm{Udd}^{5}$ \\ ${ }^{1}$ Medical Genetics, Medicum, Folkhalsan Institute \\ of Genetics, Uni Helsinki, Helsinki, FI; ${ }^{2}$ Medical \\ Genetics, Medicum, Folkhalsan Institute of \\ Genetics, Uni Helsinki, Helsinki, \\ FI; ${ }^{3}$ Neuromuscular Research Center, University \\ and University Hospital of Tampere, Tampere, \\ FI; ${ }^{4}$ Fondazione Policlinico Universitario " $a$. \\ Gemelli", Istituto di Neurologia, Università \\ Cattolica del Sacro Cuore, Rome, IT, ${ }^{5}$ Department \\ Of Neurology, Vaasa Central Hospital, Vaasa, FI
}

Background: ANO5 (TMEM16E) is an eight-pass, $107 \mathrm{kDa}$ membrane protein, possibly playing a role in membrane dynamics. Mutations in the ANO5 gene are a frequent cause of recessive limb-girdle muscular dystrophy, LGMD2L, and Miyoshi muscular dystrophy -like disease MMD3, with distal phenotype. More than 70 different mutations, dispersed all over the ANO5 gene have been identified. The most frequent mutation in European populations is the truncating c.191dupA in the N-terminal domain of the ANO5 protein, whereas in Finland, the point mutation c. $2272 \mathrm{C}>\mathrm{T}$ is the most common. In addition, there are numerous patients with phenotypes compatible with anoctaminopathy, but with just one confirmed ANO5 mutation found in genetic analysis, leaving the diagnosis unsolved with conventional approach. Methods: Studying the effects of ANO5 mutations in patient biopsies at protein level for diagnostic purposes has become possible only recently, as we developed a novel western blotting method, using isolated membrane fractions, suitable for endogenous ANO5 detection in human muscle tissue. Anoctaminopathy patients in this study were confirmed by genetic analysis. Results: Using this technique, a commercial mouse monoclonal antibody detected a single $107 \mathrm{kDa}$ ANO5 protein in normal muscle biopsy membrane fractions. The ANO5 band was clearly decreased in most anoctaminopathy patients tested, except for a patient with a very $\mathrm{N}$-terminal point mutation, located in the soluble cytoplasmic domain of ANO5. Conclusion: We conclude that ANO5 has low tolerance for mutations, leading in most cases to protein destabilization and degradation. The method described here allows direct analysis of human ANO5 protein, which can be used in diagnostics, for evaluating the pathogenicity of the potentially harmful ANO5 variants of uncertain significance.

\section{PS1Group1-054 / \#628}

Topic: Group 1 - Muscle Diseases of Genetic Origin and Acquired Myopathies: Clinical Features, Pathophysiology, Therapy

\section{CRISPR/CAS GENE EDITING IN DUCHENNE MUSCULAR DYSTROPHY CULTURES TO TEST NEW TREATMENTS FOR THE DISEASE}

$\underline{\text { Patricia Soblechero-Martin }}^{1}$, Iker García-Jiménez ${ }^{2}$, Estibaliz Ruiz-Del-Yerro ${ }^{2}$, Edurne Albiasu-Arteta ${ }^{2}$, Virginia Arechavala-Gomeza ${ }^{1}$

${ }^{1}$ Neuromuscular Disorders, Biocruces Health

Research Institute, Barakaldo, ES; ${ }^{2}$ Neuromuscular

Disorders, Biocruces Health Research Institute,

Barakaldo, ES

Background: Gene editing techniques have the possibility of becoming a permanent cure to many genetic disorders, but the delivery hurdle of these techniques will delay their practical application, particularly in neuromuscular disorders where muscle represents an extremely large and widespread target. However, these techniques have a more immediate application in the generation of better disease models in a field where not many good human myogenic cultures are available. Methods: The aim of our studies is to use gene editing to generate a specific DMD deletion in healthy control myoblasts cultures and then correct this deletion also by gene editing, the first objective will provide us with a model to test many possible mutation-specific treatments, such as 
antisense oligonucleotides, while the second is a proof of concept of a possible therapeutic gene editing option. Results: For our first objective, we have designed specific guide RNAs to edit the $D M D$ gen removing exon 52 in order to reproduce a common deletion in Duchenne patients. We have confirmed the efficacy of our designs and selected the best candidates in HEK293 cells. Once selected, the best candidate gRNAs have been transfected in a control myoblast culture to create this new "DMD-like" culture. To complete our second objective, we have designed gRNAs targeting exon 51 and followed the same methods, this time on a DMD culture missing exon 52, to restore dystrophin expression. Conclusion: Issues such as safe and efficient delivery to target regions stop gene editing from being an immediate therapeutic option, but, as shown in this poster, gene editing is a useful tool to create better disease models to help in the development of other treatments and could be a feasible option to ex vivo treatment of cultures before autologous transplant.

\section{PS1Group1-055 / \#693}

Topic: Group 1 - Muscle Diseases of Genetic Origin and Acquired Myopathies: Clinical Features, Pathophysiology, Therapy

\section{EXTRA-SKELETAL MUSCLE MANIFESTATIONS OF FACIOSCAPULOHUMERAL MUSCULAR DYSTROPHY}

\section{Cecilia Kelly, Elie Naddaf \\ Mayo Clinic, Rochester, MN, US}

Background: Facioscapulohumeral muscular dystrophy (FSHD) is the third most common cause of muscular dystrophy. It is inherited in an autosomal dominant pattern. Over $95 \%$ of FSHD cases are type 1 , due to a contraction in the D4Z4 repeats on chromosome 4p. In its classic form, FSHD is characterized by weakness of facial and shoulder girdle muscles, and can be associated with hearing loss and rarely, retinal vascular abnormalities. However, the extra-skeletal muscle manifestations of FSHD remain poorly explored. In this study, we aimed to better characterize the spectrum of such manifestations in a large, single center cohort of FSHD patients. Methods: We performed a retrospective chart review of patients seen between 2000 and 2017 carry- ing the diagnosis of FSHD. We only included patients with genetically-confirmed FSHD type 1 or 2. We reviewed medical records for evidence of extra-skeletal muscle manifestations in the following domains: 1) hearing loss, captured by audiometry and/or hearing aid use; 2) cardiac disease, captured by electrocardiogram, Holter, and/or echocardiography; 3) dysphagia, captured by video swallow study; 4) retinopathy, captured by ophthalmologic exam; and 5) respiratory dysfunction, captured by pulmonary function tests (PFTs) and/or overnight oximetry. Results: We identified 88 patients, 39 of whom (44\%) were female. At time of evaluation, median age was 44 years (range 1.5-89), and 69 patients (78\%) were adults ( $>18$ years old). Hearing loss was present in 10 of $15(67 \%)$ tested patients, 8 of whom were diagnosed in adulthood. Ocular abnormalities were found in 5 of 25 (20\%) patients; however, none were noted to have retinal telangiectasias or exudates. Electrocardiography was abnormal in 29 of 56 patients (52\%) and echocardiography was abnormal in 18 of 35 tested patients (51\%). Oropharyngeal dysphagia was documented in 8 of 17 (47\%) patients who underwent video swallow study, most commonly classified as mild to moderate. PFTs were abnormal in 25 of 27 patients (93\%): 8 had a restrictive pattern and 23 had reduced maximal respiratory pressures. Overnight oximetry showed sleep-related disordered breathing in 14 of 24 patients (58\%). A single patient had focal epilepsy diagnosed in adulthood. We will analyze the correlation between the various extra-skeletal muscle manifestations and the number of D4Z4 repeats, and present it at the time of the conference. Conclusion: Hearing loss, cardiac abnormalities, dysphagia, and respiratory insufficiency were relatively common in our cohort. However, retinal vascular abnormalities were not encountered. Increased awareness regarding the various extra-skeletal muscle manifestations of FSHD allows timely screening, and subsequently providing better patient care. 


\section{PS1Group1-056 / \#717}

Topic: Group 1 - Muscle Diseases of Genetic Origin and Acquired Myopathies: Clinical Features, Pathophysiology, Therapy

\section{ANALYSIS OF CARDIAC TROPONIN T ALTERNATIVE SPLICING IN SKELETAL MUSCLE OF DM1 PATIENTS}

Francesca Bose' ${ }^{1}$, Laura V. Renna ${ }^{1}$, Nicola Ferrari ${ }^{2}$, Valentina Labate ${ }^{3}$, Michele Cavalli ${ }^{4}$, Giovanni Meola $^{5}$, Rosanna Cardani ${ }^{6}$

${ }^{1}$ Laboratory Of Muscle Histopathology And Molecular Biology, IRCCS Policlinico San Donato, San Donato Milanese (MI), IT; ${ }^{2}$ Department Of Biomedical Sciences For Health, University of Milan, Milan, IT; ${ }^{3}$ Department Of Cardiology Heart Failure Unit, IRCCS Policlinico San Donato, San Donato Milanese (MI), IT; ${ }^{4}$ Department Of Biomedical Sciences For Health, IRCCS Policlinico San Donato, Milan, IT; ${ }^{5}$ Department Of Biomedical Sciences For Health, University of Milan, San Donato Milanese (MI), IT; ${ }^{6}$ RCCSPoliclinico San Donato, San Donato Milanese (MI), IT

Background: Myotonic dystrophy type 1 (DM1) is an autosomal dominant neuromuscular disorder caused by expanded CTG repeats in the DMPK gene. Nuclear accumulation of the mutant RNA leads to aberrant alternative splicing of different genes that have been linked to the multiorgan involvement that characterized the pathology. Alteration of cardiac Troponin $\mathrm{T}$ (cTnT) alternative splicing observed in DM1 patients leads to the coexpression of both adult (excluding-exon 5) and fetal (including-exon 5) isoform in cardiac tissue and it is considered one cause of cardiac dysfunctions in DM1. Recently, cTnT aberrant splicing pattern has been observed also in skeletal muscle of DM1 patients. The aim of this work is to study the cTnT alternative splicing in biceps brachii (BB) muscle biopsies and in tibialis anterior (TA) samples from DM1 patients and to investigate if it is related to cardiac dysfunctions observed in these patients. Methods: cTnT fetal isoform expression was evaluated by RT-PCR using primers flanking exon 5 on both $\mathrm{BB}(\mathrm{n}=12)$ and TA samples $(\mathrm{n}=11)$ from DM1 patients and healthy subjects (CTR; $n=6 \mathrm{BB}, \mathrm{n}=4 \mathrm{TA})$. Patients were divided in two main groups on the basis of cardiac evaluation performed by ECG, echo- cardiogram and 24h ECG-Holter. Patients with cardiac involvement present at least one out of four altered parameters (increased PR, QRS, QTc, reduced \%FE). Results: Both in BB and TA samples, a significantly higher expression of cTnT fetal isoform was observed in DM1 patients presenting cardiac involvement than in CTR. Moreover, in TA samples a correlation between the expression of fetal cTnT with QTc and \%FE was present. In order to verify if the alteration of cTnT splicing is related to skeletal muscle alterations, we analysed aberrant alternative splicing of muscle specific genes (CLCN1, CACNA1S, NFIX, SERCA1, TNNT3, DMD1, LDB3, $R Y R 1$ and BIN1) and histopathological parameters of muscle damage (atrophic and hypertrophic factor, $\%$ of fibers with central nuclei, $\%$ of MHC embrional and neonatal positive fibers) on both $\mathrm{BB}$ and TA muscle biopsies. No correlation was found between $\mathrm{cTnT}$ fetal isoform expression and the parameter of skeletal muscle impairment. Conclusion: In conclusion, fetal isoform expression both in $\mathrm{BB}$ and TA skeletal muscle of DM1 patients seems to be related to cardiac involvement and could represent a possible biomarker of heart dysfunction in these patients.

\section{PS1Group1-057 / \#784}

Topic: Group 1 - Muscle Diseases of Genetic Origin and Acquired Myopathies: Clinical Features, Pathophysiology, Therapy

\section{IMPACT OF IDEBENONE ON RESPIRATORY BURDEN, INCLUDING RISK OF BRONCHOPULMONARY COMPLICATIONS, IN DUCHENNE MUSCULAR DYSTROPHY}

Oscar H. Mayer ${ }^{1}$, Mika Leinonen ${ }^{2}$, Shabir Hasham³, Thomas Meier ${ }^{2}$, Thomas Voit ${ }^{4}$, Gunnar Buyse For The Delos Study Group ${ }^{5}$ ${ }^{1}$ Children's Hospital of Philadelphia, Philadelphia, PA, US: ${ }^{2}$ Santhera Pharmaceuticals, Pratteln, $\mathrm{CH} ;{ }^{3}$ Santhera Pharmaceuticals, Pratteln, $\mathrm{CH} ;{ }^{4} \mathrm{UCL}$ Great Ormond Street Institute of Child Health, London, GB; ${ }^{5}$ University Hospitals Leuven, Leuven, $B E$

Background: In Duchenne muscular dystrophy (DMD), respiratory function declines due to progressive muscle weakness, and accelerates once pa- 
tients become non-ambulatory. As well-described clinical thresholds are crossed (based on forced vital capacity, expressed as a percentage of the predicted value; $\mathrm{FVC} \% \mathrm{p}$ ), the risk and severity of respiratory complications, including bronchopulmonary adverse events (BAEs), ${ }^{1}$ increases, presenting an ever worsening disease burden. In the 52 week, Phase 3 clinical trial in DMD patients not taking glucocorticoids (DELOS), we examined the potential for idebenone to reduce the rate of respiratory function decline and the rate of BAEs. Methods: Using a combination of pre-specified and post-hoc analyses, we assessed data from the DELOS trial, which enrolled 64 DMD patients aged 10-18 years and already in respiratory decline (peak expiratory flow $\% \mathrm{p}<80 \%$ ), to determine the potential of idebenone ( $900 \mathrm{mg} /$ day) to reduce disease burden by delaying the time to reaching clinically relevant respiratory function thresholds, and the frequency of BAEs. Clinically relevant thresholds for $\mathrm{FVC} \% \mathrm{p}$, were compiled following a review of standard of care guidelines. We compared the number of patients (idebenone vs placebo) crossing these thresholds (FVC $\%$ p of $50 \%, 40 \%, 30 \%$ ) or experiencing BAEs. Results: Treatment with idebenone reduced the risk of crossing the clinically relevant $\mathrm{FVC} \% \mathrm{p}$ threshold of $50 \%$ compared to placebo (hazard ratio $=0.34$, (95\% CI: $0.10-1.18)$. A similar trend was observed for patients crossing any clinically relevant $\mathrm{FVC} \% \mathrm{p}$ threshold (50, 40 or $30 \%$ ) (hazard ratio $=0.51,95 \%$ CI: $0.23-1.14)$. Patients in the idebenone group were also at a lower risk of experiencing a BAE than those in the placebo group (hazard ratio $=0.28,95 \%$ CI: 0.12 - 0.64). Conclusion: In the DELOS trial, idebenone treatment significantly reduced the proportion of patients crossing any clinically relevant threshold of FVC and also reduced the risk of bronchopulmonary adverse respiratory events, further supporting the efficacy and utility of treatment with idebenone in patients with DMD in established respiratory function decline and not taking glucocorticoid therapy. 1. Mayer OH, et al. US Neurology 2017;13:35-41.

\section{PS1Group1-058 / \#811}

Topic: Group 1 - Muscle Diseases of Genetic Origin and Acquired Myopathies: Clinical Features, Pathophysiology, Therapy

\section{CLINICAL AND MOLECULAR FEATURES OF DISTAL MYOPATHIES IN A PORTUGUESE COHORT: REPORTING NOVEL GENE MUTATIONS}

Catarina Falcão De Campos ${ }^{1}$, Miguel Oliveira

Santos $^{1}$, Isabel Conceição ${ }^{2}$, Mamede De Carvalho ${ }^{2}$

${ }^{1} 1$ - Departamento de Neurociências e Saúde

Mental, CHLN- Hospital Santa Maria. Lisboa, Portugal 2- Instituto de Fisiologia, Universidade de Lisboa, Faculdade de Medicina, IMM. Lisboa, Portugal, Lisbon, PT; 1 1- Departamento de Neurociências e Saúde Mental, CHLN- Hospital Santa Maria. Lisboa, Portugal 2- Instituto de Fisiologia, Universidade de Lisboa, Faculdade de Medicina, IMM. Lisboa, Portugal, Lisbon, PT

Background: Distal myopathies (DM) are a clinically and genetically heterogeneous group, which selectively or disproportionally affect distal muscles. DM are classified according to age of onset, inheritance pattern, clinical features and molecular diagnosis. Recent developments in identifying the underlying gene defects have increased the number of disorders, to more than 20 entities. Some forms of DM have higher prevalence in certain regions, such as Welander Myopathy in Sweden and Udd Myopathy in Finland. We describe the phenotypic and molecular features of the first cohort of Portuguese patients with DM, reporting novel gene associations. Methods: We retrospectively assessed patients with $\mathrm{DM}$ and molecular diagnosis followed in our center. Demographic and clinical data, familiar history, neurophysiological and pathological findings as well as gene mutations were collected. Results: We identified 123 patients with myopathy followed in our center which only 9 patients $(7,3 \%)$ were classified as DM. Two thirds of the patients with DM had molecular genetic diagnosis, including four novel mutations. The demographic and clinical features are described in table 1 . Three patients were diagnosed as tibial muscular dystrophy (Udd myopathy) associated with two likely pathogenic titin (TTN) gene mutations not previously described in the literature. Two patients were father and son and presented with 


\begin{tabular}{|c|c|c|c|c|c|c|c|c|c|c|}
\hline Patient & $\begin{array}{l}\text { Gender, } \\
\text { Age (y) }\end{array}$ & Diagnosis & $\begin{array}{c}\text { Age of } \\
\text { onset }\end{array}$ & $\begin{array}{l}\text { Onset of } \\
\text { weaknes }\end{array}$ & CK & EMG & $\begin{array}{l}\text { Muscle } \\
\text { Biopsy }\end{array}$ & Other features & $\begin{array}{l}\text { Family } \\
\text { History }\end{array}$ & $\begin{array}{c}\text { Molecular } \\
\text { diagnosis }\end{array}$ \\
\hline $1+$ & M, 66 & $\begin{array}{c}\text { Udd } \\
\text { Myopathy }\end{array}$ & 54 & $\begin{array}{l}\text { Legs Anterior } \\
\text { compartment }\end{array}$ & $\begin{array}{c}101 \mathrm{U} / \mathrm{L} \\
\text { (normal) }\end{array}$ & Myopathic & $\begin{array}{l}\text { Variation in } \\
\text { fiber size, } \\
\text { splitting } \\
\text { and nuclear } \\
\text { centralization }\end{array}$ & $\varnothing$ & Yes (son) & $\begin{array}{c}\text { TTN } \\
\text { c.63652T }>\mathrm{C}^{*} \\
\text { heterozygous }\end{array}$ \\
\hline $2+$ & M, 39 & $\begin{array}{c}\text { Udd } \\
\text { Myopathy }\end{array}$ & $30-35$ & $\begin{array}{l}\text { Legs Anterior } \\
\text { compartment }\end{array}$ & N/A & $\begin{array}{l}\text { Myopathic } \\
\text { Myotonic } \\
\text { discharge }\end{array}$ & N/A & $\varnothing$ & $\begin{array}{c}\text { Yes } \\
\text { (father) }\end{array}$ & $\begin{array}{c}\text { TTN } \\
\text { c.63652T }>\mathrm{C}^{*} \\
\text { heterozygous }\end{array}$ \\
\hline 3 & $\mathrm{M}, 61$ & $\begin{array}{c}\text { Udd } \\
\text { Myopathy }\end{array}$ & 50 & $\begin{array}{l}\text { Legs Anterior } \\
\text { compartment }\end{array}$ & $\begin{array}{c}427 \mathrm{U} / \mathrm{L} \\
\text { (increased) }\end{array}$ & Myopathic & $\begin{array}{l}\text { Unspecific } \\
\text { changes }\end{array}$ & $\varnothing$ & $\begin{array}{c}\text { Yes } \\
\text { (father and } \\
\text { brother) }\end{array}$ & $\begin{array}{c}\text { TTN } \\
\text { c.74366C }>\mathrm{G}^{*} \\
\text { heterozygous }\end{array}$ \\
\hline 4 & M, 32 & $\begin{array}{l}\text { Miyoshi } \\
\text { Myopathy }\end{array}$ & 25 & $\begin{array}{c}\text { Legs } \\
\text { Posterior } \\
\text { compartment }\end{array}$ & $\begin{array}{c}9090 \mathrm{U} / \mathrm{L} \\
\text { (increased) }\end{array}$ & N/A & $\begin{array}{l}\text { Dystrofic } \\
\text { changes } \\
\text { Absence of } \\
\text { dysferlin }\end{array}$ & $\varnothing$ & No & $\begin{array}{c}\text { DYSF } \\
\text { c.3367_3368del* } \\
\text { homozygous }\end{array}$ \\
\hline 5 & $\mathrm{~F}, 61$ & IBMPFD & $50-55$ & Hands & N/A & Myopathic & $\begin{array}{l}\text { Unspecific } \\
\text { changes }\end{array}$ & $\begin{array}{l}\text { Fronto-temporal } \\
\text { dementia }\end{array}$ & No & $\begin{array}{c}\mathrm{VCP} \text { c. } 265 \mathrm{C}>\mathrm{T}^{*} \\
\text { heterozygous }\end{array}$ \\
\hline 6 & $F, 63$ & $\begin{array}{l}\text { Laing Distal } \\
\text { Myopathy }\end{array}$ & 45 & $\begin{array}{l}\text { Legs Anterior } \\
\text { compartment }\end{array}$ & $\begin{array}{c}174 \mathrm{U} / \mathrm{L} \\
\text { (normal) }\end{array}$ & $\begin{array}{l}\text { Myopathic } \\
\text { Myotonic } \\
\text { discharges }\end{array}$ & N/A & $\begin{array}{c}\text { Hypertrophic } \\
\text { cardiomyopathy } \\
\text { (HCM) }\end{array}$ & $\begin{array}{l}\text { Yed (son } \\
\text { with } \\
\text { HCM) }\end{array}$ & $\begin{array}{c}\text { MYH7 c. } 2539- \\
2542 \mathrm{del} \\
\text { c. } 3056 \mathrm{C}>\mathrm{A} \\
\text { c. } 322 \mathrm{C}>\mathrm{T} \\
\text { heterozygous }\end{array}$ \\
\hline
\end{tabular}

+ Same family; * Novel gene mutation, likely pathogenic. CK - Creatine kinase; EMG - Electromyography; N/A - Non available IBMPFD Inclusion body myopathy with Paget disease and fronto-temporal dementia TTN - Titin gene; DYSF - Dysferlin gene; VCP - Valosin-containingprotein gene; MYH7 - Myosin heavy chain 7 gene

the same TTN gene mutation. One young patient had diagnosis of Miyoshy myopathy, with a likely pathogenic novel mutation in the dysferlin gene. An older patient presented with progressive upper and lower limbs distal weakness. Five years later the patient developed cognitive deterioration suggestive of a frontotemporal dementia, without associations with Paget disease. A novel gene mutation in the valosincontain-protein (VCP) was diagnosed. Lastly, we report a patient with lower limb distal weakness and hypertrophic cardiomyopathy associated with MYH7 gene mutation. Conclusion: We summarize the first cohort of Portuguese patients with DM associated with genetic mutations, including 4 novel mutations. To our knowledge, we describe for the first time a Portuguese patient with IBMPFD diagnosis associated with a novel VCP gene mutation. Molecular genetic investigation was essential since some of our patients are sporadic cases.

\section{PS1Group1-059 / \#965}

Topic: Group 1 - Muscle Diseases of Genetic Origin and Acquired Myopathies: Clinical Features, Pathophysiology, Therapy

\section{DUCHENNE MUSCULAR DYSTROPHY AND THE HEART - HOW TO VISUALIZE BETTER? CASE SERIES REPORT}

Ruza Kalicanin Milanovic ${ }^{1}$, Ana Kosac ${ }^{2}$, Stefan Djordjevic $^{3}$, Maja Bjelic ${ }^{3}$, Milan Djukic ${ }^{3}$, Jelena Mladenovic ${ }^{2}$, Jelena Glumac ${ }^{2}$, Tamara Ristic ${ }^{2}$, Majda Kiklic ${ }^{2}$, Ida Jovanovic ${ }^{3}$, Vedrana Milic Rasic $^{2}$

${ }^{1}$ Health Centar Novi Pazar, Novi Pazar, RS; ${ }^{2}$ Neurology, Clinic of neurology and psychiatry for children and youth, Belgrade, $R S,{ }^{3}$ University children's hospital, Belgrade, RS

Background: During the progressive course of disease of Duchenne muscular dystrophy monitoring of heart function and diagnosing the very first signs of potential heart failure is of vital importance. Aim: To correlate functional measures of patient achievements to different visualized procedures of the heart made at the same time period. Methods: We per- 
formed complete cardiological assessment, echocardiography, cardiac magnetic resonance imaging, biochemical parameters of patients diagnosed with Duchenne muscular dystrophy, confirmed by molecular genetic testing, in uniform fashion by three pediatric cardiologists educated in the same manner. All neurological assessment was performed by pediatric neurologists. Results: We included 16 boys with Duchenne muscular dystrophy, age range from 6 to 12 years (mean 9,2+-1,7), from whom $87,5 \%$ were on stable doses of corticosteroid therapy and $81,2 \%$ were mobile, with six minute walk test in rage 182 to $579 \mathrm{~m}$. Normal sinus rhythm (85-124/ $\mathrm{min}$ ) and cardiac auscultatory findings were found in all patients, with QTc interval from 0,36 to $0,43 \mathrm{sec}$ onds, slightly increased R/S in V1 in $9(56,2 \%)$ patients and NT-proBNP from 7.69 to $231.6 \mathrm{pg} / \mathrm{ml}$. In echocardiography parameters shortening fraction and ejection fraction were lower than $28 \%$ and $55 \%$ only in one patient $(6,25 \%) 8$ years old, independently mobile and on corticosteroid therapy. Cardiac magnetic resonance imaging revealed thin right ventricular myocardial wall and presence of left ventricular fibrosis in $25 \%$ of our patients, all mobile, on corticosteroid therapy and without echocardiography parameters of heart failure, with one patient having unexpectedly large fields of fibrosis despite excellent motor performances and normal echocardiography findings. Conclusion: Although echocardiography is a more available and easier accessible method, cardiac magnetic resonance imaging is becoming a superior diagnostic tool in the field of visualizing the first signs of potential cardiac dysfunction and failure in patients with Duchenne muscular dystrophy.

\section{PS1Group1-060 / \#753}

Topic: Group 1 - Muscle Diseases of Genetic Origin and Acquired Myopathies: Clinical Features, Pathophysiology, Therapy

\section{RATIONALE FOR EDASALONEXENT DOSE SCHEDULE IN PHASE 2 OF THE MOVEDMD® TRIAL}

Hanlan Liu ${ }^{1}$, Richard S. Finkel ${ }^{2}$, H.Lee Sweeney ${ }^{3}$, Krista Vandenborne ${ }^{4}$, Maria Mancini ${ }^{5}$, John F. Reilly ${ }^{6}$, Pradeep Bista ${ }^{6}$, Derek Wachtel ${ }^{7}$, Sachin Chandran $^{8}$, Rafif Dagher ${ }^{9}$, Dominic Picarella ${ }^{9}$,
Angelika Fretzen ${ }^{8}$, Andy Nichols ${ }^{10}, \underline{\text { Joanne }}$ Donovan $^{5}$

${ }^{1}$ Dmpk And Clinical Pharmacology, Catabasis

Pharmaceuticals, Inc., Cambridge, MA, US; ${ }^{2}$ Nemours Children's Hospital, Orlando, FL, US: ${ }^{3}$ Myology Institute, University of Florida Health Myology Institute, Gainesville, FL, US: ${ }^{4}$ Physical Therapy, 4University of Florida Health Physical Therapy, Gainesville, FL, US, ${ }^{5}$ Clinical Operations, Catabasis Pharmaceuticals, Inc., Cambridge, MA, US; ${ }^{6}$ Biology, Catabasis Pharmaceuticals, Inc., Cambridge, MA, US, ${ }^{7}$ Biology Analytical, Catabasis Pharmaceuticals, Inc., Cambridge, MA, US: ${ }^{8}$ Product Development, Catabasis Pharmaceuticals, Inc., Cambridge, MA, US; ${ }^{9}$ Pharmacology \& Toxicology, Catabasis Pharmaceuticals, Inc., Cambridge, MA, US; ${ }^{10}$ Research And Development, Catabasis Pharmaceuticals, Inc., Cambridge, MA, US

Background: Duchenne muscular dystrophy (DMD) is a progressively debilitating and ultimately fatal inherited neuromuscular disorder caused by mutations in the gene encoding dystrophin. NF-kB is activated in DMD and drives inflammation and fibrosis, muscle degeneration and suppresses muscle regeneration. Edasalonexent is an oral small molecule that inhibits NF- $\mathrm{kB}$. In the Phase 2 and the open-label extension (OLE) portions of the MoveDMD trial enrolling 4- to 7-year-old boys with DMD, edasalonexent given $33 \mathrm{mg} / \mathrm{kg}$ three times daily (TID) $(100 \mathrm{mg} / \mathrm{kg} /$ day) demonstrated a preservation of muscle function and slowing of DMD disease progression through 60 weeks as compared to the rates of change during the control period prior to edasalonexent treatment. Methods: The MoveDMD Trial was designed to evaluate efficacy, safety/tolerability, pharmacokinetics (PK), pharmacodynamics (PD), and dose response of edasalonexent. The Phase 1 portion of the MoveDMD trial was a 1-week study to evaluate the safety, PK, and PD at 33 and 67 $\mathrm{mg} / \mathrm{kg} / \mathrm{day}$, given in 2 divided doses, and $100 \mathrm{mg} / \mathrm{kg} /$ day given in 3 divided doses. Phase 2 was a 12-week placebo-controlled study with two cohorts given 33 $\mathrm{mg} / \mathrm{kg}$ twice daily (BID) (67 mg/kg/day) and $33 \mathrm{mg} /$ $\mathrm{kg}$ TID $(100 \mathrm{mg} / \mathrm{kg} /$ day $)$. Patients in the OLE continued with either dose regimen of edasalonexent. The dose schedule for the Phase 2 of the MoveDMD trial was selected based on nonclinical and clinical data including (1) the exposure/efficacy relationship observed in animal models; (2) the Phase 1 safety, tolerability, and PD in pediatric DMD patients; and 
(3) human PK. In the $m d x$ mouse model of DMD, dose efficacy was driven by sustained systemic exposure. An oral dose of edasalonexent given TID showed greater pharmacological effects than the same dose given all at once daily despite similar AUC. Results: In the Phase 1 of MoveDMD trial, all three doses were well tolerated with no safety signals observed. Decreases in NF-kB driven gene expression were observed at total daily doses of 67 and $100 \mathrm{mg} / \mathrm{kg}$, both of which were selected for the Phase 2 of MoveDMD trial. In Phase 1 PK studies in healthy volunteers, edasalonexent exhibited increasing exposure with food. Exposure plateaued at 33 $\mathrm{mg} / \mathrm{kg}$ when administered with a low-fat meal. Patients typically eat meals three times per day, which as a practical matter limits dosing frequency to TID. To validate $33 \mathrm{mg} / \mathrm{kg}$ TID that provides sustained systemic exposure, a population PK model was developed and used to simulate the plasma concentration-time profile of $33 \mathrm{mg} / \mathrm{kg}$ TID and BID to reflect the regimen of Phase 2 of the MoveDMD trial. Coverage over the concentration range at which pharmacological activity was observed in the nonclinical model was greater with TID than with BID. Conclusion: In conclusion, data from preclinical studies highlight the importance of time above exposure threshold achieved by more frequent dosing. Consistent with nonclinical studies and human PK characteristics of edasalonexent, $33 \mathrm{mg} / \mathrm{kg}$ TID dose schedule in the Phase 2 and OLE portions of the MoveDMD trial showed positive dose response vs. $33 \mathrm{mg} / \mathrm{kg}$ BID and exhibited numerical improvements in physical function assessments vs. the offtreatment control period.

\section{PS1Group1-061 / \#816}

Topic: Group 1 - Muscle Diseases of Genetic Origin and Acquired Myopathies: Clinical Features, Pathophysiology, Therapy

\section{REPURPOSING TAMOXIFEN FOR SEVERE MYOPATHIES: FROM PRECLINICAL EVALUATION IN ANIMAL MODELS TO CLINICAL TRIALS IN PATIENTS}

$\underline{\text { Olivier M. Dorchies }}{ }^{1}$, Elinam Gayi², Hesham M. Ismail $^{2}$, Laurence A. Neff ${ }^{2}$, Xènia MassanaMuñoz ${ }^{3}$, Belinda S. Cowling 3 , Jocelyn F. Laporte ${ }^{3}$, Talya Dor ${ }^{4}$, Dirk Fischer ${ }^{5}$, Urs T. Ruegg ${ }^{2}$, Leonardo Scapozza ${ }^{2}$
${ }^{1}$ School Of Pharmaceutical Sciences, University of Geneva, Geneva, $\mathrm{CH} ;{ }^{2}$ School Of Pharmaceutical Sciences, University of Geneva, Geneva, $\mathrm{CH},{ }^{3}$ Department Of Translational Medicine And Neurogenetics, University of Strasbourg, Illkirch, FR $;{ }^{4}$ Hadassah Medical Centre, Jerusalem, IL; ${ }^{5}$ Department of Neurology, University of Basel Hospital, Basel, $\mathrm{CH}$

Background: Duchenne muscular dystrophy (DMD), X-linked centronuclear (myotubular) myopathy (XLCNM/XLMTM) and congenital muscular dystrophy type $1 \mathrm{~A}(\mathrm{MDC} 1 \mathrm{~A})$ are rare and fatal muscular diseases for which no cure exists. DMD is caused by the lack of dystrophin, a large protein that connects the extracellular matrix to the cytoskeleton in muscle fibres. DMD patients develop progressive muscle wasting, leading to cardiac and respiratory failure and death usually in their third decade. XLCNM is due to loss-of-function mutations in MTM1, a gene encoding the lipid phosphatase myotubularin. XLCNM patients show generalized hypotonia from birth and most of them die before the age of 2. The pathogenic mechanisms of XLCNM involve imbalance of phosphoinositide levels and of proteins involved in formation and trafficking of membrane vesicles. MDC1A is due to reduced levels or lack of laminin alpha-2, an extracellular matrix protein that plays critical roles in the stability of skeletal muscles and signalling to the myofibres. MDC1A patients show generalized hypotonia from birth and severe respiratory insufficiency. Methods: Tamoxifen, a selective oestrogen receptor modulator, is used for nearly 40 years to treat hormone-dependent breast cancers. We have tested the efficacy of tamoxifen in $m d x^{5 C v}$ mice, Mtm1-null mice and $d y^{W}$ mice, models of DMD, XLCNM and MDC1A, respectively. Results: We previously published that long-term tamoxifen improved the symptoms of DMD in $m d x^{5 C v}$ mice, of which force generation and fibrosis in the diaphragm and in the heart. Follow-up studies showed that tamoxifen doses as low as 0.3 $\mathrm{mg} / \mathrm{kg} /$ day were efficacious. In 2017, tamoxifen was granted an Orphan Drug Designation for DMD by the European Medicines Agency. Based on our robust pre-clinical data, Talya Dor offered tamoxifen as a compassionate treatment to $3 \mathrm{DMD}$ boys for over 24 months. Because of encouraging outcomes, she is now conducting a larger open-label trial with 30 DMD boys. In parallel, Dirk Fischer is leading "TAMDMD", a multicentre, randomized, placebocontrolled, phase 3 clinical trial for assessing tamoxifen efficacy and safety in 100 DMD boys. Using the 
murine model of XLCNM, we found that clinically relevant doses of tamoxifen $(3 \mathrm{mg} / \mathrm{kg} /$ day) corrected hallmarks of the disease, resulting in dramatic extension of the lifespan of XLCNM mice from around 40 days to $>200$ days. In $d y^{W}$ mice, the murine model of MDC1A, preliminary findings suggest that tamoxifen modestly increases the lifespan of affected mice, although it significantly enhances motor function as evaluated with a grid hanging test. Conclusion: Our work reveals the efficacy of tamoxifen in a variety of severe muscular diseases that have independent pathogenic mechanisms, as well as previously unrecognized roles of estrogen signalling as a disease modifier pathway in these fatal diseases. Our preclinical evaluation of tamoxifen in $m d x^{5 C v}$ mice paved the way to compassionate use and clinical trials for DMD boys. Hopefully, the launch of TAMDMD may increase the interest of clinicians for tamoxifen and accelerate clinical evaluation of that drug in patients with XLCNM. Further work is ongoing in our lab for evaluating the efficacy of tamoxifen for MDC1A.

\section{PS1Group1-062 / \#862}

Topic: Group 1 - Muscle Diseases of Genetic Origin and Acquired Myopathies: Clinical Features, Pathophysiology, Therapy

\section{THE CLINICAL VARIATION OF RYR1 GENE IN A LARGE FAMILY}

Stacey Jankelowitz

Central Clinical School, University of Sydney,

Sydney, $A U$

Background: RYR1 gene mutations are many and their clinical presentations are varied. There are at least 217 mutations of the gene that can result in malignant hyperthermia. The RYR1 gene codes for the ryanodine receptor which permits the passage of calcium ions in cells. Methods: This study describes the various manifestations of a single RYR1 gene mutation in a large family, including the proband, her children, her mother and her siblings. The presenting features and severity while linked and described are varied within the one family. Results: A single gene mutation may result in mild scoliosis, malignant hyperthermia and miscarriage as well as ptosis, kyphoscoliosis and weakness. The study of epigenetics will hopefully aid in the understanding of these disorders and their variable presentation.
Conclusion: While gene tests are useful in determining the mutation responsible for clinical disorders, there is more to be learnt from gene expression to explain why a single gene disorder can have such varied presentation and differ in the severity of the illness within one family.

\section{PS1Group1-063 / \#940}

Topic: Group 1 - Muscle Diseases of Genetic Origin and Acquired Myopathies: Clinical Features, Pathophysiology, Therapy

\section{CLINICAL OUTCOME STUDY OF DYSFERLINOPATHY: WHAT ARE THE BEST FUNCTIONAL AND STRENGTH OUTCOME MEASURES FOR THIS POPULATION?}

Volker Straub $^{1}$, Meredith James ${ }^{1}$, Anna Mayhew ${ }^{1}$, Marni Jacobs², Jia Feng', Simone Spuler ${ }^{3}$, Kate Bushby $^{1}$, John Day ${ }^{4}$, Kirsti J. Jones ${ }^{5}$, Diana X. Bharucha-Goebel $^{6}$, Emmanuelle Salort-Campana ${ }^{7}$, Alan Pestronk ${ }^{8}$, Maggie C. Walter ${ }^{9}$, Carmen Paradas $^{10}$, Tanya Stojkovic ${ }^{11}$, Madoka MoriYoshimura' $^{12}$, Elena Bravver ${ }^{13}$, Jordi Diaz-Manera ${ }^{14}$, Elena Pegoraro ${ }^{15}$, Jerry Mendell ${ }^{16}$

${ }^{1}$ John Walton Muscular Dystrophy Research Centre, Newcastle University, Institute of Genetic Medicine, Newcastle Upon Tyne, GB; ${ }^{2}$ Division Of Biostatistic And Study Methodology, Children's National Health System, Centre for Translational Science, DC, WA, US, ${ }^{3}$ Charite Muscle Research Unit, Experimental and Clinical Research Center, Berlin, DE: ${ }^{4}$ Department Of Neurology, Stanford University, Palo Alto, CA, US; ${ }^{5}$ Institute For Neuroscience And Muscle Research, Children's Hospital At Westmead, University of Sydney, Sydney, AU; ${ }^{6}$ Department Of Neurology, Children's National Health System, Washington DC, US: ${ }^{7}$ Neuromuscular And Als Center, La Timone Hospital, Aix-Marseille Universite, Marseille, FR $;^{8}$ Department Of Neurology, Washington University School of Medicine, St. Louis, MO, US: ${ }^{9}$ Dept. Of Neurology, Ludwig-maximiliansuniversity, Friedrich-Baur-Institute, Munich, $D E ;{ }^{10}$ Neuromuscular Unit, Department Of Neurology, Hospital U. Virgen del Rocio/Instituto de Biomedicina de Sevilla, Sevilla, ES, ${ }^{11}$ Ap-hp, G.h. Pitié-salpêtrière 47-83, Boulevard De L'hôpital, Institut de Myologie, Paris, FR; ${ }^{12}$ Department Of 
Neurology, National Center Hospital, National Center of Neurology and Psychiatry, Tokyo, $J P,{ }^{13}$ Carolinas Healthcare System Neurosciences Institute, Charlotte, NC, US: ${ }^{14}$ Neuromuscular Disorders Unit, Hospital de la Santa Creu i Sant Pau, Barcelona, ES, ${ }^{15}$ Department Of Neuroscience, University of Padova, Padova, IT; ${ }^{16}$ Center For Gene Therapy, Nationwide Children's Hospital, Columbus, OH, US

Background: Dysferlinopathy, an autosomal recessive form of limb girdle muscular dystrophy, clinically presents with a variable spectrum of muscle weakness. The Clinical Outcome Study is an international 3 year natural history study of dysferlinopathy patients, which aims to gain a better understanding of the disease and to improve clinical trial readiness. Diagnostic criteria for inclusion in this study were a) two predicted pathogenic mutations or $b$ ) one predicted pathogenic mutation and absent mutation or c) one predicted pathogenic mutation and dysferlin protein level less than $20 \%$ as determined by blood monocyte testing. The cohort is $52 \%$ female $48 \%$ male. At baseline the ration of ambulant to non-ambulant was 3:1. The mean age was 40 years (range 12 to 88 years). The median years disease duration was 17 years (range 3-52 years). Here we aim to describe changes in functional outcome measures and muscle strength over a 2 year period in individuals with dysferlinopathy. Methods: 197 subjects across 15 sites in 8 countries underwent physiotherapy assessments including muscle strength (manual muscle testing, MMT; hand held dynamometry, HHD); and functional performance using the North Star Assessment for Dysferlinopathy (NSAD), Brooke score and Performance of Upper Limb, as well as timed tests (rise from floor, 10 metre walk / run, four stair climb and descend; Timed Up and Go, TUG; Six Minute Walk Distance), and respiratory function testing (forced vital capacity, FVC). Change was defined as significant difference in paired medians by Wilcoxon Signed Rank-Sum test $(p<0.05)$. Results: Change is captured in this population consistently from baseline to year 1 and from year 1 to year 2 across a range of functional outcome measures. Motor performance assessed by NSAD demonstrated slow but consistent and significant deterioration in scores and confirmed NSAD as a dysferlin specific scale. All timed tests except rise from floor showed significant consistent decline between year 1 and year 2 . Of the muscle groups assessed by HHD that showed significant change, the plantar flexors were the muscles with the highest mean differences $(3.14 \mathrm{lbs}, \mathrm{SD}$ : 8.22). We detected a mild but significant decrease in FVC (mean difference 0.09L) Conclusion: Progression in dysferlinopathy can be demonstrated using functional outcomes and dynamometry as measured by physiotherapists over two years. Change between baseline, year 1 and year 2 were slow but relatively consistent and significant. Results will help to power future clinical trials.

\section{PS1Group1-064 / \#294}

Topic: Group 1 - Muscle Diseases of Genetic Origin and Acquired Myopathies: Clinical Features, Pathophysiology, Therapy

\section{BREATHING IS LIFE! INSPIRATORY MUSCLE TRAINING IN CHILDREN AND ADOLESCENTS LIVING WITH NEUROMUSCULAR DISEASES}

\author{
$\underline{\text { Anri Human }}^{1}$, Jennifer Jelsma ${ }^{2}$, \\ Brenda M. Morrow ${ }^{3}$ \\ ${ }^{1}$ Physiotherapy, Sefako Makgatho Health Sciences \\ University, Garankuwa, ZA; ${ }^{2}$ Division \\ Physiotherapy, University of Cape Town, \\ Observatory, ZA, ${ }^{3}$ Paediatrics And Child Health, \\ University of Cape Town, Rondebosch, ZA
}

Background: People with neuromuscular diseases (NMD) have a high risk of morbidity and mortality caused by underlying respiratory muscle weakness and an ineffective cough. Inspiratory muscle training (IMT) aims to preserve or improve respiratory muscle strength; reduce morbidity; optimise ventilation and ultimately improve health-related quality of life (HRQoL). Inspiratory muscle training among children and adolescents with NMD is controversial, owing to differences in pathophysiology and potential risk of muscle damage in some conditions. Despite reports of potential benefits, there is insufficient evidence to guide clinical practice regarding the use of IMT in this sub-population. Methods: A pre-experimental, observational pre-test post-test study was used to determine changes in measures of spirometry, peak expiratory cough flow (PECF), inspiratory muscle strength, upper limb function and coordination (using the Motor Function Measurement (MFM) scale), adverse events and HRQoL (using the PedsQL tool). Eight participants ( $\mathrm{n}=4$ boys; 
mean age $12.71 \pm 3.53$ years) with a variety of NMD were included. Training consisted of 30 breaths, twice daily, five days a week, for six weeks with an electronic threshold device (Powerbreathe K3, HaB International Ltd, Southam, UK). Results: There were no significant changes in spirometry measures, PECF or HRQoL. Upper limb function and coordination (MFM score) improved from pre-to post-intervention $(p<0.04)$. Measures of inspiratory muscle strength also improved significantly: maximal inspiratory mouth pressure (Pimax) $(\mathrm{p}<0.02)$, strengthindex $(p<0.02)$ and peak inspiratory flow $(p<0.03)$. No adverse events occurred during the intervention period. Overall patient satisfaction with the IMT programme, on a 10-point visual analogue scale, was extremely high, with a median (IQR) of 9 (910). Conclusion: This study suggests that short term IMT may improve inspiratory muscle strength; as well as improving upper limb function and coordination. IMT may be a safe and effective adjunct intervention for children and adolescents with NMD, but larger, long-term randomised controlled trials are recommended.

\section{PS1Group1-065 / \#490}

Topic: Group 1 - Muscle Diseases of Genetic Origin and Acquired Myopathies: Clinical Features, Pathophysiology, Therapy

\section{PERSONALIZED MOLECULAR THERAPY IN A NEW TRANSLATIONAL LARGE ANIMAL MODEL FOR DUCHENNE MUSCULAR DYSTROPHY}

Tina Donandt ${ }^{1}$, Simone Reichert ${ }^{1}$, Maria Schmuck ${ }^{1}$, Claudia Kalbe ${ }^{2}$, Barbara Kessler ${ }^{3}$, Andreas Blutke ${ }^{4}$, Eckhard Wolf ${ }^{5}$, Sabine Krause ${ }^{1}$, Maggie C. Walter ${ }^{1}$ ${ }^{1}$ Dept. Of Neurology, Ludwig-maximiliansuniversity, Friedrich-Baur-Institute, Munich, DE; Institute Of Muscle Biology And Growth, Leibniz Institute for Farm Animal Biology (FBN), Dummerstorf, DE: ${ }^{3}$ Department Of Biochemistry, Molecular Animal Breeding And Biotechnology, Ludwig-Maximilians-University, Munich, DE: ${ }^{4}$ Institut Für Tierpathologie, LudwigMaximilians-University, Munich, DE: ${ }^{5}$ Gene Center And Department Of Biochemistry, Molecular Animal Breeding And Biotechnology, LudwigMaximilians-University, Munich, DE
Background: The development of efficient and safe gene therapy approaches in muscular dystrophies is a major challenge. Cell culture models derived from our large animal model, the DMD pig, provide an in vitro system to qualitatively and quantitatively evaluate therapeutic restoration of dystrophin. The DMD pig carries an exon 52 deletion in the dystrophin gene and mimics the most frequent human $D M D$ mutation. A major hurdle is the low degree of myogenic differentiation in porcine satellite cells. As compared to other species, terminal differentiation of porcine myoblasts is very inefficient. Moreover, full-length dystrophin (Dp427) is not expressed until after the myoblasts begin myogenic differentiation. Methods: Primary satellite cells were isolated from pig skeletal muscle by consecutive enzymatic digestion. The fusion medium and the surface coating were optimised to facilitate myotube formation. Differentiation medium containing Ultroser $G$ and matrigel/gelatin coated culture dishes promoted myotube surface adherence and maturation. Low levels of dystrophin protein in differentiating porcine cells demanded also an improvement of the Western blot technique. To enrich for myotubes, differential trypsination was successfully employed to yield sufficient differentiated cells for dystrophin Western blot detection. Results: Among key factors which affect myogenesis in vitro are the extracellular matrix proteins chosen to coat the culture plates and the choice of growth and differentiation media. Notably, a preplating step for $1-2 \mathrm{~h}$ did not improve the yield of myogenic cells. By means of optimised fusion media and culture dish coating, we significantly improved myogenic differentiation in terms of fusion index and dystrophin protein expression. It is well established that dystrophin is a low-abundance protein and constitutes less than $0.01 \%$ of total striated muscle protein. For protein extraction and Western blot detection in the porcine cell culture model, we enriched for myotubes to increase the dystrophin specific signal. Conclusion: Differentiated dystrophin-deficient porcine myoblasts provide a primary exon 52-deficient cell culture model ideally suited for evaluation of targeted RNA- or DNA-based molecular therapy. The efficiency of dystrophin restoration, potential toxicity, and additional side effects may be assessed by our preclinical approach and provides excellent translational impact for future treatment options in affected DMD patients. 


\section{PS1Group1-066 / \#605}

Topic: Group 1 - Muscle Diseases of Genetic Origin and Acquired Myopathies: Clinical Features, Pathophysiology, Therapy

\section{DILATED CARDIOMYOPATHY AND LIMB-GIRDLE MUSCULAR DYSTROPHY- DYSTROGLYCANOPATHY REVEALING NEW DPM3 GENE MUTATIONS}

Juliette Svahn ${ }^{1}$, Pascal Laforet ${ }^{2}$, Christophe Vial $^{3}$, Nathalie Streichenberger ${ }^{4}$, Norma Romero ${ }^{5}$, Céline Bouchet-Séraphin ${ }^{6}$, Nathalie Seta ${ }^{7}$, Rita Menassa ${ }^{8}$, Françoise Bouhour ${ }^{3}$, Guillemette Jousserand ${ }^{3}$, Tanya Stojkovic ${ }^{2}$

${ }^{1}$ Electroneuromyography And Neuromuscular Department, Hospices Civils de Lyon, Bron, FR: ${ }^{2}$ Sorbonne Universités, UPMC Univ Paris 06, INSERM UMRS974, CNRS FRE3617, Center of Research in Myology, Myology Institute, Paris, FR $;^{3}$ Electroneuromyography And Neuromuscular Department, Hospices Civils de Lyon, Bron, FR $;{ }^{4}$ Centre de Neuropathologie Est - Hospices Civils de Lyon, Université Claude Bernard Lyon1, Institut NeuroMyogène - CNRS UMR 5310 INSERM U1217, Bron, FR, ${ }^{5}$ Unité De Pathologies Neuromusculaires, Institut De Myologie, UMRS974, Institut de Myologie, Paris, FR $;{ }^{6}$ Département De Biochimie Et De Génétique, AP-HP, Hôpital Bichat, Paris, FR, ${ }^{7}$ Biochemistry Laboratory, AP-HP, Hôpital Bichat, Paris, $F R ;{ }^{8}$ Department Of Molecular Endocrinology And Rare Diseases, Hospices Civils de Lyon, Bron, FR

Background: DPM synthase catalyzes the formation of DPM from Dolichol-phosphate and GDPmannose(Man). The deficiency of Dolichol-P-mannose (DPM) synthase subunit 3 (DPM3) affects two particular glycosylation pathways: N-glycosylation and O-mannosylation which are respectively involved in congenital disorders of glycosylation (CDG) and alpha-dystroglycanopathies. Aberrant glycosylation of alpha-dystroglycan $(\alpha \mathrm{DG})$ leads to alpha-dystroglycanopathies. We describe novel pathogenic DPM3 mutations previously unreported in the literature in two unrelated male patients presenting limb-girdle muscular dystrophy (LGMD) and dilated cardiomyopathy. Methods: Clinical examination, cardiac and biologic investigations, muscle imaging, muscle biopsy and transferrin isoelectric focusing were performed. Next generation sequencing of 18 alpha-dystroglycanopathies genes was performed (B3GALNT2, B3GNT1, DAG1, DOLK, DPM1, DPM2, DPM3, FKRP, FKTN, GMPPB, ISPD, LARGE, POMGNT1, POMGNT2, POMK, POMT1, POMT2, TMEM5). Results: The two patients presented early and severe dilated cardiopathy, which turned out to be the main symptom in one of our patient. They developed limb-girdle muscular dystrophy predominating in pelvic girdle, in childhood and adulthood respectively. Laboratory investigations revealed elevated CK level. The deltoid muscle biopsies of both patients revealed moderate muscle dystrophy with fiber-size variation, multiple internal nuclei, and interstitial fibrosis. Immunolabelling of $\alpha \mathrm{DG}$ (VIA4-1 antibody, Millipore) showed reduced alpha-dystroglycan immunostaining in patient 1 and thin and irregular sarcolemmal staining in patient 2. Muscle imaging displayed diffuse fatty degeneration in gluteus muscles, rectus femoris muscles and lower limb muscles with severe fatty replacement in the adductors, hamstrings and triceps surae muscles. Transferrin isoelectric focusing of both patients revealed an abnormal profile suggesting a congenital disorder of glycosylation (CGD) type 1 pattern, consistent with defective N-glycosylation (DPM3CGD1). Next generation sequencing uncovered in the two patients a hemizygous deletion of DPM3 and a hemizygous pathogenic missense mutation in exon 2 c.41T $>$ C, p.Leu14Pro, predicted to be pathogenic by in silico tools. Conclusion: Previously, defects in Nglycosylation and O-mannosylation were considered to cause separate groups of diseases but cases have been since described which linked congenital disorders of glycosylation (CDG) type I with dystroglycanopathies. DPM3 mutations have been previously reported in two patients. Lefeber et al., described a female child with mild LGMD2 and a homozygous missense mutation, who developed dilated cardiomyopathy in early adulthood (Lefeber et al., 2009). Recently, an adult female patient with isolated pelvic girdle muscle weakness and a homozygous missense mutation was reported (Van Den Bergh et al., 2017). In conclusion, patients with DPM3 mutations may present with a particular phenotype: mild to severe limb-girdle muscular dystrophy predominating in the pelvic girdle, early dilated cardiomyopathy and biochemical features of deficient protein N-glycosylation. These observations underline the importance of considering a DPM synthase defect in unsolved alpha-dystroglycanopathy patients and of performing serum N-glycosylation screening. 


\section{PS1Group1-067 / \#637}

Topic: Group 1 - Muscle Diseases of Genetic Origin and Acquired Myopathies: Clinical Features, Pathophysiology, Therapy

\section{DYSFERLINOPATHY: PHENOTYPIC ASPECTS IN 24 MOROCCAN PATIENTS AND BENEFITS FROM MUSCLE EXERCISE AND CORTICOIDS IN SOME CASES}

\author{
Nazha Birouk ${ }^{1}$, Sana Sefiani², Bouchra Kably ${ }^{3}$, \\ Halima Belaïdi ${ }^{3}$, Reda Ouazzani ${ }^{3}$ \\ ${ }^{1}$ Clinical Neurophysiology Rabat, Speciality \\ Hospital, Rabat, MA $;{ }^{2}$ Neuropathology, Speciality \\ Hospital, Rabat, MA, ${ }^{3}$ Clinical Neurophysiology, \\ Speciality Hospital, Rabat, MA
}

Background: Dysferlin deficiency is responsible for different phenotypes of muscle dystrophies including limb girdle syndrome and distal myopathies. This work aims to give a phenotype description of dysferlin deficient patients belonging to 22 Moroccan families and to describe the benefit from regular muscle exercise and corticosteroid treatment in some patients. Methods: All patients underwent clinical evaluation for muscle impairment distribution and orthopedic signs, CK dosage, electroneuromyography and muscle biopsy with immunocytochemistry for dystrophin, sarcoglycans and dysferlin. 3 patients received oral prednisone and 5 patients had a regular follow up with muscle exercise. Results: Twenty four patients aged 14 to 57 years were examined. There were 15 males and 9 females. Age at onset was 20,2 $\pm 6,7$ years (range: 12 to 35$) .16$ families had an established consanguinity, 11 families had more than 1 affected member. The phenotypes were as follows: Myoshi type in 7 patients, limb girdle syndrome was isolated in 5, associated to lower limbs distal muscles involvement in 7, to biceps brachialis muscles deficit in 4 and to tibialis anterior impairment in 1 patient. CK level was 1.5 to $6 \mathrm{X}$ normal value. 3 patients had myalgia and inflammatory pattern on muscle biopsy. 1 patient had asymptomatic high CKemia. All patients had dystrophic changes on muscle biopsy with absence of dysferlin where as dystrophin and sarcoglycans were normal on immunocytochemistry. Functional disability was variable, 3 patients were wheel chair bound at the ages of 23,25 and 38 years respectively. 3 patients received treatment with oral prednisone with a drastic benefit on functional disability. 5 patients had a sustained functional benefit from regular muscle exercise that was adapted to their deficits. Conclusion: Dysferlin deficiency was identified in $41.5 \%$ of muscle dytrophy families with limb girdle and or distal muscles impairment in our series. It seems to represent the second cause of muscle dystrophies in Morocco after sarcoglycanopathies. A screening for the responsible dysferlin gene mutations is necessary to identify a probable founder mutation in our population. We should consider the potential benefit from corticosteroids and regular muscle exercise in some patients.

\section{PS1Group1-068 / \#614}

Topic: Group 1 - Muscle Diseases of Genetic Origin and Acquired Myopathies: Clinical Features, Pathophysiology, Therapy

\section{LGMD DUE TO $\gamma$-SARCOGLYCAN DEFICIENCY (LGMD2C): STUDY OF NATURAL HISTORY IN 77 PATIENTS BELONGING TO 69 MOROCCAN FAMILIES}

Nazha Birouk $^{1}$, Armel Ghislain Mpandzou ${ }^{1}$, Bouchra Kably ${ }^{2}$, Sana Sefiani ${ }^{3}$, Abdelaziz Sefiani ${ }^{4}$, Halima Belaïdi ${ }^{2}$, Reda Ouazzani ${ }^{2}$

${ }^{1}$ Clinical Neurophysiology Rabat, Speciality Hospital, Rabat, MA; ${ }^{2}$ Clinical Neurophysiology, Speciality Hospital, Rabat, MA ${ }^{3}$ Neuropathology, Speciality Hospital, Rabat, MA ${ }^{4}$ Genetic, National Health Institut, Rabat, MA

Background: Sarcoglycanopathies (SGP) are autosomal recessive progressive limb-girdle muscular dystrophies, due to $\alpha$-, $\beta$-, $\gamma$ - and $\delta$-sarcoglycan gene mutations, respectively leading to LGMD (limb girdle muscular dystrophy) 2D, E, C and F. In North Africa, $\gamma$-SG is the most common. We report a study of 77 patients from 69 Moroccan families with highlights to the disease progression. Methods: Throughout a 21-year time lapse, 77 patients, among 133 SGP, were regularly followed-up during an average time of $4.1 \pm 3.4$ years (SD) [Max:Min; 1-14]. Diagnosis of $\gamma$-SGP was established on protein immunostaining and/or genetic analysis $(n=69)$ and by findings obtained from siblings $(\mathrm{n}=8)$. Disease progression was assessed annually in all patients by frequency, age and delay of the occurrence of the main 
functional incapacities, skeletal deformities, cardiac and respiratory impairments and by survival curve of the loss of ambulation. Functional scores (Research Medical Council, Vignos, modified Gardner-Medwin-Walton, walking distance and climbing stairs) were calculated in 35 patients regularly followed-up during five years. Results: Consanguinity rate was 69.6\% (48/69 families), 40 (51.9\%) patient were sporadic cases and $37(48.1 \%)$ had positive familial history. Sex ratio was 0.8 . Immunostaining analysis showed isolated $\gamma$-SG deficiency in 57/67 (85.1\%) patients investigated and combined $\gamma$-SG deficiency with reduction of one or more other SG in 10/67 (14.9\%). Genetic analysis showed $4525 T$ mutation in $15 / 23(65.2 \%)$ patients investigated. Mean age at onset was $7.2 \pm 2.8$ years [1-19]. Loss of ambulation occurred after $7.5 \pm 4.6$ years [2-22] of disease duration; almost concomitant with scoliosis. From Kaplan Meier survival curve, median of loss ambulation was $8 \pm 0.5$ years $(95 \%$ CI $7.03-8.96$ years) with probability of $44.4 \%$. Comparison tests of delay of the loss of ambulation showed no significant difference for gender, family history, parental consanguinity, and expression profile of $\gamma-\mathrm{SG}$ deficiency. Cardiac impairment was found in 10/53 (18.8\%) patients at a mean age of $18.8 \pm 10.6$ years [8-45]. Main cardiac anomalies were left ventricular dysfunction either isolated or associated with dilated cardiomyopathy in $7 / 10$ patients, incomplete right bundle branch block in 5/10, premature ventricular contraction in $3 / 10$, and right ventricular dysfunction in $1 / 10$. Restrictive respiratory syndrome was found in $21 / 24(87.5 \%)$ patients at a mean age of $18.9 \pm 8.2$ years [9-43]; this syndrome became severe requiring non-invasive ventilation in 10 patients, at a mean age of $21.5 \pm 9.4$ years [11-43]. In the 35 patients selected for the functional assessment, scores measures changes were most significant in patients with loss of ambulation or in patients with early age at onset between 5-9 years. Conclusion: The analysis of SG natural history highlights disease prognosis and can thus help in anticipating medical management to improve patient's quality of life.

\section{PS1Group1-069 / \#659}

Topic: Group 1 - Muscle Diseases of Genetic Origin and Acquired Myopathies: Clinical Features, Pathophysiology, Therapy

\section{DOES A NORMAL DYSTROPHIN STAINING IN MUSCLE BIOPSY RULE1 OUT THE MOLECULAR DIAGNOSIS OF DUCHENNE / BECKER MUSCULAR DYSTROPHY?}

Mira Ginzberg, Tally Lerman-Sagie, Dorit Lev, Ron Dabby, Menachem Sadeh, Esther Leshinsky-Silver E. Wolfson M.D, Holon, IL

Background: Molecular genetic testing of the Dystrophin gene can confirm the clinical diagnosis of a dystrophinopathy without muscle biopsy in most individuals with Dhchenne uscular Dystrophy / Becker Muscular Dystrophy (DMD/BMD). The multiplex ligation-dependent probe amplification (MLPA) assay is the most powerful tool in screening for deletions and duplications in the dystrophin gene in patients with (DMD/BMD). Deletions of one or more exons account for about $65 \%$ of DMD and about $85 \%$ of BMD cases. MLPA is a quantitative method enabling the detection of deletions and duplications of all 79 DMD exons. MLPA can determine the size of the deletion or duplication and predict, whether the mutation disrupt the reading frame Methods: We describe two male siblings, born to healthy non consanguineous parents. Both have suffered since a young age from muscle weakness and recurrent episodes of rhabdomyolisis with high CK values. The muscle weakness has been improving constantly and now as grownup males, they have minimal selective proximal girdle muscle weakness. However, the older brother experiences recurrent episodes of severe rhabdomyolysis and atrial fibrillation. The younger brother, experiences fatigability and lately dilated cardiomyopathy was diagnosed. Their maternal grandfather, who died at the age of 39 years, because of cardiomyopathy, did not suffer from myopathy. Results: We describe two male siblings, born to healthy non consanguineous parents. Both have suffered since a young age from muscle weakness and recurrent episodes of rhabdomyolisis with high CK values. The muscle weakness has been improving constantly and now as grownup males, they have minimal selective proximal girdle muscle weakness. However, the older brother experiences 
recurrent episodes of severe rhabdomyolysis and atrial fibrillation. The younger brother, experiences fatigability and lately dilated cardiomyopathy was diagnosed. Their maternal grandfather, who died at the age of 39 years, because of cardiomyopathy, did not suffer from myopathy. Conclusion: Exon 1 deletion in the Dystrophin gene is the cause for BMD in these two brothers and probably the cause for cardiomyopathy in their grandfather. If the clinical presentation and the pattern of inheritance is suspicious enough, only molecular analysis of the dystrophin gene by sequencing and MLPA can lead us to the correct diagnosis. However, there are some pitfalls of MLPA in detecting DMD single exon deletion or duplication, because of false-positives due to sequence variations.

\section{PS1Group1-070 / \#820}

Topic: Group 1 - Muscle Diseases of Genetic Origin and Acquired Myopathies: Clinical Features, Pathophysiology, Therapy

\section{ESYN- AND CK9- PROMOTORS DRIVE MUSCLE-SPECIFIC EXPRESSION OF TRANSGENES IN VITRO AND IN VIVO}

Jinhong Meng ${ }^{1}$, John Counsell ${ }^{2}$, Simon

Waddington $^{3}$, Francesco Muntoni ${ }^{4}$, Jennifer

Morgan $^{5}$

${ }^{1}$ UCL Great Ormond Street Institute of Child

Health, London, GB; ${ }^{2} U C L$ Great Ormond Street

Institute of Child Health, London, $G B ;^{3} E G A$

Institute for Women's Health, London, GB: ${ }^{4}$ Dubowitz Neuromuscular Unit, UCL Great Ormond Street Institute of Child Health, London, $G B ;{ }^{5}$ University College London, London, GB

Background: Lentivirally-mediated dystrophin expression is a promising therapeutic approach for Duchenne muscular dystrophy (DMD). Lentivirus coding either full-length or mini- dystrophin can transduce patient-derived muscle stem cells, providing a valuable cell source for autologous transplantation. However, due to the large size of the dystrophin gene and the limited packaging capacity of the lentiviral vector, the promoter driving the transgene expression needs to be small. Furthermore, as dystrophin is mainly expressed in muscle fibres, the promoter must be active in differentiated muscle cells. Methods: In the present study, we tested the expression of a reporter gene driven by either ESyn- (547bp) or CK9- (429bp) promoters in comparison to the ubiquitous SFFV promotor, by transducing lentiviruses coding luciferase driven by the ESyn-, CK9- or SFFV- promoter into cultured human cells derived from lung, liver, kidney, neuroblastoma and skeletal muscle. Furthermore, these viruses were injected systemically into newborn outbred mice and the expression of luciferase was examined one month later using the IVIS Lumina III in vivo imaging system. Results: We found that cells transduced with ESyn- and CK9- lentivirus express significantly lower levels of luciferase in comparison to those transduced with SFFV- lentivirus. Interestingly, the expression of luciferase was dramatically increased in Esyn- and CK9- lentivirus-transduced muscle cells when they were induced to undergo myogenic differentiation. In mice injected with SFFV- lentivirus, luciferase was expressed in organs including liver, spleen and skeletal muscle, while in mice injected with Esyn- or CK9- lentivirus, luciferase was expressed mainly in skeletal muscles. Conclusion: our data show that both ESynand CK9- promoters drive skeletal muscle specific expression of a transgene, indicating that they are good candidates for driving expression of proteins within skeletal muscle.

\section{PS1Group1-071 / \#354}

Topic: Group 1 - Muscle Diseases of Genetic Origin and Acquired Myopathies: Clinical Features, Pathophysiology, Therapy

\section{E-CADHERIN IS ECTOPICALLY EXPRESSED IN THE MUSCLE FIBER OF INCLUSION BODY MYOSITIS}

Chiseko Ikenaga, Hiroyuki Ishiura, Hidetoshi Date, Akatsuki Kubota, Shoji Tsuji, Tatsushi Toda, Jun Goto, Jun Shimizu

Department Of Neurology, The University of Tokyo, Tokyo, JP

Background: Both inclusion body myositis (IBM) and polymyositis (PM) are characterized by the endomysial infiltrates of CD8+ cytotoxic T-lymphocytes invading or surrounding major histocompatibility class 1 expressing non-necrotic 
muscle fibers (CD8-MHC-1 complex). Patients with IBM have rimmed vacuoles or abnormal protein accumulation and they are refractory to treatments, while patients with PM generally respond well to treatments. It seems to be important to clarify genes or pathways, which contribute to the difference of these two disease's phenotypes. Methods: We analyzed frozen muscle biopsy samples from 62 patients with CD8-MHC-1 complex (43 IBM, 9 PM, and 10 unclassifiable) and 9 normal controls (NCs) by RNA-sequencing (Illumina Hiseq 2500). We detected the differentially expressed genes (DEGs) and performed pathway analysis by edgeR. In addition, we compared the gene expressions of cell surface antigens (CD1 CD371) among these groups. In order to evaluate the protein level of these DEGs and cell surface antigens, we performed immunohistochemistry on frozen sections of muscle biopsy samples from patients with IBM, PM, dermatomyositis (DM), immune-mediated necrotizing myopathy (IMNM), and NCs, respectively. Results: Compared with NCs, there were 8588 DEGs in IBM $(F D R<0.05)$ and the sets of genes concerning cancer, immune system, and NFkB/TNF/PI3K-Akt/JAK-STAT/ TGF- $\beta$ signaling pathways were upregulated, while the sets of genes concerning translation, transcription, and mitochondrial function were downregulated in IBM. Compared with NCs, there were 4744 DEGs in PM $($ FDR $<0.05)$ and the sets of genes, which were upregulated or downregulated in PM, were the same as those in IBM. When we compared IBM with PM, there were 1419 DEGs (FDR $<0.05$ ) and the gene sets of the innate and adaptive immune systems were more upregulated in IBM than those in PM. Among these DEGs, there were 28 genes, in which the expressions of IBM were twice as much as those of PM $($ FDR $<0.05)$ and 12 genes of cell surface antigens including E-cadherin $(\mathrm{FDR}<0.01)$. In immunohistochemistry, the muscle fibers of IBM, which were diffusely positive for p62, were also positive for E-cadherin. On the other hand, the muscle fibers, which were positive for dense p62 aggregates, were not stained with E-cadherin antibody. Although there were some muscle fibers, which were diffusely positive for $p 62$ in the samples of IMNM and DM, they were not stained with E-cadherin antibody, except for the partial staining of perifascicular fibers with atrophy in DM. Conclusion: IBM and PM shared most of the upregulated/downregulated pathways. Among them, the gene sets of the innate and adaptive immune systems were upregulated in the muscle of IBM, compared with those of PM. Among the DEGs, E-cadherin, which is known to be expressed in the epidermis, was highly expressed in the muscle of IBM. Considering the ectopical expression and the unique staining pattern of E-cadherin in the muscle of IBM, it may have some roles in the pathogenesis of IBM.

\section{PS1Group1-072 / \#399}

Topic: Group 1 - Muscle Diseases of Genetic Origin and Acquired Myopathies: Clinical Features, Pathophysiology, Therapy

\section{CARE EVALUATION OF DUCHENNE MUSCULAR DYSTROPHY PATIENTS IN BRAZIL}

Karine C. Turke, Alzira A.S. Carvalho, David Feder Faculdade de Medicina do ABC, Santo André, BR

Background: The Duchenne Muscular Dystrophy (DMD) is the most frequent genetic lethal disease in early childhood of boys. International protocols for surveillance and treatment are broadly diffused and our objective was to assess if the patients with DMD in Brazil receive adequate treatment accordingly to those protocols. Methods: Through social networks, parents and responsible person were invited to answer to an online form. The questionnaire addressed conditions of the diagnosis, exams and treatments. Results: 54 patients were included in the study. The average age was $13,0 \pm 1,2$ years. $79,62 \%$ of the boys was medicated with corticosteroids, where $76,7 \%$ used deflazacort; $18,1 \%$ prednisolone and 4,65\% prednisone. In the diagnosis, $31,4 \%$ were submitted to a skeletal muscle biopsy, and $79,6 \%$ knew the genetic mutation. $40,7 \%$ had cardiac alteration. The first electrocardiogram (ECG) and echocardiogram (Echo) happened at 8,4 and 8,8 years old respectively. The ECG was not performed yearly in only $23,5 \%$ of the cases, and the Echo in $26,4 \% .50 \%$ of the patients went through the holter, and $9,2 \%$ was submitted to a cardiac magnetic resonance image. Only $31,4 \%$ performed a strength test every 6 months. Just $22,11 \%$ of the studied cases attended rehabilitation programs. $79,5 \%$ of the patients over 6 years of age performed spirometry, with an average of 10,5 years old. In the assessment of coughing effort only $34,7 \%$ of the patients over 6 years old submitted to the exam. The annual spirometry was performed by $48,1 \%$ of the participants. $37 \%$ had an appointment with occupational therapist and $31,4 \%$ with a nutritionist in the 
last year. 59,2\% of the patients had done bone densitometry, and $37,5 \%$ of those were diagnosed with osteoporosis. Only $57,4 \%$ of the participants was vaccinated against influenza in the last year. Conclusion: It is concluded that the international protocols of surveillance and management of the patients with DMD are not being followed in Brazil. This could contribute to a greater mortality and morbidity. Enhancing medical education about DMD through disclosure protocols as well as providing patient access to specialized health care and medication adherence would be meaningful to improve the quality of life of DMD patients.

\section{PS1Group1-073 / \#891}

Topic: Group 1 - Muscle Diseases of Genetic Origin and Acquired Myopathies: Clinical Features, Pathophysiology, Therapy

\section{GENOTYPE AND PHENOTYPE FEATURES OF BRAZILIAN PATIENTS WITH MCARDLE DISEASE}

\author{
Alzira A.S. Carvalho' ${ }^{1}$, Vinicius G. Silva ${ }^{2}$, Miriam \\ E. $\mathrm{Koch}^{3}$, Pamela O. Delgado ${ }^{4}$, Bruno M. \\ Bertassoli $^{2}$, Roseli Corazzini ${ }^{4}$, Karine C. Turke ${ }^{4}$, \\ Denise M. Christofolini ${ }^{5}$, Itatiana Rodart ${ }^{5}$, Fernando \\ L.A. Fonseca ${ }^{6}$, David Feder ${ }^{2}$ \\ ${ }^{1}$ Neurology, Faculdade de Medicina do ABC, Santo \\ Andre, BR; ${ }^{2}$ Pharmacology, Faculdade de Medicina \\ do ABC, Santo Andre, BR $;{ }^{3}$ Faculdade de Medicina \\ do ABC, Santo Andre, BR $;{ }^{4}$ Faculdade de Medicina \\ do ABC, Santo André, BR, ${ }^{5}$ Genetics, Faculdade de \\ Medicina do ABC, Santo Andre, BR, ${ }^{6}$ Laboratory \\ Analysis, Faculdade de Medicina do ABC, Santo \\ Andre, BR
}

Background: Glycogenosis Type V(GTV) also known as McArdle disease, is a rare autosomal recessive disorder characterized by exercise intolerance, myalgia and painful cramping since childhood or adolescence associated to persisted elevated creatine kinase(CK). Mutations in the gene cause the disorder causing inability to produce the enzyme known as phosphorylase or myophosphoryase in muscle. The objective was to analyze clinical, epidemiological and genetic aspects as well as, to demonstrate the morphological muscular findings in 18 patients with McArdle disease. Methods: The clini- cal parameters evaluated were: cramps, myalgia, fatigue and dark urine. $\mathrm{CK}$, modified ischemic exercise test (TI), hand-grip strength (using dynamometer) were also analyzed. The muscular morphological aspects analyzed were the percentage of internal nuclei, vacuoles and Feret's using H\&E stain. The PYGM gene mutation was analyzed by screening exons 1, 15 and 17 followed by next generation sequencing (TruSight Inherited Disease panel from Illumina Inc.) when the mutation was not found. Results: The total of 18 patients were analyzed: performed muscle biopsy: $n=15$; CK: $n=18$; ischemic exercise test(IET) $n=16$; hand-grip strength: $n=16$; genetic test: $n=14$ (the remaining four patients were siblings belonging to three distinct families and presented the same symptoms, elevated CK and muscle biopsy compatible with GTV the four). 56\% men; $44 \%$ women. Considering clinical symptoms, $66,7 \%$ of them refer cramps, $72.2 \%$ myalgia, $100 \%$ fatigue and $66,7 \%$ dark urine. Fibers with internalized nuclei (FIN): $10 \%$; vacuoles: $16,1 \%$; type 2 fiber predominance (T2FP): 62,3\%. Feret's diameter ranged from 21.5 to 83.5 um (type 1) and 34.6-78.2 um (type 2). CK at rest: $1767 \mathrm{U} / \mathrm{L}$ (median). IET was consisted with GTV in $100 \%$ of patients without side effects. Hand-grip strength was $44.3 \mathrm{~kg}$ (median). The morphological parameters vacuoles and FIN were not statistically significant when correlated. The T2FP and Feret's diameter were significant, $p=0.0000$ and $p=0.0007$ respectively). The most frequent mutation was R50X in homozygous (50\%). The second most frequent was association of R50X and c. $1967 \mathrm{~A}>\mathrm{C}(17 \%)$. One patient had 4 mutations that "in silica" showed to be pathogenic. There was no correlation among the parameters evaluated, even among members of the same family. Conclusion: 1 . $\mathrm{CK}$ is not related to the histological alterations analyzed. 2. IT is a good screening test in the differential diagnosis of patients with IE and without risks. 3. The T2FP suggests a compensatory mechanism in the use of fast fiber energy. 4. The R50X Arg50Ter mutation was the most frequent in our sample. Our study was the pioneer in the analysis of the histological phenotype versus genotype. 5. The results regarding the heterogeneity of the parameters analyzed suggest that GTV presents a clinical, histological and molecular individual phenotype. Therefore, genotype and histological phenotype are not prognostic factors of the disease. 


\section{PS1Group1-074 / \#571}

Topic: Group 1 - Muscle Diseases of Genetic Origin and Acquired Myopathies: Clinical Features, Pathophysiology, Therapy

\section{PROPOSED CUT-OFF FOR}

REACTIVITY OF ANTI-HMGCR AND ANTI-SRP ANTIBODIES IN PATIENTS STATIN-EXPOSED AND STATIN-UNEXPOSED

\author{
Alzira A.S. Carvalho ${ }^{1}$, Thaiane F. Vieira ${ }^{1}$, Miriam \\ E. Koch ${ }^{1}$, Vinicius G. Silva ${ }^{1}$, Roseli Corazzini ${ }^{1}$, \\ Pamela O. Delgado ${ }^{1}, \underline{K a r i n e ~ C . ~ T u r k e ~}^{1}$, Fernando \\ L.A. Fonseca ${ }^{2}$, David Feder ${ }^{3}$ \\ ${ }^{1}$ Faculdade de Medicina do ABC, Santo André, \\ $B R ;{ }^{2}$ Laboratory Analysis, Faculdade de Medicina \\ do ABC, Santo Andre, BR, ${ }^{3}$ Pharmacology, \\ Faculdade de Medicina do ABC, Santo Andre, BR
}

Background: Introduction: Few reports have been published demonstrating that anti-HMGCR and anti-SRP are specific to immune-mediated necrotizing myopathy (IMNM). The therapeutic approach with statins is widely used in the control of dyslipidemias. However, there is no laboratory evaluation to elect patients to make use of this class of therapeutic drugs. Aim: To analyze the prevalence of anti-SRP and anti-HMGCR antibodies in a heterogeneous cohort of 85 patients to determine cut-off reference values for these antibodies. Methods: Serum samples from 85 patients were screened for the presence of anti-HMGCR and anti-SRP autoantibodies by ELISA. The demographic, clinical and morphological features were also correlated with anti-HMGCR and anti-SRP antibodies. The patients were divided in two groups; A: statin-exposed and B: statin-unexposed. Results: The mean age of groups A and B were 61.1 and 39.5 years, respectively. AntiHMGCR and anti-SRP antibodies were present in $12.6 \%$ and $14.1 \%$ of patients, respectively. 57 statinexposed and 28 statin-unexposed. There were no significant associations of anti-HMGCR and antiSRP titers with age, gender, exposure to statins or symptoms; however, significantly more symptomatic patients had been exposed to statins $(\mathrm{p}=0.003)$. There was no significant difference in anti-HMGCR levels between asymptomatic and symptomatic patients $(p=0.994)$. Conclusion: Our results defined antibody cut-off points for a heterogeneous population with different diagnoses. We also demonstrated that anti-HMGCR and anti-SRP antibodies are not $100 \%$ specific for IMNM. Eleven (12.9\%) patients were positive for anti-HMGCR antibodies and 12 $(14.1 \%)$ were positive for anti-SRP antibodies in this heterogeneous cohort.

\section{PS1Group1-075 / \#573}

Topic: Group 1-Muscle Diseases of Genetic Origin and Acquired Myopathies: Clinical Features, Pathophysiology, Therapy

\section{NECROTIZING MYOPATHY AFTER DENGUE: CASE REPORT}

\author{
Alzira A.S. Carvalho ${ }^{1}$, Karine C. Turke ${ }^{1}$, David \\ Feder $^{2}$ \\ ${ }^{1}$ Faculdade de Medicina do ABC, Santo André, \\ $B R ;{ }^{2}$ Pharmacology, Faculdade de Medicina do \\ $A B C$, Santo Andre, BR
}

Background: Muscle involvement is rare in dengue. Usually just myalgia and rarely as myositis, hypokalemic paralysis, or rhabdomyolysis. Methods: We report a case of necrotizing myopathy occurring in a 38-year-old Caucasian woman was referred to emergency unit care complaining of low grade fever for 2 days and severe myalgia in lower limbs followed by a skin rush and thrombocytopenia. Results: The diagnosis of the dengue viral infection was confirmed on the basis of the positive serum IgM antibody. Creatine kinase levels persisted elevated after 1 year from diagnosis with minimal complains. Her first assessment revealed muscle weakness, which was proximal and asymmetric in the lower limbs accompanied by mild muscle atrophy of left lower limb. A muscle biopsy of the deltoid showed myopathic changes, including moderate variation in fiber size and necrosis, suggesting a necrotizing myopathy. Protein immunohistochemistry studies showed normal immunoexpression except a diffuse MHC class I over-expression. A genetic panel of Next Generation Sequencing containing ANO5, DYSF, GAA, SGCB, SGCG, CAPN3, FKRP, SGCA, SGCD, and TCAP genes was done and with no mutations found. Conclusion: This report is the first one to describe dengue associated with necrotizing myopathy. We should to think in infection disease in patients presenting persisted creatine kinase levels with almost no symptoms. We also suggest that reviews of creatine levels and muscle biopsy in a large cohort of patients with dengue fever would be necessary to confirm this impression. 


\section{PS1Group1-076 / \#492}

Topic: Group 1 - Muscle Diseases of Genetic Origin and Acquired Myopathies: Clinical Features, Pathophysiology, Therapy

\section{EXPRESSION OF DYSTROPHIN ISOFORMS IN NEW DUCHENNE MUSCULAR DYSTROPHY MICE MODEL}

Tatiana Egorova ${ }^{1}$, Dmitry Vlodavets $^{2}$, Denis Reshetov $^{3}$, Evgenia Zotova ${ }^{3}$, Anna Polikarpova ${ }^{3}$, Svetlana Vassilieva ${ }^{3}$, Alexei Deikin ${ }^{1}$ ${ }^{1}$ Core Facility, Institute of Gene Biology RAS, Moscow, RU; ${ }^{2}$ Russian Children Neuromuscular Center, Veltischev Scientific Research Clinical Pediatric Institute, Pirogov Russian National Research Medical University, Moscow, $R U,{ }^{3}$ Research Department, Marlin Biotech LLC, Moscow, $R U$

Background: New Duchene muscular dystrophy mice model was created in Russia using CRISPR/ Cas9 gene editing system. Two sgRNAs targeted to introns 7 and 34 respectively were injected into mice zygotes with Cas9 protein. Cas9 introduces doublestrand breaks which are repaired by cell machinery with random indel formation. As a result of microinjections into zygotes 20 mice were born with desired deletion of exons 8-34 (DMDdel8-34). Sequence verification of deletion borders revealed several groups with different mutations. Methods: Three mice representing different deletion borders with 3 , 8 and 18 base pair gaps between target sites were used for breeding. For all of them sgRNAs targeted intronic regions and they have same mutations on mRNA level. Third and fourth mice generations after their breeding were analyzed using histology, physiology and molecular biology methods. Results: Here we present some features of the model. New model demonstrates representative dystrophy symptoms on muscle histology such as central nucleation in myofibers, fibrosis and calcinosis formation in striated muscles. Great difference was shown between control (CBA/C57B16) and experimental group (DMDdel8-34) in hanging test. Quantitative RT-PCR was used to check dystrophin mRNA presence in knockout mice. It was expected that in this case dystrophin-coding RNA should be destroyed by nonsense-mediated decay because deletion of exons 8-34 leads to frameshift in 35 exon. Surprisingly there was no difference in mRNA expression level between control and experimental groups when using primers to dystrophin exons 1-7. Dystrophin mRNA expression can be explained by alternative splicing. To verify that there is no dystrophin protein expression western blotting assay was performed on primary myoblasts derived from murine model muscles using polyclonal antibodies raised against Cterminus of dystrophin. Absence of full-length $(427 \mathrm{kDa})$ dystrophin form was verified but $70 \mathrm{kDa}$ band was detected which corresponds to Dp71 dystrophin C-terminal isoform normally expressed in non-muscle tissues such as lung or brain. Therefore, we can suggest the presence of alternative dystrophin isoforms in DMDdel8-34 muscle cells. Conclusion: The finding needs further investigation because any treatment strategy tested on the model require functional restoration of dystrophin. Short dystrophin isoforms with noncanonical expression profile existing in model muscles can occupy dystrophin binding sites on cell membrane. Even in the case of restored expression of proper muscular isoform, functionality of the protein should be investigated.

\section{PS1Group1-077 / \#759}

Topic: Group 1 - Muscle Diseases of Genetic Origin and Acquired Myopathies: Clinical Features, Pathophysiology, Therapy

\section{MYOTONIC DYSTROPHY TYPE 2 AS A MULTISYSTEM DISEASE}

Ivo Bozovic ${ }^{1}, \underline{\text { Stojan Peric }}{ }^{1}$, Jovan Pesovic ${ }^{2}$, Bogdan Bjelica ${ }^{1}$, Milos Brkusanin², Ivana Basta ${ }^{1}$, Ana Marjanovic ${ }^{1}$, Marija Brankovic ${ }^{1}$, Aleksandra Kacar $^{1}$, Dusanka Savic-Pavicevic ${ }^{3}$, Vidosava Rakocevic-Stojanovic ${ }^{1}$ ${ }^{I}$ Neurology Clinic, Clinical Center of Serbia, School of Medicine, University of Belgrade, Belgrade, Serbia, Belgrade, RS; ${ }^{2}$ Faculty of biology, Belgrade, RS:; Faculty of Biology, Center for Human Molecular Genetics, University of Belgrade, Belgrade, RS

Background: Myotonic dystrophy type 2 (DM2) is a multisystem disorder, mostly presented with mild but heterogeneous spectrum of symptoms. The aim of this research was to provide detailed sociodemographic, clinical and laboratory data of a large DM2 cohort from the Serbian registry. Methods: In 2008, 
we started to prospectively enter data of all DM patients. We also retrospectively collected data of patients hospitalized from 1990 until 2008. At the end of 2017, Serbian registry comprised 87 (68\%) of 128 genetically confirmed DM2 patients in Serbia, i.e. 1.2 registered cases per 100,000 inhabitants. Results: Female subjects were more prevalent in the registry $(63 \%)$. The diagnostic delay was $12 \pm 11$ years and mean age at entry in the Registry was $51 \pm 13$ years. The most common first symptom in our patients was leg weakness, followed by handgrip myotonia and pain, although some patients presented with cataracts or extrapyramidal syndrome. In $75 \%$ of patients lens opacities were present. Severe ECG abnormalities were noted in $8 \%$ and pacemaker was implanted in 5\% of DM2 subjects. Pulmonary restriction was observed in $10 \%$ of DM2 patients. Insulin resistance and diabetes mellitus were frequent in our DM2 cohort $(21 \%$ and $17 \%$, respectively). Majority of them had hyperlipidemia. Male subjects more frequently had snoring, baldness, sterility and polyneuropathy, while waddling gait and brisk muscle reflexes were more common in females. Conclusion: This registry offers a spectrum of different DM2 features presented in the Serbian population, which could be at service of earlier diagnosis and better follow-up of these patients.

\section{PS1Group1-078 / \#672}

Topic: Group 1 - Muscle Diseases of Genetic Origin and Acquired Myopathies: Clinical Features, Pathophysiology, Therapy

\section{HEART INVOLVEMENT IN MYOTONIC DYSTROPHY TYPE 2}

\author{
Bogdan Bjelica ${ }^{1}$, Stojan Peric ${ }^{1}$, Edita Cvijan ${ }^{1}$, \\ Gorana Mandic-Stojmenovic ${ }^{1}$, Masa Kovacevic ${ }^{1}$, \\ Ksenija Aleksic ${ }^{1}$, Jovan Pesovic ${ }^{2}$, Dusanka Savic- \\ Pavicevic $^{2}$, Ivana Basta ${ }^{1}$, Dragana Lavrnic ${ }^{1}$, \\ Vidosava Rakocevic-Stojanovic ${ }^{1}$ \\ ${ }^{1}$ Neurology Clinic, Clinical Center of Serbia, \\ School of Medicine, University of Belgrade, \\ Belgrade, Serbia, Belgrade, RS:;2Faculty of Biology, \\ Center for Human Molecular Genetics, University \\ of Belgrade, Belgrade, RS
}

Background: Myotonic dystrophy type 2 (DM2) is a slowly progressive, autosomal-dominant disease. This is a multisystemic disorder that affects the heart, which may be one of the main causes of morbidity and mortality in DM2. The aim of this study was to define cardiac impairments in patients with DM2 and its association with sociodemographic and clinical features of the patients. Methods: This retrospective study comprised 62 patients with genetically confirmed DM2, hospitalized at the Neurology Clinic, Clinical Center of Serbia, from 2013 until 2018. All patients were analyzed by electrocardiography (ECG) and transthoracic echocardiography. Results: Hypertension was observed in $42 \%$ of DM2 patients. One fifth of the DM2 patients had bradycardia while other conduction and rhythm impairments were rare, including left bundle branch block (3\%), ventricular extrasystoles (5\%), and supraventricular extrasystoles $(2 \%)$. Only one patient had implanted pacemaker because of the first degree AV block and incomplete left bundle branch block. Valve fibrosis was common: aortic $64 \%$, tricuspid $30 \%$, and mitral 19\%. Mitral regurgitation was seen in $27 \%$ of DM2 subjects. Diastolic dysfunction was observed in $44 \%$ of patients, while systolic dysfunction was found in $4 \%$. Cardiomyopathy was observed in $18 \%$ of patients, of whom three fourths had dilated type. Conclusion: Cardiac conduction and rhythm defects were rare in DM2, but sometimes even pacemaker implantation is needed. Diastolic dysfunction of the left ventricle was common. This suggest that regular ECG and echocardiography screening is needed in DM2. We suppose that early treatment may prevent development of cardiac complications and early death.

\section{PS1Group1-079 / \#673}

Topic: Group 1 - Muscle Diseases of Genetic Origin and Acquired Myopathies: Clinical Features, Pathophysiology, Therapy

\section{VARIANT REPEATS STABILIZE EXPANSION AND MODIFY AGE AT ONSET IN MYTONIC DYSTROPHY TYPE 1}

Jovan Pesovic ${ }^{1}$, Stojan Peric $^{2}$, Milos Brkusanin ${ }^{1}$, Goran Brajuskovic ${ }^{1}$, Vidosava RakocevicStojanovic $^{3}$, Dusanka Savic-Pavicevic ${ }^{1}$ ${ }^{1}$ Faculty of biology, Belgrade, $R S ;{ }^{2}$ Neurology Clinic, Clinical Center of Serbia, School of Medicine, University of Belgrade, Belgrade, Serbia, Belgrade, RS; ${ }^{3}$ Neurology Clinic, Belgrade, RS

Background: Myotonic dystrophy type 1 (DM1) is a variable multisystem disease with age at onset 
ranged from in utero to late decades. Underlying mutation is the expansion of CTG repeats at the $3 \phi$ untranslated region of $D M P K$ gene, characterized by a pronounced somatic instability biased towards further expansions over time. Expansion size and level of somatic instability explain $\sim 60 \%$ of variation in age at onset. Variant repeats at DMPK expansions, observed in $3-5 \%$ of patients, have been associated with later age at onset. We aimed to study an effect of variant repeats on dynamics of somatic instability at $D M P K$ locus and age at onset in seven patients. Methods: Four patients with pure CTG repeats served as controls. Repeat-primed PCR profiles and Sanger sequencing indicated the stable structure of different patterns of CCG (CCGCTG hexamers, small and large CCG blocks) and CTC repeats in blood cells over time ranging the period of two and a half to four years. We performed single cell smallpool PCR to size approximately 5000 individual alleles in analysed samples and thus quantify somatic instability of variant $D M P K$ expansions as whole tracks. Results: By using the published correlation models and data set of individually sized alleles in the reference group of patients [Martorel et al. 1998; Morales et al. 2012] we obtained the following results: (1) a lower level of somatic instability of variant expansions then expected based on the expansion size and age at sampling $(\mathrm{V}=0, \mathrm{p}=0.016$ for the first time point and $\mathrm{V}=0, \mathrm{p}=0.008$ for the second time point), and a less mean residual variation in somatic instability not accounted for by the expansion size and age at sampling in patients with variant expansions than in the reference group ( $\mathrm{W}=871, \mathrm{p}=2.2 \mathrm{e}^{-}$ ${ }^{4}$ for the first time point and $\mathrm{W}=1004, \mathrm{p}=6.135 \mathrm{e}^{-5}$ for the second time point); (2) a lower expansion increment over time than expected based on the expansion size and time interval between sampling $(\mathrm{V}=0$, $\mathrm{p}=0.016$ ); (3) a less mean residual variation in age at onset not accounted for by the expansion size and level of somatic instability in patients with variant expansions than in the reference group $(\mathrm{W}=172$, $\mathrm{p}=0.04$ for the first time point and $\mathrm{W}=192, \mathrm{p}=0.02$ for the second time point). Conclusion: Our results provide a firm evidence for a general stabilizing effect of different patterns of variant repasts at the $D M P K$ locus in blood cells. This feature of variant repeats most likely positively modulate age at onset of DM1 symptoms and, therefore, has the clinical importance in terms of disease course prediction and new potential therapeutic approaches.

\section{PS1Group1-080 / \#674}

Topic: Group 1 - Muscle Diseases of Genetic Origin and Acquired Myopathies: Clinical Features, Pathophysiology, Therapy

\section{FEATURES OF THE SERBIAN COHORT OF PATIENTS WITH CALPAINOPATHY}

$\underline{\text { Stojan Peric }}^{1}$, Ana Kosac ${ }^{1}$, Marija Brankovic ${ }^{1}$, Ana Marjanovic $^{1}$, Bojan Banko², Sanja Milenkovic ${ }^{3}$, Milena Jankovic ${ }^{1}$, Dragana Lavrnic ${ }^{1}$, Ruzica Maksimovic ${ }^{2}$, Vedrana Milic Rasic ${ }^{4}$, Vidosava Rakocevic-Stojanovic ${ }^{1}$ ${ }^{1}$ Neurology Clinic, Clinical Center of Serbia, School of Medicine, University of Belgrade, Belgrade, Serbia, Belgrade, $R S ;{ }^{2}$ Center for Radiology and Magnetic Resonance Imaging, Clinical Center of Serbia, Belgrade, Serbia, Belgrade, RS, ${ }^{3}$ Department of Pathology, Clinical Hospital Centre Zemun, Belgrade, Serbia, Belgrade, RS: ${ }^{4}$ Clinic for Neurology and Psychiatry for Children and Youth, Belgrade, Serbia, Belgrade, $R S$

Background: In majority of countries the most common form of limb-girdle muscular dystrophy is a type $2 \mathrm{~A}(\mathrm{LGMD} 2 \mathrm{~A})$ caused by mutation in CAPN3 gene coding calpain 3 protein. Aim of this research was to analyze genetic and phenotypic features of Serbian patients with calpainopathy. Methods: This study comprised 19 patients (42\% males) from 18 families with genetically confirmed LGMD2A. Here we describe their clinical and laboratory features, magnetic resonance imaging (MRI) of lower legs and histopathological (HP) findings in muscle biopsy samples. Results: Eighteen (95\%) patients in this cohort had c.550delA mutation, with nine being homozygous. The age at onset ranged between 7 and 40 years (median 14 years). Only one patient had disease onset after age of 22 (at age of 40). Symptoms usually started as a proximal leg weakness and in three (16\%) patients as a proximal arm weakness. Majority of patients were symptomatic at time of diagnosis, but in two of them the disease was discovered during analysis of asymptomatic hyperCKemia, while one was diagnosed as an asymptomatic family member. Serum CK levels were elevated in all patients from about four to 100 times higher than normal. EMG showed a myopathic pattern in all subjects. Mean age at the last examination was $25 \pm$ 10 years with mean disease duration of $12 \pm 5$ years. 
Proximal leg and (to lesser degree) proximal arm muscle wasting and weakness was the most prominent feature. Two patients started to use wheelchair at the age of 19 and 27, one used a walker and one cane. Scapular winging was present in $16(84 \%)$ patients. Ankle contractures were present in all CAPN3 subjects, while three (16\%) also had elbow contractures. Muscle hypertrophy was detected in ten (53\%) patients: seven had calf, two proximal leg and one lower arm hypertrophy. The facial and bulbar muscles were not affected. None of the patients had significant conduction defects nor cardiomyopathy. Four $(21 \%)$ patients exhibited spirometry restriction (FVC $<90 \%)$. Muscle MRI showed more severe affection of the proximal leg muscles, with triceps surae muscle being the most affected among distal muscles. Muscle HP showed dystrophic pattern with eosinophilic infiltrations in some patients. Conclusion: Calpainopathy is a common form of LGMD in Serbia and it has specific clinical, laboratory, MRI and HP features.

\section{PS1Group1-081 / \#408}

Topic: Group 1 - Muscle Diseases of Genetic Origin and Acquired Myopathies: Clinical Features, Pathophysiology, Therapy

\section{MALIGNANT HYPERTHERMIA AND MH-LIKE REACTIONS IN NEUROMUSCULAR DISEASES}

\author{
Bram De Wel $^{1}, \underline{\text { Kristl G. Claeys }}{ }^{2}$ \\ ${ }^{1}$ Neurology, University Hospitals Leuven And Ku \\ Leuven, Leuven, BE; ${ }^{2}$ University Hospitals Leuven \\ And Ku Leuven, Leuven, BE
}

Background: Malignant Hyperthermia $(\mathrm{MH})$ is a life-threatening hypermetabolic reaction in susceptible patients caused by an excessive calcium release from the sarcoplasmic reticulum in skeletal muscles in response to primarily volatile halogenated anesthetics and the depolarizing muscle relaxant succinylcholine. Mutations in the gene encoding the ryanodine receptor $1(R Y R 1)$ are the most frequently identified underlying genetic defects, but mutations in other genes have also been described such as CACNA1S, STAC3, SCN4A and CACNL2A. Over the years, case reports have linked an increasing number of muscular phenotypes to MH susceptibility. This can cause uncertainty in the clinical neurological practice, sometimes leading to excessive labeling of patients as MH susceptible, or even worse, missing the diagnosis. MH-like reactions in response to succinylcholine (and possibly volatile anesthetics) also exist and are associated with severe hyperkalemia and rhabdomyolysis, but are thought to be of a different etiology than true MH. The aim of our study is to explore all muscular disorders that can possibly be linked with $\mathrm{MH}$ or $\mathrm{MH}-$ like reactions and to suggest an appropriate approach to interpret the accompanying risks. Methods: We performed a systematic and comprehensive search using Pubmed for studies published from the 1960's up to January 2018. Search terms such as 'Malignant Hyperthermia', 'Malignant Hyperthermia susceptibility', 'Malignant Hyperthermia genetics' and 'RYR1 myopathies' were used. We included case reports, reviews and retrospective cohort studies. In addition, we incorporated data published on the 'European Malignant Hyperthermia Group' website. Results: Throughout the literature ( $\mathrm{n}=1012$ papers), all patients with myopathies caused by RYRl mutations are assumed MH susceptible, unless an in vitro contracture test has proven otherwise. This is a good practical assumption, although theoretically it is estimated that only about $30 \%$ of these patients are actually $\mathrm{MH}$ susceptible. The spectrum of myopathies and clinical syndromes linked to MH through RYR1 mutations now includes - amongst others - central core disease, multiminicore myopathy, congenital myopathy with cores and rods, King-Denborough syndrome, centronuclear myopathy, and nemaline myopathy. In addition, idiopathic hyperCKemia and exertional rhabdomyolysis are also part of the RYR1disease spectrum. Mutations in CACNA1S mainly cause periodic paralysis and mutations in STAC3 are known to be the cause of Native American myopathy. Finally, there are muscle diseases that were long presumed to be at an increased risk of developing $\mathrm{MH}$, such as Myotonic Dystrophy type I, that are now known to be at an equal risk of developing $\mathrm{MH}$ in response to volatile anesthetics compared to the general population. However, succinylcholine should still be avoided in these patients as there can occur unpredictable muscle contractures after administration and sometimes even an exaggerated hyperkalemic response (i.e. MH-like reaction). The muscular dystrophies such as Duchenne and Becker muscular dystrophy can also be associated with a MH-like reaction to succinylcholine. Conclusion: We provide a summary of current evidence linking certain neuromuscular diseases to $\mathrm{MH}$ or $\mathrm{MH}$-like 
reactions peri-operatively. Our study results in a guide for the practicing clinician, in order to know when peri-operative precautions need to be taken or advised in patients with diverse muscular diseases.

\section{PS1Group1-082 / \#493}

Topic: Group 1 - Muscle Diseases of Genetic Origin and Acquired Myopathies: Clinical Features, Pathophysiology, Therapy

\section{ORO-PHARYNGEAL DYSPHAGIA IN MYOTONIC DYSTROPHY TYPE 1 (DM1): IDENTIFICATION OF SENSORY CHANGES IMPACTING SWALLOWING FUNCTION}

\author{
$\underline{\text { Jodi E. Allen }}{ }^{1}$, Chris Turner ${ }^{2}$ \\ ${ }^{1}$ Therapy And Rehabilitation, The National \\ Hospital for Neurology and Neurosurgery, 3BG, \\ $G B ;^{2}$ Institute Of Neurology, University College \\ London Hospital MRC Centre for Neuromuscular \\ Diseases, $B G, G B$
}

Background: Aspiration pneumonia is the leading cause of mortality in adults living with Myotonic Dystrophy Type 1 (DM1). Swallowing disturbance (oro-pharyngeal dysphagia, OPD) contributes to this profile of recurrent respiratory infection, hospital admission and premature death. Patients with DM1 rarely report alterations in their swallowing despite its high prevalence. It is currently unclear as to why this lack of complaint occurs but absence of patient report does have implications for identification of OPD and its management. This study explored the possibility of sensory alterations in DM1 as a reason for poor patient reporting by studying patient responses to post-swallow residues, aspiration and/or penetration which normally provoke an overt set of clinical signs and symptoms. Methods: A retrospective review of swallowing reports was undertaken for all adult patients with genetically confirmed DM1 referred for instrumental evaluation of swallowing via Videofluoroscopy (VFS) or Fibreoptic Endoscopic Evaluation of Swallowing (FEES) from a tertiary neuromuscular centre between May 2015-February 2018. Reports were analysed for presence of pooled secretions (FEES only), post swallow residues, awareness of residues and presence and response to aspiration and penetration. Results: A total of 39 VFS reports and 8 FEES reports were reviewed. Videofluoroscopy: 38/39 (97\%) patients had post-swallow residues in the pharynx; 9 (24\%) were not aware, 21 (55\%) were partially aware, 7 (18\%) had good awareness and 1 was not reported. $6 / 39(15 \%)$ aspirated, 5 showed no response. $12 / 39$ (31\%) had food and/or drink penetrating the airway, 9 showed no response. FEES: $7 / 8$ $(88 \%)$ had pooled saliva at rest, 5 of whom had secretions in the laryngeal vestibule. $8 / 8(100 \%)$ had post-swallow residues; 2 showed no awareness, 4 showed partial awareness, 2 were not reported. 2/8 aspirated, both without response. $5 / 8$ penetrated without response. Conclusion: Post-swallow residues occur frequently as part of the OPD profile in DM1. In this study, over three quarters of patients with residues were not fully aware of them. Reduced awareness of these residues plus absence of response to aspirated and penetrated material strongly suggests an underlying sensory deficit. Altered sensation has not previously been explored in DM1 but could provide one explanation for poor reporting of OPD symptoms. Altered sensation is of clinical significance in both the identification and management of OPD. Reliance on patient report, in the presence of sensory deficits, is likely to lead to delayed or inaccurate diagnosis; putting patients at risk of unexpected chest infections and unplanned hospital admissions. Effective OPD management frequently relies on good sensory awareness in the pharynx and larynx. In the absence of this, patients may not recognise the benefits of swallow management advice, nor self-monitor its effectiveness. This is the first step in better understanding non-motor changes in OPD associated with DM1. Further research will explore the nature and aetiology of these sensory changes as well as relationships between OPD and other clinical markers with the aim of identifying more reliable tools for detection of OPD in this population. In the meantime clinicians should exercise caution when relying solely on patient report in the detection and management of swallowing problems. 


\section{PS1Group1-083 / \#609}

Topic: Group 1 - Muscle Diseases of Genetic Origin and Acquired Myopathies: Clinical Features, Pathophysiology, Therapy

\section{HMGCR-MYOPATHY. A RARE BUT STILL A SEVERE DISEASE}

$\underline{J o s e p ~ M . ~ G r a u ~}^{1}$, Pedro J. Moreno ${ }^{1}$, Sergio PrietoGonzález', Albert Selva-O'Callaghan², Odette Viñas $^{3}$, José C. Milisenda ${ }^{1}$

${ }^{I}$ Internal Medicine, Hospital Clinic de Barcelona, Barcelona, ES: ${ }^{2}$ Internal Medicine, Hospital Vall d'Hebrón, Barcelona, ES; ${ }^{3}$ Immunology, Hospital Clinic de Barcelona, Barcelona, ES

Background: The term HMGCR-myopathy has been recently coined in order to define the cases presenting with subacute proximal muscle weakness, high values of $\mathrm{CK}$, necrotizing myopathy with minimal or no inflammation in muscle biopsy and the detection of the anti HMGCR antibodies. Nevertheless the clinical picture, associated manifestations as well as its management remain controversial.
Methods: Our aim was to present our series of HMGR-myopathy patients observed in a single Center at the Muscle Research Group In the Hospital Clínic of Barcelona. Results: Fourteen cases were identified. They came from a series of 1,200 muscle biopsies in the past 10 years. Relevant information is expressed in table 1. Table 1. Patient's relevant data. CMP: cardiomyopathy; PR:partial reponse: CR: complete response. Conclusion: After a median follow-up of 38,7 months, only 4 patients are in complete remission without any immunosuppressive drug. Fifty percent required IV Ig and 30\% RTX for their refractority to prednisone plus an immunosuppressive agent such as MTX, AZA or TK. All patients but one were exposed to statins. Of note was the severe dysphagia in two patients and the simultaneous and reversible cardiomyopathy in other two cases. Although necrotizing immunomediated myopathy was the pathological diagnosis in 12 cases, one case exhibited only a few regenerating fibers (untreated patient) and typical features of dermatomyositis were found in another case. The poorest outcome was observed in younger patients.

\begin{tabular}{|c|c|c|c|c|c|c|c|c|c|c|}
\hline Muscle patholoy & Gender & Age & Associations & CK & $\begin{array}{l}\text { Follow-up } \\
\text { (months) }\end{array}$ & $\begin{array}{l}\text { Treatment } \\
\text { PDN }\end{array}$ & AZA/MTX/TC & IG. IV. & RTX & Outcome \\
\hline IMNM & M & 57 & Paravertebral & 6200 & 42 & Yes & Yes & $\begin{array}{c}6 \\
\text { cycles }\end{array}$ & Yes & PR \\
\hline IMNM & M & 79 & No & 7420 & 18 & Yes & Yes & No & No & $\begin{array}{l}\text { Dead.Unrelated } \\
\text { cause }\end{array}$ \\
\hline IMNM & F & 71 & No & 4230 & 72 & Yes & Yes & No & No & PR \\
\hline IMNM & $\mathrm{F}$ & 81 & No & 620 & 16 & Yes & No & No & No & PR \\
\hline IMNM & $\mathrm{F}$ & 70 & No & 1240 & 20 & Yes & Yes & No & No & PR \\
\hline IMNM & $\mathrm{F}$ & 84 & No & 579 & 15 & Yes & No & No & No & $\mathrm{CR}$ \\
\hline IMNM & $\mathrm{F}$ & 51 & No & 5340 & 12 & Yes & Yes & $\begin{array}{c}6 \\
\text { cycles }\end{array}$ & Yes & PR \\
\hline IMNM & M & 66 & Dysphagia & 5204 & 13 & Yes & Yes & $\begin{array}{c}6 \\
\text { cycles }\end{array}$ & No & PR \\
\hline Regeneration & M & 77 & No & 2890 & 8 & No & No & No & No & $\mathrm{CR}$ \\
\hline DM & M & 48 & Dysphagia & 7660 & 108 & Yes & Yes & $\begin{array}{c}12 \\
\text { cycles }\end{array}$ & Yes & Poor response \\
\hline IMNM & $\mathrm{F}$ & 64 & CMP & 5980 & 50 & Yes & Yes & $\begin{array}{c}8 \\
\text { cycles }\end{array}$ & Yes & Poor response \\
\hline IMNM & M & 60 & No & 11330 & 72 & Yes & Yes & $\begin{array}{c}12 \\
\text { cycles }\end{array}$ & Yes & PR \\
\hline IMNM & M & 66 & No & 1650 & 60 & Yes & Yes & No & No & CR \\
\hline IMNM & M & 73 & $\begin{array}{c}\text { Dysphagia and } \\
\text { CMP }\end{array}$ & 6600 & 36 & Yes & Yes & $\begin{array}{c}6 \\
\text { cycles }\end{array}$ & No & $\mathrm{CR}$ \\
\hline
\end{tabular}




\section{PS1Group1-084 / \#639}

Topic: Group 1 - Muscle Diseases of Genetic Origin and Acquired Myopathies: Clinical Features, Pathophysiology, Therapy

\section{VALUE OF DIAGNOSTIC ALGORITHM IN ASYMPTOMATIC HYPER-CK-EMIA AND ITERATIVE RHABDOMYOLYSIS}

\author{
Josep M. Grau ${ }^{1}$, José C. Milisenda ${ }^{2}$, Francesc \\ Cardellach $^{2}$, Judith García ${ }^{3}$ \\ ${ }^{1}$ Internal Medicine, Hospital Clínic, Barcelona, \\ ES; ${ }^{2}$ Internal Medicine, Hospital Clinic de \\ Barcelona, Barcelona, ES $;^{3}$ Biochemistry, Hospital \\ Clinic de Barcelona, Barcelona, ES
}

Background: Asymptomatic HiperCkemia (AHCK-emia) as well as iterative rhabdomyolysis (IR) still represent a diagnostic challenge. After the comprobation (three different samples, a week apart) of the raised CK values, and after rulling out the common causes of raised CK (strenuous exercise, illicit drugs, alcoholism, hypothyroidism, acute viral infection, seizures, surgery, trauma, dyselectrolitemia and apnea-hypopnea syndromes, among others) we propose a diagnostic algorithm. Methods: The first step in the algorithm includes a simultaneous triple approach. First the Ischemic forearm test (grip test), second, dried blood spot for excluding Pompe disease and third acylcarnitines profile (betaoxidative dysfunctions). If the diagnosis is not yet reached, the next steps include whole-body muscle MRI and muscle biopsy, with genetic studies, mitochondrial respiratory chain activity and biochemical studies in fibroblasts culture, when indicated. Results: From January 2016 to December 2017, 25 well-selected patients were included for either AHCK-emia or IR. After sequential tests as indicated a definite diagnosis was obtained in 13 cases. The diagnosis were as follows: muscle dystrophies 5, glucogenosis 3, acylcoA multiple dehydrogenase deficiency 2 , congenital myopathies 2 and myoadenilate deficiency 1 . In the remaining 12 cases, all the tests, including genetics were normal. Conclusion: The proposed algorithm allows the diagnosis in only $50 \%$ of the cases. Of note is that in near $50 \%$ of the cases a definite diagnosis was not achieved.
PS1Group1-085 / \#760

Topic: Group 1 - Muscle Diseases of Genetic Origin and Acquired Myopathies: Clinical Features, Pathophysiology, Therapy

\section{NEXT GENERATION SEQUENCING OF DYSTROPHIN GENE IN A COHORT OF NON-DELETION/ DUPLICATION DMD/BMD EGYPTIAN PATIENTS}

\author{
Nagia Fahmy $^{1}$, Nasser Elhawary ${ }^{2}$, Nermin Elsayed ${ }^{1}$, \\ Sabin Schröder ${ }^{3}$, Martin Werber ${ }^{3}$ \\ ${ }^{1}$ Ain Shams University, Ain Shams University, \\ Cairo, EG ; ${ }^{2}$ Umm El-qura University, Umm \\ El-Qura University, Makkah, SA, ${ }^{3}$ Centogene Ag, \\ Centogene AG, Rostock, DE
}

Background: The diagnosis of Duchenne/Becker muscular Dystrophy (DMD/BMD) is still challenging owing to the large size of the gene and the occurrence of many mutations. The clinical phenotype, $\mathrm{X}$-linked inheritance and high creatine kinase (CK) serum level are the corner stones in the diagnosis of DMD/BMD patients. The confirmed genetic diagnosis and accurate detection of the type and site of mutation of dystrophin gene became mandatory for the recent therapies and genetic interventions. Methods: We studied 32 patients with DMD/BMD phenotype (including 8 families with 2 affected members of each family) that had no deletion/duplication of MLPA of dystrophin gene. Patients were selected from those attending the outpatient myology and Neurology clinics, Neuromuscular unit, Faculty of Medicine, Ain Shams University, Cairo, Egypt. They had laboratory assessment included serum creatine kinase, nerve conduction and electromyography of both upper and lower limbs, Echocardiography and Multiplex Ligation-dependent Probe Amplification (MLPA) of dystrophin gene to diagnose large deletion/duplication. Muscle biopsy with histochemistry and immunohistochemistry was done to 6 patients and other patients preferred to continue further genetic study without biopsy. The dystrophin gene was analyzed in Centogene laboratories by PCR and next generation sequencing of DNA strands of the entire coding region and the highly conserved exon-intron splice junctions. Results: We found 11 patients with non-sense mutation (four of them were brothers; three of these mutations were not described before). A missense variant was detected in two 
brothers that were not described before, Small deletions were present in 8 patients, four of them were not described before and a patient with small duplication that was not described before. We found one patient with a hemizygous deletion encompassing intron 11 that was not covered by MLPA and another patient with a pathogenic variant predicted to disrupt the highly conserved donor splice site of exon 55 . Another patient showed failure of sequencing exons 48-50, so MLPA was repeated and showed deletion of those exons. No clinically relevant variants were found in 7 patients, 3 of them had the BMD phenotype and diagnosed by immunohistochemistry and western blot study. Conclusion: Next generation sequencing of dystrophin gene is mandatory to all DMD/BMD patients with no deletion/duplication of MLPA. Muscle biopsy and immunohistochemistry of dystrophin protein and dystrophin associated proteins is still highly recommended before doing detailed genetic study. RNA sequencing is needed for those patients who are pathologically proved to have dystrophinopathy and their DNA sequencing didn't show any mutations.

\section{PS1Group1-086 / \#823}

Topic: Group 1 - Muscle Diseases of Genetic Origin and Acquired Myopathies: Clinical Features, Pathophysiology, Therapy

\section{NATURAL HISTORY OF DISEASE- RELATED COMPLICATIONS IN PATIENTS WITH DUCHENNE MUSCULAR DYSTROPHY}

Jessie Lynch, Katherine Tsai, John Lu, Chris Mix, Andre M. York

Sarepta Therapeutics Inc, Cambridge, MA, US

Background: Duchenne muscular dystrophy (DMD) is a rare, $\mathrm{X}$-linked recessive, fatal disorder in which $D M D$ gene mutations inhibit production of dystrophin, leading to progressive muscle weakness. A current therapeutic approach for DMD involves use of phosphorodiamidate morpholino oligomers (PMOs) for exon skipping. These neutrally charged oligonucleotides target specific exons within the $D M D$ pre-messenger ribonucleic acid (mRNA), induce skipping and exclusion of the targeted exon, and restore the $D M D$ mRNA reading frame. Collectively, these actions facilitate translation and produc- tion of an internally shortened dystrophin protein. Clinical trials are ongoing to evaluate PMOs for use in DMD. Individuals diagnosed with DMD develop numerous complications throughout disease progression. Within the clinical trial setting, it is critical to differentiate complications associated with the disease and use of concomitant medications, such as corticosteroids, from adverse events potentially related to treatment. Methods: A review of the published literature was conducted to identify relevant articles evaluating the natural history of disease-related complications in DMD patients. Results: A majority of the literature focused on complications representing the two primary causes of death in DMD: cardiac failure, with cardiomyopathy as a significant factor, and respiratory failure, which stems from functional declines in the diaphragm and other respiratory muscles. The prevalence of cardiomyopathy (shortening fraction $<28 \%$ and/or ejection fraction $<55 \%$ ) increased with age and disease progression, impacting $5 \%$ of patients aged $0-5$ years, $38 \%$ of patients aged $14-17$ years, and $>60 \%$ of patients aged $\geq 18$ years. Age was an important predictor of cardiomyopathy presence, regardless of corticosteroid use. Similarly, impairments in respiratory function also worsened with DMD progression; the majority of data showed a linear decline of $\geq 5 \%$ annually in percent predicted forced vital capacity (FVC\%p). In some studies, decline in pulmonary function was largely unaffected by corticosteroid treatment regimen or ambulatory status. Clinical evidence suggested that DMD patients were also prone to numerous other complications, including nephrolithiasis, low bone mass density, rhabdomyolysis, and comorbid psychiatric conditions. In a logistic regression analysis, DMD was an independent risk factor for development of symptomatic nephrolithiasis (odds ratio, 14.26 vs nonambulatory, nonDMD control patients). Increased risk of low bone mass density or fracture was frequently reported and related to progressive muscle weakness, inflammatory cytokine release, continuous corticosteroid regimens, immobility, and vitamin D insufficiency, among other factors. Rhabdomyolysis is a less common but possibly fatal complication that may result from use of specific types of anesthesia in DMD patients. However, unprompted, acute episodes of rhabdomyolysis also occur in DMD patients, and many clinicians perceive this complication as a component. Finally, DMD progression was linked to comorbid psychopathologies, including intellectual disability, autistic spectrum disorder, attention defi- 
cit hyperactivity disorder, and behavioral problems. Conclusion: In conclusion, numerous comorbid conditions parallel DMD disease progression. Thorough understanding and awareness of these diseaserelated comorbidities is critical to differentiation from potential side effects of disease-modifying therapies, especially in the context of clinical investigation.

\section{PS1Group1-087 / \#790}

Topic: Group 1 - Muscle Diseases of Genetic Origin and Acquired Myopathies: Clinical Features, Pathophysiology, Therapy

\section{GOLODIRSEN LEADS TO SARCOLEMMAL DYSTROPHIN EXPRESSION IN PATIENTS WITH GENETIC MUTATIONS AMENABLE TO EXON 53 SKIPPING}

Francesco Muntoni ${ }^{1}$, Diane Frank ${ }^{2}$, Jennifer Morgan $^{3}$, Joana Domingos ${ }^{3}$, Frederick Schnell ${ }^{2}$, J. G. Dickson ${ }^{4}$, Michela Guglieri ${ }^{5}$, Andreea Seferian ${ }^{6}$, Mauro Monforte ${ }^{7}$, Laurent Servais ${ }^{6}$, Volker Straub ${ }^{8}$, Eugenio Mercuri ${ }^{7}$, Skip-Nmd Study Group ${ }^{9}$ ${ }^{1}$ Dubowitz Neuromuscular Unit, UCL Great Ormond Street Institute of Child Health, London, GB; ${ }^{2}$ Sarepta Therapeutics Inc, Cambridge, MA, US: ${ }^{3}$ University College London, London, $G B ;{ }^{4}$ Royal Holloway, University of London, London, GB:5 ewcastle University and Royal Victoria Infirmary Hospital, Newcastle upon Tyne, GB, Institute of Myology, Paris, FR ${ }^{7}$ Catholic University of the Sacred Heart, Rome, $I T ;{ }^{8}$ Newcastle University, Institute of Genetic Medicine, Newcastle Upon Tyne, GB $;{ }^{9}$ SKIP-NMD Study Group, Cambridge, MA, US

Background: Golodirsen (formerly SRP-4053) is a phosphorodiamidate morpholino oligomer (PMO) that binds to dystrophin mRNA, and targets and excludes exon 53 during mRNA processing, facilitating the production of internally shortened dystrophin protein. A 2-part, first-in-human, multicenter trial (ClinicalTrials.gov Identifier: NCT02310906) is evaluating the safety, tolerability, and efficacy of golodirsen in patients with Duchenne muscular dystrophy (DMD) and genetic mutations amenable to exon 53 skipping. We report dystrophin production, exon 53 skipping, and dystrophin cellular localiza- tion at baseline and after 48 weeks of treatment with golodirsen. Methods: Part 1 of this trial, which has been completed, included a randomized, doubleblind, placebo-controlled, 12-week dose escalation of golodirsen. Part 2, which is ongoing, is a 168week open-label evaluation of once-weekly golodirsen $30 \mathrm{mg} / \mathrm{kg}$. In preplanned interim analyses, eligible patients had paired muscle biopsies of the biceps brachii at baseline (part 1) and at Week 48 of once-weekly treatment with golodirsen (part 2). Patients newly enrolled in part 2 had the same biopsies at baseline of part 2 and at Week 48. A validated Western blot method quantified dystrophin production (primary biological end point). Exon 53 skipping was evaluated using reverse transcription polymerase chain reaction (RT-PCR). Immunohistochemistry assessed dystrophin localization and sarcolemma fiber intensity. Results: Biopsies were obtained and analyzed from all 25 patients. Mean percent of normal dystrophin protein increased from $0.095 \%$ at baseline to $1.019 \%$ at Week 48 , a significant mean change of $+0.924 \%(P<0.001)$. Muscle biopsy samples from all patients displayed a significant increase from baseline in exon 53 skipping via RT-PCR at Week $48(P<0.001)$, demonstrating the intended mechanism of action. A positive correlation between exon 53 skipping and de novo dystrophin production was observed (Spearman $\mathrm{r}=0.500$; $P=0.011$ ). Mean fiber intensity analysis showed a significant increase from baseline in de novo dystrophin production $(P<0.001)$ and confirmed correct dystrophin localization to the sarcolemma. Conclusion: Increased mean dystrophin protein production, exon 53 skipping, and correct sarcolemmal localization of dystrophin were observed in muscle biopsies from golodirsen-treated patients. Golodirsen is the second PMO shown to increase dystrophin protein expression and demonstrate membrane localization following initiation of exon skipping. These findings add to the growing body of evidence of a role for the PMO technology platform in the treatment of DMD. 


\section{PS1Group1-088 / \#945}

Topic: Group 1 - Muscle Diseases of Genetic Origin and Acquired Myopathies: Clinical Features, Pathophysiology, Therapy

\section{POMPE DISEASE IN FRANCE: MOLECULAR FEATURES, EPIDEMIOLOGY AND CLINICAL CORRELATIONS FROM A FOURTY- FIVE YEAR NATIONWIDE STUDY}

Claudio Semplicini ${ }^{1}$, Pascaline Letard ${ }^{2}$, Marie De Antonio $^{3}$, Nadjib Taouagh ${ }^{4}$, Barbara Perniconi $^{4}$, Françoise Bouhour ${ }^{5}$, Andoni Echaniz-Laguna ${ }^{6}$, David Orlikowski ${ }^{7}$, Sabrina Sacconi ${ }^{8}$, Emmanuelle Salort-Campana ${ }^{9}$, Guilhem Solé ${ }^{10}$, Fabien Zagnoli ${ }^{11}$, Dalil Hamroun ${ }^{12}$, Roseline Froissart ${ }^{13}$, Catherine Caillaud $^{14}$, Pascal Laforet ${ }^{15}$

${ }^{1}$ University of Padova - Azienda Ospedaliera di Padova, Padova, IT, ${ }^{2}$ Laboratoire de Biochimie Métabolomique et Protéomique, Hôpital Necker Enfants Malades, Paris, France, FR, ${ }^{3}$ Centre de référence des maladies neuromusculaires, $\mathrm{CHU}$ Henri-Mondor, AP-HP, Créteil, France., Paris, FR: ${ }^{4}$ Institut de Myologie, Hôpital La PitiéSalpétrière, AP-HP, Paris, France, Paris, FR, ${ }^{5}$ Service ENMG et pathologies neuromusculaires, Hôpital Neurologique Pierre Wertheimer, Hospices Civils de Lyon, Lyon, FR; ${ }^{6}$ Department of Neurology, University Hospital Strasbourg, Strasbourg, FR, ${ }^{7}$ Pôle de ventilation à domicile, AP-HP, Hopital Raymond Poincaré,, Garches, $F R{ }^{8}$ Centre de référence des Maladies Neuromusculaires, Hôpital Archet, France. CNRS UMR7277, INSERM U1091, IBV - Institute of Biology Valrose, UNS Université Nice SophiaAntipolis, Faculté de Médecine, Nice,

FR, ${ }^{9}$ Reference Center for Neuromuscular Diseases and ALS, La Timone University Hospital, AixMarseille University, Marseille, FR, ${ }^{10}$ Department of Neurology, Nerve-Muscle Unit, CHU Bordeaux (Pellegrin Hospital), University of Bordeaux, Bordeaux, FR, ${ }^{11}$ CHRU Cavale-Blanche,, Brest, FR; ${ }^{12}$ Direction de la Recherche et de l'Innovation, CHRU de Montpellier, Hôpital Arnaud de Villeneuve, Montpellier, FR, ${ }^{13}$ Service de Biochimie et Biologie Moléculaire, Centre de Biologie et Pathologie Est, Hospices civils de Lyon, Lyon, FR: ${ }^{14}$ INSERM U1151, Institut Necker Enfants Malades, and Université Paris Descartes, Sorbonne Paris Cité, Paris, FR, ${ }^{15}$ Centre de référence des maladies neuromusculaires Nord-Est-Ile de France, Service de Neurologie, CHU Raymond Poincaré, AP-HP, Garches, France. INSERM U1179, ENDICAP, équipe Biothérapies des Maladies du Système Neuromusculaire, Université Versailles Saint, Garches, FR

Background: Pompe disease (PD) is an autosomalrecessive disorder caused by the deficiency of the lysosomal enzyme acid $\alpha$-glucosidase (GAA) due to mutations in GAA gene. The clinical spectrum of disease ranges from rapidly fatal multisystemic disorder (classical PD) to a milder adult onset myopathy (late onset PD). There are no straightforward correlations between clinical features, rate of enzymatic reduction and type of mutations. Methods: Aim of the study was to identify all patients diagnosed with non classical PD in France since 1970s, in order to characterize molecular diagnoses and clinical features in a large nationwide study. This was possible by collecting data from the two main French biochemical laboratories (Paris and Lyon) and from the French Pompe registry, that includes since 2004 virtually all French PD patients in a structured long-term observational study. Results: We included 246 late onset PD patients diagnosed since 1972 (130F, 116M; mean age at diagnosis $43.1 \pm 17.5$ years). Eighty-three different variants were identified, spread all over the sequence and including all types of mutations, and 28 (34\%) were novel. The common c.-32-13T $>\mathrm{G}$ mutation was detected in 151/170 index cases (89\%). Other recurrent mutations included deletion exon 18 (7.5\%), c.1927G $>$ A, p.Gly643Arg (3.7\%), c.655G $>$ A, p. Gly219Arg (2.8\%) and the small deletion c.525del, p.Glu176fs*15 (2.5\%). The incidence of non-classic PD in France could be estimated around 1/80,000. Correlations between genotype and age at disease onset were weak. Patients with IVS1 mutation $(\mathrm{n}=131)$ presented a later onset compared to 18 nonIVS1 patients $(36.2 \pm 13,9$ vs $24,7 \pm 14,8 ; \mathrm{p}<0,01)$. However, patients presenting identical genotype could manifest at very different ages. Conclusion: In conclusion, in this study we present the molecular and epidemiologic data from the largest described cohort of non classic PD patients, in a nationwide study covering more than 40 years of this challenging diagnosis. The high molecular and clinical variability we described suggest the need of more population studies to identify potential disease modifiers. 


\section{PS1Group1-089 / \#987}

Topic: Group 1-Muscle Diseases of Genetic Origin and Acquired Myopathies: Clinical Features, Pathophysiology, Therapy

\section{A SPANISH MYOTONIC DYSTROPHY TYPE I FAMILY CARRYING INTERRUPTIONS SHOWING A MILDER AND ATYPICAL PHENOTYPE}

\author{
Emma A. Koehorst ${ }^{1}$, Alfonsina Ballester-Lopez ${ }^{2}$, \\ Miriam Almendrote ${ }^{3}$, Alba R. Fransi ${ }^{3}$, Alicia \\ Martínez-Piñeiro ${ }^{3}$, Giuseppe Lucente ${ }^{3}$, Ian Linares ${ }^{3}$, \\ Judith Nuñez ${ }^{3}$, Nicolau Guanyabens ${ }^{4}$, Alejandro \\ Lucia $^{5}$, Antoni Cano 5 , Teresa Casadevall ${ }^{6}$, Agustí \\ Rodríguez-Palmero ${ }^{3}$, Laura Monlleó-Neila ${ }^{7}$, \\ Guillem Pintos-Morell ${ }^{3}$, Jaume Coll-Cantí ${ }^{3}$, Gisela \\ Nogales-Gadea ${ }^{3}$ \\ ${ }^{1}$ Neuromuscular And Neuropaediatrics Group, \\ IGTP Research Center, Badalona, ES: ${ }^{2}$ Centro De \\ Investigación Biomédica En Red De Enfermedades \\ Raras (ciberer), Instituto de Salud Carlos III, \\ Madrid, ES, ${ }^{3}$ Neuromuscular And Neuropaediatrics \\ Group, IGTP research center, Badalona, \\ ES; ${ }^{4}$ Neurology Unit, Neuroscience Department, \\ Hospital de Mataró, Barcelona, ES: ${ }^{5}$ Universidad \\ Europea, Instituto de Investigación Hospital 12 de \\ Octubre (i+12), Madrid, ES; ${ }^{6}$ Neuromuscular Unit. \\ Neurology Service. Neuroscience Department, \\ Hospital Comarcal Sant Jaume de Calella, \\ Barcelona, ES: ${ }^{7}$ Neuropediatric Unit. Pediatric \\ Service., Hospital Universitari Germans Trias $i$ \\ Pujol, Barcelona, ES
}

Background: In recent years, patients with Myotonic Dystrophy type I (DM1) carrying variant repeat patterns in the CTG expansion, have been described. The phenotypical consequences of the interrupted CTG expansion are still highly controversial. The variant repeat patterns have been associated with a complex co-segregated neurological phenotype, including an intermediate Charcot-Marie-Tooth neuropathy, deafness and encephalopathy. While others have found a milder phenotype with a later age of onset, absence of muscle atrophy and CNS involvement or an atypical phenotype with symptoms more resembling Myotonic Dystrophy type II. The number of DM1 patients described with these interruptions is low, with an estimated prevalence of 3-5\%. In this study, a cohort of 50 Spanish DM1 patients has been analyzed with the aim of finding new patients carrying interruptions and determining their clinical phenotypes. Methods: Blood DNA was obtained from the participants and triplet-primed PCR (at the 5' and 3' sites), Acil digestion and sequencing was used to determine the presence of these interruptions and the type of interruption present (CCG, CTC or GGC). A patients' registry was built, containing their clinical information. Results: Out of our fifty DM1 patients, four patients were found to have CCG interruptions in the 3' end of the CTG expansion. Our clinical database revealed that these patients belong to the same family, in which patient 1,2 and 3 are sisters, who paternally inherited the disease, and patient 4 is the son of patient 3 . Mother and son showed the exact same interruption pattern, while the other 2 sisters both had a different interruption pattern. The male interrupted patient ( $\mathrm{P} 4$, aged 37 years old) was completely asymptomatic upon clinical examination, despite the 108 CTG progenitor allele. The 3 sisters showed most of the typical features of DM1, although predominant distal weakness was absent. The most remarkable thing is that in the 3 sisters' symptoms started at a later age ( $>50$ y.o.), classifying them as late-onset DM1, while their symptoms resemble more of a classic/adult phenotype. Conclusion: Here, we report the presence of four interrupted cases in a cohort of $50 \mathrm{DM} 1$ patients, with a prevalence of $3.4 \%$ in our DM1 families. Inheritability of the interruption pattern was shown in our cohort, with an exact replica on maternal transmission. Our study contributes to the observation of a milder and/or atypical clinical phenotype of variant repeat carrying DM1 patients, with a later age of onset than expected and predominantly proximal weakness. The latter resembles more a DM2 phenotype.

\section{PS1Group1-090 / \#977}

Topic: Group 1 - Muscle Diseases of Genetic Origin and Acquired Myopathies: Clinical Features, Pathophysiology, Therapy

\section{MERRF CLASSIFICATION: IMPLICATIONS FOR DIAGNOSIS, AND CLINICAL TRIALS}

$\underline{\text { Josef Finsterer }}^{1}$, Sinda Zarrouk-Mahjoub ${ }^{2}$, John M. Shoffner ${ }^{3}$

${ }^{1}$ Krankenanstalt Rudolfstiftung, Vienna, AT; ${ }^{2}$ Pasteur Institute Of Tunis, University of Tunis El Manar and Genomics Platform, Tunis, TN; ${ }^{3}$ Molecular Genetics, Neurology, Biochemical Genetics, Atlanta, US 
Background: Given the etiologic heterogeneity of disease classification using clinical phenomenology, we employed contemporary criteria to classify variants associated with myoclonic epilepsy with ragged-red fibers (MERRF) syndrome and to assess the strength of evidence of gene-disease associations. Standardized approaches are used to clarify the definition of MERRF which is essential for patient diagnosis, patient classification, and clinical trial design. Methods: Systematic literature and database search with application of standardized assessment of gene-disease relationships using modified Smith criteria and of variants reported to be associated with MERRF using modified Yarham criteria. Results: Review of available evidence supports a gene-disease association for two MT-tRNAs and for POLG. Using modified Smith criteria, definitive evidence of a MERRF gene-disease association is identified for MT-TK. Strong evidence gene-disease evidence is present for MT-TL1 and POLG. Functional assays that directly associate variants with oxidative phosphorylation impairment were critical to mtDNA variant classification. In silico analysis was of limited utility to the assessment of individual MT-tRNA variants. Using contemporary classification criteria, several mtDNA variants previously reported as pathogenic/possibly pathogenic are reclassified as neutral variants. Conclusion: MERRF is primarily a MT-TK disease with pathogenic variants in this gene accounting for $\sim 90 \%$ of MERRF cases. Although MERRF is phenotypically and genotypically heterogeneous, myoclonic epilepsy is the clinical feature that distinguishes MERRF from other categories of mitochondrial disorders. Given its low frequency in mitochondrial disorders, myoclonic epilepsy is not explained simply by an impairment of cellular energetics. Although MERRF phenocopies can occur in other genes, additional data is needed to establish a MERRF disease-gene association. This approach to MERRF emphasizes standardized classification rather than clinical phenomenology, thus improving patient diagnosis and clinical trials design.

\section{PS1Group1-091 / \#978}

Topic: Group 1-Muscle Diseases of Genetic Origin and Acquired Myopathies: Clinical Features, Pathophysiology, Therapy

\section{MITOCHONDRIAL MULTI-ORGAN DISORDER SYNDROME SCORE GENERATED FROM DEFINITE MITOCHONDRIAL DISORDERS}

\author{
$\underline{\text { Josef Finsterer }}^{1}$, Sinda Zarrouk-Mahjoub ${ }^{2}$ \\ ${ }^{1}$ Krankenanstalt Rudolfstiftung, Vienna, \\ AT; ${ }^{2}$ Pasteur Institute Of Tunis, University of Tunis \\ El Manar and Genomics Platform, Tunis, TN
}

Background: Mitochondrial disorders(MIDs) frequently present as mitochondrial multi-organ disorder syndrome(MIMODS) already at onset or evolve into MIMODS during the course. This study aimed to find which are the organs/tissues most frequently affected in MIMODS, which are the most frequent abnormalities within an affected organ, if there are typical MIMODS patterns, and to generate a MIMODS-score to assess the diagnostic probability for a MID. Methods: Retrospective evaluation of clinical, biochemical, and genetic investigations of adult, definite MIDs. Results: Included were 36 definite MID patients, 19 males, 17 females, aged 29-82y. The diagnosis was based on genetic testing $(n=21)$, on biochemical investigations $(n=17)$, or on both $(n=2)$. The number of organs most frequently affected was four ranging from 1-9. MIMODS was diagnosed in $97 \%$ of patients. The organs most frequently affected were the muscle $(97 \%)$, central nervous system (CNS) $(72 \%)$, endocrine glands $(69 \%)$, heart $(58 \%)$, intestines $(55 \%)$, and peripheral nerves (50\%). The most frequent CNS abnormalities were leucencephalopathy, prolonged visually-evoked potentials and atrophy. The most frequent endocrine abnormalities included thyroid dysfunction, short stature, and diabetes. The most frequent cardiac abnormalities included arrhythmias, systolic dysfunction, and hypertrophic cardiomyopathy. The most frequent MIMODS patterns were encephalomyopathy, encephalo-myo-endocrinopathy, and encepalomyo-endocrino-cardiopathy. The mean $\pm 2 \mathrm{SD}$ MIMODS score was $35.97 \pm 27.6$ (range: 11-71). A MIMODS score $>10$ was regarded as indicative of a MID. Conclusion: Adult MIDs manifest as MIMODS in the vast majority of the cases. Organs most frequently affected in MIMODS are the muscle, CNS, endocrine glands, and heart. A MIMODS score $>10$ suggests a MID. 


\section{PS1Group1-092 / \#238}

Topic: Group 1 - Muscle Diseases of Genetic Origin and Acquired Myopathies: Clinical Features, Pathophysiology, Therapy

\section{CLINICAL PRACTICE WITH STEROID THERAPY FOR DUCHENNE MUSCULAR DYSTROPHY, A CLINICIAN SURVEY IN ASIAN AND OCEANIA}

Fumi Takeuchi $^{1}$, Harumasa Nakamura ${ }^{2}$, Hirofumi Komaki ${ }^{2}$, Ichizo Nishino ${ }^{2}$, Shin'Ichi Takeda ${ }^{2}$, Ikuya Nonaka $^{2}$, Naohiro Yonemoto ${ }^{3}$

${ }^{1}$ The John Walton Muscular Dystrophy Research Centre, Newcastle University, Newcastle upon Tyne, GB $;^{2}$ National Center of Neurology and Psychiatry, Tokyo, JP, ${ }^{3}$ Department Of Biostatistics, Kyoto University School of Public Health, Kyoto, $J P$

Background: Several studies investigating current clinical practice for Duchenne muscular dystrophy (DMD) have been conducted in Western countries. However, there have been few studies in Asia and Oceania. This study aims to elucidate clinical practice for DMD related to steroid therapy among local experts in Asia and Oceania. Methods: The Asian and Oceanian Myology Center (AOMC) was established in 2001 to facilitate scientific communication and collaboration in neuromuscular disease field in Asian and Oceanian countries. As of 2016, the AOMC executive board members comprised 37 experts from 15 countries in Asia and Oceania (Australia, China, Hong Kong, India, Japan, Malaysia, Myanmar, New Zealand, Pakistan, Philippines, Singapore, South Korea, Taiwan, Iran, and Thailand). We enrolled local experts and/or clinicians who manage DMD patients from the regions by asking AOMC executive board members to nominate potential participants who may meet our inclusion criteria. We included clinicians who: 1) had experience in treating DMD patients; 2) were able to reply to the questionnaire in English. Responses were collected electronically with SurveyMonkey or by mail between December 2015 and June 2016. Responses by mail were inputted into SurveyMonkey on receipt. Data analysis was conducted in Microsoft Excel. The survey consisted of 17 questions regarding the respondent's personal information (name, gender, age, speciality, and working medical site) and their usual clinical practice for patients with DMD. Results: Among the 15 countries, the executive board members in 13 countries agreed with the study and nominated 148 clinicians. We sent our questionnaire to the 148 clinicians via email in December 2015, and 87 clinicians (Australia: 3, China: 5, Hong Kong: 8, India: 13, Japan: 20, South Korea: 5, Malaysia: 2, Myanmar: 8, Pakistan: 8, Philippines: 2, Singapore: 6, Taiwan: 5, and Thailand: 2 ) replied by July 2016 (total response rate 62\%). Among the 87 clinicians, 79 clinicians (91\%) were aware of DMD care recommendations from Bushby et al. (2010). Moreover, 83 clinicians (95\%) reported currently managing patients with DMD (i.e., between 2011 and 2015) and 4 clinicians (5\%) reported managing patients with DMD only before 2011. The number of DMD patients appeared to differ depending on clinicians, as well as on country. In China, there were more patients with DMD at ages 0-9 and 10-19 years old, while there were more patients $\geqq 20$ years old in Japan. Daily prednisolone/prednisone was the most popular treatment regimen at initiation $(\mathrm{n}=45,64 \%)$, but was less popular at maintenance $(n=39,56 \%)$. Steroid therapy for patients after loss of ambulation and medication for bone protection seemed to be controversial. Conclusion: Our results from an international survey of clinicians increase our knowledge of the epidemiology of DMD, as well as current clinical practice for DMD as reported by local experts in Asia and Oceania. It is important to expand registries of patients with DMD in Asia and Oceania and to accumulate real-world longitudinal patient data. These strategies will aid the study of the epidemiology and natural history and could improve treatment and care for patients with DMD worldwide.

\section{PS1Group1-093 / \#570}

Topic: Group 1-Muscle Diseases of Genetic Origin and Acquired Myopathies: Clinical Features, Pathophysiology, Therapy

\section{EXPERIENCES WITH BARIATRIC SURGERY IN PATIENTS WITH FSHD AND DM1}

Nicol Voermans

Neurology, Radboudumc, Nijmegen, $N L$

Background: Overweight and obesity are common in patients with facioscapulohumeral dystrophy (FSHD) and myotonic dystrophy type 1 (DM1). 
Lifestyle change is often challenging for patients with neuromuscular diseases, especially to increase physical activity. Bariatric surgery is a treatment option for adults with severe obesity in whom lifestyle interventions have not been effective. However, very little is known about the benefits and risks in patients with neuromuscular disorders. This study therefore aims to obtain insight into the patients' perspectives and experiences, the outcome, effects and risks of bariatric surgery in patients with FSHD and DM1. This is expected to improve counseling of patients with FSHD and DM1 who consider bariatric surgery. Methods: A qualitative study, consisting of 14 in-depth interviews with six patients (three FSHD and three DM1), four relatives, three bariatric surgeons and one general practitioner. The study used a qualitative descriptive method. Thematic analysis was used to analyze the data. Results: Four themes were formulated: 1) Overweight as burden; 2) Bariatric surgery as last option; 3) Not your standard patient; and 4) A different life, a different me. Conclusion: This qualitative study shows that bariatric surgery has beneficial physical and mental effects for most patients with FSHD and DM1, and does not influence the muscular disease course. It shows that bariatric surgery is feasible in patients with FSHD and DM1, but specific precautions and a suitable follow-up including tailored dietary and training advices are required. A guideline for the patient and the procedure would be welcome.

\section{PS1Group1-094 / \#612}

Topic: Group 1 - Muscle Diseases of Genetic Origin and Acquired Myopathies: Clinical Features, Pathophysiology, Therapy

\section{AN UNUSUAL PRESENTATION OF GNE MYOPATHY WITH PROMINENT AXIAL MUSCLE WEAKNESS}

\author{
$\underline{\text { Jin-Mo Park }}^{1}$, Jin-Sung Park ${ }^{2}$ \\ ${ }^{1}$ Neurology, Dongguk University Gyeongju \\ Hospital, Gyeongju, KR; ${ }^{2}$ Department Of \\ Neurology, Kyungpook National University Chilgok \\ Hospital, Daegu, KR
}

Background: GNE myopathy is an autosomal recessive myopathy which is previously known as distal myopathy with rimmed vacuoles (OMIM
605820) or hereditary inclusion body myopathy (OMIM 600737). It is caused by biallelic mutation in $G N E$ which is a rate-limiting, bifunctional enzyme in the sialic acid pathway (Eisenberg I 2001). GNE myopathy is clinically characterized by early involvement of tibialis anterior muscle leading to early foot drop, with relatively spared quadriceps muscles. Pathologic study of GNE myopathy frequently shows "rimmed vacuoles" with fiber size variation (Argov Z 1984). However, diagnosis of GNE myopathy can be challenging due to phenotypic variability (Park YE, de Dios JK). We report a GNE myopathy patient with axial muscle involvement, probably triggered by repeated usage of truncal muscles. Methods: A twenty-three year-old man presented with back pain. He was serving in the military service for 6 months and his back pain started after strenuous military activities that require usage of back muscles. He had no past medical history or delay in the milestones. Results: The initial physical examination revealed axial muscle weakness, and mild ankle dorsiflexion weakness. He had hypoactive deep tendon reflexes and no pathologic reflexes. The initial laboratory data were unremarkable except for elevated creatinine kinase level of 1146 IU/L. The electromyography showed evidence of active myopathy with active denervation potentials observed in right tibialis anterior, medial gastrocnemius and thoracic paraspinal muscles. The T1weighted muscle MRI scans revealed mild fatty infiltrative and asymmetric atrophic changes in bilateral hamstring and soleus muscles. There were marked fatty infiltrations in bilateral lumbar paraspinal muscles. The muscle pathology in the left tibialis anterior muscle showed mild fiber size variation without significant inflammatory infiltrates, but showed rimmed vacuoles that were suggestive of GNE myopathy. The genetic test revealed a pair of compound heterozygous missense mutations in GNE gene, p.V572L and p.A591T, previously reported (NP_005467.1), that are one of the most common GNE mutations found in Korean and Japanese ethnicities. Conclusion: The decreased sialic acid synthesis due to bialleic mutation in UDP N-Acetylglucosamine 2-epimerase/N-Acetylmannosamine kinase leads to decreased GNE activity is known to cause GNE myopathy. A recent study reported a young male patient who presented with asymmetric hand weakness (De Dios JK, 2014). This report suggested that muscle overuse may be a potential environmental factor that can influence atypical clinical presentation in GNE myopathy. Our patient showed 
not only unusual onset of symptom but only unique manifestations. The most severe damage site was lumbar paraspinal muscles that is almost complete fatty replaced and atrophied. Meanwhile, anterior compartment of lower legs including tibialis anterior muscle was normal. Several reported axial involvements of GNE myopathy (Argov Z, Chu CC, Park YE). A subset of myopathies that show significant involvement of the axial muscles are known as axial myopathy currently. GNE myopathy is not a disease that is addressed for differential diagnosis (Witting 2016). GNE myopathy has phenotypic variability. In GNE myopathy, environmental factor such as repeated muscle overuse might contribute disease onset and onset site.

\section{PS1Group1-095 / \#761}

Topic: Group 1 - Muscle Diseases of Genetic Origin and Acquired Myopathies: Clinical Features, Pathophysiology, Therapy

\section{HIGHER MRI MUSCLE FAT} FRACTION AT SIMILAR AGE IS ASSOCIATED WITH EARLIER LOSS OF AMBULATION IN DUCHENNE MUSCULAR DYSTROPHY

Karin J. Naarding ${ }^{1}$, Harmen Reyngoudt ${ }^{2}$, Erik W. Van Zwet ${ }^{3}$, Melissa T. Hooijmans ${ }^{1}$, Brenda L. Wong ${ }^{4}$, Cuixia Tian ${ }^{4}$, Irina Rybalsky ${ }^{4}$, Karen C. Shellenbarger ${ }^{4}$, Julien Le Louër ${ }^{2}$, Pierre G. Carlier ${ }^{2}$, Hermien E. Kan ${ }^{5}$, Erik H. Niks ${ }^{6}$

${ }^{1}$ Neurology, Leiden University Medical Center, Leiden, NL; ${ }^{2}$ Aim \& Cea Nmr Laboratory, Neuromuscular Investigation Center, Institute of Myology, Paris, FR ${ }^{3}$ Department Of Biostatistics, Leiden University Medical Center, Leiden, NL; ${ }^{4}$ Neurology, Cincinnati Children's Hospital Medical Center, Cincinatti, OH, US, ${ }^{5}$ C.j. Gorter Center For High Field Mri, Leiden University Medical Center, Leiden, $N L ;{ }^{6}$ Neurology, Leiden University Medical Center, Leiden, $N L$

Background: Duchenne muscular dystrophy (DMD) is characterized by progressive replacement of muscle with fat. Full market-approved drugs are lacking. Clinical trials are challenging because of the low prevalence and practical and ethical issues in performing long placebo-controlled studies in children. Muscle fat fraction (FF), measured by quantitative
MRI, is considered a potential surrogate endpoint in trials since it is objective and non-invasive. However, such biomarkers are only acceptable as endpoints to regulatory agencies if they are related to clinically meaningful milestones. Therefore, we aimed to assess the additive predictive value of vastus lateralis (VL) FF to age on the loss of ambulation (LoA). Methods: 3-point gradient-echo Dixon images of the right upper leg of DMD patients were acquired at two centers, LUMC and CCHMC, between 2013 and 2016. At the LUMC, images were acquired at baseline, 12 and 24 months on a $3 \mathrm{~T}$ MR scanner. At CCHMC, scans were acquired on a $1.5 \mathrm{~T}$ MR scanner at baseline, 6, 12 and 18 months. Weighted mean VL FF was calculated using five slices at the mid-upper leg. FF's were fitted to a logistic growth-model to predict FF from 0-20 years of age. Slopes of FF curves differed per patient based on FF. Month and year of LoA were determined through a detailed interview with patients and parents, either during regular clinical follow-up, or by telephone between July 2017 and February 2018. This phone call also defined last follow-up for ambulant patients. We fitted a Cox model for the time to LoA with predicted VL FF as the only (time-varying) covariate. Results: At least one VL datapoint was available for 19 LUMC DMD patients (median baseline age 8.9 years, range 5.6-16.1, seven non-ambulant), and for 16 from CCHMC (median baseline age 11.2 years, range 7.615.1, all ambulant). Between first MRI and February 2018 LoA occurred in ten DMD patients. VL FF data in relation to age of 35 DMD patients and their superimposed logistic growth-curves are presented in Figure 1. The hazard ratio of a percent increase in VL FF for the time to LoA was estimated at $1.13(95 \%$ confidence interval 1.07-1.18) which was highly sig-

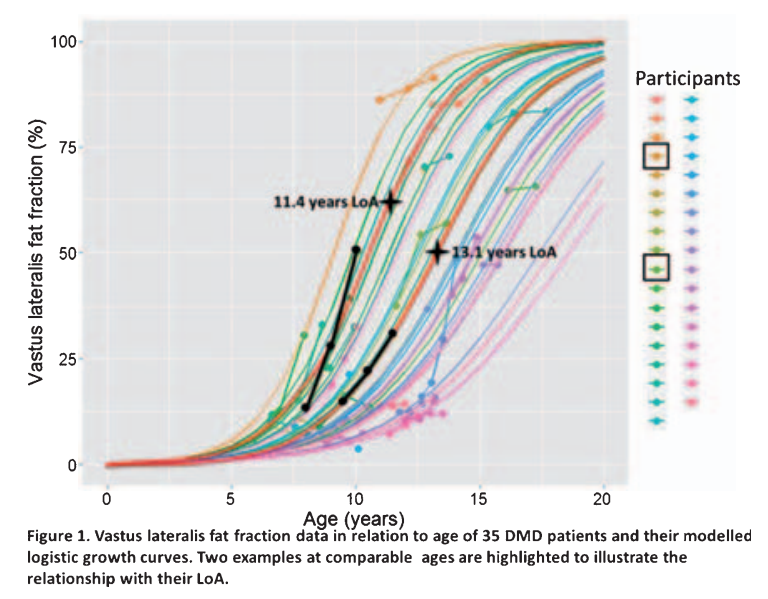


nificant (Wald test, $\mathrm{p}<0.001$ ). Conclusion: Vastus lateralis fat fraction has a significant additive predictive value to age on the date of loss of ambulation. This direct relation supports the use of quantitative MRI as an outcome measure for future clinical trials in DMD.

\section{PS1Group1-096 / \#1018}

Topic: Group 1-Muscle Diseases of Genetic Origin and Acquired Myopathies: Clinical Features, Pathophysiology, Therapy

\section{TOR1A VARIANTS CAUSE A SEVERE ARTHROGRYPOSIS WITH DEVELOPMENTAL DELAY, STRABISMUS AND TREMOR}

Ariana Kariminejad ${ }^{1}$, Martin Dahl-Halvarsson², Gianina Ravenscroft ${ }^{3}$, Fariba Afroozan ${ }^{1}$, Elham Keshavarz ${ }^{4}$, Hayley Goullée ${ }^{3}$ ${ }^{1}$ Kariminejad-Najmabadi Pathology \& Genetics Center, Tehran, IR; ${ }^{2}$ Department Of Pathology, University of Gothenburg, Sahlgrenska University Hospital, Gothenburg, SE, ${ }^{3}$ Centre For Medical Research, The University of Western Australia and the Harry Perkins Institute for Medical Research, Nedlands, AU; ${ }^{4}$ Department Of Radiology, Mahdieh Hospital, Shahid Beheshti University of Medical Science, Tehran, IR

Background: Autosomal dominant torsin dystonia 1 is a disease with incomplete penetrance most often caused by an in-frame GAG deletion (p.Glu303del) in the endoplasmic reticulum luminal protein torsinA encoded by TOR1A. Methods: 25 cases of AMC with congenital contractures in more than two joints and in multiple body areas were collected through international collaboration and medical history, physical examination and imaging were performed. Next-generation sequencing was performed on DNA from patients. Results: We report an association of the homozygous dominant disease-causing TOR $1 A$ p.Glu303del mutation, and a novel homozygous missense variant (p.Gly318Ser) with a severe arthrogryposis phenotype with developmental delay, strabismus and tremor in three unrelated families. All parents who were carriers of the TORIA variant showed no evidence of neurological symptoms or signs, indicating decreased penetrance similar to families with autosomal dominant torsin dystonia 1 . The results from cell assays demonstrate that the
p.Gly318Ser substitution causes a redistribution of torsinA from the endoplasmic reticulum to the nuclear envelope, similar to the hallmark of the p.Glu303del mutation. Conclusion: Our study highlights that TOR1A mutations should be considered in patients with severe arthrogryposis and further expands the phenotypic spectrum associated with TOR1A.

\section{PS1Group1-097 / \#877}

Topic: Group 1-Muscle Diseases of Genetic Origin and Acquired Myopathies: Clinical Features, Pathophysiology, Therapy

\section{NDUFAF3 VARIANTS THAT DISRUPT MITOCHONDRIAL COMPLEX I ASSEMBLY CAUSE ASSOCIATE WITH CAVITATING LEUKOENCEPHALOPATHY}

Akihiko Ishiyama $^{1}$, Kazuhiro Muramatsu ${ }^{2}$, Shunpei Uchino $^{3}$, Chika Sakai ${ }^{3}$, Yuichi Matsushima ${ }^{3}$, Nishiki Makioka $^{2}$, Tomomi Ogata ${ }^{2}$, Eriko Suzuki², Hirofumi Komaki ${ }^{1}$, Masayuki Sasaki ${ }^{1}$, Masakazu Mimaki $^{4}$, Yu-Ichi Goto ${ }^{3}$, Ichizo Nishino ${ }^{5}$ ${ }^{1}$ Department Of Child Neurology, National Center Hospital, National Center Of Neurology And Psychiatry, National Center Hospital, National Center of Neurology and Psychiatry, Kodaira, $J P,{ }^{2}$ Department Of Pediatrics, Gunma University Graduate School Of Medicine, Gunma University Graduate School of Medicine, Maebashi, $J P ;{ }^{3}$ Department Of Mental Retardation And Birth Defect Research, National Institute Of Neuroscience, National Center Of Neurology And Psychiatry, National Institute of Neuroscience, National Center of Neurology and Psychiatry, Kodaira, JP; ${ }^{4}$ Department Of Pediatrics, Faculty of Medicine, Teikyo University, Faculty of Medicine, Teikyo University, itabashi, JP, ${ }^{5}$ Department $O f$ Neuromuscular Research, National Institute Of Neuroscience, National Center Of Neurology And Psychiatry, National Institute of Neuroscience, National Center of Neurology and Psychiatry, Kodaira, JP

Background: Genetic abnormalities in mitochondrial complex assembling factors are associated with leukoencephalopathy. However, genotype-phenotype correlations remain elusive. Methods: We present a one-year-old girl with consciousness disturbance after a respiratory infection. Brain MRI re- 
vealed leukoencephalopathy with bilaterally symmetrical hyperintensity in the substantia nigra, medial thalamic nuclei, and basal nuclei, as well as cavities in the cerebral white matter and corpus callosum. Lactate levels in the spinal fluid were high, while magnetic resonance spectroscopy of the cerebral white matter and basal nuclei showed high peak lactate levels, suggesting mitochondrial dysfunction. After 2 months of hospitalization, her developmental status returned to that before admission. She acquired the ability to speak meaningful words at 2 years and 6 months and walked independently at 3 years. However, there was no deterioration in language comprehension, but her motor function deteriorated: her sitting posture became slightly unstable, and she could not walk without assistance at 4 years. Results: A muscle biopsy was performed on the biceps brachii at 3 years, revealing no pathological findings characteristic of mitochondrial-related diseases. Mitochondrial respiratory chain complex activities were analyzed in the skeletal muscle. Complex I activity levels, normalized to the activities of complex II and citrate synthase, were $17 \%$ and $21 \%$, respectively, but complex III and V activity levels were mildly decreased. Whole exome sequencing identified compound heterozygous variations in NDUFAF3, involved in the assembly of mitochondrial complex I (c.342_343insGTG:p.117 Valdup, c.505C $>$ A:p.Pro169Thr). Two-dimensional blue native polyacrylamide gel electrophoresis (PAGE) and sodium dodecyl sulfate-PAGE revealed reductions in Q-module (NDUFS2, NDUFS3, and NDUFA9) and P-module (NDUFB10 and NDUFB11) subunits, indicating disruption of mitochondrial complex I assembly. Conclusion: Few studies on the assembly of mitochondrial respiratory chain complexes have been conducted, and the phenotypes and pathologies of associated diseases are not completely understood. Our result suggests leukoencephalopathy with cavities associates with NDUFAF3 mutations and expands the clinical phenotypes associated to impaired assembly factor for mitochondrial complex I.
PS1Group1-098 / \#979

Topic: Group 1 - Muscle Diseases of Genetic Origin and Acquired Myopathies: Clinical Features, Pathophysiology, Therapy

\section{RESTRICTION ENZYME CLEAVAGE OF PCR PRODUCTS ALLOWS GENOTYPING MDX3CV, MDX4CV AND MDX5CV ALLELES WITHOUT SEQUENCING}

\author{
Laurence A. Neff, Elinam Gayi, Leonardo \\ Scapozza, Olivier M. Dorchies \\ School Of Pharmaceutical Sciences, University of \\ Geneva, Geneva, $\mathrm{CH}$
}

Background: Duchenne muscular dystrophy (DMD) is due to the absence of dystrophin. It is characterized by progressive muscle wasting and premature death. The exploration of the pathogenic mechanisms and the evaluation of therapeutic options rely mostly on dystrophic mice. The $m d x$ mouse originates from a spontaneous mutation that arose in a colony of C57BL/10 mice in the 1980's. Since then, it has become the most commonly used animal model of DMD. In the 1990's, several allelic variants of the $m d x$ mouse, namely the $m d x^{2 C v}, m d x$ ${ }^{3 C v}, m d x^{4 C v}$, and $m d x^{5 C v}$, have been recovered from chemical mutagenesis in the $\mathrm{C} 57 \mathrm{BL} / 6 \mathrm{~J}$ background. The different strains are characterized by differential expression of naturally occurring shorter isoforms (C-terminus truncated) of dystrophin in a tissue-dependent manner: The $m d x^{2 C v}, m d x^{3 C v}$ and $m d x^{4 C v}$ strains are useful for studying the roles of dystrophin and dystrophin-associated glycoprotein complex in non-muscle tissues such as the retina, kidney, and brain. The mutations carried by the $m d x^{4 C v}$ and $m d x$ ${ }^{5{ }^{C v}}$ mice are characterized by the absence of revertant fibres, an advantage for evaluating gene-, virus, or cell-mediated dystrophin restoration. Until now genotyping of this strains requires sequencing of PCR product. Here we propose a simple restriction base method allowing genotyping without sequencing. Methods: Sequence analysis revealed that the $m d x$ ${ }^{3 C v}$ and $m d x^{5 C v}$ alleles bear restriction sites for AluI, and HphI, respectively, which are absent from the wild type alleles. The $m d x^{4 C v}$ mutation disrupts a restriction site for Alw26I (BsmAI), which is normally present on the wild type allele. Based on these findings, we designed new strategies for genotyping these dystrophic strains using PCR amplification 
followed by enzymatic restriction of the PCR products. Results: Here we show that PCR followed by restriction digestion provides an easy mean to discriminate wt, heterozygous and homozygous mice bearing $m d x^{3-5 C v}$ alleles. We demonstrated the excellent stability to freeze-thaw cycles of the PCR and restriction enzyme master mixes and we optimized a number a protocol steps. The resulting protocols are user-friendly and allow straightforward genotyping at minimum cost and hands-on time. Conclusion: The $m d x^{3 C v}, m d x^{4 C v}$, and $m d x^{5 C v}$ strains all carry point mutations. Conventional genotyping involves PCR amplification, yielding amplicons of same sizes, followed by allele identification by sequencing, a step that is often outsourced, causing extra cost and delay in analysis. By contrast, our procedure is time and cost effective: genotyping is achieved within a day using standard molecular biology techniques and eliminates extra cost for sequencing.

\section{PS1Group1-099 / \#462}

Topic: Group 1 - Muscle Diseases of Genetic Origin and Acquired Myopathies: Clinical Features, Pathophysiology, Therapy

\section{CLINICO-PATHOLOGICAL CORRELATIONS IN IDIOPATHIC INFLAMMATORY MYOPATHIES}

Endre Pál $^{1}$, Katinka Gulyas², Tünde Minier ${ }^{2}$, Timea Berki $^{3}$, Laszlo Czirjak ${ }^{2}$, Cecilia Varju ${ }^{2}$ ${ }^{1}$ Neurology, University of Pécs, Pécs, $H U ;{ }^{2}$ Rheumatology And Immunology, University of Pécs, Pécs, HU; ${ }^{3}$ Immunology And Biotechnology, University of Pécs, Pécs, $H U$

Background: Idiopathic inflammatory myopathies (IIM) are heterogeneous systemic autoimmune diseases, characterised by muscle weakness, various organ involvements and the presence of specific autoantibodies. Our aims were to classify patients according to the clinical features, muscle histology findings and autoantibody profiles. Methods: 82 patients with muscle biopsy proven IIM were included in the study. Muscle biopsy, myositis specific (MSA) and myositis associated (MAA) antibody tests were performed in each patient before starting the immunomodulatory treatment. The MSAs and MAAs (Jo-1, PL-7, PL-12, Mi-2, SRP, Pm-Scl, Ku, ribosomal, AMA-M2) were tested with Western-blot kit. The ANA, dsDNA, ENA, Sm, Sm/RNP, SS-A and SS-B antibody examinations were performed with ELISA tests. Dermatomyositis/DM/, polymyositis /PM/, juvenile PM/DM, inclusion body myositis /IBM/, overlap myositis /OM/ and immune mediated necrotizing myopathy /IMNM/ were identified based on clinical data, immunserology and muscle biopsy. Survival analysis was perfomed by Kaplan Meier test. Results: 59 women and 23 men with a mean age of $49.3 \pm 14.6$ years were included. Mean follow-up of the patients was $7.5 \pm 4.5$ years. The distribution of myositis subsets was: $30.5 \%$ $(\mathrm{n}=25) \mathrm{DM}, 26.8 \%(\mathrm{n}=22) \mathrm{PM}, 22 \%(\mathrm{n}=18) \mathrm{OM}$, $11 \%(\mathrm{n}=9)$ IMNM, 8.5\% $(\mathrm{n}=7) \mathrm{IBM}$, and $1.2 \%$ $(n=1)$ juvenile PM/DM. Lung fibrosis $(51.2 \%)$, arthritis (51.2\%), Raynaud's phenomenon (42.7\%), skin symptoms (45.1\%), dysphagia (24.4\%) and significant cardiac involvement $(15.9 \%)$ were the most prevalent disease-manifestations. Fifteen cases were associated with malignancies, mjority of them was classified as IMNM ( 7 out of 9 patients). 18 patients died of myositis related events: 8 patients died of malignancies ( 4 in the IMNM, 2 in the PM and 2 in the DM group), 6 died related to cardiac conditions, 2 in lung fibrosis and 2 caused by infections. The worst prognosis with a 10 year survival of approximately $31.1 \%$ of patients was seen in the IMNM subgroup, followed by patients with PM (68.4\%) and DM (85.3\%). Patients with antisynthetase antibody-positivity had worse prognosis compared with patients with other antibodies or no identifiable antibodies. The worst prognosis was seen in patients with both MSA and MAA positivity and the best in patients with no identifiable MSA and MAA. Conclusion: Complex clinicopathologic assessment is recommended for classification and management of idiopathic inflammatory myopathy cases. Muscle histology is still an important diagnostic tool in IIMs. IMNM and PM are associated with worse prognosis compared to other types of IIMs. 


\section{PS1Group1-100 / \#537}

Topic: Group 1 - Muscle Diseases of Genetic Origin and Acquired Myopathies: Clinical Features, Pathophysiology, Therapy

\section{FIRST REPORTED VARIANT AT THE TRIM32 RING DOMAIN IN A PEDIGREE WITH LIMB-GIRDLE MUSCULAR DYSTROPHY AND BARDET-BIEDL SYNDROME}

$\underline{\text { Ana L. Pelayo-Negro }}^{1}$, Miguel A. FernándezGarcía $^{2}$, Patricia Blanco-Arias ${ }^{2}$, Elena GallardoAgromayor ${ }^{3}$, Gerardo Gutierrez-Gutierrez ${ }^{4}$, José Berciano $^{1}$

${ }^{1}$ Neurology, University Hospital Marqués de Valdecilla, Santander, ES, ${ }^{2}$ Neurology, Health in Code, A Coruña, ES ${ }^{\cdot}$ Radiology, University Hospital Marqués de Valdecilla, Santander, ES: ${ }^{4}$ Neurology, University Hospital Infanta Sofia, Madrid, ES
Background: Pathogenic variants in TRIM32 have been related to limb-girdle muscular dystrophy type 2H (LGMD2H, OMIM\#254110). To date, most reported variants lead to a truncated protein and are clustered at the C-terminal NHL-repeat domain. Whole gene deletions have also been described. Additionally, a missense change in the B-box motif was found in a family with Bardet-Biedl syndrome (BBS11, OMIM\#615988). Methods: A 60-year-old woman began in the 4th decade with LL weakness, inability to run or stand from sitting or lying position and slow progression with difficulties in walking, fatigue and frequent falls. She had mild atrophy of pelvic and thigh muscles, CK 250UI/L, myopathic EMG pattern; not willing to perform muscle biopsy to date. She was born of healthy consanguineous parents and had two affected siblings with a similar phenotype and hypogonadism. Additionally, she had a healthy brother and a daughter, aged 37 , that recently complained of fatigue and clumsiness with normal EMG and CK levels. Next-generation sequencing of 34 LGMD-related genes identified a homozygous

(i)

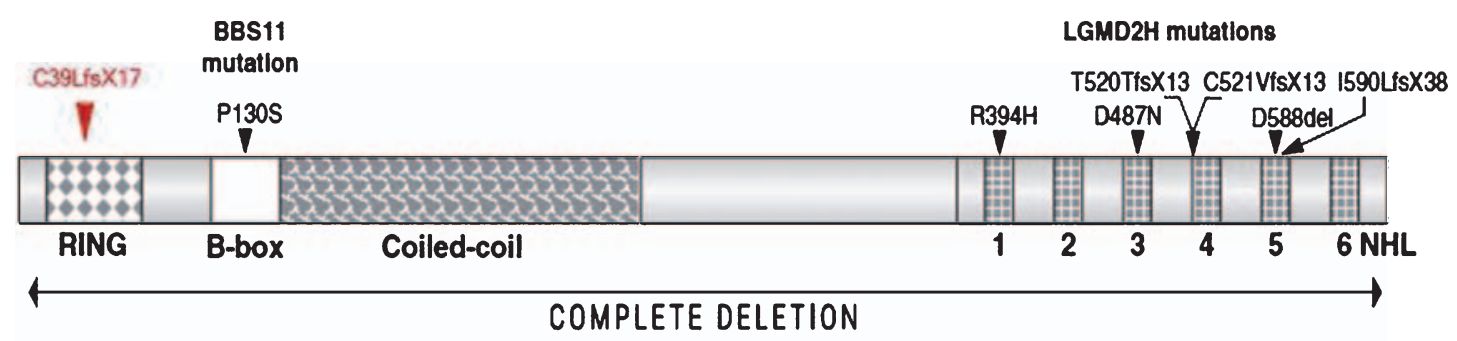

(ii)

\section{TRIM32}

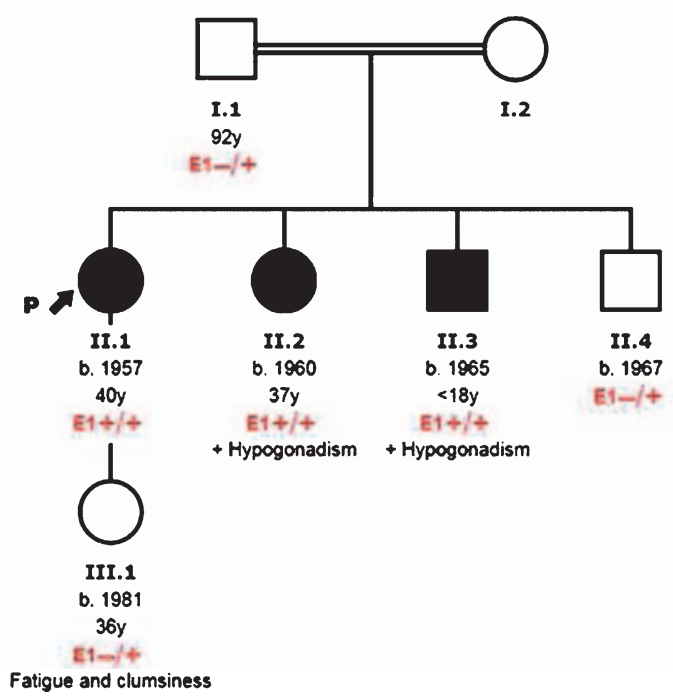

Figure1. (i) Schematic diagram of the TRIM32 protein domains, modified from Shieh et al. (2011), depicting the variants identified in $\mathrm{LGMO} 2 \mathrm{H}$ and BBS11 patients, including our variant (in red). (ii) Family tree, where black figures represent affected LGMD2H patients. 
1bp-insertion in TRIM32, predictably originating a frameshift (NM_012210.3:c.115_116insT; NP_036342.2:p.Cys39Leufs*17). Both affected siblings were also confirmed as homozygous; father, healthy brother and daughter were heterozygous carriers. Results: This is the first reported pathogenic variant at the TRIM32 N-terminal RING domain. Homozygous whole gene deletions confirmed that a total loss of protein function resulted in LGMD2H without greater clinical severity. Despite TRIM32 recessive behaviour, some descriptions of heterozygous carriers found mild clinical symptoms and vacuolar changes in muscle biopsies, suggesting a mild dominance pattern. In our family, father and brother of the index case (aged 92 and 50, respectively) had no neuromuscular abnormalities; in contrast, the daughter (37yo) complained about mild symptoms, suggesting the possibility of some light dominancy of the TRIM32 variant. On the other hand, TRIM32 has been linked to BBS11: a syndromic form of obesity with pigmentary retinopathy, renal anomalies, cognitive problems and hypogonadism. To date, only a Bedouin family carrying the $p$. Pro130Ser missense variant, located at the N-terminal B-box motif, has been described. However, none of the patients with complete TRIM32 deletion showed systemic features. In our family, two siblings have hypogonadism, suggesting the possibility of an association with BBS11. Conclusion: This would be the first report of BBS features in a LGMD2H affected family and should highlight the importance of a thorough phenotypic evaluation of TRIM32 patients to further delineate this association, particularly if other RING-domain pathogenic variants shall be identified.

\section{PS1Group1-101 / \#572}

Topic: Group 1 - Muscle Diseases of Genetic Origin and Acquired Myopathies: Clinical Features, Pathophysiology, Therapy

\section{MUSCLE SPECIFIC KINASE PROTECTS MDX MOUSE MUSCLES AGAINST ECCENTRIC CONTRACTION-INDUCED LOSS OF STRENGTH}

William D. Phillips, Joanne Ban, Sofie Trajanovska Physiology \& Bosch Institute, The University Of Sydney, Sydney, NSW, AU
Background: Muscles of mdx mice lack dystrophin, providing a mouse model for Duchenne muscular dystrophy (DMD). Eccentric contractions applied to mdx muscles cause an acute drop in force, reflecting the vulnerability of dystrophin-deficient muscles to injury. It has been reported that mdx muscles express less Muscle Specific Kinase (MuSK) and that this might predispose their neuromuscular junctions to eccentric contraction-induced damage. Methods: To increase the level of expression of MuSK we injected the tibialis anterior muscles of 8-week old male mdx and C57BL10 (wild-type background) mice with recombinant adeno-associated viral vector (AAV) encoding MuSK fused to green fluorescent protein (MuSK-GFP). Contralateral control muscles were injected with empty AAV vector. One month later the mice were anaesthetized with 2-3\% inhaled isoflurane/oxygen and maximum tetanic force was recorded in response to direct muscle stimulation or stimulation via the nerve. Eccentric contractions (forced lengthening to 1.25 time optimal length) were imposed during maximum (directly evoked) activation of the muscle in situ. Maximum isometric force was recorded before, and one hour after, a series of four such eccentric contractions. Results: Consistent with previous studies mdx muscles were more vulnerable to injury compared to C57BL10 wild-type mice. After the series of four eccentric (stretch) contractions, mdx muscles retained only $73 \pm 2 \%$ (mean \pm SEM) of their original (pre-stretch) maximum isometric force. In contrast, mdx muscles expressing MuSK-GFP retained significantly more of their original force after identical ECs $(84 \pm 1 \%$; $p<0.01)$. Rapsyn, an established downstream effector of MuSK signaling, conferred similar protection to $\mathrm{mdx}$ muscles. The protective effect was evident even when isometric force was elicited by direct muscle stimulation. Conclusion: These results indicate that enhanced MuSK signaling can protect dystrophin-deficient muscle fibers from acute eccentric contraction-induced loss of force. Moreover they suggest that the MuSK/rapsyn signaling pathway can act downstream of neuromuscular transmission to protect the sarcolemma of from tension-induced injury. 


\section{PS1Group1-102 / \#645}

Topic: Group 1 - Muscle Diseases of Genetic Origin and Acquired Myopathies: Clinical Features, Pathophysiology, Therapy

\section{NOVEL LMNA GENE MUTATION PRESENTING WITH DILATED CARDIOMYOPATHY AND LIMB- GIRDLE MUSCULAR DYSTROPHY TYPE 1B}

\author{
Martha Spilioti ${ }^{1}$, Konstantinos Notas ${ }^{1}$, George \\ Stavropoulos $^{2}$, Georgios Efthimiadis ${ }^{3}$, Kleita \\ Michaelidou ${ }^{4}$, Ioannis Zaganas ${ }^{4}$, Maria Moschou \\ Marianthi Arnaoutoglou ${ }^{1}$, Magdalini Tsolaki ${ }^{1}$ \\ ${ }^{1} 1$ st Department Of Neurology, Ahepa General \\ Hospital, Aristotle University of Thessaloniki, \\ Thessaloniki, GR;22nd Cardiology Department, \\ Hippokration General Hospital, Aristotle \\ University of Thessaloniki, Thessaloniki, GR: ${ }^{3} 1$ st \\ Cardiology Department, Ahepa University \\ Hospital, Aristotle University of Thessaloniki, \\ Thessaloniki, GR: ${ }^{4}$ Department Of Neurology, \\ School Of Medicine, University of Crete, \\ Heraklion, Crete, GR
}

Background: Lamins A and C, encoded by the lamin $\mathrm{A} / \mathrm{C}$ gene ( $L M N A)$, are major components of the nuclear lamina, a fibrillar network that sustains the structural integrity and stability of the nuclear envelope. Additionally, lamins are involved in multiple cellular processes, such as chromatin organization, DNA replication and gene regulation. LMNA gene mutations have been shown to cause several inherited diseases known as laminopathies, which include cardiac and skeletal myopathies, lipodystrophy, metabolic disorders, peripheral neuropathy and premature aging syndromes. Heart involvement counts as the most common feature, regardless of the presence of a myopathy and is characterized by conduction system disorders, arrhythmias, dilated cardiomyopathy and arrhythmogenic right ventricular cardiomyopathy. Many LMNA mutation carriers are at risk of sudden cardiac death, due to ventricular arrhythmias, high-degree atrioventricular block and end-stage heart failure. LMNA-related myopathies represent a subgroup with different phenotypes, based on the distribution of muscle weakness or age at onset: limb-girdle muscular dystrophy type 1B (LGMD1B), autosomal dominant Emery-Dreifuss muscular dystrophy and congenital muscular dystrophy.
LGMD1B typically causes progressive muscle weakness in the proximal muscles of the lower limbs. Methods: We herein present a 47 years old female patient, with a medical history of arrhythmias, second degree atrioventricular block, dilated cardiomyopathy (confirmed via magnetic resonance imaging) and weakness of the proximal muscles, with distinct myopathic features in the electromyography. Cardiac and skeletal muscle involvement developed gradually during her third decade of life. Family history was positive for sudden cardiac deaths and dilated cardiomyopathy (Figure 1), indicating a possible autosomal dominant manner. Genomic DNA was extracted from patient's peripheral blood and Whole Exome Sequencing (WES) was performed on Illumina HiSeq 2000/2500 platform. A comprehensive bioinformatics analysis for data interpretation was done using Ingenuity software (Qiagen).

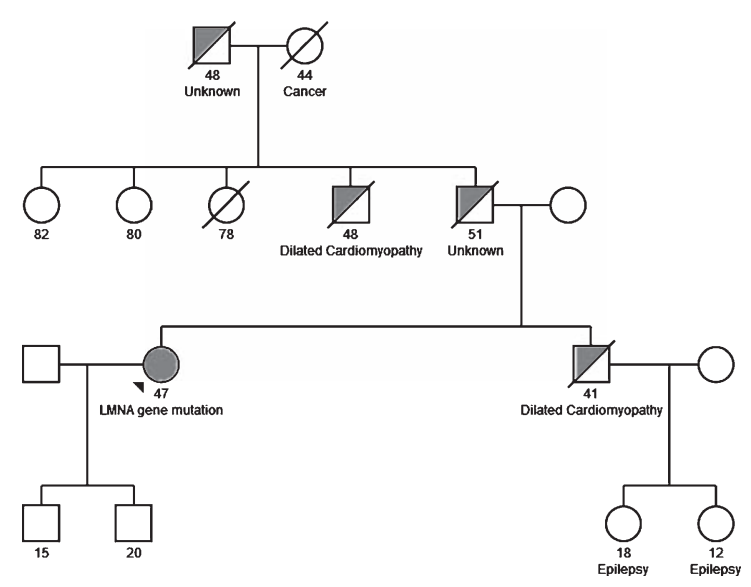

Results: We identified a novel splice site variant (c.1272+2T>C) in LMNA gene in the heterozygous state in our patient. This genetic variant results in splice site loss and in silico analysis using different bioinformatic tools predicted that it exerts a pathogenic effect. In order to verify segregation of this genetic variant in $L M N A$ gene with disease, several affected and unaffected members of the family were tested with pending results. Conclusion: Laminopathies can present with a vast clinical spectrum and should be suspected in patients with conduction system disorders, dilated cardiomyopathy and muscle weakness. We report a novel LMNA gene mutation in a female patient, presenting with dilated cardiomyopathy and myopathy, underlining the genetic diagnostic value and cost effectiveness of WES. 


\section{PS1Group1-103 / \#498}

Topic: Group 1 - Muscle Diseases of Genetic Origin and Acquired Myopathies: Clinical Features, Pathophysiology, Therapy

\section{LONGTERM APPLICATION OF HUMAN IMMUNOGLOBULIN G FOR EXPERIMENTAL TREATMENT OF DUCHENNE MUSCULAR DYSTROPHY}

Jana Zschüntzsch ${ }^{1}$, Pia V. Jouvenal ${ }^{1}$, Yaxin $Z_{\text {hang }}^{2}$, Florian Klinker ${ }^{3}$, Malte Tiburcy ${ }^{4}$, David Liebetanz ${ }^{3}$, Heinrich Brinkmeier ${ }^{2}$, Dörthe Malzahn ${ }^{5}$, Jens

Schmidt ${ }^{1}$

${ }^{I}$ Neurology, University medical Center Göttingen, Göttingen, DE:²Institute Of Pathophysiology, University Medicine Greifswald, Karlsburg, $D E ;{ }^{3}$ Clinical Neurophysiology, University medical Center Göttingen, Göttingen, DE; ${ }^{4}$ Institute Of Pharmacology And Toxicology, University medical Center Göttingen, Göttingen, DE; ${ }^{5} \mathrm{mzB}$ Biostatistics, Göttingen, DE

Background: Duchenne muscular dystrophy (DMD) is a progressive, muscle wasting, inherited $\mathrm{X}$-linked muscle disease caused by mutations in the dystrophin gene. The pathology consists of structural instability of the dystrophin-glycoprotein-complex (DGC), muscle degeneration, and various degrees of myoinflammation depending on the disease stage. Current treatments have targeted the genetic defect or the inflammatory response. So far, the standard treatment by glucocorticosteroids is limited because of numerous side effects. A previous study addressed the early disease phase of the well-established mdx mouse model for DMD and demonstrated an improvement of clinical and paraclinical parameters upon treatment with human immunoglobulins (IgG). The aim of the present study was to confirm the effectiveness of human IgG in a longterm preclinical study in mdx mice. Methods: Human IgG (Privigen, CSL Behring) at a dosage of $2 \mathrm{~g} /$ $\mathrm{kg} \mathrm{BW}$ or saline $(\mathrm{NaCl})$ as control was administered monthly by intraperitoneal injections in $\mathrm{mdx}$ mice over 18 months beginning at 4 weeks of age. Advanced voluntary wheel running parameters were recorded continuously over the entire study period. Cardiac ultrasound was performed every three months and grip strength measurements every week. After 18 months, animals were sacrificed. Blood and muscle were sampled for ex vivo muscle contraction tests, quantitative PCR and histological analysis. Results: IgG compared to $\mathrm{NaCl}$ significantly improved the voluntary running performance and grip strength. The muscle fatigability and specific tetanic force in an ex vivo muscle contraction test remained unchanged upon IgG. A significant benefit of cardiac function was evidenced by improved fractional area shortening and ejection fraction upon human $\mathrm{IgG}$. These results were supported by a reduced cardiac fibrosis and infiltration of T- cells as well as macrophages. Despite an unchanged mRNA expression of the relevant inflammatory markers TGF-beta, SPP1 and MCP1, a significant reduction of T- cells and macrophages in representative muscles (diaphragm, M. tibialis anterior, M. gastrocnemicus and M. quadriceps) was observed by immunohistochemistry in mdx mice treated with human IgG compared to controls. These findings were underlined by reduced myopathic changes upon human IgG in the same muscles. Conclusion: Taken together, this study demonstrates a beneficial effect of 18 months of IgG treatment in mdx mice, evidenced by improved voluntary wheel running parameters, cardiac function and reduced histopathological changes of the skeletal muscle, diaphragm and heart. Our long- term study underlines the importance of myoinflammatory contribution to the disease progression of DMD/ $\mathrm{mdx}$ and shows the therapeutic effectiveness of human IgG in the mdx mouse. Human IgG is usually well tolerated and might be a useful treatment option beside mutation-based therapies for DMD patients. The data call for a clinical trial with IgG in DMD. 


\section{PS1Group1-104 / \#675}

Topic: Group 1-Muscle Diseases of Genetic Origin and Acquired Myopathies: Clinical Features, Pathophysiology, Therapy

\section{BODY COMPOSITION ANALYSIS IN PATIENTS WITH MYOTONIC DYSTROPHY TYPE 1 AND 2}

$\underline{\text { Stojan Peric }}^{1}$, Milorad Vujnic ${ }^{2}$, Tanja Nisic ${ }^{3}$, Marija Banovic $^{4}$, Bogdan Bjelica ${ }^{5}$, Ivo Bozovic ${ }^{5}$, Jovan Pesovic $^{6}$, Dusanka Savic-Pavicevic ${ }^{6}$, Ivana Basta ${ }^{5}$, Zorica Stevic ${ }^{5}$, Dragana Lavrnic ${ }^{5}$, Vidosava Rakocevic-Stojanovic ${ }^{5}$

${ }^{1}$ Neurology Clinic, Clinical Center of Serbia, School of Medicine, University of Belgrade, Belgrade, Serbia, Belgrade, RS:²Faculty of Medicine, Banja Luka, BA, ${ }^{3}$ Center for Obesity, Clinic of Endocrinology, Diabetes and Metabolic Disease, Belgrade, $R S ;{ }^{4}$ School of Medicine, Belgrade, RS; ${ }^{5}$ Neurology Clinic, Clinical Center of Serbia, School of Medicine, University of Belgrade, Belgrade, Serbia, Belgrade, RS; ${ }^{6}$ Faculty of biology, Belgrade, $R S$

Background: Myotonic dystrophies (DM1) are inherited, slowly progressive, multisystem diseases. There are only several reports about body composition in DM1 and no data for DM2. The aim of this research was to analyze the body composition of patients with DM1 and DM2, and also to examine the association between body composition and sociodemographic/clinical features of patients. Methods: Body composition was assessed by DEXA (DualEnergy X-ray Absorptiometry) in 22 DM1 and 12 DM2 patients. A tree compartment model was used: bone mineral content (BMC), fat mass (FM) and lean tissue mass (LTM). Results: Patients with DM1 and DM2 had similar total body mass, BMC, FM and LTM. Patients with DM1 had higher trunk limb fat index (TLFI) that indicated higher accumulation of fat mass in abdominal area (visceral obesity) $(1.16 \pm 0.32$ for DM1 vs. $0.87 \pm 0.23$ for DM2, $\mathrm{p}<0.05)$. Right ribs bone mineral density was worse in DM2 group $\left(0.68 \pm 0.07 \mathrm{~g} / \mathrm{cm}^{2}\right.$ vs. $0.61 \pm 0.09 \mathrm{~g} /$ $\left.\mathrm{cm}^{2}, \mathrm{p}<0.05\right)$. The percentage of FM in legs of DM1 subjects showed negative correlation with the strength of proximal leg muscles $(\rho=-0.47, p<0.05)$. The strength of muscles in DM2 was in correlation with bone density $(\rho=+0.62, p<0.05$ for upper arm muscles, $\rho=+0.87, p<0.01$ for lower arm muscles, $\rho=+0.72, p<0.05$ for lower leg muscles). Conclusion: Patients with DM1 had visceral obesity and percentage of fat mass correlated with a degree of muscle weakness. In patients with DM2 lower bone mineral content was noticed. Higher degree of muscle weakness was in correlation with lower bone density in DM2.

\section{PS1Group1-105 / \#795}

Topic: Group 1 - Muscle Diseases of Genetic Origin and Acquired Myopathies: Clinical Features, Pathophysiology, Therapy

\section{EVALUATING THE EFFECTS OF BASELINE VARIABLES ON THE RESPIRATORY FUNCTION BENEFIT OF IDEBENONE IN DUCHENNE MUSCULAR DYSTROPHY}

$\underline{\text { Shabir Hasham }}^{1}$, Thomas Meier ${ }^{1}$, Mika Leinonen ${ }^{1}$, Thomas Voit ${ }^{2}$, Oscar H. Mayer ${ }^{3}$, Gunnar Buyse For The Delos Study Group ${ }^{4}$

${ }^{1}$ Santhera Pharmaceuticals, Pratteln, $\mathrm{CH} ;{ }^{2} \mathrm{UCL}$ Great Ormond Street Institute of Child Health, London, GB; $;^{3}$ Children's Hospital of Philadelphia , Philadelphia, PA, US: ${ }^{4}$ University Hospitals Leuven, Leuven, BE

Background: Respiratory function decline is a major cause of morbidity and mortality in patients with Duchenne muscular dystrophy (DMD). The randomized, placebo-controlled Phase 3 DELOS trial assessed the safety and efficacy of idebenone in DMD patients already in respiratory decline and not taking concomitant glucocorticoids (GCs). This trial demonstrated that idebenone significantly slowed respiratory function loss, measured as peak expiratory flow (expressed as a percentage of predicted, PEF\%p) - the primary endpoint. The objective of this analysis is to evaluate the effect of idebenone in various patient subgroups of the DELOS trial. Methods: Respiratory function data were prospectively collected from 64 DMD patients aged 10-18 years and already in respiratory decline $(\mathrm{PEF} \% \mathrm{p}<80 \%)$. The consistency of the effect of idebenone in DELOS was investigated by analyzing change in PEF\%p from baseline to week 52 (study end) in subgroups defined by prior GC use (naïve vs previous user), age ( $<14$ years vs $\geq 14$ years), baseline PEF\%p ( $<50 \%$ vs $\geq 50 \%)$, weight $(<58 \mathrm{~kg}$ vs $\geq 58 \mathrm{~kg}$ ) and ambulatory status. Each subgroup was analyzed using a mixed model for repeated measures, a similar approach to that used in the 
primary analysis. Results: The observed treatment difference in PEF\%p at week 52 between idebenone and placebo was $6.3 \%$ in the total intention to treat population $(p=0.03)$. Similar $P E F \% p$ treatment differences in favor of idebenone were observed regardless prior GC use, age, baseline $\mathrm{PEF} \% \mathrm{p}$, weight or ambulatory status - varying from $4.2 \%$ to $7.3 \%$. Of note, the treatment difference in prior GC users was $6.73 \%$ compared to $6.19 \%$ in GC-naïve patients. No significant interactions between the treatment group and subgroup factors were observed. Conclusion: The previously reported treatment effect of idebenone on $\mathrm{PEF} \% \mathrm{p}$ was consistent in a broad population of DMD patients, regardless of previous GC use, age, weight, ambulatory status or severity of respiratory decline at treatment initiation. These results suggest that none of these factors significantly influence the respiratory function benefits of idebenone in patients with DMD.

\section{PS1Group1-106 / \#984}

Topic: Group 1 - Muscle Diseases of Genetic Origin and Acquired Myopathies: Clinical Features, Pathophysiology, Therapy

\section{NOVEL RYR-CALSTABIN}

STABILIZERS WITH

\section{THERAPEUTICAL POTENTIAL FOR DUCHENNE MUSCULAR DYSTROPHY}

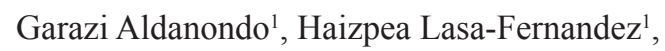
Jaione Lasa-Elgarresta ${ }^{1}$, Jose Ignacio Miranda ${ }^{2}$, Jesus Maria Aizpurua², Adolfo López De Munain¹, Ainara Vallejo-Illarramendi ${ }^{1}$

${ }^{1}$ Biodonostia Institute, Donostia-San Sebastian, ES:²UPV/EHU, Donostia-San Sebastian, ES

Background: Several studies indicate that calcium is dysregulated in the muscles from patients with Duchenne and Becker muscular dystrophy. In the mdx mouse model of Duchenne muscular dystrophy (DMD), the sarcoplasmic reticulum ryanodine receptor RyR1 and RyR2 are abnormally nitrosylated and this leads to calstabin depletion from the protein complex and subsequent calcium leak through these channels. RyR modulators enhance RyR-Calstabin binding preventing calcium leak, reducing muscle damage and improving muscle function. Methods:
We have generated a family of small molecules, namely Ahulken compounds (AHK), which bind RyRs and modulate their activity. In this work we have analyzed the in vivo and in vitro effect of the novel RyR stabilizers AHK1 and AHK2, in the mdx mouse model and in human immortalized myotubes. Results: Treatment of human myotubes with AHK compounds for 12 hours enhanced RyR-calstabin binding, and normalized resting cytosolic calcium levels. Moreover, no toxicity was observed in myotubes treated with high concentration $(2 \mathrm{mM})$ of AHK compounds. Treatment of $\mathrm{mdx}$ mice with AHK modulators during 5 weeks normalized intracellular calcium levels in muscle fibers, reduced biochemical and histological evidence of muscle damage and improved muscle function. Moreover, AHK2 also improved specific CNS and cardiac muscle deficiencies observed in mdx mice. Conclusion: Our study shows that AHK modulators ameliorate dystrophic phenotype in cellular and animal models of Duchenne muscular dystrophy. In addition, our results consolidate RyR-calstabin complex as a useful therapeutic target for drug development against muscular dystrophies as well as other disorders with subjacent nitro-oxidative stress.

\section{PS1Group1-107 / \#501}

Topic: Group 1 - Muscle Diseases of Genetic Origin and Acquired Myopathies: Clinical Features, Pathophysiology, Therapy

\section{THE EFFECT OF UNCARIA TOMENTOSA IN DIAPHRAGM MUSCLE OF MDX DYSTROPHIC MICE}

David Feder ${ }^{1}$, Alzira A.S. Carvalho' ${ }^{2}$, Bruno M. Bertassoli ${ }^{1}$, Lucas P. Giordani ${ }^{1}$, Giuliana Petri ${ }^{1}$, Fabio F. Perazzo ${ }^{3}$, Beatriz C.A. Alves ${ }^{4}$, Matheus M. Perez $^{4}$, Fernando L.A. Fonseca ${ }^{4}$

${ }^{1}$ Pharmacology, Faculdade de Medicina do ABC, Santo Andre, BR $;{ }^{2}$ Neurology, Faculdade de Medicina do ABC, Santo Andre, $B R ;{ }^{3}$ Pharmaceutical Sciences, Universidade Federal de São Paulo, Santo Andre, $B R ;{ }^{4}$ Laboratory Analysis, Faculdade de Medicina do $A B C$, Santo Andre, $B R$

Background: Duchenne Muscular Dystrophy (DMD) is a severe muscle wasting disease caused by 
mutations in dystrophin gene. This deficiency leads to sarcolemma instability, inflammation, muscle degeneration and fibrosis. Uncaria tomentosa (UT) is a medicinal plant with anti-viral, anti-mutagenic, antiinflammatory and antioxidant properties. The objective of study was evaluating the effect of UT in diaphragm muscle of mdx dystrophic mices. Methods: 8 -weeks-old male mdx mice were treated, 10 mice with $200 \mathrm{mg} / \mathrm{kg}$ of UT extract orally during 30 days (UT group) and 6 mice received saline solution orally during 30 days (control group). Creatine kinase $(\mathrm{CK})$ was measured on the day of euthanasia. H\&E stained frozen section of the muscle biopsy of diaphragm (DIA) was performed in order to analyze number of internal nuclei and minimal Feret diameter. In addition, gene expression of transforming growth factor- $\beta 1$ (TGF- $\beta 1$ ), tumor necrosis factor- $\alpha$ (TNF- $\alpha$ ), osteopontin (OSP) and Myostatin (Myo) were determined by quantitative real time polymerase chain reaction (qRT-PCR). Data are presented as mean \pm standard error of mean. One-way or two-way ANOVA and Bonferroni test were used for analysis of variance to determine the statistical significance, followed by the least significant difference (LSD) analysis, using Prism Statical software (Graphpad, San Diego, CA). P $<0.05$ was considered statistically significant. Results: CK levels were significantly higher in UT group. UT treatment did not prevent muscle damage, indicated by increase of central nucleated fibers with a concomitant increase in number of small fibers $(0-30 \mu \mathrm{M})$. By other side, It had an effect on the inflammatory process demonstrated by decrease in TNF- $\alpha$, TGF and osteopontin expression (figure 1)

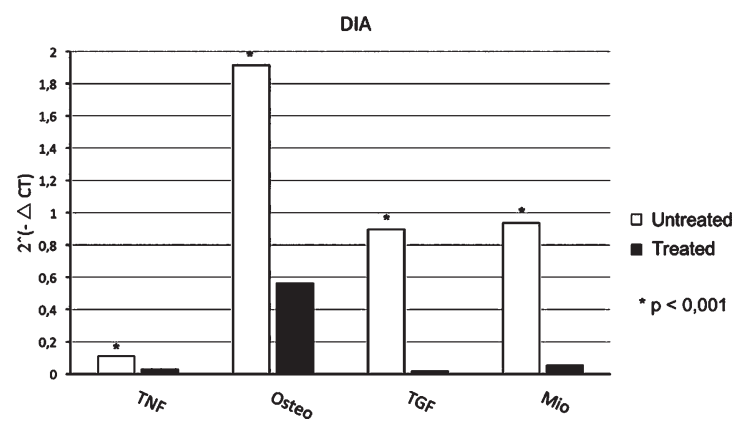

Conclusion: So, in this study, we evaluated the antiinflammatory properties of Uncaria tomentosa against myonecrosis and inflammatory response in the diaphragm muscle of $\mathrm{mdx}$ mice. Uncaria tomentosa downregulates the TNF- $\alpha$, TGF and osteopontin expression indicating improving of inflammatory role however, there was a worsening of the dystrophic phenotype in the diaphragm muscle, indicated by increase of CK level, number of central nucleated fibers and small fibers diameter. Perhaps, as many inflammatory pathways are triggered in muscular dystrophy, it is possible that Uncaria tomentosa, alone, is insufficient to halt the whole process.

\section{PS1Group1-108 / \#620}

Topic: Group 1 -Muscle Diseases of Genetic Origin and Acquired Myopathies: Clinical Features, Pathophysiology, Therapy

\section{MYTOBLOTS FOR THE EVALUATION OF NEW TREATMENTS IN NEUROMUSCULAR DISORDERS}

\author{
Estibaliz Ruiz-Del-Yerro ${ }^{1}$, Patricia Soblechero- \\ Martin $^{2}$, Irene Larrañaga ${ }^{1}$, Edurne Albiasu-Arteta ${ }^{1}$, \\ Virginia Arechavala-Gomeza ${ }^{3}$ \\ ${ }^{1}$ Neuromuscular Disorders, Biocruces Health \\ Research Institute, Barakaldo, ES ${ }^{2}$ Neuromuscular \\ Disorders Group, Biocruces Helath Research \\ Institute, Barakaldo, ES: ${ }^{3}$ Neuromuscular \\ Disorders, Biocruces Health Research Institute, \\ Barakaldo, ES
}

Background: New therapies for neuromuscular disorders often require to be studied in patient's cell cultures. Many potential therapies for Duchenne Muscular Dystrophy (DMD) aim to alter the expression of key proteins, such as dystrophin or utrophin, but, as muscle cell cultures from DMD patients are scarce and do not grow or differentiate well, only a limited number of candidate drugs are tested. Moreover, dystrophin and utrophin quantification by western blotting requires a large number of cultured cells; so fewer compounds are evaluated as thoroughly as it is desirable. Methods: We have developed a quantitative assessment tool using fewer cells to contribute in the screening of better drug candidates: an "in-cell-western", also known as cytoblot, is a quantitative immunofluorescence assay performed in microplates that makes possible the quantification of proteins directly in cell culture, combining the specificity of western blotting with the reproducibility and throughput of ELISA. Results: Our group has recently optimized the assay ('myoblot') to quantify several muscle proteins in differentiated myoblast cultures and we are using 
this method to assess dystrophin restoration treatments, drugs that aim to increase the expression of utrophin and others that alter the differentiation of cultures. Conclusion: We expect that the use of myoblots will accelerate the development and validation of new therapies.

\section{PS1Group1-109 / \#622}

Topic: Group 1-Muscle Diseases of Genetic Origin and Acquired Myopathies: Clinical Features, Pathophysiology, Therapy

\section{PHENOTYPE-GENOTYPE RELATIONS IN FACIOSCAPULOHUMERAL MUSCULAR DYSTROPHY TYPE 1}

$\underline{\text { Karlien Mul }}^{1}$, Richard J. Lemmers ${ }^{2}$, Corinne G. Horlings $^{3}$, Patrick J. Van Der Vliet ${ }^{2}$, Marianne A. Jonker ${ }^{4}$, Nicol C. Voermans ${ }^{3}$, Silvere M. Van Der Maarel$^{2}$, Baziel G. Van Engelen ${ }^{3}$

${ }^{1}$ Neurology, Radboud University Medical Center, Nijmegen, NL, ${ }^{2}$ Human Genetics, leiden University medical Center, Leiden, NL, ${ }^{3}$ Neurology, Radboud University Medical Center, Nijmegen, $N L ;{ }^{4}$ Health Sciences, Radboud University Medical Center, Nijmegen, $N L$

Background: Facioscapulohumeral muscular dystrophy (FSHD) is characterized by large variability in disease severity and penetrance, that is only partially explained by the current knowledge on the disease mechanism. This study integrates previous findings on phenotype-(epi)genotype relations to assess how much of the clinical variability can be explained. Additionally, clinical scores per body region are used to refine the interpretation of (epi)genetic data. Methods: We included 148 FSHD1 patients from 49 different families and performed genetic testing (D4Z4 repeat array size, haplotype, and D4Z4 methylation), extensive clinical evaluation (clinical severity scores, manual muscle testing, motor function measure, facial weakness score), and muscle MRI of the leg muscles. Results: The explained variability in disease severity by familial factors, including the D4Z4 repeat array size, was approximately $40 \%$. Asymptomatic gene carriers had longer repeat sizes compared to symptomatic individuals (7.3 vs 6.2 units, $\mathrm{p}=0.001$ ). The D4Z4 methylation (Delta1 value) was not useful as a prognostic factor for disease severity and penetrance on an individual level. The D4Z4 repeat array size explained approximately $29 \%$ and $15 \%$ of the variability in the involvement of the facial muscles and upper extremity muscles respectively. Involvement of the leg muscles was influenced by age, but only to a very limited extent by the repeat array size (explained variance approximately 3\%). Conclusion: This study provides additional evidence that other disease modifying factors play an important role in the clinical variability of FSHD patients, since only $40 \%$ of the variability can be attributed to familial factors. Possibly, the facial muscles are more sensitive to DUX4, whereas involvement of the leg muscles is highly dependent on disease modifying factors.

\section{PS1Group1-110 / \#708}

Topic: Group 1 - Muscle Diseases of Genetic Origin and Acquired Myopathies: Clinical Features, Pathophysiology, Therapy

\section{SCN4A CAN ACT AS MODIFIER GENE IN PATIENTS WITH MYOTONIC DYSTROPHY TYPE 2}

Anna Binda ${ }^{1}$, Laura V. Renna ${ }^{2}$, Francesca Bose'2, Elisa Brigonzi ${ }^{3}$, Annalisa Botta ${ }^{4}$, Rea Valaperta ${ }^{5}$, Barbara Fossati ${ }^{6}$, Ilaria Rivolta ${ }^{1}$, Giovanni Meola ${ }^{3}$, Rosanna Cardani $^{7}$

${ }^{1}$ School Of Medicine And Surgery, University of Milano Bicocca, Monza, IT; ${ }^{2}$ Laboratory Of Muscle Histopathology And Molecular Biology, IRCCS Policlinico San Donato, San Donato Milanese (MI), IT; ${ }^{3}$ Department Of Biomedical Sciences For Health, University of Milan, San Donato Milanese (MI), IT; ${ }^{4}$ Department Of Biomedicine And Prevention, Tor Vergata University of Rome, Rome, IT; ${ }^{5}$ Research Laboratories, IRCCS Policlinico San Donato, San Donato Milanese (MI),

IT; ${ }^{6}$ Department Of Neurology, IRCCS Policlinico

San Donato, San Donato Milanese (MI),

IT; ${ }^{7}$ Laboratory Of Muscle Histopathology And Molecular Biology, IRCCS-Policlinico San Donato, San Donato Milanese (MI), IT

Background: Myotonic dystrophy type 2 (DM2) is caused by a CCTG repeat expansion in intron 1 of the CNBP/ZNF9 gene located on chromosome 3q21. The mutant RNA transcripts containing CCUG repeats alter the activity of specific RNA binding proteins involved in alternative splicing regulation leading to splicing defects that are considered the 
primary cause of DM1 symptoms. Usually myotonia is mild and inconsistent in DM2 even by electromyography and it has been correlated with the disruption of the alternative splicing of the muscle chloride channel $C L C N 1$. It is noteworthy that mutations in CLCN1 and SCN4A can act as modifier genes in patients with DM2 disease leading to an intensification of their myotonia. We report a 32 years old DM2 patient who presented an atypical phenotype characterized by an early severe myotonia since he was 12 years old. Mexiletine treatment resulted ineffective in reducing myotonia. While no mutation on $C L C N 1$ gene was found, the genetic analysis of $S C N 4 A$ gene showed a c. $2717 \mathrm{G}>\mathrm{C}$ base exchange in exon 14 predicting an S906T substitution. This variant is considered a benign polymorphism however electrophysiological studies revealed that it affects the fast and slow gating processes. Methods: To investigate the atypical DM2 phenotype of our patient, the combined effects of DM2 mutation and S906T substitution have been studied performing wholecell patch-clamp in voltage and current clamp mode in myoblasts and myotubes obtained from the skeletal muscle biopsy of the patient. Results have been compared to those obtained in muscle cells derived from his mother, who is also affected by DM2, but did not present the S906T polymorphism. Results: A faster decay of the inactivation kinetics and a $+5 \mathrm{mV}$ shift in the availability curve $(\mathrm{p}<0.05)$ were found in the sodium current recorded in myoblasts derived from the proband compared to his mother. In myotubes showing a stable resting membrane potential, instead, the rheobase current was significantly lower in the proband respect to the mother while the overshoot and the maximum slope of the depolarizing phase of the action potential were higher in the proband respect to the mother. Conclusion: These findings suggest that SCN4A polymorphism may be responsible for a higher excitability of DM2 patients sarcolemma, supporting the severe myotonic phenotype observed. We suggest that SCN4A may be considered a modifier factor and its screening should be performed in DM2 patients with uncommon clinical features.

\section{PS1Group1-111 / \#834}

Topic: Group 1 - Muscle Diseases of Genetic Origin and Acquired Myopathies: Clinical Features, Pathophysiology, Therapy

\section{AAV-SERCA2A EXPRESSION AMELIORATED CARDIOMYOPATHY IN THE MDX MOUSE MODEL OF DUCHENNE MUSCULAR DYSTROPHY}

Yongping Yue ${ }^{1}$, Nalinda Wasala ${ }^{1}$, William Lostal ${ }^{1}$, Lakmini Wasala $^{1}$, Nandita Niranjan ${ }^{2}$, Roger Hajjar ${ }^{3}$, Gopal Babu², Dongsheng Duan ${ }^{1}$

${ }^{1}$ Molecular Microbiology And Immunology (medical Science Bldg), University of Missouri, Columbia, MO, US $;{ }^{2}$ Cell Biology And Molecular Medicine, New Jersey Medical School, Rutgers, The State University of New Jersey, Newark, US, ${ }^{3}$ Mount Sinai School of Medicine, New York, US

Background: Loss of dystrophin leads to Duchenne muscular dystrophy (DMD). Increased cytosolic calcium levels play a critical pathogenic role in the development DMD cardiomyopathy. Cytosolic calcium can be removed by the sarco/endoplasmic reticulum calcium ATPase (SERCA). SERCA activity is reduced in dystrophic muscle. Methods: To test whether SERCA gene transfer can improve calcium recycling and mitigate dystrophic phenotype, we delivered an adeno-associated virus (AAV)-9 SERCA2a vector to $3-\mathrm{m}$-old $\mathrm{mdx}$ mice at the dose of $6 \mathrm{E} 12$ viral genome particles/mouse via the tail vein. Whole-body performances were assessed at 8 and 18 months post-treatment using treadmill running and forelimb grip force assays. Serum creatine kinase $(\mathrm{CK})$ activity and heart function (ECG and left ventricular catheterization) were examined at 18 months post-treatment. Results: Western blots and immunostaining confirmed robust SERCA2a expression in heart and skeletal muscle. Forelimb grip strength and treadmill running were significantly improved in treated mice. AAV-9 SERCA2a treatment also reduced the serum CK level, a marker of muscle damage. Importantly, SERCA2a treatment significantly improved heart function. Specifically, all ECG parameters were normalized to the wildtype levels. Several measures of systolic function were normalized in the catheter assay. Treatment also significantly improved ejection fraction, the rate of cardiac relaxation and the end diastolic volume. 
Conclusion: Our results suggest that AAV-9 SERCA2a gene therapy is a promising approach to treat DMD cardiomyopathy

\section{PS1Group1-112 / \#623}

Topic: Group 1 -Muscle Diseases of Genetic Origin and Acquired Myopathies: Clinical Features, Pathophysiology, Therapy

\section{QUANTITATIVE ANALYSIS OF THIGH MUSCLE BUNDLES OF PATIENTS WITH MYOTONIC DYSTROPHY TYPE 1 (DM1), USING CT IMPAIRMENT RATIO}

Takahiro Nakayama $^{1}$, Satoshi Kuru ${ }^{2}$

${ }^{1}$ Department Of Neurology, Division Of

Neuromuscular Diseases, Yokohama Rosai

Hospital, Yokohama, JP, ${ }^{2}$ Neurology, National

Hospital Organization Suzuka National Hospital,

Suzuka, JP

Background: It was reported that the atrophy of cervical and thoracic paravertebral, vastus lateral and medial head of gastrocnemius muscle were commonly affected in the patients with myotonic dystrophy type 1 (DM1), however, that the time dependent deterioration had not been confirmed until now. We wanted to know the affected pattern of their thigh muscle and estimating function of their deterioration. Methods: Subjects: CT images of 7 DM1 patients ( 3 males and 4 females), who were examined once a year, enrolled in this study. Their ages at first visit to hospitals were from 17 to 43 , and the number of CTG repeats were from 100 to 1200 . Their CT data was collected from 3 to 11 years. Method: The Impairment ratios of each muscle bundle were plotted in each patient as long as possible. The muscle impairment ratio is the ratio of affected muscle fibers in muscle bundles, and calculation formulas were developed by our group. The ratio was determined by following formula, and we used the previously reported CT values of 58 and $-98 \mathrm{HU}$ for muscle and fat, respectively. impairment ratio=("muscle CT value" - "pixel value")/("muscle CT value" - "fat CT value") This investigation was carried out in accordance with the Human Research Guidelines of the Institutional Ethics Review Board of Yokohama Rosai Hospital. Results: In DM1 patients, firstly there is a period of slight muscle impairment. After this period, the muscle bundles deteriorate, and this deterioration was well estimated by cumulative normal distribution function. At the end of deterioration stage, we deduced the intermediate vastus thigh muscle is most commonly severely impaired. Most cases felt difficulty to walk at the age when the most affected muscle was impaired 10$20 \%$. There was no correlation between the CTG repeats and the deterioration. Conclusion: We inferred the muscle will begin to be impaired after the increase of CTG repeats in muscles. The intermediate vastus muscle will be more commonly and more severely affected than surrounding thigh muscles. We concluded that the cumulative normal distribution function was suitable for estimating their muscle deterioration.

\section{PS1Group1-113 / \#740}

Topic: Group 1-Muscle Diseases of Genetic Origin and Acquired Myopathies: Clinical Features, Pathophysiology, Therapy

\section{LIMB-GIRDLE MUSCULAR DYSTROPHY IN THE CZECH REPUBLIC}

Kristyna Stehlikova ${ }^{1}$, Jana Zidkova ${ }^{2}$, Kamila Reblova $^{3}$, Radim Mazanec ${ }^{4}$, Stanislav Vohanka ${ }^{5}$, Jana Haberlova $^{6}$, Lenka Fajkusova ${ }^{7}$

${ }^{1}$ Centre Of Molecular Biology And Gene Therapy,

University Hospital Brno, Brno, CZ; ${ }^{2}$ Centre Of

Molecular Biology And Gene Therapy, University

Hospital Brno, Brno, CZ; ${ }^{3}$ Ceitec, Masaryk

University Brno, Brno, CZ; ${ }^{4}$ Neurology, Second

Faculty of Medicine, Charles University and

University Hospital Motol, Prague, CZ, ${ }^{5}$ Neurology,

University Hospital Brno, Brno, CZ;66 Department

Of Child Neurology, Second Faculty of Medicine, Charles University and University Hospital Motol, Prague, CZ; ${ }^{7}$ Masaryk University Brno, Brno, CZ

Background: Limb-girdle muscular dystrophy (LGMD) is a group of disorders, which first affect muscles of the hip and shoulder areas. Genes associated with LGMD normally encode proteins that play vital roles in the muscle function, cell signalling, cell membrane repair, or removal of potentially toxic wastes from cells. 32 genes have been identified so far, 7 with autosomal dominant inheritance (LGMD1) and 25 with autosomal recessive inheritance (LGMD2). LGMD1 is relatively rare and represents less than $10 \%$ of all LGMD. LGMD2 is 
much more common, having a cumulative prevalence of 1:15,000, with some differences among countries. Methods: We perform molecular genetic diagnostics of neuromuscular disorders (NMD). At present, it is based on gene panel sequencing, pulsedfield gel electrophoresis and Southern analysis (for diagnostics of FSHD1), and repeat-primed PCR (for diagnostics of myotonic dystrophy) in case of muscular dystrophies and myopathies. Differential diagnosis of the LGMD remains quite large and may include LGMD, FSHD, Emery-Dreifuss muscular dystrophy, manifesting carriers of DMD, congenital myasthenic syndromes, congenital myopathies presenting later in childhood or adulthood, and others. Genes associated with mentioned diseases (except FSHD1 which is tested separately) are analysed in parallel with LGMD obligatory genes. Results: We present results of molecular genetic diagnostic of Czech LGMD patients determined by gene panel sequencing (used since 2012) or Sanger sequencing within the gene-by-gene approach (by 2011). In LGMD, we have 166 patients with confirmed genetic diagnosis - 94 patients with variants in CAPN3, 17 patients with variants in FKRP, 14 patients with variants in ANO5, 14 patients with variants in SGCA, 8 patients with a variant in LMNA, 6 patients with variants in DYSF, 4 patients with a variant in CAV3, 3 patients with variants in POMT2, 2 patients with variants in TRIM32, and 1 patient with variants/variant in each of ISPD, SGCG, SGCB, and TNPO3. Conclusion: Besides patients with genetically confirmed LGMD, we have LGMD patients without genetic diagnosis although all so far known muscular dystrophy/myopathy related genes were analysed. These patients are candidates for whole exome sequencing. This study was supported by the project of the Technology Agency of the Czech Republic (TE2000058) and the project of the Grant Agency of the Czech Republic(GA16-11619S/2016).
PS1Group1-114 / \#837

Topic: Group 1 - Muscle Diseases of Genetic Origin and Acquired Myopathies: Clinical Features, Pathophysiology, Therapy

\section{PULMONARY FUNCTION IN ADVANCED DUCHENNE MUSCULAR DYSTROPHY: ETEPLIRSEN-TREATED PATIENTS VERSUS A NATURAL HISTORY COHORT}

Heather Gordish-Dressman ${ }^{1}$, Erik Henricson ${ }^{2}$, Lixin $\mathrm{Han}^{3}$, Ashish Dugar ${ }^{3}$, Craig Mcdonald ${ }^{2}$, Cooperative International Neuromuscular Research Group (Cinrg) ${ }^{4}$

${ }^{1}$ George Washington University School of Medicine and Health Sciences, Washington, DC, US: ${ }^{2}$ University of California Davis, Davis, CA, US, ${ }^{3}$ Sarepta Therapeutics, Inc., Cambridge, MA, $U S ;{ }^{4} . U S$

Background: Eteplirsen is a phosphorodiamidate morpholino oligomer that binds to and excludes exon 51 from dystrophin pre-mRNA, allowing translation and production of internally shortened dystrophin protein. It is approved in the US for treatment of patients with Duchenne muscular dystrophy (DMD) with a confirmed genetic mutation amenable to exon 51 skipping. We evaluated pulmonary function in patients with advanced DMD in a phase 2, open-label, multicenter study of eteplirsen. Methods: Eligible males aged 7-21 years with advanced DMD were enrolled and received once-weekly intravenous eteplirsen $30 \mathrm{mg} / \mathrm{kg}$ for 96 weeks. Eligible patients must have been receiving either no corticosteroids or a stable oral corticosteroid dose for $\geq 24$ weeks prior to study entry, with no change in dosage anticipated. Pulmonary function, a predefined exploratory outcome, was assessed by mean change from baseline in percent predicted forced vital capacity (FVC\%p). Safety assessments related to pulmonary function included mean change from baseline in respiratory rate (breaths/min) by Week 96. FVC\%p outcomes were also evaluated in a Cooperative International Neuromuscular Research Group (CINRG) DMD Natural History Study cohort (patients aged 8-19 years) receiving standard of care treatment with glucocorticoids. Results: Of 24 patients enrolled, 23 (96\%) completed 96 weeks of eteplirsen treatment (baseline median age, $13.0 \mathrm{y}$ 
[range: 8-19]; mean FVC\%p, 68.7). Eteplirsentreated patients had a mean (standard error [SE]) change from baseline in $\mathrm{FVC} \% \mathrm{p}$ of -6.4 (2.16) at Year 1. Mean (SE) change in FVC \%p from Year 1 to Year 2 for eteplirsen-treated patients was -0.5 (2.07). Mean respiratory rates were 19.0 at baseline, 20.5 at Week 48, and 20.5 at Week 96 for eteplirsentreated patients. A comparable CINRG natural history subset $(\mathrm{n}=10)$ had similar demographic and clinical characteristics, with mean change in $\mathrm{FVC} \% \mathrm{p}$ of -8.2 over a time frame of 1 year. Conclusion: Eteplirsen treatment slowed the decline of $\mathrm{FVC} \% \mathrm{p}$ in patients with advanced DMD relative to a comparable natural history cohort. The rate of decline for eteplirsen-treated patients slowed most appreciably in Year 2, suggesting a greater impact on disease progression with prolonged eteplirsen treatment.

\section{PS1Group1-115 / \#931}

Topic: Group 1 - Muscle Diseases of Genetic Origin and Acquired Myopathies: Clinical Features, Pathophysiology, Therapy

\section{CLINICAL OUTCOME STUDY OF DYSFERLINOPATHY: TEENAGE EXERCISE AS A POTENTIAL MODIFIER OF DISEASE SEVERITY}

$\underline{\text { Claudio Semplicini }}^{1}$, Ursula Moore ${ }^{2}$, Marni Jacobs ${ }^{3}$, Roberto Fernández-Torrón ${ }^{4}$, Jiji Jang ${ }^{3}$, Meredith James $^{2}$, Anna Mayhew ${ }^{2}$, Laura Rufibach ${ }^{5}$, Plavi Mittal $^{5}$, Michele Eagle ${ }^{2}$, Avital Cnaan ${ }^{6}$, Pierre G. Carlier ${ }^{7}$, Andrew Blamire ${ }^{8}$, Heather Hilsden ${ }^{2}$, Hanns Lochmuller ${ }^{2}$, Ulrike Grieben ${ }^{9}$, Simone Spuler $^{10}$, Carolina Tesi Rocha ${ }^{11}$, John W. Day ${ }^{11}$, Kirsti J. Jones ${ }^{12}$, Diana X. Bharucha-Goebel ${ }^{13}$, Emmanuelle Salort-Campana ${ }^{14}$, Matthew Harms ${ }^{15}$, Alan Pestronk ${ }^{15}$, Sabine Krause ${ }^{16}$, Olivia SchreiberKatz $^{16}$, Maggie C. Walter ${ }^{16}$, Carmen Paradas ${ }^{17}$, Jean-Yves Hogrel ${ }^{18}$, Tanya Stojkovic ${ }^{19}$, Shin'Ichi Takeda $^{20}$, Madoka Mori-Yoshimura' ${ }^{21}$, Elena Bravver $^{22}$, Susan Sparks ${ }^{22}$, Jordi D. Manera ${ }^{23}$, Luca Bello $^{24}$, Elena Pegoraro ${ }^{1}$, Jerry R. Mendell ${ }^{25}$, Kate Bushby $^{2}$, Volker Straub ${ }^{26}$

${ }^{1}$ University of Padova - Azienda Ospedaliera di Padova, Padova, IT; ${ }^{2}$ The John Walton Muscular Dystrophy Research Centre, Newcastle Hospitals Trust, Newcastle University, Newcastle Upon Tyne, $G B ;{ }^{3}$ Division Of Biostatistic And Study
Methodology, Children's National Health System, Centre for Translational Science, DC, WA, US: ${ }^{4}$ Neuromuscular Area, Biodonostia Health Research Institute, Neurology Service, Donostia University Hospital, Donostia University Hospital, Donostia-San Sebastian, ES, ${ }^{5}$ Jain Foundation, Seattle, US: ${ }^{6}$ Pediatrics, Epidemiology And Biostatistics, George Washington University, Washington DC, US $;^{7}$ Aim \& Cea Nmr Laboratory, Neuromuscular Investigation Center, Institute of Myology, Paris, FR, ${ }^{8}$ Magnetic Resonance Centre, Institute For Cellular Medicine, Newcastle University, Newcastle Upon Tyne, GB $;^{9}$ Charite Muscle Research Unit, A Joint Cooperation Of The Charité Medical Faculty And The Max Delbrück Center For Molecular Medicine, Experimental and Clinical Research Center, Berlin, DE $:{ }^{10}$ Charite Muscle Research Unit, Experimental and Clinical Research Center, Berlin, DE; ${ }^{11}$ Department Of Neurology And Neurological Sciences, Stanford University School of Medicine, Stanford, CA, US; ${ }^{12}$ Institute For Neuroscience And Muscle Research, Children's Hospital At Westmead, University of Sydney, Sydney, AU: ${ }^{13}$ Department of Neurology, Children's National Health System, Washington DC, US; ${ }^{14}$ Neuromuscular And Als Center, La Timone Hospital, Aix-Marseille Universite, Marseille, FR $;{ }^{15}$ Department $O f$ Neurology, Washington University School of Medicine, St. Louis, MO, US: ${ }^{16}$ Friedrich-baur Institute, Departement Of Neurology, LudwigsMaximilians University of Munich, Munich, DE: ${ }^{17}$ Neuromuscular Unit, Department Of Neurology, Hospital U. Virgen del Rocio/Instituto de Biomedicina de Sevilla, Sevilla, ES; ${ }^{18}$ Institut De Myologie, Boulevard de l'Hôpital, Paris, FR: ${ }^{19} \mathrm{Ap}$ hp, G.h. Pitié-salpêtrière 47-83, Boulevard De L'hôpital, Institut de Myologie, Paris, FR, ${ }^{20}$ National Center of Neurology and Psychiatry, Tokyo, JP; ${ }^{21}$ Department Of Neurology, National Center Hospital, National Center of Neurology and Psychiatry, Tokyo, JP, ${ }^{22}$ Carolinas Healthcare System Neurosciences Institute, Charlotte, NC, US:;2Neuromuscular Unit, Neurology Department, Hospital de la Santa Creu i Sant Pau, Barcelona, ES:24 Department Of Neuroscience, University of Padova, Padova, IT; ${ }^{25}$ Center for Gene Therapy Nationwide Children's Hospital, Columbus, US; ${ }^{26}$ John Walton Muscular Dystrophy Research Centre, Newcastle University, Institute of Genetic Medicine, Newcastle Upon Tyne, GB 
Background: The Clinical Outcome Study of Dysferlinopathy $(\mathrm{COS})$ is an international 3 year natural history study of dysferlinopathy patients. Dysferlinopathy, a genetic muscular dystrophy caused by $D Y S F$ mutations, demonstrates a variable age of presentation and progression rate, suggesting disease modifying factors. Here we investigate teenage exercise intensity and subsequent disease progression in 182 patients. Methods: Participants were asked about the type, level and frequency of all physical activity prior to symptom onset. Self-reported age of first symptoms, first wheelchair use and full-time wheelchair use was taken from screening questionnaires. Exercise was classified based on metabolic equivalents (METs) as moderate (MET 3-6) or vigorous (MET $>6$ ). Participants were coded, based on the maximum frequency of activity reported between ages 10 and 18 years, as 0 - no physical activity; 1-vigorous activity occasionally/monthly, or moderate activity once weekly; 2 - moderate activity multiple times per week or vigorous activity once weekly; and 3-vigorous activity multiple times per week. Age of symptom onset was compared by analysis of variance (ANOVA) with least squares means for individual group differences. Risk of symptom onset, occasional wheelchair use and full-time wheelchair requirement over time were compared for exercise groups 1, 2 and 3 against group 0 using Cox proportional hazards regression. Interaction between teen exercise level, gender and clinical diagnosis was also assessed by two-way ANOVA. Subgroups of limb girdle muscular dystrophy 2B (LGMD2B), Miyoshi myopathy (MM) or 'other' (all genetically confirmed dysferlinopathies) were used for analysis. Results: Estimated mean age of symptom onset differed by group $(\mathrm{P}=0.03)$ and was later in group 0 (mean 24.8 years; $95 \%$ CI 22.3 to 27.2 years) compared with groups 2 (mean 20.2 years; $\mathrm{CI}$ 18.1 to 22.3 years, $\mathrm{P}=0.006$ ) and 3 (mean 20.6 years, CI 18.4 to 22.8 years, $\mathrm{P}=0.01$ ), but not group 1 (mean 21.7 years; CI 17.7 to 25.7 years, $\mathrm{P}=0.20$ ). Cox regression analysis suggested that groups 2 (Hazard ratio $1.5695 \%$ CI 1.06 to 2.30 )) and 3 (Hazard ratio 1.54 (1.04 to 2.30)) were at increased risk of earlier symptom onset than group 0 (figure 1). This was not significant for group 1 (Hazard ratio R 1.38 (0.78 to 2.45)). Conclusion: This study raises implications for early diagnosis and clinical care, however as we did not look at the effects of exercise once symptoms began, we would not advocate that symptomatic patients stop exercising.

\section{PS1Group1-116 / \#507}

Topic: Group 1 - Muscle Diseases of Genetic Origin and Acquired Myopathies: Clinical Features, Pathophysiology, Therapy

\section{GLYCOGENOSIS TYPE V (MCARDLE DISEASE): THERAPY WITH VITAMIN B6}

\author{
Annika Saak, Heinz Reichmann, Jochen Schaefer \\ Neurology, University Hospital Dresden, \\ Dresden, $D E$
}

Background: The glycogen storage disease type V or McArdle disease is one of the metabolic myopathies and is characterized by a stress intolerance with myalgia, myopathy and muscle cramps. The autosomal-recessive disorder is in Europe usually caused by a stop mutation (P-R50X) in the PYGM gene. There is no causal form of therapy so far, so that patients are advised of symptomatic therapy with creatine or cane sugar before physical exertion. Positive effects of vitamin B6 on the subjective muscular resilience have been described in individual case reports. Our patient, in which a previously undescribed genetic defect (c. 1456G > A) could be detected in the PYGM gene, was treated with vitamin B6 for over 4 years. The subjective improvement reported by the patient should be clinically and laboratorychemically objectified. Methods: We performed recurrent clinical examinations to find a subjective symptom improvement. In addition, the laboratorychemical determination of the lactate and ammonia with and without vitamin B6 therapy was carried out during the work test with 2-minute ischemia. Results: The patient reports a progressive improvement in muscular strength and endurance and negates myalgia under the vitamin B6 therapy. A doubling of the walking distance could be achieved under vitamin B6. Under the vitamin B6 substitution occurred a drawdown of the creatine kinase. In the work test with vitamin B6 supplementation exposed a normal increase in lactate and ammonia, while without vitamin B6 only a slight increase in lactate was recorded. Conclusion: We report on a patient with a previously undescribed homozygous, pathogenic mutation in the PYGM gene, which is located in the vicinity of the PLP binding site of the myophosphorylase and thus explains the good response to vitamin B6. The daily substitution of vitamin B6 could help to improve clinical and laboratory chemical muscular symptoms. In our view, a therapy attempt seems 
justified in the case of low costs and side effects of vitamin B6 in the case of proven McArdle disease, particularly in the case of a mutation in the immediate vicinity of the PLP binding site of the myophosphorylase.

\section{PS1Group1-117 / \#782}

Topic: Group 1 - Muscle Diseases of Genetic Origin and Acquired Myopathies: Clinical Features, Pathophysiology, Therapy

\section{MASSIVE INCREASE IN CARDIAC TROPONIN T WITHOUT CARDIAC INVOLVEMENT IN NECROTIZING MYOPATHY WITH ANTI-HMGCR- ANTIBODIES}

Annika Saak $^{1}$, Jochen Schaefer ${ }^{2}$, Sandra Jackson ${ }^{2}$

${ }^{1}$ Neurology, University Hospital Dresden, Dresden, $D E ;{ }^{2}$ Neurology, University Hospital Dresden, Dresden, $D E$

Background: Immune-mediated necrotizing myopathies may be caused by antibodies to anti-SRP or anti-HMG CoA reductase. They are characterized by muscle weakness, pronounced hyperCKemia and myalgias and may be associated with malignancies. Therapeutically, various immunosuppressants such as steroids, immunoglobulins or cytotoxic drugs are used. Methods: A 19-year-old statin-naïve patient developed acute tetraplegia with muscle tenderness and high antibody titers to HMG-CoA reductase. Besides marked hyperCKemia (up to $308 \mu \mathrm{Cat} / \mathrm{L}$; norm $<3.2$ ) a massive increase in cardiac troponin $\mathrm{T}$ (up to $2580 \mathrm{ng} / \mathrm{L}$; norm $<14$ ) was noted in the absence of overt cardiac involvement. A musculoskeletal origin of cardiac troponin $\mathrm{T}$ was suspected. We determined cardiac troponin $\mathrm{T}(\mathrm{cTnT})$ and cardiac troponin I (cTnI) under various therapeutic regimens and investigated for cardiac involvement due to the underlying necrotizing myopathy. In addition, the expression of cTnT and cTnI is currently being analyzed in undifferentiated (myoblasts) and differentiated (myotubes) cultured muscle cells. Results: Extensive cardiac diagnostic investigations (ECG, TTE, cardiac CT and MRI, heart rate variability) were unremarkable. However, despite massively elevated cTnT levels, cardiac troponin I values always remained normal. Alongside the clinical improvement under intravenous immunoglobu- lin therapy, there was a concomitant decrease in creatine kinase, cTnT and anti-HMG-CoA reductase antibody titers. Conclusion: We report a statin-naive patient with severe anti-HMGCR-positive necrotizing myopathy, who had massively elevated cTnT levels. After exclusion of cardiac involvement, which was corroborated by normal concentrations of the entirely cardiospecific cTnI, we interpret the massive increase of cTnT in the patient as of exclusively musculoskeletal origin. Although cTnT may be mildly elevated (10fold) in various myopathies, it has - to our knowledge - not been described to rise to such an extent (200fold) in myopathies without cardiac involvement. Compared to mature myocytes, HMGCR expression is up-regulated in regenerating muscle fibres which may therefore undergo an accelerated turnover in an anti-HMGCR-positive necrotizing myopathy. Moreover, some evidence suggests that cTnT which is expressed only in low amounts in mature myocytes, is expressed in much higher amounts in regenerating skeletal muscle cells, thus explaining particularly high levels of cTnT in our patient. This is to be verified in muscle cell cultures at various stages of differentiation.

\section{PS1Group1-118 / \#688}

Topic: Group 1 - Muscle Diseases of Genetic Origin and Acquired Myopathies: Clinical Features, Pathophysiology, Therapy

\section{RESULTS OF NORTH STAR}

\section{AMBULATORY ASSESSMENTS IN THE ACT DMD TRIAL IN IN PATIENTS WITH NMDMD}

Francesco Muntoni ${ }^{1}$, Panayiota Trifillis ${ }^{2}$, Gary L. Elfring $^{2}$, Stuart W. Peltz ${ }^{3}$, Craig Mcdonald ${ }^{4}$ ${ }^{1}$ Dubowitz Neuromuscular Unit, UCL Great Ormond Street Institute of Child Health, London, GB, ${ }^{2} P T C$ Therapeutics, Inc, South Plainfield, NJ, US, ${ }^{3} P T C$ Therapeutics Inc, South Plainfield, NJ, US: ${ }^{4}$ Physical Medicine And Rehabilitation, UC Davis Medical Center, Sacramento, CA, US

Background: Ataluren is the first drug to treat the underlying cause of nonsense mutation Duchenne muscular dystrophy (nmDMD) by promoting readthrough of a premature stop codon to produce full-length functional dystrophin. ACT DMD was a phase 3, randomized, double-blind, placebo-con- 
trolled trial of ataluren that enrolled males aged 7-16 years with nmDMD and baseline six-minute walk distance $(6 \mathrm{MWD}) \geq 150 \mathrm{~m}$ and $\leq 80 \%$-predicted. Methods: Eligible patients were randomized 1:1 to receive ataluren $10,10,20 \mathrm{mg} / \mathrm{kg}$ or placebo orally three times daily for 48 weeks. The NSAA is a validated functional scale to measure disease progression specifically in ambulant boys with DMD. It consists of 17 activities ranging from standing from a chair to jumping. Each activity is scored as 0,1 , or 2; the sum of these 17 scores forms the total score, which is linearized to a 0 (worst)-100 (best) score. The intent-to-treat population of ACT DMD consisted of 228 patients (ataluren, $\mathrm{n}=114$; placebo, $\mathrm{n}=114)$. Results: Overall, patients who received ataluren gained a 1.5-point advantage in NSAA observed score compared with patients who received placebo (mean NSAA scores, ataluren: -7.0; placebo: $-8.5 ; p=0.270$ ). In addition, fewer patients who received ataluren shifted from a score of 1 or 2 (able to perform function) to 0 (unable to perform function) across all 17 activities compared with those who received placebo. Between-group differences across each of the 17 activities consistently favored ataluren, ranging from $1 \%$ (lift head) to $11 \%$ (jump) to $12 \%$ (rise from chair). Conclusion: In conclusion, ataluren is the first drug to demonstrate a benefit to patients with nmDMD compared with placebo as assessed by NSAA scores.

\section{PS1Group1-119 / \#932}

Topic: Group 1 - Muscle Diseases of Genetic Origin and Acquired Myopathies: Clinical Features, Pathophysiology, Therapy

\section{NONSENSE AND SINGLE NUCLEOTIDE FRAMESHIFT MUTATIONS IN BECKER MUSCULAR DYSTROPHY}

Monica Traverso ${ }^{1}$, Chiara Panicucci ${ }^{1}$, Michela Catteruccia ${ }^{2}$, Paolo Broda ${ }^{1}$, Francesca Madia ${ }^{3}$, Giulia Pozzolini ${ }^{3}$, Maria Derchi ${ }^{4}$, Annalaura Torella ${ }^{5}$, Claudio Bruno ${ }^{6}$, Federico Zara ${ }^{3}$, Vincenzo Nigro $^{5}$, Carlo Minetti ${ }^{1}$, Adele D’Amico ${ }^{2}$, Chiara $\underline{\text { Fiorillo }}^{7}$

${ }^{1}$ Pediatric Neurology And Muscular Disorders, Gaslini Children Hospital, Genoa, IT; ${ }^{2}$ Unit Of Neuromuscular Disorders, Laboratory Of Molecular Medicine, Bambino Gesu' Children's
Hospital,, Rome, IT; ${ }^{3}$ Neurogenetic Laboratory, University of Genoa-Gaslini Children Hospital, Genoa, IT: ${ }^{4}$ Cardiology, University of GenoaGaslini Children Hospital, Genoa, IT; ${ }^{5}$ Precision Medicine, Università degli Studi della Campania "Luigi Vanvitelli", Naples, IT; ${ }^{6}$ Centre Of Myology, University of Genoa-Gaslini Children Hospital, Genoa, IT; Neuroscience, University of Genoa, Genoa, IT

Background: Nonsense and frameshift mutations in $D M D$ gene potentially determine the interruption of protein synthesis and degradation of a truncated dystrophin, for this reason they are usually associated with severe Duchenne phenotype (DMD). Conversely, in-frame mutations lead to reduced or abnormal protein synthesis and are associated to milder Becker muscular dystrophy (BMD). Exceptions of this rule are found in a small percentage of cases. In these uncommon cases, the exact location of the mutation is considered an important factor. Nevertheless other factors, such as presence of splicing regulatory elements, can influence the ultimate outcome and they are largely unexplored in routine diagnostic procedures. Methods: We present clinical, molecular and immunohistochemistry study of a sample of 9 Italian BMD patients (age range 7-30 years) carrying nonsense or single nucleotide mutations in $D M D$ gene. Patients were evaluated with North-Star Ambulatory Assessment (NSAA) and muscle MRI. Muscle biopsies were obtained from 8 cases. Routine histological procedures, immunofluorescence and western blot were performed. We also analysed the specific transcripts involving the different mutations via RTPCR. Results: Most mutations are nonsense, localised between exon 1-29 and in exon 74. One mutation is a splice site variant leading to absence of exon 11 and subsequent loss of reading frame. Another mutation is a single nucleotide duplication leading to downstream frameshift in exon 73. Six mutations are described and have been associated either with DMD or BMD. Three variants are novel. From a clinical point of view, all patients are still ambulant, however 4 presented moderate phenotype (NSAA below 30) and the oldest patient was not able to rise from the floor autonomously. CK was significantly elevated (range 3000-15000 U/1, normal value $<200$ ). The 2 oldest patients also displayed signs of heart involvement and were in therapy with ACE inhibitors. No patient was taking corticosteroids. Muscle sections showed variable degree of necrosis and degeneration of muscle fibres, which correlates with 
age at biopsy and CK level. Dystrophin expression was only partially reduced with immunofluorescence from moderate severe, to mild reduction. Interesting in most patients staining for alpha-dystroglycan was also markedly reduced. Western blot showed either absent dystrophin or reduced amount of protein of normal size. In three cases a lower weight dystrophin band was detected with Western Blot. RT-PCR documented presence of normal size products in nonsense mutations and of shorter products in the case of splice site mutation. Conclusion: In this small cohort, we observed nonsense and single nucleotide frameshift mutations in BMD patients, few presenting a moderate phenotype when compared with the most common BMD patient with multiple exon deletions. Additionally, we showed that dystrophin mRNA and protein are still retained in affected muscles, suggesting an altered function of the protein. We argue that this can be highlighted by marked reduction of the distrophyn-dystroglycans complex. These cases underscore the importance of a complete analysis of $D M D$ gene and its transcript in order to provide better prognostic information and genotypephenotype correlation. Additionally presence of nonsense mutations in BMD patients raise question on the possible therapeutic approaches.

\section{PS1Group1-120 / \#959}

Topic: Group 1 - Muscle Diseases of Genetic Origin and Acquired Myopathies: Clinical Features, Pathophysiology, Therapy

\section{DEVELOPMENT OF A PROGNOSTIC MODEL FOR 1-YEAR CHANGE IN TIMED 4 STAIR-CLIMB IN DUCHENNE PATIENTS}

Nathalie Goemans $^{1}$, Marleen Vanden Hauwe ${ }^{1}$, James Signorovitch², Gautam Sajeev², Zhiwen $\mathrm{Yao}^{2}$, Madeline Jenkins ${ }^{2}$, Erin Mcdonald ${ }^{2}$, Imbrahima Dieye ${ }^{2}$, Susan J. Ward ${ }^{3}$

${ }^{1}$ Neuromuscular Reference Centre, Department Of

Paediatrics And Child Neurology, University

Hospitals Leuven, Leuven, BE;'Analysis Group,

Boston, US: ${ }^{3}$ cTAP, Cambridge, US

Background: Longitudinal progression of disease is inherently heterogeneous among individuals with Duchenne muscular dystrophy (DMD). The resulting variation in outcome measures can complicate clinical trial design and potentially cloud interpretation of results. The collaborative Trajectory Analysis Project (cTAP) is a pre-competitive coalition of academic clinicians, drug developers, and patient foundations; cTAP was formed in 2015 to identify biostatistical approaches to overcome the challenges of high variation in clinical trials in DMD. We previously reported a prognostic score that explained $60 \%$ of variation in the change in 6 minute walk distance (6MWD) over 1 year, and significantly improved upon prognosis from baseline age, 6MWD and steroid use by also incorporating timed function tests, height and weight [Goemans N, vanden Hauwe M, Signorovitch J, Swallow E, Song J, CollaborativeTrajectory Analysis Project (cTAP) (2016). Individualized Prediction of Changes in 6-Minute Walk Distance for Patients with Duchenne Muscular Dystrophy. PLoS ONE 11(10): e0164684. doi:10.1371/ journal.pone.0164684] The aim of the present study was to develop a prognostic model for 1-year change in 4-stair climb (4SC) among DMD patients, and to assess the additional predictive value of the model compared to commonly used factors (i.e., age, baseline 6MWD and steroid use). Methods: Natural history data were collected from DMD patients approximately every 6 months over the course of 2 to 5 years during routine clinical practice at the Universitaire Ziekenhuizen pediatric neurology clinic in Leuven, Belgium. Patient demographics, treatment experience and ambulatory outcomes were recorded at each visit. Annualized changes in 4SC were studied between all pairs of visits separated by $\sim 1$ year (8-16 months). Prediction models were developed using multivariable regression for repeated measures. Generalized estimating equations (GEE) with an exchangeable covariance structure were used to account for the use of multiple pairs of visits from individual patients. Results: A total of $n=235 \sim 1$ year follow-up intervals from $n=81$ boys were included. Mean age was 9.1 years and mean 4-SC was $3.84 \mathrm{~s}$ at the start of these intervals; average duration of steroid use was 29.11 months. During the subsequent $\sim 1$-year, mean annualized change in $4 \mathrm{SC}$ was $0.71 \mathrm{~s}$ with a standard deviation (SD) of 2.20. Predictions based on age, baseline 4SC and steroid use explained $13 \%$ of variation in annualized $4 \mathrm{SC}$ changes $(\mathrm{R}$-squared $=0.13)$. A broadened prognostic model, adding timed 10-meter walk/run, and rise from supine, as well as height and weight, significantly improved prediction, explaining $34 \%$ of variation in annualized 6MWD changes (R-squared $=0.34$ ). In the broadened model, parameters that were most 
strongly correlated with 1 year change in $4 \mathrm{SC}$ were steroid use $>1$ year $(p<0.001)$, baseline $4 \mathrm{SC}(\mathrm{s})(\mathrm{p}<$ $0.001)$, baseline rise from supine $(\mathrm{s})(\mathrm{p}<0.001)$ and baseline 10 meter walk run $(\mathrm{s})(\mathrm{p}<0.05)$. Conclusion: A prognostic model incorporating timed function tests significantly improved prediction of 1-year changes in 4SC. Explained variation was more than doubled compared to predictions based only on age, baseline 4SC and steroid use, indicating significant potential for broader prognostic models to inform clinical trial design and interpretation in DMD

\section{PS1Group1-121 / \#989}

Topic: Group 1 - Muscle Diseases of Genetic Origin and Acquired Myopathies: Clinical Features, Pathophysiology, Therapy

\section{AMBULATORY \\ ELECTROCARDIOGRAPHIC LONGITUDINAL STUDY IN THE GRMD DOG MODEL OF DUCHENNE MUSCULAR DYSTROPHY}

Inès Barthélémy, Xavier Cauchois, Stephane Blot U955 - Imrb, Inserm, Ecole Nationale Vétérinaire D'alfort, Upec, U955 - IMRB, Inserm, Ecole

Nationale Vétérinaire d'Alfort, UPEC, MaisonsAlfort, FR

Background: Duchenne muscular dystrophy (DMD) is a progressive muscle disease leading to motor, respiratory and cardiac disabilities. Electrocardiographic (ECG) monitoring is part of the standards of care for DMD patients, and reveals tachycardia, elongated QT, deep Q-waves, premature ventricular beats (PVBs), and early decreased heart rate variability (HRV). The preclinical research of treatments targeting DMD is supported by a translational chain, which ultimate link is the GRMD dog (Golden Retriever Muscular Dystrophy), since it faithfully reproduces the DMD disease course in a large animal context. This includes the occurrence of a cardiomyopathy, with onset of myocardial fibrosis in the 6-12 months of age period, followed by a decrease of heart contractility, and ultimately in some dogs a death from decompensated dilated cardiomyopathy. Since this model is used at the very last preclinical step, there is a need to evaluate the effect of candidate treatments using methods analog to those used in patients. Few studies have focused on ECG in GRMD, but no one longitudinally using ambulatory Holter recordings, and investigating HRV. We aimed to describe the sequential ECG alterations in GRMD dogs in order to provide relevant indices to be used in preclinical studies. Methods: Holter ECG recordings were performed monthly overnight in 15 GRMD and 4 healthy littermates from the age of 2 months until the age of 24 months. The lead II was used for quantitative analysis, which first focused on four timepoints: 6, 12, 18, and 24 months of age. The heart rate, QT interval, Q/R ratio, and the occurrence of arrythmias were quantified. A HRV analysis was also performed including time-domain analysis and frequency domain analysis. Results: One of the 4 healthy dogs presented an increased heart rate, a markedly reduced HRV, and had transiently elevated serum cTpnI. We thus chose to exclude this dog from the analysis. The heart rate was found increased in GRMD dogs, this difference being significant from the age of 12 months. There was a trend towards elongated QT at 24 months. We found a significantly increased $\mathrm{Q} / \mathrm{R}$ ratio only at the age of 6 months. PVBs were observed in 5 over the 8 dogs at 24 months of age. Two of them already presented such arrhythmias at 6 months of age, and most of the others started to exhibit PVBs between 12 and 18 months of age. These PVBs were polymorphic in two dogs, and could be seen either isolated, in doublets or triplets; transient episodes of ventricular tachycardia could also be detected. HRV analysis revealed significantly decreased pNN50 at 12 months of age, and a decrease of the LF/HF ratio from the age of 12 months. Conclusion: This study provides new indices to monitor the cardiac disease in GRMD dogs, and again demonstrates similarities between GRMD and DMD cardiomyopathies. These similarities and the availability of a comprehensive evaluation set including ECG, echocardiography, cardiac NMRI, paralleling the one used in DMD patients, make the GRMD dog an optimal context to anticipate therapeutic effects expectable in DMD patients, measured with the same tools and indicators. 
PS1Group1-122 / \#652

Topic: Group 1-Muscle Diseases of Genetic Origin and Acquired Myopathies: Clinical Features, Pathophysiology, Therapy

\section{MIMICS OF INCLUSION BODY MYOSITIS: CASE PRESENTATIONS AND IDENTIFICATION OF TYPICAL PITFALLS}

Rachel Zeng, Stefanie Glaubitz, Karsten Schmidt, Per-Ole Carstens, Jens Schmidt, Jana Zschüntzsch Department Of Neurology, University of Göttingen, Göttingen, DE

Background: Inclusion body myositis (IBM) is an idiopathic inflammatory myopathy with typical clinical symptoms und histological features. However, due to a range of acquired or genetically determined myopathies with similar presentations, IBM is often missed and diagnosed in error as another neuromuscular disease. Methods: Along with dermatomyositis, polymyositis, overlap myositis and (immune mediated) necrotizing myopathy, IBM belongs to the group of idiopathic inflammatory myopathies (IIM). The typical clinical presentation of IBM involves slowly progressive muscle atrophy and weakness with a preference of long finger flexors, knee and foot extensors, often with an asymmetric distribution. Other symptoms may include myalgia and dysphagia, the latter reported in over the half of the patients with IBM. The diagnosis of IBM is currently based on a defined composition of clinical, serological and muscle histologic criteria. Additionally, electromyography, muscle MRI and muscle ultrasound may help to confirm the diagnosis. A muscle biopsy is required to diagnose a clinico-pathologically defined IBM, showing the co-existence of myodegenerative and inflammatory pathologic features. Nevertheless, the diagnosis of IBM or the differentiation from other neuromuscular diseases can be difficult due to atypical clinical presentations. Histopathological findings can also lead to confusion since inflammatory infiltrates can also be found in hereditary myopathies like dysferlinopathy or facioscapulohumeral dystrophy. The myodegenerative characteristics of myofibrillar myopathies like vacuoles and abnormal accumulations of filamentous proteins can also be mistaken. Only a correct and reliable diagnosis of IBM allows for recruitment to clinical studies and helps to find novel, exploratory treatment modalities and prevent unnecessary treatments. Results: Here, we provide typical case reports of hereditary inclusion body myopathy (hIBM), titinopathy (TMD/LGMD2J), facioscapulohumeral muscular dystrophy (FSHD), polymyositis, chronic inflammatory axonal polyneuropathy (CIAP) and other neuromuscular diseases that can mimic IBM. Conclusion: The data are carefully discussed and typical clinical pitfalls are summarized.

\section{PS1Group1-123 / \#690}

Topic: Group 1 - Muscle Diseases of Genetic Origin and Acquired Myopathies: Clinical Features, Pathophysiology, Therapy

\section{LONG-TERM PULMONARY FUNCTION IN NON-AMBULATORY PATIENTS WITH NMDMD TREATED WITH ATALUREN}
Craig Mcdonald ${ }^{1}$, Eugenio Mercuri ${ }^{2}$, Francesco $^{2}$ Muntoni $^{3}$, Kathryn Selby ${ }^{4}$, Fengbin Jin $^{5}$, Gianina Panaghie-Meltzer ${ }^{6}$, Panayiota Trifillis ${ }^{5}$, Marcio Souza $^{6}$, Stuart W. Peltz ${ }^{6}$, Mar Tulinius ${ }^{7}$ ${ }^{1}$ Physical Medicine And Rehabilitation, UC Davis Medical Center, Sancramento, CA, US $;^{2}$ Università Cattolica del Sacro Cuore, Rome, IT, ${ }^{3}$ Dubowitz Neuromuscular Unit, UCL Great Ormond Street Institute of Child Health, London, GB; ${ }^{4}$ British Columbia Children's Hospital, Vancouver, BC, CA, ${ }^{5}$ PTC Therapeutics, Inc, South Plainfield, NJ, US: ${ }^{6}$ PTC Therapeutics Inc, South Plainfield, NJ, US $;^{7}$ Gothenburg University, Gothenburg, SE

Background: Duchenne muscular dystrophy (DMD), an X-linked, recessive disease affecting $\sim 1$ in every $3600-6000$ live male births, is caused by dystrophin gene mutations. Dystrophin is critical for myofiber structural stability and function; its absence leads to progressive muscle dysfunction, loss of ambulation, and early death from respiratory/cardiac failure. Forced vital capacity (FVC) $<1 \mathrm{~L}$ raises mortality risk in DMD patients. About $10-15 \%$ of patients have a dystrophin gene nonsense mutation (nonsense mutation DMD [nmDMD]), where a premature stop codon halts translation to generate truncated, nonfunctional dystrophin. Ataluren promotes ribosomal readthrough of the premature stop codon to produce a full-length dystrophin protein, treating the underlying cause of nmDMD. Methods: FVC data from non-ambulatory patients at study entry (un- 
able to run/walk $10 \mathrm{~m}$ in $\leq 30$ seconds) aged 9 to 18 years receiving oral ataluren $(40 \mathrm{mg} / \mathrm{kg} /$ day $[10,10$, and $20 \mathrm{mg} / \mathrm{kg}$ for morning, midday and evening doses, respectively]) were obtained from Study 019 (NCT01557400; begun in 2012; data cut-off Jan. 31, 2017), an international, multicenter, open-label trial that enrolled patients from previous ataluren PTCsponsored trials at non-US study sites. Data for agematched,non-ambulatorypatients(wheelchair-bound) in subjects receiving SOC (not ataluren) were obtained from an ongoing natural history study (CINRG, NCT00468832; from 2012 through Nov.18, 2016) and compared to 019 FVC < 1L by KaplanMeier analysis. Results: Subgroups included 38 ataluren- and 58 SOC-treated patients. At data cut-off fewer ataluren-treated vs SOC-treated patients had FVC $<1$ L ( $7.9 \%$ vs $39.7 \%$, respectively). FVC $<1 \mathrm{~L}$ was reached by $50 \%$ of SOC-treated patients by age) age 19.3 years (95\% CI, 18.8-22.6). At age 19.3 years, only $16 \%$ of ataluren-treated patients had FVC $<1 \mathrm{~L}$. FVC $<1 \mathrm{~L}$ was reached by $50 \%$ of SOC-treated patients after 7.1 years (5.3-9.4) with SOC (log-rank test $p=0.067) ; 10 \%$ of ataluren-treated patients had FVC $<1 \mathrm{~L}$ after 4 years. Most adverse events (AEs) on ataluren were mild $(29.5 \%$ of patients) or moderate (31.8\% of patients). The most common AEs were nasopharyngitis $(45.5 \%)$, headache $(27.3 \%)$, vomiting (27.3\%), and gastroenteritis $(22.7 \%)$. Conclusion: Findings suggest that ataluren preserves lung function in non-ambulatory patients with nmDMD. Safety and tolerability were consistent with previous findings.

\section{PS1Group1-124 / \#701}

Topic: Group 1 - Muscle Diseases of Genetic Origin and Acquired Myopathies: Clinical Features, Pathophysiology, Therapy

\section{USE OF A $>/=$ 5-SECOND}

\section{THRESHOLD IN BASELINE TIME TO STAND FROM SUPINE TO PREDICT PROGRESSION IN DMD}

Craig Mcdonald ${ }^{1}$, Marcio Souza ${ }^{2}$, Gary L. Elfring ${ }^{3}$, Panayiota Trifillis ${ }^{3}$, Joseph Mcintosh ${ }^{2}$, Stuart W. Peltz $^{2}$, Eugenio Mercuri ${ }^{4}$ ${ }^{1}$ Physical Medicine And Rehabilitation, UC Davis, Sacramento, CA, US: ${ }^{2} P T C$ Therapeutics Inc, South Plainfield, NJ, US, ${ }^{3} P T C$ Therapeutics, Inc, South Plainfield, NJ, US, ${ }^{4}$ Università Cattolica del Sacro Cuore, Rome, IT
Background: Time to stand from supine is a predictor for loss of clinically meaningful Duchenne muscular dystrophy (DMD) milestones. A $\geq 5$-second threshold in this functional test can predict disease progression over 48 weeks; conversely, a $<5$-second threshold suggests functional stability. This analysis examined the usefulness of $\mathrm{a} \geq 5$-second threshold as a subset analysis for DMD trials. Methods: A post hoc analysis was conducted using data from the subset of patients with a baseline time to stand from supine $\geq 5$ seconds from two 48-week, randomized controlled trials examining the efficacy and safety of ataluren in patients with nonsense mutation DMD: a phase $2 \mathrm{~b}$ (Study 007/NCT00592553; boys $\geq 5$ years) and a phase 3 trial (Study 020/NCT01826487; boys $\geq 7-\leq 16$ years). Results: In Study 007, 37 atalurenand 29 placebo-treated patients met the $\geq 5$-second threshold. Their respective mean (standard deviation, SD) baseline 6-minute walk distances (6MWD) were 311.3 (91.1) and 312.2 (82.2) m. In Study 020, 56 ataluren- and 46 placebo-treated patients met the $\geq 5$-second threshold: mean (SD) baseline 6MWD, 349.9 (60.2) vs 336.6 (63.8) m, respectively. A beneficial ataluren response vs placebo was observed across multiple endpoints in both trials in the $\geq 5$-second threshold subgroup. Study 007 (ataluren vs placebo, respectively); 48-week change in: 6MWD $(-35.9$ vs $-85.6 \mathrm{~m}, p=0.01), 10-\mathrm{m}$ run/walk $(2.5 \mathrm{vs}$ 5.1 seconds, $p=0.10)$, 4 -stair climb ( 3.5 vs $7.5 \mathrm{sec}$ onds, $p=0.02$ ). Study 020 (ataluren vs placebo, respectively); 48-week change in: 6MWD ( -48.7 vs $-100.1 \mathrm{~m}, p<0.01), 10-\mathrm{m}$ run/walk (2.3 vs $4.8 \mathrm{sec}-$ onds, $p=0.03$ ), 4-stair climb (4.7 vs 7.6 seconds, $p=0.04$ ). Conclusion: Consistent with published natural history data, placebo-treated patients with a baseline time to stand from supine $\geq 5$ seconds showed declining ambulatory ability over 48 weeks. $A \geq 5$-second threshold may represent a useful subset analysis for DMD trials; this post hoc analysis highlights an ataluren treatment benefit vs placebo. 


\section{PS1Group1-125 / \#780}

Topic: Group 1 - Muscle Diseases of Genetic Origin and Acquired Myopathies: Clinical Features, Pathophysiology, Therapy

\section{NOVEL THERAPEUTIC PERSPECTIVES FOR SARCOGLYCANOPATHY, IN VITRO AND IN VIVO STATE OF THE ART}

\author{
Dorianna Sandona $^{1}$, Roberta Sacchetto ${ }^{2}$, Elisa \\ Bianchini $^{2}$, Marcello Carotti ${ }^{2}$, Michela Soardi ${ }^{3}$, \\ Chiara Fecchio ${ }^{4}$ \\ ${ }^{1}$ University of Padova, Padova, IT; ${ }^{2}$ university of \\ padova, Padova, IT; ${ }^{3}$ university of padova, padova, \\ IT; ${ }^{4}$ Biomedical Science, University of Padova, \\ Padova, IT
}

Background: Sarcoglycans (SG) are glycosylated proteins (alpha-, beta-, gamma- or delta-SG) forming a key structural complex, essential for the sarcolemma integrity of striated muscles during contraction. In sarcoglycanopathies, defects in any one of the sarcoglycan genes lead to the strong reduction or even the loss of the SG-complex. Most of the reported cases are due to missense mutations originating a full length but folding-defective proteins. We proved that the primary pathological event in sarcoglycanopathy occurs in the Endoplasmic Reticulum, where the quality control system, by proofreading newly synthesized sarcoglycans, recognizes and delivers to proteasomal degradation the foldingdefective mutants. This results in the secondary loss of the wild-type partners. We also observed that many missense mutants retain their function as the entire complex can be properly rescued by reducing the mutant degradation. These findings opened new therapeutic perspectives for this neglected disease allowing to design small molecule-based approaches aimed either to inhibit sarcoglycan mutants degradation, or to help their folding so that, skipping disposal, they can assemble and traffic at the proper site of action. Methods: Culture and differentiation of primary myogenic cells of LGMD2D patients. Biochemical and molecular analysis of sarcoglycan expression. Primary myotubes treatment with small molecules. AAV transduction of newborn alpha-SG KO mice. CRISPR-Cas9 genome editing of zebrafish. Results: We tested several small molecules known as CFTR correctors which successfully recovered different mutants of alpha-sarcoglycan in cellular models and, notably, the whole SG-complex in primary myotubes from a patient suffering of alpha-sarcoglycanopathy. Moreover, we performed a functional test by measuring CK release from patient's myotubes under stressful conditions, showing membrane stabilization when cells were pretreated with CFTR correctors. Interestingly, cytotoxicity tests highlighted absent or low toxicity of these compounds on cells. To confirm in vivo this successful strategy we need animal models expressing foldingdefective sarcoglycans. As the available SG-KO and KI mice are unsuitable to our purposes, and considering the large number of reported sarcoglycan missense mutants, our aim is now the generation and characterization of novel and unconventional alphasarcoglycanopathy vertebrate models. To this purpose, we have planned to generate transiently "humanized" mice, by the transduction of the null mice with rAAVs (recombinant adeno associated viruses) expressing different missense mutants of the human alpha-SG. On the other hand, we are focusing on the small vertebrate zebrafish, also considering that the available SG KI mice failed to develop a dystrophic phenotype. Taking advantage of state of the art genome editing techniques such as the CRISPR-cas9 system, we have obtained the beta- and delta-SG KO lines, at present under characterization, whereas the generation of SG KI zebrafish is ongoing. Conclusion: While mice are usually the vertebrate model of election for pharmacological studies, zebrafish represents a valuable and recognized vertebrate for modelling muscle diseases and presents advantages over mammals especially for quicker and wider drug screening procedures. Together, these two complementary approaches will allow us to evaluate efficacy and safety of the promising molecule identified in vitro and will foster the development of a cure for sarcoglycanopathy.

\section{PS1Group1-126 / \#807}

Topic: Group 1 - Muscle Diseases of Genetic Origin and Acquired Myopathies: Clinical Features, Pathophysiology, Therapy

\section{ATALUREN IN PATIENTS AGED $\geq 2$ TO <5 YEARS WITH NMDMD: 28- WEEK RESULTS FROM A PHASE 2 STUDY}

Cuixia Tian ${ }^{1}$, Robert Kong ${ }^{2}$, Fengbin $\mathrm{Jin}^{3}$, Edward O’Mara $^{3}$, Panayiota Trifillis ${ }^{3}$, Joseph Mcintosh ${ }^{2}, \mathrm{~J}$ B. Renfroe ${ }^{4}$ 
${ }^{1}$ Neurology, Cincinnati Children's Hospital Medical Center, Cincinatti, OH, US, ${ }^{2} P T C$ Therapeutics Inc, South Plainfield, NJ, US, ${ }^{3} P T C$ Therapeutics, Inc, South Plainfield, NJ, US: ${ }^{4}$ Child Neurology Center of NW Florida, Gulf Breeze, FL, US

Background: Nonsense mutation Duchenne muscular dystrophy (nmDMD) is a rare, X-linked genetic disorder that relts in a progressive decline in function, loss of ambulation and early death due to respiratory or cardiac complications. Ataluren enables production of full-length dystrophin protein by promoting ribosomal readthrough of a premature stop codon in the mRNA transcribed from the dystrophin gene. The goal of dystrophin restoration therapy is to slow or stabilize disease progression in patients with nmDMD. Ataluren is conditionally approved by the European Medicines Agency for the treatment of ambulatory patients aged $\geq 5$ years with nmDMD. Initiation of ataluren therapy for dystrophin restoration at a younger age, prior to substantive muscle loss, may maximize its therapeutic benefit. Methods: Ataluren study 030 was an observational, open-label Phase 2 study designed to evaluate the safety and pharmacokinetics (PK) of ataluren $(10,10$, and $20 \mathrm{mg} / \mathrm{kg})$ in patients aged $\geq 2$ to $<5$ years with nmDMD. The study included a 4-week treatment period, a 48-week extension period, and a 4-week follow-up period. Secondary objectives in Study 030 evaluated changes in timed function tests (TFTs) and the 3-part and 8-part North Star Ambulatory Assessment (NSAA) scales, adapted for children $<5$ years of age. All patients were male $(\mathrm{N}=14)$ with genotypic confirmation of $\mathrm{nmD}$ MD. Results: Two patients were excluded from the current analysis: one patient did not have reported functional assessment at Week 28; and one patient did not have baseline measurement all for post line evaluations, resulting in $\mathrm{N}=12$. Seven out of the fourteen patients in the safety population $(50 \%)$ reported $\geq 1$ treatment-emergent adverse event (TEAE) during the extension phase, all of which were deemed unrelated to the study drug; there were no serious TEAEs or discontinuations due to a TEAE. Pyrexia, ear infection, and nasopharyngitis were the most common TEAEs, each occurring in 2 patients $(14.3 \%)$. The interim analysis presented (Table) represents a preliminary assessment of ataluren's efficacy after 28 weeks of treatment. Conclusion: These interim data represent preliminary assessments of ataluren's safety and efficacy after 28 weeks of treatment in patients with nmDMD aged $\geq$ 2 to $<5$ years.

Table: Summary of Change in (A) TFTs and (B) NSAA 8-Item and 3-Item Scores Baseline to Week 28 in Study 030 Evaluable Population ( $N=12)$

\begin{tabular}{|l|c|}
\hline \multicolumn{2}{|c|}{ A. TFTs } \\
\hline Time to run/walk 10meters, s & \\
\hline Baseline, mean (SD) & $6.7(2.46)$ \\
\hline Decrease from Baseline to Week 28, mean(SD) & $-0.6(1.44)$ \\
\hline Time to climb 4 stairs, & $7.4(7.49)$ \\
\hline Baseline, mean (SD) & $-2.1(5.07)$ \\
\hline Decrease from Baseline to Week 28, mean (SD) & $7.6(7.79)$ \\
\hline Time to Stand from a Supine Position, s & $-3.3(6.70)$ \\
\hline Baseline, mean (SD) & \\
\hline Decrease from Baseline to Week 28, mean(SD) & \\
\hline \multicolumn{1}{|c|}{ B. NSAA } & $10.42(2.75)$ \\
\hline NSAA 8-item Score & $1.50(1.45)$ \\
\hline Baseline, mean (SD) & \\
\hline Increase from Baseline to Week 28 & $5.33(0.65)$ \\
\hline NSAA 3-item Score & $0.50(0.80)$ \\
\hline Baseline, mean (SD) & \\
\hline Increase from Baseline to Week 28 & \\
\hline
\end{tabular}




\section{PS1Group1-127 / \#456}

Topic: Group 1-Muscle Diseases of Genetic Origin and Acquired Myopathies: Clinical Features, Pathophysiology, Therapy

\section{A FEMALE CARRIER OF BECKER MUSCULAR DYSTROPHY PRESENTING WITH A MYOPATHY WITH PIPESTEM CAPILLARIES}

\author{
Giuseppe Cosentino $^{1}$, Filippo Brighina ${ }^{2}$, Brigida \\ Fierro $^{2}$, Laura Pilati ${ }^{2}$, Massimiliano Mirabella ${ }^{3}$, \\ Carmelo Rodolico ${ }^{4}$ \\ ${ }^{1}$ University of Palermo, Palermo, IT; ${ }^{2}$ University of \\ Palermo, Palermo, IT; ${ }^{3}$ Cattolica University, Roma, \\ IT; ${ }^{4}$ University of Messina, Messina, IT
}

Background: Myopathy with pipestem capillaries represents a very rare entity of uncertain classification with a poor prognosis, characterized by necrosis of muscle fibers, minimal cellular infiltration, and vessel wall thickening with luminal narrowing. Methods: We describe the case of a carrier woman of Becker muscular dystrophy (BMD), who presented at the age of $61 \mathrm{yr}$ with acute onset of neck and shoulder pain followed by proximal weakness of the upper limbs and the neck. Over the next 4 years, weakness progressively worsened also involving the distal muscles of the upper limbs and the pelvic girdle muscles. The electromyographic evaluation showed myopathic signs with minimal denervation in the affected muscles. Serum levels of creatine kinase were slightly increased, and a myositis-specific antibody panel was negative. A muscle biopsy of the right tibialis anterior muscle showed abnormal variation in fiber size with thin atrophic fibers, central nuclei, presence of numerous pipestem capillaries, and minimal necrosis of muscular fibers. Some cytochrome-oxidase (COX) negative muscle fibers compatible with slight mitochondrial abnormalities were also observed. As autoimmunity is considered to play a role in the development of myopathy with pipestem capillaries, patient was treated with oral corticosteroids, which determined a slight improvement (especially as regards pain). Patient also underwent a trial with azathioprine, that was stopped after several months due to inefficacy, and subsequently a trial with methotrexate, which was also soon discontinued due to side effects. At the moment the patient refuses any immunosuppressive therapy except for prednisone, and its clinical status is quite stable. Results: This case suggests that, albeit rarely, a necrotizing myopathy with pipestem capillaries could occur in previously asymptomatic patients with dystrophin gene mutations. Conclusion: The present case has having a more favorable outcome than previously reported in the literature. The role of dystrophinopathy as a triggering factor for this rare form of myopathy and/or as a disease modifier could be hypothesized though it remains questionable.

\section{PS1Group1-128 / \#459}

Topic: Group 1-Muscle Diseases of Genetic Origin and Acquired Myopathies: Clinical Features, Pathophysiology, Therapy

\section{URINARY TITIN IS A NON-INVASIVE BIOMARKER TO DIAGNOSE DUCHENNE MUSCULAR DYSTROPHY EVEN IN ADVANCED STAGE}

Hiroyuki Awano $^{1}$, Yuka Ishikawa ${ }^{2}$, Yukitoshi Ishikawa $^{2}$, Masaaki Matsumoto ${ }^{3}$, Masashi Nagai ${ }^{3}$, Taku Shirakawa ${ }^{4}$, Nobuhiro Maruyama ${ }^{5}$, Yo-Ichi Nabeshima $^{6}$, Kazumoto Iijima ${ }^{3}$, Masafumi Matsuo ${ }^{4}$ ${ }^{1}$ Pediatrics, Kobe University Graduate School of Medicine, Kobe, JP,2 Yakumo National Organization Hospital, Yakumo, JP:3 Pediatrics, Kobe University Graduate School of Medicine, Kobe, JP ${ }^{4}$ Physical Therapy, Kobe Gakuin University, Kobe, JP; ${ }^{5}$ Immuno-Biological Laboratories Co., Ltd., Fujioka, JP;'Laboratory Of Molecular Life Science, Foundation for Biomedical Research and Innovation, Kobe, JP

Background: Duchenne muscular dystrophy (DMD) is a fatal muscle-wasting disease caused by lack of dystrophin. As the disease progresses, muscle mass decreases. Loss of muscle mass is supposed to reduce leaking enzymes including creatine kinase (CK), a most common-used biomarker for DMD. Therefore, reliable biomarker regardless of muscle loss is required to evaluate the status of dystrophic muscles, especially in advanced stage of DMD patients Titin/connectin is the largest protein in human and functions as molecular spring in the sarcomere. We previously reported titin-fragment in urine was a novel disease progression biomarker correlated with serum CK in young DMD patients (Awano et al. 2017). Urinary titin in advanced DMD remains unknown. In this study, we measured urinary titin level 
of advanced DMD patients and examined the association between urinary titin and serum CK. Methods: Sixty-two DMD patients from aged 21 to 50 years and 15 healthy male controls were enrolled in this study. Titin was quantified using Titin N-Fragment Assay Kit - IBL (Immuno-Biological Laboratories Co, Ltd., Fujioka, Japan). Titin was normalized to urine creatinine concentration and was expressed as $\mathrm{pmol} / \mathrm{mg}$ creatinine $(\mathrm{Cr})$. Statistical analysis was analyzed by Mann-Whitney U test, ANOVA, and logistic regression analysis. Receiver operating characteristic (ROC) curve was generated and area under the curve (AUC) was calculated by GraphPad PRISM 7.02 (Graphpad Software, CA, USA). Values of $p<0.05$ were concidered statistically significant. The protocol of this study was approved by the ethical committee. Results: Urinary titin was determined in 62 and 15 samples in advanced DMD patients and controls, respectively. In advanced DMD patients, median concentration of urinary titin was $57.7 \mathrm{pmol} / \mathrm{mg}$ Cr. This was significantly higher than those of controls $(1.8 \mathrm{pmol} / \mathrm{mg} \mathrm{Cr})(\mathrm{p}<0.0001)$. The AUC in the ROC curve was 0.99 , indicating that urinary titin was a diagnostic biomarker in progressed stage of DMD. We previously reported that urinary titin decreased by aging and was significantly correlated to serum CK concentration in DMD patients (Awano et al.2017). An association between concentration of urinary titin, and age or serum CK was investigated by liner regression analysis. The analysis revealed that urinary titin showed a significant decline with age $(p<0.0001)$ and a positive correlation to serum CK $(p<0.001)$. It was noted that 46 out of 62 patients (74.2\%) had normal or low CK level. On the other hand, all except 1 patients showed higher titin concentration than controls. Conclusion: Urinary titin was first disclosed to be higher than healthy males in advanced DMD patients and demonstrated to be an accurate biomarker for DMD diagnosis even in elder patients. Notably, urinary titin was elevated in DMD patients who had normal or low CK level. This suggested that titin fragmentation was still active in the progressed-stage patients. It was indicated that urinary titin was a better biomarker than serum CK in advanced DMD patients. Reference; Awano H, et al. Diagnostic and clinical significance of the titin fragment in urine of Duchenne muscular dystrophy patients. Clin Chim Acta. 476.111-116.2018
PS1Group1-129 / \#830

Topic: Group 1 -Muscle Diseases of Genetic Origin and Acquired Myopathies: Clinical Features, Pathophysiology, Therapy

\section{A NOVEL MUSCLE PHENOTYPE IN A PATIENT WITH TROPOMYOSIN- RECEPTOR KINASE-FUSED GENE (TFG) DISEASE}

Nicolas N. Madigan $^{1}$, Jennifer A. Tracy ${ }^{1}$, William J. Litchy $^{1}$, Zhiyv $\mathrm{Niu}^{2}$, Margherita Milone ${ }^{1}$

${ }^{1}$ Neurology, Mayo Clinic, Rochester, MN, US, ${ }^{2}$ Laboratory Genetics \& Genomics, Mayo Clinic, Rochester, MN, US

Background: The tropomyosin-receptor kinasefused gene (TFG) protein is ubiquitously expressed across tissues, including muscle, and is predominantly present in neural tissue. Autosomal dominant $T F G$ mutations were first identified in Okinawa and Kansai prefecture patients with proximal weakness and distal sensory loss, who were diagnosed with a hereditary sensorimotor neuropathy (HSMNO). Pathological studies later demonstrated that HSMNO is a motor neuronopathy with associated sensory ganglionopathy. Limited muscle pathological findings showing fiber size variability and fiber type grouping have been reported. TFG-related neurological disorders are now understood to encompass a spectrum of phenotypes which include Charcot-Marie-Tooth disease type 2 (CMT2) and hereditary spastic paraplegia. Recently there have been several other genes that are recognized to cause motor neuron disease, neuropathy and myopathy. Methods: Review of clinical and laboratory data in a patient with $T F G$-related neurological disease. Results: A 44-year-old Caucasian male presented with a 7 year history of upper and lower limb muscle fasciculation and cramps, progressive asymmetric proximal more than distal weakness, muscle atrophy and lengthdependent sensory loss. He was areflexic. He had bilateral pes cavus. He could not walk on either his toes or heels. Serial measurements of serum creatine kinase ranged between 1897-2400 U/L (normal $<336$ ). Neurophysiologic studies demonstrated findings suggestive of a diffuse disorder of motor neurons in association with a sensory neuronpathy. Muscle biopsy of the quadriceps showed fiber type grouping and atrophic fibers of either histochemical type, but also several fibers with rimmed and nonrimmed vacuoles, increased numbers of internalized 
nuclei, fiber splitting, and necrotic and regenerating fibers. In addition there were several foci of perivascular inflammatory cells. Next generation exome sequencing detected a known pathogenic heterozygous missense variant in exon 8 (c.854C $>$ T, p.Pro285Leu) of theTFG gene, affecting the carboxy-terminal proline-glutamine rich $(\mathrm{P} / \mathrm{Q})$ protein domain. Conclusion: Our patient presented with a classic clinical phenotype of HSMNO and a known pathological genotype. Prominent rimmed and unrimmed vacuoles were present in several muscle fibers, which suggests an associated myopathic component separate from neurogenic changes. Of interest, the function of the TFG protein relates to vesicle trafficking of unfolded and misfolded proteins through the endoplasmic reticulum and the ubiquitin-proteasome system (UPS). The $\mathrm{P} / \mathrm{Q}$ domain specifically regulates the interaction with the UPS, and disruption of this domain may lead to accumulation of abnormal protein in motor neurons as well as in muscle.

\section{PS1Group1-130 / \#264}

Topic: Group 1 - Muscle Diseases of Genetic Origin and Acquired Myopathies: Clinical Features, Pathophysiology, Therapy

\section{GLYCOGEN STORAGE DISEASE TYPE IV PRESENTING AS CONGENITAL MYOPATHY WITH CONTRACTURES AND RIGID SPINE}

Maggie C. Walter ${ }^{1}$, Stephan Wenninger ${ }^{1}$, Angela Abicht $^{2}$

${ }^{1}$ Dept. Of Neurology, Ludwig-maximiliansuniversity, Friedrich-Baur-Institute, Munich, DE; ${ }^{2}$ Medical Genetic Center, Munich, DE

Background: Glycogen storage disase type 4 (GSD4) ist an autosomal recessive disorder caused by branching enzyme deficiency. The phenotype is very heterogeneous, ranging from a congenital or infantile neuromuscular disorder to late-onset polyglycosan body disease. We report on a 5-year-old girl from Ukraine, presenting with elongated facial bones, proximal weakness, atrophy of the shoulder girdle muscles, severe hip, knee and ankle contractures, hyperlaxity of elbow joints and rigid spine. First symtoms were noted at birth in form of hypotonia and congenital arthrogryposis with bilateral hip luxation, requiring splint treatment for six months. Motor milestones were mildly delayed, turning around at 5 months of age, sitting at 7 months, crawling at 2,5 years, standing on knees from age 3; however, walking was never achieved. The parents are non-consanguineous, the patient is the only child, there are no other affected family members. Methods: We perfomed a clinical and electrophysiological examination, timed function test, oximetry, lung function test, cranial MRI, cardiac and abdominal ultrasound, along with a blood test for CK and multigene panel diagnostics for congenital myopathies. Results: Clinical testing revealed normal cranial nerves, no facial weakness, no high palate, but elongated facial bones, no macroglossia, atrophy of shoulder girdle muscles and proximal paresis of arm and leg muscles. The arms could be elevated up to $45^{\circ}$. Severe contractures in hips, knees and ankles along with pes equinus was detected, while elbow, hand and finger joints showed hyperlaxity. Manual muscle strength showed bilaterally MRC $3 / 5$ in arm elevation, biceps brachiii $4+/ 5$, triceps $4+/ 5$. Head flexor and extensor $4-/ 5$, quadriceps right $4-/ 5$, left $3+/ 5$, iliopsoas $5-/ 5$ and gluteus $4+/ 5$ bilaterally. Hand grip dynamometry revealed $2 \mathrm{~kg}$ bilaterally. The patient can sit independently, and gets up from lying position with the help of the arms. Independent standing or walking is not possible. Bilateral scapular winging, hyperlordosis, and rigid spine, but no scoliosis or stiff neck was seen. Tiimed test $6.43 \mathrm{sec}$. from lying to sitting position, displaying beevor's sign. Coordination and sensibility was intact, nerve conduction velocities were normal. Electromyography in proximal and distal leg muscles showed mild myopathic changes if any, CK levels were normal. Cranial MRI, cardiac and abdominal ultrasound showed normal results. Puls oximetry was normal, while vital capacity was reduced with 0.571 and $66 \%$ of normal in sitting, and 58\% of normal in lying position. However, the little patients cooperation was compromised by the language barrier. At age 5, height was $115 \mathrm{~cm}$, weight $15 \mathrm{~kg}$. Multigene panel diagnostics for congential myopathies, including collagen-6 deficiency, which we had initially suspected, did not reveal any pathogenic mutations. Therefore, we broadened the panel and detected two mutations in the GBE1 gene: c. $691+2 \mathrm{~T}>\mathrm{C}$ and c. $708 \mathrm{G}>\mathrm{C}$, both previously described as pathogenic in GSD4. Segregation analysis in the parents was in line with the autosomal recessive trait. Conclusion: Our studies provide new insights in the heterogeneity of genotype/phenotype correlations in glycogen 
storage diseases and congenital myopathies. Even though GSD4 represents a rare condition, testing should be included into the diagnostic work-up of congenital myopathies with contractures, rigid spine and/or joint hyperlaxity.

\section{PS1Group1-131 / \#475}

Topic: Group 1 - Muscle Diseases of Genetic Origin and Acquired Myopathies: Clinical Features, Pathophysiology, Therapy

\section{STATIN-ASSOCIATED AUTOIMMUNE NECROTIZING MYOPATHY}

$\underline{\text { So Hyun Ahn }}{ }^{1}$, Jung-Joon Sung 2 , Yoon-Ho Hong ${ }^{3}$, Kyoung Hyun Kwun ${ }^{1}$, Jin Ah Kim ${ }^{1}$, Je-Young Shin $^{4}$, Sung Min Kim${ }^{1}$, Ah Won Kim ${ }^{1}$

${ }^{1}$ Neurology, Seoul National University Hospital, Seoul, KR $;^{2}$ Neurology, Seoul National University College of medicine, Seoul University Hospital, Seoul, KR, ${ }^{3}$ Seoul National University College of medicine, SMG-SNU Boramae Medical Center, Seoul, KR; ${ }^{4}$ Seoul National University Hospital, Seoul, KR

Background: Necrotizing autoimmune myopathy presents with subacute proximal limb muscle weakness and a high serum creatine kinase (CK) level. Statins are generally well tolerated, but recently, progressive necrotizing myopathy leading to profound weakness has been directly linked to statin therapy. Methods: Case1 : A 63-year-old woman presented to our hospital with progressive weakness on both upper and lower extremities. She had a history of taking statin medication 10 months ago. Needle electromyography demonstrated features consistent with an active myopathy. Her serum CK level was $13237 \mathrm{IU} / \mathrm{L}$. She treated with IV steroid pulse therapy suspected as r/o dermatomyositis or polymyositis. After then, initially she showed responsiveness of steroid therapy, but her symptom was exacerbated while steroid tapering. At January 2016, She presented dysphagia and newly appeared both arm weakness. Muscle biopsy showed some necrotizing and regenerating myofibers without infiltration of inflammatory cells in endomysium or perivascular area. She treated with oral steroid and immunosuppressive agents, and her symptoms was improved and serum CK level was also decreased.

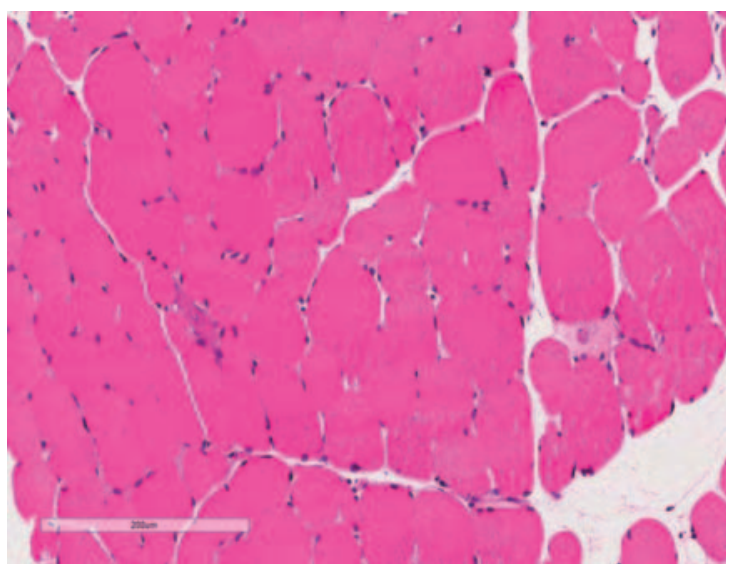

Results: Case 2: A 65-year-old woman presented to the neurology department with progressive weakness on both legs. She took stains for two years for preventing atherosclerosis. The weakness of both legs worsened and she could not climb the stairs and could not walk without assistance. Serum CK level was 1994 IU/L. Muscle biopsy showed some necrotizing and regenerating myofibers without infiltration of inflammatory cells in endomysium or perivascular area. She treated with IV methylprednisolone, and followed by oral prednisolone and tacrolimus.

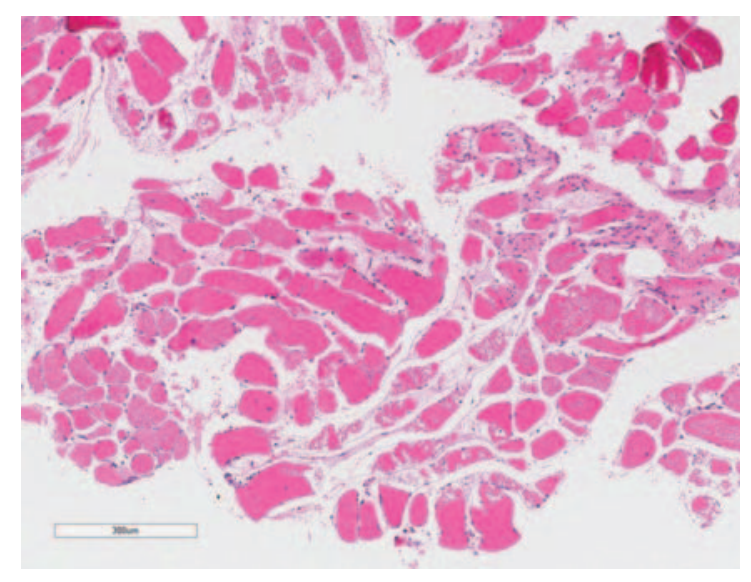

Conclusion: We reported two cases of statin-associated autoimmune necrotizing myopathy. The distinct histologic profile consists of profound necrotic, degenerating, or regenerating muscle fibers without inflammation. It may occur anytime and even long after initial exposure or statin cessation. Proper awareness of this rare complication is important to prevent further damage from statin use and treatment plan. 


\section{PS1Group1-132 / \#881}

Topic: Group 1-Muscle Diseases of Genetic Origin and Acquired Myopathies: Clinical Features, Pathophysiology, Therapy

\section{PHENOTYPIC HETEROGENEITY IN THREE PATIENTS WITH M.3243A>G MUTATION}

\author{
Birute Burnyte $^{1}$, Kristina Grigalioniene ${ }^{1}$, Arunas \\ Vaitkevicius ${ }^{2}$, Donatas Petroska ${ }^{3}$, Loreta \\ Cimbalistiene ${ }^{1}$, Vaidutis Kucinskas ${ }^{1}$, Algirdas Utkus ${ }^{1}$ \\ ${ }^{1}$ Institute Of Biomedical Sciences Of The Faculty \\ Of Medicine, Vilnius University, Vilnius, \\ LT: ${ }^{2}$ Institute Of Clinical Medicine Of The Faculty \\ Of Medicine, Vilnius University, Vilnius, \\ LT; ${ }^{3}$ Department Of Pathology, Forensic Medicine \\ and Pharmacology of the Faculty of Medicine of \\ Vilnius University, Vilnius, $L T$
}

Background: Mitochondrial myopathy, encephalopathy, lactic acidosis and stroke-like episodes (MELAS) syndrome is the most frequent syndromic manifestation of the m.3243A $>$ G mutation. We aim to describe the phenotypic variability of three adult patients harboring the m.3243A $>$ G mutation. Methods: Patients were identified in the research project during years 2015-2017. All three unrelated adult patients underwent extended clinical, laboratory, imaging and neurophysiological evaluations. An open muscle biopsy was performed in two patients for diagnostic purposes prior to the molecular study. Mitochondrial DNA (mtDNA) was investigated to determine disease mechanisms. The whole-length Sanger sequencing for detection of pathogenic point variants and long PCR and Multiplex Ligation-dependent Probe Amplification (MLPA) methods for detection of mtDNA deletions/duplications were applied. Results: We identified three female patients with the m.3243A $>$ G mutation. The average age at onset was 21.3 and at diagnosis 40.3 years. All three patients had short stature, ptosis, hearing impairment, dysarthria, dysphonia, feeding difficulties, exercise intolerance and muscle weakness as well gastrointestinal dysmotility, diarrhoea, constipation and abdominal distension. One patient manifested with ataxic syndrome, one with acute severe weakness after exposure to chemical agents at work, and one with slowly progressive exercise intolerance. Pigmentary retinopathy was detected in one patient. Liver impairment was observed in one patient, and liver biopsy showed macrovesicular steatosis. Only one patient showed persistent mild lactic acidosis. Endocrine dysfunction was observed in two patients, and was represented by diabetes mellitus type I and hypogonadism, and diabetes type II, respectively. Cardiac involvement was detected in one patient. Respiratory involvement was not observed in all three patients. No one referred a migraine. Sudden neurological deterioration was observed in two patients. Electroneurography in two patients showed decreased axonal amplitudes of sensory nerves. Electromyography was myopathic in two patients. Muscle biopsies were performed in two patients and showed ragged red fibers and COX deficiency until $10 \%$ of myocites only in one patient's biopsy (from m. guadriceps femori). Neuroimaging showed diffuse cerebral and cerebellar atrophy in one patient, and subcortical focal abnormalities in two patients. EEG abnormalities were not observed in all patients. One patient presented lack of social interactions and emotional instability. Pathogenic variant m.3243A $>G$ was detected in all the patients and heteroplasmy levels were less than $50 \%$ for pathogenic variant. Largescale mtDNA deletions or duplications were not detected in all three cases. Conclusion: We report detailed phenotypic and genetic characteristics of three unrelated Lithuanian patients with the m.3243A $>$ G mutation. Although the patients harbor the same mutation, they demonstrate a heterogeneous phenotype. Since the heteroplasmy level is not the main consideration explaining the phenotypic variability, our study supports the previous research findings that nuclear genomic factors also contribute to the phenotypic expression of MELAS. Supported by grant TAP LLT-02/2015.

\section{PS1Group1-133 / \#485}

Topic: Group 1-Muscle Diseases of Genetic Origin and Acquired Myopathies: Clinical Features, Pathophysiology, Therapy

\section{DOUBLE TROUBLE: A CHILD WITH DUCHENNE MUSCULAR DYSTROPHY AND NOONAN SYNDROME}

Yun Jeong Lee $^{1}$, Heamin Jang ${ }^{2}$, Ji Young Park ${ }^{3}$, Jin Sung Park ${ }^{4}$

${ }^{1}$ Pediatrics, School of Medicine, Kyungpook

National University, Daegu, KR $;^{2}$ Pediatrics,

Kyungpook national university children's hospital,

Daegu, KR $;{ }^{3}$ Pathology, School of Medicine,

Kyungpook National University, Daegu,

KR; ${ }^{4}$ Neurology, School of Medicine, Kyungpook

National University, Daegu, KR 
Background: Duchenne muscular dystrophy (DMD) is an X-linked disorder, caused by mutation in the $D M D$ gene, which encodes the dystrophin protein and affects 1 in 3600-6000 live male births. Noonan syndrome (NS) is a multiple congenital anomaly syndrome with a prevalence of $1: 1000$. It is clinically characterized by facial dysmorphism, growth retardation and congenital heart defects. In addition skeletal, genitourinary and skin manifestations can be also observed. Recent literatures show that approximately $50 \%$ of NS is caused by heterozygous missense mutations in PTPN11 gene. Herein, we describe a rare case of a child with coinheritance of DMD and NS based on pathology study and genetic analysis using the next generation sequencing. Methods: 8 year-old boy was referred to our hospital due to elevated serum creatine kinase level (CK level; $8250 \mathrm{U} / \mathrm{L}$ ) and progressive proximal limb weakness. He had a past history of pulmonary stenosis and aterial septal defect diagnosed at 1 year of age. The neuropsychological test reflected language, and cognitive developmental delay compared to his age. The patient showed waddling gait, Gower sign and calf muscle hypertrophy. The morphology of the patient was typical of NS, including short stature and dysmorphic facial features such as webbed neck, low posterior hair line, triangle shaped face, small chin, wild forehead, prominent nasolabial fold, low set posteriorly rotated ear and down slanting palpebral fissures. The needle electromyography study showed short amplitude, polyphasic motor unit potentials that were compatible with a myopathy. However clinical feature of proximal limb weakness and hyperCKemia are not common features of NS. Results: Therefore, we proceeded with the muscle biopsy that showed severe dystrophic alterations with marked size variation and perimysial fibrosis in the Hematoxylin-Eosin stain and complete loss of dystrophin was observed in dystrophin staining, that were consistent with dystrophinopathies. DNA analysis by multiplex ligation-dependant probe amplification (MLPA) analysis of the DMD gene was negative. Therefore, next generation sequencing (NGS) analysis was utilized and hemizygous c.3433$13 \mathrm{~T}>\mathrm{A}$ in $D M D$ gene was found and this was predicted to disrupt the highly conserved acceptor splice site of exon 26. In addition, a known pathogenic heterozygous missense mutation in c.236 $>\mathrm{G}$ in PTPN11 gene was also detected. With the above clinical, pathologic features and genetic results, the patient was diagnosed to have a double trouble of DMD and NS. Conclusion: There are reports on double trouble of two different monogenetic diseases. Previous studies have described coexistence of NS with 22q11.2 deletion syndrome, retinitis pigmentosa, neurofibromatosis and Charcort-marietooth disease. A recent report described a double trouble found in Becker muscular dystrophy and NS via muscle biopsy and genetic testing in accordance to our study. Our study is of significance as this is the first report of double trouble of DMD and NS and currently we have proceeded with the functional study to verify the pathogenicity of our novel mutation.

\section{PS1Group1-134 / \#926}

Topic: Group 1 - Muscle Diseases of Genetic Origin and Acquired Myopathies: Clinical Features, Pathophysiology, Therapy

\section{THE HETEROZYGOUS R155C VCP MUTATION: TOXIC IN HUMANS, HARMLESS IN MICE}

Christoph Clemen $^{1}$, Lilli Winter ${ }^{2}$, Karl-Heinz

Strucksberg ${ }^{3}$, Carolin Berwanger ${ }^{1}$, Matthias Türk ${ }^{4}$, Ludwig Eichinger ${ }^{3}$, - The German Mouse Clinic Consortium $^{5}$, Rolf Schröder ${ }^{6}$

${ }^{1}$ Department Of Neurology, Heimer Institute For

Muscle Research, Ruhr-University Bochum,

Bochum, DE; ${ }^{2}$ Center For Anatomy And Cell

Biology, Medical University of Vienna, Vienna,

AT; ${ }^{3}$ Institute Of Biochemistry, Medical Faculty, University of Cologne, Cologne, DE: ${ }^{4}$ Department

Of Neurology, Friedrich-Alexander University

Erlangen-Nürnberg (FAU), Erlangen, DE, ${ }^{5}$ The

German Mouse Clinic, Munich,

DE: ${ }^{6}$ Neuropathology, University Hospital

Erlangen, Erlangen, DE

Background: VCP, a very abundant and evolutionarily highly conserved member of the Triple-A ATPase family, is involved in a plethora of cellular processes such as membrane dynamics, protein quality control, cell cycle, apoptosis, and DNA damage response. Mutations in the VCP gene cause Inclusion Body Myopathy associated with Paget disease of bone and Fronto-temporal Dementia (IBMPFD). Moreover, four more neurodegenerative disorders, Amyotropic Lateral Sclerosis (ALS), Parkinson's disease, Hereditary Spastic Paraplegia, and CharcotMarie-Tooth disease type 2 (HMSN2) have been attributed to VCP missense mutations. The exact 
molecular mechanisms by which VCP mutations cause these late-onset disorders remain elusive. In the present study, we report on the generation and comprehensive analysis of a R155C VCP knock-in mouse model, which harbors the ortholog of one of the most frequently occurring human pathogenic VCP mutations. Methods: i) generation of R155C VCP knock-in mice; ii) comprehensive phenotypic and morphological analyses at the German Mouse Clinic Consortium in Munich; iii) VCP mRNA and protein expression analyses; iv) comparative myopathological analysis of human and murine R155C VCP mutant skeletal muscle tissue. Results: We successfully generated a R155C VCP knock-in mouse strain (B6J.B6J-Vcp tm2(R155C)Cers) using an appropriate targeting vector for homologous recombination. To restore the original VCP gene structure, mice were bred to $\mathrm{C} 57 \mathrm{BL} / 6 \mathrm{~J}$ mice expressing Cre recombinase for removal of the neomycin selection cassette. Correct gene targeting was confirmed by Southern blotting, PCR genotyping, and sequencing of both genomic and complementary DNA. Breeding of heterozygous R155C VCP knock-in mice did not yield in the generation of mice homozygous for the R155C VCP mutation. VCP immunoblotting indicated unchanged VCP protein levels in skeletal muscle and brain tissue derived from heterozygous mice as compared to wild-type littermates. Notably, our comprehensive phenotypic and morphological analyses of heterozygous R155C VCP knock-in mice did not reveal any signs of a previously described mutant VCP-related striated muscle, bone or central nervous system pathology. In comparison to wild-type littermates, heterozygous mice showed aberrant parameters for plasma lactate, fasting cholesterol, serum albumin, oxygen consumption, platelets, CD $8+/ \mathrm{Ly} 6 \mathrm{C}+\mathrm{T}$-cells, and liver weight. The pathological significance of these findings is currently unclear. Conclusion: While the heterozygous R155C VCP mutation has been shown in several reports to cause IBMPFD and ALS in humans, our analysis of corresponding R155C VCP knock-in mice did not detect any signs of a striated muscle, bone or central nervous system pathology. Though the failure to generate mice homozygous for the R155C VCP missense mutation clearly denotes a toxic effect not compatible with life, the lack of any clear-cut VCP-related pathology in heterozygous $\mathrm{R} 155 \mathrm{C}$ knock-in mice is somewhat unexpected and may be attributed to an R155C VCP expression level below a disease-causing threshold. This issue is currently addressed in further experiments.
PS1Group1-135 / \#497

Topic: Group 1 -Muscle Diseases of Genetic Origin and Acquired Myopathies: Clinical Features, Pathophysiology, Therapy

\section{ADAPTIVE PROTEIN QUALITY CONTROL RESPONSE IN DESMINOPATHY SKELETAL MUSCLE CELLS AND TISSUE}

\author{
Christoph Clemen $^{1}$, Lilli Winter ${ }^{2}$, Carolin \\ Berwanger $^{3}$, Marina Spörrer ${ }^{4}$, Ursula Schlötzer- \\ Schrehardt $^{5}$, Rolf Schröder ${ }^{6}$ \\ ${ }^{1}$ Department Of Neurology, Heimer Institute For \\ Muscle Research, Ruhr-University Bochum, \\ Bochum, DE; ${ }^{2}$ Center For Anatomy And Cell \\ Biology, Medical University of Vienna, Vienna, \\ AT; ${ }^{3}$ Department Of Neurology, Heimer Institute \\ For Muscle Research, Ruhr-University Bochum, \\ Bochum, DE: ${ }^{4}$ Department Of Physics, Friedrich- \\ Alexander-University Erlangen-Nuremberg, \\ Erlangen, DE; ${ }^{5}$ Department Of Opthalmology, \\ University Hospital Erlangen, Erlangen, \\ DE: ${ }^{6}$ Neuropathology, University Hospital \\ Erlangen, Erlangen, DE
}

Background: Mutations of the human desmin gene on chromosome 2q35 cause autosomal-dominant and -recessive myopathies and cardiomyopathies with marked clinical variability. For pathophysiological and therapeutic studies, we generated and characterized hetero- and homozygous R349P desmin knock-in mice, which serve as patient-mimicking disease models for dominant and recessive desminopathies with maintained protein expression. By crossbreeding our desminopathy mouse strain with p53 knock-out mice, we now generated immortalized desminopathy myoblast cell lines. We used these immortalized desminopathy myoblasts in conjunction with skeletal muscle tissue from the corresponding desminopathy mice to study the effects of R349P mutant desmin on the ubiquitin-proteasome system, bulk autophagy, chaperone-assisted selective autophagy, and heat shock proteins. Methods: i) R349P desmin knock-in mice; ii) p53 knock-out mice; iii) immortalized R349P desmin knock-in skeletal muscle myoblasts and myotubes; iv) indirect immunofluorescence microscopy; v) determination of proteasomal activity; vi) immunoblotting addressing autophagy rate, chaperone-assisted selective autophagy (CASA), and heat shock protein levels. Results: We crossbred our R349P desmin 
knock-in mouse line with p53 knock-out mice, prepared muscles from offspring with the following genotypes, hom R349P desmin knock-in/hom p53 knock-out, het R349P desmin knock-in/hom p53 knock-out, and wild-type littermates/hom p53 knock-out, and finally generated immortalized satellite cell-derived myoblast cultures. Desmin immunoblots and immunostains of differentiated myotubes revealed that these desminopathy cell models closely mirror central aspects of the desmin pathology observed in our desminopathy mice. Proteasomal activity measurements in muscle tissue lysates revealed a 1.23-fold and a 1.47-fold increase in heteroand homozygous R349P desmin knock-in mice, respectively, as compared to the wild-type. Notably, treatment of the desminopathy myotubes with the proteasome inhibitor MG132 did not alter the levels of both wild-type and R349P mutant desmin protein. Immunoblotting of tissue and myotube lysates demonstrated markedly increased levels of the serin/ threonin protein kinase Ulk-1 and sequestosome 1 (p62/SQSTM1) as well as of p62/SQSTM1 and LC3-II, which are classical autophagy-related markers. Furthermore, we detected increased protein levels of filamin-C and BAG-3, which are essential components of the CASA system. Finally, we assessed the protein levels of $\alpha \mathrm{B}$-crystallin, Hsp27, and Hsp90, and found increased signal intensities in skeletal muscle homogenates from homozygous mice, while only $\alpha \mathrm{B}$-crystallin and Hsp27, but not Hsp90, were increased in both desminopathy myotube genotypes. Conclusion: Our analyses in the newly generated immortalized desminopathy muscle cell cultures in conjunction with their corresponding desminopathy mouse models revealed a complex pattern of adaptive protein quality control response to the presence of R349P mutant desmin. Key findings are the upregulation of proteasomal activity, autophagy, CASA, and heat shock protein levels, which were predominantly seen in the homozygous and, to a lesser extent, in the heterozygous R349P desmin knock-in skeletal muscle tissues and cultured immortalized muscle cells.
PS1Group1-136 / \#514

Topic: Group 1 -Muscle Diseases of Genetic Origin and Acquired Myopathies: Clinical Features, Pathophysiology, Therapy

\section{CLINICAL BACKGROUND OF 94 ADULT PATIENTS WHO RECOGNIZED NEMALIN RODS IN MUSCLE TISSUE}

\author{
Akihiro Hashiguchi ${ }^{1}$, Kento Kodama $^{2}$, Itsuro \\ Higuchi $^{3}$, Hiroshi Takashima ${ }^{4}$ \\ ${ }^{1}$ Neurology, Neurological Disease Center, \\ Kagoshima University Hospital, Kagoshima city, \\ $J P ;{ }^{2}$ Department Of Neurology And Geriatrrics, \\ Kagoshima University Graduate School of Medical \\ and Dental Sciences, Kagoshima, JP, ${ }^{3}$ Physical \\ Therapy Foundation Course, Kagoshima University \\ School of Health, Kagoshima, JP; Department Of \\ Neurology And Geriatrrics, Kagoshima University \\ Graduate School of Medical and Dental Sciences, \\ Kagoshima, JP
}

Background: It is natural to recognize nemaline rods in muscle tissue in congenital nemaline myopathy patients. On the other hand, we sometimes find unexpected nemaline rods in adult muscle tissue. From the muscle biopsy record of the past 40 years, we will clarify the background of adult patients with nemaline rods. Methods: We extracted adult cases with nemaline rods from the muscular pathological reports analyzed in our facility over the past 40 years. We compared the clinical background and pathology of cases. Results: We extracted 94 adult cases with nemaline rods. In the pathological classification of 94 cases, myopathy was 72 cases, neuropathy was 16 cases, and merger of both was 6 cases. The age at examination was widely distributed in the 20 s to 80 s, and no characteristic was seen by age. In myopathy 72 cases, inflammatory myopathy was included in 13 cases (18\%).Eight cases of 16 cases of neuropathy were motor neuron disease such as amyotrophic lateral sclerosis, hereditary motor neuropathy, Kugelberg-Welander disease. Many cases coexisting with changes of mitochondrial abnormality, rimmed vacuole and cores. Conclusion: There is no disease specificity in the adult neemarin rod, not only in various myopathies but also in neuropathy. 
PS1Group1-137 / \#720

Topic: Group 1-Muscle Diseases of Genetic Origin and Acquired Myopathies: Clinical Features, Pathophysiology, Therapy

\section{P62 IMMUNOSTAINING COULD HELP IN DIFFERENTIATING POLYMYOSITIS FROM SIBM}

$\underline{\text { José C. Milisneda }}^{1}$, Cristina Jou², Iago PinalFeranndez $z^{3}$, Josep M. Grau ${ }^{4}$

${ }^{1}$ Internal Medicine, Hospital Clínic de Barcelona, Barcelona, ES, ${ }^{2}$ Hospital Sant Joan de Déu, Barcelona, ES, ${ }^{3}$ National Institute Of Arthritis And Musculoskeletal And Skin Diseases, National Institutes of Health, Bethesda, Maryland, AL, US; ${ }^{4}$ Internal Medicine Service, Hospital Clinic de Barcelona, Barcelona, ES

Background: The sensitivity and specificity of the existing criteria for sIBM have been reviewed recently, with high specificity but variable sensitivity. In consequence major problems arise for the diagnosis in early stages or to predict when a polymyositis is going to become a SIBM. Our objective was to investigate the utility of p62 immunostaining to predict when a PM is going to become a sIBM. Methods: This retrospective study enrolled a sample from the longitudinal muscle unit cohort of Hospital Clínic de Barcelona. We compared 4 muscle biopsies from (clinico-pathologically well-defined sIBM), with seven patients classified as having PM, based on European Neuromuscular Center (ENMC) criteria, and with normal muscle biopsies (control group). The PM cases were divided in two groups, because four of them had an associated disease (autoimmune or cancer) and the rest had an isolated PM. Clinical data was review from clinical charts, and p62 histopathological analysis was made with double immunofluorescence. In addition the amount of p62 in muscle biopsies was quantified using "Image J" software. Results: We observed a significant increase of p62 expression (expressed in \% of muscle area) in the isolated PM group, compared with the other PM cases (associated to a SLE, systemic sclerosis, HLAB27+ artrhopathy, and non-Hogkin lymphoma) and normal group. Also the expression of p62 was higher in the sIBM group. Of note was the poor response to immunesuppresive therapy in the four isolated PM cases, and furthermore one of them developing a typical clinical phenotype of sIBM, confirmed by a second muscle biopsy six

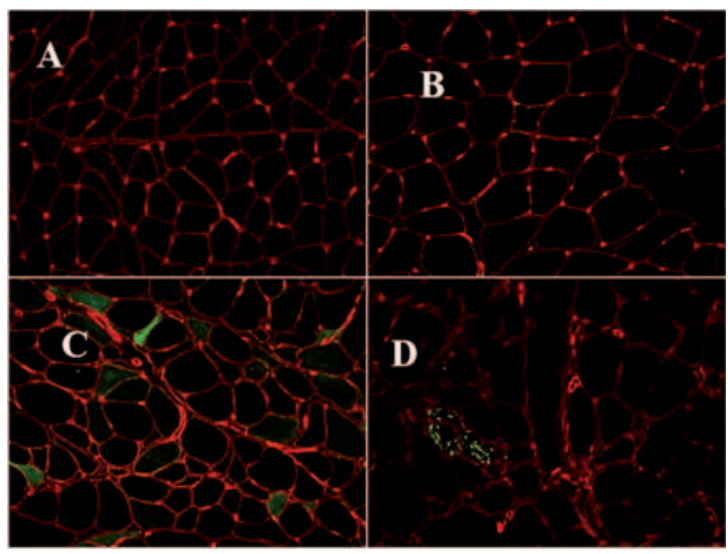

years later. Picture. Double immunofluorescence for p62. A) Normal patient. B) PM with associated disease. C) Isolated PM. D) sIBM. Conclusion: Despite the limitations of the present study, it seems that $\mathrm{p} 62$ positivity immunostaining could help in the identification of refractory isolated PM cases, with high probability to evolve to classical sIBM.

\section{PS1Group1-138 / \#347}

Topic: Group 1-Muscle Diseases of Genetic Origin and Acquired Myopathies: Clinical Features, Pathophysiology, Therapy

\section{HOMOZYGOSITY OF THE}

\section{AUTOSOMAL DOMINANT VCP P. ARG159HIS MUTATION}

$\underline{\text { Willem De Ridder }}^{1}$, Tine Deconinck ${ }^{2}$, Peter De Jonghe $^{2}$, Katherine Johnson ${ }^{3}$, Ana Töpf ${ }^{3}$, Marta Bertoli $^{3}$, Lauren Phillips ${ }^{3}$, Daniel Macarthur ${ }^{4}$, Jonathan Baets ${ }^{2}$

${ }^{1}$ Neurogenetics Group, Vib-uantwerp Center For Molecular Neurology, University of Antwerp, Antwerp, BE; ${ }^{2}$ Neurogenetics Group, Vib-uantwerp Center For Molecular Neurology, University of Antwerp, Antwerp, BE:3 John Walton Muscular Dystrophy Research Center, Newcastle University, Newcastle upon Tyne, GB; ${ }^{4}$ Broad Institute of Harvard and MIT, Cambridge, MA, US

Background: Mutations in valosin containing protein $(V C P)$ are linked to a dominantly inherited multisystem proteinopathy (MSP1) with inclusion body myopathy (IBM), Paget disease of bone (PDB) and frontotemporal dementia (FTD). Additional phenotypes have been described recently. More than 40 different missense mutations have been reported to 
date. Many gaps remain in our knowledge of this intriguing disorder with regard to molecular mechanisms of the disorder, genotype-phenotype correlations and penetrance. Methods: We studied a family in which the currently 33-year-old male index patient and his father exhibited progressive limbgirdle weakness. Whole-body muscle magnetic resonance imaging (MRI) was performed on a 1.5T MRI platform. Muscle biopsies were obtained from quadriceps muscle and analysed following standard histologic and immunohistochemical techniques for light microscopy. Whole exome sequencing of leucocyte DNA of the index patient was performed. Genes known to be associated with limb-girdle weakness, including $V C P$, were analysed for pathogenic variants. A candidate variant in $V C P$ was confirmed by Sanger sequencing and segregation analysis was performed with available DNA-samples of family members. Results: We describe clinical, radiological and anatomopathological details for the index patient and his father. Intriguingly, on the maternal side of the family, many individuals had been diagnosed with a dementia phenotype in the past. To our surprise, the index patient was homozygous for the known p.Arg159His mutation in VCP. When studying the familial history in detail, we identified distant consanguinity between the parents of the index patient. Comprehensive baseline studies were performed to evaluate potential subclinical bone or central nervous system involvement. Conclusion: We identified the known $V C P$ p.Arg159His mutation in homozygosity in a patient exhibiting a MSP1 phenotype. The phenotype of the index patient does not seem to be strikingly worse compared to heterozygous patients. Homozygous mutations in inherited autosomal dominant disorders are rare events. The case yields insightful and intriguing observations and incites to reflect on the presumably complex molecular mechanisms of $V C P$-linked disease.
PS1Group1-139 / \#742

Topic: Group 1 - Muscle Diseases of Genetic Origin and Acquired Myopathies: Clinical Features, Pathophysiology, Therapy

\section{GENOTYPE CHARACTERIZATION OF CZECH PATIENTS WITH FACIOSCAPULOHUMERAL DYSTROPHY}

Jana Zidkova $^{1}$, Stanislav Vohanka ${ }^{2}$, Radim

Mazanec $^{3}$, Lenka Fajkusova ${ }^{4}$

${ }^{1}$ Centre Of Molecular Biology And Gene Therapy,

University Hospital Brno, Brno, CZ; ${ }^{2}$ Neurology,

University Hospital Brno, Brno, CZ; $;^{3}$ Neurology,

Second Faculty of Medicine, Charles University

and University Hospital Motol, Prague,

CZ; ${ }^{4}$ University Hospital Brno and Masaryk

University Brno, Brno, $C Z$

Background: Facioscapulohumeral dystrophy (FSHD) is an autosomal dominant muscle disorder characterized by progressive weakness of facial, scapular and humeral muscles. FSHD is commonly associated with contraction of the D4Z4 macrosatellite repeat on chromosome 4q35 (FSHD1) or with mutations in the SMCHD1 or DNMT3B gene (FSHD2). Both situations result in a partial opening of the D4Z4 chromatin structure and transcription of D4Z4-encoded DUX4 mRNA in muscles. A specific haplotype is necessary for stabilization of the DUX4 transcript by providing the polyadenylation signal and is called 4qA haplotype. Methods: The number of D4Z4 macrosatellite repeats was determined by pulsed-field gel electrophoresis, Southern blotting and hybridization with specific probes, and analysis of genes $S M C H D 1$ and DNMT3B by gene panel sequencing. Results: The aim of our study was to confirm FSHD on molecular level. We tested FSHD1 in 400 patients and FSHD1 was confirmed in 210 of them. We correlated the available clinical data with detected number of repeats and found out that small number of repeats $(1-3 ; 21 \%$ of probands) is connected with earlier onset and more severe phenotype. Most FSHD1 patients (69\%) have medium number of repeats (4-8 repeats) and border number of repeats was detected only in $10 \%$ of probands. Conclusion: We detected two pathogenic variants in the SMCHD1 gene and so confirmed FSHD2. Both patients have 13 D4Z4 repeats and 4qA haplotype. This study was supported by the project of the Technology Agency of the Czech Republic (TE02000058) and the project of the Grant Agency of the Czech Republic (GA16-11619S/2016). 


\section{PS1Group1-140 / \#362}

Topic: Group 1-Muscle Diseases of Genetic Origin and Acquired Myopathies: Clinical Features, Pathophysiology, Therapy

\section{OCULOPHARYNGEAL MUSCLE WEAKNESS AFTER TREATMENT WITH CHECKPOINT INHIBITORS: PATHOLOGY IS BEYOND THE NEUROMUSCULAR JUNCTION}

\author{
Anahit C. Mehrabyan ${ }^{1}$, Aiesha Ahmed ${ }^{2}$, \\ Charles Specht ${ }^{3}$ \\ ${ }^{1}$ Neurology, University of North Carolina at Chapel \\ Hill, Chapel Hill, US: ${ }^{2}$ Neurology, PennState \\ Hershey Medical Center, Hershey, PA, \\ US ${ }^{3}$ PennState Hershey Medical Center, Hershey, \\ $P A, U S$
}

Background: Oculopharyngeal weakness related to checkpoint inhibitors became a well-known phenomenon over the last several years. The condition has been reported in patients with pre-existing myasthenia gravis, as well as de novo diagnosis after the initiation of treatment with checkpoint inhibitors. It has been reported after the use of programmed-death 1 (PD-1) and cytotoxic T-lymphocyte-associated protein 4 (CTLA-4) inhibitors, and combination of these agents. Methods: We report a case of progressive oculopharyngeal muscle weakness with detailed clinical follow up over the two months after the initial clinical presentation, laboratory, electrodiagnostic, immunologic and pathologic findings. Results: 69 yo man was diagnosed with recurrence of malignant melanoma with metastasis and was treated with combination of checkpoint inhibitors: Nivolumab (PD-1 inhibitor) and Ipilimumab (CTLA-4 inhibitor). He presented with an acute

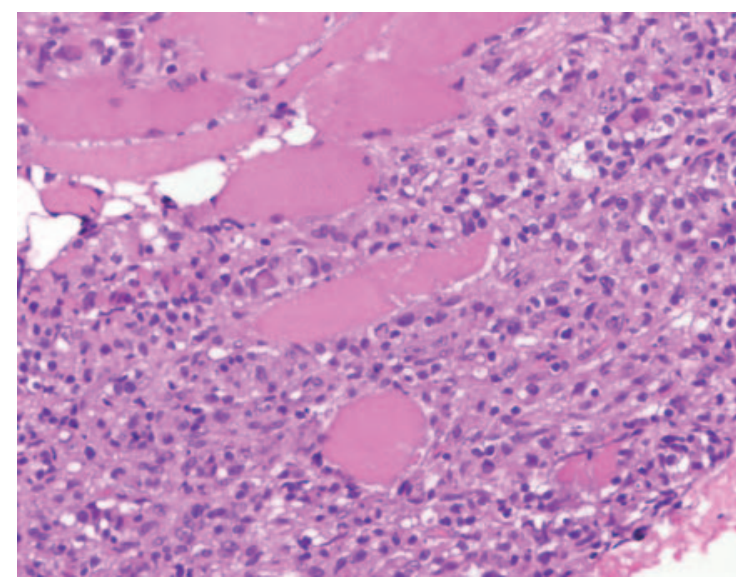

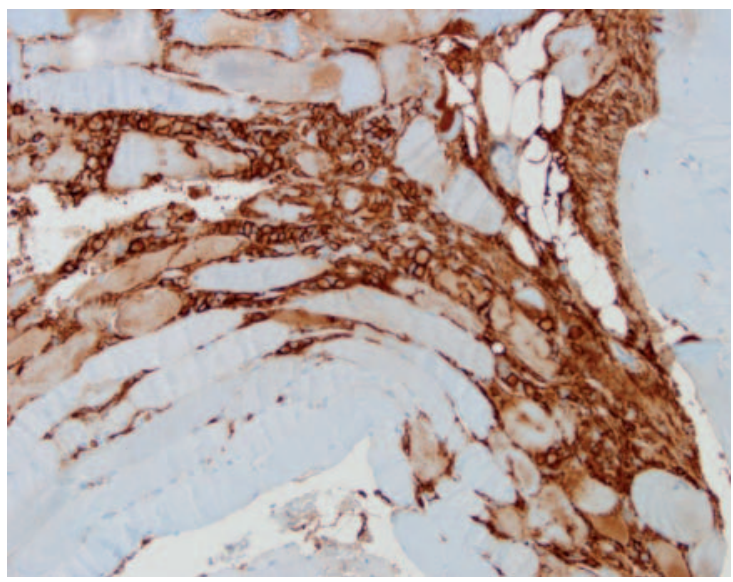

onset of right proximal leg weakness, which progressed to extraocular muscle weakness, asymmetric bilateral ptosis, pharyngeal, tongue and low facial muscle weakness, with subsequent resolution of right leg weakness and progression of oculopharyngeal symptoms. He was diagnosed with immune mediated myopathy based on clinical, laboratory, electrodiagnostic and histopathology findings. Conclusion: An isolated subacute acquired oculopharyngeal weakness in an adult population is the most common presentation of autoimmune mediated dysfunction of neuromuscular junction, myasthenia gravis. Here we present an unusual case of subacute onset of severe oculopharyngeal weakness due to autoimmune myopathy secondary to checkpoint inhibitors. Despite of unusual clinical presentation the cases of severe progressive muscle weakness with high suspicion for primary muscle pathology, including elevated creatine kinase (CK), negative/normal acetylcholine receptor antibodies, progression of symptoms despite immunotherapy, need thorough evaluation to exclude immune mediated myopathies, including detailed electrodiagnostic testing and muscle biopsy. 


\section{PS1Group1-141 / \#755}

Topic: Group 1-Muscle Diseases of Genetic Origin and Acquired Myopathies: Clinical Features, Pathophysiology, Therapy

\section{A CASE OF CENTRAL CORE DISEASE WITH NOVEL RYR1 MUTATION IN KOREAN PATIENT}

\author{
Je-Young Shin ${ }^{1}$, Chanmi Huh², So Hyun Ahn', \\ Kyoung Hyun Kwun ${ }^{4}$, Jin Ah Kim${ }^{4}$, Ah Won Kim \\ Yoon-Ho Hong ${ }^{5}$, Jung-Joon Sung ${ }^{6}$ \\ ${ }^{1}$ Neurology, Seoul National University Hospital, \\ Seoul, KR:²Seoul National University Hospital, \\ Seoul, KR; ${ }^{3}$ Neurology, Seoul National University \\ hospital, Seoul , KR; ${ }^{4}$ Neurology, Seoul National \\ University Hospital, Seoul, KR, ${ }^{5}$ Seoul National \\ University College of medicine, SMG-SNU \\ Boramae Medical Center, Seoul, KR; ${ }^{6}$ Neurology, \\ Seoul National University College of medicine, \\ Seoul University Hospital, Seoul, KR
}

Background: Central core disease (CCD), or central core myopathy, is a congenital myopathy with nonprogressive muscle weakness, mainly inherited in an autosomal dominant pattern. The gene responsible for CCD is type I ryanodine receptor (RYR1). The C-terminal domain mutation of RYR1 is relatively well known for its genotype-phenotype correlation due to its typical clinical and pathologic features. Methods: In this case report, we present a Korean patient with de novo missense mutation of a C-terminal domain of RYR1 gene. The purpose of this study is to broaden genetic mutational spectrum and understanding of genetic-phenotype correlation in CCD. Results: A 31-year-old man presented with non-progressive bilateral gait disturbance which lasted for about fifteen years. The patient showed musculoskeletal problems. Serum creatinine phosphokinase level was normal. There was no specific finding in other laboratory tests including creatinine phosphokinase. Electromyography (EMG) findings showed myopathic patterns. The muscle biopsy revealed the typical histopathologic features of CCD. The sequence analysis showed de novo missense mutation [c.14815G>T (p.Asp4939Tyr)] of RYR1 gene in the proband. We found no genetic variant or mutation among other family members. Conclusion: We report one Korean patient with CCD in whom de novo missense mutation in the $\mathrm{C}$-terminal transmembrane region of RYR1 gene was identified. This mutation is expected to be the '(likely) pathogenic variants.' Although mutations in the C-terminal of RYR1 are known to have significant muscle weakness since neonatal period, the index case did not present muscle symptoms until his adolescent period. On the other hand, the patient exhibited non-progressive muscle symptoms with proximal weakness and musculoskeletal deformities; his histopathologic findings were consistent with typical CCD. In this sense, even though patients do not show clinical symptoms of typical CCD, we still need muscle biopsy and genetic analysis, especially when we cannot find other causes. We hope that this case report helps us to broaden our clinical spectrum of CCD in association with RYR1 gene mutation.

\section{PS1Group1-142 / \#999}

Topic: Group 1 - Muscle Diseases of Genetic Origin and Acquired Myopathies: Clinical Features, Pathophysiology, Therapy

\section{MYOADENILATE DEAMINASE DEFICIENCY IN PATIENTS WITH MYALGIA}

\author{
Gabriele Siciliano, Costanza Simoncini, \\ Giulia Ricci \\ Department Of Clinical And Experimental \\ Medicine, University of Pisa, Pisa, IT
}

Background: Myoadenylate deaminase deficit (MAD) is the most common cause of metabolic myopathies. Methods: In our Center, we routinely use forearm ischemic exercise testing for blood lactate and ammonium in evaluation of patients with exercise intolerance, myalgias, muscle cramps and/or hyperCKemia. Results: In the last 5 years, we have detected lack of increase in the blood ammoniun concentration during exercise in twelve unrelated patients, all affected by exercise intolerance with muscle cramps and myalgia. In these patients, genetic test confirmed the presence of mutations in $A M P D 1$ gene, suggestive of myopathy due to myoadenylate deaminase deficiency (MAD). Conclusion: It has been reported that the spectrum of the MAD deficiency can range from asymptomatic carriers to patients who manifest exercise-induced muscle pain, fatigue, idiopathic hyperCKemia and, occasionally, rhabdomyolysis. MAD deficiency is not currently considered a well proven disease entity. Nevertheless, in people with muscle symptoms, $A M P D 1$ gene mutations seem to be significantly more frequent than healthy population, suggesting a possible clinical significance of this defect. 


\section{PS1Group1-143 / \#365}

Topic: Group 1-Muscle Diseases of Genetic Origin and Acquired Myopathies: Clinical Features, Pathophysiology, Therapy

\section{A PHASE IIA STUDY OF TAS-205, A NOVEL INHIBITOR OF HEMATOPOIETIC PROSTAGLANDIN D SYNTHASE, IN DUCHENNE MUSCULAR DYSTROPHY}

Tsuyoshi Matsumura $^{1}$, Hirofumi Komaki ${ }^{2}$, Satoshi $\mathrm{Kuru}^{3}$, Takahiro Nakayama ${ }^{4}$, Shinnichi Takeda ${ }^{5}$ ${ }^{1}$ Neurology, National Hospital Organization Toneyama National Hospital, Toyonaka, JP $;{ }^{2}$ Child Neurology, National Center Hospital, National Center of Neurology and Psychiatry, Kodaira, $J P,{ }^{3}$ Neurology, National Hospital Organization Suzuka National Hospital, Suzuka, JP: ${ }^{4}$ Neurology, Yokohama Rosai Hospital, Yokohama, JP, ${ }^{5}$ National Institute of Neuroscience, National Center of Neurology and Psychiatry, Kodaira, JP

Background: Inflammation has been included in the pathological process of Duchenne muscular dystrophy (DMD). Prostaglandin $D_{2}\left(P G D_{2}\right)$ is involved in inflammation, and hematopoietic $P G D$ synthase (HPGDS) is expressed in myonecrotic areas in DMD. A phase I study demonstrated that TAS-205, a novel HPGDS inhibitor, was highly safe and tolerable in patients with DMD when given at 1.67 to 13.33 $\mathrm{mg} / \mathrm{kg}$ as a single- and repeated-doses twice daily for 7 days. TAS-205 also decreased urinary excretion of tetranor-PGDM, the pharmacodynamics marker to show HPGDS inhibition. Based on the results of a phase I study, a phase IIa study was carried out. Methods: The primary objective of this double-blind randomized study was to evaluate the efficacy of TAS-205 orally administered twice daily for 24 weeks compared with placebo in ambulatory patients with $D M D$ in an exploratory manner. The secondary objective was to evaluate the safety and dose response relationship in efficacy and safety, and also the effect on the urinary excretion of tetranor-PGDM. Subjects were randomized to one of 3 groups; low-dose group (6.67 to $13.33 \mathrm{mg} / \mathrm{kg} /$ dose), high-dose group (13.33 to $26.67 \mathrm{mg} / \mathrm{kg} /$ dose), and placebo group evenly. This study was carried out at 11 sites in Japan. Results: Thirty-six subjects were enrolled and 35 subjects were treated with the investigational product.
The full analysis set consisted of 34 subjects, and the per protocol set consisted of 32 subjects where the main efficacy evaluation was conducted. The primary endpoint was the change from baseline in the measured 6-minute walk distance (6MWD) at week 24. It was $-17.0 \pm 55.6 \mathrm{~m}$ (mean value $\pm \mathrm{SD}$ ) in the placebo group, $-3.5 \pm 67.3 \mathrm{~m}$ in the low-dose group, and -7.5 $\pm 37.3 \mathrm{~m}$ in the high-dose group. The difference of the mean value from the placebo group was $13.5 \mathrm{~m}$ $(p=0.625)$ in the low-dose group and $9.5 \mathrm{~m}(p=$ $0.646)$ in the high-dose group. The decrease in 6MWD tended to be less in the TAS-205 groups as compared with the placebo group. The muscle volume index (\%MVI) by skeletal muscle CT in the thigh and lower leg, as the secondary endpoint for efficacy, tended to be less decreased in the TAS-205 group as compared with the placebo group. Additionally, there were several subjects where muscle volume of the thigh and lower leg increased in high-dose group. The change rate in the urinary excretion of tetranorPGDM corrected by creatinine from baseline signifcantly decreased in the high-dose groups as compared with the placebo group. No adverse drug reactions specific to the TAS-205 were observed, suggesting proper tolerability. Conclusion: From the above results, it was suggested that TAS-205 had a possibility of being effective for ambulatory patients with DMD.

\section{PS1Group1-144 / \#767}

Topic: Group 1 - Muscle Diseases of Genetic Origin and Acquired Myopathies: Clinical Features, Pathophysiology, Therapy

\section{USE OF HUMAN INDUCED PLURIPOTENT STEM CELLS FOR MODELLING SKELETAL MUSCULAR DEFECTS ASSOCIATED TO MYOTONIC DYSTROPHY TYPE} 1

Julie Tahraoui $^{1}$, Lea Lesueur ${ }^{2}$, Christian Pinset $^{3}$, Xavier Nissan ${ }^{2}$, Denis Furling ${ }^{4}$, Cecile Martinat ${ }^{1}$ I-stem, Inserm Ueve Umr861, CORBEIL ESSONNES, FR $;^{2} I$-stem, CECS, CORBEIL ESSONNES, FR $;{ }^{3} I$-stem, INSERM UEVE UMR861, CORBEIL ESSONNES, FR $;{ }^{4}$ Institut De Myologie, Centre de Recherche en Myologie, UMRS974, PARIS, FR 
Background: Myotonic Dystrophy type I (DM1) is a rare neuromuscular disease that is mainly characterized by myotonia, progressive muscle weakness and wasting. DM1 is an autosomal dominant disorder caused by an expanded CTG repeat in the 3' UTR of $D M P K$ gene with the size of the expanded repeats globally correlates with disease severity. This abnormal expansion leads to a toxic gain-offunction of the mutated mRNAs which aggregate within the nucleus in association with different RNA binding proteins such as the splicing factor MBNL1. Consequently, several alternative splicing defects have been identified in skeletal muscular biopsies from DM1 patients. Up to now, cellular models available for DM1 rely mainly on primary cultures of skeletal muscle cells derived from DM1 patients. However, several difficulties, such as a limited in vitro proliferation, may limit the use of primary cells, especially considering drug screening approaches for this incurable disease. Methods: Based on recent protocols allowing the conversion of human pluripotent stem cells into skeletal muscle cells without genetic modifications, our goal was to evaluate the potential offered by DM1 specific hiPSCs to better understand the effect of DM1 mutation on the generation and the maintenance of skeletal muscle cells. Results: Altogether, our results demonstrate the potential offered by DM1 specific hiPSC-derived skeletal muscle cells to phenocopy and decipher new molecular pathophysiological mechanisms. Conclusion: Furthermore, this new cellular system holds great promises to identify new compounds capable to target the RNA toxicity associated with DM1 mutation.

\section{PS1Group1-145 / \#367}

Topic: Group 1 - Muscle Diseases of Genetic Origin and Acquired Myopathies: Clinical Features, Pathophysiology, Therapy

\section{A CASE OF \\ FACIOSCAPULOHUMERAL MUSCULAR DYSTROPHY TYPE 2 WITH NOVEL FRAMESHIFT MUTATION OF SMCHD1 GENE IN KOREA}

Jung Hwan Lee ${ }^{1}$, Moon-Woo Seong ${ }^{2}$, Chang-Seok $\mathrm{Ki}^{3}$, Young Chul Choi ${ }^{4}$

${ }^{1}$ Department Of Neurology, Ewha Womans
University Mokdong Hospital, Seoul, $K R ;{ }^{2}$ Department Of Laboratory Medicine, Seoul National University Hospital, Seoul, $K R,{ }^{3}$ Department Of Laboratory Medicine And Genetics, Samsung Medical Center, Sungkyunkwan University School of Medicine, Seoul, $K R,{ }^{4}$ Department Of Neurology, Gangnam Severance Hospital, Yonsei University College of Medicine, Seoul, KR

Background: Facioscapulohumeral muscular dystrophy 2 (FSHD2) is a genetic muscular disorder characterized by DNA hypomethylation on the 4qsubtelomeric macrosatellite repeat array, D4Z4. DNA hypomethylation is caused by heterozygous mutations in the gene encoding structural maintenance of chromosomes flexible hinge domain containing 1 (SMCHD1). In Korea, there are no reports of patient with FSHD2. We reported a first case of FSHD2 in Korea. Methods: A 40-year-old male visited to our clinic for asymmetric upper and lower extremity weakness. He said that His father, dead due to liver cirrhosis, and elder brother were similar with him. He was normal at birth and presented normal development. He presented asymmetric arm weakness after the age of 10 and leg weakness after the age of 24. And, he also presented winged scapula and Popeye arm. On neurologic examination, we could observe bilateral facial weakness, asymmetric proximal dominant arm and leg weakness. Results: First, we performed conventional Southern blot analysis but D4Z4 repeat size was 12 , which means normal. Then, we performed whole exome sequencing and found novel frameshift mutation (c.3801delG, p.Glu1267Aspfs*5) of SMCHD1 gene. Then, we re-checked size of D4Z4 repeat with 4qA haplotype and performed hypomethylation assay of D4Z4 repeat region. As a result, he has homozygous 4qA haplotype and mild reduced D4Z4 repeat size $(41 \mathrm{~kb} / 39 \mathrm{~kb})$, and reduced D4Z4 methylation ratio (14.6\%). So, we report a first case of FSHD2 in Korea. Conclusion: For rapid and accurate diagnosis of FSHD2 patients, the genetic analysis of D4Z4 haplotype and methylation is very important and needed to add for diagnostic step for FSHD. 


\section{PS1Group1-146 / \#1012}

Topic: Group 1 - Muscle Diseases of Genetic Origin and Acquired Myopathies: Clinical Features, Pathophysiology, Therapy

\section{ASYMMETRIC WEAKNESS IN HMGCOA REDUCTASE ANTIBODY NECROTIZING MYOPATHY}

\author{
Madhu Soni \\ Neurological Sciences, Rush University Medical \\ Center, Chicago, IL, US
}

Background: Objectives: To report a case of asymmetric weakness in statin-associated HMGCoA reductase antibody necrotizing myopathy, and to heighten awareness of the potential etiology. Background: Necrotizing myopathy due to statins and other drug-induced or immune-mediated triggers typically presents with symmetric, proximal weakness that can be abrupt in onset and aggressive in its course, requiring prompt recognition to stop the offending agent, institute immunotherapy if necessary and provide the appropriate supportive care. A case is described here of an elderly gentleman with prominent, asymmetric proximal upper extremity weakness that did not improve with immunotherapy. Methods: Case Report: A 74- year-old, previously active, man was brought in for progressive weakness over 6 weeks that started abruptly. Symptoms started with symmetric, painless proximal lower extremity weakness limiting ambulation and use of stairs, and contributed to falls. The weakness progressed to involve the upper extremities, starting on the left where there was self-limited pain in the shoulder associated with weakness. Weakness in the right upper extremity was painless. Dysphagia and dyspnea also developed. The patient had been on daily atorvastatin for 10 years. Exam was notable for asymmetric right arm abduction weakness of $0 / 5$ compared to $3 / 5$ contralaterally. He had moderate weakness of neck flexion and bilateral hip flexion, and was unable to stand without assistance. Work up included a left quadriceps muscle biopsy that revealed an active, necrotizing myopathy. HMGCoA reductase Ab was elevated. The patient was admitted for IVIG treatment and received prednisone $60 \mathrm{mg}$ orally, along with physical and occupational therapy. Results: After two courses of IVIG, a month apart, his strength markedly improved with resolution of weakness in the neck and left upper extremity and mild residual weakness in the proximal lower extremities. Right arm abduction remained 0/5. He denied pain, including with passive movement of the arm at the shoulder. Shoulder xray revealed no significant abnormalities. Due to the discrepancy in right arm abduction, despite improvement in the remainder of his strength, a shoulder MRI was obtained and revealed a large tear of the right supraspinatus tendon and a moderate tear in the biceps. Conclusion: Asymmetric weakness is atypical in statin-associated HMGCoA reductase necrotizing myopathy and should trigger consideration for alternative, nonneurologic causes that may warrant an alternative treatment approach. In this case, a previously undiagnosed painless rotator cuff tear was the cause of the right arm abduction weakness that was refractory to immunotherapy for the underlying necrotizing myopathy. Heightened awareness and identification of potential co-exising orthopedic conditions and complications is essential to guide appropriate treatment and avoid unnecessary additional immunotherapy in immune-mediated myopathies.

\section{PS1Group1-147 / \#809}

Topic: Group 1 - Muscle Diseases of Genetic Origin and Acquired Myopathies: Clinical Features, Pathophysiology, Therapy

\section{CAN NECK FLEXION WEAKNESS PREDICT CHANGES IN SWALLOWING AND COUGH PEAK FLOW IN PATIENTS WITH MYOTONIC DYSTROPHY TYPE 1?}

\author{
Charlotte Massey $^{1}$, Jodi E. Allen ${ }^{2}$, Umesh \\ Vivekananda $^{3}$, Nikoletta Nikolenko ${ }^{4}$, Cecilia \\ Jimenez-Moreno ${ }^{4}$, Chris Turner ${ }^{5}$ \\ ${ }^{1}$ Therapy And Rehabilitation, The National \\ Hospital for Neurology and Neurosurgery, $B G$, \\ $G B ;{ }^{2}$ Therapy And Rehabilitation, The National \\ Hospital for Neurology and Neurosurgery, $3 B G$, \\ $G B,{ }^{3}$ University College London Hospitals, $B U$, \\ GB; John Walton Muscular Dystrophy Research \\ Centre, BZ, GB; $;^{5}$ Institute Of Neurology, University \\ College London Hospital MRC Centre for \\ Neuromuscular Diseases, BG, GB
}

Background: Recurrent pneumonia secondary to bulbar and respiratory muscle weakness is the leading cause of morbidity and mortality in patients with Myotonic Dystrophy Type 1 (DM1). Early detection 
of cough and swallow impairment enables prophylactic measures such as cough augmentation and swallow management to be put in place to prevent acute respiratory decline. Measuring neck flexor strength using the MRC muscle score is a simple, efficient and inexpensive measure that can be used in the outpatient or domicillary setting without specialist equipment. We hypothesised that alterations in neck flexion strength could be used to predict deterioriation in swallowing and cough ability and therefore has potential utility as a tool to identify the need for early physiotherapy and speech and language therapy assessment and management. Methods: 52 adult patients with genetically confirmed DM1 were enrolled as part of the PHENO DM1 natural history study. Neck flexion strength was assessed using the MRC muscle scale by a trained physiotherapist with the patient in lying. Cough Peak Flow (CPF) was assessed in sitting using a Mini-Wright peak flow meter fitted with a face mask. Swallow function was assessed using the Sydney Swallow Questionnaire (SSQ), a validated patient self-report tool of swallowing symptoms. Spearman's rank was used to establish correlations between neck flexion strength, SSQ and CPF. Results: Significant correlations were found between MRC neck scores and CPF $(\mathrm{p}=0.01)$ and MRC neck and SSQ ( $\mathrm{p}=0.04)$. Conclusion: This is the first study to consider relationships between neck flexion strength, swallow function and cough. Correlations have been identified. It is possible that MRC neck flexion scores could be used to help predict changes in swallowing and cough function; however further research needs to explore this in more detail, including analysis of instrumental measures of swallowing ability, respiratory and mobility parameters. The ongoing search for tools to support identication of patients at increased risk of respiratory decline secondary to bulbar weakness and poor cough would allow prophylactic measures to be put in place to prevent unplanned hospital admissions with chest infection or pneumonia.
PS1Group1-148 / \#778

Topic: Group 1 - Muscle Diseases of Genetic Origin and Acquired Myopathies: Clinical Features, Pathophysiology, Therapy

\section{THE ADDITIONAL VALUE OF HISTOPATHOLOGICAL FASCIA EXAMINATION IN DIAGNOSING MYOSITIS}

\author{
$\underline{\text { Anneke Van Der Kooi }}$ \\ Neurology, AMC, Amsterdam, $N L$
}

Background: Early diagnosis of idiopathic inflammatory myopathies or myositis may prevent irreversible disease damage and disability, but is often complicated. Needle muscle biopsy is widely used as its diagnostic accuracy appears generally comparable to that of the more invasive en-bloc biopsy. However, en-bloc biopsy including skin, fascia and muscle allows additional histopathological examination of the fascia. We investigated whether additional histopathological fascia examination might contribute to early diagnosis of myositis. Methods: Fifty-five en-bloc biopsies from patients who were diagnosed with myositis were retrospectively reviewed: dermatomyositis (DM; $\mathrm{n}=9$ ), non-specific/ overlap myositis $(\mathrm{NM} / \mathrm{OM} ; \mathrm{n}=17)$, immune-mediated necrotizing myopathy $(\mathrm{n}=21)$, and anti-synthetase syndrome $(\mathrm{n}=8)$. Biopsies were scored for skin, muscle, and fascia involvement and re-evaluated in case of presumed isolated fascia involvement. Results: In DM and NM/OM, muscle analysis alone led to the diagnosis of myositis in $89 \%$ and $94 \%$, respectively. One patient (11\%) with DM and one $(6 \%)$ with $(\mathrm{NM} / \mathrm{OM})$ had isolated histopathological fascia involvement, which contributed to the diagnosis. Conclusion: Our findings indicate that en-bloc biopsy allowing histopathological fascia examination may contribute to an early diagnosis of DM and NM/OM. 


\section{PS2Group3-001 / \#268}

Topic: Group 3 - Peripheral Neuropathy, Cranial Nerves, Mononeuropathies: Clinical Features, Pathophysiology, Therapy

\section{A TALE OF TWO CHARCOT'S JOINTS: LONDON AND DAR-ES- \\ SALAAM}

\section{Chin Yong Kok}

Mrc Neuromuscular Centre, National Hospital of

Neurology, London, GB

Background: We report two cases of long-standing neuropathy presenting with predominantly foot deformities. Methods: Case reports. Results: Case 1: A 32 year old man from Tanzania had twisted his ankle whilst playing football. He experienced no pain but developed localised swelling. A CT of the ankle and foot revealed a shattered navicular bone with anterior subluxation. He was referred as a case of leprosy for nerve biopsy. However, further history revealed that at the age of eight while climbing Mount Kilimanjaro his father commented that he walked with bilateral foot drop. He denied any sensory symptoms. Examination showed pes cavus on the right and a Charcot's joint of the left ankle. He had evidence of a length dependant motor neuropathy with no sensory abnormalities. No thickened nerves. Neurophysiology showed a length dependant sensory- motor axonal neuropathy. The CMT2/ HSN panel revealed heterozygous mutation in Rasrelated protein Rab-7a gene. Case 2: A 74 year old lady with a 17 years history of a painful neuropathy with ulceration and Charcot's joints. She was a longstanding diabetic on oral hypoglycaemic agents. Neurophysiological studies showed $\mathrm{n}$ axonal sensory - motor neuropathy with abnormal thermal thresholds. The CMT2/HNS panel revealed heterozygous mutation in serine palmitoyltransferase, long chain base subunit 2 gene (SPTLC2). Conclusion: The use of next-generation sequencing makes it easy to undertake genetic tests. These two cases highlight the need to consider genetic diagnoses which obviate the need for nerve biopsies.
PS2Group3-002 / \#908

Topic: Group 3 - Peripheral Neuropathy, Cranial Nerves, Mononeuropathies: Clinical Features, Pathophysiology, Therapy

\section{HETEROGENEITY OF THE PATIENT PATHWAY AND CHARACTERISTICS FOR HATTR AMYLOIDOSIS: PERSPECTIVES FROM CENTRAL AND EASTERN EUROPE}

Yeșim Parman $^{1}$, Ivailo Tournev ${ }^{2}$, Daniel Coriu ${ }^{3}$, Michael Arad ${ }^{4}$, Marta Lipowska ${ }^{5}$, Sergei Nikitin 6 , Stayko Sarafov ${ }^{2}$, Janez Zidar ${ }^{7}$

${ }^{1}$ Istanbul University, Istanbul, TR; $;{ }^{2}$ University

Hospital Aleksandrovska, Sofia, $B G ;^{3}$ University of

Medicine, Bucharest, RO; $;{ }^{4}$ Chaim Sheba Medical

Center, Tel-Hashomer, IL,; Medical University of

Warsaw, Warsaw, PL; ${ }^{6}$ Institute of General

Pathology and Pathophysiology, Moscow,

$R U,{ }^{7}$ University Medical Center, Ljubljana, SI

Background: Hereditary transthyretin (hATTR) amyloidosis is an autosomal dominant disease, caused by a range of mutations in the transthyretin gene. As such, hATTR presents in many different forms and with considerable variation in signs and symptoms, particularly across geographic locations. Such heterogeneity, in combination with its rarity, can result in a sub-optimal patient pathway to diagnosis and, ultimately, management. As such, it is important that we share our experiences to understand the challenges and obstacles to enhancing the patient journey. The aim of this project was to therefore assess the current clinical picture for patients with hATTR amyloidosis across Central and Eastern Europe (CEE). Methods: In November 2017, 8 experts, from 7 countries in CEE, involved in the diagnosis and care for patients with hATTR amyloidosis, presented insights from their respective countries. The panel was asked to share their experiences in terms of: how patients enter the healthcare system; who is typically responsible for diagnosing patients with hATTR amyloidosis; common misdiagnoses; availability of multidisciplinary networks involved in diagnostic work-up; availability of, and access to, Centres of Excellence; and, capabilities for genetic testing and counselling for family members. Additionally, the typical patient profile in CEE, including genetic background, age of disease onset and rate of disease progression were discussed. Results: Through the sharing of experiences country-by-country, it 
emerged that there is a high degree of heterogeneity of clinical practice and patient characteristics in CEE. Factors that drove such diversity included: • Range of different genotypes encountered, including: Asp38Ala, Gly47Glu, Glu54Gln, Glu89Gln, Glu89Lys, Ile107Phe, Phe33Leu, Ser77Phe, Ser77Tyr, Thr40Lys, Val122Ala, Val30Met, Val71Ala, among other rare mutations - Subsequent variability in symptomatology, especially in the age of onset and presenting symptoms, which are often non-neurologic - Natural history of hATTR amyloidosis within this region - Status of disease awareness outside of Centres of Excellence, including of key redflag signs and symptoms that should raise suspicion • Availability and use of specific diagnostic tests for hATTR amyloidosis It emerged that, although there is a need for a global approach to identifying and managing patients with hATTR amyloidosis, such heterogeneity of disease and practice in CEE might necessitate a more localised approach in this region. The expert panel discussed options that might facilitate optimal patient care, including: the sharing of expertise nationally and internationally through improved communication networks; development and further optimisation of Centres of Excellence; and, improved disease awareness among clinicians who are likely to encounter patients with hATTR amyloidosis. Conclusion: Clinical practice across CEE is highly heterogeneous, partly due to the broad spectrum of hATTR amyloidosis genotypes and phenotypes, as well as the varying structures of healthcare systems. Improved communication is key to heightening the urgency of identifying and managing this complex disease for clinicians across the region; sharing of such local experiences with colleagues, nationally and internationally, may help in facilitating the delivery of optimal patient care.

\section{PS2Group3-003 / \#966}

Topic: Group 3 - Peripheral Neuropathy, Cranial Nerves, Mononeuropathies: Clinical Features, Pathophysiology, Therapy

\section{AUTONOMIC AND SENSORY NEUROPATHY: CHALLENGES IN THE ETIOLOGY AND TREATMENT OF A PEDIATRIC CASE}

\author{
Mafalda Sampaio ${ }^{1}$, Cristina Garrido ${ }^{1}$, Manuela \\ Santos $^{1}$, Ana Sousa $^{2}$, Sónia Figueiroa ${ }^{1}$ \\ ${ }^{1}$ Neuropediatrics, Centro Hospitalar do Porto, Porto, \\ $P T ;{ }^{2}$ Neurophysiology, Centro Hospitalar Porto, -, PT
}

Background: The acute presentation of severe dysautonomia, sometimes with additional neuromuscular or central nervous system manifestations, may reflect impairment of sympathetic, parasympathetic and/or enteric functions. Autoimmune autonomic ganglionopathy (AAG) is an acquired, immunomediated, dysautonomia. It has a wide clinical spectrum and antibodies against the alpha- 3 subunit of the nicotinic acetylcholine receptor $(\alpha 3-\mathrm{AChR} \mathrm{Ab})$ on autonomic ganglia have been found acutely only in about $50 \%$ of patients. The low prevalence of the disease in the pediatric population and the difficulty in recognizing the symptoms often lead to a significant delay in the diagnosis. The differential diagnosis, especially in children, encompass immune, paraneoplastic, genetic, metabolic and mitochondrial disorders. Methods: (Not applicable). Results: CASE REPORT A 15 months girl was admitted for vomiting during an acute pyelonephritis. During the first week, she developed irritability, a rash with periorbital edema and severe mucositis with loss of a primary tooth. Additionally, she developed dysphonia, hemodynamic instability, and bladder and bowel dysfunction. On the second week, she presented paroxysmal events of bradycardia and cyanosis, and developed hypotonia associated with poor spontaneous limb movements (but offering some resistance when manipulated) and arreflexia. Brain and spinal cord MRI disclosed gadolinium enhancement of the cauda equina's roots, mainly dorsal roots. Cerebrospinal fluid analysis showed albuminocytologic dissociation. Guillain-Barré syndrome (GBS) was hypothesized and IVIG treatment started. On the $4^{\text {th }}$ week, EMG revealed absent sensory potentials and normal motor potentials. Because of paroxysms of staring, cyanosis and oromandibular movements, EEG was performed and disclosed an encephalopathic pattern. Corticotherapy was started. The extensive metabolic, immune, paraneoplastic and infectious investigation was unremarkable. Throughout the first months, she had intermittent hyperthermia and bladder dysfunction. Since then, she had a slowly progressive neurological improvement, being able to walk by the age of 3 years old. She has intermittent vomiting, diarrhea, with failure to thrive associated with polydipsia and intermittent hyperthermia. Arreflexia persists. The last neurophysiological evaluation, at age 4 , was consistent with a sensory ganglionopathy, and sympathetic and parasympathetic dysautonomia. Conclusion: This clinical case illustrates an acute presentation of prominent pandysautonomia with additional clinical manifestations secondary to 
sensory ganglionopathy, leading to an initial diagnosis of a sensory variant of GBS. Like in GBS, the acute presentation, a previous infection, the albuminocytological dissociation and a partial response to immunomodulatory treatment, suggest an immune-mediated mechanism. In our case, an extensive etiology workup was unremarkable. Although very rarely described in pediatric age, we consider the hypothesis of AAG, and testing for $\alpha 3-\mathrm{AChR} A b$ is ongoing. We would also like to discuss further treatment approach, since there are case reports in which some functional improvement is reported with treatment, even in seronegative patients. Further studies will be useful to establish treatment protocols for acute autoimmune neuropathies, and to do a better characterization of seronegative cases in order to determine other relevant autonomic nervous system antibodies.

\section{PS2Group3-004 / \#228}

Topic: Group 3 - Peripheral Neuropathy, Cranial Nerves, Mononeuropathies: Clinical Features, Pathophysiology, Therapy

\section{EXTENSIVE GENETIC ANALYSIS IN A TAIWANESE COHORT WITH CHARCOT-MARIE-TOOTH DISEASE}

\author{
Kon-Ping Lin ${ }^{1}$, Yi-Chu Liao ${ }^{2}$, Yi-Chung Lee ${ }^{3}$ \\ ${ }^{1}$ Taipei Veterans General Hospital, Taipei, \\ $T W{ }^{2}$ Neurology, Taipei Veterans General Hospital, \\ Taipei, TW; ${ }^{3}$ Neurology, Taipei Veterans General \\ Hospital, Taipei, $T W$
}

Background: Charcot-Marie-Tooth disease (CMT) comprises a clinically and genetically heterogeneous group of inherited neuropathies. Mutations in at least 90 genes have been described to cause CMT. The aim of this study was to determine the frequency and spectrum of mutations in different genes in a Taiwanese CMT cohort of Han Chinese origin. Methods: We recruited 427 unrelated CMT patients from the Neurology Service of Taipei Veterans General Hospital, Taiwan. A series of genetic testing was performed, including a real-time PCR-based method to investigate $P M P 22$ duplication, sanger sequencing to check GJB1 mutations, a targeted sequencing panel covering all known CMT genes and further genes causing neuropathy (124 genes in total), and whole exome sequencing in several selected large pedigrees. Results: Mutations have been identified in 315 of the 427 patients ( $73.8 \%$ ), including 275 with demyelinating CMT $(85.7 \%$ of all demyelinating
CMT cases; 275/321), 38 with axonal CMT (36.5\% of all axonal CMT cases; 38/104) and 2 with dominant intermediate CMT $(100 \% ; 2 / 2)$. Among the 315 patients with clear genetic diagnosis, 208 were found to have a PMP22 duplication $(48.7 \%$ of total CMT cases; 208/427), 40 had a GJB1 mutation (9.4\%; 40/427), 14 had a $M P Z$ mutation $(3.3 \% ; 14 / 427), 14$ had a MFN2 mutation $(3.3 \% ; 14 / 427)$ and 8 had a NEFL mutation $(1.9 \% ; 8 / 427)$. Thirty-one patients carry a mutation in 15 different rare CMT disease genes, including LITAF, EGR2, FBLN5, SH3TC2, GARS, HSPB1, GDAP1, AARS, LRSAM1, IGHMBP2, MORC2, BSCL2, KIF5A, TFG and $G N B 4$. Fifteen novel mutations were identified in this cohort. Conclusion: This study clearly demonstrates the distribution and frequency of mutations in a Taiwanese CMT cohort of Han Chinese origin, and expands the spectrum and supports their global presence of mutations in the causative genes of CMT.

\section{PS2Group3-005 / \#566}

Topic: Group 3 - Peripheral Neuropathy, Cranial Nerves, Mononeuropathies: Clinical Features, Pathophysiology, Therapy

\section{IMPACT OF PATISIRAN ON AUTONOMIC NEUROPATHY IN HEREDITARY TRANSTHYRETIN- MEDIATED AMYLOIDOSIS PATIENTS}

$\underline{\text { Michelle Mauermann }}^{1}$, David Adams ${ }^{2}$, Alejandra Gonzalez-Duarte ${ }^{3}$, Teresa Coelho ${ }^{4}$, Chih-Chao Yang $^{5}$, Michael Polydefkis ${ }^{6}$, Arnt Kristen 7 , Ivaylo Tournev $^{8}$, Hartmut Schmidt ${ }^{9}$, John Berk ${ }^{10}$, KonPing Lin ${ }^{11}$, Pritesh Gandhi ${ }^{12}$, Marianne Sweetser ${ }^{12}$, Matthew White ${ }^{12}$, Jared Gollob ${ }^{12}$, Ole Suhr ${ }^{13}$

${ }^{1}$ Mayo Clinic, Rochester, US ${ }^{2}$ Neurology

Department, LFB, Le Kremlin Bicetre, FR: ${ }^{3}$ Instituto

Nacional de Ciencias Medicas y Nutricion,

Salvador Zubiran, MX $;^{4}$ Hospital de Santo Antonio,

Porto, PT; National Taiwan University Hostpital,

Taipei, TW; Johns Hopkins, Baltimore, MD,

US, ${ }^{7}$ Heidelberg University Hospital, Heidelberg,

$D E,{ }^{8}$ University Multiprofile Hospital for Active

Treatment, Sofia, BG, ${ }^{9}$ University Hospital

Muenster, Muenster, DE; ${ }^{10}$ Boston Universty,

Boston, MA, US: ${ }^{11}$ Neurology, Taipei-Veterans

General Hospital, Taipei, TW: ${ }^{12}$ Alnylam

Pharmaceuticals, Cambridge, MA,

US; ${ }^{13}$ Department Of Public Health And Clinical

Medicine, Umea University, Umea, SE 
Background: Hereditary transthyretin-mediated (hATTR) amyloidosis is a rare, multisystemic, rapidly progressive, life-threatening disease with heterogeneous clinical presentation including sensory, motor and autonomic neuropathy, as well as cardiac involvement. Clinical manifestations of autonomic dysfunction may include chronic diarrhea and nausea/vomiting, orthostatic hypotension, and urinary tract infections. In the Phase 3 APOLLO study, patisiran, an investigational RNAi therapeutic, showed improvement in primary, secondary and exploratory endpoints compared to placebo and was generally well tolerated in hATTR amyloidosis patients. Here, all data on measurements of autonomic symptoms from APOLLO are presented. Methods: APOLLO was a multi-center, international, randomized (2:1), double-blind study of patisiran $0.3 \mathrm{mg} / \mathrm{kg}$ or placebo IV q3W in hATTR amyloidosis patients with polyneuropathy (NCT01960348). The composite autonomic symptom score 31 (COMPASS-31): a 31-item questionnaire (range 0-100 points) used to evaluate patient reported autonomic neuropathy symptoms on the following six domains: orthostatic intolerance, vasomotor, secretomotor, gastrointestinal, bladder, and pupillomotor. Subjective perceptions of autonomic nerve function were captured within the Norfolk quality of life diabetic neuropathy (Norfolk QOL-DN), a 35-item patient-reported scale. Postural blood pressure (PBP) was captured in the modified neuropathy impairment score (mNIS+7 score). Among all 3 tools, higher scores are indicative of worsening autonomic symptoms. Results: APOLLO enrolled 225 patients: mean age 61 years (24-83), 74\% males and 43\% V30M. Overall, COMPASS-31 showed a least squares (LS) mean decrease (improvement) of -5.29 points with patisiran compared to an LS mean increase (worsening) of 2.24 points with placebo, for an overall treatment difference of -7.53 points at 18 -months $(p=0.0008)$. Patisiran treated patients experienced a benefit compared to placebo across all COMPASS-31 domains, and showed an improvement relative to baseline for the orthostatic intolerance and gastrointestinal domains (Figure 1). Similarly, autonomic improvement with patisiran and worsening with placebo was observed in autonomic domain of Norfolk QOL-DN and PBP component of mNIS+7 with an LS mean change (SEM) compared to baseline for patisiran, placebo: [-0.3 (0.19), $0.8(0.39)]$ and [-0.2 (0.06), $0.1(0.11)]$, respectively. These findings were accompanied by a lower frequency of adverse events of syncope, nausea, and urinary tract infection in the patisiran group compared to placebo group. Conclusion: In APOLLO, multiple measures of autonomic symptoms and function showed a consistent benefit of patisiran over placebo as well as an improvement compared to baseline resulting in lowering of GI symptoms (e.g. diarrhea and constipation) and orthostatic intolerance (e.g. inability to stand upright).

\section{Figure 1: APOLLO studv: Subcomponents of COMPASS-31 at 18 months}

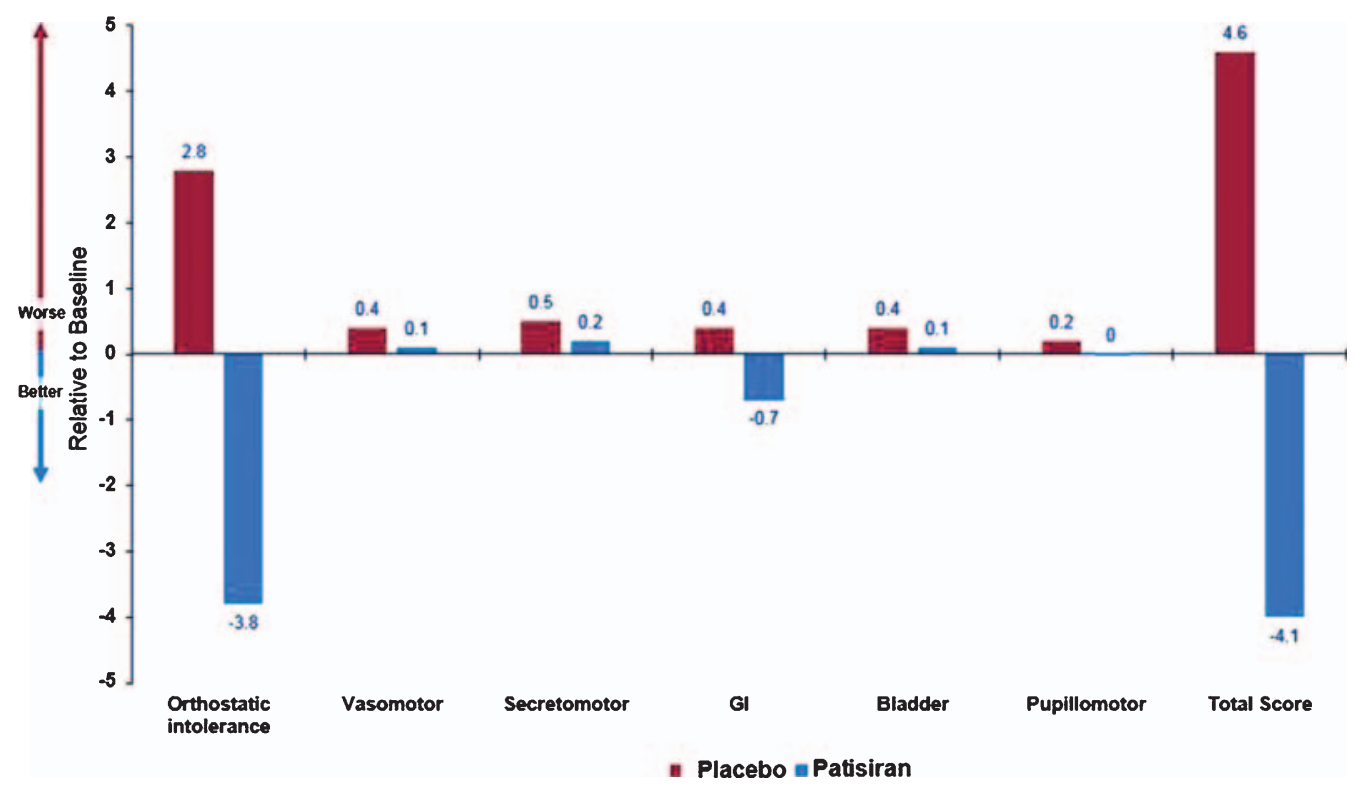


PS2Group3-006 / \#705

Topic: Group 3 - Peripheral Neuropathy, Cranial Nerves, Mononeuropathies: Clinical Features, Pathophysiology, Therapy

\section{THE FUNCTIONAL AND STRUCTURAL EVALUATION OF SMALL FIBERS IN ASYMPTOMATIC PATIENTS WITH VAL30MET MUTATION}

\author{
Ezgi Yilmaz ${ }^{1}$, Can Ebru Bekircan-Kurt ${ }^{2}$, Sibel \\ Kocabeyoglu ${ }^{3}$, Fatma Gokcem Yildiz ${ }^{1}$, Murat \\ Irkec $^{3}$, Ersin Tan ${ }^{1}$, Sevim Erdem-Ozdamar ${ }^{1}$ \\ ${ }^{I}$ Neurology, Hacettepe University, Ankara, TR $;{ }^{2}$ \\ Neurology, Hacettepe University, Ankara, TR; ${ }^{3}$ \\ Ophtalmology, Hacettepe University, Ankara, TR
}

Background: Transthyretin (TTR)-related hereditary amyloidosis usually presents as a progressive, axonal sensory autonomic and motor neuropathy called familial amyloid polyneuropathy (FAP). Distinct polyneuropathies including sensorimotor polyneuropathies, entrapment neuropathies, autonomic neuropathy and small fiber neuropathy can manifest in TTR-FAP patients. Moreover, small fiber neuropathy (SFN) could be the first manifestation of FAP. Here, we investigated the structural and functional properties of small fibers in asymptomatic carriers of Val30Met mutation. Methods: A family with Val30Met mutation, whom index case was diagnosed with FAP 20 years ago, has been followed in our department. Five asymptomatic family members with Val30Met mutation were included to the study. The neurological examination, DN4 questionnaire, body mass index (BMI), 24-hour urine parameters plasma brain natriuretic peptide levels were assessed. Nerve conduction studies, sympathetic skin response test, heart rate variability (HRV) were done. were performed to detect cardiac involvement. Quantitative sensory testing (QST) was assessed to determine cold and warm sensation thresholds. Skin punch biopsies were performed and intraepidermal nerve fiber densities (IENFD) were calculated. Moreover subbasal corneal nerve densities, long nerve fiber (LNFs) count per frame, long nerve fiber length (LNFL) and the total number of nerve branches/ frame (TNF) were evaluated by in vivo confocal microscopy (IVCM). Results: The mean age was 39.6 years (18-60) and four of the carriers were female. The mean BMI was $26 \mathrm{~kg} / \mathrm{m} 2$ (23.2-29.4). Only one carrier defined burning in her feet upon questioning and his DN4 questionnaire score was 2. The neurological examination of all carriers was normal. Quantitative sensory testing (QST), 24-hour urine parameters, plasma brain natriuretic peptide levels, echocardiogram were normal in all carriers. One had Mobitz type 1 atrioventricular block detected by Holter monitoring. The neurophysiological studies including nevre conduction studies, sympathetic skin responses and heart rate variability tests were all normal compared to age and sex matched reference values. In vivo corneal confocal microscopic evaluation revealed normal basal epithelial cell density. LNFL (mean=2315.9),LNFs (mean=3), TNF(mean=6.8) were lower in all carriers than age and sex matched controls. One carrier had grade 3 subbasal nevre tortuosity. The mean IENFD was 3.23 fibers $/ \mathrm{mm}$ (1.16-4.75) and all IENFD of the patients were lower than the age and sex matched normative values. Conclusion: In this pilot study small fiber loss was determined by skin punch biopsy and IVCM in all of our asymptomatic carriers with Val30Met mutation. However the functional diagnostic tests such as QST, sympathetic skin responses did not show any functional loss in this early stage in these asymptomatic carriers. It is open to debate if a carrier should be considered as a patient in such a condition. But it is for sure that the carriers must be followed by morphological as well as functional studies. As they develop symptoms comparison of these tests with the presymptomatic basal values will be very valuable for diagnosing a carrier as a patient and starting treatment.

\section{PS2Group3-007 / \#954}

Topic: Group 3 - Peripheral Neuropathy, Cranial Nerves, Mononeuropathies: Clinical Features, Pathophysiology, Therapy

\section{CLINICAL AND ELECTROPHYSIOLOGICAL FEATURES OF AMAN FROM THE SONORAN OUTBREAK}

Suraj A. Muley, Shafeeq Ladha

Neurology, Barrow Neurological Institute, Phoenix, AZ, US

Background: Acute motor axonal neuropathy (AMAN) is a rare variant of Guillain-Barré syndrome. Patients with AMAN have an axonal electrophysiology affecting motor nerves with sparing of 
the sensory nerves. Even though the clinical features and electrophysiology are suggestive of axonal degeneration, there is increasing evidence of a spectrum of axonal changes from reversible axonal conduction failure on the one extreme to severe axonal degeneration on the other. Patients may fall along this spectrum. Thus far, the majority of information about AMAN is based on outbreaks of this disease in China and Mexico. Given the rare nature of this disease, the pathophysiological basis of this disease can become clearer if future outbreaks of this disease are studied systematically, both clinically and electrophysiologically. Methods: There was an outbreak of AMAN in the Sonoran state of Mexico and the adjoining area of the United States in June 2011. We studied the clinical features and electrophysiological findings of 14 patients from this outbreak in August 2011 and again in a followup visit in August 2013. The clinical characteristics that were studied included antecedent illness, time from onset of symptoms to nadir, severity of disability at nadir including ability to ambulate, need for mechanical ventilation, treatments administered, time to improvement, degree of improvement in terms of independent ambulation and severity of residual deficits. Electrophysiological features that were studied included median, ulnar, peroneal and tibial motor amplitudes, conduction velocities, distal latencies and F-wave latencies. Median, ulnar and sural sensory responses were also studied. Electromyography of tibialis anterior, vastus lateralis, first dorsal interosseous and biceps brachii muscles was studied. Results: The average age of patient's was 50.3 years. All patients had a rapidly progressive course with an average of 3.15 days from symptom onset to nadir. 11 of $14(78.5 \%)$ patients became nonambulatory and 4 of $14(29 \%)$ needed mechanical ventilation. Eight patients were treateed with IVIG, 2 patients were treated with both IVIG and plasma exchange and 4 patients received no immunotherapy. Severely reduced CMAP amplitudes were seen in the motor nerves along with normal sensory responses. Four of the 11 nonambulatory patients required mechanical ventilation. Three of the 4 patients who were nonambulatory at follow-up had required mechanical ventilation. One of 4 untreated and one of 8 IVIG-treated patients were nonambulatory. All patients who received both IVIG and plasma exchange were nonambulatory. 4 of 14 patients reached nadir within 1-2 days and 10 of 14 patients within 3-6 days. From the first group 1 was intubated and 1 was nonambulatory at follow-up and from the second group 4 patients were intubated and 3 were nonambulatory at follow-up. Mean median, ulnar, peroneal and tibial CMAP amplitudes were $0.175,0.25,0.23$ and $0.66 \mathrm{mV}$ in non-ambulatory patients versus $3.11,2.98,2.17 \& 4.63 \mathrm{mV}$ in ambulatory patients. Conclusion: Despite severe axonal features most patients improved in a time course suggestive of reversible axonal failure. Poor prognostic factors included the need for mechanical ventilation and severity of CMAP amplitude reduction. Shorter time from onset to nadir may be a good prognostic sign.

\section{PS2Group3-008 / \#980}

Topic: Group 3 - Peripheral Neuropathy, Cranial Nerves, Mononeuropathies: Clinical Features, Pathophysiology, Therapy

\section{METABOLIC SYNDROME COMPONENTS AND NEUROLOGIC OUTCOMES IN A BARIATRIC SURGERY POPULATION}

\author{
Brian Christopher Callaghan, Emily Villegas- \\ Umana, Ericka Chant, Evan Reynolds, Mousumi \\ Banerjee, Eva Feldman \\ University of Michigan School of Public Health, \\ Ann Arbor, US
}

Background: Previous studies demonstrate that diabetes, pre-diabetes, and obesity are the main metabolic drivers of polyneuropathy. Whether these same factors are associated with other neurologic outcomes is unknown. The aim of this study is to investigate the association of MetS components with polyneuropathy, retinal and cognitive function in a bariatric surgery cohort prior to surgery. Methods: Patients were recruited from the Bariatric Surgery Clinic at the University of Michigan and lean controls from a research website (no MetS components based on NCEP/ATPIII definition). Participants underwent extensive metabolic phenotyping including a glucose tolerance test and fasting lipid profile. Polyneuropathy was assessed using the Toronto consensus definition of probable clinical neuropathy. Retinal function was measured with frequency doubling technology perimetry (average mean deviation), and cognitive function with the NIH Toolbox (composite score). Multivariate regression models were used to evaluate the association between MetS components and neurologic outcomes adjusted for 
age, gender, and height. Results: We have recruited 109 bariatric surgery participants and 35 lean controls. In the bariatric population, the mean (SD) age was 45.3 (11.1). In the bariatric group, $33.9 \%$ had diabetes, $38.5 \%$ pre-diabetes, and $27.5 \%$ normoglycemia. The DSP prevalence was 3\% in lean controls, $10.0 \%$ in normoglycemic, $7.1 \%$ in pre-diabetic, and $40.5 \%$ in diabetic bariatric participants $(\mathrm{p}<0.001$ for trend). Retinal function was $0.01(2.61),-0.10$ (2.68), -2.11 (4.28), and -1.62 (4.56) $(\mathrm{p}=0.01$ for trend), and cognitive function was 117.2 (14.5), 108.3 (17.5), 106.8 (18.6), 103.7 (19.3) ( $\mathrm{p}=0.001$ for trend) in these same groups. Waist circumference $(\mathrm{OR}=1.36, \quad 95 \% \mathrm{CI} \quad 1.07-1.73)$, triglycerides $(\mathrm{OR}=1.44,95 \% \mathrm{CI} 1.01-2.05)$, and systolic blood pressure $(\mathrm{OR}=2.18,95 \% \mathrm{CI} 1.12-4.27)$ were associated with polyneuropathy. Diabetes $(\mathrm{PE}=-2.5$, $\mathrm{p}=0.03)$ and Pre-diabetes $(\mathrm{PE}=-2.3, \mathrm{p}=0.03)$ were associated with retinal function, and waist circumference $(\mathrm{PE}=-1.5, \mathrm{p}<0.001)$ was associated with cognitive function. Conclusion: Polyneuropathy, retinal and cognitive function all decline with worsening glycemic status. Obesity is significantly associated with polyneuropathy and cognitive dysfunction, whereas pre-diabetes and diabetes were significantly associated with retinal dysfunction. Interestingly, while clinical DSP is common in this population, clinical retinopathy and dementia are not, indicating that DSP may be the first metabolic complication in the morbidly obese.

\section{PS2Group3-009 / \#412}

Topic: Group 3 - Peripheral Neuropathy, Cranial Nerves, Mononeuropathies: Clinical Features, Pathophysiology, Therapy

\section{A SUCCESSFUL TREATMENT OF IDIOPATHIC BRACHIAL NEURITIS WITH SONO-GUIDED INJECTION AND LOW DOSE STEROID THERAPY}

Chang-Hwan Kim

\section{Physical \& Rehabilitation Medicine, InHa University Hospital, inchon, KR}

Background: Brachial neuritis has been conventionally treated with high dose oral corticosteroid $(1 \mathrm{mg} /$ $\mathrm{kg}$ ) for relieving pain or help improve functional recovery. However, the higher therapeutic dose could have various systemic side effects. A significant number of brachial neuritis patients suffer from persistent pain, weakness, fatigue and leading to an impairment. We intended to reduce side effects and achieve better recovery with effective pain control, we have used low-dose steroid therapy with ultrasound-guided local steroid injection (brachial plexus block). Methods: Case: A fourty-one-year old righthanded woman has got a common cold and this has gradually got better. About two weeks later, she felt pain and sudden weakness on left upper arm without trauma. Her history was negative for hereditary or metabolic neuropathies. On physical examination, she could not elevate arm; shoulder flexion and abduction (MRC 2/5), elbow flexion (MRC 4/5). A laboratory study and shoulder X-rays showed no abnormalities. The nerve conduction study and electromyography showed left brachial plexopathy involving C5 radiculopathy. The brachial plexus MRI showed lesions involving left divisions and lateral, posterior cord with mild swelling on post-ganglionic level of C5 nerve root, suggesting radiculoplexus neuropathy. Results: We started low-dose steroid therapy $(0.5 \mathrm{mg} / \mathrm{kg}, 0.4 \mathrm{mg} / \mathrm{kg}$ for 3 days each following $0.2 \mathrm{mg} / \mathrm{kg}$ for maintaining dose 4 weeks course). Concurrently, 2-week interval, 3-times brachial plexus injection was performed. We used MRC
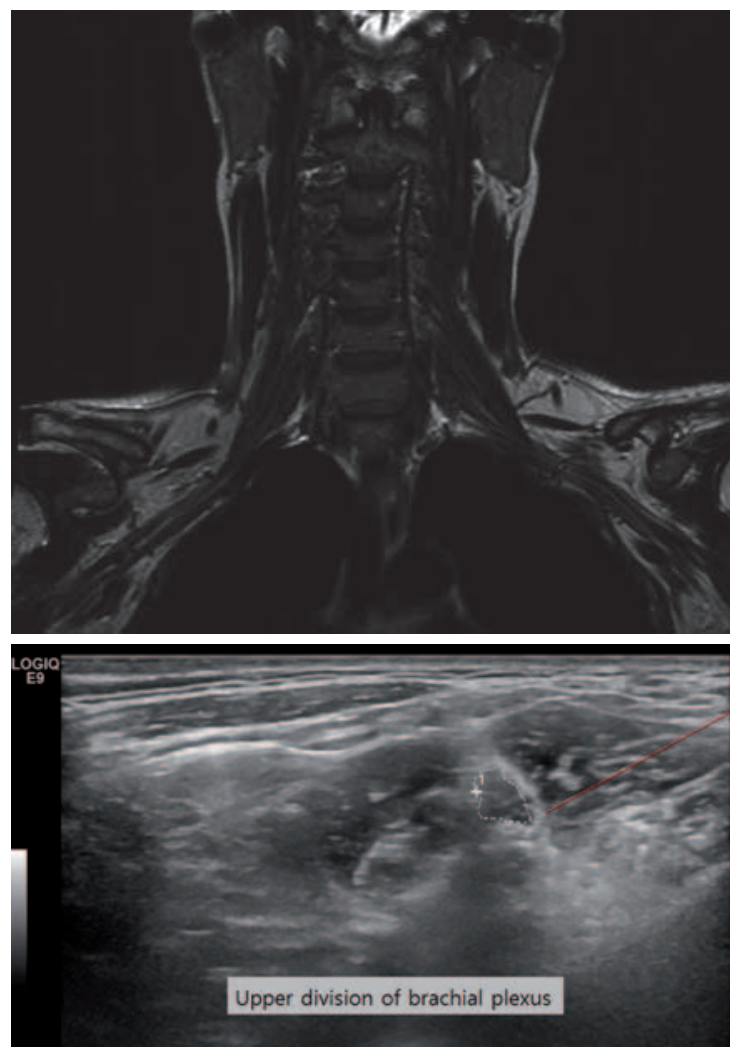
grades of following 3 muscle pairs comprising MRC sum score for brachial plexitis: the three weakest muscles (shoulder flexor, abductor and elbow flexor). The MRC sum score were increased from 8 to 13 in one month, and further improved to 15 in 2 nd month. And pain scale was graded from 5 to 2 and finally 0 in the same periods. Conclusion: Although precise pathophysiology of idiopathic brachial plexitis is thought to be associated with localized inflammatory-immune attack. Therefore, ultrasound-guided injection would reduce systemic side effects and improve therapeutic outcome.

\section{PS2Group3-010 / \#455}

Topic: Group 3 - Peripheral Neuropathy, Cranial Nerves, Mononeuropathies: Clinical Features, Pathophysiology, Therapy

\section{APOLLO PHASE 3 STUDY: IMPACT OF BASELINE NEUROPATHY SEVERITY ON RESPONSE TO PATISIRAN}

Laura Obici ${ }^{1}$, Teresa Coelho², David Adams ${ }^{3}$, Alejandra Gonzalez-Duarte ${ }^{4}$, William O'Riordan ${ }^{5}$, Chih-Chao Yang ${ }^{6}$, Arnt Kristen ${ }^{7}$, Ivaylo Tournev ${ }^{8}$, Hartmut Schmidt ${ }^{9}$, John Berk ${ }^{10}$, Kon-Ping Lin ${ }^{11}$, Pritesh Gandhi ${ }^{12}$, Marianne Sweetser ${ }^{12}$, Tim Lin ${ }^{12}$, Jared Gollob ${ }^{12}$, Ole Suhr ${ }^{13}$

${ }^{1}$ Fondazione IRCCS Policlinico San Matteo, Pavia, IT; ${ }^{2}$ Hospital de Santo Antonio, Porto, $P T ;{ }^{3}$ Neurology Department, LFB, Le Kremlin Bicetre, FR; ${ }^{4}$ Instituto Nacional de Ciencias Medicas y Nutricion, Salvador Zubiran, MX; ${ }^{5}$ eStudy Site, La Mesa, NM, US; ${ }^{6}$ National Taiwan University Hostpital, Taipei, TW; ${ }^{7}$ Heidelberg University Hospital, Heidelberg, $D E ;{ }^{8}$ University Multiprofile Hospital for Active Treatment, Sofia, BG, ${ }^{9}$ University Hospital
Muenster, Muenster, DE; ${ }^{10}$ Boston Universty, Boston, MA, US; ${ }^{11}$ Taipei Veterans General Hospital, Taipei, TW; ${ }^{12}$ Alnylam Pharmaceuticals, Cambridge, MA, US: ${ }^{13}$ Department Of Public Health And Clinical Medicine, Umea University, Umea, SE

Background: Hereditary transthyretin-mediated (hATTR) amyloidosis is a rare, multi-systemic, rapidly progressive, life-threatening disease caused by mutations in the transthyretin (TTR) gene resulting in multi-organ TTR amyloid deposition. Clinical manifestations include sensorimotor and autonomic neuropathy, gastrointestinal symptoms, and cardiomyopathy. The modified neuropathy impairment score (mNIS+7), a sensitive quantitative measure to assess somatotopic distribution of muscle weakness, sensory loss, and autonomic symptoms, has been utilized in clinical trials to evaluate polyneuropathy progression in hATTR amyloidosis patients. In the phase 3 APOLLO study, patisiran, an investigational RNAi therapeutic, resulted in a statistically significant improvement in mNIS+7 compared to placebo in patients with hATTR amyloidosis, and was generally well tolerated. Here, we present an analysis of the $\mathrm{mNIS}+7$ response by baseline NIS quartile in order to demonstrate the impact of patisiran across a broad range of neuropathy severity. Methods: APOLLO was a multi-center, international, randomized (2:1), double-blind, placebo-controlled study of patisiran $0.3 \mathrm{mg} / \mathrm{kg}$ or placebo IV q3W in adult patients with hATTR amyloidosis with polyneuropathy (NCT01960348). The primary endpoint was change in mNIS+7 from baseline to 18-months. Patients were grouped into quartiles by baseline NIS scores (Figure 1). The mean change from baseline in mNIS+7 over time was calculated for each quartile by treatment group. Results: APOLLO enrolled 225 patients: mean age 65 years (24-83), 74\% males and 43\% V30M. Compared to

\section{Figure 1. mNIS+7 LS Mean Difference by Quartile}

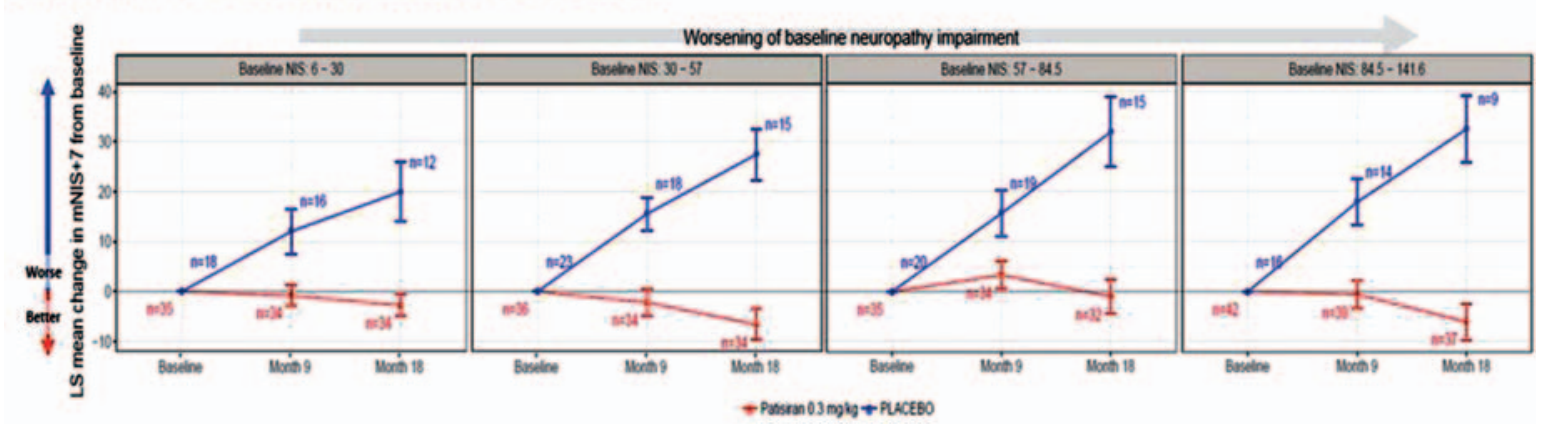


baseline, patients treated with patisiran had an improvement in neuropathy as measured by mNIS+7 (LS mean change: -6.0 points), while placebo patients worsened (LS mean change: +28.0 points) resulting in a LS mean difference of -34.0 points favoring patisiran at 18 -months $(\mathrm{p}=9.3 \times 10-24)$. Effect was seen as early as 9 months. Within each baseline NIS quartile (Figure 1), mNIS+7 worsened in the placebo group, with mean changes from baseline at 18-months of 20.0, 27.4, 31.9 and 32.5 points going from lowest (less severe disease) to highest (more severe disease) quartiles. In contrast, in the patisiran group mNIS+7 improved or remained stable within each quartile going from lowest (less severe disease) to highest (more severe disease) quartiles with $(-2.8,-6.6,-1.0$, and -6.0 .) points at 18-months (Figure 1). Conclusion: APOLLO patients on placebo presenting with either early or advanced neuropathy demonstrated significant disease progression over 18-months as measured by change in mNIS+7. These findings were consistent regardless of the severity of polyneuropathy at study entry indicating that treatment with patisiran can improve or stabilize the course of the disease.

\section{PS2Group3-011 / \#575}

Topic: Group 3 - Peripheral Neuropathy, Cranial Nerves, Mononeuropathies: Clinical Features, Pathophysiology, Therapy

\section{CLINICAL SPECTRUM OF HEREDITARY SPASTIC PARAPARESIS BY MUTATION IN KIF1A GENE}

$\underline{\text { Carlos Ortez }}^{1}$, Debora Itzep ${ }^{2}$, Laura CarreraGarcía $^{2}$, Daniel Natera De Benito ${ }^{3}$, Anna Lia Frongia $^{4}$, Delia Yubero ${ }^{2}$, Giovanni Stevanin ${ }^{5}$, Jaume Colomer ${ }^{2}$, Andres Nascimento ${ }^{2}$

${ }^{1}$ Neuromuscular Unit, San Joan de Deu Hospital, Barcelona, ES: ${ }^{2}$ Hospital Sant Joan de Déu, Barcelona, ES, ${ }^{3}$ Neuromuscular Disorders Unit, Hospital Sant Joan de Déu, Barcelona, ES: ${ }^{4}$ Neuromuscular Unit, Sant Joan de Deu Hospital, Barcelona, ES: ${ }^{5}$ Institut National de la Santé et de la Recherche Medicale (INSERM), Paris, FR

Background: KIF1A is a member of the kinesin family and functions as an anterograde motor protein that transports membranous organelles along microtubules. The phenotypic spectrum of hereditary spastic paraparesis (HSP) related to KIF1A is broad. Families with HSP with dominant and recessive inheritance have been described. Methods: Retrospective study of a cohort of patients followed in the Neuromuscular Unit of our hospital with a diagnosis of HSP with mutations in KIF1A gene. Results: Five cases of patients affected by HSP with mutations in KIF1A with an average age of 14 years (range 12-19) are reported. One of them has a pure HSP and four have complex forms, two with a family history of HSP. In the complex forms (three patients), axonal motor neuropathy was associated to mild-moderate intellectual disability, optic atrophy and cerebellar atrophy was observed in one patient. Clinical debut at an average age of 11.5 months (range: 1-18 months) in the form of hypertonia, equine gait and / or frequent falls. Progressive development of spasticity of proximal predominance of lower extremities. Currently all maintain autonomous spastic walk. All patients are carriers of heterozygous mutations in KIFIA gene. Conclusion: In our series, the HSP by mutations in KIF1A gene predominate the complex form associated with neuropathy and intellectual disability. Our data reaffirm the existence of heterozygous de novo mutations related to a more severe phenotype of complex HSP of early onset and autosomal dominant inheritance.

\section{PS2Group3-012 / \#607}

Topic: Group 3 - Peripheral Neuropathy, Cranial Nerves, Mononeuropathies: Clinical Features, Pathophysiology, Therapy

\section{PLACEBO EFFECT IN THE PATH STUDY OF SUBCUTANEOUS IMMUNOGLOBULIN IN CHRONIC INFLAMMATORY DEMYELINATING POLYNEUROPATHY}

$\underline{\text { Richard A. Lewis }}^{1}$, Vera Bril ${ }^{2}$, Nan Van Geloven ${ }^{3}$, Hans-Peter Hartung ${ }^{4}$, Gen Sobue ${ }^{5}$, Orell Mielke ${ }^{6}$, John-Philip Lawo ${ }^{6}$, Billie L. Durn 7 , David R. Cornblath $^{8}$, Ingemar Merkies ${ }^{9}$, Ivo Van Schaik ${ }^{10}$ ${ }^{1}$ Department Of Neurology, Cedars-Sinai Medical Center, Los Angeles, US, ${ }^{2}$ Department Of Medicine (neurology), University Health Network, University of Toronto, Toronto, CA; ${ }^{3}$ Department Of Medical Statistics And Bioinformatics, Leiden University Medical Center, Leiden, $N L,{ }^{4}$ Department Of Neurology, Heinrich-Heine University, Duesseldorf, 
DE, ${ }^{5}$ Department Of Neurology, Nagoya University Graduate School of Medicine, Nagoya, JP, ${ }^{6} \mathrm{CSL}$ Behring, Marburg, DE, ${ }^{7}$ CSL Behring, Fuquay Varina, NC, US; ${ }^{8}$ Department Of Neurology, Johns Hopkins University, Baltimore, MD, US; ${ }^{9}$ Department Of Neurology, Maastricht University Medical Center, Maastricht, NL: ${ }^{10}$ Department Of Neurology, Academic Medical Center, University of Amsterdam, Amsterdam, NL

Background: Placebo response is a recognised variable that is important in the design of clinical trials. In most clinical trials, 'placebo response' refers to unexpected improvement while on placebo. However, in trials involving chronic inflammatory demyelinating polyneuropathy (CIDP), it can refer to a lack of clinical deterioration on placebo when such deterioration was expected. This lack of deterioration may also be in part due to the remitting-relapsing nature of CIDP. The PATH study investigated subcutaneous immunoglobulin (SCIG) versus placebo in the treatment of CIDP and had two periods in which the placebo response could be evaluated. In the openlabel Ig dependency period, intravenous immunoglobulin (IVIG) was withdrawn from subjects stated by their physicians to be IVIG-dependent. In the randomisation period, subjects were randomised to SCIG maintenance therapy or placebo. Here we detail the placebo response rates from PATH and compare these with other studies of CIDP and IVIG therapy from the literature. Methods: PATH investigated SCIG (IgPro20, CSL Behring) as maintenance therapy for CIDP. Before randomisation to SCIG or placebo, subjects underwent IVIG withdrawal and, upon clinical deterioration, were restabilised with IVIG (IgPro10, CSL Behring). IVIG restabilisation comprised an initial dose of $2 \mathrm{~g} / \mathrm{kg}$ followed by 3-4 doses of $1 \mathrm{~g} / \mathrm{kg}$ at 3 -week intervals. Subjects were then randomised to weekly SCIG maintenance therapy $(0.2$ or $0.4 \mathrm{~g} / \mathrm{kg}$ ) or placebo for 24 weeks. The primary endpoint was CIDP relapse or withdrawal (relapse: $\geq 1$ point increase in total adjusted Inflammatory Neuropathy Cause and Treatment [INCAT] score). Placebo responses in other studies were identified through a literature review of articles published on PubMed (2000-2017). Results: Of the 245 subjects who entered the Ig dependency period, 28 (11.4\%) did not deteriorate despite being thought IVIG-dependent. A further 10 subjects did not continue to the restabilisation period due to other reasons. Of the 207 who entered the IVIG restabilisation period, 172 subjects demonstrating CIDP stability were randomised to placebo $(\mathrm{n}=57)$ or to SCIG $0.2 \mathrm{~g} /$ $\mathrm{kg}(\mathrm{n}=57)$ or $0.4 \mathrm{~g} / \mathrm{kg}(\mathrm{n}=58)$. In the placebo group, $21(37 \%)$ did not relapse or withdraw, compared with $61 \%$ and $67 \%$ of the two SCIG arms, despite all having deteriorated in the Ig dependency phase. Reports in the literature of placebo-controlled trials in patients with CIDP indicate placebo response or nonrelapse rates of $10-55 \%$, using a range of outcome measures (e.g., Medical Research Council sum score, INCAT, reduction in mean dose) and a variety of active treatments (e.g., IVIG, methotrexate). Conclusion: Among CIDP patients thought to be dependent on IVIG, $11.4 \%$ were able to stop IVIG without deterioration. For those shown to be IVIG dependent, when given placebo in a blinded fashion, 37\% did not relapse or withdraw. This study has important implications for clinical practice and CIDP clinical trials in highlighting both placebo effect and the remitting-relapsing nature of CIDP as confounding factors when assessing efficacy. Comparisons with other studies raise questions as to the reasons for this effect and whether study design can help mitigate these effects.

\section{PS2Group3-013 / \#936}

Topic: Group 3 - Peripheral Neuropathy, Cranial Nerves, Mononeuropathies: Clinical Features, Pathophysiology, Therapy

\section{THE EFFECT OF REHABILITATION ON SLEEP, RESPIRATORY AND LIFE QUALITY}

Filiz Meryem Sertpoyraz ${ }^{1}$, Figen Baydan ${ }^{2}$, Mehtap Turanoglu ${ }^{1}$

${ }^{1}$ Physical Therapy And Rehabilitation Clinic, İzmir Tepecik Education and Research Hospital, İzmir, TR:²Pediatric Neurology, Tepecik Eğitim ve Araştırma Hastanesi, İzmir, TR

Background: Polynoropathy usually starts symmetrically, sensibly, in lower limb limbs. Paresthesia, pain and motor involvement are accompanied by weakness We wanted to share the developments that occurred after rehabilitation in our patient with sensoromotor mix type polyneuropathic diagnosis. Methods: A 28-year-old male patient was admitted to İzmir Hospital Neuromuscular Diseases Unit with complaints of walking, unable to use his hands, and quadriplegic weakness. The patient was born with a low birth weight $(2 \mathrm{~kg})$ in the story, and there was 
retardation in motor development compared to their peers. He walked after a 2 year old. He was evaluated orthopedic physician at the age of 4 . He underwent tendon lengthening operation due to deformity in his right foot. He was diagnosed polyneuropathy. During this period, she continued her intensive rehabilitation program. At the age of 7, his long-legged walking device was used to mobilize. His walk was completely disturbed due to his progress and he was able to mobilize with a wheelchair at the age of 15 . 2 times orthopedic surgery. No known muscle and nervous system disease in the family. Functional Ambulance Scale: 0, using a wheelchair. Barthel index :90 Advanced dependent, Dorsolomber scoliosis present. Bilateral upper extremity elbows, wrists, flexion contractures in the hips, knees and ankles in the lower extremities. Upper limb proximal muscle branch $4 / 5$, distal $3 / 5$, lower extremity proximal muscle branch 2-3 / 5, distal 2/5. Atrophy was present in quadriceps, gastrocnemius, and hand interosseal muscles. EMG:Findings compatible with advanced stage sensorial dominance, sensoromotor axonal and demyelinating polyneuropathy in chronic process Bone Densitometry: Hip Femur neck T score: -3,2 Respiratory function test: Severe restrictive type of respiratory failure $X$ ray: Dorsolomber scoliosis Polysomnography: BIPAP was recommended due to Obstructive Sleep Apnea Syndrome in the middle Alendronate and calcium + cholecalciferol were started for osteoporosis. A regular rehabilitation program has started.The basis of the rehabilitation program was stretching, joint range of

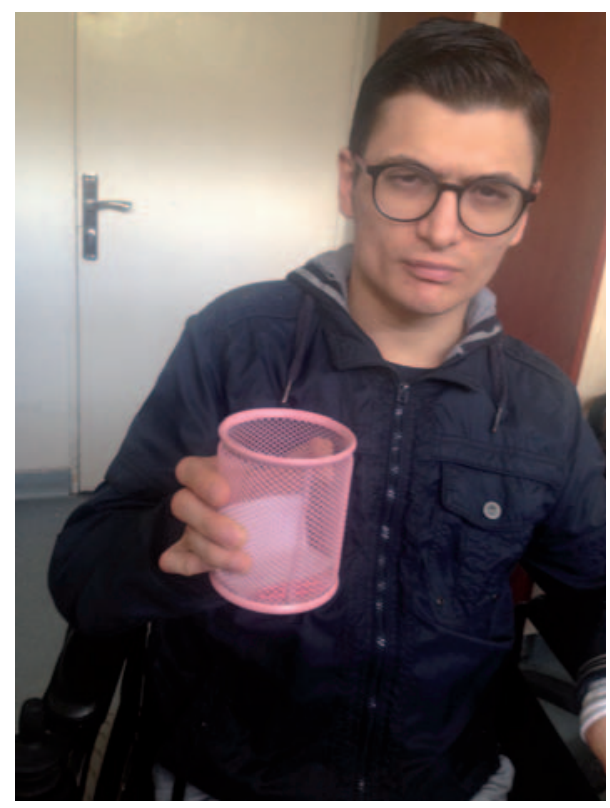

motion and breathing exercises. After rehabilitation ,In the pulmonary function test were significantly improved compared to previous findings. The findings of mild obstructive apnea syndrome were present on control polysomnography. Sleep therapy was significantly improved with rehabilitation therapy. Joint range of motion and muscle strength increased. Results: a Polyneuropathy rehabilitation is the most important method in the treatment of patients. Conclusion: It is possible for the patient to be able to perform his / her daily life activities independently, in order to participate in community life by rehabilitation.

\section{PS2Group3-014 / \#379}

Topic: Group 3 - Peripheral Neuropathy, Cranial Nerves, Mononeuropathies: Clinical Features, Pathophysiology, Therapy

\section{MULTIFOCAL MOTOR NEUROPATHY WITH CONDUCTION BLOCK: A CASE SERIES}

\author{
Aysha A. Alshareef \\ Neurology, King Abdulaziz University, jeddah, SA
}

Background: Motor neuropathies and multifocal motor neuropathy with conduction block are treatable causes of neuropathy that present with the clinical syndrome of asymmetric motor weakness with atrophy,. The diagnosis of the disorder relies on the typical clinical presentation as well as electro diagnostic findings of conduction block at non compression Site, in this article we reviewed the clinical characteristics, electrophysiological findings and treatment response of six patients with diagnosis of multifocal motor neuropathy with conduction clock in Saudi Arabia ;king Abdulaziz University hospital. Methods: Data of 6 patients of multifocal motor neuropathy with conduction block, who were diagnosed and treated from 2015 to August 2018 in Department of Neurology, king Abdulaziz university hospital at Saudi Arabia, were analyzed retrospectively, including clinical features, electrophysiological studies, and response to immunotherapy. Results: The 6 cases included 2 females and 4 males, 4 cases was above age of $50 \mathrm{y}$, the oldest case was $60 \mathrm{y}$ old and the youngest was $28 \mathrm{y}$ old. The follow-ups after treatment were between 6 months to 2 y.all from Arab population, the duration of symptoms prior presentation to our department were variable 
(10y,5y,3y,6m,2m,1m) All 6 cases were presented asymmetrically , 4 patients presented with right hand weakness predominantly finger drop ,one case presented with right claw hand, the commonest nerve affected was the radial nerve followed by ulnar nerves, and the distal part of limbs was initially affected at all patients. Only 3 patients have wasting of small muscle of the hand predominately first dorsal interosseous muscle, Two patients has mild paresthesia of the hand. Electrophysiological studies demonstrated temporal dispersion and conduction blocks on non-entrapment sites in more than one nerves in all patients, asymptomatic limb nerve conduction study revealed conduction block in five patients.sensory nerve conduction study were normal in all 6 patients, fibrillation and fasciculation were recorded in two patients, rapid firing rate, decreased recruitment and large motor unit potential were recorded in all patients, anti GM1 antibodies were done for 4 patients and I was negative, 4 cases showed significant improvement after IV Ig infusion while one patient did not improve and one missed follow up. Conclusion: asymmetrical pure motor nerve damage is the most important manifestation of multifocal motor neuropathy with conduction block, conduction block is the most important diagnostic criteria and it is found in symptomatic and asymptomatic limb ,radial nerve is the commonest nerve affected ,. IV Ig is an effective treatment for this disease

\section{PS2Group3-015 / \#560}

Topic: Group 3 - Peripheral Neuropathy, Cranial Nerves, Mononeuropathies: Clinical Features, Pathophysiology, Therapy

\section{PHARMACOKINETICS AND SAFETY OF A SELECTIVE ANTIBODY-SCAVENGING GLYCOPOLYMER FOR THE TREATMENT OF ANTI-MAG NEUROPATHY}

Delphine Demeestere $^{1}$, Butrint Aliu ${ }^{1}$, Wolf Heusermann ${ }^{1}$, Beat Ernst ${ }^{1}$, Pascal Hänggi², Ruben Herrendorff ${ }^{2}$ ${ }^{1}$ Institute Of Molecular Pharmacy, University of Basel, Basel, CH; ${ }^{2} \& \&$, Polyneuron Pharmacetuicals AG, Basel, $\mathrm{CH}$

Background: Anti-myelin-associated glycoprotein (MAG) neuropathy is a demyelinating polyneurop- athy that is caused by pathogenic monoclonal $\operatorname{IgM}$ antibodies against MAG. These autoantibodies specifically recognize the human natural killer-1 (HNK1) glycoepitope, which is highly expressed on MAG. Patients suffer from paresthesia, sensory ataxia, tremor and a varying degree of sensorimotor deficits, but no safe and efficacious treatment is available. Therefore, we developed a glycopolymer, PPSGG, which presents a mimetic of the HNK-1 epitope in a multivalent manner on a biodegradable polymer backbone, and demonstrated its therapeutic potential as a selective scavenger for anti-MAG autoantibodies in an immunological mouse model. Methods: In this study, we explored the pharmacokinetic properties of PPSGG and some toxicological parameters by using imaging tools (2-photon tomography, confocal microscopy) and immunoassays (ELISA, Luminex). Results: Tissue distribution was explored after injection of a fluorescently labeled PPSGG in wild type mice and revealed a predominant presence of the drug in the liver and spleen. The presence of PPSGG in the marginal zone of the spleen, co-localization with the macrophages marker F4/80 in the liver and the short half-life $\left(\mathrm{t}_{1 / 2}, 16.9\right.$ $\pm 5.5 \mathrm{~min}$ ) of the drug suggest a fast uptake and
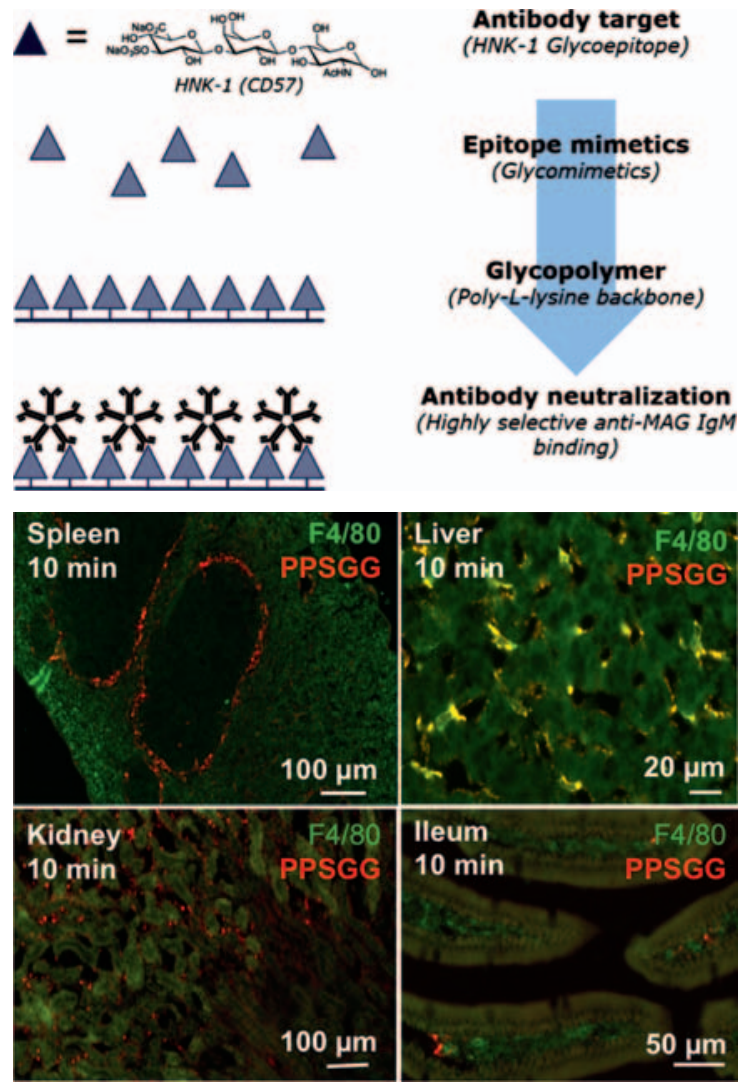
metabolism by the mononuclear phagocyte system. Additionally, PPSGG did not induce any obvious systemic inflammation or anti-drug antibody formation in vivo in mice, and no hepatoxicity in a human liver microtissue model. Conclusion: In conclusion, all obtained results so far are in favor of the clinical use of PPSGG in selective autoantibody removal.

\section{PS2Group3-016 / \#587}

Topic: Group 3 - Peripheral Neuropathy, Cranial Nerves, Mononeuropathies: Clinical Features, Pathophysiology, Therapy

\section{A NOVEL MISSENSE MUTATION OF TRANSTHYRETIN CAUSING AMYLOIDOSIS}

\author{
$\underline{\text { Hua-Chuan Chao }}^{1}$, Yo-Tsen Liu' ${ }^{2}$, Yi-Chung Lee ${ }^{3}$, \\ Kon-Ping $\operatorname{Lin}^{4}$ \\ ${ }^{1}$ Medicine, Taipei Veterans General Hospital, \\ Taoyuan Branch, Taoyuan, TW; ${ }^{2}$ Neurology, \\ Taipei-Veterans General Hospital, Taipei, \\ $T W ;{ }^{3}$ Neurology, Taipei Veterans General Hospital, \\ Taipei, TW; ${ }^{4}$ Taipei Veterans General Hospital, \\ Taipei, $T W$
}

Background: Hereditary transthyretin amyloidosis (ATTR) is a disorder caused by dominantly inherited transthyretin (TTR) mutations, and is characterized as a progressive peripheral neuropathy and autonomic neuropathy associated with systemic involvement. The Taiwanese population is unique that majority of subjects carry the Ala97Ser missense mutation in TTR. We reported a patient with generalized neuropathy and cardiac involvement, caused by TTR Glu89Asp, which is a novel variant with heterozygous missense mutation. Methods: This male patient presented with distal paresthesia from his toes without impaired moto function since his age 59. Intermittent chest tightness, diarrhea, sensitive to light while driving and erectile dysfunction developed at the age of 64 . In addition, progressively ascending numbness, tingling pain with loss of body weight up to $12 \mathrm{~kg}$ were reported in two years. Progressively weakness and muscular atrophy developed, resulting wheelchair-bound at his age 68. The neurological evaluation including nerve conduction study, SSR/ RRIV and HUT were obtained, which revealed axonal polyneuropathy and autonomic dysfunction. Echocardiography and Tc-PYP scan were collected for cardiac evaluation, and cardiac amyloidosis was documented. He received colonoscopy and rectum biopsy, and the focal amyloid deposition was confirmed pathologically. Results: Transthyretin (TTR) gene analysis showed one point mutation $(\mathrm{G}>\mathrm{T})$ in the third base of codon 327 with the corresponding amino acid substitution of Glu for Asp. Glu89Asp (c.327G $>$ T) was absent in the public database Mutations in Hereditary Amyloidosis (http://amyloidosismutations.com/cdna-attr.html) and Genome Aggregation Database (gnomAD). This putative pathogenic variant was also absent in 997 Taiwanese control exomes from Taiwan biobank database (http://taiwanview.twbiobank.org.tw/index). In silico predictions of pathogenicity via MutationTaster (http://www.mutationtaster.org), and PolyPhen-2 (http://genetics.bwh.harvard.edu/pph2), Glu89Asp (c.327G $>$ T) was predicted as a disease-causing variant. According to American College of Medical Genetics and Genomics (ACMG) standards and guideline, Glu89Asp was considered as a likely pathogenic variant. Conclusion: We reported here a patient with Glu89Asp TTR mutation, who presented with painful peripheral neuropathy, early autonomic dysfunction and cardiac involvement. This is a novel missense change at the 89 amino acid residue which is different from the previously reported variant.

\section{PS2Group3-017 / \#619}

Topic: Group 3 - Peripheral Neuropathy, Cranial Nerves, Mononeuropathies: Clinical Features, Pathophysiology, Therapy

\section{CHRONIC MOTOR AXONAL NEUROPATHIES: A CHALLENGING DIAGNOSIS}

\author{
Yaacov Anziska $^{1}$, Ilana Lasner ${ }^{2}$ \\ ${ }^{1}$ Neurology, SUNY-Downstate Medical Center, \\ Brooklyn, NY, US; ${ }^{2}$ Neurology, Kings County \\ Hospital, Brooklyn, AL, US
}

Background: Chronic motor axonal neuropathies (CMAN) are rarely described in the literature and are usually thought to be immune-mediated, associated with anti-GM antibodies. Typically, these neuropathies are treated with immunomodulatory therapies, including IVIG or plasma exchange (PLEX), with varying degrees of success. However, there are instances of CMAN which are not primarily immune-mediated and are associated with various systemic diseases. Methods: In our case series, 
we reviewed the inpatient charts for four inpatients diagnosed with CMAN. Data included nerve coduction studies/Electromyography, laboratory tesing, and clinical examination, as documented on daily notes. Results: We describe 4 cases of chronic motor axonal neuropathies, in which neither anti-GM1 antibodies nor conduction block were present on laboratory and electrophysiologic testing. One of the cases was a patient with newly diagnosed lupus, one with newly diagnosed sacrocidosis, one with multiple myeloma (discovered 8 months after onset of motor weakness), and one with a chronic IgG gammopathy. Only the multiple myeloma patient responded temporarily to PLEX, eventually relapsing, and none of the other patients responded to various immunomodulatory therapies, including IVIG, PLEX, or high-dose steroids. Three of the patients had severe ventilatory weakness, bulbar symptoms (dysphagia, dysarthria), and vocal cord paralysis/ dysfunction. Only the multiple myeloma patient recovered permanently after receiving a combination of Bendamustine and Rituximab treatment; the lupus patient improved mildly on Plaquenil, Cytoxan, Prednisone, and Imuran. Conclusion: We propose that for patients with CMAN, besides testing for various antibodies (GM-1, GD1a, GD1b, and GQ1b), a broader workup should include rheumatologic, oncologic, and infiltrative processes. Unfortunately, clinical recovery may still be limited for these patients, but might be improved if treatment could focus on the underlying systemic disease, rather than a general immunomodulatory approach administering only IVIG or PLEX.

\section{PS2Group3-018 / \#730}

Topic: Group 3 - Peripheral Neuropathy, Cranial Nerves, Mononeuropathies: Clinical Features, Pathophysiology, Therapy

\section{QUALITY OF LIFE IN A CLINICAL STUDY OF MAINTENANCE TREATMENT OF CIDP WITH IGPRO20: THE PATH STUDY}

Hans-Peter Hartung ${ }^{1}$, Vera Bril², Ingemar Merkies ${ }^{3}$, Ivo Van Schaik ${ }^{4}$, Richard A. Lewis ${ }^{5}$, Nan Van Geloven $^{6}$, David R. Cornblath ${ }^{7}$, Gen Sobue ${ }^{8}$, Orell Mielke $^{9}$, Rajiv Mallick ${ }^{10}$, Billie L. Durn ${ }^{11}$, JohnPhilip Lawo ${ }^{9}$, Alphonse Hubsch ${ }^{12}$ ${ }^{1}$ Department Of Neurology, Heinrich Heine University, Düsseldorf, DE; ${ }^{2}$ Department Of
Medicine (neurology), University Health Network, University of Toronto, Toronto, ON, CA, ${ }^{3}$ Maastricht University Medical Center, Maastricht, NL, ${ }^{4}$ Department Of Neurology, Academic Medical Center, University of Amsterdam, Amsterdam, $N L,{ }^{5}$ Department Of Neurology, Cedars-Sinai Medical Center, Los Angeles, CA, US $;{ }^{6}$ Department Of Medical Statistics And Bioinformatics, Leiden University Medical Center, Leiden, NL; ${ }^{7}$ Department Of Neurology, Johns Hopkins University, Baltimore, MD, US; ${ }^{8}$ Department Of Neurology, Nagoya University Graduate School of Medicine, Nagoya, $J P ;{ }^{9} \mathrm{CSL}$ Behring, Marburg, DE: ${ }^{10} \mathrm{CSL}$ Behring, King of Prussia, PA, US, ${ }^{11}$ CSL Behring, Fuquay Varina, NC, US, ${ }^{12}$ CSL Behring, Bern, $\mathrm{CH}$

Background: Subcutaneous immunoglobulin (SCIG) offers an alternative administration option to intravenous immunoglobulin (IVIG), with anticipated improvements in patient convenience and autonomy. As part of an international randomized clinical study evaluating two doses of IgPro20 (a $20 \%$ SCIG solution) versus placebo in the maintenance treatment of patients with CIDP, we evaluated patient-reported quality of life (QoL). Methods: Following stabilization on IgPro10 (a 10\% IVIG solution), patients received 0.2 or $0.4 \mathrm{~g} / \mathrm{kg}$ IgPro20 weekly, or placebo. The primary outcome was percentage of patients with CIDP relapse (determined by adjusted Inflammatory Neuropathy Cause and Treatment score) or withdrawal during 24 weeks of treatment. Patient-reported quality of life was assessed using the EuroQoL (EQ)-5D instrument. The EQ-5D comprises five health dimensions: mobility, self-care, usual activities, pain/discomfort, and anxiety/depression, each rated in terms of three possible health status levels: No problems (assigned 0 points), Some/Moderate problems (1 point), and Extreme problems ( 2 points). A change from baseline to last post-dose observation on each of the health dimensions was classified in terms of improvement (decline of 1-2 points), maintenance (no numeric score change) and deterioration (increase of 1-2 points). Consistent with the study's primary outcome, the 'maintenance' and 'improvement' categories, were collapsed, post-hoc, to show improvement/ maintenance versus deterioration. In addition to the five health dimensions, the EQ-5D also assessed overall patient-reported health status on a Visual Analog Scale (VAS) ranging from 0 (worst possible health) to 100 (best possible health). Results: A higher proportion of SCIG treated subjects improved 


\begin{tabular}{|c|c|c|c|c|c|c|c|c|c|c|}
\hline \multirow{4}{*}{$\begin{array}{l}\text { Observed data, no } \\
\text { imputation ( } n=151 \text { ) } \\
\text { Mobility }\end{array}$} & \multirow[b]{3}{*}{$\begin{array}{l}\text { Maintained or } \\
\text { improved }\end{array}$} & \multirow{2}{*}{\multicolumn{2}{|c|}{ Placebo }} & \multirow{2}{*}{\multicolumn{2}{|c|}{$0.2 \mathrm{~g} / \mathrm{kg}$}} & \multirow{2}{*}{\multicolumn{2}{|c|}{$0.4 \mathrm{~g} / \mathrm{kg}$}} & \multicolumn{3}{|c|}{$\begin{array}{l}\text { p value (Fisher's exact test } \\
\text { no adjustment for multiplicity) }\end{array}$} \\
\hline & & & & & & & & \multirow{2}{*}{$\begin{array}{c}0.2 \mathrm{~g} / \mathrm{kg} \\
\mathrm{vs} \mathrm{PIb}\end{array}$} & \multirow{2}{*}{$\begin{array}{c}0.4 \mathrm{~g} / \mathrm{kg} \\
\mathrm{vs} \mathrm{PIb}\end{array}$} & \multirow{2}{*}{$\begin{array}{c}0.4 \text { vs } \\
0.2 \mathrm{~g} / \mathrm{kg} \\
0.724\end{array}$} \\
\hline & & 32 & $72.7 \%$ & 47 & $87.0 \%$ & 45 & $84.9 \%$ & & & \\
\hline & Worsened & 12 & $27.3 \%$ & 7 & $13.0 \%$ & 8 & $15.1 \%$ & & & \\
\hline \multirow[t]{2}{*}{ Self-care } & $\begin{array}{l}\text { Maintained or } \\
\text { improved }\end{array}$ & 35 & $79.5 \%$ & 47 & $87.0 \%$ & 47 & $88.7 \%$ & 0.234 & 0.169 & 0.515 \\
\hline & Worsened & 9 & $20.5 \%$ & 7 & $13.0 \%$ & 6 & $11.3 \%$ & & & \\
\hline \multirow[t]{2}{*}{ Usual activities } & $\begin{array}{l}\text { Maintained or } \\
\text { improved }\end{array}$ & 30 & $68.2 \%$ & 46 & $85.2 \%$ & 48 & $90.6 \%$ & 0.039 & 0.006 & 0.290 \\
\hline & Worsened & 14 & $31.8 \%$ & 8 & $14.8 \%$ & 5 & $9.4 \%$ & & & \\
\hline \multirow[t]{2}{*}{ Pain/discomfort } & $\begin{array}{l}\text { Maintained or } \\
\text { improved }\end{array}$ & 37 & $84.1 \%$ & 50 & $92.6 \%$ & 48 & $90.6 \%$ & 0.158 & 0.256 & 0.765 \\
\hline & Worsened & 7 & $15.9 \%$ & 4 & $7.4 \%$ & 5 & $9.4 \%$ & & & \\
\hline \multirow[t]{2}{*}{ Anxiety/depression } & $\begin{array}{l}\text { Maintained or } \\
\text { improved }\end{array}$ & 36 & $81.8 \%$ & 47 & $87.0 \%$ & 47 & $88.7 \%$ & 0.332 & 0.252 & 0.515 \\
\hline & Worsened & 8 & $18.2 \%$ & 7 & $13.0 \%$ & 6 & $11.3 \%$ & & & \\
\hline
\end{tabular}

\begin{tabular}{|c|c|c|c|c|c|c|c|c|c|c|}
\hline $\begin{array}{l}\text { Observed plus imputed } \\
\text { data }(n=167)\end{array}$ & & \multicolumn{2}{|c|}{ Placebo } & \multicolumn{2}{|c|}{$0.2 \mathrm{~g} / \mathrm{kg}$} & \multicolumn{2}{|c|}{$0.4 \mathrm{~g} / \mathrm{kg}$} & $\begin{array}{l}0.2 \mathrm{~g} / \mathrm{kg} \\
\text { vs Plb }\end{array}$ & $\begin{array}{l}0.4 \mathrm{~g} / \mathrm{kg} \\
\text { vs Plb }\end{array}$ & $\begin{array}{l}0.4 \mathrm{vs} \\
0.2 \mathrm{~g} / \mathrm{kg}\end{array}$ \\
\hline \multirow[t]{2}{*}{ Mobility } & $\begin{array}{l}\text { Maintained or } \\
\text { improved }\end{array}$ & 33 & $60.0 \%$ & 48 & $85.7 \%$ & 46 & $82.1 \%$ & 0.002 & 0.009 & 0.780 \\
\hline & Worsened & 22 & $40.0 \%$ & 8 & $14.3 \%$ & 10 & $17.9 \%$ & & & \\
\hline \multirow[t]{2}{*}{ Self-care } & $\begin{array}{l}\text { Maintained or } \\
\text { improved }\end{array}$ & 36 & $65.5 \%$ & 48 & $85.7 \%$ & 48 & $85.7 \%$ & 0.011 & 0.011 & 0.606 \\
\hline & Worsened & 19 & $34.5 \%$ & 8 & $14.3 \%$ & 8 & $14.3 \%$ & & & \\
\hline \multirow[t]{2}{*}{ Usual activities } & $\begin{array}{l}\text { Maintained or } \\
\text { improved }\end{array}$ & 31 & $56.4 \%$ & 47 & $83.9 \%$ & 49 & $87.5 \%$ & 0.001 & $<0.001$ & 0.394 \\
\hline & Worsened & 24 & $43.6 \%$ & 9 & $16.1 \%$ & 7 & $12.5 \%$ & & & \\
\hline \multirow[t]{2}{*}{ Pain/discomfort } & $\begin{array}{l}\text { Maintained or } \\
\text { improved }\end{array}$ & 38 & $69.1 \%$ & 51 & $91.1 \%$ & 49 & $87.5 \%$ & 0.003 & 0.016 & 0.820 \\
\hline & Worsened & 17 & $30.9 \%$ & 5 & $8.9 \%$ & 7 & $12.5 \%$ & & & \\
\hline \multirow[t]{2}{*}{ Anxiety/depression } & \begin{tabular}{|l|} 
Maintained or \\
improved
\end{tabular} & 37 & $67.3 \%$ & 48 & $85.7 \%$ & 48 & $85.7 \%$ & 0.019 & 0.019 & 0.606 \\
\hline & Worsened & 18 & $32.7 \%$ & 8 & $14.3 \%$ & 8 & $14.3 \%$ & & & \\
\hline
\end{tabular}

or maintained health status in each of the 5 dimensions of the EQ-5D, compared to placebo (Table 1). Additionally, compared to a decline in median VAS scores in the placebo group ( -10.0 points; Q1, Q3: $-25.0,0.0$ points), median VAS score declined marginally in the $0.2 \mathrm{~g} / \mathrm{kg}$ IgPro20 group ( -5.0 points; Q1, Q3: - 15.0, 6.0 points) and was stable in the 0.4 $\mathrm{g} / \mathrm{kg}$ IgPro20 group (0.0 points, Q1, Q3: -7.5, 5.5) $(\mathrm{p}<0.005$, across treatment groups). Table 1: Numbers and percentages of patients maintained or improved versus worsened on the five health dimensions of the EuroQol-5D: IgPro20 dose groups and placebo. Conclusion: Patient-reported QoL was maintained in a higher proportion of patients treated with either dose of subcutaneous IgPro20, compared to placebo. Differences with placebo were greatest for the domains of mobility and usual activities, consistent with corresponding differences in prevention of neuromuscular relapse impacting physical function in CIDP. 
PS2Group3-019 / \#651

Topic: Group 3 - Peripheral Neuropathy, Cranial Nerves, Mononeuropathies: Clinical Features, Pathophysiology, Therapy

\section{FEASIBILITY OF SWITCHING FROM INTRAVENOUS TO SUBCUTANEOUS IG THERAPY IN CIDP: PATH TRIAL RESULTS VERSUS CLINICAL EXPERIENCE}

Dario Cocito ${ }^{1}$, Erdita Peci ${ }^{1}$, Alberto Romagnolo ${ }^{1}$, Vera Bril' ${ }^{2}$, Nan Van Geloven ${ }^{3}$, Hans-Peter Hartung ${ }^{4}$, Richard A. Lewis ${ }^{5}$, Gen Sobue 6 , John-Philip Lawo ${ }^{7}$, Orell Mielke ${ }^{7}$, Billie L. Durn ${ }^{8}$, David R. Cornblath ${ }^{9}$, Ingemar Merkies ${ }^{10}$, Ivo Van Schaik ${ }^{11}$

${ }^{1}$ University of Turin, Turin, IT; ${ }^{2}$ Department Of Medicine (neurology), University Health Network, University of Toronto, Toronto, CA, ${ }^{3}$ Department Of Medical Statistics And Bioinformatics, Leiden University Medical Center, Leiden, NL; ${ }^{4}$ Department Of Neurology, Heinrich-Heine University, Duesseldorf, DE, ${ }^{5}$ Department $O f$ Neurology, Cedars-Sinai Medical Center, Los Angeles, US; ${ }^{6}$ Department Of Neurology, Nagoya University Graduate School of Medicine, Nagoya, JP, ${ }^{7} C S L$ Behring, Marburg, DE, ${ }^{8}$ CSL Behring,
Fuquay Varina, NC, US; ${ }^{9}$ Department Of Neurology, Johns Hopkins University, Baltimore, MD, US: ${ }^{10}$ Department Of Neurology, Maastricht University Medical Center, Maastricht, NL; ${ }^{11}$ Department Of Neurology, Academic Medical Center, University of Amsterdam, Amsterdam, NL

Background: In chronic inflammatory demyelinating polyneuropathy (CIDP), immunoglobulin (Ig) therapy is typically administered intravenously (IVIG). Subcutaneous Ig administration (SCIG) enables independence from hospitals and increased convenience. The phase 3 PATH study showed efficacy of SCIG in CIDP. Here we evaluate the feasibility of switching from IVIG to SCIG in CIDP patients by comparing PATH data with clinical experience. Methods: In PATH, subjects with CIDP were switched to SCIG (0.2 or $0.4 \mathrm{~g} / \mathrm{kg} /$ week) or placebo after IVIG induction. Adverse events (AEs), quality of life (QoL; EuroQol - 5 Dimension questionnaire) and patient preference were assessed. Observational studies of switching from IVIG to SCIG in CIDP and multifocal motor neuropathy (MMN), with assessment of safety, QoL (Life Quality Index [LQI] questionnaire) and patient preference are detailed. Results: The percentage of PATH subjects who ex-

Table 1. Data from PATH and observational studies.

\begin{tabular}{|c|c|c|c|c|c|}
\hline Study & $\begin{array}{c}\text { van Schaik et al, } \\
2018 \text { (PATH) } \\
\end{array}$ & Cocito et al, 2014 & $\begin{array}{c}\text { Cocito et al, } \\
2013 \\
\end{array}$ & Cocito et al, 2012 & $\begin{array}{c}\text { Markvardsen et al, } \\
2013 \\
\end{array}$ \\
\hline Population & 207 CIDP & $66 \mathrm{CIDP}, 21 \mathrm{MMN}$ & $10 \mathrm{CIDP}$ & 5 CIDP, 5 MMN & $\begin{array}{c}29 \text { CIDP (14 SCIG, } \\
115 \text { placebo) }\end{array}$ \\
\hline IVIG to SCIG dose & $\begin{array}{l}\text { Fixed dose of } 0.2 \text { or } \\
0.4 \mathrm{~g} / \mathrm{kg} \mathrm{SCIG}\end{array}$ & $1: 1$ & $1: 1$ & $1: 1$ & $1: 1$ \\
\hline SCIG infusion details & $\begin{array}{c}\text { 1-2 infusions per } \\
\text { week }\end{array}$ & $\begin{array}{c}\text { 1-3 infusions per } \\
\text { week; } \\
\text { 5-10 days after } \\
\text { last IVIG dose }\end{array}$ & NR & $\begin{array}{c}5 \text { weekly } \\
\text { infusions; } \\
7-15 \text { days after } \\
\text { last IVIG dose }\end{array}$ & $\begin{array}{l}\text { 2-3 weekly } \\
\text { infusions; } 2 \text { weeks } \\
\text { after last IVIG dose }\end{array}$ \\
\hline Quality of Life findings & $\begin{array}{l}\text { 88\% found SCIG } \\
\text { easier to use than } \\
\text { IVIG; SCIG } \\
\text { maintained or } \\
\text { improved E5-QD QoL }\end{array}$ & $\begin{array}{l}\text { Increased LQI in } \\
\text { CIDP patients for } \\
\text { therapeutic } \\
\text { setting, } \\
\text { interference with } \\
\text { daily activities, } \\
\text { problems with } \\
\text { administration } \\
\end{array}$ & $\begin{array}{l}\text { Increase in all } \\
\text { LQI domains }\end{array}$ & $\begin{array}{l}\text { LQI I, II and III } \\
\text { increased with } \\
\text { SCIG; } \\
90 \% \text { preferred } \\
\text { SCIG to IVIG }\end{array}$ & $\begin{array}{l}\text { 20/29 preferred } \\
\text { SCIG to IVIG }\end{array}$ \\
\hline Adverse events & $\begin{array}{l}48 \% \text { of IVIG subjects } \\
\text { reported AE vs } 52- \\
58 \% \text { of SCIG; } \\
\text { local reactions most } \\
\text { common AE in SCIG } \\
\text { vs headache in IVIG }\end{array}$ & $\begin{array}{l}21.8 \% \text { reported } \\
\text { systemic AEs with } \\
\text { IVIG vs } 1.1 \% \text { with } \\
\text { SCIG }\end{array}$ & NR & $\begin{array}{l}\text { AEs reported in } \\
60 \% \text { of IVIG } \\
\text { patients vs } 0 \% \text { of } \\
\text { SCIG patients }\end{array}$ & $\begin{array}{l}\text { 6/14 SCIG patients } \\
\text { reported AEs; all } \\
\text { AEs were local }\end{array}$ \\
\hline
\end{tabular}


perienced $\geq 1$ AE with IVIG was $48 \%$ (rate: 0.175 / infusion). Corresponding percentages for SCIG-0.2 and SCIG-0.4 were 58\% and 52\% (0.08 and $0.05 /$ infusion). The most common AE was headache for IVIG (16\%, 0.033/infusion), and local infusion site reactions for SCIG (19\% [0.03/infusion] for SCIG$0.2 ; 29 \%$ [0.02/infusion] for SCIG-0.4). Most subjects $(88 \%)$ felt SCIG was easier to use versus IVIG. Significantly more subjects (P-values $<0.005$ ) improved/maintained QoL health status with SCIG versus placebo. In observational studies, switching from IVIG to SCIG was associated with increased QoL and reduced systemic AEs (Table 1). Conclusion: The randomised PATH study and observational studies comprising large cohorts of subjects have documented the feasibility, safety and efficacy of SCIG therapy in CIDP.

\section{PS2Group3-020 / \#924}

Topic: Group 3 - Peripheral Neuropathy, Cranial Nerves, Mononeuropathies: Clinical Features, Pathophysiology, Therapy

\section{TWO NOVEL VARIANTS IN THE SLC25A46 GENE CAUSING OPTICAL ATROPHY AND PERIPHERAL NEUROPATHY IN CZECH SIBLINGS}

\author{
Marie Šedivá ${ }^{1}$, Petra Laššuthová2 ${ }^{2}$ Jiřina Kaňáková ${ }^{3}$ \\ Jana Haberlova ${ }^{4}$, Lucie Sedláčková2 ${ }^{2}$ Markéta \\ Vlčková ${ }^{5}$, Pavel Seeman ${ }^{6}$ \\ ${ }^{1}$ Department Of Paediatric Neurology, 2nd Faculty \\ of Medicine, Charles University in Prague and \\ Motol University Hospital, Prague, \\ CZ; ${ }^{2}$ Department Of Paediatric Neurology, Dna \\ Laboratory, 2nd Medical Faculty, Charles \\ University and University Hospital Motol, Prague, \\ CZ; ${ }^{3}$ Paediatric Department, Hospital Klatovy, \\ Klatovy, CZ; ${ }^{4}$ Department Of Paediatric Neurology, \\ 2nd Faculty of Medicine, Charles University in \\ Praue and Motol University Hospital, Prague, \\ CZ; ${ }^{5}$ Department Of Biology And Medical Genetics, \\ 2nd Faculty of Medicine, Charles University in \\ Prague and Motol University Hospital, Prague, \\ CZ; ${ }^{6}$ Department Of Paediatric Neurology, Dna \\ Laboratory, 2nd Medical Faculty, Charles \\ University and University Hospital Motol, Prague, \\ Prague, $C Z$
}

Background: Hereditary polyneuropathies are a heterogeneous group of diseases with all modes of inheritance and variable phenotype. There are 2 main groups according to impairment of either myelin sheets (HMSN I) or axons (HMSN II). Polyneuropathy is sometimes connected with optical neuropathy. SLC25A46 gene was identified to be responsible for autosomal recessive hereditary optic atrophy in combination with peripheral axonal neuropathy and cerebellar atrophy. (1) The patients from the original study belonged to 4 families and were all compound heterozygotes for variants of SLC25A46, different in each family. ${ }^{(1)}$ Methods: We present a clinical case of a 19-year-old male with primarily axonal motor and sensitive peripheral polyneuropathy and optical atrophy who has a similarly affected sister, and a healthy mother. The father is not in contact with the family. The patient was examined by whole exome sequencing. Results: By whole exome sequencing (WES), two novel variants in compound heterozygous state where found in the patient and his older sister. One is a nonsense variant in the SLC25A46 (NM_138773.2):c.1208T $>$ G (p.Leu403*) and the second is a missense variant c.1075G $>$ A (p.Val359Met), affecting a highly-conserved amino-acid residue and predicted as pathogenic. The healthy mother is a heterozygous carrier of the variant $p$. Leu403*, the father was not available for testing. The patient was born from $2^{\text {nd }}$ uneventful pregnancy to healthy parents, with no perinatal complications. He first presented with impairment of visual function and atrophy of optic nerves at the age of 3 years. Since the age of 6 years he had walking difficulties and progressive deformities of feet (pedes excavates). Since the age of 10 years ataxia was noticed. The condition is slowly progressive, with a similar course in both affected siblings. Brain and spine MRI were normal. EEG was with abnormal basal activity, without any specific findings. EMG showed peripheral axonal motor and sensory polyneuropathy. Genetically there was coincidental cytogenetic finding of karyotype 47,XXY. No variants in MFN2 and GDAP genes, neither in the gene panel associated with hereditary polyneuropathies, that could explain the cause of the disease, were found. Finally, the two above-mentioned variants were detected by WES. Conclusion: To conclude, two novel likely pathogenic variants of SLC25A46 were found in 2 siblings presenting with optic neuropathy associated with polyneuropathy and ataxia, with autosomal recessive inheritance pattern. So far, the association of variants in this gene has been described in only four families by Abrams (2015). ${ }^{(1)}$ Our case supports the role of SLC25A4 gene in etiology of hereditary optic 
atrophy associated with peripheral neuropathy. References: ABRAMS, Alexander J, Robert B HUFNAGEL, Adriana REBELO, et al. Mutations in SLC25A46, encoding a UGO1-like protein, cause an optic atrophy spectrum disorder. Nature Genetics [online]. 2015, 47(8), 926-932 [cit. 2018-03-15]. DOI: 10.1038/ng.3354. Supported by: AZV 1630206, MH CZ - DRO, University Hospital Motol, Prague,Czech Republic 00064203

\section{PS2Group3-021 / \#950}

Topic: Group 3 - Peripheral Neuropathy, Cranial Nerves, Mononeuropathies: Clinical Features, Pathophysiology, Therapy

\section{GENOME-WIDE DNA METHYLATION PROFILING OF HUMAN DIABETIC PERIPHERAL NEUROPATHY}

Eva Feldman

\section{University of Michigan, Ann Arbor, US}

Background: DNA methylation is an important epigenetic regulator in both health and disease. However, to date there are no studies investigating possible alterations in DNA methylation in the peripheral nerves of human patients with diabetic neuropathy. Methods: In the current study, we assessed DNA methylation profiles of 12 patients with diabetic peripheral neuropathy (DPN). Examination of sural nerve biopsies from these individuals revealed that 6 patient biopsies had significant nerve regeneration whereas 6 had significant nerve degeneration over a period of 52 weeks. These samples underwent reduced representation bisulfite sequencing (RRBS) for analysis of DNA methylation between patients who exhibited sural nerve regeneration and those who exhibited degeneration. Results: There were a total of 3,460 differentially methylated CpGs (DMCs) and 246 differentially methylated regions (DMRs) between the two patient cohorts. Analyses of genes associated with the DMCs identified pathways highly enriched in neuron development and differentiation, as well as pathways associated with cancer. Conclusion: These results suggest that DNA methylation has an important role in regulating nervous system development and cellular proliferation in the progression or regression of DPN. Additionally, these novel results provide insights into possible epigenetic regulation of DPN and offer useful information for future DPN research.
PS2Group3-022 / \#948

Topic: Group 3 - Peripheral Neuropathy, Cranial Nerves, Mononeuropathies: Clinical Features, Pathophysiology, Therapy

\section{TRANSCRIPTIONAL SIGNATURE OF DIABETIC PERIPHERAL NEUROPATHY CONSERVED ACROSS HUMAN AND MOUSE}

\author{
Eva Feldman \\ University of Michigan, Ann Arbor, US
}

Background: Aims/hypothesis: Diabetic peripheral neuropathy (DPN) is one of the most common complications of diabetes. Despite extensive research, the underlying mechanisms leading to DPN are not fully understood. While multiple murine models and patient samples have been examined, a unified systematic approach has yet to compare observed changes between groups which may help identify a possible conserved mechanism of DPN. In this study, we aimed to identify DPN-related transcriptional pathways conserved across human and various murine models of diabetes by using a systems biology approach. Methods: Eight microarray datasets were collected on peripheral nerve samples from murine models of type 1 (streptozotocin-treated) and type $2(\mathrm{db} / \mathrm{db}$ and $o b / o b)$ diabetes of different ages and human subjects with non-progressive and progressive DPN. Differentially expressed genes (DEGs) were identified between control and diabetic samples in murine models, and non-progressive and progressive human samples using a unified analysis pipeline. A transcriptional network for each DEG set was constructed based on literature-derived gene-gene interaction information and shared sub-networks between the human and murine networks were identified using a network-comparison program. Seven pairwise human-vs-murine comparisons resulted in sub-networks including 46 to 396 genes, which were further merged into a single network of 688 genes. Results: Functional analyses on the identified shared networks between murine and human datasets revealed that genes involved in LXR/RXR activation and adipogenesis were highly affected by diabetes and conserved across both species. Centrality analysis detected the most highly connected genes that may play important roles in this cross-species DPN shared network, including PIK3CA, MAPK8, CD44, MAPK1, CREB1, LEP, CCL2, JUN, ESR1, FOS, CD36, IL1B, HGF, and PLAT. These genes indicate enrich- 
ment of different pathways with the most significant including glucocorticoid receptor signaling, multiple cytokine pathways, and chemokine signaling. Conclusion: s/interpretations: Our systems biology approach identified highly conserved pathways across human and murine models such as LXR/RXR activation and multiple immune signaling pathways. These pathways likely play a role in DPN pathogenesis and provide new possible mechanism-based targets for DPN therapy.

\section{PS2Group3-023 / \#260}

Topic: Group 3 - Peripheral Neuropathy, Cranial Nerves, Mononeuropathies: Clinical Features, Pathophysiology, Therapy

\section{RESTABILISATION TREATMENT AFTER IVIG WITHDRAWAL IN CHRONIC INFLAMMATORY DEMYELINATING POLYNEUROPATHY: THE PATH STUDY RESULTS}

Orell Mielke1, Vera Bril'2, Nan Van Geloven ${ }^{3}$, Hans-Peter Hartung ${ }^{4}$, Richard A. Lewis ${ }^{5}$, Gen
Sobue $^{6}$, John-Philip Lawo ${ }^{1}$, Billie L. Durn ${ }^{7}$, David R. Cornblath ${ }^{8}$, Ingemar Merkies ${ }^{9}$, Amgad Shebl ${ }^{1}$, Ivo Van Schaik ${ }^{10}$

${ }^{1}$ CSL Behring, Marburg, DE; ${ }^{2}$ Department Of Medicine (neurology), University Health Network, University of Toronto, Toronto, CA, ${ }^{3}$ Department Of Medical Statistics And Bioinformatics, Leiden University Medical Center, Leiden, $N L,{ }^{4}$ Department Of Neurology, Heinrich-Heine University, Duesseldorf, DE; ${ }^{5}$ Department Of Neurology, Cedars-Sinai Medical Center, Los Angeles, US; ${ }^{6}$ Department Of Neurology, Nagoya University Graduate School of Medicine, Nagoya, JP, ${ }^{7} \mathrm{CSL}$ Behring, Fuquay Varina, NC, US, ${ }^{\circ}$ Department Of Neurology, Johns Hopkins University, Baltimore, MD, US: ${ }^{9}$ Department Of Neurology, Maastricht University Medical Center, Maastricht, NL; ${ }^{10}$ Department Of Neurology, Academic Medical Center, University of Amsterdam, Amsterdam, NL

Background: In patients with chronic inflammatory demyelinating polyneuropathy (CIDP), the dose or frequency of intravenous immunoglobulin (IVIG) administration is recommended to be periodically reduced to assess the need for ongoing therapy. Little is known about the effectiveness of IVIG restabilisa-

Figure 1. Efficacy of IgPro10 IVIG restabilisation therapy
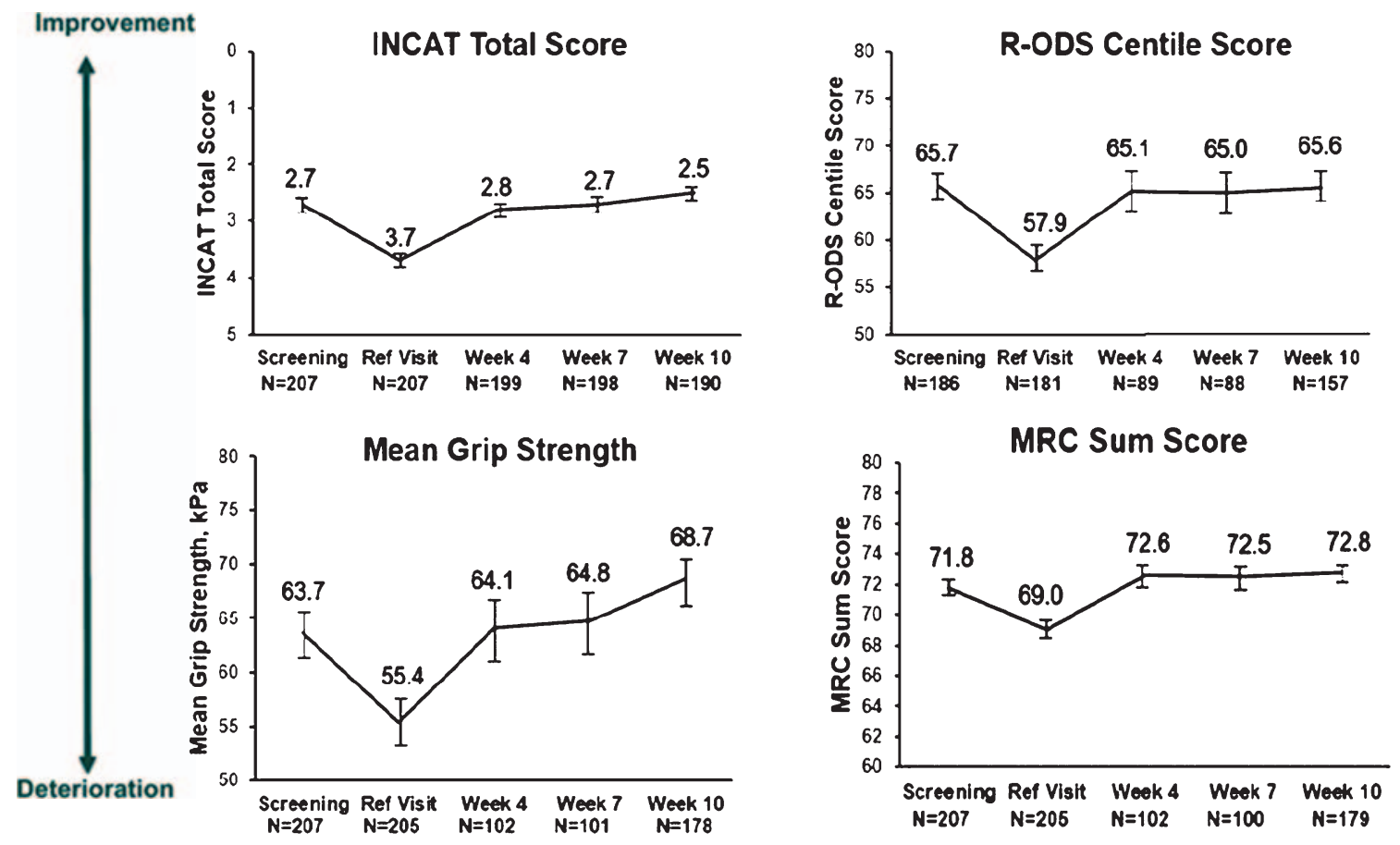

INCAT, Inflammatory Neuropathy Cause and Treatment; MRC, Medical Research Council; N, number of patients; Ref, reference; R-ODS. Rasch-built Overall Disability Scale. Error bars represent standard error of the mean. 
tion in patients who worsen after IVIG withdrawal. Methods: In PATH, a randomised, double-blind study of subcutaneous immunoglobulin in CIDP, IVIG therapy was withdrawn before randomisation. Subjects not deteriorating within 12 weeks of IVIG withdrawal discontinued the study as Ig dependency was not confirmed. Upon deterioration (increase in Adjusted Inflammatory Neuropathy Cause and Treatment [INCAT] score), subjects received IVIG restabilisation with IgPro10 (CSL Behring): induction dose $2 \mathrm{~g} / \mathrm{kg}$ bw, maintenance doses $1 \mathrm{~g} / \mathrm{kg}$ every 3 weeks for up to 10-13 weeks. Results: Of 245 subjects in whom IVIG was withdrawn, 28 (11.4\%) did not deteriorate within 12 weeks. Another 10 subjects withdrew for other reasons, leaving 207 in the restabilisation phase. Of these $91 \%$ improved in at least 1 efficacy measure (improvement: 1 point in adjusted INCAT score, 4 points in RODS score, $8 \mathrm{kPa}$ in mean grip strength or 3 points in MRC score) by $10-13$ weeks. Adjusted INCAT score improved in $72.9 \%$ with $21 \%$ of subjects improving beyond their status at study entry. Improvements were seen in all secondary scores (Figure 1). Post-study follow-up of non-improving subjects revealed most subjects had improved. Conclusion: IVIG withdrawal was effective in detecting subjects not requiring IVIG therapy. For IVIG-dependent subjects, restabilisation with IgPro10 was effective in reversing observed deteriorations within 12 weeks.

\section{PS2Group3-024 / \#625}

Topic: Group 3 - Peripheral Neuropathy, Cranial Nerves, Mononeuropathies: Clinical Features, Pathophysiology, Therapy

\section{EFFICACY AND SAFETY OF INTRAVENOUS IMMUNOGLOBULIN IGPRO10 IN CIDP: COMBINED ANALYSIS OF THE PRIMA AND PATH STUDIES}

$\underline{\text { Ingemar Merkies }}^{1}$, Jean-Marc Léger ${ }^{2}$, Vera Bril ${ }^{3}$, Nan Van Geloven ${ }^{4}$, Hans-Peter Hartung 5 , Richard A. Lewis ${ }^{6}$, Gen Sobue ${ }^{7}$, John-Philip Lawo ${ }^{8}$, Billie L. Durn ${ }^{9}$, David R. Cornblath ${ }^{10}$, Jan De Bleecker ${ }^{11}$, Claudia Sommer ${ }^{12}$, Wim Robberecht ${ }^{13}$, Mika Saarela $^{14}$, Jerzy Kamienowski ${ }^{15}$, Zbigniew Stelmasiak $^{16}$, Bjorn Tackenberg ${ }^{17}$, Orell Mielke ${ }^{8}$, Ivo Van Schaik ${ }^{18}$ ${ }^{1}$ Department Of Neurology, Maastricht University Medical Center, Maastricht, $N L,{ }^{2}$ National Referral
Center For Neuromuscular Diseases, University Hospital Pitié-Salpêtrière, Paris, FR; ${ }^{3}$ Department Of Medicine (neurology), University Health Network, University of Toronto, Toronto, CA $;{ }^{4}$ Department Of Medical Statistics And Bioinformatics, Leiden University Medical Center, Leiden, NL; ${ }^{5}$ Department Of Neurology, HeinrichHeine University, Duesseldorf, DE; ${ }^{6}$ Department $O f$ Neurology, Cedars-Sinai Medical Center, Los Angeles, US; ${ }^{7}$ Department Of Neurology, Nagoya University Graduate School of Medicine, Nagoya, $J P ;{ }^{8} \mathrm{CSL}$ Behring, Marburg, DE $;{ }^{9} \mathrm{CSL}$ Behring, Fuquay Varina, NC, US: ${ }^{10}$ Department Of Neurology, Johns Hopkins University, Baltimore, MD, US; ${ }^{11}$ Neurology, AZ St-Lucas, Ghent, BE, ${ }^{12}$ Neurologische Klinik Und Poliklinik, Universitätsklinikum Würzburg, Würzburg, DE: ${ }^{13}$ UZ Leuven, Leuven, BE, ${ }^{14}$ Department Of Neurology, Helsinki University Central Hospital, Helsinki, FI; ${ }^{15}$ Dolnoślaski Szpital Specjalistyczny, Wroclaw, PL; ${ }^{16}$ Samodzielny Publiczny Szpital Kliniczny, Lublin, PL; ${ }^{.17}$ Department Of Neurology, Philipps University, Marburg, DE: ${ }^{18}$ Department of Neurology, Academic Medical Center, University of Amsterdam, Amsterdam, NL

Background: Efficacy and safety of intravenous immunoglobulin IgPro10 (CSL Behring) were investigated in CIDP subjects in two studies: PRIMA and PATH. Methods: PRIMA was a prospective, open-label, single-arm study in 28 CIDP subjects investigating IgPro10 for induction $(2 \mathrm{~g} / \mathrm{kg})$ and maintenance therapy ( $1 \mathrm{~g} / \mathrm{kg}$ every 3 weeks for 21 weeks). This regimen was also used in 207 IVIG-pretreated subjects during the 10-13 week restabilisation period of PATH (before randomisation to subcutaneous immunoglobulin vs placebo). Both studies investigated response (defined as $\geq 1$-point decrease in adjusted Inflammatory Neuropathy Cause and Treatment [INCAT] score), changes in mean grip strength and Medical Research Council (MRC) score, and safety. We analysed separate and pooled results from both studies. Results: INCAT response rate at last observation was $76.9 \%$ (95\% confidence interval [CI]: 49.7-91.8) in PRIMA IVIG-pretreated subjects (60.7\% in all PRIMA subjects) and 72.9\% (95\% CI: 66.5-78.5) in PATH. In the pooled cohort $(\mathrm{n}=235)$, INCAT response rate was $71.1 \%(95 \% \mathrm{CI}$ : 65.0-76.5; Table 1). Most responders improved by week 4; additional responses occurred up to week 13 (Table 1). No clear differences in characteristics were noticed between early (responding before 
Table 1. Overall INCAT response rate and response rate by week from PRIMA and PATH as separate and pooled cohorts

\begin{tabular}{|c|c|c|c|c|c|}
\hline & $\begin{array}{l}\text { PRIMA IVIG- } \\
\text { pretreated } \\
\text { (n=13) }\end{array}$ & $\begin{array}{c}\text { PRIMA IVIG- } \\
\text { naïve } \\
\text { (n=15) }\end{array}$ & $\begin{array}{l}\text { PRIMA total } \\
(n=28)\end{array}$ & $\begin{array}{c}\text { PATH (all pre- } \\
\text { treated) } \\
\text { (n=207) }\end{array}$ & $\begin{array}{l}\text { PATH and } \\
\text { PRIMA } \\
\text { pooled } \\
(\mathrm{N}=235)\end{array}$ \\
\hline $\begin{array}{l}\text { Response rate by } \\
\text { adjusted INCAT, \% }\end{array}$ & 76.9 & 46.7 & 60.7 & 72.9 & 71.1 \\
\hline \multicolumn{6}{|c|}{ Number of responders by adjusted INCAT by week, $\%$} \\
\hline Week 1 & 0.0 & 0.0 & 0.0 & $n / a$ & 0.0 \\
\hline Week 4 & 53.8 & 13.3 & 32.1 & 49.8 & 47.7 \\
\hline Week 7 & 61.5 & 40.0 & 50.0 & 65.2 & 63.4 \\
\hline Week 10 & 76.9 & 40.0 & 57.1 & 72.0 & 70.2 \\
\hline Week 13 & 76.9 & 40.0 & 57.1 & 72.9 & 71.1 \\
\hline Week 16 & 76.9 & 40.0 & 57.1 & $n / a$ & $n / a$ \\
\hline Week 19 & 76.9 & 53.3 & 64.3 & $n / a$ & $n / a$ \\
\hline Week 22 & 76.9 & 53.3 & 64.3 & $n / a$ & $n / a$ \\
\hline Week 25 & 76.9 & 53.3 & 64.3 & $n / a$ & $n / a$ \\
\hline
\end{tabular}

Table 2. Median change from baseline to last observation carried forward in efficacy endpoints in PRIMA and PATH

\begin{tabular}{|l|c|c|c|c|}
\hline & $\begin{array}{c}\text { PRIMA IVIG- } \\
\text { pretreated } \\
(\mathbf{n}=13)\end{array}$ & $\begin{array}{c}\text { PRIMA IVIG-naïve } \\
(\mathbf{n}=15)\end{array}$ & $\begin{array}{c}\text { PRIMA total } \\
(\mathbf{n}=28)\end{array}$ & $\begin{array}{c}\text { PATH (all pre- } \\
\text { treated) } \\
(\mathbf{n}=207)\end{array}$ \\
\hline $\begin{array}{l}\text { Adjusted INCAT } \\
\text { score }\end{array}$ & -2.0 & -1.0 & -1.0 & -1.0 \\
\hline MRC score & 5.0 & 6.0 & 5.0 & 3.0 \\
\hline $\begin{array}{l}\text { Grip strength, } \mathbf{k P a} \\
\text { (dominant hand)* }\end{array}$ & & & & \\
& 5.0 & 5.0 & 5.0 & 9.4 \\
\hline
\end{tabular}

"Grip strengthrecorded as maximal value of 3 readings in PRIMA and mean value of 3 readings in PATH

Week 7) and late responders. Change from baseline to last observation in efficacy parameters is shown in Table 2. In the pooled cohort, 271 adverse drug reactions (ADRs) were reported in 105 subjects (44.7\%), a rate of 0.144 ADRs per infusion.

Conclusion: Pooled PRIMA/PATH data showed improvement in disability with IgPro10 in a large cohort of CIDP subjects. Improvement was seen at up to $10-13$ weeks, suggesting some subjects may need multiple Ig doses to respond.
PS2Group3-025 / \#538

Topic: Group 3 - Peripheral Neuropathy, Cranial Nerves, Mononeuropathies: Clinical Features, Pathophysiology, Therapy

\section{A FATAL CASE OF HEPATITIS E-ASSOCIATED GUILLAIN-BARRE SYNDROME}

Ho Ming June Wong ${ }^{1}$, Wai Sze Katie Kwan², Chun Yu Say ${ }^{2}$, Wing Yin Winnie Wong ${ }^{2}$

${ }^{1}$ Medicine And Geriatrics, Caritas Medical Centre, Hong Kong, $H K,{ }^{2}$ Medicine \& Geriatrics, Caritas Medical Centre, Hong Kong, HK 
Background: Extra-hepatic manifestations of hepatitis $\mathrm{E}$ infection have been increasingly recognised. Guillain-Barre syndrome (GBS) is one of the most commonly reported neurological complication. The mechanism of neurological involvement was believed to be due to molecular mimicry. Favorable neurological outcome was generally observed in hepatitis E-associated GBS while fatality was uncommonly encountered. Methods: We hearby report an 85-year-old Chinese lady who presented with classical form of GBS and acute hepatitis E infection. Results: An 85-year-old Chinese lady presented with unsteady gait and numbness of the four extremities for one day. She also reported nausea and reduced appetite for five days. Her past medical history was notable for hypertension, type 2 diabetes, hyperlipidemia and ischemic heart disease. On examination, she was fully conscious and oriented. She had jaundice and was apyretic. Her blood pressure was $172 / 63 \mathrm{mmHg}$ and the pulse was $84 / \mathrm{min}$. Cranial nerve examination was unremarkable. There were symmetrical distal weakness over the upper and lower limbs with Medical Research Council (MRC) muscle grading of 4/5. Generalised areflexia and glove-and-stocking sensory loss were noted. Cardiovascular, respiratory and abdominal examinations were unremarkable. Laboratory investigation revealed elevated liver enzymes (alkaline phosphatase (ALP) 292U/L, alanine transferase (ALT) $1085 \mathrm{U} / \mathrm{L}$, aspartate aminotransferase (AST) 333U/L) and elevated total bilirubin 74umol/L (direct bilirubin $70 \mathrm{umol} / \mathrm{L}$ ). Complete blood picture, renal function test, electrolytes, glucose and clotting profile were unremarkable. Anti-hepatitis A virus (HAV) IgM, hepatitis B surface antigen and antihepatitis $\mathrm{C}$ antibody were negative. Anti-hepatitis $\mathrm{E}$ virus (HEV) IgM was positive. Cerebrospinal fluid (CSF) analysis revealed albuminocytological dissociation with protein of $0.70 \mathrm{~g} / \mathrm{L}$ and white blood cell (WBC) of $6 / \mathrm{mm}^{3}$ while red blood cell (RBC) was $2000 / \mathrm{mm}^{3}$. Nerve conduction study was suggestive of sensorimotor polyneuropathy with demyelinating features. A diagnosis of hepatitis E-associated GBS was made. Intravenous immunoglobulin (IVIg) was started since the second day of admission. The patient's condition deteriorated, however, with progressive ascending weakness, bulbar dysfunction, dysautonomia and type II respiratory failure requiring invasive mechanical ventilation on day 5 . Two days later, she developed ventricular fibrillation requiring repeated defibrillation and cardiopulmonary resuscitation. Despite maximal support, she finally succumbed after 7 days of hospitalization. Conclusion: Severe GBS with autonomic dysfunction may occur in the setting of acute hepatitis E infection and can be fatal. Any neurological symptoms in patients with concurrent hepatitis E infection should be carefully looked into to ensure timely diagnosis and effective treatment. Whereas in patients who are diagnosed with GBS, deranged liver function may indicate an associated hepatitis E infection.

\section{PS2Group3-026 / \#899}

Topic: Group 3 - Peripheral Neuropathy, Cranial Nerves, Mononeuropathies: Clinical Features, Pathophysiology, Therapy

\section{PUTATIVE DIGENIC INHERITANCE OF CHARCOT-MARIE-TOOTH DISEASE INVOLVING TWO NOVEL HETEROZYGOUS MUTATIONS IN MARS AND HARS}

\author{
Martin Krenn $^{1}$, Anna Grisold ${ }^{1}$, Gudrun Zulehner ${ }^{1}$, \\ Jakob Rath ${ }^{1}$, Hakan Cetin ${ }^{1}$, Matthias Tomschik ${ }^{1}$, \\ Bojan Zagrovic ${ }^{2}$, Lukas Bartonek ${ }^{2}$, Tomislav \\ Kokotovic ${ }^{3}$, Vanja Nagy ${ }^{3}$, Matias Wagner ${ }^{4}$, Tim M. \\ Strom $^{4}$, Fritz Zimprich ${ }^{1}$ \\ ${ }^{1}$ Department Of Neurology, Medical University of \\ Vienna, Vienna, $A T ;{ }^{2}$ Department Of Structural And \\ Computational Biology, Max F. Perutz \\ Laboratories, University of Vienna, Vienna, \\ AT; ${ }^{3}$ Ludwig Boltzmann Institute for Rare and \\ Undiagnosed Diseases, Vienna, AT; Institute Of \\ Human Genetics, Technical University Munich, \\ Munich, DE
}

Background: Charcot-Marie-Tooth disease is a clinically and genetically highly heterogeneous disorder. All subtypes together represent the most prevalent form of inherited polyneuropathies. The genetic spectrum of Charcot-Marie-Tooth disease currently involves more than 70 causative genes. Amongst many others, mutations in genes encoding various aminoacyl-tRNA synthetases have been reported to be involved in the pathogenesis of the disease. In recent years, the two genes HARS (histidyl-tRNA synthetase) and MARS (methionyltRNA synthetase) have been shown to cause inherited peripheral neuropathies in few cases with variable clinical phenotypes. Methods: We provide a detailed clinical characterization and the findings of diagnostic whole-exome sequencing in a patient 
with Charcot-Marie-Tooth disease. Results: This female patient first noticed a weakness for toe dorsiflexion and then developed a bilateral weakness for plantar and dorsiflexion of the foot at the age of 24 years. The symptoms were reported to progress slowly. On neurological examination at the age of 30 , she revealed a mild weakness of hand muscles (MRC grade 4 for wrist extension), whereas distal strength in both lower limbs was severely impaired (MRC grade 2 for foot and MRC grade 1-2 for great toe dorsiflexion, MRC grade 3-4 for plantar flexion). Deep tendon reflexes were absent throughout. The lower limbs were distally affected by symmetric paraesthesia and reduced sensation for light touch and vibration. There was also a significant atrophy of the calf muscles. Nerve conduction studies indicated a severe sensory and motor polyneuropathy. CSF analysis showed mildly increased protein levels (44 $\mathrm{mg} / \mathrm{dl}$ ), while the cell count was normal. Serum electrophoresis and testing for ganglioside antibodies (anti-GM1, anti-GQ1b) as well as serological testing for autoimmune antibodies (e.g. ANA, ENA, ANCA) was all negative. The family history was unremarkable for any neurological disorders. After having excluded acquired causes, whole-exome sequencing was conducted as the next diagnostic step and showed two novel mutations in the two aminoacyl-tRNA synthetase genes HARS and MARS, each present in a heterozygous carrier state. The missense mutation in HARS (NM_002109.4): c.1488G>T, p. (Glu496Asp) was predicted to lead to an exchange of an evolutionary conserved amino acid. The detected MARS variant (NM_004990.3): c.181_183del, p.(Ser61 del) causes a deletion of a highly conserved amino acid. Both mutations were absent from the inhouse database including more than 9,000 exomes as well as from more than 120,000 alleles of the Exome Aggregation Consortium (ExAC)-Browser (http://exac.broadinstitute.org) and therefore considered to be pathogenic. Conclusion: To our knowledge, this is the first case suggesting a digenic inheritance pattern in Charcot-Marie-Tooth disease involving two new mutations in the functionally related genes MARS and HARS. However, the extent of pathogenicity of each individual mutation remains unclear and highlights the need for functional analyses beyond the clinical application of wholeexome sequencing.
PS2Group3-027 / \#960

Topic: Group 3 - Peripheral Neuropathy, Cranial Nerves, Mononeuropathies: Clinical Features, Pathophysiology, Therapy

\section{A RANDOMIZED, BLINDED, LIFESTYLE INTERVENTION STUDY IMPROVES THE EXPIRATION:INSPIRATION RATIO IN DIABETIC NEUROPATHY}

\author{
James Russell ${ }^{1}$, Lindsay Zilliox ${ }^{2}$, Pranith Kumar ${ }^{1}$ \\ ${ }^{1}$ University of Maryland School of Medicine, \\ Baltimore, US: ${ }^{2}$ University of Maryland School of \\ Medicine, Baltimore, MD, US
}

Background: Diabetic autonomic neuropathy is a common complication of type 2 diabetes mellitus (T2DM) that may affect up to $73 \%$ of patients. Currently, there is a need for therapies that will reduce progression or reverse diabetic autonomic neuropathy. The expiration:inspiration (EI) ratio is one of the most sensitive and reproducible measures of cardiac autonomic neuropathy (CAN). Methods: A blinded, randomized, intention to treat, parallel group intervention study was performed to examine the effect of a yearlong lifestyle intervention program in subjects with impaired glucose tolerance or T2DM and neuropathy. The lifestyle intervention program consisted of a dietary weight loss intervention that was tailored to the participants' caloric expenditure and a graded, moderate intensity aerobic physical activity intervention that was tailored to the participants' baseline physical activity (TDPA). The TDPA intervention group was compared to a standard care (SC) group receiving general dietary and exercise advice. Results: Data was obtained from 30 subjects in the TDPA and 31 on the SC group. Most participants had impaired glucose tolerance (89\% -duration $6.91 \pm 1.31$ months) and $11 \%$ had $\mathrm{T} 2 \mathrm{DM}$ (duration $20.70 \pm 8.26$ months). Both groups were matched at baseline for age, gender, BMI, and glycemic control. Gender distribution was $69 \%$ male and $31 \%$ female). There was an interval improvement in the EI ratio in $80 \%$ of the subjects in the TDPA, compared to SC group, over the duration of the intervention $(\mathrm{P}<0.05)$. The mean relative improvement in the EI ratio was greatest between 0 and 6 months in the TDPA group (0.15) compared to a decline in the SC group (-0.04). Conclusion: In this randomized, blinded, intention to treat study of a lifestyle intervention in diabetic neuropathy, there 
is improvement in CAN in the intervention group. This is a very important finding because of the strong association between impaired CAN and sudden death.

\section{PS2Group3-028 / \#668}

Topic: Group 3 - Peripheral Neuropathy, Cranial Nerves, Mononeuropathies: Clinical Features, Pathophysiology, Therapy

\section{PREDICTORS OF EARLY \\ RETIREMENT IN PATIENTS WITH CHRONIC INFLAMMATORY DEMYELINATING POLYRADICULONEUROPATHY}

Bogdan Bjelica ${ }^{1}$, Ivo Bozovic ${ }^{1}$, Ivana Basta ${ }^{1}$, Aleksandra Kacar ${ }^{1}$, Ana Nikolic ${ }^{1}$, Aleksandra Dominovic-Kovacevic ${ }^{2}$, Zoran Vukojevic ${ }^{2}$, Vesna Martic $^{3}$, Aleksandar Stojanov ${ }^{4}$, Gordana Djordjevic $^{4}$, Milutin Petrovic ${ }^{5}$, Miroslav Stojanovic $^{5}, \underline{\text { Stojan Peric }}^{1}$

${ }^{1}$ Neurology Clinic, Clinical Center of Serbia, School of Medicine, University of Belgrade, Belgrade, Serbia, Belgrade, RS: ${ }^{2}$ Neurology Clinic, Clinical Center Banja Luka, Banja Luka, Republic of Srpska, Bosnia and Herzegovina, Banja Luka, BA, ${ }^{3}$ Neurology Clinic, Military Medical Academy, Belgrade, Serbia, Belgrade, RS; ${ }^{4}$ Neurology Clinic, Clinical Center Nis, Nis, Serbia, Nis, RS, ${ }^{5}$ Neurology Clinic, Clinical Center Kragujevac, Kragujevac, Serbia, Kragujevac, $R S$

Background: It has been previously shown that being unemployed or retired is one of the independent predictors of worse quality of life (QoL) in chronic inflammatory demyelinating polyradiculoneuropathy (CIDP). The aim of this study was to assess predictors of early retirement in CIDP subjects. Methods: We included 107 patients diagnosed with CIDP. Following measures were used: questionnaire on employment status, Medical Research Council (MRC) Sum Score, Inflammatory Neuropathy Cause and Treatment (INCAT) disability and sensory scores, Beck Depression Inventory (BDI), and Krupp's Fatigue Severity Scale (FSS). Results: Fifteen percent of CIDP patients were employed, nine percent were unemployed due to CIDP, while $28 \%$ were retired early. Mean age when patients got retired due to CIDP was $50.0 \pm 8.2$ years. Mean time from CIDP onset to retirement was $2.7 \pm 2.3$ years.
Older age at onset, lower education, and more severe motor/sensory deficit appeared as significant independent predictors of being retired due to CIDP. Retired patients compared to employed patients were in 12 times higher risk to suffer from depression $(\mathrm{OR}=12.2,95 \% \mathrm{CI}=1.41-100)$, and in eight-time higher risk of having fatigue $(\mathrm{OR}=8.2,95 \% \mathrm{CI}=1.89$ 35.82). Conclusion: Older patients with lower education, more severe weakness and sensory impairment were most likely to be retired due to CIDP. Early-retirement was highly associated with depression and fatigue. Therefore, maintaining employment should be important aim in treatment of CIDP patients.

\section{PS2Group3-029 / \#699}

Topic: Group 3 - Peripheral Neuropathy, Cranial Nerves, Mononeuropathies: Clinical Features, Pathophysiology, Therapy

\section{NEUROPATHY AS AN ADVERSE EFFECT OF VASCULAR ENDOTHELIAL GROWTH FACTOR TYROSINE KINASE INHIBITORS, A META-ANALYSIS}

\author{
Bhaskar Roy ${ }^{1}$, Avash Das ${ }^{2}$, Abhishek Maiti ${ }^{3}$, \\ Dhrubajyoti Bandyopadhyay ${ }^{4}$, Kumar Ashish ${ }^{3}$ \\ ${ }^{\prime}$ Neurology, Yale University, New Haven, CT, \\ US, ${ }^{2}$ Cardiovascular Medicine, Massachusetts \\ General Hospital, Boston, MA, US $;^{3}$ MD Anderson \\ Cancer Center, Houston, TX, US; ${ }^{4}$ St Luke \\ Roosevelt, New York, NY, US
}

Background: Targeted therapies like vascular endothelial growth factor tyrosine kinase inhibitors (VEGF-TKI) is the first-choice treatment in several types of cancers. Neuropathy as an adverse effect of VEGF-TKI is not yet established. In this pairwise and network meta-analysis, we explored neuropathy with VEGF-TKI use. Methods: Published data search up to March 2017 identified 26 randomized clinical trials (RCTs) reporting neuropathy in cancer patients treated with VEGF-TKI. The primary outcome was presence of neuropathy at the end of the trial. VEGF-TKI was compared either with combination chemotherapy or with placebo. Meta-analysis was performed using frequentist's approach to estimate Relative Risk (RR) of individual treatment. Network meta-analysis based on random effects model estimating RR was performed. Results: 
Twenty-six RCTs including 11,971 cancer patients were included in this analysis. Six studies compared VEGF-TKI with placebo and others in combination. VEGF-TKI was associated with higher risk of neuropathic events (RR 1.12; 95\% CI, 1.01-1.24, $\mathrm{p}<0.01$ ). High grade (III and above) neuropathy (RR 1.23 ; 95\% CI, 1.01-1.48) and sensory neuropathy (RR 1.23; 95\% CI, 1.05-1.31) were noted more frequently with VEGF-TKI but did not reach statistical significance. When compared against placebo, VEGF-TKI was strongly associated with neuropathy (RR 1.88; 95\% CI, 1.16-3.06, $\mathrm{p}=0.01$ ). Network meta-analysis did not show increased risk of neuropathy with any particular VEGF-TKI therapy compared to others. There was moderate heterogeneity among the included studies $\left(\mathrm{I}^{2}=44 \%\right)$.

Conclusion: VEGF-TKI is possibly associated with increased risk of neuropathy.

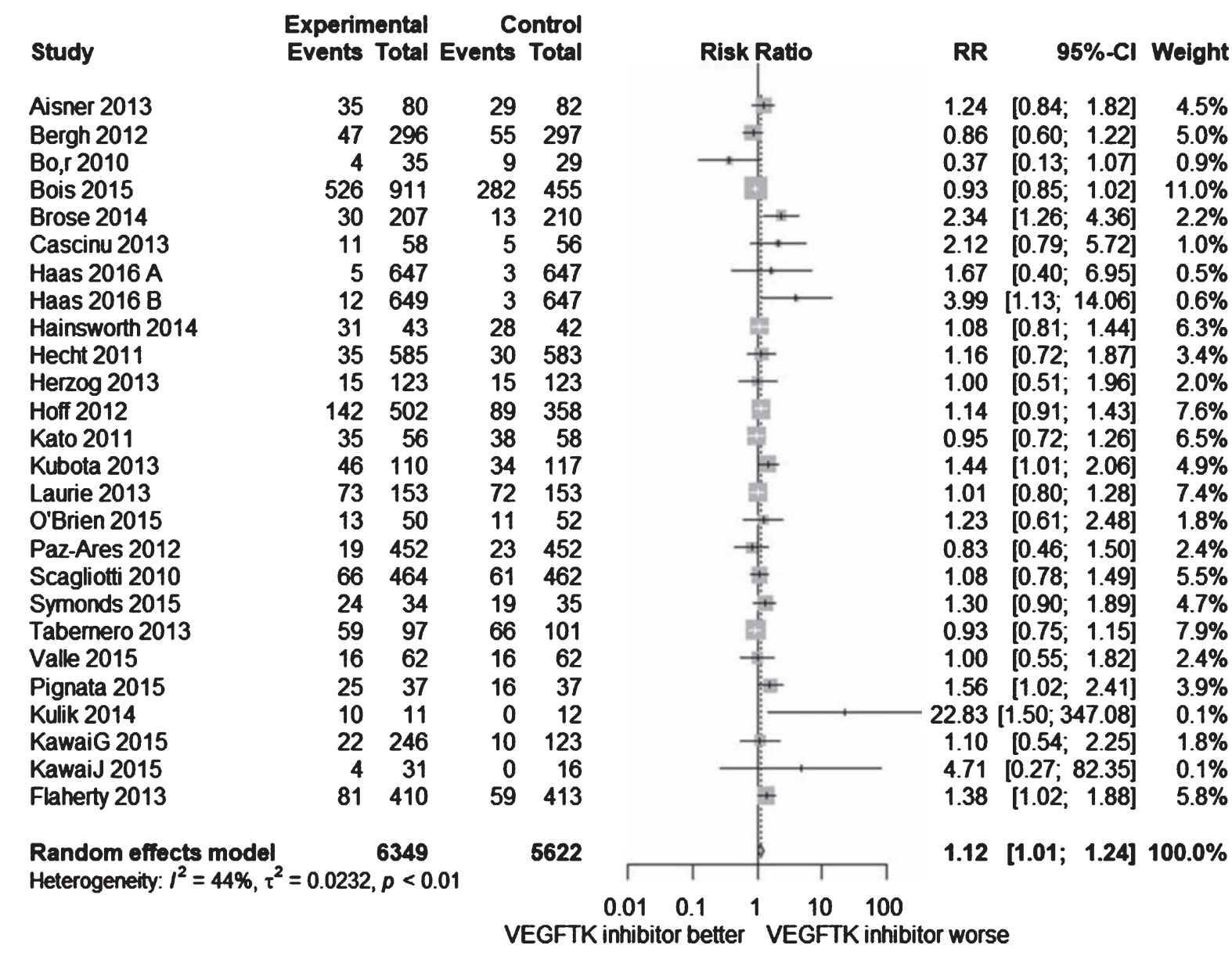

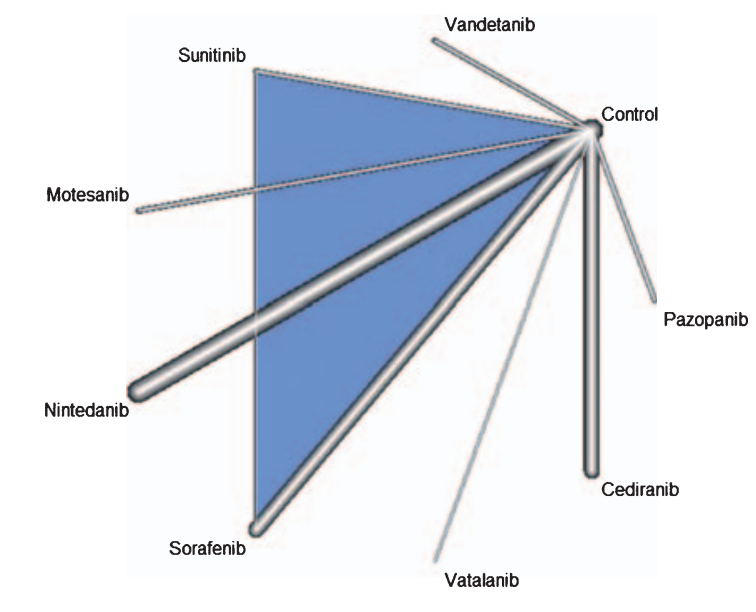




\section{PS2Group3-030 / \#727}

Topic: Group 3 - Peripheral Neuropathy, Cranial Nerves, Mononeuropathies: Clinical Features, Pathophysiology, Therapy

\section{AXONAL FUNCTION PREDICTS RESPONSE TO SUBCUTANEOUS IMMUNOGLOBULIN IN CIDP: THE PATH STUDY}

Vera Bril ${ }^{1}$, Hans-Peter Hartung ${ }^{2}$, Gen Sobue ${ }^{3}$, John-Philip Lawo ${ }^{4}$, Orell Mielke ${ }^{4}$, Billie L. Durn ${ }^{5}$, Ingemar Merkies ${ }^{6}$

${ }^{1}$ Department Of Medicine (neurology), University Health Network, University of Toronto, Toronto, CA; ${ }^{2}$ Department Of Neurology, Heinrich-Heine University, Duesseldorf, DE; ${ }^{3}$ Department $O f$ Neurology, Nagoya University Graduate School of Medicine, Nagoya, JP; ${ }^{4} \mathrm{CSL}$ Behring, Marburg, $D E,{ }^{5} C S L$ Behring, Fuquay Varina, $N C$, US, ${ }^{6}$ Department Of Neurology, Maastricht University Medical Center, Maastricht, $N L$

Background: Chronic Inflammatory Demyelinating Polyneuropathy (CIDP) is an immune mediated disease starting with functional impairment and demyelination; in later stages, axonal degeneration may occur. Methods: PATH was a randomised, double-blind study investigating 0.2 (low) and $0.4 \mathrm{~g} /$ $\mathrm{kg}$ (high) weekly doses of maintenance SCIG IgPro20 (Hizentra, CSL Behring) versus placebo $(\mathrm{N}=172)$. After Ig dependency testing, and IVIG restabilisation, patients were randomised to SCIG or placebo for 25 weeks or until early termination. Nerve conduction studies (NCS) were performed before study drug administration and at end of study visit. Exploratory comparisons of relapse rates (defined as a $>=1$ point increase by adjusted Inflammatory Neuropathy Cause and Treatment score) were undertaken on patients with assumed non-axonal damage (amplitude $>1$ ) versus assumed axonal damage (amplitude $<=1$ ) at the peroneal ankle stimulation site. Results: Patients with assumed non-axonal damage who received placebo had a $73 \%$ relapse rate versus $39 \%$ on low-dose and $19 \%$ on high-dose SCIG. Patients with assumed axonal damage had relapse rates of $25 \%, 30 \%$ and $19 \%$ for placebo, low-dose and high-dose SCIG, respectively (Table 1). Conclusion: CIDP patients with assumed non-axonal damage had a high relapse rate when switched from IVIG to placebo that was significantly lower in patients switched to SCIG therapy. Relapse rates were lower in assumed axonal damage patients and were not influenced by SCIG. These findings could help in redesigning future trials including maintenance regimens based on NCS categorisation of patients.

Table 1. Frequency of relapsers by assumed axonal damage status in PATH

\begin{tabular}{|c|c|c|c|c|c|c|}
\hline & \multicolumn{3}{|c|}{$\begin{array}{c}\text { Relapse \% } \\
\text { (95\% Wilson CI) } \\
\text { Number of patients }\end{array}$} & \multicolumn{3}{|c|}{$\begin{array}{c}\text { Difference \% } \\
\text { (unadj. } 95 \% \text { Wilson CI) }\end{array}$} \\
\hline & $\begin{array}{l}\text { Placebo } \\
(\mathrm{N}=57)\end{array}$ & $\begin{array}{c}\text { Low-dose SCIG } \\
(N=53)\end{array}$ & $\begin{array}{l}\text { High-dose SCIG } \\
(\mathrm{N}=57)\end{array}$ & $\begin{array}{l}\text { Low-dose } \\
\text { vs } \\
\text { placebo }\end{array}$ & $\begin{array}{l}\text { High-dose } \\
\text { vs } \\
\text { placebo }\end{array}$ & $\begin{array}{l}\text { High-dose } \\
\text { vs } \\
\text { low-dose }\end{array}$ \\
\hline $\begin{array}{c}\text { Assumed } \\
\text { non-axonal } \\
\text { damage } \\
\mathrm{N}=86\end{array}$ & $\begin{array}{c}73.0 \\
(57.0 \text { to } 84.6) \\
27 \text { of } 37\end{array}$ & $\begin{array}{c}39.1 \\
(22.2 \text { to } 59.2) \\
9 \text { of } 23\end{array}$ & $\begin{array}{c}19.2 \\
\text { (8.5 to } 37.9) \\
5 \text { of } 26\end{array}$ & $\begin{array}{c}-33.8 \\
(-54.4 \text { to }-8.2) \\
* * *\end{array}$ & $\begin{array}{c}-53.7 \\
(-69.9 \text { to }-29.2) \\
* * *\end{array}$ & $\begin{array}{c}-19.9 \\
(-42.7 \text { to } 5.3) \\
\text { NS }\end{array}$ \\
\hline $\begin{array}{l}\text { Assumed } \\
\text { axonal } \\
\text { damage } \\
\mathrm{N}=81\end{array}$ & $\begin{array}{c}25.0 \\
(11.2 \text { to } 46.9) \\
5 \text { of } 20\end{array}$ & $\begin{array}{c}30.0 \\
(16.7 \text { to } 36.3) \\
9 \text { of } 30\end{array}$ & $\begin{array}{c}19.4 \\
(9.2 \text { to } 36.3) \\
6 \text { of } 31\end{array}$ & $\begin{array}{c}+5 \\
(-20.6 \text { to } 27.6) \\
\text { NS }\end{array}$ & $\begin{array}{c}-5.6 \\
(-29.8 \text { to } 16.2) \\
\text { NS }\end{array}$ & $\begin{array}{c}-10.7 \\
(-31.2 \text { to } 10.9) \\
\text { NS }\end{array}$ \\
\hline
\end{tabular}

Cl, confidence interval; NS, not significant; ***, significant, assessed as exclusion of 0 from unadjusted $95 \% \mathrm{CI}$ 
PS2Group3-031 / \#722

Topic: Group 3 - Peripheral Neuropathy, Cranial Nerves, Mononeuropathies: Clinical Features, Pathophysiology, Therapy

\section{ELECTROPHYSIOLOGICAL} TESTING IN PATIENTS WITH CIDP TREATED WITH SUBCUTANEOUS IMMUNOGLOBULIN: THE PATH STUDY

$\underline{\text { Vera Bril }}^{1}$, Ivo Van Schaik², Nan Van Geloven ${ }^{3}$, Hans-Peter Hartung ${ }^{4}$, Richard A. Lewis ${ }^{5}$, Gen Sobue $^{6}$, John-Philip Lawo ${ }^{7}$, Orell Mielke ${ }^{7}$, Billie L. Durn $^{8}$, Ingemar Merkies ${ }^{9}$ ${ }^{1}$ Department Of Medicine (neurology), University Health Network, University of Toronto, Toronto, CA; ${ }^{2}$ Department Of Neurology, Academic Medical Center, University of Amsterdam, Amsterdam, $N L,{ }^{3}$ Department Of Medical Statistics And Bioinformatics, Leiden University Medical Center, Leiden, NL; ${ }^{4}$ Department Of Neurology, HeinrichHeine University, Duesseldorf, DE; ${ }^{5}$ Department Of Neurology, Cedars-Sinai Medical Center, Los Angeles, US; ${ }^{6}$ Department Of Neurology, Nagoya University Graduate School of Medicine, Nagoya, $J P,{ }^{7} C S L$ Behring, Marburg, DE, ${ }^{8}$ CSL Behring, Fuquay Varina, NC, US; ${ }^{9}$ Department Of Neurology, Maastricht University Medical Center, Maastricht, $N L$

Background: Nerve conduction studies (NCS) form an essential part of the diagnostic criteria for chronic inflammatory demyelinating polyneuropathy (CIDP) and are objective measures of peripheral nerve function independent of confounders that may interfere with clinical scores. The objective of this analysis was to determine whether NCS remain stable in patients with CIDP during study drug administration with either low or high dose subcutaneous immunoglobulin (SCIG) or placebo. Methods: This was a randomised, double-blind trial investigating 0.2 (low) and $0.4 \mathrm{~g} / \mathrm{kg}$ (high) weekly doses of maintenance SCIG IgPro20 (CSL Behring) versus placebo in 172 subjects. After testing for dependency on Ig therapy, patients were randomised to SCIG or placebo, and NCS were performed at the start and end of the study drug administration period at 25 weeks or early termination. Electrophysiological data from each patient was averaged to give average proximal latency, conduction velocity, conduction block (30, 40 and 50\%), compound muscle action potential amplitude, and we compared the mean changes in these averaged parameters from baseline to completion visit. Results: The majority of NCS parameters did not change; however, averaged proximal latency increased in the placebo group $(+1.1 \mathrm{~ms})$ but remained stable in both IgPro20 groups (low dose: $+0.1 \mathrm{~ms}$; high dose: $-0.1 \mathrm{~ms}$ ). The averaged motor nerve conduction velocity (MNCV) decreased in the placebo group $(-1.6 \mathrm{~m} / \mathrm{s})$ but increased in both IgPro20 groups (low dose: $+0.2 \mathrm{~m} / \mathrm{s}$; high dose: $+1.0 \mathrm{~m} / \mathrm{s}$ ). Conclusion: Most NCS parameters did not change during the SCIG treatment phase of the PATH study aside from those reflecting speed of conduction (averaged proximal latency and MNCV) which went in a positive direction in SCIG treated patients compared with placebo patients.

\section{PS2Group3-032 / \#921}

Topic: Group 3 - Peripheral Neuropathy, Cranial Nerves, Mononeuropathies: Clinical Features, Pathophysiology, Therapy

\section{CHRONIC RELAPSING INFLAMMATORY OPTIC NEUROPATHY (CRION): A MANIFESTATION OF MYELIN OLIGODENDROCYTE GLYCOPROTEIN ANTIBODIES}

Haeng-Jin Lee ${ }^{1}$, Boram Kim², Patrick Waters ${ }^{3}$, Sarosh Irani ${ }^{3}$, Mark Woodhall ${ }^{3}$, So Hyun $\mathrm{Ahn}^{2}$, Jung-Joon Sung ${ }^{2}$, Seong-Joon Kim ${ }^{1}$, Sung Min $\underline{\mathrm{Kim}}^{2}$

${ }^{1}$ Ophthalmology, Seoul National University Hospital, Seoul, KR $;{ }^{2}$ Neurology, Seoul National University Hospital, Seoul, KR; ${ }^{3}$ Nuffield Department Of Clinical Neurosciences, John Radcliffe Hospital, Oxford, GB

Background: The key clinical features of chronic relapsing inflammatory optic neuropathy (CRION) include relapsing inflammatory optic neuritis $(\mathrm{ON})$ and steroid-dependency, both of which also have been reported among patients with myelin oligodendrocyte glycoprotein antibodies (MOG-Abs). We aimed to investigate the relevance of the presence of serum MOG-Ab with the current diagnostic criteria for CRION among patients with idiopathic inflammatory optic neuritis (iON). Methods: Retrospective reviews of the prospectively collated database between 2011 and 2017 were performed in a tertiary referral multiple sclerosis / neuromyelitis optica cen- 
ter. Sixty-four patients with iON, who did not meet the diagnostic critera for multiple sclerosis, neuromyelitis optica (NMO) spectrum disorder with/without NMO-IgG, or acute disseminated encephalomyelitis, and who had no symptomatic central nervous system (CNS) lesion other than the optic nerve were included from the cohort of 615 patients with inflammatory demyelinating diseases of the CNS. Fulfillment for the current diagnostic criteria of CRION, assay result for the serum IgG1 MOG$\mathrm{Ab}$, and the characteristics of CRION patients compared to those of non-CRION with MOG-Ab. Results: Twelve patients with iON fulfilled the current diagnostic criteria for CRION all of whom tested positive for MOG-Ab. Among the other 52 patients, 14 had relapsing disease courses and 38 had monophasic course, of which group the MOG-Ab positivity were $0 \%$ and $29 \%$, respectively. Though the CRION patients with MOG-Ab had more relapsing disease courses and poorer OCT outcomes at follow up than non-CRION patients with MOG-Ab, they did not differ in terms of age at onset, sex, duration of the steroid treatment after initial attack. The CRION patients with MOG-Ab experienced their first steroiddependent worsening/relapse in 2.2 (ranged from $0.43-6.97)$ months from their disease onset. Conclusion: The CRION, according to the current diagnostic criteria, appears to be mostly a manifestation of the relapsing optic neuritis associated with MOG$\mathrm{Ab}$. Among iON patients with MOG-Ab, absence of the steroid-dependent attack in an early stage of the disease can be associated with a long term non-relapsing disease courses and better outcomes.

\section{PS2Group3-033 / \#304}

Topic: Group 3 - Peripheral Neuropathy, Cranial Nerves, Mononeuropathies: Clinical Features, Pathophysiology, Therapy

\section{PREVALENCE AND RISK FACTORS OF CARPAL TUNNEL SYNDROME IN OYSTER-SHUCKERS}

$\underline{\text { Kee Hong Park }}{ }^{1}$, Rock Bum Kim², Eun Bin $\mathrm{Cho}^{3}$, Ki-Jong Park ${ }^{3}$

${ }^{1}$ Neurology, Gyeongsang National University

Hospital, Jinju, KR; ${ }^{2}$ Regional

Cardiocerebrovascular Center, Gyeongsang

National University Hospital, Jinju, KR, ${ }^{3}$ Neurology, Gyeongsang National University Changwon

Hospital, Changwon, KR
Background: To investigate the prevalence of carpal tunnel syndrome (CTS) in oyster-shuckers and to reveal the risk factors of CTS. Methods: One hundred and forty-seven oyster-shuckers were recruited. All the subjects had worked for more than 10 years. CTS was diagnosed based on nerve conduction study. Subjects with abnormal ulnar nerve study were excluded to rule out polyneuropathy $(n=17)$. Degree of CTS was graded as normal, mild (decreased sensory nerve conduction velocity with normal distal motor latency), moderate (decreased sensory nerve conduction velocity with prolonged distal motor latency), and severe (absent sensory nerve action potential with prolonged distal motor latency). Risk factors analyzed included age, sex, BMI, medical history, work time, exercise time, and laboratory data. Results: CTS was seen in $66.9 \%$ (n $=87$ ) of the whole population $(\mathrm{n}=130)$. In a multivariate logistic regression analysis, risk of CTS was increased with longer work time (odds ratio, 1.43; $95 \%$ confidence interval, 1.18-1.73) and higher BMI (odds ratio, 1.27; 95\% confidence interval, 1.05-1.53). Degree of CTS showed statistically significance only with work time $(p=0.005)$. Conclusion: CTS is prevalent in oyster-shuckers. In order to reduce the risk of CTS, it may be necessary to adjust the working time and reduce the BMI.

\section{PS2Group3-034 / \#469}

Topic: Group 3 - Peripheral Neuropathy, Cranial Nerves, Mononeuropathies: Clinical Features, Pathophysiology, Therapy

\section{NEXT GENERATION SEQUENCING TECHNOLOGIES IN THE GENETIC DIAGNOSIS OF EARLY ONSET HEREDITARY SPASTIC PARAPLEGIAS}

Laura Carrera-García ${ }^{1}$, Daniel Natera De Benito ${ }^{1}$, Debora Itzep $^{1}$, Anna Lia Frongia ${ }^{1}$, Andrea Sariego ${ }^{1}$, Delia Yubero $^{2}$, Elena Maqueda ${ }^{1}$, Loreto Martorell ${ }^{3}$, Carlos Ortez ${ }^{1}$, Jaume Colomer ${ }^{3}$, Giovanni Stevanin $^{4}$, Andres Nascimento ${ }^{1}$ ${ }^{1}$ Neuromuscular Disorders Unit, Hospital Sant Joan de Déu, Barcelona, ES, ${ }^{2}$ Department Of Genetics, Hospital Sant Joan de Déu, Barcelona, ES: ${ }^{3}$ Hospital Sant Joan de Déu, Barcelona, ES; ${ }^{4}$ Institut National de la Santé et de la Recherche Medicale (INSERM), Paris, FR 
Background: Hereditary spastic paraplegias (HSP) are a genetically heterogeneous group of diseases that are characterized by progressive symmetric spasticity of lower extremities. Mutations in SPAST represent the most frequent molecular etiology. Today, more than 60 genes related to hereditary spastic paraplegias are recognized. The aim of our work is to analyse the utility of Next-generation-sequencing (NGS) diagnostics in patients with HSP and describe the clinical and molecular genetic findings associated with specific genes. Furthermore, we also describe phenotypes and distinguish clinical signs associated with the various HSP-related genes, which might help to guide diagnosis. Methods: A cohort of children followed-up in our hospital with clinical diagnosis of HSP was recruited. Metabolic or structural origin was discarded in all of them. A NGS-based gene panel of 74 genes involved in HSP was performed. Demographic and phenotype data were collected from medical records. The study complies with the ethical guidelines of the institutions involved. Results: Sixty-seven unrelated index cases were analysed by the NGS-HSP panel. In some of them, several genetic tests, including SPAST, ALT1, MFN2, MPZ, GDAP1 sequencing, had previously been performed with negative results. The NGS-HSP panel diagnostic yield was $31.3 \%$, since 21 out of 67 patients reached a molecular genetic diagnosis. Among index cases with a confirmed genetic diagnosis, 7 presented with pure and 14 with complicated HSP. The mean age at first symptoms was 26 months; a male/female ratio was 15/6. Mutations were found in 12 different genes. The genes most frequently involved were KIFIA (5/21 patients), SPAST (4/21), and ALT1 (3/21). Additionally, 9 cases were detected in family members. Conclusion: The use of next generation sequencing technologies has increased the number of genes known and has expanded both the phenotype and genotype of HSP. Molecular diagnosis was obtained in a third of patients using a NGS-HSP panel. Knowledge of the genes involved in HSP is now opening possibilities to build the functional pathways related with these disorders and could hopefully lead to a potential treatment for HSP patients.
PS2Group3-035 / \#710

Topic: Group 3 - Peripheral Neuropathy, Cranial Nerves, Mononeuropathies: Clinical Features, Pathophysiology, Therapy

\section{A CASE OF SEROPOSITIVE NEUROMYELITIS OPTICA IN A PATIENT WITH CO-EXISTING MYASTHENIA GRAVIS AND SYSTEMIC LUPUS ERYTHEMATOSUS}

\author{
$\underline{\text { Il-Han Yoo }}^{1}$, So-Hyun Park ${ }^{1}$, Suk-Won Ahn ${ }^{2}$ \\ ${ }^{1}$ Chung-Ang University Hospital, Heukseok-ro, \\ Dongjak-gu, Seoul, KR; ${ }^{2}$ Neurology, ChungAng \\ University Hospital, Seoul, KR
}

Background: Neuromyelitis optica (NMO) is an immune related disease involving central nervous system (CNS) which has been previously reported in association with other autoimmune diseases. Herein, we report an unusual case of a 51-year-old female with NMO, myasthenia gravis (MG) and systemic lupus erythematosus (SLE) who initially presented with optic neuritis, transverse myelitis and abnormality in the brain magnetic resonance image (MRI). Methods: A 51-year-old female presented with visual disturbance, gait disturbance, slurred speech and dysesthesia at lower limbs which has developed over several times. Results: Brain MRI revealed high signal intensity lesion at bilateral hemispheres, pons and both optic nerve sheath with focal gadolinium enhancement. The serum aquaporin-4 (APQ4) receptor antibody level was positive; therefore she was initially diagnosed as NMO by the NMO diagnostic criteria and has been treated with high-dose steroid and immunosuppressants. Furthermore, the immune-serologic studies yielded positive titers for acetylcholine receptor (AchR) antibodies, antinuclear antibodies (ANA), anti-double stranded DNA antibodies (anti-dsDNA), lupus anticoagulant and anti-cardiolipin antibodies. And repetitive nerve stimulation test (RNST) revealed typical decremented CMAP amplitude by the low-frequency nerve stimulation. Collectively, in addition to NMO, she was also diagnosed as seropositive MG and SLE on the basis of diagnostic criteria. Conclusion: We consider that this patient had a seropositive neuromyelitis optica (NMO) spectrum disorder which was associated with systemic autoimmune disease including MG and SLE, therefore the possibility of NMO should be considered in patients with autoimmune diseases who have similar clinical manifestations, and anti-AQP4 antibody should be assessed. 


\section{PS2Group3-036 / \#275}

Topic: Group 3 - Peripheral Neuropathy, Cranial Nerves, Mononeuropathies: Clinical Features, Pathophysiology, Therapy

\section{NERVE ULTRASOUND ASSESSMENT IN A NOVEL MUTATION C.379DELG (PALA127LEUFS*52) IN DRP2 GENE}

\author{
$\underline{\text { Stephan Wenninger }}^{1}$, Ralf Jankovits ${ }^{2}$, Beate \\ Schlotter-Weigel ${ }^{1}$ \\ ${ }^{1}$ Dept. Of Neurology, Ludwig-maximilians- \\ university, Friedrich-Baur-Institute, Munich, \\ $D E ;{ }^{2}$ Neurozentrum Prien, Prien, DE
}

Background: Mutations in the gene "Dystrophinrelated protein 2" (DRP2) cause an X-linked intermediate Charcot Marie Tooth (CMT) neuropathy. The protein contributes to the periaxin-DRP2-dystroglycan (PDG) complex that leads to the creation of Cajal bands. Disruption of the PDG-complex is followed by hypermyelination and destabilization of the Schwann cell-axon. This report is about a 67year old man who noticed first neuropathic symptoms at age of 65. The symptoms included numbness, tingling and burning in the feet, a slight gait disturbance and recurrent muscle cramps in distal legs. There were no similar affected individuals in his family. A sensorimotor, predominantly demyelinating polyneuropathy in lower limbs was diagnosed previously and a slight elevation of proteins in cerebrospinal fluid was found. Suspecting CIDP, the patient received immunotherapy with oral corticosteroids and one course of IVIg $0,2 \mathrm{mg} / \mathrm{kg}$. Both therapies did not improve symptoms convincingly. Methods: A clinical and electrophysiological examination, nerve ultrasound, along with immunological tests for antibodies and multigene panel diagnostics for hereditary neuropathies were performed. Results: Clinical examination in our clinic revealed a predominant symmetric numbness in lower limbs and a slight sensory ataxia. Walking on tiptoes was normal, but walking on heels was mildly impaired, combined with decent distal muscular atrophy. Distal muscle tendon reflexes on lower limbs were absent and tendon reflexes of proximal muscles of lower limbs as well as tendon reflexes on upper limbs were normal.Sensory deficits of all qualities were present in a stocking distribution distal symmetric. CMTNSv2-Score was 6 , indicating a mild clinical affection. Electrophysiological examination revealed an intermediate, mainly axonal sensorimotor neuropathy of predominantly lower limbs with slowed NCVs and severely reduced amplitudes. There was no conduction block or temporal dispersion. For both sural nerves there were no responses. EMG revealed a chronic neurogenic pattern and a reduction in recruitment in distal muscles and slight neurogenic changes in lateral vastus and first dorsal interosseous muscles. Nerve ultrasound showed generalized thickened peripheral nerves in upper and lower limbs as well as plexus brachialis, measured as cross-sectional area (CSA) and diameter. Concerning the combination of severely affected peripheral nerves with thickening of peripheral and proximal nerves including plexus brachialis in ultrasound, remarkably good clinical compensation and barely notably improvement after immunotherapy, we performed a genetic testing for demyelinating polyneuropathies. A novel hemizygous stop mutation c.379delG (pAla127Leufs*52) in the DRP2 gene was found. This mutation was not described in the literature so far. Conclusion: Combined examinations in electrophysiology and high-resolution nerve ultrasound can help to identify and characterize hereditary neuropathies in a cost-effective manner. Characteristically, a higher peripheral nerve CSA can be found in patients with CMT 1A, but there are also reports of mild to moderate CSA elevations in CMT 2 and X-linked CMT patients. Based on the patient's data and presentation, we conclude that this patient has a DRP2-related intermediate CMT with late onset and mild clinical affection. This case contributes to the knowledge of DRP2associated neuropathies with new insights in nerve ultrasound in intermediate CMT.

\section{PS2Group3-037 / \#370}

Topic: Group 3 - Peripheral Neuropathy, Cranial Nerves, Mononeuropathies: Clinical Features, Pathophysiology, Therapy

\section{PHRENIC NERVE DEMYELINATION CAUSING ACUTE RESPIRATORY FAILURE IN MADSAM}

\author{
Chantal Baldwin ${ }^{1}$, Karl Ng$^{2}$ \\ ${ }^{1}$ Neurology, Royal North Shore Hospital, Sydney, \\ NSW, AU; ${ }^{2}$ Neurology, Royal North Shore Hospital, \\ ST LEONARDS, NSW, AU
}

Background: A 72-year-old man with an eight year history of Multifocal acquired demyelinating sensory and motor neuropathy (MADSAM) presented with acute worsening of left sided weakness and 
new right upper limb weakness in an asymmetrical pattern. He was managed with an induction dose of intravenous immunoglobulin (IVIG) $2 \mathrm{~g} / \mathrm{kg}$ over five days. Initial nerve conduction studies demonstrated a generalised demyelinating neuropathy affecting the upper limbs to a greater degree than the lower limbs, of note motor conduction block was seen in the right median forearm segment. Methods: He developed acute type 2 respiratory failure requiring intubation and ventilation. IVIG was continued with marked improvement in respiratory parameters and limb weakness. Repeat nerve conduction studies demonstrated resolution of the previously seen motor conduction block and phrenic nerve studies showed prolonged latencies: right $16.3 \mathrm{~ms}$ and left $14.8 \mathrm{~ms}$ with amplitudes of 0.7 and $0.9 \mathrm{mV}$ respectively (normal latency $5.53-7.72 \mathrm{~ms}$ and amplitude $0.66-1.46 \mathrm{mV}$ ). Results: Respiratory involvement is considered atypical in CIDP and rarely presents acutely. A review of 67 consecutive CIDP patients by Gorson et al. suggests this perception may be inaccurate. Nine percent of their cohort developed respiratory failure at some point during the course of their illness requiring ventilatory support. Stojkovic et al subsequently reported four cases of respiratory failure secondary to phrenic nerve palsy in CIDP. Phrenic nerve involvement was confirmed on neurophysiological testing of the phrenic nerve which revealed reduced CMAP amplitudes or prolonged latencies. Conclusion: Macia et al. performed a prospective study evaluating the phrenic nerve involvement in 14 patients with multifocal motor neuropathy $(\mathrm{MMN})$ and 14 patients with CIDP. Electrophysiological phrenic nerve involvement was common in CIDP and rare in MMN. Specifically relating to MADSAM Verschueren et al reported in their retrospective cohort of 13 MADSAM patients, two presenting with a relapsing remiting course developed respiratory failure requiring intensive care unit admission.

\section{PS2Group3-038 / \#545}

Topic: Group 3 - Peripheral Neuropathy, Cranial Nerves, Mononeuropathies: Clinical Features, Pathophysiology, Therapy

\section{BACLOFEN, NALTREXONE AND SORBITOL ALL CONTRIBUTE TO THE EFFICACY OF PXT3003 IN CMT1A RATS}

Thomas Prukop ${ }^{1}$, Stephanie Wernick ${ }^{1}$, David

Ewers $^{1}$, Anthony Brureau ${ }^{2}$, Julien Laffaire ${ }^{3}$, Nathalie Cholet ${ }^{2}$, Klaus A. Nave ${ }^{1}$, Rodolphe Haji ${ }^{2}$, Daniel Cohen ${ }^{4}$, Michael W. Sereda ${ }^{1}$

${ }^{1}$ Neurogenetics, Max-Planck-Institute of

Experimental Medicine, Göttingen,

DE; ${ }^{2}$ Experimental Biology And Pharmacology,

Pharnext, Issy Les Moulineaux, FR; ${ }^{3}$ Biostatistics, Pharnext, Issy Les Moulineaux, FR $;^{4}$ Pharnext, Issy Les Moulineaux, FR

Background: The most common type of CharcotMarie-Tooth disease is caused by a duplication of $P M P 22$, leading to dysmyelination, axonal loss and progressive muscle weakness (CMT1A). Currently, no approved treatment for CMT1A patients is available. The novel polytherapeutic drug PXT3003, a low-dose combination of Baclofen, Naltrexone and Sorbitol, was previously shown to correct Pmp22 overexpression, improve dysmyelination and axonal loss in Pmp 22 transgenic rats, a known animal model of CMT1A. Here, we performed a long-term treatment study in adult CMT1A rats with PXT3003 to test the superior effect of PXT3003 compared to its three binary combinations of Baclofen/Naltrexone, Baclofen/Sorbitol and Naltrexone/Sorbitol. Additionally, CMT1A and wildtype rats were included as placebo controls. Methods: Treatment was performed in adult rats starting at postnatal week 6 , after the manifestation of symptoms commonly observed in CMT1A rats, and was continued until week 18 . Behavioral analyses were performed at the age of 6,10, 14 and 18 weeks (grip strength, bar test and inclined plane test). Furthermore, electrophysiological measurements and a hot plate test were carried out at the end of the study. Post-mortem analyses included mRNA, protein and histological analyses of peripheral nerves. Results: No significant effect on motor endpoints was observed with any of the binary combinations of Baclofen/Naltrexone, Baclofen/Sorbitol or Naltrexone/Sorbitol. In contrast, PXT3003 proved its superior efficacy to the binary 
combinations on the behavioral level in CMT1A rats. Conclusion: This data suggests a synergistic effect of the three drug components contained in PXT3003 for CMT1A in vivo and clearly demonstrates the contribution of baclofen, naltrexone and sorbitol to the overall positive efficacy of PXT3003. These findings highlight the value of pleiotropic combinational repurposing of drugs at low doses for rapid development of highly safe combinations.

\section{PS2Group3-039 / \#638}

Topic: Group 3 - Peripheral Neuropathy, Cranial Nerves, Mononeuropathies: Clinical Features, Pathophysiology, Therapy

\section{IDEAL TIME FOR A REPEAT CONDUCTION TO SUBTYPE VARIANTS IN GUILLAIN BARRE SYNDROME}

\author{
A K. Meena ${ }^{1}$, Ramesh Chepuru ${ }^{2}$, Sailaja Sarva ${ }^{2}$, \\ Sireeesha Yareeda ${ }^{2}$, Niharika Mathukomali ${ }^{2}$ \\ ${ }^{1}$ Neurology, Nizam's Institute of Medical Sciences, \\ HYDERABAD, IN; ${ }^{2}$ Neurology, Nizam's Institute of \\ Medical Sciences, HYDERABAD, IN
}

Background: Guillain Barre Syndrome (GBS) is the most common severe form of peripheral neuropathy with rapid onset and progression. There are several variants of GBS. Many studies have suggested that serial electrophysiology is essential for accurate diagnosis of GBS. We aim to study the usefulness of serial nerve conduction studies (NCS) and at what time a repeat conduction is needed to subtype GBS. Methods: We reviewed the records of patients discharged with the diagnosis of GBS from our institute retrospectively from Jan 2011 to Dec 2016. We included patients with at least 2 electrophysiological studies. At least 4 motor nerve conduction and 3 sensory nerve conduction studies were done. We applied 3 sets of criteria at various time period in disease course: Hadden et al (1988), Rajabally et al (2014) and Uncini et al (2017). Approval was taken from institutional ethical board. Statistical analysis was done using Fisher's exact tests, McNemar's test, Excel 2013. Results: Most of the patients presented to our hospital at the end of 1 st (50.56\%) and 2nd week (34.18\%) of disease onset. Out of 354 patients 136 had complete data with repeat NCSs. Hadden's and Uncini's criteria were almost equally sensitive (37\% vs $35 \%$ ) in diagnosing AIDP as compared to Rajabally's criteria (26\%) in the 1st week of the illness amongst 77 patients. Equivocal findings not fulfilling any criteria was seen more frequently with Rajabally's criteria ( $38 \%$ vs $30 \%$ ). The 3 criteria were equally sensitive to diagnose axonal GBS in first week of illness in all 77 patients. Repeat conduction study showed was done in 1st week of disease course. Using Hadden's and Uncini criteria 6.5\% (5 patients out of 77 patients) converted from AIDP to axonal at 2nd week of illness and equivocal to axonal 9\% (7 patients out of 77). When Rajabally criteria was used the conversion of equivocal to axonal was $13 \%$ (10 patients out of 77). Shifting rate was lower where initial NCS was done at 3 rd week and later $(2 \%$ \& $8 \%$ ). Forty three out of 53 patients who had repeat conduction at 5 weeks did not show any changes in NCS as compared to that at 3 weeks. Eleven percent(15 patients) of patients had equivocal findings at the end of 4 weeks not fulfilling any criteria. Conclusion: Single study is sufficient to subtype axonal variant of GBS if $1^{\text {st }}$ study shows axonal subtype irrespective of the time course. Repeat conduction study should be done to further subtype patients in whom initial study showed AIDP/ Equivocal/not fulfilling as $5-10 \%$ of patients with initial conduction study showing AIDP changed to axonal on repeated conduction. As there is insignificant change in electrophysiological findings at 3rd week and beyond to subtype GBS probable time to repeat conduction study is $3^{\text {rd }}$ week in disease course. A significant proportion of may not fulfill any of the criteria even after 4 weeks wherein only clinical criteria for GBS , evidence of peripheral neuropathy and exclusion of other causes is important to diagnose GBS.

\section{PS2Group3-040 / \#662}

Topic: Group 3 - Peripheral Neuropathy, Cranial Nerves, Mononeuropathies: Clinical Features, Pathophysiology, Therapy

\section{COST OF ILLNESS IN CHARCOT- MARIE-TOOTH NEUROPATHY: RESULTS FROM GERMANY}

Simone Thiele $^{1}$, Elisabeth Schorling ${ }^{2}$, Peter

Reilich$^{1}$, Sabine Krause ${ }^{1}$, Klaus Nagels ${ }^{2}$, Maggie C. Walter $^{1}$

${ }^{1}$ Friedrich-baur Institute, Departement Of Neurology, Ludwigs-Maximilians University of Munich, Munich, DE; Institute Of Management In Medicine And Health Care Sciences, University of Bayreuth, Bayreuth, DE 
Background: Charcot Marie-Tooth (CMT) neuropathies are the most common inherited neurological diseases with a prevalence of 1 in 2500 individuals. In CMT, the quality of life (QoL) is reduced compared to the general population. No causative treatment is currently available but new therapeutic strategies are being investigated and various innovative clinical trials are expected within the next years. Objective Increasing demands on efficacy and safety of newly developed drugs are likely to increase costs for the health care system. However, innovative therapies may have the potential to reduce the cost of illness (COI) by improving the clinical phenotype. Our study aimed to estimate the total annual cost of illness in CMT. Methods: The study enrolled 397 patients with genetically confirmed diagnosis of CMT; subtypes of the disease were differentiated. A micro-cost method was used to determine the COI. Direct and indirect costs were assessed to determine the economic burden of CMT. Results: The most frequent genetic diagnoses in our cohort were CMT1A (PMP22 duplication; n=246), HNPP (PMP22 deletion; $\mathrm{n}=36)$, CMT1X (GJB1 mutation; $\mathrm{n}=33)$, CMT1B (MPZ mutation; $\mathrm{n}=24), \mathrm{CMT} 2 \mathrm{~A}$ (MFN2 mutation, $\mathrm{n}=16)$ and CMT4C (SH3TC2 mutation; $n=12$ ), accounting for $92,4 \%$ of the genetically confirmed mutations. The remaining subtypes accounted for $1 \%$ each. Patients were classified as slightly, moderately or severely affected by the CMT neuropathy score (CMTNS of $<10,11-20$ or $>20$, respectively). The economic burden of CMT patients was assessed and analyzed with regard to the actual CMT standard of care. Conclusion: Our results significantly contribute to a more comprehensive insight into the current burden of CMT and quality of life status as related to CMT health services in Germany. In the light of innovative therapeutic interventions, our results suggest a notable potential for a reduction in overall COI and improvement of HRQOL if the therapeutic intervention leads to a less severe course of the disease. Our results could help accelerating the implementation of innovative $\mathrm{CMT}$ therapies in standard CMT clinical care.
PS2Group3-041 / \#697

Topic: Group 3 - Peripheral Neuropathy, Cranial Nerves, Mononeuropathies: Clinical Features, Pathophysiology, Therapy

\section{CLINICAL HETEROGENEITY OF ANTI-GM2-GANGLIOSIDE ANTIBODY SYNDROME}

\author{
Jong Seok Bae ${ }^{1}$, Sun Min Yoon ${ }^{2}$, Soon Kyung \\ Shim $^{2}$ \\ ${ }^{1}$ Neurology, Kangdong Sacred Heart Hospital, \\ Seoul, KR, ${ }^{2}$ Neurology, Kangdong Sacred Heart \\ Hospital, Seoul, KR
}

Background: Antiganglioside antibody is known to play a pathogenic role in Guillain-Barré syndrome (GBS). Either an immunoglobulin (Ig)G- or IgMtype anti-GM2 antibody is detected in rare cases in GBS patients. However, the specific pathogenic role of these antibodies in GBS has not been reported previously. This study aimed to define and characterize the clinical spectrum of GBS with anti-GM2 positivity. Methods: We reviewed the database of the DAUNIT (Dong-A University Neuroimmunology Team), which has collected sera of GBS and its variants from more than 40 general and universitybased hospitals in Korea. Detailed information about the involved patients was often obtained and corrected by the charge doctor applying additional questionnaires. Results: Four patients with acute monophasic peripheral neuropathy or cranial neuropathy with isolated IgM-type anti-GM2-antibody positivity were recruited. In addition, IgG-type antiGM2 antibody was solely detected in the sera of another four patients. The IgM-positive group comprised heterogeneous syndromes: two cases of acute motor axonal neuropathy, one of acute inflammatory demyelinating polyneuropathy, and one of isolated facial diplegia. In contrast, all of the cases enrolled in the IgG-positive group manifested with dizziness with or without oculomotor palsy due to cranial neuropathy syndrome. Conclusion: This study identified that anti-GM2 antibody can be found in various subtypes of GBS in rare cases. Compared to the clinical heterogeneity of the IgMpositive group, the IgG-positive group can be characterized by cranial-dominant GBS presenting mainly with oculomotor and vestibular dysfunctions. 


\section{PS2Group3-042 / \#798}

Topic: Group 3 - Peripheral Neuropathy, Cranial Nerves, Mononeuropathies: Clinical Features, Pathophysiology, Therapy

\section{LATE-ONSET AXONAL CHARCOT- MARIE-TOOTH ASSOCIATED WITH AUTOSOMAL RECESSIVE MME MUTATIONS}

Tania García-Sobrino ${ }^{1}$, Maria Pacifica Vidal-Lijo ${ }^{2}$, Elena Pintos ${ }^{3}$, Vicenzo Lupo ${ }^{4}$, Carmen Espinós ${ }^{5}$, Teresa Sevilla ${ }^{6}$, Julio Pardo ${ }^{7}$

${ }^{1}$ Neurology, Hospital Clínico Santiago de Compostela, Santiago de Compostela, ES, ${ }^{2}$ Neurophysiology, Hospital Clínico Santiago de Compostela, Santiago de Compostela, ES, ${ }^{3}$ Pathology, Hospital Clínico Santiago de Compostela, Santiago de Compostela, ES ${ }^{4}$ Centro de Investigación Príncipe Felipe. INCLIVA \& IIS La Fe Rare Diseases Joint Units, Valencia, ES; ${ }^{5}$ Unit Of Genetics And Genomics Of Neuromuscular And Neurodegenerative Disorders And Service Of Genomics And Traslational Genetics, Centro de Investigación Príncipe Felipe (CIPF), and Joint Unit for research on Rare Diseases, CIPF-IISLa Fe, Valencia, ES; ${ }^{6}$ Neurology, 4 Neurology Department. Hospital Universitari i Politecnic La Fe. Neuromuscular Research Unit, Instituto de Investigación Sanitaria La Fe. Centro de Investigación Biomédica en Red de Enfermedades Raras (CIBERER). Valencia. Spain, Valencia, ES; ${ }^{7}$ Neurology, Hospital Clínico Santiago de Compostela, Santiago de Compostela, ES

Background: Charcot-Marie-Tooth (CMT) disease is the most common inherited peripheral neuropathy. The disease onset usually occurs in the first two decades of life and shows a slow progression. Clinically CMT phenotype is characterized by distal weakness, wasting and sensory loss with foot deformities. Axonal CMT (CMT2) with preserved nerve conduction velocities (MMMNCV $>38 \mathrm{~m} / \mathrm{s}$ ) are a group of clinically and genetically heterogeneous subtypes. A high rate of CMT2 remain without genetic diagnosis. In $2016 M M E$ mutations were identified in patients with CMT2 linked to an autosomal dominant or autosomal recessive inheritance pattern. We report a family with an autosomal recessive lateonset CMT2 associated with MME mutations. Methods: A 57-year-old man without medical or family history and no consanguinity. He developed a progressive gait disturbance, cramps and distal lower limb weakness with foot drop at the age of 53. Neurological examination revealed pes cavus, gynecomastia, fasciculations and wasting in lower limbs, slight left hand weakness, proximal lower limb weakness (4/5) and distal lower limb weakness (3/5), areflexia and steppage gait. Clinical progression was evident in the first year of evolution and patient associated distal lower limb sensory loss. Results: Laboratory investigations showed a high serum CK (600-1500UI/L). Spinal cord MRI was normal. Nerve conduction study are consistent with an axonal motor neuropathy. Needle electromyography showed acute denervation and fasciculations in lower limbs with reduced recruitment pattern in upper and lower limb muscles. After six month a nerve conduction study revealed an axonal motor and sensory neuropathy. Lower limb muscle MRI showed a bilateral fatty infiltration of the gastrocnemius, soleus and in the anterolateral group. A myopathic and neuropathic pattern was evident in the muscle biopsy. Molecular diagnosis revealed that our patient was a homozygous for the c.466delC mutation in the $M M E$ gene. Neurological examination and nerve conduction studies were normal in his relatives. Molecular study revealed that his parents and two sisters were heterozygous for the same mutation in $M M E$ gene. Conclusion: CMT2 are a clinically and genetically heterogeneous group of neuropathies. The screening of $M M E$ mutations should be performed in patients with late-onset neuropathies with or without a family history in wich acquired causes are ruled out and the phenotype is compatible with CMT2.

\section{PS2Group3-043 / \#567}

Topic: Group 3 - Peripheral Neuropathy, Cranial Nerves, Mononeuropathies: Clinical Features, Pathophysiology, Therapy

\section{POPULATION}

PHARMACOKINETICS (PK) OF INVESTIGATIONAL PATISIRAN IN HEALTHY VOLUNTEERS AND IN PATIENTS

Varun Goel $^{1}$, Claudia Jomphe ${ }^{2}$, Nathalie Gosselin ${ }^{2}$, Husain Attarwala ${ }^{3}$, Xiaoping Zhang ${ }^{4}$, Jean-Francois Marier $^{2}$, Gabriel Robbie ${ }^{3}$

${ }^{1}$ Alnylam Pharmaceuticals, Cambridge, MA, US, ${ }^{2}$ Cetera, Montreal, QC, CA ${ }^{3}$ Alnylam

Pharmaceuticals, Cambridge, AL, US: ${ }^{4}$ Clinical Pharmacology And Pharmacometrics, Alnylam Pharmaceuticals Inc, Cambridge, MA, US 
Background: Patisiran drug product is composed of drug substance ALN-18328 (siRNA) and lipid excipients formulated in lipid nanoparticle in isotonic phosphate buffer saline. Population PK models were developed to describe plasma PK profiles of ALN18328 and the 2 novel lipids (DLin-MC3-DMA and PEG2000-C-DMG) and to identify sources of variability. Methods: PK data collected were pooled from 5 clinical studies following single or multiple doses $(0.01$ to $0.5 \mathrm{mg} / \mathrm{kg})$ to healthy volunteers $(\mathrm{N}=22)$ or patients with Hereditary TransthyretinMediated (hATTR) amyloidosis ( $\mathrm{N}=177)$. Nonlinear mixed-effects modeling was used to identify PK compartmental models. Covariate-parameter relationships were explored using a pre-specified full model approach. The clinical relevance of covariate effects was assessed based on statistical significance and impact on steady-state PK exposures. Results: PK of ALN-18328 was described with a semi-mechanistic model with linear elimination from the liver (Figure 1). PK of the 2 lipids were described by 3-compartment mammillary models with linear elimination. All models resulted in acceptable goodness-of-fit. Estimated PK parameters are shown in Table 1. For patients in phase 3 study $(\mathrm{N}=148)$, posthoc plasma clearance (geometric mean) and associated inter-patient variability (\% CV) of ALN-18328, DLin-MC3-DMA, and $\mathrm{PEG}_{2000}$-C-DMG were estimated to be $0.422 \mathrm{~L} / \mathrm{h}(47.5 \%), 0.128 \mathrm{~L} / \mathrm{h}(25 \%)$, and $0.128 \mathrm{~L} / \mathrm{h}(19.6 \%)$ respectively. None of following covariates affected PK exposures: baseline age, sex, race (Caucasian/non-Caucasian), mild and moderate renal impairment, mild hepatic impairment, anti-drug antibody status, and concomitant administration of moderate or strong CYP3A inhibitors or inducers. There was a trend of increasing PK exposures with increasing body weights; however, this did not result in differences in TTR lowering. Conclusion: Population analyses adequately described plasma PK profiles of patisiran components across a wide range of dose levels. Overall PK of patisiran components was linear, dose-proportional, time-invariant and predictable with chronic dosing of $0.3 \mathrm{mg} / \mathrm{kg}$ q3w patisiran over 2 years.

\section{PS2Group3-044 / \#788}

Topic: Group 3 - Peripheral Neuropathy, Cranial Nerves, Mononeuropathies: Clinical Features, Pathophysiology, Therapy

\section{CHARACTERIZATION OF NEURONAL MOLECULAR MECHANISMS UNDERLYING CMT2Z NEUROPATHY}

Paula Sancho' ${ }^{1}$, Luca Bartesaghi², Olivia Miossec ${ }^{2}$, Francisco García-García ${ }^{3}$, Laura Ramírez-Jiménez ${ }^{1}$, Vincenzo Lupo ${ }^{4}$, Roman Chrast ${ }^{2}$, Carmen Espinós ${ }^{1}$ ${ }^{1}$ Unit Of Genetics And Genomics Of Neuromuscular And Neurodegenerative Disorders And Service Of Genomics And Traslational Genetics, Centro de Investigación Príncipe Felipe (CIPF), and Joint Unit for research on Rare Diseases, CIPF-IISLa $\mathrm{Fe}$, Valencia, ES; ${ }^{2} 2$ department Of Neuroscience And Department Of Clinical Neuroscience, Karolinska Institutet, Stockholm, SE; $;^{3}$ Unidad De Bioinformática Y Bioestadística, Centro de

Figure 1: Schematic Representation of the Population PK Model of ALN-18328

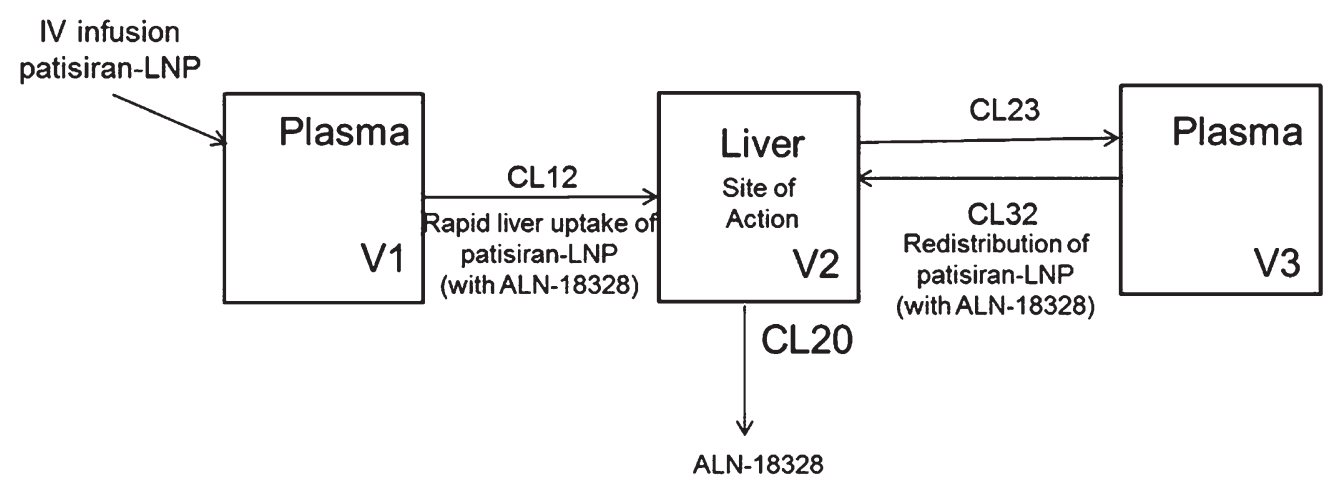

CL20 = LNP (ALN-18328) elimination clearance from liver (compartment 2); CL12 = LNP (ALN-18328) initial distribution clearance from plasma (compartment 1) to liver (compartment 2) in first phase; CL23 = LNP (ALN-18328) redistribution clearance from liver (compartment 2) to plasma (compartment 3) in second phase; CL32 = LNP (ALN-18328) distribution clearance from plasma (compartment 3) to liver (compartment 2) in second phase; IV = intravenous; LNP = lipid nanoparticle; V1 = distribution volume of compartment 1 ; V2 = distribution volume of compartment $2 ; \mathrm{V} 3=$ distribution volume of compartment 3 
Investigación Príncipe Felipe, Valencia, ES: ${ }^{4}$ Unit Of Genetics And Genomics Of Neuromuscular And Neurodegenerative Disorders And Service Of Genomics And Traslational Genetics, Centro de Investigación Príncipe Felipe (CIPF), and Joint Unit for Research on Rare Diseases, CIPF-IISLa $\mathrm{Fe}$, Valencia, ES

Background: Mutations in MORC2 lead to an axonal form of Charcot-Marie-Tooth neuropathy (CMT2Z). To date, thirty-two families have been described with mutations in MORC2, indicating that this gene is relatively frequently involved in CMT cases. While the genetic data clearly established the causative role of MORC2 in CMT2Z, its phenotypic consequences in patients and role in neuronal biology remains to be clarified. Methods: RNA and protein expression of MORC2 was tested at different development stages in several neural tissues: sciatic nerve, dorsal root ganglion (DRG) and brain. In order to determine the effect of the most common MORC2 clinical mutations, p.S87L and p.R252W in a neural context, we used an in vitro culture system based on virally mediated overexpression of MORC2 in sensory neurons. ATPase activity of MORC2 was measured by using colorimetric assay. A rat gene expression microarray was used for transcriptomics studies. Results: Here, we show that the full-length form of MORC2 is predominantly expressed in both adult and embryonic human neural tissue and that the expression of Morc2 is dynamically regulated in the developing and maturing peripheral and central nervous systems. We observed that in particular the overexpression of p.S87L mutant in sensory neurons induced significant axonal phenotype. In addition, by using a colorimetric assay we observed that p.S87L mutant has an impaired ATPase activity which may contribute to its pathogenicity. Since MORC2 has recently been identified as an essential gene required for epigenetic silencing by the human silencing hub (HUSH) complex, we decided to look with a transcriptomic approach in sensory neurons for genetic and biochemical pathways altered in presence of mutated forms of MORC2. Preliminary data from this analysis indicate complex changes affecting transcripts involved in neuronal and axonal functions. Conclusion: Collectively, our data provide an important insight into the pathomechanisms underlying CMT2Z and the role of MORC2 in nervous system.
PS2Group3-045 / \#955

Topic: Group 3 - Peripheral Neuropathy, Cranial Nerves, Mononeuropathies: Clinical Features, Pathophysiology, Therapy

\section{INCREASED DEACETYLATION OF PROTEINS BY SIRTUIN 1 PROTEIN OVER EXPRESSION REVERSES T2D PERIPHERAL NEUROPATHY}

James Russell, Krish Chandrasekaran, Cheng-Yeng Ho, Pranith Kumar, Sai Sruthi Reddy University of Maryland School of Medicine, Baltimore, US

Background: Sirtuin1 (SIRT1) protein uses NAD+ as a substrate to deacetylate transcription factors, cofactors, and histones to enhance mitochondrial function. The sirtuins use $\mathrm{NAD}^{+}$as a co-substrate to catalyze the deacetylation and/or mono-ADP ribosylation of target proteins. Overall, there is evidence that SIRT1 is neuroprotective. Furthermore, there is evidence that activation of SIRT1 by $\mathrm{NAD}^{+}$is important in axonal regeneration. In part, SIRT1 neuroprotection may be because of improved energy homeostasis. SIRT1 activity in the nucleus deacetylates histone, non-histone transcriptional and cotranscriptional factors that regulate glucose homeostasis and fat oxidation. Although SIRT1 isoforms are primarily present in the nucleus and cytosol and are very important in neuroprotection, there is a distinct $\mathrm{Mt}$ isoform. This mitochondrial regulatory function may be critical in reducing diabetic injury to mitochondria within the peripheral nervous system. Methods: We tested the hypothesis whether increased expression of SIRT1 protein in dorsal root ganglion (DRG) neurons would rescue mice from peripheral neuropathy induced by a high fat diet (HFD). Neuron-specific, doxycycline (DOX)-inducible, SIRT1 protein over expressing C57BL6 transgenic mouse (nSIRT1OE Tg) were generated. Expression of SIRT1 protein was observed in small to medium sized DRG neurons. nSIRT1OE was shut off by feeding, weaned Tg mice, with DOX in the diet for 12 weeks. The Tg mice were divided into 3 groups. Group \# 1: nSIRT1OE Tg mice fed with standard diet (STD) plus DOX for 4 months; Group \# 2: nSIRT1OE Tg mice fed with HFD plus DOX for 4 months; Group \# 3: nSIRT1OE Tg mice fed with HFD plus DOX for 2 months until they develop neuropathy and then switched to HFD minus DOX for additional 2 months. Neuropathy was determined by 
mechanical allodynia thresholds (MAT) and nerve conduction velocity (NCV) at 0,2 and 4 months. Intraepidermal nerve fiber density (IENFD) was measured at 4 months. Results: MAT, NCV and IENFD were decreased in HFD-fed (Group \# 2) mice compared to STD-fed mice (Group \# 1) at 2 and 4 months. In Group \# 3 mice, 2 months after turning on nSIRT1OE, we observed a reversal of mechanical allodynia, NCV and attenuation of IENFD (Group \# 3 compared to Group \# 2). Western blot of protein extracts from DRG neurons showed that HFD increased acetylation of proteins and SIRT1OE abolished the HFD-induced acetylation of proteins. The mitochondrial bioenergetics profile of cultured adult DRG neurons showed that hyperglycemia induced a decrease in the spare respiratory capacity in wild type (WT) DRG neurons. This was corrected in nSIRT1OE Tg DRG neurons. Conclusion: In type 2 diabetic neuropathy, altered acetylation of proteins and reduced mitochondrial function via a defective SIRT1 pathway may contribute to developing distal axonopathy. In contrast, overexpression of SIRT1 is able to reverse experimental diabetic neuropathy. SIRT1 is amenable to activation by therapies that increase $\mathrm{NAD}^{+}$in diabetic neuropathy.

\section{PS2Group3-046 / \#953}

Topic: Group 3 - Peripheral Neuropathy, Cranial Nerves, Mononeuropathies: Clinical Features, Pathophysiology, Therapy

\section{PGC-1 $\alpha$ REGULATES NEURONAL RESPITRATORY CHAIN SUPERCOMPLEXES IN MITOCHONDRIA}

James Russell, Joungil Choi, Mohammad Salimian, Sai Sruthi Reddy

University of Maryland School of Medicine, Baltimore, US

Background: Loss of PGC- $1 \alpha$ signaling is important in the development of experimental diabetic neuropathy (DN) [Choi J et al. Neurobiology of Disease, 2014. 64; 118-130]. In PGC- $\alpha$ knockout (KO) mice there is exacerbation of $\mathrm{DN}$ with loss of large and small myelinated fibers, loss of mitochondria (Mt) and DNA content, and increased protein oxidation. We have shown potential important functions of $35-\mathrm{kDa}$ PGC-1 $\alpha$, a novel PGC-1 $\alpha$ isoform in regulating Mt integrity. Methods: The focus of the present work was to analyze the respiratory chain complex integrity in neurons of PGC-1 $\alpha \mathrm{KO}$ mice. Results: In the respiratory supercomplexes of 1,030, 980 , and $840 \mathrm{kDa}$, the levels of complexes 1,030 and $980 \mathrm{kDa}$ are significantly decreased, while the level of $840-\mathrm{kDa}$ complex is significantly increased in PGC-1 $\alpha$ KO mice compared to wild type (WT) mice. Mass spectrometry analysis identifies; (1) subunits of complex I, III, and IV in the $1,030-\mathrm{kDa}$ band, 2) subunits of complex I and III in the 980$\mathrm{kDa}$ band, and (3) subunits of complex I in the 840$\mathrm{kDa}$ band. Quantification results reveal that the activities of complexes I, III, and IV within the $1,030-\mathrm{kDa}$ protein complex are significantly decreased in PGC-1 $\alpha$ KO mice. In contrast, we observe little complex I, III, and IV activities either in the 980- or $840-\mathrm{kDa}$ complex. We observed altered $\mathrm{Mt}$ morphology and impaired respiration in PGC-1 $\alpha$ $\mathrm{KO}$ mice. Co-immunoprecipitation and mass spectrometry analyses reveal that the $35-\mathrm{kDa}$ PGC- $1 \alpha$ is associated with NADH dehydrogenase (ubiquinone) flavoprotein 2 (NDUFV2), a subunit of respiratory complex I in the Mt. Immunoblot analysis combined with two-dimensional-blue native gel show the presence of both $35-\mathrm{kD} \alpha$ PGC- $1 \alpha$ and NDUFV2 proteins in the $1,030-\mathrm{kDa}$ protein band. Conclusion: These results are consistent with a cooperative role of $35-\mathrm{kDa}$ PGC-1 $\alpha$ and NDUFV2 in the organization of Mt respiratory supercomplex assembly. The study provides evidence that $35-\mathrm{kDa}$ PGC- $1 \alpha$ is present in the $\mathrm{Mt}$ where it could directly regulate $\mathrm{Mt}$ function and affect response to diabetes and in diabetic neuropathy.

\section{PS2Group3-047 / \#330}

Topic: Group 3 - Peripheral Neuropathy, Cranial Nerves, Mononeuropathies: Clinical Features, Pathophysiology, Therapy

\section{THE VALIDITY OF SUDOSCAN IN SCREENING OF UPPER EXTREMITY NEUROPATHIES: A PILOT STUDY}

Geun-Young Park, Sun Im, Hung Jung Koo, Yongjun Jang, Choong Sik Chae Rehabilitation Medicine, Bucheon St. Mary's Hospital, College of Medicine, The Catholic University of Korea, Bucheon-si, KR

Background: SUDOSCAN (Impeto Medical, France) is a recently used device in the quantitative assessment of sudomotor function to screen 
peripheral polyneuropathy (PPH), and Sudomotor function was assessed through values of electrochemical skin conductance (ESC) by two pairs of electrodes for feet and hands. Conventionally, nerve conduction study(NCS) and needle electromyography (EMG) are regarded as a golden standard in the evaluation of large nerve fiber function, but there is a limitation to evaluate small nerve fiber and sweat glands dysfunction. Interestingly, there are a few reports suggesting that neuropathies involving lower extremities, such as lumbosacral radiculopathy, can also induce sudomotor dysfunction. However, there has been no report targeting upper extremity neuropathy. The objectives of this study was to determine the diagnostic validity of SUDOSCAN in screening CTS and cervical radiculopathy, which are one of the most common pathologies of dysesthesia on upper extremities, in an outpatient clinic with suspected patients of sudomotor dysfunction. Methods: Medical record of 48 patients with dysesthesia on upper extremities, who underwent SUDOSCAN, NCS and EMG, between May 1st, 2015 and August 31 st, 2015, were analyzed retrospectively. Based on NCS and EMG results, these subjects were allocated to three different groups: Normal findings(Group A, 16 patients), CTS(Group B, 16 patients) and cervical radiculopathy(Group C, 9 patients). ANOVA and Kruskal-Wallis analysis were used to compare the differences in demographic features, average ESC values on feet and hands of SUDOSCAN and pain severity measured by Michigan neuropathy screening instrument(MNSI). Results: There were demographic differences about age and proportion on sex among those groups and no differences for the proportion of DM and alcoholics history. Group A was younger than $\mathrm{B}$ and $\mathrm{C}$, and male proportion is higher than female in only in group C. There were no demographic differences about DM and alcoholic history among those groups. ESC values on feet and hands in CTS diagnosed-group $\mathrm{B}(\mathrm{Mean} \pm \mathrm{SD}$ $61.56 \pm 14.70 \mu \mathrm{S}, \quad 52.17 \pm 15.37 \mu \mathrm{S})$ smaller than Group $\mathrm{A}(62.63 \pm 19.61 \mu \mathrm{S}, 60.43 \pm 17.43 \mu \mathrm{S})$ and group $\mathrm{C}(64.88 \pm 24.15 \mu \mathrm{S}, 61.33 \pm 25.01 \mu \mathrm{S})$, respectively. However, there were no significant differences among three groups in statistical analysis by using ANOVA. Pain severity using MNSI were each $2.88 \pm 0.99$ (group A), $3.73 \pm 1.89$ (group B) and $2.40 \pm 1.28$ (group C), all of which showed no statistical significance among these groups. Conclusion: Due to the limited number and uneven distribution of the subjects, our result failed to reveal the usefulness of SUDOSCAN in screening of CTS or cervical radiculopathy among patients with dysesthesia on upper extremities. However, because we have seen the tendency of decrease in ESC value on feet and hands by SUDOSCAN among CTS patients, large population study for SUDOSCAN should be considered in order to validate our findings.

\section{PS2Group3-048 / \#415}

Topic: Group 3 - Peripheral Neuropathy, Cranial Nerves, Mononeuropathies: Clinical Features, Pathophysiology, Therapy

\section{IPSC MOTOR NEURONS FROM AN X-LINKED CMT TYPE 6 PATIENT AS A MODEL FOR STUDYING AXONAL DEGENERATION AND DEVELOPING THERAPIES}

Gonzalo Perez Siles $^{1}$, Rebecca Screnci ${ }^{2}$, Anthony Cutrupi $^{1}$, Melina Ellis ${ }^{1}$, Carolyn Ly ${ }^{1}$, Garth A. Nicholson $^{3}$, Marina L. Kennerson ${ }^{1}$

${ }^{1}$ Northcott Laboratory (neurobiology), ANZAC Research Institute, Sydney, NSW, AU; ${ }^{2}$ School Of Life Sciences, University of Technology Sydney, Sydney, NSW, AU; ${ }^{3}$ Concord Hospital, Molecular Medicine Laboratory, Sydney, NSW, AU

Background: A missense mutation (c.G473A; p.R158H) in the pyruvate dehydrogenase kinase 3 (PDK3) gene causes an X-linked form of CharcotMarie-Tooth neuropathy (CMTX6). PDK3 negatively regulates the pyruvate dehydrogenase complex (PDC) by reversible phosphorylation. Mitochondrial PDC catalyses the oxidative decarboxylation of pyruvate to acetyl CoA and links glycolysis to the critical energy producing Krebs (TCA) cycle. We have shown the p.R158H mutation causes hyperactivity of PDK3 and hyperphosphorylation of the E1 subunit of PDC, leading to reduced PDC activity. As a result, CMTX6 patient fibroblasts show mitochondrial abnormalities, lactate acidosis and reduced ATP production Methods: We have generated a line of induced pluripotent stem cells (iPSCs) by re-programming CMTX6 patient fibroblasts carrying the PDK3 p.R158H mutation (iPSC ${ }^{\text {CMTX6). We }}$ also have engineered an isogenic control using the CRISPR/Cas9 system (iPSC ${ }^{\text {isogenic }}$ ). iPSC ${ }^{\text {CMTX6 }}$ and iPSC $^{\text {isogenic }}$ have been differentiated into spinal cord motor neurons following a 28 days differentiation protocol and L1CAM magnetic bead enrichment to increase the percentage of mature motor neurons. 


\section{wild-type PDK3}

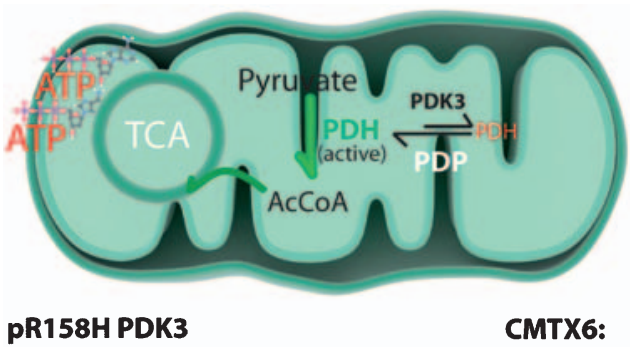

pR158H PDK3

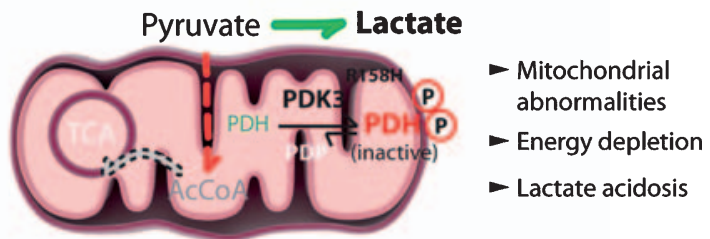

Results: Our preliminary experiments demonstrate the iPSC ${ }^{\text {CMTX6 }}$ line shows E1-hyperphosphorylation, confirming the reprogramed cells retain the pathogenic molecular signature found in the CMTX6 patient fibroblasts. Treating the iPSC ${ }^{\text {CMTX6 }}$ cells with the pan-PDK inhibitor dichloroacetate (DCA) reduced the levels of phosphorylation in the patientderived cells, demonstrating E1-hyperphosphorylation is a reversible process and an ideal pharmacological target for the development of treatment therapies. Gene editing of the mutated residue in PDK3 completely restores phosphorylation levels. This result undoubtedly indicates the $\mathrm{p}$. $\mathrm{R} 158 \mathrm{H}$ mutation is responsible of the disease phenotype. Conclusion: iPSC lines re-programmed from CMTX6 patient fibroblasts are an ideal resource to investigate molecular mechanisms underlying axonal degeneration in the relevant neuronal tissue affected in patients. Future studies culturing patient and isogenic control motor neurons will assess the therapeutic effect of compounds that can revert E1 hyperphosphorylation and ameliorate classical features of axonal degeneration affecting axon morphology and function
PS2Group3-049 / \#542

Topic: Group 3 - Peripheral Neuropathy, Cranial Nerves, Mononeuropathies: Clinical Features, Pathophysiology, Therapy

\section{PARANODAL ANTIBODIES IN AUSTRIAN PATIENTS WITH ACUTE ONSET INFLAMMATORY NEUROPATHY}

Desiree De Simoni ${ }^{1}$, Elisabeth Lindeck-Pozza ${ }^{2}$, Harald Hegen ${ }^{3}$, Wolfagang Löscher ${ }^{3}$, Friedrich Zimprich $^{4}$, Romana Hoeftberger ${ }^{5}$, Julia Wanschitz ${ }^{3}$ ${ }^{1}$ Neurology, University Hospital St. Poelten, St. Poelten, AT; ${ }^{2}$ Sozialmedizinisches Zentrum Süd, Vienna, AT; ${ }^{3}$ Medical University of Innsbruck, Innsbruck, AT; ${ }^{4}$ Department Of Neurology, Medical University of Vienna, Vienna, AT; ${ }^{5}$ Medical University of Vienna, Vienna, AT

Background: Nodal, paranodal and juxtaparanodal proteins have been identified as antigens in peripheral inflammatory neuropathies, however the frequency and clinical relevance of antibody responses against these targets remain poorly investigated in Guillain-Barré-Syndrome. Methods: Patients with acute onset inflammatory neuropathies were identified by exploration of the local databases of the Departments of Neurology and the Institute of Neurology of the Medical Universities in Innsbruck and Vienna. Patient data, electrophysiological classification and presence of anti-ganglioside antibodies were retrospectively retrieved by review of patient records. Only patients meeting Brighton criteria certainty level one or two were included in the study. We so far identified 100 patients who were admitted to the hospital between 2004 and 2017 with typical clinical presentation and electrophysiological results consistent with one of the subtypes of GBS, amongst them AIDP, AMAN, AMSAN, MFS, and pharyngo-cervico-brachial GBS variant. Some patients with the initial suspicion of AIDP had disease duration of more than 6 months and were reclassified as acute onset CIDP. Sera, and if available CSF, from all 100 patients and sera from twenty control individuals with non-inflammatory polyneuropathy were screened by an optimized tissue based assay using rat brains and teased nerve fibers for immune responses against surface antigens and by cellbased assays with transfected HEK cells for antibodies against contactin1 (CNTN1), contactin2 (CNTN2), contactin-associated-protein1 (CASPR1), 
and neurofascin-155 (NF155). Results: In the teased nerve fiber assays, some of the patients showed specific antibody staining to the node of ranvier, some of which were also positive in the brain tissue assay. None of the GBS patients' sera had antibody reaction to CNTN1, CNTN2, CASPR1 or NF155 in cellbased assays. Among the CIDP patients, two patients demonstrated reactivity against CNTN1 with similar clinical presentation as previously described. None of the controls had any antibody reaction in the performed tests. Conclusion: Our results suggest that antibody responses to CNTN1, CNTN2, CASPR1 or NF155 are absent in Austrian GBS patients, although analysis of more patients is needed to confirm these results. Furthermore, it remains to be established whether antibodies against CNTN1 may predict a chronic course in acute onset inflammatory neuropathies.

\section{PS2Group3-050 / \#695}

Topic: Group 3 - Peripheral Neuropathy, Cranial Nerves, Mononeuropathies: Clinical Features, Pathophysiology, Therapy

\section{ULNAR NERVE CONDUCTION STUDY USING THE FIRST DORSAL INTEROSSEOUS MUSCLE RECORDING IN HEALTHY KOREAN SUBJECTS}

$\underline{\text { Joong-Yang Cho }}^{1}$, Hye Jung Lee ${ }^{2}$

${ }^{1}$ Neurology, Ilsan Paik Hospital, Inje University

College of Medicine, Goyang-si, Gyeonggi-do, $K R ;^{2}$ Neurology, Ilsan Paik Hospital, Inje University College of Medicine, Goyang-si, Gyeonggi-do, KR

Background: Ulnar neuropathy at the wrist (UNW) can be difficult to localize by nerve conduction studies. The standard approach has been the demonstration of prolonged distal motor latency to first dorsal interosseous (FDI), in conjunction with a normal dorsal ulnar sensory response and denervation on needle EMG of ulnar innervated hand muscles with sparing of ulnar innervated muscles proximal to the wrist. However, none of these electrophysiologic abnormalities in isolation clearly differentiate patients with UNW from the more numerous patients with ulnar neuropathy at the elbow (UNE). Recently, conduction block (CB), along with conduction slowing, of FDI motor fibers across the wrist using a simplified version of the short segment incremental study
(SSIS) were reported in patients with UNW. The purpose of this study was to describe normative values for ulnar nerve conduction study using the FDI recording before a simplified version of the short segment incremental study (SSIS) was applied in patients with UNW. Methods: Ulnar nerve motor conduction study with FDI and abductor digiti minimi muscle (ADM) recording was performed in 132 hands of 66 healthy subjects. Ulnar NCS was performed with 2 different recording electrode montages (ADM-base of 5th finger; FDI-thumb) and differences in latency and amplitude were compared. The ulnar nerve was stimulated at the wrist $(5 \mathrm{~cm}$ proximal to the active recording electrode for ADM). Results: The maximal values for terminal motor latency to the ADM and FDI muscle were $2.5 \mathrm{msec}$ and $4.3 \mathrm{msec}$, respectively. The maximal side-to-side terminal motor latency difference to FDI was $0.4 \mathrm{msec}$. In addition, the maximal side-to-side terminal motor latency difference to ADM was also $0.4 \mathrm{~ms}$. The maximal ipsilateral latency difference between ADM and FDI was $1.9 \mathrm{msec}$. There was a tendency for the terminal motor latency of FDI to increase with advancing age. Conclusion: We obtained the normative values for ulnar nerve conduction study using the FDI recording, which is useful in applying a simplified version of the SSIS in patients with UNW.

\section{PS2Group3-051 / \#907}

Topic: Group 3 - Peripheral Neuropathy, Cranial Nerves, Mononeuropathies: Clinical Features, Pathophysiology, Therapy

\section{PATIENT ASSISTED INTERVENTION FOR NEUROPATHY: COMPARISON OF TREATMENT IN REAL LIFE SITUATIONS (PAIN-CONTROLS)}

Richard J. Barohn ${ }^{1}$, Byron Gajewski², Mamatha Pasnoor $^{1}$, Lexie Brown ${ }^{2}$, Laura Herbelin ${ }^{1}$, Kim Kimminau $^{3}$, Omar Jawdat ${ }^{1}$, Tina Liu ${ }^{1}$, Chad Parks ${ }^{1}$, Pam Shlemon ${ }^{4}$, Mazen Dimachkie ${ }^{5}$, P PainControl'S Study Team ${ }^{1}$

${ }^{1}$ Neurology, University of Kansas Medical Center, Kansas City, KS, US, ${ }^{2}$ Department Of Biostatistics, The University of Kansas Medical Center, Kansas City, US; ${ }^{3}$ Family Medicine, The University of Kansas Medical Center, Kansas City, US: ${ }^{4}$ The Foundation of Peripheral Neuropathy, Kansas City, US, ${ }^{5}$ Neurology, The University of Kansas Medical Center, Kansas City, KS, US 
Background: Cryptogenic sensory polyneuropathy (CSPN) is a common slowly progressive neuropathy that affects adults and presents with significant neuropathic pain for which multiple medications have been tried including antiepileptics, antidepressants, topicals and narcotics. A web based survey among neuromuscular experts suggested pregabalin as being more effective than other medications, however there are presently no comparative studies to assess the most effective medication. Objectives: To determine which of the 4 pharmaceutical therapies (pregabalin, duloxetine, nortriptyline or mexiletine) is most effective for neuropathic pain and best tolerated with least side effects in cryptogenic sensory polyneuropathy. Methods: We performed a prospective randomized open labelled comparative effectiveness study of CSPN patients through the Patient Centered Outcomes Research Institute (PCORI). The study used a Bayesian adaptive design, which included response adaptive randomization. At each interim analysis a decision was made to either continue enrolling patients or to stop the trial for success. CSPN patients who fulfilled the inclusion and exclusion criteria were enrolled into this study. Patients underwent a baseline neurological evaluation and randomly assigned to one of the 4 neuropathic medications for 12 weeks. The co-primary outcomes are the change in Likert-like pain scale and quit rates. The outcome measures were performed at baseline, week 4, 8 and 12. Results: There were a total of 402 CSPN patients with 134 , 126,73 , and 69 randomized to nortriptyline, duloxetine, pregabalin, and mexiletine, respectively. The posterior probability each treatment was best were $0.52,0.43,0.05$, and 0.00 , with efficacy rates $25.4 \%$, $23.0 \%, 15.1 \%, 20.3 \%$ and quit rates of $38.1 \%$, $37.3 \%, 42.5 \%, 58.0 \%$, respectively. Conclusion: Mexiletine met our criteria for being a loser, primarily due to side effects. While there was no clear winner, overall nortriptyline and duloxetine outperformed pregabalin and mexiletine.
PS2Group3-052 / \#938

Topic: Group 3 - Peripheral Neuropathy, Cranial Nerves, Mononeuropathies: Clinical Features, Pathophysiology, Therapy

\section{A NEW MUTATION IN MORC 2 CAUSES SCAPULOPERONEAL CHARCOT-MARIE-TOOTH NEUROPATHY: DAVIDENKOW SYNDROME}

\author{
Marina Frasquet ${ }^{1}$, Fernando Mas $^{2}$, Juan Francisco \\ Vázquez-Costa ${ }^{3}$, Carmen Espinós ${ }^{4}$, Vincenzo \\ Lupo $^{5}$, Teresa Sevilla ${ }^{6}$ \\ ${ }^{1}$ Neuromuscular And Ataxia Research Group, \\ Instituto de Investigación Sanitaria La Fe, \\ Valencia, ES, ${ }^{2}$ Radiology, Eresa, Hospital \\ Universitari i Polècnic La Fe, Valencia, \\ ES: ${ }^{3}$ Neurology, Hospital Universitari i Politècnic \\ La Fe, Valencia, ES; ${ }^{4}$ Unit Of Genetics And \\ Genomics Of Neuromuscular And \\ Neurodegenerative Disorders And Service Of \\ Genomics And Traslational Genetics, Centro de \\ Investigación Principe Felipe (CIPF), and Joint \\ Unit for research on Rare Diseases, CIPF-IISLa \\ $\mathrm{Fe}$, Valencia, ES:5 Unit Of Genetics And Genomics \\ Of Neuromuscular And Neurodegenerative \\ Disorders And Service Of Genomics And \\ Traslational Genetics, Centro de Investigación \\ Principe Felipe (CIPF), and Joint Unit for \\ Research on Rare Diseases, CIPF-IISLa Fe, \\ Valencia, ES: ${ }^{6}$ Neurology, Hospital Universitari i \\ Politècnic La Fe, Universitat de València and \\ CIBERER, Valencia, ES
}

Background: Davidenkow (1927) described the clinical picture of a neuropathy which was characterized by onset of weakness in legs' anteroexternal group, pes cavus, involvement of the scapular girdle, and distal hypoesthesia in limbs. He called this syndrome "scapuloperoneal amyotrophy". Our objective is to report clinical, muscle imaging, neurophysiological and molecular features of a patient with a new mutation in MORC2 (c.1031C > T; p.A344V) associated with a scapuloperoneal phenotype. Methods: Clinical evaluation, electrophysiological study and muscular magnetic resonance imaging (MRI) of a patient who presented progressive muscle weakness since the second decade of life and with a positive family history (affected father). Genetic study was reached by means of a gene panel. The proband, his affected father and two 
healthy brothers were genetically studied. Results: A 45-year-old patient presented inability to walk on heels since childhood. He developed progressive distal weakness in the lower limbs at age 12 with frequent falls, and then in upper extremities with consequent difficulty in raising arms and in fine object manipulation with hands. Clinical examination showed weakness in proximal and distal muscle of upper and lower limbs, with relative preservation of flexo-extension of elbows and knees. Pinprick and vibration sensation were reduced in distal lower and upper limbs. Electrophysiological studies were compatible with an asymmetric axonal motor and sensory neuropathy, which was in concordance with the disease severity. Muscle MRI showed fatty infiltration in muscles of the shoulder and pelvis girdle, and also in thighs, legs and feet. Fatty infiltration had a multifocal pattern with preservation of some intrinsic muscles of the feet and affecting some muscles of the shoulder girdle such as the deltoid and subscapular muscles. Conclusion: This patient presents a phenotype compatible with Davidenkow Syndrome. The clinical picture of all patients with mutations in MORC2 is reviewed. FUNDING: Instituto de Salud Carlos III (ISCIII) - FEDER [Grants no. PI15/00187 and PI12/00946]; Fundación para la Investigación del Hospital Universitari La Fe [2015/0085]. The Centro de Investigación Biomédica en Red de Enfermedades Raras (CIBERER) is an initiative from the ISCIII.

\section{PS2Group3-053 / \#522}

Topic: Group 3 - Peripheral Neuropathy, Cranial Nerves, Mononeuropathies: Clinical Features, Pathophysiology, Therapy

\section{AUTOPHAGIC NEUROMYOPATHY CAUSED BY P.R140G HEAT SHOCK PROTEIN B1 (HSPB1) MUTATION}

$\underline{\text { Marina Frasquet }}^{1}$, Nuria Muelas², Vincenzo Lupo ${ }^{3}$, Inmaculada Azorín ${ }^{4}$, Roger Vílchez ${ }^{1}$, Maria Jose Chumillas $^{5}$, Ricard Rojas-García ${ }^{6}$, Carmen Espinós ${ }^{7}$, Teresa Sevilla ${ }^{8}$, Juan J. Vílchez ${ }^{8}$ ${ }^{1}$ Neuromuscular And Ataxia Research Group, Instituto de Investigación Sanitaria La Fe, Valencia, ES: ${ }^{2}$ Neurology, Hospital Universitari i Politècnic La Fe and CIBERER, Valencia, ES, ${ }^{3}$ Unit Of Genetics And Genomics Of Neuromuscular And Neurodegenerative Disorders And Service Of Genomics And Traslational Genetics, Centro de
Investigación Príncipe Felipe (CIPF), and Joint Unit for Research on Rare Diseases, CIPF-IISLa $\mathrm{Fe}$, Valencia, ES $;{ }^{4}$ Neuromuscular And Ataxia Research Group, Instituto de Investigación Sanitaria La Fe and CIBERER, Valencia, ES $;{ }^{5}$ Clinical Neurophysiology, Hospital Universitari $i$ Politècnic La Fe and CIBERER, Valencia, ES; ${ }^{6}$ Neuromuscular Diseases Unit, Hospital de la Santa Creu i Sant Pau and Universitat Autònoma de Barcelona, Barcelona, ES $;{ }^{7}$ Unit Of Genetics And Genomics Of Neuromuscular And Neurodegenerative Disorders And Service Of Genomics And Traslational Genetics, Centro de Investigación Príncipe Felipe (CIPF), and Joint Unit for research on Rare Diseases, CIPF-IISLa Fe, Valencia, ES; ${ }^{8}$ Neurology, Hospital Universitari i Politècnic La Fe, Universitat de València and CIBERER, Valencia, ES

Background: Mutations in the heat shock protein B1 (HSPB1) gene are responsible of a variety of neuromuscular disorders including diverse forms of neuropathies and rare cases of distal myopathy, the latter not being fully characterized yet. The mechanism responsible of these variable phenotypic manifestations reminds unrevealed. We report a large cohort of patients from different families harboring the same mutation in the HSPB1 gene. Our aim is to provide novel data that contribute to the understanding of HSPB 1 -related disorders. Methods: We analyze patients' clinical, neurophysiological and muscle MRI features. We provide pathological data from muscle and sural nerve biopsies that were studied by conventional, immune-fluorescence and electron-microscopic methods. Genetic diagnosis was performed in some cases by targeted Sanger sequencing and in others by means of an in-home designed gene panel for testing Charcot-Marie-Tooth (CMT) and distal hereditary motor neuropathy (dHMN) genes. Results: The cohort comprises 18 patients (6 families) harboring the HSPB1 p.R140G mutation and living in Eastern Spain. Twelve subjects from 5 families developed a neuropathy that started as a dHMN in 10 of them and as an axonal Charcot-Marie-Tooth (CMT2) in the other two. The additional six patients, all belonging to the same family, presented a myopathic picture that was characterized by raised serum creatine-kinase (CK) and progressive distal weakness in three cases, a longlasting asymptomatic hyperCKemia in two others, while the sixth case resulted to be an asymptomatic mutation carrier. Pathologically the myopathy was 
characterized as an autophagic vacuolar myopathy (AVM) defined by the occurrence of membrane lined vacuoles with sarcolemma features, proliferation of autophagosomes and the presence of aggregates containing the autophagic markers LC3-II and p62. The nerve pathology showed loss of myelinated and unmyelinated fibres, axonal atrophy, myelin remodeling, neurofilament disarrangement and accumulation of autophagic vacuoles in axoplasm and Schwann cell cytoplasm. Conclusion: This study gives detailed insights into the clinical presentation of HSPB1 gene mutations and expands the list of causes of AVMs. Our observations may also contribute to guide futures research approaches because of the resemblance of $H S P B 1$-related neuro-myopathy to colchicine myopathy caused by an alteration of b-tubuline, a molecule that is involved in the experimental neuropathy of HSPB1 transgenic models. FUNDING: Instituto de Salud Carlos III (ISCIII) FEDER [Grants no. PI15/00187, PI12/00946 and PI16/00316]; Fundación para la Investigación del Hospital Universitari La Fe [2015/0085]; Fundación Isabel Gemio; The Centro de Investigación Biomédica en Red de Enfermedades Raras (CIBERER) is an initiative from the ISCIII.

\section{PS2Group3-054 / \#227}

Topic: Group 3 - Peripheral Neuropathy, Cranial Nerves, Mononeuropathies: Clinical Features, Pathophysiology, Therapy

\section{HEREDITARY TRANSTHYRETIN AMYLOIODOSIS MIMICKING REFRACTORY CHRONIC INFLAMMATORY DEMYELINATING POLYRADICULONEUROPATHY}

Kyomin Choi ${ }^{1}$, Jeeyoung $\mathrm{Oh}^{2}$

${ }^{1}$ Department Of Neurology, Konkuk University

Medical Center, Seoul, KR; ${ }^{2}$ Department Of

Neurology, Konkuk University Medical Center,

Seoul, KR

Background: Transthyretin (TTR) amyloidosis is a life-threatening systemic amyloidosis caused by autosomal dominant mutation of the TTR gene. Amyloid deposition in peripheral nerves causes polyneuropathy known as familiar amyloidotic polyneuropathy (TTR-FAP). Chronic inflammatory demyelinating polyradiculoneuropathy (CIDP) is clinically defined as a 'chronically progressive, stepwise or recurrent proximal and distal weakness and sensory dysfunction of all extremities, developing over at least 2 months. Patients with CIDP were treated by immunotherapy. However, $20-33 \%$ of CIDP patients remain refractory to these therapies. The refractory CIDP is not completely understood, incorrect diagnosis is considered one of causes of therapeutic failure. To report this issue, we performed retrospective review of 3 patients who were misdiagnosed refractory CIDP and finally confirmed TTR-FAP by genetic analysis. Methods: We reviewed three patients who were considered refractory CIDP and finally confirmed alternate diagnosis of TTR-FAP. All of patients underwent intensive neurologic examination, and serial electrophysiological studies. Other types of primary and secondary amyloidosis and paraproteinemic polyneuropathy were excluded by laboratory testing of protein immune electrophoresis in serum and urine. TTR gene mutations and types were identified by polymerase chain reaction and direct sequencing of TTR gene after informed consent. Results: Patient 1. This male patient initially presented paresthesia of bilateral lower limbs at the age of 38 years. In electrophysiologic study, conduction block and temporal dispersion of compound muscle action potential (CMAP) were shown on three nerves in bilateral upper limbs. He treated with prednisolone and immunoglobulin but no improvement. After the presentation of severe autonomic symptoms, he diagnosed amyloidosis from the biopsy of colon and genetically confirmed to have Lys35Asn mutation in TTR gene. Patient 2. This female patient initially presented urinary incontinence at age of 45 years. She was referred to neurology department due to progressive generalized weakness. In electrophysiological study, delayed terminal latencies, slow nerve conduction velocities(NCVs) and extended duration of CMAP suggested demyelinating neuropathy. Despite administration of steroid, her symptoms kept worsening and she complained of decreased visual acuity. Considering cataracts caused by steroid, she was performed ophthalmologic evaluation. Vitreous opacity associated to amyloid deposit was found. Lys35Asn heterozygous mutation of TTR gene was demonstrated. Patient 3. A 46-year-old man complained weakness of distal lower limbs. His body weight decreased 11 kilograms for a year due to chronic diarrhea. Following electrophysiological study, temporal dispersion, delayed terminal latencies and slow NCVs were presented on three nerves of bilateral upper limbs. He 
was treated by steroid, but there was no clinical improvement. The gastrointestinal symptoms of amyloidosis are demonstrated by tissue biopsy performed in colon. On genetic analysis, heterozygous mutation of Ala36Pro was detected in TTR gene. Conclusion: Our findings showed that the main reason of inadequate treatment outcome might be incorrect diagnosis of CIDP. These three patients had significant features indicating clues of alternative diagnosis. First, coexisting symptoms including gastrointestinal or cardiac diseases and autonomic dysfunctions. Second, these three patients did not show elevation of CSF protein. Third, these 3 patients presented distal dominant weakness. Clinician should be aware that electrophysiological findings of TTR-FAP sometimes can mimic the demyelinating diseases.

\section{PS2Group3-055 / \#565}

Topic: Group 3 - Peripheral Neuropathy, Cranial Nerves, Mononeuropathies: Clinical Features, Pathophysiology, Therapy

\section{TRANSTHYRETIN REDUCTION WITH PATISIRAN IN THE APOLLO PHASE 3 STUDY}

$\underline{\text { Teresa Coelho }}^{1}$, David Adams ${ }^{2}$, Alejandra Gonzalez-Duarte $^{3}$, William O'Riordan ${ }^{4}$, Chih-Chao Yang $^{5}$, Michael Polydefkis ${ }^{6}$, Arnt Kristen ${ }^{7}$, Ivaylo Tournev $^{8}$, Hartmut Schmidt ${ }^{9}$, John Berk ${ }^{10}$, KonPing Lin ${ }^{11}$, Pritesh Gandhi ${ }^{12}$, Marianne Sweetser ${ }^{12}$, Christine Powell ${ }^{12}$, Jared Gollob ${ }^{12}$, Ole Suhr ${ }^{13}$ ${ }^{1}$ Hospital de Santo Antonio, Porto, PT; ${ }^{2}$ Neurology Department, LFB, Le Kremlin Bicetre, FR $;^{3}$ Instituto Nacional de Ciencias Medicas y Nutricion, Salvador Zubiran, MX; ${ }^{4}$ eStudy Site, La Mesa, NM, US, ${ }^{5}$ National Taiwan University Hostpital, Taipei, TW; ${ }^{6}$ Johns Hopkins, Baltimore, MD, US $;^{7}$ Heidelberg University Hospital, Heidelberg, $D E ;{ }^{8}$ University Multiprofile Hospital for Active Treatment, Sofia, BG; ${ }^{9}$ University Hospital Muenster, Muenster, DE: ${ }^{10}$ Boston Universty, Boston, MA, US: ${ }^{11}$ Taipei Veterans General Hospital, Taipei, TW; ${ }^{12}$ Alnylam Pharmaceuticals, Cambridge, MA, US: ${ }^{13}$ Department Of Public Health And Clinical Medicine, Umea University, Umea, $S E$

Background: Mutations in transthyretin (TTR), whose physiological roles include binding to retinol binding protein (RBP) which then binds retinol (also known as vitamin A), and thyroxin (T4), can result in multi-organ TTR amyloid deposition causing a life-threatening disease known as hereditary transthyretin-mediated (hATTR) amyloidosis. hATTR manifests in a heterogeneous manner with clinical presentations including sensorimotor and autonomic neuropathy, gastrointestinal symptoms, as well as cardiomyopathy. Based on the hypothesis that the reduction of circulating TTR levels will reduce TTR amyloid deposition leading to stabilization or improvement of disease, patisiran, an investigational RNAi therapeutic, was evaluated in patients with hATTR amyloidosis. Patisiran resulted in improvement in primary, secondary, as well as exploratory endpoints and was generally well tolerated in the APOLLO study. Furthermore, patisiran resulted in rapid, sustained and durable TTR reduction. Given the wide range of TTR genotypes observed in this patient population, we investigated the degree of reduction for TTR, as well as additional pharmacodynamic (PD) assessments RBP and vitamin A, achieved in patisiran patients in APOLLO on a mutational basis. Methods: APOLLO was a Phase 3, randomized (2:1), double-blind, study of patisiran $0.3 \mathrm{mg} / \mathrm{kg}$ or placebo IV q3W in hATTR amyloidosis patients with polyneuropathy (NCT01960348). Males or females (18 to 85 years of age) with a diagnosis of hATTR amyloidosis with a documented TTR mutation, with a NIS of 5 to 130 and a PND score of $\leq 3 \mathrm{~b}$ at baseline were eligible. Serum TTR levels were measured serially by ELISA and serum RBP was quantified using nephelometry. Results: APOLLO enrolled 225 patients with a mean age of 61 years; $45 \%$ and $55 \%$ had FAP Stage 1 , or 2 , respectively; $76 \%$ had PND $>1 ; 56 \%$ had cardiac involvement. Enrolled patients had one of 39 different mutations: $43 \% \mathrm{~V} 30 \mathrm{M}$ and $58 \%$ non-V30M mutations. At 18 months, mean TTR reduction was $4.8 \%$ $(n=47)$ in the placebo group and $84.3 \%(n=130)$ for the patisiran group. Among patients with the V30M and non-V30M mutation groups, mean TTR reduction in the patisiran groups was $79.7 \%(\mathrm{n}=56)$ and $76.5 \%(n=92)$, respectively. Further, a similar effect on TTR was observed across the 29 different nonV30M mutations evaluated in the patisiran group. As expected based on the mechanism of action, trends at 18 months for RBP and vitamin A were consistent with patisiran TTR reduction in each population for RBP: overall $45.3 \% \quad(n=146)$, V30M $60.2 \%,(n=56)$, non-V30M $36.0 \%(n=90)$ and for vitamin A: overall $62.4 \%(\mathrm{n}=145), \quad \mathrm{V} 30 \mathrm{M} \quad 68.9 \%$ 
$(\mathrm{n}=56)$, non-V30M 58.3\% ( $\mathrm{n}=89)$. Safety events were similar between treatment arms with no clinical manifestations of vitamin A deficiency or thyroid disorder. Detailed data, including earlier timepoints, to be presented. Conclusion: Patisiran resulted in robust and durable serum TTR reduction throughout 18 months of dosing in V30M and non-V30M patients. These data show that patisiran had a similar effect on TTR in patients with hATTR amyloidosis regardless of TTR genotype.

\section{PS2Group3-056 / \#589}

Topic: Group 3 - Peripheral Neuropathy, Cranial Nerves, Mononeuropathies: Clinical Features, Pathophysiology, Therapy

\section{INFLAMMATORY NEUROPATHY AFTER IMMUNE CHECKPOINT THERAPY}

Min-Xia Wang ${ }^{1}$, Jeannette Lechner-Scott ${ }^{2}$, Toan Nguyen ${ }^{1}$, Judith M. Spies ${ }^{1}$

${ }^{1}$ University of Sydney, Royal Prince Alfred Hospital, Sydney, NSW, AU; 22. University of Newcastle, John Hunter Hospital, Newcastle, NSW, AU

Background: The immune checkpoint inhibitor therapies, anti-programmed cell death-1 (PD-1) and anti-CTLA4 monoclonal antibodies, are among the most promising new therapies for a variety of cancers. Despite significant clinical benefits, these therapies can lead to immune-related adverse events in a small number of patients. These include gastrointestinal, dermatologic, hepatic, endocrine, pulmonary and neurological complications. Severe neurological complications, although rare, require early recognition and management to prevent irreversible neurological deficit. A variety of peripheral neuropathies have been described after checkpoint inhibitor therapy, including inflammatory demyelinating neuropathies. Here we present the nerve pathology in two cases of inflammatory axonal neuropathy following anti-PD1 therapy. Methods: Case 1. A 64 year-old woman developed subacute asymmetric weakness and sensory disturbance in the lower limbs, and paraesthesia at hands after 4 cycles of anti-PD1 (pembrolizumab) treatment for mesothelioma. MRI showed no evidence of lumbosacral root or plexus pathology. A sural nerve biopsy showed a marked reduction in myelinated fibre density, scattered nerve fibres undergoing active axonal degeneration and an epineurial vessel obliterated by intense inflammatory cell infiltrates (figure), suggestive of a vasculitic neuropathy. Case 2. A 73 year-old man presented with myalgia in lower limbs, left foot drop, speech and swallowing difficulties after the second dose of pembrolizumab for metastatic melanoma. He had a left foot drop, symmetric mild proximal upper limb weakness and distal loss of pin and temperature sensation in the feet. Electrophysiology revealed an axonal peripheral neuropathy with widespread denervation. A right sural nerve biopsy showed a chronic active axonal neuropathy with mild inflammation (inflammatory cells adjacent to endoneurial vessels). Results: Both patients responded well to cessation of anti-PD1 treatment and high dose steroid. Conclusion: A variety of immune mediated neurological complications have been described with immune checkpoint inhibitor therapy including peripheral neuropathy, myasthenia gravis, myositis, aseptic meningitis, CNS demyelination and encephalitis. Peripheral neuropathy is the most common neurological complication and is estimated to occur in perhaps $1 \%$ of patients. Distal sensorimotor neuropathy, GBS, CIDP, polyradiculitis and enteric neuropathy have been reported. In one series, $50 \%$ of cases were mild and responded to cessation of therapy $+/$ steroids. In more severe cases further immunomodulatory therapy is required. These two cases provided histopathological evidence of vasculitic/inflammatory neuropathy with ani-PD1 therapy. With increased use of immune checkpoint therapy in cancer, it is important for clinicians to remain vigilant in order to recognise symptoms of immune mediated adverse events, and to initiate prompt management. 


\section{PS2Group3-057 / \#646}

Topic: Group 3 - Peripheral Neuropathy, Cranial Nerves, Mononeuropathies: Clinical Features, Pathophysiology, Therapy

\section{BENEFIT-RISK PROFILE OF INTRAVENOUS IMMUNOGLOBULIN (IVIG) AND SUBCUTANEOUS IMMUNOGLOBULIN (SCIG) IN CIDP: THE PATH STUDY}

Amgad Shebl ${ }^{1}$, Billie L. Durn ${ }^{2}$, Vera Bril ${ }^{3}$, Ingemar Merkies $^{4}$, Hans-Peter Hartung ${ }^{5}$, Richard A. Lewis ${ }^{6}$, Gen Sobue ${ }^{7}$, John-Philip Lawo ${ }^{1}$, David R. Cornblath $^{8}$, Orell Mielke ${ }^{1}$, Ivo Van Schaik ${ }^{9}$ ${ }^{1}$ CSL Behring, Marburg, DE; ${ }^{2}$ CSL Behring, Fuquay Varina, NC, US, ${ }^{3}$ Department Of Medicine (neurology), University Health Network, University of Toronto, Toronto, CA, ${ }^{4}$ Department Of Neurology, Maastricht University Medical Center, Maastricht, NL, ${ }^{5}$ Department Of Neurology, Heinrich-Heine University, Duesseldorf, DE; ${ }^{6}$ Department $O f$ Neurology, Cedars-Sinai Medical Center, Los Angeles, US; ${ }^{7}$ Department Of Neurology, Nagoya University Graduate School of Medicine, Nagoya, $J P{ }^{8}$ Department Of Neurology, Johns Hopkins University, Baltimore, MD, US; ${ }^{9}$ Department Of Neurology, Academic Medical Center, University of Amsterdam, Amsterdam, NL

Background: PATH investigated efficacy and safety of SCIG (IgPro20, CSL Behring) as maintenance therapy for chronic inflammatory demyelinating polyneuropathy (CIDP). Before randomisation to IgPro20 or placebo, subjects underwent IVIG withdrawal and, upon clinical deterioration, were re-stabilised with IVIG (IgPro10, CSL Behring). IgPro10 and IgPro20 have the same manufacturing process with the only differences between products being the final immunoglobulin concentration and administration route. The benefit-risk profiles of IgPro10 IVIG and IgPro20 SCIG are evaluated here. Methods: IVIG re-stabilisation comprised an initial dose of $2 \mathrm{~g} / \mathrm{kg}$ followed by 3-4 doses of $1 \mathrm{~g} / \mathrm{kg}$ at 3 -week intervals. Subjects were then randomised to weekly SCIG maintenance therapy $(0.2$ or $0.4 \mathrm{~g} / \mathrm{kg})$ or placebo for 24 weeks. The adverse event (AE) profile and relapse (change in adjusted Inflammatory Neuropathy Cause and Treatment [INCAT] score) were analysed for both products. Results: A total of 207 subjects received 1894 IVIG infusions and 115 of these subjects subsequently received 4225 SCIG infusions. The most frequent AEs were headache (IVIG) and local reactions (SCIG). The majority of headaches $(65 \%)$ and local reactions $(95 \%)$ were mild. With IVIG, 9 haemolysis AEs occurred (all non-serious and resolved without transfusion); none occurred with SCIG. No thromboembolic events, renal failures or deaths were reported (Tables 1\&2). A total of $83 \%$ of subjects re-stabilised on IVIG; subsequently, $81 \%$ of subjects did not relapse on SCIG 0.4 g/kg (0.2 g/kg: 67\%; placebo: 44\%). Conclusion: Both IVIG and SCIG had the expected effectiveness reported in literature, while rates of systemic AEs were lower with both SCIG doses.

Table 1. Summary of SCIG (IgPro20) adverse events

\begin{tabular}{|c|c|c|c|c|}
\hline & \multicolumn{2}{|c|}{$\begin{array}{c}\text { IgPro20 }(0.2 \mathrm{~g} / \mathrm{kg}) \\
(\mathrm{N}=57 ; \mid=2007)\end{array}$} & \multicolumn{2}{|c|}{$\begin{array}{c}\text { IgPro20 }(0.4 \mathrm{~g} / \mathrm{kg}) \\
(\mathrm{N}=58 ; \mathrm{l}=2216)\end{array}$} \\
\hline & Subjects (\%) & $\begin{array}{c}\text { Event (rate } \\
\text { per infusion) }\end{array}$ & Subjects (\%) & \begin{tabular}{|c|}
$\begin{array}{c}\text { Event (rate per } \\
\text { infusion) }\end{array}$ \\
\end{tabular} \\
\hline Any AE & $33(58)$ & $158(0.08)$ & $30(52)$ & $114(0.05)$ \\
\hline Any serious AE & $3(5.3)$ & $5(0.002)$ & $2(3.4)$ & $5(0.002)$ \\
\hline Causally related AE & $17(29.8)$ & $71(0.035)$ & $20(34.5)$ & $64(0.029)$ \\
\hline Local reactions* & $11(19.3)$ & $54(0.027)$ & $17(29.3)$ & $49(0.022)$ \\
\hline Headache & $4(7.0)$ & $5(0.002)$ & $4(6.9)$ & $4(0.002)$ \\
\hline Nausea & $0(0.0)$ & $0(0.000)$ & $0(0.0)$ & $0(0.000)$ \\
\hline Haemolysis & $0(0.0)$ & $0(0.000)$ & $0(0.0)$ & $0(0.000)$ \\
\hline
\end{tabular}

I, infusions $N$, number of subjects

The population who entered the SCIG randomised phase were a subset of the 207 subjects in the IVGG re-stabilisation population.

"Local reactions included all AEs reported within the MedORA high level terms "Administration Site Reactions NEC", "Infusion Site Reactions", and "Injection Site Reactions". 
Table 2. Summary of IVIG (IgPro10) adverse events

\begin{tabular}{|l|c|c|c|c|}
\hline \multirow{2}{*}{} & \multicolumn{2}{|c|}{$\begin{array}{c}\text { IgPro10 (re-stabilisation) } \\
\text { (N=207; I=1620) }\end{array}$} & \multicolumn{2}{|c|}{$\begin{array}{c}\text { IgPro10 (rescue) } \\
\text { (N=60; I=274) }\end{array}$} \\
\cline { 2 - 5 } & $\begin{array}{c}\text { Event (rate } \\
\text { per } \\
\text { infusion) }\end{array}$ & $\begin{array}{c}\text { Event (rate } \\
\text { per infusion) }\end{array}$ \\
\hline Any AE & $100(48.3)$ & $284(0.175)$ & $17(28.3)$ & $39(0.142)$ \\
\hline Any serious AE & $11(5.3)$ & $11(0.007)$ & $2(3.3)$ & $4(0.015)$ \\
\hline Causally related AE & $57(27.5)$ & $115(0.071)$ & $13(21.7)$ & $24(0.088)$ \\
\hline Local reactions* & $\mathrm{NA}$ & $\mathrm{NA}$ & $\mathrm{NA}$ & $\mathrm{NA}$ \\
\hline Headache & $34(16.4)$ & $53(0.033)$ & $4(6.7)$ & $6(0.022)$ \\
\hline Nausea & $10(4.8)$ & $12(0.007)$ & $6(10.0)$ & $4(0.015$ \\
\hline Haemolysis & $7(3.4)$ & $7(0.004)$ & $2(3.3)$ & $2(0.007)$ \\
\hline
\end{tabular}

1, infusions $N$, number of subjects; NA, not applicable

The IVIG rescue populacion were a subset of the 172 subjects (induding those on the placebo arm) who entered the SCIG randomised phase of the study. "Local reactions inchuded all AEs reported wikh in the MedoRA high level terms "Administration Size Reactions NEC" "Infusion Size Reactions", and "Injection Site Reactions.

\section{PS2Group3-058 / \#703}

Topic: Group 3 - Peripheral Neuropathy, Cranial Nerves, Mononeuropathies: Clinical Features, Pathophysiology, Therapy

\section{EPIDEMIOLOGY OF HEREDITARY MOTOR-SENSORY \\ NEUROPATHIES IN THE \\ POPULATION OF THE REPUBLIC OF BASHKORTOSTAN}

Elena Saifullina $^{1}$, Rim Magzhanov ${ }^{2}$, Irina Khidiyatova $^{3}$, Elsa Khusnutdinova ${ }^{3}$

${ }^{1}$ Neurology And Medical Genetics, Bashkir State Medical University, Ufa, RU: ${ }^{2}$ Bashkir State Medical University, Ufa, $R U,{ }^{3}$ Institute of Biochemistry and Genetics, Ufa, RU

Background: Hereditary motor-sensory neuropathies (HMSN, Charcot-Marie-Tooth disease) are the most common inherited disorders of the peripheral nervous system, with an overall prevalence estimated at 9.37 - 20.1/ 100000 [Barreto L.C. et al., 2016]. The aim of our study was to estimate the prevalence of HMSN in the population of the Republic of Bashkortostan (Russian Federation). Methods: The study covers the 46-years period from 01 January 1970 to 01 January 2016 in the territory of Bashkortostan where, according to the 01.01.2016, the population was 4,071,064 $(1,907,815$ males and 2,164,249 females). Data on the patients with HMSN were obtained from registry "Hereditary motor-sensory neuropaties in the Republic of Bashkortostan". The prevalence of the disease was calculated per 100,000 population. Crude rates were standardized by age using the method of direct standardization according to the world population. Results: We registered 540 patients with HMSN in Bashkortostan: 330 males and 210 females. The most frequent type was HMSN 1 (HMSN $1:$ HMSN $2=3: 1$ ). The crude prevalence of HMSN by 01.01 .2016 was $13.3 / 100,000$ for all subtypes. Gender-specific prevalence was 17.3/100,000 for males and 9.7/100,000 for females. The highest age-specific prevalence was registered in the oldest age male group (60+years; 29.3/100,000). The highest age-specific prevalence among females was registered in the group of 40-59 years old $(14.7 / 100,000)$. Conclusion: The prevalence of HMSN in the Republic of Bashkortostan appeared higher than it was established in our previous study $(10.6 / 100,000)$. 


\section{PS2Group3-059 / \#785}

Topic: Group 3 - Peripheral Neuropathy, Cranial Nerves, Mononeuropathies: Clinical Features, Pathophysiology, Therapy

\section{INFLUENCE OF THE EXTRACT OF SUCUPIRA BRANCA (PTERODON EMARGINATUS VOGEL) IN SURAL NERVE OF DIABETIC RATS}

\author{
Bruno M. Bertassoli ${ }^{1}$, Aline S. Faiola ${ }^{1}$, José \\ Armando Jr' ${ }^{1}$, Giuliana Petri ${ }^{1}$, Jose F.R. Santos ${ }^{1}$, \\ Fernando L.A. Fonseca ${ }^{2}$, Alzira A.S. Carvalho ${ }^{3}$, \\ David Feder ${ }^{1}$ \\ ${ }^{1}$ Pharmacology, Faculdade de Medicina do ABC, \\ Santo Andre, BR; ${ }^{2}$ Laboratory Analysis, Faculdade \\ de Medicina do ABC, Santo Andre, BR; ${ }^{3}$ Neurology, \\ Faculdade de Medicina do ABC, Santo Andre, BR
}

Background: The sucupira branca plant (SB) (Pterodon emarginatus Vogel) is used in tradicional medicine treatment as antirheumatic, analgesic, anti-inflammatory and anti-parasitic agent. The tuber portion of sucupira root, also known as "sucupira potatoes" has been used as alternative treatment for diabetes mellitus (DM). The purpose of this study is to evaluate the effect of root extract of SB in the sural nerve of diabetic rats. Methods: In this study, Wistar rats (Ratus norvegicus), were selected with age ranging from 14 to 16 weeks, weighting 250-350g were used. Diabetes was induced by single, subcutaneous injection of aloxane $(120 \mathrm{mg} / \mathrm{kg})$. 7 days after induction, blood glucose was dosed. We considered diabetic rat those one with glycemia higher than $200 \mathrm{mg} / \mathrm{dL}$. The animals were divided into 3 groups. Control group (GC) $(n=7)$ : treated with physiological solution; $(n=8)$ : treated with $100 \mathrm{mg} / \mathrm{kg} /$ day of SB extract and incremental dose group (GD) $(\mathrm{n}=8)$ : treated with $100 \mathrm{mg} / \mathrm{kg} /$ day SB extract of $20 \%$ of the dose every week. All groups were treated via gavage for 6 weeks. The animals were then anesthetized followed by glycemia analysis. Then, they were euthanized by anesthetic overdose, followed by collection of right sural nerve. Semithin sections stained with toluidine blue for axonal morphometric and fiber density. The images were obtained at a magnification of $40 \mathrm{x}$ under an optical microscope using the imageJ program. For statistical analysis, the program Graph prism6 was used, with significance level $p<0.05$. Results: Glycemia levels (mg/dL): GC $744.7 \pm 110.4$; GDC 523.4 181.6; GDP $610.4 \pm 147.8$ with significant difference between GC and GDC groups. Morphometric analysis: mean fiber density of myelinazed axons - GC 3236, GDC 3454 and GDP 3535. We obtained a significant diference $(p<0,05)$ between GC and GDP group. The Feret diameter of myelinated fibers: GC 6,37+1,63um; GDC 5,36+1,73um; GDP 4,87+ 1,65um. Feret diameter of axons: GC 4,54+ 0,97um;GDC 4,02+1,18um; GDP $3,99+1,29$ um. No significant diference was observed among the groups. Conclusion: Although sucupira branca demonstrated to be hypoglycemiant agent, we did not find significant difference among the three groups in relation to Feret diameter of myelinated fibers and axons. However the fiber density was significant difference between the GC and GDP groups. That could suggest a possible positive effect of sucupira branca in peripheral nerves.

\section{PS2Group3-060 / \#925}

Topic: Group 3 - Peripheral Neuropathy, Cranial Nerves, Mononeuropathies: Clinical Features, Pathophysiology, Therapy

\section{EXPANDING THE PHENOTYPIC SPECTRUM OF GARS MUTATIONS}

Giulia Ricci, Veronika Boczonadi, Boglarka Bansagi, Rita Horvath Institute Of Genetic Medicine, Newcastle University, Newcastle, GB

Background: Mutations in the GARS gene have been associated with autosomal dominant disorders such as Charcot-Marie-Tooth 2D (CMT2D), distal hereditary motor neuropathy $\mathrm{V}((\mathrm{dHMN}-\mathrm{V})$ or distal spinal muscular atrophy $\mathrm{V}$ (dSMAV), generally starting in the second decade of life with predominant distal extremity weakness and atrophy of upper limb with slow progression. Methods: We identified two families with novel heterozygous GARS mutations, respectively c.647A $>\mathrm{G}$ p.(His $216 \mathrm{Arg}$ ) and c.1528A $>$ C p.(Lys510Gln). Results: The c.647A $>\mathrm{G}$ variant of GARS gene was detected in the index case (male, 36 years old) and in his mother (66 years old). They showed the classical clinical picture characterized by weakness and atrophy of hand muscles since teenage and, subsequently, by mild and slow progressive distal lower limb weakness, with loss of toe walking ability but without sensory impairment. Electrophysiological examination was consistent with axonal motor neuropathy. In the other family, in 
which the GARS c. $1528 \mathrm{~A}>\mathrm{C}$ variant segregated, we identified three affected members (index case, her daughter and her granddaughter). They showed a different phenotype with a predominant involvement of lower limbs started in early childhood and characterized by tiptoeing and ankle contractures. The index case, at the age of 52, complained of distal wasting in her feet and feet deformities with clawing toes and reduced deep tendon reflexes; she required a stick to aid her walk. The daughter, aged 30, also reported disabling pain Electrophysiological examination detected signs of axonal neuropathy, moderate inactive neurogenic change and neuromuscular junction defect. Conclusion: In the last years, several case descriptions have expanded the phenotypic spectrum associated with mutations in the GARS gene, ranging from almost asymptomatic carriers or patients with a late adult onset disease and mild clinical symptoms to very disabling infantile/childhood forms with diffuse weakness of both upper and lower limbs. Our cases further confirm the clinical variability of GARS mutations.

\section{PS2Group3-061 / \#262}

Topic: Group 3 - Peripheral Neuropathy, Cranial Nerves, Mononeuropathies: Clinical Features, Pathophysiology, Therapy

\section{REDDISH SKIN COLOR CHANGE IN A PATIENT WITH COMPRESSIVE RADIAL NEUROPATHY}

\author{
Byung-Nam Yoon $^{1}$, Ji-Won Yang ${ }^{2}$, Jung-Joon \\ Sung $^{3}$, Kee-Hong Park ${ }^{4}$, Suk-Won Ahn ${ }^{5}$ \\ ${ }^{1}$ Neurology, Inje University College of Medicine, \\ Seoul Paik Hospital, Seoul, KR; ${ }^{2}$ Neurology, \\ Gachon University, Gil medical Center, Incheon, \\ KR $;{ }^{3}$ Neurology, Seoul National University College \\ of medicine, Seoul University Hospital, Seoul, \\ KR $;{ }^{4}$ Neurology, Kyung Sang University Hospital, \\ Jinju, KR $;{ }^{5}$ Neurology, ChungAng University \\ Hospital, Seoul, KR
}

Background: The motor and sensory symptoms caused by compressive radial neuropathy are well known, but the involvement of the autonomic nervous system or the dermatologic symptoms is not well known. In carpal tunnel syndrome (CTS), diverse dermatologic symptoms, including ulceration, blistering, hypohidrosis, Raynaud's phenomenon, and irritant contact dermatitis, have been reported, but these symptoms are rare in radial neuropathy. Here, we report an unusual case of compressive radial neuropathy with reversible reddish skin color change. Methods: Case presentation A 42-year-old male was referred for left wrist drop, finger drop and a tingling sense over the lateral dorsum of the left hand. The patient reported that he was well until 4 days prior, when he was drunk and awoke with the symptoms. For 4 days, slight improvement of weakness occurred. Neurologic examination revealed weakness of the left wrist and finger extension (Medical Research Council grade II). Finger abduction appeared weak, but strength was much better when the hand was passively extended to the neutral position. Wrist and finger flexion was intact. On sensory examination, there was a well-demarcated area of hypoesthesia and a tingling sensation over the lateral dorsum of the left hand between the thumb and index fingers extending into the proximal phalanges of the $2^{\text {nd }}$ finger. In addition, a reddish skin color change was observed in the similar area. According to the clinical information and neurologic examination, he was diagnosed with compressive radial neuropathy. Results: In this case, the patient presented with a reddish skin color change. The reddish skin color change suggested autonomic nervous abnormality caused by vasodilation. These kinds of autonomic dysfunctions are reported in CTS but rarely reported in radial neuropathy. A reddish skin color change is also observed in patients with acute phase complex regional pain syndrome (CRPS). In CRPS, inhibition of sympathetic vasoconstrictor activity leads to vasodilation and skin warming. In our case, similar to CRPS, sympathetic dysfunction caused by radial nerve compression may induce a vasodilation and reddish color of skin innervated by the radial nerve. As the radial motor and sensory symptoms improve, the reddish skin color change would improve. With a reddish skin color change, an elevated skin temperature could be suspected but not evident, and the skin thermometer test was not performed. Conclusion: Autonomic symptoms in radial neuropathy are very unusual. Vasomotor innervation to the skin of the radial aspect of the dorsum of the hand is normally provided by the median nerve, whereas sensory and sudomotor innervation is received via the radial nerve. In a study using local anesthetic nerve block, only one subject of 18 is revealed to have vasomotor innervation via the radial nerve. To the best of our knowledge, this is the first report of compressive radial neuropathy with a reversible reddish skin color change. Compressive 
radial neuropathy is presented with not only motor and sensory symptoms but also autonomic symptoms; therefore, careful examination and inspection are needed at diagnosis.

\section{PS2Group3-062 / \#453}

Topic: Group 3 - Peripheral Neuropathy, Cranial Nerves, Mononeuropathies: Clinical Features, Pathophysiology, Therapy

\section{UNCLASSIFIED CONGENITAL AXONAL NEUROPATHY IN GIPSY FAMILLIES IN PORTUGAL}

Teresa Moreno $^{1}$, Raquel Siva², Isabel Conceição ${ }^{3}$, José Castro ${ }^{3}$, Isabel D. Castro ${ }^{3}$, Fatima Furtado ${ }^{4}$, Oana Moldovan ${ }^{2}$

${ }^{1}$ Unidade De Neuropediatria, Hospital Santa

Maria, Lisboa, PT:2Serviço Genética, Hospital

Santa Maria, Lisbon, PT; ${ }^{3}$ Laboratorio

Neurofisiologia, Hospital Santa Maria, Lisboa, PT; ${ }^{4}$ Serviço De Pediatria, Hospital Joaquim

Fernandes, Beja, PT

Background: Autosomal recessive forms of Charcot-Marie-tooth (CMT) represents around 10\% of all CMT cases and are more frequent in consanguineous populations. Gypsies are a minority with an estimated frequency of 10-14 million (8 million in Europe), with a high prevalence of intrafamilial marriages and consanguinity. Autosomal recessive forms are mainly represented by demyelinating forms of neuropathies, with 3 known genes associated (NDRG1, HK1, SH3TC2), but axonal forms are more rare and less known. Methods: In our tertiary, university hospital (specialized pediatric neuromuscular consultation), 61 patients were observed by possible hereditary neuropathies in the last 15 years, from whom 16 are from Roma origin. The neuropathy was not confirmed in 2, despite familial history. From 14 patients, 8 presented axonal forms, 7 with an unusual congenital phenotype. Results: We present 7 patients, from 3 families, all consanguineous. Both sexes were represented with no gender predominance. All presented at birth or in first weeks of life, with hypotonia, feeding difficulties, congenital hip luxation, artrogriposis and equinovarus feet. An important scoliosis was present in the first year of life. EMG showed an axonal form of predominantly sensitive neuropathy. Clinical evolution was with severe hand and feet deformities, hallux valgus and adducted thumbs, peroneal atrophy and severe scoliosis. Muscular strength was long preserved and didn't represent a major clinical feature. Absent osteotendinous reflexes. Independent walk was achieved between 4 and 10 years old. Sensitive ataxia is also present. Conclusion: From the genetic point of view, strongly suggestive of recessive hereditary transmission, only EGRD was done in one patient and recently a NGS panel was done in another - with no identified genetic cause. We are planning to pursue eventually by WES for a common genetic cause of an unusual, severe, very homogeneous phenotype.

\section{PS2Group3-063 / \#504}

Topic: Group 3 - Peripheral Neuropathy, Cranial Nerves, Mononeuropathies: Clinical Features, Pathophysiology, Therapy

\section{ETIOLOGY AND OUTCOME IN NEUROMUSCULAR PATIENTS PRIMARILY PRESENTING WITH DIAPHRAGMATIC DYSFUNCTION}

Matthias Türk $^{1}$, Irina Weber ${ }^{2}$, Gernot Vogt-Ladner ${ }^{2}$, Rolf Schröder ${ }^{3}$, Martin Winterholler ${ }^{2}$

${ }^{1}$ Department Of Neurology, Friedrich-Alexander University Erlangen-Nürnberg (FAU), Erlangen, DE: ${ }^{2}$ Department Of Neurology, Hospital Rummelsberg, Schwarzenbruck, DE: ${ }^{3}$ Neuropathology, University Hospital Erlangen, Erlangen, DE

Background: In neuromuscular disorders uni- or bilateral diaphragmatic dysfunction is caused by affection of the phrenic nerves, the neuromuscular junctions or the diaphragm muscle. Diaphragmatic dysfunction leading to respiratory problems is common in later stages of neuromuscular disorders, but there is little knowledge about its presence as the first symptom of neuromuscular disorders. The aim of this study was to analyze the etiology and clinical outcome in patients presenting with uni- or bilateral diaphragmatic dysfunction but no prior history or diagnosis of a specific neuromuscular disease. Methods: Retrospective analysis of the medical records of patients, who had been treated at the Department of Neurology of the University Hospital Erlangen (1995 - 2001), Germany, and the Department of Neurology of the Rummelsberg Hospital (2002 - 2014), Germany. Inclusion criteria were 
documented neurophysiological evidence of diaphragmatic dysfunction and absence of previous medical history or diagnosis explaining diaphragmatic dysfunction. Patients suffering from critical illness neuropathy/myopathy were excluded from this study. Medical records were analyzed in terms of medical history, neurological examination, laboratory investigations, cerebrospinal fluid analysis, pulmonary function tests, neurophysiological examinations, and clinical outcome. Results: In total, 30 patients with diaphragmatic dysfunction (17 unilateral; 13 bilateral) were included in this study. Analysis of pulmonary function tests showed that decline of the predicted forced vital capacity (FVC\%pred) in supine position, which was pathologic in all tested patients, is a very sensitive parameter to detect diaphragmatic dysfunction. Neurophysiological examination revealed phrenic neuropathy in 28 patients and disorder of the neuromuscular transmission as well as myopathy in one patient each. Extensive clinical, neurophysiological, radiologic and laboratory testing lead to a definite diagnosis in $71 \%$ of patients with phrenic neuropathy comprising iatrogenic lesion $(n=6)$, amyotrophic lateral sclerosis $(n=5)$, neuralgic amyotrophy $(n=2)$, neuroborreliosis $(\mathrm{n}=2)$, multifocal motor neuropathy $(\mathrm{n}=1)$, chronic inflammatory demyelinating neuropathy $(\mathrm{n}=1)$, post-polio syndrome $(\mathrm{n}=1)$, spondylosis affecting the nerve root $\mathrm{C} 4 / 5(\mathrm{n}=1)$ and diabetes mellitus ( $n=1)$. In $29 \%$ of patients, the etiology of phrenic neuropathy (6 unilateral, 2 bilateral) remained elusive despite extensive work-up. The patients with neurophysiological evidence of a neuromuscular transmission disorder and myopathy were diagnosed with myasthenia gravis and Pompe's disease, respectively. Outcome analysis of all patients with follow-up data revealed full recovery in $19 \%$, partial recovery in $41 \%$, stable course in $15 \%$ and progressive deterioration in $26 \%$ of patients. Latter group comprised all patients with amyotrophic lateral sclerosis. Conclusion: Our study highlights that extensive clinical, respiratory, neurophysiological, radiologic and laboratory testing is obligatory to unravel the diagnosis and etiology of diaphragmatic dysfunction especially in consideration of treatable conditions.
PS2Group3-064 / \#540

Topic: Group 3 - Peripheral Neuropathy, Cranial Nerves, Mononeuropathies: Clinical Features, Pathophysiology, Therapy

\section{IQYMUNE® IS EFFECTIVE AS MAINTENANCE TREATMENT FOR MMN: A RANDOMISED, DOUBLE- BLIND, CROSS-OVER STUDY VERSUS KIOVIG®}

\author{
Rabye Ouaja ${ }^{1}$, Ousmane Alfa Cissé ${ }^{2}$, Eduardo \\ Nobile-Orazio $^{3}$, Jean-Marc Léger ${ }^{4}$ \\ ${ }^{1}$ Immunology Franchise, LFB, LES ULIS , \\ FR ${ }^{2}$ Global Medical Affairs Unit, LFB, Les Ulis, \\ FR, ${ }^{3}$ Humanita Research Hospital, Neurology Unit, \\ Rozzano, IT; ${ }^{4}$ National Referral Center For \\ Neuromuscular Diseases, University Hospital \\ Pitié-Salpêtrière, Paris, FR
}

Background: To demonstrate the non-inferiority of Iqymune $^{\circledR}$ versus Kiovig ${ }^{\circledR}$ in the maintenance therapy of MMN, LFB conducted a phase III, multicenter, randomized, double-blind, active-comparator-controlled, cross-over study. Methods: Twenty-two adult MMN patients, treated with any IVIg (except Kiovig $^{\circledR}$ or Iqymune ${ }^{\circledR}$ ) at an individualized and stable maintenance dose within the range of 1 to $2 \mathrm{~g} / \mathrm{kg}$ every 4 to 8 weeks, were enrolled. They were randomized to receive either Kiovig first and then Iqymune (sequence A) or Iqymune first, then Kiovig (sequence B). Each product was administered for 24 weeks. The primary endpoint was the difference between Iqymune ${ }^{\circledR}$ and Kiovig ${ }^{\circledR}$ in the mean assessments of MMRC 10 sum score (strength of 5 muscle groups in upper limbs and 5 muscle groups in lower limbs, both sides, making a score range from 0 to 100) during the evaluation period (non-inferiority margin of $\Delta=2$ ). Secondary efficacy endpoints were assessed through 3 other MRC sum scores, INCAT disability score, grip strength, clinical global impression and discontinuation of study treatment. Exploratory objectives were assessed through MMN-Rasch-built Overall Disability Scale (RODS), Total serum IgG trough levels, IgM anti-GM1 and anti-GM2 antibodies levels and change in grip strength 2 weeks after the last course compared to the grip strength just before this specific course. Safety was also assessed. Results: Based on a linear mixed-model, the non-inferiority of Iqymune compared to Kiovig was demonstrated, independently from any of the covariates (value at baseline, treatment period and treatment sequence. The difference 
'Iyqymune - Kiovig' estimate was -0.01 with a $95 \%$ CI of [-0.51 to 0.48$]$ that was centered around zero and entirely above the non- inferiority margin of $-\Delta$ $=-2$. No difference was found between products for all secondary endpoints. For categorical efficacy endpoints, analysis was purely descriptive (no statistical test). The number of adverse reactions (AR) and the percentage of affected patients were similar for both products: $39 \mathrm{AR}$ in $45.5 \%$ of patients with Iqymune $^{\circledR}$ vs $32 \mathrm{AR}$ in $52.4 \%$ of patients with Kiovig $^{\circledR}$. No thromboembolic event was observed. Biological tests were performed before and after each treatment course. No IVIg-associated hemolysis or renal function impairment was observed. A marked decrease $(-25 \%)$ in mean neutrophils count was observed after courses with both products, causing sometimes mild and transitory neutropenia. Conclusion: This is the first clinical trial comparing the efficacy and safety of two IVIg in the maintenance treatment of MMN. Iqymune, a novel 10\% IVIg, was found non-inferior to the comparator with regards to primary and secondary endpoints.

\section{PS2Group3-065 / \#361}

Topic: Group 3 - Peripheral Neuropathy, Cranial Nerves, Mononeuropathies: Clinical Features, Pathophysiology, Therapy

\section{ANTI-MAG TITERS PRE/POST DEGLYCOSYLATION IN PATIENTS WITH IGM NEUROPATHY: CORRELATION WITH CLINICAL PHENOTYPE IN 8 CASES}

Katherine Beadon ${ }^{1}$, Jean Neil ${ }^{2}$, Emilien Delmont ${ }^{3}$, B Haghi ${ }^{4}$, L Musset ${ }^{2}$, S Attarian ${ }^{3}$, José Boucraut ${ }^{3}$, Jean-Marc Léger $^{4}$

${ }^{1}$ Neurology, University Hospital Pitié-Salpêtrière, Paris, FR, ${ }^{2}$ Immunology, University Hospital Pitié-Salpêtrière, Paris, FR $;^{3}$ University Hospital Timone, Marseille, FR $;{ }^{4}$ Neurology, University Hospital Pitié-Salpêtrière, Paris, FR

Background: Anti myelin associated glycoprotein (MAG) demyelinating neuropathy associated with IgM monoclonal gammopathy (MG) is a dysimmune disorder resulting in symmetric, distal, sensory neuropathy with variable degrees of ataxia and tremor. There is evidence that anti-MAG antibodies are pathogenic including the induction of demyelination in animal models. However, titers of IgM and anti-MAG antibodies do not seem to correlate with clinical severity, evolution or response to treatment in some cases. MAG is a transmembrane glycoprotein located on periaxonal Schwann cells. The target antigen in anti-MAG neuropathy is HNK-1, a carbohydrate epitope on MAG. MAG shows a high affinity for alpha-2,3-linked sialic acid (2,3-SA). Human monoclonal IgM has 5 heavy chain glycosylation sites. IgM may bind to MAG via these glycan epitopes without clinical effect, resulting in overestimation of anti-MAG titers in patient sera. By removing the clinically neutral interactions, there may be an improvement in the correlation with clinical phenotype and evolution. Methods: Serum was analysed in 8 patients with anti-MAG neuropathy and also in 2 patients with IgM MG and anti-MAG antibodies without anti-MAG neuropathy (Non anti-MAG Neuropathy). Clinical features, Overall Neuropathy Limitations Scale (ONLS) and response to treatment were recorded. For 3 patients, serial measurements of anti-MAG activity were performed 1 or more years apart. IgM was extracted and purified by affinity chromatography. Digestion of IgM was performed by Jack bean alpha-mannosidase. Digestion was assessed using lectin blot with Concanavalin A. IgM extracts were then tested for anti-MAG activity pre and post-deglycosylation using ELISA and Indirect Immunofluorescence (IIF). Results: For the 8 first patients, mean duration of illness was 9 years (1-25). Seven had pure sensory and 1 sensorimotor deficits. All had demyelinating neuropathy. Seven out of 8 had an IgM MG and all anti-MAG antibodies. Five patients received immunosuppressant treatment and 3 showed clinical improvement after treatment. Deglycosylation resulted in decrease in anti-MAG activity in all but 1 patient. Only 1 of 6 patients $(12.5 \%)$ showed a saturation of activity $(>70,000 \mathrm{BTU})$ after deglycosylation vs 6 of 8 patients before $(75 \%)$. In the two patients with non anti-MAG Neuropathy, deglycosylation resulted in a decrease in titers by a factor of 4.1-6.5 (mean 5.4, 20,500-39,000 BTU pre and 3700-8400 BTU post). No correlation was seen between anti-MAG titers pre or post-deglycosylation and ONLS score or stability of the disease. For the patient with IgM MG, neuropathy and serial serum samples, post-deglycosylation results correlated with IgM titers (IgM $3.7 \rightarrow 7.5 \mathrm{~g} / \mathrm{L}$, anti-MAG $22,000 \rightarrow 55,000 \mathrm{BTU}$ ). Conclusion: No correlation between clinical phenotype and anti-MAG activity before or after deglycosylation was found in patients with anti-MAG neuropathy. However, deglycosylation showed interesting results in patients with anti-MAG activity without anti-MAG neuropathy. The activity of 
anti-MAG antibodies is multifactorial and also depends on the affinity and avidity of the antibodytarget interaction. After deglycosylation, the titers may better represent pathogenic activity and help to follow a given patient's clinical status prospectively.

\section{PS2Group3-066 / \#606}

Topic: Group 3 - Peripheral Neuropathy, Cranial Nerves, Mononeuropathies: Clinical Features, Pathophysiology, Therapy

\section{ULTRASONOGRAPHY IS USEFUL IN EVALUATION FOR ULNAR NEUROPATHIES WITH NO LOCALIZATION IN ELECTROPHYSIOLOGICAL STUDIES}

Jae Young An ${ }^{1}$, Dae Woong Bae ${ }^{2}$

${ }^{1}$ Department Of Neurology, St. Vincent Hospital, The Catholic University of Korea, Suwon, KR $;{ }^{2} S t$. Vincent Hospital, The Catholic University of Korea, Suwon, KR

Background: Ulnar neuropathy is the second most common peripheral nerve entrapment neuropathy. Most common compression site of the nerve is within cubital tunnel. Nerve conduction studies (NCS) are the gold standard for localization of nerve compression site. However, there may be false negative findings of NCS. Mild nerve involvement, improper position, nerve dislocation or Martin-Gruber anastomosis (MGA) may explain some false negative results of NCS. Methods: We describe NCS and ultrasonography (US) findings in two patients with pain and numbness in their right $4^{\text {th }}$ and $5^{\text {th }}$ fingers. They complained of minimal weakness of fourth and fifth finger flexion. Results: Nerve conduction studies showed no definite results about localization of ulnar neuropathy except MGA at the forearm. Electromyography revealed high amplitude in Adductor digiti minimi. US showed an increased crosssectional area of right ulnar nerve just below and at the medial epicondyle. Conclusion: This case indicates that US may be another important diagnostic tool for evaluation compressive neuropathy, especially in ulnar neuropathy with MGA.
PS2Group3-067 / \#990

Topic: Group 3 - Peripheral Neuropathy, Cranial Nerves, Mononeuropathies: Clinical Features, Pathophysiology, Therapy

\section{CLINICAL AND GENETIC ANALYSIS OF HEREDITARY PERIPHERAL NEUROPATHY IN EGYPTIAN POPULATION}

Nourelhoda A.A. Haridy ${ }^{1}$, Jana Vandrovcova ${ }^{2}$, Mohamed A. Abd El-Hamed ${ }^{3}$, Sherifa A. Hamed ${ }^{3}$, Henry Houlden ${ }^{2}$

${ }^{1}$ Department Of Neurology And Psychiatry, Assuit University Hospitals, Assiut, EG: ${ }^{2}$ Department Of

Molecular Neuroscience, UCL Institute of neurology, London, GB; ${ }^{3}$ Department Of Neurology And Psychiatry, Assuit university hospitals, Assiut, $E G$

Background: Hereditary neuropathies are the most common inherited neurological diseases. They encompass a range of diseases from those in which the neuropathy is the predominant feature as in CharcotMarie-Tooth (CMT) and related neuropathies, to those in which the neuropathy occurs as part of a multisystem disease as in Friedreich's ataxia and leukodystrophy. With the advent of next-generation sequencing, more than 80 genes and loci have been described in causing inherited neuropathies. A lot of studies for inherited neuropathies were carried out worldwide with no studies so far from Egypt. The aim of our study is to characterize the clinical and genetic features of Egyptian patients with hereditary neuropathy. Methods: We recruited 79 patients from 45 unrelated Egyptian families with a clinical diagnosis suggestive of hereditary neuropathy. After an informed consent, all patient were examined neurologically and systemically. Nerve conduction studies were performed to determine the type of neuropathy (Demylinating or axonal). Acquired causes of neuropathies as nutritional were excluded. Blood samples from all probands and first degree relatives (parents and siblings) were obtained when available. Genomic DNA was extracted according to the standard protocol. Multiplex ligation detection probe amplification (MLPA) was performed as the first genetic testing to exclude the commonest type (CMT1A). Whole exome sequencing was performed for negative MLPA results. Validation of variants was carried out by traditional Sanger sequencing and the detected mutations were segregated in other fam- 
ily members when available. Results: Interestingly, no case with CMT1A was diagnosed in our cohort in spite of the clear autosomal dominant pattern in some families along with confirmed and demyelinating features in the nerve conduction studies. We have reached the molecular diagnosis for 24 patients from 12 families out of 45 so far $(30.37 \%$ patients, $26.66 \%$ families). All detected variants were novel in known neuropathy genes like; NEFL, SH3TC2, FGD4, MFN2, KIF1B, FBLN5, ABHD12, LMNA and GDAP1 with one known variant in SPTLC1. Conclusion: From our study, we conclude that PMP22 duplication is not a common cause for Egyptian inherited neuropathy. Other causes like NEFL, $M F N 2$, and SPTLC1 were found as a cause for autosomal dominant neuropathies. SH3TC2 mutations were detected to be the cause of neuropathy in three families so far which suggest that this gene mutation is a common cause of CMT4 in Egypt. Rare gene mutations like FGD4 were described. Work is still progressing for diagnosis of the other families.

\section{PS2Group3-068 / \#427}

Topic: Group 3 - Peripheral Neuropathy, Cranial Nerves, Mononeuropathies: Clinical Features, Pathophysiology, Therapy

\section{THE USEFULNESS OF NERVE CONDUCTION STUDIES IN CHEIRALGIA PARESTHETICA}

In Soo Joo

Neurology, Ajou University School of Medicine, Suwon, KR

Background: Cheiralgia paresthetica (CP) is an isolated sensory mononeuropathy of the superficial radial nerve. The SRN could be easily damaged by prolonged external compression or diverse procedures. The diagnosis of $\mathrm{CP}$ basically relies on the characteristic sensory symptoms, but nerve conduction studies (NCS) may be useful for identifying the exact site or the extent of nerve injury. Methods: Sixteen CP patients having a relevant clinical history and typical physical findings were recruited for last 7 years. The sensory nerve action potentials and sensory nerve conduction velocities were recorded bilaterally using a modified Shirali's method. Results: Of the 16 patients, $13(81 \%)$ were female. Mean age of onset was 47.7 years. Left side was more commonly involved (left : right : both $=8,6$,
2). Symptom duration was 1 day to 1 year. The most common cause of $\mathrm{CP}$ was venipuncture $(7,44 \%)$, followed by prolonged external compression (4, $25 \%$ ). Only 5 patients $(31.3 \%)$ revealed abnormal NCS findings. The prognosis of CP was relatively good, and did not show any significant correlation with NCS findings. Conclusion: For the diagnosis of $\mathrm{CP}$, taking a careful history and performing a thorough physical examination are more important than the NCS, at least in such case of external compression or minor nerve injury.

\section{PS2Group3-069 / \#728}

Topic: Group 3 - Peripheral Neuropathy, Cranial Nerves, Mononeuropathies: Clinical Features, Pathophysiology, Therapy

\section{BACLOFEN, NALTREXONE AND SORBITOL ALL CONTRIBUTE TO PXT3003-INDUCED MYELINATION IN CMT1A DRG CO-CULTURES}

Nathalie Cholet $^{1}$, Julien Laffaire ${ }^{2}$, Rodolphe Hajj ${ }^{3}$, Daniel Cohen ${ }^{4}$

${ }^{1}$ Experimental Biology, Pharnext, Issy-lesMoulineaux, FR; ${ }^{2}$ Biostatistics, Pharnext, Issy Les

Moulineaux, FR ${ }^{3}$ Experimental Biology,

PHARNEXT, Issy-les-Moulineaux, FR; ${ }^{4} C e o$, PHARNEXT, Issy-les-Moulineaux, FR

Background: Charcot-Marie-Tooth type 1A disease (CMT1A) is due to the duplication of the gene encoding the peripheral myelin protein of $22 \mathrm{kDa}$ (PMP22). CMT1A is characterized by a dys/demyelination of peripheral nerves associated with a reduced nerve conduction velocity of both motor and sensory fibers, all leading to a progressive muscle weakness and loss of proprioception. Although CMT1A is the most common inherited neuropathy, there is currently no approved treatment. We previously showed that a combination of 3 drugs, baclofen, sorbitol and naltrexone (PXT3003) was effective in pre-clinical CMT1A models as well as in a Phase 2 clinical study in CMT1A patients. The aim of this work was to i) demonstrate the ability of PXT3003 to induce myelination of CMT1A neurons, and ii) prove that baclofen, naltrexone and sorbitol all contribute to this myelination induction by comparing the activity of PXT3003 to the drugs alone or combined two by two (duos). Methods: Transgenic rodent models based on Pmp22 overexpression de- 
velop a demyelinating neuropathy mimicking the human disease and offer the possibility to modelize CMT1A features in vitro. Hence, we developed an in vitro model of sensory neurons and Schwann cells co-culture derived from CMT1A transgenic rats that was validated and adapted to 96-well culture plates for drug testing. Total myelin length was quantified with an automatic image analyzer after PMP22 immunostaining. This model allows us to mimic the physiological process of in vivo myelination. Results: We confirmed myelination impairment of CMT1A neurons, as assessed by a decrease of total myelin length in CMT1A cultures compared to wildtype. We subsequently performed a full dose-response analysis of single drugs and showed their own promyelinating dose-dependent activities. We then tested 27 combinations of the three drugs at their sub-active concentrations to assess their synergistic activity when put all together (PXT3003). We found that 5 out of these 3 drug combinations induced myelination whereas none of the 3 possible $2 \mathrm{X} 2$ combinations revealed a significant effect at the same sub-active concentrations, suggesting a synergy between the 3 drugs. Conclusion: We validated a sophisticated co-culture system mimicking the pathophysiological myelination process in CMT1A disease. We confirmed PXT3003 activity on myelination induction and clearly demonstrated the contribution of the 3 drugs to the effect of PXT3003 for the treatment of CMT1A. These findings highlight the value of combinational repurposing of drugs at low doses to act synergistically on pleiotropic pathways and represent an important novel approach for rapid drug development and safety improvement.

\section{PS2Group3-070 / \#922}

Topic: Group 3 - Peripheral Neuropathy, Cranial Nerves, Mononeuropathies: Clinical Features, Pathophysiology, Therapy

\section{AN AUTOSOMAL DOMINANT FAMILY WITH UNCOMPLICATED SPASTIC PARAPLEGIA DUE TO A STOP MUTATION IN HARS}

\author{
Nicolas Dubuisson ${ }^{1}$, Michel Dupuis $^{2}$, Laurent \\ Servais $^{3}$, V Benoit ${ }^{4}$, Christine Verellen-Dumoulin ${ }^{4}$ \\ ${ }^{1}$ Neurology, Avenue Hippocrate 10, Bruxelles, \\ $B E ;{ }^{2}$ Clinique Saint-Pierre Ottignies, Ottignies, \\ BE; ${ }^{3}$ Institute of Myology, Paris, FR; $;{ }^{4}$ Institut de \\ Pathologie et de Génétique (IPG) Gosselies, \\ Gosselies, BE
}

Background: The hereditary spastic paraplegias (HSPs) are clinically and genetically heterogeneous disorders. HSPs are classified as «uncomplicated» if neurological impairment is limited to lower extremity spastic weakness and as «complicated» if the impairment of the uncomplicated HSP is accompanied by other systemic or neurologic abnormalities such as peripheral neuropathy. Methods: A 54 years old woman has been suffering since her thirties from HSP and one of her sons developed the same clinical condition before 10 years of age. The mother is still able to walk after 20 years of evolution. Both patients have brisk reflexes with clonus and Babinski sign. There are no clinical nor electrophysiological signs of peripheral involvement. Results: For the 2 patients, various sets of HSP associated genes were tested negative by Sanger sequencing and NGS. Mendeliome sequencing of 2742 genes (Capture SureSelect Inherited Disease , Agilent followed by sequencing Illumina) performed on the mother ,her husband and their son has shown a stop mutation in HARS (NM_002109.05) to the HSP patients. This mutation c. $499 \mathrm{C}>\mathrm{T}$ p. $\left(\operatorname{Arg} 167^{*}\right)$ has not been described yet. Conclusion: Hars mutation has been reported in 4 families harbouring autosomal dominant axonal and demyelinating motor and sensory neuropathies (HMSN) (Brozkova et al 2015). This gene encodes a histidyl-tRNA synthetase and all the described variations were missense variants. This family is the fifth described family with a HARS variant and the uncomplicated HSP clinical presentation is unique and expands the clinical symptoms from HMSN to autosomal dominant SP. For genetic counseling this gene should be added to the known 41 HSP related genes.

PS2Group3-071 / \#259

Topic: Group 3 - Peripheral Neuropathy, Cranial Nerves, Mononeuropathies: Clinical Features, Pathophysiology, Therapy

\section{CONDUCTION STUDIES OF PHRENIC NERVE IN HEALTHY CHILDREN}

Vladislav Voitenkov ${ }^{1}$, Evgenia Ekusheva ${ }^{2}$, Natalia

Skripchenko ${ }^{3}$

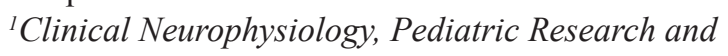

Clinical Center for Infectious Diseases, Saint-

Petersburg, RU; ${ }^{2}$ Advanced Training Institute of the

Federal Medical Biological Agency, Moscow,

$R U ;{ }^{3}$ Pediatric Research and Clinical Center for

Infectious Diseases, Saint-Petersburg, $R U$ 
Background: In adult population conduction studies parameters of phrenic nerve (its CMAP latency and amplitude) are quite well established. In children, on the contrary, these parameters change with the age and tend to disperse widely. Our goal was to establish our own set of normative data for latency and amplitude of phrenic nerve CMAP in healthy children of different age. Methods: 48 healthy children (mean age 9,19 $\pm 5,43$, range 1-18 years, 28 females, 20 males) were enrolled. Conduction studies were performed with active electrode at xiphoid process and reference at the VII intercostal space, with stimulation at the external edge of the lower third of sternocleidomastoid muscle, with maximum stimulation at $40 \mathrm{~mA}$. Additional electrodes were placed on m.Deltoideus and m.Serratus Anterior to control the amount of ecitatory output to the ce rvical plexus (Fig.1). Results: Mean CMAP latency in the group was $5,64 \pm 1,25 \mathrm{~ms}$ and amplitude was $0,66 \pm 0,34 \mu \mathrm{V}$. As the group was subdivided on age subgroups as follows: $1-2$ years $(n=7), 3-5$ years $(n=9), 6-12$ years $(\mathrm{n}=14)$ and 13-18 years $(\mathrm{n}=17)$, CMAP latencies were $4,96 \pm 1,94 ; \quad 5,01 \pm 1,13 ; \quad 5,42 \pm 0,84$ and $6,44 \pm 1,43 \mathrm{~ms}$ and CMAP amplitudes were $1,01 \pm 0,37 ; 0,87 \pm 0,31 ; 0,61 \pm 0,24$ and $0,45 \pm 0,21 \mu \mathrm{V}$. CMAP amplitudes in very young children aged 1-2

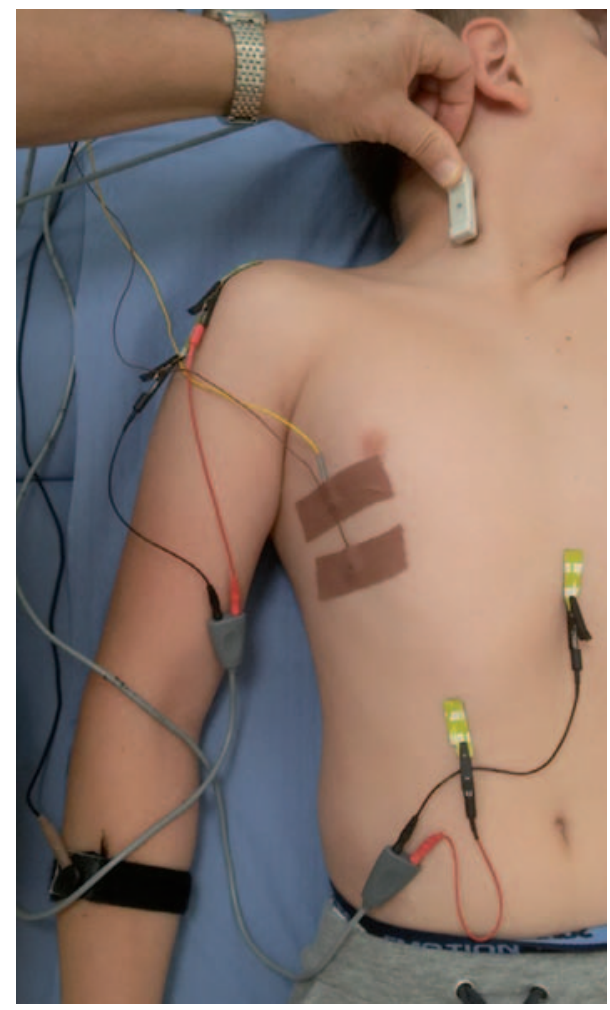

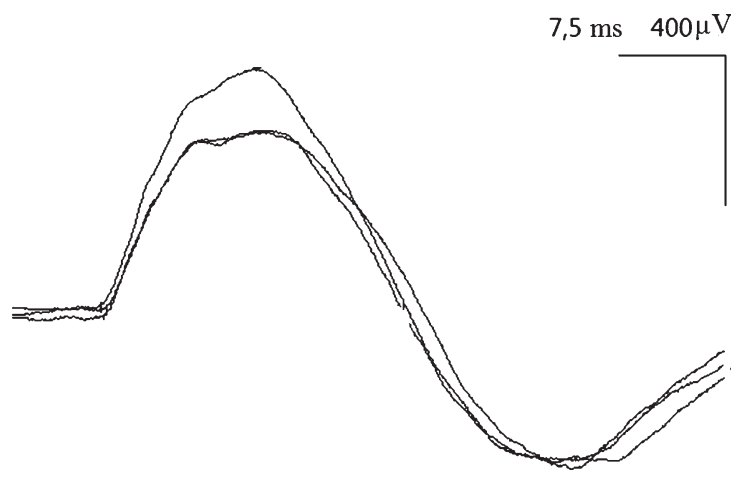

years were significantly $(\mathrm{p}<0.05)$ higher than in children aged 6-12 and 13-18 years. Typical CMAP is presented on Fig. 2. Conclusion: Conduction study of the phrenic nerve, if performed properly, is a simple and reliable method. In pediatric population, though, age dynamic and wide range of normative parameters has to be taken into the consideration. In children CMAP amplitude is significantly higher at the age under 2 years than in older ones (6-18 years).

\section{PS2Group3-072 / \#310}

Topic: Group 3 - Peripheral Neuropathy, Cranial Nerves, Mononeuropathies: Clinical Features, Pathophysiology, Therapy

\section{FIBER TRACTOGRAPHY OF FACIAL NERVE: IMPLICATIONS FOR HEMIFACIAL SPASM}

\author{
Yeow Hoay Koh${ }^{1}$, Ling Ling $\mathrm{Chan}^{2}$, Ee Wei $\mathrm{Lim}^{2}$ \\ ${ }^{1}$ Neurology, Singapore General Hospital, \\ Singapore, $S G ;^{2}$ Singapore General Hospital, \\ Singapore, $S G$
}

Background: Diffusion tensor imaging and fiber tractography of the facial nerve is particularly challenging due to its slenderness and anatomic position in an area fraught with susceptibility and CSF pulsation artifacts. Nevertheless, there is potential utility in surgical navigation and semi-quantitative disease monitoring of response to treatment in various $7-8^{\text {th }}$ $\mathrm{CN}$ pathologies including hemifacial spasm. Methods: Sub-mm inplane resolution DTI scans over the facial nerves were acquired. 3D MR angiography and heavily T2-weighted high resolution scans were also obtained. Fiber tracking of the $7-8^{\text {th }}$ nerves was performed using commercially available software. The FA and MD of the $7-8^{\text {th }}$ nerves tracked are recorded and the DTI tractography quality is 
compared to that reported in the literature. Results: 21 subjects ( 9 men, 12 women) with mean age of 61 years (37-84) were scanned. The $7-8^{\text {th }}$ nerves were successfully tracked in 11 subjects. The $7^{\text {th }}$ nerve can be seen separately from the $8^{\text {th }}$ nerve in 5 subjects. The mean DTI parametrics are: fractional anisotropy $0.301 \pm 0.026(0.271-0.341)$ and mean diffusivity $4820 \pm 492 \times 10^{-6} \mathrm{~mm}^{2} / \mathrm{sec}\left(4000-5600 \times 10^{-6} \mathrm{~mm}^{2} /\right.$ $\mathrm{sec})$. The DTI tractography is compatible with the expected DTI findings of the $7-8^{\text {th }}$ nerves, supporting the validity of our imaging methodology. Conclusion: Our initial data demonstrate that DTI fiber tracking of the facial nerve is clinically feasible and would be useful for evaluation of clinical conditions such as hemifacial spasm.

\section{PS2Group3-073 / \#860}

Topic: Group 3 - Peripheral Neuropathy, Cranial Nerves, Mononeuropathies: Clinical Features, Pathophysiology, Therapy

\section{NEUROMYOPATHY CAUSED BY LONG TERM COLCHICINE THERAPY}

Min Young Chun, Jung Hwan Lee, Kee-Duk Park Neurology, Mokdong Hospital, Ewha Womans University College of Medicine, Seoul, KR

Background: Colchicine is commonly used for the treatment of gout, familial Mediterranean fever, Behcet's disease, and recurring pericarditis with effusion. The use of colchicine is usually safe, but several adverse effects such as gastrointestinal intolerance are known. Neuromyopathy is rare complication and can develop in the setting of acute overdose or chronic administration in therapeutic doses. Two cases of colchicine induced neuromyopathy has been previously reported as acute toxicity in Korea. We reported on a case of neuromyopathy after long term colchicine therapy in Korea. Methods: A 72-year-old man was referred to our institution due to 5 months history of proximal muscle weakness and myalgia. He had angina pectoris and Behcet's disease, leading to the treatment of colchicine (1.2mg daily for about 6 years), cyclosporine, methylprednisolone, simvastatin, and aspirin. Neurologic examination revealed proximal muscle weakness and muscle tenderness on palpitation. Hypesthesia was only present in the tiptoes. Deep tendon reflexes are absent. Results: Laboratory studies for common causes of neuropathy were normal. The serum cy- closporine level was low $(45.10 \mathrm{ng} / \mathrm{mL})$. The serum creatine kinase was elevated (1130 IU/L). The serum creatinine level was in normal rang. Chest and abdominopelvic CT scans did not show any malignant lesions. Nerve conduction studies showed axonal sensorimotor polyneuropathy and needle electromyography revealed a myopathic pattern. Muscle biopsy of biceps brachii demonstrated myofibers with mild variation of size and shape and occasionally noted degenerated or necrotic myofibers. On electron microscopic examination, degenerative or necrotic myofibers show disruption of intermyofibrillar pattern and degenerated organelles with frequently noted autophagic vacuoles. Myofibers with prominent subsarcolemmal accumulation of autophagic vacuoles were also noted. In addition, there were myofibers with smeared $\mathrm{Z}$ line material and myofilament disruption. He was initially suspected with inflammatory myopathy and was treated with steroid pulse therapy. However, muscle weakness did not improve. So, we considered colchicine-induced neuromyopathy and discontinued the colchicine. Then, muscular weakness and myalgia improved steadily, and serum creatine kinase level became decreased into normal range $(25 \mathrm{IU} / \mathrm{L})$. Three months later, his muscle power improved, and he could walk without a cane. Conclusion: Colchicine induced neuromyopathy is a rare toxic complication. However, we should consider 'colchicine induced neuromyopathy' for the patients, who present muscular weakness of unknown origin in a condition of treatment with colchicine because that clinical suspicion is the most important for the diagnosis and treatment is just only the termination of colchicine. In conclusion, this is the first report of the neuromyopathy after long term colchicine therapy in Korea, and we should be cautious to use colchicine. 


\section{PS2Group3-074 / \#372}

Topic: Group 3 - Peripheral Neuropathy, Cranial Nerves, Mononeuropathies: Clinical Features, Pathophysiology, Therapy

\section{CHARCOT MARIE TOOTH DISEASE TYPE 2CC DUE TO A MUTATION IN THE NEUROFILAMENT-HEAVY GENE IN A GERMAN FAMILY}

\author{
Elena Ikenberg ${ }^{1}$, Peter Reilich², Corinna Heller ${ }^{3}$, \\ Angela Abicht ${ }^{4}$, Benedikt Schoser ${ }^{2}$, Maggie C. \\ Walter ${ }^{4}$ \\ ${ }^{1}$ Friedrich-baur Institute, Departement Of \\ Neurology, Ludwigs-Maximilians University of \\ Munich, Munich, DE; ${ }^{2} F r i e d r i c h-b a u r$ Institute, \\ Departement Of Neurology, Ludwigs-Maximilians \\ University of Munich, Munich, DE: ${ }^{3} \mathrm{Ce} G a T \mathrm{GmbH}$ \\ und Praxis für Humangenetik, Tübingen, DE; ${ }^{4}$ Dept. \\ Of Neurology, Ludwig-maximilians-university, \\ Friedrich-Baur-Institute, Munich, DE
}

Background: Neurofilaments are structural components of motor axons. Accumulation of neurofilament occurs in various pathological conditions. Recently different variants resulting in translation of a cryptic amyloidogenic element (CAE) in the $3^{\prime}$ untranslated region (UTR) of the neurofilament-heavy polypeptide (NFH) gene have been described to cause charcot marie tooth disease type $2 \mathrm{CC}$ (CMT2CC) by forming amyloidogenic toxic protein aggregation. Here we describe the first German pedigree suffering from CMT2CC and give an overview about the features of CMT2CC described so far. Methods: Patients: This study examined a German autosomal dominant CMT2 family with two affected members. Patient 1: Around the age of 8 years the patient experienced difficulties in running and squatting. At the age of 16 a muscle biopsy showing myopathic changes in line with muscular atrophy was performed when he underwent Achilles tendon surgery due to a neurogenic clubfoot. Neurological examination at age 34 displayed marked symmetric atrophy and weakness of the ventral thighs and extensor and flexor muscles of the feet. Tendon reflexes were absent. A distal hypoesthesia from the ankles to the toes was noted. Electromyography revealed a severe neuropathic pattern and pseudomyotonic runs in tibialis anterior muscle. Nerve-conduction-velocity studies were consistent with a motor and sensory axonal neuropathy of the lower limbs. Patient 2: The father of the patient developed a weakness of the lower limbs when he was 16. At the age of 34 he noticed a mild weakness of the upper limbs. The father is 74 years old and wheelchair bound since 64 . A muscle biopsy in 1978 showed features suggestive of denervation. The electromyogram of the lower limbs showed a chronic neuropathic pattern,. Nerveconduction-velocity studies of the motoric medianus were mildly elongated. Genetic analysis: Genomic DNA was purified from peripheral blood. Next generation sequencing identified a heterozygous mutation c.3057dupG (p.K1020Efs*43) in the NFH gene. Results: We identified a mid-adult onset family of two generations carrying a mutation of c.3057dupG (p.K1020Efs*43) in NFH. Until now, only five pedigrees have been reported worldwide. Our patients presented with a similar symptom profile to that of the previously reported. Clinical features include progressive muscle weakness and muscle wasting, predominantly affecting the lower limbs. Mild sensory deficits and absent deep tendon reflexes are also common. Electrophysiological studies show a symmetrical, distal and proximal sensorimotor axonal neuropathy and can be pathological even before clinical symptoms appear. In addition to classic CMT features some patients are also reported to have increased serum creatine kinase, myopathic changes in electromyographic studies and pyramidal signs. Conclusion: Here we report of the first German pedigree suffering from CMT2CC due to an autosomal dominant mutation of c.3057dupG (p. K1020Efs*43) in NFH. The father of our patient was misdiagnosed with spinal muscular atrophy, while our patient initially presented with a distal myopathy phenotype; therefore, the clinical spectrum can mimic motoneuron and muscle phenotypes. Our observation further expands the phenotypic spectrum of NFH mutations and indicates that NFH mutations should be considered in the differential diagnosis of patients presenting with gait disturbances, muscular weakness and muscle wasting predominantly affecting the lower limbs. 
PS2Group3-075 / \#550

Topic: Group 3 - Peripheral Neuropathy, Cranial Nerves, Mononeuropathies: Clinical Features, Pathophysiology, Therapy

\section{ISOLATED ABDUCENS PALSY AS TOLOSA-HUNT SYNDROME IN A SYSTEMIC LUPUS ERYTHEMATOSUS PATIENT}

$\underline{\text { Valeria Serban }}$

Neurology Clinic, Cluj, RO

Background: Tolosa Hunt Syndrome (THS) is a rare occurrence (one case per million per year) first described by Tolosa in 1954 and explored further by Hunt publishing six cases of remittent unilateral retro-orbital pain and extra-ocular nerve palsies (third, fourth, sixth) leading to diplopia, responding well to steroids. Frontal parenthesis can be present as well due to fifth cranial nerve involvement. It is caused by idiopathic granulomatous inflammation. We report a rare case of THS presenting as new onset isolated sixth nerve palsy in a young patient known with systemic lupus erythematosus (SLE). Methods: Twenty seven year-old right-handed lady presented with new onset frontal headache and diplopia persisting for the past several days. No previous similar symptoms. She had no ptosis, limb weakness nor parenthesis. She carried a diagnosis of lupus treated with Plaquinil and low dose steroids for several years On exam there was isolated sixth nerve palsy on the right. However, third, fourth and fifth nerves on the right were not clinically affected.

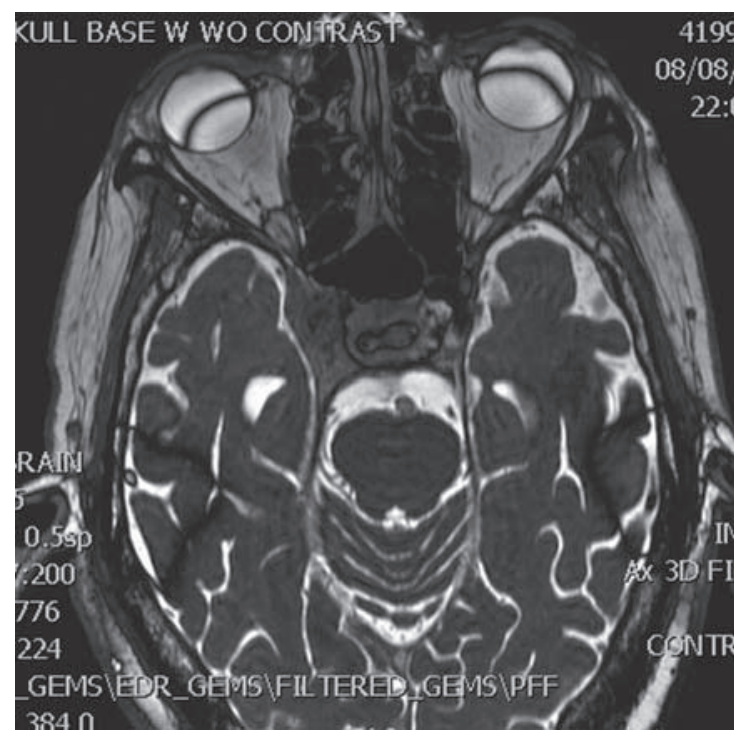

The rest of the exam was unremarkable. Results: AAN was positive in high titers as well as anti-phospholipid antibodies. Auto-antibodies to AchR, MuSK, titin, striated muscle were all absent. In addition, ANCA and rheumatoid factor were not present. TSH was normal. Cerebrospinal fluid (CSF) had normal values and infectious etiology was ruled out. MRI brain and MRA head with and without contrast revealed increased density in the right cavernous sinus suggestive for inflammatory tissue. There was a fair clinical response to high dose steroids. Diplopia improved in several days. Plaquinil was continued, as well. Repeat MRI and MRA head revealed partial resolution of the inflammation present in the right cavernous sinus. Conclusion: This case is unique due to several features. First, the clinical presentation was not the classic painful ophthalmoplegia involving third, fourth, sixth nerves plus frontal parenthesis due to fifth cranial nerve involvement. Instead patient presented with isolated sixth nerve palsy and frontal headaches. Second, the response to steroids was not as spectacular as previously reported. And third, our patient already had an auto-immune condition (SLE) treated with steroids and immunosuppression. Possible lupus to have a role in the pathogenesis of the inflammation developed in the right cavernous sinus. Infectious component was ruled out. Further investigation is needed for a better understanding.

\section{PS2Group3-076 / \#751}

Topic: Group 3 - Peripheral Neuropathy, Cranial Nerves, Mononeuropathies: Clinical Features, Pathophysiology, Therapy

\section{MULTIFOCAL MOTOR NEUROPATHY IN AUSTRIA: A NATIONWIDE SURVEY}

Eva-Maria Oberreiter ${ }^{1}$, Stefan Quasthoff ${ }^{2}$, Marcus Erdler $^{3}$, Raffi Topakian ${ }^{4}$, Susanna Grinzinger ${ }^{5}$, Friedrich Zimprich ${ }^{6}$, Karl Stieglbauer ${ }^{7}$, Julia Wanschitz ${ }^{1}$, Stephan Höger ${ }^{8}$, Klaus Berek ${ }^{9}$, Claudia Thaler-Wolf ${ }^{10}$, Norbert Embacher ${ }^{11}$, Julia Jecel ${ }^{12}$, Isolde Heß-Eberle ${ }^{13}$, Waltraud Kleindienst ${ }^{5}$, Michael Huemer ${ }^{14}$, Eva Laich ${ }^{15}$, Dierk Oel ${ }^{16}$, Wolfgang Oertl ${ }^{17}$, Eva Lenzenweger ${ }^{17}$, Wolfagang Löscher $^{1}$

${ }^{1}$ Medical University of Innsbruck, Innsbruck, AT; ${ }^{2}$ Medical University Graz, Graz, AT; ${ }^{3} S M Z$ Ost, Vienna, AT; ${ }^{4}$ Neurology, Klinikum Wels- 
Grieskirchen, Wels, AT; ${ }^{5}$ Paracelus University Salzburg, Salzburg, AT; ${ }^{6}$ Department Of Neurology, Medical University of Vienna, Vienna, AT; ${ }^{7}$ Private Practice, Linz, AT; ${ }^{8}$ LKH Graz Süd-West, Graz, AT; ${ }^{9} B K H$ Kufstein, Kufstein, AT; ${ }^{10}$ Private Practice, Hall iT, AT; ${ }^{11}$ University Clinic St. Pölten, St. Pölten, AT; ${ }^{12}$ Krankenhaus Hiezing, Vienna, AT; ${ }^{13}$ Private Practice, Salzburg, AT; ${ }^{14}$ Kardinal Schwarzenberg Klinikum, Schwarzach im Pongau, AT; ${ }^{15}$ LKH Steyr, Steyr, AT; ${ }^{16}$ Neurology, Klinikum Wels-Grieskirchen, Wels, AT: ${ }^{17}$ Kepler-Universitätsklinikum Linz,

Linz, $A T$

Background: Multifocal Motor Neuropathy (MMN) is a rare immune-mediated neuropathy, which leads to slowly progressive, asymmetric muscle weaknesses. An electrophysiological motor conduction block $(\mathrm{CB})$ is the hallmark of this disorder. With early diagnosis and treatment with intravenous immunoglobulins (IVIG) MMN is a treatable disorder, at least during the first years of disease. Due to its rarity, data on clinical features and response to longterm treatment are sparse and limited to a few studies. The aim of this study was to provide a survey of Austrian MMN patients and a comprehensive review of epidemiological and clinical features of MMN cases in Austria and their response to treatment. Also, we compared our results with previously published studies. Methods: Austrian neurologists were contacted via the Austrian Neurological Society (ÖGN) asked to provide anonymized data on MMN patients under their care. A total of 57 patients were reported in Austria which have been diagnosed between 1993 and 2017. We performed an exploratory analysis of clinical, electrophysiological and laboratory data, as well as IVIG use and treatment outcome. Results: The point prevalence of MMN in Austria is $0,65 / 100.000$. The male to female ratio was 2,2:1 and the median age of symptom onset was 45 years. Mean diagnostic delay was 5,4 $\pm 8,33$ ys., but there was a decline in diagnostic delay in relation to symptom onset; i.e. mean diagnostic delay was 17 ys, when symptoms began between 1982-86 and was 0,9 ys when symptom onset was between 20122017. MMN typically began in the upper extremity $(77,2 \%)$ and in distal muscles $(87,7 \%)$. CB was found in $95 \%$ of the patients, most frequently in the ulnar and median nerves. $43 \%$ showed IgM antibodies against GM1. 51 of 57 patients were treated with IVIG at median intervals of 4 weeks with a mean dosage of $1,55 \mathrm{~g} / \mathrm{kg}$. High muscle strength was a favorable prognostic factor, while elevated serum igM
anti-GM1 antibody titer, muscle atrophy and long intervals of treatment were negative prognostic factors. Duration of treatment correlated with outcome: the longer the treatment, the worse the outcome. IVIG improved muscle strength, but frequently lost its effectiveness after treatment duration of $>7$ years. Conclusion: These results show that the prevalence of MMN in Austria is similar to the few reports from other countries. Also, the present study corroborates previous results on clinical, electrophysiological and laboratory features in MMN. Intravenous Immunoglobulins are still the most effective treatment; however, deterioration of symptoms during long-term treatment though occurs in most cases.

\section{PS2Group3-077 / \#973}

Topic: Group 3 - Peripheral Neuropathy, Cranial Nerves, Mononeuropathies: Clinical Features, Pathophysiology, Therapy

\section{AUTOSOMAL RECESSIVE CHARCOT MARIE TOOTH DISEASE: CLINICAL, ELECTROPHYSIOLOGY AND GENETIC SPECTRUM IN A TUNISIAN SERIES}

Imen Kacem ${ }^{1}$, Emna Ellouz ${ }^{2}$, Mouna Ben Djebara ${ }^{1}$, Eric Leguern ${ }^{3}$, Amina Gargouri ${ }^{1}$, Riadh Gouider ${ }^{4}$ ${ }^{1}$ razi hospital, tunis, TN; ${ }^{2}$ Neurology, Gabes Hospital, ibn khaldoun street gabes, TN, ${ }^{3}$ salpetriere, paris, FR $;{ }^{4}$ Neurology, Razi Hospital, Manouba, TN

Background: Charcot Marie Tooth disease is a genetically heterogeneous group of hereditary motor and sensory neuropathies. Autosomal Recessive forms (AR CMT) are known to be the least common forms worldwide but still frequent in the Mediterranean basin because of high rate of consanguinity. Objectives We aimed to investigate clinical, electrophysiological and genetic features of AR CMT in a Tunisian cohort. Methods: Patients and Methods We performed a prospective study of a cohort of AR CMT Tunisian patients followed in the neurology department of Razi Hospital, Tunisia from June 2002 to December 2017. Clinical and electrophysiological data were analyzed. Neuropathy was classified in axonal or demyelinating form according to nerve conduction velocities in upper limb motor nerves NCVs. Disability was assessed by the Overall 
Neuropathy Limitations Scale (ONLS) and CMT neuropathy scale (CMTNS). Genetic study was made to all probands in Neurogenetic Department of Pitié Salpêtrière-France. Written informed consent was obtained from all of the participants. Results: We included 65 patients belonging to 43 families. Mean age at diagnosis was 20.8 years. Consanguinity was found in $94 \%$. Mean age of onset was 3.7 years. Delay of milestone was found in $11 \%$ of cases. Classical phenotype was found in the majority of patients and uncommon presentation as pyramidal syndrome or glaucoma were found in two families. Axonal neuropathy was found in $48.5 \%$ cases and demyelinating one in $51.5 \%$ cases. Mean ONLS score was 4.7 and mean CMTNS one was $12.2 \%$. Axonal polyneuropathy was more frequently associated to an early onset $(90.9 \%)(\mathrm{p}<0.001)$, with more severe weakness $(\mathrm{p}=0.04)$ and more severe ONLS score. Gene mutations were identified in 15 families with predominance of demyelinating forms. The GDAP1gene mutations were the most frequent (26.3\%).Four novel mutations were detected and characterized phenotypically. Conclusion: Discussion We reported a large AR CMT cohort with a high consanguinity rate. Recessive CMT forms, are more severe and have an earlier onset than the dominant forms. They usually show pure CMT clinical phenotype; however, some forms can be associated to cyphoscoliosis, deafness or glaucoma. Demyelinating forms (CMT 4) were more frequent but axonal ones (AR-CMT 2) were more severe with rapid progress. In concordance with literature, Mutations in GDAP1 are a frequent cause of AR CMT. Conclusion AR CMT are common in Tunisia. Comparatively to dominant forms, AR ones were characterized by an earlier onset, more severe clinical features and with important handicap. Electrophysiological results and associated signs must be considered to guide molecular study. GDAP1 gene mutations still the most frequent mutation in these cases.

\section{PS2Group3-078 / \#257}

Topic: Group 3 - Peripheral Neuropathy, Cranial Nerves, Mononeuropathies: Clinical Features, Pathophysiology, Therapy

\section{COLCHICINE INDUCED} NEUROMYOPATHY IN A PATIENT USING CONCOMITANT DIURETICS

Young-Do Kim

Neurology, Incheon St. Mary's Hospital, The

Catholic University of Korea, Incheon, KR
Background: Colchicine is commonly used to treat gout. The common side effects of colchicine include gastroenteritis, blood dyscrasias, and dermatitis; the neuromuscular toxicity associated with colchicines is under-recognized. The characteristic neuromuscular complication is a neuromyopathy, presenting with subacute proximal lower extremity weakness, elevated serum creatine kinase (CK) and electromyography (EMG) showing an "irritable myopathy" and a length-dependent sensorimotor peripheral neuropathy. We present a patient with colchicineinduced neuromyopathy using comcomitant diuretics. Methods: Case : An 70-year-old woman with a significant past medical history including hypertension, heart failure with pericardial effusion, liver cirrhosis with ascites, hypothyroidism and hyperuricemia presented to our clinic for evaluation of general weakness for 1 month. The weakness started in the both thighs and lower legs about 4 weeks prior to presentation and progressively worsened. She complained of myalgia in the arms and legs with mild paresthesia and hypoesthesia. She denied cranial nerve deficits or bladder and bowel difficulty. But, she compained of continous diarrhea for 3 weeks. She was on colchicine $1.2 \mathrm{mg}$ per day, which she had been taking for several months for pericardial effusion and hyperuricemia. Also, she had been taking diuretics (furosemide $80 \mathrm{mg}$, spironolactone $25 \mathrm{mg}$ ) for 2 months due to generalized edema and pleural effusion. Results: On examination, cognition and cranial nerves were normal. She had both proximal and distal upper limb weakness (Medical Research Council [MRC] grade 3/5); severe hip flexor and dorsiflexor weakness (MRC grade 2-3/5) and diffuse lower limb weakness otherwise (MRC grade $3 / 5$ ). She had graded sensory loss to pain and cold sensation up to the knees,reduced vibration sensation at the ankles and reduced joint position sensation at the toes. Laboratory investigations revealed high creatinine $(1.3 \mathrm{mg} / \mathrm{dL}$; normal range 0.6-1.1). Serum CK (1581 U/mL; normal range 52-336) and aldolase $(9.4 \mathrm{U} / \mathrm{mL}$; normal range 1-7.5) were both elevated. Nerve conduction studies revealed sensorimotor polyneuropathy of axonal type. Needle electrode examination demonstrated fibrillation, postitive sharp and myotonic potentials in distal extremity muscles; motor unit potentials were of varying duration and amplitudes in various muscles diffusely. These clinical, laboratory and electrophysiologic findings were consistent with colchicine-induced neuromyopathy aggravated by diuretics. Colchicine was discontinued. Two months later the patient had no weakness in the arms 
with mild weakness in the lower limbs (MRC grade 4/5). Conclusion: The neuromuscular complications associated with colchicine are commonly under-recognized. Chronic renal dysfunction or diuretic use makes patients more susceptible to this neuromuscular complication at the usual dose range. Co administration of any drug that is metabolized by the CYP3A4 system can induce colchicine myopathy. Laboratory investigations usually show mild to marked elevation of CK. The characteristic electrodiagnostic findings include myopathic motor unit potentials with features of "membrane irritability" as may be seen in inflammatory myopathy and a coexistent sensorimotor axonal polyneuropathy. The common diagnostic consideration in patients with colchicine myoneuropathy is polymyositis because of predominant proximal weakness, elevation of CK and irritable myopathy on EMG.
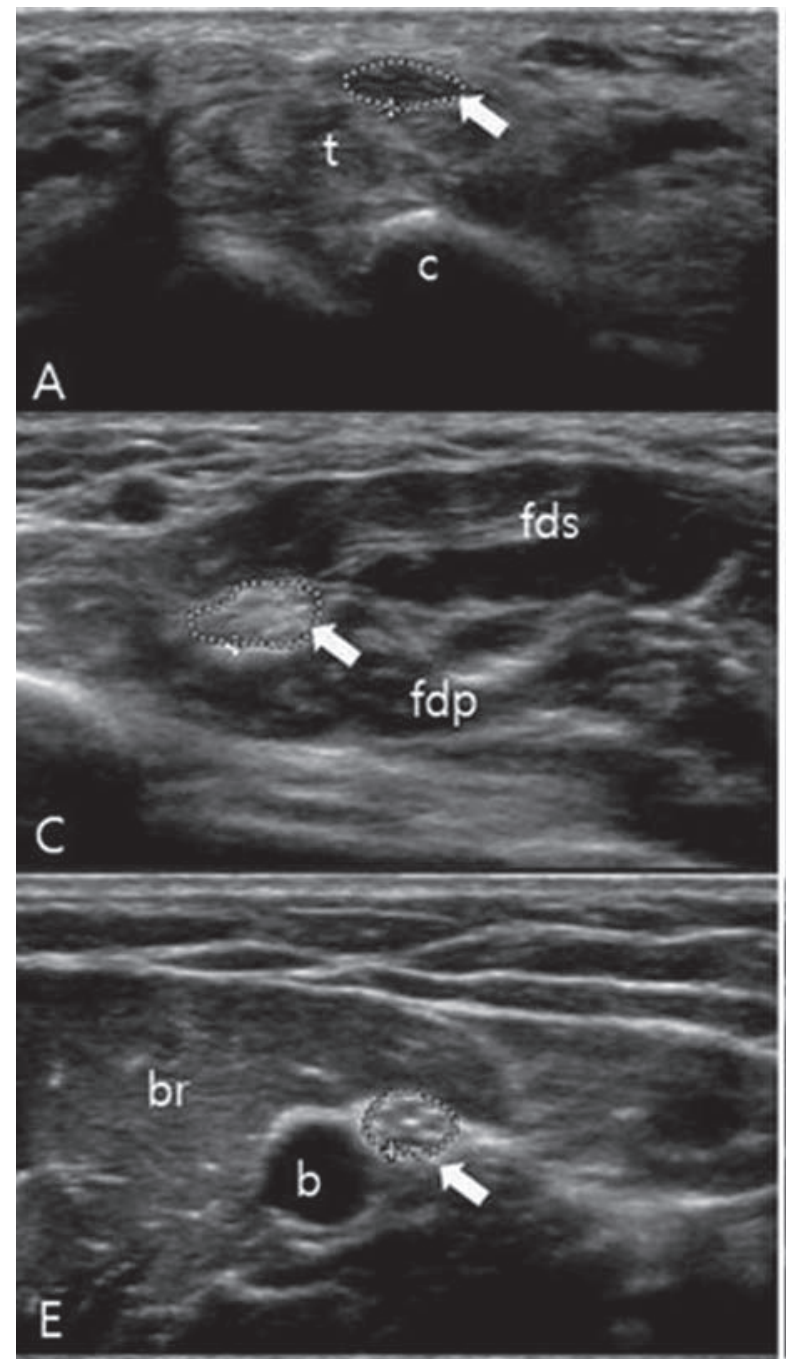

PS2Group3-079 / \#389

Topic: Group 3 - Peripheral Neuropathy, Cranial Nerves, Mononeuropathies: Clinical Features, Pathophysiology, Therapy

\section{A CASE OF MULTIFOCAL MOTOR NEUROPATHY: COMPLEMENTARY ROLE OF ULTRASOUND}

Jung Im Suk ${ }^{1}$, Hae Joo Rha ${ }^{2}$

${ }^{1}$ Neurology, Catholic University of Daegu, School of Medicine, Daegu, KR; $;{ }^{2}$ Catholic University of Daegu, School of Medicine, Daegu, KR

Background: Multifocal motor neuropathy (MMN) is an uncommon, asymmetric motor neuropathy. As MMN is a treatable disorder, its differentiation from lower motor neuron disease is important. Evidence of conduction block (CB) or positive IgM anti-GM1

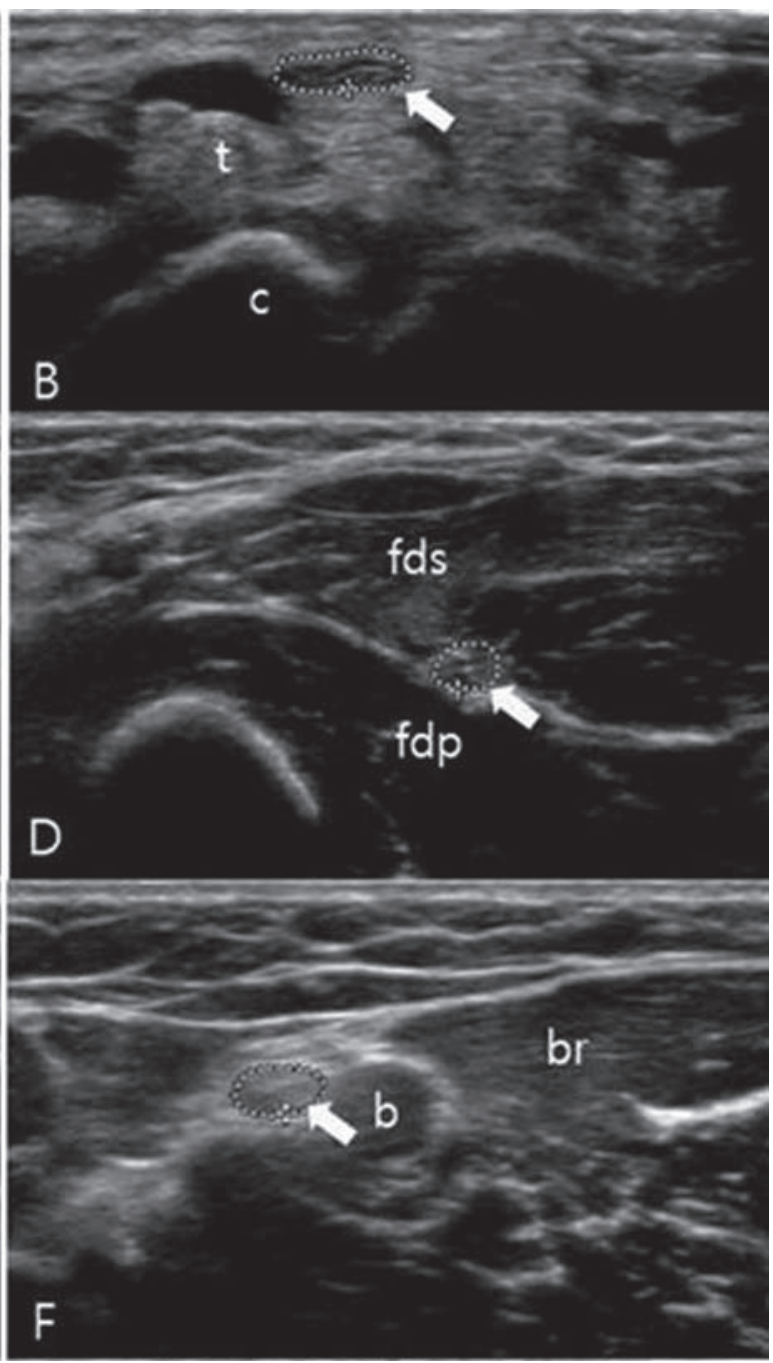




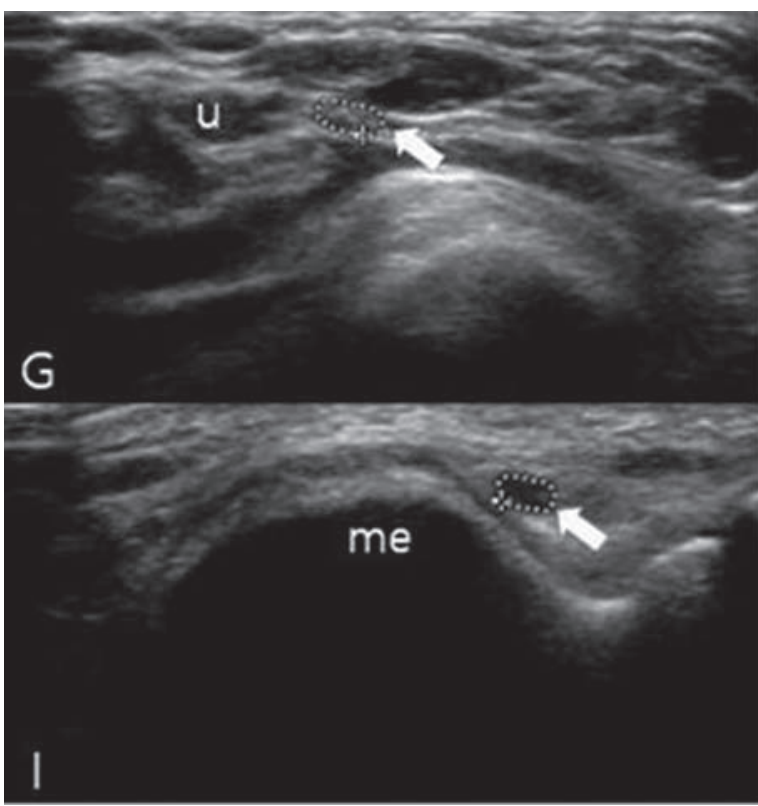

is considered one of important markers for the diagnosis. However, some patients with atypical MMN have no detectable CB or anti-GM1 antibodies.

Methods: A 40-year-old man presented with twoyear history of progressive left-sided weakness without sensory symptoms. We experienced a case of MMN with focal nerve enlargement on ultrasound. Results: Nerve conduction study revealed partial conduction block in two nerves. Anti-GM1 antibody is negative and other laboratory studies were unremarkable. Ultrasound showed focal nerve enlargement of right median nerve at the forearm and left ulnar nerve at the elbow (Fig. 1). Weakness improved completely after intravenous immunoglobulin. Ultrasound image shows focal enlargement of median and ulnar nerve (arrow). (A and B) The cross-sectional area (CSA) of right (A) and left (B) median nerve at the wrist is normal. (C and D) The CSA of right $(\mathrm{C})$ median nerve at the forearm is increased at $15.2 \mathrm{~mm}^{2}$ and left (D) median nerve is normal $\left(5.9 \mathrm{~mm}^{2}\right)$. (E and $\left.\mathbf{F}\right)$ The CSA of right (E) and left $(\mathrm{F})$ median nerve at the upper arm is normal. (G and H) The CSA of right $(\mathrm{G})$ and left $(\mathrm{H})$ ulnar nerve at the wrist is normal. (I and J) The CSA of right (I) ulnar nerve at the elbow is normal (3.5 $\mathrm{mm}^{2}$ ), but left (J) ulnar nerve is increased at 11.1 $\mathrm{mm}^{2}$. $\mathrm{t}$; tendon, c; carpal bone, fds; flexor digitorum superficialis, fdp; flexor digitorum profundus, b; brachial artery, br; brachialis, u; ulnar artery, me; medial epicondyle. Conclusion: Ultrasound can be a valuable tool in supporting the diagnosis of MMN.

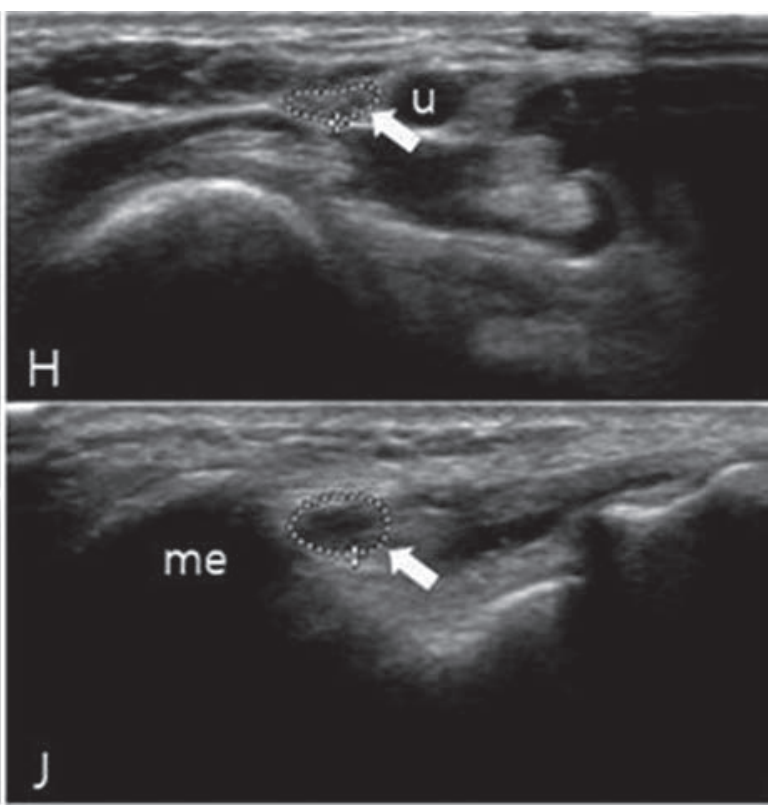

PS2Group3-080 / \#568

Topic: Group 3 - Peripheral Neuropathy, Cranial Nerves, Mononeuropathies: Clinical Features, Pathophysiology, Therapy

\section{PHARMACOKINETICS OF PATISIRAN IN PATIENTS WITH HEREDITARY TRANSTHYRETIN- MEDIATED AMYLOIDOSIS}

\section{Xiaoping Zhang, Varun Goel, Gabriel Robbie} Alnylam Pharmaceuticals, Cambridge, MA, US

Background: Patisiran is an investigational RNA interference therapeutic in development for the treatment of hereditary transthyretin-mediated amyloidosis (hATTR). Early clinical development has demonstrated that patisiran was generally well tolerated and elicited robust, rapid, and sustained lowering of TTR protein levels in healthy volunteers and in patients. Here, we report pharmacokinetic (PK) data from the long-term, open-label Phase 2 study in patients (NCT01961921). Methods: Adult patients $(\mathrm{N}=27)$ received patisiran $0.3 \mathrm{mg} / \mathrm{kg}$ intravenously (IV) q3w over 24 months. Intensive plasma PK sampling was performed on Weeks 1, 34, and 106 for the characterization of PK properties of ALN-18328 (siRNA) and 2 novel lipid excipients (DLin-MC3-DMA and PEG2000-C-DMG). Plasma PK parameters were computed using non-compartmental analysis. Achievement of steady state was also assessed by measuring maximum plasma con- 
centration at the end of infusion (Cmax) and predose concentration (Ctrough) over 24 months of treatment. Results: After IV infusion of patisiran in patients, all 3 analytes exhibited multiphasic plasma profiles (Figure 1).PK parameters are summarized in Table 1. PK profiles from all 3 analytes were characterized by a short half-life $\left(\mathrm{t}_{1 / 2 \mathrm{a}}\right)$ in the first phase and a relatively long terminal half-life $\left(\mathrm{t}_{1 / 2 \mathrm{~b}}\right) . \mathrm{C}_{\max }$ and/or $\mathrm{C}_{\text {trough }}$ increased over time and approached steady state by week 24 . Accumulation of area under the curve at steady state compared to the first dose
$\left(\mathrm{AUC}_{\text {tau }}\right)$ was 3.2- fold for ALN-18328 and 1.7- fold for DLin-MC3-DMA, whereas no accumulation for $\mathrm{PEG}_{2000}$-C-DMG. The mean volume of distribution $\left(\mathrm{V}_{\mathrm{ss}}\right)$ for ALN-18328, DLin-MC3-DMA, and $\mathrm{PEG}_{2000}$-C-DMG was 3.43-, 6.33-, and 1.75-fold higher, respectively, than the blood volume in a typical 70-kg human, indicating distribution outside the vasculature. Conclusion: After chronic dosing of patisiran $0.3 \mathrm{mg} / \mathrm{kg} \mathrm{q} 3 \mathrm{w}$, steady state was reached by week 24 with moderate accumulation. PK of patisiran components were stable over time.

Figure 1: Mean ( \pm SEM) Plasma Concentration-Time Profiles of ALN-18328, DLin-MC3-DMA, and PEG $2000-C-D M G$ on Weeks 1, 34 , and 106 After Administration of $0.3 \mathrm{mg} / \mathrm{kg}$ q3w Patisiran over 24 Months in Patients
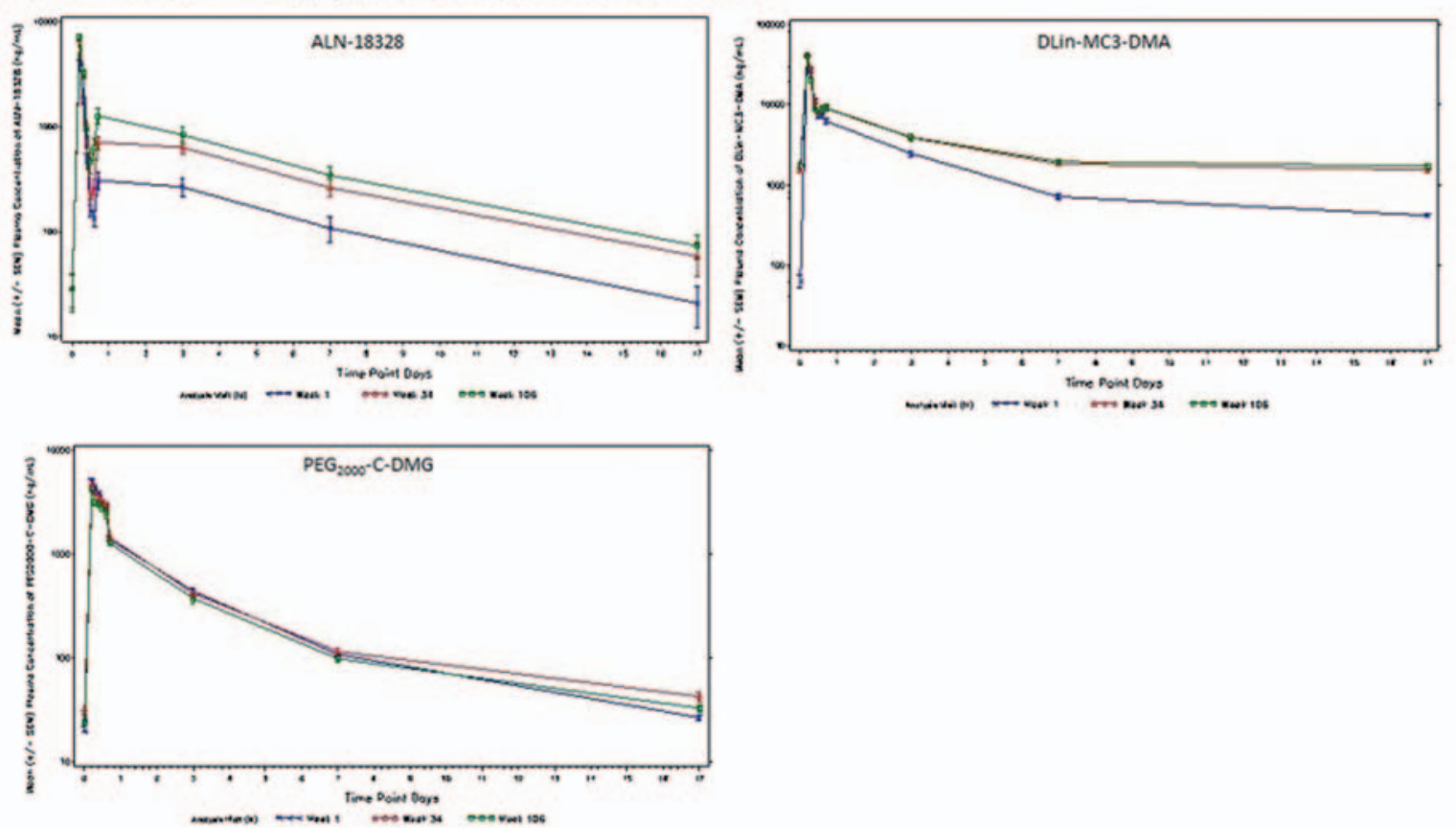
Table 1: MeantSD (\%CV Plasma PK Parameters of ALN-18328, OLin-MC3-OMA and PEG2000-CDMG After Administration of Patisiran-LNP $0.3 \mathrm{mg} / \mathrm{kg}$ q3w in Patients

\begin{tabular}{|c|c|c|c|}
\hline PK Parameter & $\begin{array}{l}A L N-18328 \\
N=27\end{array}$ & $\underset{N=27}{\text { DLin-MC3-DMAA }}$ & 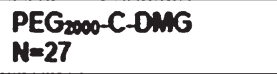 \\
\hline \multicolumn{4}{|l|}{ Week 1} \\
\hline $\mathrm{C}_{\max }, \mu g / \mathrm{mL}$ & $4.10 \pm 1.28(31.2)$ & $42.5 \pm 13.1(30.9)$ & $5.07 \pm 1.31(25.9)$ \\
\hline Crown, $\mu g / m L$ & $0.0650 \pm 0.00281(225)$ & $0.628 \pm 1.10(176)$ & $0.0179 \pm 0.00133(38.6)$ \\
\hline $\mathrm{C}_{m=0}, \mu \mathrm{g} / \mathrm{mL}$ & $0.367 \pm 0.30$ & $7.94 \pm 2.30$ & NA \\
\hline AUC $C_{\text {ow, }} \mu g \cdot h / m L$ & $57.5 \pm 57.4(99.8)$ & $796 \pm 292(36.7)$ & $179 \pm 168(93.7)$ \\
\hline 2nd phase AUC', \% & 81.7 & 71.0 & NA \\
\hline$t_{\max }^{2}, n$ & $1.25(1.03-2.67)$ & $1.25(1.03-2.67)$ & $1.27(1.03-3.42)$ \\
\hline \multicolumn{4}{|l|}{ Week 34} \\
\hline $\mathrm{C}_{\max }, \mu \mathrm{g} / \mathrm{mL}$ & $6.12 \pm 1.69(27.7)$ & $41.1 \pm 9.25(22.5)$ & $4.84 \pm 1.42(29.3)$ \\
\hline Crougn, $\mu \mathrm{g} / \mathrm{mL}$ & $0.0211 \pm 0.0377(179)$ & $1.51 \pm 0.367(24.4)$ & $0.0296 \pm 0.0085(28.7)$ \\
\hline 2nd phase AUC', \% & 91.5 & 82.2 & NA \\
\hline$t_{\max }, h$ & $1.23(1.15-2.10)$ & $1.23(1.15-2.10)$ & $1.30(1.15-3.00)$ \\
\hline Crese, $\mu \mathrm{g} / \mathrm{mL}$ & $0.781 \pm 0.492$ & $9.65 \pm 2.49$ & NA \\
\hline \multicolumn{4}{|l|}{ Week 106} \\
\hline$C_{\text {mexes, }}$ Ng/mL & $7.15+2.14(30.0)$ & $40.2 \pm 11.5(28.7)$ & $4.22 \pm 1.22(28.9)$ \\
\hline Crougn.se, $\mu \mathrm{g} / \mathrm{mL}$ & $0.0210 \pm 0.0442(210)$ & $1.75 \pm 0.698(39.9)$ & $0.0236 \simeq 0.00930(39.3)$ \\
\hline AUCou, $\mu g \cdot h / m L$ & $184 \pm 159(86.4)$ & $1403 \pm 105(36.6)$ & $145 \pm 64.7(44.6)$ \\
\hline 2nd phase AUCc, \% & 83.3 & 84.4 & NA \\
\hline$t_{\max } x^{2} \cdot h$ & $1.30(1.17-2.10)$ & $1.30(1.17-2.10)$ & $1.30(1.17-3.10)$ \\
\hline $\mathrm{C}_{\text {mex }} \mu \mathrm{g} / \mathrm{mL}$ & $1.57 \pm 2.04(129)$ & $9.90 \pm 4.10(41.4)$ & NA \\
\hline ixo, $n$ & $0.789 \pm 0.170(21.6)$ & $0.875 \pm 0.112(12.8)$ & $17.1 \pm 5.35$ (31.3) \\
\hline tses, d & $3.16 \pm 1.75(55.3)$ & $10.9 \pm 1.75(72.7)$ & $3.76 \pm 1.05(28.0)$ \\
\hline$V_{n, ~ L / k g}$ & $0.255 \pm 0.198(77.9)$ & $0.470 \pm 0.238(50.5)$ & $0.130 \pm 0.050(38.7)$ \\
\hline CLes, mL/kg/n & $3.03 \pm 2.50(82.6)$ & $2.08 \pm 0.771(37.1)$ & $2.08 \pm 0.586(28.2)$ \\
\hline Rac for Croup & 3.23 & 2.79 & 1.32 \\
\hline$R_{a c}$ for $C_{\max }$ & 1.74 & 0.95 & 0.812 \\
\hline$R_{a c}$ for $A U C_{a s}$ & 3.20 & 1.76 & 0.832 \\
\hline
\end{tabular}

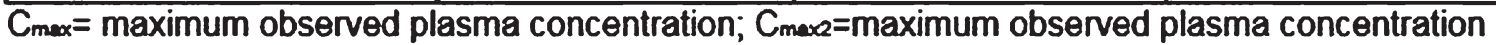
for the second phase; Crough= minimum observed plasma concentration; AUCtru = Area under the plasma concentration time curve for a dosing interval: $C_{2 s}=$ steady state clearance; $V_{s s}=$ Volume of distribution at steady state; $t_{12 \Omega}=$ apparent distribution half-life ; $t_{1220}=$ apparent elimination half-life; $\% C V=$ coefficient of variation; $q 3 w=$ once every 3 weeks; $S D=$ standard deviation; $A U C_{p 1}=A U C$ from the start of infusion to the trough concentration (i.e, Peak 1); $A \cup C_{P 2}=A \cup C$ from the trough concentration to the last quantifiable concentration (i.e, Peak 2); RAc= Accumulation ratio calculated as Week 106 Week $1 ; \mathrm{NC}=$ not calculated.

- Median (minimum - maximum).

- Calculated as (mean $\left.A U C_{p 1}\right)\left(\right.$ mean $A U C_{p 1}+$ mean $\left.A U C_{p 2}\right) \times 100 \%$ 


\section{PS2Group3-081 / \#735}

Topic: Group 3 - Peripheral Neuropathy, Cranial Nerves, Mononeuropathies: Clinical Features, Pathophysiology, Therapy

\section{ROLE OF THE ER STRESS TRANSCRIPTION FACTOR XBP1 IN CHARCOT-MARIE- TOOTH DISEASE TYPE 1B}

Thierry Touvier ${ }^{1}$, Cinzia Ferri ${ }^{2}$, Rosa Mastrangelo ${ }^{2}$, Cristina Rivellini ${ }^{3}$, Laurie Glimcher ${ }^{4}$, Lawrence Wrabetz ${ }^{5}$, Maurizio D'Antonio ${ }^{2}$

${ }^{1}$ Division Of Genetics And Cell Biology, Ospedale San Raffaele, Milan, IT; ${ }^{2}$ Division Of Genetics And Cell Biology, Ospedale San Raffaele, Milan, IT; ${ }^{3}$ Division Of Neuroscience, Ospedale San Raffaele, Milan, IT; ${ }^{4}$ Department Of Cancer Immunology And Virology, Dana-Farber Cancer Institute, Boston, MA, US: ${ }^{5}$ Hunter James Kelly Research Institute, Hunter James Kelly Research Institute, Buffalo, NY, US

Background: Mpz protein is the most abundant protein in myelin of peripheral nerves. The mutant MpzS63del causes Charcot-Marie-Tooth (CMT) 1B disease in humans and a similar demyelinating neuropathy in transgenic mice. MpzS63del protein provokes an endoplasmic reticulum (ER) stress in myelinating Schwann cells, which mount an unfolded protein response (UPR) characterized by activation of PERK, ATF6 and XBP1 pathways. We have previously reported that activation of $\mathrm{CHOP}$ and GADD34, two downstream mediators of PERK, is pathogenetic in MpzS63del mice (Pennuto, 2008; D'Antonio, 2013) but the role of the other UPR branches remains to be investigated. Methods: We generated a new mouse model with Schwann cellsspecific ablation of XBP1 and in parallel we exploited MpzS63del dorsal root ganglia (DRG) explant cultures in which XBP1 signaling is modulated by gain/loss of function approaches. Results: We observed that absence of XBP1 dramatically worsens hypomyelination and electrophysiological/locomotor parameters in young and adult S63del neuropathic animals. Interestingly we observed that a strong upregulation of PERK and IRE1-mediated RIDD signalings in neuropathic animals lacking XBP1. This suggests that activation of XBP1 targets has an essential role in limiting MpzS63del toxicity, which cannot be compensated by other stress responses. Moreover, we demonstrated in S63del DRG cultures that inhibition of XBP1 pathway decreases myelination whereas activation of XBP1 signaling slightly ameliorates myelination. Conclusion: Altogether, these data demonstrate that the XBP1 pathway has a critical adaptive role in MpzS63del neuropathy and suggest that activating this pathway may be beneficial for CMT1B and perhaps for other neuropathies characterized by UPR activation.

\section{PS2Group3-082 / \#1032}

Topic: Group 3 - Peripheral Neuropathy, Cranial Nerves, Mononeuropathies: Clinical Features, Pathophysiology, Therapy

\section{OBINUTUZUMAB, A NEW ANTI- CD20 ANTIBODY, IS ACTIVE AND EFFECTIVE IN ANTI-MAG ANTIBODY POLYNEUROPATHY}

\author{
Chiara Briani $^{1}$, A Visentin ${ }^{2}$, A Salvalaggio ${ }^{1}, \mathrm{M}$ \\ Ruiz $^{1}$, M Cacciavillani ${ }^{3}$ \\ ${ }^{1}$ Neurology Unit, Department Of Neuroscience, \\ University of Padova, Padua, IT; ${ }^{2}$ Hematology And \\ Clinical Immunology Unit, Department Of \\ Medicine, University of Padova, Padua, \\ IT; ${ }^{3}$ CEMES, Data Medica Group, Padova, IT
}

Background: Rituximab, a chimeric anti-CD20 monoclonal antibody (mAb), has been used in polyneuropathy associated with anti-myelin-associatedglycoprotein (MAG) antibodies with controversial results. Obinutuzumab, a new glycoengineered humanized anti-CD20 mAb, in combination with chemotherapy, induces longer progression-free survival in chronic lymphocytic leukemia (CLL) and follicular lymphoma, as compared with rituximab. We describe two patients with anti-MAG antibody neuropathy who were successfully treated with Obinutuzumab. Patient 1. A 82-yr-old man presented with severe demyelinating sensory-motor neuropathy. At our first evaluation, he used wheelchair to travel outdoors, was incapable of standing and walked few steps only with bilateral support. He had distal weakness at lower limbs (2/5 MRC), tactile hypoestesia and loss of vibration up to knees, areflexia. He had a clonal B lymphocytosis CD5+ CD23+, compatible with CLL, IgM lambda paraprotein and anti-MAG titre was $>70,000 \mathrm{BTU}$. The presence of favorable CLL prognostic markers (mutated IGHV gene and absent of TP53 deletions or mutations) prompt us to use chlorambucil+obinutuzumab 
(obinutuzumab iv at $1000 \mathrm{mg}$ on day 1,8 and 15 of cycle 1 and day 1 of cycles 2-6; chlorambucil os at $0.5 \mathrm{mg} / \mathrm{kg}$ on day 1 and 15 of cycles 1-6). At cycle 6 the patient was able to stand, gait was possible with monolateral support, tactile hypoestesia was limited to feet, distal strength and vibration improved. Mprotein decreased from $15.8 \mathrm{~g} / \mathrm{L}$ to 11.61 at cycle 6 . Similarly, IgM level $(14.8 \mathrm{vs} 8.7 \mathrm{~g} / \mathrm{L})$, lambda freelight chain $(145 \mathrm{vs} 59 \mathrm{mg} / \mathrm{L})$ and lymphocytes $(6,340 \mathrm{vs} 900 / \mu \mathrm{l})$ decreased. Anti-MAG still was $>70,000$ BTU. Patient 2. A 84-yr-old female presented mild demyelinating sensory-motor neuropathy complained of distal sensory impairment at four limbs (painful paresthesia) and subjective gait instability. Areflexia at lower limbs was observed. She had clonal B lymphocytosis compatible with CLL and with IgM kappa paraprotein at immunofixation. Anti-MAG titre was $>70,000 \mathrm{BTU}$. Chlorambucil + obinutuzumab (obinutuzumab iv at $1000 \mathrm{mg}$ on day 1,8 and 15 of cycle 1 and day 1 of cycles 2-3; chlorambucil os at $0.5 \mathrm{mg} / \mathrm{kg}$ on day 1 and 15 of cycles 1-3) were administered. After 3 cycles monoclonal component was absent and the patient reported dramatic improvement of symptoms with reduction of pain and gait normalization. Neurological evaluation was unremarkable, except for absent Achilles reflexes. Our data indicate that Obinutuzumab may be effective in anti-MAG antibody polyneuropathy. CLL might have had a role in the response to therapy, but a possible role of chlorambucil+obinutuzumab in anti-MAG antibody polyneuropathy, regardless of the associated hematological condition, should be considered in future trials.

\section{PS2Group5-001 / \#544}

Topic: Group 5 - Novel Diagnostic Methods in Neuromuscular Diseases and Basic Sciences in Neuromuscular Diseases

\section{LATE-ONSET POMPE DISEASE ASSOCIATED WITH POLYNEUROPATHY}

Marta Lamartine Monteiro ${ }^{1}$, Gauthier Remiche ${ }^{2}$ ${ }^{1}$ Neurology, Erasme Hospital, Brussels, BE; ${ }^{2}$ Centre De Référence Neuromusculaire, Service De Neurologie, Hôpital Erasme, Université Libre de Bruxelles, Brussels, BE

Background: This study aimed to assess if late-onset Pompe disease (LOPD) could be associated with polyneuropathy. LOPD is a rare autosomal recessive disease caused by alpha-glucosidase lysosomal enzyme deficiency. This strikes mainly striated muscle but also involves many other tissues. To the best of our knowledge an involvement of large nerve fibers was never depicted. However it has been showed in mice models of Pompe's disease, glycogen accumulation in Schwann cells and in the perineurium of peripheral nerves. Moreover two LOPD patients were reported as having a small fiber neuropathy. Methods: We retrospectively assessed medical records of LOPD patients followed in our center in order to assess potential indicative clinical and neurophysiological data suggesting a polyneuropathy. Moreover we systematically looked for the presence of potential etiologies for polyneuropathy. LOPD and polyneuropathy international diagnostic criteria were applied and ethic committee agreement was obtained. Results: Four of the six LOPD patients found in our database had clinical evidence for polyneuropathy and it was confirmed by electroneuromyography for three of them. None had other common known cause of polyneuropathy. Conclusion: Polyneuropathy seems overrepresented in LOPD patients. Pathophysiological basis may be evoked to explain this potential disabling additional condition in these patients.

\section{PS2Group5-002 / \#825}

Topic: Group 5 - Novel Diagnostic Methods in Neuromuscular Diseases and Basic Sciences in Neuromuscular Diseases

\section{CORRELATION BETWEEN ELECTRICAL IMPEDANCE MYOGRAPHY AND TWO QUANTITATIVE ULTRASOUND PARAMETERS IN DUCHENNE MUSCULAR DYSTROPHY}

Bhaskar Roy ${ }^{1}$, Basil Darras ${ }^{2}$, Craig Zaidman ${ }^{3}$, Jim $\mathrm{Wu}^{4}$, Kush Kapur ${ }^{2}$, Seward Rutkove ${ }^{5}$ ${ }^{1}$ Neurology, Yale University, New Haven, CT, US, ${ }^{2}$ Boston Children's Hospital, Harvard Medical School, Boston, MA, US ${ }^{3}$ Neurology, Washington University at St Louis, St Louis, MO, US: ${ }^{4}$ Neurology, Beth Israel Deaconess Medical Center, Boston, MA, US; ${ }^{5}$ Neurology, Beth Israel Deaconess Medical Center, Harvard Medical School, Boston, MA, US 
Background: Quantitative ultrasound (QUS) parameters, such as grayscale levels (GSL), quantitative backscatter analysis (QBA) and electrical impedance myography (EIM) are potential non-invasive biomarkers of muscle health in Duchenne muscular dystrophy (DMD). This study explores correlation between GSL and QBA values with EIM parameters. Methods: GSL, QBA values along with EIM parameters, $50 \mathrm{kHz}$ phase, $100 \mathrm{kHz} / 300 \mathrm{kHz}$ phase ratio, and least-squares fit of 100 to $500 \mathrm{kHz}$ phase (slope) at baseline, 6 months, and 12 months from 6 muscles were recorded from an ongoing study of QUS and EIM in 36 DMD and 29 healthy boys, aged 2-14 years. Results: Among the DMD patients, GSL values showed significant correlation with all EIM parameters in biceps brachii. Particularly, strong correlation was noted with EIM 100/300 ratio and EIM-slope at 12-months; spearman rho was -0.67 and 0.7 respectively, p-values $<0.01$. Weak-to-moderate but consistent correlation was noted between GSL and all EIM parameters in deltoid except at baseline for EIM-slope (p-value was 0.06). Significant correlation was noted between GSL values with EIM-50kHz at every time point in the forearm muscles. Among lower extremity muscles, quadriceps showed significance or near significance at 6 months and 12 months with every EIM parameters. In gastrocnemius, weak correlation was noted with EIM 100/300 ratio reaching significance or near significance. No consistent correlation was noted in tibialis anterior. QBA values showed a similar trend. Consistently significant correlation was noted between QBA and all EIM parameters in biceps and deltoid. Correlation was strong for biceps at 12 months; rho for EIM 100/300 ratio, EIM-slope were -0.716 , and 0.731 respectively ( $p$-values $<0.01)$. Strong correlation was also noted at baseline and 6-months with EIM-50kHz (rho values -0.72 and -0.78 respectively, p-values $<0.01$ ). Forearm muscles showed moderate to strong correlation with EIM-50kHz at every time points. Among lower extremity muscles, gastrocnemius showed consistent correlation most of the time. In quadriceps, significance was reached at 6 months with all EIM parameters. Again, tibialis anterior did not show any consistent correlation. When considered in groups, proximal muscles, upper extremity muscles, and all six-muscles grouped together showed significant correlation throughout with all EIM parameters for both GSL and QBA values. In majority of the occasions, spearman rho was $>0.5$ and $\mathrm{p}$-values were $<0.05$. Correlations noted in the group of distal muscles showed statistical significance or near significance most of the time. Lower extremity muscles taken together did not show significant correlation consistently. No consistent correlation between the QUS and EIM parameters was noted, either in individual muscle or group of muscles among the healthy subjects. Changes in GSL and QBA values from baseline to 12 months were correlated with changes in different EIM parameters from baseline to 12 months. No consistent correlation was noted when these changes were considered in individual muscles, except in gastrocnemius. However, when averaged muscle groups were considered, more consistent correlation pattern emerged. Conclusion: Moderate correlation between GSL and QBA with EIM parameters probably reflects on the distinct nature of the muscle-health information provided by these non-invasive methods.

\section{PS2Group5-003 / \#868}

Topic: Group 5 - Novel Diagnostic Methods in Neuromuscular Diseases and Basic Sciences in Neuromuscular Diseases

\section{PHYSIOLOGICAL DIFFERENCES IN SARCOLEMMAL EXCITABILITY MEASUREMENTS BETWEEN HUMAN MUSCLES}

\author{
Robert Boland-Freitas, James Lee, Karl Ng \\ Neurology, Royal North Shore Hospital, ST \\ LEONARDS, AU
}

Background: The sarcolemmal resting membrane potential (RMP) affects muscle excitability, contractility and force generation[1]. There is however limited in vivo data on the normal RMP of the human sarcolemma. We hypothesize that the in vivo RMP may differ between human muscles with different physiological roles. Methods: EMG recording of muscle velocity recovery cycles (MVRC) allows direct in vivo measurement of sarcolemmal excitability measures. MVRC were recorded from a proximal antigravity muscle, rectus femoris (RF), and compared to paired recordings from a distal non-antigravity muscle, tibialis anterior (TA) in thirty normal individuals. Results: Significant differences in muscle relative refractory period (TA:3.97ms vs RF:3.67ms, $P=0.006$ ), early supernormality (TA: $10.4 \%$ vs RF: $14.3 \%, P<0.001)$ and late supernormality (TA:3.35\% vs RF:5.46\%, $P<0.001$ ) were 
observed. Conclusion: The results are suggestive of a relatively depolarized RMP in tibialis anterior versus rectus femoris and differences in t-tubule function between these muscles. This novel result lends support to inter-muscle differences in normal excitability and physiology. Mechanisms underlying this include differences in fiber type make-up[2] and physiological function[3] between muscles in normal individuals. References 1 . Clausen, T., $\mathrm{Na}+-\mathrm{K}+$ pump regulation and skeletal muscle contractility. Physiol Rev, 2003. 83(4): p. 1269-324. 2. Bottinelli, R. and C. Reggiani, Human skeletal muscle fibres: molecular and functional diversity. Prog Biophys Mol Biol, 2000. 73(2-4): p. 195-262. 3. Tirrell, T.F., et al., Human skeletal muscle biochemical diversity. J Exp Biol, 2012. 215(Pt 15): p. 2551-9.

\section{PS2Group5-004 / \#384}

Topic: Group 5 - Novel Diagnostic Methods in Neuromuscular Diseases and Basic Sciences in Neuromuscular Diseases

\section{LABEL-FREE IMAGING OF ABNORMAL LIPID ACCUMULATION IN PERIPHERAL NERVES FROM FABRY DISEASE PATIENTS USING RAMAN SPECTROSCOPY}

Yu Nagashima ${ }^{1}$, Atsushi Iwata ${ }^{1}$, Kosuke Yoshioka $^{2}$, Junko Omachi ${ }^{3}$, Jun Shimizu', Tatsuhi Toda ${ }^{1}$, Junji Yumoto $^{3}$, Makoto K. Gonokami ${ }^{4}$
${ }^{1}$ Department Of Neurology, The University of Tokyo Hospital, Tokyo, JP; ${ }^{2}$ School Of Engineering, The University of Tokyo, Tokyo, JP, ${ }^{3}$ School Of Science, The University of Tokyo, Tokyo, JP:4 The University of Tokyo, Tokyo, JP

Background: Fabry disease is an X-linked genetic disorder resulting from deficient activity of alphagalactosidase A. It causes systemic accumulation of globotriaoslyceramide (Gb3) in the plasma and cellular lysosomes of tissues and organs throughout the body. In routine histopathological studies, toluidine blue staining usually demonstrates abnormal lipid accumulation, but it is not molecular specific and the other lipids in addition to $\mathrm{Gb} 3$ are potentially stained at the same time. The problem is that there is no guarantee that the accumulated lipid is really Gb3. In this study, we measured molecular vibrational spectra of Gb3. Vibrational spectra obtained using Raman spectroscopy provides vibrational information characteristic of chemical groups or bonds in a molecule without any labeling procedures. Measuring vibrational spectra enables us to locate and quantitate $\mathrm{Gb3}$ within tissues in a molecular specific manner. Methods: Vibrational spectra of a $10 \mu \mathrm{m}$ thick frozen sections of sural and femoral nerves biopsied or autopsied from three Fabry disease patients were obtained using spontaneous Raman microspectroscopy and compared with the spectra of $\mathrm{Gb3}$, phosphatidylcholine (PC) and sphingomyelin (SM) in vitro. We also measured sural nerve sections from vasculitis patients as disease control using the same procedure. Results: The Raman shift in $1064 \mathrm{~cm}^{-1}$ (a). Raman spectra of PC, Gb3 and SM

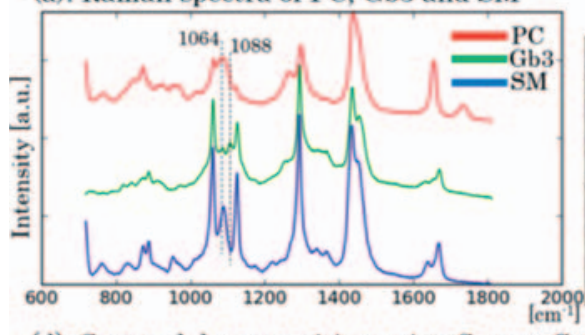

(b). Transmitted light image (Femoral N)

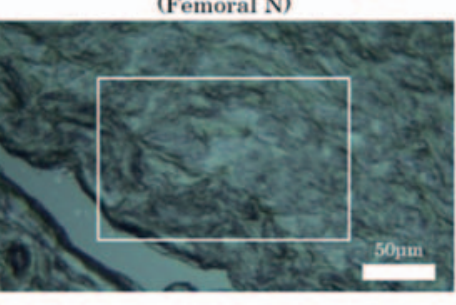

(c). Ratio of Raman shifts $\left(\mathrm{P}\left[1088 \mathrm{~cm}^{-1}\right] / \mathrm{P}\left[1064 \mathrm{~cm}^{-1}\right]\right)$

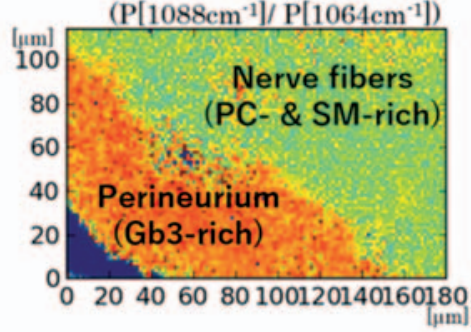

(d). Spectral decomposition using Sparse Coding
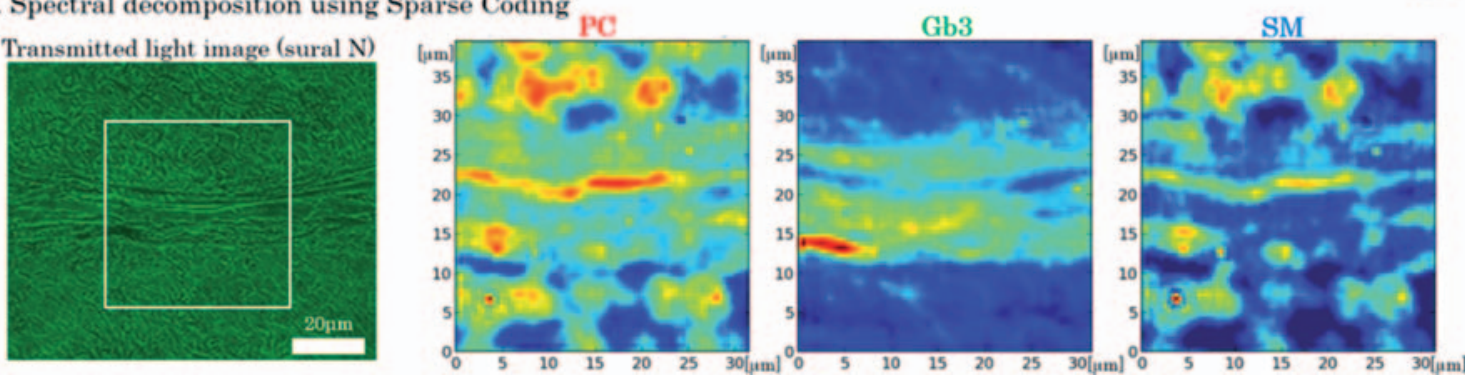
was more prominent in $\mathrm{PC}$ and $\mathrm{SM}$ than in $\mathrm{Gb} 3$, whereas the Raman shift in $1088 \mathrm{~cm}^{-1}$ only existed in Gb3 (Figure 1(a)). The ratio of them $\left(1088 \mathrm{~cm}^{-1}\right.$ to $\left.1064 \mathrm{~cm}^{-1}\right)$ enabled us to contrast Gb3 in the perineurium with PC- and SM-rich nerve fibers in Fabry disease patients (Figure 1(c)). This ratio was not elevated in the sections from the disease control cases. Next, in order to visualize spatial distribution of these lipid species quantitatively, the spectral image data were linearly decomposed into 16 independent bases using sparse coding. The most highly correlated basis with Gb3, PC and SM spectra in vitro were selected and spatially mapped. These mappings visualized a streak-like accumulation of Gb3 within the perineurium, whereas PC and SM showed relatively ubiquitous distribution including the myelinated nerve fibers (Figure 1(d)). Conclusion: The Raman shifts worked as a molecular specific marker of Gb3 within the peripheral nerves from the Fabry disease patients. This is a label-free and lipid-specific visualization technique of $\mathrm{Gb3}$ and is potentially useful even within the tissues other than peripheral nerves.

\section{PS2Group5-005 / \#433}

Topic: Group 5 - Novel Diagnostic Methods in Neuromuscular Diseases and Basic Sciences in Neuromuscular Diseases

\section{MODULATING EFFECTS OF UNSATURATED FATTY ACIDS ON GENE EXPRESSION OF MYOSIN HEAVY CHAIN CLASS I AND II ${ }_{B}$ IN C2C12 MYOCYTES}

Junko Yamaji ${ }^{1}$, Yoshiaki Mori ${ }^{2}$

${ }^{1}$ Department Of Nutrition Sciences, Kansai University of Welfare Sciences, Kashiwara, JP;2 Department Of Rehabilitation Sciences, Kansai University of Welfare Sciences, Kashiwara, JP

Background: Oleic acid (OA) and linoleic acid (LA) belong to long-chain unsaturated fatty acids and present in nuts, seeds, fishes, and other plants. It has been reported that OA and LA had act as a calcineurin activator, but the effects on skeletal muscle still remains almost elusive. Interleukin-6 (IL-6) is a cytokine from immune cells and is also known as a myokine, an anti-inflammatory cytokine from muscle. Recently, we reported that IL-6- and/or calcineurin-activation increased messenger RNA
(mRNA) expression of myosin heavy chain class I (MyHC I), IL-6 and heat shock protein (HSP) 70 during the myotube differentiation of $\mathrm{C} 2 \mathrm{C} 12$ cells, murine skeletal muscle myoblast cell line. In the present study, we examined the effects of unsaturated fatty acids including oleic acid and linoleic acid on expression levels of MyHC I, myosin heavy chain class $\mathrm{II}_{\mathrm{b}}\left(\mathrm{MyHC}_{\mathrm{b}}\right.$ ) and IL-6 mRNA in C2C12 cells. Methods: $\mathrm{C} 2 \mathrm{C} 12$ cells were used after induction of differentiation from myoblast cells to myotubes. At the beginning of differentiation of $\mathrm{C} 2 \mathrm{C} 12$ cells, the cells were incubated in D-MEM containing $2 \%$ serum with or without agents, which are oleic acid and linoleic acid and a calcineurin inhibitor, namely cyclosporine A, and IL-6 and anti-IL-6 antibody as a IL-6 receptor antagonist. After 24-hr incubation, the medium supplemented with agents was removed from cell culture dish and cells were maintained in D-MEM containing 2\% serum for 3 days. The mRNA expression levels were measured by quantitative RT-PCR method using Taqman probes. Quantitative RT-PCR method was performed by a LightCycler Nano system (Roche Applied Science). Quantitative RT-PCR data was analyzed by the comparative CT method. The mRNA expression levels of MyHC I, MyHC II ${ }_{b}$, and IL-6 mRNAs expressed as the cycle time $(\mathrm{Ct})$ values normalized by the $\mathrm{Ct}$ of $18 \mathrm{~S}$ as reference gene expression level. Statistical analyses were performed by the Tukey's test. Results: First, we assessed the effect of unsaturated fatty acids and calcineurin inhibitor on MyHC I and MyHC II mRNA expression. MyHC I and MyHC $\mathrm{II}_{\mathrm{b}}$ mRNA expression levels were upregulated by unsaturated fatty acids such as oleic acid or linoleic acid. These mRNA levels of MyHC I and MyHC II were inhibited by cyclosporine A with oleic acid or linoleic acid. Second, we tested the effects of unsaturated fatty acids, and anti-IL-6 antibody on MyHC $\mathrm{I}$ and $\mathrm{MyHC} \mathrm{II}_{\mathrm{b}}$ mRNA expression. The upregula-

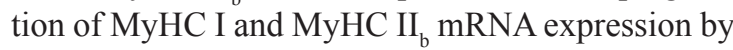
oleic acid and linoleic acid was decreased by antiIL-6 receptor antibody. Third, we examined the effects of unsaturated fatty acids and calcineurin inhibitor on IL-6 mRNA level. Then, the expression level of IL-6 mRNA were significantly upregulated by oleic acid and linoleic acid. The upregulation of IL-6 mRNA expression by oleic acid and linoleic acid was decreased by cyclosporine A. Conclusion: These results indicated that secretion of IL-6 induced by calcineurin activation increases MyHC I and $\mathrm{MyHC}_{\mathrm{b}}$ mRNA in $\mathrm{C} 2 \mathrm{C} 12$ cells. COI:No 
PS2Group5-006 / \#716

Topic: Group 5 - Novel Diagnostic Methods in Neuromuscular Diseases and Basic Sciences in Neuromuscular Diseases

\section{ELECTROPHYSIOLOGICAL} DIAGNOSTIC OF

\section{NEUROMUSCULAR DISEASES IN NEW-BORNS, INFANTS AND TODDLERS}

Philip J. Broser ${ }^{1}$, Oswald Hasselmann², Oliver Maier $^{3}$, Jürg Lütschg ${ }^{3}$

${ }^{1}$ Neuropediatrics, Children 's Hospital of Eastern Switzerland, St Gallen, $\mathrm{CH} ;{ }^{2}$ Neuropediatrics, Children 's hospital of Eastern Switzerland, St. Gallen, $\mathrm{CH} ;{ }^{3}$ Neuropediatrics, Children 's hospital of Eastern Switzerland, St Gallen, $\mathrm{CH}$

Background: Recognizing neuromuscular disease in newborns, infants and toddlers is even for the experienced neuro-pediatrician a challenging task. Many neuromuscular diseases present in the beginning only with very minor symptoms, other cases are quite severe with i.e. generalized muscular hypotonia requiring mechanical ventilation and making a proper clinical assessment difficult. However, early treatment of neuromuscular conditions can alter the prognosis significantly. Therefore precise diagnosis is important. Methods: The children's hospital of Eastern Switzerland has become a major centre for neurophysiological diagnostic in children. We have established a workup to precisely assess the neuromuscular system in newborns and toddlers. Our assessment protocol starts with the basic study of sensory and motor nerves and typically a EMG of the M. tibialis anterior. Depending on the results of the basic study the examination is extended based on the clinical picture. In the case of a Floppy infant with a suspicion of a myasthenia a single fibre EMG is conducted typically at one of the muscles innervated by the cranial nerves. If an asymmetrical weakness is present the examination focuses on the affected muscle and its contralateral counterpart. Results: Based on this assessment protocoll we can diagnose a significant number of neuromuscular diesease. In this presentation we will show examples how spinal muscular atrophy can be diagnosed early by recording pathological discharges during wake and sleep, how a congenital myasthenic syndrome can be diagnosed by single fibre EMG techniques and how especially in clinical apparent myotonia an examination of the mother can show myotonic discharges and lead to the diagnosis of myotonic dystrophy in the child. Conclusion: We conclude our presentation with an outlook of the current developments in pediatric electorneuromyography and especially foucs on latets developments in the application of single fibre EMG techniques in the pediatric population.

\section{PS2Group5-007 / \#964}

Topic: Group 5 - Novel Diagnostic Methods in Neuromuscular Diseases and Basic Sciences in Neuromuscular Diseases

\section{DISCOVERY OF PROTEIN BIOMARKERS FOR DYSFERLINOPATHY}

Laura Rufibach $^{1}$, Zsuzsanna Hollander ${ }^{2}$, Darlene Ly Dai $^{2}$, Virginia Chen ${ }^{2}$, Amrit Singh ${ }^{2}$, Douglas Albrecht ${ }^{1}$, Bradley Williams ${ }^{1}$, Hillarie Windish ${ }^{1}$, Elaine Lee ${ }^{1}$, Plavi Mittal ${ }^{1}$, Anna Mayhew ${ }^{3}$, Marni Jacobs $^{4}$, John W. Day ${ }^{5}$, Kirsti J. Jones ${ }^{6}$, Diana X. Bharucha-Goebel ${ }^{7}$, Matthew Harms ${ }^{8}$, Alan Pestronk $^{8}$, Maggie C. Walter ${ }^{9}$, Tanya Stojkovic ${ }^{10}$, Susan Sparks ${ }^{11}$, Elena Bravver ${ }^{11}$, Jordi DiazManera $^{12}$, Elena Pegoraro ${ }^{13}$, Carmen Paradas ${ }^{14}$, Jerry Mendell ${ }^{15}$, Hanns Lochmuller ${ }^{3}$, Kate Bushby ${ }^{3}$, Volker Straub ${ }^{3}$, Sara Assadian'2, Janet E. WilsonMcmanus $^{16}$, Derek S. Smith ${ }^{17}$, Christoph H. Borchers ${ }^{18}$, Bruce Mcmanus ${ }^{2}$, Raymond $\mathrm{Ng}^{19}$ ${ }^{1}$ Jain Foundation, Seattle, WA, US:2Prevention of Organ Failure (PROOF) Centre of Excellence, Vancouver, BC, CA, ${ }^{3}$ John Walton Muscular Dystrophy Research Centre, Newcastle University, Institute of Genetic Medicine, Newcastle Upon Tyne, GB ${ }^{4}$ Division Of Biostatistic And Study Methodology, Children's National Health System, Centre for Translational Science, DC, WA, US: ${ }^{5}$ Department Of Neurology And Neurological Sciences, Stanford University School of Medicine, Stanford, CA, US: ${ }^{6}$ Institute For Neuroscience And Muscle Research, Children's Hospital At Westmead, University of Sydney, Sydney, AU; ${ }^{7}$ Department of Neurology, Children's National Health System, Washington DC, US; ${ }^{8}$ Department Of Neurology, Washington University School of Medicine, St. Louis, MO, US: ${ }^{9}$ Friedrich-baur Institute, Departement Of Neurology, Ludwigs-Maximilians University of Munich, Munich, DE; ${ }^{.10}$ Institut de Myologie, Paris, FR: ${ }^{11}$ Carolinas Healthcare System Neurosciences Institute, Charlotte, NC, 
US: ${ }^{12}$ Neuromuscular Disorders Unit, Hospital de la Santa Creu i Sant Pau, Barcelona, ES, ${ }^{13}$ Department Of Neuroscience, University of Padova, Padova, IT; ${ }^{14}$ Neuromuscular Unit, Department Of Neurology, Hospital U. Virgen del Rocio/Instituto de Biomedicina de Sevilla, Sevilla, ES: ${ }^{15}$ Center For Gene Therapy, Nationwide Children's Hospital, Columbus, OH, US: ${ }^{16}$ Netcad, Canadian Blood Services, Vancouver, BC, $C A,{ }^{17}$ Genome $B C$ Proteomics Centre, University of Victoria, Victoria, BC, CA: ${ }^{18}$ Proteomics Centre, Segal Cancer Centre, Lady Davis Institute, Jewish General Hospital, McGill University, Montreal, QC, CA $;{ }^{19}$ Department Of Computer Science, University of British Columbia, Vancouver, BC, CA

Background: Dysferlinopathy is a rare neuromuscular disease caused by deficiency in the dysferlin protein and results in progressive skeletal muscle weakness of the limbs, which often leads to the inability to walk. The most common clinical presentations of dysferlinopathy are limb-girdle muscular dystrophy type 2B (LGMD 2B) and Miyoshi Myopathy type 1 (MMD1). Due to the rare nature of the disease, the molecular signatures that differentiate patients from healthy people, as well as different levels of severity, have not been defined. Methods: Blood-based biomarker discovery was performed to identify proteins that can be used to assess disease state and progression, with the goal of eventually using these signatures to assess the efficacy of emerging therapies. For the pilot phase, patients were split into highly ambulant $(\mathrm{N}=25)$ and non-ambulant $(\mathrm{N}=25)$ groups to assess profiles associated with disease progression and to also identify profiles when compared to healthy age and sex matched controls $(\mathrm{N}=25)$. Ensembling was applied to combine protein biomarkers to improve upon the performance of individual proteins. In the technical replication phase, the best performing protein panels were recalibrated in the same subjects using a targeted platform called multiple reaction monitoring mass spectrometry (MRM-MS). In the external replication phase, the MRM panels with the best performance were analyzed in 145 baseline visit samples of the dysferlinopathy natural history study called the Clinical Outcome Study for Dysferlinopathy (COS). In the longitudinal phase, MRM data and clinical metrics from 3 timepoints from COS (baseline, year 1, and year 2) are being evaluated in 120 samples per visit to explore whether longitudinal MRM protein data can be used to stratify dysferlinopathy patients with respect to their future clinical outcomes. Results: In the patients versus healthy control comparison, the best performing protein biomarker panel using MRM had an area under the receiver operating characteristics curve (AUC) performance of $\mathrm{AUC}=0.92$ with $92 \%$ sensitivity, and $76 \%$ specificity. When this panel was tested in the external COS replication cohort it had an $A U C=0.89$. In the ambulatory versus non-ambulatory comparison, the best performing panel using MRM had an AUC $=0.91$ with $92 \%$ sensitivity and $79 \%$ specificity. The external replication performance of this MRM panel in the COS subjects yielded an AUC of 0.77. Conclusion: We identified protein biomarkers that show promise for evaluating and monitoring disease states in dysferlinopathy, and potentially, to predict the effect of therapeutics during clinical trials. The upcoming analysis of the longitudinal COS samples will help us correlate clinical outcomes with protein biomarkers.

\section{PS2Group5-008 / \#992}

Topic: Group 5 - Novel Diagnostic Methods in Neuromuscular Diseases and Basic Sciences in Neuromuscular Diseases

\section{NERVE ULTRASOUND FOR THE IDENTIFICATION OF TREATMENT- RESPONSIVE CHRONIC NEUROPATHIES WITHOUT NERVE CONDUCTION ABNORMALITIES}

$\underline{\text { Stephan Goedee }}^{1}$, Ingrid Herraets ${ }^{1}$, Leo Visser ${ }^{2}$, Thies Van Asseldonk ${ }^{2}$, Hessel Franssen ${ }^{1}$, Ludo Van Der Pol ${ }^{1}$, Leonard Van Den Berg ${ }^{1}$

${ }^{1}$ Neurology, Brain Centre Rudolph Magnus, UMC Utrecht, Utrecht, NL; ${ }^{2}$ Neurology, Elisabeth-

Tweesteden Hospital, Tilburg, NL

Background: Diagnostic consensus criteria for inflammatory neuropathies primarily rely on NCS results that indicate multifocal demyelination. However, NCS can be inconclusive or even normal in patients with neuropathies that respond to immune-modulating treatment. Methods: We analysed 240 incident patients with a clinical phenotype compatible with chronic inflammatory demyelinating polyneuropathy (CIDP), Lewis Sumner Syndrome (LSS) or multifocal motor neuropathy (MMN). Work-up consisted of routine ancillary investigations recommended in the diagnostic consensus guidelines and a standardized sonographic protocol 
assessing arm nerves and brachial plexus. All patients without any demyelinating features on NCS, but with sonographic abnormalities fitting inflammatory neuropathy underwent standard treatment with IVIg. Treatment effect was evaluated by routine clinical examination and hand grip or myometry. Results: We found 17 (7\%) patients with a clinical phenotype compatible with CIDP $(n=10)$, $\operatorname{LSS}(n=1)$ and MMN ( $n=6$, with 3 positive for antiGM1 auto-antibodies), sonographic nerve enlargement of proximal median nerve segments and/or cervical (nerve) roots, yet negative NCS ( 2 only axonal loss, 15 normal). In contrast, MRI of brachial plexus was normal in 8/17 (47\%) and protein content of cerebrospinal fluid in 6/17 (35\%). All 17 patients showed objective improvement on IVIG treatment. Conclusion: Our study suggests that nerve ultrasound may help to improve detection of treatment-responsive chronic neuropathies, even in patients not fulfilling the diagnostic NCS and other supportive criteria, including MRI. Therefore, future revisions of the diagnostic consensus criteria may include sonography as complementary technique to NCS and MRI.

\section{PS2Group5-009 / \#810}

Topic: Group 5 - Novel Diagnostic Methods in Neuromuscular Diseases and Basic Sciences in Neuromuscular Diseases

\section{MOTOR-UNIT NUMBER} ESTIMATION IN THE ABDUCTOR POLICIS BREVIS MUSCLE OF PATIENTS WITH CARPAL TUNNEL SYNDROME

Bahram Haghiashtiani ${ }^{1}$, Fahimeh Akhondi ${ }^{2}$, Zahra Mirza Asgari², Mostafa Almasi², Babak Zamani², Mohammad Reza Motamed ${ }^{2}$, Masood Mehr Pour ${ }^{2}$ ${ }^{I}$ Neurology, Iran University of Medical Sciences, $I R ;{ }^{2}$ Iran University of Medical Sciences, Tehran, IR

Background: Median nerve entrapment at wrist is the most common of all entrapment neuropathies and, consequently, one of the most frequent reasons for referral for an electrodiagnostic (EDX) study. In any individual patient with CTS, there may be little correlation between the frequency and severity of clinical symptoms and signs and the abnormalities seen on routine nerve conduction studies. Also, decreased compound Muscle action potentials
(CMAPs) is only a late finding in the EDX study of CTS patients and becomes apparent when the thenar eminence is actually atrophic. Motor Unit Number Estimation (MUNE) is a unique EDX technique that enables us to estimate the number of axons innervating a given muscle. A motor unit (MU) is defined as a single motor neuron, and all muscle fibers innervated by it. Activation of a motor neuron causes contraction of all the muscle fibers innervated by it; all of the motor units that innervate a single muscle are considered to be its motor unit pool. A number of MUNE methods have been described that include the Incremental Technique, Multiple Point Stimulation Spike Triggered Statistical method, F wave MUNE, and MUNIX. In this study we opted for the incremental technique to evaluate the number and amplitude of active motor units in the Abductor Policis Brevis (APB) muscle of patients presenting with symptoms and signs typical of CTS. Methods: We evaluated 42 hands of 21 patients ( 2 males and 19 females), who presented with clinical features indicative of CTS. We performed routine Median nerve conduction studies, and divided our subjects to mild, moderate, and severe CTS based on routine Electrodiagnostic (EDX) findings. We estimated the number and the amplitude of motor units by the Incremental stimulation technique, and compared our findings to normal values. For MUNE, we used the Incremental stimulation technique originally described by McComas. The recording and stimulating electrodes were placed as described above. First, we set the gain at 50 microvolts $(\mu \mathrm{Vs})$ and the sweep at 5 milliseconds, and using supramaximal stimulation, we measured the distal Median CMAP amplitude in two ways: Base-to-Peak (from the baseline to the first negative peak) and Peak-to-Peak (from the first negative peak to the following positive peak). Finally, we divided the supramaximal CMAP amplitude to the mean amplitude of a single motor unit to yield a MUNE value. Results: MUNE values were as follows: $95.5 \pm 28.9$ in males and $100.1 \pm 32.4$ in females by the Base-to-Peak method, and $98.7 \pm$ 20.6 in men and $91.3 \pm 40.9$ in women by the Peakto-Peak way. These values were significantly lower than the normal population. We also observed a significant correlation between MUNE values and the severity of CTS. Conclusion: MUNE is a valid tool to assess CTS patients grading even in the early stages of the disease. 


\section{PS2Group5-010 / \#739}

Topic: Group 5 - Novel Diagnostic Methods in Neuromuscular Diseases and Basic Sciences in Neuromuscular Diseases

\section{HIGH RESOLUTION NEUROSONOGRAPHY IN PATIENTS WITH CARPAL TUNNEL SYNDROME AND NORMAL CONTROLS}

Atchayaram Nalini ${ }^{1}$, Saraswati Nashi ${ }^{2}$, Veeramani Preethish-Kumar ${ }^{1}$, Nishtha Yadav ${ }^{3}$, Kajari Bhattacharya $^{3}$, Kiran Polavarapu ${ }^{1}$, Seena Vengalil ${ }^{1}$ ${ }^{1}$ Neurology, National Institute of Mental Health and Neurosciences, Bengaluru, IN; ${ }^{2}$ NIMHANS, Bengaluru, IN; ${ }^{3}$ Neuro Imaging And Interventional Radiology, National Institute of Mental Health and Neurosciences, Bengaluru, IN

Background: Carpal tunnel syndrome (CTS) is the commonest entrapment neuropathy with a prevalence of $3.8 \%$. Ultrasonography (USG) measurements for the diagnosis of CTS has sufficient accuracy and allows differential diagnosis and supports the surgeon. The objective was to compare the sensitivity of electrodiagnosis and high resolution nerve US in CTS. Methods: Nerve US was performed with Philips Epiq 7G, linear 5 - 18Hz transducer. Median nerve (MN) cross sectional area (CSA) was recorded transversely at level of pisiform and scaphoid bones parallel to each other (carpal tunnel inlet).Distal to proximal ratio was calculated $4 \mathrm{~cm}$ from the distal crease in forearm. Nerve conductions were performed as per standard procedure. MR Neurography (axial and sagittal images using a temporomandibular surface coil, fat saturation, and flow suppression). Results: Total number of patients studied were 30. F:M=4:1. Mean age at presentation was $41.6 \pm 10.5(27-61)$ years. Clinically, 28(93.3\%) had bilateral CTS [symmetrical-4; asymmetrical-24] and two had unilateral involvement. Duration of illness varied from 2 months to 8 years(mean $35.8 \pm$ 25.5). All had sensory complaints while 9 patients $(30 \%)$ also had motor deficits. Sensitivity of Tinel sign / Phalen's maneuver was $40 \%$ and $63.3 \%$ respectively. By USG, cross sectional area (CSA) at pisiform bone level was $>0.1 \mathrm{~cm}^{2}$ in 27 patients. Distal/proximal ratio was $\geq 1.5$ bilaterally in 16 patients and unilaterally in 5(right -3 , left -2 ). Mean distal CSA of $\mathrm{RMN}=1.44 \pm 0.42 \quad \mathrm{~cm}^{2} \quad(0.8-2.5)$, left $=1.31 \pm 0.3 \mathrm{~cm}^{2}(0.7-2.0)$. By comparison, in age and sex matched controls[37 cases; 75 hands, $\mathrm{F}=$ $39 ; \mathrm{M}=6)$ ], the mean distal CSA was $0.79 \pm 0.13 \mathrm{~cm}^{2}(0.59-0.11)$ and $0.77 \pm 0.1 \mathrm{~cm}^{2}(0.53-$ 0.1 ) in right and left median nerves respectively. Mean CSA of patients compared to controls was significantly higher. Nerve conduction studies in 27 patients revealed absent evoked motor response in 4 patients (right-3, left -1 ). Mean distal motor latency and compound muscle action potential of right median nerve $(\mathrm{RMN})$ in 24 cases was $5.8 \pm 1.4 \mathrm{~s}(4.2$ $11.4)$ and $8.65 \pm 3.0 \mathrm{mv}(3.3-10.3)$ respectively. On the left(26 cases), it was $5.5 \pm 1.8 \mathrm{~s}(3.3-10.3)$ and $8.3 \pm 3.7 \mathrm{mv}(0.6-15.8)$. Median nerve SNAPs were absent in 16 patients (bilateral -10 , right -4 , left-2). Mean sensory latencies were $4.5 \pm 2.4 \mathrm{~s}(2.5-12)$ and $3.4 \pm 0.7 \mathrm{~s}(2-4)$ in right(13) and left(14) sides respectively with corresponding mean sensory amplitudes[Right: $\quad 7.2 \pm 5.3 \mathrm{mv}(1.5-23) \quad$ Left: $9.9 \pm 5.9 \mathrm{mv}(2.3-22.1) \quad$ and conduction velocities[Right: $35.6 \pm 11 \mathrm{~m} / \mathrm{s}(11.7-\quad 58)$ Left: $43.7 \pm 13 \mathrm{~m} / \mathrm{s}(22.2-68.6)]$. MRI in 19 patients showed mean distal CSA of RMN of $1.55 \pm 0.49 \mathrm{~cm}^{2}$ (range, $1-2.7$ ) and $L M N=1.57 \pm 0.4 \mathrm{~cm}^{2}$ (range, 1-2.7). Conclusion: This study emphasizes the importance of USG as sensitive, cheaper and effective alternate to MRI in the confirmation of CTS. CSA of the proximal MN could be used as a predictor of severity of CTS. Moreover, the mean CSA and inlet CSA may be valid and easy-to-acquire parameters for routine clinical use in confirming CTS.

\section{PS2Group5-011 / \#793}

Topic: Group 5 - Novel Diagnostic Methods in Neuromuscular Diseases and Basic Sciences in Neuromuscular Diseases

\section{CAN WE EARLY DETECT CARDIAC DYSFUNCTION IN PATIENTS WITH DUCHENNE MUSCULAR DYSTROPHY?}

Michal Marchel, Janusz Kochanowski, Agnieszka Serafin, Bozena Truszkowska, Grzegorz Opolski Department Of Cardiology, Medical University of Warsaw, Warsaw, PL

Background: Duchenne muscular dystrophy (DMD) is characterized by musculoskeletal abnormalities due to the mutations in dystrophin coding gene (dystrophinopathy), frequently accompanied by cardiac dysfunction. Methods: The aim of the study was to 
analyse both ventricular function in adult DMD patients (pts) with the use of among other techniques global longitudinal strain assessed by speckle-tracking echocardiography. The group of 33 adult male pts with genetically confirmed dystrophinopathy as well as 25 healthy volunteers were subjected to echocardiography with the assessment of global longitudinal strain (GLS) [Philips Epiq 7]. Results: The mean left ventricular ejection fraction (LVEF) was 48,0 +/$15,3 \%$ and $65,3+/-3,7$ for DMD pts and for controls respectively $(p<0,05)$. We identified three subgroups of DMD patients: 1 - significant systolic dysfunction - $\mathrm{LVEF}<40 \%(\mathrm{n}=12), 2$ - mid-range and preserved systolic function $-\mathrm{LVEF}>=40 \%(\mathrm{n}=21)$ as well as 3 - preserved systolic function $-\mathrm{LVEF}>=50 \%(\mathrm{n}=19)$ accompanied by group 4 (healthy controls, $n=25$ ). In group 1 we observed lower GLS as compared to group $4(11,8+/-2,6$ vs. $20,6+/-3,4 ; p=0,01)$, but also pts with persistent LVEF (group 2) had lower GLS than controls $(16,6+/-2,0$ vs. $20,6+/-3,4$; $\mathrm{p}=0,01$ ). Pts with preserved LVEF (group 3, mean EF $59,4+/-7,3)$ had also lover GLS compering to control group $(16,8+/-2,1$ vs. $20,6+/-3,4 ; p=0,02)$. Conclusion: Severe left ventricular systolic dysfunction is common in DMD pts, (12/33, 36\%). The quantitative assessment of global longitudinal myocardial deformation can be helpful in detection of systolic abnormalities in DMD pts with normal LVEF. Subclinical systolic dysfunction is frequent $(13 / 19,66 \%)$ in DMD pts with LVEF still in normal range.

\section{PS2Group5-012 / \#197}

Topic: Group 5 - Novel Diagnostic Methods in Neuromuscular Diseases and Basic Sciences in Neuromuscular Diseases

\section{PERIPHERAL NERVOUS SYSTEM DISORDERS IN MULTIPLE MYELOMA: RABAT CLINICAL NEUROPHYSIOLOGY DEPARTMENT EXPERIENCE}

Laila Belarabi ${ }^{1}$, Nezha Birouk ${ }^{2}$, Bouchra Kably², Leila Errguig' ${ }^{2}$, Halima Belaïdi², Reda Ouazzani² ${ }^{1}$ Clinical Neurophysiology, Speciality Hospital, Rabat, MA $;{ }^{2}$ Clinical Neurophysiology, Speciality Hospital, Rabat, MA

Background: Multiple myeloma is a cancer formed by malignant plasma cells. Peripheral neuropathy has been considered as mainly secondary to the plasma cell dyscrasia itself, or following a direct compression. The association of neuropathy with multiple myeloma apart of these mecanisms is rare, often asymptomatic and discovered during the prechemotherapy assessment. With the advent of new drugs such as thalidomide and bortezomide for chemotherapy, the iatrogenic neurotoxicity has become the leading cause of peripheral neuropathy in multiple myeloma. Methods: We conducted a retrospective study from January 2014 to September 2017 including all patients referred to the department for an electroneuromyogram (ENMG) as part of a prechemotherapy assessment or in post-thalidomide follow-up, whether the patient was symptomatic or not. Results: We collected a serie of 32 cases, which we divided into two groups : patients before treatment with thalidomide (18 cases) and under thalidomide (14 cases). In the first group before treatment, the mean age was 58.6 years, with a sex ratio of 1.6. Four axonal sensory motor polyneuropathy (25\%), and one demyelinating sensory motor polyneuropathy was discovered at the ENMG without any clinical manifestation. In the second group, the mean age was 61 years with a sex ratio of 1.3. Eight patients $(57 \%)$ had peripheral neuropathy predominant to lower limb in six cases and painful in five cases. Conclusion: Polyneuropathy in multiple myeloma is well recognized, most often with compressive involvement, secondary to plasma cell dyscrasia or autoimmune mechanism. In subclinical neuropathies, it is often a sensory motor axonal polyneuropathy, predominant in distal and sensitive. Thalidomide is responsible for length-dependent sensory axonal polyneuropathies requiring close clinical and ENMG follow-up, in order to stop treatment before causing irreversible damage. Finally, early detection of peripheral neuropathy and the use of dose adjustment algorithms should help reduce the side effects while maintaining anti-tumor efficacy

\section{PS2Group5-013 / \#343}

Topic: Group 5 - Novel Diagnostic Methods in Neuromuscular Diseases and Basic Sciences in Neuromuscular Diseases

\section{CRAMPS, FASCICULATIONS AND MUSCLE FATIGABILITY DUE TO VITAMIN D DEFICIENCY}

Laila Belarabi, Nazha Birouk, Bouchra Kably, Reda Ouazzani Clinical Neurophysiology, Ibn Sina Hospital, Rabat, $M A$ 
Background: The clinical signs of vitamin D deficiency are well known, dominated by the manifestations of hypocalcemia and secondary hyperparathyroidism. In adults, this syndrome is mostly represented by proximal muscle weakness, associated in some cases with cramps, fasciculations, and paresthesia. Patients can be misdiagnosed as having a motor neuron disease or muscle intolerance to effort, the ENMG being often normal or showing only fasciculations or neurogenic pattern.

Methods: We report the clinical and outcome data in 5 patients with muscles cramps, fasciculation's and fatigability. Results: 5 patients were included in this study, 2 women and 3 men, aged from 20 to 59 years. All patients suffered from muscle weakness and exercise induced muscle pain, with a reduction in the walking distance in 2 cases, cramps in 3 cases, and fasciculations in 2 cases. The usual biological investigations were normal in all cases, except elevated CK $(3 \mathrm{xN})$ in 1 case. The ENMG was normal in 3 cases, and revealed diffuse fasciculations in 1 patient. 2 patients underwent muscle biopsy that was normal. Vitamin D level was reduced in all patients. After correction with vitamin D supplementation, 3 patients are totally asymptomatic, with a reduction of the symptoms in the others. Conclusion: Vitamin D deficiency, despite its classic manifestations, must be included in the causative diagnosis of muscle fatigability, cramps and fasciculations, in order to prevent the patient from delayed diagnosis and in some cases from invasive examinations.

\section{PS2Group5-014 / \#617}

Topic: Group 5 - Novel Diagnostic Methods in Neuromuscular Diseases and Basic Sciences in Neuromuscular Diseases

\section{NEXT-GENERATION SEQUENCING FOR MOLECULAR DIAGNOSIS IN NEUROMUSCULAR DISORDERS. RESULTS FROM 151 PATIENTS}

Daniel Natera De Benito ${ }^{1}$, Jessica Exposito

Escudero $^{2}$, Delia Yubero ${ }^{2}$, Lidia González

Quereda $^{3}$, Pía Gallano Petit ${ }^{3}$, Ana Töpf $f^{4}$, Carlos

Ortez $^{5}$, Laura Carrera-García ${ }^{2}$, Anna Lia Frongia ${ }^{6}$, Cristina $\mathrm{Jou}^{2}$, Cecilia Jimenez Mallebrera ${ }^{2}$, Anna Codina $^{2}$, Jaume Colomer ${ }^{2}$, Andres Nascimento ${ }^{2}$ ${ }^{1}$ Neuromuscular Disorders Unit, Hospital Sant Joan de Déu, Barcelona, ES: ${ }^{2}$ Hospital Sant Joan de Déu, Barcelona, ES, ${ }^{3}$ Hospital Sant Pau, Barcelona, ES:4 John Walton Muscular Dystrophy Research
Center, Newcastle University, Newcastle, GB; ${ }^{5}$ Neuromuscular Unit, Hospital Sant Joan de Déu, Barcelona, ES, ${ }^{6}$ Hospital Sant Joan de Déu, Barcelona, ES

Background: Inherited neuromuscular disorders (NMD) are chronic genetic diseases posing a significant burden on patients and the health care system. Despite tremendous research and clinical efforts, the molecular causes remain unknown for a significant proportion of patients, due to genetic heterogeneity and the use of a conventional gene-by-gene approach until relatively recently. Massively parallel sequencing using next generation sequencing (NGS) technologies has emerged as a successful approach to interrogate multiple genes simultaneously. It has proven to be an efficient and cost-effective tool to accelerate patient diagnosis. Herein, we describe the results obtained in an extensive study of 151 genetically undiagnosed patients presenting clinical signs of muscular dystrophies, congenital myopathies, or other neuromuscular conditions. We report the huge improvement observed after NGS aplication into the clinical field. Methods: Patients with clinical criteria of a neuromuscular disease and that had been studied using different NGS strategies were collected. The 151 patients had been evaluated with three different methodologies, due to the quick evolution of NGS in this field. The first group of patients $(\mathrm{n}=58)$ was studied with a custom panel (Nextera Rapid Capture, Illumina) including 118 genes, sequenced on a MiSeq instrument (Illumina) and analysed with the Variant Studio and DNA Nexus softwares. The second group $(n=40)$ had sequenced 4813 genes associated to human disease (commercial TruSight One Sequencing Panel (Illumina) and Illumina sequencing on a NextSeq500), with an inhouse bioinformatic analysis developed in the bioinformatics unity. The third group $(n=53)$ followed a broader strategy, performing an Illumina exome capture (38 $\mathrm{Mb}$ baited target) to interrogate the whole exome, and after sequencing and bioinformatics analysis, only 169 genes associated with neuromuscular disorders were studied to check for candidate mutations. Data interpretation and candidate variants selection was performed according to the ACMG guidelines, and sanger sequencing in cases and progenitors was done in cases where strong candidates appeared. Results: Patients were classified, according to their clinical phenotype, as affected by congenital myopathy (52\%), muscular dystrophies (29\%), congenital myasthenic syndromes (6\%), neuropathies $(5 \%)$, or other clinical conditions $(8 \%)$, 
including, among others, metabolic myopathy and hereditary spastic paraplegia. Most patients were sporadic cases. The majority of samples collected had previously been tested unsuccessfully, according to the observed phenotype. Eighty cases (53\%) obtained a diagnosis, showing known disease-associated variants, or variants of a likely pathogenicity, or variants predicted to affect function in genes corresponding to clinical suspicions. Genetic cause of the disease was elucidated in $24 / 40$ samples $(60 \%)$ by the clinical exome, in $30 / 58$ samples $(51 \%)$ by the custom panel, and in 26/53 samples (49\%) by the selected-WES analysis. Conclusion: In recent years, NGS has totally transformed the approach to the study of neuromuscular disorders. Our experience indicates that about half of the patients with a suspected neuromuscular disease are solved with NGS technology. Despite this, the molecular causes remain unknown for $47 \%$ of patients. Some of the unsolved cases could in fact be attributable to the kind of NGS strategy applied, to previously undescribed causative genes, or to genetic variation in areas that are out of the rule.

\section{PS2Group5-015 / \#258}

Topic: Group 5 - Novel Diagnostic Methods in Neuromuscular Diseases and Basic Sciences in Neuromuscular Diseases

\section{TRANSCRANIAL MAGNETIC STIMULATION AS A DIAGNOSTIC AND PROGNOSTIC TOOL IN CHILDREN WITH SEQUELAE OF ACUTE TRANSVERSE MYELITIS}

\author{
Vladislav Voitenkov $^{1}$, Natalia Skripchenko ${ }^{2}$ \\ ${ }^{1}$ Clinical Neurophysiology, Pediatric Research and \\ Clinical Center for Infectious Diseases, Saint- \\ Petersburg, RU, ${ }^{2}$ Pediatric Research and Clinical \\ Center for Infectious Diseases, Saint-Petersburg, $R U$
}

Background: Diagnostic process and especially prognosis in acute transverse myelitis in children may be challenging. Diagnostic TMS may be useful additional tool in this process. Goal of our study was to evaluate motor pathways in children with viral myelitis using TMS. Methods: 20 healthy children (7-14 years old, average 12) without signs of spinal disorders were enrolled as controls. Main group consisted of 24 patients (8-16 years old, average 11) with acute viral transverse myelitis, in 4 cases with cervical localization of the lesion, in 7 - thoracic and in 13 - lumbar. Etiology was enterovirus $(n=20)$ Epstein-Barr $(\mathrm{n}=4)$ viruses. 18 children were unable to walk, lower paresis was seen in other 6 cases; ventilation was not required in any cases. We used single-pulse TMS protocol. Neiro-MS D magnetic stimulator was used. Neiro-MVP EMG/EP system was used for data registration. Results: Three neurophysiologic patterns were observed in the myelitis group: Presence of both cortical MEP (although disperse and low-amplitude) and spinal MEP $(n=15)$. Presence of only spinal MEP without cortical one $(n=6))$. Total absence of both spinal and cortical MEP (n=3). (Fig. 1). In the follow-up period (2-5 years) 10 children (42\%) were unable to walk 50 meters and required a walker, $11(46 \%)$ had normal motor function and $3(13 \%)$ had total paraplegia without any movement of the lower limbs, no signs of recovery. Among those 11 who fully recovered only the first TMS pattern (presence of both cortical and spinal MEPs) was registered. Those who recovered with sequelae had first or second pattern and in 3 patients who did not recover only the third pattern was seen. ROC-analysis data revealed significant correlation between the long-term (3-5 years) recovery of walk in patients with consequences of myelitis and CMCT value $\geq 28,7 \mathrm{~ms}$. Conclusion: Acute transverse myelitis in $96 \%$ of the cases causes neurophysiologic changes which may be detected by diagnostic TMS. Method may be used as a predicting tool: absence of the cortical and spinal MEP may be considered as a sign of highly probable poor clinical outcome.

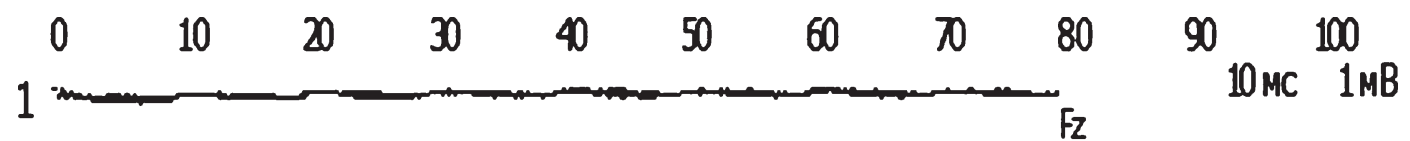




\section{PS2Group5-016 / \#762}

Topic: Group 5 - Novel Diagnostic Methods in Neuromuscular Diseases and Basic Sciences in Neuromuscular Diseases

\section{TARGETED METHYL-SEQ QUANTIFICATION BY NGS TECHNOLOGY FOR ROUTINE DIAGNOSTIC OF FSHD}

$\underline{\text { Stefanie Bulst }}{ }^{1}$, Florentine Scharf ${ }^{1}$, Anna BenetPagès $^{1}$, Peter Reilich ${ }^{2}$, Sibylle Jakubiczka ${ }^{3}$, Martin Zenker $^{3}$, Maggie C. Walter ${ }^{4}$, Elke Holinski-Feder ${ }^{1}$, Angela Abicht ${ }^{1}$

${ }^{1}$ Medical Genetic Center, Munich, DE, ${ }^{2}$ Friedrichbaur Institute, Departement Of Neurology, Ludwigs-Maximilians University of Munich, Munich, DE: ${ }^{3}$ Institut Für Humangenetik, Otto-vonGuericke-University of Magdeburg, Magdeburg, DE: ${ }^{4}$ Dept. Of Neurology, Ludwig-maximiliansuniversity, Friedrich-Baur-Institute, Munich, DE

Background: Facioscapulohumeral Muscular Dystrophy (FSHD) is associated with hypomethylation of the D4Z4 microsatellite, leading to an increased expression of the DUX4 gene which is suggested to cause progressive muscle atrophy and ultimately FSHD pathology. Chromatin changes are caused by contractions to less than 11 units of the D4Z4 repeat (FSHD1) or by pathogenic variants in chromatin regulatory proteins (FSHD2). We developed a novel NGS-based method in an attempt to diagnose FSHD and distinguish FSHD1 from FSHD2 in a single approach. Methods: Enrichment of 4qA (A) and 4qAL $(\mathrm{AL})$ associated D4Z4 repeats as well as all $4 \mathrm{q} 35$ D4Z4 repeat units (DUX4) was performed using nested PCRs after bisulfite conversion of genomic DNA (EpiTect). DNA libraries were prepared with the NEBNext Ultra DNA Library Prep Kit and sequenced on an Illumina MiSeq System. Reads were mapped to A, AL and DUX4 using bwameth. Mean methylation levels were calculated with an in-house script. Results: We analyzed 21 patients with a confirmed FSHD diagnosis and 21 negative controls. We could confirm the hypomethylation status in all patients. Low methylation levels tend to correlate with a more severe clinical disease status. Due to a chromatin dysregulation DUX4 is hypomethylated in FSHD2 as well; as expected we could confirm lower methylation levels in DUX4 in FSHD2-patients compared to FSHD1. Conclusion: In conclusion, sensitive detection of methylation levels in the
D4Z4 array may give insight into the clinical progression levels more exactly than currently available methods. Additionally, a much higher yield and throughput in FSHD diagnostics may be achieved.

\section{PS2Group5-017 / \#579}

Topic: Group 5 - Novel Diagnostic Methods in Neuromuscular Diseases and Basic Sciences in Neuromuscular Diseases

\section{FACIAL NERVE ULTRASOUND IN BELL'S PALSY}

$\underline{\text { Seol-Hee Baek }}^{1}$, Ye-Ji Kwon ${ }^{1}$, Yoo Hwan Kim², Hung Youl Seok ${ }^{3}$, Byung-Jo Kim ${ }^{1}$ ${ }^{1}$ Department Of Neurology, Korea University Medical Center, Korea University College of Medicine,, Seoul, KR;²Department Of Neurology, Hangang Sacred Heart Hospital, Hallym University Medical Center, Seoul, KR; $;^{3}$ Department Of Neurology, Keumyung University Dongsan Medical center, Daegu, KR

Background: Bell's palsy is one of the causes of acute paralysis of the facial nerve. However, the etiology of Bell's palsy is not clearly understood. Ultrasound is the useful tool for evaluating neuromuscular disease. This study is aimed to investigate the usefulness of facial nerve ultrasound in Bell's palsy. Methods: We prospectively recruited patients with Bell's palsy who visited Korea University Anam Hospital from January 2016 and November 2017. Ultrasonography was performed using a $15 \mathrm{MHz}$ linear array transducer (HD15 system, Philips Ultrasound, Bothell, WA, USA). Facial nerves were scanned bilaterally along its longitudinal axis inside the parotid gland. The diameters of facial nerve with and without sheath were measured at the proximal and distal portion. Results: Eighty-one patients (43 males, mean age $43.9 \pm 15.9$ years) were enrolled. At the proximal portion of the facial nerve, the diameters with and without sheath on the affected side were significantly larger than the unaffected side $(0.97 \mathrm{~mm}$ vs. $0.85 \mathrm{~mm}, \mathrm{p}<0.001 ; 0.61 \mathrm{~mm}$ vs. $0.32 \mathrm{~mm} ; \mathrm{p}=0.003$, respectively). At the distal portion of the facial nerve, the diameter with sheath was significantly different between the affected and unaffected side $(0.86 \mathrm{~mm}$ vs. $0.80 \mathrm{~mm}, \mathrm{p}=0.004)$. However, the diameter without sheath was not a significant difference $(0.31 \mathrm{~mm}$ vs. $0.29 \mathrm{~mm} ; \mathrm{p}=$ 0.633). Among 81 patients, 34 patients have tested 
follow-up sonography after 2 months. At the proximal portion of the facial nerve, the diameter with sheath on the affected side was significantly decreased after 2 months later $(\mathrm{p}=0.037)$. However, there were no significant changes in diameters with the sheath at a distal portion of the facial nerve between initial and follow-up test. Also, the diameters without sheath did not significantly change at both proximal and distal portion of the facial nerve. Conclusion: The diameters of facial nerve were enlarged on the affected side, especially at the proximal portion. Ultrasonography may be helpful for assessment of facial nerve in Bell's palsy.

\section{PS2Group5-018 / \#385}

Topic: Group 5 - Novel Diagnostic Methods in Neuromuscular Diseases and Basic Sciences in Neuromuscular Diseases

\section{CGRP UPREGULATES MYOSIN} HEAVY CHAIN TYPE I MESSENGER RNA THROUGH CALCINEURIN-IL6-INDEPENDENT MANNER IN

\section{C2C12 CELLS}

\author{
Yoshiaki Mori $^{1}$, Junko Yamaji ${ }^{2}$ \\ ${ }^{1}$ Department Of Rehabilitation Sciences, Kansai \\ University of Welfare Sciences, Kashiwara, \\ $J P ;{ }^{2}$ Department Of Nutrition Sciences, Kansai \\ University of Welfare Sciences, Kashiwara, JP
}

Background: Our previous study using differentiated $\mathrm{C} 2 \mathrm{C} 12$ cells indicated that myosin heavy chain type I (MyHC I), myosin heavy chain type IIb (MyHC II $)$, and interleukin-6 (IL-6) mRNA expression levels were significantly increased by the application of calcineurin activators. In this study, we examined the effects of calcitonin gene-related peptide (CGRP) on mRNA levels of MyHC I, MyHC II and IL-6 in C2C12 cells. CGRP is a 37 amino acid peptide which belongs to a family of related peptides including calcitonin, amylin, and adrenomedullin. CGRP peptides are mainly localized in sensory and central neurons and have been implicated in a variety of physiological processes. CGRP has also been identified in spinal motoneurons of several species and in the nerve terminals of the rodent neuromuscular junction. Effects of CGRP have been studied in two skeletal muscle cell lines, rat L6 cells and mouse $\mathrm{C} 2 \mathrm{C} 12$ cells. These cell lines appear to express CGRP receptors coupled to adenylyl cyclase activity.
However, the effects of CGRP on expression of MyHC mRNA have not been reported in the skeletal muscle cells. Methods: $\mathrm{C} 2 \mathrm{C} 12$ cells were maintained until semi-confluence in D-MEM containing $15 \% \mathrm{FBS}$. The cells were incubated in D-MEM containing $2 \% \mathrm{FBS}$ with or without agents at a beginning of differentiation. After 24h. incubation, the medium was removed and the cells were maintained in differentiation medium for 3 days. MyHC I, MyHC II and IL- 6 mRNA expression levels were measured by the real-time PCR method. Results: First, we confirmed the effect of calcineurin activators, such as chlorogenic acid and oleic acid, on mRNA expression levels of MyHC I, MyHC II ${ }_{b}$, and IL-6 in C2C12 myocytes. These mRNA levels were significantly increased by the administration of calcineurin activators, and the effects of calcineurin activators on upregulation of these mRNA levels were attenuated by the co-administration of cyclosporine A. The effects of calcineurin activators on upregulation of $\mathrm{MyHC} \mathrm{I}$ and MyHC $\mathrm{II}_{b}$ mRNA levels were significantly attenuated by the co-administration of anti-IL-6 receptor antibody, although IL-6 mRNA levels were not affected by co-administration of the antibody. These results indicate that the upregulation of $\mathrm{MyHC}$ I and MyHC II $\mathrm{b}_{\mathrm{b}}$ mRA by the administration of calcineurin activators would be the result of autocrine/paracrine secretion of IL-6 in C2C12 myocytes. Second, we tried to examine the effects of CGRP on mRNA expression levels of MyHC I, MyHC II , and IL-6. Administration of CGRP to the culture medium did not affect to the MyHC II and IL-6 mRNA levels. However, MyHC I mRNA levels were significantly increased by the administration of CGRP. Conclusion: These results suggest that the effects of CGRP on upregulation of MyHC I mRNA levels do not depend on calcineurin-IL-6-mediated mechanisms. Further experiments need to be done to clarify the CGRP-mediated intracellular signaling in the upregulation of MyHC I mRNA. 


\section{PS2Group5-019 / \#600}

Topic: Group 5 - Novel Diagnostic Methods in Neuromuscular Diseases and Basic Sciences in Neuromuscular Diseases

\section{ANALYSIS OF EUROPEAN EARLY- ONSET MYASTHENIA GRAVIS GWAS SIGNALS IN AFRICAN GENOMES}

$\underline{\text { Jeannine M. Heckmann }}{ }^{1}$, Melissa $\mathrm{Nel}^{2}$, Nicola

\section{Mulder $^{3}$}

${ }^{1}$ Medicine (neurology), University of Cape Town, Cape Town, ZA, ${ }^{2}$ Neurology Research Group, University of Cape Town, Cape Town, $Z A,{ }^{3}$ Computational Biology Division, University of Cape Town, Cape Town, ZA

Background: Genome wide association studies (GWAS) in myasthenia gravis (MG) have only been performed in European populations to date. The follow-up of these putative susceptibility variants in African populations, whose genomes have substantially increased genetic diversity and shorter linkage disequilibrium blocks, has the potential to fine-map true causal variants. We performed a cross-racial analysis to determine whether previously identified European GWAS signals in individuals with earlyonset acetylcholine receptor (AChR) antibody positive myasthenia gravis (Eur-EOMG) are replicated in African genomes with the same disease phenotype. Methods: 25 individuals with African genetic ancestry from South Africa and early-onset AChR-MG (SA-EOMG), underwent whole genome sequencing. HLA allele group assignment was inferred through "best-match" alignment of reads against the IMGT/ HLA database (v3.29.0.1, 2017) using HLA Explore software (Omixon). Ancestry-matched controls were from a local dataset (SA-controls, $\mathrm{n}=94$ ) where available, or the African sample from the 1000 Genomes Project (AFR-controls, $n=1322$ ). Association signals in genetic loci, which showed positive association with Eur-EOMG from two published GWAS studies (HLA class I and II regions, TNIP1 and PTPN22), were interrogated in the SA-EOMG data. Results: An HLA class I region variant (rs7750641), which is strongly associated with Eur-EOMG (minor allele frequency (MAF) 0.41; odds ratio (OR) 6.3) and tags the $H L A-B^{*} 08$ allele in this population group, was lower in SA-EOMG compared to SA-controls (MAF $0.10 ; \mathrm{p}=0.069)$. However, the $H L A-B^{*} 08$ allele was associated with SA-EOMG (MAF 0.22; OR 4.1, $\mathrm{p}<1 \times 10^{-3}$ ) which is consistent with previous reports in EUR-EOMG (MAF 0.41, OR 6.4), despite a lower frequency amongst Africans with EOMG. This was also true for an $H L A-D Q A 1$ variant (rs601006) which was lower in SA-EOMG compared to Eur-MG, but higher compared to AFR-controls (MAF 0.40, OR $2.3, \mathrm{p}=0.007)$. Due to its proximity to $H L A-B$, we interrogated the frequency of TNFA $-308 G>A$ in SAEOMG but our data did not replicate previously reported associations with EOMG (OR 1.7, $\mathrm{p}=0.151)$. GWAS signals for Eur-EOMG outside the HLA region, were not replicated in our cohort: TNIP1 (rs2233287, OR 1.2, p=0.681), PTPN22 (rs2476601, minor allele not observed in SA-EOMG dataset and SA-control MAF 0.01). Conclusion: This cross-racial analysis using an African EOMG dataset, showed that the GWAS association signals previously found outside the HLA region among Europeans with EOMG, could not be replicated. Furthermore, the LD structure in the African HLA class I region may contribute a stronger association signal with $H L A$ $B^{*} 08$ in SA-EOMG individuals compared to variants close to $H L A-B$ such as rs7750641 (upstream) or TNFA -308 $G>A$ (downstream). These results suggest that African genomes can be an informative resource to fine map association regions. Funding: South African (SA) National Research Foundation (113416), SA MRC, AFM Telethon (20049), Novartis Africa CRG Mobility fellowship, Rare disease foundation and BC Children's Hospital Foundation (2016).

\section{PS2Group5-020 / \#648}

Topic: Group 5 - Novel Diagnostic Methods in Neuromuscular Diseases and Basic Sciences in Neuromuscular Diseases

\section{RNASEQ IN URINE-DERIVED STEM CELLS IDENTIFIED THE EXPRESSION OF 264 NEUROMUSCULAR GENE TRANSCRIPTS}

Maria Sofia Falzarano ${ }^{1}$, Hana Osman ${ }^{1}$, Rachele Rossi $^{1}$, Rita Selvatici ${ }^{1}$, Marcella Neri ${ }^{1}$, Francesca Gualandi' ${ }^{2}$, Mingyan Fang ${ }^{3}$, Zhiyuan $\mathrm{Lu}^{3}$, Andrea Grilli $^{4}$, Silvio Bicciato ${ }^{4}$, Alessandra Ferlini ${ }^{1}$ ${ }^{1}$ Medical Sciences, University of Ferrara, Ferrara, IT; ${ }^{2}$ Medical Sciences, University of Ferrara, Ferrara, IT; ${ }^{3} B G I-$ shenzhen, Shenzhen, $C N ;{ }^{4}$ Dipartimento Di Scienze Della Vita, University of Modena, Modena, IT 
Background: Urine specimen has become a valuable source for isolating urine stem cells (USCs), a novel, non-invasive and powerful modelling tool for studying human specific diseases as well as for generating pluripotent patient-specific cell lines. Few studies have investigated the applicability of USCs for studying neuromuscular diseases. In a previous work, we demonstrated that native USCs and differentiated myogenic USCs are able to recapitulate the specific DMD phenotype, and provide a suitable in vitro environment for AONs mediated dystrophin gene splicing modulation. Methods: In this study we have analyzed the whole transcriptome of native USCs in order to propose them as an in vitro model for studying other neuromuscular diseases. The expression of 398 genes involved in neuromuscular diseases (http://www.musclegenetable.fr/) was investigated by RNAseq analysis on USCs isolated from three healthy donors. Results: We found that 264 out of 398 neuromuscular interrogated genes are expressed in wild type native USCs. All considered disease groups (hereditary motor and sensory neuropathies ( 85 genes), motor neuron diseases (58 genes), hereditary paraplegias (58 genes), hereditary ataxias (51 genes), distal myopathies (16 genes), congenital myopathies (32 genes), muscular dystrophies (45 genes), congenital muscular dystrophies (34 genes), ion channel muscle diseases (13 genes), myotonic syndromes (6 genes), with the only exception of ion channel genes, are represented with a different rate of expression. Indeed 50-60\% of analyzed genes causing muscular dystrophies, congenital muscular dystrophies, distal myopathies, hereditary ataxias, hereditary paraplegias, hereditary neuropathies are detectable at an appreciable level. Up to $70 \%$ of genes associated to motor neuron diseases are expressed in USCs.Based on RNAseq data, gene specific analysis can disclose several gene transcript behaviours as novel isoforms or splicing choices. As an example, COL6A1, COL6A2 and COL6A3 messengers show novel isoforms due to skipping events. Conclusion: Our results demonstrate that USCs may represent a robust tool for studying pathogenic mechanisms underlining neuromuscular diseases, proving functional meaning of novel DNA variations, testing drug profiling, and identifying novel biomarkers. Acknowledgment A grant of The Duchenne Parent Project Italy has funded this research.

\section{PS2Group5-021 / \#706}

Topic: Group 5 - Novel Diagnostic Methods in Neuromuscular Diseases and Basic Sciences in Neuromuscular Diseases

\section{NEURONOPATHY IN SPINOCEREBELLAR ATAXIA TYPE 2: A NERVE ULTRASOUND STUDY}

$\underline{\text { Luciana Pelosi }}{ }^{1}$, Rosa Iodice ${ }^{2}$, Antonella Antenora ${ }^{2}$, Dean Kilfoyle ${ }^{3}$, Eoin Mulroy ${ }^{3}$, Miriam Rodrigues ${ }^{3}$, Richard Roxburgh ${ }^{3}$, Alessandro Filla ${ }^{2}$, Fiore

Manganelli ${ }^{2}$, Lucio Santoro ${ }^{3}$

${ }^{1}$ Neurology And Clinical Neurophysiology, Bay of

Plenty District Health Board, Auckland,

NZ; ${ }^{2}$ Neurosciences, Reproductive Sciences And

Odontostomatology, University Federico II, Naples, Italy, naples, IT, ${ }^{3}$ Neurology And Clinical

Neurophysiology, 3Auckland District Health Board (ADHB, Auckland, NZ

Background: Somatosensory impairment is not uncommon in autosomal dominant spinocerebellar ataxia (SCA), but it is unclear to what extent this is a neuronopathy from neuron degeneration in dorsal root ganglia, or a primary peripheral nerve pathology, as typically seen in length-dependent neuropathy. We have recently shown that patients with cerebellar ataxia neuropathy vestibular areflexia syndrome (CANVAS), who have sensory impairment from neuronopathy, have pathologically small nerves on ultrasound. This is significantly different from the ultrasound findings in patients with primary axonal neuropathy, whose nerves are either normal or enlarged. In this study, we wished to see if the nerve ultrasound abnormality of CANVAS neuronopathy was also present in patients with SCA type 2. Methods: The ultrasound cross-sectional area of median, ulnar, sural and tibial nerves of 18 patients (mean age 51.9, 11 females) with SCA2 (confirmed by ATXN2 gene mutation) were compared to the reference values of our control population. All patients underwent objective assessment of sensory deficit using the Inflammatory Neuropathy Cause and Treatment (INCAT) sensory sum score (ISS), ataxia assessment using the scale for the assessment and rating of ataxia (SARA) and, disability quantification using the INCAT disability scale. All patients had motor (median, ulnar, peroneal and tibial nerves) and antidromic sensory (median, ulnar, radial and sural nerves) nerve conduction studies. Results: All but one patient had clinical evidence of sensory impairment with ISS score $\geq 1$ (mean 3.88). 
The ultrasound nerve cross-sectional area was significantly reduced at three or more sites in 13 patients and increased at three sites in one patient. Electrophysiology showed reduced amplitude sensory action potentials in 11 patients. The distribution of these abnormalities was non-length dependent in eight patients who also had reduced nerve crosssectional area on ultrasound, length-dependent in two patients with normal ultrasound, and not clearly defined in one patient with reduced nerve cross-sectional area. Pearson's correlation analysis showed weak to moderate inverse correlation $(p<0.05)$ between SARA scores and nerve cross sectional area at two upper limb sites. Conclusion: By analogy with the CANVAS study, the small nerves in patients with SCA2 are likely to reflect neuronopathy with axonal loss secondary to degeneration of the nerve cells. Neuronopathy was identified by nerve ultrasound in 13 (72\%) patients, thus representing the most common type of nerve impairment in our SCA2 patient population. In addition, electrophysiology and/or ultrasound findings consistent with axonal neuropathy were present in $3(16 \%)$ patients. Nerve ultrasound had higher sensitivity and specificity than electrophysiology for neuronopathy in our patient cohort, suggesting that it can be a useful complementary tool in the investigation of neuronopathy in spinocerebellar ataxia syndromes.

\section{PS2Group5-022 / \#743}

Topic: Group 5 - Novel Diagnostic Methods in Neuromuscular Diseases and Basic Sciences in Neuromuscular Diseases

\section{NERVE ULTRASOUND IN POEMS REVEALS PROXIMAL AND DISTAL SWELLING OF NERVES SIMILAR TO CIDP}

\author{
$\underline{\text { Anne-Kathrin Peyer Kauffmann }}^{1}$, Einar Wilder- \\ Smith $^{2}$ \\ ${ }^{1}$ Neurozentrum, Luzerner Kantonsspital, Luzern, \\ $\mathrm{CH} ;{ }^{2}$ Neurologie, Universität, Bern, $\mathrm{CH}$
}

Background: The electrophysiological features of the polyneuropathy seen in POEMS syndrome are well established. Little is known about the imaging of the nerves in this rare and severe paraneoplastic polyneuropathy. Methods: We describe the clinical, electrophysiological and sonographic features of the peripheral nerves and cervical roots of a 50-year old patient according to Ultrasound Pattern Sum Score (UPSS). Results: A 50 year old female patient was newly diagnosed with POEMS syndrome according to the diagnostic criteria of the International Myeloma Working Group (IMWG). On nerve conduction studies, no motor and sensory nerve recordings were recordable at the lower extremities and upper extremities showed signs of primary demyelination. High resolution ultrasound of median, ulnar, common and superficial peroneal, tibial and sural nerve showed hypoechoic enlargement of the nerves and their fascicles of variable degree. Moreover, longitudinal diameter and cross sectional areas of cervical roots were markedly enlarged. In summary, the total UPSS was $12 / 20$ points, the distal UPSA $9 / 12$, the proximal UPSB $3 / 3$ compatible with the typical features of CIDP showing proximal and distal enlargement of the nerves. Conclusion: Sonographic findings in POEMS resemble the features seen in CIDP.

\section{PS2Group5-023 / \#796}

Topic: Group 5 - Novel Diagnostic Methods in Neuromuscular Diseases and Basic Sciences in Neuromuscular Diseases

\section{COMPARISON OF HOME-BASED VERSUS HOSPITAL-BASED SPIROMETRY MEASUREMENTS IN DUCHENNE MUSCULAR DYSTROPHY}

Christian Rummey ${ }^{1}$, Thomas Meier ${ }^{2}$, Mika Leinonen $^{3}$, Shabir Hasham ${ }^{2}$, Thomas Voit ${ }^{4}$, Oscar H. Mayer ${ }^{5}$, Gunnar Buyse For The Delos Study Group $^{6}$

${ }^{1}$ Clinical Data Science GmbH, Basel, $\mathrm{CH} ;{ }^{2}$ Santhera Pharmaceuticals, Pratteln, $\mathrm{CH} ;{ }^{3}$ Santhera

Pharmaceuticals, Pratteln, $\mathrm{CH} ;{ }^{4} \mathrm{UCL}$ Great

Ormond Street Institute of Child Health, London, $G B ;{ }^{5}$ Children's Hospital of Philadelphia , Philadelphia, PA, US, ${ }^{6}$ University Hospitals Leuven, Leuven, $B E$

Background: Respiratory function decline in Duchenne muscular dystrophy (DMD) is caused by progressive weakening of respiratory muscles and leads to a high disease burden and early mortality. Standard of care guidelines recommend routine respiratory function monitoring to guide patient management, anticipate complications and improve 
outcomes. We compared respiratory function measurements obtained using a hand-held device allowing frequent home-based measurements to hospital-based spirometry measures in patients with DMD taking part in the randomized, controlled, Phase 3 DELOS trial. Methods: Respiratory function data were prospectively collected from 64 DMD patients aged 10-18 years, and not taking concomitant glucocorticoids. All patients were required to have established respiratory function decline (peak expiratory flow $<80 \%$ p) at baseline. Patients were treated with idebenone $(900 \mathrm{mg} /$ day $)$ or placebo. Spirometry was conducted during hospital visits at baseline and at 3 month intervals thereafter over the 52 week study period. Patients also measured peak expiratory flow (PEF) and forced expiratory volume in 1 second (FEV1) weekly at home using the ASMA-1 device (Vitalograph). Data were analyzed using a mixed model for repeated measures method. Results: Patients enrolled in the DELOS study were in an advanced stage of disease, with a mean age of 14.3 years. A majority (92\%) were already non-ambulatory and showed signs of significant upper limb weakness at baseline (59\% had a Brooke's score of 5 or above). The weekly changes in PEF measured at home (expressed as percent of predicted, PEF\%p) compared well with those from hospital-based measurements, showing a $5.6 \%(p=0.002)$ overall treatment difference in favor of idebenone across all weekly visits, compared to a $6.27 \%$ difference at last visit using standard hospital spirometry $(p=0.031)$. Similarly to the hospital based data, the weekly ASMA-1 data indicated a stabilization of PEF\%p in the idebenone group versus a natural history consistent decline in the placebo group. Conclusion: Home-based recording of commonly used respiratory function measures is reliable in pediatric and adolescent patients with DMD, and may prove useful and convenient when used in combination with hospital-based monitoring. These data further support the efficacy of idebenone in slowing respiratory function decline in DMD.
PS2Group5-024 / \#867

Topic: Group 5 - Novel Diagnostic Methods in Neuromuscular Diseases and Basic Sciences in Neuromuscular Diseases

\section{AGE-RELATED DIFFERENCES IN MUSCLE MEMBRANE POLARIZATION AS ASSESSED BY VELOCITY RECOVERY CYCLES}

\author{
James Lee ${ }^{1}$, Robert Boland-Freitas ${ }^{2}, \mathrm{Karl} \mathrm{Ng}^{3}$ \\ ${ }^{1}$ Neurology, Royal North Shore Hospital, SYDNEY, \\ $A U,{ }^{2}$ Neurology, Royal North Shore Hospital, \\ Sydney, NSW, AU; ${ }^{3}$ Neurology, Royal North Shore \\ Hospital, ST LEONARDS, AU
}

Background: The exact mechanisms underlying the loss of skeletal muscle bulk and power with normal human ageing are not well established. The aim of this study was to examine the effect of age on skeletal muscle membrane properties in normal patients as assessed by muscle velocity recovery cycles (MVRC). Methods: Informed consent was obtained from 74 healthy control patients (aged 18 to 84yo, median age 35 and interquartile range 29-55yo) with intact neuromuscular strength (MRC score 5). MVRC recordings were obtained from tibialis anterior $(\mathrm{N}=74)$ and rectus femoris $(\mathrm{N}=32)$ muscles as representative distal and proximal muscles. Data was captured using QTRACS threshold tracking software employing the M3RC3 recording protocol with subsequent data analysis through the MAnal8 program. Results: Regression analysis on tibialis anterior MVRC recordings demonstrated that increasing age was associated with longer muscle relative refractory period (MRRP: $\left.r^{2}=0.38, P<0.001\right)$. There was a reduction in early supernormality $\left(r^{2}=0.38\right.$, $P<0.001)$ and a smaller reduction in additional late supernormality with multiple conditional stimuli as age increased $(r=-0.45, p<0.001)$. Analysis of rectus femoris MVRCs revealed a similar association of increased age with longer MRRP $\left(r^{2}=0.30, \mathrm{P}=0.002\right)$ and reduced early supernormality $\left(r^{2}=0.19, P=\right.$ 0.01 ), but no significant association with late supernormality was apparent. Conclusion: The changes suggest resting muscle membrane potential appears relatively more depolarized with increasing age in adulthood. Possible reasons for this finding include the increasing predominance of type 1 muscle fibres with age (1), or age-related $\mathrm{Na}^{+} / \mathrm{K}^{+}$ATPase and mitochondrial dysfunction (2). These findings may represent an important mechanism in explaining 
age-related muscle decline. REFERENCES 1. StJean-Pelletier F, Pion CH, Leduc-Gaudet JP, Sgarioto N, Zovile I, Barbat-Artigas S, et al. The impact of ageing, physical activity, and pre-frailty on skeletal muscle phenotype, mitochondrial content, and intramyocellular lipids in men. J Cachexia Sarcopenia Muscle. 2016. 2. Miljkovic N, Lim JY, Miljkovic I, Frontera WR. Aging of skeletal muscle fibers. Ann Rehabil Med. 2015;39(2):155-62.

\section{PS2Group5-025 / \#885}

Topic: Group 5 - Novel Diagnostic Methods in Neuromuscular Diseases and Basic Sciences in Neuromuscular Diseases

\section{EFFECTIVE DIAGNOSTIC AND TREATMENT METHODS IN VASCULAR PARKINSONISM AND PARKINSON'S DISEASE: TEMPORHYTHMAL CORRECTION}

\author{
Dilshoda T. Akramova \\ Neurology, Tashkent Medical Academy, Tashkent \\ city, $U Z$
}

Background: It is known that, in vascular parkinsonism and Parkinson's disease it is observed shortening of steps, bradi-, oligokinesis, and also tremor disturb such kind of patients. Methods: order to early and effective differential diagnostics, also to properly treat of tremor and short steps in vascular parkinsonism and Parkinson's disease it is used method of tempo-rythmal correction 80 patients have participated in our research and the mean age of them was $62.3 \pm 4.7$ years. All patients were divided into 4 group. 1st group - patients who have vascular parkinsonism and they have received both medicamentous treatment and temporythmal correction. In the group 2 were patients with Parkinson's disease, they have also received both medicamentous treatment and temporythmal correction. Group 3 was a group of patients with vascular parkinsonism they have received only medicamentous treatment. 4th group of patients with Parkinson's disease, they have received also only medicamentous treatment. First of all, there is measured height, weight and body mass index. Calculated and scheduled common length of steps, amount of steps passed in $500 \mathrm{~m}$ and sum of spent kcal. Patients were observed during 10 days: was selected quiet music and have measured amount of steps and length of passed distance for 3 times during the 10 day. Patients walked in the morning under quiet music, on the midday under quickened and on the evening under fast rythm music. The results were recorded while there were walking. All patients were evaluated by the Parkinson's Disease Questionnaire (PDQ-39) scale. Results: According to the results of our 10-day observations. In 1st group the maximal positive result according to PDQ-39 was on "vital activity" and was $2.87 \pm 1.36$. Patients said that they had felt easiness while dressing, bathing, eating and serving to themselves. In normal people the average length of step is $40 \%$ of the height, in the first days of temporythmal correction in all patients this index was 25 or $28 \%$ and 30 or $32 \%$ on the last day of correction. In patients of 2nd group according to PQD-39 the maximal positive outcome was on "vital activity" and is $2.23 \pm 1.16$. Patients of this group said that they felt more easiness on dressing and eating. The average length of steps in patients with Parkinson's disease was 21 or $23 \%$ on the first day of the temporythmic correction, and $22,24 \%$ in the last days of the temporythmal correction. Groups 3 and 4 received only medicamentous therapy, and correction was not conducted in group patients. The PDQ-39 consisted in 3rd group - $1.9 \pm 2.16$ and 4th group $2.03 \pm 1.37$. Conclusion: conclusion, we can say that temporythmal correction is method of treatment and rehabilitation, which is effective in each type of vascular parkinsonism and Parkinson's disease also in economical aspect that patient can use both in the hospital and in the home The average length of steps in Parkinson's disease is more shorter than in the vascular parkinsonism. Temporitmal correction is an effective and cost-effective method of differential diagnosis of vascular parkinsonism and Parkinson's disease

\section{PS2Group5-026 / \#316}

Topic: Group 5 - Novel Diagnostic Methods in Neuromuscular Diseases and Basic Sciences in Neuromuscular Diseases

KIF5A, MUTATION CAN LEAD TO SPASTIC PARAPLEGIA (SPG10); GABAARS-VESICLE TRANSPORT MOTOR INTERACTS WITH G3BP2 AND GABARAPS

Dae-Hyun Seog ${ }^{1}$, Sangjin Kim ${ }^{2}$

${ }^{1}$ Biochemistry, Inje University College of Medicine, Busan, KR; ${ }^{2}$ Neurology, Inje University College of Medicine, Busan, KR 
Background: Spastic paraplegia type 10 (SPG10) is a rare form of autosomal dominant hereditary spastic paraplegia due to mutations in kinesin superfamily proteins 5A (KIF5A). KIF5A mutations have been associated with a wide clinical spectrum, ranging from pure hereditary spastic paraplegia to isolated peripheral nerve involvement or complicated hereditary spastic paraplegia phenotypes. Most KIF5A mutations are clustered in the motor domain of the protein that is necessary for microtubule interaction. Molecular motor protein KIF5A is involved in the gamma-aminobutyric acid type A receptors (GABAAR) containing vesicle transport. Deletion in the KIF5A can impair intracellular transport of GABAA receptors. However, the binding partners or regulation proteins of KIF5A have not yet been fully identified. Methods: We were used the yeast two-hybrid system to identify the binding proteins that interact with the C-terminal 73 amino acids of KIF5A. The binding affinity was quantified by measuring $\beta$-galactosidase activity in liquid cultures of yeast transformed cells. Direct interaction between binding proteins and KIF5A in mammalian cells as well as in vitro was assayed using the co-immunoprecipitation with the antibodies. The cellular co-localization in cells was used the immunocytochemistry. Results: We revealed an interaction between the Cterminal region of KIF5A and GABARAPs, and G3BP2, which is involved in stress granules (SGs) formation and mRNA-protein (mRNP) localization. KIF5s are a heterotetrameric protein composed of two heavy chains (KIF5s), and two light chains (KLCs). The KIF5s possess a motor domain at the amino-terminal region connected by a long stalk domain to a carboxyl (C)-terminal tail domain. The motor domain, which binds to microtubules, is responsible for the motor activity of kinesin 1 , and the C-terminal tail domain is responsible for interacting to cargoes. KIF5s have been revealed to consist of three closely related subtypes: KIF5A, KIF5B, and KIF5C. KIF5B is expressed ubiquitously in many tissues, whereas KIF5A and KIF5C are expressed in nervous tissue. KIF5A can form homodimer or heterodimer with KIF5B, or KIF5C. GABARAPs and G3BP2 bound to the C-terminal 73 amino acids of KIF5A and did not interact with the other KHCs (KIF5B, and KIF5C) in the yeast two-hybrid assay. However, other G3BP isoform, G3BP1 did not interact with KIF5A, suggesting a specific interaction of KIF5A with GABARAP and G3BP2. The KIF5AGABARAP, G3BP2 interaction was also assessed in mammalian cells. When the lysate of HEK-293T cells transiently expressing myc-KIF5A and FLAGGABARAP, FLAG-G3BP2 was immunoprecipitated with anti-FLAG antibody, KLC1 and KIF5A, but not KIF3A were co-precipitated with GABAR, GABARAP and G3BP2. This result indicates that GABARAP and G3BP2 associate with kinesin 1. In order to further confirm whether KIF5A and G3BP2 co-localize in cells, KIF5A was co-expressed with EGFP-GABARAP, EGFP-G3BP2 in HEK-293T cells. KIF5A and GABAR, GABARAP and G3BP2 co-localized at the same region in cells. Conclusion: These results suggest that KIF5A may transport of GABAAR containing vesicles and SGs or mRNP granules. Furthermore, these results strengthened the link between the cargos trafficking and Spastic paraplegia phenotypes.

\section{PS2Group5-027 / \#394}

Topic: Group 5 - Novel Diagnostic Methods in Neuromuscular Diseases and Basic Sciences in Neuromuscular Diseases

\section{VANGL2, A CORE COMPONENT OF THE WNT PCP PATHWAY CONTRIBUTES TO NEUROMUSCULAR JUNCTION FORMATION}

Myriam Boex $^{1}$, Julien Messéant ${ }^{2}$, Stéphanie Bauché $^{3}$, Laure Strochlic ${ }^{4}$, Bertrand Fontaine ${ }^{4}$ ${ }^{1}$ Team "gen-phys: Neugenetics And Physiology" Umr_s1127, Brain and Spine Institute, Paris, FR:'eam "gen-phys: Neugenetics And Physiology" Umr_s1127, Brain and Spine Institute, Paris, FR $;^{3}$ Equipe Neurogenetic And Physiology, Institut du Cerveau et de la Moelle epinière, Paris, FR; ${ }^{4}$ Institut du Cerveau et de la Moelle épiniere, Paris, FR

Background: The development of the neuromuscular junction (NMJ), a cholinergic synapse between the axon terminal of a motoneuron and a specialized region of the skeletal muscle fiber requires a dynamic communication via various reciprocal signaling processes between the two elements that are only partially understood. Several studies have recently highlighted the emerging and complex role of Wnt signaling at the NMJ. Secreted Wnt ligands (notably Wnt4 and Wnt11) signal through interaction with the Frizzled-like cysteine rich domain (CRD) of the muscle-specific tyrosine kinase MuSK to activate 
both canonical/b-catenin dependent and core Planar Cell Polarity (PCP) pathways. First evidence for a role of Wnt PCP signaling during NMJ formation comes from studies in Zebrafish showing that both Wnt4a and Wnt11r cooperate to initiate NMJ formation likely by stimulating PCP-dependent MuSK endocytosis. In mice, we previously demonstrated that one of the key PCP core component, namely Van Gogh-Like protein 2 (Vangl2), is expressed in both pre- and postsynaptic compartments, interacts with extracellular Wnt4 and Wnt11 and is required to shape the neuromuscular synapse. However, the molecular determinants of Vangl2 signaling at the NMJ still largely remains unknown. Methods: Using experimental mouse models and a large array of cell biology technics we investigated Vangl2 function during NMJ development. Results: In this study, we first examined in vivo the NMJ phenotype of diaphragms from Vangl2 knock-out (KO; Vangl2 ${ }^{-/}$) mouse embryos and showed that the absence of Vang12 induces pre- and postsynaptic differentiation defects characterized by a decrease in the number and volume of acetylcholine receptors (AChRs) and overgrowth of motor axon within the target field. Moreover, loss of Vangl2 function inhibited Wnt11mediated AChRs clustering in primary muscle cells. We further demonstrated that only muscle (-HSA) but not motoneuronal (-HB9) specific conditional $\mathrm{KO}$ (cKO) of Vangl2 recapitulates both pre- and postsynaptic defects of the Vang12 ${ }^{-/}$mice and Vangl2 forms a complex with MuSK independently of its CRD. Conclusion: All together, these results demonstrate that a Wnt/PCP signaling pathway in the muscle relying on MuSK/Vangl2 interaction contributes to the establishment of mammalian neuromuscular connectivity.

\section{PS2Group5-028 / \#604}

Topic: Group 5 - Novel Diagnostic Methods in Neuromuscular Diseases and Basic Sciences in Neuromuscular Diseases

\section{A 3D SYSTEM FOR MECHANICAL CHARACTERIZATION OF ARTIFICIAL SKELETAL MUSCLE MICROTISSUE}

Marina Spörrer $^{1}$, Delf $\mathrm{Kah}^{2}$, Sandra Wiedenmann ${ }^{2}$, Ingo Thievessen ${ }^{2}$, Rolf Schröder ${ }^{3}$, Wolfgang H. Goldmann², Ben Fabry ${ }^{2}$
${ }^{1}$ Department Of Physics, Biophysics Group, Friedrich-Alexander University ErlangenNuremberg, Erlangen, DE; ${ }^{2}$ Department Of Physics, Biophysics Group, Friedrich-Alexander University Erlangen-Nuremberg, Erlangen, DE $;^{3}$ Neuropathology, University Hospital Erlangen, Erlangen, DE

Background: Artificial skeletal muscle tissues grown from cells that are isolated from patients with myofibrillar myopathies offer the prospect to study otherwise hard to measure processes such as muscle contractility, the occurrence of microlesions after exercise, or the response to drugs. However, previous attempts to grow artificial muscle have suffered from insufficient differentiation of myoblast precursor cells and poor maturation into an in-vivo-like, highly aligned, and contractile tissue. Methods: Here, we develop a method for growing muscle tissue in $4 \times 2 \times 2 \mathrm{~mm}$ microwells containing two flexible silicone pillars that serve as force sensors. Myofibroblasts are seeded in a collagen/matrigel suspension. Upon polymerization of the collagen/matrigel mixture into a soft 3-D hydrogel, the myoblasts spread, adhere, and compact the matrix, and form an aligned tissue that spans between the pillars. When cultured in a differentiation medium containing insulin, transferrin and selenium (ITS), muscle tissues start to contract upon electrical pacing 6-7 days after seeding. A Stretchable device with 18 wells. Inset: close-up view of a single well. B Top view of a microtissue containing 7500 myoblasts. Inset: staining of aligned myotubes. Green: alpha-actinin, blue: dapi. C Side view of a well with tissue. D Contractile force of a tissue in response to electrical pacing at $1 \mathrm{~Hz}$. Results: We find an optimal tissue formation and force generation (2 $\mathrm{kPa}$ static tension, $400 \mathrm{~Pa}$ contractile tension) for a collagen concentration of $0.6 \mathrm{mg} / \mathrm{ml}$ and a Matrigel concentration of $1.5 \mathrm{mg} /$ $\mathrm{ml}$. At lower or higher Matrigel concentrations, contractile tension dramatically decreases. This is attributable to absence of matrix-mediated signaling at lower Matrigel concentrations, and poor alignment of collagen fibers and hence of the differentiated myotubes at higher Matrigel concentrations. Conclusion: These findings suggest that addition of different matrix components and fibroblasts to primary myoblasts critically influence tissue formation and contraction, and thus need to be tuned for optimal function of the microtissues. 

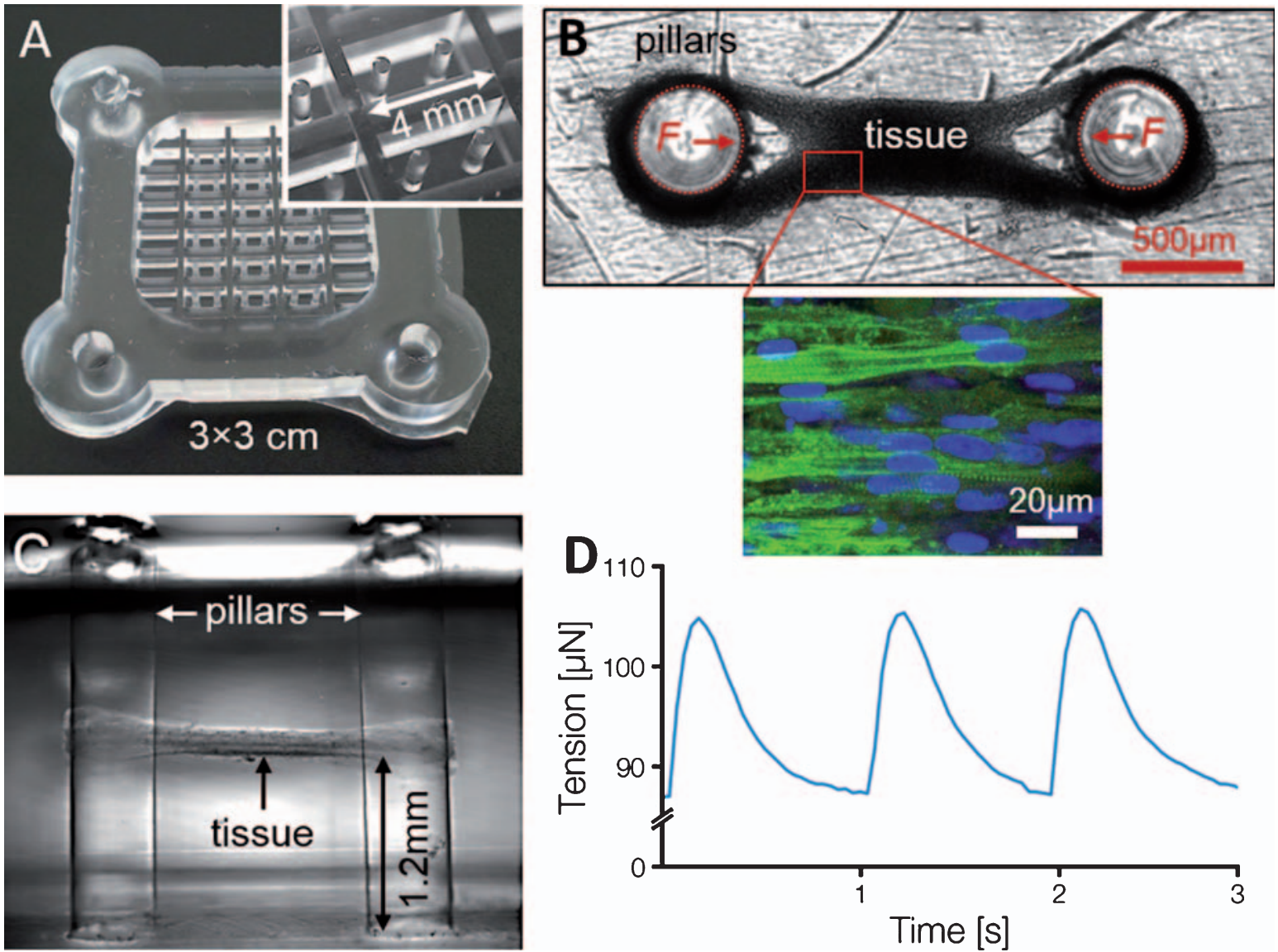

PS2Group5-029 / \#27

Topic: Group 5 - Novel Diagnostic Methods in Neuromuscular Diseases and Basic Sciences in Neuromuscular Diseases

\section{POTENTIOALLY CONFOUNDING VARIABLES OF GDF-15: \\ MITOCHONDRIA DISEASE AND OTHER NEUROLOGICAL DISEASES}

Akiko Ishii $^{1}$, Seitaro Nohara ${ }^{2}$, Zenshi Miyake ${ }^{2}$, Naoki Tozaka², Sho Okune ${ }^{2}$, Hayato Takeda ${ }^{2}$, Hiroshi Tsuji ${ }^{2}$, Yasushi Tomidokoro², Kiyotaka Nakamagoe $^{2}$, Kazuhiro Ishii ${ }^{2}$, Masahiko Watanabe ${ }^{2}$, Akira Tamaoka $^{2}$, Shuichi Yatsuga ${ }^{3}$, Yasutoshi Koga ${ }^{3}$ ${ }^{1}$ Neurology, University of Tsukuba, Tsukuba, $J P,{ }^{2}$ Neurology, University of Tsukuba, Tsukuba, $J P{ }^{3}$ Pediatrics, Kurume University, kurume, JP

Background: GDF-15 (Growth Differentiation Factor 15) is one of transforming growth factor beta superfamily and regulate inflammation and apoptosis in various disease, such as heart failure, kidney dysfunction, and tumor. Yatsuga et al. reported that

GDF-15 was a new biomarker of mitochondria diseases. The aim of this study is to clarify potentially confounding variables of GDF-15 and demonstrate as mitochondria biomarker. Methods: We used serum from 14 patients of MELAS, 15 patients of limbic encephalitis (LE), 10 patient of multiple sclerosis (MS), and 19 patients of ALS. GDF-15 and FGF-21 are measured using ELISA. Clinical features of patients are also evaluated retrospectively. Results: Average of GDF-15 and FGF-21 in mitochondria diseases are $2860.7 \mathrm{pg} / \mathrm{ml}$ and $855 \mathrm{pg} / \mathrm{ml}$, respectively. Average of GDF-15 and FGF-21 in LE are $786.2 \mathrm{pg} / \mathrm{ml}$ and 238.2 $\mathrm{pg} / \mathrm{ml}$, respectively. GDF-15 and FGF-21 in MS are $575.4 \mathrm{pg} / \mathrm{ml}$ and $384.0 \mathrm{pg} / \mathrm{ml}$, respectively. Average of GDF-15 and FGF-21 in ALS are $509.6 \mathrm{pg} / \mathrm{ml}$ and $119.3 \mathrm{pg} / \mathrm{ml}$, respectively. Although, two patients with ALS showed high FGF-21, there were no correlation with age, severity, and phenotype. GDF-15 and FGF21 are significantly elevated in mitochondria diseases. There is no correlation between GDF-15 and clinical features, such as ADL level, number of cells in cerebrospinal fluid (CSF), and brain MRI findings. No GDF-15 elevation is found in three patients having 
cancer. There are tendency of correlation between GDF-15 and protein concentration of CSF in LE patients. GDF-15 shows positive correlation between EDSS in MS patients (Pearson correlation coefficient 0.74). Conclusion: GDF-15 is a useful biomarker of mitochondria disease and potentially influenced by severity of diseases. Age is the most potentially confounding variables of GDF-15.

\section{PS2Group5-030 / \#726}

Topic: Group 5 - Novel Diagnostic Methods in Neuromuscular Diseases and Basic Sciences in Neuromuscular Diseases

\section{QUANTITATIVE ESTIMATES OF ULTRASOUND IMAGING IN MUSCLE PATHOLOGY}

$\underline{\text { Dimitra Veltsista }}^{1}$, Elisabeth Chroni ${ }^{2}$

${ }^{1}$ Neurology, University Hospital of Patras, Patras, GR ${ }^{2}$ Neurology, University of Patras, Patras, GR

Background: There is a growing tendency to employ ultrasound examination for neuromuscular investigation. This study aimed to explore potential markers for quantitative assessments of muscle ultrasound appearance in order to increase the test reliability and objectivity. Methods: Ten patients (6 men; mean \pm sd age $50.1 \pm 8.5$ years) with neurogenic weakness (NE), diagnosed with spinal muscular atrophy, amyotrophic lateral sclerosis or motor neuronopathy and 10 patients $(8 \mathrm{men} ;$ mean \pm sd age
$36.2 \pm 16.2$ years) with a primary myopathy (MY) i.e. Duchenne, Becker, limb-girdle or facioscapulohumeral muscular dystrophy were examined. The control group (C) consisted of 14 healthy subjects ( 8 men; $37.4 \pm 14.0$ years). Data were obtained from ultrasound transverse image of the biceps brachii muscle at the $2 / 3$ distance from acromion to antecubital fossa using a linear array probe. Images were exported and the entire region of muscle was manually selected and analyzed using ImageJ fiji, processing program. A histogram of echo intensity was created depicting the counts (pixels) for each bin on a gray scale from 0 (black) to 255 (white). The mean (sd) gray scale, minimum, maximum and mode value as well as the full width at half maximum value were estimated. Muscles were scored for strength on MRC and their images on Heckmatt's rating scale. Results: Group comparison, employing one-way ANOVA post hoc Dunnett t-test, showed significantly increased values of mean gray scale for the patient groups as opposed to control (NE 60.6 15.4 / MY 67.6 \pm 16.3 / C $47.6 \pm 6.9$ and corresponding pvalues $=0.032,0.001)$. Full width at half maximum was also significantly higher for patients (NE 47.3 \pm 9.2 / MY 50.6 \pm 9.5 / C 33.1 \pm 5.9 and both p-values $=0.000$ ). Figure illustrates the group histogram shifting and broadening in diseased muscles. No statistical differences between patient groups were found. There was a linear correlation of mean gray scale values with MRC score $\left(\mathrm{R}^{2}=0.5, \mathrm{p}\right.$-value $\left.=0.000\right)$ but not with Heckmatt's score. Conclusion: Our findings suggested that creation of gray scale histo-

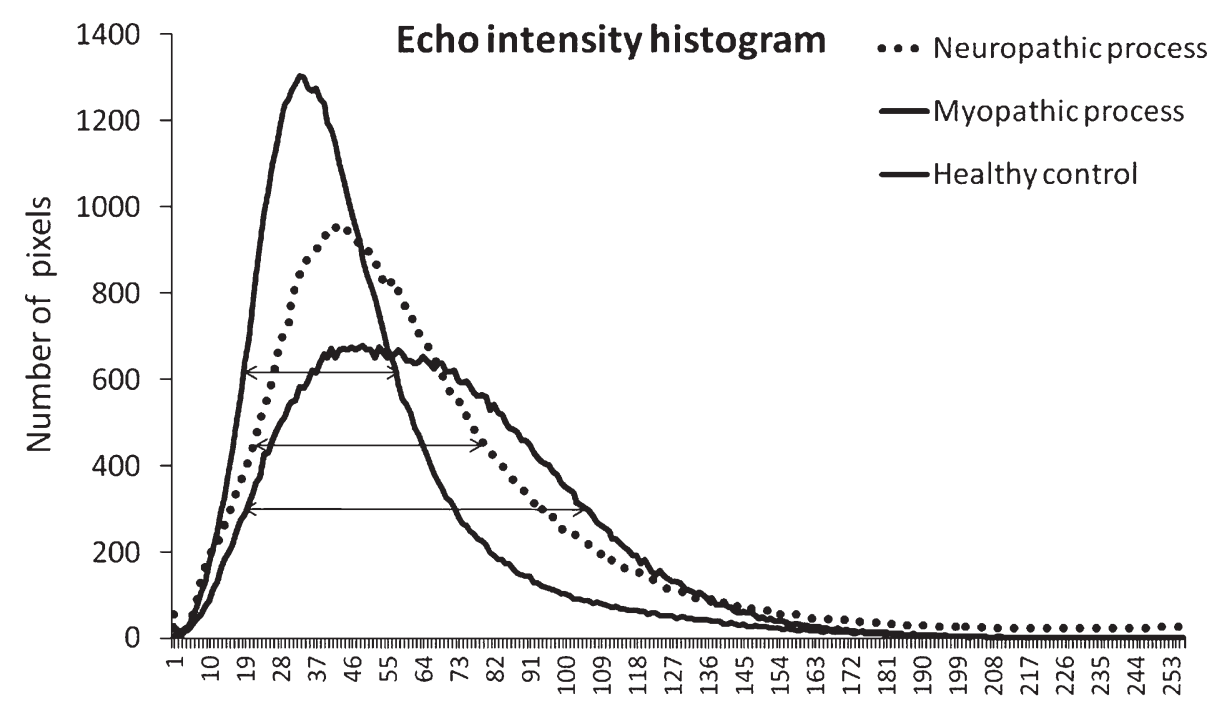

Gray scale values 
grams followed by estimation of full width at half maximum value, which seemed to be the most sensitive measurement, may serve as a reliable index of muscle pathology reflecting the severity though not the type of the primary pathogenic process.

\section{PS2Group5-031 / \#828}

Topic: Group 5 - Novel Diagnostic Methods in Neuromuscular Diseases and Basic Sciences in Neuromuscular Diseases

\section{CIRCULATING KV1.3+ CELLS IN PATIENTS WITH SPORADIC INCLUSION BODY MYOSITIS}

\author{
Tahseen Mozaffar $^{1}$, Marie Wencel ${ }^{2}$, Karissa \\ Munoz $^{3}$, Jenna Kastenschmidt ${ }^{3}$, S. Armando \\ Villalta $^{3}$ \\ ${ }^{1}$ Neurology, University of California, Irvine, \\ Orange, CA, US; ${ }^{2}$ Neurology, University of \\ California, Irvine, Orange, US, ${ }^{3}$ Physiology And \\ Biophysics, University of California, Irvine, Irvine, \\ CA, US
}

Background: We had recently shown that muscle biopsies from patients with sIBM express large numbers of $\mathrm{CD}^{+}$cells (22.4\% to $53.1 \%$ ) that co-localized Kv1.3, a voltage-gated potassium channel seen on effector memory T-cells. We examined if circulating Kv1.3 $3^{+}$cells can be detected in unstimulated samples from sIBM patients. Methods: Blood collection was done at the annual patient conference of the Myositis Association in San Diego, CA with immediate processing for PBMCs. Multichannel flow cytometry was done, with a panel containing the following markers: CD3, CD8, CD4, CD244, CD57, CD28 viability marker, and Kv1.3. Descriptive statistics and student t-tests were used for statistical purposes. Results: PBMCs were collected from 22 patients with a diagnosis of IBM, 9 patients with PM, 9 patients with DM and 5 healthy controls. $\mathrm{CD} 4{ }^{+} \mathrm{CD} 57^{+} \mathrm{CD} 28^{\text {null }}$ cells were seen in $3.8 \pm 0.9 \%$ of sIBM patients ( $\mathrm{p}=0.02$ vs. HC) while $\mathrm{CD} 8^{+} \mathrm{CD} 57^{+} \mathrm{CD} 28^{\text {null }}$ cells were seen in $50 \pm 3.6 \%$ of sIBM patients ( $\mathrm{p}=0.01 \mathrm{vs}$. HC). The percentage of these cells was not statistically different in DM and PM patients. Percentage of Kv1.3 $3^{+}$cells was statistically increased in both $\mathrm{CD}^{+}$as well as $\mathrm{CD} 8^{+}$cell populations $\left(\mathrm{CD} 4{ }^{+} \mathrm{CD} 244^{+} \mathrm{Kv} 1.3^{+}=0.53 \pm 0.15 \%\right.$; $\mathrm{p}<0.02$ vs $\mathrm{HC}$ and $\mathrm{CD} 8^{+} \mathrm{CD} 244^{+} \mathrm{Kv} 1.3^{+}=1.70 \pm$ $0.21 \% ; \mathrm{p}=0.02$ ). Conclusion: Circulating $\mathrm{Kv} 1.3^{+}$ cells were statistically increased in SIBM compared to healthy controls and other inflammatory myopathies. The numbers of these cells are small, probably representing a small percentage of the large T-cell repertoire in circulating blood. We next plan to isolate live T-cells from skeletal muscle biopsies from sIBM patients to quantitate presence of $\mathrm{Kv} 1.3^{+}$cells in diseased muscle.

\section{PS2Group5-032 / \#467}

Topic: Group 5 - Novel Diagnostic Methods in Neuromuscular Diseases and Basic Sciences in Neuromuscular Diseases

\section{ULTRASOUND IMAGING OF GENIOGLOSSUS MUSCLE FUNCTION WITH CONTRAST AGENT}

\author{
Orest M. Semeryak \\ Lviv Regaional Clinical Hospital Center of NMD \\ and Disease of PNS, Lviv, UA
}

Background: INTRODUCTION. the human upper airway has many important physiological functions including speech, swallowing and breathing. The human tongue forms an important part of the upper airway and plays a key role in the act of swallowing. Among these, the genioglossus (GG) is the largest dilator of the upper airway and main swallowing muscule. There is a large group of neuromuscular disorders, which can cause dysphagia. In patients with neuromuscular diseases, a gradual dystrophy of the muscle of the tongue with a violation of the act of swallowing, sweating and breathing disorder is observed. Therefore, in this situation it is necessary to find an adequate, safe screening method of dynamic visualization for the evaluation of structural changes and motor disorders of the muscles of the tongue. Methods: METHODS. Ultrasound does not emit radiation, is non-invasive, gives good resolution of soft tissue structures, can be used as a screening method and dynamically during the treatment process, and can display cross-sectional anatomy and tissue motion in real time. It also has been used to demonstrate dynamic upper airway motion during swallowing and speech. Ultrasonography can be used to quantify GG movement. Ultrasound images from the suprahyoid region and from the surface of the tongue were made in 25 healthy subjects aged 10 to 30 years to determine the normal structure and movements; that 
data were compared with 5 patients with motoneuron disease. To detect manifestations of dysphagia and to study the nature of the movements of the tongue when swallowing with normal and pathology, we used contrast media - a solution of sugar saturated with small gas bubbles. Results: RESULTS. All measurements were feasible in all subjects and patients with good reproducibility. Our findings suggest gradual involvement of oral muscles in motoneuron disease. The use of contrast shows the mechanism of movement of the muscles of the tongue and the movement of the epiglottis while swallowing. Conclusion: CONCLUSION. This study demonstrates a novel use of ultrasound to quantify the GG movement in awake humans during swalloving act. It is a simple, reproducible, safe technique that does not emit radiation and allows use during the treatment process without restrictions. Use the ultrasound in oral muscles is feasible in healthy children, adults and patients with neuromuscular disorders. These data show that it is possible to differentiate between healthy persons and patients with neuromuscular disorders. The use of contrast provides an additional understanding of the pathological process, gives additional data on swallowing biomechanics, and clearly illustrates problems with dysphagia.

\section{PS2Group5-033 / \#535}

Topic: Group 5 - Novel Diagnostic Methods in Neuromuscular Diseases and Basic Sciences in Neuromuscular Diseases

\section{COMPUTATIONAL SPEECH}

\section{ANALYSIS AS A TOOL FOR EARLY DETECTION OF BULBAR DYSFUNCTION IN ALS PATIENTS}

Bernat B. Recasens $^{1}$, Diego C. Lahoz ${ }^{2}$, Anna G. Solà ${ }^{3}$, Maria S. Martinez ${ }^{4}$, Francisco A. Cabrera ${ }^{5}$, Juana M. Llorens ${ }^{6}$, Ana B. Corberó ${ }^{6}$, Montserrat V. Moreno $^{7}$, Miguel Angel R. Perez ${ }^{8}$ ${ }^{1}$ Neurology, Hospital del Mar, Barcelona, Barcelona, ES: ${ }^{2}$ Hospital del Mar, Barcelona, ES, ${ }^{3}$ Physical Medicine And Rehabilitation Department, Hospital del Mar, Barcelona, ES; ${ }^{4}$ Neurology, Hospital del Mar, Barcelona, ES, ${ }^{5}$ Pompeu Fabra University, Barcelona, ES; ${ }^{6}$ Pneumology Department, Hospital del Mar, Barcelona, ES, ${ }^{7}$ Endocrinology Department, Hospital del Mar, Barcelona, ES $;^{8}$ Neurology Department, Hospital del Mar, Barcelona, ES
Background: In amyotrophic lateral sclerosis (ALS) the initial symptoms of dysarthria are subtle and there is no objective method for assessing them, which can be difficult to early detection. We propose the computational analysis of speech as a method for the early detection of bulbar impairment in ALS patients. Methods: An analysis of different speech acoustic variables using Praat software was performed. ALS patients with no apparent bulbar impairment, according to El Escorial criteria, were compared to healthy controls $(\mathrm{H})$. Lingual strength, measured with IOPI system, was also included as alternative measure of bulbar involvement expression. Results: 9 patients were evaluated and compared to 20 healthy volunteers. ALS patients compared to volunteer group presented a greater number of pauses $(\mathrm{p}=0.016)$, longer duration during the reading of a text $(\mathrm{p}=0.005)$, as well as a lower verbal fluency $(p<0.001)$. In addition, they presented a significant decrease in the lingual movement in the horizontal plane $(p<0.001)$, with a trend towards a lingual anterior position $(\mathrm{p}=0.005)$ and inferior $(p=0.003)$ during phoneme emission. The decrease in lingual strength was related $(\mathrm{r}=0.80)$ with a decrease in lingual movement $(\mathrm{p}=0.003)$. Conclusion: ALS patients without apparent bulbar dysfunction had an affectation of the lingual movements in the horizontal plane. The computational analysis of speech is a simple and objective tool that seems useful for an early detection of bulbar dysfunction in ALS and even allows to distinguish from other etiologies of dysarthria.

\section{PS2Group5-034 / \#985}

Topic: Group 5 - Novel Diagnostic Methods in Neuromuscular Diseases and Basic Sciences in Neuromuscular Diseases

\section{THE IMPORTANCE OF A NON- INVASIVE SCREENING IN PROXIMAL MYOPATHIES}

Giorgia Bruno, Lia Allegorico, Luca Lombardi, Giuseppe Di Iorio, Simone Sampaolo Second Neurology, University of Campania "Luigi Vanvitelli", Naples, IT

Background: The Late-Onset Pompe Disease (LOPD) is caused by compound mutations of the acid alpha-glucosidase gene (GAA) which lead to deficiency of GAA enzyme activity and accumulation of glycogen within autophagic vacuoles. LOPD 
affects primarily proximal muscle, as in a limb-girdle muscolar dystrophy and polymyosities. Methods: We describe unusual cases of three patients presenting hyperCKemia, proximal muscle weakness, autoimmunity nonspecific indices slightly above the reference range, that received inflammatory myopathy diagnosis. So they underwent to steroid and immunosuppressor therapy, that worsened the clinical picture. Therapeutic failure imposed clinical and instrumental revaluation. Results: The first evaluation was made with Lymphocytes on blood smears that disclosed PAS positive glycogen granula in their cytoplasm and Dried Blood Spot (DBS) that revealed reduced GAA enzyme activity. Elettromyography extended to paraspinal muscles revealed a myopathic pattern with complex repetitive discharges. For this reason we decided to repeat muscle biopsy: histological examination revealed a myopathy pattern characterized by consistent glycogen storage at intermyofibrillary and sub- sarcolemmal levels and numerous PAS and acid phosphatase positive vacuoles in many fibres. In suspect of LOPD, patients also performed an ANGIO-MRI that disclosed dolicho-ectasis of basilar trunk. Genetic investigations confirmed LOPD. Fig. 1. a) PAS positive glycogen granula in the Lymphocytes cytoplasm on blood smears b) Histology and histochemistry of quadriceps muscle biopsy revealed a myopathic pattern characterized by presence of numerous vacuolary formations of variable shape, number and extension. These findings are suggestive of vacuolar myopathy, like glycogenosis. Conclusion: Proximal muscle weakness and hyperCKemia should be screened at first, with rapid and non-inva- sive methods like peripheral blood smear and DBS to avoid, despite a rise in aspecific inflammatory markers, immunomodulatory therapies able to worse, potentially, the clinical features of a metabolic myopathy.

\section{PS2Group6-001 / \#349}

Topic: Group 6-Cancer related Disorders and General Diseases

\section{CLINICAL DIVERSITY OF P/Q-TYPE CALCIUM CHANNEL ANTIBODY- ASSOCIATED PARANEOPLASTIC DISORDERS}

Takashi Irioka $^{1}$, Yuko K. Takahashi ${ }^{2}$, Susumu Igarashi $^{1}$, Takamasa Majima ${ }^{2}$, Hirokazu Shiraishi ${ }^{3}$, Shunsuke Yoshimura ${ }^{3}$, Hiroko Kitanosono ${ }^{3}$, Takanori Yokota $^{2}$, Masakatsu Motomura ${ }^{4}$ ${ }^{1}$ Department Of Neurology, Yokosuka Kyosai Hospital, Kanagawa, JP;2Department Of Neurology And Neurological Science, Tokyo Medical and Dental University, Graduate School, Tokyo, JP; ${ }^{3}$ Department Of Neurology And Strokology, Nagasaki University Hospital, Nagasaki, JP, ${ }^{4}$ Department Of Electrical And Electronics Engineering, Faculty of Engineering, Nagasaki Institute of Applied Science, Nagasaki, JP

Background: P/Q-type voltage-gated calcium channel antibodies (P/Q-VGCC abs) are not only pathogenic in Lambert-Eaton myasthenic syndrome (LEMS) but are also associated with diverse

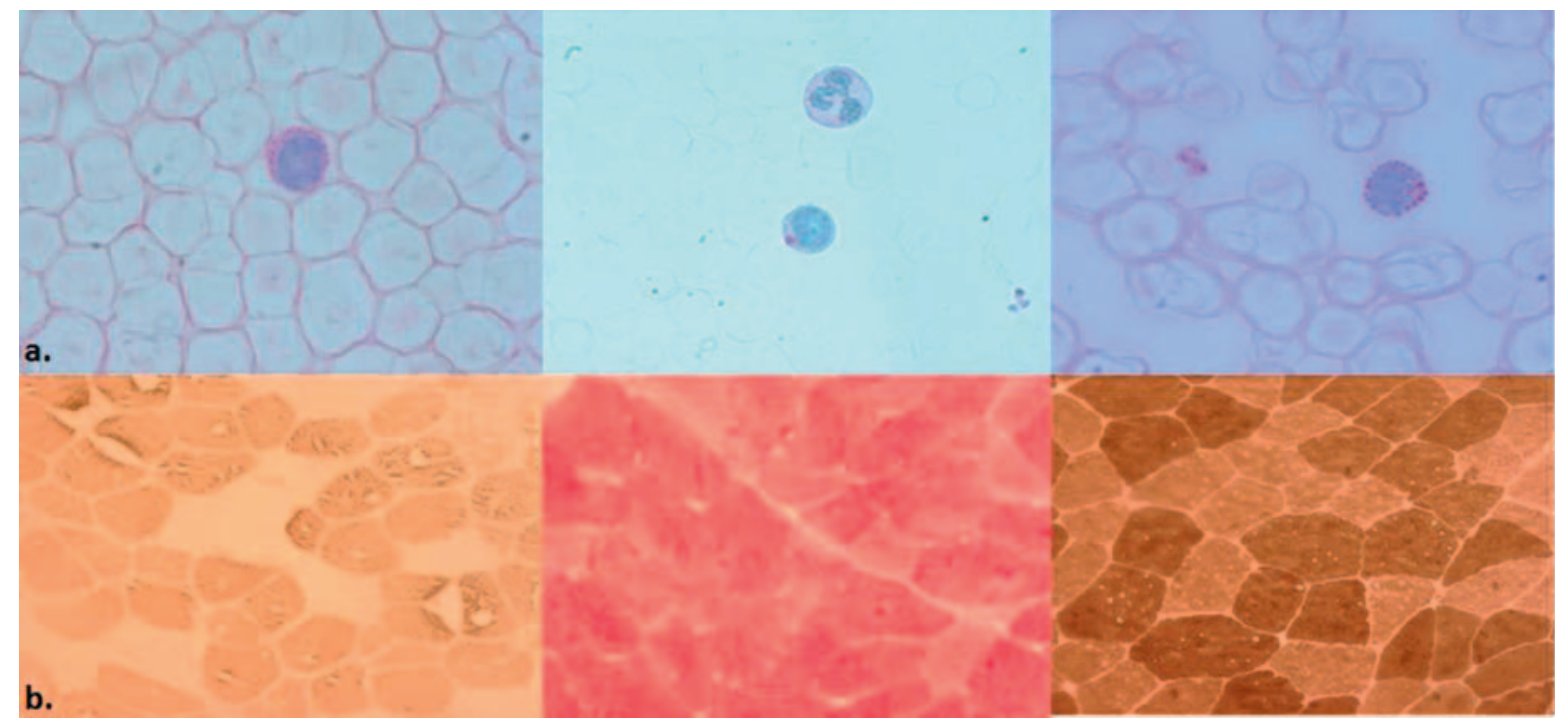


paraneoplastic neurologic disorders (PNDs). Methods: To clarify clinical characteristics of PNDs associated with small cell lung cancer (SCLC) and $\mathrm{P} / \mathrm{Q}-\mathrm{VGCC}$ abs. We encountered 3 adult male patients with SCLC- and P/Q-VGCC ab-associated PNDs. We reviewed their medical records to investigate the clinical phenotypes of the PNDs, autoantibody profiles, treatments, and therapeutic responses. Results: 1) PND phenotypes. Patient 1: A 65-year-old man suffered from dry mouth for several months. Thereafter, he presented with proximal limb weakness and bilateral ptosis. Tendon reflexes were depressed, and electrophysiologic studies confirmed LEMS. Patient 2: A 69-year-old man developed subacute pancerebellar syndrome, which was compatible with paraneoplastic cerebellar degeneration (PCD). Neurologic and electrophysiologic examinations did not reveal complicated LEMS. Patient 3: A 71-year-old man presented with unstable gait for months. Subsequently, dysesthesia in the limbs appeared. Neurologic examination revealed dysarthria and kinesthetic sensory loss with Romberg sign. Electrophysiologic studies were consistent with sensory polyneuropathy (sensory neuronopathy) without LEMS. 2) Autoantibody profiles. Radioimmunoprecipitation assays for P/QVGCC abs were positive in all 3 patients. Paraneoplastic autoantibody evaluation of the serum (Mayo Clinic) revealed N-type VGCC antibody and antiglial nuclear antibody (AGNA-1, Sox1 antibody) in Patient 2, but no coexisting autoantibodies were found in Patients 1 and 3. 3) Treatments and responses. All 3 patients were treated with chemotherapy against SCLC. The neurologic symptoms were resistant to chemotherapy in Patients 1 and 3, but the cerebellar symptoms in Patient 2 improved robustly. Improvement of the ataxia was correlated with decreases of anti-P/Q-VGCC antibody titers in the serum. Additionally, Patient 2 was treated with immunotherapies (plasma exchange [PLEX] and intravenous immunoglobulins [IVIG]) after the chemotherapy. Again, the ataxia improved immediately (the next day after the first PLEX or after IVIG infusion for 3 consecutive days). Conclusion: P/QVGCC abs are associated with various types of PNDs and can affect both the central and peripheral nervous systems. Although it remains uncertain whether P/Q-VGCC abs have pathogenic, functional effects on PCD, the immediate clinical response of Patient 2 to PLEX, which removes plasma-soluble factors including antibodies, could support such a hypothesis.
PS2Group6-002 / \#290

Topic: Group 6-Cancer related Disorders and General Diseases

\section{CLINICAL FEATURES AND TREATMENT OUTCOMES OF NEUROLYMPHOMATOSIS IN KOREA}

\author{
Duk Hyun Sung \\ Department Of Physical And Rehabilitation \\ Medicine, Samsung Medical Center, Seoul, KR
}

Background: Neurolymphomatosis (NL) is a disease entity characterized by infiltration of malignant lymphocytes into the peripheral nervous system. The aim of the present study was to analyze clinical features, radiographic evaluation, treatment and outcomes of patients with primary and secondary NL. Methods: We retrospectively investigated 10 patients with NL diagnosed at one tertiary hospital from June 2013 to October 2016. We extracted data including clinical presentations, Magnetic resolution imaging (MRI), Positron emission tomography (PET) scans, Cerebrospinal fluid (CSF) cytology findings, results of electrodiagnostic study, treatments, and outcomes. We defined response of NL to therapy as clinical improvement of neurologic deficits. Results: There were 8 men and 2 women, and mean age at onset was 54.1 years (range: 33-83). Nine patients had secondary NL and one patient had primary NL. All patients were related to non-Hodgkin lymphoma. Of presenting symptoms, pain was reported in all patients. MRI was abnormal in 7 out of 9 patients with enhancement of spinal nerve roots (9 patients), plexus (5 patients), peripheral nerve (4 patients), and cranial nerve (1 patient). PET better identified NL than MRI and was positive in all patients. Electrodiagnostic studies demonstrated plexoradiculopathy (4 patients), radiculopathy (1 patient), sensorimotor polyneuropathy (3 patient), mononeuropathy multiplex (1 patients). One patient underwent nerve biopsy which confirmed primary $\mathrm{NL}$ in the S2 root. Eight patients received systemic chemotherapy, 4 intrathecal chemotherapy. Neurological improvement was observed in 3 patients. Seven cases who underwent the multimodality treatments of cancer eventually deteriorated and died. Conclusion: NL should be considered in the differential diagnosis of any neuropathy in patients with lymphoma or leukemia as well as no previous comorbidity in the setting of a painful and motor 
weakness. Because it could be confused with other neuropathies in lymphoma and various musculoskeletal diseases, a high index of suspicion and familiarity with clinical manifestation of NL is key. The diagnosis of NL is challenging but contemporary imaging tools such as PET/CT frequently detect the relevant neural invasion.

\section{PS2Group6-003 / \#916}

Topic: Group 6-Cancer related Disorders and General Diseases

\section{PEDIATRIC EXTRADURAL COMPRESSIVE MYELOPATHY SECONDARY TO GANGLIONEUROBLASTOMA; NERVE ROOTS AND BRACHIAL PLEXUS BEWARE}

Amanda Yaworski ${ }^{1}$, Ratika Srivastava ${ }^{1}$, Vivek Mehta $^{2}$, Atilano Lacson ${ }^{3}$, Hanna Kolski ${ }^{1}$ ${ }^{1}$ Pediatric Neurology, University of Alberta, Edmonton, CA; ${ }^{2}$ Neurosurgery, University of Alberta, Edmonton, CA, $;^{3}$ Pathology, University of Alberta, Edmonton, $C A$

Background: We present a four-year-old boy with long-standing history of bilateral hand weakness, whose symptoms went unnoticed for years prior to diagnosis. Methods: Our patient presented with a one week history of abnormal gait and frequent falls. Further inquiry found lifelong bilateral hand weakness, described as weak pincer grasp since infancy and the inability to close hands around objects. These symptoms had been progressively worsening over six months. He was medically healthy and otherwise active. Developmentally he reached gross motor milestones at an appropriate time but regressed to the point of having trouble going down stairs. His fine motor skills had always been delayed using a fisted pencil grasp and was unable to use buttons or shoelaces. Physical exam showed decreased muscle bulk and atrophy in both hands and feet. A contracture at the left heel cord was noted but otherwise he had normal range of motion. He had a predominantly distal pattern of weakness with weak hand grip and ankle dorsiflexion bilaterally (left > right), with weak shoulder girdle muscles bilaterally. Reflexes were slightly brisk with upgoing plantars. His gait showed reduced left foot clearance, and he was unable to heel walk or tandem walk. Results:
An MRI brain and spine found a left hemithorax superior sulcus tumour encasing the great vessels and left brachial plexus, extending to the left side of the neck. The mass extended into the intervertebral foramina from $\mathrm{C} 3$ to $\mathrm{T} 4$ and caused cord compression and edema in the spinal cord. The patient received one round of chemotherapy (carboplatin and etoposide) for presumed neuroblastoma, and high dose dexamethasone which resulted in clinical improvement of his gait and hand weakness. The first biopsy report revealed a ganglioneuroblastoma after Canadian Oncology Group (COG) review. Follow-up MRI suggested no change in mass size. Tumour debulking took place through a multilevel laminoplasty, following which our patient showed substantial improvement in gait. The post-operative MRI also showed improvement in cord compression. The second tissue sample confirmed a ganglioneuroblastoma, intermixed. The tumour showed focal necrosis and degenerative changes among the ganglionic component with no immature neuroblastic components, which could represent therapy effect. Full tumour resection was deferred due to the precarious location surrounding the vessels and left brachial plexus. Conclusion: This case demonstrates the variation in presentation of pediatric neurological diseases. The relatively mild and slow progression of this patient's symptoms caused delay in seeking medical attention. It reminds us that a high index of suspicion is necessary for accurate localization and timely diagnosis of cervical myelopathies. Our case is unique because to the best of our knowledge, this is the first reported case of an extradural compressive myelopathy secondary to ganglioneuroblastoma in a pediatric patient. This case also highlights the need for ongoing clinical, radiographic, and pathologic correlations.

\section{PS2Group9-001 / \#417}

Topic: Group 9 - Miscellaneous

\section{PSYCHOLOGICAL ASPECTS IMPACTING QUALITY OF LIFE OF PATIENTS WITH MYOPATHY}

Amandine Rohmer Cohen ${ }^{1}$, Virginia $\mathrm{Noel}^{2}$, Michèle Mane $^{2}$, Sylvie Zorgani ${ }^{2}$, Delphine Delorme ${ }^{2}$, Juan Rangel Escribano ${ }^{2}$, Catherine Bungener ${ }^{1}$

${ }^{1}$ Université Paris Descartes, Boulogne-Billancourt, FR $;{ }^{2}$ Hopital Rothschild, Paris, FR 
Background: Literature shows that the patient's quality of life is affected by depression, fatigue, and anxiety. The aim of this study was to assess the quality of life using a new evaluation scale: the quality of life in genetic neuromuscular disease (QOL-gNMD, Dany, 2017) and its link with three psychological variables: depression, anxiety and fatigue. Methods: 31 patients with adult-form of myopathy were included: 14 patients with myotonic dystrophy type 1, 9 with facioscapulohumeral dystrophy, 4 with limb girdle muscular dystrophy, 1 with mitochondrial myopathy, 1 with Central Cores myopathy, 1 with Ullrich muscular dystrophy, 1 with inclusion body myositis (population description in table 1).

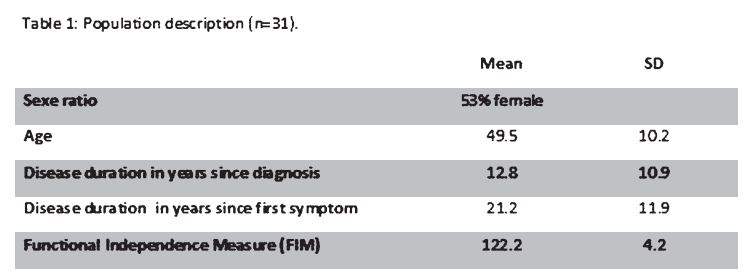

Results: Spearman correlations showed that depression and fatigue were the most correlated with the quality of life, and its three under-score. State anxiety was only correlated with "impact of physical symptom". Disease duration since diagnosis was correlated with under-score "activities and social participation". There was no correlation with trait anxiety, disease duration since first symptom, Functional Independence Measure score, age or genre.

\begin{tabular}{|c|c|c|c|c|}
\hline & BDI 21 & STAI-A & STAI-B & KFSS \\
\hline Impact of Physical Symptom & $-.476^{* *}$ & $-.471 \neq *$ & .120 & $-.495^{* *}$ \\
\hline Self-Perception & $-.625^{ \pm *}$ & -.311 & .062 & $.0404^{\circ}$ \\
\hline Activities \& Social Participation & $-4.06^{\circ}$ & -.172 & .095 & $-.431^{\circ}$ \\
\hline
\end{tabular}

Conclusion: Results are consistent with previous studies which described a strong influence of patient's mood on quality of life. Depression and fatigue affect the different aspects of the quality of life of patients with myopathy. Different ways to preserve the quality of life are discussed, for example: therapeutic workshop to prevent fatigue or regular assessment of depression.
PS2Group9-002 / \#610

Topic: Group 9 - Miscellaneous

\section{AN UNCOMMON CO-EXISTENCE OF INHERITED AND CHRONIC INFLAMMATORY DEMYELINATING POLYNEUROPATHY}

$\underline{\text { Ieva Glāzere }}^{1}$, Signe Šetlere ${ }^{2}$, Inese Kazaine², Guntis Rozentāls ${ }^{2}$

${ }^{1}$ Neurology, Pauls Stradins Clinical University Hospital, Riga, LV; ${ }^{2}$ Pediatric Neurology, Children's Clinical University Hospital, Riga, LV

Background: Chronic inflammatory demyelinating polyneuropathy (CIDP) is an autoimmune neuropathy with a low prevalence between pediatric patients. Diagnosis of CIDP can be very challenging due to its ability to mimic other peripheral nerve disorders such as Charcot-Marie-Tooth neuropathy (CMT). CMT1A is the most common subtype of CMT with highly variable phenotype and age of onset. CIDP and CMT may clinically manifest not only with similar symptoms but in rare occasions also coexist. Accurate and detailed examination is crucial to confirm both overlapping diagnosis and start the appropriate treatment. Methods: Retrospective analysis of patient's history and data. Results: We present a case of previously healthy 13 -year-old boy with slowly progressive gait difficulties, inability to lift his feet since November 2016. In January 2017 symptoms rapidly progressed until he was not able to walk properly and stand up from a sitting position. History data revealed no infections or trauma before, he has 3 healthy brothers with no clinical symptoms. His clinical examination was remarkable for severe muscle atrophy and weakness in legs (MRC grade $3 / 5$ proximally, $2 / 5$ distally), and generalized hyporeflexia. No sensory impairment. Lumbar puncture was performed, cerebrospinal fluid (CSF) workup showed elevated protein level $(0.73 \mathrm{~g} / \mathrm{L})$, with normal CSF cell counts. Further, a nerve conduction study demonstrated a severe motor - sensory axonal demyelinating polyneuropathy in lower extremities, mild in upper extremities; MRI scan of spine revealed nerve root enhancement in cervical and lumbosacral regions. $\mathrm{He}$ received intravenous immunoglobulins (IVIG) totally $2 \mathrm{~g} / \mathrm{kg}$ over 5 days. As clinically patient has pes cavus, DNA analysis was ordered and approved diagnosis of CMT1A neuropathy by detecting $17 \mathrm{p} 11.2 \pm 12$ duplication. 
Antiganglioside antibodies were negative. The patient recovered and was discharged with a mild weakness (MRC grade 4/5) in lower limbs. In October 2017 he was hospitalized again because of rapidly progressive leg weakness. The second MRI scan revealed nerve root enhancement and enlargement in cauda equina nerve roots, CSF showed elevated protein level $(0.82 \mathrm{~g} / \mathrm{L})$. He responded poorly to the second course of IVIG, he was discharged with moderate distal and mild proximal paresis in both legs. Conclusion: The main purpose of the report was to demonstrate an uncommon combination of inherited neuropathy and acquired inflammatory polyneuropathy. Both diseases were confirmed by laboratory, electrophysiological and genetic tests, as well as clinical course and MR imaging. There have been only few publications discussing a possible predisposition to develop an inflammatory neuropathy for patients with CMT. Our case could support this possibility as the patient has a negative family history and no antecedent events. By identifying this association there is a potential to determine a rate of response to immune therapy. According to the literature (Rajabally et al., 2016) approximately 50 percent of CMT1A patients respond to CIDP treatment, which is less then patients without hereditary neuropathies. To conclude, patients with hereditary neuropathies could experience a rapid clinical deterioration, in that case an overlap with inflammatory neuropathies should be considered to establish adequate treatment; further research may unveil the immune processes associated with CMT and its overlap in inflammatory neuropathy pathogenesis.

\section{PS2Group9-003 / \#797}

Topic: Group 9-Miscellaneous

\section{ASSESSING IDEBENONE'S IMPACT ON RESPIRATORY FUNCTION IN DUCHENNE MUSCULAR DYSTROPHY: META-ANALYSIS OF TWO CLINICAL TRIALS}

\author{
Christian Rummey ${ }^{1}$, Mika Leinonen ${ }^{2}$, Shabir \\ Hasham $^{2}$, Thomas Voit ${ }^{3}$, Oscar H. Mayer ${ }^{4}$, Gunnar \\ Buyse For The Delos Study Group ${ }^{5}$ \\ ${ }^{1}$ Clinical Data Science GmbH, Basel, CH; ${ }^{2}$ Santhera \\ Pharmaceuticals, Pratteln, $\mathrm{CH} ;{ }^{3} \mathrm{UCL}$ Great \\ Ormond Street Institute of Child Health, London,
}

$G B ;{ }^{4}$ Children's Hospital of Philadelphia, Philadelphia, PA, US, ${ }^{5}$ University Hospitals Leuven, Leuven, $B E$

Background: Patients with Duchenne muscular dystrophy (DMD) experience respiratory function decline that accelerates after they become non-ambulatory, leading to significant disease burden and, in many cases, death. The ability of idebenone to slow respiratory function decline in patients with DMD has been investigated in two randomized, placebo-controlled trials (the phase 2 DELPHI trial and phase 3 DELOS trial). DELPHI included patients (aged 8 to 16 years) irrespective of baseline respiratory function status, while DELOS required patients (aged 10 to 18 years) to be in the respiratory decline phase. DELPHI allowed the randomization of both glucocorticoid users and non-users, whilst DELOS included only glucocorticoid non-users. Methods: Source data from both trials were pooled and analyzed for treatment effects on peak expiratory flow and forced vital capacity, expressed as a percentage of predicted ( $\mathrm{PEF} \% \mathrm{p}$ and $\mathrm{FVC} \% \mathrm{p})$. Change in $\mathrm{PEF} \% \mathrm{p}$ and $\mathrm{FVC} \% \mathrm{p}$ from baseline to weeks 26 and 52 (end of study) were analyzed using a mixed model for repeated measures, with study as a random effect. Results: In total, 76 patients (DELPHI: 12; DELOS: 64) were in the respiratory function decline phase $(\mathrm{PEF} \% \mathrm{p}<80 \%$ ), of which 72 were not using glucocorticoids. Across all patients, the difference between the idebenone and placebo groups in PEF\%p from baseline to week 52 was $8.0 \%$ p ( $p=$ $0.003)$. When omitting glucocorticoid users, this difference was $7.7 \% \mathrm{p}(p=0.005)$. For $\mathrm{FVC} \% \mathrm{p}$, the treatment difference was 3.6\%p $(p=0.046)$ in all patients, with a comparable difference of $3.5 \% \mathrm{p}(p=$ 0.051 ) when omitting glucocorticoid users. Conclusion: Results from a meta-analysis with data from two randomized, placebo-controlled trials (DELPHI and DELOS) demonstrate that idebenone slows respiratory function decline in patients with DMD. Additionally, the magnitude of treatment difference observed was unaffected by concomitant glucocorticoid use. 
PS2Group9-004 / \#812

Topic: Group 9-Miscellaneous

\section{DEMANDS FROM THE PSYCHOLOGY OF HEALTH FOR THE CARE OF PEOPLE WITH STEINERT€}

\author{
Alejandro J. Solernou Ferrer ${ }^{1}$, Alexis L. Ruiz ${ }^{2}$, \\ Tatiana Z. Vaillant ${ }^{3}$, Carlos E. Oyola Valdizán ${ }^{4}$ \\ ${ }^{1}$ Department Of Neurogenetics, Institute of \\ Neurology and Neurosurgery, Havana, CU; ${ }^{2}$ Faculty \\ Of Psychology, University of Havana, Havana, \\ $C U ;{ }^{3}$ Institute of Neurology and Neurosurgery, \\ Havana, CU; ${ }^{4}$ Management in Health Services, \\ Lima, PE
}

Background: The psychology of the health faces the challenge from the attention to people with illnesses and its support groups to guarantee its quality of life and psychological well-being, like it is in the case of the neurodegenerative illnesses. On Steinert's disease from a literature search, it was observed that only the neuropsychological and psychopathological characteristics of psychology are described. Then, the psychosocial characteristics that these people can present are not described and the authors of this work consider that it is one more element, necessary to better understand the reality that people with this disease go through. It is not a matter of neglecting the neuropsychological and psychopathological elements, but of incorporating into this understanding the psychosocial elements so that it is a more comprehensive understanding, which can lead to an improvement in the multidisciplinary care that these people need to receive, not only from psychology. Methods: This work presents a proposal of what the authors have denominated "demands for the support boarding to people that have the illness of Steinert", starting from a series of necessities identified in a previous work with mixed methodology that consisted on the bibliographical revision, the application of a semiestructuredinterview and the familiogram, to a group of 15 patients with this illness that they are assisted in the Institute of Neurology and Neurosurgery. Results: The demands were contained in different levels according to people that contributed them and they will be good to guide the work with those same people. Among the results of the study prior to this work, some of the characteristics found were the lack of knowledge in the different levels of health care about the existence of the disease which delays the diagnosis of the disease. Also the lack of prior knowledge about the disease in these patients stands out as a worrying element, even though many of them have relatives with the same condition. Based on the characteristics found in the previous study, a series of demands were made based on the needs identified of the patients and of the different socialization groups in which they can be found. They were grouped as follows: 1. Patients with Steinert's Myotonic Dystrophy 2. Their families 3. Friends, neighbors, co-workers. 4. Institutions and social organizations 5. Society Some common demands were identified in all groups, among them is to have more information about the disease. In addition, the need to develop actions to keep as long as possible in the different activities of life in society to people with this disease, this work shows some examples. Conclusion: The characterization made evidences aspects necessary for attention with the humanistic approach that characterizes Cuban health. The elaborated demands are an integrative and systemic proposal from the Psychology of Health that should support the accompaniment of people with this disease and their relatives.

PS2Group9-005 / \#263

Topic: Group 9 - Miscellaneous

\section{POMPHOLYX AND ECZEMATOUS SKIN REACTIONS AFTER INTRAVENOUS IMMUNOGLOBULIN THERAPY: CASE REPORTS AND REVIEW OF THE LITERATURE}

\author{
Byung-Nam Yoon $^{1}$, Ji-Won Yang ${ }^{2}$ \\ ${ }^{1}$ Neurology, Inje University College of Medicine, \\ Seoul Paik Hospital, Marrenarero Gung-gu, \\ $K R,{ }^{2}$ Gachon University, Gil medical Center, \\ Incheon, $K R$
}

Background: Intravenous immunoglobulin (IVIGs) has had a major impact on the treatment of neurological disorders including Guillain-Barre syndrome, chronic inflammatory demyelinating polyneuropathy, myasthenia gravis, and inflammatory dermatositis. IVIG administration is generally considered safe and well tolerated, except for several adverse effects such as anaphylactic reactions, 
thromboembolic events and acute renal failure. Most of the adverse effects include headache, fever, flulike symptom, chest tightness, blood pressure change and dyspnea. The skin adverse reactions are very uncommon. Recently we experienced two cases with pompholyx associated with IVIG therapy. Methods: Case presentations; We report 2 cases of characteristic extensive eczematous reaction that occurred approximately 7 days after administration of IVIG for Guillain-Barre Syndrome (GBS) and Miller Fisher Syndrome(MFS). The onset was characterized by dyshidrotic lesions on the palms, rapidly followed by pruriginous maculopapular lesions involving the whole body. They were treated with topical and/or systemic steroids, and complete resolution of skin lesions was observed within 1 month. Results: Severe eczematous skin reaction with a characteristic lesions on palms that then extends to the rest of the body is a rare but characteristic adverse effect of high dose IVIG therapy. A similar report has been consistently reported. We could not fully understand the pathophysiology and the precautions for the side effects are not clear. Fortunately the side effects are easily treated. Conclusion: With the use of IVIG increasing, it is important for neurologists or dermatologists to recognize this cutaneous side effect and its clinical course.

\section{PS2Group9-006 / \#541}

Topic: Group 9 - Miscellaneous

\section{GLOBAL VS INDIVIDUAL MUSCLE FATTY DEGENERATIVE CHANGES TO MONITOR DISEASE PROGRESSION IN IMMUNE- MEDIATED NECROTIZING MYOPATHY}

Cédi Koumako ${ }^{1}$, Harmen Reyngoudt ${ }^{2}$, Jean-Marc Boisserie $^{3}$, Océane Landon-Cardinal ${ }^{4}$, Yves Allenbach $^{4}$, Pierre G. Carlier ${ }^{2}$

${ }^{1}$ Aim \& Cea Nmr Laboratory, Neuromuscular Investigation Center, Institute of Myology, Paris, FR:2Aim \& Cea Nmr Laboratory, Neuromuscular Investigation Center, Institute of Myology, Paris, $F R ;{ }^{3} \mathrm{Nmr}$ Laboratory, Neuromuscular Investigation Center, Institute of Myology, Paris, FR ${ }^{4}$ Department Of Internal Medicine And Clinical Immunology, APHP Pitié-Salpêtrière Hospital, Paris, FR
Background: Fat fraction (FF), as calculated from water-fat (Dixon) NMR images, is a largely accepted, if not fully validated, muscle imaging biomarker, which has been proposed as an outcome measure in most neuromuscular disorders these last few years. The question remains, however, as to whether specific muscle or muscle group should be taken into consideration for longitudinal evaluation in specific neuromuscular diseases. Most often all muscles are segmented and evaluated over a number of slices. Here, we looked into a cohort of patients suffering from immune-mediated necrotizing myopathy (IMNM), an inflammatory neuromuscular disease characterized by severe muscular lesions and muscle fatty replacement. The aim of this work was to compare whole segment FF with individual muscle and muscle group FF and identify the most efficient procedure to quantify disease progression. Methods: Fourteen IMNM patients (46.4 \pm 17.7 years, 18.974.8 age range, 5 male) were scanned twice within a one-year interval on a clinical 3T NMR system (Siemens). A fat/water 3-point Dixon NMR sequence was performed at the level of the thigh and the leg (right and left). FF was calculated both in the different individual muscles, muscle groups and in the central slice of the whole leg and thigh. The changes in $\mathrm{FF}(\Delta \mathrm{FF})$ using all methods (individual, group or whole) were compared using paired $t$-tests. Standardized response means (SRM) were also calculated. Results: $\triangle \mathrm{FF}$ was highly significant $(P<0.01)$ for all investigated separate muscles, muscle groups and the whole segment. In the leg, $\Delta \mathrm{FF}$ was only highly significant in gastrocnemius medialis muscle $(P<0.001)$ and non-significant in muscle groups and the whole segment. $\triangle \mathrm{FF}$ from the whole thigh segment resulted in an SRM of 0.80, which was similar when combining quadriceps and hamstring muscle groups (0.8). Lower SRMs were found when evaluating separate muscle groups (hamstring and quadriceps: 0.7 ) or individual muscles (vastus lateralis: 0.6 , rectus femoris: 0.6 , biceps femoris: 0.7 ). In leg, all SRM values were smaller than 0.5. Conclusion: Using the SRMs to evaluate the ability of various FF estimation procedures to monitor disease progression, it appeared that the simplest, mono-slice and global, estimate of thigh FF was also the most sensitive in IMNM patients. This suggests that multi-slice individual muscle segmentation might not be a mandatory tedious step in using quantitative NMR imaging to characterize muscle structural changes. There has also been a recurrent quest by investigators for the most appropriate muscle on which to 
focus the analysis. Our results indicate that this question is likely to be pointless. Other yet unpublished data in LGMD2B and LGMD2I patients showed that global approaches similar to the one we propose were also associated with superior performance. Still, generalization of the concept is premature in the present stage. Other conditions need to be revisited. Applicability to contractile tissue indices and water $\mathrm{T}_{2}$ distribution has yet to be investigated. Because the global approach takes into account the intermuscular fat, changes in patient nutritional status will likely bias the evaluation of the muscular FF and the impact has to be carefully determined.

\section{PS2Group9-007 / \#632}

Topic: Group 9 - Miscellaneous

\section{KCNK9 IMPRINTING SYNDROME WITH CONGENITAL HYPOTONIA, DYSMORPHISM AND DEVELOPMENTAL DELAY DUE TO NOVEL MUTATION P.ALA237ASP}

Pavel Seeman $^{1}$, Jana Haberlova ${ }^{2}$, Lucie Sedláčková ${ }^{3}$, Marie Šedivá ${ }^{4}$, Petra Laššuthová ${ }^{4}$ ${ }^{1}$ Child Neurology, Dna Laboratory, 2nd Medical Faculty, Charles University and University Hospital Motol, Prague, Praha, CZ; 6 Department Of Child Neurology, Second Faculty of Medicine, Charles University and University Hospital Motol, Prague, CZ; ${ }^{3}$ Child Neurology, Dna Laboratory, 2nd Medical Faculty, Charles Universityl, Prague, Praha, CZ; ${ }^{4}$ Child Neurology, Dna Laboratory, 2nd Medical Faculty, Charles University and University Hospital Motol, Prague, Praha, CZ

Background: KCNK9 imprinting syndrome also called Birk Barel mental retardation dysmorphism syndrome is a very rare neuromuscular disorder which is caused by always only one amino acid exchange p.Gly236Arg in the $K C N K 9$ gene, which is silenced on the paternal chromosome. Patients with this mutation demonstrate congenital hypotonia, cleft palate, delayed development, feeding problems and normal MRI and EEG. We report a novel cause of this syndrome in a Czech girl with congenital neurogenic hypotonia, weakness and developmental delay. Methods: Whole exome sequencing (WES) of patients DNA from blood and Sanger sequencing of the exon 2 of the KCNK9 gene were used in the
DNA sample from the patient and from the healthy father. Neurological and electrophysiological and muscle biopsy examination of the patient were done. Results: A novel mutation, only the second at the amino acid level in this gene, namely p.Ala237Asp (c. $710 \mathrm{C}>\mathrm{A}$ ) in exon 2 of $\mathrm{KCNK} 9$ gene was detected in heterozygous state by WES and later confirmed by Sanger sequencing in the patient, but not in her healthy father, the mother is not alive anymore. This mutation is not in ExAC database and affects a highly conserved aminoacid and is predicted to be deleterious/disease causing. DNA samples from maternal grandparents were not available at the time of abstract preparation. The patient is now 17 year old female, who is followed in our clinic for congenital hypotonia and generalized weakness of unknown cause since her age of 8 months. Birth weight and length were normal $(3200 \mathrm{~g} / 50 \mathrm{~cm})$. She had a cleft palate, which was corrected and the motor development was delayed: walking at the age of 4 years. The patient has also mild mental retardation - mental developmental delay, she spoke first words at the age of 5 years. There was a feeding problem and swallowing problem. EMG showed pure motor primary axonal peripheral neuropathy and decreased number of motor units. Muscle biopsy at the age of 1,5 year showed mild neurogenic changes with selective atrophy of type II fibers and hypertrophy of type I fibers. She has fasciculations on the tongue. The muscle weakness is slowly progressive, she is still able to walk several hundreds meters independently, but for longer distances she uses wheelchair since the age of 12 years. Several DNA tests were done without clarification, incl. SMN 1 gene. Brain MRI at the age of 11 was normal. We have not yet tried the therapy by flufenamic acid (FFA) in this patient, but this is now available after the clarification of the cause of the disease. Conclusion: Birk Barel mental retardation dysmorphism syndrome or KCNK9 imprinting syndrome may be caused not only by always the same mutation p.Gly236Arg, but also by the mutation affecting residue p.Ala237 and the new causal genotype is associated with the same or very similar phenotypic expression. 
PS2Group9-008 / \#655

Topic: Group 9 - Miscellaneous

\section{QUANTITATIVE NMR OUTCOME MEASURES FOR SKELETAL MUSCLE CHARACTERIZATION OF DUCHENNE MUSCULAR DYSTROPHY PATIENTS}

Teresa Gerhalter ${ }^{1}$, Lena V. Gast ${ }^{1}$, Benjamin Marty ${ }^{2}$, Regina Trollmann ${ }^{3}$, Stephanie Schüssler ${ }^{3}$, Frank Roemer ${ }^{1}$, Frederik B. Laun ${ }^{1}$, Michael Uder ${ }^{1}$, Rolf Schröder $^{4}$, Pierre G. Carlier ${ }^{2}$, Armin M. Nagel ${ }^{1}$ ${ }^{1}$ Institute Of Radiology, University Hospital Erlangen, Erlangen, DE; ${ }^{2}$ Aim \& Cea Nmr Laboratory, Neuromuscular Investigation Center, Institute of Myology, Paris, FR, ${ }^{3}$ Department Of Pediatrics, University Hospital Erlangen, Erlangen, DE: ${ }^{4}$ Neuropathology, University Hospital Erlangen, Erlangen, DE

Background: DMD is a hereditary neuromuscular disease caused by mutations in the dystrophin gene associated with inflammation, ionic homeostasis dysregulation, and exhaustion of regenerative capacities which leads ultimately to replacement of muscle by fatty and fibrotic tissue. Quantitative NMR has been used to describe the natural history of the disease and has been suggested as an outcome measure in clinical trials for DMD. Most of these studies evaluated the fat fraction (FF) and the ${ }^{1} \mathrm{H}$ transverse relaxation time (water $\mathrm{T}_{2}$ ) to assess disease severity and progression. Both methods have their limitations: water $T_{2}$ changes are non-specific events influenced by the usually prescribed steroid therapy and fatty degenerative changes, while picking up the extreme phenotypic variability, have a poor predictive value. Thus, there is a need to identify NMR variables as potential early sensitive indicators of dystrophic muscle response to treatment. Higher total sodium concentrations (TSC) and intracellular weighted sodium (ICW) signal have been measured by ${ }^{23} \mathrm{Na} \mathrm{MRI}$. Here, we evaluated the sensitivity of ${ }^{23} \mathrm{Na}$ MRI in DMD in comparison to the commonly used water $\mathrm{T}_{2}$ and fat fraction. Methods: MRI of the right calf was performed in twelve DMD patients (age 9.0 03.1 years) and twelve age-matched controls (age $9.6 \pm 2.3$ years) on a $3 \mathrm{~T}$ whole-body MR system (Siemens Healthcare). Water $\mathrm{T}_{2}$ was determined using a multi-slice-multi-echo sequence (TR 3000ms, 32 echoes: TEs 9.5-304ms, Tacqu 3 min $41 \mathrm{sec}$ ) and a tri-exponential fitting procedure.
FF was measured using a 3-point DIXON method (TR $10 \mathrm{~ms}$, TEs $2.75 / 3.95 / 5.15 \mathrm{~ms}$, Tacqu $3 \mathrm{~min} 12$ $\mathrm{sec}$ ). TSC was derived from an density-adapted 3Dradial UTE sequence (TE/TR $0.3 / 50 \mathrm{~ms}$, Tacqu $6 \mathrm{~min}$ $53 \mathrm{sec}$ ). An inversion-recovery (IR) sequence was used to achieve an ICW signal (TE/TR 0.3/124ms, TI $34 \mathrm{~ms}$, Tacqu $9 \mathrm{~min} 50 \mathrm{sec}$ ). ROIs were drawn on the gastrocnemius medialis (Gas.m.), soleus (Sol), tibialis anterior (TA) and posterior (TP) muscles. Results: Mean water $T_{2}$ and $F F$ were elevated in DMD patients compared to healthy controls $(38.6 \pm 2.8 \mathrm{~ms}$ vs $36.1 \pm 1.5 \mathrm{~ms}$ and $0.078 \pm 0.072 \mathrm{vs}$ $0.024 \pm 0.012, p=0.046$ and $p=0.007$, respectively). Patients showed an increased TSC of $24.7 \pm 4.45 \mathrm{mM}$ and an elevated ICW of $27.26 \pm 3.73$ a.u. (controls: $16.66 \pm 2.46 \mathrm{mM}$ TSC and $19.11 \pm 2.58$ a.u. ICW, both $\mathrm{p}<0.0001$ ). Moreover, the ${ }^{23} \mathrm{Na}$ indices are frequently abnormal in DMD even when water $\mathrm{T}_{2}$ or FF remained in the normal range. DMD patients at all ages exhibited abnormally elevated sodium indices, whereas FF increased with age and the water $\mathrm{T}_{2}$ fluctuated during the course of disease. Conclusion: Sodium anomalies seemed to be systematically present in patients with DMD compared to controls and precede fatty degenerative changes. Sodium alterations may also occur before water $T_{2}$ increases, however more investigations are needed to confirm this observation. Moreover, muscles that were relatively spared, such as the TA and TP, showed increased TSC or ICW in all participating DMD patients. Although this study, which is still in progress, has currently its limitation in the small number of subjects, the data supports that ${ }^{23} \mathrm{Na}$ MRI could serve as an early and sensitive biomarker to investigate ion channel leakage and membrane integrity.

\section{PS2Group9-009 / \#772}

Topic: Group 9 -Miscellaneous

\section{ASSESSMENT OF LIVER SAFETY USING AN EMERGING LIVER BIOMARKER, GLUTAMATE DEHYDROGENASE}

Sumita Chowdhury ${ }^{1}$, Ruth Osahon ${ }^{2}$, David Peters ${ }^{1}$, Gary Layton ${ }^{2}$, Anne Heatherington ${ }^{1}$, David Roblin ${ }^{2}$, Francesco Muntoni ${ }^{3}$

${ }^{1}$ Summit Therapeutics Inc., Cambridge, MA, US, ${ }^{2}$ Summit Therapeutics Inc., Abingdon, GB, ${ }^{3}$ Dubowitz Neuromuscular Unit, UCL Great Ormond Street Institute of Child Health, London, GB 
Background: To evaluate hepatic safety of ezutromid, a first-in-class small molecule utrophin modulator, using standard hepatic monitoring in conjunction with the emerging biomarker, glutamate dehydrogenase (GLDH). Thorough assessment of hepatic safety during drug development is essential; however, it is challenging in Duchenne muscular dystrophy (DMD) as traditional enzymes (ALT and AST) are not liver-specific and are markedly elevated due to muscle injury in DMD. GLDH has emerged as a potentially more sensitive and specific exploratory liver injury biomarker. With normal levels in DMD patients, it may be a better marker of ongoing acute liver injury compared to ALT. Recent consortia efforts (D-RSC) have resulted in regulatory support for GLDH as a drug induced liver injury (DILI) biomarker to complement the usual liver parameters, potentially replacing AST/ALT in assessing serious liver injury (e.g. Hy's Law case) as an indication of hepatocellular necrosis. Methods: PhaseOut DMD is a Phase 2 open-label study of ezutromid administered to 40 ambulatory patients with DMD. In addition to other safety monitoring, hepatic safety was monitored by standard liver enzymes (ALT, AST, GGT, ALP) and functional markers (bilirubin, coagulation times) as well as GLDH, evaluated at screening, baseline, and throughout ezutromid dosing to monitor for evidence of liver safety signal. The Roche analytical method used to measure serum GLDH is based on UV measurement of GLDH enzymatic activity associated with NADH oxidation in the presence of substrate with an accuracy of 0.0 to $1.8 \%$, precision of 0.6 to $1.9 \%$, sensitivity of 0.5 $\mathrm{U} / \mathrm{L}$, and reference range of 0 to $6.4 \mathrm{U} / \mathrm{L}$. The assay is robust and repeatable. Results presented include up to the Week 24 interim analysis, by which time a DILI effect would typically be observed. Results: During 24 weeks of treatment, two patients exceeded protocol-specified GLDH threshold of $\geq 3 x$ ULN prompting ezutromid to be withheld: $20 \mathrm{U} / \mathrm{L}$ at Week 4 in one case, and $22.1 \mathrm{U} / \mathrm{L}$ at Week 24 in the other. After drug withholding, GLDH in both cases dropped below threshold within 3 weeks. Ezutromid was successfully resumed in both cases. There were no recurrences of GLDH elevation despite re-challenge. The bilirubin, INR, GGT, and ALP, were in the clinically normal range with no overt clinical signs of liver disease, both at the time of GLDH elevation and upon re-initiation of ezutromid. The totality of the data suggests no hepatic safety issues in either of these subjects; both have successfully continued long-term ezutromid therapy. Implementing
Hy's Law (with GLDH $\geq 2.5 x$ ULN replacing ALT/ AST), would have prevented potentially unnecessary actions resulting from using GLDH $\geq 3 \mathrm{x}$ ULN as a stand-alone criterion for hepatic safety. Conclusion: 1) Rigorous assessment of the hepatic safety profile of ezutromid over 24 weeks has indicated no clinically meaningful concerns. 2) The emerging liver-specific biomarker GLDH may be of particular value in monitoring liver injury in DMD. However, to avoid potentially unnecessary actions, it should be evaluated in the context of functional assessment of the liver with bilirubin and/or coagulation, and not as a stand-alone parameter for hepatic safety.

\section{PS2Group9-010 / \#536}

Topic: Group 9 - Miscellaneous

\section{PERCEIVED QUALITY OF LIFE AND MOTOR SCALES OUTCOMES IN TYPE 2 AND 3 SMA PATIENTS: ARE THEY RELATED?}

Anna Lia Frongia ${ }^{1}, \underline{\text { Irene Zschaeck }}^{1}$, Macarena M.A. Alarcon Cornejo ${ }^{1}$, Daniel Natera De Benito ${ }^{2}$, Laura Carrera-García ${ }^{3}$, Joaquin F. Mata ${ }^{1}$, Nuria Padros $^{1}$, Obdulia Moya ${ }^{1}$, Judit Armas ${ }^{1}$, Julita Medina $^{1}$, Carlos Ortez ${ }^{1}$, Jaume Colomer ${ }^{4}$, Andres Nascimento $^{1}$

${ }^{1}$ Neuromuscular Disorders Unit, Hospital Sant Joan de Déu, Barcelona, ES, ${ }^{2}$ Neuromuscular Disorders Unit, Hospital Sant Joan de Déu, Barcelona, ES, ${ }^{3}$ Unidad De Patología Neuromuscular., Hospital Sant Joan de Déu, Barcelona, ES; ${ }^{4}$ Hospital Sant Joan de Déu, Barcelona, ES

Background: Spinal muscular atrophy (SMA) related to SMN1 gene mutations is the most common genetic cause of mortality in childhood. It presents with atrophy and progressive muscular weakness due to motor neuron degeneration that restricts mobility and increases physical dependence, affecting quality of life. Patients with later-onset SMA (SMA type 2 and 3) became symptomatic after 6 months and life expectancy is shortened by early onset scoliosis, joint contractures, and restrictive lung disease, requiring a multidisciplinary approach. To date, there is no cure for this neuromuscular disorder, thus it becomes important to determine how far the disease and its treatment compromise a child's 
QoL. Methods: To describe the correlation between the functional motor status with the perceived quality of life by families and patients we compare the outcomes scores from PedsQL with scores of functional motor scales (EK2, HFMSE and RULL) in a cohort of type 2 and 3 SMA patients. Results: Fiftyone patients with SMA (42 with type 2 and 9 with type 3 ) were enrolled in the study. Mean age was 10 years (range: 3-18.5). Mean score in patients and parents PedsQ1 was 67,32 (range 13-91) and 61,25 (range 22-90) respectively. HFMS score of 0 was reported in 12 SMA 2 patients and in 1 SMA 3 type, in this group mean RULL score was 10 (range 0-21). 4 out of 42 parents of children with SMA 2 and 3 out of 9 parents of SMA type 3 patients reported scores $>80$ in PedsQL. When comparing patients and parents PedsQL scores, a moderate or large discrepancy was found in 5 out of 51 families. In this group, indepth unstructured interviews were conducted and analyzed with a qualitative approach. Conclusion: Regardless of the functional motor outcome, children and adolescents with SMA, as well as their parents, have a perception of relatively good quality of life. These findings are similar to those reported in several other studies.

\section{PS2Group9-011 / \#791}

Topic: Group 9-Miscellaneous

\section{DEVELOPMENT OF A PSYCHOSOCIAL INTERVENTION PROGRAMME FOR MOTOR NEURON DISEASE}

$\underline{\text { Priya T. Thomas }}^{1}$, Manjusha Warrier ${ }^{2}$, Atchayaram Nalini $^{3}$

${ }^{1}$ Department Of Psychiatric Social Work, National Institute of Mental Health and Neuro Sciences, Bangalore, IN; ${ }^{2}$ Department Of Psychiatric Social Work, National Institute of Mental Health and Neuro Sciences, Bangalore, IN; ${ }^{3}$ Department Of Neurology, National Institute of Mental Health and Neuro Sciences, Bangalore, IN

Background: Background: Amyotrophic Lateral Sclerosis is a progressive neuromuscular disorder that can have significant and debilitating impact on the affected patient and families. Few studies have looked in to the neuropsychiatric and other psychosocial dimensions in this group especially in the so- ciocultural scenario that is unique to a developing country like India. Methods: Aim: To assess develop a structured psychosocial intervention program for persons living with Motor Neuron Disease and their families. Method A quasi experimental, pretest post-test research study was performed among the patients and family members of 80 patients diagnosed with ALS (Both Males and females) who were receiving treatment from a national tertiary referral care centre for Neurological disorders in South India. All the patients were evaluated for their complaints and were diagnosed as Definite ALS according to the El Escorial Criteria. Mean age at onset of illness in this group was 51.6. Mean duration of illness at the time of presenting to hospital was eleven months. ALSFRS was used to assess the functional limitations due to the illness. All patients were assessed with Beck's Depression Inventory (BDI), Beck's Anxiety Inventory (BAI), ALSQoL (R) and COPE. Caregivers completed Caregiver Burden Assessment (Zarit Burden Inventory) and COPE. Structured psychosocial intervention was developed, pilot tested, and was delivered by a trained Psychiatric Social Worker. All the patients and caregivers were re assessed on the same variables immediately after the intervention. Results: The preliminary analysis showed that psychosocial intervention is effective in bringing down the mental health concerns in the patients. Caregiver Burden and Quality of life showed positive changes after the intervention. Coping strategies most commonly adopted were emotional ventilation and instrumental support. Further results and the implications of the study will be presented. Conclusion: Diagnosis of a degenerative illness that is progressive and debilitative has significant impact on the mental health and coping in the affected individual. The treatment plan should take into account the various dimensions of living with the illness, the tasks that might arise during the advanced stages and periodic support during the course of the illness. 
PS2Group9-012 / \#746

Topic: Group 9 -Miscellaneous

\section{HTLV-1-SPECIFIC CTLS IN THE SPINAL CORDS OF ASYMPTOMATIC HTLV-1 CARRIERS}

\author{
Eiji Matsuura \\ Neurology And Geriatrics, Kagoshima University \\ Graduate School of Medical and Dental Sciences, \\ Kagoshima city, JP
}

Background: HTLV-1 is an exogenous human retrovirus that is the causative agent of adult T-cell leukemia/lymphoma (ATL) and chronic inflammatory neurological disease, HTLV-1-associated myelopathy/Tropical spastic paraparesis (HAM/TSP). The major route of HTLV-1 infection is from mothers by a breastfeeding. Symptoms of HAM/TSP, however, typically manifest in patients later in life (50-60 years old in age). Some patients have just urinary dysfunction for several decades without motor symptom. The etiology of the urinary dysfunction in HTLV-1 carriers remains unclear. Little is known about the inflammation in the spinal cord lesion of HTLV-1 carrier (AC) before the onset of motor symptom. Methods: Aim: To elucidate onset of inflammation in the spinal cord of $\mathrm{AC}$, we performed immunohistochemical study with the spinal cord of AC and tried to detect HTLV-1-specific CTL. Methods: We obtained autopsied spinal cords and brain from the patient with HAM/TSP and HTLV-1 carriers after obtaining written, informed consent from their family. One brain sample and four spinal cords were for use in this study. This study was approved by the Kagoshima University Ethics Committee. Results: CD8+ lymphocytes and HTLV-1-specific CTLs were detected in a brain and the four spinal cords. Interestingly, inflamed lesions of HTLV-1 carrier were distributed mainly in the lower thoracic level of the spinal cords like the spinal cord of HAM/TSP. Conclusion: Our data suggest that latent inflammatory change occurs in the spinal cords of ACs.
PS2Group9-013 / \#941

Topic: Group 9 - Miscellaneous

\section{AAV-ASSOCIATED SENSORY GANGLIONITIS AND AXONOPATHY IN LABORATORY ANIMALS: A RETROSPECTIVE STUDY}

\author{
Juliette Hordeaux, Elizabeth L. Buza, Laura \\ Richman, Christian Hinderer, Nathan Katz, Peter \\ Bell, James M. Wilson \\ Gene Therapy Program, Department Of Medicine, \\ University of Pennsylvania, Perelman School of \\ Medicine, Philadelphia, PA, US
}

Background: Adeno-associated viral (AAV) vectors are increasingly being used in clinical trials to treat neurologic disorders. Neurotropic serotypes such as AAV9 have been demonstrated to transduce spinal alpha motor neurons and dorsal root ganglia sensory neurons efficiently when administered intravenously to juvenile animals or intrathecally in both juveniles and adults. As more nonclinical safety and tolerability studies are performed using these vectors, we were confronted with unexpected toxicity targeting dorsal root ganglia neurons and their corresponding central and peripheral axons. Previous studies have reported efficient transduction of dorsal root ganglia utilizing systemic or intrathecal AAV delivery. Dorsal root ganglia are outside of the blood brain barrier, directly bathed into cerebrospinal fluid, and contain a cell body rich area that presents a high density of fenestrated capillaries. Those anatomic considerations likely explain why they are so efficiently targeted following both systemic and intrathecal administration. Methods: The toxicity was observed in adult rhesus macaques after intrathecal administration of $1-5 \times 10^{13}$ Genome Copies of AAV $\left(1-5 \times 10^{11}\right.$ Genome Copies per gram of brain) and in juvenile macaques and piglets after high dose intravenous administration $\left(2 \times 10^{14} \mathrm{GC} /\right.$ $\mathrm{kg}$ ). It was not observed in dogs, cats, or rodents dosed in the cerebrospinal fluid with up to $2 \times 10^{11}$ $\mathrm{GC} / \mathrm{g}$ brain. Results: The toxicity was characterized by neuron cell body degeneration with mononuclear cell infiltration in the dorsal root ganglia, and axonopathy with dilated myelin sheaths and occasional macrophages in the corresponding central and peripheral axons. Rhesus macaques were less severely affected with subclinical lesions only detected on histological examination whereas piglets 
demonstrated proprioceptive deficits and ataxia. Conclusion: The significance of the DRG, spinal cord and peripheral nerve nonclinical findings is not currently known and for many monogenic neurologic diseases without a standard of care, the benefit-risk profile is still in favor of AAV-mediated gene therapy. For the first-in-human trials, informed consent, electrophysiological recordings as well as careful monitoring of sensory symptoms such as paresthesias or changes in sensation of pain, temperature, touch or proprioception will be essential.

\section{PS2Group9-014 / \#813}

Topic: Group 9 -Miscellaneous

\section{OSTEOPOROSIS IN DUCHENNE MUSCULAR DISTROPHY PATIENTS}

Filiz Meryem Sertpoyraz $^{1}$, Figen Baydan ${ }^{2}$

${ }^{1}$ Physical Therapy And Rehabilitation Clinic, Izmir Tepecik Education and Research Hospital, İzmir, TR; ${ }^{2}$ Pediatric Neurology, Tepecik Eğitim ve Araştırma Hastanesi, İzmir, TR

Background: Osteoporosis is a progressive metabolic bone disease that results in increased bone fragility and fracture tendency as a result of low bone mass and disruption of the micro-architecture of bone tissue. Duchenne Muscular Dystrophy (DMD) is a progressive, junctional type of muscle disease characterized by a distroflex syndrome defect that passes through X. Muscle weakness, immobilization and steroid use in DMD patients accelerate the development of osteoporosis and are implicated in studies that lead to osteoporosis-related complications. Objective: To evaluate the status of osteoporosis in patients with DMD. Methods: Retrospective, descriptive study In the Neuromuscular Disorders unit, files of 48 male patients who had received a definite DMD diagnosis and signed the voluntary form were examined. Serum $25(\mathrm{OH})$ vitamin $\mathrm{D}$, calcium, phosphorus, alkaline phosphatase and parathormone levels were recorded in the first visit. Demographic information, functional activity levels, steroid use, fracture story, fracture site and first time measurements were recorded. Lomber and hip z scores were evaluated. The number of patients is 48 and all are male. Inclusion criteria: 1. Patients signed voluntary consent form 2. Patients between the ages of 8 and 183 Men 4. Patients with definite DMD
Exclusion criteria : 1. Patients who do not sign the voluntary consent form 2. Osteoporosis caused by endocrine, malignant disease, rheumatic disease 3. Those who do not have definite DMD diagnosis. Results: Forty eight (48) male patients with DMD were included in the study. The mean age of DMD-diagnosed patients was $14.17 \pm 4.4(8-24)$ years, The mean duration of diagnosis was $9.8 \pm 4.11(1-20)$ years. Mean DMD $25(\mathrm{OH})$ vitamin D level: $15 \pm 7,8$

\begin{tabular}{lc}
\hline $\begin{array}{l}\text { Serum } 25(\mathrm{OH}) \text { vitamin D } \\
\text { levels }\end{array}$ & $\begin{array}{c}\text { Number of patients: } \mathrm{n} \text { percent } \\
(\%)\end{array}$ \\
\hline D vitamin deficiency & $32(67 \%)$ \\
D vitamin insufficiency & $15(31 \%)$ \\
D vitamin sufficient & $1(2 \%)$ \\
\hline
\end{tabular}

There was no statistically significant difference in vitamin D levels among patients with and without steroids in DMD patients. $\mathrm{P}=0.720$ There was no significant difference between vitamin $\mathrm{D}$ levels in patients with and without fractures in DMD patients. $\mathrm{P}=0.833$ The average Lomber $\mathrm{Z}$ score in DMD patients: $-2.0 \pm 1.5$, hip $\mathbf{Z}$ score: $\mathbf{- 3} \pm \mathbf{1 , 3}$ The decrease in hip bone density was statistically significant. Conclusion: We have found vitamin D deficiencyinadequacy at a high rate in our patients. Loss of bone density in the hip is thought to be due to early gait loss. Considering high prevalence of vitamin D deficiency and osteoporosis in DMD patients, it seems vitamin D supplementation can improve vitamin D status and osteoporosis in these patients.

\section{PS2Group9-015 / \#635}

Topic: Group 9 - Miscellaneous

\section{NEUROMUSCULAR GENETIC REGISTRY IN RUSSIA}

Dmitry Vlodavets $^{1}$, Anastasya Monakhova ${ }^{1}$, Denis Reshetov $^{2}$, Svetlana Artemyeva ${ }^{1}$, Irina Shulyakova ${ }^{1}$, Olga Shidlovskaya ${ }^{1}$, Elena Belousova ${ }^{1}$ ${ }^{1}$ Russian Children Neuromuscular Center, Veltischev Scientific Research Clinical Pediatric Institute of Pirogov Russian National Research Medical University, Moscow, $R U ;{ }^{2}$ Science Advice, Moscow, $R U$

Background: Neuromuscular disorders are widespread all over the globe. In the most developed countries of Europe and North America, extensive 
work is underway to identify these patients suffering from neuromuscular diseases, aimed both at identifying individual needs and improving the quality of life, and on stimulating the development of basic science and molecular genetic technologies, which in the near future will be able to cure such patients. The main ways of working are to concentrate efforts and knowledge of patients, clinicians, scientists on a specific problem (for a particular disease). After another round of accumulation of knowledge, a breakthrough takes place - there is a technology that allows solving the problem with specific diseases. So, for example, it happened with Pompe's disease and SMA. One way of initial accumulation of knowledge is to create registers for specific diseases to unite patients into unique groups. This allows to determine the incidence and prevalence of diseases in specific populations. Methods: About 3 years ago we started to collect blood and data of the DMD and SMA patients in Russia and collect them in 2 Genetic Registers. Results: At that moment most of the patients were not provided with genetic testing. To the date we collected more than 500 patients with DMD and BMD; more than 550 patients with different types of SMA. Now most of them are genotyped and few patients with sarcoglycanopathies were surprisingly found in DMD group. During this work, we identified more than 80 new point mutations in DMD gene. About half of the SMA patients now have not only deletion of 7 exon test but also a copy number of SMN2 gene testing. And since the 2018 we started the project Neuromuscular Genetic Registry in Russia. The aim is to collect different data of patients (clinical, genetics, muscle MRI, etc.) also with collecting patient's blood. We have concentrated on several diseases that are most common in Russia: congenital muscular dystrophy type 1A, collagen 6 disorders (Bethlem and Ullrich CMD), FKRP patients with LGMD2I, CAPN3 patients with LGMD2A, patients with dysferlinopathies, patients with Landousy muscular dystrophy, patients with RYR1 mutations and other rare conditions. Conclusion: With the total population in Russia about 150 million of people there are hundreds of patients that still have no genetics and proved diagnosis. This is making them very far from the future treatment. Those patients who have been tested genetically could be included in our Neuromuscular Genetic Registry and provide additional information about the natural history of the disease that possibly in future will help scientists for better understanding pathogenesis and other factors that could influence on the course of the disease. And also collecting the representative groups of patients with different disorders will allow our site to take part in clinical trials providing to our patients advanced treatment.

\section{PS2Group9-016 / \#792}

Topic: Group 9-Miscellaneous

\section{ALTERNATIVE ANALYSES OF RESPIRATORY FUNCTION IN DUCHENNE MUSCULAR DYSTROPHY: CONSISTENT TREATMENT BENEFIT OF IDEBENONE}

\author{
Oscar H. Mayer ${ }^{1}$, Mika Leinonen ${ }^{2}$, Christian \\ Rummey $^{3}$, Thomas Meier ${ }^{4}$, Shabir Hasham ${ }^{4}$, \\ Thomas Voit ${ }^{5}$, Gunnar Buyse For The Delos Study \\ Group $^{6}$ \\ ${ }^{1}$ Children's Hospital of Philadelphia, Philadelphia, \\ PA, US: ${ }^{2}$ Santhera Pharmaceuticals, Pratteln, \\ $\mathrm{CH} ;{ }^{3}$ Clinical Data Science GmbH, Basel, \\ $\mathrm{CH} ;{ }^{4}$ Santhera Pharmaceuticals, Pratteln, $\mathrm{CH} ;{ }^{5} \mathrm{UCL}$ \\ Great Ormond Street Institute of Child Health, \\ London, GB; ${ }^{6}$ University Hospitals Leuven, \\ Leuven, $B E$
}

Background: In Duchenne muscular dystrophy (DMD), progressive weakness of respiratory muscles leads to life-threatening respiratory complications. In a randomized placebo-controlled, Phase 3 clinical trial (DELOS) in 64 DMD patients aged 1018 years and not taking concomitant glucocorticoids, idebenone $(900 \mathrm{mg} /$ day) reduced the rate of respiratory function decline, as measured by peak expiratory flow (PEF), forced vital capacity (FVC) and forced expiratory volume in $1 \mathrm{sec}$ (FEV1) - as percent predicted $(\%$ p). Additional analyses were undertaken using alternative statistical models to determine the consistency of magnitude of efficacy of idebenone. Methods: We compared the efficacy of idebenone vs placebo on $\mathrm{PEF} \% \mathrm{p}, \mathrm{FVC} \% \mathrm{p}$ and FEV $1 \%$ p in a pre-specified analysis, and also carried out two additional post hoc analyses. Analysis 1 (pre-specified): Change from baseline (BL) to last visit (week 52), was analyzed by mixed model for repeated measures, with treatment group, visit and treatment group by visit interaction as fixed factors, and the BL as a covariate. Analysis 2 (post-hoc): 
Similar to above, but taking into account all post-BL visits (weeks 13, 26, 39 and 52). Analysis 3 (post$h o c$ ): The slope across all visits was analyzed using a random coefficient regression model. Results: Using analyses 1 and 2, the estimated difference between idebenone and placebo for PEF\%p was $6.3 \%$ p $(p=0.031)$ at the last visit. The differences at weeks 13,26 and 39 were $3.9 \%$ p $(p=0.104), 8.3 \%$ p $(p<$ $0.001)$ and $7.6 \% \mathrm{p}(p=0.016)$, respectively, with an overall difference (all post-BL visits combined) of $6.5 \%(p=0.006)$. The estimated difference at last visit for FVC\% $\%$ was $3.3 \%$ p $(p=0.082)$, with week 13, 26 and 39 differences of $3.3 \%$ p $(p=0.021)$, $4.7 \% \mathrm{p}(p<0.002)$ and $5.0 \% \mathrm{p}(p=0.019)$, respectively, and an overall difference of $4.1 \%(\mathrm{p}=0.005)$. For FEV1, the results were $6.4 \%$ at the last visit, with week 13, 26 and 39 differences of $5.2 \%$ p ( $p=$ 0.010,), 9.3 \% \mathrm{p}(p<0.001$,$) and 6.9 \% \mathrm{p}(p=0.017)$, respectively, and an overall difference of $7 \%(p=$ $0.0011)$. Using analysis 3 , the estimated annual slope difference across all visits was $5.8 \% \mathrm{p}(p=$ $0.007)$ for $\mathrm{PEF} \% \mathrm{p}, 4.1 \% \mathrm{p}(p<0.001)$ for $\mathrm{FVC} \% \mathrm{p}$ and $6.9 \%$ p $(p<0.001)$ for FEV1\%p. Conclusion: Based on results from three different analyses for PEF\%p, FVC\%p and FEV1\%p data, idebenone consistently slowed the rate of respiratory function decline compared with placebo in patients with DMD.

\section{PS2Group9-017 / \#423}

Topic: Group 9 - Miscellaneous

\section{MISDIAGNOSIS OF DUCHENNE MUSCULAR DYSTROPHY}

$\underline{\text { Isabella D.L. Kalil }}^{1}$, Julia D.L. Fernandes ${ }^{2}$, Arnaldo J. Godoy ${ }^{2}$

${ }^{1}$ University City of Sao Paulo, Alto de Pinheiros São Paulo, BR $;^{2}$ UNIVERSITY CITY OF SAO

PAULO, Alto de Pinheiros - São Paulo, BR

Background: Becker muscular dystrophy (BMD) was first described 65 years ago as a benign variant of Duchenne muscular Dystrophy (DMD). BMD is clinically more heterogeneous, with a wide spectrum of clinical presentations. About $50 \%$ of the patients become symptomatic by the age of 10 . We report the case of a boy diagnosed as DMD but who was, in fact, a BMD patient. Methods: We asked permission for the mother to report her child condition. The patient was seen this February at our university clin- ic in Sao Paulo City. Results: The patient is 10 years old and is attending the fourth grade of an elementary school in the rodeo city of Barretos, State of Sao Paulo, Brazil. His mother told us he presented speech difficulty a year ago. The boy went to see a child neurologist, who found his calves enlarged and considered the diagnosis of DMD. His mother noticed a "different" way of walking and her son fell frequently during the past few months. He has been taking prednisolone for 7 months. During the first 5-6 months he was on an intermittent regimen. For about a month, he has been taking the medicine every day. In 2017, March, his molecular test showed deletion of the exons 18 to 41 . CK levels were 10,719 in May of 2017 and 4,931 in January of 2018. Echocardiogram was normal this February. Physical examination showed a cushingoid facies. Muscle strength in the upper limbs graded 4, proximally and 5 , distally and in the lower limbs graded 4 globally. Pseudohypertrophy of the calves was detected. He could walk and run for 20 meters without showing tiredness. Gowers sign was positive. It took $5.5 \mathrm{sec}-$ onds for him to stand from the supine position. We told his mother his son was a BMD patient and not DMD and recommended her to give prednisolone intermittently again and come back in 3 months. By that time, if no significant progression of the disease will be detected, we intend to interrupt the use of steroids. Conclusion: This report stresses the difficulty of making a correct diagnosis in case of rare diseases. As BMD is much less frequent compared to $\mathrm{DMD}$, the physician attending our patient considered DMD as the likely diagnosis. Because of that, the child was treated with steroids. He is already presenting significant side effects. Changing his diagnosis to BMD allowed us to reduce the amount of that medicine to evaluate in a safer manner the course of the illness. Although a combination of clinical and molecular genetic evaluations may be sufficient for accurate diagnosis of BMD and DMD, in many cases, the contribution of a histopathological test still remains an important component of evaluation. We are trying to convince the mother to accept us to do a muscle biopsy. In spite of the spread of the knowledge about neuromuscular rare diseases, it is very important to emphasize the subtle differences between similar conditions such as DMD and BMD. Otherwise, patients will be put in risk of mistreatment. 
PS2Group9-018 / \#679

Topic: Group 9 -Miscellaneous

\section{ENSURING HIGH QUALITY MUSCLE BIOPSIES AND MAGNETIC RESONANCE BIOMARKERS IN THE PHASEOUT DMD STUDY}

Jon Tinsley $^{1}$, Yalin Hasoglu ${ }^{2}$, Michael A. Boss ${ }^{3}$, Diane Frank ${ }^{4}$, Crystal Faelan ${ }^{5}$, Krista Vandenborne ${ }^{6}$, Francesco Muntoni ${ }^{7}$

${ }^{1}$ Summit Therapeutics Plc, Abingdon, GB; ${ }^{2}$ Summit

Therapeutics plc, Abingdon, GB; ${ }^{3}$ Summit

Therapeutics plc, Cambridge, MA, US; ${ }^{4}$ Sarepta

Therapeutics Inc, Cambridge, MA, US, ${ }^{5}$ Flagship

Biosciences, Westminster, CO, US; ${ }^{6}$ Department Of

Physical Therapy, University of Florida,

Gainesville, FL, US; ${ }^{7}$ Dubowitz Neuromuscular

Unit, UCL Great Ormond Street Institute of Child

Health, London, GB

Background: Utrophin is a naturally occurring protein that may act as a functional replacement for dystrophin. Increasing the production of utrophin in mature muscle fibres protects and improves outcome in preclinical models, and could modify the course of Duchenne Muscular Dystrophy (DMD). Methods: PhaseOut DMD (NCT02858362) is a Phase 2 open-label study of ezutromid administered to 40 ambulatory patients with DMD. Primary endpoints (week 48) are magnetic resonance spectroscopy (MRS) assessments, with an interim analysis conducted after 24 weeks treatment (recently reported elsewhere). Quantitative magnetic resonance imaging (spin-echo and 8 point Dixon) and localized proton spectroscopy is performed at baseline, 12, 24, 36 and 48 weeks to measure the intramuscular fat fraction (FF) and transverse relaxation time (bulk $\mathrm{T}_{2}$ and water $\mathrm{T}_{2}$ ) in lower extremity muscles. Key secondary endpoints from muscle biopsy include analyses of utrophin protein levels (estimate of target engagement) and developmental myosin heavy chain (MHCd; a biomarker for muscle fibre regeneration). Biopsies were to be collected from the biceps at baseline $(\mathrm{n}=40)$ with a second biopsy taken either after 24 weeks $(n=25)$ or 48 weeks $(n=15)$ of ezutromid dosing. Results: PhaseOut DMD sites have undergone a robust training and certification process to ensure high quality and reproducible quantitative MRI/MRS data acquisition. All images and spectra are acquired by standardized procedures and are centrally reviewed and processed. Quantification of MRS endpoints are performed by an automated blinded process with regular, scheduled data transfers. Quantification of the images is performed by 2 independent MRI experts; a $3^{\text {rd }}$ adjudicating reviewer is used in the event of discordance. All images and spectra, except 1, up to week 24 have yielded data of sufficient quality for the quantification of MRI/MRS biomarkers in the vastus lateralis and soleus. High quality muscle biopsies allow early evaluation of muscle health biomarkers and target engagement. A thorough biopsy process, including training of all sites (surgeons and histotechnicians) has been developed. Biopsy samples from all UK and US sites were shipped to a centralised laboratory for analysis. All biopsy samples taken at baseline and week 24 have been deemed of suitable quality for processing, as confirmed by a pathologist's assessment of the stained cryosection. Utrophin, MHCd, and fibre morphometrics were quantified in 3,000-15,000 fibres/section using analytically validated immunohistochemistry and image analysis. Analyses demonstrated high reproducibility between replicates, with all biopsies yielding data for at least one biomarker. Conclusion: Implementation of robust methodology for the collection of biomarkers in the PhaseOut DMD study has facilitated the evaluation of both target engagement and early indicators of disease progression at the 24 -week interim timepoint.

PS2Group9-019 / \#892

Topic: Group 9 - Miscellaneous

\section{TREAT-NMD: ADVANCING DIAGNOSIS, TREATMENT AND CARE IN NEUROMUSCULAR RARE DISEASES}

Anne Oyewole ${ }^{1}$, Joanne Lee ${ }^{1}$, Rebecca Leary $^{1}$, Katharine Bushby ${ }^{1}$, Annemieke Aartsma-Rus², Janbernd Kirschner ${ }^{3}$, Kevin Flanigan ${ }^{4}$ ${ }^{1}$ The John Walton Muscular Dystrophy Research Centre, Newcastle University, Newcastle, $G B ;{ }^{2} H u m a n$ Genetics, Leiden University Medical Center, Leiden, $N L,{ }^{3}$ Department of Neuropediatrics and Muscle Disorders, Medical Center-University of Freiburg, Freiburg, DE; ${ }^{4}$ Nationwide Children's Hospital, Columbus, US 
Background: The rarity and heterogeneity of neuromuscular disorders contributes to some of the challenges faced both for providing good quality healthcare and for conducting translational research in the neuromuscular field. TREAT-NMD (Translational Research in Europe: Assessment and Treatment of Neuromuscular Disorders) is a global network of world-class experts within the neuromuscular community. Our mission is to accelerate the process of developing new treatments and improve the health and quality of life of people around the world with NMDs, by supporting all stages of translational research. Over the last decade we have identified major barriers in the diagnosis, treatment and care for people with NMD and have developed essential 'go-to' tools and resources to overcome these challenges. Methods: Since 2007, TREATNMD has played a central role in bringing together experts within the NMD community (patients, advocacy organisations, scientists, clinicians and industry) to develop models, projects and resources to meet the challenges for NMD along the translational pathway, from the interpretation of preclinical research to resources for clinical trial readiness. Results: As part of a large scale international effort (CARE NMD project) with the involvement of many experts and patient organisations, TREATNMD has contributed to the development and dissemination of care standards for Duchenne muscular dystrophy (DMD) and spinal muscular atrophy (SMA) and the creation of 'family friendly' guides. The DMD family guide, which is now available in 35 languages, has been downloaded over 17,500 times since 2011 and continues to ensure best practice care is more widespread across the world. TREAT-NMD is now working with clinicians, physiotherapists, nutritionists and patient organisations, to begin work on updating the SMA and DMD family guides, based on the recently published standard of care recommendations for these disorders. Over the last decade the TREAT-NMD network has played a pivotal role in successfully bringing together partners for numerous grant applications. The resources that were started or supported by TREAT-NMD have contributed to more than 20 funded national and international projects, including RD-Connect, OPTIMISTIC, BIO-NMD and most recently IMI2PREFER and the European Reference Network (ERN) on NMD (EURO-NMD). Since 2009, the TREAT-NMD advisory committee for therapeutics (TACT), a unique multi-disciplinary international group of experts, have reviewed over 46 applica- tions for promising drug candidates, providing guidance on the translation and development path of therapeutics programs in NMDs. Two other important tools that have helped to accelerate trial planning and remove potential barriers to getting trials established to test potential therapies, are the TREAT-NMD affiliated Patient Registries and the Care and Trial Sites Registry (CTSR). Conclusion: TREAT-NMD has fostered an environment in which collaborative initiatives have and continue to support translational research efforts. The resources generated by TREAT-NMD have contributed heavily across several areas of activity including care standards, preclinical research and clinical trial readiness. Furthermore, TREAT-NMD Global Patient Registries, the CTSR and TACT experience has been invaluable in the field. A great many lessons have been learned and opportunities for improved care and treatments for people living with NMD are closer than ever before.

\section{PS3Group2-001 / \#336}

Topic: Group 2 - Diseases of Neuromuscular Junction: Clinical Features, Pathophysiology, Therapy

\section{THYMECTOMY LOWERS THE MYASTHENIA GRAVIS BIOMARKER MIR-150-5P}

\author{
Carl Johan Molin ${ }^{1}$, Anna R. Punga ${ }^{2}$, Liis Sabre ${ }^{2}$, \\ Tanel Punga ${ }^{2}$, Cleo-Aron Weis ${ }^{3}$ \\ ${ }^{1}$ Neuroscience, Uppsala University, Uppsala, \\ SE: ${ }^{2}$ Uppsala University, Uppsala, SE:; Pathology, \\ University Medical Centre Mannheim/University of \\ Heidelberg, Mannheim, DE
}

Background: The aim of the study was to analyze the effect of thymectomy on the proposed diseasespecific microRNA (miRNA) biomarkers miR-150$5 p$ and miR-21-5p in Myasthenia Gravis (MG) patients. For this purpose we examined serum samples from the prospective Randomized Trial of Thymectomy in Myasthenia Gravis (MGTX) and evaluated the longitudinal changes in clinical pattern compared to these miRNA levels. Methods: Serum samples were obtained from 80 acetylcholine receptor positive $(\mathrm{AChR}+) \mathrm{MG}$ patients included in the MGTX trial. 38 patients were randomized to thymectomy plus prednisone treatment and 42 patients were randomized to prednisone treatment. Serum 
samples were analyzed for the expression of miR$150-5 p$ and miR-21-5p with qRT-PCR at baseline and 1, 2 and 3 years after randomization. Results: Patients treated with thymectomy had lower levels of miR-150-5p at 1-year post thymectomy, both compared to baseline values and to the prednisone group. No change in miRNA-levels was found in the prednisone group. Levels of miR-21-5p displayed a negative correlation with prednisone dose within the prednisone group.

Conclusion: Thymectomy lowers the levels of the proposed biomarker miR-150-5p, which strengthens its position as a potential disease-specific biomarker for $\mathrm{AChR}+\mathrm{MG}$
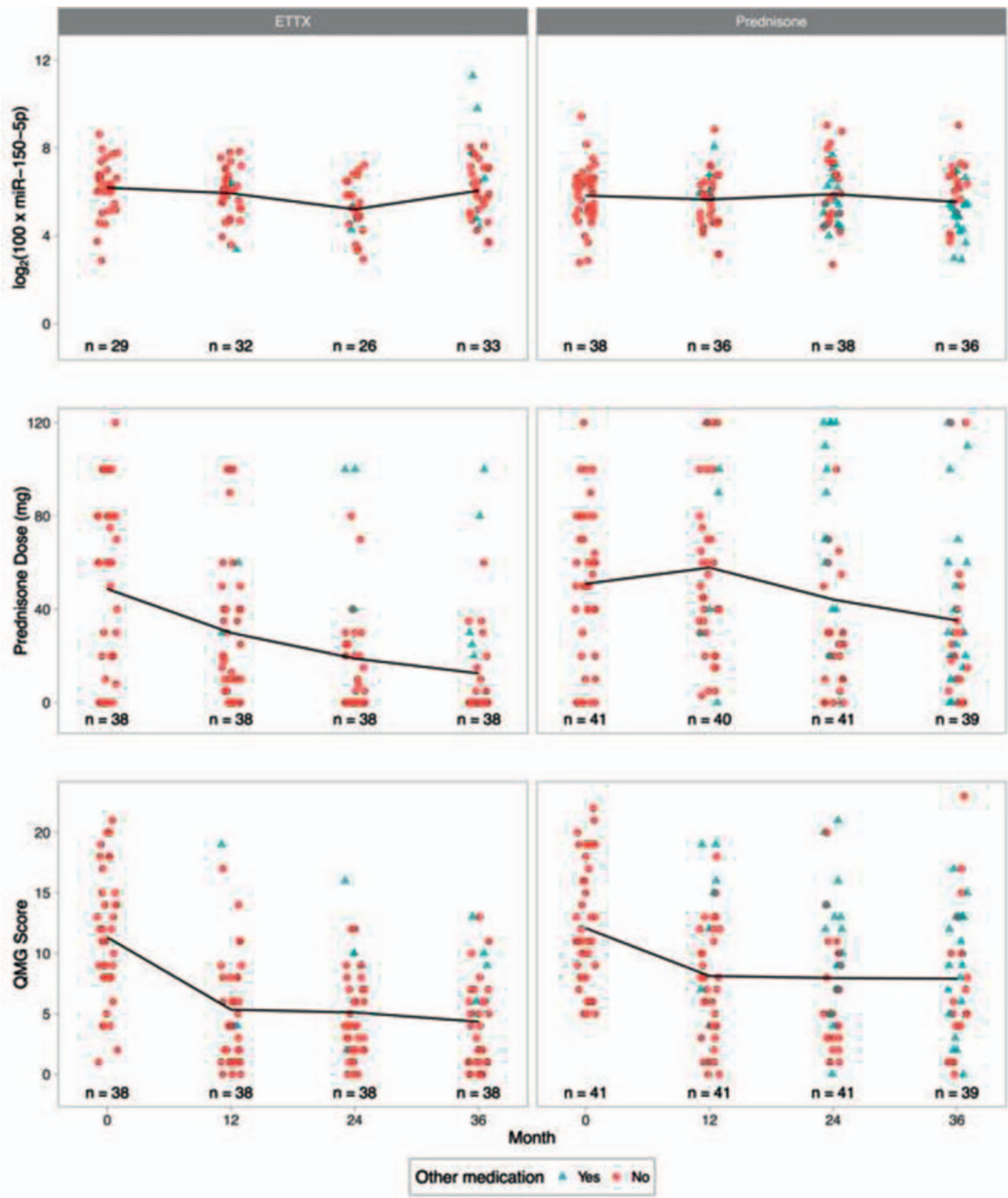

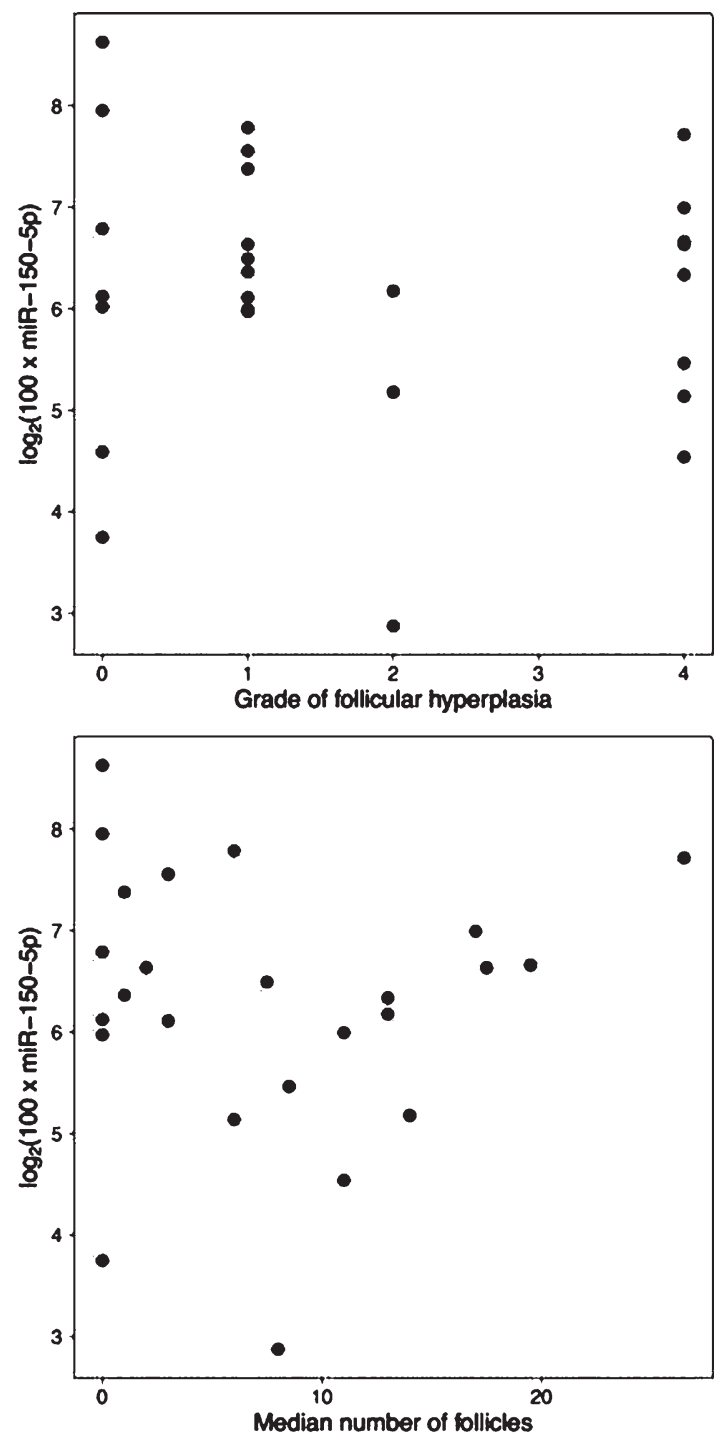

PS3Group2-002 / \#457

Topic: Group 2 - Diseases of Neuromuscular Junction: Clinical Features, Pathophysiology, Therapy

\section{EFFICACY OF SUBCUTANEOUS IMMUNOGLOBULIN IN MYASTHENIA GRAVIS EXACERBAION}

Zaeem A. Siddiqi ${ }^{1}$, Grayson Beecher ${ }^{2}$, Dustin Anderson $^{3}$

${ }^{1}$ Neurology/medicine, University Of Alberta, Edmonton, CA; ${ }^{2}$ Neurology/medicine, University of Alberta, Edmonton, $A B, C A,{ }^{3}$ University of Alberta, Edmonton, $A B, C A$

Background: Intravenous immunoglobulin (IVIg) is an effective therapy for patients with worsening
MG. Recently, the subcutaneous form of immunoglobulin (20\% SCIG; Hizentra) has been used in neuromuscular conditions amenable to IVIg therapy. SCIG appears to be associated with fewer adverse effects, comparable efficacy and the added advantage of flexibility due to self infusion by the patients. The efficacy of SCIG has not been systematically studied in patients with MG. Methods: This is an investigator-initiated, single phase, open label, phase II clinical trial in patients with moderately severe exacerbation in MG (MGFA Grade II and III). Enrolled subjects self-infused 20\% SCIg (Hizentra) at $2 \mathrm{gm} /$ $\mathrm{kg}$ over 3 to 4 weeks in a dose escalating manner dosing regimen. The primary endpoint was the change in quantitative MG (QMG) score from baseline to study end at 6 weeks. Secondary endpoints included change in manual muscle testing (MMT), MG activities of daily living (MG-ADL), and MG composite (MGC) scores. The trial was approved by Health Canada and the local institution ethics board. Results: Of the 29 patients who were screened 23 were enrolled in the trial and 22 (female $=14$; male $=8$; mean age $53.0 \pm 19.6$ years) completed the study. At the end of study period the QMG score decreased from $14.9 \pm 4.1$ to $9.8 \pm 5.6(\mathrm{p}<0.0001)$, MMT score decreased from $16.8 \pm 9.5$ to $5.2 \pm 4.5$ $(\mathrm{p}<0.0001)$, MG-ADL score decreased from 9.56 3.0 to $4.663 .0(\mathrm{p}<0.0001)$, and MGC score decreased from $17.4 \pm 5.0$ to $5.6 \pm 4.5(\mathrm{p}<0.0001)$. Transient worsening of the MG occurred in two patients initially but neither had to be withdrawn. Conclusion: SCIg is effective in the treatment of mild to moderate MG exacerbation. Comparative efficacy must be established with larger randomized controlled trials.

\section{PS3Group2-003 / \#461}

Topic: Group 2 - Diseases of Neuromuscular Junction: Clinical Features, Pathophysiology, Therapy

\section{FEASIBILITY AND}

\section{SAFETY OF SUBCUTANEOUS} IMMUNOGLOBULIN

\section{IN MYASTHENIA GRAVIS} EXACERBATION

Zaeem A. Siddiqi ${ }^{1}$, Grayson Beecher ${ }^{2}$, Dustin Anderson $^{3}$

${ }^{1}$ Neurology/medicine, University Of Alberta, Edmonton, CA, ${ }^{2}$ Neurology/medicine, University of Alberta, Edmonton, $A B, C A,{ }^{3}$ University of Alberta, Edmonton, $A B, C A$ 
Background: The University of Alberta Myasthenia Gravis (MG) program is the primary site of investigator initiated clinical trial to assess the feasibility, safety and efficacy of $20 \%$ SCIG in MG exacerbation. This study describes the results in the safety and feasibility domains, while the efficacy of SCIG is described in a separate study. Methods: A dose of $2 \mathrm{gm} / \mathrm{kg}$ of SCIG was infused in a flexible regimen over 4 weeks and outcome measures assessed at 6 weeks. The safety of infusions was assessed through patient reported adverse effects, infusion site reactions and incidence of hemolysis. Serum Ig levels were serially monitored during the study period. Patient compliance and tolerability were assessed via Treatment Satisfaction Questionnaire for Medication (TSQM; a score of 0 represents not satisfied and 100 represents fully satisfied). Results: Of the 23 patients enrolled 22 patients completed the trial, one discontinued due to MG worsening. Three patients had subsequent worsening and were re-enrolled resulting in a total of 25 SCIG infusions being analyzed. Mean total dose infused was $180.8 \mathrm{gms}$ (range: 104 to $204 \mathrm{gms}$ ), mean volume $904 \mathrm{ml}$ per patient (range 520-1020 ml). All patients were able to infuse the full-prescribed SCIG dose and needed only a single training session. Automated pump infusions resulted in better compliance than manual infusions. There was no incidence of hemolysis or any other serious adverse event. Two patients previously intolerant to IVIg were able infuse the full SCIg dose. Minor infusion related site reactions (mild redness, and itching), a mild flu like syndrome and headache were reported with some infusions. None caused discontinuation of infusions. Serum IgG levels gradually increased by about $200 \%$ at study end. The TSQM showed satisfaction scores in the domains of effectiveness, side effects, and convenience of 70.9 $\pm 19.0,94.0 \pm 13.9$, and 75.0 \pm 19.0 , respectively. Conclusion: This study provides Class IV evidence that despite its large volume SCIG appears to be well tolerated at standard doses for neuromuscular diseases i.e. $2 \mathrm{gm} / \mathrm{kg}$, when infused over four weeks. Infusion site reactions and systemic side effects appear to be mild. SCIG may be better tolerated than IVIg. Majority of patients report high levels of overall satisfaction with the effectiveness, convenience, and side effect profile of SCIG.
PS3Group2-004 / \#578

Topic: Group 2 - Diseases of Neuromuscular Junction: Clinical Features, Pathophysiology, Therapy

\section{A DOUBLE-BLIND PLACEBO- CONTROLLED STUDY TO EVALUATE SAFETY AND EFFICACY OF FCRN ANTAGONIST ARGX-113 IN GENERALIZED MG}

Jan Verschuuren ${ }^{1}$, Vera Bril², Renato Mantegazza ${ }^{3}$, Andrej Szczudlik ${ }^{4}$, Said R. Beydoun ${ }^{5}$, Francisco Rodriguez De Rivera ${ }^{6}$, Maria Rosa Rottoli ${ }^{7}$, Fredrik Piehl $^{8}$, Philip Van Damme ${ }^{9}$, Tuan $\mathrm{Vu}^{10}$, Peter Ulrichts $^{11}$, Katrien Verschuuren ${ }^{12}$, Antonio Guglietta $^{12}$, Hans De Haard ${ }^{12}$, Nicolas Leupin ${ }^{12}$, James F. Howard $\mathrm{Jr}^{13}$

${ }^{1}$ Leiden University Medical Center, Leiden,

$N L ;{ }^{2}$ University of Toronto, Toronto,

CA, ${ }^{3}$ Fondazione IRCCS Istituto Neurologico Carlo Besta, Milan, IT; ${ }^{4}$ Krakowska akademia neurologii, Krakow, PL; ${ }^{5}$ USC Keck School of Medicine, Los Angeles, CA, US: ${ }^{6}$ Hospital Universitario La Paz, Madrid, ES, ${ }^{7}$ Azienda Ospedaliera Papa Giovanni XXIII, bergamo, IT; ${ }^{8}$ Karolinska university, Stockholm, SE, ${ }^{9}$ UZ Leuven, Leuven, BE: $;{ }^{10} U S F$ health, Tampa, FL, US:"1 argenx, Zwijnaarde, $B E ;{ }^{12}$ argenx, Zwijnaarde, BE; ${ }^{13}$ University of North Carolina, Chapel Hill, NC, US

Background: Myasthenia gravis (MG) is an autoimmune disease driven by IgG autoantibodies targeting the neuromuscular junction hence leading to muscle weakness. ARGX-113 (efgartigimod) is an Fc fragment antagonizing the $\mathrm{IgG}$ salvage receptor FcRn thereby accelerating the degradation of IgGs and reducing their serum levels. This effect may be beneficial in IgG-driven autoimmune diseases such as MG. Methods: A phase 2 randomized, doubleblind, placebo-controlled, multicenter study was conducted in 24 patients with generalized MG (gMG). Primary objective of the study was to evaluate the safety and tolerability of ARGX-113 and secondary objectives included an efficacy analysis using four established clinical scales (MG-ADL, QMG, MGC and MG-QoL-15r). Adult anti-AChR+ gMG patients with $\mathrm{MG}-\mathrm{ADL} \geq 5$ were enrolled. $\mathrm{Pa}$ tients were randomized 1:1 to receive four onceweekly infusions of $10 \mathrm{mg} / \mathrm{kg}$ ARGX-113 or placebo and were monitored 8 additional weeks after last treatment. Results: ARGX-113 was well tolerated 
in all patients, with most adverse events characterized as mild and deemed unrelated to the study drug. No serious or severe adverse events were reported. Treatment with ARGX-113 resulted in rapid and strong clinical improvement over placebo lasting for the entire duration of the study as measured by all four efficacy scales (MG-ADL, QMG, MGC and MG-QoL-15r). More specifically, 75\% of patients treated with ARGX-113 had a clinically meaningful improvement in MG-ADL scores ( $\geq 2$-point reduction from baseline) for a period of at least 6 consecutive weeks versus $25 \%$ of placebo patients $(p=$ 0.0391 ). All patients in the treatment arm showed a rapid and deep reduction of their total IgG levels and disease improvement correlated with reduction in pathogenic IgG levels. Conclusion: These results validate ARGX-113 as a potential novel therapeutic option for $\mathrm{gMG}$ patients and warrant further clinical evaluation.

\section{PS3Group2-005 / \#696}

Topic: Group 2 - Diseases of Neuromuscular Junction: Clinical Features, Pathophysiology, Therapy

\section{WHEN IS BETTER GOOD ENOUGH? PATIENT ACCEPTABLE STATES IN MYASTHENIA GRAVIS}

\author{
$\underline{\text { Carolina Barnett }}^{1}$, Meg Mendoza ${ }^{2}$, Hans Katzberg ${ }^{1}$, \\ Vera Bril ${ }^{2}$ \\ ${ }^{1}$ University of Toronto, toronto, $\mathrm{ON}, \mathrm{CA} ;{ }^{2}$ University \\ of Toronto, Toronto, CA
}

Background: Clinical trials are often aimed at detecting the minimally important difference (MID) that is, the smallest change in scores that patients consider significant. While important, the MID does not reflect the overall disease impact. For example, some patients might experience a significant improvement, but still have severe disease. The Patient Acceptable Symptom State (PASS) is an absolute threshold in an outcome measure where patients in general feel well or "well enough". PASS thresholds have not been estimated for Myasthenia Gravis (MG) measures. The main objective of this study was to estimate PASS scores for some commonly used MG measures, including the MGII, MGC, QMGS and MG-ADL. Methods: We analyzed data on 257 patients with MG who participated in the validation of the MGII. As anchor of acceptable state, we used the global health VAS from the EQ$5 \mathrm{D}$, considering $95 \%$ values as cut-points to define PASS. We estimated PASS scores for each measure using the $75^{\text {th }}$ percentile method-the $75 \%$ of the distribution of patients who were PASS positive. We also built receiver operating characteristic curves to find the points of best sensitivity and specificity to classify a patient as PASS positive. Results: The 75th percentile PASS scores for the MGII, MGC, QMGS and MG-ADL were: 19, 4, 7 and 4 points respectively. The ROC method PASS scores were: $11.5,3.5,4.5$ and 2.5 points respectively. Conclusion: These PASS estimates might help inform clinical trials, to determine acceptable outcomes in MG. Further studies are needed using MG-specific anchors to determine PASS and also to understand which factors beyond disease severity- such as disease duration or age - are associated with achieving PASS.

\section{PS3Group2-006 / \#888}

Topic: Group 2 - Diseases of Neuromuscular Junction: Clinical Features, Pathophysiology, Therapy

\section{BIOMARKER DISCOVERY IN CHILDHOOD REFRACTORY OCULAR MYASTHENIA GRAVIS}

Chaiyos Khongkhatithum, Kannikar Srisuwan, Somchai Chutipongtanate, Lunliya Thampratankul, Anannit Visudthibhan

Pediatrics, Faculty of Medicine Ramathibodi Hospital, Bangkok, TH

Background: Childhood ocular myasthenia gravis $(\mathrm{OMG})$ is characterized by selective weakness of ocular muscles. It's response to treatment varies from one the other. The OMG children who were unresponsive to steroid and treated with the other immunosuppressive drug, called refractory $\mathrm{OMG}$. Refractory to therapy was observed from $15-30 \%$ of patients with OMG. Identification of the relevant biomarkers in children with OMG may be useful for determining of the prognosis and for appropriate treatment strategies. This study is aimed to identify the potential biomarkers for prediction the clinical severity and response to treatment in childhood OMG patients. Methods: This cross-sectional study was conducted from March 2016 to February 2017 at Department of Pediatrics, Faculty of Medicine 
Ramathibodi Hospital. Seven children with refractory $\mathrm{OMG}$ and seven aged-matched children with non-refractory OMG were recruited. In addition, four aged-matched children with other autoimmune neurological diseases being treated with the similar immunosuppressive drug to those with refractory OMG, were also included to the control group. Plasmas were collected and these specimens underwent proteomic analysis using two-dimensional gel electrophoresis (2-DE). Protein spots, that showed significant difference, were further analyzed by matrix- assisted laser desorption/ionization time of flight/time of flight (MALDI-TOF/TOF) mass spectrometry. Results: Four protein spots with significantly different intensity among the three groups were identified on 2-DE. All of them had increased expression (3.8, 2, 2.4 and 1.8 folds respectively) in the refractory OMG group compared to the others. Three proteins with their isoform were identified from these 4 spots, including transthyretin, apolipoprotein A-I (Figure 1) and two isoforms of fibrinogen alpha chain (Figure 2). Conclusion: Transthyretin, apolipoprotein A-I and fibrinogen alpha chain were the potential candidate biomarker of

\section{Plot of Normalized Optical Densities of Apolipoprotein \& Transthyretin (mean \pm SEM).}

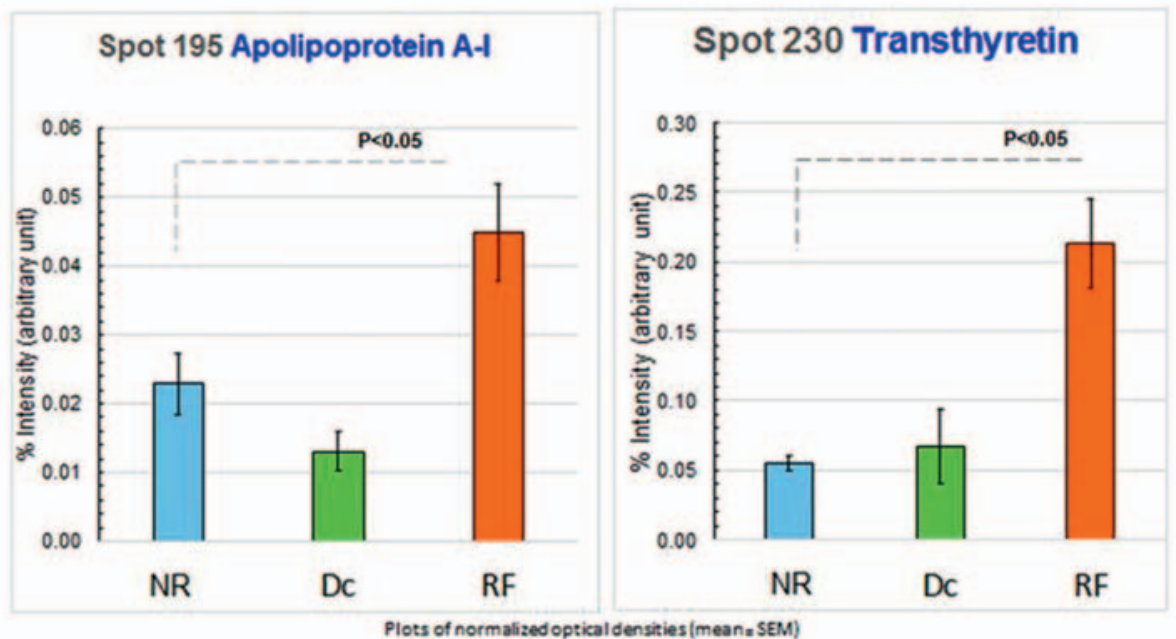

\section{Plot of Normalized Optical Densities of Fibrinogen Alpha Chain (mean \pm SEM).}
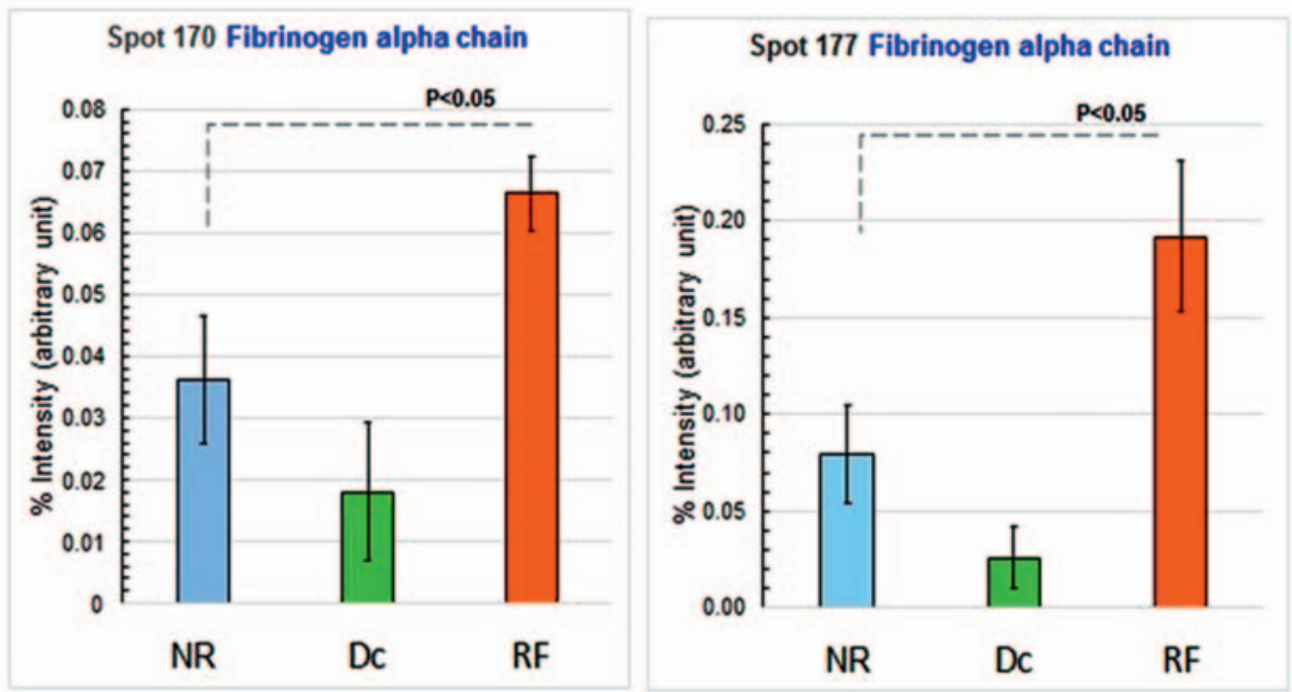

Plots of normalized optical densities (mean= SEM) 
refractory ocular myasthenia gravis in children. The validation of these proteins with immunoassay technique (western blot) and the clinical implications of these proteins will be elucidated by further studies.

\section{PS3Group2-007 / \#863}

Topic: Group 2 - Diseases of Neuromuscular Junction: Clinical Features, Pathophysiology, Therapy

\section{DIFFERENCES BETWEEN THYMOMATOUS AND NON- THYMOMATOUS MYASTHENIA GRAVIS}

Florencia Aguirre $^{1}$, Analisa Manin ${ }^{2}$, Andres Villa $^{1}$

${ }^{1}$ Neurology, Ramos Mejia Hospital, Buenos Aires, AR: ${ }^{2}$ Neurology, Ramos Mejia Hospital, Buenos

Aires City, AR

Background: Myasthenia Gravis (MG) is an autoimmune disease mediated by autoantibodies against post-synaptic components of the neuromuscular junction. The thymus gland is considered to play a rol in the pathogenesis of MG, with evidence of thymic abnormalities in a large proportion of patients. Pathological abnormalities of the thymus are described in about $80 \%$ of MG patients and thymomas are detected in around $15 \%$ of all MG cases. The aim of the study was to investigate epidemiological, clinical and serological characteristics and treatment responses of individuals diagnosed as having thymoma associated $\mathrm{MG}(\mathrm{tMG})$ and compare these parameters with non-thymomatous MG patients (non-tMG). Methods: We conducted a retrospective observational study in a population of patients with diagnosis of MG. Records of MG patients assisted at a public hospital in Buenos Aires City between 2008 and 2018 were reviewed. According chest imaging findings, patients were classified in $\mathrm{tMG}$, those patients with images compatible with thymomas, and non-tMG, those with findings suggestive of thymic hyperplasia and normal thymus. Results: Out of 166 MG patients who were evaluated for thymus involvement with a computarized tomography scan or magnetic resonance imaging scan of the chest, 117 $(70,5 \%)$ had normal thymus, $30(18.05 \%)$ had thymic hyperplasia and $20(12.05 \%)$ were compatible with thymomas. In tMG $75 \%$ were women vs $61.22 \%$ in non-tMG patients. The mean MG age of onset was 47.45 years in $\mathrm{tMG}$, older than patients with non-thymomatous $\mathrm{MG}$ (non-tMG), which mean onset age was 37,63 ( $p=0,031)$. AChR-ab seropositivity was detected in $100 \%$ of tMG. In nontMG 73\% were AChR-ab seropositivity, out of 32 AChR-ab negative patients, 5/22 (22.7\%) were MuSK-ab positive. Related to clinical severity, tMG patients showed more severe disease according to maximal MGFA score achieved, 90\% tMG patients had III, IV or V grade vs $67.35 \%$ in non-tMG ( $\mathrm{p}=$ 0.038). Also, myasthenic crisis were more frequent in tMG patients than non-tMG: $30 \%$ vs $10.88 \%$ respectively $(p=0.018)$. However, no differences were found related to immunossupresion requirements or remission rate between groups. There were no differences in the risk of autoimmune comorbidity between tMG and non-tMG patients. Autoimmune thyroid disease was the most frequent association in both groups. Conclusion: Thymoma-associated myasthenia gravis represented $12.02 \%$ Patients with tMG showed a female predominance and started their disease at an older age than non-tMG. All tMG cases were associated to AChR-antibody. tMG patient had a more severe course according to maximal grade of MGFA achieved. However, no differences were found related to immunossupresion requirements between groups.

\section{PS3Group2-008 / \#861}

Topic: Group 2 - Diseases of Neuromuscular Junction: Clinical Features, Pathophysiology, Therapy

\section{THYMOMA-ASSOCIATED MYASTHENIA GRAVIS IN ARGENTINA $€$}

Florencia Aguirre $^{1}$, Analisa Manin ${ }^{2}$, Villa Andres ${ }^{1}$

${ }^{1}$ Neurology, Ramos Mejia Hospital, Buenos Aires, AR $;{ }^{2}$ Neurology, Ramos Mejia Hospital, Buenos Aires City, AR

Background: Thymoma is a tumor originating from the epithelial cells of the thymus gland. Myasthenia Gravis (MG) is the commonest paraneoplastic disorder associated with this tumor, occurring in around 20 to $65 \%$ of the cases. Thymomas are traditionally considered to have a benign behavior, but it ranges from an indolent growth pattern to highly invasive. The aim of this study was to analyze histological characteristics, tumoral invasiveness, and prognosis of thymomas occurring in patients with diagnosis of 
MG in an argentinian population. Methods: We conducted a retrospective observational study reviewing records of patients with diagnosis of Myasthenia gravis (MG) who underwent thymectomy at the "Ramos Mejia Hospital", a public hospital in Buenos Aires City. Patients with diagnosis of thymoma and MG were analized. All pathological specimens were classified histologically according to the WHO thymoma classification (2004 revision) and pathological staging was based on the Masaoka system. We also, analized the clinical evolution using MGFA Post Interventios Status (MGFA-PIS) score at last visit. Results: Amongst $166 \mathrm{MG}$ patient routinely evaluated for thymus involvement with chest imaging, 20 had findings compatible with thymoma. Of these, fifteen patients accepted to undergo a thymectomy. The mean age at thymectomy was 45.87 years (range 27-66 years) and the mean follow-up duration post-thymectomy was 8.4 years (range 1-27 yeears). Tumor extension evaluated by Masaoka system showed $6 / 15$ in stage I, 7/15 patients in stage II, 2 patients in stage III and 1 patient in stage IV. According to histopathological examination, 6/14 patients had mixed type thymoma (WHO type AB), 5/14 had cortical type thymoma (WHO type B), and 2 patients thymic carcinoma (WHO type C). One patient had thymolipoma. Specimen of one patient was lost. After surgical resection, 5/15(27.8\%) required radiotherapy and $2 / 15(11.1 \%)$ chemotherapy. $1 / 15$ developed late recurrence of thymoma, it occurred 13 years after initial thymectomy. One patient had other extrathymic neoplasm (breast cancer). No patient died during follow-up. Concerning Clinical outcome according to MGFA-PIS score, 6 patients improved, 7 patients were on minimal manifestation stage and 2 patients in pharmacological remission. Interestingly, in $2 / 15$ patient thymoma was detected $>5$ years after the onset of MG with a normal initial thoracic imaging screening, one of them developed an invasive type $\mathrm{C}$ thymoma. Conclusion: Twenty cases of thymoma were identified in an Argentinian population of myasthenic patients. Of these, 15 underwent a thymectomy. Concerning histological subtypes we found a prodminance of mixed type thymomas, and a $13 \%$ of type $\mathrm{C}$, higher than previously reported in other series. We found a favorable clinical outcome with $86.6 \%$ of patients in pharmacological remission or minimal manifestation stage. Two patients developed thymoma many years after myasthenia gravis was diagnosed, highlighting the importance to keep searching this neoplasia in the follow up of myasthenic patients.
PS3Group2-009 / \#211

Topic: Group 2 - Diseases of Neuromuscular Junction: Clinical Features, Pathophysiology, Therapy

\section{EPIDEMIOLOGICAL FEATURE OF MYASTHENIA GRAVIS IN SOUTHERN CHILEAN POPULATION}

\author{
Mario Fuentealba $^{1}$, Manuel Padilla ${ }^{2}$, Fabian \\ Maturana $^{3}$ \\ ${ }^{1}$ Medicine, University of Concepcion, concepcion, \\ CL; ${ }^{2}$ hospital de arauco, concepcion, CL; ${ }^{3}$ university \\ of concepcion, concepcion, $C L$
}

Background: Introduction: Myasthenia gravis $(\mathrm{MG})$ is a infrequent neuromuscular disease of autoimmune origin, with a prevalence of 1.5-17.9 per 100000 around the world. In Chile there is only one epidemiologic study of estimated prevalence using the hospital record of the prescription of pyridostigmine whose clinical use is exclusive in $\mathrm{MG}$, and that through a the statistical technique of capture and recapture is obtaining a estimated prevalence $8.36 \mathrm{x}$ 1000000 (1) The Regional Hospital of Concepcion and Hospital of Arauco are the only centers of the public health system of two southern chilean provinces (Concepcion and Arauco) that dispense pyridostigmine for the patients with MG Methods: Method In Concepcion and Arauco hospitals was obtained the registry of patients who received the prescription of this drug in the 2013-2016 period. Were excluded those under 15 years of age, deceased and those residing in communes not assigned to the corresponding health service. The diagnosis of $\mathrm{MG}$ was confirmed by carrying out a review of the clinical record (capture and recapture). the prevalence was calculated using official data from the Ministry of Health with a with a population coverage older

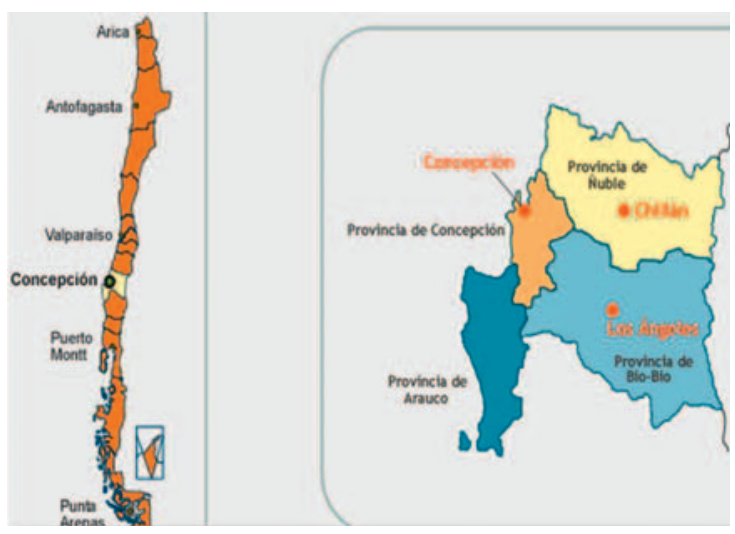


than 15 years of 511718 people in the 2014 latest year available (2). Results: 49 patients were registered who met the inclusion and exclusion criteria. reason women / men of 5.1 (41 women and 8 men) / 1 with average age of 55.23 years. the estimated prevalence was 9.6 per 100000 population Conclusion: The estimated prevalence of $\mathrm{MG}$ in these southern provinces of Chile is comparable to the national series published using the capture and recapture statistical technique (1) Journal of Neuromuscular Diseases 3 (2016) S185 (2) www. fonasa.cl

\section{PS3Group2-010 / \#210}

Topic: Group 2 - Diseases of Neuromuscular Junction: Clinical Features, Pathophysiology, Therapy

\section{MYASTHENIA GRAVIS: THYMECTOMY IN CHILE (MGTC)}

Mario Fuentealba ${ }^{1}$, Roberto Gonzalez ${ }^{2}$, Alejandra Riquelme ${ }^{2}$

${ }^{1}$ Medicine, University of Concepcion, concepcion, $C L ;{ }^{2}$ university of concepcion, concepcion, $C L$

Background: The historical controversy of the efficacy of Thymectomy in myasthenia gravis ( MG ) was resolved favorably with Randomized Trial of Thymectomy in Myasthenia Gravis (MGTX) awhat did he show a benefit of thymectomy in patients with myasthenia gravis with elevated anti nicotinic acetylcholine antibodies (AChR ) with respect to clinical outcomes and requirements and of immunosuppressive drugs. Methods: descriptive analytic study, period 1990-2015, at Regional Clinical Hospital of Concepción: Review of databases, surgical protocols, clinical records and interviews. Description perioperative clinical characteristics, anticholinesterasic treatment, anatomopatological study and immediate and remote results. Follow up to 10 years with the next classifications: Masaoka, Zielinski, DeFilippi and Myasthenia Gravis Foundation of America Post-Intervention State (MGFA-PIS). Application of poll: Myasthenia Gravis Daily Life Activities (MGADL) and Myasthenia Gravis Quality of Life scale 15 (MGQOL 15). Results: 58 patients; 42 $(72,4 \%)$ women, mean age $35,4 \pm 13,9$ years. The most frequent initial symptoms were ptosis, diplopia and dysphagia ( $67 \%, 48 \%, 39 \%$ ),Preoperative classification (Osserman) grades: I 9 (15,5\%), IIA 8
(13,8\%), IIB 40 (69\%). ETT 100\%. Anatomopathological study: thymic hyperplasia $39(67,2 \%)$. Postoperative morbidity $4(6,9 \%)$, without mortality. Follow-up at 1, 3, 5, 8 and 10 years: Masaoka palliation rate $(71,7 \% / 77,5 \% / 67,7 \% / 70,0 \% / 70,6 \%)$, remission rate $(13,0 \% / 15,0 \% / 19,4 \% / 35,0 \% / 35,3 \%)$; Zielinski positive results $(79,6 \% / 87,5 \% / 87,1 \% /$ 90,0\%/ 82,4\%); DeFilippi improved (80,4\%/87,5\%/ $87,1 \% / 90,0 \% / 82,4 \%)$; MGFA-PIS improved (67,4\%/ 77,5\%/77,5\%/ 75,0\%/ 70,6\%).(figure) Application of surveys: Myasthenia Gravis Activities of daily living (MGADL) and Myasthenia Gravis Quality of life scale 15 (MGQOL 15) group average (1,65 / 6,31) respectively. There was a significant decrease in the average dose of oral anticholinesterase (piridostigmine) between the preoperative dose and the follow-up (271mg/201mg/146mg/158mg/124mg/11 $5 \mathrm{mg}$ ). Conclusion: One limitation of the work is not to count antibodies for the selection of patients, Nevertheless our results being comparable with series that used this criterion. One possible explanation is the high frequency of thymic hyperplasia in not thymomatous MG ( $82 \%$ ) since a direct correlation has been reported between the level of AChR and the degree of thymic hyperplasia. however, it is mandatory to do so when accessibility to this diagnostic method is available Conclusion: In clinically selected patients with classical phenotype of MG, ETT has good immediate and long term outcomes

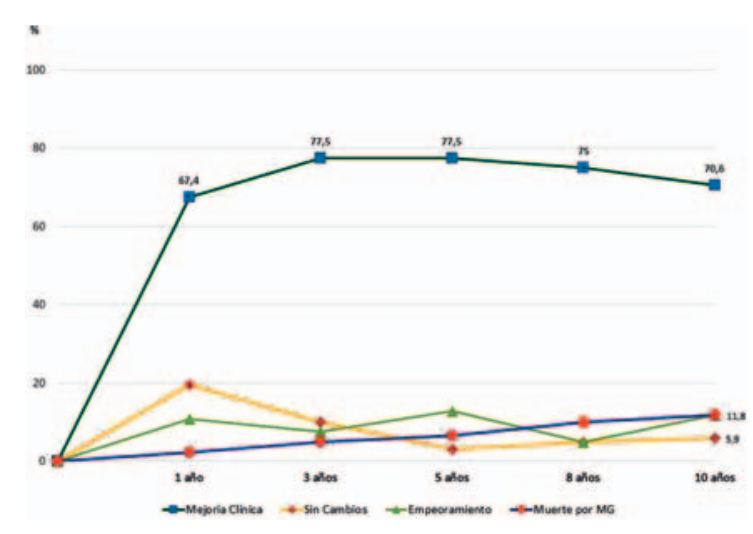




\section{PS3Group2-011 / \#430}

Topic: Group 2 - Diseases of Neuromuscular Junction: Clinical Features, Pathophysiology, Therapy

\section{RESPONSE TO ECULIZUMAB IN PATIENTS WITH ACHR+ REFRACTORY MYASTHENIA GRAVIS RECENTLY TREATED WITH CHRONIC IVIG}

$\underline{\text { Saiju Jacob }}^{1}$, Hiroyuki Murai ${ }^{2}$, Kimiaki

Utsugisawa $^{3}$, Richard J. Nowak ${ }^{4}$, Heinz Wiendl ${ }^{5}$, Kenji P. Fujita ${ }^{6}$, Fanny O’Brien ${ }^{6}$, James F. Howard $\mathrm{Jr}^{7}$

${ }^{1}$ University Hospitals of Birmingham, Birmingham, $G B ;{ }^{2}$ International University of Health and Welfare, Narita, JP; ${ }^{3}$ Hanamaki General Hospital, Hanamaki, JP; ${ }^{4}$ Yale University School of Medicine,, New Haven, CT, US:; University of Münster, Münster, DE: ${ }^{6}$ Alexion Pharmaceuticals, New Haven, CT, US, ${ }^{7}$ University of North Carolina, Chapel Hill, NC, US

Background: Patients with refractory myasthenia gravis (MG) may require chronic intravenous immunoglobulin (IVIg) to treat their disease. The phase 3, randomized, double-blind, placebo-controlled REGAIN study evaluated the efficacy and safety of eculizumab in patients with anti-acetylcholine receptor antibody-positive refractory generalized MG. This subgroup analysis investigated response to eculizumab in patients who received chronic IVIg before entry into REGAIN, compared with placebo. Methods: The use of concomitant IVIg was not permitted during REGAIN other than as rescue therapy, and patients previously treated with IVIg participated in a washout period of 4 weeks prior to randomization. Patients who had previously received chronic IVIg were included if they received IVIg $\geq 4$ times in 1 year, with at least one dose occurring during the 6 months prior to the first dose of REGAIN study drug. Results: Of the 18 patients (eculizumab $n=9$, placebo $n=9$ ) evaluated in this subgroup analysis, four experienced exacerbations during REGAIN; of these, one received eculizumab and three received placebo (Table). Eculizumab-treated patients had numerically larger clinically relevant improvements (mean [SD]: MG activities of daily living score [MG-ADL], -5.3 [4.0]; quantitative $\mathrm{MG}$ score [QMG], -4.1 [6.1]) compared with placebo (mean [SD]: MG-ADL, -2.1 [2.8]; QMG, -1.3 [3.5]) (Table). The majority of patients who received eculizumab (7/9) had clinically meaningful improvements in their $M G$ (by $\geq 3$ points for $\mathrm{MG}-\mathrm{ADL}$ and/or $\geq 5$ points for $\mathrm{QMG}$ ), compared with placebo (3/9). The safety profile of eculizumab was consistent with its known profile. An open-label

\begin{tabular}{|c|c|c|c|c|c|}
\hline \multicolumn{6}{|c|}{ Exacerbations and rescue therapy during REGAIN (eculizumab, $n=9$; placebo, $n=9$ ) } \\
\hline & $\begin{array}{l}\text { Treatment } \\
\text { group }\end{array}$ & $\begin{array}{c}\text { Patients with } \\
\text { exacerbations, } \\
\text { n (\%) }\end{array}$ & $\begin{array}{c}\text { Number of } \\
\text { exacerbations, } \\
n\end{array}$ & $\begin{array}{l}\text { Patients with } \\
\text { rescue } \\
\text { therapy, } \mathrm{n}(\%)\end{array}$ & $\begin{array}{l}\text { Number of } \\
\text { rescue therapy } \\
\text { events, } n\end{array}$ \\
\hline \multirow{2}{*}{$\begin{array}{l}\text { Exacerbations } \\
\text { and rescue } \\
\text { therapy }\end{array}$} & Eculizumab & $1(11)$ & 7 & $1(11)$ & 7 \\
\hline & Placebo & $3(33)$ & 11 & $3(33)$ & $12^{\mathrm{a}}$ \\
\hline \multicolumn{6}{|c|}{ Disease severity scores during REGAIN (eculizumab, $n=9$; placebo, $n=9$ ) } \\
\hline & $\begin{array}{l}\text { Treatment } \\
\text { group }\end{array}$ & $\begin{array}{l}\text { Baseline, } \\
\text { mean (SD) }\end{array}$ & $\begin{array}{l}\text { Week } 26^{b} \text {, mean } \\
\text { (SD) }\end{array}$ & $\begin{array}{l}\text { Change, } \\
\text { mean (SD) }\end{array}$ & $\begin{array}{c}\text { Mean } \\
\text { difference } \\
(95 \% \mathrm{Cl})\end{array}$ \\
\hline \multirow[t]{2}{*}{ MG-ADL } & Eculizumab & $10.9(3.4)$ & $5.6(5.6)$ & $-5.3(4.0)$ & \multirow{2}{*}{$\begin{array}{c}-3.2 \\
(-6.7,0.3)\end{array}$} \\
\hline & Placebo & $9.7(2.4)$ & $7.6(3.3)$ & $-2.1(2.8)$ & \\
\hline \multirow[t]{2}{*}{ QMG } & Eculizumab & $18.1(5.8)$ & $14.0(8.2)$ & $-4.1(6.1)$ & \multirow{2}{*}{$\begin{array}{c}-2.8 \\
(-7.8,2.3)\end{array}$} \\
\hline & Placebo & $18.2(5.5)$ & $16.9(7.2)$ & $-1.3(3.5)$ & \\
\hline \multirow[t]{2}{*}{ MGC } & Eculizumab & $21.7(5.6)$ & $12.0(10.0)$ & $-9.7(5.8)$ & \multirow{2}{*}{$\begin{array}{c}-5.8 \\
(-11.5,-0.1)\end{array}$} \\
\hline & Placebo & $19.1(5.9)$ & $15.2(7.2)$ & $-3.9(5.6)$ & \\
\hline \multirow[t]{2}{*}{ MG-QoL15 } & Eculizumab & $31.4(14.2)$ & $21.6(19.4)$ & $-9.9(15.2)$ & \multirow{2}{*}{$\begin{array}{c}-5.7 \\
(-18.2,6.9)\end{array}$} \\
\hline & Placebo & $32.7(10.2)$ & $28.4(12.3)$ & $-4.2(8.6)$ & \\
\hline
\end{tabular}


extension of REGAIN is ongoing, and interim data (December 2017 cut-off) will also be presented for this subgroup. Conclusion: Eculizumab treatment results in a trend toward meaningful clinical improvements and fewer disease exacerbations, compared with placebo, for patients who previously received chronic IVIg (NCT01997229, NCT02301624).

\section{PS3Group2-012 / \#432}

Topic: Group 2 - Diseases of Neuromuscular Junction: Clinical Features, Pathophysiology, Therapy

\section{ECULIZUMAB REDUCES EXACERBATION RATES IN PATIENTS WITH ACHR+ REFRACTORY GENERALIZED MYASTHENIA GRAVIS}

$\underline{\text { Saiju Jacob }}^{1}$, Jeffrey T. Guptill' ${ }^{2}$, Andreas Meisel ${ }^{3}$, Kenji P. Fujita ${ }^{4}$, Kaushik Patra ${ }^{4}$, James F. Howard $\mathrm{Jr}^{5}$

${ }^{1}$ University Hospitals of Birmingham, Birmingham, $G B ;^{2}$ Duke University School of Medicine, Durham, NC, US, ${ }^{3}$ Charité Universitätsmedizin Berlin, Berlin, DE; $;{ }^{4}$ Alexion Pharmaceuticals, New Haven, CT, US, ${ }^{5}$ University of North Carolina, Chapel Hill, NC, US

Background: Exacerbations are a major burden for patients with anti-acetylcholine receptor antibodypositive $(\mathrm{AChR}+)$ refractory generalized myasthenia gravis (gMG). In $\mathrm{AChR}+\mathrm{gMG}$, complement activation and membrane attack complex (MAC)/ terminal complement complex formation are central to neuromuscular junction pathology and contribute to chronic disease morbidities. Eculizumab uniquely targets complement and specifically inhibits MAC formation. We analyzed the effect of eculizumab on exacerbation rates for patients from the REGAIN study and interim data from the open-label extension (September 2016 cut-off). Methods: REGAIN was a 26-week phase 3, randomized, double-blind, placebo-controlled study assessing the efficacy and safety of eculizumab in patients with $\mathrm{AChR}+$ refractory gMG. Patients who completed REGAIN could enroll in the open-label extension. Patient-years- adjusted exacerbation rates were estimated for the year prior to study entry (REGAIN, $N=125$ ), during REGAIN (placebo, $N=63$ ), and during REGAIN and the open-label study (all eculizumab exposure combined; $N=123$ [56/62 eculizumab and 61/63 placebotreated patients entered the extension]). Comparisons of event rates were based on a Poisson regression model. Exacerbations during the studies were defined as all reported events of clinical worsening/ deterioration, $\mathrm{MG}$ crises or requirement for rescue therapy. Exacerbations/MG crises before REGAIN were as defined in patient records. Results: Exposure to eculizumab resulted in a $56 \%$ relative reduction in exacerbation rates compared with placebo $(p=0.0409)$, and a $66 \%$ relative reduction compared with the year prior to REGAIN entry $(p=0.0008)$ (Figure). The long-term safety profile of eculizumab was consistent with its known profile. REGAIN and updated interim (December 2017 cut-off) open-label study data will be presented. Conclusion: Eculizumab reduced exacerbation rates in patients with refractory $\mathrm{gMG}$, suggesting that elevated complement activation contributes to risk of exacerbation in this patient population (NCT01997229, NCT02301624).

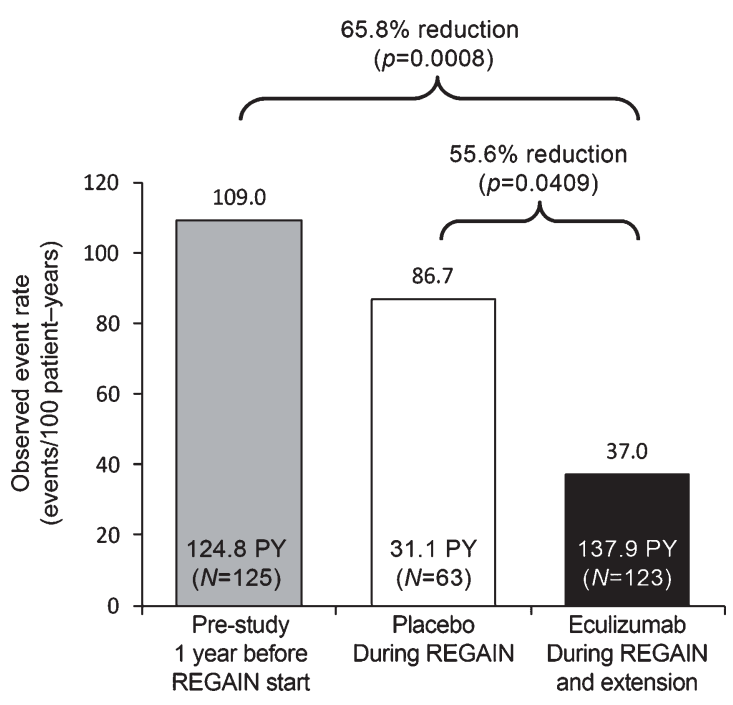




\section{PS3Group2-013 / \#486}

Topic: Group 2 - Diseases of Neuromuscular Junction: Clinical Features, Pathophysiology, Therapy

\section{NONLETHAL CHRNA1-RELATED CONGENITAL MYASTHENIC SYNDROME CAUSED BY NOVEL HETEROZYGOUS MISSENSE MUTATIONS}

\author{
Daniel Natera De Benito ${ }^{1}$, Laura Carrera-García ${ }^{2}$, \\ Anna Lia Frongia ${ }^{3}$, Lidia González Quereda ${ }^{4}$, Pía \\ Gallano Petit ${ }^{4}$, Cristina Jou ${ }^{3}$, Anna Codina ${ }^{3}$, Cecilia \\ Jimenez Mallebrera ${ }^{3}$, Carlos Ortez $z^{3}$, Jaume \\ Colomer $^{3}$, Andres Nascimento ${ }^{3}$ \\ ${ }^{1}$ Neuromuscular Disorders Unit, Hospital Sant \\ Joan de Déu, Barcelona, ES, ${ }^{2}$ Unidad De Patología \\ Neuromuscular., Hospital Sant Joan de Déu, \\ Barcelona, ES: ${ }^{3}$ Hospital Sant Joan de Déu, \\ Barcelona, ES: ${ }^{4}$ Hospital Sant Pau, Barcelona, ES
}

Background: Congenital myasthenic syndromes (CMSs) are a heterogeneous group of genetic disorders affecting neuromuscular junction (NMJ). Postsynaptic NMJ defects encompass up to $75 \%$ of CMS. Defects in the acetylcholine receptor (AChR) can be primary, resulting from a decreased expression of one of the subunits, or alternatively can result from kinetic abnormalities of the ion channel that give rise to slow and fast channel syndromes. Most cases of primary AChR deficiency involve mutations in the CHRNE gene, because the epsilon subunit can be substituted by the foetal gamma subunit, thus rescuing the phenotype. Compound heterozygous or homozygous mutations in the CHRNA1, CHRNB or CHRND gene do not benefit from the gamma subunit rescue and are frequently lethal. We herein present a nonlethal case of a severely affected CMS patient that harbors two missense mutations in CHRNA1. Methods: One patient with genetically confirmed diagnosis of CHRNA1-related CMS followed-up in our hospital is described. Medical record of the patient with demographic information, clinical data, electrophysiological studies, and muscle biopsy findings were compiled. Results: We describe a patient previously misdiagnosed as congenital myopathy. He was born from unaffected non-consanguineous parents out of an uneventful pregnancy with reduced foetal movements. Immediately after birth, the patient required orotracheal intubation due to respiratory insufficiency and was admitted to the neonatal ICU, where he stayed for a year and a half. He was discharged to continue home care with gastrostomy and tracheostomy, requiring BiPAP support. Despite having gradually improved, he remained severely impaired, being able to walk but never having acquired the ability to stand up from floor. Physical exam demonstrated bilateral ptosis with partial ophthalmoplegia, facial hypomimia, and proximal greater than distal weakness in all four limbs. His serum CK level has always remained within the normal range. Muscle biopsy showed variability in fiber size with occasional small and round fibers, a marked predominance of type 1 fibres, and a partial disruption of the intermyofibrillar network. Decremental response following repetitive stimulation at $3 \mathrm{~Hz}$ was not detected. Electromyography revealed myopathic features. Mutation analysis revealed two heterozygous missense variants in the CHRNA1 gene, c.119G $>\mathrm{A}$ (p. Arg40Gln) and c.257G $>$ A (p.Arg86His). None of these changes had been previously reported in LOVD database and they were both absent in a population of 120,000 control chromosomes. Both missense changes were predicted pathogenic or disease causing by in silico prediction tools. Both asymptomatic parents were heterozygous carriers. Based on exon trapping analysis studies, c. $257 \mathrm{G}>\mathrm{A}$ (p.Arg86His) seems to be a critical splicing mutation that causes exclusive inclusion of exon P3A in patient muscle, which is known to produce a non-functional AChR. A significant beneficial response after treatment with pyridostigmine was observed: he was able to stand from floor for the first time in his life after few days of treatment. Conclusion: Recessive CHRNA1 mutations are rare. Our case expands the number of mutations associated with CHRNA1-related CMS. Genetic comprehensive studies may be required to differentiate CMS from congenital myopathies. Reaching a diagnosis is particularly important in CMS given the existence of accessible and effective treatments. 
PS3Group2-014 / \#521

Topic: Group 2 - Diseases of Neuromuscular Junction: Clinical Features, Pathophysiology, Therapy

\section{THE FIRST KOREAN CASE OF COLQ-MUTANT MYASTHENIC SYNDROME}

Sun-Hye Jung, Jin-Hong Shin, Dae-Seong Kim Neurology, Pusan National University Yangsan Hospital, Kyung sang Nam Do, KR

Background: The COLQ gene encodes a collagenlike strand that anchors acetylcholinesterase upon MuSK molecule at the endplate of neuromuscular junction. Homozygous or compound heterozygous mutation in this gene causes reduction in acetylcholinesterase activity, which in turn leads to an overloading of cations at the synaptic space. It is also pathogenically similar to anti-MuSK myasthenia gravis. Many cases could have been missed before the advent of next generation sequencing, as the clinical presentation is often non-specific. Methods: A 34-year-old woman presented with one year of progressive limbs weakness. She has not suffered difficulties in her daily activity before, though she had not been good at sports since her childhood. She first noticed weakness in her both legs when she stands up from squatting. She also felt difficulty raising heavy objects, which she could manage. The weakness progressed in a few months that she could not keep up with the walking pace with her friend. In 6 months, she could not stand up from sitting on a chair, nor walk without aid. Repetitive nerve stimulation test revealed marked decremental response on her trapezius muscle, indicative of postsynaptic neuromuscular transmission defect. Needle electromyography showed low amplitude, short duration MUPs and rapid recruitment in several muscles, while nerve conduction study was unremarkable. Muscle imaging as well as muscle pathology showed mild myopathy. Blood chemistry findings were all within normal limits. Results: Custom panel myopathic genes detected compound heterozygous mutation in COLQ, c.1195+1G $>$ A (rs755782087) and c.1354C $>\mathrm{T}$ (p.R452C, rs368932156), which was reported to be pathogenic in Japanese patients with congenital myasthenic syndrome. She started to take bambutrol $10 \mathrm{mg}$ qd, which significantly improved her limbs weakness. Conclusion: Here we report the first Korean case of congenital myasthenic syndrome by COLQ mutations. Higher level of awareness is required for this ultra-rare disease, as some forms of congenital myasthenic syndromes including COLQ-mutant one can be alleviated by use of beta agonist.

\section{PS3Group2-015 / \#583}

Topic: Group 2 - Diseases of Neuromuscular Junction: Clinical Features, Pathophysiology, Therapy

\section{PREVALENCE AND CHARACTERISTICS OF OBSTRUCTIVE SLEEP APNEA IN KOREAN MYASTHENIA GRAVIS PATIENTS}

$\underline{\text { Jin-Sung Park }}^{1}$, Sung-Jae $\mathrm{Heo}^{2}$, Jin-Mo Park ${ }^{3}$, Yun Jeong Lee ${ }^{4}$

${ }^{1}$ Department Of Neurology, Kyungpook National University Chilgok Hospital, Daegu,

$K R ;{ }^{2}$ Department Of Otorhinolayngology-head \& Neck Surgery, Kyungpook National University, School of Medicine, Daegu, KR $;{ }^{3}$ Department Of Neurology, Dongguk University Gyeongju hospital, Gyeongju, KR: ${ }^{4}$ Pediatrics, Kyungpook national university children's hospital, Daegu, KR

Background: Myasthenia gravis (MG) is an autoimmune disease caused by antibodies that bind against nicotinic acetylcholine receptor of the postsynaptic membrane. It is characterized by progressive and diurnal muscle weakness and sometimes with respiratory difficulty. There a only few studies on obstructive sleep apnea (OSA) as one of the clinical symptom in MG which is caused by weakness of the oropharyngeal muscles, diaphragm and muscles that are involved in moving the chest wall. The prevalence of OSA between the age of 40 and 65 years was $3-7 \%$ in male and $2-5 \%$ in females in western countries. The prevalence of OSA in MG needs to be elucidated but according to a few studies, approximately $20-60 \%$ of the MG patients had OSA. The aim of our study was to find the prevalence of OSA in stable Korean MG patients and analyze the characteristics of OSA in these patients. Methods: 18 MG patients were enrolled (9 males and 9 females). All the patients consisted of MGCS 0 or 1 and all the patients had no change in their medication for at least 6 months. All the patients gave their informed consent to access their clinical 
data and perform an overnight PSG. The patients also underwent Korean Epworth sleepiness scale (ESS). The clinical data constituted of body mass index (BMI), disease follow up duration, post synaptic receptor antibody status, thymectomy status, pulmonary function test, and current medication usage. Results: The mean age of the enrolled MG patients was $49.6 \pm 11.1$ with a mean $6.7 \pm 5.3$ years of follow up. The mean body mass index was $23.7 \pm 3.4$ and all the patients showed MGFA score of less than 1. 14 patients were acetylcholine receptor antibody positive, $1 \mathrm{MuSK}$ antibody positive and 3 double seronegative patients. Only 5 patients were on low dose steroids $(<20 \mathrm{mg})$ and 8 patients $(44.4)$ underwent thymectomy. The Korean ESS showed a prevalence of $27.8 \%$ ( 5 out of 18 ). The polysomnography performed in all stable MG patients showed an OSA prevalence of 7 out of 18 patients (38.9\%) (AHI > 5 ), and of these patients 2 patients were mild, 3 patients were moderate and 2 patients were severe. $\mathrm{AHI}$ in $\mathrm{MG}$ patients correlated with disease duration $(\mathrm{r}=0.979, \mathrm{p}=0.007)$ and $\mathrm{BMI}(\mathrm{r}=0.728, \mathrm{p}=0.001)$, but it showed no correlation to acetylcholine receptor antibody level, thymectomy status, pulmonary function or steroid dosage. Conclusion: In conclusion, prevalence of OSA in stable Korean MG patients was $38.9 \%$ which was higher than the normal population. Sleep apnea showed statistically significant correlation to disease duration and BMI. Further well designed longitudinal studies are warranted to understand the pathophysiology of OSA in neuromuscular disease which is one of the under recognized clinical symptom in MG.

\section{PS3Group2-016 / \#1002}

Topic: Group 2 - Diseases of Neuromuscular Junction: Clinical Features, Pathophysiology, Therapy

\section{PHARMACOLOGICAL TREATMENT ADHERENCE IN PATIENTS WITH MYASTHENIA GRAVIS: ASSOCIATED FACTORS AND CLINICAL CONSEQUENCES}

Bruno K. Vitturi, Ada Pellegrinelli, Berenice C.O. Valério Neurology, Faculdade de Ciências Médicas da Santa Casa de São Paulo, São Paulo, BR
Background: Several recent studies are devoted to therapeutic advances of Myasthenia Gravis (MG). However, therapeutic success is impossible without a good therapeutic adherence. Medication adherence is a public health problem and this has not been previously studied in MG patients. Methods: Consecutive patients from our MG Clinics were included in a cross-sectional study. Medication adherence was assessed with the Morisky Medication Adherence Scale (MMAS) and patients were classified in "adherent" or "non-adherent". Participants were assessed for myasthenia gravis severity with the MG Composite Scale (MGC) and myasthenia gravis-related quality of life with the MG-QOL15. Patients were screened for depression with the Hospital Anxiety and Depression Scale (HADS). Univariate and multivariate statistical analysis were employed to compare clinical characteristics of the two groups of patients and regression models were used to assess predictors of adherence. Results: We enrolled 58 patients and $42(72.4 \%)$ were women. The mean age was $46.6( \pm 17.0)$ years. Only $26(44.8 \%)$ of patients were adherent to treatment. Patients who were not adherent to medication had more weakness $(\mathrm{p}<0.05)$ and had a worse quality of life $(p<0.05)$. Determinants of low adherence to treatment were: low educational level, multiple comorbidities and higher medication doses $(p<0.05)$. Duration of the disease and MGC scale were not statistically associated with adherence to MG medication. Adverse effects of medications were the cause of poor adherence only in $4(15.4 \%)$ patients. Higher likelihood of adherence was associated with female sex (OR 1.20; 95\% CI: $1.03-1.37 ; p<0.05)$ and age groups older than 38 years (ORs 1.220-1.331; $p<0.01$ ). Depression was associated with a lower likelihood of adherence (OR 0.66; 95\% CI: $0.2-0.78 ; \mathrm{p}<0.0001)$ as well. Conclusion: Medication adherence should be assessed routinely in patients with myasthenia gravis. Our study may help healthcare professionals identify patients at greatest risk of poor adherence. 


\section{PS3Group2-017 / \#1000}

Topic: Group 2 - Diseases of Neuromuscular Junction: Clinical Features, Pathophysiology, Therapy

\section{SOCIAL CONSEQUENCES AND QUALITY OF LIFE OF PATIENTS WITH MYASTHENIA GRAVIS}

\author{
Bruno K. Vitturi, Ada Pellegrinelli, Berenice C.O. \\ Valério \\ Neurology, Faculdade de Ciências Médicas da \\ Santa Casa de São Paulo, São Paulo, BR
}

Background: Several papers are devoted to the pathophysiology and treatment of Myasthenia Gravis (MG). However, very few studies have taken into consideration the social and professional consequences of MG as well as its effects on patient's quality of life and on their personal lives. Methods: Consecutive patients with MG with at least 1-year of clinical follow-up were included in a cross-sectional study. Baseline clinical characteristics were recorded, a social questionnaire was applied to all patients and the social support was assessed using Medical Outcome Study Social Support Survey (MOS-SSS). Clinical severity at the worst condition was graded according to the MG Foundation of America classification, and that at the current condition was determined according to the quantitative MG composite (MGC). Quality of life was evaluated with the 15item Myasthenia Gravis Quality of Life Scale (15-MGQoL). Statistical analysis of these variables included t-tests, Chi-square tests and linear and multivariable regression. Results: We included 54 patients in our study. The patients' mean age was 46.6 years and the mean disease duration was 10.8 years. About $52.5 \%$ of patients had experienced unemployment, $4.0 \%$ had been unwillingly transferred, 18\% had retired and $78.0 \%$ had experienced a decrease in income, $38.2 \%$ of whom reported that the decrease was $\geq 50 \%$ of their previous total income. In addition, $76.0 \%$ of the patients reported feeling reduced social positivity. Factors promoting social disadvantages were advanced age, severity of illness, dose of prednisone, low educational level and marital status $(p<0.05)$. Time of disease onset and gender didn't predict social outcomes. However, severe forms of the disease and a worse performance in MGC were correlated with a worse quality of life and increased social disadvantages $(\mathrm{p}<0.05)$. Furthermore, quality of life was directly related to social support $(\mathrm{p}<0.01)$.
Conclusion: Patients with MG are often evaluated according to the presence and the severity of neurological symptoms. However, this study shows that the disease may be accompanied by numerous social problems and by a significant reduction of quality of life. The investigation of these aspects is indispensable for an integral approach of patients with MG.

\section{PS3Group2-018 / \#682}

Topic: Group 2 - Diseases of Neuromuscular Junction: Clinical Features, Pathophysiology, Therapy

\section{HIGH EFFICACY AND SAFETY OF RITUXIMAB FOR MYASTHENIA GRAVIS: A NATIONWIDE STUDY BY AUSTRIAN ADULT NEUROLOGISTS}

$\underline{\text { Raffi Topakian }}^{1}$, Friedrich Zimprich ${ }^{2}$, Stephan Iglseder $^{3}$, Norbert Embacher ${ }^{4}$, Michael Guger ${ }^{5}$, Karl Stieglbauer ${ }^{6}$, Dieter Langenscheidt ${ }^{7}$, Jakob Rath ${ }^{8}$, Stefan Quasthoff ${ }^{9}$, Philipp Simschitz ${ }^{10}$, Julia Wanschitz $^{11}$, Petra Müller ${ }^{1}$, Dierk Oel ${ }^{1}$, Stefan Einsiedler ${ }^{1}$, Günther Schustereder ${ }^{1}$, David Windisch $^{12}$, Wolfagang Löscher ${ }^{11}$ ${ }^{1}$ Neurology, Klinikum Wels-Grieskirchen, Wels, AT; ${ }^{2}$ Department Of Neurology, Medical University of Vienna, Vienna, AT; ${ }^{3}$ Neurology, Hospital St. John of God, Linz, AT; ${ }^{4}$ University Clinic St. Pölten, St. Pölten, AT; Department Of Neurology Ii, Kepler University Hospital, Linz, AT; ${ }^{6}$ Consultant neurologist in private practice, Linz,

AT; ${ }^{7}$ Landeskrankenhaus Rankweil, Rankweil, AT; ${ }^{8}$ Department Of Neurology, Medical University of Vienna, Vienna, AT; ${ }^{9}$ Medical University Graz, Graz, AT; ${ }^{10}$ Klinikum Klagenfurt, Klagenfurt, $A T ;{ }^{11}$ Medical University of Innsbruck, Innsbruck, AT:12Landeskrankenhaus Bruck, Bruck, AT

Background: Most patients with myasthenia gravis (MG) do need long-term immunosuppressive therapy. However, conventional steroid-sparing agents may have intolerable side effects, fail, or take too long to achieve sufficient control of disease activity. Rituximab (RTX), a monoclonal antibody that rapidly depletes B cells, has emerged as an off-label treatment option. The aim of this nationwide retrospective study was to assess the use, efficacy and safety of RTX for MG. Methods: All Austrian neurologists, neuromuscular specialists and departments of neurology were contacted via the Austrian 
Neurological Society to provide anonymized data of all adult MG patients treated with RTX with a minimum follow-up of 3 months. The Myasthenia Gravis Foundation of America Clinical Classification and Postintervention Status scales were used to assess disease severity and outcomes, respectively. Results: A total of 56 patients were reported. 34 $(60.7 \%)$ patients were women. Median age at diagnosis of MG and at start of RTX were 41.5 (24.3; $65.8)$ years and $47.5(33.0 ; 71.0)$ years, respectively. Median length of follow-up after start of RTX was $20(10 ; 53.5)$ months. 39 (69.6\%) patients had antiacetylcholine receptor antibodies (AchR ab+), 14 (25\%) patients had anti-muscle-specific tyrosine kinase antibodies (MuSK ab+), and 3 patients were seronegative. Eight (14.3\%) patients had thymomaassociated MG. Protocols for RTX induction and maintenance therapy varied widely across centers. Before the start of RTX, 47 (83.9\%) patients had already received high cost therapies (plasma exchange, immune adsorption or immunoglobulins), and 33 (58.9\%) patients had "severe MG" (grade 3b or worse). Three months after RTX, 14 of 53 (26.4\%) patients had already achieved pharmacological remission. At last follow-up, 24 of 56 (42.9\%) patients had pharmacological or complete stable remission, and another $14(25 \%)$ patients had minimal manifestations. At last follow-up, remission was achieved in $71.4 \%$ of MuSK ab+ patients and $35.9 \%$ of AchR $\mathrm{ab}+$ patients, respectively. In 33 of the $39(84.6 \%)$ patients still taking steroids before RTX, steroids could be stopped altogether or the dose tapered to a dose $50 \%$ less than the initial dose. RTX was well tolerated and safe. One patient with highly refractory AchR ab+ MG died 4.5 months after RTX from a cardiac cause. Multivariate logistic regression analysis identified presence of MuSK ab as the only baseline variable independently predicting remission at last follow-up. Conclusion: In this retrospective study on RTX for MG, the largest to date on the topic, RTX was found to be safe, efficacious and relatively fast-acting. Benefit from RTX was greatest in MuSK ab+ MG.
PS3Group2-019 / \#876

Topic: Group 2 - Diseases of Neuromuscular Junction: Clinical Features, Pathophysiology, Therapy

\section{DIFFERENTIAL RESPONSE TO RITUXIMAB IN ACHR AND MUSK ANTIBODY POSITIVE MYASTHENIA GRAVIS: A SINGLE-CENTER RETROSPECTIVE STUDY}

Tess D. Litchman ${ }^{1}$, Bhaskar Roy ${ }^{1}$, Valentine Njike², Aditya Kumar ${ }^{1}$, Aditi Sharma ${ }^{3}$, Richard J. Nowak ${ }^{1}$

${ }^{1}$ Neurology, Yale University School of Medicine, New Haven, CT, US, ${ }^{2}$ Yale-Griffin Prevention Research Center, Derby, US: ${ }^{3}$ Neurology, Yale University, New Haven, CT, US

Background: Response to immunomodulatory therapies in myasthenia gravis (MG) can be variable. A subset of MG patients remains refractory to conventional agents. B-cell targeted therapy with rituximab has shown durable response in treating refractory MG. This study compares the response to rituximab therapy between acetylcholine receptor autoantibody positive $(\mathrm{AChR}+)$ and muscle-specific kinase autoantibody positive (MuSK+) MG. Methods: This retrospective study included patients with either $\mathrm{AChR}+$ or $\mathrm{MuSK}+\mathrm{MG}$, who were treated with rituximab between $05 / 2003$ to $05 / 2017$. Results: Thirty-three patients were identified (17 $\mathrm{AChR}+, 16 \mathrm{MuSK}+$; mean age $35.9 \pm 15.6$ years; 24 women and 9 men) with a mean follow-up of $1861 \pm$ 953.4 days. The mean baseline MGFA Clinical Class was $1.76 \pm 0.75$ for the entire group, which improved to $0.61 \pm 0.88$ at 12 -months and to $0.63 \pm 0.79$ at last follow-up (p-values <0.001) after rituximab treatment. The mean MFGA Clinical Class for AChR+ patients improved from a baseline of $1.7 \pm 0.77$ to $0.56 \pm 0.81$ at 12 -months (p-value 0.005 ) and $0.63 \pm$ 0.8 at last visit ( $\mathrm{p}$-value 0.008 ) after rituximab treatment. Similarly, MuSK + patients improved from a mean baseline class of $1.81 \pm 0.75$ to $0.66 \pm 0.98$ at 12 -months (p-value 0.004 ) and $0.63 \pm 0.8$ at last follow-up visit (p-value 0.003). Symptom-free state was attained at 12 -months in $62.5 \%$ and $66.7 \%$ of $\mathrm{AChR}+$ and MuSK+ patients, respectively. Twentyone patients achieved clinical remission (12 AChR+, $9 \mathrm{MuSK}+$ ) with time to remission of $441.4 \pm 336.6$ days for AChR+ versus $230 \pm 180.8$ days for MuSK+ patients ( $p$-value 0.049). The mean prednisone dose requirement decreased in both groups following 
rituximab treatment $(\mathrm{AChR}+$ : baseline $46.8 \pm 20.8$ $\mathrm{mg}$ /day, 12-months $11.8 \pm 15.3 \mathrm{mg} /$ day, last followup 17.9 \pm 15.6 ; MuSK+: baseline $30.9 \pm 24.9 \mathrm{mg}$ / day, 12-months $9.5 \pm 8.7 \mathrm{mg} /$ day, last follow-up 2.8 $\pm 3.8 \mathrm{mg}$ /day), however, there was no statistically significant difference between the two groups ( $\mathrm{p}$ values between the groups at baseline 0.057 , at 12-months 1 , and at last follow-up 0.75). The relapse rate was $58.8 \%$ and $37.5 \%$ for $\mathrm{AChR}+$ and MuSK+ patients, respectively. $\mathrm{AChR}+$ patients required more frequent hospitalization for exacerbation even after rituximab therapy (p-value 0.046). The number of maintenance IVIg or PLEX treatments were reduced in MuSK + patients post-rituximab (p-value 0.0153 and 0.0033 , respectively), but not in $\mathrm{AChR}+$ patients. Similarly, MuSK + patients required rescue therapy less frequently following rituximab ( $p$-value 0.029 ). The number of post-rituximab IVIg rescue therapy cycles in the MuSK + patients was lower than in the AChR+ group (p-value 0.0198). Conclusion: Rituximab therapy is observed to have both a clinical benefit and durable response in the majority of $\mathrm{AChR}+$ and MuSK $+\mathrm{MG}$ patients with refractory disease, however, there is no significant difference between these groups in terms of MGFA Clinical Class improvement, symptom-free state and prednisone burden. Overall, MuSK + patients may experience greater benefits, including earlier time to remission, lower relapse rate, fewer hospitalizations, and require less IVIg/PLEX therapy post-treatment.

\section{PS3Group2-020 / \#915}

Topic: Group 2 - Diseases of Neuromuscular Junction: Clinical Features, Pathophysiology, Therapy

\section{OUTCOMES IN GENERALIZED MYASTHENIA GRAVIS PATIENTS IN THE NEW MILLENIUM: DATA FROM A SINGLE-CENTER RETROSPECTIVE STUDY}

Matthias Tomschik, Jakob Rath, Gudrun Zulehner, Hakan Cetin, Eva Hilger, Anna Paul, Friedrich Zimprich

Department Of Neurology, Medical University of Vienna, Vienna, AT

Background: Myasthenia gravis (MG) is an autoimmune disorder that when generalized can lead to life threatening complications and often requires immunosuppression. Based on clinical characteristics, serologic findings and thymus pathologies, patients are divided into subgroups with differing disease characteristics, responses to treatments, and prognoses. The $21^{\text {st }}$ century has seen important discoveries in the pathophysiology of myasthenia and new immunosuppressive agents have been available for treatment. In light of these advances, we aim to provide an update on the disease course of generalized myasthenia gravis with data from the new millennium. Methods: We retrospectively analyzed electronic patient records of all MG patients diagnosed and treated after Jan. $1^{\text {st }} 2000$ until 2016 at our neurology department. Symptom severity and pharmacologic therapies were recorded at yearly follow up visits for all patients who presented with generalized myasthenic symptoms within the first year after disease onset. Additionally, we collected data on baseline characteristics, antibody status, occurrence of life threatening events, date of thymectomy, and thymus histology whenever applicable. Cases were organized into five distinct subgroups: early onset AChR antibody positive, late onset AChR antibody positive, MuSK antibody positive, double-seronegative, and thymoma associated. A dichotomized outcome variable "sustained remission" was defined as freedom from symptoms for more than two consecutive years and until last follow-up. Differences in distribution between groups were analyzed for statistical significance by Fisher's exact test. Results: We identified 153 patients matching our inclusion criteria, who were followed for one year or longer. Follow up visits at three years were recorded for 103 patients, five years for 80 and 10 years for 38 patients. Elevated AChR antibody levels were found in 129 patients $(84 \%), 52$ of which had an onset before fifty years of age and 77 thereafter. MuSK antibodies were found in seven patients $(5 \%)$ and 17 patients were double seronegative (11\%). Seventy-three patients $(47 \%)$ underwent thymectomy and thymoma was confirmed histologically in 19 patients $(12 \%)$. Twenty-four myasthenia-related life threatening events occurred in 21 patients. Sixteen of these events occurred in late onset AChR antibody positive patients. Symptom severity, treatment regimens, and intensity of immunosuppression differed significantly between subgroups over the years. MuSK antibody positive patients in this cohort were treated aggressively, including use of monoclonal antibodies, but in turn had milder disease severity and no life threatening events. Thirty of 103 patients followed for three years or longer achieved sustained 
remission. Patients with sustained remission achieved freedom from symptoms early and needed less immunosuppression, even though $40 \%$ required intensive immunosuppression in their first year. No patients with thymoma achieved sustained remission regardless of therapeutic regiment. Conclusion: We provide an update on the outcome of generalized myasthenia gravis patients and their prognostic factors from a single-center retrospective study. Use of modern immunosuppressive agents led to low morbidity in younger seropositive patients. Seronegative patients appeared more treatment resistant while late onset AChR antibody positive patients were at the highest risk for life threatening complications. Thymoma associated MG remains a therapeutic challenge and highlights the need for further expansion of treatment options.

\section{PS3Group2-021 / \#967}

Topic: Group 2 - Diseases of Neuromuscular Junction: Clinical Features, Pathophysiology, Therapy

\section{B CELL TARGETED TREATMENT IN MYASTHENIA GRAVIS (BEATMG) - A PHASE 2 TRIAL OF RITUXIMAB IN MG: TOPLINE RESULTS}

$\underline{\text { Richard J. Nowak }}{ }^{1}$, Christopher Coffey ${ }^{2}$, Jonathan M. Goldstein ${ }^{3}$, Mazen Dimachkie ${ }^{4}$, Michael Benatar $^{5}$, Safawa Huq ${ }^{6}$, Brenda Pearson 7 , Jon W. Yankey $^{2}$, Liz Uribe ${ }^{2}$, Laura Herbelin ${ }^{8}$, Ted M. Burns $^{9}$, Kevin C. O’Connor ${ }^{1}$, Robin Conwit ${ }^{10}$, John T. Kissel ${ }^{11}$, Gil I. Wolfe ${ }^{12}$, David A. Hafler ${ }^{1}$, Merit E. Cudkowicz ${ }^{13}$, Richard J. Barohn ${ }^{14}$ ${ }^{1}$ Neurology, Yale University School of Medicine, New Haven, CT, US $;{ }^{2}$ Clinical Trials Statistical Data Management Center, University of Iowa, Iowa City, US: ${ }^{3}$ Neurology, Hospital for Special Surgery, New York City, US; ${ }^{4}$ Neurology, The University of Kansas Medical Center, Kansas City, KS, US: ${ }^{5}$ Neurology, University of Miami, Miami, US; ${ }^{6}$ Massachusetts General Hospital, Boston, US: ${ }^{7}$ University of Iowa, Iowa City, US: ${ }^{8}$ Neurology, University of Kansas, Kansas City, KS, US ${ }^{9}$ Department Of Neurology, University of Virginia, Charlottesville, US: ${ }^{10}$ Neurology, National Institute of Neurological Disorders and Stroke, Bethesda, US, ${ }^{11}$ Neurology, Ohio State University, Columbus, US: ${ }^{12}$ Department Of Neurology, University of Buffalo, Buffalo, US, ${ }^{13}$ Neurology,
Massachusetts General Hospital, Boston, US: ${ }^{14}$ Neurology, University of Kansas Medical Center, Kansas City, KS, US

Background: Despite available therapies, some myasthenia gravis (MG) patients continue to have uncontrolled disease or intolerable adverse effects. Autoreactive B cells appear to play an important role in the immunopathogenesis of MG and represent a rational target for therapeutic development. Additionally, encouraging preliminary findings with rituximab strengthened rationale for this trial. The primary objective of the BeatMG study is to determine whether rituximab is a safe and beneficial therapeutic for MG that warrants further study in an efficacy trial. Methods: We conducted a randomized, double-blind, placebo-controlled, multicenter phase 2 clinical trial utilizing a futility analysis for the primary outcome. Participants 21-90 years of age, with confirmed acetylcholine receptor (AChR) antibody positive generalized MG (Myasthenia Gravis Foundation of America Class II-IV) were eligible for enrollment if they were taking $\geq 15 \mathrm{mg}$ of prednisone per day. Participants were randomized 1:1 to receive a two-cycle rituximab regimen $\left(375 \mathrm{mg} / \mathrm{m}^{2}\right.$ IV weekly x 4 weeks, repeated at Week 24) versus matching placebo; randomization was stratified based on baseline immunotherapy regimen. Study duration was 52 weeks, and enrolled a total of 52 participants. A forced steroid taper, beginning at Week 8 , was guided by clinical stability or improvement. The primary outcome was a measure of steroid sparing effect, defined as the proportion of participants achieving a $\geq 75 \%$ reduction in mean daily prednisone dose in the 4 weeks prior to week 52 and with clinical improvement or no significant worsening. Safety evaluations included adverse events, vital signs, and laboratory assessments. Secondary outcomes included MG-specific clinical assessments: MG Composite (MGC) and Quantitative MG (QMG) scores, among others. Results: Primary and secondary outcome results from the study will be available for presentation and discussion. Conclusion: We will discuss the role of rituximab in the treatment of patients with AChR antibody positive disease based on the findings of this study. 


\section{PS3Group2-022 / \#295}

Topic: Group 2 - Diseases of Neuromuscular Junction: Clinical Features, Pathophysiology, Therapy

\section{CONGENITAL MYASTHENIC SYNDROME: AN INDIAN SCENARIO}

Sunitha Balaraju ${ }^{1}$, Veeramani Preethish Kumar², Ana Töpf ${ }^{1}$, Seena Vengalil ${ }^{2}$, Kiran Polavarapu ${ }^{2}$, Saraswati Nashi ${ }^{2}$, Andreas Roos ${ }^{1}$, Rita Horvath ${ }^{1}$, Nalini A ${ }^{2}$, Hanns Lochmüller ${ }^{3}$

${ }^{1}$ John Walton Muscular Dystrophy Research Center, Newcastle University, Newcastle, GB $;^{2} N I M H A N S$, Bengaluru, IN; ${ }^{3}$ Institute Of Genetic Medicine, Newcastle University, International Centre for Life, Newcastle Upon Tyne, US

Background: Congenital myasthenic syndromes (CMS) are a heterogeneous group of inherited neuromuscular disorders, caused by mutations in genes coding for proteins involved in the maintenance of structure and function of the neuromuscular junction (NMJ). Treatment is available and the choice of medical treatment varies with the CMS subtype, thus patients can be effectively treated and prognosis can be improved only when the precise genetic cause is known. In addition, the frequency of the different CMS-causing genes varies with ethnic and geographic origin. There are only a few reports from India describing the occurrence of DOK7, RAPSN and $C O L Q$ mutations, and the prevalence of various gene mutations in Indian population is unknown, hence, limiting the effective prognosis and access to treatments in these patients. Methods: Bloods from 74 clinically suspected CMS families (total of 193 samples) were collected and DNA was isolated. Samples were subjected to screening of the four most common CMS mutations, namely DOK7 c.1124_1127dupTGCC, CHRNE c.130insG, CHRNE c.1327delG and RAPSN c.264C $>$ A. Cases where there was clinical suspicion of a specific causative gene (e.g. $C H R N E$ and $G M P P B$ ) were subjected to Sanger sequencing of the whole gene including all exons and exon-intron boundaries. Results: A total of 85 samples comprising 74 index cases and 11 affected family members were subjected to screening of common mutations. All cases were negative for the RAPSN c.264C $>$ A European founder mutation. We identified six homozygous cases with the common CHRNEc.1327delG mutation. In addition we identified seven cases with heterozygous DOK7 mutations, including the common c.1124 1127dupTGC $\quad(\mathrm{n}=5), \quad$ a $\quad$ novel c.1127_1128insTGCC (p.A378Sfs*30) and a novel c.1083_1086delCGTG (p.V362Gfs*93) variant. Since the phenotype of these patients carrying heterozygous mutations was suggestive of DOK7CMS, the full coding sequence of $D O K 7$ was Sanger sequenced but no second mutation was detected. Similarly, further sequencing of $C H R N E(\mathrm{n}=21)$ and $G M P P B(\mathrm{n}=3)$ full coding region in patients where the phenotype was suggestive of these genes identified 16/21 cases with CHRNE and 3/3 with GMPPB mutations. The overall solved rate was $37.6 \%$ and this was higher in consanguineous pedigrees $(50 \%)$. Conclusion: Mutations in CHRNE gene are the most common cause of CMS at a hot-spot screening in our large Indian patient cohort. This approach may help to provide quick genetic diagnosis in at least a third of all clinically suspected cases. Clinical diagnosis further aids in providing clues for specific gene screening. The remaining patients will undergo further testing with next-generation sequencing technologies.

\section{PS3Group2-023 / \#435}

Topic: Group 2 - Diseases of Neuromuscular Junction: Clinical Features, Pathophysiology, Therapy

\section{'MINIMAL SYMPTOM EXPRESSION' IN ACETYLCHOLINE RECEPTOR- POSITIVE REFRACTORY MYASTHENIA GRAVIS PATIENTS TREATED WITH ECULIZUMAB}

John Vissing ${ }^{1}$, Saiju Jacob ${ }^{2}$, Kenji P. Fujita ${ }^{3}$, Fanny

$\mathrm{O}^{\prime}$ Brien $^{3}$, James F. Howard $\mathrm{Jr}^{4}$

${ }^{1}$ Rigshospitalet, Copenhagen, DK $;{ }^{2}$ University

Hospitals of Birmingham, Birmingham, GB: $;^{3}$ Alexion Pharmaceuticals, New Haven, CT, US: ${ }^{4}$ University of North Carolina, Chapel Hill, NC, US

Background: REGAIN was a 26-week, phase 3, randomized, double-blind, placebo-controlled study of eculizumab versus placebo for patients with antiacetylcholine receptor antibody-positive $(\mathrm{AChR}+)$ refractory generalized myasthenia gravis (gMG). Patients completing REGAIN could participate in an open-label extension study. We evaluated achievement of 'minimal symptom expression' in REGAIN and from an interim analysis of the open-label study, 
as measured by two patient-reported outcomes. Methods: 'Minimal symptom expression' was defined as achievement of myasthenia gravis activities of daily living (MG-ADL) total score of 0-1 or myasthenia gravis quality of life 15 (MG-QOL15) total score of $0-3$. The proportions of placebo- and eculizumab-treated patients achieving 'minimal symptom expression' in REGAIN, and after 26 weeks of eculizumab treatment in the open-label study for the placebo/eculizumab and eculizumab/eculizumab groups were calculated. Results: At week 26 of REGAIN, a higher proportion of patients receiving eculizumab than placebo achieved 'minimal symptom expression': MG-ADL (21.4\% vs $1.7 \%$, respectively; difference $=19.7 \%$, 95\% CI: 8.5, 31.0); MGQOL15 (16.1\% vs 1.7\%, respectively; difference = $14.4 \%$, 95\% CI: 4.2, 24.5). After 26 weeks of eculizumab treatment in the open-label study, 'minimal symptom expression' in MG-ADL was achieved by similar proportions of the placebo/eculizumab and eculizumab/eculizumab groups $(23.6 \%$ vs $24.5 \%$, respectively; difference $=0.9 \%, 95 \%$ CI: -15.6 , 17.3). For MG-QOL15, the proportion achieving 'minimal symptom expression' in the placebo/eculizumab group increased to $12.5 \%$ (vs $23.4 \%$ in the eculizumab/eculizumab group; difference $=10.9 \%$, 95\% CI: -4.0, 25.8) (Figure). The long-term safety profile of eculizumab was consistent with its known profile. Conclusion: Eculizumab was associated with a higher proportion of patients with $\mathrm{AChR}+$ refractory gMG achieving 'minimal symptom expression' compared with placebo, assessed using MG-ADL and MG QOL15 (NCT01997229, NCT02301624).
PS3Group2-024 / \#466

Topic: Group 2 - Diseases of Neuromuscular Junction: Clinical Features, Pathophysiology, Therapy

\section{THE PLACEBO EFFECT IN MYASTHENIA GRAVIS: A META- ANALYSIS}

Elisa Frisaldi ${ }^{1}$, Aziz Shaibani ${ }^{2}$, Jan Vollert ${ }^{3}$, Bruno Ferrero $^{4}$, Roberta Carrino ${ }^{4}$, Hayan Ibraheem², Lene Vase $^{5}$, Fabrizio Benedetti ${ }^{4}$

${ }^{1}$ Department Of Neuroscience "rita Levi

Montalcini", University of Turin Medical School, Turin, IT; ${ }^{2}$ Nerve \& Muscle Center Of Texas, Nerve \& Muscle Center, Houston, TX, US, ${ }^{3}$ Center Of Biomedicine And Medical Technology Mannheim Cbtm, Heidelberg University, Medical Faculty Mannheim, Mannheim, DE; ${ }^{4}$ Department Of Neuroscience "rita Levi Montalcini", University of Turin Medical School, Turin, IT; Department Of Psychology And Behavioural Sciences, School of Business and Social Sciences, Aarhus, DK

Background: This meta-analysis aims to determine the magnitude of placebo effect for generalized myasthenia Gravis (MG) in a pool of eligible clinical trials. Depending on clinical conditions and outcome measures examined, placebo effects in neuromuscular disorders show a substantial variability and most of our knowledge about them comes from painful neuropathies. In MG, the clinical improvement attributable to a placebo effect seems to be smaller than the improvement due to active treatments. MG represents quite an interesting model to investigate
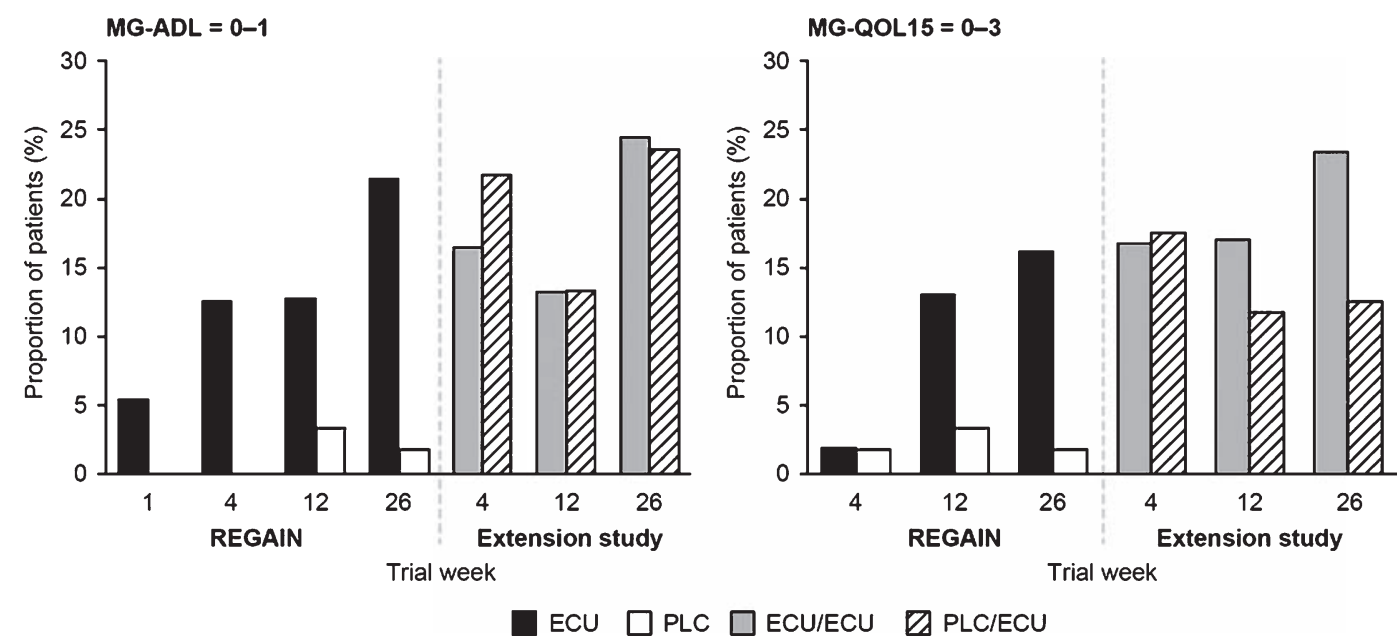
placebo effects because drug and placebo responses can be quantified by both subjective and objective measurements. Methods: A comprehensive literature search via PubMed, Scopus, Web of Science, Cochrane Controlled Trial Register and Embase provided the basis of our meta-analysis. Any restrictions for year of publication, duration of treatment, or sample size were applied. Eligible for inclusion were only randomized clinical trials (RCTs) including a placebo control group and setting the Quantitative Myasthenia Gravis (QMG) score as primary or secondary outcome, since QMG test has demonstrated high inter-rater reliability, concurrent validity, and responsiveness. Main outcome variable was number of patients improving the QMG score of $\geq 3$ points per group, or, when this information could not be retrieved, QMG change from baseline per group. On this basis, odds ratios and $95 \%$ confidence intervals or drug effect sizes were calculated. Results: Twelve studies, covering a total of 579 participants, met the inclusion criteria. Tirasemtiv, eculizimab, tacrolimus, mycophenolate mofetil, intravenous immunoglobulin, methotrexate, cyclosporine and terbutaline were the different interventions compared to placebo for the treatment of MG. Improvement in the QMG score for groups receiving placebos were generally low in most studies, but responsiveness varied broadly between studies between (from $0 \%$ to $57 \%$ ), which should be attributed to random sampling bias. The magnitude of the verum effect was consistently above these numbers (from $25 \%$ to $86 \%$ ), leading to odd's ratios and effect sizes favoring drug over placebo, as a forest plot revealed (Figure 1). Since none of the RCTs analyzed in this meta-analysis include a natural history group, it was impossible to disentangle improvements due to the active drug or to the real placebo component from improvements due to other phenomena, such as spontaneous reductions of symptoms severity. Conclusion: In accordance with previous studies, our meta-analysis showed little improvements attributable to placebo interventions in clinical trials for MG management. All active treatments show a significantly higher impact on QMG scores compared to placebos, except from one study (Wolfe 2002).

\section{*Odds ratio of number of patients improving the QMG score of $\geq 3$ points ${ }^{\circ}$ Exponentiated effect size of average QMG improvement}

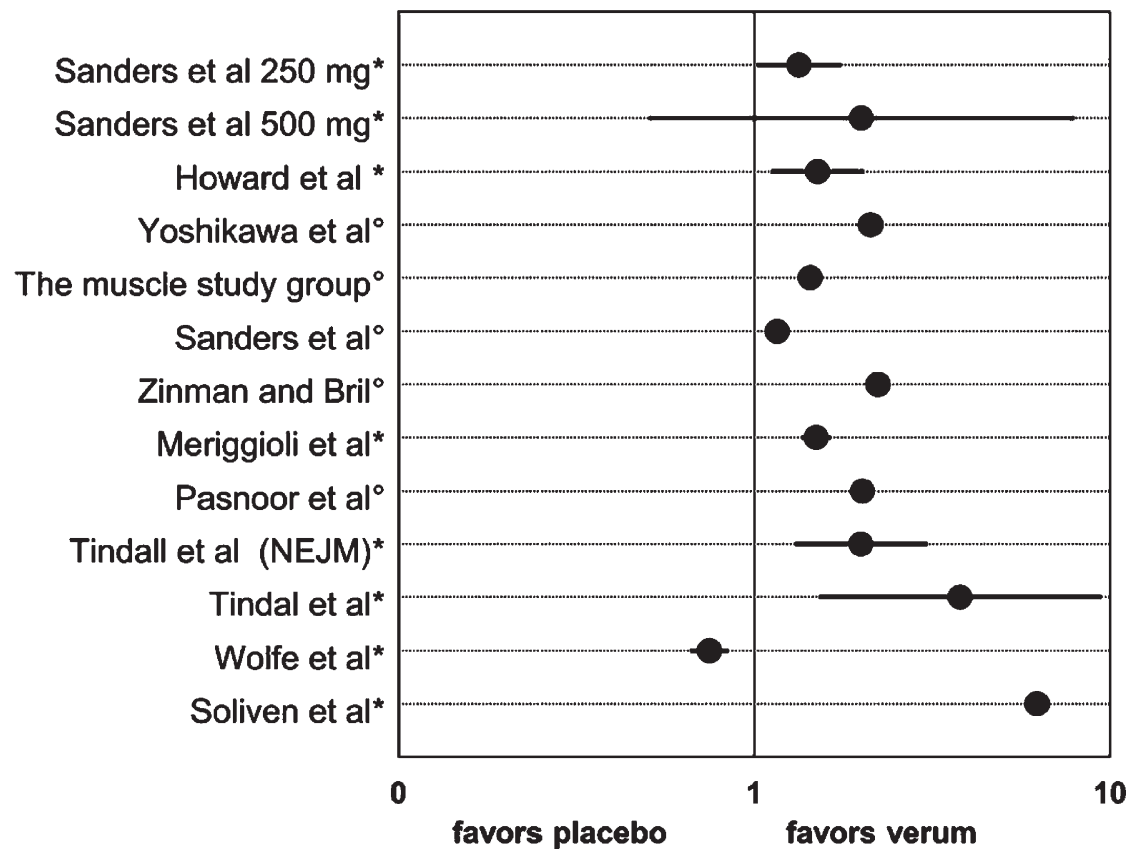




\section{PS3Group2-025 / \#678}

Topic: Group 2 - Diseases of Neuromuscular Junction: Clinical Features, Pathophysiology, Therapy

\section{CONGENITAL MYASTHENIC SYNDROMES IN A SUBPOPULATION OF THE NORTH OF PORTUGAL}

Luísa Sousa $^{1}$, Ernestina Santos ${ }^{1}$, João Martins ${ }^{2}$, Ana Sousa $^{3}$, Marcio Cardoso ${ }^{3}$, Fernando Silveira ${ }^{4}$, Goreti Nadais ${ }^{4}$, Ricardo Maré 5 , Andreia Veiga ${ }^{6}$, Catarina S. Santos ${ }^{7}$, Teresa Coelho ${ }^{3}$, Manuela Santos ${ }^{8}$

${ }^{1}$ Neurology, Centro Hospitalar do Porto, Porto, PT; ${ }^{2}$ Neurology, Hospital Pedro Hispano - Unidade Local de Saúde de Matosinhos, Matosinhos, PT; ${ }^{3}$ Neurophysiology, Centro Hospitalar Porto, Porto, PT: ${ }^{4}$ Neurology, Centro Hospitalar São João, Porto, PT; ${ }^{5}$ Neurology, Hospital de Braga, Braga, PT; ${ }^{6}$ Neurology, Centro Hospitalar Trás-os-Montes e Alto Douro, Vila Real, PT; ${ }^{7}$ Neurology, Centro Hospitalar Entre Douro e Vouga, Santa Maria da Feira, PT; ${ }^{8}$ Neuropediatrics, Centro Hospitalar Porto, -, PT

Background: Congenital myasthenias are rare diseases of the neuromuscular junction that usually present with ophthalmoparesis, ptosis and motor fatigability. Nevertheless, the clinical and genetic presentations are heterogeneous. The aim of this work was to analyse the characteristics of a group of patients with congenital myasthenic syndromes from a region of Portugal. Methods: We conducted a retrospective review of medical charts of patients diagnosed with congenital myasthenic syndromes from a convenience sample. Cases were identified from Neurology and Neuropediatrics consultations from six different hospitals in the north of Portugal. Demographic, clinical, electromyographic and genetic tests' results were reviewed and described. Results: 23 patients from 16 families were identified, including 7 pairs of siblings. Median age was 37 years old (range 13-54). The first manifestation of disease was related to ocular involvement $(\mathrm{n}=11)$, motor weakness or gait disturbance $(n=9)$, and bulbar or respiratory involvement $(\mathrm{n}=2)$. In most cases, symptoms were noted in the first two years of life, with one patient developing life-threatening symptoms in the neonatal period. The most frequent mutation was of the epsilon subunit of the acetylcholine receptor gene, CHRNE ( $\mathrm{n}=16,69 \%)$, followed by DOK-7 $(\mathrm{n}=6,26 \%)$ and rapsyn mutations $(\mathrm{n}=1)$. The median time from symptom onset to genetic testing was 37.5 years. The most severe phenotypes were observed in two brothers with DOK-7 mutations and the patient with the rapsyn mutation, with periods of severe respiratory insufficiency or dysphagia requiring a gastrostomy tube placement. Regarding treatment, 19 patients were started on pyridostigmine, out of which 14 had at least partial improvement and 3 deteriorated (all with DOK-7 mutations). All DOK-7 cases improved with salbutamol or ephedrine. One of the patients with CHRNE mutation also benefited with salbutamol. Conclusion: All cases had a postsynaptic neuromuscular junction defect. Clinical characteristics are congruent with what is described in the literature for these patients. In this convenience sample we found a high relative frequency of DOK-7 mutations, superior to what is reported in larger published cohorts. The early identification of the genetic mutation is important since it may impact treatment decisions.

\section{PS3Group2-026 / \#577}

Topic: Group 2 - Diseases of Neuromuscular Junction: Clinical Features, Pathophysiology, Therapy

\section{FETAL ACETYLCHOLINE RECEPTOR INACTIVATION SYNDROME: A RARE, BUT POTENTIALLY TREATABLE CAUSE OF FAMILIAL MYOPATHY}

Doris Nguyen ${ }^{1}$, Stephan Botez ${ }^{2}$, Cam-Tu Emilie Nguyen $^{3}$

${ }^{1}$ CHU Sainte-Justine, Montreal, QC, $C A ;{ }^{2}$ Neurology, CHUM, Montreal, QC, CA, ${ }^{3}$ Pediatric Neurology, CHU Sainte-Justine, Montreal, $Q C, C A$

Background: Fetal Acetylcholine Receptor Inactivation Syndrome (FARIS) is a recently recognized congenital myopathy due to in utero exposure to maternal acetylcholine receptor (AChR) antibodies directed against the fetal AChR gamma subunit. Unlike transient neonatal myasthenia gravis, the manifestations of FARIS extend well beyond the neonatal period. In children who survive the neonatal period, the myopathic features of FARIS typically improve. The diagnosis of FARIS is particularly challenging when the mother is not 
known for myasthenia gravis (MG). We report the second case of FARIS born to an asymptomatic mother and review the literature. Methods: Case report and review of the literature. Results: We describe a term female, with polyhydramnios, congenital weakness, hypotonia, arthrogryposis, respiratory and bulbar insufficiency requiring intubation and gastrostomy, followed by marked clinical improvement in childhood. At age 9, she is able to play tennis, but still has significant facial weakness and velopharyngeal insufficiency. Brother had a similar, but more severe phenotype and passed away at age 6 weeks. Extensive investigations in the siblings, including whole exome sequencing, were non diagnostic. The mother, who is completely asymptomatic for MG, was found to have elevated AChR antibodies and postexercice exhaustion on repetitive nerve stimulation. We found 12 other FARIS cases from 6 different families. Only one other mother was asymptomatic for MG. FARIS begins antenatally, with polyhydramnios and reduced fetal movements, and can result in lethal arthrogryposis multiplex congenita. In children who survive the neonatal period, the myopathic features of FARIS typically improve, but often there is persistent myopathic facies and velopharygeal insufficiency. The risk of recurrence in subsequent pregnancies is extremely high. More aggressive MG treatment during pregnancy appears to lessen the severity of FARIS. Conclusion: FARIS is a rare, early onset myopathy caused by in utero exposure to maternal AChR antibodies directed against the fetal AChR gamma subunit. The diagnosis of FARIS is particularly challenging when the mother is not known for myasthenia gravis (MG), but has important clinical implications for future pregnancies as $\mathrm{MG}$ treatment during pregnancy appears to impact the severity of FARIS. Therefore, FARIS should be included in the differential diagnosis of congenital myopathies.

\section{PS3Group2-027 / \#463}

Topic: Group 2 - Diseases of Neuromuscular Junction: Clinical Features, Pathophysiology, Therapy

\section{CLINICAL AND SEROLOGICAL PREDICTORS OF THYMOMA RECURRENCES IN MYASTHENIA GRAVIS}

Anna De Rosa ${ }^{1}$, Roberta Ricciardi ${ }^{2}$, Michelangelo Maestri² $^{2}$, Melania Guida ${ }^{2}$, Stefania Rizzo ${ }^{2}$, Antonio
Chella ${ }^{3}$, Franca Melfi ${ }^{4}$, Marco Lucchi ${ }^{5}$, Ubaldo

Bonuccelli $^{2}$, Gabriele Siciliano ${ }^{2}$

${ }^{1}$ Department of Clinical and Experimental

Medicine, Neurology Unit, University of Pisa, Italy,

Pisa, IT; ${ }^{2}$ Department of Clinical and Experimental

Medicine, Neurology Unit, University of Pisa, Italy,

Pisa, IT; ${ }^{3}$ Division of Pneumonology, Cardio

Vascular and Thoracic Department, University

Hospital of Pisa, Pisa, Italy, Pisa,

IT: ${ }^{4}$ Multidisciplinary Center of Robotic Surgery,

University of Pisa, Italy, Pisa, IT, ${ }^{5}$ Cardiothoracic

and Vascular Surgery Department, University of

Pisa, Italy, Pisa, IT

Background: TAMG (Thymoma-associated MG) represents one of the subtypes of MG associated with autoantibodies against the acetylcholine receptor (AChR-Ab). We analyzed the clinical and serological features of patients with thymoma and relapsed thymoma, at different time points, in order to identify a possible relationship among relapses, clinical features and changes in AChR-Ab titres. Methods: We enrolled 349 MG patients with AChR$\mathrm{Ab}$ and thymoma: 317 with TAMG and 32 (9\%) who experienced one or more recurrences of thymoma. We then retrospectively assessed: age of MG onset, MG clinical status according to MGFA (Myasthenia Gravis Foundation of America), time of thymectomy, surgical approach, post-thymectomy status and oncological features (according to histological classifications: WHO and Masaoka-Koga). AChR-Ab serum titres have been closely monitored overtime. $\mathrm{P}$-values $<0.05$ were considered statistically significant. Results: Patients with relapsed thymoma were younger than those without recurrences $(p<0,0001)$ with an average neoplastic disease-free time of about 3.7 years. In relapsed thymomas symptoms worsened immediately after the first thymectomy $(p<0,0001)$. However, MG symptoms did not worsen by the time of recurrence of thymoma. Relapsed thymomas had a Masaoka stage more aggressive than those without recurrences $(p<0,0001)$. Patients with relapses achieved a better MG status than those with thymoma $(p<0,0115)$. Overall, AChR-Ab titres in patients with thymoma decreased immediately after thymectomy (thymomas: $\mathrm{p}<0,0001$; relapsed thymomas: $p<0,02$ ) and remained unchanged overtime. There was no statistical difference in AChRAb titres before and immediately after thymectomy between patients with relapsed thymoma and patients without relapses. Conclusion: Thymoma recurrences are not associated with an increase of AChR-Ab titres or worsening of MG symptoms but with the 
histological grading. Clinical presentations are similar in thymoma and relapsed thymoma, although symptoms can get worse immediately after thymectomy in the second group of patients. In the longterm, pharmacological and complete stable remission is achieved by a high percentage of patients with relapsed thymoma. Although rare, relapsed thymoma does occur and further studies would need to be carried out to identify possible biomarkers of recurrence.

\section{PS3Group2-028 / \#631}

Topic: Group 2 -Diseases of Neuromuscular Junction: Clinical Features, Pathophysiology, Therapy

\section{THE EFFECTS OF NEUROTROPHIC FACTORS ON HUMAN MUSCLE CELLS: A COMPARISON WITH MURINE MUSCLE CELLS}

$\underline{\text { Susie Barbeau }}^{1}$, Fannie Semprez ${ }^{2}$, Claire Legay ${ }^{1}$

${ }^{1}$ CNRS UMR8119, Université Paris Descartes, Sorbonne Paris Cité, Paris cedex , FR $;{ }^{2} C N R S$ UMR8119, Université Paris Descartes, Sorbonne Paris Cité, Paris cedex, FR

Background: We aim at establishing mature human neuromuscular junction (NMJ) in vitro models representative of different neuromuscular diseases (NMD) and to understand the mechanisms of crosstalks between muscle cells and motor neurons at the synapse in normal and pathological conditions. NMD correspond to a vast group of diseases that perturbs or even progressively blocks the control and the force of muscle voluntary movement by affecting NMJs structure or activity. To date, no efficient curative treatments have been identified for NMD. Progresses towards identification of new treatments have been hampered by the incomplete understanding of the molecular mechanisms that control synapse formation and maintenance and the paucity of informations concerning the human NMJ. There are two types of NMDs, autoimmune diseases and congenital myasthenic syndromes (CMS). Our team focus on CMS and more specifically on CMS with Acetylcholinesterase (AChE) deficiciency due to mutations in ColQ, a collagen secreted by muscle cells. In the past, we have analyzed in details the phenotype of transgenic mice deficient for ColQ and although these transgenic mice represent a good model for the CMS, it remains that the entire ColQ gene is deleted in these mice when in human, point mutations or small deletions have been described. In addition, in vivo analysis are not allowing us to determine the impact of ColQ mutations on muscle cells independently of the motor neuron. Methods: Cell culture, immunocytochemistry, morphological analyses, epifluorescence and confocal imaging. Results: Therefore in a first step, we focus on human muscle cells and their sensitivity to neurotrophic factors in normal conditions in vitro. We will present the morphological characteristics of human myotubes obtain from human primary myoblasts on micropatterned culture dishes designed to optimize myoblasts fusion and myotubes differentiation. We also explored the impact of various proteins secreted at the NMJ junction on AChR clustering in these human myotubes. We compare these results with murine myotubes cultured in the same conditions. Preliminary results emphazise the differences and similarities between the 2 species. Future experiments will test the same factors in human muscle cells from patients suffering from CMS with AChE deficiency. Conclusion: These preliminary results are a necessary step to understand and characterize at the muscle cells and NMJ levels the deficiencies engender by the mutations involved in ColQ-CMS and develop new therapeutics.

\section{PS3Group2-029 / \#900}

Topic: Group 2 - Diseases of Neuromuscular Junction: Clinical Features, Pathophysiology, Therapy

\section{A PILOT STUDY OF ENGINEERED AGRIN AND ITS EFFECT ON THE SEVERITY OF EXPERIMENTAL AUTOIMMUNE MYASTHENIA GRAVIS}

Zhiguo $\mathrm{Li}^{1}$, Minshu $\mathrm{Li}^{2}$, Kristofer Wood ${ }^{1}$, Steffan Hettwer ${ }^{3}$, Suraj Muley ${ }^{4}$, Fu-Dong Shi ${ }^{2}$, Qiang Lui², $\underline{\text { Shafeeq Ladha }}{ }^{4}$

${ }^{1}$ Neurobiology, Barrow Neurological Institute, Phoenix, US; ${ }^{2}$ Tianjin Neurological Institute, Tianjin, $C N ;{ }^{3}$ Neurotune AG, Schlieren-Zurich, $\mathrm{CH} ;{ }^{4}$ Neurology, Barrow Neurological Institute, Phoenix, US

Background: Since standard immunotherapy for MG involves reducing the pathogenic effects of 
disease causing antibodies, strategies that enhance repair of these changes are attractive as potential adjunctive therapies to immunosuppression treatments. Activation of agrin and its downstream pathways may accomplish this by promoting repair and organization of the NMJ. Therefore, we attempted to determine the effects of a soluble C-terminus fragment of neural agrin (NT-1654) on the severity of experimental autoimmune myasthenia gravis (EAMG). Compared to prior agrin agonists which had poor tissue penetration, NT-1654 is resistant to degradation and highly soluble owing to induced mutations at the neurotrypsin and heparin binding sites, respectively. Methods: EAMG was induced in female Lewis rats by immunization with the Torpedo acetylcholine receptor (tAChR) and complete Freund's adjuvant. A total of forty-eight rats used in this study were assigned to three EAMG groups receiving vehicle phosphate-buffered saline (disease control), $2 \mathrm{mg} / \mathrm{kg} \mathrm{NT}-1654$, or $6 \mathrm{mg} / \mathrm{kg} \mathrm{NT}$ 1654 , and a naïve control group ( $\mathrm{n}=12$ per group). NT-1654 was dissolved in PBS and injected daily s.c. into tAChR immunized rats during the first 10 days after immunization, and then every other day for the following 20 days. Performing serum agrin antibody assays assessed the immunogenicity of NT-1654. EAMG clinical scores, body weight, repetitive nerve stimulation, and muscle histopathology were assessed as readouts. Results: NT-1654 reduced clinical severity of EAMG in the rats as evidenced by higher EAMG clinical scores and improved weight gain. Administration of NT-1654 also effectively promoted the clustering of AChRs at NMJs and alleviated the neurophysiologic impairment of NMJ transmission. Increased staining of muscle-specific kinase (MuSK) protein was seen although alterations in MuSK mRNA expression was not. Conclusion: These findings suggest that NT1654 may be a promising agent to induce repair and augment synaptic strength at NMJs in EAMG. Agrin antibodies were not seen suggesting that there is no significant immunogenicity of NT-1654. Although these findings suggest that NT-1654 could have a beneficial effect in EAMG, future investigations are needed to validate these results and test NT-1654 in the setting of treatments typically used in MG (corticosteroids) and at the time of symptom onset.

\section{PS3Group2-030 / \#375}

Topic: Group 2 - Diseases of Neuromuscular Junction: Clinical Features, Pathophysiology, Therapy

\section{MUTATIONS IN GFPT1- RELATED CMS UNDERLIE A TUBULAR AGGREGATES MYOPATHY WITH SYNAPTOPATHY}

$\underline{\text { Stéphanie Bauché }}^{1}$, Geoffroy Vellieux ${ }^{1}$, Damien Sternberg ${ }^{2}$, Maris-Josephine Fontenille ${ }^{1}$, Guy Brochier $^{3}$, Julien Messéant ${ }^{1}$, Michel Fardeau ${ }^{3}$, Norma Romero ${ }^{3}$, Emmanuel Fournier ${ }^{4}$, Nathalie Streichenberger ${ }^{5}$, Veronique Manel $^{6}$, Arnaud Lacour $^{7}$, Aleksandra Nadaj-Pakleza ${ }^{8}$, Sylvie Sukno $^{9}$, Françoise Bouhour ${ }^{10}$, Pascal Laforet ${ }^{11}$, Bertrand Fontaine ${ }^{1}$, Laure Strochlic ${ }^{1}$, Bruno Eymard $^{1}$, Frederic Chevessier ${ }^{12}$, Tanya Stojkovic ${ }^{11}$, Sophie Nicole ${ }^{1}$

${ }^{1}$ Neurogenetic And Physiology, Inserm U 1127, CNRS UMR 7225, Sorbonne Universités, UPMC Université Paris 06 UMR S 1127, Institut du Cerveau et de la Moelle épinière, ICM, Paris, $F R ;{ }^{2}$ Cardiogénétique Et Myogénétique, APHP, Paris, FR ${ }^{3}$ Unité De Pathologies

Neuromusculaires, Institut De Myologie, UMRS974, Institut de Myologie, Paris, FR; Institut $d u$ Cerveau et de la Moelle epinière, Paris, FR: ${ }^{5}$ Centre de Neuropathologie Est - Hospices Civils de Lyon, Université Claude Bernard Lyon1, Institut NeuroMyogène - CNRS UMR 5310 INSERM U1217, Lyon, FR; ${ }^{6}$ Service d'Epileptologie Clinique, des Troubles du Sommeil et de Neurologie Fonctionnelle de l'Enfant, Hôpital Femme Mère Enfant, Lyon, FR $;{ }^{7}$ Clinique neurologique et centre de référence des maladies rares neuromusculaires, Hôpital Roger-Salengro, CHRU de Lille, Lille, FR: ${ }^{8}$ Centre de Référence des Maladies Neuromusculaires Nantes-Angers, Service de Neurologie, Centre Hospitalier Universitaire d'Angers, Angers, FR; ${ }^{9}$ Centre Hospitalier Béthune Beuvry, Service des consultations externes pédiatrie, Béthune, FR: ${ }^{10}$ Hospices Civils de Lyon, Hôpital Neurologique Pierre Wertheimer, Service d'ENMG-Pathologies neuromusculaires, Bron, FR; ${ }^{11}$ Sorbonne Universités, UPMC Univ Paris 06, INSERM UMRS974, CNRS FRE3617, Center of Research in Myology, Myology Institute, Paris, FR; ${ }^{12}$ Institute of Neuropathology, UniversityHospital Erlangen, schwabachanlage 6, Erlangen, DE 
Background: Congenital myasthenic syndromes (CMS) are a clinically and genetically heterogeneous group of inherited disorders that share common clinical features characterized by fluctuation of muscle weakness and fatigability resulting from defective synaptic transmission at the neuromuscular junction (NMJ). The distribution of symptoms and treatment's efficiency diverge according to the localization of the mutated proteins at the NMJ. Mutations in GFPT1 (glutamine-fructose-6-phosphate transaminase 1), a gene encoding an enzyme involved in glycosylation of ubiquitous proteins, cause a limb-girdle congenital myasthenic syndrome (LGCMS) with tubular aggregates (TAs) characterized predominantly by affection of the proximal skeletal muscles and presence of highly organized and remodeled sarcoplasmic tubules in patients' muscle biopsies. GFPT1 is the first and rate-limiting enzyme of the hexosamine biosynthetic pathway (HBP) involved in ubiquitous glycosylation processes. Since 2011, a total of 52 patients with GFPT1 mutations have been clinically reported. In this study, we report the first retrospective clinical description and the molecular investigations of 11 individuals from 9 unrelated families with LG-CMS linked to recessively-inherited mutations in GFPT1. Interestingly, GFPT1 is expressed in various tissues, including skeletal muscle and motor nerve, raising the intriguing question of why mutations in such a ubiquitous gene would specifically lead to synaptic and muscular disorganization. Methods: Yet, none of studies was dedicated to a long-term clinical follow-up, and investigation of the endplate was only reported for 7 patients. In this study, we report the retrospective clinical description and the molecular investigations of LG-CMS individuals with GFPT1 mutations. We analyzed the synaptic features and glycosylation level in muscle biopsies from 4 patients. Results: The retrospective clinical evaluation of LG-CMS individuals stresses an evolution toward a myopathic weakness that occurs concomitantly to ineffectiveness of usual CMS treatments. Analysis of neuromuscular biopsies from 3 unrelated individuals demonstrates that the maintenance of NMJs is dramatically impaired with loss of post-synaptic junctional folds and evidence of denervation-reinnervation processes affecting the 3 main NMJ components. Moreover, molecular analyses of the human muscle biopsies confirm glycosylation defects of proteins with reduced O-glycosylation and show reduced sialylation of transmembrane proteins in extrajunctional area. Conclusion: In this study, we identified 9 new GFPT1 mutations, further stressing the high number of mutations reported in this gene associated with CMS and reinforcing that GFPT1 is the first genetic cause of ubiquitous CMS. We highlight a profound remodeling of NMJs characterized by a loss of junctional folds as well as abnormal nerve endings when GFPT1 is decreased and impaired $\mathrm{N}$ - and $\mathrm{O}$-glycosylation processes not only at the synapse but also in the extrajunctional area. Together with the clinical evolution of LG-CMS linked to GFPT1 towards a myopathic weakness phenotype with progressive ineffectiveness of usual CMS treatments, these histological and molecular results lead us to consider individuals with GFPT1 mutations as suffering from a "tubular aggregates myopathy with synaptopathy" rather than from the usual ubiquitous CMS due to reduced post-synaptic density of AChRs linked to hypoglycosylation.

\section{PS3Group2-031 / \#553}

Topic: Group 2 - Diseases of Neuromuscular Junction: Clinical Features, Pathophysiology, Therapy

THE POTENTIAL OF A NOVEL ANTIGEN-SPECIFIC GLYCOPOLYMER IN THE TREATMENT OF PATIENTS WITH MULTIFOCAL MOTOR NEUROPATHY

Delphine Demeestere $^{1}$, Horst Prescher $^{1}$, Beat Ernst ${ }^{1}$, Pascal Hänggi ${ }^{2}$, Ruben Herrendorff ${ }^{2}$

${ }^{1}$ Institute Of Molecular Pharmacy, University of

Basel, Basel, $\mathrm{CH} ;{ }^{2} \mathrm{R} \&$, Polyneuron

Pharmacetuicals AG, Basel, $\mathrm{CH}$

Background: Multifocal motor neuropathy (MMN) is a rare inflammatory peripheral neuropathy that causes asymmetric muscle weakness and atrophy. The pure motor neuropathy is characterized by conduction block and, in many cases, pathogenic autoantibodies against the glycan epitope of the GM1 ganglioside. Current treatment is unspecific, leading to severe side effects, and lacks efficacy. For this reason, we are developing an antigen-specific approach with a potentially better safety and long-term efficacy, i.e. glycopolymers that selectively bind anti-GM1 autoantibodies. These glycopolymers comprise the natural GM1 glycoepitope, or mimetic thereof, that is presented in a multivalent way on a 
biodegradable polymer backbone to increase avidity. Methods: Both serum from MMN patients and plasma from GM1 immunized and non-immunized mice were used for enzyme-linked immunosorbent assay (ELISA) analysis. Results: Serum samples from MMN patients were tested for the presence of anti-GM1 antibodies and revealed the presence of both anti-GM1 IgG and IgM in, respectively, $57 \%$ $(8 / 14)$ and $14 \%(2 / 14)$ of the cases. According to literature, mainly anti-GM1 IgM antibodies are linked to the pathogenesis of MMN, but our results indicate an underestimated role for anti-GM1 antibodies of the IgG isotype. As it is well accepted that the local glycolipid environment is important in facilitating or inhibiting antibody binding to GM1 in immunoassays, a combined GM1/galactocerebroside (GalC) assay was performed. Indeed, the percentage of patients positive for IgM antibodies increased to 36\% (5/14) of the MMN sera tested, but the opposite was true for IgG antibodies with $43 \%$ (6/14). Furthermore, some preliminary experiments performed with a glycopolymer containing the natural GM1 epitope showed the successful blocking of patient anti-GM1 IgM antibodies. Interestingly, a combination of both the GM1 and GalC epitope on the polymer backbone gave a similar inhibitory effect. An active immunization model of MMN, in which BALB/c mice were immunized with GM1, did not generate robust high anti-GM1 IgM levels. Moreover, administration of the glycopolymer carrying the natural epitope in GM1-immunized mice only lowered anti-GM1 IgM levels to a limited extent. It is well known that healthy subjects have low affinity anti-GM1 IgM antibodies in the circulation, which we also observed in non-immunized mice. When injecting these wild type mice with the same GM1 glycopolymer, the same limited response was observed. So, we believe that the presence of natural low affinity anti-GM1 IgM antibodies explains the high variability of anti-GM1 IgM antibody induction in the active immunization model and the limited response upon drug administration. Therefore, we plan to introduce a humanized mouse MMN model and a human motor neuron model to further characterize the therapeutic value of the different GM1 glycopolymers. Conclusion: In conclusion, these preliminary findings suggest a potential role for anti-GM1 IgG antibodies in MMN and support the use of GM1 glycopolymers in scavenging antiGM1 antibodies from patient sera. However, there is an urgent need for a better in vivo mouse model for MMN, in which high affinity anti-GM1 IgM antibodies are present.
PS3Group2-032 / \#584

Topic: Group 2 - Diseases of Neuromuscular Junction: Clinical Features, Pathophysiology, Therapy

\section{RELATIONSHIP OF \\ ACETYLCHOLINE RECEPTOR ANTIBODY TITER AND MYASTHENIA GRAVIS SEVERITY IN CIPTO MANGUNKUSUMO HOSPITAL}

\author{
Fika T. Widayati ${ }^{1}$, Ahmad Y. Safri $^{2}$, Fitri
}

Octaviana $^{2}$, Luh Ari Indrawati ${ }^{2}$, Dewi Wulandari ${ }^{3}$, Tony Loho ${ }^{3}$, Manfaluthy Hakim ${ }^{2}$

${ }^{1}$ Neurology, Universitas Indonesia, Faculty of Medicine, Jakarta, ID; ${ }^{2}$ Neurology, universitas Indonesia, faculty of medicine, Jakarta, ID $;{ }^{3}$ Clinical Pathology, universitas Indonesia, faculty of medicine, Jakarta, ID

Background: Acetylcholine receptor antibody (antiAChR) is main antibody in pathogenesis of myasthenia gravis $(M G)$ and important for diagnostic test. MG clinical features can be devided into ocular $M G$, generalisata $M G$ and bulbar $M G$ and each individual can have different severity assesed with $M G$ composite scale (MGCS). Aim this study was to evaluate relationship between antiAChR titer with $M G$ severity based on MGCS in Cipto Mangunkusumo Hospital,Jakarta. Methods: This was a descriptiveanalytic cross sectional study in Cipto Mangunkusumo Hospital during January 2017 to November 2017. Inclusion criteria were 18-75 years old $M G$ patients. The concentration of antiAChR was measured by ELISA method (Euroimun, Germany). MG severity was assessed using MGCS and blood sample for antiAChR was withdrawn at the same time. $M G C S$ value ranging from 0-52. Results: There were 72 subjects $M G$ met inclusion criteria and ratio between female and male was 2.5:1. MG with ocular onset was found in 57 subjects (79.2\%) and proportion of early onset $M G$ (age of onset $<50$ years old) was $77.8 \%$ (56 subjects). Median of $M G$ duration was 3 (0.25-29) years. Fifty eight (80.6\%) subjects had generalized $M G$, thirteen (18.1\%) had ocular $M G$ and only one (1.4\%) subject had bulbar $M G$. MGCS mean 7.82 3.91 and ptosis is the most common finding. About $59.7 \%$ subject had seropositif antiAChR and 3 among them had titer $>800 \mathrm{nmol} / \mathrm{L}$ (exceeded quantification limit). There were no association between antiAChR titers and MGCS ( $p$ 0.727). Additional analysis, there were no significant 
difference between seropositivity of antiAChR and gender, age of onset, type of $M G, M G F a$ classification and imunosupresant therapy. Conclusion: AntiAChR titer was not related to MG severity.

\section{PS3Group2-033 / \#833}

Topic: Group 2 - Diseases of Neuromuscular Junction: Clinical Features, Pathophysiology, Therapy

\section{THE IMPACT OF REFRACTORY MYASTHENIA GRAVIS (MG) ON PATIENT HEALTH-RELATED QUALITY-OF-LIFE (QOL)}

\author{
Audra N. Boscoe ${ }^{1}$, Haichang Xin ${ }^{2}$, Gilbert J. \\ L'Italien ${ }^{3}$, Linda Harris ${ }^{4}$, Gary R. Cutter ${ }^{5}$ \\ ${ }^{1}$ Agios Pharmaceuticals, Cambridge, MA, \\ US $;^{2}$ University of Alabama, Birmingham, $A L$, \\ US, ${ }^{3}$ Alexion Pharmaceuticals, New Haven, \\ US: ${ }^{4}$ Alexion Pharmaceuticals, New Haven, \\ US: ${ }^{5}$ Biostatistics, University of Alabama at \\ Birmingham, Birmingham, AL, US
}

Background: Myasthenia gravis (MG) is a debilitating disease that can substantially interfere with patients' activities of daily living, particularly among the subset of patients whose disease is refractory to conventional therapies. The impact of refractory $\mathrm{MG}$ on health-related quality-of-life (QOL) and disease burden has not been fully characterized. This study assessed both QOL and disease burden in patients with refractory MG compared to patients with non-refractory MG. Methods: The study population was derived from the enrollment survey of the Myasthenia Gravis Foundation of America's (MGFA) MG Patient Registry. The study included MG patients aged 18 years or older who had been diagnosed as having MG for two or more years prior to completing the enrollment survey. Patients were classified as refractory or non-refractory based on the number and type of treatments prescribed and their level of symptomatology as measured by the Myasthenia Gravis Activities of Daily Living Scale (MG-ADL) total score. Refractory was defined as those who received at least two prior and one current $\mathrm{MG}$ treatment at the time of enrollment along with an MG-ADL score of $\geq 6$. Patients who met the treatment criteria but had an MG-ADL score lower than 6 , were not included in the study. All other patients were classified as having non-refractory MG. QOL was assessed by comparing the overall and individual item scores of the Myasthenia Gravis Quality of Life 15-item Scale (MG-QOL15) between refractory and non-refractory patients. Results: This analysis included 56 refractory and 717 nonrefractory patients; 26 patients were excluded because they met the treatment criteria for refractory MG but not the MG-ADL criteria and thus could not be cleanly classified as either refractory or nonrefractory. At the time of enrollment, refractory patients were younger [48.5 years (SD: 12.1) vs. 55.4 years (SD: 14.6), $\mathrm{p}<.01]$, had a younger mean age of MG onset [36.5 years (SD: 15.7) vs. 45.8 years (SD: 17.5), $\mathrm{p}<.01]$, and were more likely to be female $(82.1 \%$ vs. $65.3 \%, p<.01)$ than non-refractory patients. Refractory patients were significantly more likely than non-refractory patients to have had 2 or more clinical exacerbations $(37.5 \%$ vs. $21.1 \%$, $\mathrm{p}<.01), \geq 1$ emergency room visit $(42.9 \%$ vs. $22 \%$, $\mathrm{p}<.01)$, and $\geq 1$ hospitalization $(37.5 \%$ vs. $18.4 \%$, $\mathrm{p}<.01)$ in the prior 6 months. Refractory patients also exhibited significantly higher mean MGQOL15 total scores (representing worse QOL) than patients with non-refractory MG [31.4 (SD 11.1) vs. 20.8 (SD: 15.0) $\mathrm{p}<0.01]$. Furthermore, all individual item scores differed significantly between refractory and non-refractory patients $(\mathrm{p} \leq .01)$. Conclusion: These findings demonstrate that refractory $\mathrm{MG}$ patients exhibit greater clinical burden compared with non-refractory MG patients and that this increased burden is correlated with a detrimental impact on patient QOL as measured by MG-QOL15 total and individual item scores. The MG-QOL15 was shown in this study to be a sensitive marker of patient-centered, QOL issues that reflect the debilitating nature of refractory MG.

\section{PS3Group2-034 / \#281}

Topic: Group 2 - Diseases of Neuromuscular Junction: Clinical Features, Pathophysiology, Therapy

\section{VOCAL CORD PARALYSIS: A RARE PRESENTATION OF MYASTHENIA GRAVIS $€$}

Vera Montes, Sandra Sousa, Rui Guerreiro, Cátia Carmona, Fernando Pita

Neurology, Cascais Hospital, Alcabideche

(Cascais) - Lisboa, PT 
Background: Involvement of vocal cords as an initial presentation of myasthenia gravis (MG) in adults is extremely rare. Methods: We describe a case of vocal cord paralysis as initial manifestation of MG and perform a review of the cases of MG presenting with vocal cord paralysis reported in literature, using the Pubmed's MeSH database to localize the MeSH terms "Vocal Cord Paralysis" and "Myasthenia Gravis". Results: A 80-year-old caucasian woman, who was admitted to the emergency department for acute respiratory failure requiring invasive mechanical ventilation. Examination revealed dysphonia, dysphagia, dyspnea and an inspiratory stridor caused by an airway obstruction because of a bilateral paralysis in vocal cord abduction, confirmed by nasolaryngofibroscopy. A tracheostomy was performed. At that time muscle strength was normal. Cranial nerve examination failed to disclose ophthalmoparesis or ptosis. Sensory modalities were intact. After one month she developed limb muscle weakness with fatigability and a bilateral ophthalmoparesis. Her history included hypertension, type 2 diabetes and hypothyroidism. She had no previous exposure to alcohol and tobacco. There was no family history of neurologic or autoimmune disorders. Laboratory investigations revealed normal renal, liver and thyroid functions. Serum antibodies against acetylcholine receptors (AchR), muscle-specific kinase (MuSK) and low-density lipoprotein receptor-related protein (LRP4) were negative. Magnetic resonance of the head and neck, and chest computed tomography were unremarkable. Edrophonium test was negative. Repetitive nerve stimulation didn't show abnormal decremental response but single fiber electromyography confirmed dysfunction of the neuromuscular junction. She received intravenous immunoglobulin therapy and corticosteroid, without clinical benefit. Pyridostigmine was also ineffective. She was discharged, tracheostomized, with prednisolone $60 \mathrm{mg}$ a day and pyridostigmine $60 \mathrm{mg}$ four times a day. For lack of improvement, she started azathioprine three months later. After two months, there was a relapse with worsening of laryngopharyngeal symptoms. She initiated plasmapheresis with a partial improvement of dysphonia and dysphagia and muscle power, despite the need to maintain tracheostomy. She repeats the AchR and MuSK antibodies that were negative. Nine months after the initial symptoms, the patient died by asphyxia caused by obstruction of the upper airway by alimentary content. From the systematic review, we found 15 published cases of MG that presented with vocal cord paraly- sis, $60 \%$ female and $40 \%$ male, with a mean age of $57.0 \pm 18.0$ years. Acute respiratory failure was the form of presentation in $87 \%$ of patients, dysphonia in $47 \%$ and dysphagia in $47 \%$ of the cases. $33 \%$ eventually developed ocular symptoms and in $27 \%$ MG was generalized. Antibodies associated with MG were detected in $33 \%$ of the cases and $53 \%$ did not respond to pyridostigmine therapy. $87 \%$ of the patients required invasive mechanical ventilation and $67 \%$ tracheostomy, of which $50 \%$ maintained tracheostomy for more than 6 months. Conclusion: Orolaryngopharyngeal manifestations cause by upper airway obstruction, such as dysarthria, dysphonia or dysphagia are uncommon in MG, especially as presenting symptoms. This case demonstrates the importance of considering neuromuscular junction disorders in the approach to new onset acute respiratory failure due to with vocal cord paralysis.

\section{PS3Group2-035 / \#581}

Topic: Group 2 - Diseases of Neuromuscular Junction: Clinical Features, Pathophysiology, Therapy

\section{SERONEGATIVE MYASTHENIA GRAVIS WITH ANTI-LRP4 ANTIBODIES}

\author{
Jee Eun Kim${ }^{1}$, Kee Hong Park ${ }^{2}$, Hye Seon $\mathrm{Seo}^{3}$, \\ Jung-Joon Sung ${ }^{4}$, Yoon-Ho Hong ${ }^{5}$ \\ ${ }^{1}$ Department Of Neurology, Seoul Medical Center, \\ Seoul, KR; ${ }^{2}$ Neurology, Gyeongsang National \\ University Hospital, Jinju, KR, ${ }^{3}$ Medical Research \\ Institute, Seoul Medical Center, Seoul, \\ KR: ${ }^{4}$ Neurology, Seoul National University College \\ of medicine, Seoul University Hospital, Seoul, \\ $K R,{ }^{5}$ Seoul National University College of medicine, \\ SMG-SNU Boramae Medical Center, Seoul, KR
}

Background: Myasthenia gravis (MG) is an autoimmune disorder characterized by fluctuating muscle weakness. $85-90 \%$ of MG patients have auto-antibodies to postsynaptic proteins against the nicotinic acetylcholine receptor (AChR) or musclespecific tyrosine kinase (MuSK). Recently, anti-lowdensity lipoprotein receptor-related protein 4 (LRP4) antibodies were known to associated with MG pathogenesis in double-seronegative (seronegative to AchR and MUSK) patients. Methods: Here, we report one Korean patient who was double-seronegative, but had anti-LRP4 antibodies. Results: The 
57-year-old man with history of hypertension and coronary artery disease was admitted for limb weakness predominantly in lower part and fluctuating binocular diplopia which aggravated when tired. A neurological examination revealed mild lower extremities weakness in proximal and distal similarly (Medical Research Council grade 4+). There were no signs of weakness in the ocular, bulbar, neck muscles. His deep tendon reflexes were preserved with no upper motor neuron sign. In the result of routine blood tests, creatine kinase is slightly increased (276U/L; normal range 24-204 U/L) but other results including thyroid function and tumor marker were normal. The serum anti-AChR antibodies and anti-MuSK antibodies were negative. A chest computer tomography (CT) showed $1 \mathrm{~cm}$ sized nodule in anterior mediastinum suspecting thymoma. Repetitive nerve stimulation test (RNST) of the right orbicularis oculi, trapezius, abductor digiti quinti and flexor carpi ulnaris muscles showed abnormal decremental responses which is consistent with MG. Nerve conduction study was normal. Electromyography showed evidence of active myopathy and muscle biopsy from the left vastus lateralis muscle was consistent with mitochondrial myopathy. AntiLRP4 antibody was tested with his serum by cell based assay based on human LRP4 expressing HEK293 cells and revealed to positive. The administration of an oral cholinesterase inhibitor and steroid was initiated, and his weakness moderately improved. Conclusion: Clinical characteristics of anti-LRP4 antibody associated MG are not well known until now. Several studies report that antiLRP4 antibody positive MG patients to have lateonset age, mild symptoms, good therapeutic response, and no thymic changes. Our case with double-seronegative, anti-LRP4 antibody positive MG patient also showed symptom onset in late age and mild weakness without ocular symptom. Testing for anti-LRP4 antibodies need to be considered when the clinical suspicion for myasthenia gravis with mild symptom, but negative conventional diagnostic tests.
PS3Group2-036 / \#666

Topic: Group 2 - Diseases of Neuromuscular Junction: Clinical Features, Pathophysiology, Therapy

\section{INHIBITION OF ACETYLCHOLINE RECEPTOR FUNCTION BY MYASTHENIA GRAVIS SERA DEPENDS ON RECEPTOR CLUSTERING}

$\underline{\text { Hakan Cetin }}^{1}$, Richard Webster ${ }^{2}$, Wei Liu ${ }^{2}$, Inga Koneczny $^{3}$, Friedrich Zimprich ${ }^{4}$, Judith Cossins ${ }^{2}$, David Beeson ${ }^{2}$, Angela Vincent ${ }^{2}$

${ }^{1}$ Department Of Neurology, Medical University of Vienna, Vienna, $A T ;{ }^{2}$ Nuffield Department Of Clinical Neurosciences, University of Oxford, Oxford, GB, ${ }^{3}$ Institute Of Neurology, Medical University of Vienna, Vienna, AT; ${ }^{4}$ Department Of Neurology, Medical University of Vienna, Vienna, AT

Background: Myasthenia gravis (MG) is an autoimmune disorder of the neuromuscular junction clinically associated with fatigue and weakness. Autoantibodies directed against the acetylcholine receptor (AChR) can be detected in about $80 \%$ of $\mathrm{MG}$ cases. Other antibodies (Abs) bind only to AChRs that are clustered on a cell surface, or to MuSK, LRP4 or unidentified antigens (i.e. seronegative MG, SNMG). Some of the sera from MG patients including those from patients with seronegative $\mathrm{MG}$ were shown to inhibit AChR function. However, all studies were performed before the identification of MuSK- or clustered AChR-Abs. Our aim here was to perform a comprehensive functional evaluation of the effects of sera with AChR-, clustered AChR- or MuSK-Abs, or of sera from SNMG patients, first on unclustered AChRs and then on clustered AChRs. Methods: The CN21 muscle cell line expresses unclustered AChR and was transfected with rapsyn to enable receptor clustering. The whole-cell patch clamp technique was used for electrophysiological analyses. Serum application and rapid AChR stimulation was achieved using a fast perfusion system. Five AChR stimulations with the cells in physiological solution were followed by five AChR stimulations in the presence of serum (with one stimulation every 25s). The reduction of AChR current amplitudes during the perfusion with MG serum was measured and compared to healthy controls (HC). For MG sera, any current reduction higher than 3 standard deviations from $\mathrm{HC}$ mean values was consid- 
ered to denote a functionally blocking serum. Results: The mean current reduction of five $\mathrm{HC}$ sera was $-8.8 \pm 1.2 \%$ in unclustered AChRs and $-8.0 \pm$ $1.9 \%$ in clustered AChRs (mean \pm SEM). Unclustered AChRs were blocked by $40 \%$ of the sera in the AChR-Ab group ( $n=5)$, by $16.7 \%$ of the sera in the clustered AChR-Ab group ( $\mathrm{n}=6)$, by $20 \%$ of the sera in the MuSK-Ab group $(n=5)$ and also by $40 \%$ of the sera in the SNMG group $(n=5)$. Clustered AChRs were only blocked by AChR-Ab sera (40\%) and clustered AChR-Ab sera (66.7\%) and by none of the MuSK or SNMG sera. Conclusion: We provide evidence that sera with clustered AChR-Abs exhibit a blocking effect predominantly on clustered AChRs, with $50 \%$ of the sera inhibiting AChR function only when the receptors are clustered. Surprisingly, a significant proportion of SNMG sera were also associated with a blocking effect but only when unclustered AChRs were examined. This suggests a yet unknown pathogenic serum factor in some of the SNMG sera.

\section{PS3Group2-037 / \#601}

Topic: Group 2 - Diseases of Neuromuscular Junction: Clinical Features, Pathophysiology, Therapy

\section{ECULIZUMAB SHOWS CONSISTENCY OF IMPROVEMENT ACROSS MUSCLE GROUPS IN PATIENTS WITH ACHR-POSITIVE REFRACTORY MYASTHENIA GRAVIS}

$\underline{\text { Renato Mantegazza }}^{1}$, Kenji P. Fujita ${ }^{2}$, Fanny

$\mathrm{O}^{\prime}$ Brien $^{2}$, James F. Howard $\mathrm{Jr}^{3}$

${ }^{1}$ Fondazione IRCCS Istituto Neurologico Carlo Besta, Milan, IT; ${ }^{2}$ Alexion Pharmaceuticals, New Haven, CT, US: ${ }^{3}$ University of North Carolina, Chapel Hill, NC, US

Background: The quantitative myasthenia gravis (QMG) test was a key prospectively-defined effica- cy measure in REGAIN, a 26-week, phase 3, randomized, double-blind, placebo-controlled study of patients with anti-acetylcholine receptor antibodypositive refractory generalized myasthenia gravis (MG). Ocular and generalized weakness have previously shown different degrees of response to various therapies (prednisone has shown the most benefit for ocular symptoms; intravenous immunoglobulin/ plasma exchange has shown most benefit for generalized symptoms). In REGAIN, eculizumab showed a consistent trend toward rapid and sustained improvement across all four domains (bulbar, respiratory, limb and ocular) in the patient-reported $\mathrm{MG}$ activities of daily living (MG-ADL) scale. We examined the effect of eculizumab on different muscle groups (bulbar, respiratory, gross motor and ocular) during REGAIN using QMG, an objective, physician-reported measure of muscle strength. Methods: Changes from baseline to week 26 in QMG domain scores were measured in patients with abnormal QMG domain scores at baseline. Repeatedmeasures analyses of QMG scores for bulbar (swallowing and speech), respiratory (forced vital capacity), gross motor (limb and axial motor items) and ocular (ocular and facial muscles) domains were performed. Results: Eculizumab-treated patients showed improvements in all QMG domain scores from baseline to week 26 in REGAIN (Figure). Repeated-measures analyses (least-squares means, standard error of mean) showed rapid, sustained improvements across all four QMG domains, with a trend towards significant differences between eculizumab and placebo at week 26 (bulbar, $p=0.0628$; respiratory, $p=0.0682$; gross motor, $p=0.0114$; ocular, $p=0.0017)$. The safety profile of eculizumab was consistent with its known profile. Conclusion: In contrast to other therapies reporting differential effects for different muscle groups, eculizumab showed a consistent clinical response across all muscle groups (bulbar, respiratory, gross motor and ocular) using QMG assessments. This is consistent with previously reported MG-ADL results (NCT01997229, NCT02301624). 

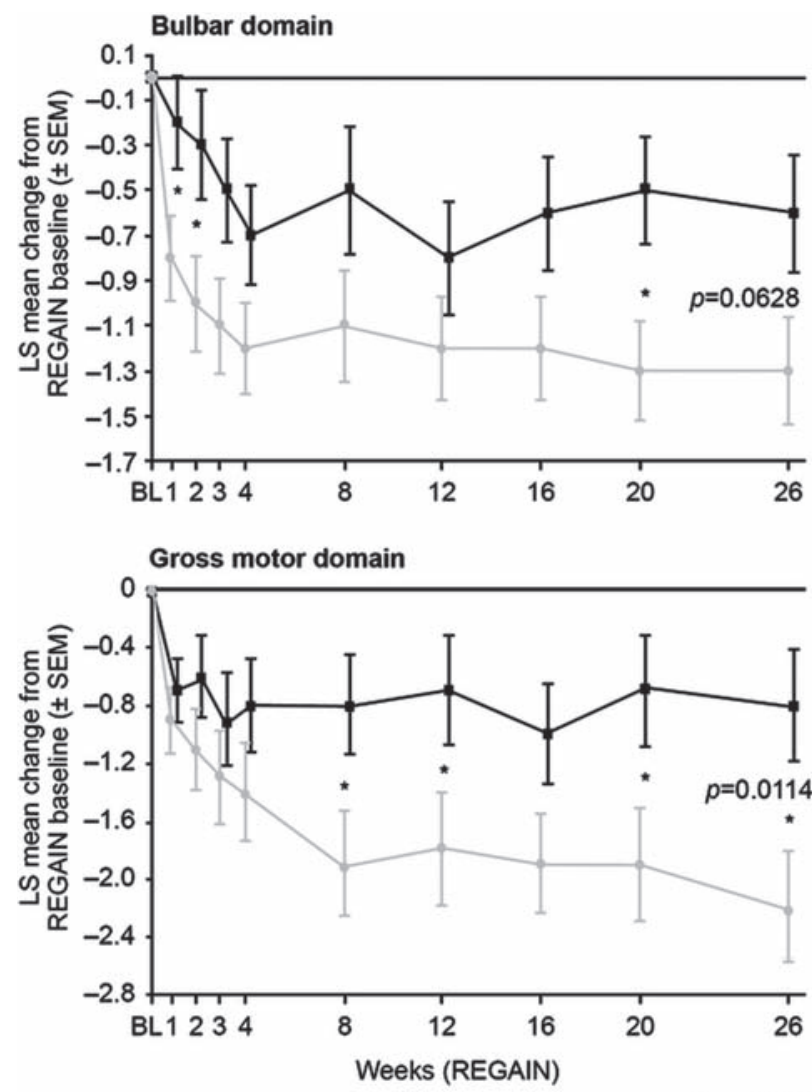

PS3Group2-038 / \#649

Topic: Group 2 - Diseases of Neuromuscular Junction: Clinical Features, Pathophysiology, Therapy

\section{ACHIEVEMENT OF MINIMAL MANIFESTATIONS IN ECULIZUMAB-TREATED ACHR- POSITIVE REFRACTORY MYASTHENIA GRAVIS PATIENTS}

Renato Mantegazza $^{1}$, Gil Wolfe ${ }^{2}$, Srikanth Muppidi $^{3}$, Heinz Wiendl ${ }^{4}$, Kenji P. Fujita ${ }^{5}$, Fanny O'Brien ${ }^{5}$, James F. Howard $\mathrm{Jr}^{6}$

${ }^{1}$ Fondazione IRCCS Istituto Neurologico Carlo Besta, Milan, IT; Jacobs School of Medicine and Biomedical Sciences, University at Buffalo, Buffalo, NY, US; ${ }^{3}$ Stanford University School of Medicine, Stanford, CA, US: ${ }^{4}$ University Hospital Münster, Münster, DE: ${ }^{5}$ Alexion Pharmaceuticals, New Haven, CT, US $;{ }^{6}$ University of North Carolina, Chapel Hill, NC, US

Background: Patients with anti-acetylcholine receptor positive $(\mathrm{AChR}+)$ refractory generalized
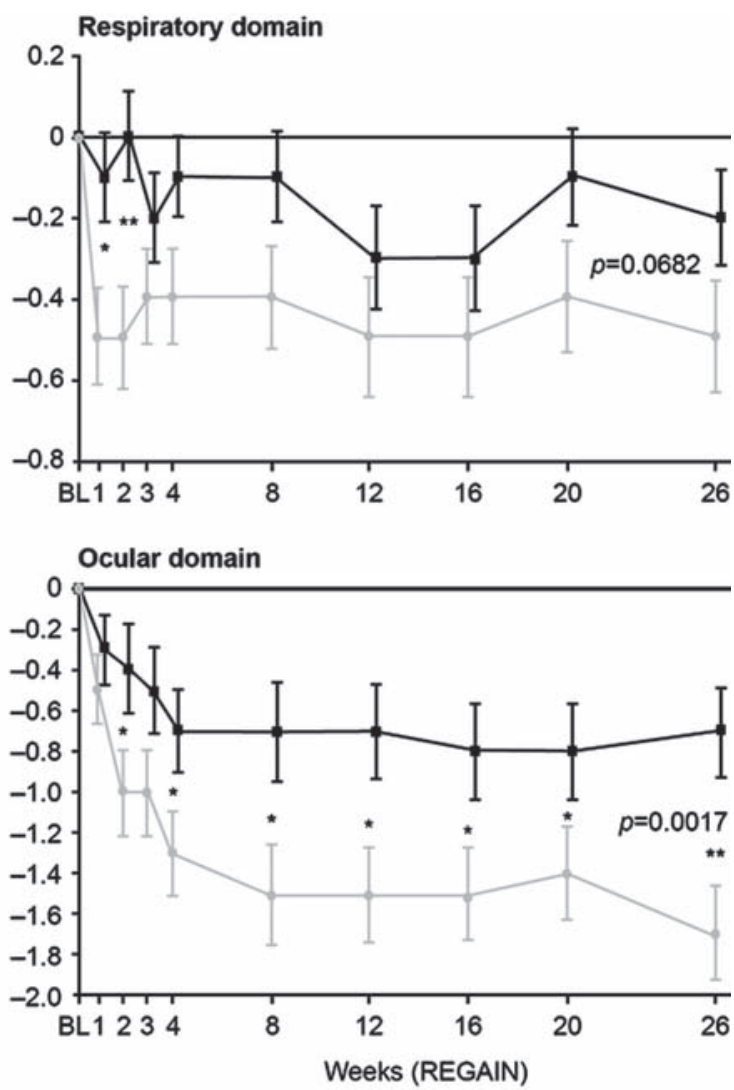

$\longrightarrow$ ECU

myasthenia gravis (gMG) continue to experience significant unresolved disease symptoms and increased risk of exacerbations and hospitalizations, despite multiple therapies. We investigated if eculizumab helps this population achieve Minimal Manifestations as evaluated by the MG Foundation of America (MGFA) Post-interventional Status.

Methods: Patients were enrolled in REGAIN, a 26week, phase 3, randomized, double-blind, placebocontrolled study of the safety and efficacy of eculizumab in patients with AChR + refractory $\mathrm{gMG}$. Patients who completed REGAIN could enroll into the open-label extension study. Post-interventional Status, including achievement of Minimal Manifestations, was assessed at visits 4, 12 and 26 of REGAIN and week 26 of the open-label study. The sub-categories of Minimal Manifestations (MM-0, 1, 2 or 3) were not used. Results: At week 26 of REGAIN, 34/56 eculizumab-treated patients (61\%) had a Post-interventional Status of Improved and 14 (25\%) achieved Minimal Manifestations; 25/59 placebo-treated patients $(42 \%)$ had a status of Improved, with $8(14 \%)$ achieving Minimal Manifestations (Figure). Improvements from REGAIN baseline to week 26 of the open-label study 


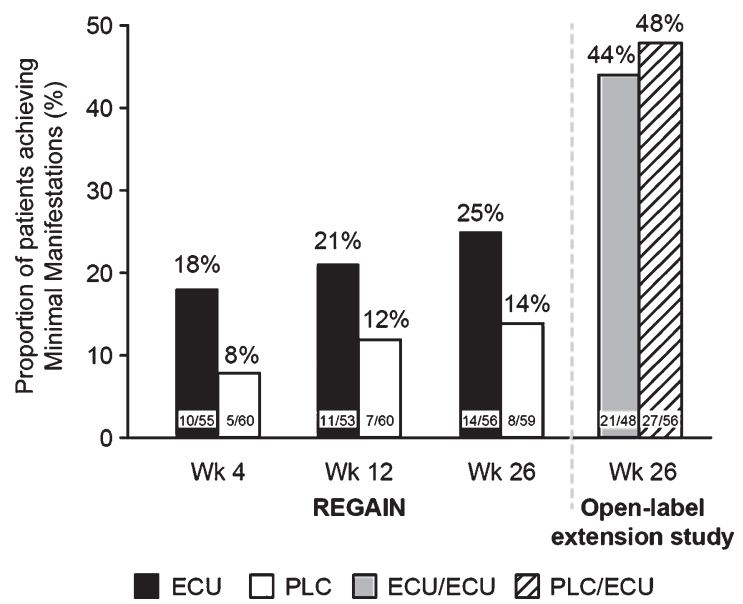

(September 2016 cut-off) were similar between the placebo/eculizumab and eculizumab/eculizumab groups. A Post-interventional Status of Improved was achieved by 75/104 (72\%) patients (eculizumab/eculizumab, 35/48 [73\%]; placebo/eculizumab, 40/56 [71\%]). Minimal Manifestations status was achieved by 48/104 (46\%) patients (eculizumab/eculizumab, 21/48 [44\%]; placebo/eculizumab, 27/56 [48\%]). The long-term safety profile of eculizumab was consistent with that previously reported. Conclusion: In REGAIN, eculizumab treatment was associated with a higher proportion of patients achieving Minimal Manifestations, compared with placebo. By week 26 of the open label study, a similar proportion of patients had achieved Minimal Manifestations in the eculizumab/eculizumab and placebo/eculizumab groups (NCT01997229, NCT02301624).

\section{PS3Group4-001 / \#512}

Topic: Group 4 - Motor Neuron Diseases: Clinical Features, Pathophysiology, Therapy

\section{INCREASED GLUCOSE}

\section{FLUCTUATION IN MOTOR NEURON DISEASE DURING ENTERAL NUTRITION}

Hiroto Takada ${ }^{1}$, Seiko Kon ${ }^{1}$, Yumiko Ogasawara ${ }^{2}$, Toshiko Ota ${ }^{3}$

${ }^{1}$ Neurology, Aomori National Hospital, National Hospital Organization, Aomori, JP: ${ }^{2}$ Medical Technology, Aomori National Hospital, National Hospital Organization, Aomori, JP, ${ }^{3}$ Nursing, Aomori National Hospital, National Hospital Organization, Aomori, JP
Background: Skeletal Muscle is a primary site for glucose uptake and storage, and the muscle loss influences glucose metabolism. Increased glucose excursion is reported during enteral nutrition (EN) therapy. Patients with motor neuron disease (MND) in advanced stage develop severe muscle atrophy and require EN. The aim of the study was to elucidate daily glucose profiles in MND patients with EN minutely using continues glucose monitoring (CGM). Methods: Five patients with MND were participated (the mean age; 63.0 years, the mean duration of disease; 10.4 years, five male patients). All patients were bedridden and under the artificial ventilator management with tracheotomy, having EN therapy with gastrogavage. None had a past history of diabetes mellitus (DM). CGM was performed for 72 hours. Oral glucose tolerance test (OGTT) was carried out at the last part of the CGM recording. Results: Three of five patients were diagnosed as $\mathrm{DM}$ and one as borderline of glucose intolerance by OGTT, in spite of normal HbA1c (the mean; 5.0\%) and fasting plasma glucose (the mean; $90.4 \mathrm{mg} / \mathrm{dl}$ ) level. Two patients without DM and one DM patient showed hypoglycemia. Regarding CGM profiles, hypoglycemia was observed like excessive reaction subsequently to the peak of the sharp rising glucose value after the infusion of enteral nutrient, frequenty from about four hours after the administration in the evening to midnight. Conclusion: In MND patients with severe muscle atrophy during EN management, glucoe fluctuation is increased, and hyperglycemia or hypoglycemia is not rare. Careful observation is necessary for the nutritional management in advanced patients with NMD.

\section{PS3Group4-002 / \#745}

Topic: Group 4 - Motor Neuron Diseases: Clinical Features, Pathophysiology, Therapy

\section{PD AND SAFETY DATA FROM JEWELFISH, A STUDY OF RG7916 IN SMA PATIENTS PREVIOUSLY ENROLLED IN A SMN2-SPLICING MODIFIER STUDY}

Claudia A. Chiriboga ${ }^{1}$, Eugenio Mercuri², Dirk Fischer $^{3}$, Dominik Kraus ${ }^{4}$, Norman Thompson ${ }^{4}$, Gillain Armstrong ${ }^{5}$, Heidemarie Kletzl ${ }^{6}$, Marianne Gerber $^{6}$, Yumi Cleary ${ }^{6}$, Tobias Bergauer ${ }^{6}$, Ksenija Gorni $^{6}$, Omar Khwaja ${ }^{6}$ 
${ }^{1}$ Department Of Neurology, Columbia University Medical Center, New York, US, ${ }^{2}$ Paediatric

Neurology And Nemo Center, Catholic University and Policlinico Gemelli, Rome, IT; ${ }^{3}$ Department of Neurology, University of Basel Hospital, Basel, $\mathrm{CH} ;{ }^{4}$ Roche Pharmaceutical Research and Early Development, Roche Innovation Center, Basel, CH; ${ }^{5}$ Roche Products Ltd, Welwyn Garden City, $G B ;{ }^{6}$ Roche Pharmaceutical Research and Early Development, Roche Innovation Center, Basel, $\mathrm{CH}$

Background: Spinal muscular atrophy (SMA) is characterized by motor neuron loss and muscle atrophy due to reduced levels of survival of motor neuron $(\mathrm{SMN})$ protein from loss of function of the SMN1 gene. RG7916 (RO7034067) is an investigational, orally administered, centrally and peripherally distributed small molecule that is being evaluated for its effect to modulate $S M N 2$ pre-mRNA splicing towards the production of full-length SMN2 mRNA and increase of SMN protein. JEWELFISH (NCT03032172) is a multi-center, open-label, exploratory study evaluating the safety, tolerability and pharmacokinetics of daily oral RG7916 in patients with SMA Type 2 or 3, aged 12-60 years, who previously participated in a study with therapies targeting SMN2 splicing. The pharmacodynamic effects on SMN2 mRNA and SMN protein are also assessed. Methods: At the time of abstract submission, 10 patients have received RG7916 for a minimum of 2 months, and up to 11 months, duration as part of the JEWELFISH study. Results: To date, no drug-related adverse events leading to withdrawal have been reported. Preliminary pharmacodynamic data in whole blood from these 10 patients receiving RG7916 showed a rapid increase in the full-length SMN2/SMN2 47 mRNA ratio after treatment initiation, with up to a 4-fold increase from baseline over 4 weeks. This resulted in an up to 4 -fold SMN protein increase versus baseline after 4 weeks of treatment. A JEWELFISH update, including safety and biomarker data, will be presented. Conclusion: In JEWELFISH, oral RG7916 treatment modulated SMN2 mRNA and increased SMN protein. While the number of patients in the JEWELFISH study is limited, the magnitude of the SMN protein increase and the safety observations thus far are comparable to what is seen in the SUNFISH Part 1 study (NCT02908685) with patients who have not previously received an SMN2-targeting therapy. JEWELFISH is currently recruiting in sites in Europe and the US. Acknowledgments The authors would like to thank all individuals enrolled in the RG7916 studies, their families and the site staff involved. Study sponsored by F. Hoffmann-La Roche.

\section{PS3Group4-003 / \#774}

Topic: Group 4 - Motor Neuron Diseases: Clinical Features, Pathophysiology, Therapy

\section{MLPA BASED SMN1 DELETION ANALYSIS: CLINICAL CORRELATION IN INDIAN PATIENTS WITH SPINAL MUSCULAR ATROPHY}

Hansashree Padmanabha ${ }^{1}$, Veeramani Preethish$\underline{\text { Kumar }}^{2}$, Kiran Polavarapu ${ }^{2}$, Saraswati Nashi ${ }^{3}$, Seena Vengalil ${ }^{2}$, Deepah Sekar $^{4}$, Atchayaram Nalini ${ }^{5}$, Priya Thomas ${ }^{6}$, Krishna Gk $^{6}$ ${ }^{1}$ Neurology, Nimhans, Bengaluru, Bengaluru, IN $;{ }^{2}$ Neurology, National Institute of Mental Health and Neurosciences, Bengaluru, IN $;^{3}$ NIMHANS, Bengaluru, IN; ${ }^{4}$ NIMHANS, Bengaluru, Bengaluru, IN: $;^{5}$ Neurology, National Institute of Mental Health and Neurosciences, IN; ${ }^{6}$ Neurology, NIMHANS, Bengaluru, Bengaluru, IN

Background: SMA is caused due to mutation of survival motor neuron $1(S M N$ 1) gene located on chromosome 5q13. About $95-98 \%$ are homozygous for SMN1 deletion or gene conversion of SMN1 to $S M N 2$ and $2-5 \%$ are compound heterozygotes or have an intragenic SMN1 mutation. The objective of this study is to describe the utility of multiplex ligation-dependent probe amplification technique (MLPA) in the genetic diagnosis of SMA and correlate it clinically in a large cohort from India. Methods: It was a retrospective study conducted from a single quaternary care center for neurological disorders. Medical records of the patients who attended the neuromuscular clinic, during October 2012 to December 2018, and genetically confirmed to have SMA based on MLPA, were included. MLPA was used to detect deletions in exon 7 and exon 8 of the SMN1 gene using SALSA MLPA P034 and P035 probe sets(MRC Holland, Netherlands) as per manufacturer's instructions. Patient's demographics, clinical features, type of SMA as per international SMA consortium criteria, and the site of deletion(exon 7, exon 8 or both) were recorded. Results: During the study period a total of 96 patients were genetically 


\begin{tabular}{lccccc}
\hline Variables & SMA 1 (N=39) & SMA 2 (N=21) & SMA 3a (N=14) & SMA 3b (N=19) & SMA 4 (N=3) \\
\hline Age at presentation in years (Mean $\pm \mathrm{SD})$ & $3.38 \pm 4.21$ & $5.52 \pm 4.45$ & $11.14 \pm 6.07$ & $18.68 \pm 9.06$ & $33.67 \pm 9.29$ \\
Age at onset in months (Mean $\pm \mathrm{SD})$ & $0.21 \pm 0.92$ & $12.05 \pm 3.95$ & $30.43 \pm 5.98$ & $104.2 \pm 43.4$ & $268 \pm 30.1$ \\
Total duration of illness (Mean $\pm \mathrm{SD})$ & $33.85 \pm 47.7$ & $56.24 \pm 51.62$ & $104.57 \pm 72.02$ & $117.47 \pm 100$ & $132 \pm 135.2$ \\
Sex (M: F) & $15: 24$ & $11: 10$ & $11: 3$ & $15: 4$ & $3: 0$ \\
Initial presentation Bulbar [N (\%)] Hypotonia & $14(36 \%) 25$ & $1(4.8 \%) 20$ & None 14 (100\%) & None 17 (89.5\%) & None None 3 \\
[N (\%)] Gait disturbances [N (\%)] & $(64 \%)$ None & $(95.2 \%)$ None & None & $2(10.5 \%)$ & $(100 \%)$ \\
Attained sitting [N(\%)] & $20(51 \%)$ & $18(85.7 \%)$ & $14(100 \%)$ & $19(100 \%)$ & $3(100 \%)$ \\
Attained walking [N(\%)] & None & $13(62 \%)$ & $14(100 \%)$ & $19(100 \%)$ & $3(100 \%)$ \\
Tongue fasciculation's [N(\%)] & $12(31 \%)$ & $7(33 \%)$ & $7(50 \%)$ & $2(11 \%)$ & None \\
Calf hypertrophy [N(\%)] & $4(10.3 \%)$ & $2(9.5 \%)$ & $5(35.7 \%)$ & $12(63.2 \%)$ & $2(66.7 \%)$ \\
Kyphoscoliosis [N(\%)] & $3(7.7 \%)$ & $3(14.3 \%)$ & $5(35.7 \%)$ & $3(15.8 \%)$ & None \\
Arthrogryposis [N(\%)] & None & $1(4.8 \%)$ & None & None & None \\
Elbow contractures [N(\%)] & $1(2.6 \%)$ & $3(14.3 \%)$ & None & $1(5.3 \%)$ & None \\
Wrist contractures [N(\%)] & $1(2.6 \%)$ & None & None & None & None \\
Hip contractures [N(\%)] & $5(12.8 \%)$ & $2(9.5 \%)$ & None & $2(10.5 \%)$ & None \\
Ankle contractures [N(\%)] & $17(43.6 \%)$ & $14(66.7 \%)$ & $11(78.6 \%)$ & $15(78.9 \%)$ & $2(66.7 \%)$ \\
Creatine kinase (Mean $\pm \mathrm{SD})$ & $177.67 \pm 74.8$ & $175.2 \pm 85.3$ & $305.9 \pm 174.4$ & $714 \pm 708.4$ & 220 \\
Isolated exon 7 deletion [N(\%)] & $7(18 \%)$ & $5(24 \%)$ & $2(14 \%)$ & $8(42 \%)$ & $2(67 \%)$ \\
Isolated exon 8 deletion [N(\%)] & None & None & None & None & None \\
Combined exon 7 plus exon 8 deletion [N(\%)] & $33(84.6 \%)$ & $16(76.2 \%)$ & $13(93 \%)$ & $11(58 \%)$ & $1(33 \%)$ \\
\hline
\end{tabular}

confirmed to have SMA based on MLPA. It comprised of 55 boys and 41 girls. Thirty-nine patients $(40.6 \%)$ belonged to type 1 ; twenty-one(22\%) to type 2 , fourteen(14.6\%) to type $3 \mathrm{a}$, nineteen $(19.8 \%)$ to type $3 \mathrm{~b}$ and three patients(3.1\%) with ages 26, 31 and 44years belonged to type 4-SMA. Consanguinity was noted in twenty-eight families, along with affected sibling in eight patients. Tongue fasciculation was prominent in type 1, type 2 and type 3 a SMA; whereas calf hypertrophy was marked in type $3 \mathrm{~b}$ and type 4 SMA patients. MLPA analysis revealed combined deletions of exon 7 and 8 in seventy-four patients (77\%), and isolated exon 7 deletions in twenty-four patients $(25 \%)$. Isolated exon 8 deletion wasn't present in any case. The detailed results are described in table 1. Conclusion: In the study cohort, majority of type 1 and type 2 SMA patients had combined deletion of exon 7 plus exon 8 . Nonetheless, in a minority of patients with type 1 and type 2 SMA, isolated exon 7 deletion was found. The mutational spectrum in type 3 SMA differed in the sense, those who had isolated exon 7 deletion had a milder type $3 \mathrm{~b}$ SMA, those with combined had type 3a SMA. Isolated exon 8 deletions probably aren't pathogenic and doesn't cause SMA.

\section{PS3Group4-004 / \#464}

Topic: Group 4 - Motor Neuron Diseases: Clinical Features, Pathophysiology, Therapy

\section{ACUTE MOTOR NEURONOPATHY ASSOCIATED TO AN INFECTION BY TICK-BORNE ENCEPHALITIS VIRUS}

Lisa Bauer ${ }^{1}$, Marie_Line Harlay ${ }^{2}$, Andoni EchanizLaguna $^{1}$, Raoul Herbrecht ${ }^{3}$, Vincent Castelain ${ }^{2}$, Jean-Baptiste Chanson ${ }^{4}$

${ }^{1}$ Neurologie, Hôpitaux Universitaires de Strasbourg, STRASBOURG, FR, ${ }^{2}$ Réanimation, Hôpitaux Universitaires de Strasbourg, STRASBOURG, FR $;{ }^{3}$ Hématologie, Hôpitaux Universitaires de Strasbourg, STRASBOURG, FR: ${ }^{4}$ Neurologie, Hôpitaux Universitaires de Strasbourg, STRASBOURG, FR

Background: The infection by the Tick-Borne Encephalitis (TBE) flavivirus is usually associated with various brain lesions. We report here a case of TBE infection resulting in a motor neuronopathy. Methods: A 60-year-old man, often bitten by ticks in 
France and Germany, was addressed for fever and headaches followed by left hemiparesis and disorder of consciousness. His medical history only included an untreated mild chronic lymphocytic leukemia. Brain MRI showed a diffuse leptomeningeal contrast enhancement and analysis of cerebrospinal fluid (CSF) found a lymphocytic meningitis (595 lymphocytes per $\mathrm{mm} 3, \mathrm{~N}<5$ ) and an increased proteinorachy $(1,51 \mathrm{~g} / \mathrm{L}, \mathrm{N}<0,45)$. IgM but not IgG antibodies against TBE virus were discovered in CSF and serum. Borrelia burgdorferi serology was negative. A few days later, a tetraparesis with abolition of tendinous reflexes but preserved sensory function appeared. A first electroneuromyographic examination (ENMG) found markedly decreased compound muscle action potential (CMAP) amplitudes in four limbs without alteration of sensory parameters. Acute denervation was present in needle examination of proximal and distal muscles. Level of consciousness progressively improved but respiratory impairment and motor deficit predominant in upper limbs persisted. A new ENMG examination one month later found a worsening of CMAP amplitudes and denervation in proximal and distal muscles of upper limbs. A spinal cord MRI showed a hypersignal of cervical and thoracic grey matter. Results: The association of purely motor clinical and ENMG abnormalities involving proximal and distal muscles suggested an acute motor neuronopathy. Inflammation of grey matter in spinal cord confirmed that the ingoing TBE infection resulted in anterior horn damages. This case was reminiscent of abnormalities caused by poliomyelitis (polio= grey) virus or West Nile virus. Anterior horn damages have been rarely reported with other viruses. Up to now, no case associated with TBE virus was described. Conclusion: TBE virus infection may be associated with an acute motor neuronopathy and should be evoked in the context of acute motor deficit with abolished reflexes during a lymphocytic meningitis.
PS3Group4-005 / \#647

Topic: Group 4 - Motor Neuron Diseases: Clinical Features, Pathophysiology, Therapy

\section{ASSOCIATION OF AMYOTROPHIC LATERAL SCLEROSIS AND MULTIPLE SCLEROSIS: A CASE REPORT}

\author{
Bruno K. Vitturi ${ }^{1}$, Charles P. Tilbery², Wilson L. \\ Sanvito $^{1}$ \\ ${ }^{1}$ Neurology, Faculdade de Ciências Médicas da \\ Santa Casa de São Paulo, São Paulo, BR $;^{2}$ Centro \\ De Atendimento E Tratamento De Esclerose \\ Múltipla (catem), Faculdade de Ciências Médicas \\ da Santa Casa de São Paulo, São Paulo, BR
}

Background: Amyotrophic lateral sclerosis (ALS) and multiple sclerosis (MS) are two different idiopathic neurological disorders. Occurrence of both ALS and MS in the same patient is described as extremely rare in literature; its likelihood is estimated to be $3: 10$ billion. Methods: We present a case of man diagnosed with concurrent multiple sclerosis and amyotrophic lateral sclerosis. Results: The case we present concerns a 48 -year-old male patient that in 2011 presented a left lower limb weakness with distal onset associated with a low back pain. Two years later the patient evolved with weakness and pain in right lower limb and finally in 2015 the patient was admitted to our hospital presenting an upper left limb paresis with a proximal onset associated with dysphagia and dysphonia. Neurological examination also found monoplegia in left lower limb and weakness grade $2 / 5$ in lower right limb. There was a substantial hand hypotrophy as well. No deficit of sensation or coordination was present. No accompanying or relevant previous diseases were described by the patient and no history of neurological disorders was known in his family. Serologies and blood analysis were normal. Brain MRI revealed a typical MS pattern with numerous inflammatory bilateral lesions characterized by T2-weighted hypersignal foci with slight post-contrast enhancement spread through fronto-parietal white matter, brain stem, midbrain, cerebellum and thalamus. Cerebrospinal fluid (CSF) analysis showed 4 leucocytes $/ \mu \mathrm{L}(88 \%$ lymphocytes), protein $49 \mathrm{mg} / \mathrm{dl}$, glucose $71 \mathrm{mmol} / \mathrm{L}$ and positive oligoclonal bands (OCB). A diagnosis of MS was entertained. EMG showed chronic signs of bilateral myotome involvement corresponding to 
bulbar, cervical, thoracic and lumbosacral segments that associated with normal sensory conduction localized the involvement of the lower motor neuron, what supported the diagnosis of ALS. The patient was treated with Riluzole and Glatiramer Acetate. Our patient developed clinical and pathological features consistent with separate diagnoses of MS and ALS. MRI abnormalities exceed substantially the signal alterations usually described in ALS. Detection of OCB and the clinical course of the neurological deficits suggest MS as well. On the other hand, our patient didn't present sensory deficits, what sustain the diagnosis of ALS since in MS it is one of the most common symptoms. In addition, high-grade peripheral paresis and amyotrophy are not supposed to be relevant in MS, as MS is considered to be limited to central nerve system. Coexistence of ALS and MS may be due to random coincidence, common pathway in both diseases or genetic and exogenous factors, leading to an increased predisposition for neurodegeneration. Conclusion: The association of multiple sclerosis and amyotrophic lateral sclerosis is very rare and represents a diagnostic and therapeutic challenge. This case may indicate that these two neurodegenerative diseases may have some point in common in pathophysiology. Further studies are needed for better understanding this association.

\section{PS3Group4-006 / \#733}

Topic: Group 4 - Motor Neuron Diseases: Clinical Features, Pathophysiology, Therapy

\section{EXPLORATION OF THE REPOSITIONING POTENTIAL OF MARKETED DRUGS FOR NEUROPATHIES}

Jacqueline Gide ${ }^{1}$, Florine Roussange ${ }^{2}$, Hélène Polvèche $^{1}$, Didier Auboeuf ${ }^{3}$, Anne Boland ${ }^{4}$, Jean-François Deleuze ${ }^{4}$, Borckman Knut ${ }^{5}$, Johanna Tournois $^{1}$, Marc Peschanski ${ }^{6}$, Cecile Martinat ${ }^{7}$, $\underline{\text { Sandrine Baghdoyan }}^{2}$

${ }^{1}$ I-stem, CECS, CORBEIL ESSONNES, FR; ${ }^{2}$ Motor Neurone Disease, INSERM/UEVE UMR 861, I-STEM, Corbeil-Essonnes, FR $;^{3}$ Laboratoire De Biologie Et Modélisation De La Cellule,, ENS de Lyon, Lyon, FR ${ }^{4}$ Centre National De Genotypage, CEA, EVRY, FR; ${ }^{5}$ Interdisciplinary Pediatric center for children with developmental disabilitiesand severe chronic disorders, Gottingen, DE; ${ }^{6}$ I-stem

Cecs, INSERM UEVE UMR861, CORBEIL

ESSONNES, FR ; ${ }^{7}$ I-Stem, INSERM UEVE UMR861, CORBEIL ESSONNES, FR

Background: Human embryonic stem cells and their progenies are powerful models for drug discovery studies. Their amplification and differentiation allow reaching a large amount of well characterized cells in cell type of interest. Taking advantage of this property, we proceed to the screeing of 50 marketed drugs in derivatives from human pluripotent stem cells in order to identfy drugs that could have a potential therapeutic effect for rare monogenic diseases. Methods: A human pluripotent stem cell line was differentiated in Mesenchymal Stem Cells (MSC) and treated with 50 marketed drugs at the dose of $10 \mu \mathrm{M}$ for 24 hours in triplicates. The modulation of genes after drug treatment was analyzed by deep RNA sequencing approach. Among the regulated genes, we focussed on those whose modulation could present a potential therapeutic effect for rare monogenic diseases. Results: In MSC, we have identified drugs that are able to induce the expression of SQSTM1 whose mutation is responsible of Amyotrophic lateral sclerosis (ALS), and to decrease the level of expression of $L M N B 1$ and PLP1 genes whose duplication is causative for Adult-onset autosomal dominant leukodystrophy (ALDL) and Pelizaeus-Merzbacher disease (PMD) respectively. We then undertook the validation of these regulations in the cell type affected in the disease and in a pathological cellular model. Conclusion: By the use of an unbiaised RNA deep sequencing approach and human pluripotent stem cell lines, we have identified two marketed drugs with a therapeutic potential for three rare diseases through the modulation of $L M N B 1, P L P 1$ and SQSTM1 genes expression. 
PS3Group4-007 / \#527

Topic: Group 4 - Motor Neuron Diseases: Clinical Features, Pathophysiology, Therapy

\section{DISSECTING UBA5 DYSFUNCTION IN NERVOUS SYSTEM DISORDERS USING THE ZEBRAFISH}

$\underline{\text { Rita Serrano }}^{1}$, Viola Oorschot ${ }^{2}$, Georg Ramm², Robert Bryson-Richardson ${ }^{1}$

${ }^{1}$ School Of Biological Sciences, Monash University, Melbourne, VIC, AU; ${ }^{2}$ Ramaciotti Centre For Cryo-electron Microscopy, Monash University, Melbourne, VIC, AU

Background: Mutations in the ubiquitin-like modifier activating enzyme 5 (UBA5) gene were recently identified to cause childhood-onset cerebellar ataxia and infantile-onset epileptic encephalopathy, disorders predominantly associated with the central nervous system. Cerebellar ataxia patients show cerebellar atrophy, motor dysfunction, and mild peripheral demyelination; whereas, the clinical symptoms associated with epileptic encephalopathy include reduced motor skills, developmental delay, intellectual disability, microcephaly, delayed brain myelination, and recurrent seizures, ultimately resulting in the death of most patients within the first 2 decades of life. The function of Uba5 in the nervous system and how mutations in $u b a 5$ result in disease remains to be elucidated. Methods: To understand the pathophysiology associated with $u b a 5$ mutation and to identify pathways that could reduce disease progression we are using the zebrafish. Using Crispr-Cas 9 methodology we generated novel $u b a 5$ alleles to investigate the consequences of $u b a 5$ loss-of-function on zebrafish locomotion, survival, and nervous system development and homeostasis. Results: We found that $u b a 5$ mutation results in a significant reduction of locomotor activity that recapitulates the mobility impairment in uba5 patients. Notably, the motor impairment is not preceded by damage to muscle structure or neuronal network, as demonstrated by immuno-labelling. However, electron microscopy reveals signs of myelin degeneration, a hallmark of neuropathies, in the nerves innervating the skeletal muscle of $u b a 5$ mutants. This observation suggests that myelin synthesis or maintenance is impaired in $u b a 5$ mutants in the peripheral nervous system and may be an initial trigger to the resulting phenotype. Finally, we show that uba 5 mutants have a reduced lifespan. Conclusion: These models provide a new tool for characterising the function of Uba5 in the nervous system and determining the role of Uba5 in causing neurological disease.

\section{PS3Group4-008 / \#702}

Topic: Group 4 - Motor Neuron Diseases: Clinical Features, Pathophysiology, Therapy

\section{BRANAPLAM IN TYPE 1 SPINAL MUSCULAR ATROPHY: RESPIRATORY SUPPORT AND FEEDING}

Nicolas Deconinck ${ }^{1}$, Ap Born ${ }^{2}$, G Baranello $^{3}$, Enrico Bertini $^{4}$, Nathalie Goemans ${ }^{5}$, R Pingili ${ }^{6}$, J Praestgaard $^{7}$, R Roubenoff ${ }^{8}$, U Schara $^{9}$, Emilie Voltz ${ }^{10}$

${ }^{1}$ Department Pediatric Neurology, HUDERF, Brussels, BE; ${ }^{2}$ Rigshospitalet, Copenhagen, $D K,{ }^{3}$ Istituto Neurologico "Carlo Besta”, Milano, IT; ${ }^{4}$ Unit Of Neuromuscular Disorders, Laboratory Of Molecular Medicine, Bambino Gesu' Children's Hospital,, Rome, IT; ${ }^{5}$ Treat-NMD Neuromuscular Network, Leuven, BE; ${ }^{6}$ Novartis Pharmaceuticals, East Hanover, NJ, US $;^{7}$ 6Novartis Pharmaceuticals, Novartis Pharmaceuticals, East Hanover, NJ, US: ${ }^{8}$ Novartis Inst for Biomedical Research, Basel, $\mathrm{CH} ;{ }^{9}$ Universitaetsklinikum, Essen, DE $;{ }^{10}$ Novartis Institutes for BioMedical Research, Basel, $\mathrm{CH}$

Background: Introduction: LMI070X2201 (NCT02268552) is an open-label, multi-part, firstin-human study of oral branaplam (formerly known as LMI070) in infants with Type 1 spinal muscular atrophy (SMA) with $2 S M N$ copies. Part 1 of this study evaluated the safety, tolerability, pharmacokinetics, pharmacodynamics, and efficacy of branaplam after 13 weeks of treatment and estimated the Maximum Tolerated Dose (MTD) and optimal dosing regimen of enterally administered branaplam in patients with Type 1 SMA. Methods: Methods: Safety and efficacy data were collected following completion of the initial 13-week treatment period for as long as the patients remained in the study. Treatment extensions and dose escalation were also allowed after the initial 13-week treatment period. This report focuses on respiratory support and feed- 
ing; updated safety information will also be presented. Results: Results: 14 patients enrolled in the trial. One patient failed screening. 13 patients were treated with branaplam starting at 2.2 to 7.6 months of age. All continued into the treatment extension period. Five patients died during the course of the trial from disease progression as assessed by the investigators and the Data Monitoring Committee. No patients withdrew from treatment for reasons other than death. Eight patients continue on branaplam with current ages of 27.5 to 34 months and they have been followed for between 24 and 31 months. No MTD has been reached; the emerging safety profile of branaplam shows adverse events that are mild, reversible and manageable. The most commonly reported events include pyrexia, pneumonia, constipation, vomiting, and diarrhea. Pulmonary events were the most frequent cause of hospital admissions. No dose dependent toxicities were observed across all the dose groups. As of the most recent evaluation on February 5, 2018, four $(50 \%)$ surviving patients required no ventilatory support and four $(50 \%)$ patients required some ventilatory support (Figure). Transition to oral administration from initial enteral tube administration has been successful; five $(62.5 \%)$ patients are exclusively orally fed and three $(37.5 \%)$ patients are managed with a combination of oral and tube feeding. Four of the 5 patients fed only by mouth also required no ventilatory support. Conclusion: Conclusions: Results from this ongoing open-label, first-in-human trial of branaplam in SMA Type 1 demonstrate good safety and tolerability. Over long-term follow-up, $50 \%$ of patients required no ventilatory support and $62.5 \%$ were exclusively orally fed. Figure

\section{PS3Group4-009 / \#502}

Topic: Group 4 - Motor Neuron Diseases: Clinical Features, Pathophysiology, Therapy

\section{NUSINERSEN TREATMENT IN LONGSTANDING ADULT SMA TYPE 3 PATIENTS}

Anne J. Stauber ${ }^{1}$, Natalie Hackel ${ }^{1}$, Eva Greckl ${ }^{1}$, Benedikt Schoser ${ }^{2}$, Maggie C. Walter ${ }^{1}$ ${ }^{1}$ Dept. Of Neurology, Ludwig-maximiliansuniversity, Friedrich-Baur-Institute, Munich, DE; ${ }^{2}$ Friedrich-baur Institute, Departement Of Neurology, Ludwigs-Maximilians University of Munich, Munich, DE

Background: Spinal muscular atrophy (SMA) is a progressive autosomal recessive motor neuron disease caused by loss of the survival motor neuron 1 gene (SMN1), and is characterized by loss of motor neurons in the spinal cord and lower brain stem, resulting in severe and progressive muscular atrophy

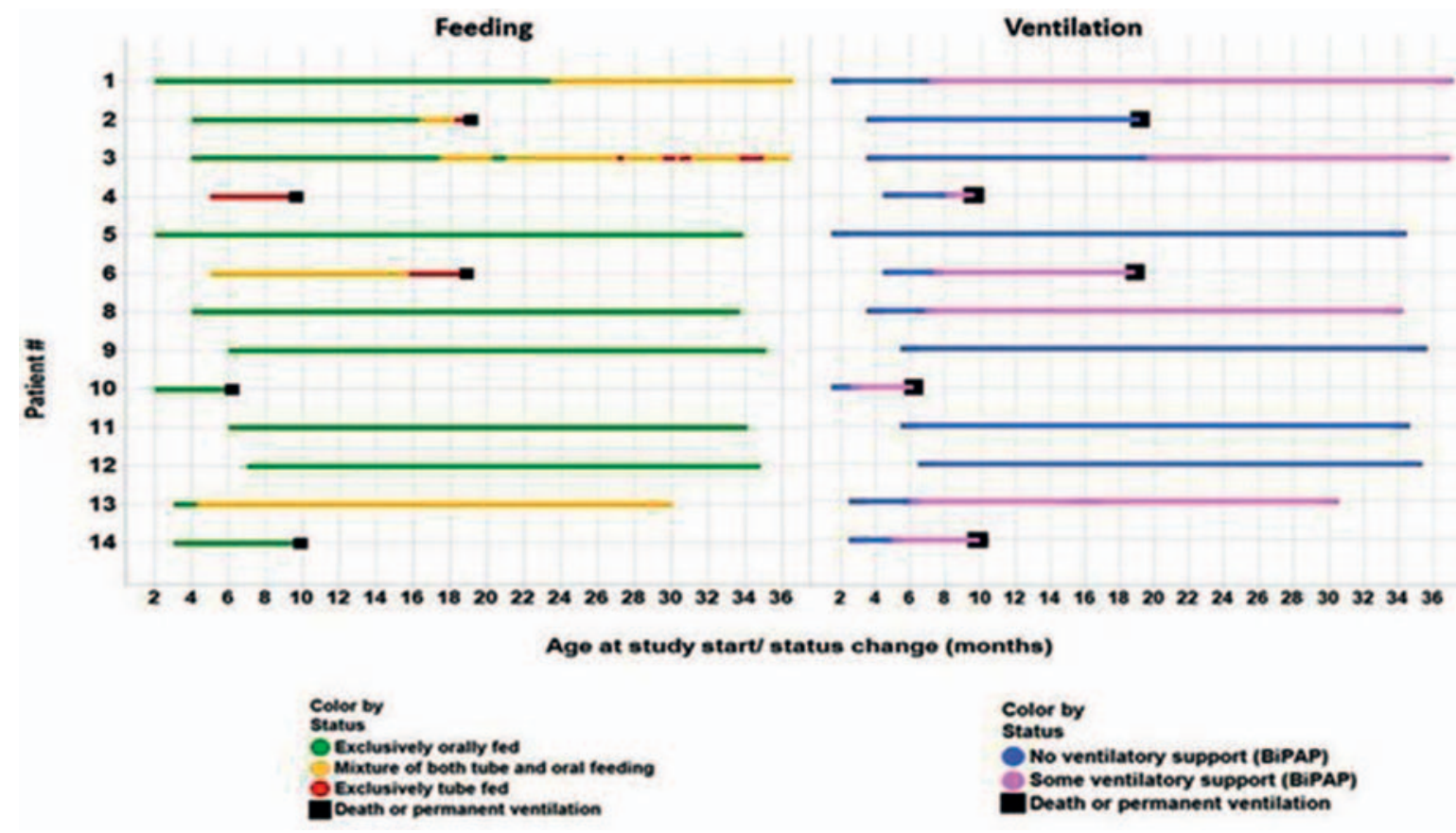


and weakness. SMA is clinically heterogeneous and has been categorized into four types (0-3) based on age of onset, severity of motor decline and life expectancy. With greater understanding of the molecular basis of SMA, a major focus of therapeutic developments has been on increasing the full-length SMN protein by increasing the inclusion of exon 7 in SMN2 transcripts, enhancing SMN2 gene expression, stabilizing the SMN protein or replacing the SMN1 gene. In June 2017, the antisense oligonucleotide Nusinersen has been approved as first treatment for all types of SMA by the EU, based on results from two pivotal multicenter, controlled studies. Nusinersen alters the splicing of SMN2 premRNA in order to increase production of full-length SMN protein. Methods: 12 adult patients with SMA type 3 between 18 and 54 years of age and a disease duration ranging from 6 to 40 years were treated with intrathecal loading doses at day 1, 14, 28 and 63 , followed by maintenance dose every 4 months. None of the patients suffered from severe scoliosis or contractures. Three Patients were wheelchair-dependent at baseline. Patients were monitored within the SMArtCARE initiative for disease progression, side effects and treatment efficacy by clinical examination, monitoring of respiratory function, assessment of the Functional Rating Scale (FRS-SMA), 6-Minute-Walk-Test (6-MWT), Revised Upper Limb Module (RULM) and Hammersmith Functional Rating Scale-Expanded (HFSME). Results: Intrathecal administration was well tolerated in general; in some patients, back pain and headache was noted. Laboratory tests did not reveal nephrotoxicity. At visit 4 (day 63), a mild clinical benefit in form of improved endurance, reduced frequency of falls and better motor function was reported by the patients. Conclusion: Although the SMA research field is rapidly expanding with all the new therapeutic opportunities, there are still several questions that remain unsolved. The timing for optimal intervention for all these approaches is not clear in the human, and in particular at which point there is irreversible pathology that precludes any meaningful therapeutic response. Early diagnosis will be crucial for therapeutic success. However, treatment response should not be based on improvement of motor function alone, but focus on preventing disease progression. The clinical benefit in patients with long-standing disease will have to be further evaluated regarding efficacy and side effects in the years to come.
PS3Group4-010 / \#770

Topic: Group 4 - Motor Neuron Diseases: Clinical Features, Pathophysiology, Therapy

\section{DIAGNOSIS AND DIFFERENTIAL DIAGNOSIS OF SPINAL MUSCULAR ATROPHY}

\author{
Bakhytkul Myrzaliyeva $^{1}$, Marzhan Lepessova ${ }^{2}$ \\ ${ }^{1}$ Medical Faculty, International Kazakh-Turkish \\ University, Turkistan, KZ; ${ }^{2}$ Child Neurology And \\ Medical Genetics, Kazakh Medical University of \\ Continuing Education, Almaty, $K Z$
}

Background: Introduction: Spinal Muscular Atrophy (SMA) - a heterogeneous group of hereditary diseases that occur with lesion / loss of motor neurons of the anterior horns of the spinal medulla and are characterized by the syndromic complex of the "floppy infant". SMA is one of the common causes of child mortality caused by hereditary diseases. Children's forms of the disease have a progressive nature and form an early disability. Methods: Patients and methods: clinical case of the syndrome "floppy infant" in the boy 9 months. Results: Results: complaints of weakness in the legs and hands, a delay in motor development: poorly poises the head, does not turn over, does not sit. Symptoms appeared after 3 months of life: poor sucking, poor weight gain, muscle weakness. Burdened obstetric mother anamnesis: antenatal fetal death in the first pregnancy at week 24, undeveloped 2 pregnancy; this pregnancy against a background of toxicosis, the threat of interruption and thrombophilia. Clinical data: fasciculation of the tongue, diffuse muscle hypotension, more pronounced in the hands, a doughlike consistency of muscles, muscle strength reduced to 1 point, areflexia, decreased abdominal reflexes, "frog" posture, chest deformation, beginning contractures in the elbow joints. Delay in motor development, psycho-emotional development does not suffer. Diagnostic findings: the level of Creatinephosphokinase is within normal limits. Genetic diagnosis: deletion of exons 7-8 of the SMN gene in the homozygous state is not detected; analysis of the number of copies of SMN: 2 copies of the SMN1 gene, 2 copies of the SMN2 gene. Analysis of the carrier of a married couple - parents are not carriers of deletions of exons 7-8 of the SMN gene. EMG: Neuronal type - signs of lesion of the anterior horns of the spinal medulla. Conclusion: Conclusion: for 
genetic confirmation of the diagnosis of spinal muscular atrophy, it is necessary to perform SMN sequencing in search of a point mutation. In the absence of a mutation, the diagnosis of proximal SMA is not confirmed. It is necessary to exclude SMA, not related to SMN-gene, distal forms, Xlinked SMA, SMARD.

\section{PS3Group4-011 / \#933}

Topic: Group 4 - Motor Neuron Diseases: Clinical Features, Pathophysiology, Therapy

\section{A CASE OF HEXANUCLEOTIDE REPEAT EXPANSION CAUSING AMYOTROPHIC LATERAL SCLEROSIS}

Priya D. Shanmugarajah, Judy O.S. Archer Neurology, Pinderfields General Hospital, Wakefield, GB

Background: Amyotrophic lateral sclerosis (ALS) is a fatal neurodegenerative condition involving large motor neurone of the brain and spinal cord, with an incidence of 1 in 100,000. Majority of ALS cases are sporadic, however autosomal dominant inheritance are seen in $5-10 \%$ of cases, with C9orf72 being the most common. This hexanucleotide repeat expansion of GGGGCC $\left(\mathrm{G}_{4} \mathrm{C}_{2}\right)$ is commonly associated with bulbar onset ALS and behavioural variant frontotemporal dementia (FTD). We describe a case of probable motor neurone disease(MND) with positive gene test for C9orf72. Methods: 69 year old right handed man presented with a year history of progressive swallowing difficulties, slurred speech, weight loss and weakness of his limbs particularly his right arm. He also noticed muscle wasting and twitching. He denied any sensory symptoms, sphincter disturbance, personality change or psychiatric symptoms in the form of hallucination or delusions. He has poorly controlled diabetes, fibromyalgia and previous shoulder fracture. His mother died from MND and his maternal aunt was affected with parkinsonism. On examination, he had wasting of the intrinsic small muscle of the hand with flattening of the thenar eminence and first dorsal interosseous, particularly affecting the right more than the left hand. Pectoralis major, thoracic paraspinal muscles and triceps were wasted with fasciculation. Deep tendon reflexes were easily obtainable. He had dysarthria and tongue fasciculation with normal jaw jerk. Sensory examination were normal with flexor plantar response. Results: His blood test was normal for thyroid function test, vitamin D, B12 and folate. Autoimmune screening bloods were negative for myelin associated glycoprotein, ganglioside antibodies, protein electrophoresis, ANA and ANCA. His $\mathrm{HbAlc}$ was elevated at $75 \mathrm{mmol} / \mathrm{mol}$. MRI brain scan showed mild cortical and cerebellar atrophy. MRI spine showed degenerative changes affecting the cervical spine. Neurophysiology showed chronic neurogenic changes on needle EMG affecting muscles from the cervical, thoracic and lumbosacral region however active denervation was only seen in thoracic paraspinal muscles and right upper limb giving the diagnosis of probable motor neurone disease (MND). Given the family history of MND and parkinsonism, genetic testing was done which showed positive C9orf72 hexanucleotide repeat expansion confirming the diagnosis of hereditary ALS Conclusion: C9orf 72 carriers are commonly associated with bulbar onset ALS and behavioural variant FTD. This heterogeneous hexanucleotide expansion of $\mathrm{G}_{4} \mathrm{C}_{2}$ is not restricted to this phenotype as it's also implicated in parkinsonism in ALS, corticobasal syndrome, Huntington-like disorder and primary lateral sclerosis. Our patient with a family history of MND and parkinsonism, presented with bulbar onset ALS without features of FTD. Neurophysiology supported a diagnosis of probable MND with 2 regions depicting active and chronic denervation changes. Awaji-shima criteria have $60.7 \%$ sensitivity in reaching a diagnosis of MND compared to the revised El-Escorial criteria of 28\%. Both criteria had specificity of $95.9 \%$. A definite diagnosis of hereditary ALS was achieved in this patient via genetic testing. Clinicians should have a low threshold for genetic testing in ALS as it has pertinent implications for patient's progeny as well as decoding the molecular mechanism of MND, allowing enhanced disease modelling to identify therapeutic targets 


\section{PS3Group4-012 / \#426}

Topic: Group 4 - Motor Neuron Diseases: Clinical Features, Pathophysiology, Therapy

\section{A DE NOVO HETEROZYGOUS POINT MUTATION IN THE DYNC1H1 GENE CAUSING SPINAL MUSCULAR ATROPHY WITH LOWER EXTREMITY DOMINANT}

\author{
$\underline{\text { Kabelo J. Thusang }}^{1}$, Huiyuan Jiang ${ }^{2}$ \\ ${ }^{1}$ Neurology, Children's Hospital of Michigan, \\ Wayne State University Scholl of Medicine, Detroit, \\ MI, US; ${ }^{2}$ Neurology, Children's Hospital of \\ Michigan, Wayne State University scholl of \\ medicine, Detroit, MI, US
}

Background: Several mutations of DYNC1H1 gene have been identified, which have been associated with variable neurological and neurodegenerative phenotypes including autosomal dominant spinal muscular atrophy with lower extremity dominant (SMA-LED), intellectual disability, Charcot-MarieTooth (CMT) disease, brain malformation and cataract. We have one patient who was found to have a novel mutation of DYNC1H1. Methods: We evaluated a 5-year-old boy who presented for evaluation of global developmental delay and hypotonia at the age 3 years. Electromyography (EMG), muscle biopsy and whole exome sequencing (WES) were performed which led to the diagnosis. Results: The patient presented with hypotonia and weakness of bilateral lower extremity and mild cognitive disability. He later developed fatigability with chewing and dysphagia. His work up showed normal chromosomal microarray, normal Fragile X DNA testing and normal serum creatine kinase levels. He had a normal nerve conduction studies and electromyogram as well as normal magnetic resonance imaging of the brain. Muscle biopsy demonstrated severely atrophic muscles with grouping and panfascicular atrophy and increased esterase staining in atrophic fibers suggestive of advanced neurogenic atrophy. He had a normal SMN1 gene test. WES revealed a de novo, heterozygous point mutation (p.L5681I) in the DYNC1H1 gene. The parents have no such mutation. Conclusion: The mutations in the DYNC1H1 gene have been associated with variable neurological and neurodegenerative phenotypes. We presented a novel de novo heterozygous mutation (p.L5681I) in the DYNC1H1 gene causing SMA-LED. This patient developed bulbar symptoms, which is also unique. It will add onto the spectrum of dyneinopathy. EMG may not be a sensitive test for the early stage of the disease. WES is a confirmative test.

\section{PS3Group4-013 / \#460}

Topic: Group 4 - Motor Neuron Diseases: Clinical Features, Pathophysiology, Therapy

\section{SMARD1: A RARE CAUSE OF HYPOTONIA AND RESPIRATORY FAILURE IN INFANCY}

$\underline{\text { Cristina Garrido }}^{1}$, Jorge Oliveira ${ }^{2}$, Ana Sousa ${ }^{3}$, Marcio Cardoso ${ }^{4}$, Rosario Santos 5 , Manuela Santos ${ }^{6}$ ${ }^{1}$ Neuropediatria, Centro Hospitalar Porto, 011, $P T ;{ }^{2}$ Genetic Service, Centro hospitalar Porto, -, PT; ${ }^{3}$ Neurofisiology Service, Centro Hospitalar Porto, -, PT; ${ }^{4}$ Centro Hospitalar Porto, -, PT; ${ }^{5}$ Genetic Service, Centro Hospitalar Porto, -, $P T ;{ }^{6}$ Neuropediatrics, Centro Hospitalar Porto, -, $P T$

Background: Spinal muscular atrophy (SMA) with respiratory distress type 1 (SMARD1) is a rare genetic condition caused by bi-allelic defects in the immunoglobulin MU-binding protein 2 (IGHMBP2) gene, leading to irreversible degeneration of motor neurons in the anterior horns and in spinal root ganglia. In contrast to the SMA associated with $5 \mathrm{q} 13.2$ locus, the sensory and autonomic nervous system can also be affected in SMARD1, manifesting as a distal predominant muscular weakness with chief phrenic nerve involvement causing diaphragmatic paralysis and early respiratory distress. Previous genotype-phenotype correlations suggest that the amount of functional protein being produced from the mutated gene may modulate the SMARD1 phenotype. The combination of different pathogenic variants in $I G H M B P 2$ gene can also give rise to an axonal Charcot-Marie-Tooth disease type 2S (CMT2S), another less severe neurologic phenotype. Methods: Review of SMARD cases of a tertiary centre in the last 10 years. Results: Clinical cases Patient P1 presented as a 2-month-old male baby with apneic events and cyanotic spells which evolved to a progressive respiratory insufficiency. On physical examination at that time, he had a weak cry and lower limb distal weakness. Severe axonal motor and sensory neuropathy were documented on 
EMG. The genetic analysis of $I G H M B P 2$ revealed two compound heterozygous pathogenic variants: c. $958 \mathrm{C}>\mathrm{T}\left(\mathrm{p} . \operatorname{Arg} 320^{*}\right)$ and c. $1060+1 \mathrm{G}>\mathrm{T}$. The variant affecting the donor spice site was further characterized at mRNA level. Two different out-of-frame transcripts (due to exon-skipping or partial intronic retention events) were identified. Clinically, he developed a global progressive muscle weakness predominantly in lower limbs and after an anesthetic procedure he became dependent on ventilator support. Currently, he is 12 month-old and had gastrostomy for feeding and a tracheostomy for invasive ventilator support (24 hours per day). Patient P2 was a 9-month-old female baby admitted to Intensive care due to a respiratory infection needing ventilatory support (apneic events). At that time, she presented early motor delay and distal lower limb weakness, axial hypotonia, and hyporeflexia. In this patient two novel compound heterozygous pathogenic variants were also identified in IGHMBP2: c.1327C $>$ T (p.Arg443Cys) and c.1757-1G $>$ A. The variant affecting splicing was shown to disrupt the native site which leads to the use of an exonic cryptic splice-site in exon 13 (r.1757_1762del, p. Gly586_Glu587del). She died in the sequence of a respiratory infection at 10 months of age. Conclusion: In our tertiary neuromuscular center only two cases of SMARD1 have been diagnosed in the last ten years confirming the rarity of this clinical entity. Electrophysiological investigation in one of the patients (P1), showed a severe axonal sensorimotor neuropathy consistent with the descriptions in literature. Respiratory distress resulting from irreversible phrenic denervation requires, as in our patient, lifelong mechanical ventilation. Unusual prolonged survival ( $>4$ years) has been reported in some patients upon mechanical ventilation. SMARD1 should be considered in infants with a distal limb predominance weakness even before evolving to a respiratory distress.

\section{PS3Group4-014 / \#640}

Topic: Group 4 - Motor Neuron Diseases: Clinical Features, Pathophysiology, Therapy

\section{NUSINERSEN EXPERIENCE IN INDIVIDUALS WITH SPINAL MUSCULAR ATROPHY TYPE III: A CASE SERIES}

Janbernd Kirschner ${ }^{1}$, Kathryn J. Swoboda ${ }^{2}$, Eugenio Mercuri $^{3}$, Basil T. Darras ${ }^{4}$, Claudia A. Chiriboga ${ }^{5}$, Richard Foster ${ }^{6}$, Ishir Bhan ${ }^{7}$, Wildon Farwell ${ }^{7}$

${ }^{1}$ Klinik für Neuropädiatrie und

Muskelerkrankungen, Freiburg, DE; ${ }^{2}$ Massachusetts

General Hospital, Boston, MA, US, ${ }^{3}$ Università

Cattolica del Sacro Cuore, Rome, IT; ${ }^{4}$ Boston

Children's Hospital, Boston, MA, US: ${ }^{5}$ Columbia

University Medical Center, New York, NY,

US; ${ }^{6}$ Biogen, Maidenhead, GB; ${ }^{7}$ Biogen, Cambridge, $M A, U S$

Background: Spinal muscular atrophy (SMA) is a hereditary neuromuscular disease associated with progressive muscular weakness and loss of motor function caused by insufficient levels of survival motor neuron (SMN) protein. Treatment with nusinersen, an antisense oligonucleotide drug that modulates splicing of $S M N 2$ pre-mRNA and promotes production of full-length SMN protein, has demonstrated significant and clinically meaningful benefits on motor function in symptomatic infants and children, and infants in the presymptomatic stage. Here, we review outcomes in children, adolescents, and young adults with SMA Type III who were treated with nusinersen. Methods: This case series includes 11 participants with SMA Type III who received their first treatment with intrathecal nusinersen (3$12 \mathrm{mg}$ ) in the Phase $1 \mathrm{~b} / 2 \mathrm{a}$ CS2 study (NCT01703988) (age at treatment initiation: $<10$ years, $\mathrm{n}=5 ; \geq 10$ years, $\mathrm{n}=6$ ), then enrolled in CS12 (NCT02052791), an open-label, $\sim 2$-year extension study of nusinersen $12 \mathrm{mg}$ administered intrathecally, and had the longest follow-up. For this analysis, we assessed changes in motor function from CS2 baseline to last visit of CS12 (Day 715, n=10; Day 624, n=1; last patient last visit, June 30, 2016) using the Hammersmith Functional Motor Scale-Expanded (HFMSE), Upper Limb Module (ULM), and 6-Minute Walk Test (6MWT). Clinically meaningful improvement in HFSME was defined as a 3-point score increase. Changes in quality of life (QOL) were assessed with 
the PedsQL Core and Neuromuscular (NM) modules. Results: At treatment initiation in CS2, median (range) age of participants was 10.9 (3.6-16.0) years and $8 / 11$ were ambulatory. Four participants with CS2 baseline HFMSE scores of 51-59 points achieved clinically meaningful improvements of 3-7 points from baseline at CS12 last visit. HFMSE scores remained stable or improved slightly (0- to 2-point change) in 6 participants (CS2 baseline values: 37-62) and declined in 1 participant (CS2 baseline value: 31 ). In the 3 nonambulatory participants, ULM scores were 18 at every study visit. In the 8 ambulatory participants, distances walked on the 6MWT increased by $50-159$ meters from CS2 baseline values of $45-550$ meters to last CS12 visit. Caregiver/parent-reported PedsQL Core scores improved or remained stable from CS2 baseline values of 33.7-69.6 in 10 participants. Caregiver/parentreported NM scores improved or remained stable from CS2 baseline values of 45-81 in 9 participants. Self-reported Core scores improved or remained stable from CS2 baseline values of 55.4-66.3 in 3 participants. Self-reported NM scores improved or remained stable from CS2 baseline values of 69-86 in 5 participants. Changes in self-reported scores were not available for 3 participants who were age $<5$ years at CS2 baseline. The most common adverse events (AEs) were those expected following lumbar puncture. The AE pattern in this cohort was consistent with clinical trial experience. Conclusion: In contrast to the expected decline in motor function over time based on natural history, in this cohort almost all children, adolescents, and young adults with SMA Type III who were treated with nusinersen showed clinically meaningful improvements or stabilization of disease and generally improved or stable QOL over time.

\section{PS3Group4-015 / \#752}

Topic: Group 4 - Motor Neuron Diseases: Clinical Features, Pathophysiology, Therapy

\section{RG7916 SIGNIFICANTLY INCREASES SMN PROTEIN IN SMA TYPE 1 BABIES}

Giovanni Baranello ${ }^{1}$, Laurent Servais ${ }^{2}$, John Day ${ }^{3}$, Nicolas Deconinck ${ }^{4}$, Eugenio Mercuri ${ }^{5}$, Andrea Klein 6 , Basil Darras ${ }^{7}$, Riccardo Masson ${ }^{8}$, Heidemarie Kletzl ${ }^{9}$, Yumi Cleary ${ }^{10}$, Gillain
Armstrong ${ }^{11}$, Timothy Seabrook ${ }^{9}$, Christian Czech $^{10}$, Marianne Gerber ${ }^{10}$, Fung Lee ${ }^{9}$, Kristina Gelblin ${ }^{9}$, Stephane Nave ${ }^{10}$, Ksenija Gorni ${ }^{10}$, Omar Khwaja ${ }^{10}$ ${ }^{1}$ Developmental Neurology Unit, Carlo Besta Neurological Research Institute Foundation, Milan, IT; ${ }^{2}$ Institute of Myology, Paris, FR $;{ }^{3}$ Department Of Neurology, Stanford University, Palo Alto, CA, US: ${ }^{4}$ Queen Fabiola Children's University Hospital, Université Libre de Bruxelles, Brussels, Belgium and Neuromuscular Reference Center UZ Ghent, Ghent, Belgium, Ghent, BE; ${ }^{5}$ Paediatric Neurology And Nemo Center, Catholic University and Policlinico Gemelli, Rome, IT; ${ }^{6}$ University Children's Hospital Basel, Basel, Switzerland and Inselspital, Bern, Switzerland, Bern, CH; ${ }^{7}$ Boston Children's Hospital, Harvard Medical School, Boston, MA, US; ${ }^{8}$ Developmental Neurology Unit, Carlo Besta Neurological Research Institute Foundation, Milan, IT; ${ }^{9}$ Roche Pharmaceutical Research and Early Development, Roche Innovation Center, Basel, $\mathrm{CH} ;{ }^{10}$ Roche Pharmaceutical Research and Early Development, Roche Innovation Center, Basel, $\mathrm{CH} ;{ }^{11}$ Roche Products Ltd, Welwyn Garden City, GB

Background: Spinal muscular atrophy (SMA) is characterized by motor neuron loss and muscle atrophy, due to reduced levels of survival of motor neuron $(\mathrm{SMN})$ protein from a loss of function of the SMN1 gene. While SMN1 produces full-length SMN protein, a second gene, $S M N 2$, produces only low levels of functional SMN protein. RG7916 (RO7034067) is an investigational, orally administered, centrally and peripherally distributed small molecule that is being evaluated for its effect on modulating SMN2 pre-mRNA splicing towards the production of full-length SMN2 mRNA and increase of SMN protein. FIREFISH (NCT02913482) is an ongoing, multi-center, open-label, 2-part, seamless study of RG7916 in babies aged 1-7 months with Type 1 SMA and two SMN2 gene copies. Methods: We present interim data, including SMN protein data, from FIREFISH Part $1(\mathrm{n}=8-24)$, an exploratory study assessing the safety, tolerability, pharmacokinetics and pharmacodynamics of RG7916 at different dose levels. Confirmatory Part $2(n=40)$ will assess safety and efficacy of RG7916, with a primary endpoint of the proportion of infants sitting without support for 5 seconds after 12 months. Results: In an interim analysis of FIREFISH Part 1, a dose-dependent increase in SMN protein levels in blood was observed, with an up to 6.5 -fold increase 
versus baseline after 4 weeks of treatment at the highest dose of RG7916 (range 2.0-6.5). To date, no protocol-defined safety-related stopping rules have been met. While follow-up has been limited in time, none of the following events have been reported: loss of the ability to swallow, tracheostomy or need for permanent ventilation. Updated survival data will also be presented. Conclusion: The up to 6.5fold increase in SMN protein observed in FIREFISH Part 1 is expected to be beneficial based on naturally occurring differences in SMN protein levels between SMA severity types (e.g., Type 2 vs. Type 1 with differences up to approximately 2 -fold). The clinical effects will be assessed in Part 2. All doses explored so far have been well tolerated. The FIREFISH study is currently recruiting globally. Acknowledgments The authors would like to thank all individuals enrolled in the RG7916 studies, their families and the site staff involved. Study sponsored by F. Hoffmann-La Roche.

\section{PS3Group4-016 / \#391}

Topic: Group 4 - Motor Neuron Diseases: Clinical Features, Pathophysiology, Therapy

\section{PATHOGENIC VARIANT OF THE REEP1 GENE IN A KOREAN FAMILY WITH AUTOSOMAL DOMINANT HEREDITARY SPASTIC PARAPLEGIA}

Dong Wook Namgung ${ }^{1}$, Young Chul Choi ${ }^{2}$, Hyung Jun Park ${ }^{3}$, Bun Chun Suh ${ }^{4}$

${ }^{1}$ Neurology, Gangnam Severance Hospital, Yonsei

University College of Medicine, Seoul, $K R ;{ }^{2}$ Neurology, Gangnam Severance Hospital, Yonsei University College of Medicine, Seoul, KR: ${ }^{3}$ Neurology, Gangneung Asan Hospital, University of Ulsan College of Medicine, Gangneung, KR: ${ }^{4}$ Neurology, Kangbuk Samsung Hospital, Sungkyunkwan University School of Medicine, Seoul, KR

Background: Hereditary spastic paraplegia (HSP) is a heterogeneous group of genetic disorders characterized by progressive lower-limb spastic paralysis due to corticospinal tract degeneration. Three genes account for up to $50 \%$ of pathogenic variants in families with autosomal dominant HSP: the causative gene is SPAST in $40 \%$; ATL1 in $10 \%$, and REEP1 in 4.5 to $6 \%$. However, pathogenic variants in REEP1 gene have not been reported in Korea.

Methods: To identity a pathogenic variant, whole exome sequencing was performed. Results: The proband in the HSP family (an 8-year-old boy; Fig. 1A, III-8) presented to our clinic with gait disturbance. To identity a pathogenic variant, whole exome sequencing was performed on the proband (III-8). He first noticed an unstable gait at an age of 5 years, and his gait disturbance had progressed slowly since then. When we examined him at the age of 8 years, he could still ambulate independently. A neurological examination demonstrated muscle weakness and brisk reflexes of the lower extremities. His ankle joints were affected by contracture. However, motor function of the upper extremities was normal, and he did not exhibit cognitive decline, sensory deficits, or bladder dysfunction. Magnetic resonance images of brain and spinal cord were normal. Electrophysiological studies were normal. The family history showed that HSP was inherited in an autosomal dominant manner (Fig. 1A). His mother (a 45-yearold woman, II-10) and oldest aunt (a 52-year-old woman, II-4) also showed spastic paraplegia and ankle contracture. His younger brother (a 5-year-old boy,III-10) did not complain of gait disturbance, but showed mild spasticity and hyperreflexia of the lower extremities. However, his younger sister (a 5-yearold girl, III-11) did not show any abnormal neurologic deficits. Whole exome sequencing was performed on the proband (III-8). After screening of HSP-related genes, we identified a heterozygous pathogenic variant (c.337C >T) (p.Arg113*) in REEP1 (NM_022912). This variant was previously reported as the pathogenic variant in complicated HSP presented with both spastic paraplegia and lower motor neuron signs including pes cavus and motor neuropathy. (Hewamad-duma, et al. Neurogenetics 2009). However, the present patients did not show any electrophysiological evidence of peripheral neuropathy. In addition, the rate of clinical disease progression was relatively slow, and two adults (II-4 and II-10) could still ambulate independently at an age of 52 and 45 years, respectively. Conclusion: This is the first report of the pathogenic variant of the REEP1 gene in a Korean family with an autosomal dominant HSP using whole exome sequencing. 
(A)

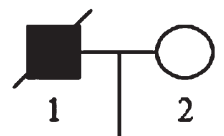

II

III

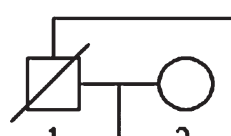

1

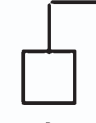

1

$\mathrm{C} / \mathrm{C}$

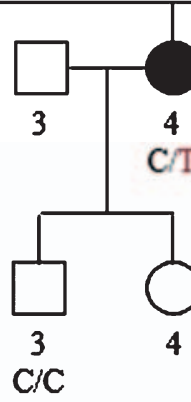

4

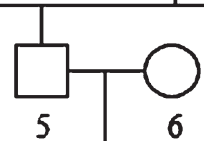

(B)

Wild allele

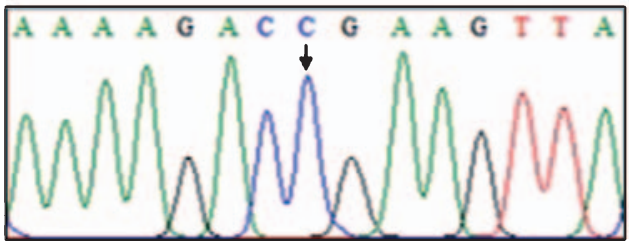

\section{PS3Group4-017 / \#626}

Topic: Group 4 - Motor Neuron Diseases: Clinical Features, Pathophysiology, Therapy

\section{NEUROFILAMENT LIGHT CHAIN AS A POTENTIAL BIOMARKER IN SPINAL MUSCULAR ATROPHY}

$\underline{T h o m a s ~ P e t e r s ~}^{1}$, Hervé Jullien De Pommerol ${ }^{2}$, Diethilde Theil ${ }^{3}$, Marie-Anne Valentin ${ }^{3}$, Agnieszka Kieloch $^{3}$, David Leppert ${ }^{2}$, Emilie Voltz ${ }^{3}$

${ }^{1}$ Translational Medicine / Biomarker Development, Novartis Institutes for BioMedical Research, Basel, $\mathrm{CH},{ }^{2}$ Novartis Pharma AG, Basel, CH; ${ }^{3}$ Novartis Institutes for BioMedical Research, Basel, CH

Background: Biomarker assessments in patients with degenerative neurologic diseases have demonstrated that neurofilament light chain (NfL) is a sensitive, blood-based, exploratory marker that can identify patients, aid in prediction of long-term outcomes, and be used for assessing effects of treatment. At present, little is known about blood NfL levels in patients with spinal muscular atrophy (SMA). Methods: Blood samples for NfL assessment were obtained from 30 healthy adult volun-

\section{Mutated allele $(c .337 C>\mathrm{T})$}

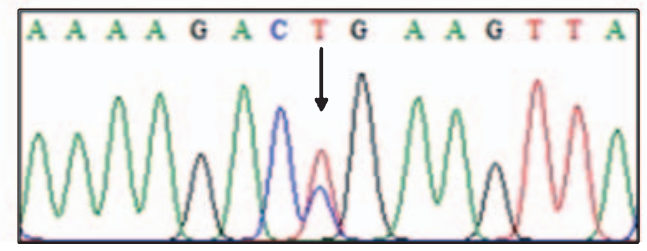

teers (24-55 years old), 21 healthy children (1-4 years of age), and 18 healthy children (8-10 years old). Samples were also obtained from 18 patients with SMA Type III and 2 with SMA Type II (6 males and 14 females, 21-66 years of age), and from 12 patients with SMA Type I (2.2-7.6 months of age) enrolled in an open-label, multi-part, first-in-human, proof of concept study of branaplam, a small-molecule RNA splicing modulator. Serum NfL levels were measured using the ultra-sensitive Simoa HD-I Analyzer. The lower limit of quantification was 3.38 $\mathrm{pg} / \mathrm{mL}$ and the upper limit was $1128 \mathrm{pg} / \mathrm{mL}$. Interrun accuracy was $98.5 \%$ to $115.2 \%$ and inter-run precision was $7.0 \%$ to $16.1 \%$. Results: The geometric mean serum level of NfL in healthy adults was $13.4 \mathrm{pg} / \mathrm{mL}$ (reference range $=4.6-38.9 \mathrm{pg} / \mathrm{mL}$ ), that for healthy pediatric subjects $8-10$ years old was $2.5 \mathrm{pg} / \mathrm{mL}$ (measured range $=1.7-6.9 \mathrm{pg} / \mathrm{mL}$ ), and that for healthy pediatric subjects $1-4$ years old was $4.0 \mathrm{pg} / \mathrm{mL}$ (measured range $=1.7-22.9 \mathrm{pg} / \mathrm{mL}$ ). Adults with SMA Type II or III had lower NfL levels $(7.2 \mathrm{pg} / \mathrm{mL}$, range $=3.9-23.2 \mathrm{pg} / \mathrm{mL})$ vs healthy adults, but there was substantial overlap in measurements for the two groups. The mean pre-treatment NfL level in pediatric patients with SMA Type I was $443 \mathrm{pg} / \mathrm{mL}($ range $=177-983 \mathrm{pg} / \mathrm{mL})$ and did not 


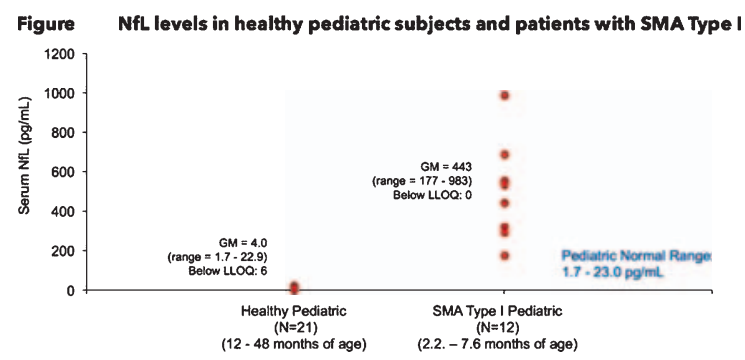

overlap with values from healthy pediatric subjects (Figure). There also appeared to be an inverse correlation between pre-treatment $\mathrm{NfL}$ levels and CHOP INTEND scores for the patients with SMA Type I. Conclusion: Blood NfL levels in adult SMA Type II/III patients seem to be within the normal reference range while those for pediatric SMA Type I patients are higher than those for healthy pediatric subjects. There is also a trend for an inverse relationship between pre-treatment blood NfL levels and CHOP INTEND scores. These conclusions are limited by the small number of samples evaluated and the lack of data in an age-matched healthy children. The dynamic course of blood NfL levels in SMA requires further study.
PS3Group4-018 / \#337

Topic: Group 4 - Motor Neuron Diseases: Clinical Features, Pathophysiology, Therapy

\section{ENHANCEMENT OF BULBAR FUNCTION IN ALS: LESSONS LEARNED FROM THE NUEDEXTA TREATMENT TRIAL}

$\underline{\text { Richard Smith }}^{1}$, Kathleen Myers ${ }^{1}$, Eric Macklin ${ }^{2}$

${ }^{1}$ Center for Neurologic Study, La Jolla, CA, US ${ }^{2}$ Massachusetts General Hospital, Neurological Clinical Research Institute and Biostatistics Center, Boston, MA, US

Background: Following up anecdotal reports, a multi-centered placebo-controlled treatment trial was conducted to determine if Nuedexta could enhance bulbar function in ALS patients. The drug, a combination product containing dextromethorphan (DM) and quinidine, was originally approved for the treatment of emotional lability. The effect on emotionality was thought to be mediated by the action of DM on sigma-1 receptors which have recently been reported to decorate motor neurons in the brain stem. Methods: Whereas most trials in ALS are conducted over long intervals, we designed a 70-day study, placing patients on either drug or placebo for 30 days followed by a washout period and subsequent place-

Table 1: Primary and Secondary Efficacy Endpoints

\begin{tabular}{|c|c|c|c|c|c|}
\hline Measure & $\begin{array}{l}\text { Active } \\
\text { Mean }\end{array}$ & $\begin{array}{l}\text { Placebo } \\
\text { Mlean }\end{array}$ & $\begin{array}{l}\text { Mean Differ } \\
\text { ence Active } \\
\text { Minus Placebo }\end{array}$ & $\begin{array}{l}\text { Mean } \\
\text { Differeace } \\
\text { SE }\end{array}$ & 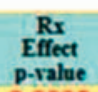 \\
\hline CNS-BFS Total & 53.45 & 59.31 & -5.85 & 1.49 & 0.0003 \\
\hline $\begin{array}{l}\text { CNS.BFS Si- } \\
\text { alorthea }\end{array}$ & 14.28 & 15.81 & -1.52 & 0.51 & 0.0041 \\
\hline $\begin{array}{l}\text { CNSBFS } \\
\text { Speech }\end{array}$ & 22.22 & 24.57 & -2.35 & 0.74 & 0.0027 \\
\hline $\begin{array}{l}\text { CNSSBFS } \\
\text { Swallowing }\end{array}$ & 17.14 & 18.92 & -1.77 & 0.65 & 0.0092 \\
\hline$\underset{t}{\text { ALSFRSR To- }}$ & 34.15 & 33.7 & 0.45 & 0.38 & 0.2478 \\
\hline$\underset{a f}{\text { ALSFRS Bulb- }}$ & 7.39 & 6.79 & 0.6 & 0.19 & 0.0026 \\
\hline ALSFRS Motor & 16.63 & 16.8 & -0.16 & 0.22 & 0.4579 \\
\hline $\begin{array}{l}\text { owords read } \\
\min \end{array}$ & 107.12 & 103.37 & 3.75 & 2.54 & 0.1466 \\
\hline $\begin{array}{l}\text { Avg swallow } \\
\text { water time } \\
\text { (sec) }\end{array}$ & 12.16 & 13.11 & -0.95 & 0.96 & 0.3288 \\
\hline $\begin{array}{l}\text { Avg swallow } \\
\text { solids time } \\
\text { (sec) }\end{array}$ & 18.53 & 19.45 & -0.92 & 1.66 & 0.5821 \\
\hline
\end{tabular}


Figure 1: Effect of Nuedexta vs. placebo on changes in the Center for Neurologic Study Bulbar Function Scale (CNS-BFS), the primary outcome measure.

Mean CNS-BFS scores for each treatment arm (i.e., patients treated with placebo first and then switched to Nuedexta vs. patients placed on Nuedexta initially and then switched to placebo) were calculated during the course of the clinical trial. Measurements were obtained at baseline, and during three subsequent clinical visits. For the group treated with placebo initially, Nuedexta treatment was initiated following Visit 2 and the drug treatment effect measured at Visit 3 . For the group treated with Nuedexta initially, drug treatment began immediately following the baseline visit and the effect of treatment was measured at Visit 1. The crossover effect is apparent: CNS-BFS scores declined following the period of Nuedexta treatment.

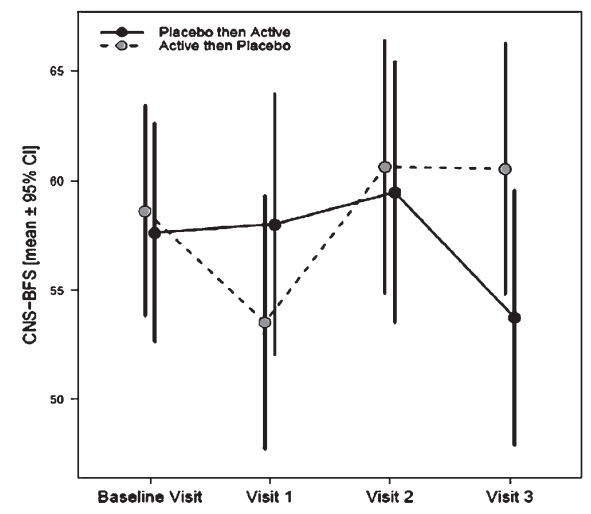

ment in the remaining limb of the trial. Another unique aspect of the study was the use of a recently validated self-report scale, the Center for Neurologic Study Bulbar Function Scale (CNS-BFS), as the primary endpoint. Secondary endpoints in the study included traditional measures, such as the ALSFRS-R, its bulbar subscale, and timed speech and swallowing. Results: The CNS-BFS proved to be a robust endpoint. Bulbar functions with Nuedexta treatment were significantly enhanced, with pvalues for the overall scale as well as its speech, swallowing, and salivation subdomains ranging from 0.0003-0.009. All other measures assessing bulbar function except for the bulbar component of the ALSFRS-R failed to reach statistical significance (see Table 1). The effect of Nuedexta is dramatically illustrated in Figure 1, which compares the two treatment periods (placebo vs. drug). Conclusion: While speech, swallowing and salivation responded to drug treatment, no effects were seen on other motor functions such as breathing or walking, implying that a useful therapy may only target a subpopulation of motor neurons. Additional studies need to be undertaken to understand the duration of this treatment effect and to discern whether Nuedexta has an effect on survival in ALS patients.
PS3Group4-019 / \#533

Topic: Group 4 - Motor Neuron Diseases: Clinical Features, Pathophysiology, Therapy

\section{SALBUTAMOL TREATMENT IN TYPE 2 SMA PATIENTS: 18 MONTHS ASSESSMENT}

\author{
Anna Lia Frongia $^{1}$, Daniel Natera De Benito ${ }^{2}$, \\ Macarena M.A. Alarcon-Cornejo ${ }^{1}$, Laura Carrera- \\ García $^{3}$, Ariadna Borras ${ }^{1}$, Nuria Padros ${ }^{1}$, Obdulia \\ Moya $^{1}$, Judit Armas ${ }^{1}$, Julita Medina ${ }^{1}$, Meritxel \\ Vigo $^{4}$, Cristina Jou ${ }^{1}$, Cecilia Jimenez Mallebrera ${ }^{1}$, \\ Carlos Ortez ${ }^{1}$, Jaume Colomer ${ }^{1}$, Andres \\ Nascimento $^{1}$ \\ ${ }^{1}$ Neuromuscular Disorders Unit, Hospital Sant \\ Joan de Déu, Barcelona, ES, ${ }^{2}$ Neuromuscular \\ Disorders Unit, Hospital Sant Joan de Déu, \\ Barcelona, ES, ${ }^{3}$ Unidad De Patología \\ Neuromuscular., Hospital Sant Joan de Déu, \\ Barcelona, ES $;{ }^{4}$ Hospital Sant Joan de Déu, \\ Barcelona, ES
}

Background: Spinal muscular atrophy (SMA) is an autosomal recessive disease characterized by motor neuron degeneration, resulting in several major phenotypes defined by age at disease onset and maximal motor capacity. Patients with SMA type 2 became symptomatic between 6 and 12 months, typically they achieve the ability to sit without support, but not independent walk. Data on natural history showed a slow decline in motor milestones over time and life expectancy is shortened by early onset scoliosis, joint contractures, and restrictive lung disease. Different studies have demonstrated that salbutamol may have a beneficial effect on muscle function in patients with SMA type 2 and 3. Methods: To describe the response to salbutamol in SMA type 2 patients motor function assessment was performed before starting treatment and every 6 months. Hammersmith Functional Motor Scale Expanded (HFMSE), Revised Upper Limb Module (RULM), and Egen Klassifikation (EK2) were used. Motor scales were longitudinally evaluated over an 18-month period. Results: Fourty eight children with a mean age of 10 years (range: 2-21 years) took oral salbutamol during a period of at least 6 month. Children $<6$ years presented a functional improvement during the first 6 months of treatment (HFMSE $+4,29)$ that reduced in intensity after 12 months (HFMSE $+1,14)$. However, amelioration in upper 
extremity tended to stabilize (RULM $+3,67$ ). Patients $>6$ years showed a slow decrease in motor function scales similar to that described in SMA natural history. $77 \%$ of families $(92 \%<6$ years and $71 \%$ in $>6$ years) referred a subjective positive effect. Conclusion: Salbutamol treatment may be effective in AME 2 patients, particularly during the first months of therapy, and in younger than 6 years. An apparent higher effect on upper limb is observed. Absence of a control group is the main limitation in our study.

\section{PS3Group4-020 / \#510}

Topic: Group 4 - Motor Neuron Diseases: Clinical Features, Pathophysiology, Therapy

\section{VERY LATE-ONSET AMYOTROPHIC LATERAL SCLEROSIS IN A PORTUGUESE COHORT: WHICH DIFFERENCES?}

\author{
Miguel Oliveira Santos ${ }^{1}$, Marta Gromicho ${ }^{2}$, Susana \\ Pinto $^{2}$, Mamede De Carvalho ${ }^{1}$ \\ 1. Institute of Physiology Unit, Instituto de \\ Medicina Molecular, Faculty of Medicine, \\ University of Lisbon, Portugal. 2. Department of \\ Neurosciences and Mental Health, Hospital de \\ Santa Maria, Centro Hospitalar Lisboa Norte, \\ Lisbon, Portugal., Lisbon, PT; Institute of \\ Physiology Unit, Instituto de Medicina Molecular, \\ Faculty of Medicine, University of Lisbon, \\ Portugal., Lisbon, PT
}

Background: Amyotrophic lateral sclerosis (ALS) is a rapidly progressive and fatal motor neuron disorder characterized by degenerative changes in upper and lower motor neurons. Although its incidence has been stable among Western countries, population-ageing effect will probably increase the proportion of very-old ALS patients. On the other hand, this particular population has been excluded from clinical trials. Therefore, its characterization is sparse. We aim to raise awareness of demographic and clinical profile of oldest ALS patients, including their survival. Methods: A retrospective study was performed, including 1162 ALS patients followed longitudinally at our ALS unit from January 1995 to December 2017. The patients were divided in two groups, according to whether disease onset was before or after 80 years. Demographic, clinical and survival data were compared between groups. ALS diagnosis was established based on the revised $E l$ Escorial criteria and supported by the Awaji criteria since 2008. Frontotemporal dementia (FTD) clinical diagnosis was established by personality and social behaviour changes, lack of insight for the on going clinical condition, with preserved memory, visuospatial abilities and praxis. Comparison between the very-old and younger patients was performed using chi-square and t-student tests, as appropriate. Survival analysis was done by Kaplan-Meier log-rank test and the multivariate Cox proportional hazards model. A $p$-value of $<0.05$ was considered statistically significant. Results: 54 of 1162 patients $(4.65 \%)$ were aged 80 or over. From those, 29 $(53.70 \%)$ were men and the mean age of onset was 83 years $(\mathrm{SD} \pm 2.63)$. Contrasting with the younger group, bulbar onset was remarkably the most common presentation form in the oldest patients $(55.56 \%, p<0.001)$, but with no gender preference $(p=0.54)$. Clinical criteria for FTD were met in 4 ( $7.41 \%, p=0.36)$ and only $1(1.85 \%, p=0.10)$ had a positive family history. All patients were taking riluzol, and non-invasive ventilation was started in 28 $(51.85 \%, p=0.11)$. Significantly shorter mean disease duration before first visit was disclosed in the very-old group (13.38 \pm 9.24 vs. $19.98 \pm 25.0$ months, $p<0.001)$. Survival was shorter in the oldest group ( $28.8 \pm 23.5$ vs. $44.1 \pm 41.9$ months, $p<0.001$ ), which was significantly dependent on disease duration and onset form, both of each showed no interaction impacting on their survival. Conclusion: Very-old patients represented a minor but distinctive ALS group. A predominant bulbar presentation (with no gender dependence) was disclosed, and it could probably explain the shorter disease duration before first visit. Despite being oldest, it was not an exclusion factor for good health care practises, in particular multidisciplinary treatment, non-invasive ventilation and riluzol prescription. 


\section{PS3Group4-021 / \#773}

Topic: Group 4 - Motor Neuron Diseases: Clinical Features, Pathophysiology, Therapy

\section{ATAXIN-2 IN RUSSIAN ALS PATIENTS ATAXIN-2 IN RUSSIAN ALS PATIENTS}

Vera S. Demeshonok ${ }^{1}$, Tatiana Alekseeva ${ }^{2}$, Vladimir Lapin $^{3}$, Vladimir Nazarov ${ }^{3}$

${ }^{1}$ Neurology, North-Western State Medical

University named after I.I. Mechnikov, Russia, $R U ;{ }^{2} V$.A.Almazov NMRC, Санкт-Петербург, $R U ;{ }^{3}$ Laboratory Of Autoimmune Diagnostics, Center For Molecular Medicine, First St. Petersburg I. Pavlov State Medical University, St-Petersburg, $R U$

Background: Amyotrophic lateral sclerosis is a fatal progressive neurodegenerative disorder, which is characterized by the loss of motor function, including bulbar and spinal involvement. Mutations in SOD1 and C9orf72 are the most common genetic causes of the familial and sporadic ALS and it is more often noticed in patients with the early onset of the disease. The polyQ intermediate expansions in ataxin-2 is also increased risk for ALS. Methods: We investigated the polyQ expansions in ataxin-2 in 70 patients ( 33 males; 37 females) with sporadic ALS and 40 unrelated controls without history of neurodegenerative disorders from St-Petersburg (Russia). There are 11 patients with early onset of symptoms (up to 46 years old), and they were tested for the presence of mutations in SOD1 and C9orf72 genes. The patients were hospitalized in Neurology Departments of V.A. Almazov NMRC or NWSMU from May to November 2017.All patients were examined and included according to El Escorial (2015). Results: We did not detect mutations in SOD1 and C9orf72 genes in the examined patients with early onset of the disease. We defined higher intermediate PolyQ repeat length expansions (2732 ) for ataxin- 2 in 6 patients $(8,57 \%): 2$ men with a spinal onset and 4 women ( 2 with a spinal and 2 with a bulbar onset of the disease). It should be noted that all 6 patients were characterized by slow progression on a ALSFRS-R Scale. PolyQ intermediate expansions were not determined in none of the control group. Conclusion: Ataxin-2 PolyQ intermediate expansions are associated with ALS in Russia.
PS3Group4-022 / \#824

Topic: Group 4 - Motor Neuron Diseases: Clinical Features, Pathophysiology, Therapy

\section{AVXS-101 PHASE 1 GENE THERAPY CLINICAL TRIAL IN SMA TYPE 1: EVENT-FREE SURVIVAL AND ACHIEVEMENT OF DEVELOPMENTAL MILESTONES}

Jerry R. Mendell ${ }^{1}$, Samiah Al-Zaidy ${ }^{1}$, Richard Shell ${ }^{1}$, W. D. Arnold ${ }^{2}$, Louise Rodino-Klapac ${ }^{1}$, Thomas W. Prior ${ }^{3}$, Linda Lowes ${ }^{1}$, Lindsay Alfano ${ }^{1}$, Katherine Berry ${ }^{1}$, Kathleen Church ${ }^{1}$, John T. Kissel $^{4}$, Sukumar Nagendran ${ }^{5}$, James L'Italien ${ }^{5}$, Douglas M. Sproule ${ }^{5}$, Courtney Wells ${ }^{5}$, Arthur H.m. Burghes $^{2}$, Kevin Foust ${ }^{5}$, Kathrin Meyer ${ }^{1}$, Shibi Likhite $^{1}$, Brian Kaspar ${ }^{1}$

${ }^{1}$ Center for Gene Therapy Nationwide Children's Hospital, Columbus, OH, US, ${ }^{2}$ Department of Neurology, Ohio State University, Columbus, $\mathrm{OH}$, US, ${ }^{3}$ Department of Pathology, Ohio State University, Columbus, OH, US, ${ }^{4}$ Department of Pediatrics, Ohio State University, Columbus, $\mathrm{OH}$, US, ${ }^{5}$-, Bannockburn, IL, US

Background: Spinal muscular atrophy (SMA) is a devastating, monogenic neurodegenerative disease. Children with its most severe form, SMA Type 1 (SMA1), will never sit unassisted or maintain head control. A natural history study of SMA1 reported that none achieved an Infant Test of Neuromuscular Disorders (CHOP-INTEND) score of $\geq 40$ by 6 months of age (1 transient exception) and $92 \%$ died or required permanent ventilation by 20 months. This trial explores safety and efficacy of a single intravenous administration of gene replacement therapy in SMA1. AVXS-101 delivers the survival of motor neuron (SMN) gene in a single intravenous dose via the adeno-associated virus (AAV) 9 viral vector, which crosses the blood-brain barrier. Methods: In this phase 1 trial, 15 patients with SMA1 confirmed by genetic testing (with $2 \mathrm{x} S M N 2$ copies) were enrolled. Patients received an intravenous dose of AVXS-101 at the low dose (Cohort $1, \mathrm{n}=3$ ) or proposed therapeutic dose (Cohort 2, $n=12$ ). The primary objective was safety and secondary objectives included survival (avoidance of death/permanent ventilation) and ability to sit unassisted (video confirmed by external independent reviewer). CHOPINTEND scores and other motor milestones were 
additional objectives. Results: AVXS-101 appeared to have a favorable safety profile and to improve survival (as of August 7, 2017). All 15 patients were alive and event-free at 20 months of age and did not require permanent mechanical ventilation. Patients in Cohort 2 demonstrated improvements in motor function: $11 / 12$ had achieved CHOP-INTEND scores $\geq 40$ points and a mean increase of 24.6 points from a mean baseline of 28.2 points; $11 / 12$ were able to sit unassisted for at least 5 seconds, 10 for at least 10 seconds, and 9 for at least 30 seconds; 11/12 achieved head control and 9 could roll over. Two patients were able to crawl, pull to stand, stand independently, and walk independently. Asymptomatic elevated serum aminotransferase levels occurred in 4 patients and were attenuated by prednisolone. Conclusion: In contrast with the natural history, a one-time intravenous administration of AVXS-101 appeared to demonstrate a positive impact on the survival of both cohorts and a dramatic, sustained impact on motor function of Cohort 2: 11/12 patients achieved CHOP-INTEND scores and motor milestones rarely or never seen in this population. No waning of effect or clinical regression in motor function was reported up to the August 7, 2017 data cut. A clinical update of the 24-month safety follow-up will be given at the time of presentation.

\section{PS3Group4-023 / \#714}

Topic: Group 4 - Motor Neuron Diseases: Clinical Features, Pathophysiology, Therapy

\section{AN AUTOMATED ANALYSIS OF REPEATER F-WAVES IN ALS}

Dimitra Veltsista $^{1}$, Elisabeth Chroni ${ }^{2}$

${ }^{1}$ Neurology, University Hospital of Patras, Patras, GR; ${ }^{2}$ Neurology, University of Patras, Patras, GR

Background: An increased frequency of repeater F-waves (Freps) has been demonstrated in neurogenic abnormalities such as amyotrophic lateral sclerosis (ALS). Analysis of repeater F-waves is rarely performed as part of routine studies, primarily because of difficulty in identifying them by simple visual inspection. The purpose of this study is to present the results of a computerized analysis of Freps and explore its clinical usefulness in ALS. Methods: Forty consecutive F-waves were recorded following supramaximal stimulation of the ulnar and peroneal nerve in 52 patients with ALS (33 males, mean age $64.1 \pm 10.2$ years, mean height $172.3 \pm 8.1 \mathrm{~cm})$ and 52 , age- and height-matched, healthy subjects (33 males, mean age $63.8 \pm 11.9$ years, mean height $172.5 \pm 8.1 \mathrm{~cm})$. ALS patients were further divided into 3 groups based on the location of their initial symptoms (upper limbs, lower limbs, bulbar). The F-waves were analyzed using an automated computerized system which identifies Freps and groups them (Figure). Parameters of Freps and non repeater F-waves (NRs) were studied and compared. Results: In the ALS group, Index Freps (100xFreps/total number of F-waves) were significantly higher than the control group $(52.9 \pm 25.3 \mathrm{vs}$ $7.3 \pm 7.8$ in ulnar nerve and $75 \pm 20.5$ vs $23.6 \pm 18.4$ in peroneal nerve) $(P<0.001)$. Likewise, the total number of repeating neurons (RNs) and Freps persistence (100xFreps/40stimuli) were significantly different between the groups. In healthy subjects, the peroneal nerve has significantly more Freps compared to the ulnar nerve $(P<0.001)$. None of the healthy subjects had more than 5 repetitions of any $\mathrm{RN}$ in the ulnar nerve, compared to ALS patients $(P$ $<0.001)$. No significant differences in RNs and NRs were identified between the subgroups of the ALS patients. Conclusion: Indexes of Freps and RNs are significantly increased in ALS patients, even in non advanced stages of disease, when compound muscle action potential (CMAP) values remain normal. These measures possibly reflect the excitability state of motor neurons and might be useful in the evaluation of early motor neuron dysfunction and followup of disease progression. The automated analyzing system improves accuracy and facilitates repeater F- wave identification and is less time consuming, thus suitable for routine neurophysiological studies. 

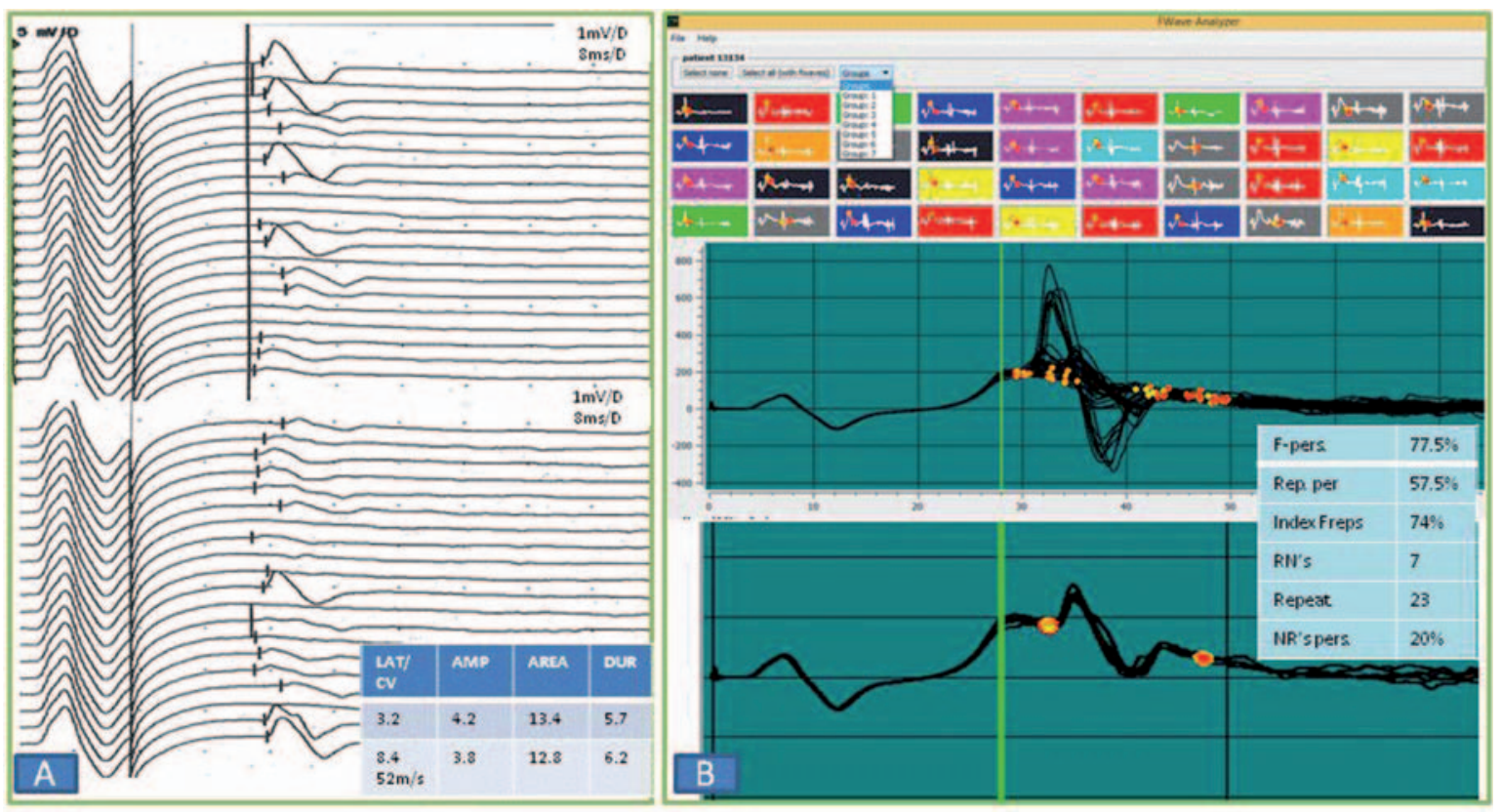

Figure. A. Forty consecutive $F$ wave recordings from the study of the ulnar nerve in a 66 year-old female patient with lower limb onset ALS. Note that CMAP values are within normal limits. B. Analysis of the $F$ wave using the $F$ wave analyzer. A total of 23 Freps are recognized (Freps persistence: 57,5\%, Index Freps: 74\%) grouped into 7 RN's. Each group is depicted on the interface in a different colour.

\section{PS3Group4-024 / \#771}

Topic: Group 4 - Motor Neuron Diseases: Clinical Features, Pathophysiology, Therapy

\section{THE OLEOS TRIAL: A LONG-TERM FOLLOW-UP OF OLESOXIME- TREATED TYPE 2 AND NON- AMBULATORY TYPE 3 SMA PATIENTS}

Francesco Muntoni ${ }^{1}$, Sabine F. Recktenwald ${ }^{2}$, Enrico Bertini ${ }^{3}$, Eugenio Mercuri ${ }^{4}$, Janbernd Kirschner $^{5}$, Muna El-Khairi ${ }^{6}$, Anna Lusakowska ${ }^{7}$, Giacomo P. Comi ${ }^{8}$, Jean-Marie Cuisset ${ }^{9}$, Jane Ives ${ }^{6}$, W Ludo Van Der $\mathrm{Pol}^{10}$, Carole Vuillerot ${ }^{11}$, Ksenija Gorni $^{12}$, Paulo Fontoura ${ }^{2}$

${ }^{1}$ Dubowitz Neuromuscular Centre, UCL Great Ormond Street Institute of Child Health and Great Ormond Street Hospital for Children, London, $G B ;{ }^{2}$ Neuroscience Product Development, F. Hoffmann-La Roche Ltd, Basel, CH; ${ }^{3}$ Department Of Neurosciences And Neurorehabilitation, Bambino Gesù Children's Research Hospital IRCCS, Rome, IT; ${ }^{4}$ Paediatric Neurology And Nemo Center, Catholic University and Policlinico
Gemelli, Rome, IT; ${ }^{5}$ Department Of Neuropediatrics And Muscle Disorders, Medical Center, University of Freiburg, Freiburg, DE; ${ }^{6}$ Roche Products Limited, Welwyn Garden City, GB; ${ }^{7}$ Department of Neurology, Medical University of Warsaw, Warsaw, $P L ;{ }^{8}$ Dino Ferrari Center, Department of Pathophysiology and Transplantation, University of Milan, Neurology Unit, I.R.C.C.S. Foundation Ca' Granda Ospedale Maggiore Policlinico, Milan, IT; ${ }^{9}$ Department Of Neuropediatrics, Neuromuscular Disease Reference Centre, Roger Salengro Hospital, Regional University Teaching Hospital (CHRU), Lille, FR $;{ }^{10}$ Department Of Neurology And Neurosurgery, Brain Center Rudolf Magnus, University Medical Center Utrecht, Utrecht, NL; ${ }^{11}$ Department Of Paediatric Physical Medicine And Rehabilitation, Hôpital Femme Mère Enfant, Centre Hospitalier Universitaire de Lyon, Lyon, FR $;{ }^{12}$ Roche Pharmaceutical Research and Early Development, Roche Innovation Center, Basel, CH

Background: Olesoxime is an oral, daily administered compound that supports the function of mitochondria. In a previous randomized, double-blind, Phase 2 study (NCT01302600) in patients aged 3-25 years with Type 2 or non-ambulatory Type 3 spinal 
muscular atrophy (SMA), olesoxime maintained motor function over 24 months, whilst the placebo group declined. OLEOS (NCT02628743) is an open-label extension of the Phase 2 study assessing the long-term safety and efficacy of olesoxime in patients with Type 2 or non-ambulatory Type 3 SMA. Methods: One hundred and twenty-nine patients with Type 2 or non-ambulatory Type 3 SMA from the previous Phase 2 study were enrolled and treated with olesoxime $(10 \mathrm{mg} / \mathrm{kg})$, and the majority have been followed for 12 months $(n=104)$. Primary endpoint is safety and secondary endpoints include change in Motor Function Measure (MFM) D1+D2 from baseline up to 5 years. OLEOS baseline visit occurred 2.4-5.1 years (median 3 years) after study drug discontinuation in Phase 2. Results: Consistent with previous studies, olesoxime was generally safe and well tolerated at the dose assessed. Maintenance of motor function observed over 2 years in the Phase 2 study was followed by a substantial decline in MFM D1+D2 ( $>2$ points/year) after drug discontinuation. However, the $\sim 2$-point MFM treatment difference between olesoxime and placebo at the end of Phase 2 was maintained at OLEOS baseline. Furthermore, olesoxime open-label treatment stabilized motor function (mean change in MFM D1 + D2 from baseline: 6 months, -0.03 [standard deviation (SD), 4.79; $\mathrm{n}=124] ; 12$ months, -0.22 [SD, 4.74, $\mathrm{n}=104]$ ). These data support the long-term stabilization of motor function observed in the Phase 2 study. A study update including novel 18-month data will be presented. Conclusion: Olesoxime was generally safe and well-tolerated. Data from the OLEOS openlabel extension study showed that olesoxime maintained motor function over 12 months in the treated patients. A study update (including novel 18-month data) will also be presented. These data suggest that olesoxime offers the potential to provide meaningful clinical benefit to patients with SMA by preventing loss of motor function, and may play a role in the future therapeutic management of SMA. Acknowledgments The authors would like to thank all the individuals enrolled in OLEOS and the previous Phase 2 study, their families and the site staff involved. Study sponsored by F. Hoffmann-La Roche.
PS3Group4-025 / \#794

Topic: Group 4 - Motor Neuron Diseases: Clinical Features, Pathophysiology, Therapy

\section{SMA-RTER? COGNITIVE ASSESSMENT IN SPINAL MUSCULAR ATROPHY TYPE 1-2 USING EYE TRACKING}

$\underline{\text { Lionel Paternoster }}^{1}$, Nicolas Deconinck ${ }^{2}$, Simon

Baijot $^{1}$, Gaetane Deliens ${ }^{3}$

${ }^{1}$ Department Of Pediatric Neurology, Queen

Fabiola Children's University Hospital, Brussels, $B E ;{ }^{2}$ Department Pediatric Neurology, HUDERF,

Brussels, BE: ${ }^{3}$ Ulb Neurosciences Institute, Université Libre de Bruxelles (ULB), Brussels, BE

Background: Spinal muscular atrophy is a devastating inherited disorder caused by ubiquitous deficiency in the SMN protein, with an incidence of around 1 to $6.000-10.000$ live births. It is the second most common autosomal recessive disorder and the most frequent genetic cause of infant death. SMA main feature is the progressive loss of motor activity caused by motor neurons degeneration and functional and structural dysfunction of neuromuscular synapses, leading to muscle atrophy. Recent evidence suggests that SMA is a systemic disease. In the brain SMN is expressed in many cell types in addition to motoneurons, brain morphology changes have been described, circadian dysregulation, abnormal fatty acid metabolism and abnormal glucose homeostasis have been observed. Symptoms of signs of a systemic disease may likely become increasingly apparent in treated children treated, where neuromuscular symptoms are alleviated and life extended. Literature on cognitive aspects of SMA type 1-2 is poor and divergent: von Gotard et al [2002] supported a normal cognitive function of SMA patients while Polido et al [2017] demonstrated matching pairs difficulty in children with SMA type 1 . Indeed, physical impairments make usual IQ tests often unusable in many SMA children. Methods: We develop an eye tracking system (Tobii ${ }^{\circledR}$ ) for the assessment of cognitive functions in SMA patients belonging to the severe end of the spectrum. The set of tests contains the following subtests: - Matrix subtest of Weschler non-verbal scale of ability for the study of fluid reasoning and perceptual reasoning. - Recognition subtest of Weschler non-verbal scale of ability for the study of visuo-spatial memory. - Peabody picture vocabulary test for the study of 
receptive vocabulary (French). - The chimeric animal stroop test to study inhibition. - Face recognition subtest of NEPSY-II to study face encoding, discrimination and recognition. - Picture complement test to study the spatial reasoning. - Matching pair test. Fourty children from 2 to 12 years old have been enrolled: 10 SMA type 1, 10 SMA type 2, and 20 age and sex matched control patients. Tests will take place in two sessions of 30 minutes for children from 2 to 4 years and in 1 session of 30 to 60 minutes for children from 5 to 12 years. The methodology and first results will be presented. Results: The developed protocol aims at validating eye tracking assessment tool for cognitive functions in SMA type 1 and 2. Conclusion: Cognitive assessment in spinal muscular atrophy type 1-2 using eye tracking system will help to adapt the overall management of those patients and to evaluate the impact on cognition of future SMA treatments.

\section{PS3Group4-026 / \#642}

Topic: Group 4 - Motor Neuron Diseases: Clinical Features, Pathophysiology, Therapy

\section{LONGER-TERM ASSESSMENT OF NUSINERSEN SAFETY/EFFICACY IN INFANTILE-ONSET SPINAL MUSCULAR ATROPHY: INTERIM ANALYSIS OF SHINE}

\author{
$\underline{\text { Richard S. Finkel }}^{1}$, Diana Castro ${ }^{2}$, Michelle A. \\ Farrar $^{3}$, Mar Tulinius ${ }^{4}$, Kristin J. Krosschell ${ }^{5}$, \\ Kayoko Saito ${ }^{6}$, Yiwei Zhang 7 , Ishir Bhan 7 , Wildon \\ Farwell $^{8}$, Sandra P. Reyna ${ }^{7}$ \\ ${ }^{1}$ Nemours Children's Hospital, Orlando, FL, \\ US; ${ }^{2} U T$ Southwestern Medical Center, Dallas, TX, \\ US, ${ }^{3}$ UNSW Medicine, UNSW Sydney and Sydney \\ Children's Hospital, Randwick, NSW, \\ $A U ;{ }^{4}$ Gothenburg University, Gothenburg, \\ SE; ${ }^{5}$ Northwestern University, Chicago, $I L$, \\ US; ${ }^{6}$ Tokyo Women's Medical University, Tokyo, \\ $J P ;^{7}$ Biogen, Cambridge, MA, US $;{ }^{8}$ Clinical \\ Development, Biogen, Cambridge, MA, US
}

Background: Nusinersen is an antisense oligonucleotide approved for the treatment of SMA. It has demonstrated a favorable benefit:risk profile and shown significant and clinically meaningful efficacy on motor function across a broad spectrum of SMA populations, and event-free survival (time to death or permanent ventilation) in infantile-onset SMA. The objective of the current analysis was to report interim results from the SHINE study (NCT02594124) for patients with infantile-onset SMA (most likely to develop Type I) who transitioned from ENDEAR. Methods: SHINE is an open-label extension study for infants/children who participated in the ENDEAR, CHERISH, CS12, or CS3A nusinersen trials. Nusinersen doses were administered according to the regimen and participant's cohort from the previous trial. The primary endpoint is safety/tolerability; secondary endpoints include achievement of Hammersmith Infant Neurological Examination - Section 2 (HINE-2) motor milestones and event-free survival defined as time to death or permanent ventilation (tracheostomy or $\geq 16$ hours ventilation/day continuously for $>21$ days in the absence of acute reversible event). Results: The cutoff date was June 30, 2017; 89 patients transitioned from ENDEAR, 65/81 previously randomized to nusinersen and 24/41 to sham-control. Within SHINE only, 83 patients had an adverse event (AE). There were no treatment-related serious AEs. The most frequent AEs were pyrexia and upper respiratory tract infection. Mean $(95 \% \mathrm{CI})$ change in HINE2 total score from nusinersen initiation to last observed visit was $1.1(0.20-1.90)$ for patients who received sham-control in ENDEAR and nusinersen in SHINE $(n=20 / 24)$ and 5.8 (4.58-7.04) for those who received nusinersen in ENDEAR and SHINE $(\mathrm{n}=74 / 81$; pooled ENDEAR/SHINE data). Median $(95 \% \mathrm{CI})$ event-free survival time among patients treated with sham-control in ENDEAR was 22.6 (13.6-31.3) weeks versus 73.0 (36.3-NA) weeks among those who received nusinersen in ENDEAR and SHINE. Conclusion: Improvements in motor function and event-free survival continued among patients who initiated nusinersen in ENDEAR and motor function improved among those who initiated nusinersen in SHINE. Further analysis of SHINE data will provide additional information on the longterm safety/tolerability and efficacy of repeated nusinersen doses across multiple SMA populations. 
PS3Group4-027 / \#712

Topic: Group 4 - Motor Neuron Diseases: Clinical Features, Pathophysiology, Therapy

\section{A CASE OF KENNEDY'S DISEASE IN A PATIENT INITIALLY PRESENTED WITH RECURRENT PERIODONTITIS AND OROMANDIBULAR PAIN}

\author{
$\underline{\text { Il-Han Yoo }}{ }^{1}$, So-Hyun Park ${ }^{1}$, Suk-Won Ahn ${ }^{2}$ \\ ${ }^{1}$ Chung-Ang University Hospital, Heukseok-ro, \\ Dongjak-gu, Seoul, KR; ${ }^{2}$ Neurology, ChungAng \\ University Hospital, Seoul, KR
}

Background: Kennedy disease (KD), also known as $\mathrm{X}$-linked spinal bulbar muscular atrophy, is a lower motor neuron degenerative disease with onset in adulthood and is characterized by slowly progressive muscular weakness and sensory neuropathy. Typically, affected patient develop symptoms and findings in the bulbar muscles with weakness, atrophy, tongue and facial muscle fasciculation and may develop difficulty managing secretions with the risk of aspiration pneumonia. Here we present a case of SBMA initially presented as recurrent periodontitis and oromandibular pain. Methods: A 45-year-old man visited our neurological department through dental clinics and presented with chronic and recurrent periodontitis accompanied by oromandibular pain over the course of 7 years. Results: Neurological examinations revealed mildly slurred speech, gait disturbance, facial fasciculation, decreased sensory function and proximal muscle weakness with Medical Resource Council (MRC) grade 4/5 in lower limbs. Laboratory investigations revealed significantly increased a creatine kinase (CK) level and nerve conduction study (NCS) and electromyography $(E M G)$ revealed sensory polyneuropathy with wide spreading denervation potentials. Furthermore, he showed an expanded cytosine-adenine-guanine (CAG) repeat in the androgen receptor (AR) gene analysis; therefore he was confirmatively diagnosed as Kennedy disease. Systemically, bulbar symptoms including dysphagia, orofacial muscle dysfunction, drooling and aspiration can predispose to malnutrition, social embarrassment and social isolation in addition to intraoral food retention, oral hygiene avoidance secondary to aspiration fear and perioral infections. Therefore these discomforts might result in the patient's inability to maintain ideal oral hy- giene. Conclusion: Here we present a case of SBMA initially presented as recurrent periodontitis and oromandibular pain. It is important to consider neuromuscular diseases backgrounds to severe periodontitis cases, as this may eventually be relevant to treatment and to understanding the pathogenesis of periodontitis.

\section{PS3Group4-028 / \#737}

Topic: Group 4 - Motor Neuron Diseases: Clinical Features, Pathophysiology, Therapy

UPDATED PHARMACODYNAMIC AND SAFETY DATA FROM SUNFISH PART 1, A STUDY OF ORAL RG7916 IN PATIENTS WITH TYPE 2 OR

\section{TYPE 3 SMA}

Eugenio Mercuri ${ }^{1}$, G Baranello², Janbernd Kirschner $^{3}$, Laurent Servais ${ }^{4}$, Nathalie Goemans ${ }^{5}$, Maria C. Pera ${ }^{1}$, Anne Marquet ${ }^{6}$, Gillain Armstrong ${ }^{7}$, Heidemarie Kletzl ${ }^{6}$, Marianne Gerber ${ }^{6}$, Christian Czech $^{6}$, Yumi Cleary 6 , Margaret Chan ${ }^{8}$, Stephane Nave $^{6}$, Ksenija Gorni ${ }^{6}$, Omar Khwaja ${ }^{6}$

${ }^{1}$ Paediatric Neurology And Nemo Center, Catholic University and Policlinico Gemelli, Rome, IT; ${ }^{2}$ Developmental Neurology Unit, Carlo Besta Neurological Research Institute Foundation, Milan, IT, ${ }^{3}$ Department of Neuropediatrics and Muscle Disorders, Medical Center-University of Freiburg, Freiburg, DE: ${ }^{4}$ Institute of Myology, Paris, FR, ${ }^{5}$ Neuromuscular Reference Centre, Department Of Paediatrics And Child Neurology, University Hospitals Leuven, Leuven, BE; ${ }^{6}$ Roche Pharmaceutical Research and Early Development, Roche Innovation Center, Basel, $\mathrm{CH} ;{ }^{7}$ Roche Products Ltd, Welwyn Garden City, GB; ${ }^{8}$ Roche Pharmaceutical Research and Early Development, Roche Innovation Center New York, New York, NY, US

Background: Spinal muscular atrophy (SMA) is characterized by motor neuron loss and muscle atrophy, due to reduced levels of survival of motor neuron (SMN) protein from loss of function of the SMN1 gene. While SMN1 produces full-length SMN protein, a second gene, SMN2, produces only low levels of functional SMN protein. RG7916 (RO7034067) is an investigational, orally administered, centrally and peripherally distributed small 
molecule that is being evaluated for its effect on modulating SMN2 pre-mRNA splicing towards the production of full-length $S M N 2$ mRNA and increase of SMN protein. SUNFISH (NCT02908685) is a multi-center, double-blind, placebo-controlled trial (randomized 2:1, RG7916:placebo) in patients with Type 2 or 3 SMA aged 2-25 years. Methods: SUNFISH comprises two parts: Part 1 evaluates the safety, tolerability and pharmacokinetics/ pharmacodynamics of different RG7916 dose levels $(n=51)$; the pivotal Part 2 assesses the efficacy and safety of the RG7916 dose level selected from Part 1 $(n=168)$. Results: We have previously presented an early analysis of SUNFISH Part 1, which showed that RG7916 administration resulted in a dose-dependent increase in full-length SMN2 mRNA and a concomitant decrease in SMN2 47 mRNA. SMN protein levels in whole blood increased in a dosedependent manner up to a median of 2.5 -fold. To date, adverse events were mostly mild, resolved despite ongoing treatment and were reflective of the underlying disease. There have been no drug-related safety findings leading to withdrawal. The safety, tolerability and pharmacokinetic/pharmacodynamic data from Part 1 were sufficient to inform the selection of a dose level for SUNFISH Part 2, to evaluate the efficacy and safety of RG7916. We will provide a more recent and detailed SUNFISH update with biomarker, pharmacokinetic and safety results from patients included in Part 1. To date, all Part 1 patients have been treated with RG7916 for a duration of a minimum of 3 months (in the last placebo patient who switched to active treatment) and up to 16 months. Conclusion: In SUNFISH Part 1, RG7916 treatment modulated SMN2 mRNA and increased SMN protein in a dose-dependent manner. The clinical benefit of the selected dose level is being assessed in SUNFISH Part 2, which is currently recruiting globally. Acknowledgments The authors would like to thank all individuals enrolled in the RG7916 studies, their families and the site staff involved. Study sponsored by F. Hoffmann-La Roche.
PS3Group4-029 / \#799

Topic: Group 4 - Motor Neuron Diseases: Clinical Features, Pathophysiology, Therapy

\section{MORPHINE FOR DYSPNEA IN ALS}

$\underline{\text { Lev Brylev }}^{1}$, Vadim Parshikov ${ }^{2}$, Yaroslava

Krasnaya $^{2}$, Ekaterina Dikhter ${ }^{2}$, Egor Larin ${ }^{3}$, Yana

Batmanova $^{2}$, Vasiliy Shtabnitskiy ${ }^{4}$, Sergey

Avdeykin ${ }^{5}$, Anastasia Ataulina ${ }^{5}$, Daria Puzanok ${ }^{2}$, Anna Kasianova ${ }^{2}$

${ }^{1}$ Buyanov City Hospital, Moscow, RU; ${ }^{2} A L S$ center

Moscow, Moscow, $R U,{ }^{3}$ Center of palliative

medicine, Moscow, $R U,{ }^{4}$ Chaika, Moscow,

$R U,{ }^{5}$ Buyanov city hospital, Moscow, $R U$

Background: Dyspnea affects more than half patients with amyotrophic lateral sclerosis (ALS). Management of this distressing symptom can be challenging. The most effective drug for dyspnea is morphine, but it is underused for this indication, due to lack of evidence of safety of morphine in patients with ALS and lack of information about it's effectiveness. Methods: In ALS centre Moscow we followed 430 patients with mean age 59,3 $\pm 10,8$ years, with median ALSFRS-R 26 (17;32) and median vital capacity $51,0 \%(38 ; 80)$ on admission. 25 ALS patients with mean age $66.3 \pm 12,1$ years, 14 males and 11 females recieved morphine for dyspnea. We used Borg scale to measure dyspnea. In five patients we measured safety of morfine by measuring $\mathrm{pCO} 2$ in arterial blood during morfine infusion. Patients were divided as respontenders to morfine if they had good dyspnea control on morphine, partial responders if they had 3 points desrease on Borg scale and nonresponders. Results: Morphine didn't cause elevation of $\mathrm{pCO} 2$ in arterial blood of our patients. $50 \%$ of patients were good responders, $40 \%$ - partial responders and $10 \%$ - nonresponders. Dose of morphine in the group of responders $(23 \pm 8,2) \mathrm{mg}$ per day was higher, than in partial responders - $(11,8 \pm 3.4$ $\mathrm{mg})$ and nonresponders $(10 \pm 2.7 \mathrm{mg}) .48 \%$ of patients used lorasepam for concominant laringospasms. Conclusion: Morphine is highly effective in $50 \%$ of ALS patients with dyspnea and doesn't supress respiration in doses, that are prescribed to control dyspnea. Morphine should be more widely used to control dyspnea in ALS patients. 
PS3Group4-030 / \#944

Topic: Group 4 - Motor Neuron Diseases: Clinical Features, Pathophysiology, Therapy

\section{EXTRACELLULAR VESICLES FROM ALS SPINAL CORD AND BRAIN CONTAIN DYSREGULATED MIRNAS}

Eva Feldman

University of Michigan, Ann Arbor, US

Background: Aims/hypothesis: Amyotrophic lateral sclerosis (ALS) is a terminal neurodegenerative disease. Clinical and molecular observations indicate that motor neuron death and muscle atrophy in ALS proceeds in a patterned manner suggesting that ALS develops as a non-infectious prion-like disease $^{1,2}$. Extracellular vesicles (EVs) maybe play a role in the spread of ALS as they facilitate transfer of molecules from cell-to-cell ${ }^{3-5}$ In this project we aim to determine the role of EVs cargo in ALS pathology. Aberrant epigenetic mechanisms such as DNA methylation and miRNAs levels have been reported in $\mathrm{ALS}^{6}$. We hypothesize that EVs may be involved in the transfer of epigenetic alterations in ALS. Methods: Methods: We used an immuno-affinitybased microfluidic device, the ExoChip, for isolation and quantification of $\mathrm{EVs}^{7}$ from frontal cortex $(\mathrm{Ctrl}=8, \mathrm{ALS}=12)$, spinal cord $(\mathrm{Ctrl}=6, \mathrm{ALS}=12)$, and plasma ( $\mathrm{Ctrl}=9, \mathrm{ALS}=7$ ). Electron microscopy was used for morphological characterization of EVs. EV-contained miRNAs were profiled with NanoString technology and analyzed by nSolver and NanoStringDiff. Results: Results: Analysis of plasma, frontal cortex, and spinal cord indicates there is no significant increase in the availability of EVs in ALS, when compared to control subjects. However, we detected alterations in the levels of mature miRNAs contained in EVs from all examined tissues. Conclusion: Conclusions/interpretations: We have identified altered miRNAs packaged in EVs from ALS tissue. These miRNAs are important in biological functions and pathways previously associated with neurodegeneration. Identification of altered EVs-contained miRNAs in ALS have both diagnostic and therapeutic potential, as EVs can represent biomarkers and may be used for the delivery of drugs or genetic molecules including miRNAs.
PS3Group7-001 / \#590

Topic: Group 7 - Patient Related Issues

\section{THE IMPACT OF PARTICIPATION IN CLINICAL TRIALS ON PSYCHOSOCIAL WELLBEING OF CHILDREN WITH DMD AND THEIR PARENTS}

$\underline{\text { Sam Geuens }}^{1}$, Joanna Willen ${ }^{2}$, Corine Antonis ${ }^{2}$, Nathalie Goemans ${ }^{3}$, Liesbeth De Waele ${ }^{2}$ ${ }^{1}$ Child Neurology, UZ Leuven, Leuven, BE; ${ }^{2}$ Child Neurology, UZ Leuven, Leuven, BE: ${ }^{3}$ Treat-NMD Neuromuscular Network, Leuven, BE

Background: Approximately 35-40 per 100000 children are diagnosed with a neuromuscular disorder, but for most of those diseases no treatment is available. However, more and more clinical trials (CT) are experimenting with promising therapies. Participation in a CT is for most patients and their parents the only hope to receive a treatment that can have a significant positive impact on the disease. The burden of participation in a CT, however, should not be underestimated: frequent time investment, impact on family activities, absence of work and school, side effects of the medication,... Studies investigating this burden are scarce and the costs for participation for individual families are not known. The aim of this study is to measure the psychosocial impact and the burden of participation in a CT for patients with Duchenne Muscular Dystrophy (DMD). Methods: A cross-sectional research design was constructed with a group of patients who participated in a CT and an age- and gender-matched control group of patients with DMD, but not participating in a CT. Both parents of patients in both groups were asked to fulfill a Child Behavior Checklist (CBCL) reporting the wellbeing of their child and an Adult Self Report (ASR) reflecting their own wellbeing. Furthermore they were asked to complete a pediatric quality of life inventory (PedsQL). All children were asked to report their quality of life by filling in an age appropriate version of the PedsQL. If they were older than 11 years an extra questionnaire was given, namely the Youth Self Report (YSR), reflecting their own wellbeing. In the CT-group an extra explorative survey, the Clinical Trial Survey (CTS), was conducted. This 
instrument was developed to measure the burden of participation to a CT for patients and their families. This new instrument includes 24 questions divided into 6 different domains. Results: 25 children with DMD who participated in a CT are included. The control group consisted of 20 age-matched children. At the moment of abstract submission only preliminary data was available. 25 mothers and 22 fathers of boys with DMD who participated in a CT completed the CTS. This survey will provide us with explorative information and will allow us to estimate and quantify the impact of participating in a clinical trial for both children and parents. Secondly, we have data from behavioral questionnaires and quality of life questionnaires of boys and parents in a clinical trial and a control group and therefor able to detect differences between groups. Moreover, because we also have self-report scores from both mothers and fathers reporting on themselves, we can illustrate what the impact is on the parents and if there is a difference between them. Finally, we want to see if we can identify some factors contributing to the impact of a CT. Conclusion: Definitive results and conclusions areexpected at the end of April 2018

\section{PS3Group7-002 / \#452}

Topic: Group 7 - Patient Related Issues

\section{PHYSICAL ACTIVITY IN PEOPLE WITH LIMB-GIRDLE MUSCULAR DYSTROPHY AND CHARCOT- MARIE-TOOTH DISEASE IN NORWAY}

Aristomo Andries, Marleen R. Van Walsem, Jan Frich

Institute Of Health And Society, University of Oslo, Oslo, NO

Background: Active lifestyle is recommended to promote health and well-being, including in people living with disabilities. People with neuromuscular diseases (NMD), particularly limb-girdle muscular dystrophy (LGMD) and Charcot-Marie Tooth disease (CMT), may have challenges to do certain physical activities. While other studies have demonstrated benefits of physical activity in the people with NMD, there is a lack of knowledge about physical activity level in people with LGMD and CMT. The aim of this study is to investigate the self-report- ed physical activity levels in people with LGMD and CMT. Methods: This is a cross-sectional observational study. People who were registered in the national database for neuromuscular conditions (University Hospital of North Norway) were selected randomly and invited to participate in the study. Invitations were sent by postal mail. People who fulfilled the inclusion criteria and consented to participate filled out a set of paper-based questionnaires or online-based questionnaire in University of Oslo's database system. Physical activity level was investigated using self-assessed International Physical Activity Questionnaire short form (IPAQ-sf). Participants with vigorous-intensity activity for at least 3 days and achieved at least 1500 metabolic equivalent (MET)-minutes/week or 7 days of any other activities with at least 3000 MET-minutes/ week were classified as "health-enhancing physical activity" (HEPA). Participants outside the HEPA group were classified as either "inactive" or "minimally active" according to IPAQ-sf protocol. Socioeconomic-demographic factors were also included in the questionnaire. Other factors which may influence physical activity level such as functional ability, health related quality of life, and fatigue were investigated using validated questionnaires. Data were analyzed using descriptive statistics. Researchers used IBM SPSS statistical program version 25 to perform the analysis. Results: Out of 236 potential participants were invited to the study, 145 (61.4\%) participants responded to the study and filled out questionnaire either paper-based or online. 79 respondents $(54.5 \%)$ had been diagnosed with CMT and $66(45.5 \%)$ respondents had been diagnosed with LGMD. Of the respondents $61(42.1 \%)$ were male and $84(57.9 \%)$ were female. The median of MET-minutes/week was 1180.50 (IQR=2643.63). Mean sitting time was 518.56 minutes ( $\mathrm{SD}=231.86$ ). $48(45.3 \%)$ respondents were classified as "inactive", $30(28.3 \%)$ as "minimally active", and 28 $(26.4 \%)$ as "HEPA". The odds ratio (OR) of respondents who have HEPA compared to inactive/minimally active was 5.5-fold (95\% CI 1.51-20.10, $\mathrm{p}=0.005$ ) in people with normal activity daily living/ ADL (measured by Barthel's index) compared to respondents with decreased ADL. Conclusion: More than a quarter of study participants have fulfilled the HEPA category, while the majority of respondents were unable to achieve this. Participants with normal ADL showed greater odds of being physically active compared to those with decreased ADL. To our knowledge, this study is the first to investigate 
self-reported physical activity level among people with LGMD and CMT in Norway. The study findings are important to understand how to achieve optimum physical activity level for beneficiaries. Furthermore, factors which may influence physical activity need to be thoroughly examined. It may also be interesting to compare these findings to other group of neuromuscular diseases.

\section{PS3Group7-003 / \#822}

Topic: Group 7 -Patient Related Issues

\section{THE JAIN FOUNDATION DYSFERLIN PATIENT REGISTRY: ACCELERATING THE PACE OF RESEARCH AND TREATMENT}

Laura Rufibach, Sarah Shira, Laurie Long, Bradley Williams, Douglas Albrecht

Jain Foundation, Seattle, US

Background: Dysferlinopathy is a recessively inherited form of muscular dystrophy caused by mutations in the DYSF gene. The most common clinical forms are limb girdle muscular dystrophy type $2 \mathrm{~B}$ (LGMD2B) and Miyoshi Myopathy type 1 (MMD1). Onset of muscle weakness generally occurs in young adulthood with loss of ambulation typically within 10-20 years. The prevalence of this disease is estimated to be between 5-8/million. The Dysferlin Registry is an important tool to facilitate and accelerate clinical research through identification of information and recruitment of participants. It also is an interactive registry that allows individuals with dysferlinopathy to communicate with one another and the members of the Jain Foundation. Aims: Identify and recruit patients for clinical studies and trials - Empower and cultivate a trial ready patient community through education and engagement - Encourage communication and expedite our understanding of challenges patients are navigating in daily living, ultimately assisting in targeting possi- ble outcome measures for trials - Provide patients with up to date information on research progress Support and encourage research by providing access to data. Methods: The Dysferlin Registry is exclusively for individuals who have been confirmed to have a dysferlinopathy through the evaluation of their clinical symptoms, dysferlin protein levels, and genetic analysis. Diagnosed patients are invited to join and after accepting, they enter data by creating a password-protected account through an online portal. The patient also chooses their level of engagement within the community. These levels range from private (allowing only the registry administrators to see their presence in the registry) to fully active (where they set up an online profile; allowing for communication with other members). The registry also features a portal that allows clinicians to enter patients into the registry after obtaining the necessary consent. The registry collects contact, demographic, clinical, genetic, protein, phenotypic and familial information. Results: As of February 2018, there are currently 654 people registered from 41 countries, with the largest number residing in the United States. The ages of the people registered ranges from $10-79$, with $67 \%$ being between the ages of 25-50. The average age of onset was 26 years of age with $79 \%$ reporting onset of muscle symptoms between $15-30$. The majority (75\%) of the registrants indicate they are still ambulant, but with differing levels of ambulation from running to walking only with assistance. The Dysferlin Registry has already supported recruitment and pre-trial design for multiple clinical studies and trials. Conclusion: Patient registries are invaluable tools in the field of rare diseases because with so few patients every one counts. Please encourage all your dysferlinopathy patients to register. The Jain Foundation Dysferlin Registry is seeking to cultivate an informed, trial ready community that has the potential to accelerate clinical trials and support research into possible treatments. Please visit www.dysferlinregistry.jainfoundation.org and if you have a patient who may be eligible, please email us at patients@jain-foundation.org. 
PS3Group7-004 / \#618

Topic: Group 7 -Patient Related Issues

\section{PATIENT PERSPECTIVES ON THE SIDE EFFECT BURDEN OF TREATMENTS FOR MYASTHENIA GRAVIS (MG) AND THEIR IMPACT ON DAILY LIFE}

\author{
Elizabeth D. Bacci ${ }^{1}$, Karin S. Coyne ${ }^{1}$, Linda \\ Harris $^{2}$, Jiat-Ling Poon ${ }^{3}$, Audra N. Boscoe ${ }^{4}$ \\ ${ }^{1}$ Business Unit Of Ppd, Evidera, Bethesda, MD, \\ US:2Alexion Pharmaceuticals, New Haven, \\ US: ${ }^{3}$ Lilly Corporate Center, Eli Lilly and Company, \\ Indianapolis, IN, US; ${ }^{4}$ Agios Pharmaceuticals, \\ Cambridge, MA, US
}

Background: Standard treatments for patients with myasthenia gravis are associated with side-effects; however, the frequency, severity and impact of these side effects on patients' lives are not fully understood. The objective of this study was to characterize the side effects of standard treatment for generalized $\mathrm{MG}$ and their associated impact on health-related quality of life (HRQL) from the patient perspective. Methods: MG Foundation of America patient registry (managed by the coordinating center at the University of Alabama Birmingham and the MGFA Registry Committee) members were invited to participate in a web survey. Participants were asked to brainstorm side effects (Step 1) experienced due to treatments for $\mathrm{MG}$ and the impact of those side effects on HRQL using a group concept mapping approach. The statements provided by participants were synthesized, and participants were resurveyed to rate the tolerability and severity of side effects (Step 2) based on the statements, and the frequency and severity of HRQL impacts on a 1 (least) to 4 (most) scale. Participants also completed sociodemographic questions, the MG Quality of Life Scale (MGQOL15), and the Morisky Medication Adherence Scale (MMAS-8). Descriptive statistics were used to characterize the study sample and sum- marize participants' ratings. Results: A total of 246 participants contributed to the brainstorming exercise ( $63 \%$ female; mean \pm SD age $58.4 \pm 13.4$ years); $158(64 \%)$ participated in the rating exercise. Nearly all $(92 \%)$ participants confirmed that their $\mathrm{MG}$ symptoms were consistent with generalized $\mathrm{MG}$ and not limited to ocular symptoms. The majority reported currently being on pyridostigmine $(69 \%)$ and/or immunosuppressive treatment (68\%). Just under half $(46 \%)$ reported having previously used at least two immunosuppressive treatments and one-third reported having received IVIg and/or plasmapheresis on more than four separate occasions over the past year. The brainstorming exercise produced 874 statements, which were condensed to 35 side effects and 23 HRQL impact statements. Blood clots, infections/decreased immunity, weight gain, and diarrhea were the least tolerable and most severely rated side effects. The most frequent and severe HRQL impacts were sleep interference and reduction in physical and social activities. Subgroup analyses compared rating results between participants who had used multiple prior therapies (refractory patients) and had prior experience with side effects to non-refractory patients, and determined that the severity and tolerability of the side effects experienced were similar regardless of treatment refractory status. However, HRQL impacts were more frequent and severe for refractory patients compared to nonrefractory patients. MGQOL15 results indicated moderate impact on HRQL, with most participants (79\%) reporting either some or quite a bit of frustration with their MG and many (57\%) reporting severe to very severe impacts on their occupational skills and job status. Per the MMAS- $8,36 \%$ of patients had low adherence and 33\% had medium adherence to their MG medications. Conclusion: MG patients experience high burden of treatment side effects with existing treatment options, which potentially impacts treatment adherence and their health outcomes. Based on these findings, there appears to be a need for better and more tolerable treatment options for this patient population. 



\section{Author Index}

Aartsma-Rus A.

Aban I.B.

Abd El-Hamed M.A.

Abicht A.

Adams D.

Afroozan F.

Aguirre F.

Ahmed A.

Ahn S.H.

Ahn S.-W.

Aizpurua J.M.

Akhondi F.

Akramova D.T.

Alarcon Cornejo M.M.A.

Albiasu-Arteta E.

Albrecht D.

Aldanondo G.

Aleff R.A.

Alekseeva T.

Aleksic K.

Alfa Cissé O.

Alfano L.

Aliu B.

Allegorico L.

Allenbach Y.

Allen J.E.

Almasi M.

Almendrote M.

Alshareef A.A.

Alves B.C.A.

Al-Zaidy S.
PS2Group9-019, S323

SS 23.2, S39

PS2Group3-067, S270

PS1Group1-130, S204, PS2Group3-074, S275, PS2Group5-016, S295

PS2Group3-005, S220, PS2Group3-010, S225, PS2Group3-055, S261

PS1Group1-096, S178

PS3Group2-007, S330, PS3Group2-008, S330 PS1Group1-140, S212

PS1Group1-131, S205, PS1Group1-141, S213, PS2Group3-032, S244 PS2Group3-035, S246, PS2Group3-061, S266, PS3Group4-027, S378 PS1Group1-038, S132, PS1Group1-106, S186 PS2Group5-009, S290 PS2Group5-025, S301 PS2Group9-010, S317 PS3Group4-019, S371 PS1Group1-054, S146, PS1Group1-108, S187 PS2Group5-007, S288, PS3Group7-003, S382 PS1Group1-038, S132, PS1Group1-106, S186 PS1Group1-011, S110 PS3Group4-021, S373 PS1Group1-078, S164 PS2Group3-064, S268 PS3Group4-022, S373, SS 13.4, S25

PS2Group3-015, S229 PS2Group5-034, S307 PS2Group9-006, S314, WS 13.3, S58

PS1Group1-082, S167, PS1Group1-147, S216 PS2Group5-009, S290 PS1Group1-089, S173 PS2Group3-014, S228 PS1Group1-107, S186 PS3Group4-022, S373, SS 13.4, S25
Amato A.

Ambawat S.

A N.

Anderson D.

Andres V.

Andries A.

Angelini C.

An J.Y.

Annousamy M.

Antenora A.

Antonini G.

Antonis C.

Anziska Y.

Aparicio J.

Apreleva A.

Arad M.

Arahata H.

Archer J.O.S.

Arechavala-Gomeza V.

Argov Z.

Armando Jr J.

Armas J.

Armstrong G.

Arnaoutoglou M.

Arnold W.D.

Artemyeva S.

Ashish K.

Ashoorian Y.

Ashtari F.

Assadian S.

Ataulina A.

Attarian S.

Attarwala $\mathrm{H}$.

Auboeuf D.

Auer-Grumbach M.

Auradé F.

Avdeykin S.
SS 19.2, S31, WS 4.1, S48, WS 7.3, S53

PS1Group1-016, S114, PS1Group1-044, S138 PS3Group2-022, S342 PS3Group2-002, S326, PS3Group2-003, S326 PS3Group2-008, S330 PS3Group7-002, S381

OA 3.1, S91, PS1Group1-051, S144, TC 1.3, S75 WS 16.4, S64 PS2Group3-066, S270 PS1Group1-004, S104 PS2Group5-021, S298 PS1Group1-051, S144 PS3Group7-001, S380

PS2Group3-017, S230 PS1Group1-013, S112 WS 3.4, S47

PS2Group3-002, S218 PS1Group1-029, S125 PS3Group4-011, S364 PS1Group1-054, S146, PS1Group1-108, S187 OA 2.2, S90, SS 1.1, S10 PS2Group3-059, S265 PS2Group9-010, S317, PS3Group4-019, S371 PS3Group4-002, S356, PS3Group4-015, S367, PS3Group4-028, S378 PS1Group1-102, S183 PS3Group4-022, S373, SS 13.4, S25

PS2Group9-015, S320 PS2Group3-029, S241 PS1Group1-043, S137 PS1Group1-043, S137 PS2Group5-007, S288 PS3Group4-029, S379, WS 3.4, S47

PS2Group3-065, S269 PS2Group3-043, S251 PS3Group4-006, S360 SS 9.4, S19, TC 3.1, S79 PS1Group1-007, S107 PS3Group4-029, S379, WS 3.4, S47 
Awano H.

Azorín I.

Azuma Y.

Babacic H.

Babu G.

Bacci E.D.

Bae D.W.

Bae J.S.

Baek S.-H.

Baets J.

Baghdoyan S.

Baijot S.

Balaraju S.

Balcarek P.

Baldwin C.

Ballester-Lopez A.

Bandyopadhyay D.

Banerjee M.

Ban J.

Banko B.

Banovic M.

Bansagi B.

Baranello G.

Barbeau S.

Barnett C.

Barohn R.J.

Bartesaghi L.

Barthélémy I.

Bartnik E.

Bartonek L.

Basiri K.

Basta I.

Batmanova Y.

Battaglia D.

Bauché S.

Baudin P.Y.

Bauer L.

Baydan F.

Beadon K.

Bechtold C.

Bednarik J.
PS1Group1-052, S145,

PS1Group1-128, S202

PS2Group3-053, S259

SS 24.2 , S40

PS1Group1-002, S102

PS1Group1-111, S189

PS3Group7-004, S383

PS2Group3-066, S270

PS2Group3-041, S250

PS2Group5-017, S295

PS1Group1-022, S119,

PS1Group1-138, S210

PS3Group4-006, S360

PS3Group4-025, S376

PS1Group1-044, S138,

PS3Group2-022, S342

PS1Group1-025, S122

PS2Group3-037, S247

PS1Group1-089, S173

PS2Group3-029, S241

PS2Group3-008, S223

PS1Group1-101, S182

PS1Group1-080, S165

PS1Group1-104, S185

PS2Group3-060, S265

PS3Group4-008, S361,

PS3Group4-015, S367,

PS3Group4-028, S378

PS3Group2-028, S347

PS3Group2-005, S328

PS2Group3-051, S257,

PS3Group2-021, S341,

SS 11.2, S21, SS 22.2, S37,

SS 23.1, S38, TC 6.1, S83

PS2Group3-044, S252

PS1Group1-005, S105,

PS1Group1-007, S107,

PS1Group1-008, S108,

PS1Group1-121, S197

PS1Group1-036, S131

PS2Group3-026, S239

PS1Group1-043, S137

PS1Group1-077, S163,

PS1Group1-078, S164,

PS1Group1-104, S185,

PS2Group3-028, S241

PS3Group4-029, S379,

WS 3.4, S47

PS1Group1-027, S123

PS2Group5-027, S302,

PS3Group2-030, S348

PS1Group1-004, S104

PS3Group4-004, S358

PS2Group3-013, S227,

PS2Group9-014, S320

PS2Group3-065, S269

PS1Group1-014, S112

PS1Group1-037, S132
Beecher G.

Beeson D.

Bekircan-Kurt C.E.

Beladi Moghadam N.

Belaïdi $\mathrm{H}$.

Belarabi L.

Belaraju S.

Bello L.

Bell P.

Belousova E.

Benatar M.

Ben Djebara M.

Benedetti F.

Benet-Pagès A.

Bennett D.

Benoit V.

Berardinelli A.

Berciano J.

Berek K.

Bergauer T.

Berini S.

Berki T.

Berk J.

Bernert G.

Berry K.

Bertassoli B.M.

Bertini E.

Bertoli M.

Berwanger C.

Beydoun S.R.

Bhan I.

Bharucha-Goebel D.X.

Bhattacharya I.

Bhattacharya K.

Bianchini E.

Bicciato S.

Bieri O.

Binda A.

Birouk N.
PS3Group2-002, S326,

PS3Group2-003, S326

PS3Group2-036, S353, SS

24.1, S40, WS 14.2, S59

PS2Group3-006, S222

PS1Group1-043, S137

PS1Group1-067, S157,

PS1Group1-068, S157,

PS2Group5-012, S292

PS2Group5-012, S292,

PS2Group5-013, S292

SS 24.2, S40

PS1Group1-115, S192

PS2Group9-013, S319

PS2Group9-015, S320

PS3Group2-021, S341

PS2Group3-077, S277

PS3Group2-024, S343

PS2Group5-016, S295

SS 15.3, S27

PS2Group3-070, S272

PS1Group1-051, S144

PS1Group1-100, S181

PS2Group3-076, S276

PS3Group4-002, S356

PS1Group1-011, S110

PS1Group1-099, S180

PS2Group3-005, S220,

PS2Group3-010, S225,

PS2Group3-055, S261

SS 5.1, S13, TC 4.1, S81

PS3Group4-022, S373

PS1Group1-047, S141,

PS1Group1-073, S161,

PS1Group1-107, S186,

PS2Group3-059, S265

PS1Group1-027, S123,

PS3Group4-008, S361,

PS3Group4-024, S375

PS1Group1-138, S210

PS1Group1-134, S207,

PS1Group1-135, S208

PS3Group2-004, S327

PS3Group4-014, S366,

PS3Group4-026, S377

PS1Group1-063, S153,

PS1Group1-115, S192,

PS2Group5-007, S288

PS1Group1-019, S117

PS2Group5-010, S291

PS1Group1-125,

PS2Group5-020, S297

PS1Group1-017, S116

PS1Group1-110, S188

PS1Group1-067, S157,

PS1Group1-068, S157,

PS2Group5-012, S292,

PS2Group5-013, S292,

SS 8.2, S16, SS 21.3, S35 
Bischoff C.

Bista $P$.

Bjelica B.

Bjelic M.

Blamire A.

Blanchard-Gutton N.

Blanco-Arias P.

Blot S.

Blutke A.

Boczonadi V.

Boex M.

Boisserie J.-M.

Boland A.

Boland-Freitas R.

Bonati U.

Bönnemann C.G.

Bonuccelli U.

Boostani R.

Borchers C.H.

Born A.

Borras A.

Boscoe A.N.

Bose' F.

Boss M.A.

Botez S.

Botta A.

Bouchet-Séraphin C.

Boucraut J.

Bouhour F.

Bozovic I.

Brajuskovic G.

Brankovic M.

Bravver E.

Briani C.

Brighina F.

Brigonzi E.

Bril V.
TC 5.1, S82

PS1Group1-060, S151

PS1Group1-077, S163,

PS1Group1-078, S164, PS1Group1-104, S185, PS2Group3-028, S241

PS1Group1-059, S150

PS1Group1-009, S108,

PS1Group1-115, S192

PS1Group1-007, S107,

PS1Group1-008, S108

PS1Group1-100, S181

PS1Group1-005, S105,

PS1Group1-007, S107,

PS1Group1-008, S108,

PS1Group1-121, S197

PS1Group1-065, S155

PS2Group3-060, S265

PS2Group5-027, S302

PS2Group9-006, S314

PS3Group4-006, S360

PS2Group5-003, S285,

PS2Group5-024, S300

PS1Group1-017, S116

PS1Group1-035, S130,

PS1Group1-039, S133

PS3Group2-027, S346

PS1Group1-043, S137

PS2Group5-007, S288

PS3Group4-008, S361

PS3Group4-019, S371

PS3Group2-033, S351,

PS3Group7-004, S383

PS1Group1-042, S136,

PS1Group1-056, S148,

PS1Group1-110, S188

PS2Group9-018, S323

PS3Group2-026, S345

PS1Group1-110, S188

PS1Group1-066, S156

PS2Group3-065, S269

PS1Group1-066, S156,

PS1Group1-088, S172,

PS3Group2-030, S348

PS1Group1-077, S163,

PS1Group1-104, S185,

PS2Group3-028, S241

PS1Group1-079, S164

PS1Group1-077, S163,

PS1Group1-080, S165

PS1Group1-063, S153,

PS1Group1-115, S192,

PS2Group5-007, S288

PS2Group3-082, S283

PS1Group1-127, S202

PS1Group1-042, S136,

PS1Group1-110, S188

PS2Group3-012, S226,

PS2Group3-018, S231,
Brinkmeier $\mathrm{H}$.

Brkusanin M.

Brochier G.

Broda P.

Broser P.J.

Brown L.

Bruno C.

Bruno G.

Brureau A.

Brusse E.

Brylev L.

Bryson-Richardson R.

Bucci E.

Buchbjerg J.

Bulst S.

Bungener $\mathrm{C}$.

Burden S.

Burghes A.H.M.

Burns T.M.

Burnyte B.

Bushby K.

Buyse For The Delos Study

Group G.

Buza E.L.

Byrne B.

Cabrera F.A

Cacciavillani M.

Caillaud C.

Callaghan B.C.

Camerino G.M.

Cano A.

Cao M.

Capogrosso R.F.

Capone A.

Cardani R.
PS2Group3-019, S233, PS2Group3-023, S236, PS2Group3-024, S237,

PS2Group3-030, S243, PS2Group3-031, S244, PS2Group3-057, S263, PS3Group2-004, S327, PS3Group2-005, S328, SS 10.1, S19

PS1Group1-103, S184 PS1Group1-077, S163, PS1Group1-079, S164 PS3Group2-030, S348 PS1Group1-119, S195

PS2Group5-006, S288 PS2Group3-051, S257 PS1Group1-119, S195

PS2Group5-034, S307

PS2Group3-038, S248

PS1Group1-030, S126, PS1Group1-040, S134

PS3Group4-029, S379, WS 3.4, S47

PS3Group4-007, S361

PS1Group1-051, S144

PS1Group1-014, S112

PS2Group5-016, S295

PS2Group9-001, S310

PL 4.1, S6

PS3Group4-022, S373,

SS 13.4, S25

PS3Group2-021, S341,

SS 20.1, S32

PS1Group1-132, S206

PS1Group1-009, S108,

PS1Group1-063, S153,

PS1Group1-115, S192,

PS2Group5-007, S288,

PS2Group9-019, S323

PS1Group1-057, S148, PS1Group1-105, S185, PS2Group5-023, S299, PS2Group9-003, S312, PS2Group9-016, S321

PS2Group9-013, S319

PS1Group1-014, S112

PS2Group5-033, S307

PS2Group3-082, S283

PS1Group1-088, S172

PS2Group3-008, S223,

WS 20.4, S71

PS1Group1-028, S124

PS1Group1-089, S173

PS1Group1-051, S144

PS1Group1-028, S124

PS1Group1-017, S116

PS1Group1-042, S136, 
Cardellach F.

Cardoso M.

Carlier P.G.

Carmes E.R.

Carmona C.

Carnesecchi S.

Carotti M.

Carrera-García L.

Carrera L.

Carrino R.

Carrozzo R.

Carstens P.-O.

Carvalho A.A.S.

Casadevall T.

Castelain V.

Castro D.

Castro I.D.

Castro J.

Catteruccia M.

Cauchois X.

Cavaletti G.

Cavalli M.

Cetin $\mathrm{H}$.

Chae C.S.

Chandran S.

Chandrasekaran K.

Chan L.L.

Chan M.

Chanson J.-B.

Chant E.

Chao H.-C.

Charleston J.S.

Chella A.

Chen V.
PS1Group1-056, S148,

PS1Group1-110, S188

PS1Group1-084, S169

PS3Group2-025, S345,

PS3Group4-013, S365

PS1Group1-004, S104, PS1Group1-005, S105,

PS1Group1-009, S108,

PS1Group1-095, S177,

PS1Group1-115, S192,

PS2Group9-006, S314,

PS2Group9-008, S316

PS1Group1-006, S106

PS3Group2-034, S351

PS1Group1-004, S104,

PS1Group1-005, S105

PS1Group1-125,

PS2Group3-011, S226,

PS2Group3-034, S245, PS2Group5-014, S293,

PS2Group9-010, S317,

PS3Group2-013, S335,

PS3Group4-019, S371

PS1Group1-013, S112

PS3Group2-024, S343

PS1Group1-027, S123

PS1Group1-122, S198

PS1Group1-047, S141, PS1Group1-072, S160, PS1Group1-073, S161, PS1Group1-074, S162, PS1Group1-075, S162,

PS1Group1-107, S186,

PS2Group3-059, S265

PS1Group1-089, S173

PS3Group4-004, S358

PS3Group4-026, S377

PS2Group3-062, S267

PS2Group3-062, S267

PS1Group1-119, S195

PS1Group1-121, S197

SS 6.3, S14

PS1Group1-056, S148

PS2Group3-026, S239,

PS3Group2-020, S340,

PS3Group2-036, S353, SS 24.3, S40

PS2Group3-047, S254

PS1Group1-060, S151

PS2Group3-045, S253

PS2Group3-072, S273

PS3Group4-028, S378

PS3Group4-004, S358

PS2Group3-008, S223

PS2Group3-016, S230

PS1Group1-049, S143

PS3Group2-027, S346

PS2Group5-007, S288
Chepuru R.

Chê V.

Chevessier F.

Chiriboga C.A.

Cho E.B.

Choi J.

Choi K.

Choi Y.C.

Cho J.-Y.

Cholet N.

Chowdhury S.

Chrast R.

Christofolini D.M.

Chroni E.

Chumillas M.J.

Chun M.Y.

Church K.

Chutipongtanate S.

Ciet P.

Cimbalistiene L.

Cirino R.H.D.

Claeys K.G.

Cleary Y.

Clemen C.

Cloutier M.

Cnaan A.

Cocito D.

Codina A.

Coelho T.

Coffey C.

Cohen D.

Coll-Cantí J.

Colomer J.

Comi G.P.

Conceição I.

Conte E.
PS2Group3-039, S249

PS1Group1-004, S104

PS3Group2-030, S348

PS3Group4-002, S356, PS3Group4-014, S366 PS2Group3-033, S245 PS2Group3-046, S254

PS2Group3-054, S260

PS1Group1-145, S215, PS3Group4-016, S368

PS2Group3-050, S257

PS2Group3-038, S248,

PS2Group3-069, S271

PS2Group9-009, S316

PS2Group3-044, S252

PS1Group1-073, S161

PS2Group5-030, S305, PS3Group4-023, S374

PS2Group3-053, S259

PS2Group3-073, S274

PS3Group4-022, S373,

SS 13.4, S25

PS3Group2-006, S328

PS1Group1-040, S134

PS1Group1-132, S206

PS1Group1-006, S106

PS1Group1-081, S166,

WS 16.2, S62

PS3Group4-002, S356, PS3Group4-015, S367, PS3Group4-028, S378

PS1Group1-134, S207, PS1Group1-135, S208

PS1Group1-020, S118 PS1Group1-115, S192 PS2Group3-019, S233 PS2Group5-014, S293, PS3Group2-013, S335 PS2Group3-005, S220, PS2Group3-010, S225, PS2Group3-055, S261, PS3Group2-025, S345,

WS 10.3, S56

PS3Group2-021, S341

PS2Group3-038, S248, PS2Group3-069, S271 PS1Group1-089, S173 PS1Group1-013, S112, PS2Group3-011, S226, PS2Group3-034, S245, PS2Group5-014, S293, PS2Group9-010, S317, PS3Group2-013, S335, PS3Group4-019, S371 PS3Group4-024, S375 PS1Group1-058, S149, PS2Group3-062, S267 PS1Group1-028, S124 
Conwit R.

Cooperative International

PS3Group2-021, S341

(Cinrg) PS1Group1-114, S191

Neuromuscular Research Group

Coppenrath E.M.

Corazzini R.

Corberó A.B.

Coriu D.

Cornblath D.

Cornblath D.R.

Cosentino G.

Cossins J.

Cossu G.

Counsell J.

Cowling B.S.

Coyne K.S.

Cozzoli A.

Crha I.

Csimma C.

Cudkowicz M.E.

Cuisset J.-M.

Cutrupi A.

Cutter G.R.

Cvijan E.

Czech C.

Czirjak L.

Dabbous O.

Dabby R.

Da Cunha C.L.P.

Dagher R.

Dahl-Halvarsson M.

Dai D.L.

D'Amico A.

D’Angelo M.G.

D'Antonio M.

Darras B.

Darras B.T.

Das A.

Date $\mathrm{H}$

Davies K.

Davis M.

Day J.

Day J.W.

De Antonio M.
PS1Group1-009, S108

PS1Group1-073, S161, PS1Group1-074, S162

PS2Group5-033, S307

PS2Group3-002, S218

PL 2.3, S5

PS2Group3-012, S226, PS2Group3-018, S231, PS2Group3-019, S233, PS2Group3-023, S236, PS2Group3-024, S237, PS2Group3-057, S263

PS1Group1-127, S202 PS3Group2-036, S353

TC 9.3, S86

PS1Group1-070, S159

PS1Group1-050, S143,

PS1Group1-061, S152

PS3Group7-004, S383

PS1Group1-028, S124

PS1Group1-037, S132

TC 9.1, S86

PS3Group2-021, S341

PS3Group4-024, S375

PS2Group3-048, S255

PS3Group2-033, S351, SS 23.2, S39

PS1Group1-078, S164

PS3Group4-015, S367,

PS3Group4-028, S378

PS1Group1-099, S180

PS1Group1-020, S118

PS1Group1-069, S158

PS1Group1-006, S106

PS1Group1-060, S151

PS1Group1-096, S178

PS2Group5-007, S288

PS1Group1-027, S123,

PS1Group1-119, S195

PS1Group1-051, S144

PS2Group3-081, S283

PS2Group5-002, S284,

PS3Group4-015, S367

PS3Group4-014, S366

PS2Group3-029, S241

PS1Group1-071, S159

PS1Group1-019, S117

PS1Group1-096, S178

PS1Group1-063, S153,

PS3Group4-015, S367

PS1Group1-009, S108, PS1Group1-115, S192, PS2Group5-007, S288 PS1Group1-088, S172
De Bellis M.

De Bleecker J.

De Bruijne M.

De Carvalho M.

Deconinck N.

Deconinck T.

De Fornel P.

De Haard H.

Deikin A.

De Jonghe P.

Deleuze J.-F.

Delgado P.O.

Deliens G.

Della Valle C.

Delmont E.

Delorme D.

De Luca A.

Demeestere D.

Demeshonok V.S.

De Paepe B.

Derchi M.

De Ridder W.

De Rosa A.

De Rover M.

De Simoni D.

De Stefano N.

Devigili G.

De Visser M.

De Waele L.

De Wel B.

Dias Henriques S.F.

Diaz-Manera J.

Dickson J.G.

Dieye I.

Di Iorio G.

Dikhter E.

Dimachkie M.

Di Muzio A.

Diodato D.

Djordjevic G.

Djordjevic S.

Djukic M.

Do $\mathrm{H}$.

Domingos J.
PS1Group1-028, S124

PS1Group1-045, S139,

PS2Group3-024, S237

PS1Group1-040, S134

PS1Group1-058, S149,

PS3Group4-020, S372

PS3Group4-008, S361,

PS3Group4-015, S367,

PS3Group4-025, S376

PS1Group1-138, S210

PS1Group1-008, S108

PS3Group2-004, S327

PS1Group1-076, S163

PS1Group1-138, S210

PS3Group4-006, S360

PS1Group1-073, S161,

PS1Group1-074, S162

PS3Group4-025, S376

PS1Group1-021, S119

PS2Group3-065, S269

PS2Group9-001, S310

PS1Group1-028, S124,

TC 9.5, S86

PS2Group3-015, S229,

PS3Group2-031, S349

PS3Group4-021, S373,

WS 3.4, S47

PS1Group1-045, S139

PS1Group1-119, S195

PS1Group1-022, S119,

PS1Group1-138, S210

PS3Group2-027, S346

PS1Group1-034, S129

PS2Group3-049, S256

OA 3.2, $\mathrm{S} 92$

TC 8.2, S85

OA 2.1, S90, SS 1.3, S10,

SS 16.1, S28, TC 1.1, S74

PS3Group7-001, S380

PS1Group1-081, S166

PS1Group1-015, S113

PS1Group1-004, S104,

PS1Group1-063, S153,

PS2Group5-007, S288

PS1Group1-087, S171

PS1Group1-120, S196

PS2Group5-034, S307

PS3Group4-029, S379,

WS 3.4, S47

PS2Group3-051, S257,

PS3Group2-021, S341,

TC 6.2, S84

PS1Group1-051, S144

PS1Group1-027, S123

PS2Group3-028, S241

PS1Group1-059, S150

PS1Group1-059, S150

PS1Group1-021, S119

PS1Group1-087, S171 
Dominovic-Kovacevic A.

Donandt T.

Doneddu P.

Donovan J.

Doorenweerd N.

Dorchies O.M.

Dor T.

Dowling J.J.

Drexler W.

Duan D.

Dubuisson N.

Ducci R.D.

Dugar A.

Dupuis M.

Durn B.L.

Dworzak J.

Dyck P..J.B.

Eagle M.

Echaniz-Laguna A.

Efthimiadis G.

Egorova T.

Eichhorn P.

Eichinger L.

Einsiedler S.

Ekusheva E.

Elfring G.L.

Elhawary N.

El-Khairi M.

Ellis M.

Ellouz E.

Elsayed N.

Embacher N.

Engel A.G.

England J.D.

Erdem-Ozdamar S.

Erdler M.

Ernst B.

Errguig L.

Espinós C.
PS2Group3-028, S241

PS1Group1-065, S155

WS 5.1, S49

PS1Group1-060, S151

OA 3.3, S92

PS1Group1-034, S129

PS1Group1-026, S122,

PS1Group1-050, S143,

PS1Group1-061, S152,

PS1Group1-098, S179

PS1Group1-061, S152

PS1Group1-039, S133,

TC 4.3, S82

SS 14.3, S26

PS1Group1-111, S189

PS2Group3-070, S272

PS1Group1-006, S106

PS1Group1-114, S191

PS2Group3-070, S272

PS2Group3-012, S226,

PS2Group3-018, S231,

PS2Group3-019, S233,

PS2Group3-023, S236,

PS2Group3-024, S237,

PS2Group3-030, S243,

PS2Group3-031, S244,

PS2Group3-057, S263

PS1Group1-049, S143

TC 3.4, S81, WS 13.2, S58, WS 19.2, S69

PS1Group1-009, S108, PS1Group1-115, S192 PS1Group1-088, S172, PS3Group4-004, S358 PS1Group1-102, S183

PS1Group1-076, S163

PS1Group1-002, S102

PS1Group1-134, S207

PS3Group2-018, S338

PS2Group3-071, S272

PS1Group1-118, S194,

PS1Group1-124, S199

PS1Group1-085, S169

PS3Group4-024, S375

PS2Group3-048, S255

PS2Group3-077, S277

PS1Group1-085, S169

PS2Group3-076, S276,

PS3Group2-018, S338

PS1Group1-011, S110

SS 21.1, S34

PS2Group3-006, S222

PS2Group3-076, S276

PS2Group3-015, S229,

PS3Group2-031, S349

PS2Group5-012, S292

PS2Group3-042, S251,

PS2Group3-044, S252,
Evangelista T.

Ewers D.

Exposito Escudero J.

Eymard B.

Fabry B.

Faelan C.

Fahmy N.

Faiola A.S.

Fajkusova L.

Falcão De Campos C.

Falzarano M.S.

Fang M.

Fardeau M.

Farrar M.A.

Farrugia M.E.

Faruq M.

Farwell W.

Fatehi F.

Fathi D.

Faust A.

Fecchio C.

Feder D.

Feldman E.

Felice K.

Feng J.

Ferlini A.

Fernandes J.D.L.

Fernández-García M.A.

Fernández-Torrón R.

Ferrari N

Ferrero B.

Ferri C.

Fierro B.

Figà Talamanca L.

Figueiroa S.

Filla A.

Filosto M.

Finkel R.S.
PS2Group3-052, S258, PS2Group3-053, S259

SS 24.2, S40

PS2Group3-038, S248

PS2Group5-014, S293

PS3Group2-030, S348

PS2Group5-028, S303

PS1Group1-019, S117,

PS2Group9-018, S323

PS1Group1-085, S169

PS2Group3-059, S265

PS1Group1-113, S190,

PS1Group1-139, S211

PS1Group1-058, S149

PS2Group5-020, S297

PS2Group5-020, S297

PS3Group2-030, S348

PS3Group4-026, S377

PS1Group1-033, S128

PS1Group1-016, S114, PS1Group1-044, S138

PS3Group4-014, S366,

PS3Group4-026, S377

PS1Group1-041, S136, PS1Group1-043, S137

PS1Group1-043, S137

PS1Group1-025, S122

PS1Group1-125,

PS1Group1-047, S141, PS1Group1-072, S160, PS1Group1-073, S161, PS1Group1-074, S162, PS1Group1-075, S162, PS1Group1-107, S186, PS2Group3-059, S265

PS2Group3-008, S223,

PS2Group3-021, S235,

PS2Group3-022, S235,

PS3Group4-030, S380,

SS 10.3, S20

PS1Group1-032, S128

PS1Group1-021, S119,

PS1Group1-063, S153

PS2Group5-020, S297

PS2Group9-017, S322

PS1Group1-100, S181

PS1Group1-009, S108,

PS1Group1-115, S192

PS1Group1-056, S148

PS3Group2-024, S343

PS2Group3-081, S283

PS1Group1-127, S202

PS1Group1-027, S123

PS2Group3-003, S219

PS2Group5-021, S298

PS1Group1-051, S144

PS1Group1-060, S151,

PS3Group4-026, S377 
Finsterer J

Fiorillo C.

Fischer D.

Flanigan K.

Fominyh M.

Fominyh V.

Fonseca F.L.A.

Fontaine B.

Fontenille M.-J.

Fontoura P.

Fonzino A.

Fossati B.

Foster R.

Fournier E.

Foust K.

Frank D.

Fransi A.R.

Franssen $\mathrm{H}$.

Frascella M.

Frasquet M.

Freeman R.

Fretzen A.

Frich J.

Frisaldi E.

Froissart R.

Fromes Y.

Frongia A.L.

Fuentealba M.

Fujita K.P.

Funato $\mathrm{M}$.

Furling D.

Furtado F.

Fusco L.

Gajewski B.

Gallano Petit P.
PS1Group1-090, S173, PS1Group1-091, S174

PS1Group1-119, S195

PS1Group1-017, S116, PS1Group1-026, S122, PS1Group1-061, S152, PS3Group4-002, S356 PS2Group9-019, S323 WS 3.4, S47

WS 3.4, S47

PS1Group1-047, S141, PS1Group1-073, S161, PS1Group1-074, S162, PS1Group1-107, S186, PS2Group3-059, S265 PS2Group5-027, S302, PS3Group2-030, S348 PS3Group2-030, S348 PS3Group4-024, S375 PS1Group1-028, S124 PS1Group1-110, S188 PS3Group4-014, S366 PS3Group2-030, S348 PS3Group4-022, S373, SS 13.4, S25

PS1Group1-049, S143, PS1Group1-087, S171, PS2Group9-018, S323 PS1Group1-089, S173 PS2Group5-008, S289 PS1Group1-021, S119

PS2Group3-052, S258, PS2Group3-053, S259 SS 9.5, S19

PS1Group1-060, S151 PS3Group7-002, S381

PS3Group2-024, S343 PS1Group1-088, S172 PS1Group1-005, S105 PS2Group3-011, S226, PS2Group3-034, S245, PS2Group5-014, S293, PS2Group9-010, S317, PS3Group2-013, S335, PS3Group4-019, S371

PS3Group2-009, S331, PS3Group2-010, S332 PS3Group2-011, S333, PS3Group2-012, S334, PS3Group2-023, S342, PS3Group2-037, S354, PS3Group2-038, S355 PS1Group1-029, S125 PS1Group1-144, S214 PS2Group3-062, S267 PS1Group1-027, S123

PS2Group3-051, S257 PS2Group5-014, S293, PS3Group2-013, S335
Gallardo-Agromayor E. Gandhi P.

Garcia A.

García-García F.

García J.

García-Jiménez I.

García-Sobrino T.

Gargouri A.

Garrido C.

Gast L.V.

Gayi E.

Geist J.

Gelblin K.

Genge A.

Gerber M.

Gerevini S

Gerhalter T.

Geuens S.

Ghaleh B.

Ghezzi D.

Ghislain Mpandzou A.

Gicquel E.

Gidaro T.

Gide J.

Gilhus N.E.

Ginzberg M.

Giometto B.

Giordani L.P.

Gioulbasanis I.

Gk K.

Glaubitz S.

Glāzere I.

Glimcher L.

Gloor M.

Glumac J.

Gocheva V.

Godoy A.J.

Goedee S.

Goel V.

Goemans N.

Goh K.-J.

Goldmann W.H.

Goldstein J.M

Gollob J.

Gomis Coloma C.
PS1Group1-100, S181

PS2Group3-005, S220, PS2Group3-010, S225, PS2Group3-055, S261 PS1Group1-021, S119 PS2Group3-044, S252 PS1Group1-084, S169 PS1Group1-054, S146 PS2Group3-042, S251 PS2Group3-077, S277 PS2Group3-003, S219, PS3Group4-013, S365 PS2Group9-008, S316

PS1Group1-050, S143, PS1Group1-061, S152, PS1Group1-098, S179

PS1Group1-035, S130 PS3Group4-015, S367

SS 11.4, S23

PS3Group4-002, S356, PS3Group4-015, S367, PS3Group4-028, S378

PS1Group1-004, S104

PS2Group9-008, S316

PS3Group7-001, S380

PS1Group1-005, S105

PS1Group1-027, S123

PS1Group1-068, S157

PS1Group1-015, S113

PS1Group1-004, S104

PS3Group4-006, S360

WS 18.1, S67

PS1Group1-069, S158

SS 3.1, S12

PS1Group1-107, S186

WS 7.1, S52

PS3Group4-003, S357

PS1Group1-122, S198

PS2Group9-002, S311

PS2Group3-081, S283

PS1Group1-017, S116

PS1Group1-059, S150

PS1Group1-017, S116

PS2Group9-017, S322

PS2Group5-008, S289,

TC 8.1, S84

PS2Group3-043, S251, PS2Group3-080, S280

PS1Group1-120, S196, PS3Group4-008, S361, PS3Group4-028, S378, PS3Group7-001, S380,

SS 13.3, S25

PS1Group1-031, S127

PS2Group5-028, S303

PS3Group2-021, S341

PS2Group3-005, S220,

PS2Group3-010, S225,

PS2Group3-055, S261

PS1Group1-046, S140 
Gonokami M.K.

Gonzalez-Duarte A.

González Quereda L.

Gonzalez R.

Gorbachev K.

Gordish-Dressman H.

Gorman G.S.

Gorni K.

Gosselin N.

Goto J.

Goto Y.-I.

Gotschall R.

Gouider R.

Goullée H.

Govi M.

Grau J.M.

Gray J.

Greckl E.

Grieben U.

Grigalioniene K.

Grilli A.

Grimm A.

Grinzinger S.

Grisold A.

Grisold W.

Gromicho M.

Grouse C.K.

Groves K.

Gruber A.

Gualandi F.

Guanyabens N.

Guerin A.

Guerreiro R.

Gueven N.

Guger M.

Guglieri M.

Guglietta A.

Guida M.

Gulyas K.

Guptill J.T.

Gutierrez-Gutierrez G.

Haberlova J.

Hackel N.

Hafler D.A.
PS2Group5-004, S286

PS2Group3-005, S220,

PS2Group3-010, S225,

PS2Group3-055, S261

PS2Group5-014, S293,

PS3Group2-013, S335

PS3Group2-010, S332

WS 3.4, S47

PS1Group1-114, S191,

TC 9.4, S86

PS1Group1-033, S128

PS3Group4-002, S356,

PS3Group4-015, S367,

PS3Group4-024, S375,

PS3Group4-028, S378

PS2Group3-043, S251

PS1Group1-071, S159

PS1Group1-097, S178

PS1Group1-021, S119

PS2Group3-077, S277

PS1Group1-096, S178

PS1Group1-051, S144

PS1Group1-083, S168,

PS1Group1-084, S169,

PS1Group1-137, S210

PS1Group1-004, S104

PS3Group4-009, S362

PS1Group1-115, S192

PS1Group1-132, S206

PS2Group5-020, S297

TC 5.4, S83

PS2Group3-076, S276

PS2Group3-026, S239,

SS 8.1, S16, SS 17.4, S29,

WS 13.1, S57, WS 20.2, S70

SS 8.1, S16, SS 17.4, S29,

WS 13.1, S57

PS3Group4-020, S372

WS 11.1, S56

PS1Group1-004, S104

PS1Group1-011, S110

PS2Group5-020, S297

PS1Group1-089, S173

PS1Group1-020, S118

PS3Group2-034, S351

PS1Group1-017, S116

PS3Group2-018, S338

PS1Group1-087, S171

PS3Group2-004, S327

PS3Group2-027, S346

PS1Group1-099, S180

PS3Group2-012, S334

PS1Group1-100, S181

PS1Group1-113, S190, PS2Group3-020, S234, PS2Group9-007, S315

PS3Group4-009, S362

PS3Group2-021, S341
Hafner P.

Haghiashtiani B.

Haghi Ashtiani B.

Haghi B.

Hajjar R.

Hajj R.

Hakim M.

Hamed S.A.

Hamilton L.E.

Hamroun D.

Hänggi $\mathrm{P}$.

Han L.

Hanna M.

Hardiman O.

Haridy N.A.A.

Harlaar L.

Harlay M.

Harms M.

Harris L.

Hartung H.-P.

Hasegawa S.

Hasham S.

Hashiguchi A.

Hasoglu Y.

Hasselmann O.

Hattori A.

Hausner T.

Hayhurst Bennett M.

Heatherington A.

Heckmann J.M.

Hegen $\mathrm{H}$.

Heller C.

Hendriksen J.G.M.

Henricson E.

Heo S.-J.

Herbelet S.

Herbelin L.

Herbrecht R.

Hercher D.
PS1Group1-017, S116, PS1Group1-026, S122

PS2Group5-009, S290

PS1Group1-043, S137

PS2Group3-065, S269

PS1Group1-111, S189

PS2Group3-038, S248,

PS2Group3-069, S271

PS3Group2-032, S350

PS2Group3-067, S270

PS1Group1-011, S110

PS1Group1-088, S172

PS2Group3-015, S229,

PS3Group2-031, S349

PS1Group1-114, S191

TC 6.4, S84

WS 12.2, S57

PS2Group3-067, S270

PS1Group1-030, S126,

PS1Group1-040, S134

PS3Group4-004, S358

PS1Group1-115, S192,

PS2Group5-007, S288

PS3Group2-033, S351,

PS3Group7-004, S383

PS2Group3-012, S226,

PS2Group3-018, S231,

PS2Group3-019, S233,

PS2Group3-023, S236,

PS2Group3-024, S237,

PS2Group3-030, S243,

PS2Group3-031, S244,

PS2Group3-057, S263

PS1Group1-052, S145

PS1Group1-057, S148,

PS1Group1-105, S185,

PS2Group5-023, S299,

PS2Group9-003, S312,

PS2Group9-016, S321

PS1Group1-136, S209

PS2Group9-018, S323

PS2Group5-006, S288

PS1Group1-029, S125

SS 2.2, S11, WS 9.3, S55

PS1Group1-023, S120,

PS1Group1-024, S121

PS1Group1-019, S117,

PS2Group9-009, S316

PS2Group5-019, S297

PS2Group3-049, S256

PS2Group3-074, S275

PS1Group1-034, S129

PS1Group1-114, S191

PS3Group2-015, S336

PS1Group1-045, S139

PS2Group3-051, S257,

PS3Group2-021, S341

PS3Group4-004, S358

SS 2.3, S12 
Herraets I.

Herrendorff R.

Heß-Eberle I.

Hettwer S.

Heusermann W.

Higuchi I.

Hilger E.

Hilsden $\mathrm{H}$.

Hilz M.J.

Hinderer C.

Hiroyuki A.

Hobson E.

Ho C.-Y.

Hoeftberger R.

Hofer M.

Höger S.

Hogrel J.-Y.

Hoke A.

Holinski-Feder E.

Hollander Z.

Hollingsworth K.G.

Holvoet B.

Hong Y.-H.

Hooijmans M.T

Hordeaux J.

Horlings C.G.

Horn S.

Horvath R.

Houlden $\mathrm{H}$.

Howard Jr J.F.

Hubsch A.

Huemer M.

Huh C.

Hulova M.

Human A.

Huq S.

Ibraheem $\mathrm{H}$.

Igarashi S.

Iglseder S.

Iijima $\mathrm{K}$.

Ikenaga C.

Ikenberg E.

Ilchenko M.
PS2Group5-008, S289

PS2Group3-015, S229,

PS3Group2-031, S349

PS2Group3-076, S276

PS3Group2-029, S347

PS2Group3-015, S229

PS1Group1-136, S209

PS3Group2-020, S340

PS1Group1-115, S192

SS 9.3, S19, TC 3.3, S80

PS2Group9-013, S319

PS1Group1-003, S103

SS 16.2, S28

PS2Group3-045, S253

PS2Group3-049, S256,

SS 3.2, $\mathrm{S} 12$

WS 19.1, S68

PS2Group3-076, S276

PS1Group1-115, S192

SS 6.2, S14, WS 9.2, S55

PS2Group5-016, S295

PS2Group5-007, S288

PS1Group1-034, S129

PS1Group1-008, S108

PS1Group1-131, S205,

PS1Group1-141, S213,

PS3Group2-035, S352

PS1Group1-095, S177

PS2Group9-013, S319

PS1Group1-001, S102,

PS1Group1-010, S109,

PS1Group1-109, S188

SS 22.1, S36

PS1Group1-044, S138, PS2Group3-060, S265, PS3Group2-022, S342, SS 24.2, S40

PS2Group3-067, S270

PS3Group2-004, S327,

PS3Group2-011, S333, PS3Group2-012, S334, PS3Group2-023, S342, PS3Group2-037, S354, PS3Group2-038, S355 PS2Group3-018, S231 PS2Group3-076, S276 PS1Group1-141, S213

PS1Group1-037, S132

PS1Group1-064, S154

PS3Group2-021, S341

PS3Group2-024, S343

PS2Group6-001, S308

PS3Group2-018, S338

PS1Group1-003, S103,

PS1Group1-128, S202

PS1Group1-071, S159

PS2Group3-074, S275

WS 3.4, S47
Imanishi $\mathrm{T}$.

Im S.

Inashkina I.

Indrawati L.A.

Inoue $\mathrm{S}$.

Iodice R.

Irani S.

Irastorza A.

Irioka $\mathrm{T}$.

Irkec M.

Ishigaki K.

Ishii A.

Ishii $\mathrm{K}$.

Ishikawa Y.

Ishiura $\mathrm{H}$.

Ishiyama A.

Ismail H.M.

Itzep D.

Ivanova $\mathrm{M}$

Ivanov $\mathrm{T}$.

Ives J.

Iwata A.

Jackson S.

Jacob S.

Jacobsen L.

Jacobs M.

Jakubiczka S.

James M.

Jang H.

Jang J.

Jang Y.

Jankelowitz S.

Jankovic M.

Jankovits R.

Jawdat O.

Jaworski M.

Jecel J.

Jelsma J.

Jenkins M.

Jiang $\mathrm{H}$.

Jimenez Mallebrera C.

Jimenez-Moreno C.

Jin F.
PS1Group1-003, S103

PS2Group3-047, S254

PS1Group1-035, S130

PS3Group2-032, S350

PS1Group1-052, S145

PS2Group5-021, S298

PS2Group3-032, S244

PS1Group1-038, S132

PS2Group6-001, S308

PS2Group3-006, S222

PS1Group1-029, S125

PS2Group5-029, S304

PS2Group5-029, S304

PS1Group1-128, S202,

PS1Group1-128, S202

PS1Group1-071, S159

PS1Group1-097, S178

PS1Group1-050, S143,

PS1Group1-061, S152

PS1Group1-013, S112,

PS2Group3-011, S226,

PS2Group3-034, S245

WS 3.4, S47

WS 3.4, S47

PS3Group4-024, S375

PS2Group5-004, S286

PS1Group1-035, S130, PS1Group1-117, S194

PS3Group2-011, S333,

PS3Group2-012, S334,

PS3Group2-023, S342

PS1Group1-014, S112

PS1Group1-063, S153, PS1Group1-115, S192, PS2Group5-007, S288

PS2Group5-016, S295

PS1Group1-009, S108, PS1Group1-063, S153,

PS1Group1-115, S192

PS1Group1-133, S206

PS1Group1-115, S192

PS2Group3-047, S254

PS1Group1-062, S153

PS1Group1-080, S165

PS2Group3-036, S247

PS2Group3-051, S257

PS1Group1-048, S142

PS2Group3-076, S276

PS1Group1-064, S154

PS1Group1-120, S196

PS3Group4-012, S365

PS1Group1-013, S112,

PS2Group5-014, S293,

PS3Group2-013, S335,

PS3Group4-019, S371

PS1Group1-147, S216

PS1Group1-123, S198,

PS1Group1-126, S200 
Johnson K.

Jomphe C.

Jones K.J.

Jonker M.A.

Joo I.S.

Joshi A.

Jou C.

Jousserand G.

Jouvenal P.V.

Jovanovic I.

Juergen D.

Jullien De Pommerol H.

Jung S.-H.

Kably B.

Kacar A.

Kacem I.

Kah D.

Kaji R.

Kalbe C.

Kalf J.G.

Kalicanin Milanovic R.

Kalil I.D.L.

Kaliszewska M.

Kamienowski J.

Kaminska A.M.

Kaminski H.J.

Kaňáková J.

Kan H.E.

Kapur K.

Kariminejad A.

Karussis D.

Kasianova A.

Kaspar B.

Kastenschmidt J. Katzberg H.

Katz N.

Kaur S.

Kay C.S.K.

Kazaine I.

Kelly C.

Kelly M.

Kennerson M.L.
PS1Group1-138, S210

PS2Group3-043, S251

PS1Group1-009, S108, PS1Group1-063, S153, PS1Group1-115, S192, PS2Group5-007, S288 PS1Group1-001, S102, PS1Group1-109, S188 PS2Group3-068, S271 PS1Group1-016, S114, PS1Group1-044, S138 PS1Group1-013, S112, PS1Group1-137, S210, PS2Group5-014, S293, PS3Group2-013, S335, PS3Group4-019, S371 PS1Group1-066, S156 PS1Group1-103, S184 PS1Group1-059, S150 PS1Group1-014, S112 PS3Group4-017, S369 PS3Group2-014, S336

PS1Group1-067, S157, PS1Group1-068, S157, PS2Group5-012, S292, PS2Group5-013, S292 PS1Group1-077, S163, PS2Group3-028, S241 PS2Group3-077, S277 PS2Group5-028, S303 TC 7.1, S84

PS1Group1-065, S155

PS1Group1-010, S109

PS1Group1-059, S150

PS2Group9-017, S322

PS1Group1-036, S131

PS2Group3-024, S237

PS1Group1-036, S131

SS 23.2, S39

PS2Group3-020, S234

PS1Group1-034, S129, PS1Group1-095, S177 PS2Group5-002, S284

PS1Group1-096, S178

OA 1.3, S90

PS3Group4-029, S379, WS 3.4, S47

PS3Group4-022, S373, SS 13.4, S25

PS2Group5-031, S306 PS3Group2-005, S328 PS2Group9-013, S319 PS1Group1-025, S122 PS1Group1-006, S106 PS2Group9-002, S311

PS1Group1-055, S147

TC 9.1, S86

PS2Group3-048, S255
Keshavarz E.

Kessler B.

Khanna R.

Khidiyatova I.

Khongkhatithum C.

Khusnutdinova E.

Khwaja O.

Ki C.-S.

Kieloch A.

Kierdaszuk B.

Kiernan M.C.

Kiklic M.

Kilfoyle D.

Kim A.W.

Kim B.

Kim B.-J.

Kim C.-H.

Kim D.-S.

Kim J.A.

Kim J.E.

Kimminau K.

Kim R.B.

Kim S.

Kim S.-J.

Kim S.M.

Kimura T.

Kim Y.-D.

Kim Y.H.

Kirschner J.

Kissel J.T.

Kitanosono H.

Klein A.

Kleindienst W.

Kletzl H.

Klinker F.

Knut B.

Kobayashi M.

Kocabeyoglu S.

Kochanowski J.

Koch M.E.

Kodama K.

Koehorst E.A.

Koga Y.
PS1Group1-096, S178 PS1Group1-065, S155 PS1Group1-021, S119 PS2Group3-058, S264

PS3Group2-006, S328

PS2Group3-058, S264 PS3Group4-002, S356, PS3Group4-015, S367, PS3Group4-028, S378 PS1Group1-145, S215 PS3Group4-017, S369

PS1Group1-036, S131

SS 15.2, S27, SS 18.1, S30,

SS 19.3, S32

PS1Group1-059, S150 PS2Group5-021, S298

PS1Group1-131, S205, PS1Group1-141, S213 PS2Group3-032, S244 PS2Group5-017, S295

PS2Group3-009, S224

PS3Group2-014, S336

PS1Group1-131, S205,

PS1Group1-141, S213

PS3Group2-035, S352

PS2Group3-051, S257

PS2Group3-033, S245

PS2Group5-026, S301

PS2Group3-032, S244

PS1Group1-131, S205,

PS2Group3-032, S244

PS1Group1-029, S125

PS2Group3-078, S278

PS2Group5-017, S295

PS2Group9-019, S323,

PS3Group4-014, S366, PS3Group4-024, S375, PS3Group4-028, S378, SS 13.1, S24

PS3Group2-021, S341, PS3Group4-022, S373, SS 13.4, S25

PS2Group6-001, S308 PS1Group1-017, S116, PS3Group4-015, S367 PS2Group3-076, S276 PS3Group4-002, S356, PS3Group4-015, S367, PS3Group4-028, S378 PS1Group1-103, S184 PS3Group4-006, S360 PS1Group1-029, S125 PS2Group3-006, S222 PS2Group5-011, S291 PS1Group1-073, S161, PS1Group1-074, S162 PS1Group1-136, S209

PS1Group1-089, S173 PS2Group5-029, S304 
Koh Y.H.

Kok C.Y.

Kokotovic T.

Kolb S.

Kollmer J.

Kolski H.

Komaki H.

Koneczny I.

Kong R.

Kon S.

Kontrogianni-

Konstantopoulos A.

Koo H.J.

Korneychuk S.

Kosac A.

Kostera-Pruszczyk A.

Kosuga M.

Koumako C.

Kovacevic M.

Kralickova E.

Krasnaya Y.

Kraus D.

Krause S.

Krenn M.

Krishnan M.

Kristen A.

Kroon H.M.J.M.

Krosschell K.J.

Kubota A.

Kucinskas V.

Kumar A.

Kumar P.

Kumar V..P.

Kuntz N.

Kuo H.-C.

Kuru S.

Kwan W.S.K.

Kwon Y.-J.

Kwun K.H.

Kyriakides T.

Labarge S.

Labate V.

Labolle D.

Labove L.
PS2Group3-072, S273

PS2Group3-001, S218

PS2Group3-026, S239

SS 13.4, S25

SS 14.1, S26, WS 6.3, S52, PS2Group6-003, S310

PS1Group1-029, S125, PS1Group1-052, S145, PS1Group1-092, S175, PS1Group1-097, S178, PS1Group1-143, S214

PS3Group2-036, S353

PS1Group1-126, S200

PS3Group4-001, S356

PS1Group1-035, S130

PS2Group3-047, S254

WS 3.4, S47

PS1Group1-059, S150, PS1Group1-080, S165

PS1Group1-036, S131

PS1Group1-029, S125

PS2Group9-006, S314

PS1Group1-078, S164

PS1Group1-037, S132

PS3Group4-029, S379,

WS 3.4, S47

PS3Group4-002, S356

PS1Group1-065, S155,

PS1Group1-115, S192,

PS2Group3-040, S249

PS2Group3-026, S239

PS1Group1-014, S112

PS2Group3-005, S220,

PS2Group3-010, S225,

PS2Group3-055, S261

PS1Group1-010, S109

PS3Group4-026, S377

PS1Group1-071, S159

PS1Group1-132, S206

PS3Group2-019, S339

PS2Group3-027, S240,

PS2Group3-045, S253

PS3Group2-022, S342

PS1Group1-039, S133

SS 23.2, S39

PS1Group1-029, S125,

PS1Group1-112, S190,

PS1Group1-143, S214

PS2Group3-025, S238

PS2Group5-017, S295

PS1Group1-131, S205,

PS1Group1-141, S213

TC 1.4, S75

PS1Group1-024, S121

PS1Group1-056, S148

PS1Group1-004, S104

PS1Group1-048, S142
Lace B.

Lacour A.

Lacson A.

Ladha S.

Laffaire J.

Laforet $\mathrm{P}$.

Lahoz D.C.

Laich E.

Laing N.

Lamartine Monteiro M.

Lamperti C.

Landon-Cardinal O.

Langenscheidt D.

Lapin V.

Laporte J.F.

Larin E.

Larrañaga I.

Lasa Elgarresta J.

Lasa-Elgarresta J.

Lasa Fernandez $\mathrm{H}$.

Lasa-Fernandez $\mathrm{H}$.

Lasner I.

Laššuthová P.

Laugel V.

Laun F.B.

Lauria G.

Laveille C.

Lavrnic D.

Lawlor M.W.

Lawo J.-P.

Layton G.

Leary R.

Lechner-Scott J.

Lee E.

Lee F.

Lee H.-J.

Lee H.J.

Lee J.

Lee J.H.
PS1Group1-035, S130

PS3Group2-030, S348

PS2Group6-003, S310

PS2Group3-007, S222,

PS3Group2-029, S347

PS2Group3-038, S248, PS2Group3-069, S271

PS1Group1-066, S156, PS1Group1-088, S172, PS3Group2-030, S348

PS2Group5-033, S307

PS2Group3-076, S276

PS1Group1-096, S178

PS2Group5-001, S284

PS1Group1-027, S123

PS2Group9-006, S314

PS3Group2-018, S338

PS3Group4-021, S373

PS1Group1-050, S143,

PS1Group1-061, S152

PS3Group4-029, S379,

WS 3.4, S47

PS1Group1-108, S187

PS1Group1-038, S132

PS1Group1-106, S186

PS1Group1-038, S132

PS1Group1-106, S186

PS2Group3-017, S230

PS2Group3-020, S234,

PS2Group9-007, S315

PS1Group1-017, S116

PS2Group9-008, S316

WS 19.3, S69

PS1Group1-004, S104

PS1Group1-078, S164, PS1Group1-080, S165, PS1Group1-104, S185

PS1Group1-039, S133

PS2Group3-012, S226, PS2Group3-018, S231, PS2Group3-019, S233, PS2Group3-023, S236, PS2Group3-024, S237, PS2Group3-030, S243, PS2Group3-031, S244, PS2Group3-057, S263 PS1Group1-019, S117, PS2Group9-009, S316 PS2Group9-019, S323 PS2Group3-056, S262 PS2Group5-007, S288 PS3Group4-015, S367 PS2Group3-032, S244 PS2Group3-050, S257 PS2Group5-003, S285, PS2Group5-024, S300, PS2Group9-019, S323

PS1Group1-145, S215, PS2Group3-073, S274 
Lee Y.-C.

Lee Y.J.

Legay C.

Léger J.-M.

Leguern E.

Lehmann D.

Leinonen M.

Le Louër J.

Lemmers R.J.

Lenzenweger E.

Leontyeva $\mathrm{T}$.

Lepessova M.

Leppert D.

Lerman-Sagie T.

Leshinsky-Silver E.

Lesueur L.

Letard P.

Leupin N.

Lev D.

Lewis R.A.

Lewis S.L.

Liao Y.-C.

Liberatore G.

Liebetanz D.

Likhite S.

Li M.

Lim E.W.

Linares I.

Lindeck-Pozza E.

Lin K.-P.

Lin T.

Lipowska M.

L'Italien G.J.

L'Italien J.

Litchman T.D.

Litchy W.J.

Liu $\mathrm{H}$.
PS2Group3-004, S220, PS2Group3-016, S230

PS1Group1-133, S206, PS3Group2-015, S336

PS3Group2-028, S347

PS2Group3-024, S237,

PS2Group3-064, S268,

PS2Group3-065, S269,

WS 5.2, S50

PS2Group3-077, S277

PS1Group1-012, S111

PS1Group1-057, S148, PS1Group1-105, S185, PS2Group5-023, S299, PS2Group9-003, S312, PS2Group9-016, S321

PS1Group1-095, S177

PS1Group1-001, S102,

PS1Group1-109, S188

PS2Group3-076, S276

WS 3.4, S47

PS3Group4-010, S363

PS3Group4-017, S369

PS1Group1-069, S158

PS1Group1-069, S158

PS1Group1-144, S214

PS1Group1-088, S172

PS3Group2-004, S327

PS1Group1-069, S158

PS2Group3-012, S226, PS2Group3-018, S231, PS2Group3-019, S233, PS2Group3-023, S236, PS2Group3-024, S237, PS2Group3-031, S244, PS2Group3-057, S263

WS 11.2, S56

PS2Group3-004, S220

WS 5.1, S49

PS1Group1-103, S184 PS3Group4-022, S373, SS 13.4, S25

PS3Group2-029, S347

PS2Group3-072, S273

PS1Group1-089, S173

PS2Group3-049, S256,

SS 17.4, S29

PS2Group3-004, S220, PS2Group3-005, S220, PS2Group3-010, S225, PS2Group3-016, S230, PS2Group3-055, S261

PS2Group3-010, S225

PS2Group3-002, S218

PS3Group2-033, S351

PS3Group4-022, S373, SS 13.4, S25

PS3Group2-019, S339

PS1Group1-129, S203

PS1Group1-060, S151
Liu T.

Liu W.

Liu Y.-T.

Li Z.

Llorens J.M.

Lochmüller $\mathrm{H}$.

Loho T.

Lombardi L.

Long L.

Longman C.

López De Munain A.

Lorenzl S.

Lorenzoni P.J.

Löscher W.

Lostal W.

Lowes L.

Lucas K.

Lucchi M.

Lucente G.

Lucia A.

Ludolph A.

Ludo Van Der Pol W.

Lui Q.

$\mathrm{Lu} \mathrm{J}$.

Lulé D.

Lun Y.

Lupescu T.

Lupo V.

Luque $\mathrm{H}$.

Lusakowska A.

Lütschg J.

$\mathrm{Lu}$ Z.

Ly C.

Lynch J.

Lyon K.

Lysogorskaya E.

Macarthur D.

Macklin E.

Madia F.

Madigan N.N.

Maeda N.

Maestri M.

Maggi L.

Magzhanov R.

Maier O.

Maioli M.A.

Maiti A.
PS2Group3-051, S257

PS3Group2-036, S353

PS2Group3-016, S230

PS3Group2-029, S347

PS2Group5-033, S307

PS1Group1-115, S192,

PS2Group5-007, S288

PL 1.3, S3, PS1Group1-044, S138, PS3Group2-022, S342,

SS 24.2, S40, TC 2.4, S78

PS3Group2-032, S350

PS2Group5-034, S307

PS3Group7-003, S382

PS1Group1-033, S128

PS1Group1-038, S132,

PS1Group1-106, S186

WS 3.3, S47

PS1Group1-006, S106

PS2Group3-049, S256,

PS2Group3-076, S276,

PS3Group2-018, S338

PS1Group1-111, S189

PS3Group4-022, S373,

SS 13.4, S25

PS1Group1-049, S143

PS3Group2-027, S346

PS1Group1-089, S173

PS1Group1-089, S173

PL 3.1, S5, SS 13.2, S25,

TC 7.2, S84

PS3Group4-024, S375

PS3Group2-029, S347

PS1Group1-086, S170

TC 7.3, S84

PS1Group1-021, S119

WS 20.1, S70

PS2Group3-042, S251,

PS2Group3-044, S252,

PS2Group3-052, S258,

PS2Group3-053, S259

PS1Group1-053, S146

PS3Group4-024, S375

PS2Group5-006, S288

PS2Group5-020, S297

PS2Group3-048, S255

PS1Group1-086, S170

PS1Group1-023, S120

WS 3.4, S47

PS1Group1-138, S210

PS3Group4-018, S370

PS1Group1-119, S195

PS1Group1-129, S203

PS1Group1-052, S145

PS3Group2-027, S346

PS1Group1-051, S144

PS2Group3-058, S264

PS2Group5-006, S288

PS1Group1-051, S144

PS2Group3-029, S241 
Majima T.

Makioka N.

Maksimovic R.

Mallick R.

Malzahn D.

Mammen A.

Mancini M.

Mandic-Stojmenovic G.

Manel V.

Mane M.

Manera J.D.

Manganelli F.

Manin A.

Manji H.

Mantegazza R.

Mantuano P.

Maqueda E.

Marchel M.

Maré R.

Maresh K.

Marier J.-F.

Marini-Bettolo C.

Marin T.A.

Marjanovic A.

Marosi C.

Marquet A.

Marsolier J.

Martens K.

Martic V.

Martinat C.

Martinez M.S.

Martínez-Piñeiro A.

Martins J.

Martí P.

Martorell L.

Marty B.

Maruyama N.

Marx A.

Mas F.

Masrori P.

Massana-Muñoz X.

Massey C.

Masson R.

Mastrangelo R.

Mata J.F.

Mathukomali N.

Mathur A.

Matsumoto M.
PS2Group6-001, S308

PS1Group1-097, S178

PS1Group1-080, S165

PS2Group3-018, S231

PS1Group1-103, S184

PL 1.2, S3, SS 3.3, S13, WS 4.2, S49

PS1Group1-060, S151

PS1Group1-078, S164

PS3Group2-030, S348

PS2Group9-001, S310

PS1Group1-009, S108,

PS1Group1-115, S192

PS2Group5-021, S298

PS3Group2-007, S330,

PS3Group2-008, S330

PS2Group3-001, S218,

WS 17.2, S65

PS3Group2-004, S327, PS3Group2-037, S354, PS3Group2-038, S355

PS1Group1-028, S124

PS2Group3-034, S245

PS2Group5-011, S291

PS3Group2-025, S345

PS1Group1-004, S104

PS2Group3-043, S251

PS1Group1-034, S129

PS1Group1-047, S141

PS1Group1-077, S163,

PS1Group1-080, S165

WS 7.4, S53

PS3Group4-028, S378

PS1Group1-015, S113

PS1Group1-011, S110

PS2Group3-028, S241

PS1Group1-144, S214,

PS3Group4-006, S360

PS2Group5-033, S307

PS1Group1-089, S173

PS3Group2-025, S345

PS1Group1-046, S140

PS2Group3-034, S245

PS1Group1-004, S104,

PS2Group9-008, S316

PS1Group1-128, S202

SS 23.2, S39, SS 23.3, S40,

WS 18.3, S68

PS2Group3-052, S258

PS1Group1-022, S119

PS1Group1-050, S143,

PS1Group1-061, S152

PS1Group1-147, S216

PS3Group4-015, S367

PS2Group3-081, S283

PS2Group9-010, S317

PS2Group3-039, S249

PS1Group1-016, S114,

PS1Group1-044, S138

PS1Group1-128, S202
Matsumura T.

Matsuo M.

Matsushima Y.

Matsuura E.

Maturana F.

Mauduit D.

Mauermann M.

Mayer O.H.

Mayhew A.

Mazanec R.

Mcdermott C.

Mcdonald C.

Mcdonald E.

Mcfarland R.

Mcgovern V.

Mcintosh J.

Mcmacken G.

Mcmanus B.

Medina J.

Meena A.K.

Mehrabyan A.C

Mehr Pour M.

Mehta V.

Meier T.

Meisel A.

Mele F.

Melfi F.

Menassa R.

Mendell J.

Mendell J.R.

Mendoza M.

Meng J.

Meng S.
PS1Group1-029, S125,

PS1Group1-143, S214

PS1Group1-003, S103,

PS1Group1-052, S145, PS1Group1-128, S202

PS1Group1-097, S178

PS2Group9-012, S319

PS3Group2-009, S331

PS1Group1-008, S108

PS2Group3-005, S220,

SS 17.3, S29, WS 5.3, S51

PS1Group1-057, S148,

PS1Group1-105, S185,

PS2Group5-023, S299,

PS2Group9-003, S312,

PS2Group9-016, S321

PS1Group1-009, S108,

PS1Group1-063, S153,

PS1Group1-115, S192,

PS2Group5-007, S288,

TC 9.8, S87

PS1Group1-037, S132,

PS1Group1-113, S190,

PS1Group1-139, S211

TC 7.4, S84, WS 3.2, S47

PS1Group1-114, S191,

PS1Group1-118, S194,

PS1Group1-123, S198

PS1Group1-124, S199

PS1Group1-120, S196

PS1Group1-033, S128

SS 13.4, S25

PS1Group1-124, S199,

PS1Group1-126, S200

SS 24.2, S40

PS2Group5-007, S288

PS2Group9-010, S317,

PS3Group4-019, S371

PS2Group3-039, S249

PS1Group1-140, S212

PS2Group5-009, S290

PS2Group6-003, S310

PS1Group1-057, S148,

PS1Group1-105, S185,

PS2Group5-023, S299,

PS2Group9-016, S321

PS3Group2-012, S334

PS1Group1-051, S144

PS3Group2-027, S346

PS1Group1-066, S156

PS1Group1-063, S153,

PS2Group5-007, S288,

SS 13.4, S25

PS1Group1-115, S192,

PS3Group4-022, S373

PS3Group2-005, S328

PS1Group1-070, S159

SS 8.1, S16, SS 14.2, S26, SS

17.4, S29, WS 6.1, S51, WS

13.1, S57 
Mensova L.

Meola G.

Mercuri E.

Merkies I.

Meshram C.

Messéant J.

Meyer K.

Michaelidou K. Mielke O.

Milenkovic S.

Milic Rasic V.

Milisenda J.C.

Milisneda J.C.

Miller T.

Milone M.

Mimaki M.

Minetti C.

Minier $\mathrm{T}$.

Minisman G.

Miossec O

Mirabella M.

Miranda J.I.

Mirza Asgari Z. Mittal P.

Mix C.

Miyake Z.

Mladenovic J.
PS1Group1-037, S132

PS1Group1-042, S136, PS1Group1-056, S148, PS1Group1-110, S188 PS1Group1-087, S171, PS1Group1-123, S198, PS1Group1-124, S199, PS3Group4-002, S356, PS3Group4-014, S366, PS3Group4-015, S367, PS3Group4-024, S375, PS3Group4-028, S378, TC 4.2, S82

PS2Group3-012, S226, PS2Group3-018, S231, PS2Group3-019, S233, PS2Group3-023, S236, PS2Group3-024, S237, PS2Group3-030, S243, PS2Group3-031, S244, PS2Group3-057, S263, TC 8.3, S85, WS 8.3, S54

SS 21.4, S36

PS2Group5-027, S302, PS3Group2-030, S348 PS3Group4-022, S373, SS 13.4, S25

PS1Group1-102, S183

PS2Group3-012, S226, PS2Group3-018, S231, PS2Group3-019, S233, PS2Group3-023, S236, PS2Group3-024, S237, PS2Group3-030, S243, PS2Group3-031, S244, PS2Group3-057, S263 PS1Group1-080, S165 PS1Group1-059, S150, PS1Group1-080, S165 PS1Group1-083, S168, PS1Group1-084, S169

PS1Group1-137, S210

SS 18.2, S30

PS1Group1-011, S110, PS1Group1-129, S203 PS1Group1-097, S178 PS1Group1-119, S195 PS1Group1-099, S180 SS 23.2, S39

PS2Group3-044, S252

PS1Group1-127, S202 PS1Group1-038, S132, PS1Group1-106, S186 PS2Group5-009, S290 PS1Group1-115, S192, PS2Group5-007, S288 PS1Group1-086, S170 PS2Group5-029, S304 PS1Group1-059, S150
Moggio M.

Moldovan O.

Molin C.J.

Monakhova A.

Monforte $\mathrm{M}$.

Mongini T.

Monlleó-Neila L.

Montagnese F.

Montes V.

Moore U.

Moreno M.V.

Moreno P.J.

Moreno T.

Morgan J.

Morgese M.G.

Mori Y.

Mori-Yoshimura M.

Morrow B.M.

Morrow J.M.

Moschou M.

Motamed M.R.

Motomura M.

Moya O.

Mozaffar T.

Muchart J.

Muelas N.

Mueller O.

Mukhametov T.

Mulder N.

Muley S.

Muley S.A.

Mul K.

Müller-Felber W.

Müller P.

Mulroy E.

Muni Lofra R.

Munoz K.

Muntoni F.

Muppidi S

Murai $\mathrm{H}$.

Muramatsu K.

Musset L.

Myers K.

Myrzaliyeva B.
PS1Group1-051, S144 PS2Group3-062, S267

PS3Group2-001, S324

PS2Group9-015, S320

PS1Group1-087, S171

PS1Group1-051, S144

PS1Group1-089, S173

PS1Group1-002, S102

PS3Group2-034, S351

PS1Group1-115, S192

PS2Group5-033, S307

PS1Group1-083, S168

PS2Group3-062, S267

PS1Group1-070, S159, PS1Group1-087, S171

PS1Group1-028, S124

PS2Group5-005, S287,

PS2Group5-018, S296

PS1Group1-063, S153,

PS1Group1-115, S192

PS1Group1-064, S154

WS 4.3, S49

PS1Group1-102, S183

PS2Group5-009, S290

PS2Group6-001, S308

PS2Group9-010, S317,

PS3Group4-019, S371

PS2Group5-031, S306,

TC 6.3, S84

PS1Group1-013, S112

PS1Group1-046, S140,

PS2Group3-053, S259

WS 15.4, S62

WS 3.4, S47

PS2Group5-019, S297

PS3Group2-029, S347

PS2Group3-007, S222

PS1Group1-109, S188

PS1Group1-039, S133

PS3Group2-018, S338

PS2Group5-021, S298

PS1Group1-009, S108

PS2Group5-031, S306

PS1Group1-004, S104,

PS1Group1-019, S117,

PS1Group1-070, S159,

PS1Group1-087, S171,

PS1Group1-118, S194,

PS1Group1-123, S198

PS2Group9-009, S316,

PS2Group9-018, S323,

PS3Group4-024, S375

PS3Group2-038, S355

PS3Group2-011, S333

PS1Group1-097, S178

PS2Group3-065, S269

PS3Group4-018, S370

PS3Group4-010, S363 
Naarding K.J.

Nabeshima Y.-I.

Nadais G.

Nadaj-Pakleza A.

Naddaf E.

Nafissi S.

Nagai M.

Nagaraju K.

Nagashima Y.

Nagel A.M.

Nagels K.

Nagendran S.

Nagy V.

Nair A.

Nakamachi Y.

Nakamagoe K.

Nakamura H.

Nakayama T.

Nalini A.

Namgung D.W.

Narayanappa G.

Narayanaswami P.

Nascimento A.

Nashi S.

Natali Sora M.G.

Natera De Benito D.

Nave K.A.

Nave S.

Nayak S.

Nazarov V.
PS1Group1-095, S177

PS1Group1-128, S202

PS3Group2-025, S345

PS3Group2-030, S348

PS1Group1-011, S110,

PS1Group1-055, S147

PS1Group1-041, S136,

PS1Group1-043, S137

PS1Group1-128, S202

SS 1.2, S10

PS2Group5-004, S286

PS2Group9-008, S316

PS2Group3-040, S249

PS3Group4-022, S373,

SS 13.4, S25

PS2Group3-026, S239

PS1Group1-021, S119

PS1Group1-003, S103

PS2Group5-029, S304

PS1Group1-092, S175

PS1Group1-112, S190,

PS1Group1-143, S214

PS1Group1-016, S114,

PS1Group1-018, S116,

PS1Group1-044, S138,

PS2Group5-010, S291,

PS2Group9-011, S318,

PS3Group4-003, S357

PS3Group4-016, S368

PS1Group1-016, S114

SS 11.3, S22

PS1Group1-013, S112, PS1Group1-026, S122, PS2Group3-011, S226, PS2Group3-034, S245, PS2Group5-014, S293, PS2Group9-010, S317, PS3Group2-013, S335, PS3Group4-019, S371

PS1Group1-016, S114, PS1Group1-018, S116, PS1Group1-044, S138, PS2Group5-010, S291, PS3Group2-022, S342, PS3Group4-003, S357 PS1Group1-004, S104

PS1Group1-013, S112, PS2Group3-011, S226, PS2Group3-034, S245, PS2Group5-014, S293, PS2Group9-010, S317, PS3Group2-013, S335, PS3Group4-019, S371 PS2Group3-038, S248 PS3Group4-015, S367, PS3Group4-028, S378 PS1Group1-016, S114, PS1Group1-044, S138 PS3Group4-021, S373
Neff L.A.

Neil J.

Nel M.

Neri $M$.

Neuronext Nn103 Beatmg Study Team .

$\mathrm{Ng} \mathrm{K}$.

$\mathrm{Ng}$ R.

Nguyen C.-T.E.

Nguyen D.

Nguyen T.

Ng Y.S.

Nichols A.

Nicholson G.A.

Nicole $\mathrm{S}$.

Nigro V.

Nikitin S.

Nikolenko N.

Nikolic A.

Niks E.H.

Nilipour Y.

Niranjan N.

Nishino I.

Nisic T.

Nissan X.

Niu Z.

Njike V.

Nobile-Orazio E.

Noel V.

Nogales-Gadea G.

Nohara S.

Nonaka I.

Notas K.

Noursalehi M.

Nowak R.J.

Nuñez J.

Oberreiter E.-M.

Obici L.

O’Brien F.

O'Connor E.

O'Connor K.C.
PS1Group1-050, S143, PS1Group1-061, S152,

PS1Group1-098, S179

PS2Group3-065, S269

PS2Group5-019, S297

PS2Group5-020, S297

PS3Group2-021, S341

PS2Group3-037, S247, PS2Group5-003, S285, PS2Group5-024, S300 PS2Group5-007, S288

PS1Group1-048, S142, PS3Group2-026, S345

PS3Group2-026, S345

PS2Group3-056, S262

PS1Group1-033, S128

PS1Group1-060, S151

PS2Group3-048, S255

PS3Group2-030, S348

PS1Group1-119, S195,

WS 16.3, S63

PS2Group3-002, S218

PS1Group1-147, S216

PS2Group3-028, S241

PS1Group1-034, S129,

PS1Group1-095, S177

PS1Group1-041, S136,

PS1Group1-043, S137

PS1Group1-111, S189

PS1Group1-092, S175,

PS1Group1-097, S178

PS1Group1-104, S185

PS1Group1-144, S214

PS1Group1-011, S110,

PS1Group1-129, S203

PS3Group2-019, S339

PS2Group3-064, S268,

WS 5.1, S49, WS 8.1, S53

PS2Group9-001, S310

PS1Group1-089, S173

PS2Group5-029, S304

PS1Group1-092, S175

PS1Group1-102, S183

PS1Group1-039, S133

PS3Group2-011, S333,

PS3Group2-019, S339,

PS3Group2-021, S341

PS1Group1-089, S173

PS2Group3-076, S276

PS2Group3-010, S225,

WS 10.1, S55

PS3Group2-011, S333, PS3Group2-023, S342, PS3Group2-037, S354, PS3Group2-038, S355

SS 24.2, S40

PS3Group2-021, S341 
Octaviana F.

Oel D.

Oertl W.

Ogasawara Y.

Ogata K.

Ogata $\mathrm{T}$.

Oh J.

Ohno K.

Okune S.

Okuyama T.

Oliveira J.

Oliveira Santos M.

Oliver D.J.

Oliver K.R.

Omachi J.

O’Mara E.

Oorschot V.

Opolski G.

O'Riordan W.

Orlikowski D.

Orsini A.-L.

Ortez C.

Osahon R.

Osman H.

Ota T.

Ouaja R.

Ouazzani R.

Oyewole A.

Oyola Valdizán C.E.

Ozasa S.

Padberg G.W.

Padilla M.

Padmanabha $\mathrm{H}$.

Padros N.

Pain-Control'S Study Team P.

Pajusalu S.

Palace J.

Pál E.

Panaghie-Meltzer G.

Panicucci C.

Papetti L.

Paradas C.
PS3Group2-032, S350

PS2Group3-076, S276,

PS3Group2-018, S338

PS2Group3-076, S276

PS3Group4-001, S356

PS1Group1-029, S125

PS1Group1-097, S178

PS2Group3-054, S260

PL 4.2, S6

PS2Group5-029, S304

PS1Group1-029, S125

PS3Group4-013, S365

PS1Group1-058, S149,

PS3Group4-020, S372

OA 2.3, S91, PL 3.3, S5,

WS 3.1, S46

SS 11.1, S21

PS2Group5-004, S286

PS1Group1-126, S200

PS3Group4-007, S361

PS2Group5-011, S291

PS2Group3-010, S225,

PS2Group3-055, S261

PS1Group1-088, S172

PS1Group1-026, S122

PS1Group1-013, S112,

PS2Group3-011, S226,

PS2Group3-034, S245,

PS2Group5-014, S293,

PS2Group9-010, S317,

PS3Group2-013, S335,

PS3Group4-019, S371

PS1Group1-019, S117,

PS2Group9-009, S316

PS2Group5-020, S297

PS3Group4-001, S356

PS2Group3-064, S268

PS1Group1-067, S157,

PS1Group1-068, S157, PS2Group5-012, S292,

PS2Group5-013, S292

PS2Group9-019, S323

PS2Group9-004, S313

PS1Group1-029, S125

PS1Group1-001, S102,

WS 2.3, S45

PS3Group2-009, S331

PS3Group4-003, S357

PS2Group9-010, S317,

PS3Group4-019, S371

PS2Group3-051, S257

PS1Group1-035, S130

SS 24.4, S41

PS1Group1-099, S180

PS1Group1-123, S198, S198

PS1Group1-119, S195

PS1Group1-027, S123

PS1Group1-063, S153,

PS1Group1-115, S192,
Pardo J.

Pareyson D.

Park G.-Y.

Park H.J.

Park J.-M.

Park J.-S

Park J.S.

Park J.Y.

Park K.-D.

Park K.-H.

Park K.H.

Park K.-J.

Parks C.

Park S.-H.

Parman Y.

Parmova O.

Parshikov V.

Pasnoor M.

Paternoster L.

Paternostro-Sluga T.

Patra K.

Paul A.

Pearson B.

Peci E.

Peduto A.

Pegoraro E.

Pelayo-Negro A.L.

Pellegrinelli A.

Pelnena D.

Pelosi L.

Peltier A.

Peltz S.W.

Penttilä S.

Pera M.C.

Perazzo F.F.

Perez M.A.R.

Perez M.M.

Perez Siles G.

Peric S.

Perlingeiro R.

Perniconi B.

Peschanski M.
PS2Group5-007, S288

PS2Group3-042, S251

SS 7.3, S15, TC 3.2, S79,

WS 10.2, S55

PS2Group3-047, S254

PS3Group4-016, S368

PS1Group1-094, S176,

PS3Group2-015, S336

PS1Group1-094, S176,

PS3Group2-015, S336

PS1Group1-133, S206

PS1Group1-133, S206

PS2Group3-073, S274

PS2Group3-061, S266

PS2Group3-033, S245,

PS3Group2-035, S352

PS2Group3-033, S245

PS2Group3-051, S257

PS2Group3-035, S246,

PS3Group4-027, S378

PS2Group3-002, S218

PS1Group1-037, S132

PS3Group4-029, S379,

WS 3.4, S47

PS2Group3-051, S257

PS3Group4-025, S376

WS 1.2, S44

PS3Group2-012, S334

PS3Group2-020, S340

PS3Group2-021, S341

PS2Group3-019, S233

PS1Group1-009, S108

PS1Group1-051, S144,

PS1Group1-063, S153,

PS1Group1-115, S192,

PS2Group5-007, S288

PS1Group1-100, S181

PS3Group2-016, S337,

PS3Group2-017, S338

PS1Group1-035, S130

PS2Group5-021, S298

WS 20.3, S71

PS1Group1-118, S194, PS1Group1-123, S198,

PS1Group1-124, S199

PS1Group1-053, S146

PS3Group4-028, S378

PS1Group1-107, S186

PS2Group5-033, S307

PS1Group1-107, S186

PS2Group3-048, S255

PS1Group1-077, S163,

PS1Group1-078, S164,

PS1Group1-079, S164,

PS1Group1-080, S165,

PS1Group1-104, S185,

PS2Group3-028, S241

OA 1.1, S90

PS1Group1-088, S172

PS3Group4-006, S360 
Pesovic J.

Pestronk A.

Peters D

Peters T.

Petri G.

Petroska D.

Petrovic M.

Petty R.K.

Peyer Kauffmann A.-K.

Pfeffer G.

Phillips L.

Phillips W.D.

Picarella D.

Piehl F.

Piétri-Rouxel F.

Pilati L.

Pinal-Feranndez I.

Pingili R.

Pinset $\mathrm{C}$.

Pinto $S$.

Pintos E.

Pintos-Morell G.

Piras R.

Pita F.

Pitchforth J.

Pittaro A.

Pitt M.

Pivneva I.

Podborska M.

Podnar S.

Polavarapu K.

Polikarpova A.

Polvèche $\mathrm{H}$.

Polydefkis M.

Ponery A.S.

Pontifex C.S.

Poon J.-L.

Porte Thome F.

Powell C.

Poyatos Garcia J.

Pozzolini G.

Praestgaard J.

Prasad S.
PS1Group1-077, S163, PS1Group1-078, S164,

PS1Group1-079, S164, PS1Group1-104, S185

PS1Group1-063, S153, PS1Group1-115, S192, PS2Group5-007, S288,

SS 18.3, S31, WS 7.2, S52

PS2Group9-009, S316

PS3Group4-017, S369

PS1Group1-047, S141, PS1Group1-107, S186, PS2Group3-059, S265

PS1Group1-132, S206

PS2Group3-028, S241

PS1Group1-033, S128

PS2Group5-022, S299

PS1Group1-011, S110

PS1Group1-138, S210

PS1Group1-101, S182,

TC 2.1, S76

PS1Group1-060, S151

PS3Group2-004, S327

PS1Group1-007, S107

PS1Group1-127, S202

PS1Group1-137, S210

PS3Group4-008, S361

PS1Group1-144, S214

PS3Group4-020, S372

PS2Group3-042, S251

PS1Group1-089, S173

PS1Group1-051, S144

PS3Group2-034, S351

PS1Group1-004, S104

PS1Group1-040, S134

TC 4.4, S82

PS1Group1-020, S118

PS1Group1-037, S132

TC 5.3, S83

PS1Group1-016, S114, PS1Group1-018, S116, PS1Group1-044, S138, PS2Group5-010, S291, PS3Group2-022, S342, PS3Group4-003, S357 PS1Group1-076, S163 PS3Group4-006, S360 PS2Group3-005, S220, PS2Group3-055, S261

PS1Group1-021, S119

PS1Group1-011, S110

PS3Group7-004, S383

PS1Group1-004, S104, PS1Group1-005, S105 PS2Group3-055, S261

PS1Group1-046, S140 PS1Group1-119, S195 PS3Group4-008, S361 PS1Group1-039, S133
Preethish-Kumar V.

Prescher $\mathrm{H}$

Previtali S.

Previtali S.C

Prieto-González S.

Prior T.W.

Prukop T.

Punga A.R.

Punga T.

Punzón I.

Puzanok D.

Quasthoff S.

Querol L.

Rabbia M.

Rakocevic-Stojanovic V.

Ramirez A.

Ramírez-Jiménez L.

Ramm G.

Rangel Escribano J.

Rao S.N.

Rath J.

Ratnasingam R..J.

Ravenscroft G.

Rawla J.

Reblova K.

Recasens B.B.

Recktenwald S.F.

Reddy S.S.

Reichert S.

Reichmann H.

Reilich P.

Reilly J.F.

Reilly M.

Relaix F.

Remiche G.

Renfroe J.B.

Renna L.V.
PS1Group1-016, S114, PS1Group1-018, S116,

PS1Group1-044, S138, PS2Group5-010, S291,

PS3Group4-003, S357

PS3Group2-031, S349

PS1Group1-004, S104

PS1Group1-051, S144

PS1Group1-083, S168

PS3Group4-022, S373,

SS 13.4, S25

PS2Group3-038, S248

PS3Group2-001, S324,

SS 20.2, S32, WS 18.2, S67

PS3Group2-001, S324

PS1Group1-007, S107,

PS1Group1-008, S108

PS3Group4-029, S379,

WS 3.4, S47

PS2Group3-076, S276, PS3Group2-018, S338

SS 12.3, S24

PS1Group1-014, S112 PS1Group1-077, S163, PS1Group1-078, S164, PS1Group1-079, S164, PS1Group1-080, S165, PS1Group1-104, S185 PS1Group1-013, S112 PS2Group3-044, S252 PS3Group4-007, S361 PS2Group9-001, S310 PS1Group1-018, S116 PS2Group3-026, S239, PS3Group2-018, S338, PS3Group2-020, S340 PS1Group1-031, S127 PS1Group1-096, S178

PS1Group1-114, S191 PS1Group1-113, S190

PS2Group5-033, S307

PS3Group4-024, S375

PS2Group3-045, S253, PS2Group3-046, S254 PS1Group1-065, S155 PS1Group1-116, S193 PS2Group3-040, S249, PS2Group3-074, S275, PS2Group5-016, S295 PS1Group1-060, S151

PL 2.1, S4, SS 19.1, S31 PS1Group1-007, S107 PS2Group5-001, S284

PS1Group1-126, S200

PS1Group1-042, S136, PS1Group1-056, S148, PS1Group1-110, S188 
Reshetov D.

Reyna S.P.

Reyngoudt H.

Reynolds E.

Rha H.J.

Ricciardi R.

Ricci G.

Richard I.

Richman L.

Rickard A.

Rico S.

Riquelme A.

Ristic T.

Rivellini C.

Rivolta I.

Rizzo S.

Robberecht W.

Robbie G.

Roblin D.

Rocha M.

Rodart I.

Rodino-Klapac L.

Rodolico C.

Rodrigues M.

Rodriguez A.

Rodriguez De Rivera F.

Rodríguez-Palmero A.

Roemer F.

Rohani M.

Rohmer Cohen A.

Rojas-García R.

Romagnolo A.

Romero N.

Roos A.

Rosa H.

Rossi R.

Rottoli M.R.

Roubenoff R.

Roussange F.

Roxburgh R.

Roy B.

Rozentāls G.

Rubino D.

Rubino-Nacht D.

Rudolf $\mathrm{R}$.
PS1Group1-076, S163, PS2Group9-015, S320

PS3Group4-026, S377

PS1Group1-095, S177, PS2Group9-006, S314 PS2Group3-008, S223 PS2Group3-079, S279 PS3Group2-027, S346

PS1Group1-051, S144, PS1Group1-142, S213, PS2Group3-060, S265 PS1Group1-015, S113, TC 9.2, S86

PS2Group9-013, S319

PS1Group1-023, S120

PS1Group1-039, S133

PS3Group2-010, S332

PS1Group1-059, S150

PS2Group3-081, S283

PS1Group1-110, S188

PS3Group2-027, S346

PS2Group3-024, S237

PS2Group3-043, S251, PS2Group3-080, S280

PS1Group1-019, S117, PS2Group9-009, S316

PS1Group1-012, S111

PS1Group1-073, S161

PS3Group4-022, S373,

SS 13.4, S25

PS1Group1-051, S144, PS1Group1-127, S202 PS2Group5-021, S298

WS 6.2, S52

PS3Group2-004, S327

PS1Group1-089, S173

PS2Group9-008, S316

PS1Group1-043, S137

PS2Group9-001, S310

PS2Group3-053, S259

PS2Group3-019, S233

PS1Group1-066, S156,

PS3Group2-030, S348

PS1Group1-044, S138,

PS3Group2-022, S342,

SS 24.2, S40

PS1Group1-012, S111

PS2Group5-020, S297

PS3Group2-004, S327

PS3Group4-008, S361

PS3Group4-006, S360

PS2Group5-021, S298

PS1Group1-032, S128, PS2Group3-029, S241, PS2Group5-002, S284, PS3Group2-019, S339 PS2Group9-002, S311

PS1Group1-017, S116

PS1Group1-026, S122

WS 14.3, S59
Rüegg M.

Ruegg U.T.

Rufibach L.

Ruggiero L.

Ruiz A.L.

Ruiz-Del-Yerro E.

Ruiz M.

Rummey C.

Russell J.

Rutkove S.

Rybalsky I.

Saak A

Saarela M.

Sabre L.

Sacchetto R.

Sacconi S.

Sadeh M.

Saegusa J.

Safri A.Y.

Said G.

Saifullina E.

Saito K.

Sajeev G.

Sakai C.

Salimian M.

Salort-Campana E.

Salvalaggio A.

Sampaio M.

Sampaolesi M.

Sampaolo S.

San Antonio Arce V.

Sanarica F.

Sancho P.

Sanders D.

Sandona D.

Sandroni P.

Santhosh R.

Santoro L.

Santos C.S.

Santos E.

Santos J.F.R.

Santos M.
WS 14.1, S59

PS1Group1-061, S152

PS1Group1-115, S192,

PS2Group5-007, S288, PS3Group7-003, S382

PS1Group1-051, S144

PS2Group9-004, S313

PS1Group1-054, S146, PS1Group1-108, S187

PS2Group3-082, S283

PS2Group5-023, S299, PS2Group9-003, S312, PS2Group9-016, S321

PS2Group3-027, S240,

PS2Group3-045, S253, PS2Group3-046, S254, SS 10.2, S19

PS2Group5-002, S284

PS1Group1-095, S177

PS1Group1-035, S130,

PS1Group1-116, S193,

PS1Group1-117, S194

PS2Group3-024, S237

PS3Group2-001, S324

PS1Group1-125,

PS1Group1-088, S172,

TC 1.2, S74

PS1Group1-069, S158

PS1Group1-003, S103

PS3Group2-032, S350

SS 21.2, S34, WS 17.1, S64

PS2Group3-058, S264

PS3Group4-026, S377

PS1Group1-120, S196

PS1Group1-097, S178

PS2Group3-046, S254

PS1Group1-063, S153, PS1Group1-088, S172,

PS1Group1-115, S192

PS2Group3-082, S283

PS2Group3-003, S219

PS1Group1-008, S108

PS2Group5-034, S307

PS1Group1-013, S112

PS1Group1-028, S124

PS2Group3-044, S252

PS1Group1-049, S143

PS1Group1-125, S200

SS 9.6, S19

PS1Group1-016, S114

PS1Group1-051, S144,

PS2Group5-021, S298

PS3Group2-025, S345

PS3Group2-025, S345

PS1Group1-047, S141,

PS2Group3-059, S265

PS2Group3-003, S219,

PS3Group2-025, S345,

PS3Group4-013, S365 
Santos R.

Sanvito W.L.

Sarafov S.

Sariego A.

Saroja M.K.

Sarva S.

Sasaki M.

Savarese M.

Savic-Pavicevic D.

Sawyer A.M.

Say C.Y.

Scapozza L.

Scarlato M.

Schaefer A.M.

Schaefer J.

Schara U.

Scharf F.

Schlotter-Weigel B.

Schlötzer-Schrehardt U.

Schmidhammer R.

Schmidt $\mathrm{H}$.

Schmidt J.

Schmidt K.

Schmidt S.

Schmidt U.

Schmuck M.

Schnell F.

Schorling E.

Schoser B.

Schreiber-Katz O.

Schröder R.

Schröder S.

Schüssler S.

Schustereder G.
PS3Group4-013, S365

PS3Group4-005, S359

PS2Group3-002, S218

PS2Group3-034, S245

PS1Group1-018, S116

PS2Group3-039, S249

PS1Group1-097, S178

PS1Group1-053, S146, WS 16.3, S63

PS1Group1-077, S163, PS1Group1-078, S164, PS1Group1-079, S164, PS1Group1-104, S185

PS1Group1-009, S108 PS2Group3-025, S238

PS1Group1-050, S143, PS1Group1-061, S152, PS1Group1-098, S179

PS1Group1-051, S144

PS1Group1-033, S128

PS1Group1-035, S130, PS1Group1-116, S193, PS1Group1-117, S194

PS1Group1-026, S122, PS3Group4-008, S361 PS2Group5-016, S295 PS2Group3-036, S247 PS1Group1-135, S208

WS 9.1, S54

PS2Group3-005, S220, PS2Group3-010, S225, PS2Group3-055, S261

PS1Group1-025, S122, PS1Group1-045, S139, PS1Group1-103, S184, PS1Group1-122, S198

PS1Group1-025, S122, PS1Group1-122, S198 PS1Group1-017, S116,

PS1Group1-026, S122

PS1Group1-023, S120, PS1Group1-024, S121

PS1Group1-065, S155 PS1Group1-049, S143, PS1Group1-087, S171 PS2Group3-040, S249 PS1Group1-002, S102, PS2Group3-074, S275, PS3Group4-009, S362 PS1Group1-115, S192 PS1Group1-134, S207, PS1Group1-135, S208, PS2Group3-063, S267, PS2Group5-028, S303, PS2Group9-008, S316,

WS 15.2, S60, WS 19.1, S68

PS1Group1-085, S169

PS2Group9-008, S316

PS3Group2-018, S338
Scola R.H.

Screnci R.

Seabrook T.

Sebastião A.M.

Šedivá M.

Sedláčková L.

Seeman P.

Seferian A

Sefiani A.

Sefiani S.

Sekar D.

Selby K.

Selva-O'Callaghan A.

Selvatici R.

Semeryak O.M.

Semina N.

Semplicini C.

Semprez F.

Seog D.-H.

Seo H.S.

Seok H.Y.

Seong M.-W.

Sera F.

Serafin A.

Serban V.

Sereda M.

Sereda M.W.

Serjensen T.

Serrano R.

Sertpoyraz F.M.

Servais L.

Seta N.

Šetlere S.

Sevilla T.

Shaibani A.

Shakir R.

Shamshiri H.

Shanmugarajah P.D.

Sharma A.

Shebl A.

Shellenbarger K.C.

Shell R.

Shidlovskaya O.
PS1Group1-006, S106 PS2Group3-048, S255 PS3Group4-015, S367

TC 2.3, S77

PS2Group3-020, S234, PS2Group9-007, S315 PS2Group3-020, S234, PS2Group9-007, S315 PS2Group3-020, S234, PS2Group9-007, S315 PS1Group1-087, S171 PS1Group1-068, S157 PS1Group1-067, S157, PS1Group1-068, S157 PS1Group1-018, S116, PS3Group4-003, S357 PS1Group1-123, S198, S198 PS1Group1-083, S168 PS2Group5-020, S297

PS2Group5-032, S306 WS 3.4, S47

PS1Group1-088, S172, PS1Group1-115, S192

PS3Group2-028, S347

PS2Group5-026, S301

PS3Group2-035, S352

PS2Group5-017, S295

PS1Group1-145, S215

PS1Group1-051, S144

PS2Group5-011, S291

PS2Group3-075, S276

SS 7.2, S15

PS2Group3-038, S248

SS 5.4, S14

PS3Group4-007, S361

PS2Group3-013, S227, PS2Group9-014, S320

PS1Group1-004, S104, PS1Group1-039, S133, PS1Group1-087, S171, PS2Group3-070, S272, PS3Group4-015, S367, PS3Group4-028, S378 PS1Group1-066, S156 PS2Group9-002, S311 PS2Group3-042, S251, PS2Group3-052, S258, PS2Group3-053, S259

PS3Group2-024, S343

SS 22.3, S38

PS1Group1-043, S137

PS3Group4-011, S364

PS3Group2-019, S339

PS2Group3-023, S236, PS2Group3-057, S263

PS1Group1-095, S177 PS3Group4-022, S373, SS 13.4, S25

PS2Group9-015, S320 
Shieh P.B.

Shi F.-D.

Shimizu J.

Shim S.K.

Shin J.-H.

Shin J.-Y.

Shiraishi H.

Shirakawa T.

Shira S.

Shlemon P.

Shoffner J.M.

Shtabnitskiy V.

Shulyakova I.

Siciliano G.

Siddiqi Z.A.

Signorovitch J.

Silani V.

Silva V.G.

Silveira F.

Simoncini C.

Simschitz P.

Singh A.

Siva R.

Skripchenko N.

Slater C.

Smith B.

Smith D.S.

Smith F.

Smith R.

Snadden L.

Soardi M.

Soblechero-Martin P.

Sobue G.

Solà A.G.

Solana-Guizar E.

Solé G.

Solernou Ferrer A.J.

Sommer C.

Sonett J.R.

Soni M.

Soska R.
PS1Group1-039, S133

PS3Group2-029, S347

PS1Group1-071, S159,

PS2Group5-004, S286

PS2Group3-041, S250

PS3Group2-014, S336

PS1Group1-131, S205,

PS1Group1-141, S213

PS2Group6-001, S308

PS1Group1-128, S202

PS3Group7-003, S382

PS2Group3-051, S257

PS1Group1-090, S173

PS3Group4-029, S379,

WS 3.4, S47

PS2Group9-015, S320

PS1Group1-051, S144,

PS1Group1-142, S213,

PS3Group2-027, S346

PS3Group2-002, S326,

PS3Group2-003, S326

PS1Group1-120, S196

SS 16.3, S29

PS1Group1-047, S141, PS1Group1-073, S161, PS1Group1-074, S162

PS3Group2-025, S345

PS1Group1-142, S213

PS3Group2-018, S338

PS2Group5-007, S288

PS2Group3-062, S267

PS2Group3-071, S272,

PS2Group5-015, S294

TC 2.2, S77

PS1Group1-039, S133

PS2Group5-007, S288

PS1Group1-009, S108

PS3Group4-018, S370

PS1Group1-033, S128

PS1Group1-125,

PS1Group1-054, S146, PS1Group1-108, S187

PS2Group3-012, S226, PS2Group3-018, S231, PS2Group3-019, S233, PS2Group3-023, S236, PS2Group3-024, S237, PS2Group3-030, S243, PS2Group3-031, S244, PS2Group3-057, S263

PS2Group5-033, S307

PS1Group1-024, S121

PS1Group1-088, S172

PS2Group9-004, S313

PS2Group3-024, S237,

SS 15.1, S27

SS 23.2, S39

PS1Group1-146, S216

PS1Group1-021, S119
Sousa A.

Sousa L.

Sousa S.

Souza M.

Sparks S.

Specht C.

Spies J.M.

Spilioti M.

Spinty S.

Spörrer M.

Sproule D.M.

Spuler S.

Srisuwan K.

Srivastava R.

Srotova I.

Staff N.

Statland J.M.

Stauber A.J.

Stavropoulos G.

Stavusis J.

Steck A.

Stehlikova K.

Stelmasiak Z.

Sternberg D.

Stevanin G.

Stevic Z.

Stieglbauer K.

Stojanov A.

Stojanovic M.

Stojkovic T.

Stradalova P.

Straub V.

Streichenberger N.

Strochlic L.

Strom T.M.

Strucksberg K.-H.

Struhal W.
PS2Group3-003, S219, PS3Group2-025, S345, PS3Group4-013, S365

PS3Group2-025, S345

PS3Group2-034, S351

PS1Group1-123, S198,

PS1Group1-124, S199

PS1Group1-115, S192,

PS2Group5-007, S288

PS1Group1-140, S212

PS2Group3-056, S262

PS1Group1-102, S183

PS1Group1-026, S122

PS1Group1-135, S208,

PS2Group5-028, S303

PS1Group1-020, S118, PS3Group4-022, S373, SS 13.4, S25

PS1Group1-063, S153, PS1Group1-115, S192

PS3Group2-006, S328

PS2Group6-003, S310

PS1Group1-037, S132

SS 6.1, S14, WS 11.3, S57

WS 2.2, S45

PS3Group4-009, S362

PS1Group1-102, S183

PS1Group1-035, S130

WS 8.2, S54, WS 17.3, S66

PS1Group1-113, S190

PS2Group3-024, S237

PS3Group2-030, S348

PS2Group3-011, S226,

PS2Group3-034, S245

PS1Group1-104, S185

PS2Group3-076, S276,

PS3Group2-018, S338

PS2Group3-028, S241

PS2Group3-028, S241

PS1Group1-009, S108,

PS1Group1-063, S153,

PS1Group1-066, S156,

PS1Group1-115, S192,

PS2Group5-007, S288,

PS3Group2-030, S348

PS1Group1-037, S132

PL 1.1, S3, PS1Group1-009, S108, PS1Group1-034,

S129, PS1Group1-063,

S153, PS1Group1-087,

S171, PS1Group1-115, S192,

PS2Group5-007, S288

PS1Group1-066, S156,

PS3Group2-030, S348

PS2Group5-027, S302,

PS3Group2-030, S348

PS2Group3-026, S239

PS1Group1-134, S207

SS 9.2, S18 
Study Group S.-N.

Sugie K.

Suh B.C.

Suhr O.

$\mathrm{Su}$ J.

Suk J.I.

Sukno S.

Sung D.H.

Sung J.-J.

Sutherland $\mathrm{H}$.

Suzuki E.

Svahn J.

Sweeney H.L.

Sweeney L.

Sweetser M.

Swoboda K.J.

Szczudlik A.

Tackenberg B.

Tahraoui J.

Tajsharghi H.

Takada H.

Takahashi M.P.

Takahashi T.

Takahashi Y.K.

Takashima H.

Takeda H.

Takeda S.

Takeshita E.

Takeuchi F.

Tamaoka A.

Tanaka N.

Tan C.-Y.

Tan E.

Tan H.-T.

Taouagh N.

Tapscott S.J.

Tasca G.

Taylor R.W.

Telese R.

Tesi Rocha C.

Thaler-Wolf C.

Thampratankul L.

The German Mouse Clinic

Consortium -

Theil D.

Thibaud J.-L.
PS1Group1-087, S171

PS1Group1-029, S125

PS3Group4-016, S368

PS2Group3-005, S220,

PS2Group3-010, S225,

PS2Group3-055, S261

PS1Group1-005, S105

PS2Group3-079, S279

PS3Group2-030, S348

PS2Group6-002, S309

PS1Group1-131, S205,

PS1Group1-141, S213,

PS2Group3-032, S244,

PS2Group3-061, S266,

PS3Group2-035, S352

PS1Group1-009, S108

PS1Group1-097, S178

PS1Group1-066, S156

PS1Group1-060, S151

PS1Group1-014, S112

PS2Group3-005, S220,

PS2Group3-010, S225,

PS2Group3-055, S261

PS3Group4-014, S366

PS3Group2-004, S327

PS2Group3-024, S237

PS1Group1-144, S214

PS1Group1-096, S178

PS1Group1-029, S125,

PS3Group4-001, S356

PS1Group1-029, S125

PS1Group1-029, S125

PS2Group6-001, S308

PS1Group1-136, S209

PS2Group5-029, S304

PS1Group1-092, S175,

PS1Group1-115, S192,

PS1Group1-143, S214

PS1Group1-029, S125

PS1Group1-092, S175

PS2Group5-029, S304

PS1Group1-029, S125

PS1Group1-031, S127

PS2Group3-006, S222

PS1Group1-031, S127

PS1Group1-088, S172

WS 2.1, S45

PS1Group1-053, S146

PS1Group1-012, S111,

PS1Group1-033, S128

PS1Group1-051, S144

PS1Group1-009, S108,

PS1Group1-115, S192

PS2Group3-076, S276

PS3Group2-006, S328

PS1Group1-134, S207

PS3Group4-017, S369

PS1Group1-008, S108
Thiele S.

Thievessen I.

Thomas P.

Thomas P.T.

Thömke F.

Thompson N.

Thusang K.J.

Tian C.

Tiburcy M.

Tiddens H.A.W.M.

Tilbery C.P.

Timmerman V.

Tinsley J.

Tirucherai G.

Toda T.

Tomelleri G.

Tomidokoro Y.

Tomschik M.

Tonska K.

Topakian R.

Topaloglu H.

Töpf A.

Torella A.

Toscano A

Tournev I.

Tournois $\mathrm{J}$.

Touvier T.

Tozaka N.

Trabace L.

Tracy J.A.

Trajanovska S.

Traverso M.

Trifillis P.

Trollmann R

Truszkowska B.

Tsai K.

Tsolaki M.

Tsuji H.

Tsuji S.

Tulinius M.

Tupler R.

Turanoglu M.
PS2Group3-040, S249

PS2Group5-028, S303

PS3Group4-003, S357

PS1Group1-018, S116,

PS2Group9-011, S318

SS 8.3, S17

PS3Group4-002, S356

PS3Group4-012, S365

PS1Group1-095, S177,

PS1Group1-126, S200

PS1Group1-103, S184

PS1Group1-040, S134

PS3Group4-005, S359

SS 7.1, S15

PS1Group1-019, S117, PS2Group9-018, S323

PS1Group1-014, S112

PS1Group1-071, S159,

PS2Group5-004, S286

PS1Group1-051, S144

PS2Group5-029, S304

PS2Group3-026, S239,

PS3Group2-020, S340

PS1Group1-036, S131

PS2Group3-076, S276,

PS3Group2-018, S338

PS1Group1-026, S122

PS1Group1-044, S138,

PS1Group1-138, S210,

PS2Group5-014, S293,

PS3Group2-022, S342,

SS 24.2, S40

PS1Group1-119, S195,

WS 16.3, S63

SS 4.1, S13

PS2Group3-002, S218, PS2Group3-005, S220, PS2Group3-010, S225, PS2Group3-055, S261

PS3Group4-006, S360

PS2Group3-081, S283

PS2Group5-029, S304

PS1Group1-028, S124

PS1Group1-129, S203

PS1Group1-101, S182

PS1Group1-119, S195

PS1Group1-118, S194,

PS1Group1-123, S198,

PS1Group1-124, S199,

PS1Group1-126, S200

PS2Group9-008, S316

PS2Group5-011, S291

PS1Group1-086, S170

PS1Group1-102, S183

PS2Group5-029, S304

PS1Group1-071, S159

PS1Group1-123, S198,

PS3Group4-026, S377

PS1Group1-051, S144

PS2Group3-013, S227 
Turke K.C.

Türk M.

Turnbull D.M.

Turner C.

Uchino S.

Udd B.

Uder M.

Ulrichts P.

Uncini A.

Uribe L.

Urtizberea A.

Utkus A.

Utsugisawa $\mathrm{K}$.

Vaillant T.Z.

Vaitkevicius A.

Valaperta R.

Valentin M.-A.

Valenzano K.J.

Valério B.C.O.

Vallejo Illarramendi A.

Van Alfen N.

Van Asseldonk T.

Van Damme P.

Van Den Bergh P.

Van Den Berg L.

Vandenborne K.

Vanden Hauwe M.

Van Der Beek N.A.M.E.

Van Der Kooi A.

Van Der Kooi E.L.

Van Der Maarel S.M.

Van Der Ploeg A.T.

Van Der Pol L.

Van Der Vliet P.J.

Van Doorn P.A.

Vandrovcova J.

Van Engelen B.G.

Van Geloven N.
PS1Group1-072, S160, PS1Group1-073, S161, PS1Group1-074, S162, PS1Group1-075, S162 PS1Group1-134, S207, PS2Group3-063, S267 PS1Group1-012, S111, PS1Group1-033, S128 PS1Group1-082, S167, PS1Group1-147, S216

Van Kooten H.A.

Van Schaik I.

PS1Group1-097, S178

PS1Group1-053, S146,

SS 4.3, S13

PS2Group9-008, S316

PS3Group2-004, S327

SS 12.1, S23

PS3Group2-021, S341

WS 16.1, S62

PS1Group1-132, S206

PS3Group2-011, S333

PS2Group9-004, S313

PS1Group1-132, S206

PS1Group1-110, S188

PS3Group4-017, S369

PS1Group1-021, S119

PS3Group2-016, S337,

PS3Group2-017, S338

PS1Group1-038, S132,

PS1Group1-106, S186

TC 5.2, S82

PS2Group5-008, S289

PS3Group2-004, S327

TC 8.4, S85

PL 3.2, S5, PS2Group5-008, S289, WS 12.1, S57

PS1Group1-019, S117, PS1Group1-060, S151, PS2Group9-018, S323 PS1Group1-120, S196 PS1Group1-030, S126, PS1Group1-040, S134

PS1Group1-148, S217

PS1Group1-001, S102

PS1Group1-001, S102,

PS1Group1-109, S188

PS1Group1-030, S126,

PS1Group1-040, S134

PS2Group5-008, S289

PS1Group1-109, S188

PL 2.2, S4, PS1Group1-030, S126, PS1Group1-040, S134 PS2Group3-067, S270

SS 4.2, S13, PS1Group1-001, S102, PS1Group1-010, S109, PS1Group1-109, S188

PS2Group3-012, S226, PS2Group3-018, S231, PS2Group3-019, S233,

PS2Group3-023, S236, PS2Group3-024, S237, PS2Group3-031, S244

PS1Group1-030, S126, PS1Group1-040, S134 PS2Group3-012, S226, PS2Group3-018, S231, PS2Group3-019, S233, PS2Group3-023, S236, PS2Group3-024, S237, PS2Group3-031, S244, PS2Group3-057, S263

Van Walsem M.R.

Van Zwet E.W.

Varju C.

Vase L.

Vassilieva S.

Vázquez-Costa J.F.

Veiga A.

Vellieux G.

Veltsista D.

Vengalil S.

Vercelli L.

Verellen-Dumoulin C.

Verrigni D.

Verschuuren $\mathrm{J}$.

Verschuuren K.

Vial C.

Vidal-Lijo M.P.

Vidal N.

Vieira T.F.

Vigo M.

Vihola A.

Vílchez J.J.

Vílchez R.

Villa A.

Villa L.

Villalta S..A.

Villegas-Umana E.

Vilquin J.-T.

Viñas $\mathrm{O}$.

Vincent A.

Vincent A.E.

Visentin A.

Visser L.

Vissing J

Visudthibhan A.

Vitturi B.K.

Vivekananda U.
PS3Group7-002, S381

PS1Group1-095, S177

PS1Group1-099, S180

PS3Group2-024, S343

PS1Group1-076, S163

PS2Group3-052, S258

PS3Group2-025, S345

PS3Group2-030, S348

PS2Group5-030, S305,

PS3Group4-023, S374

PS1Group1-016, S114, PS1Group1-018, S116, PS1Group1-044, S138, PS2Group5-010, S291, PS3Group2-022, S342, PS3Group4-003, S357 PS1Group1-051, S144 PS2Group3-070, S272 PS1Group1-027, S123 PS3Group2-004, S327, SS 3.4, $\mathrm{S} 13$

PS3Group2-004, S327 PS1Group1-066, S156 PS2Group3-042, S251

PS1Group1-004, S104

PS1Group1-074, S162 PS3Group4-019, S371

PS1Group1-053, S146 PS1Group1-046, S140, PS2Group3-053, S259

PS2Group3-053, S259

PS3Group2-007, S330

PS1Group1-051, S144

PS2Group5-031, S306

PS2Group3-008, S223

PS1Group1-008, S108

PS1Group1-083, S168

PL 4.3, S6, PS3Group2-036,

S353

PS1Group1-012, S111

PS2Group3-082, S283

PS2Group5-008, S289

PS3Group2-023, S342

PS3Group2-006, S328

PS3Group2-016, S337,

PS3Group2-017, S338,

PS3Group4-005, S359

PS1Group1-147, S216 
Vlckova E.

Vlčková $\mathrm{M}$.

Vlodavets D.

Voermans N.C.

Vogt-Ladner G.

Vohanka S.

Voitenkov V.

Voit T.

Vollert J

Volov A.

Voltz E.

Vorobyeva A.

Vroom E.

Vuillerot C.

Vujnic M.

Vukojevic Z.

$\mathrm{Vu} \mathrm{T}$.

Wachtel D.

Waddington S.

Wagner K.R.

Wagner M.

Walter M.C.

Wang M.-X.

Wanschitz J.

Ward S.J.

Warrier M.

Wasala L.

Wasala N.

Watanabe M.

Waters P.

Weber I.

Webster R.

Weis C.-A.
PS1Group1-037, S132

PS2Group3-020, S234

PS1Group1-076, S163,

PS2Group9-015, S320

PS1Group1-001, S102,

PS1Group1-093, S175,

PS1Group1-109, S188

PS2Group3-063, S267

PS1Group1-037, S132,

PS1Group1-113, S190,

PS1Group1-139, S211

PS2Group3-071, S272,

PS2Group5-015, S294

PS1Group1-057, S148, PS1Group1-105, S185, PS2Group5-023, S299, PS2Group9-003, S312, PS2Group9-016, S321, SS 5.2, S13

PS3Group2-024, S343

WS 3.4, S47

PS3Group4-008, S361, PS3Group4-017, S369

WS 3.4, S47

TC 9.9, S87

PS3Group4-024, S375

PS1Group1-104, S185

PS2Group3-028, S241

PS3Group2-004, S327

PS1Group1-060, S151

PS1Group1-070, S159

PS1Group1-014, S112,

TC 9.7, S87

PS2Group3-026, S239

PS1Group1-009, S108,

PS1Group1-063, S153,

PS1Group1-065, S155,

PS1Group1-115, S192,

PS1Group1-130, S204, PS2Group3-040, S249,

PS2Group3-074, S275, PS2Group5-007, S288, PS2Group5-016, S295, PS3Group4-009, S362

PS2Group3-056, S262

PS2Group3-049, S256, PS2Group3-076, S276, PS3Group2-018, S338

PS1Group1-120, S196

PS2Group9-011, S318

PS1Group1-111, S189

PS1Group1-111, S189

PS2Group5-029, S304

PS2Group3-032, S244

PS2Group3-063, S267

PS3Group2-036, S353

PS3Group2-001, S324, SS

23.2, S39
Wells C.

Wencel M.

Wenninger S.

Wenning G.

Werber M.

Wermelinger A.C.C.

Werneck L.C.

Wernick S.

Whitaker C.

White M.

Whittaker R.

Wiche G.

Widayati F.T.

Wieben E.

Wiedenmann S.

Wielopolski P.A.

Wiendl $\mathrm{H}$.

Wilder-Smith E.

Willen J.

Williams B.

Willison H.J.

Wilson J.M.

Wilson-Mcmanus J.E.

Wimmer K.

Windebank A.

Windisch D.

Windish $\mathrm{H}$.

Winterholler M.

Winter L.

Wohlgemuth M.

Wolf E.

Wolfe G.I.

Wong B.L.

Wong H.M.J.

Wong W.Y.W.

Woodhall M.

Wood K.

Wrabetz L.

Wright N.

Wu E.Q.

$\mathrm{Wu}$ J.

Wulandari D.

Wu Q.

Xin $\mathrm{H}$.

$\mathrm{Xu} \mathrm{S}$.

Yadav N.

Yamaji J.
PS3Group4-022, S373, SS

13.4, S25

PS2Group5-031, S306

PS1Group1-130, S204,

PS2Group3-036, S247

SS 9.1, S18

PS1Group1-085, S169

PS1Group1-006, S106

PS1Group1-006, S106

PS2Group3-038, S248

PS1Group1-032, S128

PS2Group3-005, S220

SS 24.2, S40

WS 15.1, S59

PS3Group2-032, S350

PS1Group1-011, S110

PS2Group5-028, S303

PS1Group1-040, S134

PS3Group2-011, S333,

PS3Group2-038, S355

PS2Group5-022, S299

PS3Group7-001, S380

PS2Group5-007, S288,

PS3Group7-003, S382

SS 12.2, S24

PS2Group9-013, S319

PS2Group5-007, S288

SS 17.1, S29

SS 2.1, S11, WS 1.1, S44

PS3Group2-018, S338

PS2Group5-007, S288

PS2Group3-063, S267

PS1Group1-134, S207,

PS1Group1-135, S208,

WS 15.3, S61

PS1Group1-001, S102

PS1Group1-065, S155

PS3Group2-021, S341, PS3Group2-038, S355,

SS 20.3, S33, SS 23.2, S39

PS1Group1-014, S112,

PS1Group1-095, S177

PS2Group3-025, S238

PS2Group3-025, S238

PS2Group3-032, S244

PS3Group2-029, S347

PS2Group3-081, S283

PS1Group1-035, S130

PS1Group1-020, S118

PS2Group5-002, S284

PS3Group2-032, S350

PS1Group1-032, S128

PS3Group2-033, S351

PS1Group1-021, S119

PS2Group5-010, S291

PS2Group5-005, S287,

PS2Group5-018, S296 
Yamamoto T.

Yang C.-C.

Yang J.-W.

Yankey J.W.

Yao Z.

Yareeda S.

Yatsuga S.

Yaworski A.

Yildiz F.G.

Yilmaz E.

Yokota $\mathrm{T}$.

Yonemoto N.

Yoo I.-H.

Yoon B.-N.

Yoon S.M.

York A.M.

Yoshimura S.

Yoshioka K.

Yubero D.

Yue Y.

Yumoto J.

Zadeh G.

Zaganas I.

Zagnoli F.

Zagrovic B.

Zaidman C.
PS1Group1-003, S103

PS2Group3-005, S220,

PS2Group3-010, S225,

PS2Group3-055, S261

PS2Group3-061, S266,

PS2Group9-005, S313

PS3Group2-021, S341

PS1Group1-120, S196

PS2Group3-039, S249

PS2Group5-029, S304

PS2Group6-003, S310

PS2Group3-006, S222

PS2Group3-006, S222

PS2Group6-001, S308

PS1Group1-092, S175

PS2Group3-035, S246,

PS3Group4-027, S378

PS2Group3-061, S266,

PS2Group9-005, S313

PS2Group3-041, S250

PS1Group1-086, S170,

PS1Group1-087, S171

PS2Group6-001, S308

PS2Group5-004, S286

PS1Group1-013, S112,

PS2Group3-011, S226,

PS2Group3-034, S245,

PS2Group5-014, S293

PS1Group1-111, S189

PS2Group5-004, S286

SS 17.2, S29

PS1Group1-102, S183

PS1Group1-088, S172

PS2Group3-026, S239

PS2Group5-002, S284
Zakharova M.

Zakroyshikova I.

Zamani B.

Zambon A

Zara F.

Zarrouk-Mahjoub S.

Zatz M.

Zeng R.

Zenker M.

Zhang X.

Zhang Y.

Zidar J.

Zidkova J.

Zierz S.

Zilliox L.

Zimprich F.

Zorgani S.

Zotova E.

Zschaeck I.

Zschüntzsch J.

Zulehner G.

Zumbrunn T.
WS 3.4, S47

WS 3.4, S47

PS1Group1-043, S137, PS2Group5-009, S290

PS1Group1-004, S104

PS1Group1-119, S195

PS1Group1-090, S173,

PS1Group1-091, S174

OA 1.2, S90

PS1Group1-122, S198

PS2Group5-016, S295

PS2Group3-043, S251,

PS2Group3-080, S280

PS1Group1-103, S184,

PS3Group4-026, S377

PS2Group3-002, S218

PS1Group1-113, S190,

PS1Group1-139, S211

PS1Group1-012, S111

PS2Group3-027, S240

PS2Group3-026, S239,

PS2Group3-049, S256,

PS2Group3-076, S276,

PS3Group2-018, S338,

PS3Group2-020, S340,

PS3Group2-036, S353,

SS 8.4, $\mathrm{S} 18$

PS2Group9-001, S310

PS1Group1-076, S163

PS2Group9-010, S317

PS1Group1-103, S184,

PS1Group1-122, S198

PS2Group3-026, S239,

PS3Group2-020, S340

PS1Group1-017, S116 